\title{
Imagining Chinese Medicine
}

四第論序圆莪註經鹳一十八帝黄



Edited by

VIVIENNE LO 羅維前AND PENELOPE BARRETT 
Imagining Chinese Medicine 


\title{
Sir Henry Wellcome Asian Series
}

\author{
Edited by \\ Dominik Wujastyk \\ Paul U. Unschuld \\ Charles Burnett
}

Editorial Board

Donald J. Harper

Ch. Z. Minkowski

Guy Attewell

Nikolaj Serikoff

VOLUME 18 


\title{
Imagining Chinese Medicine
}

\author{
Edited by \\ Vivienne Lo 羅維前 \\ Penelope Barrett \\ with the help of \\ David Dear \\ Lu Di 蘆笛 \\ Lois Reynolds \\ Dolly Yang 楊德秀
}



B R I L L

LEIDEN | BOSTON 
This is an open access title distributed under the terms of the prevailing CC-BY-NC License at the time of publication, which permits any non-commercial use, distribution, and reproduction in any medium, provided the original author(s) and source are credited.

front cover: Li Jiong's Neijing tu 内景圖 (Chart of the Inner Landscape). Huangdi bashiyi nanjing zuan tu jujie in Zhengtong Daozang 1436-49, Hanfen Lou, Shanghai. (The Ming edition of the Canon). Wellcome Library, London, Loo34715.

back cover: Heche niliu tu (Illustration of the Water Wheel against the current) from Che sheng ba bian neijing (The Penetrating Mirror of the Interior in Eight Books), by Liu Sijing, Qing, Kangxi reign period (1662-1722), illustrating the flow and circulation of Genuine Qi. China Academy of Chinese Medicial Sciences, copyright Wellcome Images Loo38694.

The realization of this publication was made possible by the support of the Wellcome Trust (Seed Award, grant number 201616/Z/16/Z).

Chapters not originally written in English were translated by Penelope Barrett and Vivienne Lo.

Design, layout and copy-editing: Josephine Turquet. Additional typography: Akio Morishima.

The Library of Congress Cataloging-in-Publication Data is available online at http://catalog.loc.gov LC record available at http://lccn.loc.gov/2017964171

Typeface for the Latin, Greek, and Cyrillic scripts: "Brill”. See and download: brill.com/brill-typeface.

ISSN 1570-1484

ISBN 978-90-04-36216-1 (hardback)

ISBN 978-90-04-36618-3 (e-book)

Copyright 2018 by Koninklijke Brill NV, Leiden, The Netherlands.

This work is published by Koninklijke Brill Nv. Koninklijke Brill NV incorporates the imprints Brill, Brill Hes \& De Graaf, Brill Nijhoff, Brill Rodopi, Brill Sense and Hotei Publishing.

Koninklijke Brill NV reserves the right to protect the publication against unauthorized use and to authorize dissemination by means of offprints, legitimate photocopies, microform editions, reprints, translations, and secondary information sources, such as abstracting and indexing services including databases. Requests for commercial re-use, use of parts of the publication, and/or translations must be addressed to Koninklijke Brill NV.

Brill has made all reasonable efforts to trace all rights holders to any copyrighted material used in this work. In cases where these efforts have not been successful the publisher welcomes communications from copyright holders, so that the appropriate acknowledgements can be made in future editions, and to settle other permission matters.

This book is printed on acid-free paper and produced in a sustainable manner. 


\section{Contents}

List of Plates $\quad$ viii

List of Tables $\quad$ xviii

Dedication and Acknowledgements $\quad$ xix

Vivienne Lo 羅維前

Introduction

Vivienne Lo 羅維前

1 A Survey of Images from the Chinese Medical Classics

Wang Shumin 王淑民 and Gabriel Fuentes

PART 1: Mapping the Body: Space, Time and Gender

2 Picturing the Body in Chinese Medical and Daoist Texts from the Song to the Qing Period (1oth to 19th Centuries)

Catherine Despeux

3 Imagining Practice: Sense and Sensuality in Early Chinese Medical Illustration

69 Vivienne Lo 羅維前

4 The Iconography of Time: What the Visualisation of Efficacious Movement (Shi 勢)

Tells Us about the Composition of the Yijin Jing 易筋經 (Canon for Supple Sinews)

Elisabeth Hsu

5 Nurturing the Foetus in Medieval China: Illustrating the 1o Months of Pregnancy in the Ishimpo $\overline{\text { 醫心方 }}$

Sabine Wilms

6 The Gendered Medical Iconography of the Golden Mirror, Yuzuan Yizong Jinjian 御纂醫宗金鑑, 1742

$Y i-L i W u$ 吳一立

\section{PART 2: Effective Representation}

7 The Limits of Illustration: Animalia and Pharmacopeia from Guo Pu to Bencao Gangmu 本草綱目

Roel Sterckx

8 Observational Drawing and Fine Art in Chinese Materia Medica Illustration ZhengJinsheng 鄭金生

9 Reading Visual Imagery and Written Sources on Acupuncture and Moxibustion Huang Longxiang 黃龍祥

10 The Fine Art of the Tongue 
11 Diagnostic Images of the Tongue: Aetiology and Pathology Made Visible

Liang Rong 梁喍

12 A Brief Introduction to Illustration in the Literature of Surgery and Traumatology

183 in Chinese Medicine Hu Xiaofeng 胡曉峰

\section{PART 3 Imagining Medical Practice}

13 Polychrome Illustrations in the Ming Bencao Literature Cao Hui 曹暉

14 Illustrations of Drug Collection and Preparation in Buyi Lei Gong Paozhi Bianlan 補遺雷公炮製便覽

Xiao Yongzhi 肖永芝

15 The Relationship between Chinese Erotic Art and the Art of the Bedchamber: A Preliminary Survey Sumiyo Umekawa 梅川純代 and David Dear

16 The Vital Role of Illustration in the Literature of Childhood Smallpox Wan Fang 萬芳

17 Picturing Medicine in Daily life: Court and Commoner Perspectives in Song Era Paintings

TJ Hinrichs

\section{PART 4 Imagining Travelling Medicine}

18 Images of Healing, Hygiene and the Cultivation of the Body in the Dunhuang Cave Murals

Wang Jinyu 王進玉, translated with Lu Di 蘆笛

19 Travelling Light: Sino-Tibetan Moxa-Cautery from Dunhuang

Vivienne Lo 羅維前 and Ronit Yoeli-Tlalim

20 Chasing the Vermilion Bird: Late Medieval Alchemical Transformations in

The Treasure Book of Ilkhan on Chinese Science and Techniques

Vivienne Lo 羅維前 and Wang Yidan 王一丹

21 Fanciful Images from Abroad: Picturing the Other in Bencao Pinhui Jingyao

本草品彙精要

Chen Ming 陳明

22 Chinese Horse Medicine: Texts and Illustrations

Paul D. Buell, Timothy May and David Ramey

23 Korean Anatomical Charts in the Context of the East Asian Medical Tradition Shin Dongwon 신 동원, translated by Kim Yuseok 김유석 
24 Imagining Acupuncture: Images and the Early Westernisation of Asian Medical Expertise

Roberta Bivins

\section{PART 5 Esoteric Contexts and Knowledge Transmission}

25 The Body of Laozi and the Course of a Taoist Journey Through the Heavens

Patrice Fava

26 Clinical Medicine Texts: The Earliest Stone Medical Inscription Zhang Ruixian 張瑞賢,WangJiakui王家葵and Michael Stanley-Baker 徐源

27 Embodying Animal Spirits in the Vital Organs: Daoist Alchemy in Chinese Medicine Zhang Qicheng 張其成, translated and edited by WangJing 王晶 and David Dear

28 A Phoenix Amid the Flames: Mount Emei Big Dipper Finger-Point Method,

Daoyin and Qigong

Liao Yuqun 廖育群

29 Moving towards Perfection: Physical Culture in Dzogchen as Revealed in Tibet's

Lukhang Murals

Ian A. Baker

3 o A Tibetan Image of Divination: Some Contextual Remarks

Ronit Yoeli-Tlalim

\section{PART 6 Imagining Modern Medicine}

31 Places and Traces: Selections from Professor Ma Kanwen’s 馬堪溫 Ethnography of 1955443 Abridged by Penelope Barrett with an Introduction by Vivienne Lo

32 Visualisation in Parasitological Research: Patrick Manson and his Chinese Assistants Shang-Jen $L i$ 李尚仁

33 Marketing Medicine to Koreans

Soyoung Suh 서소영

34 The Visual Language of Medicine Advertisements in The Ladies'Journal Chang Che-chia 張哲嘉

35 Beauty and Health: Images of Health and Illness from 2oth-Century China Zhou Xun 周遜

36 Sketching the Dao: Chinese Medicine in Modern Cartoons 


\section{List of Plates}

The earliest extant Taiji tu, Rashīd-al-Dīn, 1313. 3

Illustration of 'Technique for cultivating Original Spirit' (yuan shen), 1875. 4

Yuanmen maijue neizhao tu (Internal Visualisation Charts from the 'Primordial Portal' Secrets of the Pulse), attr. Hua Tuo (3rd century CE). Qing woodcut. 6

The earliest extant diagram of the vulva. Mawangdui Tomb 3, closed 168 BCE. 8

'Looms of Life' conference poster, UCL, March 2017. Design by Akio Morishima. 9

Neijing tu (Chart of the Inner Landscape), 19th century. 14

Daoyin tu (Guiding and Pulling Chart). Mawangdui Tomb 3, closed 168 BCE. 29

Diamond Sūtra, 868. 30

Illuminated Hall chart showing the loci of the head and shoulders from Dunhuang, ms. P.2675. 31

a and b. Making sea salt, Jingshi zhenglei beiji bencao, ed. Liu Jia, $1185 . \quad 32$

a and b. Salt from Shanxi Province. 32

'Diet therapy for all diseases',Yinshan zhengyao (Principles of Correct Diet), 1330. 35

Illustration of well water, Lu He, Shiwu bencao (Materia Dietetica), 1571. 37

Drug processing with Lei Gong at the centre, Buyi Lei Gong paozhi bianlan (Lei Gong's Guide to Drug Preparation with Addenda), 1591. 37

Nanzi wulao qishang (Five Wearinesses and Seven Damages in Men) from Dunhuang, ms. Or.8210/S.6168. 41 Standing Bronze Man, anterior view, Gao Wu, 1519. 44

Lingmen chuanshou tongren zhixue (Finger Point Bronze Man of the Lingmen Transmission), early Qing copy. 46

Rear view of the Viscera, Man-ampō (Remedies for Absolute Peace of Mind), 1331. 47

Illustrations of the Lungs, Yifang leiju (Collection of Classified Medical Remedies), 1445. 48

Gudai yijia huaxiang (Portraits of Ancient Doctors), 1816. 48

Gymnastic pose, from Shouyang congshu (Collected Texts on Self-Cultivation and Longevity) by Hu Wenhuan, late 17 th century. 54

Moxibustion points, from Jiu jing (Moxibustion Classic), anon., Song period. 54

Jing fahui (Exposition of the 14 Channels) by Hua Shou (13th century). 55

Composite chart of the channels and tracts, Zhenjiu dacheng (Compendium of Acupuncture and Moxibustion) by Yang Jizhou, $1601 . \quad 55$

Model representation of the body used in forensic medicine, Yuandian zhang (Compendium of Statutes and Sub-Statutes of the Yuan Dynasty), 1322. 56

Representation of the trunk and its organs according to Yanluozi, Xiuzhen shishu (Ten Books on Cultivating Perfection), c. 1250. $\quad 5^{8}$

Representation of the trunk and its organs, Hua Tuo xuanmen neizhao tu (Hua Tuo's Images for Internal Visualisation According to the Mystery School), 13th century. 59

Representation of the Lung, Hua Tuo xuanmen neizhao tu (Hua Tuo's Images for Internal Visualisation), 13th century. 6o

Xiyuan lu jizheng (Treatise on the Washing Away of Wrongs with Collected Evidence), Wang Youhuai, 1796. 62

Taiji tu according to Chen Zhixu, Shangyang zi jindan dayao tu (Images of the Golden Elixir of the Mabster of Upper Yang), 13th century. 63

Lianxing mijue tu (Secret art of cultivating the body), Taiji hunyuan zhixuan tu (Images Illustrating the Mysteries of the Chaotic Origin of the Great Ultimate), Xiao Daoxun, 13th century. 64

Daode zhenjing jiyi dazhi tuxu, DZ 723. 64

Image of the body as a mountain showing alchemical processes, Daoyuan yiqi (The One Breath of Daoyuan),

Cao Yuanbai, late 16th century. 65 
2.14 Xiuzhen tu (Image for Cultivating Perfection), 19th century. 66

3.1 Daoyin tu (Guiding and Pulling Chart). Mawangdui, Tomb 3, closed 168 BCE. 70

3.2 Lacquer figurine from Tomb 2 at Shuangbaoshan, Mianyang, Sichuan, latest date 118 BCE. 71

3.3 Detail of the Shuangbaoshan figurine. 73

3.4 Juxtaposition of Daoyin tu (Guiding and Pulling Chart) with Yinyang shiyimaijiujing (Cauterisation Canon of the Eleven Yin and Yang Channels). Mawangdui Tomb 3, closed 168 BCE. 73 Prohibitions of the Huangdi hamajing (Yellow Emperor's Toad Classic). 77

Moxa-cautery prohibitions related to the position of the shen spirit in the body, Huangdi hamajing. $\quad 78$ a and b. Dunhuang moxa-cautery charts, mid-gth century. $\quad 78$

Renzi diagram from a hemerological almanac of bamboo slips. Shuihudi, late 3rd century BCE. 80

The lines on the Shuangbaoshan figurine cluster and meet around the sense organs. 81

Detail of the Shuangbaoshan figurine. 81

Back of the Shuangbaoshan figurine. 81

The Bear Ramble, Daoyin tu (Guiding and Pulling Chart). Mawangdui Tomb 3, closed 168 BCE. 84

Monkey Bawling to Pull Internal Hotness, c. 168 BCE. 85

Dragon Ascending, c. 168 BCE. 85

Depiction of Tibetan pulses along a linear trajectory of time. Parfionovitch et al. 1992. 89

Depiction of Chinese mai 脈 in an iconic-indexical manner. 90

'Weituo offers the vajra' (Weituo xian gan). 96

'Make a bow' (da gong) and 'Wag the tail' (diao wei). 97

'Pluck the stars and reverse the dipper' (zhaixing huan). 97

Table of Contents of Ishimpō, vol. 22 (982 CE). 101

Drawings of foetal development during months $6-8$ of the 10 months of pregnancy, Ishimpō, 982 CE. 106 Drawing of the pregnant female body in the 1oth month of pregnancy, Ishimpō, Seikido Library Scroll, 1145 CE. 107

Huangdi hama tu suiyue shenghui bi jiupan fa (Yellow Emperor's Toad Chart: method for avoiding cautery and pain according to the waxing and waning of the moon). 108

5.5 Drawings of the female body in the 3rd and 1oth months of pregnancy, Ishimpō, Cabinet Library Scroll, 1791 CE. 109

6.1 'Image of the form of the heart', Imperial Encyclopaedia. Reproduced from Chen Menglei et al., 1986. 113

'The heart organ of the lesser Yin channel', Golden Mirror. Reproduced from Wu Qian (ed.), 1742. 113

'Vessels of the chest and abdomen', Golden Mirror. Reproduced from Wu Qian (ed.), 1742. 115

'The chengshan point', Golden Mirror. Reproduced from Wu Qian (ed.), 1742. 116

Portrait of Zhang Chengye. Reproduced from Jin Guliang, 1961. 116

'Pox pustules' from the Golden Mirror. Reproduced from Wu Qian (ed.), 1742. 117

'Points on the Yang springing vessel', Golden Mirror. Reproduced from Wu Qian (ed.), 1742. 119

'Deep abscess of the breast', Golden Mirror. Reproduced from Wu Qian (ed.), 1742. 120

6.10 Detail of 'Winnowing', from a Song edition of Pictures of Tilling and Weaving. Reproduced from Franke, 1913. 120

6.11 'Point to cauterise for treating difficult childbirth', Golden Mirror. Reproduced from Wu Qian (ed.), 1742. 121

6.12 Ban Zhao. Reproduced from Jin Guliang, 1961. 121

6.13 'Acupoints on the Heart channel', Golden Mirror. Reproduced from Wu Qian (ed.), 1742. 122

6.14 Kapimala (Jia-pi-mo-luo), Wondrous Traces of Immortals and Buddhas. Reproduced from Hong Yingming, 1983. 122 
7.3 'Strange animal transformations' from Yinshan zhengyao (Ming edn). Source: Zhongguo gudai banhua congkan erbian. 143

7.4 a. Determining the age of a horse by dental record; b. Horse scrotum, from Yuan heng liao ma ji (16o8). 147 a. Leech (shui zhi) versus earthworm (qiu yin); b. 'Giant mussel' (ma dao) versus 'small' clam (xian); c. Cow bezoar (niu huang, calculi bovis) and dog bezoar (gou bao, calculi canis), from Bencao gangmu (1885 edn). 148

8.1 Pharmaceutical illustrations from Bencao tujing (Illustrated Canon of Materia Medica), 1062. 151

8.2 Mineral drugs, from Li Shizhen, Bencao Gangmu (Systematic Materia Medica), Jinling edn, 1596. 152

8.3 Shanjianghua (Alpinia japonica), from Lü Chanyan bencao (Cliff Walker's Materia Medica), 1220. 153

8.4 Astragalus (Huangqi), from Jiuhuang bencao (the Famine Relief Herbal) compiled by Prince Zhu Su, 140324. 154

8.5 Pharmaceutical illustrations, from Li Zhongli, Bencao yuanshi (Origins of Materia Medica), created 1612. 154

8.6 Pharmaceutical illustration: Epimedium (Xianlingpi) from Bencao bian fang (Everyday Remedies from the Pharmacopoeia), 1870. 156

8.7 Chengzhou lily, from Bencao pinhui jingyao (Materia Medica Containing Essential and Important Material Arranged in Systematic Order), 1505. 157

8.8 Drug preparation, from Buyi Lei Gong paozhi bianlan. 159

9.1 Replica of the Song Bronze Man, Ming Zhengtong era (1436-49). 162

9.2 Tongren shuxue zhenjiu tujing, Ming. 162

9.3 Tongren shuxue zhenjiu tujing, Song. 162

9.4 Head of the Ming Zhengtong Bronze Man, viewed from the side, showing the locations of hanyan, xuanlu, and xuanli on the back of the head. 162

9.5 Variations in the siting of acu-moxa location of weiyang (Lateral to the Crook). From left to right: Ming Zhengtong (1436-49) Bronze Man; Japanese Bronze Man, Edo period (16o3-1868); Bronze Man from Deoksugung Palace, Seoul, Korea. 163

9.6 Manuscript recovered from Dunhuang (1), showing the location of tianchuang (Celestial Window). 164

9.7 Manuscript recovered from Dunhuang (2), S.6168. 165

9.8 Suwen, Song woodblock print, showing a page from the 'Maijie' treatise. 165

9.9 Twelve Waxing and Waning Trigrams. 166

1341 edition of Aoshi shanghan jinjing lu (Scholar Ao's Golden Mirror of Cold Damage Disorders) showing Shaoyang presentation with a prescription for Xiao Chai Hu Tang. 169

Shanghan zheng zhi zhun cheng (Standards of diagnosis and treatment for Cold Damage), by Wang Kentang (Ming, 1368-1644). 169

From a hand-drawn 1445 edition of the Shanghan jinjing lu tongue text. 170

White coat and red tip, Shebian sanshiliu zhong, a 1910 hand-drawn text on tongues. $\quad 170$

1529 edition of Xue Ji's compilation of Waishang jinjing lu. $\quad 171$

Shejian bianzheng 1894, showing the mapping of internal organs on to specific areas of the tongue. $\quad 172$

Anatomical placement of the tongue. 174

White coloured tongues, Bian she zhinan, 1920. 174

10.10 Standardised mapping of internal organs onto areas of the tongue, from student handouts. 175

Tongues and Prescriptions from Shanghan diandian jin shu (Gold-dust Book of Cold Damage), Ao Jiweng (Song period, 96o-1279) and Du Ben (Yuan period, 1206-1368), 1341 (1894) edition. 179 
12.2 Holding ropes and standing on stacks of bricks, Qian Xiuchang, Shangke buyao (Supplement to Traumatology), $1808 . \quad 185$

12.3 Hu Tingguang's Shangke huizuan (completed 1815). 186

12.4 a and b. Charts of bone-length measurement in chi and cun (anterior and posterior views), from Qian Xiuchang, Shangke buyao (Supplement to Traumatology), [1808] 1976. 186

12.5 a and b. Fixation appliances for fractured kneecap (Baoxi yongfa tu), from Qian Xiuchang, Shangke buyao, 1808. 188

12.6 Bamboo curtain, orthopaedic appliance, Hu Tingguang, Shangke huizuan (completed 1815). 188

12.7 (right) Fir-wood fence, orthopaedic appliance, Qian Xiuchang, Shangke buyao, 1808. 188

12.8 Chart of the healing herb rendongteng (Japanese honeysuckle, Lonicera japonica), Waike jingyao, 1273. 188

12.9 Proportional somatic measurements, Chen Ziming, Waike jingyao, 1263. 189

12.10 Nine Mansions and Travelling Spirit, Chen Shigong, Waike zhenzhong, $1617 . \quad 189$

12.11 a and b. Charts of the Five Circulatory Phases and Six Climatic Factors; Taiji diagrams, Yangyi daquan 1760. 190

12.12 Chart of the dumai channel, Chen Shiduo, Dongtian aozhi (Profound Teachings of the Heavenly Cavern), 1694. 190

12.13 Posterior location chart, Qian Xiuchang, Shangke buyao, 1808. 191

12.14 Internal visualisation images with internal organs, Sun Zhenyuan, Yangke huicui (Treasury of Dermatology), 1802. 192

12.15 Alchemical Furnace from Waike tushuo (Pictorial Manual of External Medicine), 1856. 192

12.16 Wang Ji (1463-1539), Waike lili (Principles and Examples of External Medicine). 193

13.1 a. Dragon Bone' (Longgu); b. 'Dragon' (Long), from Bencao pinhui jingyao, c. 1503. 198

13.2 Fruit of Gomuti sugar palm, Bencao tupu (Illustrated Register of Materia Medica), 1628-44. 199

13.3 Jinshi kunchong caomu zhuang (Descriptions of Minerals, Insects and Plants), Preface by Zhao Lingjun, 1617-20. 200

13.4 Mingjie zenghe qianjia shi zhu (A Children's Reader of Poems from One Thousand Authors), Song period. 202

13.5 Wanaqi (Penis and testes of fur seal), Bencao pinhui jingyao, c. 1503. 205

14.1 Shoujiao tu (Receiving [Medical] Knowledge), Buyi Lei Gong paozhi bianlan (Supplement to Lei Gong's Guide to the Preparation of Drugs), 1591 edn. 209

14.2 Huan kun zhi (Laundry Water), Buyi Lei Gong paozhi bianlan (Supplement to Lei Gong's Guide to the Preparation of Drugs), 1591 edn. 210

14.3 Detail of the canopy bed from Renjing tu (Illustration of Human Semen [Essence]), Buyi Lei Gong paozhi bianlan (Supplement to Lei Gong's Guide to the Preparation of Drugs), 1591 edn. 210

14.4 Receptacles, Buyi Lei Gong paozhi bianlan (Supplement to Lei Gong's Guide to the Preparation of Drugs), 1591 edn. $\quad 212$

14.5 Processing asparagus root, Buyi Lei Gong paozhi bianlan (Supplement to Lei Gong's Guide to the Preparation of Drugs), 1591 edn. 213

15.1 'Pan Jinlian Enjoys a Midday Battle in the Bathtub' (detail), Jinping mei, Ming. 219

15.2 'Nami Chidori', Katsushika Hokusai, c. 1829. 219

15.3 'Wang haichao' (Watching the Rising Tide), Huaying qinrong (Variegated Positions of the Flowery Battle).

Printed from an early 17 th-century(?) woodblock. 223

15.4 Fishes Kissing, anon. 224

15.5 Houtingyan (Celebration in the Rear Courtyard), Huaying qinzhen (Variegated Positions of the Flowery Battle). Printed from an early 17th-century(?) woodblock. 224

15.6 Liting hua (Flower in the Back Garden), scene from Jinping mei, Ming. 225

16.1 Suspended Mirror pox, Yulinzhishi yusui (Yulinzhishi Chalcedony Digest). 229

16.2 Scorpion pox (right-hand page); Cauldron-lid pox (left-hand page), Yulinzhishiyusui zhaiyao (Yulinzhishi Chalcedony Digest). 229

16.3 Locked Well pox (right-hand page); Coiled Snake pox (left-hand page), Yulinzhishiyusui zhaiyao (Yulinzhishi Chalcedony Digest). 229 
16.4 Tiger-feeding pox (right-hand page); Dark Tumulus pox (left-hand page), Yulinzhishiyusui zhaiyao (Yulinzhishi Chalcedony Digest). 230

16.5 The Keys to Fire, Yulinzhishi yusui zhaiyao (Yulinzhishi Chalcedony Digest). 230

16.6 Auspicious smallpox, Yulinzhishi yusui zhaiyao (Yulinzhishi Chalcedony Digest). 230

16.7 Illustration of inauspicious smallpox,Yulinzhishiyusui zhaiyao (Yulinzhishi Chalcedony Digest). 231

16.8 The Keys to Earth, Yulinzhishi yusui zhaiyao (Yulinzhishi Chalcedony Digest). 231

16.9 The Keys to Water, Yulinzhishi yusui zhaiyao (Yulinzhishi Chalcedony Digest). 231

17.1 Hancheng tomb, north wall mural, c. 1070233

Street Scene with Pharmacy from Qingming on the River, attr. Zhang Zeduan (1085-1145). 234

Moxibustion, attr. Li Tang (c.1050-after 1130). 234

a-c. Eye contact: a. Detail of 18.1; b. detail of 18.2; c. detail of Fig. 3. 235

Map of painting production sites in the Song era, 1070-1100. 235

Hancheng tomb chamber. $\quad 236$

Hancheng tomb, east wall mural. 237

Hancheng tomb, west wall mural. 237

Hancheng Tomb, north wall, left side, detail of Fig. 1. 238

Hancheng Tomb, north wall, right side, detail of Fig. 1. 239

'Taiping Era Formulas of Sagely Grace', 'White Atractylodes', 'Rhubarb'. 240

Cinnabar Pills', detail of 18.1. $\quad 241$

Qingming on the River, detail of 18.2. 244

Compassion in Moxibustion, detail of 18.3. 245

Fields of Merit Sūtra painting. Mogao Grotto no. 296, North Zhou Dynasty (557-81). 252

Fields of Merit Sūtra painting. Mogao Grotto no. 302, Sui (581-617). 253

'Ten Wheels' Sūtra painting. Mogao Grotto no. 321, Early Tang Dynasty (618-712). 254

Lan̉kāvatāra Sūtra. Mogao Grotto no. 61, Five Kingdoms (848-907). 254

1950s reproduction of a Lotus Sūtra painting from Mogao Grotto no. 217, High Tang (705-80) 255

Lotus Sūtra Painting. Mogao Grotto no. 23, High Tang (705-80). 256

Fo Tudeng washes his intestines. Mogao Grotto no. 323, Early Tang (618-704). 257

Lotus Sūtra: The betrayal of King Deer. Mogao Grotto no. 257, Northern Wei (386-534). 257

Lutou Fan Zhi: the miracle worker with the stag's head. Mogao Grotto no. 329, Early Tang (618-712). 259

a. Sūtra painting of Bhaișajya-guru, the Medicine Buddha. Mogao Grotto no. 85, Later Tang (848-907).

b-d: Sūtra paintings of Bhaișajya-guru, the Medicine Buddha.

From left to right: b. from Mogao Grotto no. 322, Early Tang (618-712); c. from Mogao Grotto no. 310, Northern Song (96o-1036); d. illustrating The Nine Abnormal Deaths: here, death by toxic drugs. Mogao Grotto no. 231, Middle Tang (781-847). 260

18.10 e. The Nine Unnatural Deaths: death from poisonous drugs. Mogao Grotto no. 76, Northern Song Dynasty (970-1036). $\quad 261$

$18.11 \quad$ a. (top left) Vajrapāni, wielding a flower-like vajra in a gth-century painting from Dunhuang; b. (top right) Yao cha (Yakshas). Mogao Grotto no. 249, Western Wei (535-57); c. (below) Worshipping Bodhisattvas: in rapturous dance or self-cultivation? Mogao Grotto no. 272, Northern Liang (397-439). 262

a. Contest between Sariputra and Raudraksa. Mogao Grotto no. 196, Late Tang (848-907) b. Teeth cleaning and washing the head. Mogao Grotto no. 196, Late Tang (848-907); c. Teeth cleaning. Mogao Grotto no. 159,

Middle Tang (781-847). $\quad 263^{-264}$

18.13 Tonsure ritual. Mogao Grotto no. 445, High Tang (705-780). 265

18.14 Washing the feet of Sakyamuni. Mogao Grotto no. 31, High Tang (705-80) 265

18.15 Boiling milk. Mogao Grotto no. 61, Five Dynasties (907-6o). 266

18.16 Sweeping-up and the latrines. Mogao Grotto no. 29o, Northern Zhou (557-81). 266

18.17 Rāksasa (Ye Hua) sweeps the city clean and the Dragon King sends down rain. Anxi Yulin Grotto no. 25, Middle Tang (781-847). $\quad 267$

18.18 Martial arts. Mogao Grotto no. 321. Early Tang (618-712). 267

18.19 Handstand. Mogao Grotto no. 249, Western Wei (535-57). 268 
18.20 Tantric sexual union between deities. Mogao Grotto no. 465, Yuan (1271-1368) (1). 268

18.21 Tantric sexual union between deities. Mogao Grotto no. 465, Yuan (1271-1368) (2). 269

19.1 Cautery depicted in a 9th-century Latin manuscript, Plut. 73.41, f. 122 r. 272

19.2 A pile of scrolls, after removal by Stein from Cave 17 in Dunhuang. 273

19.3 Tibetan moxa-cautery chart from Dunhuang, ms. Pt.1058 274

19.4 a. Chinese moxa-cautery chart from Dunhuang, ms. Or.8210/ S.6168; b. Chinese moxa-cautery chart from Dunhuang, ms. Or.8210/S.6262. 276

19.5 a. Lei Gong (Thunder Duke or the Thunder Spirit) surrounded by drums.; b. perhaps Feng Shen (Wind Spirit), or Feng Bo (Wind Uncle) with a bag full of wind on his back. Dunhuang, Mogao Grotto 249, Western Wei (534 $-556) . \quad 278$

19.6 A Uighur moxa-cautery chart with remedies, ms. Mainz 0725. 285

19.7 The circulation of bla in the body. Tibetan Medical Painting no. 12 (Ulan Ude set). 286

19.8 The Toad and the Hare in the Moon, Hamajing (Toad Classic). 287

20.1 Stone ceiling relief with Taiyi (Grand Unity) between the emblems of the constellations of the four directions. Qilingang, Henan province. 294

20.2 Li Jiong's Neijing tu (Chart of the Inner Landscape), DZ (1436-49). 295

20.3 A selection of images from Huangdi bashiyi nanjing zuan tu jujie in DZ (1436-49), including Chart of the Inner Landscape with the Yanluozi images of the internal organs in the top right-hand corner. 297

20.4 Rashīd al-Dīn's Tansūqnāma version of Li Jiong's images in the Yanluozi tradition, Tabriz, 1313. 298

20.5 a. (left) Qing manuscript illustration of the Yuanmen maijue neizhao tu (Internal Visualisation Charts from the 'Primordial Portal' Secret Art of the Pulse); b. (right) Rashīd al-Dīn's version of the qihai (Sea of Qi), 1313. 298

20.6 Chart of the Heart Connections, Tansūqnāma, 1313. 299

20.7 Shangqing huangting neijing wuzang liufu zhenren yuzhou (Precious Scroll of the Zhenren on the Six Receptacles and Five Viscera of the Yellow Court of Shangqing), DZ 1402. 302

20.8 Cosmic birds representing the stars and planets. Mogao Grotto no. 35. 302

21.1 Citragandha (Zhihan, a composite drug), Bencao pinhui jingyao, Rome ms (16th century). 306

21.2 Acronychia pedunculata (Jiangzhen xiang), Bencao pinhui jingyao, Rome ms. 306

21.3 Borneol (Longnao xiang), Bencao pinhui jingyao, Rome ms. 306

21.4 Citragandha (Zhihan, a composite drug), Buyi Lei Gong paozhi bianlan, 1591. 307

21.5 Acronychia pedunculata(Jiangzhenxiang), 1591. 307

21.6 Borneol (Longnao xiang), 1591. 307

22.1 Map of Indentations. Simu anji ji (Collections for Pacifying Stallions when Administering Flocks), $1384 . \quad 317$

22.2 German map for horse bleeding, cauterisation ('hot needling'), and branding, or minor surgery (using scalpels, 'white needles'). 318

22.3 Bo Le hualuo tu ge jue (The Song Secrets of the Diagram of Bo Le's Branding) Branding Diagram. 319

22.4 Illustration of horse colic Xinbian jicheng mayifang, (Newly Printed and Collected Recipes for Horse Medicine), 1399. 320

22.5 Hoof Problems from Xinbian jicheng mayifang, (Newly Printed and Collected Recipes for Horse Medicine), 1399. 320

22.6 Horse suffering from Chill Pain, Yuan Heng liaoma ji (Yuan and Heng's Collection for Treating Horses), 1608. 321

22.7 Horse suffering from Chill Pain.,Yuan Heng liaoma ji (Yuan and Heng's Collection for Treating Horses), 1608. 321

22.8 Horse suffering from Chill Pain, Yuan Heng liaoma ji (Yuan and Heng's Collection for Treating Horses), 16o8. $\quad 322$

22.9 Horse suffering from Chill Pain, Yuan Heng liaoma ji (Yuan and Heng's Collection for Treating Horses), 16o8. $\quad 322$

22.10 Horse suffering from prolapse of the Anus, ms., c. 19th century. 323

$22.11 \quad a$ and b. Horse physiognomy, detail from Yuan Heng liaoma ji, 16o8. 324 
23.1 Image of the lung, Kim Yemong et al. 1477. 327

$23.2 \quad$ Image of the organs in the body, Yu Seongnyong $1600 . \quad 327$

23.3 Image of the form of the body and the zang and fu organs, Heo Jun 1630. 328

23.4 Lateral view of the body, Gong Tingxian (Ming). 328

23.5 Five images of the five zang organs, Heo Jun, Dong'ui'bo'gam (Treasured Collections of an Eastern Physician), 1613. 334

24.1

24.2

24.3

24.4

24.5

24.6

24.7

24.8

25.1

25.2

$25 \cdot 3$

25.4

$25 \cdot 5$

25.6

$25 \cdot 7$

$25 \cdot 7.2$

$25 \cdot 7 \cdot 3$

$25 \cdot 7 \cdot 4$

$25 \cdot 7 \cdot 5$

25.8

details. $\quad 363-37$

25.8.1 The master kneels in front of the altar. The Vermilion Bird leads the way through the clouds. 363

25.8.2 At the entrance of Heaven, the Crystal Gate. 364

25.8.3 Beginning of the ascent through the 32 Heavens. The master arrives before Donghua Gate, the second Gate of Heaven. 365

25.8.4 Across the 28 mansions and the four Brahma Heavens. 366

25.8.5 Arrival at the third Gate of Heaven. 367

25.8.6 Beyond the 28 mansions are the constellations of the Five Agents and the stars of Ursa Major. 368

25.8.7 The seven stars of Ursa Major have the Pole star at their top (ziwei). Beyond are the Palace of the Three Masters, the Four Offices of the Stellar Lords and the Palace of the Four Saints. 369

25.8.8 Traversing the last palaces among which are those of the Three Officers of Water, Earth and Heaven. 370

25.8.9 Arrival at the court of the Jade Emperor and the Palaces of the Three Pures. 371

26.1 Panoramic view of the Longmen Caves in present-day Henan. 373

26.2 Seven Buddhas shrine, stele with medical formulas below. Medical Recipes Cavern, Longmen.

26.3 Votive statue and opening inscription. Medical Recipes Cavern, Longmen, north wall. 374

26.4 Buddhist statuary. Medical Recipes Cavern, Longmen. 381

27.1 Fei Shen (Lung Spirit), DZ 1402. $\quad 390$ 
27.2 Xin Shen (Heart Spirit), DZ 1402. 391

27.3 Gan Shen (Liver Spirit), DZ 1402. 392

27.4 Pi Shen, Spleen Spirit, DZ 1402. 393

27.5 Shen Shen (Kidney Spirit), DZ 1402. 394

27.6 Dan Shen, (Gallbladder Spirit), DZ 1402. 395

28.1 Cover of Emei shan tiangang zhixue fa (Mt Emei's Big Dipper Finger-point Method), 1985. 397

28.2 Two illustrations by the author's father, from Emei shan tiangang zhixue fa, 1985. 397

28.3 Hezui jin (The Crane's Beak). 399

28.4 Chongtian chu jin (Soaring Pestle). 399

29.1 Padmasambhava accepting obeisance from an elemental nature spirit called a Lu (Skt: naga). Lukhang Temple. 404

29.2 Practitioners of 'Great Perfection' (Dzogchen). Lukhang Temple. 408

29.3 A disembodied 'soul' in the process of incarnating in a woman's womb. Lukhang Temple, north wall. 410

29.4 Pema Lingpa's 'Secret Key to the Channels and Winds' (Rtsa rlung gsang ba'i lde mig) with 23 yogic exercises ('khrul 'khor). 411

29.5 Pema Linga publicly extracting treasure texts (gter) from 'Burning Lake'. Lukhang Temple. 412

29.6 Tsangyang Gyatso, the Sixth Dalai Lama, as a 'Bodhisattva king'. Lukhang Temple, southeast corner. 413

29.7 Dzogchen yogin acting out interior states of consciousness in a process called Korde Rushen (v'khor'das ru shan). Lukhang Temple, west wall. 414

29.8 23 yogic exercises that prepare the mind and body for meditation and interior yogic practices. Lukhang Temple, north wall. 417

29.9 Contemporary demonstration of the yogic exercises ('khrul 'khor) central to Pema Lingpa's 'Secret Key to the Channels and Winds'. 417

29.10 "The Dzogchen 'leaping over the skull' (thod rgal) postures of elephant and sage. Lukhang Temple, western wall. 418

29.11 Processes of physical and psychological illumination in which the practitioner of Dzogchen awakens to his or her indwelling Buddha Nature (1). 419

29.12 Processes of physical and psychological illumination in which the practitioner of Dzogchen awakens to his or her indwelling Buddha Nature (2). 419

29.13 A 2015-16 exhibition at London's Wellcome Collection entitled 'Tibet's Secret Temple: Body, Mind and Meditation in Tantric Buddhism'. 420

29.14 A mahāsiddha sits in a posture associated with the practice of Fierce Heat (gtum mo). 425

29.15 Three principal energy channels cultivated in Tibetan yoga. Lukhang Temple. $\quad 426$

29.16 Buddha Samantabhadra, 'All Pervading Goodness', on the cover of Pema Lingpa's 'Compendium of Enlightened Spontaneity', 17th-century copy. $\quad 428$

30.1 A Tibetan Protection painting containing the main elements of Tibetan divination. Tibet, 19th century. 429

30.2 Detail of the tortoise used in Tibetan divination. 433

30.3 A Tibetan Frog/Turtle divination chart for the purpose of finding lost objects. Dunhuang, 9th-10th century. 434

30.4 Tibetan medical painting no. 65: Urine divination using Turtle charts. 435

30.5 A sipaho (srid pa ho) protective chart. 434

3o.6 Luo shu: Chinese magic square of three. 436

30.7 Representation of the Nine Palace diagram in Xingde B, Mawangdui tombs (2nd century BCE). 437

30.8 The Tansūqnāma of Rashīd al-Dīn. Tabriz, 1313. 438

31.1 Professor Ma Kanwen 1927-2016. 443

31.2 Ruins of Yuchen Daoist temple, on the site of Tao Hongjing's mountain retreat, Maoshan, Jiangsu province. 444

31.3 'Cinnabar Well' of Tao Hongjing, in front of Dongxu Daoist temple, near Tao village, Jintan county, Jiangsu province. 444 
31.4 Tomb of Zhu Zhenhong in Dongzhu village, Yiwu, Zhejiang province. 445

31.5 Tomb relief showing, in the top right-hand corner, Bian Que, the human-headed bird, administering acupuncture to waiting patients, Eastern $\operatorname{Han}\left(25^{-220} \mathrm{CE}\right) .446$

31.6 Entrance gates ('Mountain Gates') of the Three Kings temple complex, Renqiu county, Hebei province. 447

31.7 Temple of Bian Que, Shentou village, Neiqiu county, Hebei province. 450

31.8 Fragment of a stele, temple of Bian Que, Shentou village, Neiqiu county, Hebei province. $\quad 45^{2}$

32.1

32.2

32.3

32.4

32.5

32.6

32.7

Filaria immitis, from Manson, $1877 . \quad 458$

Filaria hominis sanguinis, Manson, $1877 . \quad 458$

Filaria sanguinolenta, Manson, 1877.458

Filaria hominis sanguinis: selection of Manson's drawings of the metamorphosis of Filaria bancrofti in the mosquito. Reproduced from Manson-Bahr and Alcock, 1920. 458

A Chinese patient suffering from Elephantiasis, Manson, 1898. 461

Manson's Chart of Filarial Periodicity, Manson, 1882. 462

Portrait of Thomas Colledge, by William Daniell after George Chinnery, 1834. 463

Portrait of Patrick Manson experimenting with filaria sanguinis hominis on a human subject in China, by

Ernest Board, c. 1912. 464

Advertisement for 'Jintan' (Humane Elixir), Maeil Sinbo (Daily News), 1911. 467

Various advertisements for 'Humane Elixir', Daily News, 1910s. 468

Advertising 'Ch’ŏngsim pomyŏngdan' (Pill for Clearing the Heart and Guarding Life). 472

Advertisement for 'G-U-CIDE', 1939. 474

Advertisement for 'Mansu paekpohwan' (Longevity Pill of Nourishment with One Hundred Ingredients),

Mansŏn ilbo (Daily News of Manchuria and Korea), 1940. 477

Chūshōtō advertisement, The Ladies'Journal. 481

Bayer Aspirin advertisement 1, The Ladies'Journal. 482

Bayer Aspirin advertisement 2, The Ladies'Journal. 482

Handwritten testimonial by actor Shang Xiaoyun, The Ladies'Journal. 483

Dr Williams' Pink Pill for Pale People, The Ladies'Journal. 483

Take home gymnastics for women, Young Companion Pictorial, 1930s. 487

Swimming Pool Beauty, Young Companion Pictorial, 1939. 487

Healthy baby photos, Saturday Magazine, 193os. 488

'Hygienic habits for children'. Public health poster for the Republican Health Bureau, 1931. 488

'Summer Life at Home', Young Companion Pictorial. 489

'Summer Life at Home', Young Companion Pictorial. 489

Lacovo Malt drink advertisement. 490

Popular advertisement for luxury fabric, 1930s. 490

'Bayer medicines keep me healthy and beautiful'. Advertisement for Bayer company's Western medicine. 491

Advertisement for Pinkettes, 1930s. 491

Body builder. 491

'Go to the Seaside', Arts and Life, 5 (August 1934). 492

Film star Bai Yang (1920-96) doing calisthenics. 493

'To strengthen the nation, you must first strengthen its people. To strengthen the people, you must first make your children strong.' Advertisement for baby milk from the USA, c. 1930. 494

Healthy Baby Contest from Republican period (from Second Historical Archive of Nanjing) (1). 495

Healthy Baby Contest from Republican period (from Second Historical Archive of Nanjing) (2). 495

'Healthy Babies are China's Future'. Republican government poster (from Second Historical Archive of Nanjing). 495

Cover of Xiaorenshu, 'A Strange Herbal Prescription'. 499

Two pages from 'A Strange Herbal Prescription'. 499

The Yellow Emperor and his teacher Qi Bo. $\quad 501$ 
36.4 Adjusting to Summer weather is both an ancient and a modern problem. $\quad 5^{\mathrm{O} 2}$

36.5 'I just took the baby for his shot.' $5^{\mathrm{O} 2}$

36.6 The 'Twelve Officials' of the visceral systems. 503

36.7 The Comic Yellow Emperor's Inner Canon 504

36.8 Systematic method. 505

36.9 Systematic method followed by the ineffable dragon. 505 


\section{List of Tables}

1.1 The Earliest Materia Medica $\quad 33$

1.2 Illustrated acu-moxa texts recorded in The History of The Sui 40

1.3 Works containing acu-moxa illustrations, Jin and Yuan periods 43

1.4 Formula works containing acu-moxa illustrations, 1oth-16th centuries

1.5 Works containing acu-moxa illustrations, Ming and Qing periods 47

5.1 Textual variations in accounts of gestation $\quad 105$

6.1 Distribution of human figures by subject matter and gender 115

9.1 Comparison of three acu-moxa locations $\quad 163$

12.1 Differentiation of haemorrhoids in Waike Qixuan $\quad 187$

15.1 Positions, effects and number of insertions of the Eight Benefits 221

15.2 Therapeutic sexual intercourse for the Seven Disadvantages 222

27.1 Characteristics of the embodied animal spirits: The White Beast/Lion in the Lung 390-91

27.2 Characteristics of the embodied animal spirits: The Vermilion Bird in the Heart 391

27.3 Characteristics of the embodied animal spirits: The Dragon in the Liver 392

27.4 Characteristics of the embodied animal spirits: The Phoenix in the Spleen 393

27.5 Characteristics of the embodied animal spirits: The Deer in the Kidneys 394

27.6 Characteristics of the embodied animal spirits: The Turtle and Snake in the Gallbladder 


\section{Dedication and Acknowledgements}

Vivienne Lo 羅維前



This book is dedicated to the life and work of Professor Ma Jixing 馬繼興, a researcher and teacher whose combination of academic achievements and spirit of generosity make him the scholar who has exerted the greatest influence worldwide on generations of historians of Chinese medicine. On 15-17 September 2005, I.M. Pei's iconic Fragrant Hills Hotel in the mountains to the north-west of Beijing was the setting for a conference, convened by Ma Jixing's former student Wang Shumin 王淑民 and me, held in honour of the 8oth birthday of the venerable professor.
The conference was a part of a longer-term, and ongoing, collaborative project on the visual cultures of medicine sponsored by UCL's former Wellcome Trust Centre for the History of Medicine and the Zhongguo Zhongyi yanjiu yuan 中國中醫研究院 (Academy of Chinese Medicine, now the China Academy of Chinese Medical Sciences). ${ }^{1}$ Since the inauguration of the Academy in the early 195os, when the Chinese government endorsed a wide-ranging

1 Zhongguo Zhongyi kexueyuan 中國中醫科學院. 
programme of research into local medicines, Professor Ma has specialised in understanding the ways in which medical knowledge was constructed by analysing the ever-increasing quantities of early and medieval manuscript sources re-discovered through archaeological projects in 2 oth to 21st century China. At over 9o years old now he is still publishing prolifically, with his most recent magnum opus being the three-volume, 2, ooo-page, Zhongguo chutu guyi shu 中國出土古醫書 (Ancient Medical Texts Excavated in China), complete with photographs and full transcriptions, published in 2015 .

While at medical school in north China during the second Sino-Japanese war (1936-45), Professor Ma chose to specialise in traditional medicine. As a medical graduate in revolutionary China, he was then allocated a position teaching physiology in Peking Medical College (Beiyi Xueyuan 北醫學院), which allowed him ample time for reading the medical classics, a pursuit that he found suited him better than clinical work. Trained in both modern and traditional Chinese medicine and self-educated in reading ancient texts, he was well placed thereafter to join the first team of historians at the Institute for the History of Chinese Medicine and Medical Literature at the China Academy of Traditional Chinese Medicine.

Life wasn't always easy at the Academy, and during the years of the Cultural Revolution, he found himself denounced, criticised and sentenced to periods of isolation and self-examination. There was little freedom, but a lot of time to further his reading and to wait for the moment when he could resume his research. Throughout, he was working on multiple projects. In 199o he was finally able to publish his bibliographical work on the history of Chinese medical writing, which remains a first port of call for contemporary researchers into pre-modern medicine. ${ }^{2}$ When I first visited his office in the Dongzhimen district of central Beijing in the early 199os, he proudly showed me how one whole wall was lined with notebooks for an unfinished history of acupuncture that he was still busy researching. What was holding him up was the ancient manuscripts excavated in the previous decade from tombs along the Yangzi river, manuscripts which were revolutionising people's understanding of the history of the formation of a 'Chinese' medical identity and the grounding of that identity in unique styles of medicine: acupuncture, pharmacotherapy, nutrition and self-care.

In the gos Professor Ma published the first annotated transcript of the most significant of those medical manuscripts that were known at that time, excavated from

\footnotetext{
$2 \quad$ Ma Jixing 1988.
}

the Mawangdui tombs, and it was his commentaries that helped me translate and analyse contemporary manuscripts from other Han dynasty tombs during my PHD years at the School of Oriental and African Studies. ${ }^{3}$ Soon after graduating, my attention turned to some 100 and more medical manuscripts that had been discovered among the tens of thousands of religious texts in Grotto 17 at the Dunhuang Mogao caves at the eastern end of the Silk Roads, only to find that Professor Ma and his colleagues had been working on those same manuscripts for 10 years and had just produced the first, and still the only, comprehensive volume of transcripts of that material in 1998. ${ }^{4}$

As the balance of global power moves south, and central Asia is mined for its rich mineral resources, the imagination of the Silk Roads connecting north-eastern China to the Mediterranean and north Africa has become synonymous with the political intention to foster future commerce and scientific knowledge exchange. Dunhuang with its religious, military and commercial networks is historically and philosophically at the centre of the project to position China once more as Zhongguo 中國 (the Middle Kingdom). An open-minded and welcoming man, despite his modest, unassuming disposition, Professor Ma has always been at the cutting edge of successive waves of academic interest in the historical creation of China's national medicine.

It is no wonder then that from as early as 1985 , he pioneered the use of medical images as an important source in the writing of medical history. The contribution he made to our 2005 conference to celebrate his work, 'Historical Images from the Pharmaceutical Culture of the Emperor Yan', represented an early stage in his project to collate all the iconography relating to Shen Nong 神農 (The Divine Farmer), selfless empiricist and legendary patron of the pharmacological arts. ${ }^{5}$ Professor Ma's work includes all manner of material culture, taking in stone, wood and ivory sculptures, clay and wooden effigies with depictions of the viscera, ceramic and bronze statuary, illustrated poetry and essays, temple iconography, painted scrolls and eponyms of Shen Nong that stretch to media logos for drugs, and trademarks for food and beverage packaging. All of these media are represented by authors in the chapters of this edited volume.

Our conference in Beijing was convened at the end of a Wellcome Trust project for which Wang Shumin, now retired Professor of the History of Medicine at the Academy, and I had bought the digital rights to 1,400

\footnotetext{
$3 \quad$ Ma Jixing 1992.

$4 \quad$ Ma Jixing 1988; Ma Jixing, Wang Shumin et al. 1998. See also Despeux (ed) 2010. Ma Jixing 2012.
} 
medical illustrations from the library at the Academy on behalf of the Wellcome Collection, fully catalogued and described them, translated them with Penelope Barrett, and put them online for Wellcome Images. From there now you can download high-resolution digital copies free of charge to use in academic and educational publications. ${ }^{6}$ In 2007 we published the conference proceedings with the People's Medical Publishing House - a book that, according to Professor Li Jianmin 李建民 at Academia Sinica, Taibei, has become a mainstay of the reading lists of history of medicine courses throughout the Sinophone world. ${ }^{7}$ It is currently being translated into Korean.

The current English language edited volume is based on the earlier proceedings, but much expanded now to 35 chapters and two long introductory pieces. ${ }^{8}$ It has been accomplished with the help of numerous patient individuals with whom we have translated, edited, and reorganised and laid out the chapters, as well as all those who inspired, participated in, funded and ran the conference in the first place. These include Professors Wang Shumin, Liu Changhua 柳長華, Zheng Jinsheng 鄭金生, Francesca Bray, Hal Cook, Shigehisa Kuriyama, Andrew Wear and Geoffrey Lloyd, librarians and researchers John Moffett, Lois Reynolds, Lu Di 蘆笛, David Dear, Emma Whittaker, Josephine Turquet, Akio Morishima and Gu Man 顧漫. I am also very grateful for the help received from the Wellcome Collection and specifically Catherine Draycott and her team at Wellcome Images. Last, and far from least, I am indebted to the Wellcome Trust, who made the project and conference possible, and continue to fund my work on image-making in medicine. We are grateful for the support of the Wellcome Trust for this volume, which has enabled it to be Open Access from the point of publication.

This has been a labour of love for us all. Not only were the original conference papers submitted in five different languages, but there were an equivalent number of academic styles to weave into a coherent volume. The original submissions were also extended from conference drafts into full academic articles and another eight chapters were commissioned especially for this volume. I have also learnt

6 https://wellcomeimages.org (Search on Vivienne Lo).

$7 \quad$ Wang and Lo (eds) 2007.

8 Some of the chapters in this volume have also been published in earlier versions in the Brill journal of which I was founder editor, Asian Medicine: Tradition and Modernity. These include Despeux (Chapter 2): Vol. 1.1, 2005, 10-52; Holroyde-Downing (Chapter 10): Vol. 1.2 (2005), 432-461; Fava (Chapter 25): Vol. 4.2 (2008), 515 -547; Sterckx (Chapter 7): Vol. 4.2, (2008), 357 -394; Wu (Chapter 6): Vol. 4.2 (2008), $45^{2}$-491; Shin (Chapter 23): Vol. 5.1 (2009), 186 - 207; and Baker (Chapter 29): Vol. 7.1 (2012), $225-264$. a lot in the interim period, and have grown to appreciate more fully the value of this collection in ways that I will elaborate in the introduction.

Like Ma Jixing's complete history of acupuncture, our work will never be done. There are constantly new excavations that recover artefacts and manuscripts, which promise to enrich our knowledge and understanding, but also call into question previous findings. In light of the multiple sources that, we argue in these pages, can be analysed as the visual cultures of medicine, there are many lifetimes of research to contemplate. I hope the reader of this volume will appreciate the passion that exists in Chinese studies for the History of Medicine, the extraordinary visual record that survives from the ancient world onwards, and the contemporary significance of all that has been achieved, and all that remains to be done.

\section{Bibliography}

Despeux, C. (ed.) 2010, Médecine, religion et société dans la Chine médiévale: Étude de manuscrits chinois de Dunhuang et de Turfan (3 vols), Paris: Collège de France, Institut des hautes études chinoises.

Ma Jixing 馬繼興 1985, 'Yixinfang zhong de gu yixue wenxian chutan', 醫心方中的古醫學文獻初探 (Preliminary Investigation of the Ancient Medical Records in the Ishimpō), Nihon yishigaku zasshi 31.1, 326-71.

1988, Dunhuang guyiji kaoshi 敦煌古医籍考釋 (Ancient Medical Manuscripts of Dunhuang, examined and explained), Nanchang: Jiangxi kexue jishu.

1992, Mawangdui guyishu kaoshi 馬王堆古醫書考釋, Hunan: Hunan kexue jishu chubanshe.

1993, Zhenjiu tongrenyu tongren xuefa 針炎銅人與銅人穴 法, Beijing: Zhongyiyao chubanshe.

2003, 'Bencao gangmu chuban de kaocha' 本草綱目出版的 考察, in Qian Chaochen and Wen Changlu (eds), 16-27. 2005, Chutu wangyi gu yiji yanjiu 出土亡佚古醫籍研究 (A Study of Archaeologically Recovered Medical Texts), Beijing: Zhongyi guji chubanshe.

2012, Shen Nongyaoxue wenhuayanjiu 神农药学文化研究 (Research into the Divine Farmer's drug culture), Beijing: Renmin weisheng chubanshe.

1990, Zhongyi wenxianxue 中医文献学 (The Study of Chinese Medical Literature), Shanghai: Shanghai kexue jishu chubanshe.

Ma Jixing, Wang Shumin 王淑民 et al. 1998, Dunhuangyiyao wenxian jijiao 敦煌醫藥文獻輯校 (The Dunhuang Medical Texts, edited and collated), Nanchang: Jiangsu guji chubanshe.

Qian Chaochen 钱超尘 and Wen Changlu 文长路 (eds) 2003, Li Shizhen yanjiu jicheng 李时珍研究集成 (Collected Studies on Li Shizhen), Beijing: Zhongyi guji chubanshe.

Wang Shumin 王淑敏 and Vivienne Lo (eds) 2007, Xingxiang Zhongyi 形象中醫 (Imagining Chinese Medicine, Chinese edn), Beijing: Renmin weisheng chubanshe. 



\title{
Introduction
}

\author{
Vivienne Lo 羅維前
}

The true structure and workings of the human body are, we casually assume, everywhere the same, a universal reality. But then we look into history, and our sense of reality wavers... (Kuriyama 1999). ${ }^{1}$

The bricolage of medical images assembled and discussed in this volume will disturb the more prosaic realities we share about our bodies, inciting curiosity, incredulity and wonder in equal measure. Our authors have defined their subject matter widely. They write about ancient tomb figurines which bear complex configurations of the body, medieval cave paintings of healers at work, how-to charts for diagnosing diseases from the colour and shape of the tongue, visual instructions for cautery and bone-setting, diagrams of smallpox-like symptoms, talismans against the demons of illness, beautifully illustrated Materia Medica books, and even comic strips about the Yellow Emperor, preeminent and mythical patron of the Chinese medical arts. Each chapter analyses how writers and illustrators have thought about, sustained, enhanced and cured their own and other people's and animals' bodies - and how they have communicated that knowledge and skill through visual imagery. All the images presented here have something to tell us about healing or strengthening the body, and the people who have been involved in that process.

Medical practitioners 'have always done a lot of looking, as they learn, diagnose, operate, and heal generally; because people respond to each other through seeing their bodies, and hence also to their states of health and illness as these manifest themselves visually'.2 The assembled chapters include - and are much more than - a series of internal analyses of how images facilitated medical practice in China. They also look at how the worlds of medical practitioners interacted, and use medical imagery to reflect more widely on China's socio-political and cultural condition, and its position in the world. As a group of writer-researchers who have taken images as our subject, we have to thank those who, since the 1980s (with a few notable early exceptions), have made the study of visual culture a respectable academic pursuit for medical historians, a pursuit which challenges traditional narratives that bifurcate a modern West and an East stuck in a remote and moribund past. ${ }^{3}$ There have been comprehensive longue



durée studies of Western medical art, ${ }^{4}$ and exhibition and library catalogues that mine medical images for what they tell us about the historical practice of medicine, ${ }^{5}$ as well as studies that choose to analyse visual styles from the Renaissance to the present day since

the concept of anatomy, and the method and practice of human dissection, are ... not only the basis of medical science, but also... emblematic of all Western human thought and activity. ${ }^{6}$

The divergence of Greek and Chinese medicine on the basis of dissection and anatomy, as we shall see, has been very much overstated. For China, we have had many illustrated histories of medicine, and images have been used as sources of information, but it is only lately that we are beginning to see studies that go beyond essentialising China and the West to offer serious reflection upon the epistemological role of images that might counter established discourses.?

An intellectual transition between medieval craft and Renaissance fine art, often focused around the figure of Da Vinci and his imaging of the anatomical human body, has been integral to commonplace narratives of the history of a Western bio-science. However, there is an increasingly nuanced body of work that traces 'a more complex interplay between classical and post-classical ways of knowing about the body' and emphasises continuities with visual cultures of the past rather than the radical rupture imagined to lie at the origins of a scientific revolution in the $15^{\text {th }}$ and 16 th centuries. ${ }^{8}$ With an eye trained to complexity in the currents and flows that lead into innovation, and a sensitivity to the ways in which history is always fashioned to the present, it has become possible to take up the visual culture of Chinese medicine as a corrective to Eurocentric histories of science and medicine and simplistic comparisons between East and West, as a multi-faceted and transcultural phenomena deserving of serious attention. ${ }^{9}$

Kuriyama 1999; Daston and Galison 2007; Cooter and Stein 2007; Clunas 1997.

Herrlinger 1967 [tr. 1970]; Kemp and Wallace 2001.

Anderson et al. 2011.

Kemp and Wallace 2001, p. 158, referring to the work of the artist John Isaacs.

Kuriyama 1999; Bray et al. 2007; Heinrich 2008.

Givens, Reeds and Touwaide 2006, p. xviii.

Levi 1989; Hinrichs and Barnes 2013; Bray et al. 2007; Heinrich 2008 ; Despeux 2005; Berlekamp, Lo and Wang 2015. 
The last two decades have also seen ground-breaking research into the epistemological role of images in medicine in constructing concepts and practices related to disease, as not only the geographic but also the material range of visual sources has expanded to include medical ephemera, and material culture more generally. Many of the objects analysed in this volume would have been judged peripheral in traditional art history and medical history, yet nowadays one hardly has to justify the value of visual media, broadly defined, and the material culture of healing and curing bodies, for our understanding of the processes through which medical knowledge was constructed in the past. The playing field has been levelled, and comics, posters and bone-setting manuals, or medieval Buddhist images of cleaning the teeth, are at least as valuable for a social history of medicine as acupuncture charts or fine art manuscript paintings of herbs and drugs produced at court for the pleasure of the emperors and lofty scholars. ${ }^{10}$

As medical historians, we have long been accustomed to situating our sources within their unique social and cultural contexts, ever mindful of their distribution and changing audiences. Medical texts made up a large percentage of literary production in the early Chinese imperial world; and they were deployed in the formation of a classical tradition, and redeployed at critical moments in the subsequent and ever-emerging traditions of Chinese medicine. The circumstances of their production and consumption in China are key to many of the best recent studies. ${ }^{11}$ It is, however, all too easy to fall back on familiar habits and 'read' images as if they were ocular testimony to support our historical narratives, without analysing them for their independent value, either as members of a discrete visual genre, or as individual images in their own right representative only of their particular local settings.

In the second introductory piece that follows, Wang Shumin 王淑民 and Gabriel Fuentes survey images from the Chinese medical classics and establish how the images function in categories of Chinese medical knowledge and practice, namely materia medica (bencao 本草) ${ }^{12}$ and acu-moxa (zhenjiu 針尒). These are images that are mostly text dependent, which illustrate knowledge previously articulated in writing. Wang and Fuente's careful analysis

$10 \quad$ Gilman 1995, pp. 9-32.

$11 \quad$ Harper 1998; Lo and Cullen 2005; Unschuld 1985; Unschuld et al. 2003, 2011; Kuriyama 1999; Bray et al. 2007.

12 The term bencao 本草 refers to both the body of knowledge regarding materia medica, and to specific Materia Medica or Pharmacopeia texts, a distinction that is not always absolutely clear in the original Chinese, but is drawn out in our interpretation by the use of upper or lower case and italicisation. of the provenance of the images, their content, and the chronologies and lineages of image production sheds light on genres that are both literary and visual. There are also fascinating accounts of cross-fertilisation as images are reinvented for variant purposes, including in lavish early modern printed and manuscript works which are led more by the image than by the text.

It has proved a challenge to tease out the limits of this intertextuality (taking images as a kind of text). As Jordanova has pointed out, 'for some time now, art-historians have been developing ways of studying the mundane in art without neglecting its more transcendental qualities'. ${ }^{13}$ The nature of an image's 'transcendental qualities' can be determined in many ways, especially when it is instrumental in the fields of science and medicine. We see the same images, or similar images, recurring throughout the chapters of this volume at different times and in different places, insouciantly crossing medical, religious and social boundaries. It is tempting to attribute to them universal and constant meanings that transcend their original and local circumstances of production, rather than to focus on the virtue inherent in their translatability, their plasticity of signification. But to what extent do Chinese medical images really demand to be read in light of an authentic or ultimate source, either textual or visual? Do the images achieve an independent afterlife, and if so when and where? And how do old images participate in the creation of new forms of knowledge-making?

The ways in which imaging-making shaped scientific cultures in Europe has been the subject of important new histories of science concerned with ideas and practices of 'empiricism' and 'objectivity' and the powerful communities that promoted, and continue to promote those concepts as the cornerstone of a scientific modernity. ${ }^{14}$ During the 2oth century, the politics of these cultures influenced the modernisers of traditional Chinese medicine, and therefore also the ways in which both practitioners and academics tend to look at historical images. ${ }^{15}$ Daston and Galison's studies of the evolution of 'objectivity', and its enduring claims to being able to establish scientific truth, attributes a critical role to new styles of looking that emerged in the mid-19th century. Communities of scientists, they argue, developed new and special theories about how they could

\footnotetext{
13 Jordanova 199o, p. 90.

14 Notably Daston and Galison 2007.

15 Notably in the ways in which the channels of acupuncture have been mapped on to the pathways of nerves and the 'head zones' or 'dermatomes'. See for example Lu and Needham [1980] 2002, pp. 206-7.
} 
image their objects without prejudice, skill, fantasy or judgement. ${ }^{16}$

This practice (or skill), which aimed to remove the subjectivity of the scientist from the act of recording, was a reaction to the immediately previous, and overlapping, generations of knowledge-makers whose observing and recording of the natural world had been an integral part of the trading and scientific activities associated with the growth of informal empire. Naturalists, from the late 18 th century, had begun to categorise all the new flora and fauna they encountered on their travels into magnificent atlases, an endeavour that was concomitant with mapping and taking colonial possession of unfamiliar territories. ${ }^{17}$

Painstaking attempts were made to capture the essence of the particular object of enquiry, and image the core characteristics of a thing for taxonomic purposes - a process that involved new epistemologies of the eye, and aspirations to specific forms of 'trained judgement'. 18 In doing so, these scientists created a culture that separated themselves from a newly-conceived world of nature and created a transcendental position for humanity, in the subject-object/nature-culture divide that became part and parcel of the export of a European modernity, along with its Christianity....$^{19}$

The subsequent and quasi-religious faith in the contradictory possibility of unfettered vision, in the 'blind sight' of the untrained and empirical eye, has achieved an enduring level of transcendence for the notions of objectivity and modern science. ${ }^{20}$ Just as a focus on the binaries of "art" and "science" as discrete products ignores... the commonalities in the practices that produce them, ${ }^{21}$ so the contradistinction of a Western science and Chinese traditional knowledge is blind to their joined-upness. ${ }^{22}$ The notion of accurate observation is fundamental to the narrative about 'objectivity' and science, but it is also, precisely, what is at stake in many of the papers that make up this volume about Chinese medicine. Scientific facts arise in communities that agree about the nature of the world. Activities now associated with the culture of objectivity can be located in scientific communities that predate the 19th century, as much in ancient and medieval

16 Ibid., pp. 19-27.

17 Daston and Galison 2007, pp. 55-155.

18 Ibid., pp. 309-64.

19 On differences between Chinese and European concepts of nature, see Métailié 2010, pp. 345-67. Cf. Elman 2002, pp. 209-32; Elman 2010, pp. 368-99; Elman 2005, pp. 283-6; Cunningham and Andrews-Minehan 1997, pp. 1-23. Daston and Galison 2007, pp. 17-18. Jones and Galison 1998, p. 2. Italics in the original. Elvin in Needham et al. 2004, p. xxv.



Figure 0.1 The earliest extant Taiji tu 太極圖 (Diagram of the Grand Ultimate) comes from a Persian source. 'On the greatest and the loftiest that nothing is greater than Him. There was nothing existing before Him. He created a thing, to which He gave the name Tai Ji. From the motion of Tai Ji, Yang comes into being and from the rest of Tai Ji, Yin comes into being. They are the origin of beinglessness. Nothing in the world, neither existing nor decayed, can be separated from Yin and Yang. The formation of all things in the world originated from Yin and Yang'. Rashid-al-Din 1313. Collected Works of Rashid-al-Din Fadlallah, vol. 2, p. 105

Chinese medical initiatives as in Europe. Premodern attempts to make faithful records of pathologies associated with smallpox, for example, or the projects to standardise botanical illustrations across the empire, or to depict the vital organs revealed through human dissection, all contain important elements of what were to become markers of a European modernity. These histories underline the $a$ priori elements of the practice of objectivity that Daston and Galison have so vividly described, and therefore the transcendental nature of modern medicine.

The fact that a medical image can cross barriers of place, time and culture, and remain meaningful, provides another locus around which our authors have interrogated the liminal and transcendental qualities of Chinese medical images as they are translated for new audiences. Here the ubiquitous 'long' histories of China have been helpful, facilitated by the background chronology of this volume, which spans the 2,0oo years of imperial history and beyond. Perhaps the most transcendental images, which have survived multiple epistemic transformations, 
are those associated with the binary technologies of Yin and Yang, and with the body lineally constructed that we now know as the acupuncture body (Fig. 1$) \cdot{ }^{23}$

When we understand the degree to which early conceptions of the physiology of the body were linked to the contemporary imperial administration, or consider that Yin (normally associated with the dark, the soft and the female) was also a euphemism for the penis, our modern assumptions become deeply unsettled. Throughout the volume we will see early medical images modelled on a vision of the empire reinterpreted in works on Daoism, and then re-appropriated in more secular medical works. Follow, for example, the channels and vessels of the body themselves; the Vermilion Bird (zhu que 朱雀) as representative of the animal spirits and human officials that live within the organs of the body; or the images of the vital organs. They all cross similar boundaries of state-sponsored dissection, alchemy and religion, and travel along the trade routes to central Asia.

Foucault might have done well to focus his analysis on the ways state power worked through the regulation of the imperial Chinese body from the first empire (221 ВСE) onwards, the homologies of state and physiological function, the moral condition of their mutual excesses and deficiencies, the micro-managing of daily and seasonal regimens, the normalising of sexual behaviour; rather than exoticising, as he did, a Chinese ars erotica, using it as a foil for his construction of the uniquely modern and repressive European scientia sexualis. ${ }^{24}$ But with limited knowledge of Chinese history, it is easy to essentialise China as a distant 'other' to serve local purposes. For sure, there is a glorious techne to enhance sexual pleasure as part of a unique Chinese bodily knowledge, but when that is taken in context, it becomes abundantly clear that the (male) epistemic self involved in the enhancing of that pleasure was garnering power to himself, and to himself as a member of a political and cultural elite. All of this history is abundantly illustrated, as we will see.

Images, whether from fine art or from the wider fields of cultural production, not only reflect medical practices and their social and political realities, but simultaneously constitute them in different ways. Images of the medical body produced in both text and artefact 2,0oo years ago were continuously reinvented by different groups variously representing the controlling interests of the Chinese state,

23 As those images travelled to Persia, see Lo and Wang 2013; Berlekamp et al. 2015, 53-86; as they travelled to Europe, Africa, the Americas and elsewhere, see Barnes (ed.), in Hinrichs and Barnes 2013, pp. 284-38o.

24 Rocha 2011; Foucault 1976, vol. 1, tr. Hurley 1978, pp. 57-8.



Figure 0.2 Illustration of 'Technique for cultivating Original Spirit' (yuan shen 元神), finely executed colour painting from Daoyin tu (Diagrams of Therapeutic Movement [daoyin]), by Kun Lan (Qing period, 1644-1911), ms. dated 1875 (1st year of the Guangxu reign period of the Qing dynasty). Wellcome Library, London: Loo3978o

but equally by those that contested its authority: religious institutions and sects, revolutionaries and 'other' ethnic groups that intersected politically and culturally with those that styled themselves Chinese. The same images of the anatomical and the acupuncture body, and the bureaucrats and spirits that inhabited the body and were part of its control and surveillance, and of self-cultivators practising therapeutic exercises, repeat throughout this volume in different guises and were reproduced at different times to serve radically different purposes: bodies of state, of moral and spiritual cultivation, negotiated across regions and different ethnicities (Fig. 2). These images, as they emerge in medical, religious and cross-cultural contexts (many of them popular in the sense that they are pervasive and do not know barriers of social hierarchy and ethnicity), are 
set within complex interactive landscapes. So the question of who was reinventing the images, and what changing audiences they served, is constantly brought to mind.

My own interest in medical illustration originally took off in 2001 when I attended a conference held at the Collège de France on $t u$ 圖 - charts or maps, printed alongside text, which have served to define the territory of a place, people, subject or skill, often to establish ownership or to guide actions of one kind or another. A group of the presentations devoted to techne were later published together in Bray, Métailié and Dorofeeva-Lichtmann (eds), The Warp and the Weft: Graphics and Text in the Production of Technical Knowledge in China. I am very grateful to Francesca Bray for her ongoing support of my work, and especially for her extensive comments on this volume. Her own introduction to The Warp and the Weft has proved a most valuable resource. It historicises the concept of $t u$, charting the development of the earliest paradigmatic maps and designs that integrated space and time for the purposes of divination, planning an itinerary, ${ }^{25}$ or scheduling events according to ritual priorities. The Warp and the Weft provides analyses of how the codes embedded spatially in the $t u$ might be interpreted performatively, how two-dimensional renderings might translate into action. ${ }^{26}$ While there is increasing manuscript evidence of the ways in which cosmic diagrams and the written word were integrated in the structure of everyday life in the Warring States period (475-221 BCE), the quintessential political model in which they coalesced was the Mingtang 明堂 (Illuminated Hall, or Bright Hall). This was an idealised ritual space, 'political system as architecture', modelled on the transformations of the universe within which the ruler would, in theory, embody the divine principles of good government. ${ }^{27}$ As similar cosmic plans - based on schemes of the world codified according to the Five Agents: Wood, Fire, Earth, Metal and Water - were applied to the administrative world of the Han empire, so the designs became ever more embedded in enduring and pervasive practices, empire wide. Many of the designs first evidenced in Han times such as the Mingtang designs also became integral to the shape of Chinese medical practice, and therefore recur throughout this book.

Many of the authors of the chapters in this volume, like myself, have been practitioners of Chinese medicine and/or the martial arts at one time or another. Those who

25 On the reading of jing 經, in which the spatial arrangements of texts, served as a kind of mapless mapping to guide itineraries, see Dorofeeva-Lichtmann 2007.

26 See also Ames 1990, pp. 229-31.

$27 \quad$ Wu Hung 2007, p. 193. trained and practised in China, particularly at the China Academy of Chinese Medical Sciences, have experienced the unique survival of local medicines in China as part of a state-sponsored project, project; and also, in more recent years, the global commercial success story of Chinese materia medica both for individual entrepreneurs and for multinational companies trading in pharmaceuticals and lifestyle nutrition and medicine. For those of us outside China, Chinese medicine has also been experienced in a multiplicity of different ways, often seen as an 'alternative' to a 'Western' medicine (despite sharing many similarities regarding the nature of empiricism and evidence) and represented in contradistinction to Western medicine as 'holistic' and non-reductionist (with many anachronistic assumptions). ${ }^{28}$ In various ways, all these experiences shape our interests in the visual, as well as our own academic aspirations. At its most simple, the substantial interest in how images facilitate practice reflects a practitioner's sensibility, which is often unmistakably teleological and redolent of the positivism inherent in the re-creation of any tradition. The practitioner's gaze ineluctably gravitates towards analysing how particular techniques got better over time: more convenient, more accessible, more effective. Those who challenge Eurocentric histories of the world inevitably take pleasure in announcing that people in China did modern things better and earlier. But why does it matter, for example, that there is visual evidence that people cleaned their teeth with toothbrushes in medieval Dunhuang, or that people were at least as knowledgeable about human anatomy (see Fig. 3) and proficient in skindeep surgery in ancient China as they were in ancient Greece, that they were the first to image foetal development, ${ }^{29}$ or that Chinese medical texts were printed and distributed widely in late-medieval times creating new public spheres? ${ }^{30}$ For me it is of no consequence who,

28 Scheid 2016, pp. 66-86.

29 For medieval European illustrations of foetal positions see the 15th-century The Sekenesse of Wymmen. British Library, London, Sloane ms. 249, fol. $197 r^{\circ}$. This set of illustrations is first testified as illustrations to a 9th-century edition of the Gynaecia of Muscio, a treatise on gynaecology.

30 'Ancient' refers to the period spanning the rule of the Shang people (traditional dates: 1766-1122 BCE) and the earliest extant written records, through the political unification of a geographic entity identifiable as China (221 BCE), to the Eastern Han period $(-220 \mathrm{CE})$ and the failure of the early bureaucratic administration. The subsequent period of political fragmentation saw the rise of the rule of a northern aristocracy, and the growth of Buddhism and its alternative cultural and economic institutions. These were the centuries before maritime trade took over from the land-routes, before the rise of a cash economy, and the 




Figure 0.3 Yuanmen maijue neizhao tu 元門脈訣內照圖 (Internal Visualisation Charts from the 'Primordial Portal' Secrets of the Pulse), attributed to Hua Tuo 華佗, a renowned physician of the 3 rd century CE, but printed in the Qing dynasty. This chart shows the internal distribution of various viscera and organs from the back view. They include the stomach cavity, the lung, the stomach, the liver, the spleen, the [left] kidney, the Mingmen (right kidney considered as 'Portal of Life'), the small and large intestine, etc. Woodcut incorporated into a manuscript, from Qing tai yiyuan (Qing, Imperial Academy of Medicine). Wellcome Library, London, Loo38696

Chinese or Europeans, did innovative things sooner except in that it upsets the apple cart - in that we can deploy such observations as correctives to the multiple and powerful historical narratives of modernity that place Europe at the centre of the world, narratives that steal categorisations of time, modernities and post-modernities, as part of the ongoing colonising project of English-language history. ${ }^{31}$

The chapters, in combination, inevitably reflect our own complex world of competing and complementary practitioners, both men and women as far as it has proved possible. And naturally we have attempted not to privilege the interests of orthodox scholarly medical practitioners

decisive impact of the invention of printing. This period, from the fall of the Han ruling house to the end of the cosmopolitan era of the Tang in the early 1oth century, is generally thought of distinctive for these reasons and is referred to as 'medieval' in Chinese history. over lay practitioners working with manual therapies, religious and magical healers, or the doctors of minority peoples, within the limitations set by the sources available to us. Many of the chapters represent religious imaginations of the body, and the kinds of hygienic, dietary and meditational regimens that were used in the past to cultivate it. Interestingly, in our own times, when the epidemic of non-communicable diseases demands that the major public health initiatives for the future must concern themselves with diet, exercise and hygiene, we have to take heed of the continued success of quasi-religious practices to promote effective behaviour change, through the inculcation of moderation, vegetarianism and various forms of ritual purity.

So, what then, do we mean by medicine, in the title of this book? Medicine is often presented as distinct from ritual and religious ways of healing the body by groups of practitioners who appeal to state or professional authorities to regulate and police its boundaries. But who decides who has the right to practise medicine or to regulate it, and what is an acceptable medical education that will prepare an individual for membership? The implicit and exclusive association of secular authority with the practice of 'medicine' would, however, frame these questions all together as part of a modernising project, along with discourses of 'professionalism' as a feature of modernity. Legal ethics, learned societies and journals are attributes of the professions in this kind of specialist disciplinary knowledge creation, and therefore form a feature of the last section of the book, which also describes deliberate 2oth-century attempts to re-create the visual cultures of traditional medicine as a part of the intangible heritage of a nation. ${ }^{32}$

For the analysis of ancient worlds, with which we begin, this kind of distinction between religion and 'medicine' is not useful. There were indeed various styles of understanding and healing the body that were more or less secular or religious in character. The medicine enshrined in early medical compilations - compilations that, with state-sponsored printing, were to become canonical 1,0oo years later - appears to have been more secular. But scratch beneath the surface, and there is pervasive evidence of divination, ritual and magic in early medical art, institutions and texts, images and illustrations, all of which blurs any clear-cut boundaries that people have chosen to see between medicine and religion. Doctors used ritual techniques and incantations alongside acupuncture and the medicine of Yin and Yang and the Five Agents. Scholars have tended to see the latter as a form of medicine based on 'natural

32 Friedson $135-57$. 
philosophy', analogous to Greek humoral medicine. But nowadays, those working on the Hippocratic corpus and the Huangdi neijing 黃帝內經 (Yellow Emperor's Inner Canon corpus) alike are revisiting ancient medical history and finding a legacy that has been modified by successive editors over the millennia, with a general tendency towards eliminating ritual and magical aspects of medicine. The attribution of disease to specific spirits and demons was not necessarily in conflict with the philosophic worldviews of early China, or of ancient Greece for that matter. There were concomitant and integrated descriptions of diseases caused by spirit entities together with pathologies of Qi, Yin and Yang, or the humours.

From the beginning of empire in $221 \mathrm{BCE}$, the Chinese state also supported the healing practices of religious people, and indeed, engaged in religious practices for the state's own health and legitimacy. The human body and the body of state were linguistically and practically entangled and intertwined, requiring therapy which was often framed in the same terminology. Strategies aimed at supporting the health of vulnerable populations were integral to the larger aspects of peaceful governance. War, famine and epidemics are all examples of state concerns that affect population health, then as now. Responses to these calamities might have been to open the state granaries, or distribute land, welfare or medicine, 'taking the surplus to supplement the insufficiency', ${ }^{33}$ as when a doctor distributes Qi around the acupuncture body. Not only were these imperial concepts of proper distribution imaged in maps charting the free flow of bodily Qi (acupuncture charts); the maps were also evidence of an emperor's divine right to rule. The well-being and good conduct of the citizens of the state were also, in themselves, indicators of the incumbent ruler's virtue, his position mandated by an all-seeing Heaven. This aggregation of good government, moral and religious virtue, and medical charity is not something just of Asia or of the past. One only has to think of the Rockefeller family missions, Sir Henry Wellcome's legacy or Bill Gates' power over medical governance to find neoliberal narratives of science and progress grounded in a Christian morality underpinning our contemporary assumptions of beneficence.

As the identity of religious organisations took more recognisable shape in medieval times, leaving records of their institutions, knowledge and practices, we can see

33 Huan Kuan 桓寬 81 BCE, Yantie lun 鹽鐵論, juan 14. This text equates a doctor's redistribution of the body's vital essences with the proper distribution of resources in the empire. See also the translations in Gale, 1967, pp. 85-91. Cf. Zhangjiashan Maishu c. 186 вСE, nos 57-8, in Jiangling Zhangjiashan Hanjian zhengli xiaozu 1989 . classical medical ideas transforming as they become an integral part of religious alchemy, of the rituals involved in shamanic journeys and the pursuit of transcendent states. By contrast it is also true to say that, by the 6th century, one can identify concerted attempts by the state to define and produce a more secular medical corpus. ${ }^{34}$ Since the topics that authors in this volume have chosen to pursue are concerned both with state-sponsored medicine and with healing in other, more popular (in the sense of socially pervasive, rather than folk) and institutional religious contexts, the use of the term 'medicine' in this book is de facto inclusive. This inclusivity, for example, mirrors the use of the term $y i$ 醫 (medicine/physician) alongside $w u$ 巫 (variously translated 'spirit medium' or 'shaman'), its use in Buddhist contexts, where 'medicine' stands for the salvation of souls, and in Daoism, whose doctors specialised in exorcism. After all, an intervention can have a 'medical' effect whether or not the intervention itself is considered an orthodox, legitimate medical treatment; one just needs to be able to describe that effect in a medical language. The medical language that we regularly encounter as 'Chinese', which speaks of physiological processes in terms of Yin, Yang, Qi and the Five Agents, began as (and remains) a part of the technologies of everyday life and religious healing, just as much as a specialist scientific discourse. Chapters in this book explore this language as it was used to set out the intended effects of both classical medical practice and ritual healing, but also to describe some of the most basic techniques for understanding and fixing the broken body that generally do not make it into more scholarly medical culture either in China or elsewhere, such as bone-setting or forensic medicine. ${ }^{35}$

The next term in our title that we need to consider is hardly less complex. For the moment, suffice it to say that 'China', on the one hand, connotes the imagination of a state centred historically on the Yellow River valley, which acts as a powerful centrifugal political and cultural force, drawing in and assimilating to itself knowledge and culture from far-flung places, from Tibet, Mongolia, Persia, Greece, Korea and Japan, while simultaneously beaming out its unifying beneficence. At the same time, 'China' denotes a geographic mass, home to an enormous variety

Zhubing yuanhou lun. Is the first systematic aetiology of disease in China compiled in the Sui court under the direction of Chao Yuanfang 巢元方 in 610. While there is certainly evidence of religious and ritual healing, there is an overall secular quality to the editing and presentation of the source material.

35 For an excellent recent study of texts and images associated with trauma medicine and forensic science, its history and evolution, and its place in the broader medical and political culture see $\mathrm{Wu}$ Yi-li 2015. 
of people and a diversity of fragmented political entities and religious communities that constantly integrate and disintegrate within ever-changing and permeable borders. Both perspectives on China are vividly represented in this volume, as the body and its healing technologies are mapped and re-mapped over the millennia.

Finally, what are the imaginations of Chinese medicine of which we speak? The earliest sources the reader will encounter in this volume appropriately date to the centuries around the beginning of the Chinese empire (221 BCE), when the state of Qin conquered the Warring States, unifying a land mass that began to look something like China as we know it - albeit not a very stable political entity beyond its central core. Given the antiquity and the quantity of writing on the healing arts that survives from the early imperial period, it is strange that until relatively recently, systematic visual depiction of the ideas and techniques of medicine did not seem to have a long history in China. Woodblock prints of the early classical works of medicine such as the Song editions of the Huangdineijing 黃帝內經 (the Yellow Emperor's Corpus) are not lavishly illustrated, and illustrated medical texts only proliferated in late Ming times, when printing techniques got better and cheaper.

It is only thanks to the material culture of Chinese tombs excavated over the last few decades that we have recovered some of the wonderful images of the body and its care that are presented at the beginning of this volume. And with the building boom that has accompanied the rapid urbanisation of China, have come chance discoveries of burial sites that continuously offer up ancient manuscripts and artefacts of medical interest - sources that will keep medical historians busy for a long time to come.

A small number of surviving manuscripts from the Western Han tomb (206 BC - 9 CE) at Changsha Mawangdui 馬王堆 (c.168 BCE), in the old Han kingdom of Chu 楚 south of the Yangzi river, ${ }^{36}$ are the best known of these recently rediscovered treasures. They testify to how medical text and graphic elements were used together in early Chinese manuscripts in a medical context nearly 1,00o years before the invention of printing. The importance of these manuscripts for any consideration of technical illustration cannot be underestimated. Here are diagrams of the body with explanatory text, as well as evidence of an emerging visual culture that depicts the body and its care, complemented

36 The Mawangdui burial mound was excavated in the early 1970s. It contains three tombs. Tombs nos 1 and 2 belonged to the Marquis of Dai (軑侯), Li Cang 利蒼 (died 186 BCE), and his wife (tomb no. 1). Tomb no. 3, from which the manuscripts were excavated, was occupied by their son, who died in 168 BCE. at the age of about 3 .



Figure 0.4 The earliest extant diagram of the vulva, Mawangdui tomb 3 , closed 168 вСE. Photographed from Ma Jixing, Zhongguo chutu guyi shu 中國出土古醫書 (Ancient Medical Texts Excavated in China), 2015, vol. 3, p. 486.

by quite separately produced textual accounts that now serve us as a guide to reading the images.

The illustrated scroll from Mawangdui which has been given the title of Daoyin tu 導引圖 (Chart of Guiding and Pulling) by modern editors is well known, and is alluded to in several chapters of this book. It shows a series of 44 figures involved in physical exercise. In the first of my own chapters, I use the chart to discuss early Chinese relationships between the animal and human worlds, and the pedagogical power of animals to translate images into action. ${ }^{37}$ The use of animal imagery to train and condition the body is a device repeated in the literature of the arts of the bedchamber (fangzhong shu 房中術), and in many techniques aimed at increasing Qi in oneself or in patients.

Less well studied than the Daoyin tu, but significant for its representation of gender, power and techniques of the body, is another Mawangdui manuscript bearing an illustration that I claim is the earliest map of the vulva (Fig. 4). ${ }^{38}$ Given its proximity to some of the earliest manuscript testimony to literature of the arts of the bedchamber, I read the image as representational of gendered power relations.

$37 \quad$ Lo 2011, pp. 67-85.

$38 \quad$ Lo and Re'em 2017. 
The pursuit of knowledge and power involved in these arts resonates beyond the bedchamber. The gendered language of complementary opposition framed in terms of Yin (cool, soft, passive, dark, female) and Yang (hot, hard, active, light, male) used in the bedchamber literature makes it clear that practitioners, in addition and perhaps incidentally to making babies, performed the act of cosmogenesis through their sexual union, bringing together the creative potential of Heaven and Earth.

The image in question, a schematic diagram drawn in black ink on silk, is attached to another Mawangdui manuscript containing aphrodisiac remedies, and certainly the combination of text and illustration enhances its performative value. ${ }^{39}$ The diagram provides detailed observation of an idealised anatomy ( $v i z$. it is not a life drawing of an individual body), aimed at increasing knowledge of the female sexual body and its pleasuring. It consists of a line drawing outlining the topography of the vulva, showing the vagina, with annotations marking pubic hair and the chizhu 赤珠 (red pearl', a euphemism for the clitoris), and locating vaginal aromas, (the choushu 臭鼠 the smelly rat). Together with its annotations and the surrounding texts, the diagram encodes technical knowledge about bringing the woman to orgasm in a quintessential map for guiding female sexual response - it is a kind of control panel ultimately aimed at enhancing the male partner's jing 精, his finest essence and semen, with the benefits of sensory acuity that this was thought to bring. Those benefits are described as a keenness of hearing, clarity of the eyes, the sense of bodily strength and the brilliance of the spirits: a condition consistent with long life, transcendence, and potentially immortality (Fig. 4 ). ${ }^{40}$

Rather than cultivating emotional detachment, as has been the prescription for objective and unfettered observation since the mid-19th century, early Chinese scholars trained themselves as knowledge gatherers through training their bodily Qi, a term which came to codify and communicate inner body sensations. This self-cultivation of learned recorders of the world, their epistemic virtue, meant that the self, the perceiver, embodied what was perceived. ${ }^{41}$ This diagram therefore demands further analysis from the point of view of the history of scientific observation as well as from technical and gender perspectives. Taken together with the surrounding text, it testifies to the range of senses, including sight, touch, smell, hearing, and the sensory experience of the inner body, through which information about eliciting sexual response was hypoth-

\footnotetext{
$39 \quad$ Li Ling and McMahon 1992.

40 Tr. and introduced in Harper 1998.

41 Daston and Galison 2007, pp. 39-41.
}



Figure 0.5 Poster of the workshop 'Looms of Life' IAS, UCL, March, 2017. Poster designed by Akio Morishima. Laoguanshan 老官山 lacquer figurine. Photograph courtesy of China Academy of Chinese Medical Sciences

esised and recorded in early China. That few paintings of the human body survive from the ancient Chinese world, and even fewer naked ones, makes this illustration of the vulva even more remarkable.

Nudity has not been thought of as a common feature of early Chinese art. Nevertheless, recent excavations have allowed us to see examples of the naked body in the form of partially clothed tomb figurines depicting servants and entertainers. ${ }^{42}$ Explicit nudity of the body revealing reflections on its inner workings is also a feature of two Western Han dynasty tomb figurines with red lines running the length of their bodies. Both were discovered in modern Sichuan, the first in 1993 at Shuangbaoshan, Mianyang 綿 陽 and the second more recently excavated in 2012-13 from tomb M3 at the Tianhui 天回 Laoguanshan 老官山 tomb site near Chengdu (Fig. 5). Since the Laoguanshan discovery, we now know that the Shuangbaoshan figurine was not unique (and who knows how many more remain to be found?), and together they may collectively represent some more significant reality about changing conceptions of the

42 Chengdu wenwu kaogu yanjiusuo 2014. 
body in China. But equally the two examples are also not identical and their differences are extremely important for understanding innovation in the Han world. The Mianyang figurine is notable for its absence of therapeutic points and any sign of organs, both of which seem to feature in some sense on the Tianhui model.

Both figurines provide three-dimensional visual clues to the origins of a body schema structured lineally. Both are clearly concerned with mapping the topography of the body, its blood vessels and muscular definition. They may serve as a guide to medical action. Most analyses have taken the two figurines as precursors to the classical medical body, and specifically to the Song (96o-1279) bronze acupuncture figures that were used for pedagogical purposes 1,ooo years later. ${ }^{43}$ That is to say, the diachronic processes of standardisation have been taken as the main axes for analysis. For sure, we can count the number of red lacquer lines. We can compare them to contemporary manuscript descriptions of the Yin and Yang lines of the body and their associated illnesses. Are there nine or 10, and how do they relate to the 12 bilateral channels of classical acupuncture? Why does only one of the figurines record organs and - seemingly - acupuncture points, which ones, and why? ${ }^{44}$ But for an analysis that is more revealing about the processes and contexts in and through which the figurines were first conceived, it is essential to resist the teleological pressures of seeing the early evidence as representing deficient and primitive versions of what was to come. Most interestingly, the latest finds date to between the reign periods of the emperors Jingdi (r. 157-141 BCE) and Wudi (r. 141-88 BCE), and a nearby tomb includes the earliest extant drawloom models. ${ }^{45}$ This was exactly the moment at which new practices of weaving on innovative 24-pattern shaft, mechanical drawlooms, and the construction of a new medicine came together in new and creative ways. ${ }^{46}$ The material culture of the tomb has allowed us to revisit the well-known homology between medical and weaving technologies as it relates to visual culture, and structures images evoked in early Chinese medical treatises.

Given that these are tomb figurines, how do they, and the knowledge that they represent, relate to older mortuary beliefs and practice about the body? Much of my own work has been concerned with evaluating perceptive styles used in the study of the body in the ancient world. This has meant reading my materials, both text and illustration, as a map of the sensory worlds within which their authors

\footnotetext{
43 Zhao et al. 2016.

44 Ma Jixing 1996, pp. 55-65.

45 Zhao et al. 2016

46 Ibid.
}

and creators lived. Read in this way, both figurines seem to record ideas about vision, hearing, sight, taste and touch; they variously represent an image of sensory acuity, and an architecture of bodily pain. The placing of the Mianyang figurine next to the corpse surely invites us to imagine it as part of a project to mimic and sustain the qualities of life into the afterworld for the benefit of the deceased.

Using the sensory lens in this way to look at artefacts and illustrations, we can go beyond thinking about the way socio-political realities inscribed themselves on the body, as if the body were a passive repository of knowledge and experience, and look at how the body itself participates in the creation of culture. By this I mean that I agree with Bourdieu that we can study the agency that the body has in its own right, and the ways in which it leaves its mark on material culture and textual records. So when looking at medical images and mapping their functionality, we can also get a deeper impression of how sensory knowledge operates in practice. ${ }^{47}$

Charts like the early Daoyin tu invite analysis from art historical and critical perspectives, for what they can tell us about the social history of the living body and its care - this will be the approach taken by other chapters in this volume, especially those that analyse cave and stone murals, silk paintings, figurines, and portraits and illustrations of people going out the business of healing. These material objects and paintings are not necessarily an integral part of text-based productions unlike the majority of $t u$ and encourage us to look beyond historical sources to how material culture can be analysed as a source for medical history.

Beautiful illustrated manuscripts, such as those discovered in the tombs of these Han aristocrats, were luxury objects. It is clear that many of the texts, artefacts and illustrations were designed for an exclusive market as prestige goods that could be used in everyday life. However, where they were placed meant that they were also for the eyes of the mourners, perhaps the dead, and to impress onlookers in the afterworld.

In contrast, the archaeology of the Silk Roads provides a window onto a medieval world that dealt in roughly-illustrated medical manuscripts, as part of widespread and popular medical traditions of the living. The largest manuscript discovery was in Grotto 17 of the Mogao Caves 莫 高窟 (also known as the Thousand Buddha Caves 千佛洞) near the town of Dunhuang. Now a World Heritage site for their extraordinary Buddhist murals, the caves are situated at the far north-western edge of the modern political map of China, on one of the land routes for those travelling to Bourdieu 1990a, p. 190 
and from Central Asia. Foreign archaeological activity in Chinese Central Asia around the turn of the 19th and 2oth centuries meant that after the monk Wang Yuanlu 王圓 鉒 first discovered the Dunhuang manuscripts, Sir Aurel Stein, the first foreigner to arrive in 1907 , acquired about 7,000 of some 20,000 scrolls. These scrolls are now mostly divided between the British Library (written material) and the British Museum in London (paintings), where conservation work is being carried out at the International Dunhuang Project.

The manuscripts include a Chinese text of the Diamond Sütra, the world's earliest known complete survival of a dated printed text, as well as secular records and treatises, including many of medical interest. ${ }^{48}$ These manuscripts deftly introduce our theme of travelling medicine and the ways in which illustrations assisted in communicating medical ideas across linguistic and geographic boundaries. We know, regarding this collection of manuscripts, that at least one of the texts was a handwritten copy of a text already in print at the capital, and this helps us to understand the distribution networks and cultures of text production that connected centre and periphery. ${ }^{49}$

A far cry from the magnificent art in the cave murals, the Dunhuang charts that depict moxa-cautery seem to be a rough-and-ready medieval version of the practical illustrated medical charts that circulated independently of medical texts: they prove that the more you pare things down to their bare essentials, providing shortcuts from symptoms to treatment, and the more copies you put out, the more easily medical ideas disseminate. One set of illustrations (S.6168 and S.6262) is an invaluable resource for understanding the acupuncture and moxa-cautery body, but it tells us quite a different story from the figurines and manuscripts, which have to be assessed in relation to their funerary contexts. These Dunhuang charts are quintessentially performative, and inclusive, since they required only basic literacy and circulated on their own without accompanying texts apart from the short captions integrated into the images themselves. These images do not illustrate a text; just like their contemporary counterparts in medieval Europe, 'the text explains how to interpret the image..$^{50}$ Where all the details of medical procedures are provided in one set of moxa-cautery manuscripts, avoiding complex, culturally specific theories and specialist language, with quick and easy ways of moving from patient's illness to treatment, it

48 Barrett describes how printing was invented in late-7th century China within a Buddhist context, and flourished under the patronage of Empress Wu Zetian. Barrett 2008a and 2008b.

49 Lo 2005, pp. 232-4. Seo Tatsuhiko 2003.

50 Jones 2006, p. 5 . also becomes easier to translate those procedures from one language to another, negotiating multiple cultural realities.

Dated to a time around, or just after, the advent of woodblock printing, the Dunhuang moxa-cautery manuscript charts help to explain the relative paucity of illustration in the early printed classical works of medicine, since they raise technical questions about transferring illustrations between the different media. The woodblock print of Sun Simiao's (comp. 650-9) Beiji qianjin yaofang 備急千金 要方 (Essential Formulas Worth a Thousand in Gold for Emergencies) leaves a tantalising trace of where charts were originally placed when the text was in manuscript form..$^{51}$ No doubt economies of scale entailed in re-using single Chinese character woodblocks as movable type did not extend easily to reproducing manuscript images. Probably charts were technically difficult to recycle and were thought to be a waste of valuable resources. Images and type required different skills, and illustrations meant more work for the publishers. We are therefore lucky to have these Dunhuang moxa-cautery charts as rare witnesses to the pre-print tradition of medical illustration.

The serendipity of manuscript discovery in the ancient and medieval world gives cause to doubt that the extant pictures and diagrams are unique, and suggests that there were many more than have yet met, or will ever meet, our eyes. In the manuscripts described here, we have already encountered many of the themes that will emerge as critical for analysing medical illustration after the arrival of printing and book production: these include the standardisation of medical maps and ideas, the aesthetics of effective representation, and the gendered body. Some of these ancient images, like the diagram of the vulva and the quick and easy Dunhuang moxa-cautery charts, are schematic maps that intend to translate image directly into effective action. Not long after the Dunhuang manuscripts were sealed in the 'library cave', there was an explosion of printing, with printed ephemera, private almanacs and calendars, and manuals of many kinds, all producing different kinds of charts and diagrams. ${ }^{52}$

As Francesca Bray has observed, as far back as the 12th century, the Song writer, Zheng Qiao 鄭樵 (1104-62) was insisting 'that charts played as essential a part as written records in the techniques or arts of learning'; 53 and technical works were usefully arranged as a sequence of rubrics where, for each item, a graphic illustration was paired with explanatory text. Such Chinese charts were 'functional';

$5^{1} \quad$ Sun Simiao, Beiji qianjin yaofang (comp. 650-9), edn: 1995, Shanghai guji, pp. 508, 513. See also Despeux 1987, pp. 47-8.

$5^{2} \quad$ Chia 2002, p. 145.

53 Bray 2007, p.1 
others were diagrammatic schemes for interpreting and mobilising cosmic forces and had talismanic qualities; yet others were more representational, as we will see. These are not, however, exclusive categories.

The larger part of this collected volume is concerned with images that post-date the advent of the woodblock print and draws primarily on higher quality Ming and Qing book productions. By the end of the Ming, textual descriptions of almost every kind of 'productive' technology would be profusely illustrated, often with a stock of stereotypical images. Contemporary developments in fine art, however, had not favoured realism: as machines became more complex, for example, investment in technical drawing did not keep up with the demands of accurate representation, and the issues of perspective that were entailed. ${ }^{54}$ But there were many reasons apart from guiding technique for illustrating technical texts.

It wasn't until the late-Ming period that illustrated volumes enjoyed a burgeoning market, on account of increasing literacy and in part due to a new and accessible calligraphic style of woodblock print. Interactions between oral, manuscript and print traditions gave rise to a wide range of texts targeted at geomancers and diviners, merchants, doctors and farmers. Upwardly mobile landowners and merchants were motivated to purchase books for social status, as part of the accoutrements of the aspirant scholar family. At that time illustrated books were instructional, recreational, a way of promoting medical lineages; but as much as anything else, the acquisition of beautiful volumes demonstrated wealth and culture. By the Ming dynasty it was de rigeur for anyone aspiring to better things to collect beautiful block printed productions, and those surviving from Song times fulfilled an antiquarian sensibility that prevailed in the Ming. ${ }^{55}$

\section{The Structure of the Volume}

Beyond the illustrations themselves, what draws the collection together is the inclusive approach to the term 'medicine' described above, and an open attitude to what has constituted China, and China's relationship with other centres of power. Together the chapters explore the many ways in which medicines within China have been connected with other places and their cultures. The subsections of the book which model this diversity include Mapping the Body: Space, Time and Gender, Effective Representation, Imagining Medical Practice, Travelling Medicine, Esoteric
Contexts and Knowledge Transmission, and Imaging Modern Medicine. In what follows, I will introduce the chapters of this volume section-by-section, drawing out all the themes covered above - although the ways in which I have established their multiple linkages for the purposes of this introduction means that they will not necessarily emerge in the following analyses exactly according to their sequence in the main text.

Part I Mapping the Body: Space, Time and Gender I have argued that early Chinese medical illustration played a unique role in modelling the sensory aspects of early Chinese bodies, and that this was a collective and learned pursuit at the heart of scientific observation in the ancient Chinese world. In the first section of the book, our authors demonstrate how medical illustration structures space, time and gender, with the intention of rendering these domains amenable to effective therapeutic action. In this sense, medical representations may be more or less effective as route maps through social and body landscapes, establishing medical hierarchies and setting out body parts or functions according to therapeutic intention. How then can medical maps or charts ( $t u$ 圖 ) be read for their agency in establishing power relations and ownership, in determining who controlled whose bodies, in matching medical theory and technology? Or in choosing when and where healing should take place?

What exactly were $t u$ ? The term itself tends to be translated as 'charts', but the ways in which people have thought about and used charts or $t u$ have clearly changed over the millennia. When the (untitled) Mawangdui silk therapeutic exercise chart was given the title Daoyin tu by 2 oth-century scholars, for example, the term represented contemporary usage. The secondary scholarship on early artefacts designated as $t u$ suggests that they were mainly concerned with mapping territory or the stars. All tu style charts are arguably concerned with guiding one's attention, but as the term $t u$ was used in the ancient world, it seems to relate to military and divinatory maps, to land ownership and the manipulation of the Heavens. The ownership of a map itself had symbolic power. As Francesca Bray says, 'giving up your map... was tantamount to giving up your state': how else could one imagine possessing one's dominion? ${ }^{56}$

Maps, as we will see unfolding throughout this volume, have also been deployed as a way of appropriating the body; and in the early empires that was very clearly a form of appropriation to the imperial process. It is not, in my opinion, coincidental that through the 4 oo years of the Han period, the second Chinese empire, a model of a unifying

$56 \quad$ Bray 2007, p. 20. 
physiology of Qi - bringing together the body's internal workings with the cosmos, the river networks, free-flowing communication pathways and the function of the organs as government officials - came to be codified, in language and image, in the same terminology as the workings of the healthy state, and vice versa. ${ }^{57}$

From the time that the classical compilations of the Huangdi neijing (Yellow Emperor's Inner Corpus to 1st century CE) first enshrined an orthodox knowledge about the body, we see state and medicine interacting in the production of medical knowledge. Various government medical bureaus from the Sui period (581-618 CE) onwards sponsored medical writing in a kind of royal religious philanthropy that reached a peak in the first centuries of the Song period (96o-1279 CE): on the initiative of the Buddhist emperors, large-scale editing and printing of medical texts began with 16 authoritative editions of medical classics and many new medical texts. ${ }^{58}$ This project was a form of merit publishing. Reproducing medical texts, like sūtras and talismans, could bring good karma to the sponsor, and in this case to the Song imperial house. Building on the basis of this period of government book sponsorship, processes of technical standardisation, and the standardisation of content began, which paved the way for the illustrated medical volumes that are the source for many of the authors writing of the Ming period in this volume.

Lamenting that few charts survive from pre-Song times, Catherine Despeux remarks that medical illustration served either to enhance the learning process, or to standardise knowledge. Her chapter surveys three domains that produced visual representations of the body in China from the Song to the Qing period (1644-1912 CE): medicine, forensics and Daoism. These domains represent three distinct ways of conceiving, visualising and practising the care of the body. Each set of representations emphasised separate functions and intentions within its respective and unique sphere of knowledge and practice, so that while sharing many fundamental features, each came to be associated with a specific aspect of the body. Each type of image displays particular formulations of the body, concerned with different cognitive functions, and is accompanied by a text written in a specialist language. For Despeux, 'medicine' becomes a less all-embracing category than I have hitherto articulated in this introduction. The term 'medical illustration' is confined to the description of the viscera and the channels and tracts through which Qi flows. In contrast, forensic medicine focuses on the depiction of the skeleton, and Daoism on the presentation of the body

$57 \quad$ Unschuld 1985, pp. 77-83.

58 Hinrichs 2011. as the spatio-temporal articulation of connections with the cosmos and the spirit world mediated by the system of correspondences. Despite the codified language and representations within which each of these genres was realised, there were large areas of overlap and shared understandings, so that we might think of them as different registers and not as completely separate approaches to the body. Throughout this edited volume we will see these fluid boundaries in operation with a constant cross fertilisation between genres.

It is ever clearer, as Despeux's analysis progresses, that what has become commonplace knowledge in Chinese studies - that understanding the inner dimension of the medical body was hampered by a ban on dissection - is wrong; and she dwells at some length on the various ways in which knowledge derived from dissection was incorporated by medical experts into evolving medical models.

Apart from the editorial work involved in the preparation of this volume, which has been substantially shared with Penelope Barrett, the next chapter is the first of three here that I have authored or co-authored. It is intended as a contribution to the history of embodiment in China.59 This history cuts across two of Despeux's three domains, those of anatomy and Daoism, and demonstrates the important contribution to medical knowledge made by early Chinese practices and practitioners of self-cultivation. It is self-evidently less relevant to Despeux's third domain, simply because embodiment relates to practices and perceptions of the lived body and is therefore, not an aspect of forensic medicine in any straightforward sense. Self-cultivation practices are a major theme of a number of the chapters that follow. The dynamic between the terminology of inner body experience and medical technology is therefore foundational to the persistent dialectic between the epistemologies of the physician and those of the self-cultivation practitioners (often one and the same person).

Concepts of embodiment have had a major influence on history of medicine, and the medical humanities, and have played a critical part in the turn away from histories of the body based on its representations, towards exploring the ways in which those representations were constructed. ${ }^{60}$ One of the principle architects of embodiment, Thomas Csordas, dryly noted that 'if there is an essential characteristic of embodiment, it is indeterminacy'.61 That is, perhaps, inevitable since the term is used to capture the way our perceptions mediate all of human experience,

59 First published in Bray et al. 2007, pp. 383-423.

6o Feher et al. 1989, pt 1, 11-17.

$61 \quad$ Csordas 1994, p. 5 . 
the way they shape and transform our subjectivity, and the fact that those perceptions are rooted in the body. ${ }^{62}$ Merleau-Ponty described the body as a symbolic object, not something that ultimately belongs to us as we might assume, but as representative of our relationship to the totality of what surrounds us. ${ }^{63}$ Our identity is, however, not just a mass of symbols or a silent body inscribed with metaphors, but is constructed through an ever-emerging bodily process where the lived and connected body itself participates actively in its own formation. ${ }^{64}$ In 2001 I commented: 'Observations about the body made in yangsheng 養生 'nurturing life' literature reflect a realm of human experience that, for obvious reasons, is not evident in medical literature that describes the illness and cure of other bodies. Where the eyes cease to organise and control their environment, visualisation may free our physical space through the practice of meditations. Breath cultivation may bring increased vitality and a clarity of the spirit, while feelings elicited in sexual arts may landscape the body with mountains, rivers, and spurting seas. In this liminal world, the body may become a universe, a temple, or a continent'.65 What happens then when such visualisations are committed to stand-alone images or illustrations in medical texts?

What I hope to historicise in this chapter is an imagination of the body where bodily senses and passions were transformed into the essences for strengthening and prolonging life. Ancient and medieval self-cultivation imagery can describe a sequence that proceeds from sensory experience through image to interpretation and finally to action. Early images of self-cultivation techniques depict a distinct, lyrical body, imagined, measured and mobilised through, for example, vivid animal homologies like that of the howling gibbon or lumbering bear. Thus images of the animal world, as much as the geography, and politics and administration of empire, described above, became an integral part of an early Chinese anthropocene with lasting consequences for both medicine and self-care. Subsequent chapters take up the same themes of how artists illustrated bodies that were linked to the environment, as much in practices of anatomy as in the arts of alchemy, meditation, therapeutic exercise and the martial arts (Fig. 6).

\footnotetext{
62 Carel 2008, pp. 13, 22.

63 Merleau-Ponty 1945, tr. Smith 1962.

64 See also Yasuo Yuasa 1993 for an, albeit anachronistic, analysis of how embodied practice undoes the subject-object/mind-body dualism.

65 Lo, 2001, p. 22. Schipper 1993, pp. 100-12.
}



Figure o.6 Neijing tu 內景圖 (Chart of the Inner Landscape). 19th century. China Academy of Chinese Medical Sciences

Sabine Wilms and Yi-Li Wu 吳一立 both continue continue our theme of body-mapping with an analysis of the gendered body. Early and medieval Chinese medical authors produced, preserved, and transmitted medical information on 'nurturing the foetus' as an important aspect of the literature on yangsheng 養生 that ensured the continuation of the family lineage. Wilms' chapter demonstrates the origin and development of a textual tradition from the Mawangdui manuscripts in the early 2nd century вСE, to early medieval formularies such as the Beiji qianjinyaofang 千金要方 (Essential Formulas Worth a Thousand in Gold for Emergencies), and Chinese texts reproduced in the 1oth-century Japanese compendium Ishimpo 醫心方 (Formulas of the Heart of Medicine) as well as later Japanese manuscripts. In this process, early descriptions of the month-by-month development of the foetus and corresponding instructions for the mother were preserved intact, yet gradually supplemented by elements that reflected changes in medical theory and practice. 
These include correlations between months, the Five Agents, and the internal and external organs according to the theory of systematic correspondences; detailed descriptions of acupuncture channels and points prohibited during each month of pregnancy; medicinal formulas for the prevention and treatment of disorders of pregnancy; and, lastly, 10 line drawings that depict the monthly changes in the naked body of a pregnant woman and her foetus, as well acupuncture channels and points that were subject to prohibition during each month. Texts on 'nurturing the foetus' thus show the influence of cosmology and Yin-Yang theory, formulary literature, acu-moxa charts and prohibitions, and vessel and visceral theory, but most importantly, a growing attention to the gender-specific medical needs of female bodies in the context of 'formulas for women'.

Yi-Li Wu argues that a visual androcentrism underlies the 484 images of the body published in the Yuzuanyizong jinjian 御纂醫宗金鑑 (Imperially-Commissioned Golden Mirror of Medical Learning) of 1742, and explains some of the factors that shaped that visual focus on the masculine body. In this context, medical imagery simply reiterated the broader cultural tendency to use male figures as normative. So far this is hardly surprising for the period, but there are striking ways in which ideals of spiritual enlightenment and longevity accumulate to the partially robed scholar figure in Chinese culture, and to images of the male doctor decorated with auspicious and Daoist iconography.

With reference to a Daoist iconography of the auspicious, Wu draws our attention to cultures of prediction. From antiquity, determining lucky and unlucky times formed the basis of many types of chart concerned with knowing the future. Calendrical and astrological knowledge was critical, for example, to prognosis. It was vital for a doctor to understand how an illness might develop over days, lunar cycles or seasons, and where it would manifest in the body. Initially indistinguishable from the more general management of everyday life, complex complementary and competing systems of calculating time were codified in tables and charts and frequently had to be reconciled. Much more work is required to achieve a good historical understanding of how these systems were made to work together in medicine, and whether or how they were translated across cultures.

Picturing divisions of time is not only a feature of calendrical charts. We noted above how mimicking animals in therapeutic exercise could be used to convey subtleties of time and motion; and some figures on the Mawangdui chart give us a sense of how these subtleties might be rendered visually. Elisabeth Hsu's chapter carries these considerations of mapping the timing of movement and gesture into the sequences of images of a martial arts manual, the
Yijin jing 易筋經 (The Sinews Transformation Classic), of uncertain date. Drawing on François Jullien's analysis of shi 勢 (posture) in the Propensity of the Things (1995), she describes how the 12 images call on us to extend former understandings of $t u$. In the sequence that we are presented with, we are not so much concerned with how the images might mediate social or geographic power or position, or with using them as a guide to where one's hands and feet go (martial arts are notoriously difficult to learn from books): what Hsu shows is how the image-text relationship helps us learn and appreciate the aesthetics of physical expression - not just the techniques but also the 'mood' of the movement. However we categorise the illustrations then, as charts, maps, or paintings, the underlying question, which we arrive at next, has been: how effective are these text-illustration combinations in translating image into action? This question recurs throughout the book, but is particularly germane to the chapters in the next section.

\section{Part 2 Effective Representation}

Huang Longxiang 黃龍祥 must be the most prolific Chinese author to write on images of the acupuncture body. ${ }^{66} \mathrm{He}$ points out that Song images (whether three-dimensional like the Song Bronze figures, or two-dimensional woodblock prints like the numerous, mostly later, illustrations in acupuncture books) were indeed intended to standardise, ever since the first national system of acu-moxa locations was established in 1026. However, the precise locations of acu-moxa points was, and remains, a notoriously vague science. Regardless of these attempts to fix the acupuncture positions, it was quite common for important acu-moxa points to change location within quite short periods of time, or across regions and schools. The bronze acupoint models are in fact a great source for demonstrating the failure to standardise. But does this matter? And if so, why?

Regardless of imperial intent, state sponsorship of the Song bronzes and of printing may not have directly brought about the levels of standardised knowledge that many scholars have assumed. There are different opinions about when mass printing had a significant impact on increasing readership to a wider public, and all agree that manuscript culture continued to flourish. ${ }^{67}$ There were also 'ephemera - broadsheets, private almanacs and calendars, thin divination texts, instruction manuals of all sorts'.68 As Despeux wisely notes, 'secret', 'personal' and 'standardised' models of medical fact operate to a greater

\footnotetext{
66 See, for example, Huang Longxiang 2003.

67 McDermott 2006, pp 25-31, 43-81. Chia 2002, p. 145.

68 Brokaw, p. 26o.
} 
or lesser extent in any medical culture. One or other of these pathways may dominate the transmission process for any particular kind of medical knowledge, but will rarely, if ever, operate exclusively.

Zheng Jinsheng 鄭金生 tells us that effective representation was at the heart of an influential 11th-century Materia Medica project, the Northern Song Bencao tujing 本草圖 經 (Illustrated Classic of Materia Medica, 1061). Illustrators of the earlier Tang Xinxiu bencao 新修本草 (Newly Edited Pharmacopoeia) had not been required to draw from life, but observational drawing became an important feature of the Bencao tujing, whose illustrators worked from drawings done in situ by local experts in pharmacy. They had samples and detailed images of roots, stems, leaves, flowers and fruits sent from 150 administrative districts. The resulting illustrations became a major source for many subsequent works. But the enterprise of drawing from life for a state sponsored Bencao was never repeated. Authors and publishers reproduced earlier drawings, and many artists' impressions were created without direct reference to the originals. Some luxury texts, however, continued to be drawn from life, including the sumptuous Bencao pinhui jingyao 本草品彙精要 (Classified Treasury of Materia Medica), 1515, which was produced by court artists in meticulous gongbi 工筆 style. Some $50 \%$ of its images were copied or adapted from Bencao tujing, but the rest were especially commissioned for the book. Observational drawing of the essential characteristics of the plants was enhanced, from the 17 th century, by separate depictions of the parts used in the pharmacy, with as many as 1,805 illustrations and 1,500 drawn from life in Wu Qijun's 吳其 濬Zhiwumingshitukao 植物名實圖考 (Illustrated Survey of Plants and Plant Names, 1846).

Roel Sterckx focuses on the depiction of animals in the Chinese pharmacopoeia. By contrast with other authors, he questions to what extent such illustrations served a medical or pharmaceutical purpose. The first part of his chapter discusses the contexts in which animal species were depicted in the ancient world before the emergence of Bencao literature. The second part analyses the use of illustrations in Li Shizhen's 李時珍 Bencao gangmu, first printed posthumously in 1593 (Jinling edition), with illustrations probably provided not by the author himself but by his sons. Sterckx questions whether such illustrations were aimed to reflect zoological, botanical, or pharmaceutical information not already present in the text and argues that, instead, their composition is best understood within the context of Ming visual culture, the print economy, and naturalist collectanea produced at the time. He comments that, 'Li Shizhen personally expressed misgivings about the illustrations he saw' in Bencao publications. Li wrote of a mismatch between text and illustration, of illustrations without explanations and of drugs being described with missing illustrations. It's not clear that Li would have approved of his sons' work at all, even though their illustrations were 'mostly drawn from life'. Very possibly, he did not deem illustrations essential for understanding the text, or for that matter for identifying plant, animal, or substance. What characterises the text-illustration relationship here is that the illustrations added information in a way that was arguably consistent with a naturalist's inquiry in the Confucian epistemological spirit in the tradition of zhengming 正名 'the rectification of names', or gewu 格物 'the investigation of things'. It is concerned with the appropriate identification of materia medica or animals, but not with exploring ways in which images might address issues of identification such as details of animal anatomy. ${ }^{69}$

A tension between fine-art depiction of materia medica and mass production in the form of woodblock print permeates the whole history of the Bencao. After all, since the Northern Song, great masterpieces of painting had been based on observational drawings, in particular of seasonal changes in plants and trees. We know that Wang Jie 王介 (Hangzhou, early 13th century) employed artistic techniques to emphasise a plant's distinguishing features - but elaborate paintings were not easily translated into print media at the time, so the work of artists like Wang could not have had any great impact on the illustrations in printed Bencao, which remained schematic and constrained by space. However, given the multi-modal methods of production in the Bencao tradition, it is difficult to draw more than flexible lines between what might seem to be strictly observational drawing and fine art, or to say any more than Métailié, who suggests that it is possible to conclude that paintings can give a deeper feeling of a living plant'70

A number of chapters are concerned with the effective representation of Chinese medicine and the martial arts as a guide to practice, there being seven or so practitioners among the authors. I myself have been a practitioner of acupuncture for 40 years as well as more recently an academic historian, and much longer a practitioner of the martial arts, so it's a history from which I cannot fully abstract my own historical gaze as a practitioner. But perhaps that is not something we need to do. Since the 'affective' turn in history, the researcher's aesthetic and strategic relationship

\footnotetext{
69 Métailié 2001, pp. 240-2.

$70 \quad$ Métailié 2007, p. 499.
} 
to their subjects requires description and analysis. ${ }^{71}$ The onus upon us is to articulate how practical experience shapes a different approach to text and image. Who we are shapes what we see. So can we use our experience of practice to train the academic gaze on the 'performative' aspects of the text-illustration relationship? I am concerned in my own work with how images enhance the ability of text to effect the embodiment of knowledge; how, for example, they convey fine detail of movement. A focus on the performative is a way of judging how utilitarian an image is, how it might facilitate the acquisition of skill, or the adaptation of knowledge to particular situations and patients.

In one key context that is important to the effective transmission of knowledge and practice to larger numbers of people, images act as a shortcut to effective medical practice: that is, tongue diagnosis. The theme of simplifying, standardising and streamlining medical practice is a feature of the next two chapters.

Liang Rong 梁喍 first presents us with an internalist history of tongue diagnosis, critical to understanding a comparative history of diagnosis in China. The immediate cause of the rise of the new technique, she determines, was a difficulty in distinguishing between certain acute cold or heat disorders based on pulse alone. Accurate pulse-taking demanded complex and long-term training; and the subtleties of identifying the transformations of a Yin disease into a Yang disease, and vice versa, purely on the basis of the pulse required expert judgement. The degree of expertise involved is shown in hagiographical accounts of doctors in Ming period case histories. In modern times pulse diagnosis has become vulnerable to the critique that it is too subjective, with consequent difficulties in verification by a third party. In the Jin-Yuan period (Jurchen and Mongol dynasties, 1115-1368), the tongue became a convenient window for observing the thermodynamic processes of disease. We learn about the evolution of tongue diagnostics into a coherent and increasingly detailed system of classification, and how it was integrated into Chinese medical theories. Liang portrays, in admirable detail, a process of standardisation of the associations between the outside

71 Robinson 2010. At one time, what anthropology offered to history was 'to remain sensitive to non-variable factors and symbolisms'. Loux in Porter and Wear 1987, pp. 82-3. Yet, post-Bourdieu, and the anthropology of discourses and practices as they apply to the body, precisely the opposite seems to be true inasmuch as we are cautioned to be self-reflective about academic practice and the multiple ways in which our experience continuously shapes our objects. See also Farquhar 2002, pp. 3-10 and Halttunen in Bonnell and Hunt 1999, p. 166. and the inside of the body. The assumption here is that the process of standardisation, at the level of fine detail, is a necessary requirement for overall coherence and therefore for the transmissibility of a system, an assumption revisited in various ways throughout this volume.

Holroyde-Downing, on the other hand, pays careful attention to locating specific schools of tongue diagnostics within place, time and tradition as far as this can be known, and locating where the tongue illustrations became standardised. She provides an analysis of how and why it was that tongues became a key to fast and effective practice, tying tongue diagnosis firmly to the febrile diseases that ravaged southern China and the emergence of a theory of warmth-factor diseases during the Ming period. During fever, the tongue's colour and surface changed rapidly and provided a new systematic way of charting the progress of the disease.

It seems that there was something about certain symptoms that marked them out for illustration by authors or publishers. We could equally have chosen breast tumours or haemorrhoids. These body pathologies, however, never rank as diagnostic categories in their own right. The surge of tongue illustrations/diagnostics coincided with the arrival of medicine from Europe, particularly in the 2oth century. Holroyde-Downing hypothesises that standardisation and objectivity, being a requirement of modern 'scientific medicine', was much easier to achieve in illustrations of the tongue than of the pulses. Famous illustrations of the pulses never gained momentum in the clinic, whereas images of tongue diagnosis seem to have been assimilated directly into the emerging medical school curriculum in Europe to tangible effect. ${ }^{72}$

The last chapter in this section, by Hu Xiaofeng 胡曉 峰, focuses our attention on the close integration, and particular effectiveness, of words and graphics, in conveying practical knowledge. Chinese skin-deep surgical and orthopaedic therapies associated with waike 外科, literally 'external medicine', and specifically for shangke 傷科, the treatment of injury and trauma to the body, demanded dexterity and spatial awareness in its practitioners, and only to a lesser extent, training in the theoretical aspects of medicine. The visual medium therefore assumed a different kind of pedagogical importance, not so much in establishing medical cultures, as in communicating the knowledge of exactly where and how to manipulate a patient's body with one's own. Nevertheless, even in this genre of medical literature, there are many illustrations that link the manual therapies to more abstruse alchemical and medical theories about when and where to treat

$72 \quad$ Holroyde-Downing 2017. 
the body with acupuncture, or take the pulse; there are also portraits which serve to establish the authority of particular individuals.

\section{Part 3 Imagining Medical Practice}

The third section, Imaging Medical Practice, turns our gaze towards the conduct of state medical practice and the state's relationship with medical practitioners themselves. In the pictures we see many figures busying themselves about the process of gathering and preparing drugs, or engaged in the practice of medicine. TJ Hinrichs, in 'Imaging the reach of Song state medicine', analyses three paintings that depict the conduct of medicine in different ways: a portrait from the tomb of a merchant, and two scrolls produced at court, one depicting a village scene and the other a rural medical treatmentt; and she considers what they can tell us about the normative function of illustration as it codifies relations between the state and ordinary people. Hinrichs contends that these three very different scenarios share one underlying aim in that they illustrate the social ideals and identities that should bond groups and individuals in medical practice. Through detailed compositional analysis, she highlights how the skill of the artist draws our attention to the vitality and complexity of those interactions. Fine details of eye contact and shop signage referencing the ever-present role of the state subtly work together to create a vision of a happy, well-cared-for people, who have no need for resort to unorthodox medical care. Hinrichs shows how the combined effect serves specifically to undermine the work of shamans who were vilified at that time by central authority.

The reach of the state and its contact with the outside world is also celebrated in Ming dynasty illustrated Materia Medica works. Cao Hui 曹暉 analyses the contexts which stimulated the production of the illustrated pharmacopoeia: an enlightened ruler, a prosperous state, and a perceived need to bring about more efficient delivery of drugs. To tell this story, Cao provides a comprehensive introduction to the dozen or more manuscript editions and fragments of the Bencao pinhui jingyao 本草品彙 精要 (Classified Treasury of Materia Medica), and their current whereabouts around the globe. We also hear of new research on the reproduction of some of the same illustrations as they are co-opted into book form, notably in the form of Shiwu bencao 食物本草 (Materia Dietetica) and Buyi Lei Gong paozhibianlan 補遺雷公炮製便覽 (Lei Gong's Guide to Drug Preparation with Addenda). Key to the production of the new court-sponsored Ming dynasty medical manuscripts and printed books, we hear, was the climate of exploration and the new products brought back by the naval expeditions of Admiral Zheng He 鄭和
(1371-1433), Director of Court Eunuchs. It was the literary productions stimulated by these discoveries, encyclopaedic projects such as the creation of the Yongle dadian 永樂大 典 (the Yongle Encyclopaedia or Yongle Canon) that set the model for the new Materia Medica publications, and their incorporation of materials from the new worlds discovered.

The next chapter moves from imagining the context for the production of the illustrated Materia Medica to an analysis of the illustration of drug collection and preparation in just one of these Ming court-sponsored Materia Medica, Buyi Lei Gong paozhi bianlan, which inherits many of its figures from Bencao pinhuijingyao. Xiao Yongzhi 肖 永芝 introduces the enormous variety of figures depicted in the book, 866 individuals altogether, from the mythical Yellow Emperor himself to those involved in pulverising substances, hammering, grinding, pounding, milling and sifting. It is tempting to imagine that we have here a cross section of medical society and their relationships, from the mythical to those working in the field - until we hear that the court artists probably had no experience of the pharmacy and realise we must be circumspect about the verisimilitude of what we see. We can, however, imagine them imagining practice, and acknowledge that they were much closer to their subjects than we can get today. Equally, we can feel convinced of the verisimilitude of the household arrangements and furniture within which the drugs and drug making are placed, and the equipment used, to which the author pays detailed attention.

Sumiyo Umekawa 梅川純代 and David Dear imagine medical practice via copulating male and female bodies as they engage in yangsheng, a culture of self-cultivation, that we have already mentioned, shares technical vocabulary with orthodox medicine, and indeed was the original context within which much of that vocabulary initially developed. ${ }^{73}$ They hypothesise that the terminology and techniques that we encountered briefly above in the highly codified language of the arts of the bedchamber, the fangzhong shu literature, as first seen in Han dynasty tomb manuscripts, survived to have a direct bearing on the codification of late imperial erotic art. Set in a broad survey of the bedchamber literature and its history, the authors present a convincing argument that late imperial Chinese erotic art represents a re-working of the bedchamber arts adapted to the changing social, technological and commercial atmosphere of Ming and Qing times. Through an analysis of the performative composition of the figures, as situated within their social and domestic settings, they convincingly demonstrate Chinese philosophy in action, in the training and attenuating of human desire. Despite

$73 \quad$ Lo 1998. 
the new appetite for illustrated erotica, the authors argue that the Chinese bedchamber literature continued to represent the cultivation of male epistemic virtues. In this respect, the historical legacy permits a contrast with the more aggressively pornographic and humorous Japanese shunga of the same period.

The next chapter describes how treating critically ill children drove some innovations in the imagination of pathology. Like Liang Rong in the last section, Wan Fang 萬芳 writes another upbeat internalist account of the ways in which the illustration of smallpox developed in printed medical texts that were widely distributed, focusing on the Yuan Yulinzhishi yusui zhaiyao 毓麟芝室玉髓 摘要 (Yulinzhishi Calcedony Digest). She describes the integration of medical theory with the newly-observed categorisation of symptoms in differential diagnosis. At one level, we understand that it is practicality that drives the development of new illustrated systems of diagnosis. With the tongue, it was the ease of detecting and reproducing febrile transformations visually; with childhood smallpox, the pulse and questioning the patient were relegated to a minor role compared to visual inspection, given the difficulty of working with small children with these methods. Greater attention was therefore paid to the patterns of the lesions, their colour, density and distribution pattern, and the presence or absence of vesicular fluid. Crucially these were signs used to evaluate whether there was likely to be an auspicious outcome or not and, no doubt, regardless of that outcome, the doctor's ability to predict accurately increased his prestige.

\section{Part 4 Imagining Travelling Medicine}

Moving away from the luxuries of court painting to the dusty limits of the Chinese empire, we continue in this section with the theme of Travelling Medicine. Dunhuang, lying at the Western edge of the Taklamakan desert, was a gateway to the world, where travellers, monks and traders came and went from China to Persia, India, Tibet and Mongolia. Wang Jinyu 王進玉 collects together images that depict health, hygiene, healthcare and the martial arts in the Dunhuang caves. These images serve as a medieval foil for the later, lavish, illustrations that our authors have correctly identified as subtly indoctrinating the viewer with Sinocentric attitudes towards medicine. The murals in the Dunhuang caves are hardly less lavish, with gold leaf and expensive lapis lazuli pigments brought from afar, but they seduce their audiences with very different stories. They are most likely to have been viewed by the Buddhist faithful, and local sponsors of the murals themselves. They depict the Buddha and Bodhisattvas, their entertainers and devotees, historical scenes and everyday life in the locality.
As such they have very underlying different assumptions and priorities from the court paintings; they record and celebrate Buddhist stories, and the acts of the patrons, the viewing itself likely forming a core part of devotional practice. Wang Jinyu has discovered some 30 murals with scenes of medical interest. These include portraits of scholar physicians, local healers plying their trade, and Buddhist miracle workers, as well as images of sick people, hygienic practices such foot washing and teeth cleaning. Religious iconography of the Buddhist road to enlightenment also uses illustrated allegories of healing the body. The work of the Medicine Buddha, Yaoshi fo 藥師佛 - king of the Buddhist Pure Land - was to cure people of the sickness of ignorance. The practice of reciting sūtras is likened to a sick person finding a doctor; and Buddhist narratives of the Bodhisattva Guanyin guiding people on the path, are rendered in illustrations such as the 'Parable of the medicinal herbs' (Yaocai yupin 藥草喻品). Redolent of ideas and practices from distant lands, the visual culture of the Buddhist caves at Dunhuang enshrines the notion of pilgrimage and travel as a medical quest concerned with healing both body and soul.

We have already described the importance of the manuscripts from Dunhuang above. What we didn't mention there is the strong evidence that a version based on the Chinese model S.6168/S.6222 was rendered in Tibetan Pt.1058, one of four Dunhuang medical manuscripts in Tibetan that are likely the earliest extant Tibetan medical manuscripts in the world. "Travelling light: Sino-Tibetan moxa-cautery from Dunhuang' is Lo and Ronit YoeliTlalim's contribution to this volume. Their comparative analysis of these two manuscripts considers what elements translate and what resists being adopted and adapted cross-culturally. We have to be wary of accounts that describe a 'natural' flow of knowledge and practice from an apparently dominant political or cultural centre to a less developed periphery, and interrogate motives for the uptake of ideas and technologies, being sensitive to transformation and adaptation. As people across the geographic boundaries of China adopt Chinese symbols of knowledge, should we assume assimilation or acculturation? As likely as not, something quite different is true. That is, China's neighbouring people may appropriate exotic symbols of medical power as if displaying a kind of trophy in a manner that serves to increase their own power and prestige rather than their subservience to the other, in a world that has its own centre.

'Chasing the Vermilion Bird', by Lo and Wang Yidan 王一丹, is a study of the cultural translation of Chinese medical concepts and texts to the late medieval Persian manuscript Tansūqnāma (1313). The chapter argues that, 
while there were many intellectual synergies between Beijing and Tabriz, in northern Persia, there were pressing religious realities which ensured that some elements of the original Chinese work were less acceptable than others to the author/compiler, Grand Vizier in the Mongolian controlled Islamic courts, Rashīd al-Din. The Mongol empire (1206-1368) ruled much of Eurasia and precipitated the largest scale movement of peoples, ideas, and practices in the premodern world. Composed of four Mongol khanates at its height - the Yuan in China (1271-1368), the Golden Horde in Russia (1240s-1502), the Chagatai in Central Asia (1225-16o7), and the Ilkhans in Iran (1245-1343) - it fostered diverse cultural, political, religious, and commercial exchanges. Within these exchanges, the production of art and knowledge thrived on a dynamic tension between, on the one hand, the drive to develop art and knowledge that would be meaningful across the whole Empire, and on the other, the enduring cultural identities of the regions within each of the khanates. In this chapter, the authors demonstrate how elements like Chinese sphygmology and concepts of circulation were very attractive to Persian physicians; however, writing about the multitude of spirits of the body that were a core part of Chinese alchemical practices could not be tolerated in the new monotheistic Muslim regime.

Taking the theme of travelling medicine, Chen Ming 陳明 focuses on illustrations of 'other' practitioners and traders from afar who were involved in medicine. Seven pictures in Bencao pinhuijingyao (also copied into Buyi Lei Gong paozhi bianlan) depict human figures deliberately connoted as ethnically distinct from the Chinese of the central plains. Identifying the clothing and stereotypical features of people portrayed as transporting exotic drugs from the Persian and Arabic worlds - aromatics, theriac and icons that associate their trade with Daoist alchemy - Chen tracks multiple medical exchanges between China and central Asia. The 'fanciful images' seem doubly remarkable in these court-produced fine art Materia Medica when we are told that the two major works on food and drugs recording large quantities of substances from beyond China, the Huihui yaofang 回回藥方 (Recipes from the Huihui people) and Yinshan zhengyao 飲膳正 要 (Principles of Correct Diet), do not obviously illustrate foreign folk. This begs the question of the purpose of illustrating 'the other'. Perhaps when the general idea is to domesticate the foreign, to make foreign substances acceptable and marketable to the Chinese majority, it would be counterproductive to exoticise, more effective perhaps to make a product as familiar as possible. Just as Hinrichs identified ways in which state-citizen relations are represented and established through composition in fine art illustration, so Chen shows us in the case of the court Materia Medica that, regardless of the evidence of trade afforded by the presence of these individuals and their drugs, their minority 'otherness' simply serves to emphasise that it is Chinese medicine that occupies the position of centrality.

The authors of 'Chinese horse medicine: Texts and illustrations', Paul Buell, Timothy May and David Ramey, contend that the Ming printed tradition of veterinary works also owes a great deal to the connections established by the Mongol rulers across Asia, and they speculate about earlier Indian influence at the time of the northern rule of the Toba Wei dynasty (386-535). Using visual evidence from illustrated printed horse charts and manuscripts, they seek to demonstrate, from close similarities in the techniques of bleeding, therapeutic branding, and minor surgery, that a Western, perhaps European expertise has been added to local practices of horse physiognomy and herbal medicine, which have a demonstrable early Chinese origin. The authors make it clear that the science of Yin, Yang and the Five Agents that has been grafted on to the horse medicine texts barely disguises a deeper Chinese tradition rooted in magic and empiricism. These printed editions clearly gathered material from many sources, and this is evident from the less than consistent structure of the texts and the text-illustration relationship, the illustrated charts perhaps once having circulated independently much as we have seen with the Dunhuang moxa-cautery charts.

Turning towards East Asia, we find a medical tradition inclined to absorb Chinese alchemical concepts. Shin Dongwon's 신동원 chapter examines the characteristics of the illustrations in Heo Jun's 許浚 Dong'ui'bo'gam 東 醫寶鑑 (Treasured Collections of an Eastern Physician), which are uniquely Korean pictorial representations in the history of Korean medical texts. Those anatomical images differ from earlier East Asian anatomical charts in three important ways. First, they embody the view that Daoist practices for preserving health and vitality (yangsheng) were closer to the essence of life than was medicine. Second, unlike preceding medical texts, which mainly focused on the organs inside the body and the channels on the surface of the body, they emphasise building up systematic outer 'bodily form'. Third, they reflect Heo Jun's regard for the anatomical content of the earlier Inner Canon and the Classic of Difficult Issues rather than the contributions of positivistic anatomy from and after the Song and Yuan Dynasties, and his diagrams of the five zang-organs are devised in accord with such a view. These three points in Treasured Collections of an Eastern Physician, the most influential medical book in Korea since its publication, provides clues to understanding the very conservative 
character of traditional Korean medicine in the $17^{\text {th cen- }}$ tury and thereafter.

Staying in the 17th century, Roberta Bivin's reflection on the role and impact of medical images and image-making in the early reception of acupuncture in Europe speaks of the 'translation' of images, and their adaptation in response to the visual norms and medical politics of the receiving culture. Images, as compared to text, seem more immediately accessible, even if their original meanings are lost in that translation. When re-drawn they become more pliable, and therefore, in their turn, such images shaped early responses to Chinese medical expertise and practices in Europe, just as they had done in late medieval Tabriz. The history of the production and reception of Wilhelm Ten Rhijne's 1683 Mantissa Schematica: De Acupunctura demonstrates as much the difficulties of transmission as it does its successes. Acupuncture encountered 'intransigence' as the images were adapted but the knowledge and skills were never appropriated into Western practice. European authors and practitioners were also selective: even as they ignored the Chinese body-maps, de-emphasising the importance of the Chinese acupuncture body, a curiosity with surgical procedures meant that they often included images of the implements of acupuncture. As in Lo and Wang Yidan, what is being analysed here is partial and unsuccessful transmission, despite acupuncture having its champions in the West.

\section{Part 5 Esoteric Contexts and Knowledge Transmission}

Developing the theme of travelling medicine, Ronit YoeliTlalim takes on the subject of our next section, Esoteric Contexts and Knowledge Transmission, with a reflection on cross-cultural iatromancy through a Tibetan image of medical divination held in the Wellcome Library. Divination was used for many aspects of medicine and often relied on an ability to reconcile different calendrical and numerical schemes in order to locate the source of an illness and its prognosis. The divinatory icon that holds this chapter together is the magic square, as realised in the central element of the Tibetan chart, and in its earliest form by the Hetu 河圖 River Diagram in ancient China. While Tibetans have been slow to acknowledge the Chinese origins of key parts of their culture (and vice versa), citing Indian Buddhist origins for greater religious authority, China was always the land of divination' and a source for Tibetan calendrical calculations. Yoeli-Tlalim traces the magic square as it and other Chinese divinatory diagrams appear right across Eurasia, including an interesting case where Tibetans attribute the origin of certain of their ideas to Chinese texts that may never have existed. Despite its global traction, the myths and stories around the origin of the magic square are more potent than the ways in which it was integrated into medical practice, although we hear from Yoeli-Tlalim about its application in Tibetan urine diagnosis. This may suggest that it garnered more symbolic power through its representation of esoteric knowledge, and was used for the ways in which it referenced the ability to manipulate cosmological forces rather than for practical application among ordinary doctors.

China's earliest stone medical inscription invokes our inclusive attitude towards categories of medicine in an intriguing way. Zhang Ruixian 張瑞賢, Wang Jiakui 王 家葵 and Michael Stanley-Baker's 徐源 new analysis of the carved and inscribed images at the Longmen 龍門 caves uses a combination of philological and art historical methods to demonstrate two points. First, one should be cautious of assuming a direct connection between images and contiguous text, and second, the salvation of souls was interconnected with the practice of pharmacy - both being, as we have already seen with the image of the Medicine Buddha and the bodhisattvas at Dunhuang, features of Buddhist medicine. The Medical Recipes Cave at Longmen contains a carved and inscribed image (for the arousing of faith) as well as a number of herbal formulas. The image is dated to $575^{-6}$, and its inscription contains a phrase alluding to the planting of medicinal herbs. This has led many scholars to presume that image and formulas go together. However in the first part of this chapter, the authors examine styles of carving, calligraphy, and character taboos to argue that the formulas were added nearly a century later, during the Tang (between $65^{\circ}$ and 653). Testimony to the dissemination of these recipes comes in the various scrolls and manuscripts from Dunhuang that share recipes, and therefore presumably a source or sources, with the Longmen recipes. The carving of these recipes was an early form of merit publishing, since it is clear that rubbings could easily be taken from the stone carvings with the intention of disseminating knowledge that could provide medical relief in acts of compassion for the sick. Thus, while image and text are temporally and thematically disassociated in this chapter, both ultimately form an integrated whole, at least in the minds of those that added the carved recipes, and those that viewed them together as expression of Buddhist medical compassion. Once again, as with the symbolic power vested in icons of esoteric knowledge such as the magic square, regardless of their ultimate efficacy in disseminating herbs to the populace at large, the characters and texts of the recipes themselves are in a sense images and should be analysed together with the carved image for how they might be received in combination as an art form. 
Returning to one of the themes set up already by Hsu and Lo, Ian Baker provides a Tibetan Buddhist example of the way texts are enhanced by illustration in traditions of physical movement. Captioned with passages from Pema Lingpa's (1450-1521) treasure text the 'Compendium of Enlightened Spontaneity' (Rdzogs chen kun bzang dgongs 'dus), 23 hațha yoga-related movements illustrated on a late 17th-century mural on the northern wall of the Lukhang temple in Lhasa clearly portray the pro-somatic practices (rtsa rlung 'khrul 'khor) aimed at manifesting the adept's 'Buddha Nature' (de gshegs snying po, Skt: tathāgatagarbha) as enshrined in the text. Baker provides a comprehensive account of the Great Perfection or Dzogchen (rdzogs chen) teachings of Tibet, which add physical exercises to meditations aimed at liberating the self from worldly constraints, pushing the body - and thereby consciousness - beyond its conventional limits. A section of the beautiful polychrome images of figures captured in mid movement is reproduced in this chapter with accompanying text, to draw the reader's attention to their pedagogical value. Together the text-illustration combination is rather effective as a tool in conveying the Dzogchen practice. Subtleties of posture, timing, details of stretch, sequence, gaze and the general mood of the movement are all portrayed simultaneously. The once secret temple in Lhasa, built during the reign of the Fifth Dalai Lama, functioned as a personal meditation chamber. These illustrations were therefore not for public viewing, but for the private instruction of the most privileged of initiates. It is remarkable, then, that of the many figures depicted on the murals, all but those directly involved in the Tibetan Dzogchen lineages or the Tibetan imperial house are of Indian origin; the yogic practitioners, for example, are captioned as the Vedic seers of ancient India. Together with the Indian mahāsiddha (grub thob chen po), progenitors of the tantric lineages, there is an overwhelming visual identification of an Indian source for the origins of Dzogchen, which underlines the prestige that this exotic authority would have brought to the tradition at that time.

Another set of esoteric charts, and this time linked to a living tradition, traces a Daoist (Taoist) shamanic journey through the heavens. Patrice Fava introduces us to a map of the body of Laozi himself and shows us how it is an integral part of the performative aspects of a journey to be taken by the Daoist adept. These charts form part of the liturgical texts of the lineage of a Daoist master of the Orthodox Zhengyi 正一 sect, Master Yi Songyao 易松堯, from the Hunan/Jiangxi border. Such liturgical texts were (and still are) transmitted as a stage in establishing and protecting the lineage and as a part of the rite of ordination. The body of the Daoist initiate is then cultivated with formal instruction strictly sanctioned by ordination ceremonies.

The secrecy with which the illustrated liturgical texts were passed down from master to acolyte in the Daoist tradition contrasts with three illustrated texts in the Daoist Canon analysed by Zhang Qicheng 張其成. These are examples of illustrations already discussed in Despeux's chapter, where she opines that established fields such as classical medicine, forensic science and Daoism were different registers with overlapping concepts and terminologies rather than discrete fields. What Zhang shows is that the visual cultures of Daoism were adapted to existing classical medical techniques for adjusting the five zang-organs and six fu-organs (roughly equivalent to the solid internal organs and the hollow digestive bowels and bladders) so as to create an entirely new practice. Images of spirits of six animals which resided in six of the organs (five zang and one $f u$ organ) were the object of Daoist meditation. Later on, the images were included in standard medical compilations, proving our contention about the cross-fertilisation of classical medical treatises with Daoist imagery. All three texts contain charts including Daoist-inspired imagery that influenced late imperial editions of standard medical compilations.

To round off our section on esoteric knowledge transmission we have an intimate account of participant observation in the story of Emei tiangang zhixue fa 峨眉 山天罡指穴法 or (Mount Emei's Big Dipper Finger-point Method) as published by Zhou Qianchuan 周潜川 (1905-71), and its relationship to daoyin and Qigong. Liao Yuqun 廖 育群 tells the story of his father's acquisition of medical training, and specifically 28 different hand techniques for manipulating Qi, from Zhou Qianchuan, a physician and martial artist from Sichuan province. This chapter picks up on a number of themes recurring throughout the volume, from the use of animal imagery to convey subtleties of movement to the key role that Daoism and Buddhism continued (and continue) to play in medical care into the early 21st century. Zhou was a celebrated practitioner of both Chinese medicine and the martial arts, and we follow his fortunes as he rises to treat high-level members of the CCP, and then his fall, and untimely death in captivity, during the Cultural Revolution. In his life and that of his students, who recorded and shared his lectures among themselves, and have published his works posthumously, we have an invaluable resource for analysing the convoluted route through which secret arts have become a part of the public domain, taught and transmitted through schools and universities - and what has been gained and lost in the process. The latter point brings us to the last section of the book that we have called Imagining Modern Medicine. 


\section{Part 6 Imagining Modern Medicine}

This final collection of chapters takes us to the end of the 19th and into the 20 th and 21st centuries. It begins with a charming account of an adventure by one of our treasured, and much missed, colleagues at the Wellcome Trust Centre for the History of Medicine, Professor Ma Kanwen 馬堪溫. Professor Ma provides us with a very personal account of, and photographs from, his field trip in 1954 into rural Shandong, where he went in search of religious sites associated with medical grandees, both legendary and historical. His evocation of the mountainous landscapes of that journey, the sounds and smells, the qualities of light and chance encounters with local people who recalled the medico-religious festivities and pilgrimages, is grounded in an extensive historical knowledge of his subjects. We have extracted his ethnography of sites in modern Shandong associated with Bian Que 扁鵲 or Qin Yueren 秦越人 (6th-5th century BCE), the legendary physician associated with the auspicious magpies which are his namesake, from a much longer draft and collated it with the previously published account in Xinxiang zhongyi 形象中醫. That draft deserves detailed analysis in the future, not to speak of a new project, to retrace his steps and document whether any of the material culture which he described so affectionately survived the political events of the last 60 years. This chapter also provided me with an opportunity to sketch out, in an introduction to the ethnography, the background of important social and political events that shaped modern Chinese medicine, through a chronology of Professor Ma's life and times, and to consider briefly how they relate to the politics of heritage in today's PRC.

Li Shang-Jen's 李尚仁 chapter, 'Visualisation in parasitological research: Patrick Manson and his Chinese assistants', is, however, the only chapter in our collection to approach the new styles of seeing that have been deemed characteristic of the rise of a modern science in the 19th century. This new science, and its romantic infatuation with the practice of 'objectivity' demanded a new culture of knowledge-gathering, and critically, a change in the conduct and culture of the scientist. Both these subjects are addressed by $\mathrm{Li}$ in this chapter.

Li describes what was involved as Manson, working in Amoy (Xiamen 厦門), south China, masterminded ground-breaking projects on the role of insects in the transmission of parasitic diseases. 'Seeing' parasites through a microscope required experience, experiment, specialised equipment and the careful coordination of hand and eye. It also required the production of the kind of images that were playing a central role in the identity of the new science worldwide: these images gave form and direction to the 'gaze' on the microscopic life of the body. The process involved styles of 'trained judgement' as exemplified in the supervision and discipline that underpinned Manson's trust in the accuracy of his assistants' observations. Li notes that visual corroboration by patients was also a factor in the co-production of evidence. The description of the shaping of this new scientific community, the four-, six-, or even eight-eyed practice (Manson, the microscope, the assistants, and the patients), of its collective empiricism, makes fascinating reading, particularly when we hear that these assistants were not as 'invisible' in Manson's accounts of his work as we might have imagined, given that he was one of the chief medical advisors to the Colonial Office.

From visual cultures that are constitutive of the new medicine, we move to two chapters on the use of images to market foreign medical products in early 2oth-century East Asia. Soyoung Suh 서소영 and Chang Che-chia 張哲 嘉 analyse the production of medical advertisements for drugs in Korea, Edo Japan, and China. Chang Che-chia tells us about 'The Visual Language of Medicine Advertisements in The Ladies' Journal', a Shanghai Republican publication, where from $1915^{-31}$, we find copious evidence of a unregulated trade in foreign patent medicines. That these remedies were enduringly popular is evidenced by the advertisements themselves, but also clear from the eager letters to the journal's doctor requesting further information on the products - a rare resource that Chang finds for charting consumer reception. Chang shows us sophisticated localisation techniques in China and Edo Japan, such as Bayer's deployment of Daoist symbols and stories of clever strategy from the Romance of the Three Kingdoms in the marketing of aspirin. ${ }^{74}$ This use of cultural symbols to enhance the authority of a medicine parallels the use of well-known icons of auspiciousness that we saw decorating figures of the scholar-physician in Yi-Li Wu's chapter. Traditional forms of endorsement such as testimonials from grateful patients, which were formerly seen only by clients entering the doctor's consulting room, now penetrated freely into private homes.

We might think of this use of local cultural symbols as a way of naturalising imported drugs, making them familiar, and therefore acceptable, to new customers. But in the Korean context, Soyoung Suh finds commercial drugs being consciously nationalised in complex negotiations with Korea's changing political status: under Japanese colonial authority and a nominal Chinese rule in Manchuria, Koreans had to forge new identities. The heightened atmosphere of competing cultural, and national

74

See Unschuld 2000, pp. 52-61 for how similar advertising strategies were sometimes used by yaotang to encourage custom in analogous ways. 
interests is an important feature of advertising in a colonial context, and impacts on marketing strategies. There was an urgent need to take cultural control of a situation in which Japan was dominating the drug networks, markets and professionalisation of medicine in Korea, while Koreans were living and trading among Chinese communities, and pining for home. This inevitably required a special linguistic and cultural competence that could serve the liminal politics of Korean "in-betweenness"'. Koreans used a new multi-linguistic vocabulary and visual register to define their illnesses and shape their treatments. And as a part of the natural geography of nationalism, they selected from the medical traditions and indigenous herbs from the complex worlds which they inhabited: from Mongolia, to Japan, China and Korea. ${ }^{75}$

The early 2oth-century advertisements are redolent with images of health which the drugs draw on for their promises of enhanced youth, vitality, longevity and exuberant progeny. As both the authors so elegantly describe, they play on the articulations between cultural and national identity, and new patterns of medical consumption. The popularity among magazine editors of images depicting health, and evidence of changed ideas among the general public, also speaks of a new aesthetic of the body, concerned with underlying theories of eugenics and preoccupied with notions of hygiene - inherited directly from Europe but also via Japan.

The next chapter, Zhou Xun's 周遜 'Beauty and health: medical imagery from 2oth-century China', collects together images that represent new ideals of Republican beauty and health as they were made to collude in visions of a Chinese hygienic modernity. This is in part a feature of the same changing consumer patterns we met in the chapters on advertising. These images are aimed at a new urban elite, who could distance themselves from the perils of racial degeneration that haunted the reformers of the previous generation, their fear of squalor, thin under-nourished bodies and lack of stamina. This was the class who would become the guardians of the future, displacing the weak and discouraging the birth of inferior human beings. They were the privileged who could demonstrably afford to use soap. The women exercised and showed off their welldressed, plump babies, had them weighed and measured to prove that they were the healthiest specimens. In this sense, Zhou's images of beauty and health reflect the new priorities of public health medicine in Republican times.

75 See also Shapiro 1998 for another example of how national image comes to the fore in the conceptualisation of health problems in Republican times.
Sticking to cultures of everyday healthcare, but this time those that derive authority from the past, anthropologists Judy Farquhar and Lai Lili 賴立里 ask how useful are the cartoon books that depict information taken from classical medical texts, which are displayed, not with the other cartoons and graphic novels, but in those sections of bookshops concerned with everyday healthcare. Are the images just too abstract to be of use, or is the abstraction itself useful to convey what are essentially abstract conceptions? The answer comes from her Beijing informants: everyone assembles their understandings of health, medicine, and everyday life from experiences that exceed all forms of representation, from family, friends, personal experience, as much as from the authority of books and images. The cartoons provide a kind of scholarly shortcut to a distant past made relevant through familiar updated images; they are an amusement. Their readers instinctively understand the failings of representation, but in the end, everything is only 'grist for the experintial mill'.

I'll leave the final word on the chapters of this book to the legendary Chinese sage Lao $\mathrm{Zi}$, as channelled by Farquhar and Lai, twisting to our purposes the famous first lines of Daode jing 道德經 (The Way and its Virtue) 道可道非常道,名可名非常名 on the ephemeral nature of words and their meanings: 'The picture that can be drawn is not the lasting picture'.

\section{Conclusion}

Categories that can be populated with chapters are also not lasting categories. Just as iconographic codes transcend the boundaries of genre, so there are also many important questions which overlap the boundaries of the sections that structure this book. My personal focus on the history of embodiment is a case in point, where throughout we see political and administrative change, gender dynamics, and religious realities impacting on images of the body and its medical and healing technologies. Other, less linear, juxtapositions of these chapters draw out controversial contradictions which require further research than is possible in this volume. Chapters pertinent to the history of performative bodies, such as those by Hsu, Fava and Sterckx, for example, appear in different sections, and visual shortcuts to abstract knowledge are shared in Farquhar, Despeux, and Umekawa and Dear. The iconography of animals is everywhere used in performative and pedagogical treatises concerned with healing and augmenting the body.

Other instances of themes that transcend our categories include, but are not limited to, the critical issue of 
effective representation, where accuracy is essential to the communication of effective practice. Contextual discussions of the 'accuracy' of specific images or artefacts, and/or of how closely the information purveyed by images corresponded to that of the text have come up throughout the volume. Certain conditions have been identified, for example, which facilitated the production of images effective for therapeutic action: the treatment of children and animals suffering from acute illness, and the urgency to take action in a context where there was no hope of drawing out accurate information directly from the sufferer, being two such examples.

Closely related to the issue of accurate representation is that of medical standardisation or the lack thereof. Processes of standardisation always require establishing authority on the subject in question, and the ability and power to ensure conformity. Certainly, state initiatives to standardise faithful representations of medical substances, or of the locations of acupuncture points, were driven by the same imperial desire to control knowledge and practice in the empire as was the standardisation of coinage and weights and measures. One cannot doubt the philanthropy of the Song emperors and their drive to ensure better and universal medical provision, but the same projects involved the control of medical plurality, and the disciplining of practitioners, and were also about weeding out drugs and healers perceived as anomalous.

One common assumption about the standardisation of medical imagery is that establishing fixed, authoritative models facilitates accurate knowledge transmission. Underlying this assumption is the idea that there is a fixed and transcendent science, equivalent to the ultimate God-truth, and that human authorities, institutions and practices are capable of delivering it. But in more culturally relative contexts, a failure to achieve standardisation has facilitated knowledge transmission. That images can travel independently of their textual embedding suggests that too much local cultural baggage can weigh them down, limiting flexibility and ease of reception across cultural spheres. We saw diagrams of the organs and acu-moxa charts travelling outside China in the 14th and 17th centuries, over land and maritime routes, losing the coherence that they might have had for a Chinese audience, but gaining new meanings for new audiences in Persia and Europe respectively. In both cases, adaptation of the Chinese image, or set of images, was essential to ease of reception and acceptability.

With this flexible model of knowledge transmission, the simpler the image, the more effective it is. Thus the early cautery charts, with their peremptory and perfunctory line drawings of the human body and terse to-the-point annotations, calling for immediate and efficient action, enjoyed widespread traction around the medieval world. This suggests that they were successful pedagogical tools. No one among our authors has researched the universal traction of the Taiji tu, with its equally essentialised diagrams. While its schematic renderings of the relationship between infinity and the dualistic philosophy of Yin and Yang are less obviously didactic, those esoteric concerns allow greater freedom of interpretation. So now we find the Taiji tu as the preeminent logo of Chinese health and beauty institutions and products around the world, in philosophic and scientific treatises, and in tattoo and meditation parlours alike, simultaneously connoting holistic, alternative lifestyles, and forms of spirituality that transcend religious orthodoxy.

This holistic image of the body and its physiology, based on universal principles of Yin and Yang, and the cycles of the Five Agents - Wood, Fire, Earth, Metal and Water - has resonated with the new wave of interest in the anthropocene. For those concerned about recent human impacts on the environment and the future of the planet, logos such as the Taiji tu signal a desire for new non-human centred initiatives. ${ }^{76}$ Located as one aspect of a new consciousness of the perils of industrial progress and globalisation, a philosophy of Asian medical systems is easily deployed as a corrective to reductionist thought and a 'Western' biomedicine that does not consider 'the whole person', let alone the health of the planet. Apparently, when the human body is configured as just one centre of a holistic or 'organismic' universe, where all phenomena are interconnected, and each and every level of existence is an exact homologue of every other, the whole cosmos looks like one integrated being. It is this image that can now be imagined as a global 'ecological body', by historians and dance therapists alike, newly empowered by the concerns of environmentalism. ${ }^{77}$

While the organismic body of Chinese medicine, articulated so well by the late, great Joseph Needham, is fertile territory for this research, such historical interests are fashioned by our own circumstances, and speak as truly about the present as they do about the past. The early chapters of this volume make it abundantly clear that an historical analysis of Chinese holism requires greater sensitivity to the imperial and other local processes that structured the classical medical traditions and their iconography. There is then plenty to be found in these pages about the local circumstances of innovation that tailored those traditions over the last two millennia in China, and the imagery that was produced throughout that process.

\footnotetext{
$76 \quad$ Moore 2015, p. 27; Clarke 20oo; Latour 2014.

$77 \quad$ Frizell 2015; Horden and Hsu 2013.
} 
With change and the causes of change in mind, one key theme which emerges from between the lines of the individual chapters is how successive political changes in the PRC have affected the historiography of image production in Chinese medicine, its scope, funding and boundaries, whether as a national or international field of research, or as a field newly illuminated both by archaeological findings and by the revival of religions and schools of self-cultivation. Some of the grand historiographical projects in which authors like Cao Hui and Xiao Yongzhi are engaged involve transnational compilation of editions and lavish resources for research and publication; they mirror the scale and ambition of imperial projects discussed in their and other chapters, projects that produced the great medical encyclopaedias or state-commissioned Materia Medica. As massive state investment is being mobilised through the Yidai yilu 一帶一路 (One Belt, One Road) concept of South-South knowledge exchange and trade networks through central Asia, it will be fascinating to see how images of Chinese medicine and the body, as a critical element of China's intangible cultural heritage, establish the new style of sinocentrism. With images their power, trade and travel from ancient China through medieval central Asia - guiding key parts of our analysis, this volume surely promises to remain relevant for a long time to come.... .

This volume is a preliminary offering made in the hope that others will pick up the challenge to use visual culture as a way of understanding China's medical past, and how China's past might contribute to contemporary knowledge. The authors are largely historians or anthropologists. Some of us have a longstanding interest in working with visual material; for some it was a new, and fascinating, departure. Art historians, artists and visual culture researchers will have a great deal more to say about the circumstances of image production, and the relationship of artists and illustrators, painters, sculptors, and woodblock carvers to writers, editors and the sponsors of their work described so well in these pages, and they will be able to offer finer and more critical visual analyses of the material that we present. We look forward with interest and excitement to seeing what new insights those interdisciplinary conversations will bring.

\section{Bibliography}

\section{Primary Sources}

Beiji qianjin yaofang (comp. 650-9), Sun Simiao. Modern edn: 1995, Shanghai guji.

Ishimp $\bar{o}$ 醫心方 (Formulas of the Heart of Medicine), compiled by Tamba no Yasuyori 丹波康頼 (984).

Tansūqnāma-i İlkhān dar funūn-i 'ulüm-i Khatā'̄ (The Treasure Book of the Ilkhan on Chinese Science and Technique), Rashīd al-Dīn Fażl Allāh AH 713 (1313), Süleymaniye.

Library, Aya Sofya. Image references to Collected Works of Rashid-alDin Fadlallah, vol. 2, published by the University of Tehran in 1972. Yantie lun 鹽鐵論 (Treatise on Salt and Iron), Huan Kuan 桓寬 (81 BCE). Tr. in E.M. Gale 1967, Discourses on Salt and Iron, Taibei, Ch'eng-Wen.

Zhubing yuanhou lun 諸病源候論 (Treatise on the Origin and Symptoms of the Various Diseases), Chao Yuanfang 巢元方 (610).

\section{Secondary Sources}

Akasoy, A., C. Burnett and R. Yoeli-Tlalim (eds) 2013, Rashìd al-Dīn: Agent and Mediator of Cultural Exchanges in Ilkhanid Iran, London: Warburg Institute.

Ames, R.T. 1990, 'Meaning as imaging: prolegomena to a Confucian epistemology', in Deutsch (ed.), 227-44.

Anderson, J., E. Barnes and E. Shackleton, 2011, The Art of Medicine: Over 2, ooo Years of Images and Imagination, Chicago: University of Chicago Press and Lewes: Ilex.

Barnes, L. (ed.), 'A World of Chinese Medicine and Healing', Pts 1 and 2', in Hinrichs and Barnes 2013, 284-380.

Barrett, T.H. 2008a, Rise and Spread of Printing: A New Account of Religious Factors, London: Minnow.

2008b, The Woman Who Discovered Printing, New Haven: Yale University Press.

Berlekamp, P., V. Lo and Wang Yidan 王一丹 2015, 'Administering art, history, and science in the Mongol empire', in Landau (ed.), 53-85.

Bonnell, V.E. and L.A. Hunt (eds) 1999, Beyond the Cultural Turn: New Directions in the Study of Society and Culture, Berkeley: University of California Press.

Bourdieu, P. 199oa, 'Lecture on the lecture', in Bourdieu 199ob, 177-98. 199ob, In Other Words: Essays Towards a Reflexive Sociology, tr. M. Adamson, Cambridge: Polity Press.

Bray, F. 2007, 'Introduction: the powers of $t u$, in Bray et al. (eds), 1-78.

Bray, F., G. Métailié and V. Dorofeeva-Lichtmann (eds) 2007, The Warp and the Weft: Graphics and Text in the Production of Technical Knowledge in China, Leiden: Brill.

Brokaw, C.J. 2007, 'Book history in premodern China: the state of the discipline', Book History 10, 253-9o.

Carel, H. 2008, Illness: The Cry of the Flesh, Stocksfield: Acumen.

Chengdu wenwu kaogu yanjiusuo, and Jingzhou wenwu baohu zhongxin 2014, 'Chengdu shi Tianhui zhen, Laoguanshan Hanmu' 成都市天 回鎮老官山漢墓, Kaogu 4, 59-70.

Chia, L. 2002, Printing for Profit: The Commercial Publishers ofJianyang, Fujian (11th-17th Centuries), Cambridge, MA.: Harvard University Asia Center.

- (ed.) 2011, Knowledge and Text Production in an Age of Print: China, 900-1400, Leiden: Brill.

Clarke, J.J. 2000, The Tao of the West: Western Transformations of Taoist Thought, London: Routledge.

Clunas, C. 1998. Pictures and Visuality in Early Modern China, London: Reaktion Books; Princeton: Princeton University Press.

Cooter, R. and C. Stein 2007, 'Coming into focus: posters, power, and 
visual culture in the history of medicine', Medizinhistorisches Journal 42.2, 180-209.

Csordas, T. 1994, Embodiment and Experience, Cambridge: CuP.

Cunningham, A.R. and B.J. Andrews-Minehan (eds) 1997, Western Medicine as Contested Knowledge, Manchester: Manchester University Press.

Daston, L. and P. Galison 2007, Objectivity, New York: Zone.

Despeux, C. 1987, Prescriptions d'acupuncture valant mille onces d'or, Paris: Guy Trédaniel.

Deutsch, E. (ed.) 199o, Culture and Modernity, Honolulu: University of Hawai'i Press.

Dorofeeva-Lichtmann, F. 2007, 'Mapless mapping', in Bray et al. (eds), 191-214.

Elman, B.A. 2002, 'Jesuit scientia and natural studies in late imperial China, 1600-180o', Journal of Early Modern History 6.3, 209-32 2005, On Their Own Terms: Science in China, 1550-1900, Cambridge, MA: Harvard University Press.

2010, 'The investigation of things (gewu 格物), natural studies (gezhixue 格致學), and evidential studies (kaozhengxue 考證學) in late imperial China, 160o-180o', in Vogel and Dux (eds), 368-99.

Elvin M. 2004, 'Vale atque ave', in Needham et al., vol. 7, pt 2, xxiv-xliii.

Farquhar, J. 2002, Appetites: Food and Sex in Postsocialist China, Durham, NC: Duke University Press.

Farquhar, J. and Zhang Qicheng 張其成 2012, Ten Thousand Things: Nurturing Life in Contemporary Beijing, Cambridge, MA: MIT Press.

Feher, M., R. Naddaf and N. Tazi (eds) 1989, Fragments for a History of the Human Body, New York: Zone.

Foucault, M. 1976, Histoire de la sexualité, 1: La Volonté de savoir, Paris: Gallimard. Tr. R. Hurley 1978, The History of Sexuality, 1: An Introduction, London: Penguin.

Friedson, E. 1970, Profession of Medicine: A Study of the Sociology of Applied Knowledge, New York: Dodd Mead and Company.

Frizell, C. 2014, 'Discovering the language of the ecological body', Self \& Society, An International Journal for Humanistic Psychology $41.4,15^{-21 .}$

Furth, C. 1994, 'Rethinking van Gulik: sexuality and reproduction in traditional Chinese medicine', in Gilmartin, Hershatter, Rofel and White (eds), 125-46.

Gale, E.M. 1967, 'Discourses on salt and iron: a debate on state control of commerce and industry in ancient China', Taibei, Ch'eng-Wen.

Gilman, S. 1995, Health and Illness: Images of Difference, London: Reaktion.

Gilmartin, C.K., G. Hershatter, L. Rofel and T. White (eds) 1994, Engendering China: Women, Culture, and the State, Cambridge, MA: Harvard University Press.

Givens, J.A., K. Reeds and A. Touwaide (eds) 2006, Visualizing Medieval Medicine and Natural History, 1200-1500, Aldershot: Ashgate.

Golas, P. 2007, “'Like obtaining a great treasure”: the illustrations in Song Yingxing's The Exploitation of the Works of Nature', in Bray et al. (eds), 569-614.

Goody, J. 2006a, 'Feudalism: a transition to capitalism or the collapse of Europe and the domination of Asia', in Goody, 67-98.

Goody, J. 20o6b, The Theft of History, Cambridge: cuP.

Halttunen, K. 1999, 'Cultural history and the challenge of narrativity', in Bonnell and Hunt (eds), 165-81.

Hanson, M. and J. Farquhar (eds) 1998, Empires of Hygiene, Durham, NC: Duke University Press.

Harper, D. 1998, Early Chinese Medical Literature: The Mawangdui Medical Manuscripts, London/New York: Kegan Paul International.

Heinrich, A.L. 2008, The Afterlife of Images: Translating the Pathological Body Between China and the West, Chapel Hill, Duke University Press.

2012, 'The dissection controversy', in Barnes and Hinrichs (eds), 220-7.

Herrlinger, R. 1967, Geschichte der medizinischen Abbildung, 1: Von der Antike bis um 160o. Tr. G. Fulton-Smith 1970, History of Medical Illustration, 1: From Antiquity to A.D. 16oo, London: Pitman Medical.

Hinrichs, TJ 1998, 'New geographies of Chinese medicine', Osiris 13, $287-325$.

2011, 'Governance through medical texts and the role of print', in Chia (ed.), 217-38.

Hinrichs, TJ and L. Barnes, (eds) 2013, Chinese Medicine and Healing: An Illustrated History, Cambridge ma: Belknap Press.

Holroyde-Downing, N. 2017, 'Tongues on fire', PHD diss., University College London.

Horden, P. and E. Hsu (eds) 2013, The Body in Balance:Humoral Medicines in Practice, New York/Oxford: Berghahn.

Howes D. (ed.) 2004, Empire of the Senses: The Sensual Culture Reader, Oxford/New York: Berg Publishers.

Hsu, E. (ed.) 2001, Innovation in Chinese Medicine, Cambridge: Cup.

Huang Longxiang 黃龍祥 2003, Zhongguo zhenjiushi tuijian 中國 針炎史圖鑒 (Illustrated History of Chinese Acupuncture and Moxibustion), Qingdao: Qingdao chubanshe.

Jiangling Zhangjiashan Hanjian zhengli xiaozu 江陵張家山漢簡整 理小組 1989, 'Jiangling Zhangjiashan Hanjian (Maishu) shiwen' 江陵張家山漢簡脈書釋文, Wenwu 7, 75-81.

Jones, C.A. and P. Galison (eds) 1998, Picturing Science, Producing Art, New York: Routledge.

Jones, P.M. 2006, 'Word and image in medieval medicine', in Givens, Reeds and Touwaide (eds), $1-24$.

Jordanova, L. 199o, 'Medicine and visual culture', Social History of Medicine 3, 89-99.

Kemp, M. and M. Wallace 2001, Spectacular Bodies: The Art and Science of the Human Body from Leonardo till Now, Berkeley: University of California Press and Hayward Gallery.

Kuriyama, Shigehisa 栗山茂久 1999, The Expressiveness of the Body, New York: Zone.

Landau, A. (ed.) 2015, Pearls on a String: Artists, Patrons, and Poets at the Great Islamic Courts, Baltimore: Walters Art Museum and University of Washington Press.

Latour, B. 2014, 'Anthropology at the time of the Anthropocene. A personal view of what is to be studied', Distinguished lecture at the American Anthropologists Association meeting in Washington, December 2014. Available at: http://www.bruno-latour.fr/sites/ default/files/139-AAA-Washington.pdf (accessed o8/o8/2017).

Levi, J. 1989, 'The body: the Daoists' coat of arms', in Feher et al. (eds), pt 1, 105-12.

Li Ling and K. McMahon, 1992, 'The contents and terminology of the Mawangdui texts on the Arts of the Bedchamber', Early China 17, $145^{-85}$.

Lloyd, G.E.R. and J. Zhao Jingyi 趙靜一 2017 (forthcoming), Ancient Greece and China Compared: Interdisciplinary and Cross-Cultural Perspectives, Cambridge: CuP.

Lo, V. 1998, 'The influence of Yangsheng culture on early Chinese medical theory', PHD diss., SOAs, University of London.

1999, 'Tracking the pain:Jue and the formation of a theory of circulating qi through the channels', Sudhoffs Archiv 83.2, 191-211.

2001, 'The influence of Nurturing Life Culture on the development of Western Han acumoxa therapy', in Hsu (ed.), 19-5o.

2005, 'Quick and easy Chinese medicine: the Dunhuang moxibustion charts', in Lo and Cullen (eds), 207-51.

2007, 'Imagining practice: sense and sensuality in early Chinese medical illustration', in Bray et al. (eds), 383-423.

Lo V. and C. Cullen (eds) 2005, Medieval Chinese Medicine, London: RoutledgeCurzon. 
Lo, V. and E. Re'em 2017 (forthcoming), 'Recipes for love in the Ancient World', in Lloyd and Zhao (eds).

Lo, V and Wang Yidan 王一丹 2013, 'Blood or Qi circulation? On the nature of authority in Rashīd al-Dīn's Tānksūqnāma sهمان قوسكنات كىاتخ مولع نونف رد ناخلىا (The Treasure Book of Ilqān on Chinese Science and Techniques)', in Akasoy, Burnett and Yoeli-Tlalim (eds), 127-72.

López-Cajún, C. and M. Ceccarelli (eds) 2016, Explorations in the History of Machines and Mechanisms. Proceedings of the Fifth IFTомм Symposium on the History of Machines and Mechanisms, Switzerland: Springer.

Loux, F. 1987, 'Popular culture and knowledge of the body: infancy and the medical anthropologist', in Porter and Wear (eds), 81-97.

Ma Jixing 馬繼興 1996, 'Shuangbaoshan Hanmu chutu de zhenjiu jingmai qimu renxing' 雙包山漢墓出土的針炎經脈漆木人形, Wenwu 4, 55-65.

Ma Jixing 2015, Zhongguo chutu guyishu kaoshiyuyanjiu (Studies and Textual Analysis of Ancient Medical Texts Excavated in China) 中國出土古醫書考釋與研究, 3 vols, Shanghai: Shanghai kexue jishu chubanshe.

Ma Jixing 馬繼興, Wang Shumin 王淑民 et al. 1998, Dunhuang yiyao wenxian jijiao 敦煌醫藥文獻輯校, Nanchang: Jiangsu guji chubanshe.

McDermott, J.P. 2006, The Social History of the Chinese Book: Books and Literati Culture in Late Imperial China, Hong Kong: Hong Kong University Press.

Mawangdui Hanmu boshu zhengli xiaozu 馬王堆漢墓帛書整理小 組 (eds) 1985, Mawangdui Hanmu boshu 馬王堆漢墓帛書, vol. 4, Beijing: Wenwu chubanshe.

Merleau-Ponty, M. 1945, Phénoménologie de la perception, Paris: Gallimard, 1945. Tr. C. Smith 1962, Phenomenology of Perception, New York: Humanities Press, and London: Routledge \& Kegan Paul.

Métailié, G. 2001, 'The Bencao gangmu of Li Shizhen: an innovation in natural history?', in Hsu (ed.), 262-92.

2007, 'The representation of plants: engravings and paintings', in Bray et al. (eds), 487-520.

2010, 'Concepts of nature in traditional Chinese materia medica and botany (sixteenth to seventeenth century), in Vogel and Dux (eds), 345-67.

Moore, A. 2015 'Anthropocene anthropology: reconceptualizing contemporary global change', Journal of the Royal Anthropological Institute 22, 27-46.

Needham J. et al. 2004, Science and Civilisation in China, vol. 7, pt 2, Cambridge: CuP.

Petherbridge, D. and L. Jordanova 1998, The Quick and the Dead: Artists and Anatomy: Berkeley/Los Angeles/London: University of California Press.

Porter, R. and A. Wear (eds) 1987, Problems and Methods in the History of Medicine, London: Croom Helm.

Roberts, K.B. and J.D.W. Tomlinson 1992, The Fabric of the Body: European Traditions of Anatomical Illustration, Oxford: OUP.

Robinson, E. 2010, 'Touching the void: affective history and the impossible', Rethinking History 14.4, 502-2O.

Rocha, L.A. 2011, 'Scientia sexualis versus Ars erotica: Foucault, van Gulik, Needham', Studies in History and Philosophy of Biological and Biomedical Sciences 42, 328-33.

Scheid, V. 2016, 'Holism, Chinese Medicine and Systems Ideologies: rewriting the past to imagine the future', in Whitehead et al. (eds), 66-86.

Schipper, K. 1993, The Taoist Body, Berkeley: University of California Press.

Seo Tatsuhiko 2003, 'The printing industry in Chang'an's eastern market in the Tang dynasty', Memoirs of the Research Department of the Toyo Bunko 61, 1-42.

Shapiro, H. 1998, 'The puzzle of spermatorrhea in Republican China', in Hanson and Farquhar (eds), 551-96.

Unschuld, P.U. 1985, Medicine in China: A History of Ideas, Berkeley/ Los Angeles/London: University of California Press.

2000, Medicine in China: Historical Artifacts and Images, tr. S. Wilms, Munich/New York/London: Prestel.

Unschuld, P.U. with H. Tessenow 2011, Huang DiNeiJing Su Wen. Huang Di's Inner Classic. Basic Questions, Berkeley/Los Angeles/London: University of California Press.

Unschuld, P.U. with Zheng Jinsheng 鄭金生 and H. Tessenow 2003, Huang Di NeiJing Su Wen. Nature, Knowledge, Imagery in an Ancient Chinese Medical Text, Berkeley; Los Angeles; London: University of California Press.

Vogel, H.Ụ. and G. Dux, (eds) 2010, Concepts of Nature: A ChineseEuropean Cross-Cultural Perspective, Leiden: Brill.

Whitehead, A. et al. (eds) 2016, The Edinburgh Companion to the Critical Medical Humanities, Edinburgh: Edinburgh University Press.

Wu Hung 2007, 'Picturing or diagramming the universe', in Bray et al. (eds), 191-214.

Wu, Yi-Li 2015, 'Between the living and the dead: trauma medicine and forensic medicine in the mid-Qing', Frontiers of History in China 10.1, 38-73.

Yasuo Yuasa 1993, The Body, Self-Cultivation, and Ki-Energy, Albany: State University of New York.

Zhao F. et al. 2016, 'Mechanism of Laoguanshan pattern looms from late 2nd century вСE, Chengdu, China', in López-Cajún and Ceccarelli (eds), 209-21. 


\title{
1 Chinese Medical Illustration: Chronologies and Categories
}

\author{
Wang Shumin 王淑民 and Gabriel Fuentes
}

This chapter is concerned with illustrations that were included in medical texts prior to the end of the Qing dynasty (1644-1911): it establishes two key genres of illustration for both manuscripts and block-printed texts, and points out essential features and controversial issues. Many of the earliest texts were written on slips of bamboo or wood or pieces of silk. After the invention of papermaking techniques, texts began to be written on scrolls; subsequently paper productions were bound in concertina or sūtra-fold format (jingzhe zhuang 經摺裝), which paved the way for various types of folded-leaf binding. The illustrations within these texts included sketches, diagrams, and coloured drawings. There are also manuscript genres that are primarily pictorial such as selfstanding charts. ${ }^{1}$

The earliest printed books were produced using carved wooden blocks, generally made of pear or date wood. This technique allowed for a relatively fast production of duplicate copies. There were also a small number of lithographic prints, produced with stone blocks. The images in these books are known in Chinese as muke banhua 木刻版畫 (xylographic images) and shiban hua 石版畫 (lithographic images).

\section{The Earliest Extant Illustrations in Chinese Medical Texts}

\section{Illustrated Manuscripts}

In 1973, a cache of manuscripts was found in Tomb 3 at the Han-dynasty Mawangdui 馬王堆 ${ }^{2}$ burial site in Changsha (capital of Hunan province). The cache yielded some 30 manuscripts containing 45 texts on a variety of subjects ranging from astronomy and cosmology to ritual and philosophy, and including many maps, diagrams and pictures. ${ }^{3}$ They were buried in the time of the Han emperor Wendi 漢文帝 (202-157 BCE), although some appear to have been copied at an earlier date. Seven manuscripts, all concerned with healing and enhancing the body, are stored in a separate box, indicating that they were considered to belong to a distinct category. Among them is the earliest extant set of images illustrating daoyin 導引, literally 'leading and guiding', a form of therapeutic exercise discussed in several chapters in this volume (Fig. 1). ${ }^{4}$

This kind of exercise was in some contexts referred to as a form of yangsheng 養生 - a term for practices concerned with the cultivation of human life in all its myriad forms,



Figure 1.1 Daoyin tu 導引圖 (Guiding and Pulling Chart), Mawangdui, tomb 3 (closed 168 вCE). Silk, reconstruction. (C) Wellcome Library, London, Loo36oo7

1 See Bray, Dorofeeva-Lichtmann and Métailié (eds) 2007. An important genre that emerged later was the tupu 圖譜 (illustrated register), where text and image were complementary and closely integrated; Bray, Dorofeeva-Lichtmann and Métailié (eds) 2007, pp. 1, 38-40 et passim.

2 For transcripts see Mawangdui boshu zhengli xiaozu vol. 4, 1985. The tomb was closed in 168 вСE.

3 Harper 1998, pp. 14-36.

4 During the excavation process the daoyin chart sustained severe damage, and the illustration in Figure 1 is a reconstruction; Mawangdui Hanmu boshu zhengli xiaozu 1985, vol. 1. 




Figure 1.2 Dunhuang Vajracchedikā Prajñāpāramitā Sūtra 金剛般若波羅密經 (Diamond Sūtra), 868. @ The British Library Board Or. 8210/P.2, frontispiece and text

which is still used today in reference to traditional ways of nurturing the body. The polychrome chart is painted on a piece of silk measuring approximately $100 \mathrm{cms} \times 5^{\circ}$ cms. It shows 44 figures bending, stretching and pulling their bodies into different positions. They are young and old, women and men, some with naked upper bodies, others clad in long or short robes, perhaps designed to facilitate the performance of the exercises. Many of the postures mimic the movements of animals and birds, and they include simple chest expansions, leg and arm raises, and stretching postures. Some of the adepts can be seen crossing one leg over another, twisting and contorting their torsos, jumping or swinging their limbs. Exercise aparatus such as staffs, sandbags or balls aid a regimen that, as we know from contemporary texts, aimed at resolving pain and strengthening the body to enhance longevity. ${ }^{5}$ Scholars have associated these movements with ritual dances dating to the Spring and Autumn (770-476 BCE) and Warring States (475-221 BCE) periods, ${ }^{6}$ but by the time of this Mawangdui evidence, it is clear that the practice had become more pervasive, at least in aristocratic circles; and although contemporaneous texts describe the movements in relation to the cycles of Heaven and Earth, they have a distinctly secular feel to them. From the Han (202

$5 \quad$ Lo 2014: http://www.nri.cam.ac.uk/yinshu.pdf.

6 Sterckx 2002, pp. 128-37.
BCE-22O CE) to the Northern and Southern Song dynasties (96o-1279), a large number of daoyin works circulated, but due to the ravages of political fragmentation during much of this time, most of those works have been lost. Only a small number have survived, without illustrations, and often just as citations in later works. Looking at the Mawangdui chart, one can see clear relations between these Han-dynasty depictions and later textual accounts of daoyin exercises, such as those described in Hua Tuo's 華陀 Wuqin xi 五禽 戲 (Five Animals Frolics), the Eight-section Brocades, and even the modern practice of Taiji quan.?

\section{Earliest Block Print Illustrations}

There is much controversy about the origin of woodblock print illustrations, with some scholars giving very early dates, but the earliest extant evidence is famously the Dunhuang Vajracchedikā Prajñāpāramitā Sūtra 金剛般若 波羅密經 (Diamond Sūtra), which dates to $868 \mathrm{CE}$ in the Xiantong 咸通 reign period of the Tang dynasty (Fig. 2).

However, it is probable that the first printed Chinese medical illustrations date to just before this time. In the Dunhuang 'Library Cave'8, a work was found that describes

$7 \quad$ Wang and Barrett 2006

8 Mogao Grotto 17, the so-called 'Library Cave', was a monastic repository of some kind, which was walled up in the 11th century for reasons still unknown, and not rediscovered until 1900. 




Figure 1.3 Illuminated Hall chart showing the loci of the head and shoulders, ms. P.2675. @ courtesy of Bibliothèque nationale de France

methods of moxa-cautery or moxibustion: ${ }^{9}$ it is known by the modern title Xinji beiji jiujing 新集備急尒經 (Newly Compiled Canon of Emergency Moxibustion Remedies)..$^{10}$ Although the Dunhuang exemplar is in manuscript form, it includes a passage stating that it was printed in the eastern market of the capital by the Li family, so we know that it was hand-copied from a woodblock printed version. The colophon attributes the copying to Fan Ziying 範子盈 and 'Yin-Yang' (Daoist adept) Fang Jingxun 氾景詢, and tells us that they executed the manuscript in close collaboration, completing their work on the $25^{\text {th }}$ day of the 12 th month in the 2nd year of the Xiantong reign period (i.e. $861 \mathrm{CE}) .{ }^{11}$ As well as copying the text of the Canon of Emergency Moxibustion Remedies, Fang and Fan copied illustrations, including an illustration showing the moxibustion locations of the upper body in frontal view (Fig. 3). Above the head, the word 'Mingtang' 明堂 (Illuminated Hall or Bright Hall)

It was found to contain a cache of some $5^{0}$,ooo manuscripts as well as various sacred objects. The Library Cave is located off the the northern wall of the entrance passageway of Grotto 16.

9 Throughout this volume the terms moxa-cautery and moxibustion are used to refer to heat treatments applied to the surface of the body. The latter term mainly refers to burning ai 艾 (Artemisia vulgaris) on the skin, and sometimes on acu-moxa locations. Now held in the Bibliothèque nationale de France, cat. no. P.2675.

$11 \quad$ Lo in Lo and Cullen 2005, p. 233. is written in characters that are noticeably larger than those of the acupoint terms also contained in the chart, marking it out as a title. This is a significant discovery, for it is the earliest extant diagram of the human body identified as 'Mingtang', a designation formerly known only through received texts. The lower half of the body is no longer present because of damage to the manuscript. It may be conjectured that there were originally also back and side views, but no trace of those remain. ${ }^{12}$ Although the illustrations are incomplete, from this evidence it may be inferred that the earliest Chinese medical woodblock illustrations had begun to appear by $861 \mathrm{CE}$.

Rare illustrations from Liu Jia's 劉甲 1185 edition of Jingshizhengleibeijibencao 經史證類備急本草 (Classified Pharmacopoeia for Emergency Use, Based on Classics and Historical Sources) present a visual narrative sequence of the entire process involved in collecting and preparing edible sea salt for distribution. Of two interrelated illustrations (Fig. 4), the first depicts a scene of workers in an inlet on the seashore, collecting ocean water. The second sets forth an action-packed scene of subaltern workers boiling salt; in the middle ground a workman is bringing firewood loaded into baskets which hang on the ends of a shoulder pole, while others are carrying off containers presumably loaded with sea salt. A man sitting in the

12 Wang Shumin in Lo and Cullen 2005, pp. 413-4. 




Figures 4a, 4b Making sea salt. Liu Jia's 劉甲 edition of Jingshi zhenglei beiji bencao 經史證類備急本草 (Classified Pharmacopoeia for Emergency Use, Based on Classics and Historical Sources), block-print edition 1185 . (c) Library of the Needham Research Institute offices in the background must be the salt official, while those facing the reader are clearly overseers. One of the overseers appears to be shouting and gesticulating, and we are caught up in the heightened mood of wrangling about the manufacturing process.

Since the Han dynasty (2O2 BCE-22O CE), there had been government monopolies of the mining, manufacture and sale of salt in China. Conscripts worked the mines. Private salt mines tended to complicate the state tax base by employing large numbers of men.13 The Song dynasty (96o-1279) government established salt officials in each region to control production; this allowed the State to centralise and monopolise the purchase of salt. The officials in charge of the transportation and distribution of salt were called guanyan 官鹽. This monopolisation of edible salt facilitated the levying of salt taxes. 'Salt from Xiechi 解池 Lake' is an illustration that finds Song-dynasty officials going about their business in the market as they transport and trade in salt for the government. Various lifelike characters busy themselves with the different activities crucial to the trade: we can detect in the picture the yanguan 鹽 官 'salt officials', yanshang 鹽商 'salt merchants', chayi 差役 'runners', ganlüren 趕驢人 'donkey handlers', and yangong 監工 'overseers'. This is a very important historical image for the study of the salt industry during the Song dynasty (see Fig. 5).



Figures 5a, 5b Salt from Shanxi Province. In the original sequence, there were four depictions of sea salt manufacture illustrating Xieyan 解鹽 (Salt from Xiechi Lake 解池, Shanxi province). Here they are combined into two. This not only made the images more compact, but also saved carving additional wood blocks.@ Library of the Needham Research Institute 


\section{Distribution of Ancient Chinese Illustrated Medical Texts}

The illustrations in ancient Chinese medical texts have many functions: they aid the theoretical understanding of medicine, distinguish the appearance of medicinals, reveal the channels and point locations, explain diagnosis and treatment methods, describe characteristics of disease patterns, and illustrate the movements of the exercise methods for yangsheng 養生 'nourishing life'. Most pre-modern Chinese medical illustrations are to be found in the genre of Materia Medica, in treatises on acu-moxa, the morphology of the viscera and diagnostic methods, and in practical texts of various kinds; there are also numerous illustrated volumes on the practice of yangsheng.

Due to limitations of space, this chapter will only give a general introduction to illustration in the following two genres: Materia Medica and acu-moxa. In Shen Nong bencao jing 神農本草經 (The Divine Farmer's Canon of Materia Medica), there are no written descriptions of the morphology or appearance of medicinals, a feature which gradually begins to appear in later Materia Medica texts. Given the lack of technical data to assist readers who might have been interested in precisely what drugs were being classified, mistakes in identification were inevitable. To facilitate the differentiation of animal, plant, and mineral medicinals, illustrated Materia Medica began to emerge around the time of the Sui dynasty (581-618) or just prior to it.

\section{Materia Medica Illustration}

From a survey of medical texts in the received traditions, it is clear that none of the earliest Materia Medica, as listed in the table below, included any illustrations of medicinals. These texts are extremely difficult to date as the authorship is often obscure and it is quite likely that they were written down from oral transmissions and/or compiled from multiple remedy collections.

The Earliest Materia Medica

\begin{tabular}{|l|l|l|}
\hline $\begin{array}{l}\text { Shen Nong ben- } \\
\text { caojing }\end{array}$ & 神農本草經 & $\begin{array}{l}\text { Shen Nong's Canon of } \\
\text { Materia Medica }\end{array}$ \\
\hline $\begin{array}{l}\text { TongJun caiyao } \\
\text { lu }\end{array}$ & 桐君採藥錄 & $\begin{array}{l}\text { Lord Tong's Records of } \\
\text { Drug Collection }\end{array}$ \\
\hline Lei Gongyao dui & 雷公藥對 & $\begin{array}{l}\text { Lei Gong's Medicinal } \\
\text { Pairings }\end{array}$ \\
\hline $\begin{array}{l}\text { Li Dang zhiyao } \\
\text { lu }\end{array}$ & 李當之藥錄 & $\begin{array}{l}\text { Li Dangzhi's Drug } \\
\text { Records }\end{array}$ \\
\hline
\end{tabular}

\begin{tabular}{|l|l|l|}
\hline Wu Pu bencao & 吳普本草 & $\begin{array}{l}\text { Wu Pu's Materia } \\
\text { Medica }\end{array}$ \\
\hline Mingyi bielu & 名醫別錄 & $\begin{array}{l}\text { Supplementary Records } \\
\text { of Eminent Physicians }\end{array}$ \\
\hline Bencaojing jizhu & 本草經集注 & $\begin{array}{l}\text { Materia Medica Canon } \\
\text { Variorum Edition }\end{array}$ \\
\hline $\begin{array}{l}\text { Lei Gong paozhi } \\
\text { lun }\end{array}$ & 雷公炮采論 & $\begin{array}{l}\text { Lei Gong's Guide to } \\
\text { Drug Preparation }\end{array}$ \\
\hline
\end{tabular}

The bibliographical treatise of the official history of the Sui dynasty (Suishu jingji zhi 隋書經籍志), compiled between 641 and 656 , records 28 Materia Medica works, but since they are no longer extant, we are unable to determine definitively whether or not they contained any illustrations. However, from the titles of two items on the list, Yuan Pingzhong's 原平仲 six-juan ${ }^{14}$ Lingxiu bencao $t u$ 靈秀本草圖 (Exquisitely Illustrated Materia Medica), and a single juan entitled Zhicao tu 芝草圖 (Illustrations of Zhicao - possibly synonymous with lingzhi 靈芝 'Ganoderma'), it is likely that these two, at least, did incorporate visual images of medicinal substances. Nothing is known about the life of Yuan Pingzhong, but from the records of his publications in Suishu 隋書 (History of the Sui Dynasty, 636), we can surmise that he lived in the Sui period (581-618), or perhaps earlier. As these two items are the earliest recorded illustrated Materia Medica works, we can infer that this genre of illustrated text began to appear around the Sui Dynasty.

Tang Dynasty (618-907) Materia Medica Illustrations In 657, the second year of the Xianqing 顯慶 era of his reign, Gaozong, third emperor of the Tang dynasty, approved the compilation of Xinxiu bencao 新脩本草 (Newly Revised Materia Medica) on the recommendation of Su Jing 蘇敬 (599-674), then an aide in the Right Palace Gate Guard. The emperor appointed Changsun Wuji 長孫無忌, Li Ji李 勣 and Su Jing 蘇敬 himself to lead the project, together with an editorial team of 22 scholars. The entire book was completed two years later and comprised 54 juan divided into three sections: the Bencao 本草 (Materia Medica), the Yaotu 藥圖 (Medicinal Illustrations), and the Tujing 圖 經 (Illustrated Canon of Materia Medica). The Medicinal Illustrations section was a kind of pictorial album containing no fewer than an estimated 85 o polychrome paintings depicting the morphology of medicinal substances, which

$14 J u a n$ 卷 (literally 'scroll', often translated as 'fascicle') was the basic textual and/or physical unit of pre-modern Chinese books, long after scrolls had been superseded by page-book formats. The juan often, but not always, coincided with chapter or section divisions. 
formed a separate volume to the textual descriptions. A government edict required the illustrators to work from real-life samples collected from all over the empire, according to their actual appearance. This was China's first state-sponsored album of Materia Medica illustrations. Because of practical issues in transmitting and reproducing colour illustrations, by the early Song period ( $c$. 1oth century) the illustrations were all lost.

The existence of other notable illustrated Materia Medica texts dating to the Tang period is borne out by Zhang Yanyuan's 張彥遠 (c. 815-75) Lidai minghua ji 歷 代名畫記 (Record of Famous Paintings through the Ages), which has left us a tantalising note of the titles of work by the celebrated artist Wang Ding 王定 (58o-668): Bencao xunjie tu 本草訓誡圖 (Instructional Illustrations for the Materia Medica), and Shen Nong bencao litu 神農本草例圖 (Illustrative Examples from Shen Nong's Canon of Materia Medica), the latter in one juan. These publications, dating to the Zhenguan reign period of Emperor Taizong, Li Shimin (627-49), are no longer extant. ${ }^{15}$

Tianbao danfang yaotu 天寶單方藥圖 (Illustrations of Single-Ingredient Remedies of the Tianbao era [742-56]) was a Materia Medica work that integrated text and illustration. It was compiled under the sponsorship of the Tang Emperor Xuanzong, Li Longji 李隆基 (685-762). According to research by Zheng Jinsheng 鄭金生 (1993), the illustrations of the medicinal herbs shuiying水英, lichun cao 麗春草, and zijian 紫藏 in the 'Waicao' 外草 16 category of Zhenglei bencao 證類本草 were based on originals in Tianbao danfang yaotu. One juan of this work survived into the Northern Song period (96o-1127), and Su Song 蘇 頌 is known to have referred to it when compiling Bencao tujing 本草圖經 (Illustrated Canon of Materia Medica), the subject of the next section. However, it was subsequently completely lost to posterity.

\section{Song (96o-1279) and Yuan (1279-1368) Dynasty Illustrated Materia Medica}

When Su Song 蘇頌 compiled Bencao tujing 本草圖經 (Illustrated Canon of Materia Medica), he included 932 line drawings. These illustrations originated in sketches of medicinal substances submitted to the court from all over the empire, as well as drawings kept in the imperial library (Neifu 內府). Bencao tujing was first published in the 7 th year of the Jiayou reign period (1062), probably with block-printed illustrations. The original book was subse-

15 Zhang Yanyuan [847] 1987, Lidai minghua ji, pp. 313-14.

16 'Waicao' 外草 refers to medicinals that were used by physicians, at the time of compilation, but had not been recorded in previous medical classics. quently lost. Fortunately however, both the illustrations and the text were partially preserved by Tang Shenwei 唐慎微 (1056-93) in Jingshi zhenglei beiji bencao 經史證 類備急本草 (Classified Pharmacopoeia for Emergency Use, Based on Classics and Historical Sources), Zhenglei bencao for short. Different editions of the latter work incorporated different numbers of images from the source text: there are 922 drug illustrations in the extant edition of the Daguan bencao 大觀本草 (Pharmacopoeia of the Daguan Era), published in 1108, 932 in the extant Zhenghe bencao 政和本草 (Pharmacopoeia of the Zhenghe Era), and 801 in the extant fragments of Shaoxing bencao 紹 興本草 (Pharmacopoeia of the Shaoxing Era, 1162-87). ${ }^{17}$

\section{Lü Chanyan Bencao 履㟴巌本草 (Cliff Walker's} Materia Medica)

Lü chanyan bencao 履㟴嚴本草 (Cliff Walker's Materia Medica), compiled and illustrated by the Southern Song dynasty artist Wang Jie 王介 (1163-1224), is the earliest extant Chinese Materia Medica illustrated in colour. The book contains 206 entries on individual drugs with the same number of colour images, mostly of herbal medicines.

Wang Jie had assimilated the landscape painting style of Ma Yuan 馬遠 (c. 116o-1225) and Xia Gui 夏圭 (also written 夏珪) (c. 1180-c. 1230), of the so-called Ma-Xia 馬夏 school, which he applied in his Materia Medica illustrations. Those influential Southern Song period artists were renowned for their detailed attention to layering trees and plants in their mountain landscapes to create new perspectives, and using wide empty spaces to de-centre and de-emphasise the human subject within the larger scale of the whole scene. For Wang Jie, this meant nuancing the scale of his medicinal plants and isolating sections so as to represent the whole specimen in different ways. This helped to detail and distinguish the special characteristics of each plant. The shortcomings of this work are mostly due to the author's limited medical knowledge, which results in inconsistencies in the illustrations (See Zheng, Chapter 8 in this volume).

Chongguang Buzhu Shen Nong Bencao Bing Tujing 重廣補注神農本草并圖經 (Shen Nong's Canon of Materia Medica with Illustrations, Extensively Supplemented, 1092)

This illustrated Song-dynasty work by Chen Cheng 陳承 combined Jiayou buzhu Shen Nong bencao 嘉祐補注神農 本草 (Expanded and Annotated Edition of Shen Nong's Materia Medica, Commissioned in the Jiayou Era), 1061, and Bencao tujing 本草圖經 (Illustrated Canon of Materia Medica), with the addition of the author's own annotations

17 Shang Zhijun et al. 1989, pp. 213, 265, 286. 
and commentaries. Its distinguishing feature was a close correspondence between text and illustrations, which made the work easy and logical to read. Unfortunately this book is no longer extant in its entirety, but sections have survived and can still be seen scattered throughout Tang Shenwei's Zhenglei bencao, compiled in the late 11th-12th century).

Yinshan Zhengyao 飲膳正要 (Principles of Correct Diet), Compiled by the Yuan Dynasty Scholar, Hu Sihui 忽思慧, 133 O

This is the first specialist text on dietary medicine in Chinese to combine information on the therapeutic values of foodstuffs with recipes. Compiled for the Mongolian court, it represents foodways that have been sinicised on the basis of a Mongolian culinary aesthetic. While many of the recipes are clearly of Central and West Asian, if not Turkic, origin, they also introduce distinctively Chinese medical ideas about techniques for healing and enhancing the body. It is an invaluable text for anyone interested in culinary terminology as well as the cross-cultural history of nutrition in China. The text records a wide range of dietary taboos during pregnancy and wet nursing, and traditions of liquor consumption.

It is illustrated throughout with exquisite woodcuts, which not only aid in identifying substances and their preparation and understanding dietary practices, but critically, situate them within - albeit elite - social settings in the homestead, in lavishly furnished and ornamented courtyards and interiors (Fig. 6). The images are meticulous in their representation of people, their clothing, and their everyday surroundings, thereby providing a rich resource for analysing healthcare practices in the context of everyday life at the end of the Song and the beginning of the Yuan dynasty. ${ }^{18}$

Ming Dynasty (1368-1644) Illustrated Materia Medica The Ming dynasty Materia Medica genre represents one of the greatest undertakings in China's medical history. New versions of the pharmacopoeia Qin Shi Huangdi continuously emerged, and many of these included exquisite multi-coloured illustrations created by imperially sponsored court artists.



Figure 1.6 Yinshan zhengyao 飲膳正要 (Principles of Correct Diet), 1330. Diet therapy for all diseases. (C) Library of the China Academy of Chinese Medical Sciences

\section{Ming Block-Print Materia Medica Illustrations}

Jiuhuang Bencao 救荒本草 (Materia Medica for Famine Relief), Compiled by Zhu Su 朱橚, Engraved in the 4th Year of the Yongle Period, 1406

The author selected 414 types of edible plants, flowers, roots, and barks, and provided simple introductions pertaining to their place of origin, morphology, therapeutic nature and flavour, toxicity, place of harvesting and methods of culinary preparation. The selection represents plants that could theoretically be consumed during years of famine, hence its title. The 414 exquisite illustrations in the text were apparently all first drawn from life, from plants in Zhu Su's botanical garden. This work is thought to have had an important influence on the later development of agriculture, botany, and pharmacology. It also identifies a certain number of highly potent plants, and traditions of gathering based on specific botanical principles ${ }^{19}$ (See Zheng, Chapter 8 in this volume, Fig. 8.4). Shang Zhijun et al. 1989, pp. 213, 265, 286. 
Diannan Bencao 滇南本草 (The Southern Yunnan

Materia Medica), Compiled by Lan Mao 蘭茂 Diannan bencao is a regional Materia Medica recordingmedicinals from the Diannan 滇南 region, the ancient territory corresponding to parts of modern-day Yunnan. The extant Kunming Wubentang edition, which dates to the 3 oth year of the Guangxu 光緒 reign period (1887), has more than 6 o drug illustrations.

Bencao mengquan 本草蒙筌 (Beginner's Guide to Materia Medica), compiled by 陳嘉謨 Chen Jiamo The illustrations and accompanying textual entries of the Bencao mengquan are of comparatively high quality and make for informative reading. The first volume includes 14 illustrations taken from Xiong Zongli's 熊宗立Lidaimingyi $t u$ 歷代名醫圖 (Portraits of Eminent Physicians through the Ages). The book contains an illustration for each entry, which, according to figures given in Lidai zhongyao wenxian jinghua 歷代中藥文獻精華 (Essential Chinese Drug Literature Through the Ages), adds up to 559 illustrations, of which 30 depict crude drugs (yaocai 藥材). ${ }^{20}$ Worthy of notice is the portrait of Bian Que 扁鵲, who is represented in mundane human form, and not, as was habitual, with a bird's body (See Ma, Chapter 31 in this volume, Fig. 31.5).

\section{Bencao Gangmu 本草綱目 (Classified Materia}

Medica), c. 21st Year of the Wanli 萬曆 Period, 1593,

by Li Shizhen 李時珍 (1518-93)

The text, in 52 juan, is divided into 16 parts and 6 o categories, and contains a total of 1,892 medicinals and 1,109 block-print illustrations. The first edition was engraved by $\mathrm{Hu}$ Chenglong 胡成龍 in Jinling 金陵 (today's Nanjing) in 1593, and is commonly referred to as the 'Jinling edition' (Jinling ban 金陵版). According to Shang et al. (1989), there are differing views as to the creation and dating of the illustrations in the Jinling edition, and some people doubt that Li Shizhen had anything at all to do with preparing them. ${ }^{21}$ Since there is no form of list or key to the illustrations in the main text of Bencao gangmu, and there are some significant discrepancies between text and images, it seems at all events that Li Shizhen was not concerned with the issue of illustrations when composing the text.

The list of attributions in the first juan of illustrations in the Jinling edition notes that the images were compiled by Li Shizhen's eldest son, Li Jianzhong 李建中, gentlemanliteratus (wenlin lang 文林郎) and district magistrate of Lianxi 蓮溪 (in today's Sichuan province), and drawn by two other sons: prefectural student Li Jianyuan 李建元 Ibid. pp. 284-96. and district student Li Jianmu 李建木. These attributions make it clear that Li Shizhen's descendants were responsible for the illustrations in Bencao gangmu, in their final form at least.

Other Ming period Materia Medica included large numbers of block-printed illustrations: for example, Wang Wenjie's 文潔 Taiyixian zhiben caoyao xing daquan 太乙仙製本草藥性大全 (Transcendent Great One's Comprehensive Compendium of the Properties of Medicinal Herbs); Li Zhongli's 李中立 Bencao yuanshi 本 草原始 (Origins of Materia Medica), and Ni Zhumo's 倪 朱謨 Bencao huiyan 本草彙言 (Collected Remarks on the Materia Medica). The numerous illustrations of medicinal substances contained in these works, and others from the Ming period, vary widely in style, technique and quality, a phenomenon that has not been thoroughly studied.

\section{Ming Painted Polychrome Materia Medica Illustrations}

Bencao Pinhui Jingyao 本草品彙精要 (Classified

Treasury of Materia Medica), 1503

At the imperial decree of the Xiao Zong 孝宗 emperor, Zhu Youcheng 朱祐樘, Liu Wentai 劉文泰, an administrative assistant (yuanpan 院判) at the Tai Yiyuan 太醫院 (the Imperial Academy of Medicine), took charge of compiling a Materia Medica in 22 juan entitled Yuzhi bencao pinghui jingyao 禦製本草品彙精要 (Classified Treasury of Materia Medica by Imperial Appointment), more commonly known simply as Bencao pinhui jingyao. It recorded 1,815 medicinals and contained 1,358 lavishly coloured high-quality medicinal illustrations by eight named artists: Wang Shichang 王 世昌, Wang Fu 王輔, Zhao Xian 趙賢, Zheng Xuan 鄭宣, Liu Yuan 劉緣, Zhao Duo 趙鐸, Zhao Hai 趙海 and Wu Zan 吳瓚. The manuscript was completed in spring of 1505 , but then met with an unforeseen disaster. In the summer, Liu Wentai was summoned to treat the emperor who had apparently contracted a heat disease. Unfortunately for Liu, the emperor subsequently died and Liu was accused of having misdiagnosed his condition. Consequently the book met with censure and was never committed to woodblocks for printing. Instead it was stored away in the imperial library, where in theory only a small number of officials could set eyes on it, or make copies. Nevertheless, its fine illustrations proved irresistible to contemporary artists, who managed to gain access, and made and circulated several copies. According to Zheng Jinsheng (2003), there are nine extant copies of the Bencao pinhui jingyao. The finest is currently held at the Kyo-U Library of the Takeda Science Foundation, Osaka. It is a Ming copy dating to the 




Figure 1.7 Lu He 盧和, Shiwu bencao 食物本草 (Materia Dietetica): Illustration of well water. 1571. (C) Library of the China Academy of Chinese Medical Sciences

18th year of the Hongzhi 弘治 reign period, 1505 (See Cao, Chapter 13 in this volume, pp. 198 and 204-6).

\section{Shiwu Bencao 食物本草 (Materia Dietetica).}

The Ming edition of Shiwu bencao is a polychrome production in four juan, currently held at the National Library of China. The text lacks any preface, postscript or notes on authorship or the artists. In its format, dimensions, pigments, calligraphy and artistic style, it resembles Bencao pinhui jingyao, and it could likewise be the work of artists from the Ming Imperial Academy. ${ }^{22}$ Textual research shows that nearly half of the illustrations are copied from Bencao pinhuijingyao. It contains entries on 386 medicinal foods, and 492 colour illustrations.

Of special interest is the section that deals with the subject of 'Water', in which the artists give full play to their imagination when presented with the fascinating challenge of differentiating the nourishing value of 33 different kinds of water, including 'well water' (Fig. 7), 'thousand league water', 'autumn dew water', 'December snow water', 'water gathered from stalactites', 'cold spring water', 'summer ice', 'hot spring water', 'water from a mud hole', hot [bathing] water, 'dew from a leaf', 'water from a waterfall', 'winter frost water', 'hail water'.

Ultimately these drawings do not have a direct function in helping to identify medicinals, but they are an important resource for research into everyday life in Ming China. The pictures are certainly aesthetically beautiful; however, due to the artists' limited knowledge of materia medica they contain errors in the depiction of some items. For exam- ple, the term meiyushui 梅雨水 'plum rain water' denotes rainwater from the rainy season. The artist interpreted this expression literally, and was inspired to depict the gathering of rainwater from plum blossom petals.

\section{Buyi Lei Gong Paozhi Bianlan 補遺雷公炮製便覽 \\ (Lei Gong's Guide to Drug Preparation with Addenda)}

Buyi Lei Gong paozhi bianlan is conserved in the rare book collection of the library of the China Academy of Chinese Medical Sciences. It was published in the 19th year of the Wanli reign period (1591) of the Ming dynasty. It contains an exquisite collection of painted illustrations. There is no preface or postscript, and no information about the identity of the creators. But from the style of the paintings, we can assume that it was the work of imperial court artists. The complete book runs to $14 \mathrm{ce}$ 冊 (separately bound volumes).

According to Xiao Yongzhi 肖永芝, there are altogether 1133 colour illustrations. While the primary focus is on morphology, many of them also depict the location, cultivation, collection and processing of medicinals (Fig. 8),



Figure 1.8 Buyi Lei Gong paozhi bianlan 補遺雷公炮製便覽 (Lei Gong's Guide to Drug Preparation with Addenda). Drug processing with Lei Gong at the centre of the image. 1591. 
as well as folkways, myths and legends (See Xiao, Chapter 14, in this volume, p. 209).

Bencao Tupu 本草圖譜 (Illustrated Register of Materia Medica), Illustrated by Zhou Hu 周祜, and Zhou Xi 周禧 at the End of the Ming, Captions Provided by Zhou Rong 周榮 (160o-86)

The library of the China Academy of Chinese Medical Sciences holds two ce 冊 (volumes), which contain 14 and 15 meticulously painted colour illustrations respectively. The 29 extant illustrations are mainly of plant and animal medicinals. The National Library of China possesses three $c e$ 冊, containing 43 illustrations altogether. According to Zheng Jinsheng, the illustrations in this work were copied from originals in Jinshi kunchong caomuzhuang 金石昆蟲草木狀 (Description of Minerals, Insects and Plants).

Jinshi Kunchong Caomu Zhuang 金石昆蟲草木 狀 (Description of Minerals, Insects and Plants), 1620. Illustrated by Wen Chu 文俶. Taiwan National Central Library.

According to Zheng Jinsheng (2003), this book is divided into $12 \mathrm{ce}$, without juan numbers. The categories are identical to those in Bencao pinhui jingyao. Except for the tables of contents and picture captions, there is no written text. Altogether it contains 1,315 exquisitely painted illustrations, among the finest to survive from the late Ming.

\section{Qing Dynasty (1644-1912) Materia Medica Illustrations}

After the publication of Li Shizhen's 李時珍 Bencao gang$m u$, there were no further comprehensive Materia Medica publications in the Qing period, other than those aimed at a popular readership. In the mid to later Qing a considerable quantity of mediocre Materia Medica were published, largely without illustrations. Perhaps the most important work of the latter half of the dynasty was Wu Qijun's 吳其 濬Zhiwu mingshi tukao 植物名實圖考 (Illustrated Survey of Plants and Plant Names).

Zhiwu mingshi tukao 植物名實圖考 (Illustrated Survey of Plants and Plant Names) in 38 juan.

Twenty-eighth year of the Daoguang 道光 reign period of the Qing dynasty (1848)

The text includes 1,714 medicinals, mostly with one-to-one paired texts and illustrations. A small number of entries have multiple illustrations. According to Shang Zhijun et al. (1989), the book contains a total of 1,805 illustrations. The vast majority were taken from sketches made directly from life, and were therefore much more accurate than those in previous Materia Medica works, which frequently copied from one another.

Other notable illustrated Materia Medica from the Qing include Guo Peilan's 郭佩蘭 Bencao hui 本草匯 (Anthology of Materia Medica), 1655, Zhang Yunzhong's 張雲中 Jinghuiwuse tu zhu bencao gangmu 精繪五色圖注本草綱 目 (Bencao gangmu with Fine Five-Colour Illustrations), 1655, and the Yaohui tukao 藥會圖考 (Illustrated Survey of Drugs) printed by Yu Fengtang 裕豐堂.

\section{Acu-Moxa Therapy Illustrations}

There are many legends about the origins of acupuncture and moxibustion therapy. Some attribute the creation of these therapies to the legendary culture bringer and first of the Three August Sovereigns Fu Xi 伏羲, who, like Sheng Nong 神農 the Divine Farmer, is said to have 'tasted the flavours of the 100 medicinal drugs [to determine their efficacy], and manufactured the Nine Needles'. ${ }^{23}$ The Yellow Emperor corpus, the locus classicus for a culturally distinct Chinese medicine, places another of these legendary culture bringers, the Yellow Emperor himself, in consultation over technical matters in medicine with Qi Bo 岐伯 or Lei Gong 雷公 (Duke Thunder) and other interlocutors. Bo Gao 伯高 and Shao Yu 少俞 are also recorded as debating partners of the Yellow Emperor in the preface to the Zhenjiu jiayi jing (AB Canon of Acupuncture and Moxibustion). ${ }^{24}$ We understand their rhetoric to have a normative function in establishing standard meanings in medical theory and practice, rather than reflecting a process of contestation, although it is clear there were many competing ideas at the time that these works were written.

By the Eastern Han period $\left(25^{-220} \mathrm{CE}\right)$, there were many attempts to systematise medical theory and some consensus was emerging about the acupuncture and moxibustion body among scholars in different centres of learning throughout the empire. This is evident in the three recensions of the Huangdineijing 黃帝內經 (Yellow Emperor's Inner Canon). The received versions of the Suwen and Lingshu recensions of this text were, however, edited extensively, and were not printed until almost 1000 years later, so we cannot be certain about the exact shape of the early compilations. These texts record a network of body channels and vessels, and contain descriptions of some 300 strategic locations for the application of needles and moxa, as well as various surgical-style instruments for

\footnotetext{
23 Xu Zongyuan (ed.) [3rd century] 1963, Diwang shiji 帝王世紀, p. 5. On the figure of Fu Xi, see Lewis 1999, pp. 197-209.

24 Huangfu Mi [3rd century] 1996, Zhenjiu jiayijing, 'Preface', p. 16.
} 
treatment. There is also an extensive literature aimed at prohibiting treatment at certain locations on the body at certain times.

Excavations of Han dynasty tombs over the past 40 years have provided a new set of sources with which we have been able to move beyond legend, and piece together the medical history of the early imperial period. In 1972 medical manuscripts dating to the Eastern Han dynasty were discovered at Hantanpo 旱灘坡 in modern Wuwei 武威 prefecture, Gansu province. They are the earliest extant testimony to the therapeutic use of fine needles at strategic points on the body. They also provide contemporary evidence of the importance of ideas about the movement of the spirits in the body, and the need to protect their free flow. The safeguarding of this delicate physiology of the body was at the origin of many of the subsequent and enduring medical prohibitions (See Lo and Tlalim, Chapter 19 in this volume). In 1973 silk manuscripts excavated from the Mawangdui tomb site, in modern Changsha, Hunan province, provided new evidence from the earlier Western Han dynasty of a system of 11 channels of the body, and their relation to a method of taking the pulse at various parts of the body to diagnose the condition of those channels. Some similar texts were discovered at a near-contemporary tomb at the Zhangjiashan 張家山 site, north of the Yangzi river, closed 18 years earlier than the Mawangdui tomb. In these texts, we first see evidence of the practice of normalising the movement of Qi in the body using a needling stone. Recent finds at the Tianhui 天回 tomb site at Laoguanshan 老官山, in the suburbs of the city of Chengdu in Sichuan, have provided much more evidence of the circulation of medical texts in subsequent decades. It is noteworthy that none of these manuscripts contains any illustration of the channels. However, material culture evidence from tombs in Sichuan includes two black lacquered figurines covered with fine lines painted in red, which have stimulated much speculation about their significance in the development of channel theory. One of those two figurines, found in Tianhui tomb $\mathrm{M}_{3}$, uniquely lists the viscera on its back. ${ }^{25}$

\section{The Earliest Records of Acu-Moxa Illustrations}

With so many manuscripts from Qin, Han, Sui and Tang dynasties lost to posterity, it is hard to establish the date of the earliest illustrated acu-moxa manuscripts with any accuracy. Nevertheless, evidence from catalogues and bibliographies leaves tantalising testimony to the existence of illustrations. The bibliographic treatise of the imperial library included in Hanshu 漢書, the standard history of the Eastern Han period, lists seven medical classics (Yijing

25 See Lo, Introduction and Chapter 3 in this volume. qijia 醫經七家), totalling 216 scrolls/juan; however, it does not explicitly list any illustrations. All of the works on the list are now lost, except for the aforementioned Yellow Emperor's Inner Canon, although it is far from clear how the Han title relates to the surviving recensions.

During the period we know as the Wei, Jin and Northern and Southern Dynasties (220-589), there is clear evidence of illustrated manuscripts. In the inaugural year of the Yuanhui 元徽 reign period of the Liu-Song dynasty (473), Wang Jian 王儉, aide to the director of the Palace Library, submitted to the emperor a privately produced catalogue, the Qizhi 七志 (Seven-category bibliography), which included Tupu shu 圖譜書 (illustrated registers), a rubric that likely referred to sets of illustrations with explanatory texts. This catalogue is no longer extant. A subsequent catalogue of texts compiled by Ruan Xiaoxu 阮孝緒, the Qi lu 七錄 (Seven-Part Catalogue), lists tupu in both the inner and the outer sections of the records. The original text is now lost, but portions were appended to the bibliographical treatise of Suishu 隋書 (History of the Sui Dynasty), 636. From the latter we know that Ruan Xiaoxu's catalogue contained the following acupuncture and moxibustion illustrations:

Kongxue hama tu 孔穴蛤蟆圖 (Illustrated Toad Canon with [Acupoint] Cavities) in three juan;

Yance tu 偃側圖 (Supine and Lateral Charts) in eight juan;

another copy of Yance tu in twojuan.

The Kongxue hama jing illustrations are no longer documented with this title by the Liang Dynasty (502-57), but surviving Japanese illustrations testify to the endurance of the tradition. ${ }^{26}$ (See Lo and Tlalim, Chapter 19 in this volume, pp. 286-8) The latter two texts survive in fragmentary form.

The 'Zaying' 雜應 (Miscellaneous Responses) chapter of Ge Hong's 葛洪 Jin period (265-420) Baopuzi 抱朴子 mentions the book Mingtang liuzhuyance tu 明堂流注偃 側圖 (Illuminated Hall Supine and Lateral Human Figure Chart of the Flow), which states:

Many doctors treat illnesses with acupuncture but in their moxibustion [sic] practice, they don't have a clear understanding of the measurements of the locations. In this they cannot compare with the former doctors who studied the Illuminated Hall Supine and Lateral Human Figure Chart of the Flow, so what can they know about it?

These texts are the earliest recorded illustrated works on acupuncture and moxibustion.

The bibliographic treatise included in the History of the Sui is a summative work recording texts from the Eastern

$26 \quad$ Lo 2001. 
Han dynasty to the early Tang. Of illustrated acu-moxa texts it records the following:

\begin{tabular}{|c|c|c|}
\hline $\begin{array}{l}\text { Mingtang kongxue tu } \\
\text { 明堂孔穴圖 }\end{array}$ & $\begin{array}{l}\text { The Illuminated Hall } \\
\text { Acupoint Chart }\end{array}$ & $\begin{array}{l}3 \\
\text { juan }\end{array}$ \\
\hline $\begin{array}{l}\text { Huangdi mingtangyan } \\
r e n t u \text { 黃帝明堂偃人圖 }\end{array}$ & $\begin{array}{l}\text { The Yellow Emperor's } \\
\text { Illuminated Hall Supine } \\
\text { Man chart }\end{array}$ & $\begin{array}{l}12 \\
\text { juan }\end{array}$ \\
\hline $\begin{array}{l}\text { Mingtang hama tu } \\
\text { 明堂蝦蟆圖 }\end{array}$ & $\begin{array}{l}\text { The Illuminated Hall } \\
\text { Toad Chart }\end{array}$ & 1 juan \\
\hline $\begin{array}{l}\text { Zhenjiu tu yaojue } \\
\text { 針尒圖要訣 }\end{array}$ & $\begin{array}{l}\text { Illustrated Rhymes on } \\
\text { Acu-Moxa }\end{array}$ & 1 juan \\
\hline Zhenjiu tujing 針尒圖經 & $\begin{array}{l}\text { Illustrated Canon of } \\
\text { Acu-Moxa }\end{array}$ & $\begin{array}{l}11 \\
\text { juan }\end{array}$ \\
\hline Shier ren tu 二人人圖 & 'The 12' Human Chart & 1 juan \\
\hline $\begin{array}{l}\text { Bian Que yance zhenjiu tu } \\
\text { 扁鵲偃側針多圖 }\end{array}$ & $\begin{array}{l}\text { Bian Que's Supine and } \\
\text { Lateral Acu-Moxa Charts }\end{array}$ & $\begin{array}{l}3 \\
\text { juan }\end{array}$ \\
\hline $\begin{array}{l}\text { Yance ren jing 偃側人經 } \\
\text { by Qincheng zu 秦承祖 }\end{array}$ & $\begin{array}{l}\text { Supine and Lateral Hu- } \\
\text { man Canon }\end{array}$ & $\begin{array}{l}2 \\
\text { juan }\end{array}$ \\
\hline $\begin{array}{l}\text { Huangdi shi'er jingmai } \\
\text { Mingtang wuzang ren tu } \\
\text { 黃帝十二經脈明堂五 } \\
\text { 藏人圖 }\end{array}$ & $\begin{array}{l}\text { Yellow Emperor's Chart } \\
\text { of the 12 Jingmai and the } \\
\text { Illuminated Hall Five } \\
\text { Viscera }\end{array}$ & 1juan \\
\hline
\end{tabular}

Tragically, all these acupuncture illustrations have disappeared. From the Baopuzi and Ruan Xiaoxu's 阮孝緒 Qi $l u$, we know that by the Wei, Jin and Northern and Southern Dynasties period (220-589), there were already many illustrated acupuncture and moxibustion manuscripts and charts in circulation. But since understanding the distribution of manuscripts and books and their readership is a work in progress, each work remains to be studied in its own right in order to gauge the time between its first publication and its reaching a wider audience, if indeed it ever did. From the historical records we can deduce that acupuncture illustrations had already appeared around the Han period (2O2 BCE-22O CE), and by the Jin period $(265-420)$ at the latest, but await much more research to know what impact they had.

\section{Acu-Moxa Illustrations from the Tang Dynasty}

Huangdineijing mingtang 黃帝內經明堂(Yellow Emperor's Inner Canon: Illuminated Hall), also known as Huangdi neijing mingtang leicheng 黃帝內經明堂類成 (Yellow Emperor's Inner Canon: Illuminated Hall Classified) was compiled by Yang Shangshan 楊上善 ( $f l$. Sui-Tang) by imperial order. It was published in 619, on the cusp of the Sui and Tang dynasties. In his preface, Yang Shangshan states:

I have taken each of the 12 channels [as the theme] for the chapters [juan], and the qujing ba mai 奇經八脈 (Eight Extraordinary Vessels) for one chapter, totalling 13 chap- ters... the Taisu 太素 (Great Basis recension of the Yellow Emperor's Canon) states its purpose and the Mingtang 明 堂 makes its form visible.

The first chapter, on 'Hand Tai Yin', survives only in fragmentary form, but according to the 1826 Keiseki hōkoshi 經 籍訪古志 (Bibliography of Rare Chinese Classics in Japan):

This Hand Great Yin channel of the Lung, the image of the lung organ, its route via the channel to the acupoints are all described and annotated, explanations of the point terminology, illness treated, extremely fine and detailed. ${ }^{27}$

Qian Chaochen 錢超塵 has made an exhaustive study of Huanggdineijing mingtang, but has been unable to discover any extant acu-moxa illustrations. ${ }^{28}$

\section{Mingtang Renxing Tu 明堂人形圖 (Illuminated} Hall Charts of the Human Body) and the Mingtang Zhenjiu Tu 明堂針炎圖 (Illuminated Hall Acu-Moxa Charts)

The Mingtang renxing tu was composed during the early Tang dynasty by Zhen Quan 甄權, and published during the Wude 武德 reign period (618-26). From a simple analysis of the title it is clear that the subject of the charts was channels and points, and that it included both illustrations and text, but the most significant part of the work was its illustrations. This was a popular production, which circulated widely between the Sui and early Tang dynasties. Sun Simiao's 孫思貌 (581-682) Qianjin yifang千金翼方 (Supplementary Prescriptions Worth a Thousand Gold Pieces), 682, records the circumstances of transmission. Government officials regularly transcribed Zhen Quan's Illuminated Hall charts, and recorded the circumstances of their circulation throughout China. The original text was lost early on, but much of its content was included in Sun Simiao's 孫思遥 Qianjin yifang千金翼方, juan 26, which contains the text but not the illustrations.

The Mingtang zhenjiu tu was based on Zhen Quan's Mingtang renxing $t u$. It was put together by a state-appointed editorial team comprising Director of Palace Provisions (shaofu 少府) Li Xiyu 李襲譽, Gentleman for Rendering Service (chengwu lang 承務郎) Sima Deyi 司馬 德逸, Imperial Physician (taiyi ling 太醫令) Xie Jiqing 謝 季卿, and Zhen Liyan 甄立言, an official from the Court of Imperial Sacrifices (taichang cheng 太常丞). The complete work with its illustrations intact was lost early on, but the text is partially preserved in the first section of juan 29 , 'Mingtang sanren tu' 明堂三人圖 (Illuminated Hall Three Figure Chart), of Sun Simiao's Qianjin yaofang (Essential Prescriptions Worth a Thousand Gold Pieces), 652. When 
describing the illustrations (which are not preserved in extant versions of juan 29), Sun Simiao states:

According to the Mingtang zhengjing 明堂正經 (Regular Channels of the Illuminated Hall), the human body is 7 chi 尺, 6 cun 寸, and 4 fen 分 in stature and in making the chart now this was halved so that the length of the body [represented on the chart] is 3 chi, 8 cun and 2 fen. The points are therefore half the [real] distance apart from each other so that 5 fen are equivalent to 1 cun. The 12 channels are done in five different colours; and the Eight Extraordinary Vessels are coloured in green. The three figures altogether have $65^{\circ}$ points, and the pictures are appended to facilitate understanding. The supine figure has 282 points, the posterior has 194 points, the lateral has 174 points. In total there are 349 point names, of which 48 are single acupoints, and 301 are paired acupoints.

From Sun Simiao's description, it is evident that the Mingtang zhenjiu tu contained polychrome painted illustrations. It is the earliest known coloured acu-moxa chart. In addition to Sun Simiao's account, the official preface to the chart is cited in juan 39 of Wang Tao's 王壽 Waitai miyao, 'Mingtang xu' 明堂序 (Preface to the Illuminated Hall). The publication of the officially compiled Illuminated Hall Acu-Moxa Chart influenced the conduct of Tang corporal punishment. The 'Record of Punishments' of the Xin Tangshu 新唐書 recorded that:

In the second year of the Zhen Guan 貞觀 reign period (628), Taizong 太宗, having viewed the Mingtang zhenjiu $t u$, saw that the five viscera were all close to the back [of the body]... and decreed that offenders should not be flogged on the back. ${ }^{29}$

\section{Acu-Moxa Illustrations in the Dunhuang Manuscripts $^{30}$}

Perhaps the earliest extant Mingtang acu-moxa illustration is preserved in a fragmentary manuscript from Dunhuang designated by the modern title Xinji beijijiujing 新集備急 炎經 (Newly Compiled Canon of Emergency Moxibusion Remedies) and held at the Bibliothèque nationale de France under the accession number Pelliot $2675 \cdot{ }^{31}$ As noted above, it was copied by Fan Ziying 範子盈 and 'Yin-Yang' Fang Jingxun 汇景詢 in 861.

Also from Dunhuang and currently held in the British Library under catalogue nos Or.821o/S.6168, S.6262 are two further moxibustion chart fragments which have no title, authors, or date of copying (Fig. 9). This work cannot be matched exactly to anything recorded in the received

29 Ouyang Xiu [106o] 1986, Xin Tangshu (56), 428o.

$30 \quad$ Ma Jixing 1994; Lo in Lo and Cullen 2005; Despeux 2005.

$31 \quad$ Zhang Nong 1994.



Figure 1.9 A section of Dunhuang Or.8210/S.6168 showing a popular treatment for Nanzi wulao qishang 男子五勞七傷 (Five Wearinesses and Seven Damages in Men)

tradition. In the text describing the seventh figure, the character xie 泄 has missing strokes. This was a way of complying with the taboo against recording the name of the reigning emperor, in this case Li Shimin 李世民, and may date the copy to the first year of the Tang dynasty, although dating on this basis is far from reliable. The date of the original production of the charts is unknown. Of the set of images, only five are undamaged, although there appear to be the remains of nine more. By collating the images with the texts that were written alongside them, one can determine that there were originally 18 figures. The texts record names of illnesses, moxa point locations and the number of moxibustions to be administered. Anterior and posterior views of the whole body record the exact bilateral locations of the treatment spots so one can visualise the characteristic point combinations. It appears that the charts represent popular remedies and perhaps the summative experience of individual practitioners. The texts record many names of moxibustion locations that are not known in the received tradition, and point names not yet seen in acu-moxa texts, for example banmei 板 眉 (Wooden Brow), jiao wuzhou 腳五舟 (Foot Five Boats) wumen 天門 (Heaven's Portal), nieshu 聶俞 (Connection to $\mathrm{Nie}$ ). ${ }^{32}$ These non-standard aspects of the charts hint at the broad spectrum of Tang-period moxibustion traditions that are not to be found preserved in received texts.

Song,Jin and Yuan Dynasties Acu-Moxa Illustrations The acu-moxa illustrations in the Song Taiping shenghui fang 太平聖惠方 (Taiping ['Great Peace' Era] Formulas of Sagely Grace) are some of the most important depictions

32 Lo in Lo and Cullen 2005, pp. 237-9; Lo 2010. 
of acu-moxa channel and network vessel theory from that period.

During the early Northern Song period, Hanlin 翰林 Medical Officer Wang Huaiyin 王懷隱 received an imperial decree from Taizong 太宗, second emperor of the Song dynasty, to direct Wang You 王佑 and Zheng Qi 鄭奇 in the compilation of the Taiping shenghui fang, the first official formulary of the Song dynasty. The complete text consists of 10o juan, of which the last two contain a large number of acu-moxa illustrations. It has been argued that the 12 figures depicted in the 99th volume were based on information in Zhen Quan's 甄權 Zhenjing chao 針經鈔 (Collected Writings on Acupuncture), and Shan Tiao's 山眺 Zhenjiujing 針尒經 (Acu-Moxa Canon). ${ }^{33}$ Figures 1-4 are of supine figures; $5^{-8}$ are images of a person lying prostrate and 9-12 are lateral images. These 12 figures showing the supine, prostrate and lateral positions are a further development in the Illuminated Hall genre of illustration. In the 12 human figures, the acupuncture points are linked to their names by single black lines. The style shares many similarities with that of the moxibustion illustrations from the Dunhuang manuscripts, particularly the second figure, where the hair is painted as two buns, one on either side of the head, just as it is in the Pelliot 2675 fragment.

\section{Tongren Shuxue Zhenjiu Tujing 銅人腧穴針炎圖 經 (Illustrated Bronze Man Canon of Acupuncture and Moxibustion).}

This title is recorded in the Medical Category of the 'Zibu' 子部 section of the bibliographical treatise of the History of the Song (Songshu 宋書, comp. 488), juan 207. It records the name Wang Weiyi 王惟一 against the entry:Xin zhu tongren yuxue zhenjiu tujing 新鑄銅人腧穴針尒圖經 (Newly Cast Bronze Man Illustrated Canon of Aupoints and Acu-Moxa) in three juan. Starting from the appearance in the Jin dynasty of the Xinkan buzhu tongren shuxue zhenjiu tujing 新刊補注銅人腧穴針多圖經 (New Bronze Man Acupoint and Acu-Moxa Illustrated Canon with Supplementary Annotations), we begin to see the emergence of a Tongren shuxue zhenjiu tujing 銅人腧穴針炎圖經 (Bronze Man Acupoint and Acu-Moxa Illustrated Canon).

In the first year of the Tiansheng 天聖 reign period of the Northern Song period (1023), the Emperor Renzong

because the transmission of acupuncture techniques had not been unified, commanded Wang Weiyi 王惟一, chief steward of the Palace medical service (shangyao fengyu 尚 藥奉禦), to clarify where the Qi points and the channels came together, to cast two bronze figures, compiling and correcting the narratives of old and correcting errors, and

Liu Lianlan and Sang Xiaoning 1999. to compile Tongren shuxue zhenjiu tujing 銅人腧穴針炎 圖經 (Bronze Man Acupoint and Acu-Moxa Illustrated Canon) in three juan. ${ }^{34}$

The three juan of Xin zhu tongren yuxue zhenjiu tujing were duly completed in 1026, and the casting of the two bronze figures was completed in the following year. The Xin zhu tongren yuxue zhenjiu tu actually consists of notes and explanations of the Tiansheng Bronze Man 天聖銅人 in texts and graphics. In the historical study of acu-moxa, this text was the first to provide independent introductions to the renmai 任脈 (Controller Vessel) and dumai 督脈 (Governor Vessel). It was thus of great importance in establishing the basic theories of the 14 channels.

The complete Xin zhu tongren yuxue zhenjiu tujing has long since vanished, but it is partially extant in a Ming woodblock print from Shulin zongwen tang 書林宗文堂. The book contained illustrations of figures in supine and in prone positions, and further anterior and prostrate views showing the zangfu 臟腑 (organs and bowels), as well as charts of the 14 channels. The illustrations in this text are examples of relatively well-preserved acu-moxa illustrations.

\section{BeijiJiufa 備急炎法 (Moxibustion Techniques for Emergencies)}

Beiji jiufa is a well known text compiled by Wenren Qinian 聞人耆年 in the second year of the Baoqing 寶慶 reign period of the Southern Song period (1226).

Biographical data on Wenren Qinian tells us that having studied medicine since childhood, when he began to practise he employed techniques both for nourishing life and for treating disease. In his latter years he felt dissatisfied with methods for curing acute diseases, deeming acu-moxa the most beneficial and effective. Given that acupuncture skills were not easy to impart, he was a proponent of moxibustion as the easiest and most effective technique to learn. He dedicated his work to discovering simple but efficacious moxibustion formulas for treating acute diseases. The text attributed to him is a compilation of moxibustion techniques, which contains illustrations of point locations. 'Beiji jiufa' is included in an edition of Zhenjiu zeri bianji 針炎擇日編集 (Compilation of the Selection of Auspicious Days for Acu-Moxa) published by Shanghang Luoshi 上杭羅氏 (Mr Luo of Shanghang) in the 16th year of Emperor Guanxu's reign (189o), which is now held in the library of the China Academy of Chinese Medical Sciences. According to that edition, Wenren Qinian's text contained 16 illustrations of point selections for the treatment of various conditions including chang yong 腸 tongkao 文獻通考. 
癰 (intestinal abscess), ding chuang 疔瘡 (boils and sores), pifu zhong dufeng 皮膚中毒風 (wind toxin striking the skin), cubao xintong 卒暴心痛 (sudden heart pain), nishui 溺水 (drowning), ziyi 自縊 (self-strangulation), houbi 喉痹 (throat impediment), biniu 鼻血丑 (nosebleed), nan chan 難 產 (difficult delivery) and yaotong 腰痛 (lower back ache).

Shisi Jing Fahui 十四經發揮 (Explanations of the 14 Channels)

Shisijing fahui, edited by the Yuan physician Hua Shoubian 滑壽編, was completed in the first year of the Zhizheng 至正 reign period of the Yuan dynasty (1341), and printed in three juan at the beginning of the Ming period.
During the compilation of this text Hua Shoubian consulted a range of sources on the 14-channel system including Hu Tai's 忽泰Jinlan xunjing 金蘭循經 (Golden Orchid Tracing the Channels), as well as two treatises from different recensions of the Yellow Emperor's Inner Canon: the 'Ben shu' 本輸 (Fundamental acupoints), Lingshu 2 and 'Gukong lun' 骨空論 (Treatise on bone cavities), Suwen 6o. Hua Shoubian added detailed instructions to this received knowledge. The text contains illustrations of supine and prostrate measurements, as well as black and white woodcut print illustrations of the 14 channels. Other works containing illustrations of acu-moxa published during this period included the following:

\begin{tabular}{|c|c|c|c|}
\hline Wang Zhizhong王執中 & $\begin{array}{l}\text { Zhenjiu zisheng jing } \\
\text { 針炎資生經 }\end{array}$ & Life-Promoting Acu-Moxa Canon 1220 & 1220 \\
\hline Zhuang Chuo 莊綽 & $\begin{array}{l}\text { Jiu gaohuang shuxue fa } \\
\text { 㞺膏育腧穴法 }\end{array}$ & $\begin{array}{l}\text { Acupoint Method for Moxibustion on } \\
\text { the gaohuang point } 1128\end{array}$ & 1128 \\
\hline Dou Guifan賔桂芳 & Zhenjiu sishu 針尒四書 & The Four Books of Acu-Moxa 1312 & 1312 \\
\hline $\begin{array}{l}\text { He Ruoyu } \\
\text { 何若愚 }\end{array}$ & $\begin{array}{l}\text { Ziwu liuzhu zhenjing } \\
\text { 子午流注針經 }\end{array}$ & $\begin{array}{l}\text { Stem and Branch Flow Acupuncture } \\
\text { Canon }\end{array}$ & $\begin{array}{l}\text { Jin period } \\
\left(1115^{-1234}\right)\end{array}$ \\
\hline
\end{tabular}

A number of formula works synthesised entire medical texts and also contained acu-moxa illustrations.

\begin{tabular}{|c|c|c|c|c|c|}
\hline $\begin{array}{l}\text { Tamba no } \\
\text { Yasuyori } \\
\text { 丹波康賴 }\end{array}$ & Ishimpō & 醫心方 & $\begin{array}{l}\text { Formulas of the Heart of } \\
\text { Medicine }\end{array}$ & $\begin{array}{l}\text { Citing the Chanjing 產經, 'Shimai tu'十 } \\
\text { 脈圖 (Illustration of the } 10 \text { vessels) }\end{array}$ & 984 \\
\hline Zhao Ji 趙佶 & Shengji zonglu & 聖濟總錄 & $\begin{array}{l}\text { Comprehensive Record of } \\
\text { Sagely Beneficence }\end{array}$ & & 1118 \\
\hline \multirow[t]{4}{*}{$\begin{array}{l}\text { Yan Yonghe } \\
\text { 嚴用和 }\end{array}$} & $\begin{array}{l}\text { Yanshijisheng } \\
\text { fang }\end{array}$ & $\begin{array}{l}\text { 嚴氏濟生 } \\
\text { 方 }\end{array}$ & $\begin{array}{l}\text { Mr Yan's Lifesaving Rem- } \\
\text { edies }\end{array}$ & & 1253 \\
\hline & $\begin{array}{l}\text { Wan'anfang } \\
\text { (Man-ampō })\end{array}$ & 萬安方 & $\begin{array}{l}\text { Remedies for Absolute } \\
\text { Peace of Mind }\end{array}$ & $\begin{array}{l}\text { Moxibustion method of the Four Flower } \\
\text { points (Jiu sihua xue fa 炎四花穴法) }\end{array}$ & 1331 \\
\hline & $\begin{array}{l}\text { Yongju shenmiao } \\
\text { jiujing }\end{array}$ & $\begin{array}{l}\text { 癰疽神妙 } \\
\text { 炎經 }\end{array}$ & $\begin{array}{l}\text { Classic of Marvellous } \\
\text { Moxibustion for Carbun- } \\
\text { cles and Abscesses }\end{array}$ & & 1561 \\
\hline & $\begin{array}{l}\text { Dan liao jiyan } \\
\text { mifang }\end{array}$ & $\begin{array}{l}\text { 澹寮集驗 } \\
\text { 秘方 }\end{array}$ & $\begin{array}{l}\text { Tranquil Hut Collection } \\
\text { of Tried and Tested Secret } \\
\text { Formulas }\end{array}$ & & 1283 \\
\hline $\begin{array}{l}\text { Chen Ziming } \\
\text { 陳自明 }\end{array}$ & Waike jingyao & 外科精要 & $\begin{array}{l}\text { Essentials of External } \\
\text { Medicine }\end{array}$ & $\begin{array}{l}\text { Including 'Riding the bamboo horse' } \\
\text { moxibustion method }\end{array}$ & 1263 \\
\hline
\end{tabular}


Acu-Moxa illustrations in the Ming and Qing periods Many newly composed acu-moxa texts dating to the Ming and Qing periods contained illustrations.

\section{Zhenjiu Daquan 針炎大全 (Complete Compendium} of Acu-Moxa).

Zhenjiu daquan was compiled by Xu Feng 徐鳳 in six juan in the fourth year of the Zhengtong 正統 reign period (1439), or according to Huang Longxiang 黃龍祥, between the Chenghua 成化 and Zhengde 正德 reign periods (1464-1521). ${ }^{35}$ The work is also known by the alternative titles of Xushi zhenjiu da quan 徐氏針炎大全 (Mr Xu's Complete Compendium of Acupuncture and Moxibustion) and Zhenjiu jiefa daquan 針炎捷法大全 (The Complete Compendium of Rapid Acu-Moxa Methods). This acumoxa text was compiled from a wide range of historical sources up to the middle Ming period, including acupuncture illustrations. If we go by the Ming woodblock print edition of Tongren Xushizhenjiu heke 銅人徐氏針尒合刻 (Mr Xu's Collected Bronze Man Sets, Sanduozhai 三多齊 Press, Jinling city, i.e. present day Nanjing), it contained the following illustrations:

Juan 3

'Zhoushen cun quzhi liangfa tu' 周身寸屈指量法圖, an illustration of a body measuring method using a bent finger;

'Zhoushen cun shenzhi liangfa tu' 周身寸伸指量法圖, another chart of a body measuring method using an outstretched finger.

Juan 5

acupoint chart of the flow of the 12 channels around the four limbs.

Juan 6

Mr Cui's 崔 chart of the four flower acupoints;

chart for the technique of treating the Gaohuang point;

two charts of the technique to treat the Kidney acupoint;

chart for the 'riding the bamboo horse' moxibustion technique;

chart for the technique of applying moxibustion to the Heart Qi acupoint.

\section{Zhenjiu Juying 針尒聚英 (A Glorious Anthology}

of Acupuncture and Moxibustion), in Four Juan

Compiled by Gao Wu 高武, 14th year of the

Zhengde 正德 Reign Period (1519)

Also known as Zhenjiujie yao juying fahui 針尒節要聚英 發揮 (Explanations of 'Collected Gems of the Essentials of Acu-Moxa'), edition engraved and published in Japan



Figure 1.10 Standing Bronze Man, Anterior View. Zhenjiu juying 針炎 聚英 (A Glorious Anthology of Acupuncture and Moxibustion), in four juan, compiled by Gao Wu (1519), juan 1. (c) Wellcome Library, London, Loo37496

(copy held in the library of the China Academy of Chinese Medical Sciences).

In the introduction to the book, the author states that he has compared many texts, including the Suwen recension of the Yellow Emperor's Inner Canon and the Nanjing 難經 (Classic of Difficult Issues), and that he has analysed different perspectives, taking into account the similarities and discussing the contradictions, hence the title 'A Glorious Anthology'. He also points out that the book's illustrations are based on Hua Shou's Shisi jing fahui and Hu Gongtai's Jinlan xun jing (see above). The first juan contains the following illustrations:

a chart of the viscera and bowels;

three charts of the anterior, posterior and lateral views of the Bronze Man;

body measurements in supine and prone positions;

middle finger body measurement;

14 charts of the acu-moxa points of the channels.

The three Bronze Man charts in this work display various unusual features. For example, the Tongren zhengmian $z h i l i$ 銅人正面直立 (Standing Bronze Man Anterior View) chart (Fig. 10) displays seven trajectories in black lines on 
the surface of the body of a single figure: the Hand Greater Yin Lung Channel, the Hand Jue Yin Heart Channel, the Foot Lesser Yin Kidney Channel, the Foot Greater Yin Spleen Channel, the Foot Jue Yin Liver Channel, and the Foot Yang Brightness Stomach Channel.

Zhenjiu Dacheng 針尒大成 (The Great Compendium of Acupuncture and Moxibustion), Also Known as Zhenjiu Daquan 針炎大全, Originally Compiled by the Ming-Dynasty Scholar Yang Jizhou 楊繼洲 in 10 Juan and Edited and Amended by Jin Xian 靳賢. The Woodblock Print Dates to the 29th Year of the Wanli 萬曆 Reign Period (1601)

The author compiled this text on the basis of a book that had been transmitted through a family lineage, Weisheng zhenjiu xuanjimiyao 衛生針炎玄機秘要 (Mysterious and Secret Essentials of Acupuncture and Moxibustion for Safeguarding Life); he also consulted 20 pre-Ming works on acu-moxa and combined them with his own experience as a practitioner. Apart from the tubiao 圖表 (illustrations and tables) there are a total of some 70 images inserted into the text, which are made up as follows:

over 20 images of segments of the body with their acumoxa point locations;

40 images of the whole body showing the acu-moxa point locations;

nine images of acupuncture instruments.

The illustrations include visualisations of the jing 井 'well' points of the 12 channels of the body, the yuan 原 (original) and luo 絡 (network) acupoint systems that treat the symptoms that lodge in the main channels, the acupoints on the qijing bamai 奇經八脈 (Eight Extraordinary Vessels) for treating illness, the anatomical positions of the organs and bowels, the channels displayed on supine and prostrate figures, and the essential acupoints that protect infants.

Leijing Tuyii 類經圖翼 (Illustrated Appendix to the [Yellow Emperor's Inner] Canon Arranged by Topic), Compiled in 11 Juan by Zhang Jiebin 張介賓 (1563-1640) in the 4th Year of the Tianqi 天啟 Reign Period (1624)

The content of juan 3 to 11 mainly relates to acu-moxa. The whole text contains over 5 o illustrations (not including tables) with most concentrated in juan 3 and 4. They comprise the following woodblock print charts:

whole body channel and network vessels;

segments of the body with their acu-moxa point locations the viscera and bowels; charts of the Neijing 內景 (inner landscape, see next section);

facial diagnosis;

the nine needles.

The illustrations are innovative in one respect. Whereas the limbs were normally drawn in outline and the acu-moxa point terms placed on top or linked with a line to the text, in Leijing tuyi the skeletal structure is also revealed. Four charts are notable for this feature: those of the three Hand Yin channels, the three Hand Yang channels, the three Foot Yin channels, and the three Foot Yang channels. While the focus is not on the anatomical structure of the body for its own sake (for what purpose could that have had in the $17^{\text {th }}$ century?), it is an effect that gives extra clarity to the location of the acupoints.

\section{Lingmen Chuanshou Tongren Zhixue 淩門傳授銅} 人指穴 (Finger Point Bronze Man of the Lingmen

Transmission), Channel Chart. Anon.

From the title we might surmise that the chart was associated with the lineage teaching associated with the Ming dynasty acupuncturist Ling Yun 淩雲 (style name Hanzhang 漢章; literary name Woyan 臥巖) from Gui'an 歸安 Shuanglin 隻林, in modern Zhejiang province.

During the reign of Emperor Xiaozong (1488-1505) Ling Yun responded to a proclamation inviting doctors to go to the capital and, having had his knowledge examined against the Bronze Man, was awarded the rank of imperial physician. ${ }^{36}$ Generations of Ling Yun's descendants inherited his knowledge though family apprenticeships and some later became famous acupuncture doctors: those we know about include Ling Xuan 淩宣, Ling Zaohu 淩藻 湖, Ling Chenhu 淩振湖, Ling Zhenhou 淩貞候 and Ling Qianyi 淩千一.

Currently the only extant manuscript copy of the chart is conserved in the library of the China Academy of Chinese Medical Sciences. It was produced just before the reign of the Kangxi emperor (1661-1722) of the Qing dynasty. The text contains 36 hand-drawn images, 14 of which are illustrations of the channels. The text also contains explanations and pedagogical tools such as the Liushiliu xue liuzhuge 六十六穴流注歌 (Chant of the Flow of the 66 Acupoints).

36 Mingshi 明史 (History of the Ming), Zhejiang tongzhi 浙江通 志 (Annals of Zhejiang), Guian xian zhi 歸安縣志 (Annals of Gui'an county). 
Yuzuan Yizong Jinjian 禦纂醫宗金鑒 (ImperiallyCommissioned Golden Mirror of Medical Learning), a Comprehensive Text on, and Practical Guide to, Medicine, Produced at the Imperial Academy of Medicine in the 7 th Year of the Qianlong 乾隆

Reign Period (1742) at the Request of the Emperor, Edited by Wu Qian 吳謙 in 15 Chapters (Pian 篇) Yuzuan zizongjinjian is an exquisitely illustrated compilation which deals with many branches of medicine. The acumoxa channel and point charts are mainly concentrated in the following three chapters, which contain more than 140 illustrations: 'Dingzheng shanghan lun zhu' 訂正傷寒 論注 (Revised and Annotated Treatise on Cold Damage), 'Waike xinfa yaojue' 外科心法要訣 (Chant of Skills and Knowledge for the Treatment of Trauma), 'Cijiu xifa yaojue' 刺炎心法要訣 (Chant of Skills and Knowledge for Piercing and Moxibustion).

This work contains the following charts:

six charts of the three Yang and three Yin channels;

eight charts of the six channel acu-moxa points for piercing and moxibustion techniques;

14 charts of the flow of the 14 channels;

12 charts of the 12 channels, their exterior and interior aspects and yuan and luo acupoints;

eight charts of the exchange and confluence acupoints of the eight channels;

16 charts of the flow of the eight channels;

36 charts of the form, flow routes and acupoints of the viscera and bowels;

16 charts of the segments [of the body] with their acu-moxa point locations;

two charts of the beginning and endings of various channels in supine and prostrate views;

28 illustrations on the treatment of body points for moxibustion for various kinds of diseases.

It is interesting that the costume of the characters illustrated in the images reflects a traditional style that pre-dates the Qing and Ming dynasties.

Yuzuanyizong jinjian is perhaps the first work to differentiate the two genres of channel-network charts and acupoint charts, identifying their unique features separately, so that we can begin to grasp the relationship between the two. ${ }^{37}$ This begs further analysis of what purposes the two styles of charts served (See Wu, Chapter 6 in this volume, Figs 6.7 and 6.13).

37 Huang Longxiang 2003, ch. 6, no. 5, 'Qingdai zhenjiu de jianyuhua' 清代針炎的簡約化 (Simplification of acupuncture in the Qing period), pp. 665-75.



Figure 1.11 Lingmen chuanshou tongren zhixue 淩門傳授銅人指穴 (Finger Point Bronze Man of the Lingmen Transmission), channel chart. Anon, early Qing copy. ( ) Library of the China Academy of Chinese Medical Sciences

Zhenjiu YiXue 針炎易學 (Acu-Moxa Made Simple) in Two Juan, Edited by Li Shouxian 李守先 and Published in the Third Year of the Jiaqing 嘉慶 Reign Period (1798)

The first juan contains eight illustrations of the qijing $b a$ mai 奇經八脈 (Eight Extraordinary Vessels), a chart of the Governing and Controlling channels of the head, one of the acupoints of the back, one of the acupoints of the abdomen, and 13 charts of the main acu-moxa points for ophthalmology. The second juan contains a chart of the 12 channels, and 24 charts of the Eight Extraordinary Vessels.

Zhengjiu Fengyuan 針尒逢源 (Encountering the Origins of Acu-Moxa) in Six Juan, Edited by Li Xuechuan 李學川 in the 22nd Year of the Jiaqing 嘉慶 Reign Period (1817)

The fourth juan of this text contains two charts of figures in supine and prone positions, 12 illustrations of the 12 channels, and eight illustrations of the Eight Extraordinary Vessels. The library of the China Academy of Chinese Medical Sciences has an original Qing dynasty block-print edition of this work. 
RentiJingmai $\mathrm{Tu}$ 人體經脈圖 (Chart of the Channels of the Human Body). Anon. This Volume Contains Polychrome Illustrations Dating to the Kangxi 康熙 Reign Period (1661-1722)

The text contains 24 coloured images. Twenty-two of them illustrate the flow of the channels and the remaining two are anatomical drawings. These are finely executed depictions of human figures. Their robes are draped so as to expose the body where the pathways of the channels are marked in red, with black characters recording the names of the acu-moxa points. This rare set of Qing Dynasty coloured images of the channels is currently held in the library of the China Academy of Chinese Medical Sciences.

Apart from medical compendia and large compilations which are not introduced here, Ming and Qing period works that contain acu-moxa illustrations include the following:

\begin{tabular}{|l|l|l|l|}
\hline Anon?* & $\begin{array}{l}\text { Yang Jingzhai } \\
\text { zhenjiu quan shu } \\
\text { 楊敬齋針炎 } \\
\text { 全書 }\end{array}$ & $\begin{array}{l}\text { The Complete Yang } \\
\text { Jingzhai Collection } \\
\text { of Acu-Moxa }\end{array}$ & 1591 \\
\hline $\begin{array}{l}\text { Xia Ying } \\
\text { 夏英 } \\
\text { Lingshujing mai } \\
\text { yi 靈樞經脉翼 }\end{array}$ & $\begin{array}{l}\text { Appendix on the } \\
\text { Channels to the } \\
\text { Numinous Pivot } \\
\text { Canon }\end{array}$ & 1497 \\
\hline $\begin{array}{l}\text { Zhang Sanxi } \\
\text { 張三錫 }\end{array}$ & $\begin{array}{l}\text { Jingluo kao } \\
\text { 經絡考 }\end{array}$ & $\begin{array}{l}\text { An Examination of } \\
\text { the Channels and } \\
\text { Networks }\end{array}$ & 1609 \\
\hline $\begin{array}{l}\text { Chen Hui } \\
\text { 陳會 }\end{array}$ & $\begin{array}{l}\text { Shenyingjing } \\
\text { 神應經 }\end{array}$ & $\begin{array}{l}\text { Classic of the Spirit } \\
\text { Responses }\end{array}$ & 1425 \\
\hline $\begin{array}{l}\text { Wu Yiding } \\
\text { 吳亦鼎 }\end{array}$ & $\begin{array}{l}\text { Shen jiujing lun } \\
\text { 神炎經綸 }\end{array}$ & $\begin{array}{l}\text { Treatise on the } \\
\text { Classic of Spirit } \\
\text { Moxibustion }\end{array}$ & 1851 \\
\hline $\begin{array}{l}\text { Jin Zhitian } \\
\text { 金治田 }\end{array}$ & $\begin{array}{l}\text { Jiufa michuan } \\
\text { 炎法秘傳 }\end{array}$ & $\begin{array}{l}\text { Secret Transmitted } \\
\text { Methods of Moxi- } \\
\text { bustion }\end{array}$ & 1883 \\
\hline
\end{tabular}

* The authorship of this text is controversial. The title means that Zhenjiu quanshu 針尒全書 was owned and passed down by Yang Jingzhai 楊敬齋; i.e. Yang was not the author, but the owner of a rare copy of this book.

\section{Conclusion}

There are numerous categories of pre-modern Chinese medical texts. The China Academy of Chinese Medical Sciences Library alone has a collection of more than 5,500 rare books, which are made up of more than 6o,ooo ce m (sections and treatises bound separately), many of which are illustrated. If one includes libraries both in and outside China, the rich variety of pre-modern texts, as described in this chapter, is estimated at some 10,00o. This chapter has simply given a brief introduction to the categories and chronologies of two of the most significant genres of medical illustration in China as a taster of the rich variety of subjects that are introduced in the pages that follow: diagrams for nurturing life, diagnostic illustrations, tongue diagnosis, pulse diagnosis, paediatric diagnosis, external medical treatments, illustrations of instruments for petty surgery and much more.

In the next chapter we will see how pre-modern black and white images of the viscera outline bowels and organs, placing them in relation to each other and giving depth and solidity to the imagination of their physiological roles and theoretical functions. The Lingshu recension of the Yellow Emperor's corpus makes it clear that:

The skin and flesh of a shi 士 (gentleman) who is 8 chitall can be measured at the exterior by palpating [the pulse]; upon his death it is permissible to dissect and observe him. ${ }^{38}$

夫八尺之士, 皮肉在此, 外可度量切循而得之, 其死, 可 解剖而視之.

Contrary to what is commonly presumed in teleological histories of medicine, there were no absolute prohibitions on cutting up and displaying the corporeal depths; and so the human being is laid bare in this volume, both for the purposes of gathering and transmitting scientific knowledge throughout the world, and for culturally specific religious meditations (see Figs 12, 13, 14).



Figure 1.12 Rear View of the Viscera in Man-ampō 萬安方 (Remedies for Absolute Peace of Mind), 1331, compiled by the Japanese author Kajiwara Seizen (1266-1337) after Yang Jie's (fl. 12th century) Cunzhen huanzhong tu 存真環中圖. (C) Library of the Needham Research Institute

38 Lingshu 12 (6 'Jingshui' 經水). 




Figure 1.13 Illustrations of the Lungs in Yifang leiju 醫方類聚 (Collection of Classified Medical Remedies), 1445. These images of the lungs most likely originate in the late Tang Dynasty Daoist school of Hu Yin's 胡愔 Huangting neijing wuzang liufu buxie tu 黃庭內景五藏六腑補瀉圖 (Diagram for the Replenishing and Draining of the Five Viscera and Six Bowels in the Inner Landscape of the Yellow Court). The fifth juan includes images of the Lungs, Heart, Liver, Spleen, Kidney, and Gallbladder. Each illustration skillfully depicts the form of the internal organs and describes Daoist medical ideas about how the six viscera guard the spirit and the beasts. For example, the illustration of the Lungs depicts six lobes linked to the trachea, and the guardian spirits include seven young boys and 14 jade maidens. The guardian animal is the Tiger. (c) Library of the China Academy of Chinese Medical Sciences



Figure 1.14 Gudaiyijia huaxiang 古代醫家畫像 (Portraits of Ancient Doctors) 1816. This work contains a set of portraits of six famous physicians and includes one juan of the Mingtang yangfu zangfu tu 明堂仰伏藏腑圖 (Illuminated Hall Prostrate and Prone Diagram of the Viscera and Bowels in the Body). This illustration represents a combination of the acu-moxa Illuminated Hall tradition of illustration described in the previous section, drawn together with diagrams of the five viscera and the bowels, depicting the Heart, Liver, Spleen, Lung, and Kidneys (viscera), and the Gallbladder, Stomach, Large Intestine, Small Intestine, Bladder and Triple Burner (san jiao 三角) (bowels). (C Library of the China Academy of Chinese Medical Sciences

The visual techniques and media described here facilitated new levels of global exchange, transcending language barriers. They open new windows on to the great diversity of ways in which physicians, artists, and administrators have imagined their contemporary realities and a Chinese medical past, inviting us to new encounters with ancient subjectivities, experiments with objectivity and understandings of realism, as well as fantastic extraterrestrial journeys into the unknown. 


\section{Bibliography}

\section{Primary Sources}

Anonymous 1661-1722, Renti jingmai tu 人體經脈圖 (Chart of the Channels of the Human Body). Held at Zhongguo zhongyi kexueyuan, Beijing.

Anonymous [1591] 2005, Buyi Lei Gong paozhi bianlan 補遺雷公炮 製便覽 (Lei Gong's Guide to Drug Preparation with Addenda), Shanghai: Shanghai cishu chubanshe.

Anonymous [17th century] 2015, Lingmen chuanshou tongren zhixue 淩門傳授銅人指穴 (Finger Point Bronze Man of the Lingmen Transmission), Beijing: Zhongyi guji chubanshe.

Chen Jiamo 陳嘉謨 [1565] 1988, Bencao mengquan 本草蒙鉒 (Beginner's Guide to Materia Medica), ed. Wang Shumin 王淑民 et al., Beijing: Renmin weisheng chubanshe.

Gao Wu 高武 [1519] 2006, Zhenjiu juying 針尒聚英 (A Glorious Anthology of Acupuncture and Moxibustion), Beijing: Renmin weisheng chubanshe.

Gui'an xian zhi 歸安縣志 (Annals of Gui'an County), compiled by Lu Xinyuan 陸心源 et al., finalised in 1882, press unknown.

Hu Sihui 忽思慧 [1330] 1986, Yinshan zhengyao 飲膳正要 (Principles of Correct Diet), Beijing: Renmin weisheng chubanshe.

Hua Shou 滑壽 [1341] 2011, Shisijing fahui 十四經發揮 (Explanations of the 14 Channels), ed. Li Yuqing 李玉清, Beijing: Zhongguo yiyao keji chubanshe.

Huangfu Mi 皇甫䍀 (3rd century), Diwang shiji 帝王世紀 (Genealogical Records of the Emperors and Kings). Modern edn: Xu Zongyuan 徐宗元 (ed.) 1964, Diwang shiji jicun 帝王世紀輯存 (Collected Edition of Genealogical Records of the Emperors and Kings), Beijing: Zhonghua shuju.

Huangfu Mi 皇甫䍀 [3rd century] 1996, Huangdi sanbu zhenjiu jiayijing $x u$ 黃帝三部鍼炎甲乙經序 (Preface to the Yellow Emperor's AB Canon of Acupuncture and Moxibustion in Three Parts), in Zhang Canjia 張燦玾 and Xu Guoqian 徐國仟 (eds), Zhenjiu jiayi jing jiaozhu 針炎甲乙經校注 (The Yellow Emperor's AB Canon of Acupuncture and Moxibustion, edited with notes), Beijing: Renmin weisheng chubanshe.

Ji Yun 紀昀 et al. (eds) 200o, Siku quanshu zongmu tiyao 四庫全書總 目提要, Shijiazhuang: Hebei renmin chubanshe.

Lan Mao 蘭茂 (1397-1470) 2000, Diannan bencao 滇南本草 (The Southern Yunnan Materia Medica), ed. Yu Naiyi 於乃義 et al., Kunming: Yunnan keji chubanshe.

Li Shouxian 李守先 (ed.) 1798, Zhenjiuyixue 針炎易學 (Acu-Moxa Made Simple). Modern edn: Gao Xiyan 高希言 et al. (eds) 2014, Zhenjiuyi xue jiaozhu 針尒易學校注 (Acu-Moxa Made Simple, edited with notes), Zhengzhou: Henan kexue jishu chubanshe.

Li Xuechuan 李學川 (ed.) [1817] 2012, Zhengjiu fengyuan 針炎逢源 (Encountering the Origins of Acu-Moxa), Beijing: Zhongguo yiyao keji chubanshe.

Liu Jia 劉甲 (ed.) 1185 , Jingshizhenglei beiji bencao 經史證類備急本草 (Classified Pharmacopoeia for Emergency Use, Based on Classics and Historical Sources), Tongzhou: Printed for Liu Jia.

Liu Wentai 劉文泰 [1503] 1982, Bencao pinhui jingyao 本草品彙精要 (Classified Treasury of Materia Medica), Beijing: Renmin weisheng chubanshe.

Ma Duanlin 馬端臨 [1307] 2011, Wenxian tongkao 文獻通考, Beijing: Zhonghua shuju, 2011 (14 vols).

Mingshi 明史 (History of the Ming) [1735] 1974, Beijing: Zhonghua shuju. Ouyang Xiu 歐陽修 [106o] 1986, Xin Tangshu 新唐書 (New History of the Tang), in Ershiwu shi (suoyinben) 二十五史 (縮印本) (The Twenty-Five Official Dynastic Histories [reduced edn]), Shanghai:
Shanghai guji chubanshe.

Shibue Chūsai 澀江抽齋 and Mori Risshi 森立之 [1826] 2014, Keiseki $H o ̄ k o s h i$ 經籍訪古志, ed. Du Zexun 杜澤遜 and Ban Longmen 班 龍門, Shanghai: Shanghai guji chubanshe.

Sun Simiao 孫思㝸 682, Qianjin yifang 千金翼方 (Supplementary Prescriptions Worth a Thousand Gold Pieces). Modern edn: Li Jingrong 李景荣 et al. (eds) 1998, Qianjin yifang jiaoshi 千金翼方 校釋 (Supplementary Prescriptions Worth a Thousand Gold Pieces, collated and annotated), Beijing: Renmin weisheng chubanshe.

Wang Huaiyin 王懷隱 et al. (eds.) [992] 1958, Taiping shenghuifang 太 平聖惠方 (Taiping ['Great Peace' Era] Formulas of Sagely Grace), Beijing: Renmin weisheng chubanshe.

Wen Chu 文俶 1620, Jinshi kunchong caomu zhuang 金石昆蟲草木 狀 (Description of Minerals, Insects and Plants). Held at Taiwan National Central Library, Taipei.

Wenren Qinian 闻人耆年 [1226] 2011, Beiji iiufa 備急炎法 (Moxibustion Techniques for Emergencies), Taiyuan: Shanxi kexue jishu chubanshe.

Wu Qian 吳謙 [1742] 2000, Yuzuan yizong jinjian 禦纂醫宗金鑒 (Imperially-Commissioned Golden Mirror of Medical Learning), Beijing: Renmin weisheng chubanshe.

Wu Qijun 吳其濬 [1848] 1957, Zhiwu mingshi tukao 植物名實圖考 (Illustrated Survey of Plants and Plant Names), Shanghai: Shangwu yinshuguan.

Xu Feng 徐鳳 [1439] 1987, Zhenjiu daquan 針炎大全 (Complete Compendium of Acu-Moxa), Beijing: Renmin weisheng chubanshe.

Yang Jizhou 楊繼洲 (ed.) [16o1] 2006, Zhenjiu dacheng 針炎大成 (The Great Compendium of Acupuncture and Moxibustion), Beijing: Renmin weisheng chubanshe.

Zhang Cunhui 張存惠 (ed.) 1249, Chongxiuzhenghejingshizhenglei beiyong bencao 重修政和經史證類備用本草 (Newly Revised Materia Medica of the Zhenghe Period [1111-8o], Annotated, Arranged by Categories, and Organised for Practical Use, Based on the Classics and Historical Works), Pingyang: Huiming xuan.

Zhang Jiebin 張介賓 [1624] 1965, Leijing tuyi 類經圖翼 (Illustrated Appendix to the [Yellow Emperor's Inner] Classic Arranged by Topic), Beijing: Renmin weisheng chubanshe.

Zhang Yanyuan 張彥遠 [847] 1987, Lidaiminghuaji 歷代名畫記 (Record of Famous Paintings Through the Ages), in Siku quanshu:Zibuyilei 四庫全書.子部㙯類, 812, Shanghai: Shanghai guji chubanshe.

Zhejiang tongzhi 浙江通志 (Annals of Zhejiang), compiled by Shen Yiji 沈翼機 et al., finalised in 1682, press unknown.

Zhou Hu 周祜 and Zhou Xi 周禧 (eds) 17 th century, Bencao tupu 本草 圖譜 (Illustrated Register of Materia Medica). Held at Zhongguo zhongyi kexueyuan, Beijing.

Zhu Su 朱橚 1406, Jiuhuang bencao 救荒本草 (Materia Medica for Famine Relief). Modern edn: Ni Genjin 倪根金 (ed.) 2008, Jiuhuang bencaojiaozhu 救荒本草校注 (Collation and Annotation of Materia Medica for Famine Relief), Beijing: Zhongguo nongye chubanshe.

\section{Secondary Sources}

Bray, F., V. Dorofeeva-Lichtmann and G. Métailié (eds) 2007, Graphics and Text in the Production of Technical Knowledge in China: The Warp and the Weft, Leiden: Brill.

Despeux, C., tr. P. Barrett, 2005, 'Visual representations of the body in Chinese medical and Daoist texts from the Song to the Qing period (tenth to nineteenth century)', Asian Medicine: Tradition and Modernity 1.1, 10-52.

2010 Médecine, religion et société dans la Chine médiévale: Etude de manuscrits chinois de Dunhuang et de Turfan, Paris: Institut des Hautes Etudes Chinoises, Collège de France.

Gale, E.M. (tr. and ed.) [1931] 1967, Discourses on Salt and Iron:A Debate 
on State Control of Commerce and Industry in Ancient China, chapters I-XIX, Taipei: Ch'eng-wen.

Harper, D. 1998, Early Chinese Medical Literature: The Mawangdui Medical Manuscripts, London/New York: Kegan Paul International.

Huang Longxiang 黃龍祥 2003, Zhongguo zhenjiu shi tujian 中國針炎 史圖鑒 (An Illustrated History of Acupuncture and Moxibustion in China), Qingdao: Qingdao chubanshe.

Hunansheng bowuguan 湖南省博物館 and Hunansheng wenwu kaogu yanjiusuo 湖南省文物考古研究所 (eds) 2004, Changsha Mawangdui er san hao hanmu: diyijuan tianye kaogu fajue baogao 長沙馬王堆二、三號漢墓: 第一卷田野考古發掘報告 (Han Dynasty Tombs 2 and 3 at Mawangdui, Changsha: Archaeological Field Report, vol. 1), Beijing: Wenwu chubanshe.

Jin Shiying 靳士英 1994, 'Wuzang tukao'五臟圖考 (A study of charts of the five Zang viscera), Zhonghua yishi zazhi 24.2, 68-74.

Lewis, M.E. 1999, Writing and Authority in Early China, Albany: SUNY.

Liu Lianlan 劉蓮蘭 and Sang Xiaoning 桑曉実 1999, 'Qiantan lidai jingluo tu de bianqian' 淺談歷代經絡圖的變遷' (On historical changes in channel charts), Shanghai zhenjiu zazhi 18.6, 37-8.

Lo, V. 2001, 'Huangdi Hama jing (Yellow Emperor's Toad Canon)', Asia Major 14.2, 61-99.

2005, 'Quick and easy Chinese medicine: the Dunhuang moxibustion charts', in Lo and Cullen (eds), 211-51.

2010, 'Manuscripts de Dunhuang et de Khotan sur la moxibustion', in Despeux (ed.) 2010, vol. 1, 239-84.

2014, How to do the Gibbon Walk: A Translation of the Pulling Book (c.186 BCE), Cambridge: NRI. E-book, available at: http://www. nri.org.uk/yinshu.pdf.

Lo, V. and C. Cullen (eds) 2005, Medieval Chinese Medicine:The Dunhuang Medical Manuscripts, London/New York: RoutledgeCurzon.

Ma Jixing 馬繼興 2005, Chutuwangyiguyijiyanjiu 出土亡佚古醫籍研 究 (A Study of Archaeologically Recovered Medical Texts), Beijing: Zhongyi guji chubanshe, 2005.

Mawangdui Hanmu boshu zhengli xiaozu 馬王堆漢墓帛書整理小組 (ed.) 1979, Mawangdui Hanmu boshu daoyin tu 馬王堆漢墓帛書 導引圖 (Silk Daoyin Chart from the Mawangdui Han Burial Site), Beijing: Wenwu chubanshe, including an introductory article by
Tang Lan 唐蘭, 'Shilun Mawangdui sanhao Hanmu chutu daoyin tu' 試論馬王堆三號漢墓出土導引圖 (On the daoyin chart excavated from Mawangdui Han Dynasty tomb 3). 1985, Mawangdui Hanmu boshu 馬王堆漢墓帛書 (Silk Manuscripts from the Mawangdui Han Dynasty Tombs), Beijing: Wenwu chubanshe.

Mo Gong 墨公 2000, Yingyin Shiwu bencao caihuiben $x u$ 影印《食物 本草》彩繪本序 (Preface to the photographic facsimile of Materia Dietetica with illustrations in colour), in Gongting xieben shiwu bencao 宮廷寫本食物本草 (Materia Dietetica: An Imperial Court Manuscript), Beijing: Huaxia chubanshe, $1-4$.

Qian Chaochen 錢超塵 2006a, 'Yang Shangshan “Huangdi neijing mingtang leicheng” (canjuan) kaolüe’ 楊上善《黃帝內經明堂類 成》(殘卷) 考略, Jiangxi zhongyiyao daxue xuebao 1, 20-3.

2006b, 'Yang Shangshan "Huangdi neijing mingtang leicheng” (canjuan) kaolüe (xu)', 楊上善《黃帝內經明堂類成》 (殘卷) 考略 (續), Jiangxi zhongyiyao daxue xuebao 2, 14-19.

Shang Zhijun 尚志鈞, Lin Qianliang 林乾良 and Zheng Jinsheng 鄭 金生 1989, Lidai zhongyao wenxian jinghua 歷代中藥文獻精華 (Essential Chinese Drug Literature Through the Ages), Beijing: Kexue jishu wenxiaun chubanshe.

Sterckx, R. 2002, The Animal and the Daemon in Early China, Albany: State University of New York Press.

Wang Shumin 王淑民 2005, Appendix 2, in Lo and Cullen (eds), 374-434.

Wang Shumin and P. Barrett 2006, 'Profile of a daoyin tradition: the "Five Animal Mimes"', Asian Medicine: Tradition and Modernity $2.2,225-53$.

Zhang Nong 1994, 'Zhongguo cunshi zui zao de zhenjiu tu' 中國存 世最早的針炎圖, (China's earliest extant acu-moxa chart) Sheke zongheng 4, 41-2.

Zheng Jinsheng 鄭金生 1993, 'Tianbao danfang yaotu kaolüe', 《天寶 單方藥圖》 (A brief study of the illustrations for single-ingredient remedies of the Tianbao era) 考略', Zhonghuayishizazhi 23.3, 158-61. -2003, 'Mingdai huajia caise bencao chatu yanjiu' 明代畫 家彩色本草插圖研究, (A study of polychrome Materia Medica illustrations by Ming artists), Xin shixue 14.4, 65-118. 


\section{Part 1}

Mapping the Body: Space, Time and Gender 



\section{Picturing the Body in Chinese Medical and Daoist Texts from the Song to the Qing Period (1oth to 19th Centuries)}

\section{Catherine Despeux}

This chapter considers visual images ( $t u$ 圖) of the human body created in China over the thousand years from the Song to the Qing period in the contexts of medicine and Daoist internal visualisation. In particular, it takes a closer look at the relatively neglected field of forensic medicine, and the contested issue of dissection. By focusing on the context in which body images were produced, and their textual and intertextual environment, it aims to arrive at a clearer and more nuanced understanding of the ways in which these images were intended to be read. In the process, it will challenge culture-specific assumptions about the construction of the body and the functions of illustration.

Despite much common theoretical background, bodily representation in medical and Daoist texts differs in function and intent. Different spheres of knowledge came to be associated with distinct accounts of the body. For medicine in general, this was the description of the viscera and the channels and tracts through which Qi and humours flowed; for forensic medicine, it was the description of the skeleton; for Daoism, it was the symbolic description of the body as the spatio-temporal locus of a system of significant correspondences that permeated the natural and spirit worlds.

The examples that I will discuss here include charts and diagrams as well as more figurative illustrations, embracing various aspects of the body and processes that take place within the body or relate to it. Body imagery in fine art, though clearly relevant to the discussion, lies beyond the scope of the present chapter. The choice of period is in part dictated by necessity, as very few images of the body survive from before the last millennium, but it also reflects a huge expansion in the use of graphic representations $(t u)$ that took place in the Song.

Broadly speaking, the images fall into three categories, exemplifying three different approaches to the body: images of the whole body approached from the exterior, including gymnastic postures, locations on the body, somatic measurements, channels and tracts; images of the inside of the body, i.e. the internal organs and the skeleton (which raises issues regarding dissection); and images of the symbolic body, i.e. alchemical processes within the body and the true form of the allegorical body. They are almost always accompanied by text, and like text, they require to be read according to specific cultural codes. They reveal particular mental constructions of the body. They perform multiple functions, serving as proof of knowledge, teaching material, medium of transmission, memory aid, or graphic presentation of a text; and for the Daoists, manifesting the form of the true body.

\section{Images of the Whole Body}

Representations of the whole body fall into five subcategories: 1. illustrations of gymnastic movements; 2. illustrations of landmarks on the surface of the skin, i.e. acupuncture and moxibustion locations; 3 . depictions of the paths of the channels and tracts; 4. illustrations showing the positions of the bones and somatic measurements, intended as a guide to the configuration and relative proportions of constituent elements of the body; 5 . forensic charts of the body used for post mortem examinations.

The common feature uniting all these types of images is that the body - sometimes clothed and sometimes naked - is rendered in outline only, albeit with increasing sophistication. Elements inside the outline, if any, are confined to circles, black dots and simple lines. Measurements and proportions are not accurately reproduced. Often, though not always, the images bear captions.

\section{Representations of Gymnastic Movements (Daoyin 導引)}

The earliest known representations of gymnastic movements (daoyin) are contained in a silk scroll of the Han period (prior to $168 \mathrm{BCE}$ ), discovered in a tomb at Mawangdui 馬王堆, near Changsha (Hunan province), which is described in the preceding chapter. ${ }^{1}$ Thereafter, we find no further gymnastic images for more than 10 centuries, even though we know from bibliographic catalogues that they continued to be produced.

Extant examples from the Song and Ming periods such as Zhou Lüjing's 周履靖 Chifeng sui 赤鳳髓 (Bone Marrow of the Red Phoenix), late 16 th century, ${ }^{2}$ or Hu Wenhuan's 胡文煥 Shouyang congshu 壽養叢書 (Collected Texts on Longevity and Maintaining Life), late 16th century (see Fig. 1), are undoubtedly more sophisticated and more elaborate than their Han counterparts; nonetheless they follow the

\footnotetext{
1 See Lo, Introduction, p. 8; Wang, Chapter 1, pp. 39-40, Fig. 1.1, in this volume.

$2 \quad$ Translated in Despeux 1988.
} 




Figure 2.1 Gymnastic pose. From Shouyang congshu 壽養叢書 (Collected Texts on Self-Cultivation and Longevity) by Hu Wenhuan 胡文煥, late 17th century

same conventions and are codified in the same manner. The pictures are accompanied by text, either on the same page - above, beside or below the figure - or on the facing page, and they seem to be essentially didactic in intention, i.e. they provide the literate reader with a guide to the movements recommended to treat particular illnesses or to promote longevity. Most of the poses shown are static ones; some dynamic poses are suggested by depicting a single stage in the course of a movement, but they would be impossible to execute on the basis of the visual information alone. Here, the image is ancillary to the text; its function is to provide a schema enabling the user to orient his/her body in physical space.

Representations of Acu-Moxa Locations on the Body These are labelled diagrams, consisting of a schematic outline drawing of a naked human figure, with a head, torso and four limbs. Acupuncture and moxibustion points are indicated by black or white circles, which are linked by lines to captions outside the outline, giving the name of the point and/or its location in relation to a bodily landmark (e.g. '3 cun [Chinese inches] below the navel', 'on the inside of the lower knee'). The earliest surviving images of this kind are the moxibustion charts in the Tang medical manuscripts from Dunhuang, rediscovered in the early 2oth century



Figure 2.2 Moxibustion points. From Jiujing 炎經 (Moxibustion Classic), anon., Tang period

(Fig. 2). ${ }^{3}$ They differ from the image type discussed in the following section, where the points are shown strung out along lines representing the channels and tracts. ${ }^{4}$ These diagrams form a pendant to texts describing the points themselves, their location and their therapeutic uses. So far as we can tell, they served as a visual aid, complementing and supporting the oral transmission of medical knowledge and the practical training received from a master. They helped the apprentice physician to map the points on to physical space and to memorise their location.

\section{Representations of the Channels with Acu-Moxa Points} Acu-moxa charts, showing the channels with the points located on them, are reliably attested from the 6th century CE. In tandem with canonical texts, they performed both a didactic and a normative function. The famous Tang physician Sun Simiao 孫思貌 (581-682) writes in Qianjin yaofang 千金要方 (Prescriptions Worth a Thousand Gold Pieces), c. 652:

3 Most of them are now in the Stein collection (S.5737, S.6168, S.6262, P.2675); cf. Lo 2005. See also Lo and Yoeli-Talim, Chapter 19 in this volume, pp. 276--277, Figs $19.4 \mathrm{a}$ and b.

4 Images of this type appear, for example, in Zhenjiu zisheng jing 針炎資生經 by the Song author Wang Zhizhong 王執中 and in Zhenjiu jing 針炎經, an anonymous text of the Song period. 




Figure 2.3 Jing fahui 十四經發揮 (Exposition of the 14 Channels) by Hua Shou 滑壽 (13th century). (C) Library of China Academy of Chinese Medical Sciences



Figure 2.4 Composite chart of the channels and tracts. From Zhenjiu dacheng 針炎大成 (Compendium of Acupuncture and Moxibustion) by Yang Jizhou 楊繼洲, 1601

by various other such projects in the course of the Song and Ming periods. ${ }^{8}$

The illustrations that have come down to us include both diagrams of individual channels and composite charts offering an overview of several channels, as in Zhenjiu Dacheng 針炎大成 (Compendium of Acupuncture and Moxibustion), 16o1, by Yang Jizhou 楊繼州 (see Fig. 4). ${ }^{9}$ Each figure is drawn in a posture designed to show the course of the channel with maximum clarity, according to whether it runs along the inside or outside of the limbs, for example. at the Imperial College and for official examinations. It should be noted that the Bronze Man opened up to reveal a set of wooden viscera within. ${ }^{7}$ This initiative was followed

\footnotetext{
5 Beiji qianjin yaofang, juan 29, p. 508; see also Despeux 1985, p. 134.

$6 \quad$ See also Goldschmidt 2004.

7 See Ma 1993.
}






\section{Representations Showing the Positions of the Bones and Somatic Measurements}

Whereas the three types of representations discussed above have existed since antiquity, somatographic charts providing information about the relative proportions of the parts of the body, and the distances between them, are a relatively recent phenomenon. One of the earliest known charts of this kind can be dated to the 14th century. ${ }^{10}$ From that time onward, we begin to see images of the body with inscriptions inside the outline, giving the names of the bones and their measurements according to the two relevant chapters of the Huangdineijing 黃帝內經 (The Inner Classic of the Yellow Emperor). ${ }^{11}$ Such images became increasingly common in the late Ming (17th century). ${ }^{12}$ These somatographic charts show a two-dimensional arrangement of a series of terms, which are also given in the accompanying text. They are clearly not to be read as a literal rendering of visible objects, but as a kind of mnemonic route-map facilitating the acquisition of knowledge.

\section{Images of the Body as Forensic Documents}

Forensic body charts consisted of a schematic outline drawing of a human body, to be filled in with notes on injuries, diseased parts etc. Charts showing the back and front view of the body were included in case documents, and used in drawing up death certificates. These charts were first circulated in 1211 at the suggestion of Xu Sidao 徐似道, a judge from Jiangxi. Initially, they were printed and distributed in Hunan and Guangxi. ${ }^{13}$ They were subsequently revised by the Minister of Punishment, who had them disseminated throughout the legal circuits.

Shortly after this, we find an edict in the early 14 th-century Yuandian zhang 元典章 (Compendium of Statutes and Sub-Statutes of the Yuan Dynasty) establishing a standard

10 It is entitled Yangren chicunzhi tu 仰人尺寸之圖 (Image of Measurements on the Recumbent Person) and is found in Shisi jingfahui 十四經發揮 by Hua shou 滑壽.

11 Huangdineijing, Lingshu 靈樞, ch. 14, ‘Gudu pian’ 骨度篇 (Measurements of the Bones); Suwen 素問, ch. 6o, ‘Gukong lun’ 骨空 論 (Interstices of the Bones).

12 See for example: Leijing tuyi 類經圖翼 by Zhang Jiebin 張介 賓 (1624); Sancai tuhui 三才圖會 (1607) by Wang Qi 王圻 and Wang Siyi 王思義, and the engravings edited in 1731 by Wuying dian 武英殿.

13 Cf. Jianyan zhengbei renxing 檢驗正背人形圖 by Xu Sidao 徐 思道. These models are described in the Southern Song period, in Qingyuan tiaofa shilei 慶元條法事類. They are not the earliest known representations of the corpse; this work is predated by Jianyan gemu 檢驗格目 by Zheng Guangshang 鄭光尚 (see Jia Jingtao 1983, p. 7).



Figure 2.5 Model representation of the body used in forensic medicine. From Yuandian zhang 元典章 (Compendium of Statutes and Sub-Statutes of the Yuan Dynasty), 1322

format for the documents to be completed and submitted by the coroner (Fig. 5).

For the examination of injuries to the corpse, a standard format already exists. Nowadays, the officials who govern the people have no regard for human life... . After deliberation, we have instituted two engravings of the dead body, a front view and a back view. We command the government of each circuit ( $l u$ 路) to print a reproduction of the master copy and to mark [the reproductions] with a sign. After checking that the seals and inscriptions correspond [to the original], [the government of the $l u$ ] will send the copies to the prefectures and sub-prefectures, which will set up an archive where they will keep them locked away. If there should be [a case requiring] the examination of a corpse, the precise time [of death] will immediately be established, and notification will be sent to the nearest official Bureau that it is not otherwise engaged. The latter will, as a matter of urgency, designate someone to convey the official documents and will also assign a senior official, who will be accompanied by an employee and a forensic expert; the official will bring three copies of the picture of the corpse (shizhang 屍帳) that was received previously and will proceed rapidly to the place where the corpse is laid out... . He will describe the [state of the] entire body. As for any injuries to the corpse, he will record on the previously drawn [engraving of the] body, in the places corresponding to the injured parts, whether the wounds are wide or narrow, deep or superficial. ${ }^{14}$

A master copy of these engravings, dated 1304, is preserved in the Yuan Statutes..$^{15}$ The images themselves are crudely

\footnotetext{
14 See Ratchewsky 1937, vol. 4, p. 224.

15 Yuandian zhang, juan 43 on the Minister of Punishment, pt 5 on homicide.
} 
executed and devoid of captions, but they are prefaced by a list of the main parts of the body that had to be examined and reported on. In the Qing period, the Bureau of the Ministry of Punishment had in its possession an official annotated edition of Xiyuan lu 洗冤錄 (Treatise for Washing Away Injustice), which incorporated the engravings. ${ }^{16}$ This work re-appears as an appendix to Da Qing lüli 大清 律例 (Code of the Great Qing), in a version made in 1740 with captions above the images rather than beside them. As we have seen, these forensic images served a clearly defined purpose; though they indubitably facilitated the transmission of knowledge, their primary function was as official and legal documents.

\section{Inside the Body: the Organs and Skeleton}

\section{Internal Organs and Dissection}

The late Five Dynasties (mid-1oth century) and Song periods saw the rise of an image type that does not set out to depict the body as a self-contained whole with head, trunk and limbs outlined; instead the torso and internal organs are drawn without any surrounding contours. These images are sometimes accompanied by details of individual viscera.

The torso is shown from three angles: from the front, back and side. At first sight, it is not recognisable as a human body at all, although its identity becomes clear once one notices the rather realistic internal organs. The captions and accompanying texts are very helpful, if not indispensable, in reading the image.

In most cases, we know the circumstances in which the images were produced and the purpose for which they were intended, i.e. they were created by official request in the context of dissections. In this regard they are comparable to anatomical drawings made in the West. However, one cannot but be struck by the diversity of the finished products.

In China, dissection had far less impact than it did in the West - albeit at a rather late date. In fact, the first Western anatomical illustrations showing the human body are crudely executed and rather naïve. ${ }^{17}$ Dissection only really developed with the rise of the school of Padua in the 16th century. The Church is often unjustly accused of preventing dissections, but this was by no means the case. If anything, it played the role of facilitator in the development of anatomical knowledge. In a papal bull issued in 1472 , Pope Sextus IV acknowledged anatomy as

Lüliguan jiaozheng Xiyuan lu 律例館校正洗冤錄 in juan 4, dated 1694 (ed. Fu Sinian).

17 Grmek and Bernabeo 1997, pp. 10-11. a discipline 'useful to medical and artistic practice'. The teaching of dissection, hitherto merely tolerated, was formally authorised by Pope Clement VII (r. 1523-34), after which dissection became more generally practised..$^{18}$ Thus the 16th century marked a turning point in the knowledge of the body in Europe, thanks to an increase in dissections, coupled with increasingly sophisticated representational techniques, as displayed in the drawings of da Vinci, for example. Throughout the century, 'new relationships between painting and nature, precise observation and a demand for accuracy played a vital role both in anatomy and in natural history'. ${ }^{19}$

It is often said that dissections were rarely carried out in China because Confucian society attached such high importance to keeping the body intact, considering entry into the otherworld with a mutilated body to be an act of filial impiety, which might have all manner of evil consequences. This does not seem to me to be an adequate explanation. If dissection had been seen as particularly useful, in the first place more dissections could have been performed on condemned criminals (a documented practice); and in the second place, people would have overcome their scruples, leading to a general shift in attitudes.

The earliest recorded dissection in China took place in $16 \mathrm{CE}$, during the reign of Wang Mang 王莽, when a certain Sun Qing 孫慶 was captured and executed. According to the biography of Wang Mang, he personally ordered Shang Fang 尚方, a physician of the Imperial Academy (taiyi 太 醫), to have the corpse dissected by skillful butchers, in order to measure the size and capacity of the organs, and to trace the course of the vessels ( $m a i$ 脈) with slivers of bamboo to find out where they began and ended, so as to be able to treat illnesses more effectively. ${ }^{20}$

Thus it seems that Chinese people were aware, from antiquity, of the advantages that could accrue to medicine from improved knowledge of the body, and of the progress that could thereby be achieved in treating disease. Nonetheless, we have no definite evidence of any further dissections until 1041, even though it is likely that others took place in the intervening centuries. For instance, Hua Tuo 華佗 (?-208? CE), the personal physician of Cao Cao 曹操, father of the future ruler of Wei, may well have carried out dissections, ${ }^{21}$ and if so, he would presumably have done so in an official capacity.

\footnotetext{
18 Ibid., p. 8.

19 Ibid., p. 11.

20 Hanshu, juan 99, biography of Wang Mang王芒, p. 4,145.

21 A text entitled Hua Tuo neishi 華陀內視 is listed in the Liang catalogue (see Suishu, juan 34, p. 1,041). In the opinion of Ma Jixing, it is the work of one of Hua Tuo's disciple, and neishi sig-
} 
In 1041, 56 rebels led by Ou Xifan 歐希范 were captured and put to death by Du Qi 杜杞, the Imperial Commissar (anfushi 按撫史) charged with pacifying the circuits of Guangnan and Guangxi. He had their abdomens dissected and directed an official of the Prefecture called Wu Jian 吳簡 (or Lingjian 靈簡 according to some sources) to commission artists to make drawings of the internal organs. These were published as OuXifan wuzang tu 歐希範五臟 圖 (Images of the Five Viscera of Ou Xifan). These events are described by several contemporary writers; ${ }^{22}$ and Shen Gua 沈括 (1033-97) criticises the drawings and points out an error in them, ${ }^{23}$ implying that he actually saw them. This dissection, like the one ordered by Wang Mang in the Han period, was carried out by official command on executed war criminals. The crucial difference is that it was attended not only by doctors but also by officials and artists, who were required to draw the internal organs. Regrettably the drawings have not survived.

Another series of dissections took place in the Chongning 崇寧 era (1102-6) under similar circumstances, and once again anatomical drawings were made. In Sizhou 泗州 (in the modern province of Jiangsu), a group of rebels were executed, and the Prefect Li Yuxing 李夷 行 summoned doctors and artists to examine the bodies. They dissected the tissues, removed the diaphragm and sketched the curves and meanders of the inside of the body. They were allowed to examine and draw everything in detail. Yang Jie 楊介, a famous doctor from Sizhou, ${ }^{24}$ known to have treated the Emperor Huizong (r.1101-25), ${ }^{25}$ corrected the engravings from the original drawings and pronounced them far superior to those created from the

nifies 'observe and examine the form of the internal organs', indicating the practice of dissection. See Ma Jixing 199o, p. 136.

The sources are: Dongzhai jishi, juan xia; Mengqi bitan, juan 26, art. 480, pp. 827-9; Yanxia fangyan, ed. in Shuofu, juan 29, vol. 5; Bintuilu ed. in Shuofu, p. 234, where the official is named as Lingjian 靈簡 of Yi Zhou; Jutan $l u, 3^{2-33}$. On all these sources, see Okanishi 1958, p. 297.

He writes: 'Human beings are said to have three orifices in the throat: one for fluids, one for solid food and the third one for air. This is completely wrong. The true images of the five viscera of Ou Xifan, which are widely transmitted, do indeed contain drawings of three orifices. Probably the examination was not carried out thoroughly enough. Solid and liquid foods are actually swallowed simultaneously, so how could they possibly enter the gullet separately? In fact, the human body has only two orifices [in the throat]: the larynx (yan 咽) and the pharynx ( hou 喉). The pharynx is the conduit for liquid and solid foodstuffs, and the larynx allows air to enter...'. Mengqi bitan, juan 26, art. 48 o, pp. $827-9$.

24 See Gujin yitong daquan, juan 1. Compare Xu wenxian tongkao.



Figure 2.6 Representation of the trunk and its organs according to Yanluozi. From Xiuzhen shishu 修真十書 (Ten Books on Cultivating Perfection), c. 1250. DZ 263, juan 18

Ou Xifan dissections. ${ }^{26}$ In the preface to Yang Jie's Cunzhen huanzhong tu 存真環中圖 (1118) by Jia Weijie 賈偉節 of Luoyang, we read:

Yang Jie, styled Jilao 吉老, observed and drew the actual form of the five viscera. He examined the depictions of the viscera made by Yanluozi 煙蘿子, arranged and amended them; he then added the 12 channels and entitled his work Cunzhen huanzhong 存真環中. ${ }^{27}$

These images by Yanluozi - the 'Master of the Smoke Curtain', who was active during the Tianfu era $(936-41)$ of the Later Jin dynasty, ${ }^{28}$ are preserved in the 13 th-century Daoist encyclopaedia Xiuzhen shishu 修真十書 (Ten Books on Cultivating Perfection), c. 1250 (see Fig. 6). ${ }^{29}$ There are six altogether, including, notably, a front view and a back view of the trunk and its viscera. Their titles contain the term neijing 內境 (internal view, inner landscape, inscape), and they were presumably intended for people who practised internal visualisation techniques (the images are accompanied by a text by Yanluozi on inward contemplation, neiguan 內觀). We know nothing of the background to the creation of these images, which are exactly reproduced in an illustrated commentary on Bashiyi nanjing 八十一難 經 (The Classic of 81 Difficult Issues [in the Inner Canon of the Yellow Emperor]) by Li Jiong 李駰 (1269). ${ }^{30}$ They coincide with Yang Jie's engravings in all respects but one,

26 This is recorded in Junzhai dushu houzhi 郡齋讀書後志, juan 2, 31a-b (in Siku quanshu, 674, pp. 405-6).

27 Miyashita Saburo 1968, pp. 148-9.

28 See Chen 1963 , p. 284.

29 DZ 263, Xiuzhen shishu, juan 18, 2a-3b.

30 Huangdi bashiyi nanjing zhuyi tuxu lun 黃帝八十一難經注義 圖序論 preserved in the Daoist Canon, DZ 1024. 




Figure 2.7 Representation of the trunk and its organs. From Hua Tuo xuanmen neizhao tu 華陀玄門內照圖（Hua Tuo's Images for Internal Visualisation According to the Mystery School) 13th century

which is however extremely significant: the liver is shown on the left, in keeping with the theory of correspondences with the Five Agents and Five Directions, whereby the liver corresponds to 'spring' and 'the left'. Yang Jie placed the liver on the right after attending dissections; this is one of the amendments to which Jia Weijie alludes in the preface quoted above.

It is worth noting that a text entitled Zhu tidian neijing lun 朱提點內境論 (Treatise on the Internal View [of the Body] According to Intendant Zhu) appears in Daozang directly after Yanluozi's work. The unknown author, who probably lived during the Song period, prior to 1250, seems to have seen examples of anatomical drawings of dissected bodies. In his treatise, he states that the therapeutic location for the liver is on the left, but the liver itself is located on the right (qi zhi zai zuo, qi wei zai you 其治在 左, 其位在右). He draws a comparison with the images of Yanluozi, which he says tally with his own observations. He adds that the bodies of criminals have recently been dissected and examined in the marketplaces, making it possible to ascertain that the throat does not, as erroneously supposed, contain three orifices, one for fluids, one for solid food and one for air. ${ }^{31}$

Yang Jie's images of the viscera were incorporated into a version of Hua Tuo xuanmen neizhao tu 華 陀玄門內照圖 (Hua Tuo's Images for Internal Visualisation According to the Mystery School), published by Sun Huan 孫煥 in 1273 (see Fig. 7). This work was composed in 1095 by Shen Zhu 沈銖, a proofreader in the Imperial library and archives, and subsequently appeared under various titles. Though no editions survive from before the Ming dynasty, they become numerous from that period onward, indicating that the text was widely read. Wang Huling 汪琥苓 of Wu (Suzhou) writes in his preface of 1668 :

For a long time, this work was conserved in the Palace, and few people were able to consult it. But in the Jiajing era (1522-66), an administrative assistant of the Imperial Academy of Medicine named Zhu Yuguo 周與國 made a copy of it, which he kept secretly at his home. From his grandchild Zhu Daozhou 周道州, I obtained a copy which was in poor condition and not easily legible. The book was in four juan, the first two containing Hua Tuo's work, and the second two containing additions by Guo 郭 and $\mathrm{Yu}$ 禹. ${ }^{32}$

It should be noted that Yang Jie's images were republished in Japan in 1304, in Ton-isho 頓醫鈔 by Kajiwara Seizen 椲原性全.33

In these images, unlike those of Yanluozi as reprinted in Nanjing tuyi, the liver is shown in its actual location, on the right. The first part of the text seems to have been written as a commentary on the Ou Xifan engravings, which it criticises on three main counts.

The first criticism echoes that of Shen Gua: there are only two orifices in the throat, whereas the Ou Xifan engravings show three. ${ }^{34}$ We might note here that Shen Gua justifies his assertion by reasoned argument: it would be mechanically impossible to keep solid food and liquids apart, and therefore there cannot be two separate openings for them. The second criticism relates to the position of the liver: Hua Tuo's Images for Internal Visualisation According to the Mystery School states, without further explanation, that the liver is located on the right, in front of the right kidney and beside the stomach. ${ }^{35}$ The third criticism concerns the kidneys: in the engravings, they are shown higher in the body than the traditionally accepted location - instead of being low down and close to the sides, they are underneath the diaphragm, right next to the middle of the spinal column; and they are surrounded by a membrane with two vessels, the upper one connected to the heart and the lower one joining the two kidneys together.

Sometimes quite separately and sometimes in conjunction with these global representations of the trunk, we also find depictions of the individual viscera. They are attested in the Tang period (7th-1oth centuries), i.e. even earlier than the former type of image. The bibliographic

32 See the manuscript edition preserved in Zhongyang tushuguan 中央圖書館, Taiwan. See also Daozang jinghua, vol. 14-2.

33 In ch. 44 of this text, preserved in Neikaku bunko in Kyoto, we also find images of the 12 channels and the viscera after $\mathrm{Wu}$ Jian 吳簡.

34 Hua Tuo xuanmen neizhao tu, 7a.

35 Hua Tuo xuanmen neizhao tu, 8b. 
catalogue of the History of the Tang lists the titles of five sets of illustrations of the viscera, all of which are repeated in the Song bibliographies. ${ }^{36}$ Only one of them is still extant: Huangting wuzang liufu buxie tu 黃庭五藏六腑 補瀉圖 (Illustrations of the Reinforcement and Purging of the Organs According to the Tradition of the Yellow Court) by Hu Yin 胡愔 (preface dated 848), preserved in the Ming Daoist Canon. The author, a female Daoist of the Shangqing sect, states in her preface that she has consulted various depictions of the viscera. ${ }^{37}$ The text gives a rudimentary anatomical account of the viscera, based on the ancient medical texts, chiefly the late Han Nanjing 难经 (Classic of Difficult Issues). However this merely serves as an introduction to her main subject: the spirits which reside in the organs according to the Shangqing tradition and which the adept must visualise and inwardly nurture, to maintain good health and achieve longevity. Strangely enough, although the author is at pains to point out that she has consulted several depictions of the viscera, the existing version of the text contains no images.

During the Song period, drawings of individual viscera are associated with dissections and are found in conjunction with general views of the trunk and its viscera, especially those from Hua Tuo's Images for Internal Visualisation According to the Mystery School, which were recycled without major changes throughout the Ming and the Qing (see Fig. 8). ${ }^{38}$ These images are particularly elaborate in their treatment of the connections and junctions between organs, illustrating an attempt to reflect upon and reproduce physiological mechanisms.

These same images of the viscera reappear, with a few variations, at the end of the 17 th century in Yixue yuanshi 醫學原始 (Origins of Medicine) by Wang Honghan 王宏韓

36 These are: Wuse bangtong wuzang tu 五色傍通五藏圖 by the Tang author Pei Wangting 裴王廷, catalogued in Tangshu 唐 書, medical section (juan 59, p. 1,571), Chongwen zongmu 崇文 總目, juan 3, medical section 3, Tongzhi yiwenlüe 通志・藝文略 (with the author named as Pei Guangting 裴光廷), and Songshi yiwenzhi 宋史・藝文志; Wuse bangtong mingjian tu 五色傍通 明鑒圖 by Pei Yuanling 裴元靈 in one juan, listed in Chongwen zongmu and Tongzhi yiwenlüe, with the author named as the Tang Daoist Pei Xuanling 裴玄靈; Wuzang bangtong daoyang tu 五臟傍通導養圖 by Sun Simiao 孫思俘, listed in Bishusheng que shumu 祕書省. 閣書目, Tongzhi yiwenlüe and Songshi yiwenzhi; and Huangting neijing wuzang liufu tu 黃庭 內景五藏 六腑圖, by the Tang author Hu Yin 胡愔, the only one to have survived.

37 DZ 432, Huangting wuzang liufu buxie tu 黃庭五藏六腑補瀉 圖, 2a.

38 See for example Sancai tu hui 三才圖會 (1607) and Leijing tuyi 類經圖翼 (1624) by Zhang Jiebin.



Figure 2.8 Representation of the lung. From Hua Tuo xuanmen neizhao tu 華陀玄門內照圖 (Hua Tuo's Images for Internal Visualisation) 13th century

( fl. 1688-92). Thus, despite the growth of dissection in the Ming period, and despite the spread of Western anatomical knowledge, rudimentary though this may have been, the format remained substantially unchanged right up until the beginning of the 19th century.

It was not until the early 19th century that the images were revised and various errors were amended in Yilin gaicuo 醫林改錯 (Corrections of Errors in the Forest of Medicine), 1822, in light of Western anatomical science and findings from further dissections (which had to be carried out in secret because, officially, they were still banned). The author, Wang Qingren 王清任 (1768-1831), embarked on his work only after 42 years of detailed observation. It contains a section on the viscera and their functions, illustrated by labelled diagrams. He states explicitly that greater knowledge of the internal organs is necessary to avoid clinical errors. He was present at several executions, and was able to examine the bodies. ${ }^{39}$ During a visit to Luanzhou (Hebei province) in the second year of the Jiaqing reign period (1797), he tells us that he saw the bodies of numerous children who had died in epidemics of measles and dysentery, as well as hundreds of disembowelled corpses in mass graves, their internal organs exposed to view. He says:

In 1799, he attended the execution of a woman by dismemberment. However, because of his gender, he was not allowed to approach the corpse, and had to view from afar the organs that the executioner held up to show him. In 1820 , he attended the execution of a man found guilty of matricide. Unfortunately, he writes, the diaphragm tore before he had time to examine it. See Unschuld 1985, pp. 212-13. 
I went past every day. At first, I had to hold my nose. But then, considering how the ancients committed errors in their treatment of the viscera because they had never seen them for themselves, I no longer sought to avoid the filth and stench. Early each morning, I made my way to the mass graves and closely examined the abdomens of the corpses. After the dogs had eaten their fill, there was generally nothing but the stomach and the intestines left; as for the liver and the heart, scarcely three in 10 remained intact. Thus in the course of 10 days, I was able to examine no less than 3 o corpses. ${ }^{40}$

It is significant that these dissections yielded only drawings of the viscera; there are no depictions of the skeleton, nor do we find any mention of the limbs or head. Undoubtedly, Chinese doctors tended to operate within the intellectual framework provided by the system of correspondences of the five agents, in which a crucial role is assigned to the 'five viscera and six bowels' as the functional organs which store or distribute the stuff of life. The muscles and tendons had little relevance to their clinical practice. Nor was the head of great interest, since the seat of the individual self was considered to be the heart. When, in the mid-19th century, the theory that the soul resides in the brain reached China from the West, it provoked much controversy in medical circles.

\section{The Skeleton in Forensic Medicine}

In the Chinese medical literature, there are no illustrations of bones, let alone the whole skeleton. The one exception is the spinal column; partly because it provides the armature to which the organs are attached, but partly also because it was seen as a repository of vital forces, since spinal fluid was considered to partake of the same nature as sperm and brain tissue. Therapy literature scarcely mentions the bones and skeleton, not even in relation to the treatment of fractures, which was not completely assimilated into the learned tradition until the $13^{\text {th }}$ century. Thus, references to the bones are essentially confined to the literature of acupuncture, where they function as landmarks, making it possible to locate the acu-moxa points and also, if required, to puncture the joints deeply without causing bone damage. In this context, which involved a practitioner acting upon the body from outside, some visual images were produced, but no actual drawings of the bones; as mentioned previously, bone names were simply inscribed on various parts of the body.

The first representations of the skeleton appear in the context of forensic medicine, where the examination of the skeleton figured largely. This eventually gave rise to sets of engravings of the skeleton that were incorporated into

40 Yilingaicuo. the Qing editions of the essential text of forensic medicine Xiyuan lu 洗冤錄 (Treatise for Washing Away Injustice) by Song Ci 宋慈. ${ }^{41}$ Reports from judges of fraud and negligence in the conduct of post-mortem examinations prompted the Qing government to intervene by decreeing a new official document representing the skeleton, aimed at reducing judicial errors (see Fig. 9).

Like the acupuncture charts, images of the skeleton were produced by order of the emperor. On the 19th day of the


(1770), an edict was proclaimed requiring the Minister of Punishment to have model engravings made for the forensic examination of the bones, and to arrange for them to be distributed in the various provinces. ${ }^{42}$ The resulting document consisted of a text entitled Jian guge 檢骨格 (Norm for the Autopsy of the Bones) with engravings for the autopsy of the bones (jiangutu 檢骨圖). The latter were probably printed in Wuyingdian 武英殿 (the Hall of Martial Valour) in the emperor's palace, which functioned as the imperial press. Four acupuncture charts, one including depictions of the viscera, had already been printed here in 1731; and in 1742, it saw the publication of Yizongyindian 醫宗金鑒 (The Bronze Mirror of Medicine), a medical encyclopaedia compiled by the Imperial Academy of Medicine, which is referred to in Qing commentaries on the Treatise for Washing Away Injustice.

This project was set in train by Zeng Fu 增福, a provincial judge from Anhui. Having noted that coroners' reports were often falsified, and in view of the rapid onset of putrefaction, he submitted a memorial to the emperor requesting the provision of a standard chart of the skeleton with the status of a legal document, to curb fraudulent practices. Apparently, Zeng Fu was personally entrusted with the task of devising the engravings and the accompanying text describing the skeleton, which he accomplished with the help of a team of experts:


Anhui received the imperial command to publish standard engravings for the autopsy of the bones, and to have them appended to the Treatise for Washing Away Injustice... . The plates are not the work of a single individual, and since each member of the team followed a particular master, opinions differed on some points. ${ }^{43}$

The earliest known exemplar was edited by a certain Wang Youhuai 王又槐 (1796) (Fig. 9). The bones are roughly drawn, and are separated from one another, giving the impression that they have been found scattered at a crime

\footnotetext{
41 See Xiyuan lu, ch. 29, juan 4.

42 See Chongkan buzhu xiyuan lu jizheng, juan 5, 15a-19b.

43 Preface to Xiyuan lu jie (1831), juan 6, 1.
} 




Figure 2.9 Xiyuan lu jizheng 洗冤錄集證 (Treatise on the Washing Away of Wrongs with Collected Evidence) by Wang Youhuai 王又槐 (1796). (c) Library of China Academy of Chinese Medical Sciences

scene and reassembled. There are captions written on the larger bones and beside the smaller ones, connected to them by lines. In some places, it is possible to see small white circles, which have been interpreted by later commentators as the locations of vital points, fatal or otherwise. Depictions of the skeleton were constantly refined in the course of the Qing, and from the mid-19th century, they begin to show the influence of Western styles of illustration. ${ }^{44}$

These engravings of the skeleton reappear in the 19th century in two texts on traumatology. In fact, from the late Ming onward, there was a gradual rapprochement between coroners (wuzuo 仵作) and physicians. ${ }^{45}$ Moreover, interest in precise knowledge of the skeleton began to extend beyond medical circles, to men of letters who associated with missionaries and physicians. One of these was Shen Tong 沈䑣, the author of a short treatise entitled Shigu 釋 骨 (Exposition of the Skeleton) composed between 1736 and 1782 , who was a friend of the renowned physician $\mathrm{Xu}$ Dachun 徐大椿. Nonetheless, it was forensic medicine that provided the motive force for the development of

44 On this subject, see Catherine Despeux 2007.

45 For instance, the physician Sun Yikui 孫一奎 (c. 1522-1619) incorporated the chapter from Xiyuan lu on the examination of bones into his Yizhi xuyu 醫旨緒餘 Influence in the opposite direction is shown by the increasing frequency with which Qing commentaries on Xiyuan lu cite from medical sources. descriptions and depictions of the skeleton, which made their belated appearance in the 18 th century.

The model engravings of 1770 soon came under criticism, eliciting written commentaries, exposing and correcting various errors, as well as new drawings. A gulf opened up between text and images. Over the next century, numerous commentaries on the Treatise for Washing Away Injustice were published, reproducing the engravings of 1770, while also criticising them and providing more accurate descriptions of the bones of the skeleton, based not only on the first-hand observations of coroners, but also on philological and terminological studies of the ancient texts with special reference to medicine. There was thus a constant interaction between image, personal experience and first-hand observation, and text.

\section{The Symbolic Body and Transformative Processes within the Body}

If the body is fundamental to medicine, it is just as central, if not more so, to Daoist practices. This is because the body is not only the microcosm in which the adept must create order, but also the habitation of the gods who must be mastered and meditated upon, and the site of the transformations that lead to immortality and spiritual fulfilment. Daozang 道藏 - The Daoist Canon, an anthology of Daoist texts compiled in 1445 - contains several images 
of the body, all symbolic and all dating from no earlier than the Song. ${ }^{46}$

\section{Alchemical Diagrams of Processes Taking Place Inside the Body}

I would like first of all to draw attention to a group of images involving a very familiar motif, which is however so habitually considered in a philosophical context as to have lost all associations with the body. I refer to the Taiji symbol (Taiji tu 太極圖, lit. Image of the Great Ultimate). The cosmological model of the Taiji became established in the Song period, giving rise to various types of images all based on the motif of the circle. ${ }^{47}$ In the context of Daoist internal alchemy, various images similar to the famous Taiji tu of Zhou Dunyi 周敦頣 (1017-73), though sometimes bearing different titles, are to be found incorporated into Ming and Qing texts. They show in diagrammatic form the main transformative processes that take place within the body in internal alchemy. ${ }^{48}$ One such diagram, which unmistakably refers to the body, is the Taiji shunni tu 太 極順逆圖 (Chart of the Normal and Contrary Direction of the Taiji), found in a 14th-century text on internal alchemy, Shangyangzijindan dayao tu 上陽子金丹大要圖 by Chen Zhixu 陳致虛 (see Fig. 10). ${ }^{49}$

Without entering into details, let us merely recall that Zhou Dunyi's diagram is believed to have be adapted from an image by Chen Tuan 陳摶 (c. 9o6-89), a Daoist of the Five Dynasties period, which was entitled Xiantian tu 先 天圖 (Image of Former Heaven) according to some sources or Wuji tu 無極圖 (Image of the Boundless) according to others. As no examples of this putative original have survived, we have no definite proof that Chen Tuan's image was a representation of the body and its processes, but it seems highly likely that this was the case, and that Zhou Dunyi and the Neo-Confucians turned it into a general cosmological model when they transposed it into a different context.

All the available versions of this image, whether they go by the name of Taiji tu 太極圖, Wujitu 無極圖 or Xiantian tu 先天圖, have certain features in common. First, the design

$46 \quad$ For a more detailed discussion of this subject, see Despeux 2006. 47 On the concept of Taiji in internal alchemy, see Robinet 199o.

48 The main diagrams of this kind are Taiji miaohua shenling hundong chiwenzhi tu太極妙化神靈混洞赤文之圖. They were inserted into Yuanshi wuliang duren shangpin miaojing tongyi 元始無量度人上品妙經通義 by the Ming author Zhang Yuchu 張宇初, a commentary on the Song text Yuanshi wuliang duren shangpin miaojing neiyi 元始無量度人上品妙經內義 蕭 (Scripture of Universal Salvation) by Xiao Yingsou 蕭應瞍 (Preface of 1226), DZ 89 (fasc. 41-2), 1 b.



Figure 2.10 Taiji tu 太極圖 according to Chen Zhixu 陳致虛. From Shangyang zi jindan dayao tu 上陽子金丹大藥圖 (Images of the Golden Elixir of the Master of Upper Yang) by Chen Zhixu, DZ 1068

is placed vertically on the page, although no hierarchy is thereby implied. It mingles visual elements and text, with a circle motif representing completeness and generally suggesting the idea of unity. The bipolar contrast of Yin and Yang is conveyed through the use of black and white. Written characters relate iconically to their referent - in the case of the Zhou Dunyi's image, the five agents of the five viscera, or in the Daoist images, the five ingredients of internal alchemy (cinnabar, silver, mercury, lead and earth). Connections between different elements of the image (viscera or agents) are shown by lines joining them together.

There is nothing in the title or appearance of the image to suggest that it has anything to do with the body; this transpires from the nature and composition of the texts that accompany it. The image itself shows only the main elements involved in the alchemical process: the spatio-temporal collocation, the trajectory to be followed, the dynamics of the process, and the means of setting it in motion. The images are not susceptible to interpretation without an oral commentary or supporting text. Apart from the different kinds of inscriptions that they bear, Zhou Dunyi's Taiji tu and the images from the internal alchemy tradition differ fundamentally in the direction in which they are required to be read: the former is read from top to bottom, the latter from bottom to top. This is confirmed in a commentary by Huang Zongyan 黃宗 炎 (17th century) ${ }^{50}$ on an image named as Wuji tu and attributed to Chen Tuan.

It would be a task far beyond the scope of this chapter to compile an exhaustive list of all the images from the Daoist Canon representing alchemical processes within

5o See Yixue bianhuo, juan 2, 39b. 




Figure 2.11 Lianxing mijue tu 煉形秘訣圖 (Secret art of cultivating the body) Taiji hunyuan zhixuan tu 太極混元指玄圖 (Images Illustrating the Mysteries of the Chaotic Origin of the Great Ultimate) by Xiao Daoxun 蕭道存 (13th century), in Xiuzhen shishu 修真十書, Zhentong Daozang, Taibei Xiwenfeng, vol. 7, 1977, p. 511

the body. I will confine myself to citing the most important ones, which are contained in two texts dating from the Song and Yuan periods. The schema adopted here is particularly important: once again the contours of the body are not shown. The first of these texts is Xiuzhen taiji hunyuan tu 修真太極混元圖 (Images of the Chaotic Origin of the Great Ultimate for Cultivating Perfection), with its sequel Xiuzhen taiji hunyuan zhixuan tu 修真太極混元 指玄圖 (Images Illustrating the Mysteries of the Chaotic Origin of the Great Ultimate for Cultivating Perfection), by Xiao Dacun 蕭道存, ${ }^{51}$ which contains numerous diagrams illustrating various processes, most of which take place within the body. The same representational elements are used here as in the Taiji diagram: a circled character stands for an object (internal organ, sensory organ, location on the spinal column, etc.); the contrast between black and white symbolises the duality of Yin and Yang; there are lines showing connections; and there are occasional figurative elements (see Fig. 11). The second text is Daode zhenjingjiyidazhi tuxu道德真經集義大旨圖序 (Collected Exegesis and Main Principles of the Authentic Scripture of the Dao and its Virtue with an Illustrated Preface) $)^{52}$, a collection of miscellaneous commentaries, some of them on alchemical subjects. It is certainly not earlier than the Yuan period. It contains illustrations based on maxims in Daode jing, which represent alchemical processes inside the body (see Fig. 12).

\footnotetext{
$51 \quad$ DZ 149 and 150.

$5^{2} \quad \mathrm{DZ} 723$
}



Figure 2.12 Daode zhenjing jiyi dazhi tuxu 道德真經集義大旨圖 序, DZ 723 in Daozang zhenjing jiyi dazhi 道德真經集 義大旨, Zhentong Daozang, Taibei Xiwenfeng, vol. 23, 1977, pp. 571-2

\section{The True Form of the Symbolic Body}

In Daoist ceremonies and individual practices, the body figures as a sacred space, often visualised as a mountain or cave-womb complete with labyrinths, mansions and in-dwelling spirits. It is essential that the adept should understand the true form of this symbolic body and possess maps to guide him or her through it. Daoist depictions of the body give information on the names of the principal spirits, key locations, processes and itineraries. The physical body gradually becomes the duplicate, or rather the Shadow, of the inner body, which constitutes the real or true (zhen 真) being as revealed through numinous visual imagery. ${ }^{53}$ The symbolic body is represented by the figure of a mountain, or in a form that suggests a human body seen in profile or half-profile, with an arc representing the spine on the right-hand side and an open space on the left: it is not bounded by any kind of outline, and the distinction between inside and outside is almost effaced.

Diagrams, inscriptions, scraps of text, even some figurative elements, all intermingle in a way that is richly suggestive of the ritual and magical function of visual images in Daoism. Far from being mere diagrams or maps, they possess the double force of word and image, conferring power on those who possess them or meditate upon them. They are thus akin to sacred scriptures and talismans, and to the models of the world that were entrusted to virtuous rulers, such as the Hetu 河圖 ([Yellow] River Diagram) and Luo shu 洛 書 (Luo [River] Document) (See Yoeli-Tlalim, Chapter 30 in this volume, pp. 436-8, Fig. 30.6).

We know of three representations of the body in the form of a mountain, all of them very similar. ${ }^{54}$ The body is

\footnotetext{
53 Compare Despeux 1996, pp. 87-118.

54 1) Ti xiang yin yang sheng jiang $t u$ 體象陰陽升降圖 (Chart of
} 




Figure 2.13 Image of the body as a mountain showing alchemical processes. From Daoyuan yiqi 道元一氣 (The One Breath of Daoyuan) by Cao Yuanbai 曹元白, late 16th century

shown from the side or in half-profile, and seems to be cut in half. It appears in the guise of a mountain with rivers running through it and a sea at its feet, symbolising the ocean of suffering. Two figurative elements may be noted: the 12-tiered pagoda corresponding to the trachea, and a palace with several storeys in the head, representing one of the celestial palaces (see Fig. 13).

There are also more complex drawings of the inscape of the body, including alchemical symbols and depictions of the spirits residing in the body. One of the earliest examples is by Yanluozi 煙羅子, of the Five Dynasties period, and it accompanies his chart of the viscera. As mentioned above, both this image and the chart were reprinted in a 13th-century commentary on the Canon of Eighty-One Problems. ${ }^{55}$

At a much later period (late 19th-early 2oth century), we see representations that combine the symbolism of the body as a mountain with that of the body as inner landscape. Chief among these is Neijing tu 內景圖 (Image

the Rise and Fall of Yin and Yang in the Image of the Body), taken from Yuanshi wuliang duren shangpin miaojing neiyi, in which this chart was preceded by a design similar to the Taiji tu. 2) Yuanqi tixiang tu 元氣體象圖 (Image of the body of Original Qi), from Shangyangzi jindan dayao tu, which also included a design similar to the Taiji tu. 3) A representation of the Daoyuan yiqi by Cao Yuanbai (17th century), which offers a more detailed illustration of the energetic processes that take place within the body at different stages in the alchemical procedure. of the Internal Landscape). The extant versions of this image date from the end of the 19th century at the very earliest, and are therefore too recent to provide information about the context in which it was originally created. One engraving conserved in Baiyun guan 白雲觀 (White Cloud Temple) in Beijing was made in 1886 on the instructions of Liu Chengyin 劉誠印, 2oth abbot of the temple, who is supposed to have discovered the image on an old silk scroll that he saw at the house of a certain Gao Songshan 高嵩山. ${ }^{56}$ Another important Daoist image is Xiuzhen tu 修真圖 (Image for Cultivating Perfection) (Fig. 14). Seven exemplars have been discovered so far, the earliest dating from 1812. Even more than in the schema discussed above, these images combine visual and verbal signs to represent the body as physiological locus, internal landscape and habitation of the gods and demons. ${ }^{57}$

\section{Conclusion}

As I emphasised at the outset of this chapter, although there are few extant images of the body predating the Song period, this does not mean that others never existed, but simply that they have not survived into the present. Chinese people had begun to feel the need for such images by the Han period at least. Nonetheless, the Song period clearly marks a turning point in the graphic representation of the body as in the use of graphic representations $(t u)$ in general. From this time forth, visual imagery comes to play a more significant role, not only as a record of knowledge, but also as a teaching aid, a mode of transmission, a mnemonic device, a visual translation of a text, and a representation of a certain approach to reality.

What is immediately apparent from the images presented here is that they do not set out to reproduce visible, contingent reality. They are not pictures but diagrams or emblems, in which figurative concerns are subordinate to an ordered schematic arrangement, steering us towards an interpretation - a reading. For these coded representations, composed of conventional signifying elements, are meant to be read, in the same way that one reads a Chinese character. Indeed, all the examples that we have explored so far include some form of lettering, and there is no clear demarcation between graphics and graphs. In Xiuzhen tu - the concluding example - in particular, word and image are intimately intertwined.

\footnotetext{
$56 \quad$ See Despeux 1994, pp. 44-8; Sakade 1991.

57 On these images, see Despeux 1994; Lagerwey 1991, pp. 127-30; Komjathy 2008 and 2009
} 




Figure 2.14 Xiuzhen tu 修真圖 (Image for Cultivating Perfection) 19th century. Baiyun guan 白雲觀 (White Cloud Temple), Beijing. Photo courtesy of Baiyun guan, (c) David Dear

The nature of representation is closely dependent on its function and utilisation. Thus, the human body is represented with a complete outline when the divisions and sectors of the body are considered as parts of the whole, when it is important to identify exact locations in relation to the whole body, or when movement is involved (external movement in daoyin, or internal movements through the channels and tracts).
An image of the body must be meaningful to its users, giving them a clearer understanding of their situation in space. A complete outline is useful to the practitioner, making it possible to locate the part of the body to be treated in physical space. It is required in general when the body is considered as a unit - as a self-contained universe wherein Qi, blood and other humours circulate. In these cases, there is a clear division between inside and outside. 
A bounded view of the body becomes inevitable if one sets out to describe the circulation of matter in a closed, oriented space or to locate, on a spatio-temporal map, the points at which an external action or interpretation can be brought to bear.

But these images are also the spatio-temporal mapping of a discourse, which must be read in context and in a genealogy of transmission. For instance, the first representations of the skeleton did not emerge from the field of medicine, but of forensic science; for the purposes of acupuncture treatment, it was sufficient to approach the bones from outside, and plot their approximate position on a chart of the body. Advances in knowledge of the skeleton and in techniques of illustration derived from forensics were adopted by physicians in the late Ming period. From a technical perspective, these representations are primarily memory aids. They are integral to a text and do not set out to be true to life. Chinese artists were perfectly capable of rendering physical objects faithfully and drawing an accurately proportioned human body: in the fine art tradition, virtuoso examples abound. But an artist-craftsperson required to execute a schema, a $t u$, of the skeleton for forensic purposes would operate within the conventions of the genre, producing a stylised, generic image.

With representations of the viscera, both individual and composite, the didactic function of the image takes centre stage in the Song period, not only as an instrument for the transmission of knowledge but also as a means of proving a thesis, just as dissections in Europe served to demonstrate the ability of the practitioner. No doubt viscera charts were cited in the debates on the number of openings in the throat and the position of the liver. One might however wonder how useful this information could have been in clinical practice, since diagnostic and therapeutic principles did not adapt to accommodate the revised position of the liver, for example, and the liver pulse continued to be taken on the left. Surgery, which was almost non-existent, could not have stood to benefit from such advances in anatomical knowledge. No doubt all this intellectual activity reflects a desire to increase and enhance the sum of theoretical knowledge, but it also betrays a sense of dissatisfaction with the theoretical apparatus of medicine, which was unable to respond appropriately to some pathological conditions; the same dissatisfaction is articulated in other contexts by various physicians of the Song period, a time of acute analysis and reflection for Chinese thought in general.

Whereas depictions of the body in the technical domain are an adjunct to a textual exposition (medical, divinatory or forensic as the case may be), in Daoism, the graphic representation $(t u)$ becomes the true image (xiang) or icon of the body, realised according to the systems of the various schools. It is an object of power, like the talismans and diagrams used in rituals and individual practices. ${ }^{58}$

As we have seen, images of the body are not mere representations of physical corporality. Like a written text, they act as carriers for a specific kind of knowledge and they are aimed at a specific user or readership. We lose sight of the body itself, as the spotlight falls on certain locations or landmarks in the reader's journey through the image, making sense of the inner world of the body or its relationship with the external world, and allowing the mechanisms and operations underlying physical reality to come to the fore.

When one comes to consider the way in which the body is depicted, one is struck by the absence of flesh and muscle. We should certainly take into account the different relationship to the flesh that obtains in the West, due partly to ideas from classical Greece on the role of muscle in the affirmation of personhood and nationhood, ${ }^{59}$ and partly to Christian thinking. However, this difference is a matter of degree: Western attitudes have fluctuated over the course of history. To see the truth of this, one need only look at medieval images of the body. It was not until the 16th century that flesh and the body regained the importance they were accorded in ancient Greece and Rome, in sculpture in particular. But generally speaking, in China, flesh and muscle have had far less significance than Qi, the invisible pneuma that brings life and motion. Chinese artists set out to represent, not the movement of Qi as such, but the arrangement in a given space of the viscera in which Qi was present, together with their principal characteristics.

The treatment of the dichotomy between inside and outside does not constitute a distinguishing feature in Chinese images of the body. What really matters is not the physical object we see before our eyes, but the multiple possibilities of transformation afforded by a dynamic vision of life. The body is recast in terms of motion, as the site of the circulation of Qi and blood, resonating with the transformative mechanisms of Yin and Yang and the Five Agents; the inner landscape; the world of the spirits and demons.

\footnotetext{
$5^{8} \quad C f$. Baptandier, 1994.

59 Cf. Ulmann's work (1990) work on gymnastics in the West and the role of the Olympic Games.
} 


\section{Bibliography}

\section{Primary Sources}

Beijiqianjin yaofang 備急千金要方 (Emergency Prescriptions Worth a Thousand Gold Pieces), Sun Simiao 孫思貌, late 7 th century.

Bintuilu 賓退錄, edited in Shuofu.

Chongkan buzhu Xiyuan lu jizheng 重刊補注洗冤錄集證 (Revised and enlarged edn of Xiyuan (u), late 18th century.

Daozang 道藏 (DZ) (The Daoist Canon), compilation of 1445 .

Daozang jinghua 道藏精華, 1976, Taibei: Ziyou chubanshe.

Dongzhai jishi 東齋紀事, Fan Zhen 范鎮, 1087 .

Dz, See Daozang.

Gujin yitong daquan 古今醫通大全, Xu Chunfu 徐春甫, 1556 .

Hanshu 漢書 (History of the [Former] Han Dynasty).

Hua Tuo xuanmen neizhao tu 華 陀玄門內照圖 (Hua Tuo's Chart of Inner Contemplation [of the body] According to the Mystery School), published by Sun Huan 孫煥 in 1273 .

Huangdi neijing 黃帝內經 (Inner Classic of the Yellow Emperor).

Jutan lu 劇談錄, Kang 康駢 ( fl. 875-86).

Mengqi bitan 夢溪筆談, Shen Gua 沈括, 1094, ed. Hu Daojing 胡道靜, in Mengqibitan jiaozheng 夢溪筆談校證, 1956, Shanghai: Shanghai chuban gongsi.

$S h u o f u$ 說郛, Tao Zongyi 陶宗儀 ( $f l$. 136o-8).

Siku quanshu 四庫全書, late 18th century.

Suishu 隋書 (History of the Sui Dynasty).

Sui Tangjiahua 隋唐佳話 (Fine Tales from the Sui and Tang Dynasties), comp. Liu Su 劉䬬 ( $f$ l. $742-55)$.

Xin Tangshu 新唐書 (New History of the Tang Dynasty).

Xiuzhen shishu 修真十書, Daoist encyclopaedia of c.1250.

Xiyuan lu 洗冤錄 (Treatise for Washing Away Injustice), Song Ci 宋 慈, 18th century.

Xu wenxian tongkao 續文獻統考, Wang Qi 王圻, 16 th century.

Yanxia fangyan 岩下放言, Ye Mengde 葉夢得 (1077-1148), edited in Shuofu.

Yilin gaicuo 醫林改錯 (Corrections of Errors in the Forest of Medicine), Wang Qingren 王清任, 1822.

Yixue bianhuo 易學辨惑, Huang Zongyan 黃宗炎, in Zhaodai congshu. Yuandian zhang 元典章 (the Yuan Code), ed. Shen Jiaben 沈家本, 1322. Zhaodai congshu 昭代叢書, early 17 th century.

Zhenjiu Dacheng 針炎大成 (Compendium of Acupuncture and Moxibustion), Yang Jizhou 楊繼州, 1601.

\section{Secondary Sources}

Baptandier, B. 1994, 'Le tableau talismanique de l'empereur de jade. Construction d'un objet d'écriture', L'Homme 129, 59-92.

Bray, F., V. Dorofeeva-Lichtmann and G. Métailié (eds) 2007, Graphics and Text in the Production of Technical Knowledge in China: The Warp and the Weft, Leiden: Brill.

Chen Guofu 陳國符 1963, Daozangyuanliu kao 道藏源流考 (Original Sources of the Daoist Canon), Beijing: Zhonghua Shuju.

Despeux, C. 1985, Prescriptions valant mille onces d'or, Paris: Guy Trédaniel.

[1988] 2011, Le qigong de Zhou Lüjing. La moelle du phénix rouge (Chifeng sui), $X V I^{e}$ siècle, Paris: Trédaniel.

1989, 'Gymnastics: The Ancient Tradition', in Kohn and

Sakade Yoshinobu (eds), 225-61.

Trédaniel.

1994, Taö̈sme et corps humain. Le Xiuzhen tu, Paris: Guy

1996, 'Le corps en Chine, champ spatio-temporel, souche d'identité', l'Homme 137, 87-118.

2004, 'La gymnastique dans la Chine antique', Études chinoises $23,45^{-81}$

2006, 'Métaphores et processus d'intégration: La symbolique du corps dans l'alchimie interne de la Chine des Song $\left(\mathrm{X}-\mathrm{XIII}{ }^{\mathrm{e}}\right.$ siècles)', in Kappler and Thiolier-Méjean (eds), 291-314.

2007 , 'The body revealed. The contribution of forensic medicine to knowledge and representations of the skeleton in China', in Bray, Dorofeeva-Lichtmann and Métailié, 637-84.

2010, Taoïsme et connaissance de soi. La Carte de la culture de la perfection (Xiuzhen tu), Paris: Guy Trédaniel.

Goldschmidt, A. 2005, 'The Song discontinuity: rapid innovation in Northern Song dynasty (96o-1127 CE) medicine', Asian Medicine 1.1, 53-9o.

Grmek, M. (ed.) 1997, Histoire de la pensée médicale, vol. 2. De la renaissance aux lumières, Paris: Seuil.

Grmek, M. and R. Bernabeo 1997, 'La machine du corps', in Grmek (ed.), $7-36$.

Harper, D. 1998, Early Chinese Medical Literature: The Mawangdui Medical Manuscripts, (Sir Henry Wellcome Asian Series), London/ New York: Kegan Paul International.

Jia Jingtao 贾静涛 1983, 'Liangbu zhongyaode zuguo fayixue wenxian yanshi gemu jie'anshi de faxian' 兩鄴重要的法醫學文獻一驗屍格 目與結案式的發現 (Discovery of two major documents of Chinese forensic medicine: legal checklists and engravings of the skeleton), Fayi tongxun 4.1.

Kappler C. and S. Thiolier-Méjean (eds) 2006, Alchimie Occident-Orient. L'Alchimie dans les arts et les littératures du Moyen Age et de la Renaissance, Paris: L'Harmattan.

Kohn, L. and Sakade Yoshinobu (eds) 1989, Taoist Meditation and Longevity Techniques, Ann Arbor: Center for Chinese Studies, University of Michigan.

Komjathy, L. 2008, 'Mapping the Daoist body (1): the text of the Neijing tu', Journal of Daoist Studies 1, 67-92.

2009, 'Mapping the Daoist body (2): the Neijing tu in history', Journal of Daoist Studies 2, 64-108.

Lagerwey, J. 1991, Le continent des esprits. La Chine dans le miroir du taoïsme, Bruxelles: La renaissance du livre.

Lo, V. 2005, 'Quick and easy Chinese medicine: the Dunhuang moxibustion charts', in Lo and Cullen (eds), 207-51.

Lo, V. and C. Cullen (eds) 2005, Medieval Chinese Medicine: The Dunhuang MedicalManuscripts, London/New York: RoutledgeCurzon.

Ma Jixing 馬 繼興 1993, Zhenjiu tongren yu tongren xuefa 針炎銅人 與銅人穴法, Beijing: Zhongyiyao chubanshe.

Miyashita Saburô 宮下三郎 1968, 'Sogennô iryô' (Medical theory under the Song and the Yuan), in Yabuuchi Kiyoshi (ed.).

Okanishi Tameto 岡西為人 1958 , Song yiqian yiji kao 宋以前醫籍考 (Study of the medical documents through the Song period), Beijing: Renmin weisheng chubanshe.

Ratchnevsky, P. 1937, Un Code des Yuan, Paris: E. Leroux.

Robinet, I. and P.A. Wissing 1990, 'The place and meaning of the notion of Taiji in Taoist sources prior to the Ming dynasty', History of Religions 29.4, 373-411.

Sakade Yoshinobu, 1991, 'Neikeito to sono enkaku', in Chugoku kodai kagaku shiron besatsu, Kyoto: Kyoto daigaku, Jinbun kagaku kenkyosho.

Ulmann, J. 199o, De la gymnastique aux sports modernes. Histoire des doctrines de l'éducation physique, Paris: Vrin.

Unschuld, P. 1985, Medicine in China, Berkeley/Los Angeles/London: University of California Press.

Yabuuchi Kiyoshi (ed.) 1968, Sôgen jidaino kagaku gijitsu shi, Kyoto: Kyoto digaku jimbun kagaku kenkyusho. 


\title{
3 Imagining Practice: Sense and Sensuality in Early Chinese Medical Illustration
}

\author{
Vivienne Lo 羅維前*
}

If we define medical imagery as any representation of the body that has a function in medical praxes, then we have but a handful of examples extant from the early imperial and medieval periods in China. Even the few images that survive from the western Han tombs at Changsha Mawangdui 馬 王堆 (tomb closed c. 168 BCE), ${ }^{1}$ of the old Han kingdom of $\mathrm{Chu}$ 楚, and on the figurine excavated at Yongxing 永興, Shuangbaoshan 雙包山 (latest date 118 вCE) ${ }^{2}$ in the southwest of China do not easily translate into therapy - especially if we take medical practice in a limited way according to the interaction between a practitioner and their patient in the treatment of illness. Making sense of the images may demand a prior knowledge of a select corpus of written texts: with the help of the Mawangdui textual accounts and those from another tomb at Jiangling Zhangjiashan 江凌 張家山 (closed c. 186 BCE), ${ }^{3}$ as well as some contemporary

This paper was written and researched while I was a fellow of the history department of SOAS, London University. I am very grateful for the generous research grant provided by the Wellcome Trust during that time. My thanks are also due to the countless scholars who gave me invaluable help and advice. In the preparation of this chapter I am particularly indebted to Donald Harper, Roel Sterckx and John Moffett.

1 The Mawangdui burial mound is located in the north-eastern section of Changsha 長沙, Hunan, formerly the Western Han Kingdom of Changsha, and was excavated in the early 1970s. It contains three tombs. Tombs no. 1 and no. 2 belonged to the Marquis of Dai 軑侯, Li Cang 利蒼, and his wife (who was buried in tomb no. 1). Tomb no. 3 , from which the manuscripts were excavated, was occupied by their son, who died in 168 BCE at the age of about 3o. For the excavation report see Hunansheng bowuguan and Zhongguo kexueyuan kaogu yanjiusuo 1974, pp. 39-48. Cf. Harper 1998 for a complete translation of all the medical manuscripts as well as a comprehensive and scholarly discussion of the manuscripts. Cf. Sichuansheng wenwu kaogu yanjiusuo 1996, pp. 13-29 for the excavation report.

3 Judging by his mortuary goods, the owner of tomb 247 at the Jiangling, Zhangjiashan site in Hubei was probably not of status equivalent to the Lord and Lady of Dai, of Mawangdui, nor the owner of the Shuangbaoshan tomb. A register, buried in the tomb, records that the anonymous tomb owner was bing mian 病免 'absent from court because of illness' for a period taken from the first year of Hui Di (194 BCE). But the two boxes found in the coffin room contained, after Mawangdui, the second richest cache of medical manuscripts recovered from the end century всE. The first description of the contents of the Zhangjiashan bamboo manuscripts appeared in 1985 , followed by a transcript accounts of practice, ${ }^{4}$ this chapter begins by examining the Shuangbaoshan figurine as a response to the body in illness and, specifically, as evidence in our understanding of the development of ' 'jiu' 炎 moxibustion and cautery treatments. In contrast, medieval moxa-cautery loci charts discovered in the cache of manuscripts at Dunhuang, and those in the Hamajing 蝦蟆經 (Toad Canon) bring together simple textual instructions with practical illustration. ${ }^{5}$ Together, image and text seem to demonstrate the transmission of knowledge in popular medical practice. The proximity of text and image in the latter cases may suggest a relatively direct relationship with practice, but we must be wary of searching for a simple healing function for those less obviously explicated images of the body.

Recent scholarship demands that we pay attention to how changing socio-political realities impacted upon the construction of the early Chinese body, or how the theoretical priorities of astro-calendrical computations configured its surfaces, such as in the Mawangdui and Shuihudi drawings designed for foretelling the fortunes of a newborn baby. ${ }^{6}$ The latter are examples of the body

of Maishu 脈書 (The Channel Book), published in 1989 and Yinshu 引書 (The Pulling Book) in 199o. See Jiangling Zhangjiashan Hanjian zhengli xiaozu 1989, p. 74 and Zhangjiashan Hanjian zhengli zu 1990, pp. 82-6 (hereafter, 'Yinshu shiwen'). As the binding thread that tied the slips had rotted away, the slips were scattered and had to be reordered. Yinshu and Maishu, are written in lish $u$ 隸書, Han clerical script, and each is marked with a title on the back in the hand of a single scribe, probably in the process of cataloguing. But as the script pre-dates graphic standardisation, many terms remain unknown and positive identifications are often speculative. Some of the graphs were also mutilated to the extent that it is not possible to establish an accurate graph count. Three texts, however, are more complete editions of those texts that describe the mai also found at Mawangdui and can be used to restore lacunae in the latter. In the course of this study I will also refer to the biographies of Chunyu Yi 淳于意 and of Bian Que 扁鵲 in Sima Qian's Shiji 史 記 (Records of the Historian), Shiji 105. References to Zhonghua shuju 1962, pp. 2,785-820.

$5 \quad$ The Dunhuang charts in question are S.6168 and S.6262, held in the British Library. A transcript of most of the medical manuscripts including copies of the charts is in Ma Jixing, Wang Shumin et al. 1998.

$6 \quad$ Mawangdui Hanmu boshu zhengli xiaozu (ed.) 1985, p. 133. Also reproduced in Harper 1998, pp. 372-3. See also Shuihudi Qin mu zhujian zhengli xiaozu 199o, pp. 89-141. 




Figure 3.1 Daoyin tu 導引圖 (Guiding and Pulling Chart), Mawangdui, tomb 3 (closed 168 вCE). Silk. @ C Wellcome Library, London, Loo4O263

as a passive repository of knowledge and experience. In addition, the following analysis, in the wake of those who are more interested in how the body itself participates in culture, offers another radically different approach, examining some of the same source material as an expression of sensory experiences associated with both health and illness. Rather than mapping the body's functionality, some of these images portray and convey aesthetic knowledge where aesthetic refers to its earlier meaning of 'things perceptible to the senses.' ${ }^{7}$ And it is this codification and instruction of the body's sensual nature that is the main theme of this chapter.

Vivid images of the body can be found on the well-known Mawangdui silk chart styled the Daoyin tu 'guiding and pulling chart' 導引圖 (Fig. 1), ${ }^{8}$ illustrating a form of medical gymnastics designed to rejuvenate aging and stiffening bodies and transform pain and discomfort. Daoyin tu sets out 44 robed and semi-robed figures engaged in different exercises - some upright, some bending, with arms and legs stretched and contorted in different postures. Some of the diagrams have captions such as Long deng 龍登 'Dragon Ascending' and Yin xi tong 引膝痛 'Pulling Knee Pain'. Zhangjiashan Yinshu 引書 (The Pulling Book), a recently

7 I use aesthetic in contrast to anaesthetic in its earlier meaning of 'things perceptible to the senses' rather than the 'criticism of taste'.

8 The title was given by the editors of Mawangdui Hanmu boshu. See Tang Lan 唐蘭: 1-10 in Mawangdui Hanmu boshu zhengli xiaozu (ed.) 1979. A photographic reproduction and transcription of the extant captions on the Daoyin tu can be found in Mawangdui Hanmu boshu zhengli xiaozu (ed.) 1985, pp. 49-52. Ma Jixing 1992, pp. 849-66 provides explanatory notes. See also Li Xueqin 1991, pp. 7-9. excavated manual describing similar exercises provides further help to translate the images into action. From Yinshu we know that the art of daoyin was not simply a therapy for illness, but also a year's health regimen in four parts that adjusted personal hygiene, grooming, exercise, diet, sleep and sexual behaviour to the changing qualities of the four seasons. Seven or more of the Daoyin tu captions and some 12 Yinshu exercises relate to movements associated with animals, and the last part of this chapter will discuss how these animal images serve to convey a specialised form of knowledge. Far from representing carnal passions full of shame, such as the beasts of Christian gnosticism of the 1st to 3 rd centuries $\mathrm{CE}$, use of animal imagery in early Chinese self-cultivation served to measure and transform bodily passion into the essences for strengthening and prolonging life. ${ }^{9}$

\section{The Mai 脈}

During this first section of this chapter I will keep returning to a discussion of the phenomenon of the mai, what the term referred to and what it meant in practical medical terms. Until recently, the problem posed by the mai has been to reconcile apparently conflicting meanings of 'blood vessel,' 'channel' and 'pulse' where contexts seem to demand different translations. Various solutions are proposed: Harper translates 'vessel', which draws out early associations with the arteries and veins, Unschuld favours 'conduit', Sivin 'tract'; I prefer a more neutral 'channel', which also embraces muscular and topographical ele-

$9 \quad$ See Williams 1989 
ments of the concept that are evident in the early texts and images. ${ }^{10}$ Kuriyama points out that the divisions of structure and function differentiated by the terms vessel and pulse may be an artefact of translation - of the inseparable development of anatomy and the theory of blood circulation in Western medical culture, beginning with the Greek experience. His challenge, then, is to give a positive account of the mai. ${ }^{11} \mathrm{Li}$ Jianmin has begun to meet that challenge, maintaining that early concepts in mai theory do not simply organise observations of 'empirical experience', but are attempts to inscribe the movement of the heavens onto the body, opening it out as 'a field of temporal spaces. ${ }^{\prime 2}$ The mai were also physically located within the body - a part of its corporeal reality. Simultaneously, and perhaps within the same social milieu, those involved in the theory and practice of self-cultivation used the concept of mai to explain the movement of Qi and the experience of circulating the breath. ${ }^{13}$

If we simply assume that these mai were a primitive version of the mature jingluo system of acupuncture, we will miss a great deal about the process and context within which they were conceived. How did the mai translate into medical practice? What were the processes linking the art of pulse-taking to the instruction to apply cautery or needle to open the mai? What was the space of the body defined by the mai? Can we learn anything about therapy from medical illustration? Concentrating attention on the practical application of image and text will provide new insights to deepen our understanding of the development of this most fundamental concept in classical Chinese medicine.

See Harper, 1998, pp. 77-9o. I prefer to follow the contemporary analogy with $d u$ 瀆 'channel' or 'canal' found in the Maishu 脈 書 (The Channel Book). See Jiangling Zhangjiashan Hanjian zhengli xiaozu 1989, p. 74. The translation 'channel' also serves to emphasise the relationship of the mai to the superficial anatomical channels as defined by muscle and bone, as they were understood before the more elaborate theories of the jingluo and jingmai found in the Huangdi neijing 黃帝內經 (Yellow Emperor's Inner Canon). Jingluo or Jingmai has been variously translated as 'conduit', 'meridian', 'circulation tract' etc. See Sivin 1987, pp. 34, 122 n. 11; and Unschuld 1985, pp. 75, 81-3.

11 Kuriyama 1986, pp. 211-12.

$12 \quad$ Li 2001, pp. 107-40.

13 This was the subject of my PHD diss. See Lo 1999. See also Lo 2000, pp. $15^{-65}$.



Figure 3.2 Lacquer figurine from Tomb 2 at Shuangbaoshan, Mianyang 綿陽, Sichuan (ht $28.1 \mathrm{~cm})$. Photo kind permission of He Zhiguo

\section{Shuangbaoshan, Mianyang 綿陽, Sichuan}

Excavations during 1993 of a tomb at Shuangbaoshan, two man-made burial mounds in an area which is well known for its Han and Six Dynasty period tombs, yielded an artefact that adds yet another facet to the history of the concept of the mai (see Fig. 2). ${ }^{14}$ The tomb was shallow in comparison to the three at Mawangdui, but from its size and mortuary goods we can tell that the tomb owner was of high rank. The mortuary goods in the antechamber were arranged by theme. Most notable were the stable goods, chariots and cavalry, which include 100 large lacquerware horses $(74 \mathrm{~cm}$ high $\mathrm{x} 71 \mathrm{~cm}$ long). From these and the 20 chariots both single and double harnessed, the cavalry and the quivers, it would seem that the tomb owner held a

14 I have discussed the figurine in detail in He and Lo 1996, pp. 81-124. See also He 1994, p. 4 for the first description of the discovery of the figurine. A detailed discussion of the figurine can be found in He 1995, pp. 116-20. 
significant position in the army. The tomb is also situated on the Shudao 蜀道 - the way between the kingdoms of Shu and Qin - site of many battles during the Warring States period, and is likely to have been a military outpost of the Han court.

Of the 5 o lacquerware items recovered at Yongxing, Shuangbaoshan, the forms of the cups, jugs, plates and bowls closely resemble those found at Hubei, Jiangling Fenghuangshan 鳳凰山 tomb 168. We can assume that the owners of these tombs along the course and hinterland of the Yangzi river shared a common burial culture. The red design on a black background is also similar to lacquerware found at Mawangdui, as are the motifs of paired birds and cloud configurations. But at $28.1 \mathrm{~cm}$-high, the lacquered wood carving of the human body is unique. It was wrapped in a red woven fabric and placed in the outer coffin compartment. The figurine, unusually, is naked, and it is carved with some concern for anatomical accuracy. It bears nine red lacquer lines which extend from the extremities to the head, and one which follows the mid-line of the posterior surface of the torso over the spine traversing the spine to the bridge of the nose.

I have, elsewhere, compared and contrasted the anatomical pathways of the figurine to routes of the mai 'channels' as they are described in four texts excavated from tombs at Zhangjiashan and Mawangdui, which were closed at earlier dates in the Western Han period..$^{15}$ The many inconsistencies described in that chapter suggest that text and figurine may reflect different theoretical constructions of the body. In particular I have noted the absence of the Yin channels of the leg, and the addition of a medial posterior line that might resemble the most Yang channel known later as the dumai. The lines on the figurine differ in number and route from those described in the texts, and although both clearly share a fundamental spatial awareness of the body, we should be cautious about attributing to them a common source. In this chapter, I will revisit the relationship between text and artefact, concentrating on their relationship to practice.

We can think of the figurine as a visual aid to guide medical intervention, and this will be the first concern of this chapter. But whether intervention is styled in relation to a model of breath cultivation, to contemporary theories of pathological physiology or simply patterns of symptoms is as yet unclear. Secondly, we must be wary of how the excitement of textual corroboration of the detail on the figurine can dominate our interpretation and blind us to the information offered to us simply by the mute presence and position of the figurine in the Han tomb. We

15 He and Lo 1996, pp. 93-105. should consider how the figure and the knowledge that it represents relate to or mediate older mortuary beliefs and practices. Was the inclusion of the figurine part of a project to mimic and sustain real life in the afterworld for the benefit of the deceased or was it self-consciously representational? In form and structure, the figurine parallels the Chu interest in real human form, insomuch as it uniquely attempts to convey details of superficial anatomy, but this is not a figure that we can say is a replica of the real world.

\section{Sense}

\section{Blood Vessels}

A first approach to interpreting the Shuangbaoshan figurine is to relate it to the structure and function of the $\mathrm{mai}$ as we understand them from both received and excavated texts. Zhangjiashan Maishu 脈書 (The Channel Book), comprises 65 slips. Harper divides the manuscript into six core texts, which he describes as 'Ailment List', 'Eleven Vessels', 'Five Signs of Death', 'Care of the Body,' 'Six Constituents' and 'Vessels and Vapor'. His titles indicate well the content of each text. I shall adopt his divisions, numbering the texts (1) to (6). ${ }^{16}$ Three of those texts identified by Harper are editions of texts also found in the Mawangdui medical manuscripts.

Maishu (1) associates the mai 'channels' with holding, or, at least, controlling the movement of blood. It is a lexicon of illnesses, which begins to give an elementary differential diagnosis. In the section on illnesses of the bowel it states: when the illness is... in the bowel - when there is pus and blood, and pain in the perineum, the spleen, the buttocks and lower abdomen, this is bowel flushing. When on eating it immediately comes out, this is diarrhoea. When blood comes out everywhere first, this is mai 脈. ${ }^{17}$

Mai in this context is a technical name for an illness which is characterised by bleeding from the bowel. In Wushi'er bingfang 五十二病方, one of the Mawangdui medical texts, maizhe 脈者 refers to a type of haemorrhoid. ${ }^{18}$ Given the common symptoms accompanying such haemorrhoids, the maizhe may also refer to a small blood vessel protruding from the anus, which will inevitably tend to bleed. Both

16 Harper 1998, p. 31. Ma Jixing establishes only five texts (unpublished paper, Changsha 2001), while Gao, using a different schema, conflates the last three. See Gao 1992.

17 Jiangling Zhangjiashan Hanjian zhengli xiaozu (eds) 1989, hereafter 'Maishu shiwen', p. 72.

18 Mawangdui Hanmu boshu zhengli xiaozu (ed.) 1985, p. 53. 




Figure 3.3 Detail of the channels from the Shuangbaoshan figurine

these early illness names may then be understood to connect 脈 mai with blood or with blood vessels.

Referring to the figurine we can see that, on the back of the hand, the forearm and at the lateral edge of the head, there are patterns of lines, which obviously model the superficial arteries. The lines on the forearm, groin and neck clearly pass through areas where one could easily draw blood. They simultaneously pass through the places at the wrist, elbow, neck and temple where the pulses are most easily felt, but do not pass the deep pulses at the abdomen or the superficial pulse at the ankle. The figurine evidently demonstrates a knowledge about and concern for the superficial arteriovenous pathways in their simple spatial relationship to the places where one can detect the sensation of pulsation. (See Fig. 3.)

\section{Image: Pain and Muscular Definition}

The 11 mai of Maishu and the editions of Zubi shiyi mai jiujing 足臂十一脈炎經 (Cauterisation Canon of the 11 Foot and Arm Channels) and Yinyang shiyimai jiujing 陰 陽十一脈炎經 (Cauterisation Canon of the 11 Yin and Yang Channels) of Mawangdui are not simply blood vessels. Like the figurine they have a more vertical arrangement running from limbs to torso and head. A theory, once advanced by Bridgman, is that the mai were not based upon a knowledge of blood vessels, but on routes of pain as it is



Figure 3.4 The juxtaposition of Daoyin tu 導引圖 (Guiding and Pulling Chart) with Yinyang shiyimai jiujing 陰陽十一脈 炎經 (Cauterisation Canon of the Eleven Yin and Yang Channels), Mawangdui 馬王堆, tomb 3 (closed 168 BCE). Silk. (C) Wellcome Library, London, Loo4O263

transmitted around the body. ${ }^{19}$ Huangdi neijing lingshu 13, in the treatise entitled 'Jingjin', describes a map of the body that is similar to the jingmai of acupuncture theory, but which is also more focused on a somatography of the body defined by its muscular definition. Many features of Maishu and Yinshu also demonstrate an awareness of pain and muscular definition as the guiding structure of their architecture of the body. ${ }^{20}$ Well over half of the symptoms associated with the 11 mai described in Maishu (2), for example, are of pain or discomfort along the mai in question.

The juxtaposition of Daoyin tu, chart of therapeutic gymnastics, with Yinyang shiyimaijiujing (Fig. 4), a channel text which does not mention cautery, suggests alternative physical therapy for disorders of the mai. With the added testimony of Yinshu, the Zhangjiashan daoyin manual, we can see that the type of illnesses treated with daoyin match closely those associated with each of the mai. Over $60 \%$ are pain and discomfort or motor problems associated with co-ordination of the limbs. Thus this early Western Han movement therapy, which we shall see is also a form

19 See Bridgman 1981, p. 10. During the centuries around the turn of the millennia in China, the widespread medical theorising about the physiology of the body, and in particular that leading up to the formation of ideas about the circulation of Qi, could have taken in all of these ideas, even if at any one particular time or in one text they were not all assimilated to the concept of the mai itself.

20 See for example the following quote from Maishu, p. 74, 'So bone pain is as if being hacked at, muscle pain is as if being bound, blood pain is as if saturated, channels pain is as if flowing, flesh pain is as if floating and when the Qi is agitated there is chaos'. 
of 'inner alchemy', was concerned with alleviating pain and discomfort and was related to a body organised around mai. Working solely from the figurine, we can see that many of the lines follow the natural planes and valleys of the body. In particular the lines of the spine, the lower edge of the ribs, the lower jaw etc., mark muscle and bone boundaries on the body. ${ }^{21}$

\section{Interpretation: Diagnosis}

In the descriptions in the channel texts, the mai 'rises' shang 上 and 'falls' xia 下, 'enters' $r u$ 入 and 'comes out' $c h u$ 出. Significantly, the channels tend to 'come out' at the major points at which one can feel the pulse. On the one hand, the mai are like roads, which paradoxically, without moving, travel in certain directions, and on the other, they emerge from the body in the form of pulsation. While it is through the quality of pulsation at different points of the body that early medical practitioners determined which of the mai were in disorder, it seems that the mai were thought to move in a variety of different ways.

Treatises in the later canonical medical compilations, such as Huangdi neijing suwen 4.14 and Lingshu 1.1 speak similarly of the shen 'spirit' 神 and jing 'essence' 精 moving through the guan 關, strategic passes and junctures of the body, just as if they are discrete entities. ${ }^{22}$ We will see in the course of this chapter that knowledge of the whereabouts of these entities was an important feature of the development and control of moxa-cautery practice.

After each of the 11 descriptions of the mai in Maishu there is a list of symptoms that is introduced with the phrase 'Shi dong ze bing' 是動則病 (when it 'moves' then there will be illness). The last section of Maishu elucidates the question of movement most clearly:

The way to examine the channel is to press it with the left पि口०; place the right hand directly on the ankle bone and palpate there. If the other channel is overflowing and this one alone is depleted then this controls where the illness resides. If the other mai are slippery and this one alone is rough, then this is where the illness resides. If the other mai are quiet and this one alone moves (in agitation), then it generates illness. Now as for the certain movement in the mai 脈固有動者: the Lesser Yin of the Shin, the Great Yin and Lesser Yin of the Forearm are where the illness

21 I have discussed the role of pain in the construction of the early Chinese medical body in Lo 1999, pp. 191-210.

22 Suwen, juan 4, pian 14. SSBY, vol. SBBY vol. 205, fasc. 1, 1. 204, fasc. 1, 5 b and Lingshu, juan 1, pian 1. Lingshu, juan 1, pian 3. SBBY vol. 205, fasc. 1, 8. also notes the difference between guan and ji in crude and sophisticated attention to the joints. It adds a distinction between zhengqi 正氣 'proper Qi' and xieqi 邪氣 ‘deviant Qi', the former associated with guarding the shen 神 'spirit' and the latter with $k e$ 客 (unwelcome) 'guests'. resides. If it becomes fast then there will be illness. This is how one discerns mai that have guo 過 'excess', - as for the rest - carefully observe the excess on the relevant channel. ${ }^{23}$ [Author's italics]

The term mai in this passage refers to the route of the channel and, simultaneously, to pulsation emanating from the channel. It is no coincidence that 'certain movement' is on the three Yin channels that pass through the major pulses at the carotid, the ankle, the wrist and the femoral artery. If these pulses speed up, it signifies a pathology on the relevant channel. Where dong contrasts with jing 靜 'quiet', the text implies a pathological speed of the pulse and we should understand 'agitation'. This is followed by the observation that a fast pulse is related to illness. So mai that is in a pathological state moves in the temporal sense - it speeds up - while it also travels in a spatial sense around the body and out from a site beneath the surface of the body to the surface.

Determining the condition of a channel being guo 過 (in excess) is the stated aim of diagnosis. Apart from causing excessive speed of the pulse on the given three Yin, mai guo reveals itself on the other channels through certain types of pathology, listed in relation to each channel. So this last passage from Maishu also defines the relationship between temporal and spatial agitation by matching symptomatology on the site of the channel with the excessive speed of the pulse.

Chunyu Yi, the Western Han physician active around 154 BCE whose professional life was set out in a biography by Sima Qian, records case histories that are roughly contemporary with Maishu. ${ }^{24}$ His pulse diagnosis is also concerned with detecting pulses that are in excess, but he adds many layers of complexity to interpreting the pulse in its relationship to the dynamic between Yin and Yang. For example if the Yin and Yang of one patient are bing 并 (aligned in some way), the prognosis is good, but if they jiao 交 (cross, or encroach upon each in other in some way), then the condition is fatal. ${ }^{25}$ Whether this transgression is understood as the relative speed or movement of individual pulses, an invasion of the space of the channels, lesions or something more esoteric remains a fascinating mystery to me. Chunyu Yi also treats channels and pulses that are more closely linked to some concept of the Liver, Lung, Spleen,

23 This penultimate statement provides a clue to the practice. The channels that have pulses located along their route can be assessed by the quality of that pulse - those that don't, have to be assessed by the symptoms along the whole course of the channel.

24 See n. 5 .

25 Shiji,juan 105, p. 2,80o et passim. 
Heart and Kidney and the Qi 氣 (the fundamental stuff of life) of those viscera. ${ }^{26} \mathrm{He}$ can detect certain syndromes and the exact position of symptoms from the relative speed, regularity, strength, sise and movement of the pulse. The qualities that he feels are far more intricate than the four qualities recorded in the Maishu passage translated above.

It is not possible to find a unitary concept of mai, one that is represented equally in the excavated texts and in the Shuangbaoshan figurine: not in the structure of the mai, be that a formalisation of the muscular body or the arteries, veins or the pulse; nor is it in a circulatory tract for the movement of a discrete entity such as mai, Qi, shen 神 'spirit' or jing 精 'essence.' ${ }^{27}$ It may be safer to assume that the term mai is flexible and has popular, technical and specialised usages, all of which come to bear on one

26 Qi (sometimes rendered ch'i) is a complex and changing concept which defies simple lineal histories. In the mid-Warring States, references to Qi tend to refer to atmospheric and environmental conditions, especially moist vapours - clouds and mists - and, by analogy, to formless, clustering qualities that can be discerned with careful observation, like smoke, ghosts or the vibrant, martial aura of an army. By the mid-4th century Qi often indicates the fundamental stuff in nature which both promotes and indicates vitality in the phenomenal world. It may enter the body in various ways - through the orifices and the skin - but its movement within the body is not formalised. Some historians translate Qi as 'vapour' and, in doing so, underline the amorphous watery qualities of steam and mist, which are formative influences both in the early period and as an enduring feature of the concept. As Qi begins to be applied to the phenomena of the inner body, the ideas, although never totally distinct from the early versions, go through significant transformations. Rather than replacing the old meanings, the range of meanings grows incrementally - a process that is continuous to the present day. I have not translated the term - and shall refrain from doing so because of the substantive changes that take place as Qi itself begins to figure in the inner body.

27 Jing 精 is a term which is frequently found in medicine and self-cultivation in a triad with shen 神 'spirit' and Qi 氣 (see below). In sexual cultivation, Jing may refer directly to semen. At other times, and when in combination with Qi, it seems to refer to the finest quality of Qi which is the universal vitality out of which things condense and into which they dissolve. Shen in Warring States literature, such as Guanzi 管子, Zhuangzi 莊子 and the Zuozhuan 左傳, often designates spirits and divine entities that dwell outside the human body. Elsewhere shenming 神明 comes to mean characteristics of divine beings that allow them a spirit-like wisdom, a sharpness and clarity of perception rather than a mechanical or analytical intelligence. In Warring States and Early Imperial self-cultivation the shen began to refer to the spirit in-dwelling in the human body, conceived as an individual entity. The term continued to convey the qualities of a mysterious and radiant intelligence as represented in its earlier meaning. another at different times. For diagnostic purposes, for example, the significant elements are knowledge of the relationship of the pulse to symptoms, and of the symptoms to the physical layout of the mai. The figurine may not be a representation of any of these individual elements, but we might adduce elements of all of these phenomena. Both texts and figurine, after all, share a common conceptual framework in the way they contour the body with their parallel structure of lines running from head to toe, and little evidence of pathways leading to the inner organs. Together they form a distinctive image that contrasts with the integrated system of jingluo 經絡, fundamental to acupuncture theory that we know from a later time.

\section{Action: Treatment}

Can we know any more of the treatments that were applied to the mai? If a condition seemed to be fatal, Chunyu Yi might avoid medical intervention. Where a patient's prognosis was good his favoured therapy was pharmacological, although he also used stone needles and cautery. But these were not the only methods to treat the mai.

Long ago, Epler (1980) explored a link between the origins of the jingluo system and blood vessels as well as bloodletting, as an associated therapy. He cited many examples from Suwen to suggest the importance of bloodletting in the formation of foundational acupuncture practice. ${ }^{28} \mathrm{~A}$ reading of Maishu (6) will reveal an instruction to qi mai 'open the mai' and thus one immediate interpretation might be that this is confirmation of the practice of bloodletting in the earliest medical treatise associated with the mai. ${ }^{29}$ On close examination there is no further evidence to corroborate such a reading. In fact, this particular passage uniquely refers to opening the mai in order to correct a pathological movement of Qi and concludes with a very practical description of how to lance abscesses. While the juxtaposition of lancing abscesses and moving Qi suggests a rather random arrangement of practical interventions around a theme of the mai, there is no hint of bloodletting here. In fact, to understand the concept of mai in this text, we look to ideas of Qi and the cultivation of the inner body already well developed in the Mawangdui and Zhangjiashan sexual and breath-cultivation literature, which I will explore later in this chapter. ${ }^{30}$

Chunyu Yi favours treating a particular symptom or syndrome with lancing stones from a number of different locations. On two occasions he pierces the body in three places in a single treatment. In case 11 he treats a condition

\footnotetext{
28 Epler Jr. 1980, pp. 337-67.

29 'Maishu shiwen', p. 74.

30 See also Lo 2000, pp. 15-65.
} 
of the foot by piercing the sole in three places and in case 16 he treats rising heat and head pain with cold water and by piercing the Yangming channel in three places. ${ }^{31}$ Neither condition includes abscesses, or requires bloodletting. Nor are Chunyu Yi's treatments applied to the type of named acupuncture locations that we know from the time of Huangdi neijing onwards. ${ }^{32}$ They refer to a concept of the channels quite separate from that system, such as we find on the figurine.

Thus we have five possibilities for medical intervention aimed at the mai: herbs and mineral drugs, cautery, lancing stones for superficial surgery, bloodletting and normalising the movement of Qi. From Yinshu we can also find evidence of daoyin and massage therapy as different methods of treating mai. But of all these methods evident in Maishu it is cautery that is the most clearly stated treatment, and we have to consider the possibility that the lines on the figurine were framed in order to establish related locations for moxa-cautery.

Features of the Popular Practice of Cautery It is difficult to ascertain the extent of cautery with mugwort (moxa-cautery) in Han times. Yamada gives good

$31 \quad$ Shiji,juan 105, pp. 2,804, 2,806.

$32 \quad$ Huangdi neijing is the work most famous for its exposition of classical acu-moxa theories, although the extent to which its combined treatises reflect medical orthodoxy of the Han dynasty is questioned by Akira Akahori, who compares the Huangdi neijing material with medical ideas in the work of the contemporary author Wang Chong. See Akira Akahori, pp. 171-89. Huangdi neijing is a corpus now extant in three recensions, the Taisu (Great Basis), the Suwen (Basic Questions, hereafter Suwen) and the Lingshu (Numinous Pivot). Each of these is a compilation of small texts dealing with separate topics which may, or may not, reflect the thinking in a distinct medical lineage. It is thought that the earliest texts were set down during the ist or at the earliest the 2nd century BCE. Collectively they represent the kind of debate through which classical medical concepts matured. In this respect they act as a convenient marker against which to assess the form and content of the excavated texts which form the source material for this chapter. For an extended discussion of the development of medical theories in China, based on a clarification of the formation of the Huangdi neijing, see Yamada 1979, pp. 67-89; for a reassessment of the origins of acu-moxa see Unschuld 1985 , pp. 93-9. Since the discovery of the excavated manuscripts, most medical historians now agree that an essential fusion of the technical and theoretical elements at the foundation of acu-moxa therapy could not have happened much before the first two centuries BCE. See Sivin 1993, pp. 196-215. The canons of acu-moxa must also include Huangfu Mi's Zhenjiu Jiayijing (A and B Canon of Acu-Moxa) (Wu, Jin, 256-82 CE) and the Nanjing (Classic of Difficult Issues) (1st or 2nd century CE); translated in Unschuld 1986. evidence that the development of moxa-cautery with mugwort was related to an earlier use of mugwort to ward off inauspicious elements and drive away demons. The people of Chu in particular wore mug wort dolls at their waist. He also describes the use of a related plant, xiao 蕭, as a form of incense to attract the gods, concluding that 'mugwort must originally have been more than just a medicinal herb. $A i$ and xiao belong to the same mugwort genus, but one was used as incense to beckon the gods, whereas the other was used to drive away demons that caused illness'. These represent two contrasting approaches to ensuring that the body only played host to benign entities. ${ }^{33}$ Yamada raises the possibility that the channel system was discovered by those using moxa-cautery for 'magical therapies' because:

The routes or areas of illness caused by gods of illness invading the body is highly compatible theoretically with the concept of the vessels [channels]. ${ }^{34}$

The use of cautery is attested in literary analogies of the Warring States period: Zhuangzi puts the idea of 'cauterising where there is no sickness' into Confucius' mouth as an analogy for useless effort; Mengzi likens inadequate preparation in government to the futility of using insufficiently mature ai 艾 ('moxa punk' or mugwort) to treat chronic illness; ${ }^{35}$ in the Shiji biography Chunyu Yi also criticises other people for wrongly applying moxa-cautery to the channels.Jiu 炎 was also a technique to stimulate Qi in self-cultivation. Tianxia zhidao tan describes how the gentleman who has over-indulged in sexual activity would 'cauterise his body to bring forth his Qi' in a programme which included taking special medicines and diet. ${ }^{36}$

After listing symptoms associated with each mai, Zubi shiyi mai jiujing recommends cautery. ${ }^{37}$ We can quite

Harper 1998, p. 244 and Yamada 1985a, pp. 58-63. See also Yamada 1998, pp. 66-78. Yamada compares the stages in one Wushi'er bingfang prescription for hernia which involve minor surgery, the applications of paste and alcohol and fumigation, to the stages in a ritual ceremony described in Liji 禮記. The fumigation, he believes, was intended to drive away the god of sickness and prevent it from entering the wound. Here we have a repetition of that theme where openings in the body, here a wound but elsewhere the orifices, are both vulnerable to malevolent activity and pivotal in methods to support and protect the body. 34 Yamada 1998, p. 78.

35 Zhuangzi, juan 9, pian 29. SBBY, vol. 176, fasc. 4, 21b. Mengzi, juan 7. 9. SBвY vol. 8 , fasc. 3,8 .

$36 \quad$ Mawangdui Hanmu boshu zhengli xiaozu (ed.) 1985, p. 164.

37 Ibid., pp. 3-6. Ma Jixing 1992, pp. 9-10 notes that because Zubi jiujing and the other texts on the same manuscript are written in a script that is in between seal and clerical script, they are more accurately dated to the Qin period. 
safely assume that cautery was applied to the symptoms themselves as well as to selected parts of the extended areas associated with the location of the symptom according to the framework of the mai. Since a majority of symptoms associated with the mai were pain and discomfort along its course, we can easily understand the value of heat therapy. The lines on the figurine might then be a way of training the eye to pick out those planes of the body associated for the purpose of applying moxa.

One Eastern Han bamboo slip from the archives of Jiaqu Defence Company Headquarters 甲渠部候 records a case where the Commanding officer of a beacon unit along the line of defences in the north-west of China treated a soldier with moxa-cautery in two places on his back. This was a form of self-help medicine applied in the absence of more expert medical advice. Drugs had to be prescribed by a physician from the Commandant's office on his rounds of the military outposts. Cauterising a number of moxa locations is reminiscent of Chunyu Yi's treatments, although the officer did not work specifically on the channels (See Hinrichs, Chapter 17 in this volume, Fig. 17.3).

Private soldier attached to Dangqu Beacon Unit, Qu Fanzi 屈樊子 [person's name?], in the first month $\square$ day fell ill for four days, the office did not $\mathrm{Cu}_{\mathrm{C}}$, three days later, $\square$, Officer in Command of Wansui Beacon Unit, applied cautery to his back $\square \square$ in two places, after $\square \square$ within several days the physician at the Commandant's Office came, and he drank one dose of drug and disposed $\square \square .{ }^{38}$

One indication of the popularity of cautery is the existence of sources which tell of prohibitions, cautioning against needling and applying moxa-cautery to the parts of the body through which the spirits and souls of the human body, hun 魂, shen 神 and Qi passed in calendrical cycles. ${ }^{39}$

38 Xie Guihua, Li Junming, Zhu Guozhao 1987, pp. 49.31, 49.13 and 圖 22 and 23. See also Xie Guihua 2005, p. 97.

39 Lingshu 8 describes the relationship between the internal organs, emotion and other aspects of the human consciousness and entities that we might approximate to spirit and souls. See Lingshu 8, 'Benshen' 本神, 2.152-3. The hun 魂, and the $p o$ 魄, for example, are aspects of human being that scholarship has traditionally seen as separating at death and as the object of early funerary practice. In a recent article Brashier has shown that hun/po dualism is not at the foundation of Han burial practice and is more faithfully described as a scholastic convention. He suggests that the pair are more closely linked to medical states of anxiety and illness. Brashier 1996, pp. 125-58. In Lingshu 8 the hun is said to reside in the blood, the po in the lungs. The zhi 志 'will' resides in the kidneys. The shen 神, originally a term for 'spirit' entities external to the body - and, in the Lingshu, a somatisation of the qualities of brightness and spontaneous perception that the spirits represented (which I translate 'spirit') is



Figure 3.5 The prohibitions of the Huangdi hamajing. From Huangdi hama jing 黃帝蛤蟆經, 1984. Beijing: Zhongyi guji chubanshe

In a similar vein, medical texts excavated from a tomb at Wuwei 武威 (c.1st century CE) provide us with the earliest datable acupuncture treatment detailing the site of needle insertion and manipulation technique. ${ }^{40}$ Following this passage there is also a list of prohibitions which restrict cautery on specified parts of the body according to the age of the patient. The prohibitions set out in the Hamajing (see Fig. 5), a series of images of the human body marked with acupuncture points according to the waxing and waning of the moon, suggests that moxa-cautery should not be used on the right-hand side during the day for fear of disturbing the Yang channels and driving the patient mad. ${ }^{41}$ The phases of the moon are emphasised by the emergence and disappearance of the toad and the hare, drawn upon its surface. In general Qi was thought to travel on the Yang planes of the body in the days before and after the moon became full and on its Yin planes when the moon was darkest. One had to avoid applying moxa-cautery to the place that lodged the Qi. When the moon was at its fullest it was also inadvisable to heyinyang 合陰陽, a euphemism for having sexual relations, lest the woman suffer an attack of wind. It is not uncommon to find self-cultivation and moxa-cautery treatments contained in the same manuscripts.

The Hamajing chart and Wuwei text share moxa-cautery prohibitions related to the position of the shen within the body although the precise times and locations do not

said to reside in the mai 'the channels'. Lingshu 8 'Benshen' 本 神, pp. 2.152-3. 




Figure 3.6 Moxa-cautery prohibitions related to the position of the shen spirit within the body. Huangdi hamajing 黃帝蛤 蟆經, 1984. Beijing: Zhongyi guji chubanshe



Figure 3.7a, b Dunhuang moxa-cautery charts (mid-gth century CE). (c) The British Library: a) Or.8210/S.6168,

b) Or.8210/S.6262

as the Wuwei texts, the Hamajing, and the moxa-cautery prohibitions of Waitai miyao, the texts seem to inform us, in different ways, about the concepts and procedures of a distinctive popular moxa-cautery tradition.

No doubt these originate as part of the larger tradition of prohibition and selection of auspicious times. The existence of a tradition of prohibition associated with cautery corroborates the view that the techniques associated with cautery were many and varied. It seems that there was a perception that popular practice should be regulated according to more informed ideas about the relationship of the physiology of the body to the movement of the heavens.

For concrete evidence of popular practice, rather than its restrictions, we have the Dunhuang moxa-cautery charts held at the British Library. ${ }^{43}$ A series of sketches of the posterior and anterior aspects of the human body illustrate the relationship between strategic anatomical locations and the treatment of specific symptoms and illnesses with moxa-cautery in a medical tradition of the medieval period (S.6168 and S.6262, see Fig. 7). They are the earliest extant moxa-cautery charts, pre-dating the Northern Song bronzes that lay out the acupuncture points, by some three or four centuries.

Each diagram in S.6168 and S.6262 seems to mark between three and six locations on the body, by drawing a line from the location to the side of the figure, where there

The charts and accompanying texts are reproduced in Ma Jixing, Wang Shumin et al. 1998, pp. 477-512. See also Lo 2005, pp. 227-51. 
is a short textual description. The text records the name of the location, detailed instructions explaining how to find it, illnesses it treats and the number of cauterisations to perform. On three of the diagrams this information is summarised in a paragraph at the side of the figure. Each paragraph begins with the phrase jiu 尒 X 'cauterise X' where $\mathrm{X}$ is sometimes a specific ailment such as 'cauterising floating wind in a person's face', 'cauterising the five exhaustions and the seven harms/injuries' or 'cauterising various human crazinesses' and at other times is the name of a specific location on the body and is followed by related symptoms. Each figure represents a discrete package detailing how to recognise and treat a distinct disease, or collection of symptoms together with a guide to finding the location and how to perform the treatment.

There are a number of unique features of the charts that deserve our attention: the moxa-cautery points are not systematically associated with channels, with internal organs or with an elaborated system of correspondences; despite five anterior views, very few locations are given on the parts of the body that had for a long time been associated with its Yin planes (anterior, inner, lower) and a significant number of locations are given which are not cited in earlier or later acu-moxa literature.

The particular combinations of text and image in the charts suggest a practical orientation, perhaps reflecting a tradition of moxa-cautery that was available to a lay readership rather than exclusive to professional physicians. A view of the charts as representative of a self-treatment tradition is also corroborated by the fact that the symptoms listed are similar to those treated in other self-cultivation traditions such as daoyin - 'leading and guiding', the tradition of therapeutic gymnastics and, to some extent, in sexual cultivation.

\section{Action: Space}

Geomancy or the science of siting has a complex history in China with competing theories and is mainly concerned with the selection of a topological site with good influences for positioning ancestral tombs, houses or other enterprises. ${ }^{44}$ Western Han texts excavated at the Yinqueshan 銀雀山 burial site in Shandong, confirm that Yin and Yang were fundamental to topographical considerations when initiating military intervention. ${ }^{45}$ Similarly the

44 A general introduction to siting theories can be found in Bennet 1978, pp. 1-26.

45 The Yin and Yang texts from the Yinqueshan burial site are invaluable for studying some of the earliest extant work of Yin and Yang theorists who were concerned with philosophic and military texts. The military texts show an elaborate concern with Yin
Mawangdui text known as the Taichanshu 胎產書 (Book of the Generation of the Foetus) uses Yin and Yang as an indication of where to bury the afterbirth to ensure good influences on a child throughout its lifetime. ${ }^{46}$ Bennet, writing of the difference between intuitive or analytical siting techniques, notes that 'the difference between siting techniques was probably in some ways analogous to the difference between medical practices'. The selection of one method for locating the source of an illness over another may well have been a matter of the social group to which the practitioner belonged and the social status of his clients. ${ }^{47}$ In fact the difference in early Chinese medical practices is, to quite a large extent, defined by how each one described the site of the illness and the time and location for auspicious intervention. In Lingshu 58 Qi Bo 岐白 emphasises the importance of knowing exactly where the illness comes from:

Qi Bo stated, 'This also has its reason. When perverse (influences) flow (within) yet do not break out, this is because in the zhi 志 "inner intention" there is that which detests and that which envies. Blood and Qi are chaotic within and the two Qi attack each other. Qisuo cong lai zhe 其所 從來者 "The place where it comes from" is subtle, if you look you do not see it and if you listen you do not hear it, so it seems as if it were ghosts and spirits'.

The Yellow Emperor said, 'as for simply using spells, how could this be?'

Qi Bo said, 'as for the $w u$ 巫 of old, it is because they knew how to overcome the 100 illness: formerly they knew bing zhi suo cong sheng 病之所從生 "the place which generated the illnesses" and could simply use spells'.

When you know where the illness arises, the cure follows easily. On the face of it, the Shuangbaoshan figurine looks like a map, but what kind of territory does it represent?

Much detail on the figurine and in the excavated mai 'channel' texts is significantly different. What the two sources share is a common structuring of bodily space along vertical lines that travel through torso and limbs. Yin and Yang, as they appear in the titles of each channel, are clearly evident in the division of superficial anatomical space. Yin is understood in its most basic sense of the softer, dark, inner, and lower aspects of the body (anterior, under the arms and legs) as opposed to the harder, light, and upper parts (posterior, superior and visible), which are Yang. There is evidence of more elaborate correspondences to Yin and Yang in Maishu (3), where the meaning

and Yang terrain and how to align military strategy auspiciously. See Yates 1994, pp. 96-7.

46 Mawangdui Hanmu boshu zhengli xiaozu (ed.) 1985, pp. 133-41. See also Li Jianmin 1994, pp. 65.4, 725-83o.

47 Bennet 1978, p. 4. 




Figure 3.8 Renzi 人字 diagram from a hemerological almanac of bamboo slips, Shuihudi 睡虎地 (late 3rd century BCE)

is extended to take in heaven and earth and match it to internal pathology, but the references in Maishu (2) remain basically topographical. Yin and Yang as representations of divisions of time or phases are altogether missing from Maishu, apart from through the temporal implications of light and dark spaces.

As they divided the body into hills and valleys of Yin and Yang and marked out the routes of the channels, the authors of Maishu created a landscape into which they could locate illness from the experience of discomfort or the discovery of pathology and lesion along the channel. Much Han medical material is similarly preoccupied with locating the site and depth of an illness. The biography of Chunyu Yi, for example, shows a constant concern with whether the illness has penetrated nei 內 'inside' or to the zhong 中 'centre,' or whether it is generated in a channel or an organ. He frequently asks about bing suo zai 病所 在 'the whereabouts of the illness' and expects to know whether it is an illness of the 'inner' or 'outer' aspect of the body, to what extent Yin has invaded Yang space, or vice versa, and where the illness is 客 $k e$ 'seated' or 'lodged' in an organ, such as the Heart or Bladder.

Equally we have seen an elaborate tradition of moxa-cautery prohibition matching physical locations on the body with the passage of time, according to age or the phases of the moon. Motifs of time applied to the body are first seen in the Shuihudi hemerological texts and the Mawangdui Taichanshu (Fig. 8), and it is in these earlier images that we first find the calendrical sequences that are developed in the Eastern Han Wuwei text, the Hamajing and finally in the Dunhuang cycles of renshen 人神 'human spirit' circulation. ${ }^{48}$ The Taichanshu has two images side by side surrounded by partially extant Branch signs arranged in

48 Mawangdui Hanmu boshu zhengli xiaozu (ed.) 1985, p. 133. Harper 1998, pp. 372-84. Shuihudi Qin mu zhujian zhengli xiaozu, p. 101. a way that indicates spring and summer, and autumn and winter respectively. The child's fortune is told according to where the Branch sign related to the date and season of their birth is located, with readings given in an associated text.

Despite applying the notion of temporality and phase to the body, the Mawangdui and Shuihudi hemerological texts do not describe any form of 'circulation', such as we have seen in the later texts. But together with the more topographical divisions of Yin and Yang laid down in Maishu and the Mawangdui channel texts (which inherently suggest the light and dark of day and night, winter and summer), by the end of the Western Han period the foundations are set for various constructions of spatio-temporal movement within the body. When these concepts of circulation finally began to mature, they do so in quite distinctive ways, with different forms of measurement. In the past the apparently conflicting treatises on the circulation of Qi have confounded those looking for a single line of development. Wuwei, Lingshu 15, Hama jing and the Dunhuang manuscripts posit the circulation of a bounded entity, such as shen and Qi, that moves along a pre-defined course and at a regular speed. In contrast more allegorical accounts envision the channels as a mirror of the waterways of China, exemplified in the 'Jingshui' treatise of Lingshu 12. Finally, the very different concept of Qi emerging in the self-cultivation literature also owes much to the root homology of water in Chinese culture. These sources emphasise the more amorphous, unbounded qualities of Qi as a water-like substance: water from a source flows continuously; water flows along a course; water flows downward; water is soft and weak, it is yielding and uncontending; it takes any shape and is difficult to see. Here transformations of the body's inner essences are guided by the breath, by physical and sexual education. ${ }^{49}$ The last section of this chapter turns to a discussion of the latter subject - the animation of the body in self-cultivation.

\section{Sensuality}

The absence of texts specifically relating to the Shuangbaoshan figurine make it the most opaque of all the medical imagery considered in this chapter. In fact we can only be certain of the fact that it was a map of the body considered important enough to be placed in the outer coffin com-

49 Sarah Allan finds that many of the qualities attributed to water in Warring States philosophy apply to the early conception of Qi in the body as described in different medical treatises; Allan 1997, pp. 35-54. 




Figure 3.9 The lines on the Shuangbaoshan figurine cluster and meet around the sense organs



Figure 3.10 Detail of the Shuangbaoshan figurine. The author's collection, photo kind permission of He Zhiguo partment of the tomb - and, as a map, it gives its owner power to manipulate the territory of the body.

Reading only from the image itself, without the benefit of any inner vision facilitated by the knowledge of text, the lines on the body tell us some of their own story. Significantly, the lines cluster and meet around the sense organs: three reach parts of the nose, 10 come to an end at the eyes, four reach the mouth and six box the ears, while 14 run to the hands (Fig. 9). The lines must therefore relate to their author's ideas about vision, hearing, sight, taste and touch, and are therefore likely to represent part of the human structure that bounds and mediates between the internal and external worlds. Is this then a sensory map of the body, rather than a model concerned with illness?

I have noted the absence on the figurine of any Yin channels of the legs, as described in the excavated texts. We also do not find a representation of the renmai 任脈, that crosses the Yin, soft, anterior torso, which is classically considered the most Yin of all channels, nor do the lines of the figurine seem to enter or represent the inner organs of the body. With Yin a corollary of fatal pathology in the inner organs,

In all cases the three Yin are the Qi of earth and the channels of death. They decay the viscera and rot the bowels which controls the killing. ${ }^{50}$

the figurine seems quite likely to have little to do with modelling illness.

In the absence of Yin channels, the figurine lacks the pathway to deterioration and decay and may be a model of perfect physiology, a plan for the movement of Qi in breath cultivation, so well described in the Mawangdui and Zhangjiashan treatises on breath cultivation. In the self-cultivation literature we find a familiar code for

'Maishu shiwen', pp. 73-4. There is also an edition of this text among the Mawangdui medical texts which has been given the modern title of Yinyang sihou. See Mawangdui Hanmu boshu zhengli xiaozu (ed.) 1985, p. 21.



Figure 3.11 Posterior of Shuangbaoshan figurine. Author's collection, photo kind permission of He Zhiguo

successful practice, exemplified here in the condition of shenming 神明 'spirit illumination' from the sexual cultivation literature from Mawangdui:

If there is no orgasm in the first movement, the ears and eyes will become keen and bright, with the second the voice becomes clear, with the third the skin gleams, with the fourth the back and flanks are strengthened, with the fifth the buttocks and thighs become sturdy, with the sixth the waterways flow freely, with the seventh one becomes sturdy with strength, with the eighth the patterns of the skin shine, with the ninth one gets through to an illumination of the spirit, with the 1oth the body endures: these are called the 10 movements. ${ }^{51}$

If we look again, the figurine seems self-assured, comfortable in his sense of himself and his importance: unlike the servant figurines of Han tombs who tend to lower their eyes, he is as if looking out at a world that he owns (Fig. 10). He has bright eyes and a steady gaze, his ears are keenly carved, his skin gleams with black lacquer and his shoulders, back and flanks are square and strong (Fig. 11), the patterns on his skin run freely from top to toe and he has indeed endured 2,00o years. To me this is a representation of the shenming. Beside the corpse such an image of perfection

51 Mawangdui Hanmu boshu zhengli xiaozu (ed.) 1985, p. 156. 
might aid physical immortality by ensuring the body's invulnerability to pathological processes beyond the grave.

So if this is the image of a healthy person, resplendent with free-flowing Qi and acute sensory perception, can we know more of him? Images of robust and vigorous bodies also appear in Daoyin tu where the exercises are made relatively explicit in a number of different ways. From the first text, we learn that the basic prescription is that of a health regimen, seasonally adjusted. By the third text which is a treatise on the causes of illness and on measures for maintaining good health, we have been told that changes in the climate, social class, excessive emotion, diet, maintenance of body temperature, sleep, Yin, Yang and Qi are all implicated in what amounts to a broad multi-factorial attitude to the causation of illness. But no matter how the cause of illness is construed, Yinshu usually prescribes breath cultivation and guided therapeutic exercises to invigorate the whole or parts of the body. The exercises are the practical application of a medical philosophy that understands the maintenance of good health as dependent upon embodying movements of the external world.

The images and culture of Daoyin tu and Yinshu must be understood in the context of a wider discourse of ideas about change and transformation in early China. That there is an underlying principle of transformation at the foundation of the generation of all things is a common assumption in Warring States and Han philosophic literature. Observation of continuous movement and change in the natural environment is often cited as evidence. The sage, whether ruler, noble or physician, can achieve mastery through aligning his own behaviour with the patterns that reveal themselves. Zhuangzi 20 recommends shaking off earthly relationships and responsibility to move freely with the rhythms of the universe:

without praises, without curses,

now a dragon, now a snake,

you transform together with the times.

And never consent to be one thing alone. ${ }^{52}$

In the practice of medicine, the sage practitioners apply principles learned from observation of change in the body:

As for Qi, it benefits the lower body and harms the upper; follows heat and distances coolness. So, the sages cool the head and warm the feet. ${ }^{53}$

Sagehood comes with learning and practice. Like class it is manifest in particular forms of behaviour, and cultivation. Yinshu points out that a person's class is also distinguished by the way that they get ill, and their knowledge and/or ability to cultivate good health: a common person is subject to elements beyond their control: the vicissitudes of their labour and the weather, but the guiren 貴人 'nobility' do not harmonise their joys and passions, so either their Yin or Yang Qi becomes too abundant. The way to harmony was to normalise the thermostatic environment through breath control:

If they [nobility] are joyful then the Yang Qi is in excess. If they are angry then the Yin Qi is in excess. On account of this, if those that follow the Way are joyful then they quickly exhale [warm breath], and if they become angry they increasingly puff out [moist breath], in order to harmonise it. ${ }^{54}$

That modern European and American society is obsessed with 'happiness' as the desired and normal state serves to emphasise, through contrast, this particular expression of emotional norms in Warring States and early imperial times. Here Yinshu simultaneously records the phenomena of emotion and expresses its role in the embodiment of social relations. Harmonising is represented by the graph $h e$ 和 'to bring together'. Joy and anger come together, not necessarily in an act of suppression, but in a conscious resolving or easing of extreme emotion experienced simultaneously as conditions of heat or cold. A number of scholars interpret the terms $x u$ 呴 and chui 吹 to contrast methods of exhalation. $X u$ 呴 refers to a slow exhalation of hot vaporous breath through an open mouth. In contrast chui 吹 is thought to refer to exhaling cold breath. In both methods of harmonising emotion, $x u$ 呴 and chui 吹, involve focusing on the mode of exhalation. This is the therapeutic event in which all the daoyin practitioners engage..$^{55}$

54 'Yinshu shiwen', p. 86.

55 Ma Jixing and Gao Dalun consider $x u$ 呴 a slow exhalation of hot vaporous breath through an open mouth. In contrast, chui 吹 is understood to refer to exhaling cold breath. The source of this interpretation is given in Ma Jixing 1992, p. 826 n.17 who quotes the He Shanggong commentary to the statement in Laozi 29, 'some $x u$ 呴, some chui 吹', which states, ' $X u$ is warm, chui is cold. Where there is that which warms there is certainly that which cools'. Ma also gives some evidence that $x u$ is a slow exhalation and chui is faster. Quoting Zhuangzi 6, Ma also interprets a distinction between $x u$ 呴, the out-breath that carries a lot of moisture and chui 吹 which he extrapolates is drier: 'assist $x u$ 呴 with moistness, assist moistening with spittle'. Ma also distinguishes differences in the shape of the lips and the mouth during the different types of breath control. All the relevant references to the particular qualities of breathing given by Ma Jixing, including the Heshanggong commentary, derive from at least 400 years later than the Yinshu and may reflect a re-working of the technique and terminology of breath cultivation. There is 
This deliberate concentration and directing of attention to the sensual body is a pervasive feature of daoyin. In Yinshu it is sometimes referred to as placing yi 意 'intention' on a part of the body or an activity. Two occurrences of the term can be found in consecutive techniques related to incipient illness or illness as it first emerges. These examples show that the yi may be distracted or applied. Graham, discussing the Mohist Canons, attempts a definition of $y i$ as 'forming an image of the object of attention'. Yinshu confirms the idea that yi involves the potential for concentrating attention, but the yi may also be distracted: Pulling incipient exhaustion illness. The intention is agitatedly set on pacing around, the body hurts more and more. At this moment you must treat it with the pull of the Eight Warps; quickly breathe out (dry breath) and quickly exhale (warm breath), pull Yin. Soak the space between the eyebrows in cold water for the time it takes to eat bowl of rice. Get rid of the water. With two hands hold two (two?) rush mats. Stroke between the eyebrows upwards and rock it up and down. Call out 'hu hu' through the mouth. Do it altogether 10 times and stop. ${ }^{56}$

Resolving a distraction of the $y i$ involves breath-cultivation to reduce heat and dryness, cooling and massaging the forehead. Yi may refer to concentrating attention on specific activities and parts of the body. It is located where it is placed. With Graham's discussion in mind, we can see how concentrating Qi includes the ability to form an image (in the following case probably an image that embraces a visualisation of physical comfort) at that part of the body that is threatened by the incipient illness. The full potential of placing yi on areas of the body can be appreciated from the following technique for bowel illness:

Incipient illness in the bowel. Invariably there will be swelling at the front. When there is swelling, apply the in-

sufficient evidence in the Yinshu to provide a specific interpretation which differs somewhat from Ma's analysis. The information is summarised in Donald Harper 1998: Translation: MSIIA2 n.5. The main statement is: 'If it is dry then $h u$ 呼 frequently and lie down frequently. If it is damp then chui 吹. Do not lie down, make the Yin substantial. If it is hot then concentrate on $x u$ 呴. When it is cold then work the body'. See 'Yinshu shiwen': 86. Chui 吹 is also used to remedy an excess of Yin which manifests as anger whereas $x u$ 呴 counteracts the condition of excess Yang engendered by joy. The information implies that chui 吹 is an exhalation of moist breath which resolves a Yin, possibly cold condition, while $x u$ 呴 is an exhalation of hot breath which resolves a Yang condition. $\mathrm{Hu}$ 呼 remedies dry conditions and is therefore an exhalation of dry breath. The quality of the out- breath is controlled by the depth of breathing. Breath from the bottom of the lungs is hot and moist, from the mouth is cold and dry. tention on the lesser abdomen and concentrate on puffing out (moist breath). Stop after 100 times. ${ }^{57}$

This technique presents us with a complex interaction in the internal environment of the body; firstly it identifies the site of discomfort or illness; then the same sites become the location for concentrated attention, presumably achieved with increased awareness of that area of the body; then with measured manipulation of the breath function, perhaps even with the imagination of comfort through conscious projection of Qi, after a prescribed time a sequence of therapeutic events unfolds of its own accord.

Ailing from liao 疹 $\square \square$ [liquor]. ${ }^{\square}$

The prescription for pulling it: grasp a staff in the right hand, face a wall and do not breathe; with the left foot tread on the wall, resting when tired; likewise with the left hand grasp the staff, with right foot step on the wall, likewise rest when tired. When the Qi of the head flows downwards, the foot will not be immobile (and numb), the head will not swell, and the nose will not be stuffed up. ${ }^{58}$

Many of the Yinshu exercises mirror this process, including the one above, specified for an illness probably associated with drinking alcohol, where after a certain period of exercising the Qi will begin to flow naturally and without further stimulus. The practitioner activates his intentional body into the multiplicity of bodies already linked and overlapping within this one process (the manifestation of his sick body, its pathology and its biological processes, the experience of the sick body and the imagination of a well-ordered body, its idealised social identity, its social projection - the body that can be affected by controlled breathing - a body infused with Qi). As far as intention activates the potential to influence the internal environment of the body, it allows the practitioner to participate in his own resistance to the inevitable process of decline. This following excerpt is the outcome of a technique that includes both breath and sexual cultivation:

Be careful, do not drink wine and eat the five flavours; put the Qi in order with intent and the eye will be bright the ear keen, the skin will gleam, the 100 mai will be full and the Yin will rise again. From this you will be able to stand for a long time, go a long way, and live for (ever). ${ }^{59}$

Together Daoyin tu and Yinshu provide a practical guide to daoyin through which practitioners can restore their

\footnotetext{
$57 \quad$ Ibid. p. 83 .

58 'Yinshu shiwen', p. 83; Gao Dalun, 1995, pp. 122-3.

59 Mawangdui Hanmu boshu zhengli xiaozu (ed.) 1985, pp. 147-8. I have accepted the rearrangement of the slips proposed by Harper and Qiu Xigui. Taking the Mawangdui Hanmu boshu as the starting point the slips would then be ordered 51, 41, 40. Qiu Xigui 1992, p. 535. Harper 1998, tr.: ms. V1.A.14.
} 
health, treat illness and simultaneously affirm their natural membership of an elite group. Measured physical movement and the orientation of the senses, the training of intention, all come together in the pursuit of cultivation and refinement.

The middle texts of Yinshu provide precise details of both daily practice and the treatment of specific illnesses. The second text, which is the main bulk of Yinshu, sets out 41 set exercises. In the first part it describes and names exercises, while in the second it aims at specific therapy. Thirty-seven are perfectly preserved, whilst four are damaged. Many of the exercises are given titles, some of which simply describe the exercise, usually naming the culminating posture in a sequence. Others liken the exercises to animal movements or attitudes. Another type of title refers to applying treatment to a specific illness, or part of the body. There are treatments both for pain in joints and muscles, such as knee pain or back pain, and for internal illnesses such as loose bowels, inguinal swellings or inner exhaustion. Where the treatment is directed to a specific part of the body, it is probably meant to improve the function of that part, rather than to address a specific illness.

Where modern Taiji quan manuals provide diagrams, photographs or even videos, Yinshu may have had charts like Daoyin tu to provide one more dimension to help the student. Without visual aids it is often impossible to understand how to interpret a textual account with the body. The power of the images as a mnemonic device is that they simultaneously convey whole-body movement, its posturing, gestures, attitudes and mood. Yet more powerful is the combination of image and caption, such as in Yinshu, where the brief line contextualises the image and gives it greater depth.

Consider, for example, the appeal to the imagination of animals in the context of Daoyin tu, Yinshu and more generally throughout the culture of daoyin. Where a particular posture is given the name of an animal, there are immediate, commonly understood implications for the interpretation of that image with the body. But we cannot assume that we have grasped the full implications of the designation without questioning whether our understanding of a bear, monkey, crane or dragon is stunted by removal and distance. Yinshu mentions the Bear Ramble as being good for the muscles of the back (Fig. 12). The image on Daoyin tu suggests a square, ambling walk that might well ease the back, but perhaps the bear signifies more than lumbar strength.

Roel Sterckx has written at length about the transformational powers of animals in Warring States and Han



Figure 3.12 The Bear Ramble. Daoyin tu 導引圖 (Guiding and Pulling Chart), Mawangdui, tomb 3 (closed 168 вCE). Silk. Detail from reconstruction (C) Wellcome Library, London, Loo36oo7

literature ${ }^{60}$ He cites early references to exorcistic and ritual dances with animal skins which mirrored symbolically the potential for transformation in the animal world. In the passage of time, and with proper discipline adjusted according to the passing seasons and the movements of nature, an ordinary man might reinvent himself as a noble or a sage. Observation of motion and change in the animal world constituted an important element in the development of the earliest calendars and therefore in shaping civilised human behaviour. Animals themselves were attributed the different qualities of the Yin Yang spatial and temporal cycles, allowing them universal features in the process of metamorphosis. Animals were not even limited to the boundaries of one form or construction of species: seasonal changes marked regular times for mice to transform into quails, or hawks to pigeons. The shedding of form was not just the prerogative of observably metamorphosing animals, such as the reptiles, but of many different beasts, some capable of spirit-like metamorphosis.

Here are Harper's (1998) translations of the titles of the Daoyin tu animal forms: The Crane, Dragon Ascending, Monkey Bawling to Pull Internal Hotness, Gibbon Bawling, Gibbon Shouting, Bear Ramble, Merlin. ${ }^{61}$ It is significant that only one of these, Monkey Bawling to Pull Internal Hotness (Fig. 13), is immediately related to a physical disorder - the disorder in question being the plight of the noble who, we have seen, by virtue of his class, is committed to remedying unresolved emotional and thermostatic excess.

6o Sterckx 2002, pp. 165-204.

61 Harper 1998, pp. 310-16. 




Figure 3.13 left 'Monkey Bawling to Pull Internal Hotness';

Figure 3.14 right 'Dragon Ascending'.

Two fragments from the Daoyin tu 導引圖 (Guiding and Pulling Chart), Mawangdui, tomb 3 (closed 168 BCE). Silk. (C) Wellcome Library, London, Loo4O263

If bawling like a monkey is the path to nobility, the transformational powers of the numinous animals, such as the dragon, birds and tiger, could channel a more magical efficacy into the body. We know that when Yinshu and Daoyin tu describe a dragon ascending they do so in the context that the dragon was known to be the embodiment of change. As Sterckx points out the expression longbian 龍變 'to transform like a dragon' also provided an epithet for sagehood, authority and encompassing virtue. ${ }^{62}$ Guanzi describes the dragon as a shen 'spirit' itself, able to change its size rapidly and at will and to move between heaven and earth. The likening of the dragon changing its domain to the sage moving his habitat exposes the local person for being parochial'. The movement for Dragon Ascending, here explained in Yinshu,

Dragon Ascending. Bend the front knee, extending at the back. Interlock the two hands, hold the knee and look up ${ }^{63}$ demonstrates the proud full chest of the dragon, but does not obviously tally with the Daoyin tu image of the same name (Fig. 14) where the figure is standing with both arms raised above his head. As with the different witnesses to common texts excavated from Warring States and Han texts, it may be in vain to search for an imaginary original, so we should not assume one unified tradition of daoyin. Here both witnesses to the Dragon Ascending are expansive and rising movements, and it is in the imagination of dragons that we should look for similarity.

$62 \quad$ Sterckx 2002, p. 179.

63 The animal forms are all described in 'Yinshu shiwen', pp. 82-3.
While there are numerous bird forms in Yinshu, the titles do not exactly match the Daoyin tu captions. For birds, as well as dragons, their wings are the vehicle by which transformations through space and time occur. Moving between heaven and earth, birds symbolise the transition through that liminal space where light turns to dark and distance disappears, and so it is logical that we should find that they feature significantly in the daily practice aimed at physical rejuvenation.

Here we find the rapid movements of a wild duck shaking the water out of its feathers:

Wild Duck Bathing. Interlock the hands behind the back and shake the head [a movement which no doubt eases tension in the neck and head and changes the mood].

Here is the familiar image of an owl with its head buried into its neck looking from side to side:

The Owl (Shivers?). Interlock hands behind the back, shrink the neck and shake the head.

Here the gibbon supply flexes its body:

Gibbon Hold. With the right hand hold the left foot. Raise the left hand, turning the back. Bend forward to left and right.

The tiger is more difficult to 'see' but the movement is at the least a bold forward movement:

Tiger Pull. Step one foot forward, raise one arm high and bend.

The graceful bowing of the deer is more readily understood:

Prostrate Deer. Raise the two hands, turning the back and bend forward as far as possible.

As is the jumping frog:

Leaping Frog. With hands parallel, wave them up and down to right and to left.

Such variations as exist between the nature of an exercise described in Yinshu and the movement of a Daoyin tu figure that bears the same or a similar title serve to illustrate the diverse traditions of daoyin in the Western Han period.

In the last text of Yinshu we have six animals cited in a regimen designed to tone and strengthen the body that amounts to a great celebration of the body and its relationship with the natural animal environment.

Holding the breath is good for the coital sinew; (Praying Mantis?) is good for the constant channel; Snake Shake is good for the great brain; Wild Duck bathing is good for [illnesses] of the head; Encircling Channels is good for the patterns of the skin and is good for the heels and head; Follow to the Side is good for the ear; looking in a Yang direction is good for the eyes; look up by opening the mouth is good for the nose; roaring, but not emitting a sound is good for the mouth; stroking the heart and raising the chin is good for the throat and gullet; Owl (Shivers) is good for bending the neck; Tiger Looks Back is good for 
the neck and the buttocks; Pull the Back is good for the shoulder muscles and sinews; Limbs Falling is good for the armpits; Chicken Stretch is good for the shoulders and arms; Backward Waving is for the abdomen and heart; Backwards Swirling is good for the two sides; Bear Ramble is good for the muscles of the back; Repeating Holds is good for the small of the back; the Pace of Yu is good for between the thighs; Forward Stumble is good for the thighs and knees; Rotating the Ankles Backwards is good for the sole of the feet; Squeezing the Toes is good for the Qi of the feet; Knocking the Heels is good for the chest. For these things stop after three times. ${ }^{64}$

The appeal to animal imagery is a device that daoyin shares with literature of the sexual arts where animal movements communicate sexual postures and rhythm. The following are called the shijie 十節 '10 regulations' in He yinyang, or the shishi 十勢 '1o postures' in Tianxia zhi dao tan 天下至道談:

One states: Tiger roaming. Two states: Cicada clinging. Three states: Inchworm. Four states: Waterdeer butting. Five states: Locust splayed. Six states: Gibbon squat. Seven states: Toad. Eight states: Hare bolting hither and thither. Nine states: Dragonfly. Ten states: Fish gobbling. ${ }^{65}$

He yinyang does not detail individual movements that make up the animal postures. The activities are therefore codified and simultaneously restricted, demanding tuition by an expert. Later literature eventually provides an explanation for some of the postures. In contrast daoyin in Yinshu and Daoyin tu makes its codes and activities explicit: it is therefore more likely to be available to a wide group of people without the necessity of a professional intermediary to interpret and prescribe a course of action.

Here is the Yinshu entry for the Inchworm as a daoyin exercise:

Extend the lower leg curling the toe 30 times. This is called the Inchworm. 66

The Inchworm or looper caterpillar, as other caterpillars, moves by stretching and contracting the length of its body in a curling motion. No instructions are given for the inchworm in He yinyang and Tianxia zhi dao tan, two of the sexual cultivation texts from Mawangdui, but it is easy to imagine how stretching and curling the body can be adapted to sexual posture and movement.

All these movements belong to a tradition of maintaining good health and fitness but they can also be applied prescriptively, often in combinations that are repeated many times. Unfortunately the first example below has a series of lacunae over the symptom.

\footnotetext{
64 'Yinshu shiwen', pp. 85-6.

65 Mawangdui Hanmu boshu zhengli xiaozu (ed.) 1985, p. 165.

66 'Yinshu shiwen', p. 82.
}

Do Wild Duck Bathing 30 times and Tiger Looks Back 30 times. Lie down on the back again as before. Rest after 20 times. Get up again and sit tall (on the haunches). Do Wild Duck Bathing 40 times and Tiger Looks Back 40 times. Lie down again as before. Thirty times and rest. Then get up and do the Wild Duck Bathing 50 times, Tiger Looks Back 50 times and stop.

Pulling back ache. Bear Ramble 1o times. Forward (Grasps) 10 times. With the feet apart lean forwards and backwards, touching the hands to the floor. Stop after 10 times.

Pulling lop-sided illness. If it's on the [left] cheekbone, with the right hand hold the hair at the right temple and extend the left hand and pull it with the right hand; if it's the right cheekbone pull it the same as the left. Do it altogether three times and stop. Do Follow to the Side 10 times, Looking Yang 10 times, Wild Duck Bathing 10 times. Sit straight, loosen the hair and knock the heels 300 times, Back Step 300 times and rest. ${ }^{67}$

In the structure of Yinshu we first see a dialectic between exercises to preserve and enhance health and the treatment of disorder that finds a different balance in later centuries. Where self-cultivation is a solitary practice and aims at perfecting the physiology of the body, the records naturally generate a lyrical and animated body imagined through homology, not just a passive object upon which we construct realities. By appealing to animal imagery, the daoyin forms engage all the senses of the self cultivator, not just the visual, working through imagination on both movement and mood. In contrast as the body increasingly becomes the subject of professional observation it is, inevitably, silenced and contained within its visibly perceived boundaries, or other such boundaries that are amenable to the physician's control.

\section{Conclusion}

This chapter began with a detailed consideration of the Shuangbaoshan figurine in its relationship to the concept of mai and associated medical practice. The fundamental principles expressed in the lines drawn on its body link the mai to those texts that are explicitly concerned with the early development of cautery, and to a lesser extent to petty surgery, massage, pharmacotherapy and therapeutic exercises. It is also clear that the lines bear some relationship to empirical observations of the body, to a somatography of muscles and veins and to patterns of pain. But there are many perspectives from which we can begin to interpret its shape and form. By the end of the Han period astro-calendrical ordering of the body began

$67 \quad$ Ibid., p. 84 
to impact upon ideas of physiology, shaping the mai into what Li Jianmin has called 'a field of temporal spaces' - i.e. the physical space where heaven and earth pivot and the location for learned medical intervention.

Reading from the figurine alone, without supporting text, we find significant information about early Chinese perceptions of the body, and the importance placed on developing and maintaining an acuity of the senses, even beyond the tomb. Like the bronze figure excavated from the Sanxingdui 三星堆 tomb, whose enormous ears must surely have allowed its owner a deeper, more profound reception of external events, the figurine's clearly defined and linked sense organs, its posture and texture suggest something more powerful and daemonic than a mere physician's dummy. A training and ordering of the sensual body evident in both shape and pattern, link the figurine to the practice of self-cultivation as we know it from the texts and images of other Western Han tombs in the old kingdom of Chu. The most lively and attractive of these images are the figures of the Daoyin tu.

At a glance the Daoyin tu charts a series of figures engaged in a daily exercise routine, stretching and relaxing their bodies. Yet part of the magic of this chart is due to the fact that we know it is more than that. With the more recently discovered Yinshu we can gain new insights into how the Daoyin tu images translated into action. Text and image have helped us to piece together the practice of daoyin. Building on the memories and emotions of the collective imagination, the practitioner of daoyin could do more than just exercise his body; he could effortlessly transform himself, crossing the boundaries of class, of species, of Yin and Yang, moving with the consummate ease of the dragon or the square, lumbering strength of the bear.

Each figure emphasises an individual vitality: each character is unique in dress, expression, build and deportment. These are the guiren 'nobility' in the pursuit of harmonising the Yin and Yang of their bodies, a balance evident in their moods, movement and emotion as well as the thermostatic environment of their bodies. Here, between image and text we find the body constructed most clearly as a location for social agency. In their every aspect, the figures assert their class and the freedom they gain from being able to master the physiological elements that ensure long life. They seem free from the tyranny of ghosts and demons, and are yet far from the silent, objectified bodies that are a trademark of later medical treatments of the body.

\section{Bibliography}

\section{Primary Sources}

\section{Manuscripts}

\section{Dunhuang Manuscripts}

Pelliot P.2675 (BNF)

Stein Or.8210/S.5737 (BL)

Stein Or.8210/S.6168 (BL)

Stein Or.8210/S.6262 (BL)

Wuwei 武威 Hantanpo 旱灘坡 medical manuscripts (Eastern Han). Modern edn: Wuwei Handai yijian yanjiu 武威漢代醫簡研究, Zhang Yanchang 張延昌 and Zhu Jianping 朱建平 (eds) 1996, Beijing: Yuanzineng chubanshe.

Mawangdui Manuscripts (Tomb closed c. 168 BCE).

Modern titles given by editors of Mawangdui Hanmu boshu 馬王堆 漢墓帛書, vol. 4, Beijing: Wenwu chubanshe)

Tianxia zhi dao tan 天下至道談 (The Culminant Way Under Heaven) He yinyang 合陰陽 (Harmonising Yin and Yang)

Zubi shiyi mai jiujing 足臂十一脈炎經 (Cauterization Canon of the 11 Foot and Arm Channels)

Yinyang shiyimai jiujing 陰陽十一脈炎經 (Cauterization Canon of the 11 Yin and Yang Channels)

Zhangjiashan Manuscripts (Tomb closed c. 186 BCE)

Maishu 脈書 (The Channel Book)

Yinshu 引書 (The Pulling Book)

Printed Sources

Huangdi hama jing 黃帝蛤蟆經, Anon. n.d. Reprint: Beijing: Zhongyi guji chubanshe, 1984 .

Shiji 史記 (Records of the Historian) c. 94 BCE, by Sima Qian 司馬遷 (c. 145-86 вСE) edn. Beijing: Zhonghua shuju, 1972.

Sibu beiyao 四部備要, Shanghai: Zhonghua shuju, 1927-1936.

Waitai miyao 外臺秘要 (c. 752), Wang Tao 王壽. Reprint, 1993, Gao Wenzhu 高文鑄 (ed.). Beijing: Huaxia chubanshe.

\section{Secondary Sources}

Akira Akahori 赤倔昭 1982, 'Go-kan shoki no igaku no ichi danmen' 後漢初期醫學一斷面 Toyo no Kagaku to Gijutsu, Kyoto: Dohosha.

Allan, S. 1997, The Way of Water and Sprouts of Virtue, Albany: SUNY.

Bennet, S.J. 1978, 'Patterns of the sky and earth: a Chinese science of applied cosmology', Chinese Science 3, 1-26.

Brashier, K. 1996, 'Han Thanatology and the Division of "Souls"', Early China 21, 125-58.

Bridgman, R.F. 1981, 'Les fonctions physiologiques chez l'homme dans la Chine antique', History and Philosophy of the Life Sciences 3.1, 33 o.

Epler, D. Jr, 1980, 'Blood-letting in early Chinese medicine and its relation to the origin of acupuncture', Bulletin of the History of Medicine 54, 337-67.

Feher M., R. Naddaff and N. Tazi (eds) 1989, Fragments for a History of the Human Body, New York: Zone

Gao Dalun 高大倫 1992, Zhangjiashan Hanjian Maishu jiaoshi 張家 山漢簡脈書校釋, Chengdu: Chengdu chubanshe.

1995.Zhangjiashan Hanjian (Yinshu) yanjiu 張家山漢簡引 書研究, Chengdu: Bashu shushe.

Graham, A. 1989, Chuang-tzu: the inner chapters, London: Mandala. 
Harper, D.J. 1998, Early Chinese Medical Literature, London: Kegan Paul International.

He Zhiguo 何志國 1994, 'Woguo zuizao de renti jingmai qidiao' 我國 最早的人體經脈漆雕, Zhongguo wenwubao 15 (April 17), 4. - 1995, 'Xi Han renti jingmai qidiao kao' 西漢人體經脈漆 雕, Daziran tansuo 3, 116-20.

He Zhiguo and V. Lo 1996, 'The Channels: a preliminary examination of a lacquered figurine from the Western Han period', Early China 21, 81-124.

Hunansheng bowuguan 湖南省博物館 and Zhongguo kexueyuan kaogu yanjiusuo 中國科學院考古研究所 1974, 'Changsha Mawangdui er, sanhao Hanmu fajue jianbao' 長沙馬王堆二, 三號漢墓發掘簡 報, Wenwu 7, 39-48.

Jiangling Zhangjiashan Hanjian zhengli xiaozu 江陵張家山漢簡整 理小組 1989, 'Jiangling Zhangjiashan Hanjian Maishu shiwen' 江 陵張家山漢簡脈書釋文, Wenwu 7, 75-81.

Kuriyama, S. 1986, 'Varieties of haptic experience. A comparative Study of Greek and Chinese pulse diagnosis', PHD diss., Harvard University: MA.

Li Jianmin 李建民 1994, Mawangdui Hanmu boshu yucang mai bao tu jian zheng 馬王堆漢墓帛書禹藏埋胞圖箋証, Bulletin of the Institute of History and Philology 65.4, 725-830. 2001, Sisheng zhi yu 死生之域, Taibei: Academica Sinica.

Li Xueqin 李學勤 1991, 'Yinshu yu Daoyin tu' 引書與導引圖, Wenwu tiandi 2, 7-9.

Lo, V. 199o, 'Tracking the Pain', Sudhoffs Archiv 83, 191-210. 1998, 'The influence of yangsheng culture on early Chinese medical theory', PHD diss., London University.

2000, 'Crossing the Inner Pass: a nei/wai distinction in early Chinese medicine', EASTM 17, 15-65.

Lo V. and C. Cullen (eds) 2005, Medieval Chinese Medicine, London: RoutledgeCurzon,

Loewe M. (ed.) 1993, Early Chinese Texts: A Bibliographical Guide, Berkeley: Early China Special Monograph Series 2.

Ma Jixing 馬繼興 1992, Mawangdui guyishu kaoshi 馬王堆古醫書考 釋, Hunan: Hunan kexue jishu chubanshe.

Ma Jixing, Wang Shumin 王淑民 et al. 1998, Dunhuangyiyao wenxian jijiao 敦煌醫藥文獻輯校, Nanchang: Jiangsu guji chubanshe.

Mawangdui Hanmu boshu zhengli xiaozu 馬王堆漢墓帛書整理小 組 (ed.) 1979, Mawangdui Hanmu boshu Daoyin tu 馬王堆漢墓帛 書導引圖, Beijing: Wenwu chubanshe, including an introductory article by Tang Lan 唐蘭, 'Shilun Mawangdui sanhao Hanmu chutu Daoyin tu' 試論馬王堆三號漢墓出土導引圖, 1-10.

1985, Mawangdui Hanmu boshu 馬王堆漢墓帛書, vol. 4 Beijing: Wenwu chubanshe.

Qiu Xigui 装錫圭 1990, 'Mawangdui yishu shidu suoyi' 馬王堆醫書 釋讀瑣議, in Qiu Xigui (ed.), Gu wenzi lunji 古文字論, Beijing: Zhonghua shuju.

Shuihudi Qin mu zhujian zhengli xiaozu 睡虎地秦墓竹簡整理小 組 (ed.) 1990, Shuihudi Qin mu zhujian 睡虎地秦墓竹簡, Beijing: Wenwu chubanshe.

Sichuansheng wenwu kaogu yanjiusuo 四川省文物考古研究所 1996, 'Mianyang Yongxing Shuangbaoshan erhao Xi Hanmu guomu fajue jianbao', Wenwu 10, 13-29.

Sivin, N. 1993, 'Huang ti nei ching', in Loewe (ed.), 196-215.

Sterckx, R. 2002, The Animal and the Daemon in Early China, New York: SUNY.

Unschuld, P.U. 1985, Medicine in China: A History of Ideas, Berkeley: University of California Press. 1986, Nan-Ching: The Classic of Difficult Issues, Berkeley: University of California Press.

Williams, M.A. 1989, 'Divine image - prison of flesh: perceptions of the body in ancient gnosticism', in Feher et al. (eds), vol. 1, 128-47.

Xie Guihua 謝桂花, 2005, 'Bamboo and wooden strips scattered through the ancient city and beacon sites of north-west China', in Lo and Cullen (eds), 78-106.

Xie Guihua 謝桂花, Li Junming 李均明 and Zhu Guozhao 朱國炤 1987, Juyan Hanjian shiwen hexiao 居延漢簡釋文合校, Beijing: Wenwu chubanshe.

Yamada Keiji 山天慶兒 1979, 'The formation of the Huang-ti Nei-ching.' Acta Asiatica 36, 67-89.

(ed.) 1985, Shin hakken Chugoku kagakushi shiryo no ken$k y u$ 新發現中國科學史資料研究, Kyoto: Kyoto daigaku jinbun kagaku kenkyujo. 1998, The Origins of Acupuncture, Moxibustion and Decoction, Kyoto: International Research Centre for Japanese Studies.

Yates, R. 1994, 'The Yin-Yang texts from Yinqueshan', Early China 19, $75^{-144 .}$

Zhang Yanchang 張延昌 and Zhu Jianping 朱建平 (eds) 1996, Wuwei Handai yijian yanjiu. 武威漢代醫簡研究, Beijing: Yuanzineng chubanshe.

Zhangjiashan Hanjian zhengli zu 張家山漢簡整理組 1990, 'Zhangjiashan Hanjian Yinshu shiwen’ 張家山漢簡 引書釋文, Wenwu 10, 82-6. 


\section{The Iconography of Time: What the Visualisation of Efficacious Movement (Shi 勢) Tells Us about the Composition of the Yijin Jing 易筋經 (Canon for Supple Sinews)}

\section{Elisabeth $\mathrm{Hsu}^{*}$}

\section{Introduction}

Time can be comprehended cyclically, as we do when we think of diurnal or seasonal cycles. Or it can be experienced in a polar way, as when we contrast day and night as opposites of light and dark, or as the ancient Chinese did, when they spoke of 'spring and autumn' - chunqiu 春秋-as polar Yin and Yang 陰陽 alternations between periods of expansive growth (zhang 張) and contraction (shou 收). As argued elsewhere, the bodily routines in the Yijinjing 易筋經 (Canon for Supple Sinews, also called The Sinews Transformation Classic) lead the practitioner to embody this latter experience of time as a pulsating movement of Yin Yang directional opposition. ${ }^{1}$
This chapter will discuss another common way of relating to time, namely time as a linear flow visually rendered as a vector. In the contemporary West, the vector goes from the past on the left-hand side to the future on the right. This embodied experience, like that of writing from the left to the right on a sheet of paper, may well explain its directionality. Time as linear trajectory is not particular to the West, however. It also underlies, for instance, the Tibetan medical depiction of different pulse qualities in an educative thanka of the Blue Beryl from the 17 th century. $^{2}$ Pulse qualities are shown there as curves along a vector (Fig. 1), much as pulse beats or heart beats in a biomedical ECG are visualised as sinuses along a horizontal timeline. Yet the timeline for pulse movements on this Tibetan thanka is vertical. ${ }^{3}$



Figure 4.1 Depiction of Tibetan pulses along a linear trajectory of time (Parfionovitch et al. 1992, pl. 6o, line 4)

* I am much indebted to Master Qiu and Lim Chee Han for teaching me the postures/movements they practised of the Yijin jing. Thanks go also to Meir Shahar for making substantial inroads into its textual history (particularly after I expressed this wish at the conference on 'Chinese Medicine: A Visual History', in Beijing, 14-17 September, 2005). Finally, I thank the reviewers and editing team that made this publication possible.

1 Secondary literature on the Yijin jing in western languages is to date inexistent, with the notable exceptions of Shahar (2008, pp. 16o-73) and Kohn (2008, pp. 195-7). This article is part of a trilogy, all of which compare text with practice: Hsu and Lim (2016) compare a Singaporean version of the Yijin jing with one in the People's Republic of China, and Hsu (in prep.) addresses methodological questions of historiography. 




Figure 4.2 Depiction of Chinese mai 脈 in an iconic-indexical manner. (Ma Kanwen 1983, p. 64 claims that he found these images in an Arabic treatise, which the author could not locate; they are reproduced here in the hope that a reader may identify them.)

Another iconography of time seems to have prevailed when visualising Chinese medical 'pulse images' (mai xiang 脉象), ${ }^{4}$ where each pulse image would be depicted in a round medallion (Fig. 2). Furthermore, as a detailed study of the vessel-pulses mai 脈/脉 in a text of the received tradition suggests, ${ }^{5}$ mai were comprehended as both 'iconic' and 'indexical' signs (in C.S. Peirce's terms). For instance, an 'intense' mai (jimai 急脈), in contrast to a 'slack' mai (huan mai 緩脈), would be indexical of intense urges to urinate, and of intense pain, just as smoke is indexical of fire. Simultaneously, an 'intense' pulse had an iconic resemblance to the tensed-up tendons or muscles in those situations. ${ }^{6}$ Evidently, the movement of the mai was assessed not only visually in medallions, but also linguistically through discrete iconic and indexical signs.

Likewise, Chinese writing does not aim to account for the continuous and uninterrupted flow of meaningful (phonemic) sounds as does an alphabetic script. Rather, the Chinese script consists of discrete characters, where each is an integral whole arising from a complex interplay

4 See Kuriyama 1999, pp. 84, 10o, figs 13, 15, 16, 17; see also Ma 1983, p. 364, reproduced here in Fig. 2: 'Depiction of Chinese mai 脈').

$5 \quad$ Hsu 2010, pp. 351-7.

6 Peirce (1932) aligns himself with Western science, which does not contest the reality of fire but is doubtful of the reality of Chinese medical viscera, while the above analysis uses Peirce's indexical sign to highlight a relationship given as 'real' in Chinese medical texts. of visual form, sound and meaning. ${ }^{7}$ Chinese characters have been considered 'modular' in composition, given that their basic building blocks are recurrent. ${ }^{8}$ In most cases they are made up of an element that is indexical of a sound, and of another one, two or three elements derived from an iconic semblance. Accordingly, a Chinese character may be said to be an 'indexical-iconic' entity. This would allow us to see a continuity in iconographic style between the characters of the Chinese script and the above-mentioned Chinese pulse images.

In a similar vein, a posture in a bodily routine, like one of those in the Yijin jing, may be 'modular' in composition and visualised in the above iconographic style. Even if an iconic sign may appear static it can be indexical of temporality. The Chinese authors who made use of iconic-indexical signs obviously considered them suited to capturing the temporalities of the postures in martial arts routines, which were called shi 勢.

This chapter examines the visual rendering of shi 勢, which in translation is generally approximated as both a 'bodily posture' and a 'bodily movement', their efficaciousness and affordances. Evidently, the term shi is not easily translated, and François Jullien devoted an entire book to it, The Propensity of Things (original title: La Propension des choses. Pour une histoire de l'efficacité en Chine). ${ }^{9}$ His study has greatly enhanced our understanding of Late Imperial aesthetics in Chinese artistic expression. Furthermore, his insights put into perspective recent discussions of $t u$ 圖 (diagrams, maps, pictures) as used in science, technology and medicine texts. ${ }^{10}$

In what follows, I first introduce the primary source on which this study draws, namely the Yijin jing of 1624 as transmitted to us through the Neigong tushuo 內功圖 說 (Illustrated Exegeses on Inner Alchemy) of 1882, which outlines the shi (postures/movements) of popular forms of the martial arts and so-called 'inner alchemy'. Then, we will engage with Jullien's inspiring discussion of the term shi, before examining the specific shi (postures and/or movements) of the Yijin jing. Finally, we will turn to the opening and closing shi of the Yijinjing bodily routines, and the conceptions of time that their visualisaiton in the $t u$ diagrams might imply. Based on these observations I will formulate an argument that may be of text-critical interest and propose to consider the Yijinjing as a composite assemblage, comprising distinctively Daoist routines at its core,

\footnotetext{
$7 \quad$ Karlgren 1923.

8 Ledderose 2000.

9 Jullien 1995.

$10 \quad$ See Bray et al. 2007.
} 
rather than being a syncretic amalgamate within which core elements have been transformed beyond recognition.

\section{The Yijin Jing 易筋經 (Canon for Supple Sinews) of 1624}

The Yijin jing is thought to have been compiled in the early 17 th century and is, according to Meir Shahar, an epitome of late Ming syncretism. ${ }^{11}$ It is attributed to a certain Zongheng 宗衡 from Mount Tiantai in Zhejiang, whose sobriquet was Zining daoren 訾凝道人, 'Purple Coagulation Man of the Way'. A daoren would usually be a Daoist priest but at the time, the term may also have designated a Buddhist monk. Shahar is careful to point out furthermore that the word ning in the sobriquet Zining daoren can refer to an alchemical elixir, not least as the bodily techniques he discussed were at the time likened to alchemical processes. Accordingly, the compiler of the Yijin jing might also be rendered as the 'Purple Elixir Daoist'.

Shahar notes that the Yijin jing exercises were not originally performed within the Buddhist Shaolin temple. Although he refers to them as Weituo's 韋䭾 exercises, and Weituo (Skanda) was a popular tutelary divinity in Chinese Buddhism, Shahar notes that these exercises derived at least in part from Daoist gymnastics of daoyin 導引 'guiding and pulling'. He deduces this from their naming, as for instance 'guarding the centre' (shou zhong 守中). Or, as in the case of so-called 'energy absorption' from the sun and moon, he notes that it was already being practised in Daoist circles in medieval times.

Shahar also notes that the Yijin jing was endowed with two prefaces that linked it to the martial arts. One preface, which claimed that the manual originated from the famous Boddhidharma (Damo 達摩 in Chinese) was signed by the Tang dynasty general Li Jing 李靖; the other preface was signed by the Song dynasty general Niu Gao 牛臬. On the basis of anachronisms identified in these two prefaces, scholars of the Qing already dismissed both as forgeries. ${ }^{12}$ How did these prefaces become associated with the Yijin jing text? Shahar mentions a Daoist tradition from medieval times that attributed daoyin gymnastics to Boddhidharma. Furthermore, the Shaolin monks who eventually incorporated the Yijin jing exercises into their martial routines ${ }^{13}$ certainly would have been keen to align themselves with Buddhist predecessors. We note that at one point in history these routines were transmitted in

\footnotetext{
11 Shahar 2008, pp. 160-5.

12 Ibid

13 Shahar 2008, pp. 171-2.
}

Buddhist circles. And although scholars of the Ming often point out that it makes little sense to try to disentangle Buddhist from Daoist practices in a syncretic milieu, the findings regarding the iconography of time that are presented in this study suggest that differences both existed and persisted.

\section{The Neigong Tushuo 內功圖說 (Illustrated Exegeses on Inner Alchemy) of 1882}

The textual history of the Yijin jing is complicated..$^{14}$ The version which is currently the most popular in the People's Republic of China is the one found in Wang Zuyuan's王 祖源 Neigong tushuo of 1882. It was reprinted in facsimile in 1956. The meditation routines discussed there are given in 12 full-page depictions of shi on pp. 47-58. Each page depicts, within a rectangular frame, a human figure assuming a certain posture (shi), usually enclosed to the left and right by one or two lines of text, and headed on the top by text that gives the posture's name. Although the illustrations are prints published as tushuo (exegesis by means of illustration), it seems farfetched to aim to categorise these images according to a typology which distinguishes between $t u$ as 'symbolic mediation' and $t u$ as 'technical illustration', as does Bray. ${ }^{15}$ We will see that these tushuo of inner alchemy also fulfil certain aesthetic criteria of hua 畫-ink brush paintings.

In Bray's typology, which is formulated in full awareness of it being an entirely 'etic' definition, Wang Zuyuan's illustrations would be secular and didactic, and thus would fall into her second type of $t u$ (rather than being endowed with ritual and/or symbolic power, as is her first type). However, although such a typology, which aims to separate the 'symbolic' from the 'technical', might appear useful, it carries the danger of projecting deeply engrained biases of Western scholarship into Chinese practices. Bray points here to a 'practical logic' that can be surprisingly constant across different times and cultures (other scholars speak of 'common grounds' or of the 'common sensical'). However, it is important to keep in mind the very fundamental social anthropological insight that human perception, practice and technology cannot be entirely divorced from meaning-making processes.

Bray's survey of $t u$ starts by noting that $t u$ in pre-dynastic China were thought to 'order' and to 'position in space'. She refers to Wolfgang Behr, who noted that in the

\footnotetext{
14 Shahar 2008, pp. 203-4, lists six editions, among which only the most recent is dated, being signed by a Zining daoren in 1624 .

15 Bray 2007, p. 34
} 
ancient China of the Zhou, a habit developed of writing a wei $\square$ enclosure around some words denoting places or place names. Among those was the character $b i$ 啚, known to have occurred on oracle inscriptions, not least as the predecessor of $b i$ 鄙 meaning 'border town, military base, garrison, fortress'. ${ }^{16}$ In this way, $b i$ surrounded by wei transmuted into the character $t u$ 圖 as known today. However, Behr also notes that already in Zhou China tu could be written with a heart determiner (xin 心) and remarks in that context - which concerns the making of a necropolis - that $t u$ functioned both as a plan for action and as a ritual object. ${ }^{17}$ Behr thereby made use of Bray's typology but only to show that it does not apply to $t u$ in pre-dynastic China.

The human figures depicted in the Yijin jing are given in a rectangular frame, seemingly following this early habit of framing a position in space with a wei enclosure. Since the figures adopting different postures are framed, one might be inclined to interpret them as constitutive of a $t u$. However, the text refers to them as shi. As can be gleaned from their names, they are rife with cosmogonic implications, where 'cosmogonic' means that they have the power to (re-)create the cosmos.

Jullien says shi relates to the efficacy of one's position. His discussion of shi, as 'potential born of disposition', starts with its implications in the military and in politics and ends with its importance in divination and the historical imagination. ${ }^{18}$ Here one senses some affinity between a shi and a $t u$. Both are action- and practice-oriented. Yet however action-oriented a strategic position shi may appear to be, Jullien insists on its dynamism, which goes beyond the here and now, on the tension and suspension that it evokes, and on how the physical and visible aspects of shi are oriented towards and defined by aspects that are invisible, hidden or not yet materialised. Jullien stresses that shi was the term par excellence that Chinese literati used for aesthetic valuation, particularly that of the brush stroke and of the arts performed with the brush: calligraphy, painting and literary composition.

In this context we note that the human figures in the Yijin jing were initially drawn with an ink brush; and in line with the aesthetic requirements of ink brush paintings (hua), each illustration contains a composite of figurative expression (a semi-clothed human body) and text (in plain Chinese characters). As we already know, and as Jullien tells us again, in hua paintings, figure and figurative speech together are meant to evoke an emotion.

\footnotetext{
16 Behr 2007, pp. 114-15.

17 Ibid., pp. 117-19.

18 Jullien 1995.
}

In Wang Zuyuan's Neigong tushuo we see martial arts 'postures' or 'movements', shi, but to view them merely as technical $t u$, i.e. as representations for practical instruction, might miss the point. 'A shi starts out as a painterly technique; but it also and inevitably provokes emotion.' ${ }^{19}$ Evidently, the aesthetic creativity of a shi transcends the representational and aims to convey the power, efficaciousness and/or propensity of the configuration that it depicts. So although the compilation in which these shi of the Yijin jing are recorded called itself a tushuo, and even though it may have been compiled primarily for technical and didactic reasons, the reduction of these shi to so-called 'technical' $t u$ merely reinforces a Western bias. Most importantly, it misses out on their efficacious aesthetics, which is ultimately cosmogonic.

\section{What is a Shi and What is Shi?}

In English, a 'posture' or 'movement' is a shi and the posture's efficaciousness is shi. However, in Chinese, one and the same term applies. In the contemporary martial arts there are three basic units for bodily routines: ${ }^{20}$ the shi is generally considered the most basic unit, and is often stationary, like a 'posture'; the zhao 招 is a sequence of movements ending with a strike; and the tao 套, traditionally also called $l u$ 路, comprises long sequences of movements that form a complete exercise.

Jullien discusses the connotations of shi in the martial arts in a chapter called 'Categories of efficacious dispositions. ${ }^{21} \mathrm{He}$ refers to body routines, like those of Taiji quan 太極拳 or the Yijin jing, as 'technical lists'. 'These lists constitute an altogether new type of literature for us,' he comments, saying that 'such technological codification has not been the object of much study.'.22 I could not agree more with the latter statement but take issue with his rendering as 'lists' what to practitioners are meaningful zhao or tao. The bodily routines presented in what follows have clearly identifiable openings and closings. Furthermore, the sequencing of the shi appears to be rife with meaning.. ${ }^{23}$

Shi is a 'practical efficacy', Jullien notes in his discussion of shi in warfare, and this applies also to shi in the martial arts. Furthermore, Jullien remarks, these 'physical exercises' have a 'full cosmic dimension'. In this sense, they are cosmogonic: 'When I push in this manner with my hands,




I am pushing against the Invisible in its entirety'. ${ }^{24}$ When Jullien says 'any initiate of Chinese fist boxing learns to master a whole succession of fragments of movements that cannot be equated on a one-to-one basis with the various shi, ${ }^{25}$ we are reminded that the zhao and the shi, the tao $l u$, and their components, form what Lederrose would consider parts of a 'modular pattern'. ${ }^{26}$

Although shi relates to the concrete configuration of a situation, i.e. to practical and technical matters, it is a term with aesthetic implications, which evokes awareness of the invisible and spiritual, of cosmic rhythms and potentialities and of the depth and power of human emotion. Shi describes 'a propensity' that 'emanates from its constitution' ('just as a spherical body tends to roll and a cubic one remains still'), ${ }^{27}$ a shi collapses the present tense with the future. With this latter example, Jullien implicitly likens shi to the concept of 'affordance', which in the early 2 oth century phenomenologist philosophers and psychologists developed (and which Science and Technology Studies have further elaborated). The shi does not imply a mechanical functionalism or an Aristotelian teleology; rather it has the potential to give rise to life through the actualisation of internal energy and denotes in its current configuration the effect that it will produce in the future. ${ }^{28}$ This sort of temporality and causation, as a suspended tension of present immobility, imbued with triggers that effect future movement, is central to the notion of shi.

As enlightening as Jullien's discussion of shi is in general terms, his comments on the shi of Taiji quan and their relation to time seem to rely unreflectingly on his modern understanding of time, when he says, 'it is not possible to distinguish between one individual "position" and the movement that both stems from it and leads into it'. ${ }^{29}$ Here, Jullien is clearly imposing his understanding of time as a continuous flow on the temporality implied by the bodily routines in Taiji quan. However, just as the calligrapher writes discrete yet dynamically connected units of Chinese characters when expressing the flow of his thoughts, so too the martial artist adopts a sequence of 'postures' or 'movements' that involve both the dynamism of movement and the tension of stillness. Those shi enact both the present and the future, where the meticulous attention to minute differences in bodily posture is rewarded with the overwhelming emotional, if not spiritual,

\footnotetext{
24 Jullien 1995, p. 115

25 Ibid., p. 114.

26 Ledderose 2000.

27 Jullien 1995, p. 85 .

28 Ibid., p. 78 .

29 Ibid., p. 113
}

effects that these technicalities can strategically evoke. As documented by Judith Farquhar and Zhang Qicheng, feelings of joyfulness and peacefulness arise spontaneously on those occasions. ${ }^{30}$

Shi encompasses both form and content. Although Jullien fails to see that a similar collapse of temporality applies to shi in the martial arts, he is explicit about this with regard to the literary arts:

The 'form' through which the literary shi is realised is that of a particular configuration which itself operates spontaneously to create an effect. Thus, what we customarily translate as 'form' in Chinese texts of literary criticism is not the opposite of 'content' but the end of a process of 'actualization', shi being the potentiality characterizing the actualization. ${ }^{31}$

Applied to the martial arts, a shi is the form, the posture and the movement; shi is the contents, the tension, the power and the posture's efficaciousness, which spontaneously evokes feelings and emotions, and ultimately is cosmogonic (in that it has the potency to recreate the cosmos). In the Chinese language, as already said, this distinction between a shi and shi is not made.

\section{Practising the Yijin Jing}

During ethnographic fieldwork in 1988-9 I encountered a practitioner of the Yijin jing in a popular neighbourhood of Kunming city on a street known as that of the poor (pinmin jie 貧民街). At the time this practitioner, who readily agreed to teach me what he called Qigong 氣功 (merits achieved through breathing [techniques]), did not stress that the movements he taught me were those of the Yijinjing, although he did name some of them in order to change my performance of the movement in question through imagination (the imagination of enacting a wild animal, for instance).

In 1988-9 I did not write down the names of the movements, as I was told them in the darkness of a park and I only partially understood them. Nor did I dare to ask Master Qiu (a pseudonym) to write them down for me systematically, as he treated his teaching as secret knowledge. Although I soon became aware that degrees of secrecy were negotiable, ${ }^{32}$ I hesitated to press him to give me factual information that might have unnecessarily endangered him in the then prevailing political climate. However, while writing up my doctorate, I came across

\footnotetext{
$30 \quad$ Farquhar and Zhang 2012.

$31 \quad$ Jullien 1995, p. 88.

$32 \quad$ Hsu 1999, p. $5^{2}$
} 
Wang Zuyuan's facsimile edition in the open stacks of the Needham Research Institute, Cambridge. ${ }^{33}$ I still remember vividly today how forcefully I was struck by the images of movements seemingly identical to the ones I had learnt. They captured them so well - in their very essence, one would like to say (if being 'essentialist' were not decried as the worst possible crime a contemporary social anthropologist can commit). I was strangely touched and somewhat stirred when I saw a guarded secret unfold in front of me, in black and white, as I turned the pages. However, there were 12 pictures of movements whereas I had learnt 10, and some seemed different altogether. Furthermore, their sequencing was not as I had learnt it. ${ }^{34}$ I did not further pursue this issue then, especially as I found no secondary literature on it.

Twenty-one years later, in 2009, Qigong master Qiu was one of the few in the People's Republic of China who continued to do daily Qigong meditation in his privately owned house in another popular neighbourhood. The Qigong fever of the late 8 os had long abated. When I came to visit Qiu and stayed with his family, one of the several other disciples he had taught after me happened to drop by for a visit. In this context, I reconfirmed with Qiu, as I had already done on a previous occasion in 2005, that he had taught me the Yijinjing. Yes, indeed. This time I asked Qiu the names of the 10 movements and his disciple wrote them down. ${ }^{35}$ They turned out to be very similar to the names in Wang Zuyuan's edition of 1882, which are listed below (Qiu's sequencing is given in capital letters):

1. (A) Weituo offers his vajra club, first posture (Weituo xian gan di yi shi 韋䭾獻杵第一勢)

2. (A) Weituo offers his vajra club, second posture (Weituo xian gan di er shi 韋䭾獻杵第二勢)

3. (A) Weituo offers his vajra club, third posture (Weituo xian gan di san shi 韋䭾獻杵第三勢)

4. (C) Pluck the stars and reverse the dipper posture (zhaixing huan dou shi摘星換斗勢)

5. (F) Turn around nine oxen by pulling their tail posture (dao zhuai jiu niu wei shi 倒拽九牛尾勢)

6. (B) Put forth the claws and spread the wings posture (chu zhua liang chi shi 出爪亮翅勢)

7. (E) Nine ghosts draw the horse sabre posture (jiu gui ba ma dao shi 九鬼拔馬刀勢)

8. (D) Three plates fall to the ground posture (san pan luo di shi 三盤落地勢)

9. (G) The blue-green dragon surveys its claws (qing long tan zhua shi 青龍探爪勢)
10. $(\mathrm{H})$ The crouching tiger pounces on its prey (wo hu pu shi shi 臥虎撲食勢)

11. (J) Make a bow posture (da gong shi 打躬勢)

12. (J) Wagging the tail posture (diao wei shi 工尾勢)

What I call in English a movement or posture, I was taught during a single session on one evening. It was clearly a conceptual entity for my teacher as he spaced my teaching of each movement accordingly, and if prompted, would give me a name for each. A new movement was typically initiated by changing the position of both feet. As Despeux has noted, the body is always slightly off balance, thereby containing within each shi the tension leading into the next. ${ }^{36}$ One remains rooted to the ground, while stretching, bending and twisting one's arms, legs and torso according to a clearly prescribed routine, repeating each movement, in Qiu's case, seven times. Qiu was not verbal about how to perform such a posture or movement but Chinese scholarship calls it a shi.

In this context the affect that the Neigong tushuo generated is worth noting. As already mentioned, I instantly recognised the body routines I myself had enacted when seeing the images. I also remember that one of my teachers reacted to them with endearment, in a noticeable way. I would not have mentioned this auto-ethnographic detail, if C.S. Peirce had not made a comment precisely on the impact iconic and indexical signs have on one's mood. ${ }^{37}$ Peirce says of the symbol (the 'symbolic sign'), which, like the linguistic 'sign' defined by Ferdinand de Saussure, ${ }^{38}$ is based on convention, that it is assertive and declarative. It makes a statement in the indicative mood, so to speak. By contrast, Peirce claims, the iconic sign expresses a potentiality in the subjunctive mood and the indexical sign is exclamatory and calls for attention in the imperative mood. ${ }^{39}$ With regard to the effects it evoked, the Yijin jing images provided not so much a factual statement in the indicative mood and in terms of 'visual representations'. Rather, they combined the qualities of an iconic and an indexical sign; in an exclamatory way they reached into the emotional realms of the subjunctive and inchoate possibility. This is another reason why the Yijinjing's visual rendering of a shi, and implicitly of time, might best be conceived as 'iconic-indexical'.

Despeux 1975, 42-3; see also Jullien 1995, p. 133 Peirce 1932, vol. ii, p. 169 . de Saussure 1916. Summarised more comprehensively in Hsu 2010, p. $35^{2}$. 
In what follows I will describe my embodied experience of practising the opening and closing shi of the Yijin jing, and use this experience as a key to reading the images in Wang Zuyuan's Neigong tushuo. The methods of inquiry are thus most unusual. They involve, first, 'participant experience', ${ }^{40}$ which differs from 'participant observation' in that it requires the ethnographic fieldworker to gain the proficiency of a practitioner in the subject they study, or at least the ability to practise the subject or skill independently and without supervision. At the same time 'participant experience' requires one to stay committed to the ethnographic methods of note taking and diary writing and to the ethics of a participant observer, which is to adopt a humble attitude of learning and non-interference, as far as this is possible. Second, I engage in auto-ethnographic reflections.

Both of these methods - participant experience and auto-ethnography - are typically frowned upon, as they are interpreted as relying too much on so-called 'subjective' experience. However, if one adopts, as I do, a phenomenological orientation grounded in Merleau-Ponty's Phenomenology of Perception, which emphasises that processes of perception are 'physiognomic',11 one can redefine the field of inquiry that the empiricist sciences divide into one of active 'subjects' investigating passive 'objects' during a 'cognitive process' of 'decoding' 'reality'. The suggestion that perception, and with it the meaning-making process, is physiognomic highlights that perception is a process marked by indeterminacy, ${ }^{42}$ where a perceiver's surroundings gain their meanings and distinctiveness only through repeated and renewed interactions between what the empiricist sciences call the subject and the object. ${ }^{43}$ I justify taking recourse to the above methods through the inaccessibility of the topic studied. The martial arts and Qigong meditation are still today veiled in secrecy, although the Yijin jing has gained considerable publicity in the time since I first learnt it.

\section{The Opening and Closing Movements in the Yijin Jing}

Master Qiu's Yijin jing movements comprised a zhao or a tao of 10 shi, while Wang Zuyuan's had 12 images, and their sequencing differed. I go into a detailed analysis of

\footnotetext{
$40 \quad$ Hsu 1999, p. 15.

$41 \quad$ Merleau-Ponty 1962, p. 132

42 Ibid.

43 Well-explained by Morris 2012.
}

the different versions elsewhere ${ }^{44}$ and will limit myself here to discussing the opening and the closing movements.

The opening movement that I learnt from Qigong master Qiu resembled the closing movement in that both positions consisted of straightened legs, with feet kept parallel, touching each other at the ankle. It made one feel rooted to the ground, firm and symmetrical (the legs were not crossed, as in the first three postures in the Neigong tushuo (see Fig. 3)). One had no sense in this opening posture of being slightly off balance, except at one instance, that of standing on one's toes and stretching out both arms, while pushing with the outwardly turned palms upwards. This moment of being on tiptoe within the opening and closing shi was arguably a brief moment of going slightly off balance, but not in a lateral way, to either the left or right, as in the other movements. Rather, it felt to me as though I was supposed to assume a posture of absolute verticality to prop up the sky (the Neigong tushuo text does actually speak of propping up the 'gate of heaven', tianmen 天門, but Master Qiu provided no verbal comment). This opening movement that Qiu taught me consisted of the drawing out of a circle with arms stretched out to both sides. Over the many years of practising these bodily routines almost daily, I started to experience them as any anthropologist would do who suffers a déformation professionnelle: it felt as though the boundaries of a ritual space were hereby being demarcated.

In the closing movement one also draws a circle by folding one's hands into each other such that the palms face outward and by pushing them with stretched-out arms away from one's torso. First, one does so at shoulder height, then gradually bowing downward with the torso until the stretched out arms touch the ground with the outwardly facing palms while both legs are kept still and stretched. Thereupon, with interlocked palms facing the torso, one moves them along it upwards, passing by the abdomen, chest, neck, face, until one stands upright again. As one goes up onto one's toes to prop up the heavens, the palms are again interlocked and facing outwards, and stretched out upwards, before one starts drawing another circle in front of one's torso. It felt as though the closing circle was on an axis turned to go degrees in comparison to the opening movement.

An anthropologist finds it difficult to evade the impression that this final posture has a ritual implication. It seemed to delineate the ritual space in which the transformation of the person performing the meditation routine had taken place. Qiu put an additional end point to this Wang Zuyuan's sequence lacked Qiu's penultimate shi, (I), no. 9. 




Figure 4.3 'Weituo offers the vajra' (Weituo xian gan 韋䭾獻杵). All three images bear the same title; accordingly, they depict one and the same shi. They provide 'freeze frames' of different stages of the movement that the practitioner enacts.

movement by thereafter resting his palms on his lower abdomen, the cinnabar field (dantian 丹田), into which Qi would return.

Equipped with this embodied understanding of the opening and closing movements, let us turn to Wang Zuyuan's Neigong tushuo. The first three images each give the title of the first shi, i.e. 'Weituo offers the Vajra', followed by the numbers one, two and three, (yi er san 二二三); hence those three images seem to account for one movement (see Fig. 3). So, rather than capturing the entirety of a shi in one iconic-indexical sign of psychological salience, the first three human figures seem to depict different stages of one movement, as though they were presenting 'freeze frames' along a linear trajectory of time. This is not instantly evident from the depictions on paper, but with the embodied knowledge of the bodily routine that the shi in question involves, one can appreciate them as 'snapshots' of different stages of drawing out a circle delimiting a ritual space, as is done in the first shi.

The 'cinematographic technique' 45 also presents itself as a possible iconographic style for interpreting the two last images: these two images both show a human figure seen from the same angle with feet positioned close to each other (see Fig. 4: 'Make a bow' and 'Wag the tail'). Hence one gets the impression that the sequence of these two images is used to depict one movement, the closing movement. The very last drawing has a title but no text and it looks like a humorously added endnote to this movement: one wags

45 Jullien 1995, p. 113 . one's tail, i.e. shakes one's bottom, as is still done today in another living tradition. ${ }^{46}$

By contrast, the seven shi in between account for time and temporality in an unmistakably iconic-indexical way, where the propensity for the future and its efficaciousness are contained in the configuration of the present (see Fig. 5: 'Pluck the stars and reverse the dipper'). In the sequence I learnt from Qiu, it is possible to discern a pattern of alternation of the broad postures (movements B, D, F) and the postures with crossed legs (movements $C, E, G$ ), which can be interpreted as enacting a pulsating Yin Yang pattern of a temporality understood in terms of a cosmogonic expansive Yang and contractive Yin. ${ }^{47}$ Wang Zuyuan's edition, however, sequences Qiu's shi in this order: A, C, F, B, E, D, G, $\mathrm{H}, \mathrm{J}$. It is of course possible that Wang Zuyuan's publication recorded the same pulsating Yin Yang sequence of postures as did Qiu and that it had to be read in a coded way. It is equally possible that such variations suggest a widespread predilection for playful recombination. Such cultivation of artistic expression that is playful evidently attended to both form and content, much as one does linguistically when formulating puns and rhymes.

Hsu and Lim 2016. Admittedly, the interpretation of the last two images as 'freeze frames' depicting one single shi, is not as robust as is that of the three 'freeze frames' depicting the opening shi. Hsu (in prep.) further elaborates on this. 




Figure 4.4 'Make a bow' (da gong 打躬) and 'Wag the tail' (diao wei 掉尾). Two 'freeze frames' of another single movement? Note that there is no text to the latter image.



Figure 4.5 This one image depicts in an iconicindexical manner the shi called 'Pluck the stars and reverse the dipper' (zhai xing huan dou 摘星換斗)

\section{Text-Critical Considerations}

Our analysis of the iconography of time in the images of specific postures in martial arts routines does not stop short here and proceeds to make a text-critical argument. The above finding can be used as evidence that the current bodily routines of the Yijin jing are in fact a composite. In other words, the text in Wang Zuyuan's extant edition of the Yijinjing, and the illustrations accompanying it, seem to be made of at least two textual layers that can be distinguished from each other on the basis of their different iconographies of time. Accordingly, the three opening and the two closing shi would belong to a different textual layer from the seven shi in between.

We are here reminded that in The Propensity of Things Jullien endorses a somewhat elitist stance to texts (like that of the Yijin jing) as he considers them of 'late and secondary character' 48 and refers to them as 'lists'. He translates one such 'list' of 17 'Strategic dispositions in poetry' and, commenting on their 'heterogeneity' and 'incoherency', says: 'Could such a list really reflect little more than desultory, largely whimsical rumination?'49 Jullien herewith expresses the frustration that many a Western textual researcher experiences, which may arise from the oversight of, first, the deeper meanings of the playful in Chinese aesthetics and artistic expression, as hinted at above, and second, text-critical considerations. Since the

48 Jullien 1995, p. 281.

49 Ibid., p. 123. first concern is being dealt with elsewhere, ${ }^{50} \mathrm{I}$ limit myself to text-critical considerations in the following discussion.

Throughout his works, Jullien shows little text-critical awareness. Notably, he says of these lists' that they date to the Tang dynasty, and he even speculates why this might have been so..$^{51}$ One of the two prefaces to the Yijin jing would indeed make it appear to be a Tang dynasty text. However, both prefaces of the Yijin jing that supposedly dated to the Tang and Song dynasties have been identified as 'forgeries'. Accordingly, one wonders whether the prefaces to this literature of 'lists' of 'technological codification' were meant to imbue it with the weight of a millennium-long tradition. In this context, it is noteworthy that the primary source-material Jullien relies on throughout his monograph is largely from Ming and Qing China. In particular, when he talks about the importance of shi in Song paintings ${ }^{52}$ and Chinese philosophies about historical change, ${ }^{53}$ he actually draws amply on Late Imperial source-materials. ${ }^{54}$ This could be taken as a hint that the notion of shi starts to gain centre stage in the imagination

\footnotetext{
$5^{\circ} \quad$ Hsu and Lim 2016, Hsu (in prep.).

$51 \quad$ Jullien 1995, p. 108.

$5^{2} \quad$ Ibid., ch. 5, pp. 98-105.

53 Ibid., pt iii.

54 See two works of Wang Fuzhi 王夫之 (1619-92) mentioned on p. 291, and note figs $1-5$ and 10-11, on pp. 162-70, from the Jiezi yuan huazhuan 芥子園畫傳 (Manual of the Mustard Seed Garden). It is puzzling that although Jullien (1995) begins with the discussion of shi as central to Sunzi's 孫子 militaristic strategising and the legalistic philosophical stance in the Han Fei zi 韓非子 of pre-Imperial China, the Hanyu da zidian 漢語大詞典 notes that the character shi does not exist in pre-Imperial texts.
} 
of Chinese literati only by the 17 th century. It happens, as Jullien convincingly underlines, in an all-encompassing way, thoroughly affecting philosophical, artistic and technologically-oriented literatures.

Furthermore, as established above, the visual rendering of the opening and closing shi in Wang Zuyuan's Neigong tushuo relates to time as a linear trajectory, in contrast to the seven shi at the core of the Yijin jing bodily routines, which appear to relate to time in an iconic-indexical way. Incidentally, the depiction of the flow of time along a linear trajectory is also implied by the way in which Tibetan medical doctors (who were Buddhist) depicted the pulse on the thanka of the 17th century Blue Beryl, as mentioned in the beginning of this chapter (Fig. 1). The allusion to a linear flow of time when depicting Tibetan medical pulse movements contrasts with the iconic-indexical rendering of the Chinese medical pulse/mai (Fig. 2).

In addition, the bodily movements in both the opening and the closing shi of the Yijin jing, much like the Tibetan Buddhist medical pulse movements, are marked by verticality: the practitioner stands straight, symmetrically grounded, with parallel feet, ankles touching each other (in both cases, the knees are not bent and there is no sense of being slightly off balance, as is characteristic of the Chinese martial arts).

Finally, the opening movement refers to a Buddhist figure, Weituo.

What can we deduce from this observation? Might there have been a distinctively Buddhist comprehension of time that spanned across 17 th century Tibet and 17th-19th century China? Might this Buddhist comprehension of time as a flow have led to its depiction along a linear trajectory? Herewith we find ourselves in the midst of text-critical scholarship and its concern with textual layering. We have not investigated rhyme and rhythm, graphs and writing utensils, but scrutinised the iconography of shi as a textual genre. Based on the different iconographic styles in which the images in Wang Zuyuan's edition refer to time, we can hypothesise that the opening and closing shi are of a different cultural provenance from most of those at the core of the Yijin jing sequence.

If this were the case, it instantly raises the question: why should a basically Daoist stock of daoyin practices surround itself with opening and closing shi drawn in an iconography of linear time? Might Zining daoren in fact have been a Daoist who compiled a routine of eight shi and might, only later, one of his followers have decided to add the opening and closing routines? ${ }^{55}$ Might these two have

The Singaporean version of the Yijin jing, discussed in Hsu and Lim 2016, consists of eight shi. been of Buddhist provenance? If that were the case, the Yijin jing would basically be a Daoist routine. It might even have had a closing movement of its own, as Master Qiu's penultimate shi felt very much like a closing movement too (see fn. 44), but that shi does not appear to be given in Wang Zuyuan's version of the Yijin jing sequence of postures.

If the Yijin jing is Daoist at its core but in Wang Zuyuan's version packaged as Buddhist, what are the political and social historical events in Late Imperial China that could provide a motivation for explaining this? Shahar reminds of the pre-eminent position of the Shaolin temple and notes that martial arts practised on its grounds in the 19th century were thoroughly Daoist. ${ }^{56}$ How and why did this happen?

Here an episode from another fieldwork site may prove useful. In autumn 2009, while doing three months of ethnographic fieldwork in my ancestral village (Hsuvillage in Huizhou, Anhui province), in a moment when our conversation had died away into silence after it had provoked a non-verbalised but palpable sadness over the destruction effected by the Red Guards (some of them my dear hosts), a villager had his teenage son bring me a cookbook out of a backroom of the remains of his large Ming-dynasty house. He was proud to show me that he had saved this book from the flames, and with it a tiny bit of Chinese culture and cuisine. However, both book covers, i.e. the title page and the end page, had been discarded. I did not need to ask why. It was obvious. They carried politically relevant information.

In a similar vein, the Yijinjing may at its core consist of Daoist practices that are packaged as Buddhist: it may not be a coincidence that Wang Zuyuan's edition depicts the opening and closing movements along a linear timeline, and hence according to a different iconography of time from the seven core movements. Furthermore, it is worth noting that just in the opening and closing movements the practitioner's posture is marked by verticality. Finally, we are reminded that the Yijin jing's prefaces alluded to a Buddhist provenance.

Here I defer to the social historian of Late Imperial China, yet wish to add one final note on method relevant to this book project on the visualisation of Chinese medical practices. As the above has shown, in order to interpret the visual depiction of postures, gestures and movements of the martial arts and other routinised bodily practices in historical texts and other written documents, a practical and embodied understanding of them is crucial.

$56 \quad$ Shahar 2008. 


\section{Bibliography}

\section{Primary Sources}

Wang Zuyuan 王祖源 [1882] 1956: Neigong tushuo 內功圖說 (Illustrated Exegeses on Inner Alchemy), Beijing: Renmin weisheng chubanshe, $47-58$.

Wang Gai 王概, Wang Shi 王萻, Wang Nie 王香 (vol. i, 1679; vols ii and iii, 1701) 196o, Jiezi yuan huazhuan 芥子園畫傳 (Manual of the Mustard Seed Garden), Beijing: Renmin meishu chubanshe.

\section{Secondary Sources}

Behr, W. 2007, 'Placed into the right position: etymological notes on $t u$ 圖 and congeners', in Bray, Dorofeeva-Lichtmann and Métailié (eds), 109-36.

Bray, F. 2007, 'Introduction: the powers of $t u^{\prime}$ ' in Bray, DorofeevaLichtmann and Métailié (eds), 1-78.

F. Bray, V. Dorofeeva-Lichtmann and G. Métailié (eds) 2007, Graphics and Text in the Production of Technical Knowledge in China: The Warp and the Weft, Leiden: Brill.

Despeux, C. 1975, T'ai-k'ik'uan:technique de longue vie, technique de combat, Paris: Collège de France, Institut des Hautes Etudes Chinoises.

Farquhar, J. and Zhang Qicheng 2012, Ten Thousand Things: Nurturing Life in Contemporary Beijing, Cambridge, MA: MIT Press.

Hsu, E. 1992, 'The Transmission of Knowledge, Texts and Treatment in Chinese medicine', PHD diss. in Social Anthropology, University of Cambridge.

1999, The Transmission of Chinese Medicine, Cambridge: CuP. 2010, Pulse Diagnosis in Early Chinese Medicine: the Telling Touch, Cambridge: cuP.

(in prep.), 'History in the Body: the Yijin jing 易筋經 (Canon for Supple Sinews)'.
Hsu, E. and Chee Han Lim 2016, 'Enskilment into the Environment: the Yijinjing 易筋經 Worlds of jin 筋 and q $i$ 氣'. Berlin: MPIWG (Max Planck Institut Wissenschaftsgeschichte), Preprint Series.

Jullien, F. (tr. J. Lloyd) 1995, The Propensity of Things: Toward a History of Efficacy in China, , New York: Zone Books.

Karlgren, B. 1923, Sound \& Symbol in Chinese, Oxford: Clarendon.

Kohn, L. 2008, Chinese Healing Exercises: the Tradition of Daoyin, Honolulu: University of Hawai'i Press.

Kuriyama, S. 1999, The Expressiveness of the Body, and the Divergence of Greek and Chinese Medicine, New York: Zone Books.

Lederrose, L. 2000, Ten Thousand Things: Module and Mass Production in Chinese Art, Princeton: Princeton University Press; Leiden: Brill.

Lo, V. 2007, 'Imagining Practice: Sense and Sensuality in Early Chinese Medical Illustration', in Bray, Dorofeeva-Lichtmann and Métailié (eds), 383-423.

Ma Kanwen 1983, 'Diagnosis by Pulse Feeling in Chinese Traditional Medicine', in The Institute of the Natural History of Sciences, Chinese Academy of Sciences (eds): Ancient China's Technology and Science, Beijing: Foreign Languages Press, 358-68.

Merleau-Ponty, M.M. [1945] (tr. C. Smith), 1962, The Phenomenology of Perception, London: Routledge and Kegan Paul.

Morris, K. 2012, Starting with Merleau-Ponty. London: Continuum.

Parfionovitch, Y., D. Giurme and F. Meyer (eds) 1992, Tibetan Medical Paintings: Illustrations to the 'Blue Beryl' Treatise of Sangs Rgyas Rgya mtsho (1653-1705), London: Serindia.

Peirce, C.S. (C. Hartshorne and P. Weiss eds) 1932, Collected Papers, vols. i-vi, Cambridge, MA: Harvard University Press.

de Saussure, F. [1916] 1949, Cours de linguistique générale, published by C. Bally, and A. Sechehaye, in collaboration with A. Riedlinger, Paris: Payot.

Shahar, M. 2008, The Shaolin Monastery: History, Religion, and the Chinese Martial Arts, Honolulu: University of Hawai'i Press. 



\section{Nurturing the Foetus in Medieval China: Illustrating the 1o Months of Pregnancy in the Ishimpo $\overline{\text { 醫心方 }}$}

Sabine Wilms

\section{Introduction}

This article discusses 10 depictions of the naked female body that are found in the first chapter of juan 22 in the Ishimpo 醫心方 (Yixinfang, Formulary from the Heart of Medicine). This Japanese compilation of medieval Chinese medical texts is one of the most important sources for fragments of otherwise lost early Chinese gynaecological literature. ${ }^{1}$ It was completed in 982 by Tamba no Yasuyori 丹波康賴, an acupuncturist at the Japanese court. Based on his research in early Japanese medicine, Mayanagi Makoto proposes that Tamba limited his role to compiling Chinese texts without editing or imposing his own medical ideas on the material. ${ }^{2}$ Apparently, the only elements he added to the texts are punctuation marks and minimal notes on Japanese pronunciation and the meaning of rare characters, which are easily distinguished from the main text because they are written in red ink (see Fig. 1) as opposed to the black writing of the source texts. As a reason for this, Mayanagi suggests that Japanese indigenous medicine was, at that time, still in its infancy, and texts from China were therefore treated with great reverence and transmitted faithfully. This view has also been widely accepted by contemporary Chinese historians and textual researchers who frequently consult the Ishimpō as an early source for textual variations of Chinese medical literature. In the context of formulas for women, found in juan 21 to 24 , the Ishimpō is of the greatest significance, with quotations not only from such well-known received texts as the Beiji qianjinyaofang 備急 千金要方 (Essential Formulas Worth a Thousand in Gold for Emergencies) and the Zhubing yuanhou lun 諸病源候 論 (On the Origin and Symptoms of the Various Diseases), but also from several lost texts, including the Chanjing 產 經 (Classic of Childbirth). Directly concerning the topic of this chapter, the first chapter of juan 22, titled 妊婦脈 圖月禁法 renfu maitu yuejin fa (Charts of the Channels and Method of Monthly Prohibitions During Pregnancy), is marked as a quotation from this text. I shall therefore briefly introduce this text.

\section{The Chanjing 產經 (Classic of Childbirth)}

The dating of the Chanjing is still subject to debate. The Nihonkoku Genzaisho Mokuroku 日本國見在書目 (Catalogue of Extant Books in Japan), the oldest Japanese bibliography from the late 9 th century, records a 'Chanjing in 12 juan, authored by De Zhenchang 德貞常 and with three juan of illustrations'. For a variety of reasons, this text is most likely the one referred to by Tamba no Yasuyori. It



Figure 5.1 Table of Contents of Ishimpō, vol. 22 (982 CE), Seikido Library Scroll (1145 CE). Photographic facsimile (c) Vivienne Lo

\footnotetext{
$1 \quad$ For a detailed analysis of the sources for the text, see Ma Jixing 1985 .

$2 \quad$ Pers. comm.
} 
must therefore have been composed before the end of the Tang dynasty. A Chanjing in one juan is also recorded in the bibliographic catalogue of the Sui dynasty, the Suishu jingji zhi 隋書經籍志. Unfortunately, the author of this text, De Zhenchang, is not found in the historical records and we can therefore not determine the precise date of the composition of the Chanjing at this point. However, the citations of this text in the Ishimpō contain quotations from the Geshifang 葛氏方 (Master Ge's Formulary), a text composed by the illustrious alchemist and Daoist Ge Hong 葛洪. Thus we know at least that the text must have been composed after the lifetime of Ge Hong, namely 261-341. On the collective basis of this evidence, we can conclude with Mayanagi Makoto that 'the drawings in this text constitute the earliest illustrations in the world to systematically portray the development of the foetus during the 10 months of pregnancy.' ${ }^{3}$

The chapter that is titled 妊婦脈圖月禁法 renfumaitu yuejing fa (Charts of the Channels and Method of Monthly Prohibitions During Pregnancy) consists of the above-mentioned 10 drawings of a naked woman during each of the 10 months of pregnancy, as well as an accompanying text that is identified as a Chanjing quotation. This text offers an example of literature on nurturing the foetus, yangtai 養胎, the roots of which are found in earlier Chinese medical literature.

\section{Early Chinese Literature on 'Nurturing the Foetus' (Yangtai 養胎)}

\section{The Mawangdui Taichanshu 胎產書 (Book of Gestation and Birth)}

To make sense of the drawings that form the main topic of this chapter, we need to first contextualise the accompanying text. It is composed of several textual layers that can be traced back in surprisingly similar form to earlier Chinese sources on 'nurturing the foetus', a type of literature that discussed the development and nurturance of the foetus; the affected internal organs, channels, and other parts of the mother's body; various prohibitions and recommendations regarding the mother's foods, activities, sights, sounds, and emotions; as well as supplementary, preventative, and curative medicinal prescriptions for symptoms likely to occur at specific times in pregnancy. The earliest extant example of this literature is found in a Mawangdui manuscript that the modern editors have named Taichanshu 胎產書 (Book of Gestation and Birth), dated to before 168 вCE. This silk manuscript includes the

$3 \quad$ Mayanagi 2005. earliest preserved core of yangtai literature. It is a monthby-month account of gestation with information on the phase associated with each month, features of foetal development characteristic of that month, and related to this, dietary and behavioural prescriptions for the mother. The following is a representative example:

In the fourth month, Water is bestowed on the foetus and blood first forms. Appropriate foods are rice, wheat, and mud eel, which clarify the blood and brighten the eyes.

In the fifth month, fire is bestowed on it, and Qi first forms. Rise late and wash the hair. Wear a thick layer of clothing and remain inside the house... .

After the first three months, the text associates the foetal development in each month with one of the five phases [elsewhere in this volume translated 'agents'] in the order of conquest, with the addition of Stone in the ninth month. Thus, in the fourth month, the formation of blood is related to the bestowal of Water; in the fifth month, the formation of Qi to the bestowal of Fire; in the sixth, the formation of muscle to Metal; in the seventh, the formation of bone to Wood; and in the eighth, the formation of skin to Earth. In the ninth month, finally, the formation of hair is related to Stone, and in the 1oth month, 'Qi spreads to form [?]'. Different versions of this text, sometimes in almost verbatim citations, are found in several of the standard medieval medical texts, such as the Beiji qianjin yaofang and the Zhubing yuanhou lun.

\section{Xu Zhicai Zhuyue Yangtai Fang 徐之才逐月養胎方 (Xu Zhicai's Month-by-Month Formulas for Nurturing the Foetus)}

For a second example of yangtai literature, we can consult Sun Simiao's Beiji qianjin yaofang (Qianjinfang for short), a medical encyclopaedia with over 5,00o entris in the form of medicinal formulas interspersed with acu-moxa prescriptions, household recipes, ritual instructions, and occasional short essays, composed around $65^{2} \mathrm{CE}$. An almost literal, but greatly expanded variation of the Mawangdui account of gestation is found in the second scroll, the first of three scrolls on 'women's formulas', in the third chapter on nurturing the foetus. The section is identified as a quotation and is titled Xu Zhicai zhuyue yangtai fang 徐之才逐月養胎方 (Xu Zhicai's Month-byMonth Formulas for Nurturing the Foetus). It is one of the rare instances where we can trace the information from the Qianjinfang back to an earlier source. The alleged author Xu Zhicai is known as a physician of the Northern Qi period (550-77) , who supposedly lived from 493 to 572. He is the author of several lost texts, such as the Duiyao 對 藥 (A Comparison of Medicinals), Jia chuan mi fang 家傳 
秘方 (Secret Prescriptions Handed Down in the Family), Xiao er fang 小兒方 (Paediatric Prescriptions) and others, none of which have survived except as quotations in other texts. Well versed in the medical arts, he is said to have specialised in medicinal formulas, in particular for women and children. Sun Simiao thus claims to be using a 6th-century source.

The Qianjinfang section on 'Nurturing the Foetus' begins with a short essay on the importance of proper maternal behaviour and environment in the third month of pregnancy when 'the foetus changes and transforms in response to things and its disposition and character are not yet fixed'. This is followed by a list of dietary prohibitions during pregnancy of a decidedly magical flavour. Next, the bulk of this chapter consists of a substantially enlarged version of the account of gestation found in the Taichanshu, which quotes this text almost literally but then continues for each month with a second textual layer. The following quotation illustrates Sun's additions with the example of the fifth month of pregnancy:

...In the fifth month of pregnancy, the Foot Greater Yin Vessel nourishes [the foetus], and you must not needle or burn moxa on this channel. The Foot Greater Yin [channel] is associated with the spleen.

During the fifth month, the child's four limbs are all being developed. [The woman] must avoid both great hunger and over-eating; she must not eat dried or parched food; she must not get overheated; and she must not become taxed and fatigued.

\section{Formula}

ass hide glue (阿膠 ejiao) 4 liang

inula (旋覆花 xuanfuhua) 2 ge

ophiopogon (麥門冬 maimendong) 1 sheng

ginseng (人參 renshen) 1 liang

evodia (吳茱英 wuzhuyu) $7 \mathrm{ge}$

fresh ginger (生薑 shengjiang) 6 liang

Chinese angelica (當歸 danggui)

peony (苟藥 shaoyao)

liquorice (甘草 gancao)

scutellaria (黃芩 huangqin) 2 liang each

Pound the 10 ingredients above and decoct the drugs in nine sheng of water until reduced by half. Add 3 sheng of clear liquor as well as the ass hide glue and simmer on a small flame to obtain $3^{1 / 2}$ sheng. Divide into four doses and take before meals, three times during the day and once at night. After this, [she should] recover. If there is no difference [in her condition], take another [preparation].

Another [version of this prescription]: Butcher one black hen, take the blood from its throat, and put it in the liquor. Cook the chicken in the water and then simmer the drugs in this until reduced by half. Add the liquor and ass hide glue and simmer it to obtain $3^{1 / 2}$ sheng. Divide into four doses.

In cases of any injury to the foetus during the fifth month, [the patient] should take Centre-Quieting Decoction as a precaution.

As this example shows, the information from the Taichanshu is elaborated on with several additional features, all of which appear for each of the 10 months of gestation. These textual layers reflect developments in medical knowledge that occurred between the compositions of these two texts, that is, between 168 BCE and the $5^{\text {th }}$ to 6 th centuries CE.

First, each month of gestation has become associated with a specific channel, which must not be manipulated by acupuncture or moxibustion during that month since it is responsible for nurturing the foetus. On the basis of acupuncture prohibition texts such as Dunhuang medical manuscripts from the Sui to Tang periods or the 'Method for Avoiding Cautery and Needling According to the Waxing and Waning of the Moon' in the Huangdi hamajing 黃帝 蝦蟆經 (Yellow Emperor's Toad Canon), which will be discussed in greater detail below, ${ }^{4}$ we know that the practice of acupuncture had become so widespread by early medieval times that physicians felt the need to publish entire texts on acupuncture prohibitions, to warn against the reckless practice and abuse of this therapy during specific times and occasions. I will return to this genre in greater detail in the context of acupuncture charts below.

Next, the essay offers an alternative account of foetal development, which must have stemmed from a different and later source, as it occasionally contradicts the earlier account, but reflects standard medical theory as it was canonised during the Han period, particularly in regards to the correlation of specific channels with viscera and physiological functions. For example, the Taichanshu version associates the fifth month with the phase Fire and the formation of Qi. The later version in addition associates the fifth month with the Spleen, its associated channel, the Foot Greater Yin, and with the development of the limbs. In standard classical theory, the limbs had by late Han times come to be associated with the Stomach, which was paired with the Spleen. Given the association of the Spleen/Stomach with Earth among the five phases in medical theory, this contradicts the above association of the fifth month with Fire. In early China, the five phases were predominantly arranged in the sequence of conquest: Water conquers Fire, which conquers Metal, which conquers Wood, which conquers Earth, and so on. This is 
directly reflected in the sequence of the months of gestation in the Taichanshu from the fourth to the eighth month, where Water (the fourth month) conquers Fire (the fifth month), which conquers Metal (sixth month) and so forth. This sequence of gestational development is unrelated to the progression of channels and visceral functions found in the expanded medieval version, which, from the fifth month on, follows the cycle of generation (Fire $>$ Earth $>$ Metal $>$ Water $>$ Wood) through paired Yin and Yang channels, as explained for example in the progression of pulse diagnosis in the Nanjing 難經 (Classic of Difficult Issues), a text from the ist century CE. ${ }^{5}$ Xu Zhicai's version thus combines two alternate yet unrelated sequences, both of which progress in accordance with the five-phase system of correspondences and reflect standard medical theories of their times.

Attached to the essays, each month's account contains two medicinal prescriptions, one as a preventative treatment for symptoms likely to occur during that month, and the second as 'a treatment in case of any damage to the foetus during that month'. The list of symptoms in particular illustrates nicely the sophistication and concern with which male physicians studied and observed the course of a woman's pregnancy, in order to alleviate as much of the strain of childbearing as possible. In contrast to earlier prescription literature such as the Jingui yaolüe, 金匱要 略 (Essential Prescriptions from the Golden Cabinet), the specificity of Sun Simiao's lists of symptoms, and particularly the association of individual symptoms with specific aetiologies, is remarkable. For this development, we can most likely thank Chao Yuanfang 巢元方, the author of the Zhubing yuanhou lun 諸病源候論 (On the Origins and Symptoms of the Various Diseases), which was composed around $610 \mathrm{CE}$, only decades before the Qianjinfang. As the title implies, this text discussed medicine from the perspective of aetiology and symptomology, and was the first to attempt a systematic categorisation of specifically female disorders in eight scrolls. This text contains yet another version of the month-by-month description of gestation, very similar to the Qianjinfang. By comparison to that text, it lacks the prescriptions, but instead gives advice on pulse diagnosis, beginning with the third month of pregnancy. This merely reflects the nature and intention of each text, the former being a formulary focused on therapy and the latter essentially a symptomology concerned with the classification of disease.

The parallel structure and wording of information for each month in this chapter suggests that these prescrip-

Nanjing 18. See the translation and commentaries in Unschuld, 1986 , pp. $243-58$, especially the table on p. 256 . tions were an integral part of the text called 'Xu Zhicai's Month-by-Month Prescriptions for Nurturing the Foetus', but the full extent of Sun Simiao's involvement in shaping this chapter is impossible to reconstruct since we do not have access to the original. Given the fact that the Qianjinfang is the only instance where prescriptions are added to the text, it is quite possible that Sun Simiao himself inserted the prescriptions to make the text more fitting for the framework of his book. Since the descriptions of gestation, the association with the channels and viscera, and the behavioural and dietary prohibitions are found in almost identical wording in other texts that precede the Qianjinfang, we can at least be certain that these aspects of 'Xu Zhicai's Month-by-Month Prescriptions' were adopted from an earlier source.

\section{The Renshen Mai Tu Yue Jin Fa 妊娠脈圖月禁法 (Charts of the Channels and Method of Monthly Prohibitions during Pregnancy)}

\section{The Chanjing Quotation}

We are now ready to compare the two texts discussed above, namely the Mawangdui version and the Qianjinfang version, to the Chanjing quotation that is cited side-byside with the 10 drawings in the Ishimpō: This text again includes the account of gestation from the Taichanshu. The differences between these three texts are so small as to be mostly insignificant. For example, the foetus in the fourth month is said, according to the Taichanshu, to receive the essence of water and form blood (成氣 cheng $q i$ ). Both the Zhubing yuanhou lun and the Qianjinfang add only one character, which however does not affect the meaning of the phrase: 成其氣 cheng qi qi (form its Qi). Most likely based on a scribal error, the Chanjing states that the foetus 'fills in blood and Qi' (盛血氣). This contradicts the statement in all three texts for the third month, where the foetus is said to 'fill in blood' (盛血 cheng xue, in the Taichanshu) and to 'fill the blood in the channels' (盛血脈 cheng xue mai, in the Chanjing, Qianjinfang, and Zhubing yuanhou lun). For another example of what appears to be a textual corruption in the Chanjing, the Chanjing states that the bones are completed in the fourth month, while the form is completed in the sixth month. The Qianjinfang has these developments in the opposite order, stating that the sinews and bones are established in the sixth month. This accords better with another statement in all texts that the sinews are formed in the sixth month.

Table 1 illustrates the variations in the account of gestation from the fourth to the ninth month between the texts, juxtaposing them with standard correlative theory 
Table 5.1 Variations in the Account of Gestation between the Texts

\begin{tabular}{|c|c|c|c|c|c|}
\hline $\begin{array}{l}\text { Month } \\
\text { 月 }\end{array}$ & $\begin{array}{l}\text { Phase } \\
\text { 五行 }\end{array}$ & $\begin{array}{l}\text { Standard theory: } \\
\text { - Viscus } \\
\text { - Body part }\end{array}$ & $\begin{array}{l}\text { Taichanshu } \\
\text { 胎產書 }\end{array}$ & $\begin{array}{l}\text { Qianjinfang } \\
\text { 千金方 }\end{array}$ & $\begin{array}{l}\text { Chanjing } \\
\text { 產經 }\end{array}$ \\
\hline 4 & Water & $\begin{array}{l}\text { - Kidney } \\
\text { - Bone }\end{array}$ & Blood & $\begin{array}{l}\text { Blood/channels } \\
\text { - Hand lesser Yang } \\
\text { - Triple burner }\end{array}$ & $\begin{array}{l}\text { Blood/channels } \\
\text { - Hand lesser Yang } \\
\text { - Upper burner }\end{array}$ \\
\hline 5 & Fire & $\begin{array}{l}\text { - Heart } \\
\text { - Channels }\end{array}$ & Qi & $\begin{array}{l}\text { Qi } \\
\text { - Foot greater Yin } \\
\text { - Spleen }\end{array}$ & $\begin{array}{l}\text { Blood and Qi } \\
\text { - Foot greater Yin } \\
\text { - Spleen }\end{array}$ \\
\hline 6 & Metal & $\begin{array}{l}\text { - Lung } \\
\text { - Skin and hair }\end{array}$ & Sinews & $\begin{array}{l}\text { Sinews } \\
\text { - Foot Yang brilliance } \\
\text { - Stomach }\end{array}$ & $\begin{array}{l}\text { Sinews and bone } \\
\text { - Foot Yang brilliance } \\
\text { - Stomach }\end{array}$ \\
\hline 7 & Wood & $\begin{array}{l}\text { - Liver } \\
\text { - Sinews }\end{array}$ & Bones & $\begin{array}{l}\text { Bones } \\
\text { - Hand greater Yin } \\
\text { - Lung }\end{array}$ & $\begin{array}{l}\text { Bones and marrow } \\
\text { - Hand greater Yin } \\
\text { - Lung }\end{array}$ \\
\hline 8 & Earth & $\begin{array}{l}\text { - Spleen } \\
\text { - Flesh }\end{array}$ & Skin and hide & $\begin{array}{l}\text { Skin and hide } \\
\text { - Hand Yang brilliance } \\
\text { - Large intestine }\end{array}$ & $\begin{array}{l}\text { Skin and hide } \\
\text { - Hand Yang brilliance } \\
\text { - Large intestine }\end{array}$ \\
\hline 9 & Stone & & Filament hair & $\begin{array}{l}\text { Hair on the skin } \\
\text { - Foot lesser Yin } \\
\text { - Kidney }\end{array}$ & $\begin{array}{l}\text { Hair on skin } \\
\text { - Foot lesser Yin } \\
\text { - Kidney }\end{array}$ \\
\hline
\end{tabular}

as it was settled in the Huangdineijing 黃帝內經 around the turn of the Common Era. ${ }^{6}$

From this comparison, we can deduce several facts. First, the information is largely consistent within the three citations of the account of gestation that is first found in the Taichanshu, down to an almost exact wording. This suggests that all three of them were based on a source that was transmitted in writing and therefore quoted literally. The minor deviations appear to be scribal errors of the type frequently found when comparing several versions of the same text in medieval medical literature. Second, the inclusion of the standard five phases (Water, Fire, Metal, Wood, and Earth) in this account shows that it was influenced by the theory of systematic correspondences. Moreover, the progression of phases follows the standard order according to the cycle of conquest (xiang ke 相克). Nevertheless, the significance of the five-phase system of correspondences remains superficial; the associations between phase, body constituent, and dietary and behavioural prohibitions do not yet accord with standard classical theory.

By contrast, the information on the channels prohibited for acu-moxa treatment in each month must be a later textual layer and is therefore not found in the Taichanshu quotation. The channel names and organ associations of this later layer are identical in the Qianjinfang and Chanjing versions and moreover accord directly with standard medical theory. They must therefore stem from a source that postdates the composition of the Huangdi nei jing. This coincides with the fact that both the Qianjinfang and the Ishimpō credit a post-Han text as their source. As we do not know the exact dates of composition for their source texts, it is impossible at this point to determine the original author of this textual layer on acu-moxa prohibitions. We only know that between the 4 th and 6 th centuries this text must have circulated widely enough to be quoted with slight variations by two different authors, both of whose source texts are now lost. Both the Qianjinfang and the Ishimpo quotations contain textual elements in the sections on acu-moxa prohibitions that are not found in the other source. This could mean an even earlier date for the composition of yet another original source, predating both Xu Zhicai 徐之才 (said to have lived from 493 to 572) and De Zhenchang (between 341 and 589, the beginning of the Sui dynasty). It could also indicate that the two sources came from different textual traditions, namely that Sun Simiao's source was a formulary and Tamba no Yasuyori's source an acu-moxa manual. However, given the fact that Tamba was the official acupuncturist at the imperial court and therefore had a personal interest in acupuncture over medicinal prescriptions, it is also possible that the additional acu-moxa-related information was inserted by him, whether it originated from his own hands or from yet another Chinese source. 




Figure 5.2 Drawings of the pregnant female body, here displaying foetal development during months 6-8 of the 10 months of pregnancy. Ishimpō 23:1 (982 CE). Seikido Library Scroll (1145 CE). Photographic facsimile (C) Vivienne Lo

\section{The 10 Drawings}

In addition to the text found in the other sources, the Ishimpō citation of the Chanjing contains two further elements: an exact, point-by-point description of each channel's location and, more importantly for the context of this publication, 10 line drawings. These depict the naked female body with the foetus inside the abdomen, prohibited acu-moxa points, and certain physiological features that are considered significant in each month of pregnancy. These drawings are the earliest extant visual representations of the naked female body in Chinese medicine, and of the development of the foetus worldwide. In addition to the acu-moxa point locations, the sparse line drawings also show the overall changes in the pregnant woman's body and anatomical features significant in any particular month, such as the corresponding internal organ, as described in the accompanying text. They also depict in detail the changes in the development of the foetus, from a small round lump to an increasingly humanlike form with gradually differentiated limbs, by the seventh month with distinct extremities, by the eighth month with tiny dots presumably representing the orifices in the skin that are said, in the accompanying text, to develop during this month, and finally to a fully developed monkey-like figure in the 1oth month, drawn sitting up because it is too big to fit in the abdomen lengthwise. By the 1oth month, the mother is depicted as a markedly voluptuous figure, drawn in curvy lines from the side, with partially visible but clearly enlarged breasts, a spinal cord sagging from the weight of the foetus, bulging thighs, and an abdomen that is protruding so far outward and downward as to be clearly at an uncomfortable and temporary stage, to be succeeded shortly by delivery (Fig. 3).

Several points can be noted from these drawings. First, the information conveyed in the pictures offers far more than a mere depiction of prohibited acu-moxa point locations, as one might expect the purpose of these drawings to be. They also provide a visual account of the changes in a woman's body and in the development of the foetus during the 10 months of gestation, complementing the medical advice found in the text. The illustrations can therefore be considered as yet another layer of information on prenatal care that was based on the account first found in the Taichanshu. They testify to the interest with which male physicians treated and investigated the female body in its reproductive functions.

Second, the drawings are executed with such attention to anatomical detail, while still in accordance with the accompanying text, that they must have been based on clinical experience and continued observation of the naked female body as it passed through the 10 months of pregnancy.

Third, for each month the artist depicted the entire female body, the foetus, the most important points to be avoided, as well as different anatomical features that play a special role in the nurturance of the foetus during that month. Furthermore, the points are connected through red lines that have been the subject of continuing scholarly debate. They have been interpreted as acupuncture channels by many scholars especially in Japan, which is strongly suggested by their shape and form. Nevertheless, they are not identical with the standard course of the channels that are identified in the accompanying text as responsible for nurturing the foetus during that month. By the Tang period, which is the likely date of composition for the Chanjing, the position of these channels was certainly agreed upon generally. Thus, these lines must be depicting something besides the course of the channels mentioned in the text. What exactly that might be remains unknown. In addition, it is possible that the red lines might not even 




Figure 5.3 Drawing of the pregnant female body in the 1oth month of pregnancy. Ishimpō 23: (982 CE) Seikido Library Scroll (1145 CE). Photographic facsimile (C) Vivienne Lo

have been part of the original drawing. We will return to this question below. In any case, the drawings in general and the red lines in particular suggest a notion of pregnancy as affecting the female body as a whole, in ever-changing constellations of supply and demand of vital substances from the entire body to the foetus, rather than just being limited to the provision of blood and Qi from the kidneys to the uterus. In any given month, the foetus is shown as surrounded, suspended, held in place, or even cradled by the red lines and physiological features. This visually reinforces the idea that these are nurturing and protecting the foetus in that month and may not therefore be manipulated with acupuncture or moxibustion. Before the days of ultrasound, these pictures must have been highly suggestive and formative in the reader's mind.

Lastly and perhaps most importantly, the absence of any clothing or drapes to partially conceal the body is striking, given the general modesty in early and medieval Chinese art. The stark nudity of these images is reinforced by the posture of the woman, depicted from the front in an upright position that reveals the full details of her figure (except for the last two pictures which show her from the back and side).

The early history of medical illustrations in China is full of question marks and has yet to be written. The Mawangdui manuscripts offer the earliest traces of medical 




Figure 5.4 Huangdi hamajing, sect. 1 'Huangdi hama tu sui yue shenghui bi jiupan fa 黃帝蝦蟆圖隨月生毀避炎判法’ (Yellow Emperor’s Toad Chart: method for avoiding cautery and pain according to the waxing and waning of the moon'), Beijing: Zhongyi guji, 1984

drawings of the human body in China: first, we find pictures of different body postures that complement textual information on physical cultivation by depicting exercise positions (Daoyin tu 導引圖). Second, the Mawangdui manuscript on sexual cultivation called He yinyang (合陰 陽 Conjoining Yin and Yang) contains a poorly preserved stylised depiction of the vagina that aims at providing the male reader with technical information on female anatomy to maximise his ability to stimulate the female body during sexual intercourse and thereby obtain optimal health benefits. Lastly, we find a similarly damaged picture of two figures for prognosticating the newborn child's future, in the above-mentioned manuscript titled Taichanshu, which also contains the information on nurturing the foetus discussed above. For the following centuries, evidence is too fragmentary to reconstruct a pictorial tradition. The bibliographic catalogue of the Sui dynasty is the first to contain references to illustrations, none of which have unfortunately survived to the present. The titles suggest primarily illustrations for depicting acu-moxa point locations, known generally under the title Mingtang tu 明堂圖 (Charts of the Hall of Brightness) and mentioned in such received classics as the Qianjinfang and Waitai miyao as intended to provide additional information to the textual descriptions.

For the closest stylistic relatives to the 10 Chanjing charts of the pregnant body, we have to turn to depictions of the naked male body, of which several have survived in manu- scripts: ${ }^{7}$ The earliest extant Chinese moxibustion charts, titled Jiufa tu 炎法圖 (Charts of Moxibustion Methods, S.6168 and S.6262), were found among the medical manuscripts at Dunhuang. Similar to the Chanjing charts, the bodies are depicted with simple line drawings that reveal such anatomical structures as the nipples, sternum, and ribs, show the typical medieval hairstyle of topknots on each side of the head, and mark the important acu-moxa points for treatment with dots on the figure and lines extending to the side of the image where the points are named. While the genitals in these figures are covered with tiny loincloths, another moxibustion chart (P.3589, titled Xinji beijijiujing 新集備急尒經, “Classic of Emergency Moxibustion, Newly Collected') depicts an elderly male figure in the nude, in the context of a treatise on physiognomy. All images from Dunhuang share a distinctive style of depicting the body reminiscent of images of meditating Buddhas.

For a last, even more closely related image of the naked human body in a Chinese medical text, let us turn to the Huangdi hama jing 黃帝蝦蟆經 (Yellow Emperor's Toad Classic), which exists in a single surviving copy as a Japanese woodblock print from 1823 (Fig. 4). Sharing not only pictorial, but also textual similarities with the Chanjing quotation as another example of medical prohibition literature, this text discusses the days and times during which acu-moxa treatment is contra-indicated due

$7 \quad$ For a more detailed description of these images, see Lo 2001, pp. $75^{-81 .}$ 




Figure 5.5 Drawings of the female body in the 3rd and 1oth months of pregnancy, with the foetus and prohibited acu-moxa channels. Ishimpō 23:1 (982 CE). Cabinet Library Scroll (1791 CE $)$

to astrological prohibitions. Like the Dunhuang drawings discussed here, it marks acu-moxa points on the human body and connects these with lines to names on the side, thus clearly being intended for practical application. Unlike the Chanjing charts, none of these images depict channels, if that is what the red lines are, nor do they reflect any association with vessel and visceral theory. It is therefore possible that they originated in a more popular context of medical practice than the scholarly elite tradition of texts like the Qianjinfang.

A last question concerns the authenticity of the images in the Chanjing, given the fact that the text was not printed on woodblock until 1859. The earliest version of this text currently extant is the Seikido Library Scroll 成筫堂文庫 本. This is based on a reconstruction that was made with the help of several manuscript copies after the original was destroyed in a palace fire in 1145. In this version (depicted in the images above of the pregnant woman during months six to eight and in the 1oth month), the pregnant woman is drawn in a Chinese style, with a chignon typical for the period of its composition in China. This would suggest that the copiers of the charts made every effort to remain faithful to the Chinese original. By contrast, another version of the images, found in the so-called Cabinet Library Scrolls 桂仙堂文庫本, based on a copy made in 1791 from yet another manuscript, depicts a woman with long flowing hair and pubic hair in a style clearly reminiscent of contemporaneous Japanese art.
The images we are looking at are therefore Japanese copies, multiple times removed from the early medieval Chinese original. We also need to take into account that the Chinese original of the Ishimpo version, that is, the Chanjing quotation, was already a composite of at least three textual layers that we have identified above, namely the account of gestation found in the Taichanshu, the information on the channels that is found both in the Qianjinfang and the Ishimpō, and lastly the additional information on acu-moxa point locations and the drawings, which are found exclusively in the Ishimpō. Considering the nudity of the images and the fact that the compiler of the Ishimpō, Tamba no Yasuyori, was personally invested in the practice of acupuncture, it might be tempting to dismiss the images as Japanese insertions into a Chinese original source. However, the Dunhuang charts of acumoxa locations discussed above prove not only that an indigenous Chinese tradition of depicting nudity in a medical context existed, but also that acupuncture texts were indeed used and applied in conjunction with charts very similar in style to the images in the Ishimpo, which is also confurmed by references to Mingtang tu in the received literature. In addition, numerous studies of the relationship between the Ishimpō and its Chinese sources have shown that Tamba no Yasuyori did indeed merely compile his Chinese sources without inserting additional material, with the exception of punctuation marks and short notes on pronunciation and meaning, which are clearly marked off from the main text by their red colour. It is indeed suspicious that the mysterious red lines in the drawings are also drawn in this colour. In any case, whether the red lines are a Japanese insertion, stem from yet another Chinese text, or were an integral part of the original Chanjing source, the information gained from these pregnancy charts cannot be dismissed in any discussion of medieval Chinese gynaecology.

\section{Conclusion}

In spite of the lack of primary sources, the continuity of content between the accounts of gestation and month-bymonth prohibitions during pregnancy in the Taichanshu, the Qianjinfang, and the Chanjing suggests an uninterrupted literary tradition of increasing complexity, reflecting male interest and involvement in women's reproductive processes. The context, purpose, and audience of this literature changed over the centuries, influencing the focus of a particular text, but the similarities and at times literary equivalence stand out. The Chanjing quotation in the Ishimpō adds significant information by including 
pictures of the changes in the pregnant female body, the development of the foetus, prohibited acu-moxa point locations - connected possibly by channels or simple lines - and significant physiological features in the 10 months of pregnancy, as well as more complete descriptions of the channel course in the accompanying text. The drawings mark this text as intended for direct clinical application, possibly addressing a more professionalised readership than the Dunhuang manuscripts, which were directed at popular consumption in a remote military outpost. By contrast, the readership of the Ishimpō consisted of Japanese physicians who were intent on learning the more advanced arts of Chinese medical practice, of which acupuncture was considered the most dramatic expression. The female body in these charts is depicted in stark nudity, which could certainly have been avoided with modest drapes, accessories, or positions without interfering with the purpose of the illustrations. However, it can be argued on the basis of archaeological evidence that the depiction of a nude body in the context of acu-moxa charts might not have been as rare or atypical as we might believe from the received literature. In addition, the revealing posture and lack of clothing offered interested readers a comprehensive impression not only of the prohibited acu-moxa points, but also of the general development of the female body and the foetus during the 10 months of pregnancy, certainly a topic of great relevance for a practising physician. The inclusion of elements of channel and visceral theory might mark this text as addressing an educated professional readership that was familiar with advanced medical theory, in contrast to the more popular manuals of acu-moxa therapy found at Dunhuang and in the Huangdi hama jing. In conclusion, the development of yangtai literature represents the confluence of cosmological and Yin-Yang theory, rudimentary gynaecological notions, formulary literature, acu-moxa charts, and vessel theory, all of which express a growing awareness of the special needs of women's bodies, especially in the context of reproduction.

\section{Bibliography}

\section{Primary Sources}

Beiji qianjinyaofang 備急千金要方 (Emergency Prescriptions Worth a Thousand Gold Pieces), Sun Simiao 孫思邀, late 7 th century.

Chanjing 產經 (Classic of Childbirth) n.d.

Dui yao 對藥 (A Comparison of Medicinals), Xu Zhicai 徐之才 n.d. (lost).

Geshifang 葛氏方 (Master Ge's Formulary), Ge Hong 葛洪 (283-343). Huangdi hama jing 黃帝蛤蟆經 (Yellow Emperor's Toad Canon) n.d. Modern edn, 1984, Beijing: Zhongyi guji chubanshe.


compiled by Tamba no Yasuyori 丹波康頼, 984 .

Jia chuan mifang 家傳秘方 (Secret Prescriptions Handed Down in the Family), Xu Zhicai 徐之才 n.d. (lost).

Mawangdui manuscripts (tomb closed c. 168 BCE. Modern titles given by editors of Mawangdui Hanmu boshu 馬王堆漢墓帛書, vol. 4, Beijing: Wenwu chubanshe).

Nanjing 難經 (Classic of Difficult Issues), 1st century.

Taichanshu 胎產書 (Book of Gestation and Birth).

Xiao er fang 小兒方 (Paediatric Prescriptions), Xu Zhicai 徐之才 n.d. (lost).

Zhubing yuanhou lun 諸病源候論 (Treatise on the Origin and Symptoms of the Various Diseases), Chao Yuanfang 巢元方 610.

\section{Secondary Sources}

Lo, V. 2001, 'Huangdi Hama jing', Asia Major 14.2, 61-99.

Ma Jixing 馬繼興 1985 , 'Yixinfang zhong de gu yixue wenxian chutan', 醫心方中的古醫學文獻初探 (Preliminary Investigation of the Ancient Medical Records in the Ishimpō), Nihon yishigaku zasshi 31.1, 326-71.

Mayanagi Makoto, 'A study on the illustrations between the Chanjing and the Mingtang', Unpublished paper presented at the conference on 'Chinese Medicine: A Visual History', 14-17 September 2005, Beijing, China.

Nihonkoku Genzaisho Mokuroku 日本國見在書目(Catalogue of Extant Books in Japan), 1910.

Unschuld, P.U.1986, Nan-ching. The Classic of Difficult Issues, Berkeley: University of California Press.

2003, Huang dineijing su wen. Nature, Knowledge, Imagery in an Ancient Chinese Medical Text, Berkeley: University of California Press. 


\section{The Gendered Medical Iconography of the Golden Mirror, Yuzuan Yizong Jinjian 御纂醫宗金鑑, 1742}

$Y i-L i W u$ 吳一立*

\section{Introduction}

This chapter explores the relationship between gender and medicine by analysing the illustrations that appear in the Yuzuan yizong jinjian 御纂醫宗金鑑 (ImperiallyCommissioned Golden Mirror of Medical Learning, 1742), a textbook and guide to practice compiled by the Qianlong emperor's Imperial Academy of Medicine (Taiyi yuan 太 醫院). ${ }^{1}$

My starting point is a simple yet historically significant question: why are almost all the human figures in this book depicted as men? As historians of Western medicine have amply documented, medical illustrations are never simply an objective depiction of observed reality. Rather, they are culturally and historically constructed, both encoding and creating assumptions about the innate characteristics and proper social roles of men and women, about the relationship of humans to the non-human world, and about the nature of scientific inquiry itself. As Thomas Laqueur has shown, for example, the belief that women's bodies were an imperfect variant of men's imbued the anatomical

Research for this chapter was supported by a fellowship from the Eisenberg Institute for Historical Studies at the University of Michigan, Ann Arbor, MI, and by grants from the Hewlett-Mellon Fund for Faculty Development at Albion College, Albion, MI. I would also like to thank Ellen Johnston Laing, who read the entire manuscript and provided crucial advice on interpretations and sources; Susan Erickson, Roslyn Hammers and Martin Powers for their sage suggestions; Jen-der Lee, Shigehisa Kuriyama and Asaf Goldschmidt for insightful critiques on earlier versions of this chapter; and Raymond Lum and Xiao-he Ma of the Harvard-Yenching Library for their generous assistance. Finally, I thank Vivienne Lo for her editorial support and guidance during the preparation of this chapter for publication.

Wu Qian (ed.) 1742. My analysis is based on a microfilm of the 1742 Wuying dian 武英殿 edition of the Yizong jinjian owned by the Peking Union Medical Library. I have also consulted an original copy of this edition in the rare book collection at the Harvard-Yenching Library. The library catalogues it as a 1739 edition produced by the Neifu 內府 (Palace Treasury), but it is identical to the Wuying dian edition, which includes a preface of 1739 signed by officials of the Neifu. I have also compared these two editions to the version reprinted in the Siku quanshu 四庫全書 in 1782 , which is the most widely available of the various editions of this text. While there are stylistic differences between some illustrations in the 1742 and 1782 versions, they do not affect the gender analysis presented here. diagrams of Andreas Vesalius (1514-64), epitomised by a famous drawing depicting the vagina as an immature, internal penis. ${ }^{2}$ Similarly, Londa Schiebinger found that 18th-century European anatomists began to draw female skeletons as a means of investigating the perceived differences between men and women. However, their a priori assumptions about women's natures and aptitudes led some anatomists to create exaggerated and distorted depictions of female bone structures. ${ }^{3}$

A study of medical illustrations is also vital for understanding the interplay between medicine and gender in China, serving as an important complement to existing studies that focus on textual descriptions of the body. What makes the Chinese case particularly interesting, furthermore, is that there is an apparent disjuncture between textual and visual depictions of gender. In her path-breaking work on the history of 'medicine for women' ( fuke 婦科), Charlotte Furth argued that the body of classical medicine was fundamentally androgynous. Each body embraced both Yin and Yang elements, and the essential structures and functions of male and female bodies were fundamentally the same. ${ }^{4}$ But if the body of Chinese classical doctrine was indeed androgynous, then how should we interpret the fact that medical illustrations use male figures as the standard for that human body, with female figures used in only special cases? After all, there was no intrinsic reason why a medical figure should be endowed with a recognisable gender at all. Indeed, the Golden Mirror also includes some figures without any gendered attributes, showing that this was a possible option for Chinese medical representations of the body.

To address these issues, we must first acknowledge that the conceptual boundaries between androgyny and gender difference in Chinese medicine were continually shifting according to time and context. A central theme of Furth's work, in fact, is the alternation between androgyny and female difference in Chinese medical thought. Thus, while medical doctrine was concerned with the universal attributes of human bodies, doctors focused on 'female difference' when they sought to explain the special reproductive problems of women. Here we will see that when Chinese

\footnotetext{
2 Laqueur 1990, ch. 3.

3 Schiebinger 1989, ch. 7; generally and especially her discussion of Marie Thiroux d'Arconville, pp. 195-200.

4 Furth 1999.
} 
medical illustrators sought to visually represent the universal doctrinal body, they reached first for images of men. So what cultural and epistemological factors contributed to this particular pattern? While China's medical canon - the constituent texts of Huangdineijing 黃帝內經 (The Yellow Emperor's Inner Classic) and their commentaries - contained clear descriptions of the body, they were not originally illustrated, and the illustrated editions that we currently have are the product of later compilers. A vital issue for scholars, therefore, is to determine why certain images and forms of representation became conventional in medical texts while others did not.

Using the Golden Mirror as a case study, this chapter will analyse three related dynamics that contributed to what I will call 'visual androcentrism' in Chinese medicine, namely the tendency to use male figures to represent the generic human body. The first is that medical illustrations were part of a broader visual culture, borrowing extensively from non-medical repertoires of iconographic idioms. The Golden Mirror's reliance on male figures was thus a natural outgrowth of an iconographic tradition in which men were routinely used in generic situations and women used only in contexts specifically gendered as female. Second, in visual culture there was a strong association between the semi-exposed male body and ideals of enlightenment and health. This made male figures particularly appropriate for a text on healing that wanted to show the features of different parts of the human body. Finally, the fact that classical medicine itself was a form of knowledge shaped and wielded by male practitioners also reinforced the existing tendency to favour male heuristic figures. The Golden Mirror furthermore incorporated auspicious and Daoist motifs into its images of men, thereby creating a visual metaphor that likened the enlightened male physician to a divine sage who would use his superior insights into Yin and Yang to obtain wondrous cures.

\section{The ‘Golden Mirror' and its Illustrations}

As Marta Hanson has shown, the Golden Mirror was shaped by the leading political and intellectual currents of the day. ${ }^{5}$ Compiled on the order of the Qianlong emperor, it was meant as a statement of orthodox knowledge that would serve as a model for the empire. Emulating the sage rulers of the Chinese past, the Manchu emperor sought to enhance his political authority by serving as a patron of scholarship and disseminating information to benefit his subjects. At the same time, the editorial board was domi-

5 Hanson 2003. nated by government physicians from Jiangnan (the area 'south of the Yangzi river' in eastern China) who used the Golden Mirror to promote the standards of kaozheng 考 證 evidential scholarship and to elevate the Han dynasty doctor Zhang Ji 張機 ( $f l$.196-219) as the intellectual ancestor of scholarly medical practice. To facilitate study of the Golden Mirror's principles, its compilers presented information in an easy-to-digest format, structuring the bulk of the discussion around mnemonic verses and $t u$ 圖, a term that encompassed charts, tables, diagrams and illustrations. As Francesca Bray and others have discussed, $t u$ were distinguished from other kinds of images by their intended function: to convey technical information. ${ }^{6}$ The $t u$ in the Golden Mirror included 484 images or sets of images depicting the human body in part or in whole, constituting what is probably the largest ensemble of illustrations in a single Chinese medical text. ${ }^{7}$

In general, these illustrations fall into two main categories. The first depicts the structures of the body, most notably the zangfu 藏腑 (internal viscera), the jingmai 經 脈 (circulation channels and vessels that ran through the viscera), the xue 穴 (points on these channels where needles or moxibustion could be applied), and the location of different bones. A second category of illustrations depicts specific ailments, and the vast majority of these appear in the chapters on paediatrics and 'external diseases' (waike 外科), namely diseases of the skin and flesh. The aesthetic quality of the Golden Mirror's illustrations attests to the care and skill that went into their composition and execution. Well-proportioned and even graceful, the human figures are shown from a variety of angles, many of them captured in the midst of some implied activity or movement. One man strokes the end of his long beard with his left hand, the curve of his whiskers echoed by the swinging flywhisk in his right hand..$^{8} \mathrm{~A}$ lady with a flowery chignon

$6 \quad$ Bray in Bray et al. (eds) 2007.

$7 \quad$ Strictly speaking, there are 484 pages of human images. All fullbody figures are presented one to a page, although there are a few cases in which a body part is shown more than once on a page, usually to illustrate possible variations in its appearance. Typical examples of this multiple display include drawings of the two hands or two eyes together, 20 images of children's hands showing the different lines and creases that can be used to diagnose illness (displayed four per page over five pages in juan 50 ) and a one-page array of 24 anuses, each showing a different form of haemorrhoid (juan 69). Since there are only a few instances of such multiple displays, and since the iconographic style is consistent within each page, I have considered it expedient to count each page of illustrations as one image for the purposes of this analysis.

8 'Biding tu' 鼻疔圖 (diagram of nasal carbuncle), Wu Qian (ed.) 
pauses from the book she is reading, her right hand raised as if about to turn a page or bring attention to some meaningful passage. ${ }^{9}$ A young boy in flowing trousers decorated with ribbon-like waves walks towards an unseen friend to offer a basket of flowers. ${ }^{10}$ Furthermore, it is clear that the compilers made deliberate decisions about what kind of images to include.

This is exemplified by the Golden Mirror's illustrations of the body's circulation channels and viscera, which differ notably from those in an earlier government compilation, the Qinding gujin tushu jicheng, 欽定古今圖書 集成 (Imperial Encyclopaedia, 1726-8). ${ }^{11}$ Given that both works were produced by the Imperial Printing Office, it is noteworthy that the Golden Mirror did not reuse the earlier illustrations from the Imperial Encyclopaedia. ${ }^{12}$ Instead, the Golden Mirror and Imperial Encyclopaedia drew from two different iconographic strains, representing two alternative ways of depicting the body that had co-existed since at least the early 17 th century. The Imperial Encyclopaedia incorporated images that had previously also been anthologised in Wang Qi's 王圻 Sancaituhui三 才圖會 (Assembled Diagrams of the Three Powers, 16o9), an encyclopaedic collection of stock illustrations of numerous subjects pertaining to the triad of 'heaven, earth, and man': cosmological maps, birds and flowers, scholarly



Figure 6.1 'Image of the form of the heart', Imperial Encyclopaedia, reproduced from Chen Menglei et al. (comp.) 1986, Yishu dian 藝術典 (arts and techniques section), juan 118:52515

1742, 65:20a.

'Jiu nanchan xue tu' 灭難產穴圖 (diagram of the point to cauterise for treating difficult childbirth), Wu Qian (ed.) 1742, 86:01a. 'Suochun tu' 鎖脣圖 (diagram of a pox eruption confining the lips), Wu Qian (ed.) 1742, 57:12a.

$11 \quad$ Chen Menglei et al. (comp.) 1986.

12 See Hanson 2003 for the role of the printing office in imperial medical compilations. accoutrements and activities, people from foreign lands and mythical kingdoms. The Golden Mirror, by contrast, favoured figures that had notably circulated in the work of Zhang Jiebin 張介賓 (1563-1640), native of Shaoxing and author of a famous set of commentaries on the Yellow Emperor's Inner Classic.

While some of the visual differences between these two strains might be dismissed as largely stylistic, others suggest deep, doctrinal divergences. This is epitomised by their different renderings of the heart, long defined as the seat of consciousness and the ruler of the body. The Imperial Encyclopaedia shows the heart with five tubes linked to it: one labelled feiguan 肺管 ('lung tube' or trachea) and four additional $x i$ 系 ('connectors'), each of which led to another of the zang 臟 viscera: lung, liver, spleen, kidney (Fig. 1). ${ }^{13}$ The Golden Mirror (Fig. 2), by contrast, omits the separate connector to the lung, explaining that it is the trachea which connects the lung and heart. ${ }^{14}$

Equally significant structural differences appear in these two works' renderings of the bladder, Triple Burner and pericardium. Additional research is needed to verify the earliest origins of these two styles of illustration and to understand the theoretical orientations that underlay these very different views of visceral structure. For the purposes of this present discussion, however, it is sufficient to note



Figure 6.2 'The heart organ of the lesser Yin channel', Golden Mirror, reproduced from Wu Qian (ed.) 1742, 82:01a. Chinese Collection, Harvard-Yenching Library. (C) President and Fellows of Harvard College
13 Chen Menglei et al. (comp.) 1986, 118: 52515. Compare to Wang Qi 1970, 'Shenti' 身體 (Section on the body) 1:24a.

14 Wu Qian (ed.) 1742, 82:1b. Compare to Zhang Jiebin 1965a, p. 115 . 
that the Golden Mirror's editors had an array of options to select from when creating medical images. Their choices thus bear further scrutiny.

\section{Visual Androcentrism}

Out of 484 images in the Golden Mirror, a small minority (44 images) lack any obvious gender markings. These include isolated images of internal viscera and other body parts as well as maps of circulation networks superimposed on an outline of the body that is otherwise devoid of explicitly gendered features (Fig. 3). The remaining 440 images, however, are depicted with clothing and hairstyles that allow them to be read as male or female. Of these, only 12 are female. ${ }^{15}$ The lopsided gender distribution is even more significant because of the central importance of the topics that are illustrated by male figures (Table 1). In particular, male figures are used to depict the body's basic structures: circulation channels, location of bones, and almost all acupoints. Similarly, the figures used to illustrate how different diseases manifest themselves on the body are almost all male.

In contrast to this wide use of male bodies as the standard human body, female figures are used to illustrate medical problems that are defined as being unique to or more common in women. These include four figures depicting breast ulcers and growths, one figure showing a woman suffering from a postpartum abdominal abscess, and two that show the location of acupoints for treating complications of childbirth. The remaining five female figures depict facial skin blemishes, sores and swellings: freckles and black moles, niukou feng 鈕扣風 ('button wind', an ulcer in the hollow of the throat) plus two variants of luoli

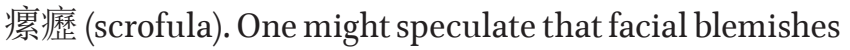
represented a greater threat to female vanity and marriageability, but the Golden Mirror's descriptions offer little clue to why these last five conditions were gendered as female.

15
External evidence, however, indicates that the Golden Mirror probably adapted these figures from earlier texts that for various reasons defined them as female diseases. As Furth has shown, the scope of Chinese fuke was quite broad before the 16th century. Although late Ming writers of $f u k e$ texts started to omit ailments not directly associated with childbearing, texts on external ailments continued to define certain kinds of skin conditions, ulcers, sores, and growths as typical of women. ${ }^{16}$ For example, Chen Shigong's 陳實功 Waike zhengzong 外科正宗, (Correct Teachings on External Ailments, 1617), categorises 'button wind' under the rubric 'various ailments of women' and illustrates this ailment with a female figure. ${ }^{17}$ The compilers of the Golden Mirror were certainly familiar with Chen Shigong's illustrations of women, for their image of postpartum abscess is a minimally modified version of Chen's image of ruyan 乳岩 (lit. 'breast mountain', a form of breast tumour). ${ }^{18}$ Shen Gongchen's 申拱辰 Waike qixuan 外科啟玄 (Dispelling the Mysteries of External Ailments, 1604) likewise uses the figure of a woman to illustrate 'scrofula' and the accompanying caption explains that 'this is a disease that women frequently have'.19 There were also distinctive female skin conditions. One female figure in Shen Gongchen's book, for example, shows fen hua chuang 粉花瘡 ('powder flower sores') defined as 'itchy pits and nodules erupting on a woman's face', and qunbian chuang 裙邊瘡 ('skirt border sores') which erupt on a woman's ankles as the result of pathogenic wind striking at the edges of her clothing. ${ }^{20}$

Viewed in historical context, therefore, it is reasonable to say that the Golden Mirror used male bodies as the standard human body, with female figures used only for diseases that were defined by earlier or contemporary doctors as being typical of women. Below we will examine more closely how the visual androcentrism of the Golden Mirror reiterated patterns in Chinese visual culture at large.

16 In particular, Ming medical writers eliminated many non-gestation related illnesses that Song dynasty fuke had associated with pathogenic female blood. Furth 1999, chs 4 and 5 .

17 Chen Shigong in Hu Xiaofeng (ed.) 1997, pp. 397-8.

18 See Wu Qian (ed.) 1742, 74:15a and Chen Shigong in Hu Xiaofeng (ed.) 1997, pp. 397-8.

19 Shen Gongchen in Hu Xiaofeng (ed.) 1997, p. 311.

$20 \quad$ Ibid., p. 313 . 


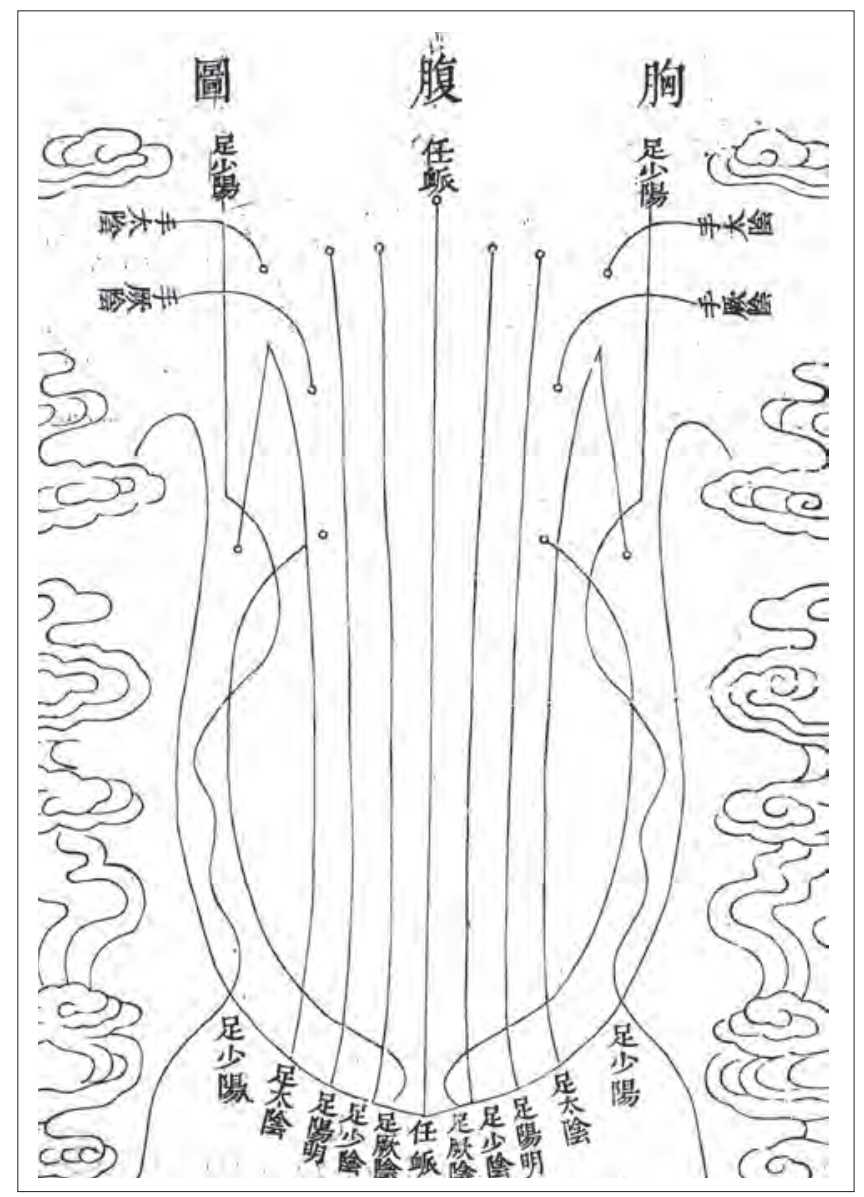

Figure 6.3 'Vessels of the chest and abdomen', Golden Mirror, reproduced from Wu Qian (ed.) 1742, 61:o6a. Chinese Collection, Harvard-Yenching Library. @ President and Fellows of Harvard College

Table 6.1 Distribution of Human Figures by Subject Matter and Gender

\begin{tabular}{|l|r|r|c|c|}
\hline Topics (juan where illustrations appear) & Male & Female & No gender & Total by topic \\
\hline $\begin{array}{l}\text { Treatise on Cold Damage Disorders } \\
\text { (juan 17) }\end{array}$ & 14 & 0 & 0 & 14 \\
\hline $\begin{array}{l}\text { Five phases and six climatic configurations } \\
\text { of Qi } \\
\text { (juan 34-5) }\end{array}$ & 1 & 0 & 2 & 3 \\
\hline $\begin{array}{l}\text { Children's diseases and poxes } \\
\text { (juan 50, 57) }\end{array}$ & 45 & 0 & 7 & 52 \\
\hline $\begin{array}{l}\text { External ailments } \\
\text { (juan 61,63-74) }\end{array}$ & 249 & 10 & 6 & 265 \\
\hline $\begin{array}{l}\text { Eye diseases } \\
\text { (juan 77) }\end{array}$ & 96 & 2 & 27 & 125 \\
\hline $\begin{array}{l}\text { Acupuncture and moxibustion } \\
\text { (juan 79-86) }\end{array}$ & 23 & 0 & 0 & 23 \\
\hline $\begin{array}{l}\text { Bone-setting } \\
\text { (juan 87-9) }\end{array}$ & $\mathbf{4 2 8}$ & $\mathbf{1 2}$ & $\mathbf{4 4}$ & $\mathbf{4 8 4}$ \\
\hline Total by gender & 0 & 2 & 2 \\
\hline
\end{tabular}






Figure 6.4 The chengshan point', Golden Mirror, reproduced from Wu Qian (ed.) 1742, 86:23b. Chinese Collection, Harvard-Yenching Library. (c) President and Fellows of Harvard College

\section{Medical Images and Chinese Visual Culture}

Historians of European medicine have documented many instances where medical images incorporated iconographic conventions from religious and secular art. ${ }^{21}$ So too, the Golden Mirror borrowed visual elements from non-medical sources. A particularly striking example consists of seven figures - six men and one woman - copied from Jin Guliang's 金古良Wu shuang pu 無雙譜 (Peerless Paragons, 169o), a famous illustrated collection of 40 historical biographies. ${ }^{22}$ The figures from Jin's work circulated widely after their initial publication, and by the 19th century they had even become incorporated into New Year's prints and porcelain designs. ${ }^{23}$ They were obviously known to the compilers of the Golden Mirror, who borrowed some to illustrate certain circulation channels and acu-moxa points. The Golden Mirror's diagram of the chengshan 承 山 point (Fig. 4), for example, is essentially an exact copy of the portrait of the Tang dynasty official Zhang Chengye 張承業 from Peerless Paragons (Fig. 5), save for the fact that Zhang's feet have been slightly repositioned to better reveal the position of the said point above the ankles. ${ }^{24}$

\footnotetext{
$21 \quad$ For examples, see Jordanova 1989, p. 45; Park 2006, ch. 6.

22 Jin Guliang 1961.

23 Claypool 2001, p. 214 and figs 3.24 and 3.27.

24 The full list of figures from Jin Guliang 1961 (and the location of their cognates in the Golden Mirror) are as follows: Yang Hu 楊 祜 (Wu Qian (ed.), 1742, 79:22a), Li Mi 李泌 Yehou (79:42a), Ban
}



Figure 6.5 Portrait of Zhang Chengye, reproduced from Jin Guliang 1961.

Additional research is needed to understand why the Golden Mirror borrowed images rather than creating new ones. However, we can speculate that this kind of visual poaching was stimulated by the need to find images for medical topics that had not previously been illustrated or for which the existing repertoire of illustrations was inadequate. This dynamic is exemplified by the Golden Mirror's chapters on pox eruptions (douzhen 痘疹), a sub-set of paediatric diseases (erke 兒科). These chapters are densely illustrated by 42 figures of children marked with different kinds of pustules and different patterns of smallpox eruption. Some precedent for these images can be found in Shen Gongchen's Dispelling the Mysteries of External Ailments. For example, one chapter of Shen's work includes a set of 10 figures showing the 10 types of red ulcers (dan 丹) in newborns caused by foetal poison (taidu 胎毒, foetal poison also being the cause of smallpox), while another chapter depicts three different patterns of smallpox eruption which augur either smooth recovery or death. ${ }^{25}$ But the Golden Mirror pox pictures are unprecedented in their number, scope and systematic nature, and it is no coincidence that this genre should emerge during the Qing

Zhao 班昭 (86:1a), Fu Sheng 伏生 (86:19a), Zhang Chengye 張 承業 (86:23b), Feng Dao 馮道 (86:25b), and Sima Qian 司馬遷 (86:28b). Jin's work does not have page numbers. Shen Gongchen in Hu Xiaofeng (ed.) 1997. The foetal poison eruptions are found in juan 8, pp. 320-2, while the smallpox eruptions are in juan 10, p. 335 . 
dynasty. This ruling house was founded by the Manchus, who swept down from north-east Asia to conquer China in the 17 th century. ${ }^{26}$ Among the challenges they now faced was smallpox, endemic among Han Chinese who enjoyed lifelong immunity if they survived the disease as children, but a disease that was deadly to Manchus who would contract it as adults. Thus, as Chang Chia-feng has shown, the Qing rulers were acutely concerned with preventing and controlling smallpox. ${ }^{27}$ During the Shunzi reign (1644-61), a specialised department of pox and rashes was even established within the imperial medical service. Although this department was later folded into the department of children's diseases, the Golden Mirror's numerous illustrations of pox and rashes were a continued expression of this special concern. ${ }^{28}$ In the absence of a well-defined tradition of pox illustrations, furthermore, the Golden Mirror created its own iconography, one that borrowed heavily from the popular visual genre of baizi tu 百子圖 ('pictures of a hundred boys'), also referred to as yingxi tu 嬰戲圖 ('pictures of playing boys'). ${ }^{29}$ Beginning in the 16 th century, such depictions of little boys at play had become standard visual devices for conveying auspicious pictorial messages, and they were commonplace in 18th-century material culture, appearing on all manner of household goods and decorative objects. As such, they provided an obvious visual resource for the compilers of the Golden Mirror, and the conventions of this genre permeate their pox pictures: the children hold lucky objects, play with toys, and two even appear to be teasing their pets (Fig. 6).

Besides borrowing from visual culture at large, the Golden Mirror also perpetuated a dominant visual convention: using men as a generic standard. Craig Clunas has documented this tendency in Ming dynasty visual

26 Although the Manchus proclaimed the beginning of their rule over China in 1644, it took another four decades for the Qing rulers to consolidate their control over the empire.

$27 \quad$ Chang Chia-feng 2002.

28 Hanson 2003.

29 I am indebted to Ari (Larissa) Heinrich for pointing out this connection to me. Auspicious images of children have been a feature of Chinese material and visual culture since at least the Tang dynasty. See Laing 1996. Terese Tse Bartholomew has shown, however, that the meaning of such images changed substantially from the Song to the Qing; see Bartholomew in Wicks (ed.) 2002. She notes that while Song art is characterised by 'naturalistic and sympathetic depictions of actual children at play... by the Qing period, images of children evolved into little more than stylised icons of good fortune' (p. 83). Bartholomew further finds that such images became a fully-developed auspicious genre by the 16th century, and that the repertoire of motifs expanded dramatically after the $17^{\text {th }}$ century.



Figure 6.6 'Pox pustules' from the Golden Mirror, reproduced from Wu Qian (ed.) 1742, 57:67a. Chinese Collection, Harvard-Yenching Library. Copyright President and Fellows of Harvard College

culture, observing that although the Chinese word ren 人 ('human, humankind') has no explicit gender marking, pictorial depictions of the triad of tian diren 天地人 ('heaven, earth, and human') assumed that men were 'the default position of humanity, and in fact the equation ren = male was largely normative in Ming pictorial usage'. ${ }^{30}$ Even a work like the Peerless Paragons, which contained an unusually high number of women for a collection of this type, was predominantly male: the 40 historical paragons include only eight women. ${ }^{31}$ Clunas remarks in passing that the dominance of male figures in physiognomy texts may reflect a concern to protect women from the gaze of unrelated males, and it has also been suggested to me that the dearth of women in The Golden Mirror might be an outgrowth of female seclusion. But this explanation becomes unsatisfactory when we remember that norms of female modesty never actually precluded the production and consumption of female images. This should come as no surprise, for as scholars of Chinese women's and gender history have shown, the precepts of the female 'inner sphere' could be negotiable and contingent. Thus women were supposed to stay at home, yet they could and did travel for reasons ranging from religious pilgrimages to escorting a dead spouse's body home for burial. ${ }^{32}$ Similarly, management of the inner sphere could include running a

\footnotetext{
$30 \quad$ Clunas 1997, p. 88.

$31 \quad$ See discussion of this issue in Claypool 2001, p. 15 o.

32 Mann in Goodman and Larson (eds) 2005.
} 
family business or overseeing household properties, all of which could take women out of the physical boundaries of the home. This fluidity of gender norms also characterised visual culture. It is true that commissioning a ritual portrait of a deceased female relative could be considered problematic: strictly speaking, a woman who avoided the gaze of unrelated men in life should also be shielded from their view after death. ${ }^{33}$ And yet, Ming-Qing print culture provided many socially-sanctioned opportunities to look at images of women in general. Ironically, after the late 16th century, the public display of women in print was greatly facilitated by the proliferation of collected, illustrated biographies of virtuous wives, widows, mothers and daughters. Such works were based on the famous didactic text Lienü zhuan 列女傳 (Biographies of Exemplary Women) by Liu Xiang 劉向 (79-8 BCE), and their late imperial redactors portrayed them as stories of virtue designed to improve female morality. As Katherine Carlitz demonstrates, however, the exquisitely illustrated editions that circulated during the late Ming in fact provided a new arena for the voyeuristic consumption of female images, a trend reinforced by an increasing resemblance between portraits of these virtuous women and the sensually-charged illustrations of female characters from works of popular fiction and drama. Most notably, the paragons of female virtue now offered up for public appraisal might even include women from a publisher's own lineage. ${ }^{34}$

In sum, to the extent that gender segregation played a role in visual androcentrism, it was not because female modesty prohibited the depiction of women. More important is the fact that gender norms endowed images of men and women with different types of cultural utility. For example, boys were depicted much more frequently than girls in Chinese visual culture. ${ }^{35}$ Because of the supreme cultural value placed on the birth of sons, who were essential for the continuation of the patrilineal family, images of boys were the most suitable iconographic medium for conveying auspicious wishes. During the Qing, furthermore, the auspicious messages that were visually encoded in images of playing boys included the specifically masculine aspirations of examination success and

Clunas 1997, pp. 90-1. The strict visual etiquette that governed images of female relatives, and the tensions inherent in such portrayals, is also discussed by Stuart in Wu and Tsiang (eds) 2005, p. 209, and pp. 218-19.

Carlitz makes this point with respect to the Huitu lienü zhuan 繪 圖列女傳 (Illustrated Biographies of Exemplary Women) compiled in the early $17^{\text {th }}$ century by a Mr Wang. Sixty per cent of the women in the collection 'are women born or married into the Wang lineage'. Carlitz 1991, p. 133. high official rank. ${ }^{36}$ In certain visual contexts, therefore, the use of male figures was positively required. As I shall show below, however, the range of situations that called for female figures was much narrower.

\section{The Gendered Meanings of Nakedness}

In medical illustration, as in other arenas of visual culture, we find factors that encouraged the generic use of male figures while relegating women to gender-specific contexts. Chief among these was the need to expose different parts of the body in order to show the site of a circulation channel, an acupoint, or the location of a pathogenic eruption on the torso, arms, legs, buttocks or feet (Fig. 7). Again, however, we must avoid assuming that gender norms would have precluded the use of exposed female figures, for six of the 12 female figures in the Golden Mirror have actually bared some part of their body: four women have let their robes fall down from the shoulder to expose one or both breasts (Fig. 8), one parts her upper garment to expose her navel and lower abdomen, and one extends a bare foot out from underneath the hem of her skirt. Furthermore, there is a long history of such images, with an early example of the bare-breasted woman appearing in Chen Ziming's 陳自明 foundational work Furen daquan liangfang 婦人大全良方 (Comprehensive Compendium of Good Prescriptions for Women, 1237). ${ }^{37}$ In Chen's work, she has pulled open her upper garment to show the site of the qimen point 期門穴, which should be needled to treat 'pathogenic heat entering the blood chamber' (re ru xueshi 熱入血室). Illustrated works on external diseases also conventionally included female figures pulling aside their garments to show various growths on their breasts. Shen Gongchen's Dispelling the Mysteries even uses a completely topless woman to illustrate 'syphilitic ulcers resembling ringworm'. ${ }^{38}$

In sum, there was a long-standing medical convention of depicting semi-exposed female figures. To explain the gender imbalance, therefore, it will be helpful to consider that in Chinese visual culture generally, the exposed male body evoked a wide range of positive meanings, most notably the pursuit of good health and longevity. By contrast, exposed female bodies evoked a much narrower and more

$36 \quad$ Bartholomew in Wicks (ed.) 2002, pp. 72-3. She observes that images of boys engaging in the four scholarly pursuits (literature, painting, music, chess) emerged as a theme in the 17 th century and that wishes for examination success became a notable theme in the Qing.

37 Chen Ziming 1992, p. 180.

38 Shen Gongchen in Hu Xiaofeng (ed.) 1997, p. 318. 




Figure 6.7 'Points on the Yang springing vessel', Golden Mirror, reproduced from Wu Qian (ed.) 1742, 84:22b. Chinese Collection, Harvard-Yenching Library. (c) President and Fellows of Harvard College

ambivalent set of meanings that made them comparatively unsuited for heuristic applications.

Representations of naked or semi-naked women historically appear in three main contexts. One is of course Chinese erotic art, notably the Ming colour prints made famous by R.H. van Gulik in 1951 (some of which are now believed to be van Gulik's own creations) and the 18thand 19th-century paintings collected by Ferdinand M. Bertholet. ${ }^{39}$ While images of the naked female body could enhance marital pleasure, they were also potentially subversive. This is exemplified by an 18 th-century woodblock print of Wang Hui's 王檅 (1632-1717) painting of Yang Guifei 楊貴妃 ('Precious Concubine Yang') emerging from her bath, published in Baimeixinyong tuzhuan 百美新詠圖傳 (New Verses on the Hundred Beauties, with Biographies and Illustrations, 1787 ). ${ }^{40}$ Yang is wrapped in a full-length cloak that ostensibly veils her nakedness, yet her breasts and nipples are visible under the fabric. This coy visual masking and signalling serve only to emphasise the charms

James Cahill has recently argued that Van Gulik may be the author of some of the exemplars of 'Ming' erotic art that he claimed to have discovered. See Cahill 2003 and Cahill in Van Gulik 2004. For the Bertholet Collection, see Bertholet 2011; Bertholet and Rev. Yimen 1998; Lefebvre (ed.) 2006.



Figure 6.8 'Shallow abscess of the breast', Golden Mirror, reproduced from Wu Qian (ed.) 1742, 66:45b. Chinese Collection, Harvard-Yenching Library. (C President and Fellows of Harvard College

of the young lady later blamed for the near-downfall of the Tang dynasty.

A second context in which we find bared female bodies is in depictions of mythical or non-human worlds, where the presence of nude women underscores the strangeness of the milieu. A stock image of the fantastical all-female 'Kingdom of Women' (nüren guo 女人國), for example, features naked women, and images of the Courts of Hell could also include disrobed female sinners being subject to various forms of torture. ${ }^{41}$

A third context for the exposure of the female body was in idealised scenes of domesticity and household activity, which could show women breast-feeding their children while performing other tasks. Some Song and Yuan dynasty paintings of 'knick-knack pedlars', for example, show a breast-feeding woman looking on as the pedlar is surrounded by chubby and playful children wanting to buy toys. ${ }^{42} \mathrm{In}$

41 For the Kingdom of Women, see Wang Qi 1970, Renwu 人物 (Section on human figures), 12:24a. Thirteenth-century paintings of the Courts of Hell with naked female sinners are reproduced in Ledderose 200o, figs 7.7, 7.8, 7.13, 7.14.

42 For exemplars of the 'knick-knack pedlar' genre painted by $\mathrm{Li}$ Song 李㠃 (fl. 1166-1243), and Ren Kangmin 任康民 (Yuan), see Laing 1975, figs 3, 10. This visual trope was sufficiently wellknown that Li Song was able to subvert it in his famous 'Skeleton 




Figure 6.9 Deep abscess of the breast', Golden Mirror, reproduced from Wu Qian (ed.) 1742, 66:45b. Chinese Collection, Harvard-Yenching Library. @ President and Fellows of Harvard College

such contexts, the nursing mother forms part of a tableau that connotes fecundity, prosperity and happiness. It was such images of breast-feeding women that provided the model for the female breast images in the Golden Mirror (Fig. 9). Although these images are not widespread, their regular appearance in government-sponsored sources attests to their symbolic importance. Song, Yuan and Qing editions of the Gengzhi tu 耕織圖 (Pictures of Tilling and Weaving), for example, all include images of breast-feeding women. Rosalyn Hammers shows that these idealised depictions of male and female agrarian production served as a form of visual propaganda depicting the harmony and prosperity that would arise from proper government policy towards rural workers. ${ }^{43}$ During the Qing, both the Kangxi and Qianlong emperors sponsored new editions of these images, demonstrating their continued utility as

Puppeteer', where the knick-knack pedlar is now represented by a skeleton who uses a marionette of a skeleton to attract a young boy. To the left and slightly behind the 'pedlar' is a breast-feeding woman. A painting that similarly uses the figure of a breast-feeding woman to comic effect is a late 13th-century scroll showing the Song dynasty official Yang Pu leaving the countryside to take up a bureaucratic post. Leading the household procession is Yang Pu's wife, nursing a naked baby boy on the back of a hulking water buffalo. Both works are reproduced and discussed in Wicks (ed.) 2002.

Hammers 2002.



Figure 6.10 Detail of 'Winnowing', from a Song dynasty edition of Pictures of Tilling and Weaving, reproduced from Franke 1913, pl. XLVIII

visual narratives of government benevolence. ${ }^{44}$ In these various editions, breast-feeding women appear as central figures in scenes of 'winnowing' (boyang 簸楊) and 'the first slumber of the silkworms' (yimian 一眠) (Fig. 10). Such images thus visually yoked the exposed, nursing female body to ideals of agricultural productivity and fertility.

In sum, domesticity was the only context in which visual depictions of exposed female bodies could serve a normative function. This also made it appropriate to show images of the female body in connection with women's diseases, a dynamic exemplified by the Golden Mirror's images of breasts. After all, diseases of the female breast were historically attributed to pathogenic imbalances arising during childbearing and breast-feeding. In this context, images of the exposed female body provided useful heuristic devices to help the doctor remember the forms and courses of different ailments, promoting proper medical care for breast diseases as part of a larger effort to preserve a woman's reproductive health.

This close relationship between domesticity and exposed female bodies also sheds light on a historically anomalous image in the Golden Mirror, entitled Jiu nanchan xue tu炎

44 For a discussion of the history of these editions, the imperial prefaces, and reproduction of the plates, see Franke 1913 and Pelliot 1913. I am indebted to Ellen Johnston Laing for providing me with these citations and to Rosalyn Hammers for providing me with additional images from the Yuan. 




Figure 6.11 'Point to cauterise for treating difficult childbirth', Golden Mirror, reproduced from Wu Qian (ed.) 1742, 86:o1a. Chinese Collection, Harvard-Yenching Library. (C) President and Fellows of Harvard College

難產穴圖 ('Acupoint to cauterise for difficult childbirth') (Fig. 11). In this image, derived from the figure of Ban Zhao 班昭 in Peerless Paragons (Fig. 12), a well-dressed, beautifully-coiffed woman sits cross-legged on a stool. Her right foot rests on the floor, unbound and bare, each slender toe clearly delineated, so as to demonstrate the position of the zhiyin 至陰 (extreme Yin) point on her little toe. The accompanying text explains exactly how to identify this point and apply moxa to resolve cases of difficult childbirth in which drugs and charms have proven ineffective. The appearance of this unbound female foot likely reflects the fact that Qing imperial doctors were responsible for treating the Manchu ruling family. ${ }^{45}$ Since Manchu women did not bind their feet, it was possible to perform interventions on their toes, something that was not necessarily true for Han Chinese women. ${ }^{46}$ However, the image of a naked female foot must have seemed jarring to Han Chinese readers, who would have expected upper-class women to

45 Unlike Ming court doctors who also treated central government officials, Qing imperial physicians treated only the members of the ruling imperial family. Chang Che-chia 1998.

46

One extant work on acupuncture, attributed to a pseudonymous Ming author named 'Qiongyao the Perfected One' and reprinted in the 19th century, discusses the challenges of performing acumoxa on women with bound feet. It suggests that a point on the woman's shoulder be substituted for a point on the foot. Qiongyao zhenren 1848 , 3:33b.



Figure 6.12 Ban Zhao, reproduced from Jin Guliang 1961.

bind their feet and then never again uncover them. Indeed, even in erotic art where the woman's body is otherwise completely naked, her feet are always covered, and this is also generally true in medical images of women. ${ }^{47} \mathrm{But}$ in the illustration from the Golden Mirror, exposure of this most private female body part was also required by its importance for resolving difficult childbirth.

\section{Male Nakedness, Enlightenment, and Health}

Female nakedness was imbued with conflicting messages: erotic desire, domesticity, but also social disruption. In contrast to this relatively narrow, ambivalent range of meanings evoked by the female figure, exposed male bodies evoked a wider spectrum of positive associations. At the most visually literal level, male nakedness appeared in depictions of the lower classes and of labourers - paintings of beggars, labourers, farmers, fishermen - people who worked in the mud or under the sun with their clothing hitched up or removed, exposing arms, legs, feet and torso. ${ }^{48}$ But when transposed to paintings of elite men, dishevelled clothing and bared bodies could figuratively

47 A medical illustration that depicts women's bound feet appears in Chen Shigong in Hu Xiaofeng (ed.) 1997, p. 397.

48 The famous example of semi-nude beggars is of course Zhou Chen 周臣, Beggars and Street Characters, 1516. See the reproductions in Cahill 1978, colour pl. 12 and figs 88 and 89. For farmers, see the images in the Pictures of Tilling and Weaving. 


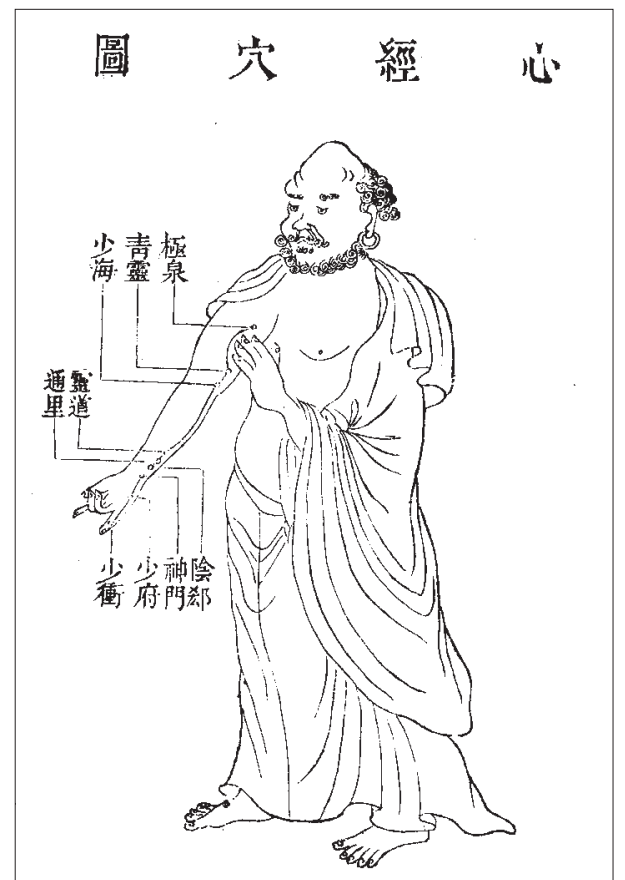

Figure 6.13 'Acupoints on the Heart channel', Golden Mirror, reproduced from Wu Qian (ed.) 1742, 82:04b. Chinese Collection, Harvard-Yenching Library. (C) President and Fellows of Harvard College

convey intellectual or creative iconoclasm and the upholding of personal integrity in defiance of social norms. ${ }^{49}$ Such visual elements were also associated with the cultural stereotype of the scholar-recluse who withdrew from official life, whether as a result of political persecution, disgust at official corruption, or refusal to serve a foreign dynasty. Retiring to a state of rural tranquillity and plain living in communion with nature, he would devote his days to spiritual and religious cultivation. This ideal appears, for example, in Du Jin's 杜堇 painting of 'Fu Sheng Transmitting the Classics' (Ming dynasty) which shows a well-known scene in the life of the man responsible for preserving Confucian teachings after the first Qin emperor's burning of books. Reciting the classics from memory to a visiting emissary, Fu Sheng 伏生 sits in his garden with his robe unloosed and falling from his shoulders, exposing his bony and gnarled chest. ${ }^{50}$

49 See, for example, Liu Guandao 劉貫道 (Yuan), 'Whiling away the summer', reproduced in Cahill 1976, pls 68 and 69. A scholar-recluse garbed as a bare-chested fisherman features in Qiu Ying 仇英, 'Fishing Boat by a Willow Bank', reproduced in Cahill 1978, figs 105 and 107 .

See the reproduction in Cahill 1978, colour pls 10 and 12. Another example of the bare-chested Fu Sheng is a painting attributed to Wang Wei 王維 (Tang dynasty). See Siren 1956, vol. 3, pl. 9 o.



Figure 6.14 Kapimala (Jia-pi-mo-luo), Wondrous Traces of Immortals and Buddhas, reproduced from Hong Yingming 1983, 5:10a

In philosophical and visual terms, these images of reclusive or iconoclastic scholars also evoked well-known portraits of male saints and religious exemplars, whose exposed bodies likewise exemplified unconventional yet enlightened behaviour. The Daoist immortal Zhongli Quan 鍾離權, for example, was conventionally depicted with a rotund, bare belly. Semi-naked Buddhist sages similarly constituted a stock visual convention, and the celebrated painters Shitao 石濤 (1642-1707) and Luo Pin 羅聘 (1733-99) were among the artists who used the exposed male torso as a visual device to construct a Buddhist persona for the lay subjects of their paintings. ${ }^{51}$

The Golden Mirror also quoted extensively from such religious imagery, including figures that circulated widely in printed collections such as the Liexian quanzhuan 列仙 全傳 (Complete Biographies of the Assembled Immortals, 16oo), conventionally attributed to Wang Shizhen 王世 貞, and Hong Yingming's 洪應明 Xian fo qi zong 仙佛奇 蹤 (Wondrous Traces of Immortals and Buddhas, 16o2). ${ }^{52}$ A good example is the Golden Mirror's illustration of the 'Acupoints of the Heart channel', which depicts a balding, bare-chested man with curly hair and beard (Fig. 13). This

\footnotetext{
$5^{1} \quad$ Vinograd 1992, p. 62, pp. 92-101.

$52 \quad$ For a description of how Liexian quanzhuan was falsely attributed to Wang Shizhen (1526-9o) after his death, see Carlitz 1991, p. 137 .
} 
resembled conventional images of foreign Buddhist monks, and is clearly related to the portrait of the 13th Buddhist patriarch Kapimala (Jia-pi-mo-lo 迦毗摩羅) from Hong Yingming's Wondrous Traces (Fig. 14). ${ }^{53}$ This infusion of religious imagery into medical illustrations is unsurprising given the long history of Buddhist and Daoist influence on Chinese medicine; indeed this particular image of the Heart channel had been circulating since at least the early 17th century. ${ }^{54}$ When the Golden Mirror borrowed images that originated in Buddhist and Daoist illustrations, it also adopted their inherent androcentrism. Here we should note that, although women were also celebrated for their religious devotion (most notably in special collections devoted to nuns and female transcendents), the most widely-circulating collections of holy figures were dominated by men, further reinforcing the associations between masculinity and enlightenment. For example, out of 107 figures in Wondrous Traces, only three were women, while the 197 Daoist immortals in Complete Biographies of the Assembled Immortals include only 20 women. Thus, despite the celebration of the 'female principle' in Daoism, the historical recognition of female Daoist practitioners, and the prominence of female deities in the Daoist pantheon, the generic face of the Daoist was still gendered as male. ${ }^{55}$

Besides evoking spiritual enlightenment, the exposed bodies of male Buddhist and Daoist adepts also visually symbolised the pursuit of immortality and mastery of the healing arts. Buddhism and Daoism promised an escape from physical suffering through spiritual enlightenment, and they also inspired a wide range of health preservation techniques and longevity practices, subsumed under the umbrella categories of nourishing life (yangsheng 養生), inner alchemy (neidan 內丹), manipulation of Qi (qigong 氣功), and martial arts. Visual depictions of such techniques conventionally depicted bare-torsoed or partially disrobed male practitioners, images that are clearly echoed in the Golden Mirror's images of semi-naked men clad only in flowing skirts or short trousers. ${ }^{56}$ Since these body practices had historically overlapped with and influenced the content of classical medicine, it is unsurprising that images from these traditions would likewise percolate into medical works. Furthermore, the visual affinities between

53 Hong Yingming 1983, 5:10a.

54 For an overview of the influence of Buddhism and Daoism on Chinese medicine, see Unschuld 1986.

55 The status of female figures in Daoism and the history of Daoist practices aimed at women is notably explored in Despeux 1990; Despeux and Kohn 2003, and Cahill 2005.

56 A standard depiction of shirtless martial artists appears in Wang Qi 1970, 'Renshi' 人事 (section on human activities), 7:9a-1ob. exposed male bodies and self-cultivation practices would have positively encouraged the use of these kinds of male figures as normative medical bodies.

\section{Auspicious Imagery and the Enlightened Male Doctor}

So far, I have argued that the Golden Mirror's visual androcentrism replicated that of Chinese culture at large and that the positive qualities associated with the exposed male body further reinforced this tendency. A third factor that made male figures more useful than female ones was that they served as a vehicle for affirming the male physician's ability and the superiority of male medical knowledge. The Golden Mirror's images were not simply male. They were male figures who were regularly portrayed with auspicious symbols, particularly those associated with Daoist immortals. The overall effect, I contend, was to liken the doctor implicitly to a Daoist sage, thus testifying to his superior mastery of the cosmic dynamics that underlay the course of disease and cure. In the context of vernacular medical practice, this appealed to beliefs in miraculous cures and remedies transmitted by transcendent beings. But it also embodied the ideals of elite scholarly doctors, who taught that one could neither diagnose nor cure without a thorough mastery of Yin and Yang.

\section{Auspicious Elements}

Of the Golden Mirror's 484 illustrations, just under 30\% (141 images) include props, background objects, and decorative motifs that conventionally carried auspicious symbolic meanings in 18th-century visual culture. I will show further below that these elements constituted a distinctive feature of the Golden Mirror's iconographic style. First, however, it will be helpful to review what these auspicious elements were. In some cases, the auspicious symbolism comes from visual puns. For example, an illustration identifying the bones of the arm shows a man with a bat ( $f u$ 蝠), a conventional visual homonym for 'good fortune' ( $f u$ 福). ${ }^{57}$ Such significant puns are a salient feature in the pox illustrations, which as we have seen were derived from the 'hundred boys' genre of auspicious imagery. Thus we find a pox-covered boy playing with a stone chime (qing 磬), the name of which was a homonym for 'celebrate' (qing

57

Wu Qian (ed.) 1742, 89:17a. There are numerous studies of these kinds of puns occurring in a wide range of material media. See, for example, Cort and Stuart 1993; Lust 1996; Cammann 20o1; Tsang 2002. 
慶). ${ }^{58}$ A boy with a toy shaped like a fish ( $y u$ 魚) evoked 'abundance' ( $y u$ 餘), while a boy with a vase (ping 瓶) suggested 'harmony' or 'peace' (ping 平)..$^{59}$ Besides visual puns, several children also carry or are shown with plants and flowers - pines, peonies, lotuses, and the like - that symbolised hoped-for attributes like long life, wealth and the birth of sons. These kinds of visual rebuses were a major motif in Qing decorative art, flourishing particularly during the reign of the Qianlong emperor, and ceramics, textiles, prints and paintings were but a few of the media that contained visually encoded wishes for prosperity, official success, a healthy old age, and the birth of many talented descendants. ${ }^{60}$

Besides objects whose names lent themselves to lucky puns, Qing visual culture included a repertoire of other objects whose auspicious meanings arose from their associations with Daoism, and in particular the Daoist pursuit of immortality. These were objects that conventionally appeared in images of Daoist immortals or adepts, and they were also integrated into portraits of living people and historical worthies to evoke the ideals and practices of Daoism. A famous example of this latter dynamic is a portrait of the Emperor Yongzheng (r. 1723-35) garbed as a Daoist magus, identified as such by a flywhisk in one hand and a pearl (or elixir of immortality) in the other. ${ }^{61}$ Yongzheng's son and successor, the Qianlong emperor (r. 1736-95), also had himself painted as a Daoist priest. ${ }^{62}$ In that image, Qianlong holds a lingzhi 靈芝 (fungus of immortality) in his right hand, while his left rests on the back of a white deer, both of these life-forms being attributes of the immortals. His boy servant carries a hoe and a basket of plants which signal that they have been herb-gathering, another stereotypical activity of Daoist worthies. ${ }^{63} \mathrm{Wu}$ Hung reads these portraits of Yongzheng and Qianlong as statements of political legitimacy, affirming inter alia their ownership and embodiment of indigenous Chinese

$5^{8} \quad$ Wu Qian (ed.) 1742, 57:52a.

59 Wu Qian (ed.) 1742, 57:37a and 57:56a

6o In addition to the works cited earlier, see Bartholomew in Wicks (ed.) 2002 .

61 This Daoist incarnation of Yongzheng appeared in a series of 'masquerade portraits' that also showed him in 14 guises ranging from Bodhisattva to European gentleman (complete with wig). The entire set of masquerade portraits appears in Wu Hung 1995 .

For a reproduction of this image, see Chou and Brown 1985, p. 29 o.

63 An earlier, famous example of this visual convention appears in a self-portrait by Chen Hongshou 陳洪綬 (1598-1652), where the role of boy servant is taken by his nephew; Vinograd 1992, pl. 8. culture and thus their right to rule the empire. ${ }^{64}$ More generally, however, Daoist-tinged portraits of artists, scholars or officials conventionally connoted the moral virtues associated with self-cultivation, closeness to nature, or disinterest in worldly affairs. Indeed, this visual topos was so commonly known that portrait painters could play with it for artistic or ironic effect. ${ }^{65}$

\section{Daoist Figures in the Golden Mirror}

Like the Qing emperors, many of the Golden Mirror's figures also masquerade as Daoist sages. For example, one figure holds a yugu 魚鼓, a percussion instrument made of bamboo and snakeskin. ${ }^{66}$ In the iconography of the Daoist Eight Immortals, the yugu was the attribute of Zhang Guolao 張果老 and images of the yugu and other immortals' attributes were recognised motifs in Qing decorative art. ${ }^{67}$ Another figure wears a loincloth made of leaves, a typical garment for Daoist immortals and adepts. ${ }^{68}$ Yet another figure sports a crown exactly like those often worn by Daoist divinities. ${ }^{69}$ Other figures in

\section{Wu Hung 1995.}

65 Richard Vinograd makes this point in his analysis of Chen Hongshou's self-portrait, 'A Tall Pine and Taoist Immortal' ('The Artist and His Nephew in a Landscape'), 1635. Here Chen subverts the conventional imagery by turning his back on the natural scene which surrounds him. Instead of celebrating 'the Taoist ideals of simplicity and modest ambition' referred to in the painting's inscription, Chen uses his Daoist self-portrait as a stage upon which to express a sense of alienation and unease; Vinograd 1992, pp. 32-3.

66 'Niucheng jian tu' 牛程蹇圖 (diagram of cow pattern lameness), Wu Qian (ed.) 1742, 71:46a.

67 The iconography of the eight immortals appears to have been fluid at the beginning of the Qing. The Liexian quanzhuan attributed to Wang Shizhen depicts the yugu in the hands of Han Xiangzi 韓湘子, while two years later, Hong Yingming's Xianfo qizong showed it in the arms of both Han Xiangzi and Zhang Guolao: Wang Shizhen (attrib.) 1961, 6:14b; Hong Yingming 1983, 1:18a and 2:16a). Ultimately the standard emblems were fixed as follows: Lü Dongbin 呂洞賓, a sword; He Xian'gu 何仙姑, a lotus; Lan Caihe 藍采和, a flower basket; Zhang Guolao, a yugu; Li Tieguai 李鐵拐, a gourd; Han Xiangzi, a flute; Cao Guojiu 曹 國舅, castanets; Zhongli Quan, a fan. See Williams 1960. A particularly impressive example of their use in Qing decorative art is a 19th-century imperial dragon robe in the collection of the Victoria and Albert Museum, London. See Wilson 1996, p. 18.

68 'Renmai xue tu' 任脈穴圖 (points on the controller vessel), Wu Qian (ed.) 1742, 84:04a.

69 Wu Qian (ed.) 1742, 88:11a. There are several other figures with similar crowns, but this is the only one where the crown includes the distinctive finial that rises up from the centre and curves forward. Many examples of this kind of crown in Daoist art can be found in Little and Eichman 200o, including the mag- 
the Golden Mirror imitate well-known portraits of Daoist immortals by holding or wearing bottle gourds (hulu 葫 蘆), a traditional receptacle for medicines that here also suggests the possession of divine remedies or elixirs of immortality. ${ }^{70}$

Among the most salient Daoist elements, however, is the lingzhi, the fungus of immortality, which appears in the hands of several figures (Fig. 7). This motif also appears in the lingzhi-shaped swirls of vapour that encircle or appear at the base of isolated body parts, particularly in diagrams of circulation channels (Fig. 3). In Chinese visual culture, such clouds were conventional elements used to denote the lands or dwellings of the Daoist immortals; similarly, the immortals could ride on or be surrounded by such clouds. ${ }^{71}$ When added to medical images, lingzhi-shaped clouds thus evoked the continued presence of an irresistible, ever-present life-force, a force inherent in the unseen body to which the depicted part is implicitly connected. The lingzhi is also suggested in figures that hold a ruyi 如 意 sceptre. The name of this object literally means 'as you wish', and in late imperial times ornate and luxurious ruyi could constitute cherished gifts, art objects, and part of an upper-class gentleman's accoutrements. By this time too, the heads of ruyi sceptres were commonly shaped like lingzhi. Besides these general auspicious connotations, however, in visual culture, the ruyi had been an attribute of Buddhist and Daoist deities since about the 1oth century. ${ }^{72}$ In particular, the ruyi appeared in visual representations of the Daoist Celestial Worthies, the triumvirate of supreme deities who rule over the realms of Jade Purity, Highest Purity and Great Purity. Conventionally, such portraits showed each of the Worthies holding a different attribute, one of which was always a ruyi (although which one is holding it seems to vary according to historical period). ${ }^{73}$

nificent cover illustration.

70 See, for example, Wu Qian (ed.) 1742, 81:02 and 89:17a. Among other things, the gourd was one of the symbols of Li Tieguai, 'who holds it in his hand while spirals of smoke ascend from it, denoting his power of setting his spirit free from his body'; Williams 1960, p. 215.

71 Laing 1998. This motif also recurs throughout the woodblock images in Wang Shizhen (attrib.) 1961 and Hong Yingming 1983.

72 In Buddhist art of the late 5 th -9 th centuries, pictorial depictions of Manjusri's famous debate with Vimalakīrti always showed the former holding a wand while the latter wielded a fan. Beginning in 1oth-century art, Manjusri's wand was visually transformed into the ruyi, and it 'became an attribute of other divinities as well'; Davidson 195 .

For variations in iconography, see Little and Eichman 2000, cat. nos 66 and 73. Cat. no. 66 has the ruyi in the hands of the Celestial Worthy of Numinous Treasure (Lingbao Tianzun 靈寶天
Portraits of Daoist immortals could show them holding ruyi, and the crowns of Daoist deities were topped with a finial shaped like a lingzhi-headed ruyi. ${ }^{74}$

In addition to images playing on the similarities between ruyi and lingzhi, the Golden Mirror also includes images playing on the iconographic continuum of fan and flywhisk. Like the ruyi, the fan could be an art object, luxury gift, and elegant accessory of the upper classes. The word for 'fan' (shanzi 扇子) was a close homonym for 'scattering sons' (san zi 散子), and thus the fan symbolised marital fecundity. The fan was also a common gift object upon which people often drew auspicious images and rebuses, thus serving as a standard vehicle for conveying good wishes from one person to another. ${ }^{75}$ At the same time, the fan was also a stock accessory for Daoist deities. One of the three Celestial Worthies, for example, was conventionally depicted holding a tasselled or fringed fan (more on this variant below), and the fan also became the standard visual emblem of the Daoist Immortal Zhongli Quan, who used it to restore life to the dead. ${ }^{76}$ The fans that appear in the Golden Mirror also include ones made of feathers that echoed fans appearing in popular images of Daoist sages. These included the one carried by Wei Boyang 魏伯陽, the legendary 3rd-century compounder of elixirs of immortality, and author of a canonical text on inner alchemy. ${ }^{77}$ The white-feathered fan was also the symbol of Zhuge Liang 諸 葛亮, the wily strategist and master of the Way whose deeds are celebrated in Sanguoyanyi 三國演義 (Romance of the Three Kingdoms), the famous 15th-century historical novel recounting the power struggles that followed the fall of the Han dynasty. ${ }^{78}$ Paintings of Daoist deities also routinely

尊), while cat. no. 73 has it in the hands of the Celestial Worthy of Primordial Being (Yuanshi Tianzun 元始天尊). See also the portrait of Song Lun 宋倫 in Wang Shizhen (attrib.) 1961, 2:8a. A typical example from painting is Wu Wei 吳 偉 (1459-1508), 'The Perfected Man of the Northern Sea'. As described by Richard M. Barnhart, 'The immortal, in windswept robes and trailing ribbons, holding a staff in the shape of the lingzhi fungus of immortality, sits serenely on the back of a giant tortoise, which sails the surface of the sea'; Barnhart in Fong and Watt (eds) 1996, p. 365. A comprehensive survey of the many social uses of fans and their symbolism is Tsang 2002.

76 For Zhongli Quan, see Williams 196o, pp. 150-1,174. For a depiction of the three Celestial Worthies and fans, see Little and Eichman 200o, cat. no. 67 .

77 Wang Shizhen (attrib.) 1961, 3:2b (text) and 3:12b (image).

78 Zhuge Liang was so closely associated with the white feather fan that scholars have found it worthwhile to refute this connection as a myth. Ka Bo Tsang points out that Zhuge Liang's accessory was actually a maoshan (literally, 'hair fan'), another name for the zhuwei (a cross between a flywhisk and an oblong stiff fan 
depict people bearing elongated feathered fans, echoing the wings of cranes on which the deities rode through the heaven s. ${ }^{79}$ Some figures in the Golden Mirror also hold objects that resemble fans with a fringe or tassel attached to the far end. These were zhuwei 塵尾 ('tail of the zhu deer'), sometimes also called maoshan 毛扇 (hairy fans). ${ }^{80}$ Visual depictions of zhuwei frequently blurred into the flywhisk, and scholars previously thought that the Daoist whisk was actually borrowed from Buddhist iconography. Liu Yang has shown, however, that the zhuwei was a historically distinct object indigenous to China. Originating in Han dynasty funerary art, which conventionally expressed desires for immortality, the zhuwei had become a standard attribute in visual representations of Daoist divinities by the 4th century CE. As in the case of the ruyi, we also find an array of hairy fans-cum-whisks in depictions of the Daoist Celestial Worthies. ${ }^{81}$ Woodblock-printed portraits of Daoist immortals also routinely show them in the act of flicking or swinging a whisk, its waving hairs evoking the transformative energy of the cosmos. ${ }^{82}$

\section{Daoist Elements as a Distinctive Iconographic Style} The Golden Mirror was not the first medical text to employ Daoist imagery. In fact, some of its Daoist-tinged figures are actually copied from or modelled after images of circulation channels that appeared in Zhang Jiebin's Leijing tuyi 類經 圖翼 (Illustrated Appendix to the [Yellow Emperor's Inner] Classic Arranged by Topic, 1624). ${ }^{83}$ But the Golden Mirror's use of auspicious and Daoist symbols was far richer and more extensive than in earlier medical works, so much so

made with the tail hair of the elk); Tsang 2002, p. 16. The association of Zhuge Liang with the feathered fan endures, however, and when I visited Tianjin in August 2005, vendors were selling white feather fans under the trade name of 'Kongming fans' (Kongming being Zhuge Liang's courtesy name).

There are several examples of this in Little and Eichman 2000, including the image on the back cover. A common representation of the Queen Mother of the West also showed her attended by a servant with a long-handled, feathered fan in the shape of a crane's wing.

Tsang 2002, p. 16.

Liu Yang 2001.

A particularly fine example is the portrait of Meng Qin 孟欽 in Liexian quanzhuan. His whisk depicted in mid-flick, Meng has just summoned a mass of lingzhi-shaped clouds that will whirl him away from the banquet hall where his enemies are about to seize him; Wang Shizhen (attrib.) 1961, 5:7b.

83 Among the 14 figures that Zhang Jiebin used to depict different circulation channels, there are five that include Daoist elements similar to those appearing in the Golden Mirror, including one figure wearing a leaf loincloth and one holding a feathered fan; Zhang Jiebin 1965a, pp. 108-27. that it constituted a distinct style of medical illustration. As I discussed earlier, the Golden Mirror created a new genre of pox pictures based on auspicious images of boys at play. The way in which the Golden Mirror incorporated auspicious imagery is also exemplified by the 265 images that illustrated its chapters on 'external diseases' (waike 外科). Within the Chinese medical tradition, works on external diseases provide some of the largest ensembles of illustrations, used to depict the position and general appearance of lesions, lumps, ulcers, rashes and the like. For example, Shen Gongchen's Dispelling the Mysteries of External Ailments of 1604 includes 194 pictures of skin diseases and various injuries, ranging from skin sores to tiger bites. Another well-known text was Chen Shigong's Correct Teachings on External Ailments of 1617 , which presents 36 illustrations of external ailments in adults and children. This genre of illustration was so standard that we also find a set of 36 external ailment illustrations collected in the pictorial encyclopaedia, Assembled Diagrams of the Three Powers of 16og. Unlike the Golden Mirror, however, none of these earlier works employ auspicious or Daoist visual elements in any appreciable way. When we compare the Golden Mirror's illustrations to those in these earlier works, we find that the Golden Mirror essentially took the well-established genre of external ailment illustration and added Daoist and auspicious imagery to it.

A comparison of illustrations of 'leaking eye ulcer' (lou jing chuang 漏睛瘡) from the Assembled Diagrams and the Golden Mirror provides a good example of this dynamic. The earlier figure from the Assembled Diagrams is empty-handed, whereas the one in the Golden Mirror cradles a handsome ruyi. ${ }^{84}$ Similar differences can be seen between analogous images of ankle abscesses from Correct Teachings on External Ailments and the Golden Mirror. The figure in the former is empty-handed, while the figure from the Golden Mirror clutches a large lingzhi fungus. ${ }^{85}$ Only Shen Gongchen's Dispelling the Mysteries of External Ailments seems to be a possible precursor of the Golden Mirror's use of Daoist imagery in external desease illustrations. Many of the 194 illustrations in Dispelling the Mysteries include props, among them a child holding a ruyi and another one wielding a lotus leaf. One figure with a crutch evokes Li Tieguai while another with a flywhisk and a large belly

\footnotetext{
84 Wu Qian (ed.) 1742, 65:13a.

85 Here I am comparing the Golden Mirror's image of 'abscesses on the interior and exterior surfaces of the ankle' to a figure from Chen Shigong that is marked up with several ailments, including 'penetrating abscess on the ankle'. See Chen Shigong in $\mathrm{Hu}$ Xiaofeng (ed.) 1997, p. 395, and Wu Qian (ed.) 1742, 71:9b.
} 
suggests Zhongli Quan. ${ }^{86}$ In general, however, most of the props and background objects in Dispelling the Mysteries are related in some way to the cause of the illness at hand. Take, for example, the figure that Dispelling the Mysteries uses to illustrate qusou chuang 蠖蠀瘡 (qusou ulcers), a condition that arises when the qusou insect 'urinates' on a person's body. This disease description is accompanied by an image of a man with a bare torso, carrying a fan, and standing under what appears to be a round spider's web. ${ }^{87}$ However, the fan does not contain any obvious message besides the fact that the weather is hot, one is walking about in a state of semi-undress, and the danger of insect urine falling on one's bare skin is thus correspondingly higher. The result is that the intermittent appearance of religious and auspicious imagery in Dispelling the Mysteries does not constitute a sustained visual style in the way that such motifs do in the Golden Mirror.

The distinctiveness of the Golden Mirror's iconography is also evident when we compare it to other 18th-century works. Shen Jin'ao's 沈金鳌 compendium Shenshizunsheng shu 沈氏尊生書 (Master Shen's Book on Revering Life, 1774) was compiled some three decades after the Golden Mirror but does not use symbolic imagery in any of its 32 external medicine illustrations. ${ }^{88}$ This suggests that the Golden Mirror's use of Daoist icons represented a deliberate choice, rather than simply reflecting some change in visual fashion over time. The Golden Mirror's images of the body's circulation channels also provide evidence that certain types of images were preferred over others. Images of the channels are among the most common of all Chinese medical illustrations, and the Golden Mirror includes 55 such illustrations appearing in six different juan. ${ }^{89}$ Eighteen of these images contain auspicious objects, such as ruyi, lingzhi, whisks, fans and medicine gourds. By contrast, none of the analogous images of bodily channels from the Imperial Encyclopaedia, published in 1726-8, includes any such motifs. This contrast is particularly significant in the case of the dumai 督脈 (Governor Vessel) and renmai 任脈 (Controller Vessel), two of the qijing ba $m a i$ 奇經八脈 (Eight Singular [or Extraordinary] Vessels). The Governor Vessel ran along the spine and into the brain and was responsible for governing all the Yang channels

\footnotetext{
86 Shen Gongchen in Hu Xiaofeng (ed.) 1997, pp. 311, 315.

87 Ibid., p. 330.

88 Shen Jin'ao 1999, pp. 473-81. Although this reprint has 33 images of external ailments, two of them are identical images used to illustrate two different diseases, so I count these as the same image.

89 These are distributed in Wu Qian (ed.) 1742, among juan 17, 61, and $81-4$.
}

of the body. The Conception Vessel ran up the centre of the front of the body, and was responsible for governing all the Yin vessels of the body. ${ }^{90}$ Thus, although all Eight Singular Vessels were important in principle, the Governor and Controller Vessels attracted the most attention, and images of these two vessels appeared more frequently than the other six. Comparing analogous pictures of these key vessels from the Golden Mirror and the Imperial Encyclopaedia, we find that the Golden Mirror included auspicious imagery while the Imperial Encyclopaedia did not. Thus, the Golden Mirror's figure of the Governor Vessel holds a flywhisk while its three illustrations of the Controller Vessel consist of one figure wearing a leaf apron, one holding a flywhisk and one holding a ruyi. ${ }^{91}$ None of these elements appear in the ImperialEncyclopaedia. As I will argue below, one reason the Golden Mirror may have favoured the use of Daoist iconography was because this imagery could readily symbolise the inherent superiority of the properly-educated male doctor.

\section{The Masculine Meanings of Auspiciousness}

In 18th-century China, material objects of all sorts incorporated lucky symbols and visual rebuses, including images of Buddhist deities and Daoist immortals. ${ }^{92}$ At the most general level, therefore, the Golden Mirror's use of ruyi, lingzhi and the like transformed the medical illustration itself into an auspicious talisman, embodying wishes for the sufferer's quick recovery and a long and healthy life. But the Golden Mirror's auspicious images were also constructed and exchanged in an environment defined by male priorities and aspirations. The Qianlong emperor was a well-known enthusiast of visual rebuses and puns, and it is reasonable to suppose that the Golden Mirror's use of auspicious symbols was at least in part a nod to the imperial patron. ${ }^{93}$ Since Qianlong saw the Golden Mirror as a tool for building moral capital and political authority, bestowing auspicious images of health on his subjects could

\footnotetext{
$90 \quad$ For a description, see Zhang Jiebin 1965a, p. 128.

91 See Wu Qian (ed.) 1742, 61:23b, 61:24b, 84:2a, and 84:4a.

92 Bartholomew in Wicks (ed.) 2002

93 Bartholomew notes: 'The Qianlong period (1736-95) was a time when the use of rebuses or pictorial puns became widespread. Many new ones were created during this time and came into common usages in the 19th and 2oth centuries... . The sudden flowering of rebuses during the Qianlong period may be attributed to the emperor himself, who delighted in the use of rebuses. One of his personal seals was a pun on his name and consisted of a circle enclosing the trigram qian, comprised of three solid horizontal lines. He often used this seal as a substitute for the character qian in his own name'. Bartholomew in Wicks (ed.) 2002, p. 75 .
} 
also reaffirm his benevolence as a ruler. At the same time, however, medical illustrations with Daoist overtones helped to affirm the efficacy of the male physicians who deployed the Golden Mirror in practice. At a general level, the use of such imagery evoked a well-known association between the Daoist sage and the bestowal of good health. Historical, medical and literary sources all attest to a long-standing belief that Daoist immortals and other divinities could reveal magical 'secret remedies' to deserving filial sons and other paragons of virtue. ${ }^{94}$ The Daoist motifs in the Golden Mirror's illustrations thus function as the visual equivalent of stock phrases that medical works had long used to describe the marvellous efficacy of a remedy or practitioner: shen $y i$ 神醫 (a divine doctor), shen xiao 神 效 (divinely efficacious), and xian chuan 仙傳 (transmitted by immortals). These auspicious connotations were probably most welcome to male physicians working in the often fraught context of the imperial medical service. As Chang Che-chia shows, the imperial physician's authority was constantly challenged both by his royal patients and by physicians summoned from outside the court to attend particularly difficult cases. An apparently bad diagnosis or failed treatment might bring a charge of treason, never mind malpractice. ${ }^{95}$ In this context, medical images with herbs of immortality, wish-granting sceptres and the like served to enrobe the government doctor in a cloud of good fortune and therapeutic success.

More specifically, the Daoist immortal served as a useful metaphor for the male physician's erudite skills. A particularly striking example of this dynamic appears on the title page to a 1579 woodblock edition of Mingyi zhizhang tu 明醫指掌圖 (The Enlightened Doctors'Guide to MedicalMastery). ${ }^{96}$ The text was first compiled in 1556 by Huang Fuzhong 皇甫中, member of a medical family, and was subsequently revised by the eminent scholar-doctor Wang Kentang 王肯堂 (1549-1613). The top of the page is occupied by a tableau of three figures. The left-hand figure, who is accompanied by a tiger, is the famous 7 th-century physician Sun Simiao 孫思曖, author of the Beiji qianjin yaofang 備急千金要方 (Prescriptions Worth a Thousand Gold Pieces). Later canonised as a god of medicine, Sun was also revered as a Daoist immortal. ${ }^{97}$ Next to him is the legendary Daoist Wei Boyang, accompanied by his

94 For a general discussion of immortals, their role in revealing 'secret formulas' and religious healing, see Wu 2000.

$95 \quad$ Chang Che-chia 1998.

$96 \quad$ Huang Fuzhong and Wang Kentang 1579.

97 For the tiger as emblem of Sun Simiao, see Unschuld 2000, pp. 208-21. For Sun as an immortal, see his biography in Hong Yingming 1983, 3:7a, and Wang Shizhen (attrib.) 1961, 5:13b. faithful dog whom he once pretended to poison with an elixir of immortality in order to test the faith of his disciples. $^{98}$ (The third person is a servant.) The appearance of Sun Simiao and Wei Boyang on the title page of this medical text visually symbolises the conflation between medicine and Daoist immortality practices. This conflation is further reinforced by the two lines of text that appear vertically one on each side of the tableau. The phrase on the right reads 'Compiling the methods of the worthies to save people's lives and benefit the world' (ji xianfang huoren jishi 輯賢方活人濟世), while the one on the left says 'Observing the methods of the sages to raise the dead and return them to life' (zun shengfa qisi huisheng 遵聖法 起死迴生). The former phrase describes a typical activity of scholarly, male medical experts, namely collecting useful medical information gleaned from textual study with the aim of benefiting humanity, while the latter phrase alludes to the famous story of Wei Boyang restoring both his dog and a dead disciple to life with an elixir of immortality. Juxtaposed in this way, the images and text together affirm that Daoist mastery of the cosmos and the 'enlightened' doctor's mastery of medicine are one and the same.

These close associations between Daoism and medical knowledge would also have framed the way in which contemporary readers interpreted the Golden Mirror's images. By pairing its male figures with Daoist imagery, the Golden Mirror essentially turned its medical illustrations into a visual metaphor that equated the male doctor's healing abilities with the transcendent powers of the Daoist immortal. During the late imperial period, fur thermore, the Daoist metaphor of medical efficacy also resonated with the central tenet of learned medicine: that scholarly mastery of cosmological patterns was the essential prerequisite for effective diagnosis and cure. Such messages, not surprisingly, were most effectively embodied by male figures.

\section{Male Medicine and Scholarly Cosmology}

As is well-known, the ideal of the male physician as cosmological expert emerged during the Song dynasty (96o-1279), the product of efforts to recast medicine as a scholarly art and moral calling. ${ }^{99}$ As the Song dynasty statesman Fan Zhongyan 范仲淹 (989-1052) famously remarked, 'if you cannot be a good minister, then become a good doctor',

98 The story of Wei Boyang and his dog was first recorded in the 4th century. For details and an account of the early filiation of this story, see Akahori Akira 1989. A widely circulating late imperial portrait of Wei and his dog is found in Wang Shizhen (attrib.) 1961, 3:12b.

99 See Goldschmidt 1999 and Chen Yuan-peng 1996 for Song dynasty transformations of medicine. 
thus portraying medicine as a worthy alternative career to officialdom. ${ }^{100}$ The Neo-Confucian 'study of the Way' (daoxue 道學) also became central to new doctrines of health and disease over the course of the Song, exemplified in the maturation of a complex medical cosmology, known as the doctrine of 'five circulatory phases and six climatic influences' (wuyun liuqi 五運六氣). ${ }^{101}$ As synthesised by Zhu Xi 朱喜(1130-1200), Neo-Confucianism called for a systematic inquiry into $l i$ 理, the patterned principles that governed all material existence and transformation in the cosmos. Scholars claimed that the ability to discern principles and to align oneself properly with the cosmic order was essential for individual morality and social harmony. Educated doctors also increasingly argued that such insights were essential for the prevention and cure of illness and they pointed out that such knowledge could be obtained only through rigorous study of properly vetted classical texts, authored by former medical sages who themselves had correctly discerned the patterns of Yin and Yang. ${ }^{102}$ From the Song to the Qing, therefore, the thrust of medical innovation centred on an ever-closer alliance between male, scholarly culture and medicine, and literate doctors developed ever more finely differentiated explanations of how the interactions of Yin and Yang produced disease and cure. Particularly influential were the doctrines of Zhu Zhenheng 朱震亨 (1281-1358), who taught that Yin tended to be in deficiency while Yang tended to be in excess. As Furth shows, Zhu Zhenheng articulated his innovative doctrines on bodily fire by interpreting the Yellow Emperor's Inner Classic through the lens of Zhou Dunyi's 周敦頣 (1017-73) interpretation of the Classic of Changes. ${ }^{103}$ The result was a 'medical body ... aligned with distinctively Neo-Confucian constructions of cosmological and moral order'. ${ }^{104}$

More research will be required to determine exactly how these kinds of doctrinal changes influenced medical illustration. However, the 17 th-century work of Zhang Jiebin suggests that the use of Daoist imagery was in fact linked to a conscious attempt to ally medicine more closely with cosmological study. A central tenet of Zhang's medical philosophy was that 'medicine and the Classic of Changes

100 For Fan Zhongyan's role in reforming government medical schools, see Goldschmidt 1999, pp. 209-12.

101 Despeux in Hsu (ed.) 2001.

102 An excellent overview of these historical developments is Scheid 2007, ch. 2. Also noteworthy is the way in which learned physicians attacked the longstanding idea that a family tradition of medical practice was an essential qualification to be a good doctor; Chao Yuan-ling 2000.

103 Furth 2006.

104 Furth 1999, p. 150. share the same origin' (yiyitongyuan 醫易同源). ${ }^{105}$ The 64 hexagrams of the Changes symbolically encapsulated all the possible patterns of Yin and Yang transformation that animated the universe. By extension, then, the essence of medicine was the ability to properly discern the workings of Yin and Yang in the human body.

In sum, understanding the human body and understanding the cosmos were a single, indivisible enterprise. Thus Zhang argued that the Classic of Changes was an essential text for all doctors, for although the Yellow Emperor's Inner Classic discussed the properties of Yin and Yang, 'there is no account of their transformations that can surpass the Changes'. ${ }^{106}$ Alongside these textual descriptions, Zhang Jiebin's works also include medical figures marked with Daoist iconography. In Zhang's Illustrated Appendix, five out of 14 figures used to depict individual circulation channels include elements such as animal pelt clothing, ruyi, feathered fans and whisks. ${ }^{107}$ As mentioned earlier, several of these images were adapted or copied by the Golden Mirror. Further research is required to determine whether such images originated with Zhang Jiebin or whether he was copying images from an earlier source. For the present, it is sufficient to note that Zhang's work allied these images to a doctrine promoting deeper medical knowledge of Yin and Yang. They thus also provide an important baseline against which to interpret the significance of the Golden Mirror's use of Daoist imagery.

For the mid-18th-century compilers of the Golden Mirror, as well as for Zhang Jiebin, mastery of cosmic transformations was the key attribute that distinguished the skilled doctor from the medical hack. Grand Secretary Ortai 鄂爾 泰, who oversaw the Golden Mirror's compilation, notably used these intersections between healing, rulership and cosmological knowledge to explain the historical significance of the Golden Mirror's compilation. In a memorial to the throne, later included in the prefatory chapter of the Golden Mirror, Ortai wrote: '

The doctors of antiquity had a thorough understanding of the regulations of Heaven and they investigated the principles of Earth, regulating their circulation and harmonising their transformations. As a result, all that was above and below was united in virtue..$^{108}$

But the transmission of medical teachings had become corrupted and irregular in subsequent ages, Ortai warned,

\footnotetext{
$105 \quad$ Zhang Jiebin 1965b, 391.

106 Zhang Jiebin laid out this argument in an essay entitled 'The Meaning of the Medical Changes' (yi yiyi 醫易義); Zhang Jiebin $1965 \mathrm{~b}, \mathrm{p} .39 \mathrm{o}$.

107 Zhang Jiebin 1965a, pp. 108-27.

108 Wu Qian (ed.) 1742, 1:1b-2a.
} 
and thus a rectification of knowledge was required. In its attempt to define correct medical practice for 18th-century doctors, the Golden Mirror also employed auspicious images of sagely men. It was not that doctors necessarily wanted to be seen as Daoists per se, but rather that Daoist images provided a handy visual shorthand for evoking the male doctor's virtuosic insights into cosmic dynamics.

\section{Conclusion}

Chinese classical medicine was historically shaped by masculine aspirations and institutions, epitomised by the government-sponsored publishing projects of the Song dynasty that created the standard versions of canonical medical texts and by male efforts to raise the status of medicine by portraying it as an extension of Neo-Confucian scholarly erudition, moral cultivation and public service. ${ }^{109}$ By the late Ming and early Qing, medicine had become a routine alternative occupation for men who could not advance in the civil service examinations, and educated physicians routinely claimed that their scholarly background made them superior to other practitioners. This alliance between masculine epistemologies and medical learning is particularly salient in the Golden Mirror, where the norms of evidential scholarship were now applied to assessing the legitimacy of ancient medical teachings. Given the androcentric history of medical learning, it is perhaps not surprising that the Golden Mirror's illustrations favour men. But the visual androcentrism of the Golden Mirror was also positively nurtured by a convergence of factors from both within and outside medicine. To facilitate the training of government doctors who studied the Golden Mirror as a guide to proper care, the text's compilers endowed it with a rich array of heuristic images. To generate the needed images, the Golden Mirror borrowed illustrations from a wide range of medical and non-medical sources, and in so doing, it also perpetuated the preference for male figures that characterised Chinese visual culture at large.

At the same time, the active visual associations between semi-naked male bodies and ideals of spiritual enlightenment and physical robustness meant that male figures were particularly ideal for representing the normative medical body. These implied links between male enlightenment and health were additionally reinforced by the use of auspicious and religious symbols that turned this impe-

109 For the role of the Song Imperial Editorial Office in compiling the received version of the Huangdi neijing suwen (Yellow Emperor's Inner Classic, Basic Questions), see Unschuld 2003, ch. 4. For other works, see Goldschmidt 1999. rial medical text into an icon of good fortune while also attesting to the expert skill of the doctors who deployed it. But while the Daoist adept harnessed Yin and Yang for his own immortality, the educated male physician would use his cosmological insights to prevail over the unruly transformations of illness, harmonising bodies as the skilled ruler harmonised all under Heaven.

\section{Bibliography}

\section{Primary Sources}

Chen Menglei 陳夢雷 et al., (comp.) 1986 [1726-8], Qinding gujin tushu jicheng 欽定古今圖書集成 (Imperially-approved Compendium of Ancient and Modern Texts and Illustrations, also referred to as 'The Imperial Encyclopaedia'), Beijing: Neifu; Reprinted Chengdu: Zhonghua shuju and Bashu shushe

Chen Shigong, 陳實功 1997 [1617], Waike zhengzong 外科正宗 (Correct Teachings on External Diseases), reprinted in Hu Xiaofeng 胡曉峰 (ed.), Zhongyi waike shangke mingzhu jicheng 中醫外科傷科名著 集成, Beijing: Huaxia chubanshe.

Chen Ziming 1992 [1237], Furen daquan liangfang (Comprehensive Compendium of Good Formulas for Women), Beijing: Renmin weisheng chubanshe.

Hong Yingming 洪應明 1983 [1602], Xian fo qi zong 仙佛奇路 (The Wondrous Traces of Immortals and Buddhas), in Haichu Daoren zushu erzhong 還初道人著書二種, Taipei: Guangwen shuju.

Huang Fuzhong 皇甫中 (author) and Wang Kentang 王肯堂 (ed.) 1579, Mingyizhizhang tu 明醫指掌圖 (The Enlightened Doctors' Guide to Medical Mastery), Anzheng tang woodblock edn in the library collection of the Peking Union Medical College.

Jin Guliang 金古良 1961 [pref. 169o], Wushuang pu 無雙譜 (Peerless Paragons) in Zhongguogudaibanhua congkan 中國古代版畫叢刊, Shanghai: Zhonghua shuju.

Qiongyao zhenren 瓊瑤真人 (1848), Qiongyao shen shu 瓊瑤神書 (Qiongyao's Divine Book), Xinyuan tang 信元堂 woodblock edn, in the library collection of the China Academy for Traditional Chinese Medicine.

Shen Gongchen 申拱辰 1997 [1604], Waike qixuan 外科啟玄 (Dispelling the Mysteries of External Ailments), reprinted in Hu Xiaofeng 胡 曉峰 (ed.), Zhongyi waike shangke mingzhujicheng 中醫外科傷科 名著集成, Beijing: Huaxia chubanshe.

Shen Jin'ao 沈金鳌 1999 [1774]. Shenshi zunsheng shu 沈氏尊生書 (Master Shen's Book on Revering Life), reprinted in Shen jin'ao yixue quanshu 沈金鳌醫學全書, Beijing: Zhongguo zhongyiyao chubanshe.

Wang Qi 王圻 1970 [1609], Sancaituhui 三才圖會 (Assembled Diagrams of the Three Powers), 6 vols, Taipei: Chengwen chubanshe.

Wang Shizhen 王世貞 (attrib.) 1961 [16oo], Liexian quanzhan 列仙 全傳 (Complete Biographies of the Exemplary Immortals), in Zhongguo gudai banhua congkan 中國古代版畫叢刊, Shanghai: Zhonghua shuju.

Wu Qian 吳謙 (ed.) 1742, Yuzuan yizong jinjian 御纂醫宗金鑑 (Imperially-commissioned Golden Mirror of Medical Learning), Beijing, Wuying dian.

Yuan Mei 袁枚 (ed.), Yan Xiyuan 顏希源 (author) and Wang Hui 王翽 (illus.) 1998 [1787], Baimei xin yong tu zhuan 百美新詠圖 傳 (New Verses on the Hundred Beauties, with Biographies and Illustrations), Jiye xuan woodblock edn; facsimile reprint Beijing: 
Zhongguo shudian.

Zhang Jiebin 張介賓 1965a [1624], Leijing tuyi 類經圖翼 (Illustrated Appendix to the [Yellow Emperor's Inner] Classic, Arranged by Topic), reprinted in Leijing tuyi, fu Leijing fuyi 類經圖翼, 附類經 附翼, Beijing: Renmin weisheng chubanshe.

1965b [1624], Leijing fuyi 類經附翼 (Supplemental Appendix to the [Yellow Emperor's Inner] Classic, Arranged by Topic), reprinted in Leijing tuyi, fu Leijing fuyi, Beijing: Renmin weisheng chubanshe.

\section{Secondary Sources}

Akahori, Akira 1989, 'Drug taking and immortality,' in Kohn (ed.) with Yoshinobu Sakade, 73-98.

Barnhart, R.M. 1996, 'The return of the Academy', in Fong and Watt (eds), 335-67.

Bartholomew, T.T. 2002, 'One hundred children: from boys at play to icons of good fortune', in Wicks (ed.), 57-83.

Bertholet, F.M. 2011, Concubines and Courtesans: Women in Chinese Erotic Art, Munich: Prestel.

Bertholet, F.M. and Rev. Yimen 1998 [1997], Dreams of Spring: Erotic Art in China from the Bertholet Collection, Amsterdam: The Pepin Press.

Bray, F. 2007, 'Introduction: The powers of Tu', in Bray, DorofeevaLichtmann and Métailié (eds), 1-78.

Bray, F., V. Dorofeeva-Lichtmann and G. Métailié (eds) 2007, Graphics and Text in the Production of Technical Knowledge in China: The Warp and the Weft, Leiden and Boston: Brill.

Cahill, J. 1976, Hills Beyond a River: Chinese Painting of the Yüan Dynasty, 1279-1368, New York: John Weatherhill.

1978, Parting at the Shore: Chinese Painting of the Early and Middle Ming Dynasty, 1368-1580, New York and Tokyo: John Weatherhill.

2003, 'Judge Dee and the vanishing Ming erotic colour prints', Orientations 34.9, 40-6.

2004, 'Introduction', in van Gulik [1951], xi-xxvi.

Cahill, S. 2005, Divine Traces of the Daoist Sisterhood, Magdalena, NM: Three Pines Press.

Cammann, S. 2001, China's Dragon Robes, Chicago: Art Media Resources.

Carlitz, K. 1991, 'The social uses of female virtue in late Ming editions of Lienü zhuan', Late Imperial China 12.2, 117-52.

Chang Che-chia 1998, "The therapeutic tug of war: the imperial physician-patient relationship in the era of Empress Dowager Cixi (1874-1908)', PHD diss., University of Pennsylvania.

Chang Chia-feng 2002, 'Disease and its impact on politics, diplomacy, and the military: the case of smallpox and the Manchus (1613-1795)', Journal of the History of Social Medicine and Allied Sciences 57.2, 177-97.

Chao Yuan-ling 20oo, 'The ideal physician in late imperial China: the question of Sanshi', East Asian Science, Technology, and Medicine $17,66-93$.

Chen Yuan-peng, 陳元朋 1996, “Liang Song de “shangyi shiren” yu “ruyi”-jianlun qi zai Jin-Yuan de liubian’ 兩宋的尚醫士人與儒 醫兼論其在金元的流變 ('Elites who esteemed medicine' and 'Confucian doctors' in the Northern and Southern Song dynasties, with a discussion of their spread and transformation during the Jin and Yuan dynasties), MA thesis, Taiwan National University.

Chou, J. and C. Brown 1985, The Elegant Brush:Chinese Painting under the Qianlong Emperor, 1735-1795, Phoenix, Az: Phoenix Museum of Art.

Claypool, L.R. 2001, 'Figuring the body: painting manuals in late imperial China', PHD diss., Stanford University.

Clunas, C. 1997, Pictures and Visuality in Early Modern China, Princeton,
NJ: Princeton University Press.

Cort, L.A. and J. Stuart 1993, Joined Colors: Decoration and Meaning in Chinese Porcelain: Ceramics from Collectors in the Min Chiu Society, Hong Kong, Washington, DC: Arthur M. Sackler Gallery, Smithsonian Institution.

Davidson, J. 1950, 'The origin and early use of the Ju-I', Artibus Asiae 13.4, 243-4.

Despeux, C. 199o, Immortelles de la Chine ancienne: Taoïsme et alchemie feminine, Puiseaux: Pardes.

2001, 'The system of the five circulatory phases and the six seasonal influences (Wuyun Liuqi), a source of innovation in medicine under the Song', in Hsu (ed.), 121-66.

Despeux, C. and L. Kohn 2003, Women in Daoism, Cambridge, MA: Three Pines Press.

Fong, W. and J. Watt (eds) 1996, Possessing the Past: Treasures from the National Palace Museum, Taipei, New York/Taipei:The Metropolitan Museum of Art, New York, and the National Palace Museum, Taipei.

Franke, O. 1913, Kêng Tschi Tu: Ackerbau und Seidengewinnung in China; Ein Kaiserliches Lehr-und Mahn-Buch, aus dem Chinesischen übersetzt und mit Erklärungen versehen, Hamburg: L. Friederichsen.

Furth, C. 1999, A Flourishing Yin: Gender in China's Medical History, 960-1665, Berkeley: University of California Press.

2006, 'The physician as philosopher of the Way: Zhu Zhenheng (1282-1358)', Harvard Journal of Asiatic Studies, 66.2, 423-59.

Goldschmidt, A. 1999, 'The transformations of Chinese medicine during the Northern Song dynasty (AD 96o-1127): the integration of three past medical approaches into a comprehensive medical system following a wave of epidemics', PHD diss., University of Pennsylvania.

Goodman, B. and W. Larson (eds) 2005, Gender in Motion: Divisions of Labor and Cultural Change in Late Imperial and Modern China, Lanham, MD: Rowman and Littlefield.

Hammers, R. 2002, 'The production of good government: images of agrarian labor in Southern Song (1127-1279) and Yuan (1272/9-1368) China', PHD diss., University of Michigan.

Hanson, M. 2003, 'The Golden Mirror in the imperial court of the Qianlong emperor', Early Science and Medicine: Special Issue: Science and State Patronage in Early Modern East Asia 8.2, 111-47.

Hsu, E. (ed.) 2001, Innovation in Chinese Medicine, Cambridge: CuP.

Jordanova, L. 1989, Sexual Visions: Images of Gender in Science and Medicine between the Eighteenth and Twentieth Centuries, Madison, wI: University of Wisconsin Press.

Kinney, A.B. (ed.) 1995, Chinese Views of Childhood, Honolulu: University of Hawaii Press.

Kohn L. (ed.) with Yoshinobu Sakade 1989, Taoist Meditation and Longevity Techniques, Ann Arbor: Center for Chinese Studies, University of Michigan.

Laing, E.J. 1975, 'Li Sung and some aspects of Southern Sung figure painting', Artibus Asiae 37.1/2, 5-38.

1996, 'Auspicious images of children in China: ninth to thirteenth century', Orientations 27.1, 47-52.

1998, 'Daoist $Q i$, clouds and mist in later Chinese painting', Orientations 29.4, 32-9.

Laqueur, T.199o, Making Sex: Body and Genderfrom the Greeks to Freud, Cambridge, MA: Harvard University Press.

Lefèbvre, E. 2006 (ed.) Le Palais du printemps: Peintures érotiques de Chine (Catalogue of Exhibition at Musée Cernuschi, Paris, 3 February-7 May 2006), Paris : Éditions Findakly.

Ledderose, L. 200o, Ten Thousand Things: Module and Mass Production in Chinese Art, Princeton: Princeton University Press.

Little, S. and S. Eichman 200o, Taoism and the Arts of China, Chicago: Art Institute of Chicago. 
Liu Yang 2001, 'Origins of Daoist iconography', Ars Orientalis, 31, 31-64. Lust, J. 1996, Chinese Popular Prints, Leiden: E.J. Brill.

Mann, S. 2005, 'The virtue of travel for women in the late empire', in Goodman and Larson (eds), 55-74.

Miller, L. 1995, 'Children of the dream: the adolescent world in Cao Xueqin's Honglou Meng', in Kinney (ed.), 219-47.

Park, K. 2006, Secrets of Women: Gender, Generation, and the Origins of Human Dissection, New York: Zone Books.

Pelliot, P. 1913, 'A Propos du Keng Tche T'ou', in Mémoires concernant L'Asie Orientale, Inde, Asie Central, Extrême-Orient, 3 vols, Paris: E. Leroux: vol. 1.

Scheid, V. 2007, Currents of Tradition in Chinese Medicine: 1626-2006, Seattle: Eastland Press.

Schiebinger, L. 1989, The Mind Has No Sex? Women in the Origins of Modern Science, Cambridge: Harvard University Press.

Siren, O. 1956, Chinese Painting: Leading Masters and Principles, New York: Ronald Press, 7 vols.

Stuart, J. 2005, 'The face in life and death: mimesis and Chinese ancestor portraits', in Wu and Tsiang (eds), 197-228.

Tsang, K.B. 2002, More Than Keeping Cool: Chinese Fans and Fan Paintings, Toronto, on: Royal Ontario Museum.

Unschuld, P.U. 1986, Medicine in China: A History of Ideas, Berkeley:
University of California Press.

2000, Medicine in China: Historical Artifacts and Images, Munich: Prestel.

2003, Huang Di Nei Jing Su Wen: Nature, Knowledge, Imagery in an Ancient Chinese Medical Text, Berkeley: University of California Press.

van Gulik, R.H. 2004 [1951], Erotic Colour Prints of the Ming Period, with an essay on Chinese Sex Life from the Han to the Chin'g Dynasty, $B C$ 206-AD 1664, Leiden: Brill.

Vinograd, R. 1992, Boundaries of the Self: Chinese Portraits, 1600-1900, Cambridge: CUP.

Wicks, A. (ed.) 2002, Children in Chinese Art, Honolulu: University of Hawaii Press.

Williams, C.A.S. 1960, Encyclopedia of Chinese Symbolism and Art Motives, New York: Julian Press.

Wilson, V. 1996 [1986], Chinese Dress, New York: John Weatherhill.

Wu Hung 1995, 'Emperor's masquerade - "costume portraits" of Yongzheng and Qianlong', Orientations, 26.7, 25-4.

Wu H. and Tsiang K.R. (eds) 2005, Body and Face in Chinese Visual Culture, Cambridge, MA: Harvard University Press.

Wu Yi-Li 200o, 'The Bamboo Grove Monastery and popular gynecology in Qing China', Late Imperial China, 21.1, 41-76. 


\section{PArt 2}

\section{Effective Representation}





\title{
7 The Limits of Illustration: Animalia and Pharmacopeia from Guo Pu to Bencao Gangmu 本草綱目
}

\author{
Roel Sterckx*
}

In The Eye of the Lynx, a study of the naturalist and founder of the Accademia dei Lincei, Federico Cesi (1585-1630), David Freedberg draws attention to the perceived limitations associated with the use of pictures to classify natural


illustrations was that they showed too much and left the naturalist caught between the desire to accurately picture everything and the desire for order. The more accurate pictures were, the more detail they had to show. Yet, at the same time, the more biological detail they revealed, the more they reflected the untidiness and disorder in nature and the less useful they were for taxonomic abstraction and classification. The ultimate tension for the Linceans, Freedberg notes, 'was between the desire to record and describe everything (on the one hand), and the need to reduce and classify (on the other)'. ${ }^{1}$

In China, about a decade after the birth of Cesi, the great naturalist Li Shizhen 李時珍 (1518-93) had just completed his landmark Materia Medica, Bencao gangmu 本草綱目. Li Shizhen did not see his own work in print (in 1596) and probably had no hand in the preparation of illustrations that came to accompany the text and were compiled and drawn by his two sons Li Jianzhong 李建中 and Li Jianyuan 李建元. ${ }^{2}$ By the 17 th century, the inclusion of illustrations in pharmacopoeia was by no means new. Bencao works were reported to have been illustrated as early as the Tang. Likewise animals, plants, rocks and other elements of nature had been depicted in various media since antiquity. Yet to the beholder of Bencao illustrations, the problem of representation sketched above by Freedberg and the inherent tension between detail and abstraction is equally relevant. What were the Bencao illustrations meant to illustrate and, more importantly, wherein lay their value for medical and pharmaceutical purposes?

To be sure, the Linceans had analytical ambitions that went far beyond the degree of biological scrutiny apparent

I would like to thank the anonymous reviewers for their comments on an earlier draft of this chapter. I am grateful to Geoffrey Lloyd, Georges Métailié, Teriyuki Kubo and Vivienne Lo for comments and suggestions, and to John Moffett of the Needham Research Institute for his help in locating materials.

$1 \quad$ Freedberg 2003, p. 366.

2 For biographical sketches of Li Shizhen, see Needham 1986, pp. 308-12; Hubei sheng zhongyi yao yanjiuyuan (eds) 1984, pp. 1-16; Wang Jian (ed.) 1996, pp. 1-37. On Li's quest to get his work published, see also Nappi 2006, pp. 9-17. in any set of botanical or zoological illustrations known in China. And the Bencao illustrators for their part operated in a different context, one that required them to design illustrations to complement an existing naturalist encyclopaedia anchored in a textual format inspired by lexicographical and commentarial conventions. These were predominantly focused on the explanation of graphs and texts rather than biological realities. A Chinese scholar's so-called 'naturalist' interest was to a great extent centred on the explanation of texts and nomenclature.

The following anecdote recounted by Yan Zhitui 顏之推, 6th-century author of the Yanshijiaxun 顏氏家訓 (Family Instructions of Master Yan), illustrates such an attitude. It suggests that the learned in medieval China were not necessarily inclined to leave their study and experiment with the observation of nature, recall observations from the past, or record contemporary hearsay:

When I first read about the two-headed hui 螝 worm in Zhuangzi 莊子 of which the Han Feizi 韓非子 says: 'Among the insects there is the hui, which has one body and two mouths, which struggle for food, gnaw each other and so kill each other', I was at a loss, as I did not know the pronunciation of this character. I asked whomever I met, but no one could explain. According to the Erya 爾雅 and works of the same nature, the larvae of silkworms are called hui, but that is not the two-headed creature with two mouths eager to harm each other. Then later on, I noticed in the Gujin zigu 古今字詁 (Dictionary of Archaic and Modern Characters) that the character is the old form of hui 虺 [a venomous snake]. So the pending problem unsolved for years cleared up like dispersed fog. ${ }^{3}$

Yan Zhitui of course was hardly a naturalist or a physician in search of drugs. The latter might have been intrigued by the biological reality of this creature and perhaps even motivated to prove its existence outside a library. Yet Yan's attitude, according to which the mystery clears once a graph is traced to its etymological or lexical origins and its status in texts, was deeply engrained in writings dealing with the natural world as early as the Han. It is hard to imagine that this textual attitude suddenly gave way to observation and accurate representation in image by the time of Li Shizhen. ${ }^{4}$ If Yan Zhitui were asked to produce an illustration of his two-headed snake, it is not unlikely

\footnotetext{
3 Wang Liqi (ed.) 1993, 3.226 ('Mian xue' 勉學).

4 I have discussed this prevalence of textual exegesis in more detail in Sterckx 2002, pp. 21-43; Sterckx 2005.
} 
that he would have to rely on imagination rather than observation. The same will apply to many of the illustrations surveyed in this chapter.

With the exception of a few short passages in prefaces to Bencao literature, next to nothing is known about the motivation behind the creation of their illustrations. Yet, this aporia of itself throws up an important question often overlooked in the treatment of illustrated Materia Medica or naturalist illustration at large in traditional China: namely, to what extent did such illustrations serve a practical - read medical or pharmaceutical - purpose at all? To develop this question, in what follows, I explore the contexts in which animal species have been depicted in traditional China leading up to and stretching into the period that saw the emergence of Bencao illustrations. My account is by no means meant to be exhaustive. It merely serves to build up an aggregate picture of the types of pictorial practices that preceded the Bencao illustrators, of the attitudes adopted towards representation in image, and of the sources that are significant stages along the way.

Generally, two styles of illustration can be distinguished across the transmitted corpus, albeit not always clearly. One could be referred to as the 'performative' or emblematic image, the other as the 'illustrative' or didactic image. The former are depictions that invite the observer or user to actively invoke the image as part of a performance, such as concentrating on an image as part of a ritual incantation, divination, or healing. By 'illustrative image', I mean images that complement an existing, mostly textual, narrative. Performative images are often accompanied by explanatory text captions, yet the latter usually remain subsidiary to the image. Illustrative images offer a type of annotation, supplement or commentary to the text. Their logical relationship to the text is usually generated through juxtaposition or implicit association and their absence does little to detract from the informative function of a text. My use of the term 'illustration' or 'picture' does not seek to distinguish pictorial media according to corresponding terms such as tu 圖, hua 畫 or xiang 像, variously rendered as 'chart', 'diagram', 'image', 'plan', 'talisman', 'map', etc. I use these English terms in a broad sense comprising any attempt at the pictorial representation of objects or, more specifically, creatures in the natural world. In short, we are concerned generally with modes in which information about the natural world was communicated visually. ${ }^{5}$

5 In a recent collection of essays on $t u$ 圖, Francesca Bray proposes a generic definition for $t u$ : 'It was a specialist term denoting only those graphic images or layouts which encoded technical knowledge: $t u$ were templates for action. This concise definition seems to us to capture the essence of $t u$ across its many vari-

\section{Animal Illustrations}

Little direct or indirect evidence survives for the pre-imperial and early imperial period of images that might have illustrated written texts or complemented performances such as songs or ritual liturgy. To be sure, pictorial evidence, including scenes that take the natural world as topic, is omnipresent in early China. Shang oracle-bone writing includes numerous pictographs representing animals; zoomorphic motifs pervade Shang and Zhou bronze vessel decor; and scenes depicting hunting expeditions, sacrificial slaughter, animal combats and games, as well as various aspects of agricultural life, abound in Han murals and on decorated ceramic bricks. ${ }^{6}$ References to officials recording landmarks and 'notable objects' (ming wu 名 物) on maps suggest that maps too could be illustrated. ${ }^{7}$ Likewise, murals have been preserved that illustrate textual narratives, often exemplifying famous episodes from the classics. The most notable examples are preserved in the shrines of the Wuliang 武梁 family (2nd century CE; Jinxiang county, Shandong). ${ }^{8}$

Most agricultural or natural scenes in Warring States and Han murals appear as decorative statements in their own right; some form part of a larger pictorial narrative or are intended to illustrate an existing textual narrative. Zoomorphic imagery appears, or was reported to appear,

ations in pre-modern China. It elegantly resolves many of the confusions about apparently fuzzy boundaries between $t u$ and other graphic categories which have puzzled historians trying to place $t u$ as an intellectually coherent category. It highlights the fact that from the Chinese perspective $t u$ was not a stylistic but a functional category: $t u$ were instructive images conveying skilled, specialist knowledge. Tu offered spatial encodings (often but not necessarily two-dimensional) of factual information, structures, processes and relationships, translating temporal or intellectual sequences into purely spatial terms, and encrypting dynamic processes as static layouts'. See Bray et al. (eds) 2007, pp. $2-3$. In addition to the applications of $t u$ discussed in this volume, see Lackner in Schmidt-Glintzer (ed.) 199o; Reiter 1992. Not discussed in this chapter are European attempts to import zoological knowledge into China through means of illustration. Ferdinand Verbiest's contributions deserve a mention in this respect. See Iannaccone in Carletti et al. (eds) 1996.

6 On the Shang origins of animal graphs, see Yang Xiaoneng 200o, pp. 90, 114; Li Haixia 2002, pp. 208-10. On the Shang royal hunt, see Fiskesjö 2001. On the hunt, see Lewis 1990, pp. 15offf; Sterckx in Knight (ed.) 2004. On zoomorphic iconography, see Sterckx in Wang (ed.), in press. Han murals with agricultural and hunting scenes are collated in Xia Henglian et al. (eds) 1996.

7 See, for example, Sun Yirang (ed.) 1987, 18.689 ('Da situ').

$8 \quad$ For the main studies, see Wu Hung 1989; Powers 1991; Liu et al. (eds) 2005 . 
predominantly on artefacts, buildings, and implements related to ritual culture, most notably, funerary art. Reconstructing the exact context and meaning behind many of these motifs remains problematic. ${ }^{9}$ A hybrid creature on a bronze is likely to contain iconographic meaning that testifies to religious, mythological or cosmological concepts (now lost to us in many cases). A hunting scene in a tomb or palace mural depicting a successful real or imaginary campaign might serve to glorify the reputation of the owner or occupant.

While relatively little direct pictorial evidence has survived, several passages in Warring States and Han texts preserve important information on the kinds of attitudes that were adopted vis-à-vis pictorial representation. The first reference to the use of apotropaic illustrations occurs in the Zuozhuan 左傳, in the story of Yu 禹 the Great's casting of nine talismanic tripods:

Anciently, when the Xia [territory] was marked by virtue, the distant regions made pictorial [representations] of the creatures [in their region], and tributes of metal [were sought from] the Nine Herdsmen. Tripods were cast with those creatures represented on them. All creatures [being thus revealed], [instructions were given] of preparations [to be made], so that the people would know these spirits and evils. Therefore when people entered rivers, marshes, hills and forests, they would not meet or follow them; and the chimei 魓魅 with them. Thus they could harmonise high and low, and enjoy the favours of heaven. ${ }^{10}$

Elsewhere, I have discussed this passage as an early example of cosmographic collection and linked it to the image of herding and shepherding as a metaphor for representing and controlling the world. ${ }^{11}$ We are not told here how the images of the unpropitious creatures were cast on to the vessels. It is also not known whether they were accompanied by inscriptions that identified their name or shape. But this brief passage set a precedent for the image of the tripod (ding 鼎) as a medium to depict the names and shapes of demonic and strange creatures. This is, for instance, reflected in titles of later treatises that were purported to be repositories of the strange, such as a text known as the 'Jiu ding ji' 九鼎記 (Record of the Nine Tripods) or another piece quoted as the 'Xia ding zhi' 夏鼎 志 (Treatise on the Xia Tripods)..$^{2}$ Although spanning too

9 On the interpretation of zoomorphic motifs, see Wang et al. 2006.

10 Yang Bojun 1995, pp. 669-71. See further Shuowen jiezi 說文解字 Shanghai: Shanghai guji, 1983, 7A.35b; Shiji 史記, Beijing: Zhonghua, 40.170o; and Liu Pansui (ed.) 199o, 26.375-76.

11 Sterckx 2002, pp. 118-20.

12 'Jiu ding ji' is quoted in Baopuzi neipian jiaoshi 抱朴子内篇 much of a time-gap to allow us to draw parallels with any confidence, the idea of images being solicited by a central authority to be represented in a lasting medium (bronze in this case) and, following an 'editorial' operation, re-issued as a guide to the unknown, recalls later imperially sponsored edicts ordering illustrations of materia medica to be submitted to editorial teams.

Several passages in Warring States texts discuss the perceived limits or benefits associated with pictorial representation. A story in the Han Feizi has a painter explain to his king that it is harder to draw dogs and horses than to depict ghosts and demons. A familiarity with domestic animals, he explains, leads to a more accurate representation. By contrast, the shapelessness of spirits leaves too much room for artistic fantasy. ${ }^{13}$ In addition to criticising the limited remit of pictorial representation, this anecdote contains an implicit warning: explaining unfamiliar creatures away as ghosts and demons deludes the observer of such images, 'imagi-nation' is the incapability to differentiate representations from actual reality. The idea is further illustrated in the story of $\mathrm{Zi} \mathrm{Gao}$ 子高 (fl. c. 523 BCE), who was so fond of dragons that he had his lodgings decorated with carvings of dragons - until one day a real dragon descended in front of his residence and he had to run away in fear. His fashioning of dragon images had prompted the arrival of a real dragon. ${ }^{14}$

In another story, the Han Feizi links the concept of imagination to a lack of familiarity or visualisation in a pun on the character xiang 象, meaning both 'elephant' and 'image'..$^{15}$ The passage presents the sage as an agent able to interpret images as a referent to a reality not visible to the common eye. The sage is also said to be able to represent what is absent in its bodily or physical form. Similar debates on the nature of visual representation continued into the Eastern Han. Zhang Heng 張衡 (78-139 CE), for instance, is on record commenting as follows on the Han Feizi an-

校, Beijing: Zhonghua, 1996, 17.308, but is also associated with a Tang authorship. See Jiu Tangshu 舊唐書, Beijing: Zhonghua, 1975, 189B.4979; and as quoted in Taiping yulan 太平御覽, Sibu congkan (ed.), 883.7a, and Tian zhong ji 天中記 (Siku quanshu ed.), 55.55a. For the 'Xia ding zhi', see Jinshu 晉書, Beijing: Zhonghua, 1947, 28.853; Songshu 宋書, Beijing: Zhonghua, 1974, 31.923; Sou shen ji 搜神記, Shanghai: Shangwu yinshuguan, 1957, 12.90; Tang Kaiyuan zhan jing 唐開元占經 (Siku quanshu ed.) 119.4a.

13 Chen Qiyou (ed.) 1991, 11.633 ('Wai chu shuo zuo shang'). See also Liu Wendian (ed.) 1992, 13.432 ('Fan lun').

14 Creel 1974, fragment 15, p. 363. See also Zhao Zhongyi (ed.) 1997, 5.173 ('Za shi'); Lunheng jiaoshi, 47.698-9 ('Luan long'); Hou Hanshu 後漢書, Beijing: Zhonghua 1965, 3oB.1082 n. 3; 52.1719 n.2.

15 Han Feizi jishi, 6.368 ('Jie Lao'). 
ecdote on why artisans prefer to depict ghosts instead of real animals: 'Truly, it is because substantial entities are difficult to represent in form, while empty counterfeits are inexhaustible' (cheng yi shishi nan xing er xu wei bu qiong $y e$ 誠以實事難形而虛偽不窮也). ${ }^{16}$

Turning to presently transmitted illustrations, a piece that deserves a mention is the famous Chu silk manuscript, recovered by tomb robbers in 1942 in Zidanku 子彈庫, Changsha (Hunan), dating to $c$. 300 BCE, which is now kept in the Arthur M. Sackler Gallery in Washington DC. The manuscript $(c .38 \times 47 \mathrm{~cm})$ was first studied in the West by Noel Barnard in the 1970s. It is designed in the shape of a divination board and contains 12 peripheral hybrid creatures that possibly represent the deities corresponding to each of the 12 calendar months described in the text. ${ }^{17}$ The link between the creatures and the text itself is debated, and although the figures carry three-character labels of their name, it is hard to establish whether its author(s) intended to design the piece as an illustrated text or whether it originated as a set of illustrations annotated with a text. ${ }^{18}$ It is noteworthy, however, that in what remains of the text captions, no direct reference is made to the shape and colour of the drawings. This suggests

16 Hou Hanshu, 59.1912. Zhang here likens those who rely on prognostication charts (instead of empirical astronomical observation) to painters preferring to draw ghosts and demons. Several occurrences are recorded in which murals, pictures and portraits were used didactically. One instance records the ageing Han Wudi 漢武帝 intimating to his Marshal of State, Huo Guang 霍光 that he should supervise the named infant successor to the throne as regent. Wudi made his will clear by commissioning a wall mural showing the Duke of Zhou carrying the infant King Cheng 成 on his back. See Hanshu 漢書, Beijing: Zhonghua, 1962, 68.2932. A passage in the Kongzi jiayu 孔子家 語 has Confucius contemplating a similar scene at the Bright Hall (Mingtang 明堂). See Kongzi jiayu (Sibu beiyao ed.), 3.1b-2a ('Guan Zhou' 觀周). In 178 CE, Cai Yong 蔡邑 (133-92 CE) painted a mural of Confucius and his 72 disciples on the wall of the imperial academy to urge on its students to study. See Hou Hanshu, 6oB.1998.

17 For a translation of the accompanying text, see Barnard 1973, pp. $175^{-203}$. In vol. 3 of this series, Barnard discusses the relevance of the Shanhaijing 山海經 and other texts for the explanation of the 12 peripheral figures. See also Li Xueqin 李学勤 1994. The cosmic board theory is set out in Li Ling 李零 2000 , pp. 89-176, 178-96. A brief summary and translation by Li Ling and Constance Cook also appears in Major and Cook (eds) 1999, pp. 171-6. Mark Lewis discusses its spatial layout as a precursor of the 'Bright Hall' (Mingtang, ming tang 明堂) model in Lewis 2006, pp. 261-2.

18 See 'Chōsa shutsudo So hakusho no jūni kami no yurai 長沙出 土楚帛書の十二神の由來, Tōhō gakuhō 42, 1971, 1-63. See Hayashi Minao 1971. that their iconography (unattested elsewhere) was part of a stock of generic imagery known to people at the time or, alternatively, that the drawings were a product of the imagination of the illustrator(s) at the time. What is clear is that the texts describe the properties of the deities represented, but it remains uncertain whether the text should be seen as primary over the image or as an explanatory commentary on the image.

Moving to early imperial times, the Mawangdui 馬王 堆 finds represent an important stage for the study of illustrated manuscripts in the early Han. ${ }^{19}$ Noteworthy for our purpose is the silk painting referred to as Bibing $t u$ 避兵圖 (Repel weapon chart) from Tomb no. 3, and the illustrations on a silk astronomical chart (Tianwen qixiang za zhan 天文氣象雜占, 'Miscellaneous astrological and astronomical divinations'). The 'Repel weapon chart' - which some scholars prefer to refer to as the 'Taiyi jiangxing tu' 太一將行圖 (Drawing of the Procession before the Departure of Taiyi) ${ }^{20}$ - depicts the high god Taiyi (Grand One) flanked by Lord Rain and Lord Thunder to his right and left respectively. Below Taiyi are four armed figures holding a knife, sword, bow and a spear. A hybrid creature rises toward Taiyi from between two dragons at the bottom. The armed figures together with the text in the right margin have led some scholars to conclude that this piece was made in support of a military campaign. ${ }^{21}$ Others have focused on Taiyi's left arm, underneath which is written the character she 社 (earth altar/soil spirit), which could indicate that the illustration was used to worship a particular deity, perhaps in a prayer for rain. Regardless of its exact function, it is clear that this illustration was meant to be visible and hence likely to be used during a ritual performance of some kind. ${ }^{22}$ The silk scroll designated 'Tianwen qixiang za zhan' illustrates celestial phenomena such as clouds and halos and figures in the shape of animals, including a well-preserved creature in the shape of a dragon-horse. The illustrations are accompanied by texts naming the portents or the event(s) they predict. The relationship between the illustrations and the accompanying divination texts continues to be debated. ${ }^{23}$

All $t u$ 'diagram' manuscripts among the Mawangdui finds are conveniently surveyed in Lai Guolong 2003.

$20 \quad$ See Chen Songchang 1993.

21 See Harper in Loewe and Shaughnessy (eds) 1999, 870-1.

22 See Zhou Shirong 1990; Li Ling 1991.

23 Harper in Loewe and Shaughnessy (eds) 1999, p. 844. In a recent article, Donald Harper, who prefers to translate qixiang as 'meteoromantic', emphasises that the drawings on this manuscript need not reflect empirical observation. He argues that they 'render phenomena using designs that, once established, predisposed the ancient observer to correlate what was seen in 
It is plausible that text and illustration came together at different stages and were compiled from different originals by several scribes. ${ }^{24}$

Illustrated texts such as the Chu silk manuscript and the Mawangdui charts, discussed above, are 2oth-century finds that add a small piece to our very fragmentary understanding of the use of illustrations in early China. And while they were evidently not available to Bencao illustrators, the creatures they depict appear cognate to the types of animalia described in a frequently adduced source in Bencao literature and commentary, namely the Shanhaijing 山海經 (Classic of Mountains and Waters). This text has variously been described as a manual on prodigies, a geographical gazetteer or explanatory notes on illustrations or maps. ${ }^{25}$ While the oldest extant illustrations that were printed independently or accompany text editions of the Shanhaijing are of Ming and Qing date, illustrations existed possibly as early as the 3rd century CE. ${ }^{26}$ Guo Pu 郭璞 $(276-324 \mathrm{CE})$ mentions illustrations in his commentary and wrote appraisals of them in a work known as the Shanhaijing tuzan 山海經圖讚 (Appraising the Illustrations to the Shanhaijing), preserved in both the Daozang 道藏 (Daoist Canon) and Sibu beiyao 四部備要 editions. The poet Tao Yuanming 陶淵明 $(365-427 \mathrm{CE})$ claims to have derived poetic inspiration from glancing at

nature with these drawings from the initial moment of observation; that is, the drawings had a determining influence on the elite's perception of nature'. In other words, the drawings here encourage people 'to see what they are looking for' and the Han reader was thus invited to 'match omen statements not recorded in writing on the manuscript with the categories represented by the drawings'. See Harper in Bray et al. (eds) 2007, pp. 169-89 (quotes pp. 172, 174).

The theory that the texts were added after the production of the illustrations is set out in detail in Chen Songchang in Wuhan daxue jianbo yanjiu zhongxin (eds) 2006. Chen concludes that the chart was copied in its current form around 196 вСE or slightly afterwards. Another case in support of a text allegedly derived from graffiti has been made by Wang Yi 王逸 (2nd century CE) who claimed that Qu Yuan 屈原 (4th century BCE) wrote down the 'Tian wen' 天問 (Heavenly Questions) on murals in ancestral temples of former kings and minsters of Chu. While not implausible, the content of the Heavenly Questions, as David Hawkes notes, appears rather abstract to be linked to a series of pictures. See Tang Bingzheng et al. (eds) 1996, pp. 80-1; Hawkes 1985, pp. 123-4.

25 For a recent, highly speculative 'zoological' reading of the Shanhaijing, see Guo Fu 2004.

26 See Fracasso 1988; Fracasso in Loewe (ed.) 1993. Guo Pu's commentarial oeuvre is surveyed by Fracasso in Carletti et al. (eds) 1996.
Shanhaijing illustrations. ${ }^{27}$ Several other titles of works, now lost to us, refer to Shanhaijing illustrations. So while it is impossible to establish whether Shanhaijing illustrations circulated in pre-Han times, continuous reference to accompanying $t u$ in the commentarial tradition suggests that they existed very early on. The oldest extant woodblock illustrations, by an unknown artist, are reproduced in a 1597 reprint of Wang Chongqing's 王崇慶 Shanhaijing shiyi 山海經釋義 (1537). ${ }^{28}$

Two other illustrated works are attributed to Guo Pu. According to the bibliographical treatise of the Suishu 隋 書, the Erya tu 爾雅圖 (Illustrated Erya) consisted of 10 scrolls. It is mentioned together with Erya tuzan 爾雅圖 讚 (Appraised Illustrations to the Erya) in two scrolls. The originals of both works were lost by Sui times. ${ }^{29}$ Guo Pu's practice of commenting on illustrations survived, at least nominally, in other works in the 'Erya tuzan 爾雅圖讚, (Appraised Illustrations) tradition. One work worth mentioning is Yang Shen's 楊慎 (1488-1559) Yi yu tu zan 異魚 圖讚 (Appraised Illustrations of Strange Fish), preserved in four scrolls in most transmitted editions but unfortunately without the illustrations. ${ }^{30}$

Despite Guo Pu's appraisals of now lost illustrations to the imaginary bestiary of the Shanhaijing, only a few examples of animal images illustrating charts or texts survive from the medieval period. ${ }^{31}$ One notably rich source is the

27 See Lu Qinli (ed.) 1979, 4.133 ('Du Shanhaijing shi-san shou' 讀山 海經十三首).

28 It is, as Vera Dorofeeva-Lichtmann has argued, very unlikely for technical reasons that illustrations of the type referred to by Guo $\mathrm{Pu}$ were part of the original text(s) circulated on bamboo slips. Incorporating illustrations into the text may only have been technically possible for versions written on silk. Guo Pu's 'Tu zan' therefore more likely existed as a reflective appendix to the text and the illustrations might have circulated separately from the text. See Dorofeeva-Lichtmann in Bray et al. (eds) 2007.

29 See Suishu (Beijing: Zhonghua, 1973), 32.937. The relationship between these two works and another work entitled Erya yin tu 爾雅音圖, printed in 1801 and based on illustrations allegedly copied in the Song, is dubious. For an art-historical perspective, see Wang Hongbin 2006.

'Yi yu tu zan' survives in several editions (and is included in the Siku quanshu and Congshu jicheng). Commentaries and supplements to the text include a Yi yu tu zan jian 異魚圖讚䇝 and a Yiyu tu zan bu 異魚圖讚補 by Hu Shi-an 胡世安 (d. 1663). The latter is also known as the author of a short work (referred to as Run ji 閏集), once described by Alexander Wylie $\left(1815^{-87}\right)$ as a 'small brochure on piscatorial monstrosities'. See Wylie 1901, p. 154. For a biographical sketch and survey of Yang Shen's literary work, see Cigliano in Carletti et al. (eds) 1996. ing is the extant version of the Huangdi hama jing 黃帝蝦蟆 
manuscripts related to divination from Dunhuang. These include the 6th-century illustrated demonography entitled Baizejingguaitu 白澤精怪圖 (Illustrations of Baize, Sprites and Oddities) (P.2682 $r^{o}$; S.6261), which contains 19 images of portentous animal spirits and creatures with brief text captions of two to four columns preceding them. They include variously coloured roosters said to call out the names of household members at night, a hybrid bird with a human head, serpents, a mouse climbing a tree trunk, a dragon and a chicken. Each is meant to be an image of the portents and demons described in the captions. Another fragment (S.6261) of the same text as P.2682 $r^{o}$. preserves six illustrations including a rabbit and a dog emerging from the soil. Possibly of late Han or early medieval date, such illustrated guidebooks were clearly popular by Tang times when various versions of a text carrying a similar title circulated.32

Other manuscripts in the Dunhuang corpus are a rich repository of animal illustrations. P.2683 contains 23 colour images of animal omens. The text names 42 fabulous animals (19 illustrations are missing) divided in three categories: tortoise, dragon, and a phoenix-like bird. The captions describe the shape of the creature, the circumstances of its appearance, and explain the omen related to its appearance. ${ }^{33}$ Most illustrations occur in so-called ruitu 瑞圖 (Illustrations of Auspicious Omens). S.2404 contains an image of one of the cyclical animals (a monkey). The cyclical animals are further found on P.4058B $v^{o}$, S.612 $r^{o}$ and S-P. $6 r^{\circ}$. Crows are depicted in a manuscript that deals with the mantic art of interpreting birdcalls (P.3479 $\left.r^{\circ}\right)$. Dragons appear in several other manuscripts not mentioned above (P.3594 $\left.r^{o}, \mathrm{P} .3602 v^{o}\right)$. The image of a bird occurs in a hemerological text used to predict a person's fate according to their date of birth in the animal zodiac (P.4881).

\section{Bencao Illustrations}

Depending on the label one wishes to attach to the Shanhaijing and its reported illustrations, it is not until the Tang period that we find references to illustrated pharmacopoeias. They were part of a work in the main

經 (The Yellow Emperor's Toad Canon), which contains illustrations of the three-legged-bird-in-the-sun and a toad-and-harein-the-moon. It only survives in an 1823 Japanese woodblock edition. Vivienne Lo has argued recently that its medical ideas might situate it as far back as the early Tang. See Lo 2001. For an excellent survey of the contents of these manuscripts, see Catherine Despeux's entry in Kalinowski 2003, pp. 436-43, 467.
Bencao tradition. ${ }^{34}$ The oldest known illustrated Bencao work is commonly referred to as the Tang bencao 唐本 草 or Xinxiu bencao 新修本草 and was compiled by an editorial committee including Su Jing 蘇敬 (c.657 CE), Li $\mathrm{Ji}$ 李漬 and others. It was published in 659 and is the first work to include drug illustrations (yaotu 藥圖, in 25 chapters) and commentaries thereon (yaojing 藥經, in seven chapters). Its illustrations are now lost but we know from the preface that its publication followed an empire-wide decree two years earlier inviting people to collect and submit locally known drugs to the editors. These were then drawn, allegedly, 'in rich colours'. The preface further reveals that part of the motivation behind this grand government-sponsored quest for materia medica was the knowledge that animals and plants showed regional characteristics that were relevant to their pharmaceutical use and hence required to be represented accurately. ${ }^{35}$ How this might have been reflected in the actual illustrations remains totally unknown, although some illustrations in later Bencao do make attempts to sketch landscape and vegetation as background to the creatures, possibly giving some account of regional variation (Fig. 1).

A more significant moment in the history of Bencao illustrations occurred in the Northern Song, when Su Song 蘇頌 (c.1020-1101) put together illustrations into what became known as the Tujing bencao 圖經本草(Illustrated Guide through Bencao, completed in 1062). This work, also known as Bencao tujing, contained over 9oo illustrations and, according to its preface, was prepared as a replacement for the lost illustrations in Xinxiu bencao. According to the preface, it was completed following an imperial order. In the same preface, the scholar-physician Zhang Yuxi 掌禹 錫 (992-1068) insists on the universal and encyclopaedic ambition of the work. It not only had to sort out 'the thousands of different drug specimens that have now been drawn in illustrations in the empire' but also to include information beyond what medical practitioners had heard and seen, including explanations that relied on previously existing commentaries and outside works ranging from historical works to novels. ${ }^{36}$ As is suggested by its title which Nathan Sivin translates 'Illustrated pharmaceutical

The most notable scholars to have worked on the Bencao tradition include Ma Jixing 馬繼興, Joseph Needham, Paul Unschuld, and Nathan Sivin. For an overview of Bencao literature, see Needham 1986, pp. 220-38. The best Western language study of the Bencao corpus and its prefaces to date is Unschuld 1986, to which I am much indebted in this section. See Unschuld 1986, pp. 45-50, which includes a translation of the preface. See also Unschuld 200o, p. 104.

36 Preface, as translated in Unschuld 1986, pp. 65-6. 




Figure 7.1
Bencao gangmu (1885): a) Fresh-water snake (shui she 水蛇) Shangwu yinshuguan, 1933), vol. 1, 30, 45 natural history'37 - the use of illustrations here appears to be central and they are presented as a genuine medium through which pharmaceutical knowledge can be transmitted. Illustrations are judged to be useful on account of the fact that they can help physicians distinguish genuinely useful plants and animals from others:

Illustrations are used to represent the appearance and colour [of the drugs], the jing [commentaries] serve to explain their similarities and differences (tuyizai qixingse, jingyi shi qi tongyi 圖以載其形色, 經以釋其同異). ${ }^{38}$

Yet implicit in this statement also is an admission that without explanatory notes, the use of illustrations is reduced to approximate recognition. The imperial decree issued in 1058 and petitioned by the authors in preparation for the work makes the same point:

Shape, colour, and size of roots, stalks, shoots, leaves, blossoms, and fruits, as well as worms, fish, birds, quadrupeds, precious stones, and minerals, if suitable for pharmaceutical use, should be drawn in illustration one by one, accompanied by an explanatory comment, describing, for every single object, the time of blooming [etc.]... . The evidence [gathered in this manner] is to serve as a basis for the creation of Bencao illustrations. In addition commentaries are to be written for the illustrations. ${ }^{39}$

$37 \quad$ See Needham 1986, p. 56.

$38 \quad$ Shang Zhijun (ed.) 1994, p. 1.

39 Unschuld 1986, p. 67, relying on the text in Okashini Tameto's 罔 西為人 Songyiqian yiji kao 宋以前醫籍考 (1969).



b) Caddis fly (shi can 石䖯). Source: Bencao gangmu (Shanghai:
The decree continues by stating that these annotated illustrations are to be circulated with the Bencao compiled during the current governmental period. Illustrations here do not appear to be valued as an independent means for the transmission of knowledge since they are expected to be accompanied by explanatory notes from the start. Likewise the involvement of an editorial team at the centre of its compilation suggests that the illustrations were standardised models derived from a copious number of drawings submitted. In other words, in the realm of the visual, as in the world of textual exegesis, standardisation is at work similar to the processes associated with the compilation of etymological lexicons and glossaries of regional dialects or calligraphies. The pharmacist appears to rely as much on methodologies inspired by textual exegesis as on practitioner's experience: ordering and rectifying the wide array of medicinal products gathered from all over the empire; juxtaposing competing explanations on the workings of the drugs; glossing together identical plants and animals that live in different regions; sorting out nomenclature, etc. In short, the most cogent steps of data-gathering appear to consist of examining and comparing the occurrence of drugs in texts from the past; in the case of Su Song and his team, nearly 200 sources. ${ }^{40}$

The original of the Tujing bencao is lost and nothing is known with certainty of its original illustrations. Its text and illustrations, however, are preserved in fragmentary form in the Daguan 大觀 edition of Tang Shenwei's 唐

40 See Gao Guangzhen in Su Kefu et al. (eds) 1991. 




Figure 7.2 Dragon bones in: a) Bencao tujing; ${ }^{*}$ b) Bencao gangmu (1885);** c) flying squirrel dung (wu ling zhi 五靈脂) (Bencao tujing). Sources: *Shang Zhijun 尚志钧 ed., Bencao tujing 本草图经 (Hefei: Anhui kexue jishu chubanshe, 1994), 423, 526. **Bencao gangmu (Shanghai: Shangwu yinshuguan, 1933), vol. 1, 42

慎微, Zhenglei bencao 證類本草 (Categorised Pharmacopoeia, collated late 11th-12th century) as well as the Bencao gangmu. In a recent reconstructed version of the fragments, it contains 814 entries, 642 of which have illustrations appended to them (Fig. 2) ${ }^{41}$ Assuming then that some of the original illustrations collated by Su Song were reproduced in some form or another in the oldest completely preserved Bencao, the Daguan bencao of 1108 $\mathrm{CE}$, the latter should be the source that holds the oldest presently transmitted animal illustrations in pharmaceutical texts. But, as Paul Unschuld points out, this is also where illustration stagnates, since little innovation seems to have followed:

In the subsequent illustrated bencao works of the Song period, there seems to have been no urge to prepare new illustrations of the plants, animals and minerals for each new edition of the Materia Medica - unless they were listed for the first time. ${ }^{42}$

One must probably allow for the fact that illustrations reproduced in printed works had to conform to some extent to the creative conventions of the schematic woodblock print. Unschuld suggests that they were created primarily for didactic purposes:

plants, plant parts, minerals, and the instruments used for their preparation, and animal and human medicinal drugs are still frequently and recognisably depicted. ${ }^{43}$

$41 \quad$ Shang Zhijun (ed.) 1994. The illustrated animal entries in this work occupy ch. 13 (qin shou 禽獸), 14 and 15 (chong yu 蟲魚). Ch. 14 starts off with an entry on dragon bones (long gu 龍骨).

42 Unschuld 1986, p. 67. In his introduction, Unschuld underlines that 'the nature of illustration and the structural patterns discernible in the herbals' serve as a caution not to assume steady linear progress from 'speculation to observation, from belief to truth'. (p. 7).
Speaking of botanical illustration in Bencao gangmu, Métailié and Haudricourt likewise speculate that the schematic nature of the illustrations might be didactic: allowing someone who already knows the plants to retrieve them easily. However, they also point out that it remains difficult to reconcile detailed emphasis on certain morphological features on the one hand, with the overall poor quality of the illustrations on the other. ${ }^{44}$ Yet while they may have been created for instructive purposes, one might also question the didactic efficiency of these illustrations. How valid could they have been to a practitioner operating in the field, centuries after the original creation of the images he is confronted with and, most likely, far removed from the social and regional environment in which those drugs were initially collected, identified and reported? Was it the case that, once a plant or animal drug had been fixed in image, it was simply copied and perpetuated in later editions in the understanding that this visual imprint had now become canonical and hence immune to new circumstances, new insights or new reports on its use as a drug? ${ }^{45}$ If so, we must assume that later Bencao compilers who were re-examining, re-editing, and correcting drug entries with a new curiosity did not attach much value to the accuracy and practical function of illustrations.

Métailié and Haudricourt 1994, pp. 386-96. Discussing the picture of a wutong 梧桐 tree in Bencao gangmu, Métailié speculates: 'It seems to me that once the text is memorised and associated with the picture, the picture can serve to tickle the memory even if it is not a true representation of the real wutong tree'. See Bray et al. (eds) 2007, p. 493

45 As much is admitted for instance in the preface to the Shaoxing bencao 紹興本草 (1159), which states: 'As to the shape and appearance [of the items discussed] we have based [our own presentation] on old illustrations'. See Unschuld 1986, pp. 79-8o. For some examples, see Karow 1956. 
In any case, this scenario could account for the possibility that the illustrations recognisable to Li Shizhen or his sons may have gone back in time as far as the original drawings in the Tujing bencao. Although nowhere in his own text does Li make reference to accompanying illustrations, he did personally express misgivings about the illustrations he saw. He speaks of a mismatch between text and illustration, of illustrations without explanations and of drugs being described with missing illustrations, or, even stronger, 'in some cases the explanations are correct but the illustration is wrong (huo shuo shitu fei 或說是圖非)'.46 And indeed Ai Cheng's 艾晟 preface to the 1108 edition of Daguan bencao partly justifies its compilation on the grounds that pharmaceutical knowledge needs constant updating; that medicines, when identified mistakenly, can be more harmful than the illnesses they try to cure; and that illustrations together with prescriptions play a vital role in assisting less experienced medical men to recognise the effectiveness of a drug. ${ }^{47}$ But although Song editors were capable of producing coloured illustrations of plants recognisable up to the present day, judging by Li Shizhen's comments, it is questionable whether such illustrations were taken to reflect zoological, botanical, or pharmaceutical information not already present in text.

The early 16 th century saw another highlight in the history of Bencao illustrations in the form of the Bencao pinhuijingyao 本草品彙精要 (completed in 1503-5), which contains 1,358 colour illustrations. Some of the illustrations in this work resemble those in the Daguan bencao but they are executed with greater precision, and include drawings that set plant and animal drugs in their natural surroundings, or illustrate the procedures of their production into drugs. The work, however, remained hidden from the public in the imperial collection for almost two centuries until Emperor Kangxi 康熙 $\left(1655^{-1723}\right)$ rediscovered its valuable existence and commissioned copies of a portion of the illustrations to be made. ${ }^{48}$

These then appear to be the main direct precursors to the Bencao gangmu illustrations. Yet other works, outside the main Bencao corpus, also contained valuable illustrations. Important in this respect is Hu Sihui's 忽思慧 (14th century) Yinshan zhengyao 飲膳正要 (Propriety and Essentials in Eating and Drinking), a Materia Dietetica and cookbook

46 Bencao gangmu (Shanghai: Shangwu yinshuguan, 1933), 5.1 ('Xu li, shang'序例上), p. 6 .

47 For the preface in translation, see Unschuld 1986, pp. 72-3.

48 For a summary of its contents and transmission, see Unschuld 1986, pp. 128-45. See also Métailié 1998. On colour illustrations in Ming Bencao, see further Zheng Jinsheng 2003, 14.4, 65-120; Xie Zongwan (ed.) 2001.



Figure 7.3 Yingshan zhengyao 'Strange animal transformations'. Source: Yinshan zhengyao (preface by Zhu Qiyu 朱祁鈺, Ming), in Zhongguo gudai banhua congkan er bian 中國 古代版畫叢刊二編 (Shanghai: Shanghai guji, 1994), vol. 2, 205

in three chapters published in 1456 and presented to the Mongol court. ${ }^{49}$ It contains well over 200 illustrations, which include not only images of isolated items but also illustrated narratives. For instance, one illustration shows variously black-and-white coloured goats with the caption 'strange transformations in animals' (qin shou bian yi 禽 獸變異) in the top right corner (Fig. 3). Captions appear to be essential to understand the illustration which otherwise would be of little informative value for the dietary physician or cook. The explanatory text to this particular illustration reads:

The shapes and species of animals originate from their basic bodily form just as their natures are divided into poisonous and non-poisonous varieties. Even more so in the case of strangely shaped creatures born through anomaly, how could these be without poison?! If one is not careful about what one eats, it will result in one becoming ill. This constitutes not looking into things. ${ }^{50}$

This Ming edition, which forms the basis of all later versions, is included in the Sibu congkan. For a full translation and study, including the original text and reproductions of the woodcut illustrations, see Buell and Anderson 2000.

50 Liu Yushu (ed.) 1989, pp. 102-3; cf. Buell and Anderson 2000, pp. 
Next follows a list of 26 anomalous animals or animal products to be avoided - a white horse with green hooves, a crab with only one claw, meat that moves when put in water, a sheep with a hole in its liver, etc. Throughout the work, illustrations are largely didactic, and given that the text invariably comments on the toxicity or lack thereof in certain foodstuffs, this makes sense. A number of captions stride away from simply giving dietetic or medicinal advice. For instance, the entry on the tiger states:

If one eats tiger meat and enters the mountains the tigers will be afraid when they see you. It wards off the 36 kinds of mei demons.

The illustration of a rhinoceros is accompanied by a statement that eating it ensures that one does not get lost in the mountains; and a broth of mandarin duck meat makes couples fall in love. ${ }^{51}$ Some illustrations show detail that seems directly related to the dietetic advice given in the text. For instance, an image of a shrimp with clearly marked whiskers illustrates an entry insisting that shrimp without whiskers should not be eaten. ${ }^{52}$

Another important category of writings, worth a separate study, is illustrated veterinary manuals. Some of these contain illustrations of anatomical animal parts; others depict animals displaying ailments and include physiognomical charts. Examples include the Yuan Heng liaoma $j i$ 元亨療馬集 (Collation of Horse Ailments by [Yu Ben 喻 本] Yuan 元 and [Yu Ben] Heng 亨, 16o8), the Xinke ma $s h u$ 新刻馬書 (Newly Cut Writings on the Horse; by Yang Shiqiao 楊時喬 et al., completed 1594), the Yuan dynasty Xinke zhushi ma niu tuo jing da quanji 新刻注釋馬牛䭾 經大全集 (Newly Cut and Annotated Comprehensive Collectanea of the Canon on Horses, Oxen, and Camels), and others. ${ }^{53}$ Anatomical illustrations or illustrations of

371-2 (illustration and text); 443-5 (translation). My translation differs.

$51 \quad$ Buell and Anderson 20oo, p. 464 (16A), p. 466 (18A), p. 475 (26B). For a summary of imagery associated with mandarin ducks in pre-Song sources, see Luo Yuan 羅願 (1136-84 CE), Erya yi 爾雅 翼 (Congshujicheng ed.), 17.187-8.

$5^{2} \quad$ Buell and Anderson 200o, p. 481 (32A).

53 A notable illustrated manuscript of Korean origins is the Xinbian jicheng mayi fang niuyi fang 新編集成馬醫方牛醫方 completed in 1399 (printed 1633). See Kim Sin-gŭn 金信根 (ed.) 1992. Xinke ma shu is readily available in a recent 1984 edition by Nongye chubanshe; Yuan Heng liaoma ji (also known as Niu ma jing 牛馬經) in a 1957 edition by Zhonghua shuju; Xinke zhushi ma niu tuo jing da quanji, with annotations by Guo Huaixi 郭懷 西 (fl. c. 1785), in a 1983 edition published by Nongye chubanshe. Some of the illustrations in these texts are attributed to or purportedly based on illustrations in the oldest transmitted veteri- body parts were not exclusive to this genre of texts. Ritual manuals and explanatory commentaries on the ritual classics also preserve some examples. For instance, the rudimentary drawings inserted in the $L i s h u$ 禮書, a text attributed to the Song scholar Chen Xiangdao 陳祥道 ( $f$ l. 1053-93), include a drawing of a sacrificial ox with names identifying the various parts of its skeleton as well as simple drawings of lungs offered up in sacrifice. ${ }^{54}$

So by the time Li Shizhen embarked on his life-time project, Bencao and related works had been illustrated for centuries. Illustrations had been copied, edited, standardised, and, in some cases, we must assume, updated with more detail. They would continue to be produced throughout the late Ming, Qing and into the 2oth century. ${ }^{55}$ Yet, unlike the successive reworking of texts and the endless accretion of commentaries, modifications to illustrations remained relatively minor to stagnant.

Reported numbers of illustrations in the early editions of Bencao gangmu range from 1109 to $1160 .{ }^{56}$ The first and original edition, also known as the Jinling 金陵 edition published in Nanjing in 1596 by Hu Chenglong 胡承龍, contains two separate chapters of illustrations from the hand of Li Shizhen's son, Li Jianyuan. Largely based on the Zhenglei bencao, they contained few actual models and were criticised for their lack of detail and regional variation. They are copied without significant innovations in the Jiangxi 江西 edition of 1603 but here they were placed at the beginning of each chapter to which they belong. ${ }^{57}$

nary manual to date, the Tang dynasty Simu an jiji 司牧安驥集. For a recent reconstruction of this text, see Pei Yaoqing 裴耀卿 (1911-71) (ed.) 2004.

54 See Li shu (Siku quanshu zhenben wuji 四庫全書珍本五集 ed., Taipei: Shangwu, 1974, 77.1b, 77.2a-2b.

55 One highlight, shortly after the publication of the Bencao gang$m u$, was the Tu xiang bencao mengquan 圖像本草冢荃 (Illustrated Abolition of Ignorance in Pharmaceutics; 1628). See Unschuld 1986, pp. 247-8.

56 For recent accounts of Bencao gangmu editions, see Hubei sheng zhong yi yao yanjiuyuan (ed.) 1988, pp. 68-100; and Ma Jixing 马继兴 in Qian Chaochen 钱超尘 and Wen Changlu 温 长路 (eds) 2003 (summarised in Wang Jian (ed.) 1996, pp. 37-8). Carla Nappi suggests that the changes in illustrations between the 1596 and 1603 editions are nevertheless significant and she lists the names of 19 entries (without the illustrations) in support. See Nappi 2006, pp. 77-8o. Clearly some illustrations were changed and other minor alterations no doubt resulted from the re-cutting of the woodblocks for the 1603 edition. Yet I remain unconvinced that these reflect a fundamental change in the modes of observation by the illustrator(s), let alone that they might be indicative of a new understanding of the relationship between text and illustration. Also, statistically, these altered illustrations make up a relatively small share in the total number 
Two later editions represent successive re-workings of the illustrations. The Hangzhou 杭州 or Qian Shi 錢氏 edition of 1640 includes three chapters totalling 1,110 illustrations from the hand of Lu Zhe 陸吉. They are the basis for the illustrations in the 1782 Siku quanshu edition. The late Qing Weiguzhai 味古齋 edition (1885) has three chapters with illustrations ( $c .1122$ in total) by Xu Gongfu 許功甫, largely based on the illustrations published in 1640. And although some changes are manifest, especially in the illustrations of plants, overall continuity and minimal variation links these different sets of illustrations. ${ }^{58}$ The majority appear to be imitations of previously transmitted illustrations and only a few appear to be based on new observations or real models. ${ }^{59}$ In comparison with Europe, as Paul Unschuld points out, the history of medical illustration in China shows greater continuities:

Changes in China between the Han period and the end of the Imperial period were always within the system, never of the system. ${ }^{60}$

\section{How to Look: Modes of Visuality}

Of course, Li Shizhen's project, as others that preceded and followed it, was more than simply a Materia Medica. It includes, among other recipes, descriptions that sometimes appear akin to what one might find in a herbal, bestiary or lapidary in the world of the Linceans. And one might argue that its taxonomic ambitions could be as rigorous, albeit conceptually different, as those of a zoologist or botanist in Renaissance Europe. Bencao illustrations therefore should not be judged solely on their pharmaceutical veracity. Yet, when Joseph Needham spoke of the 'relatively low nonsense-content' of Bencao illustrations ('what nonsense there was, never at any time got into their illustrations') are we to take his comment as a silent approval of their reliability for the practical purposes described in the recipes? ${ }^{61} \mathrm{~A}$ key question that remains is whether these illustrations are medically or pharmaceutically expedient at all. Since they were highly conventional, mimetic and not necessarily based on contemporary observations, to

of (reported) illustrations.

58 As Métailié and Haudricourt comment: 'So it took nearly three centuries and about 40 editions before some innovation appeared in the illustrations of the plants in China's most famous materia medica?. See Métailié and Haudricourt 1994, p. 403. My statistics are based on a detailed discussion in Xie Zongwan 谢宗万 in Zhongguo yao xuehui yaoxueshi xuehui (ed.) 1985 .

6o Unschuld 2000, p. 103 .

$61 \quad$ Needham 1986, p. 225 . what extent did they add to the pharmaceutical knowledge reported in the text? Would the user of an un-illustrated Bencao have less clarity about the information conveyed in its written entries?

Much depends on whether historians of medicine choose to discuss developments in so-called 'medical' illustration separately from developments in printing, painting and technical illustration that took place in the centuries from the Song to the late Ming. The utility of Bencao illustrations can be usefully evaluated in this historical and social context. First, there is the issue of artistic innovation. As Lothar Ledderose has shown, in creative techniques as varied as bronze casting and calligraphy, a salient feature of artisan production in traditional China is its reliance on modular production. 'Modular thought' implies that creative authority partly derives from combining and reworking existing models and prototypes. As in the world of the written word, visual mimesis was often the rule rather than exception. Even if artists expressed disdain for modular production, many painters, for instance, still worked along modular lines in creating their compositions by combining existing motifs. ${ }^{62}$

In the case of the naturalist, one might surmise that modularity in the realm of the visual stood in contrast to the expectations that beset the textual scholar. Whereas the compiler or editorial team of a Bencao could demonstrate encompassing scholarship through the exegetical knack of wading through and commenting on constantly accreting textually transmitted knowledge, the illustrator for his part conceived of his task as one of faithfully reproducing illustrations from the past. To do otherwise would mean that he either had to (re-)invent an image from scratch or, alternatively, go out and make observations in nature. The assumption that the illustrator went out to do observations is problematic. Many species to be illustrated would hail from regions far beyond his atelier; illustrators were first and foremost commissioned artists and not necessarily naturalists (although some were closely involved). Even to the keen observer, animals would have formed a particularly difficult category for representation since they moved, changed habitats according to the seasons, and were less 'fixable' against a real or imaginary landscape than rocks or plants.

In addition to modularity, there is the issue of abstraction in visual culture. In a study of technical drawings, Peter Golas summarises changes in painting from the early Song onwards as a trend showing 'a growing prejudice against the inclusion of accurate and detailed visual information, a clear retreat from realism'. He goes on to point out that

62 Ledderose 2000 
this reorientation away from realism in painting influenced other forms of illustration. One important element was that models for illustration were no longer real objects but instead, imitations and copies of earlier drawings:

The non-representational, sometimes almost abstract character of so much of Chinese painting and drawing meant that there was little emphasis on 'getting it right' in the sense of making what was portrayed resemble as closely and convincingly as possible what the viewer saw looking at the object itself. ${ }^{63}$

Commenting on agricultural illustrations, Francesca Bray likewise concludes that:

When it came to practical, technical matters like farming, it seems that those educated Chinese who recorded changes or improvements [to existing illustrations] were satisfied with the power of words to convey material processes, and felt no need or desire to resort to graphics. ${ }^{64}$

Unlike in the case of machines, isolated illustrations of drugs do not illustrate how a particular plant or animal drug works. In the case of animals, plants and minerals, technical information conveyed visually, as for instance in the illustrations in Bencao pinhui jingyao, is limited to representing production procedures. Isolated illustrated models, however, merely identify creatures and otherwise normal looking objects to the user as medically potent. Likewise, part of the aura and efficiency of a drug might be augmented by illustrating it. The dog depicted in a pharmacopoeia is not the guardian dog or intimate companion of the household sphere but an objectified product to be used for medicinal purposes. Yet the didactic motivation underlying the illustrations remains intimately linked to the recipes in the text. Bencao illustrators must have assumed that readers make visual connections between the creatures mentioned in the recipes and those illustrated. In this sense, the medical illustration differs from the prescriptive technical illustration. ${ }^{65}$

Readers' expectations towards Bencao gangmu illustrations should also be viewed in their historical moment, namely, the visual culture and book economy of late Ming China. Meanings associated with the term $t u$ 圖 had changed over time. By Ming times, $t u$, frequently translated as 'picture', was also used to denote a configuration of text alone. Craig Clunas suggests that 'chart' would be more accurate as a translation, and notes that the broad

63 Golas 2005, pp. 199, 200. See also the conclusion to Golas in Bray et al. (eds) 2007.

64 'Agricultural illustrations: blueprint or icon?', in Bray et al. (eds) 2007 , p. $55^{2}$.

65 On the principles underlying technological illustration, see further Golas in Arrault and Jami (eds) 2001. usage of the notion of $t u$ evolved against the background of a debate from the 16 th century onwards that focused on the adequacy of representation and 'the contested primacy of visual and purely verbal explanations of phenomena' ${ }^{66}$ Ming elites had largely lost interest in public pictorial formats such as the mural or wall painting. Yet while the world of book illustrations had unlocked a private pictorial sphere with a quantity of pictorial representations greater than ever before, Ming commentators wrote surprisingly little about them. Ming titles that announced themselves as illustrated without the pictures being essential to the text also did so for external reasons. One important motivation, Clunas highlights, was commercial, to 'add to its allure in the marketplace'. ${ }^{67} \mathrm{~A}$ logical consequence is that the illustrator's aspiration to display his artistic repertoire could detract from the goal of accurate representation. Likewise, the cost of producing richly detailed illustrations may account for the fact that only half of the nearly 2,00o drugs in Bencao gangmu were ever illustrated. ${ }^{68}$ In sum, what motivates the creation and publication of Bencao illustrations is as important a question to consider as our quality assessment of the illustrations themselves.

Charted against the background of developments in fiction and the illustrated vernacular print, Li Shizhen lived at the end of a transitional period between the use of printed illustrations to explain a text or narrative and a later phase in which illustrations mainly fulfilled a decorative and aesthetic function. ${ }^{69}$ In illustrated fiction, illustrators often repeated conventional elements. Reading habits informed the context in which pictures were judged, as Robert Hegel points out:

The quality of book illustrations was adjudged by the degree of perfection of the variations on the conventional rather than by uniqueness; repetition of familiar stock elements allowed quick recognition and ease of comprehension and appreciation. ${ }^{70}$

If illustrators of fiction crafted their images with stock ingredients, it is not impossible to conceive that Bencao illustrators could rightfully do the same. Readers of illustrated fiction may have been familiar with a storyline to the point that the relationship of illustrations to their text had become self-evident or even redundant. In the case of the illustrator of a naturalist work, one would expect that

\footnotetext{
$66 \quad$ Clunas 1997, pp. 105, 108.

67 Clunas 1997, pp. 34-5.

68 On the ascendancy of the imprint over the manuscript and shifts in production costs during this period, see McDermott 2006, ch. 1 and 2 .

69 See McLaren 1998, pp. 58-9.

$70 \quad$ Hegel 1998, p. 312.
} 



Figure 7.4 Yuan heng liao ma ji: a) Determining the age of a horse by dental record; b) Horse scrotum. Source: Yu Chuan 于船 et al. eds, Yuan heng liao majijiaozhu 校注, (Beijing: Beijing nongye daxue, 1990), 85, 127

a reader judged the quality of illustrated animal or plant drugs at least partly by his own observations, environment or previous reading record. Yet this would apply only if this reader was a practitioner intending to use the text as a manual. To others, the presence of sketchy drawings of stones, worms or bees might even have been seen as vulgar and serving a readership for whom the recipes were incomprehensible or insufficient without. The illustrated book was not infrequently condemned as a luxurious item that detracted from the quality of a text. As Clunas notes, 'pleasure, the desire to look, is not invoked in Ming texts except negatively, as a licentious act to be censured and avoided' ${ }^{71}$

\section{Naturalist Illustration}

So the doubts expressed by naturalists in Europe on the use of pictures as adequate media for the purpose of classification - from Galen to Pliny to Linnaeus ${ }^{72}$ - might have found a sympathetic ear in Li Shizhen. What unites most pictorial representations of animals in the sources discussed above is their emphasis on the outward appearance of these creatures. Whereas the texts clearly reveal that, by Li Shizhen's time, physicians had discovered the pharmaceutical workings of the insides of animals, it is equally clear that they had not transferred this knowledge with the same sense for detail into visual media. Illustrations barely touched the surface of the pharmaceutical ideas that could be expressed in text. Rarely did they invite the reader to ponder on the anatomy or internal structure of

\footnotetext{
$71 \quad$ Clunas 1997, p. 39.

$72 \quad$ Freedberg 2003, pp. 5-6.
}

animals as drugs. Instead, illustrators aligned themselves with a tradition that saw pictures as a complement to the identification of objects and, in many cases, the rectification of nomenclature. At least in this sense, natural inquiry was firmly tied to Confucian and neo-Confucian epistemological paradigms such as the 'rectification of names' (zhengming 正名) and 'investigation of things' (gewu 格 物). ${ }^{73}$ Indeed one might argue that Bencao illustrations, rather than illuminating the text, could also obfuscate and confuse the user of it. Whereas the consumer of a textual recipe - a medium suited to impart several dimensions of knowledge such as precedent, usage, circumstance, variation - is less likely to confuse one entry with another, the beholder of an illustration might not be able to distinguish between them without having recourse to a text or name caption. Without more information on the various modalities in which illustration and text came together, it remains difficult to assess their intended function.

To ascribe the lack of morphological detail in Bencao animal illustrations to a dearth of physiological or anatomical expertise is probably inaccurate. Techniques of dissection and skills in the anatomical identification of animal parts are attested in ritual texts dealing with sacrifice and engrained in dietetic culture since antiquity. An observing eye also underlies the degree of detail evinced in some animal depictions preserved in illustrated encyclopaedias that are roughly contemporaneous with Bencao gangmu such as Wang Qi's 王圻 (fl.1565-1614) Sancaituhui 三才圖

73 On Li Shizhen's recourse to 'naming' as an epistemological paradigm in a Ming context, see also Elman in Bretelle-Establet and Chemla (eds) 2007. Its antecedents are discussed in Sterckx 2005 . 





clam (xian 蜆); c) Cow bezoar (niu huang 牛黄, calculi bovis) and dog bezoar (gou bao 狗寶, calculi canis). Source: Bencao gangmu (Shanghai: Shangwu yinshuguan, 1933), vol. 1, 34, 41, 64, 65, 90

會 (Assembled Pictures of the Three Realms). ${ }^{74}$ The richly illustrated veterinary treatises mentioned above likewise contradict poor expertise. There the viewer is invited to focus on an anatomical locus or organ, often indicated by a caption and a connecting line (Fig. 4). To be sure, these texts serve a different purpose in that the illustrator tries to depict physical or behavioural abnormalities against a received model of a normal or healthy horse or ox. But it is precisely here that Bencao illustrations depart from their effectiveness as proper medical or pharmaceutical tools. Only a few depict anatomical parts or organs and many animals, stones and plants would hardly be distinguishable were it not for the name printed on the illustration (Fig. 5). Behind their arrangement lurks the principle that once the reader is able to associate the depicted drug with a primary name, the physician or reader grasps all workings and lore associated with it. Once pictured, a creature is no longer unknown. In that sense the illustration can be seen as a commentarial extension of the text, or as yet another type of 'nomenclature' that serves to circumscribe its properties. In the case of LiShizhen, the illustrations were posthumous, added later and not part of the original copy delivered to Nanjing for cutting shortly after his death. It is doubtful that he saw illustrations as an essential component for the understanding of his work.

Not much has been written on the Bencao illustrations. In the early 189os, the German missionary and sinologist Ernst Faber (1839-99) commented that its 'authors were commonly more familiar with books than with nature, and

74 For a selected survey, see Goodall 1979, pp. 95-126. Note, however, that an equal number of Sancai tuhui illustrations closely resemble the typology of Bencao illustrations, which suggests that some were sourced from the Bencao tradition. the block-cutters had no understanding of either'75 Indeed, a century later, many of the basic questions surrounding the origins, style and quality of Bencao illustrations remain. We do not know what factors determined the illustrators' choice to represent one drug over another or how to assess the results achieved by the pictorial representation of a drug. Were illustrators reconstructing or resuscitating images on the basis of their reading of the text? To what degree was the Bencao illustrator limited by his form of presentation and how did the physician plumb beneath the bodily surface of the creatures illustrated before him? If anything, such questions should encourage historians of Chinese medicine not just to explore 'what' to look at and to search for the physical referent of illustrations, but also to pose the question 'how' a reader or user of Materia Medica was meant to look at them.

\section{Bibliography}

Arrault, A. and C. Jami (eds) 2001, Science and Technology in East Asia, (xxth International Congress of History of Science), vol. 9, Liège: Brepols.

Barnard, N. 1973, The Ch'u Silk Manuscript: Translation and Commentary, Canberra: Monographs on Far Eastern History, Australian National University.

Bray, F. 2007, 'Agricultural illustrations: blueprint or icon?', in Bray et al. (eds), $55^{2}$.

Bray, F., V. Dorofeeva-Lichtmann and G. Métailié (eds) 2007, Graphics and Text in the Production of Technical Knowledge in China: The Warp and the Weft, Leiden: E.J. Brill.

Bretelle-Establet, F. and K. Chemla (eds) 2007, Qu'était-ce qu'ecrire une encyclopédie en Chine?, Extrême-Orient Extrême-Occident, hors série, Saint-Denis: Presses Universitaires de Vincennes.

Buell, P. and E. Anderson 2000, A Soup for the Qan: Chinese Dietary

75 See Fan Fa-ti 2004, p. 108 (quoting the Journal of the North China Branch of the Royal Asiatic Society). 
Medicine of the Mongol Era as seen in Hu Szu-hui's Yin-shan Chengyao, London and New York: Kegan Paul International.

Carletti, S.M., M. Sacchetti and P. Santangelo (eds) 1996, Studi in Onore di Lionello Lanciotti, Naples: Universitario Orientale.

Chen Qiyou 陳奇猷 (ed.) 1991, Han Feizi jishi 韓非子集釋, Gaoxiong: Fuwen.

Chen Songchang 陳松長 1993, "Mawangdui Han mu bohua "shenqi tu” bianzheng'馬王堆漢墓帛畫‘神祇圖'辨正, Jiang Han kaogu1, 88-92.

2006, 'Jianbo “Tianwen qixiang za zhan” yanjiu san ti' 簡 帛“天文氣象雜占”研究三題, in Wuhan daxue jianbo yanjiu zhongxin 武漢大學簡帛研究中心 (eds), Jianbo 簡帛, vol. 1, Shanghai: Shanghai guji, 449-58.

Cigliano, M. 1996, 'Yang Shen (1488-1559), un letterato in esilio e la rivalutazione delle culture minoritarie dello Yunnan', in Carletti, Sacchetti and Santangelo (eds), vol. 1, 353-76.

Clunas, C. 1997, Pictures and Visuality in Early Modern China, London: Reaktion.

Creel, H.G. 1974, 'Shenzi 申子', in Shen Pu-Hai. A Chinese Political Philosopher of the Fourth Century Bc, Chicago: University of Chicago Press.

Dorofeeva-Lichtmann, V. 2007, 'Mapless mapping: did the maps of the Shanhai Jing ever exist?', in Bray et al. (eds), 217-94.

Elman, B. 2007, 'Collecting and classifying: Ming dynasty compendia and encyclopedias', in Bretelle-Establet and Chemla (eds), 143-6.

Fan Fa-ti 2004, British Naturalists in Qing China. Science, Empire and Cultural Encounter, Cambridge, MA.: Harvard University Press.

Fiskesjö, M. 2001, 'Rising from blood-stained fields: royal hunting and state formation in Shang Dynasty China', Bulletin of the Museum of Far Eastern Antiquities 73, 48-192.

Fracasso, R. 1988, 'The illustrations of the Shan hai jing: from Yu's tripods to Qing blockprints', Cina 21, 93-104.

1993, 'Shan hai jing', in Loewe (ed.), 361-2.

1996, 'Guo Pu e lo Shanhai jing', in Carletti, Sacchetti and Santangelo (eds), vol. 2, 6o1-36.

Freedberg, D. 2003, The Eye of the Lynx. Galileo, His Friends, and the Beginnings of Modern Natural History, Chicago: University of Chicago Press, 349-66.

Gao Guangzhen 高光震 1991, 'Bencao tujing yuanliu kao' 本草图经 源流考, in Su Kefu 苏克福, Guan Chengxue and Deng Minglu (eds), 209-16.

Golas, P. 2001, 'Technological illustration in China: a post-Needham perspective', in Arrault and Jami (eds), 43-58.

2005, 'Technical drawing in Ming/Qing China and in Renaissance Europe', in Jiang Xiaoyuan 江晓原 (ed.), 199-208.

2007, "Like obtaining a great treasure": the illustrations in Song Yingxing's The Exploitation of the Works of Nature', in Bray et al. (eds), 590-3.

Goodall, J.A. 1979, Heaven and Earth: 120 Album Leaves from a Ming Encyclopedia: San-ts'ai t'u-hui, 1610, London: Lund Humphries.

Guo Fu 郭郛 2004, Shanhaijing zhu zheng 山海經注証, Beijing: Zhongguo shehui kexue chubanshe.

Harper, D. 1999, 'Warring States natural philosophy and occult thought', in Loewe and Shaughnessy (eds), 870-1.

2007, 'Communication by design: two silk manuscripts of diagrams (Tu) from Mawangdui Tomb Three', in Bray et al. (eds), 169-9o.

Hawkes, D. 1985, The Songs of the South, Harmondsworth: Penguin.

Hayashi Minao 林已奈夫 1971, 'Chōsa shutsudo So hakusho no jūni kami no yurai’ 長沙出土楚帛書の十二神の由來, Tōhō gakuhō $42,1-63$.

Hegel, R. 1998, Reading Illustrated Fiction in Late Imperial China, Stanford: Stanford University Press.
Hubei sheng zhongyi yao yanjiuyuan (ed.) 1984, Li Shizhen yanjiu 李 時珍研究, Guangdong: Guangdong keji chubanshe.

Hubei sheng zhong yi yao yanjiuyuan (ed.) 1988, Li Shizhen shishi kao 李時珍史实考, Guangdong: Guangdong keji chubanshe.

Iannaccone, I. 1996, 'Lo zoo dei Gesuiti: la trasmissione scientifica del bestiario rinascimentale europeo alla Cina dei Qing in Kunyu tushuo di Ferdinand Verbiest (1674)', in Carletti, Sacchetti and Santangelo (eds), vol. 2, 739-64.

Jiang Xiaoyuan 江晓原 (ed.) 2005, Duoyuan wenhua zhong de kexue shi 多元文化中的科学史, Shanghai: Jiaotong daxue chubanshe.

Kalinowski, M. 2003, Divination et société dans la Chine médiévale, Paris: Bibliothèque nationale de France.

Karow, O. 1956, Die Illustrationen des Arzneibuchs der Periode Shaohsing vom Jahre 1159, Leverkusen: Bayer.

Kim Sin-gŭn 金信根 (ed.) 1992, Sinpyŏn chipsŏng uma ŭibang . Umayangjŏ yŏmyŏkbyŏng chiryobang $\cdot$ Magyŏng ch'ojip ŏnhe $\cdot$ ŭnggol pang· ŭigwa sŏnseng an· ŭigwa p'alse po 新編集成牛馬醫方·牛馬 羊猪染疫病治療方·馬經抄集諺解.鷹鶻方·醫科先生案.醫科八世 譜, Seoul: Yŏgang ch'ulp'an sa 驪江出版社.

Knight, J. (ed.) 2004, Wildlife in Asia: Cultural Perspectives, London: RoutledgeCurzon.

Lackner, M. 199o, 'Die “Verplanung” des Denkens am Beispiel der T’u', in Schmidt-Glintzer (ed.), $133-56$.

Lai Guolong 2003, 'The diagram of the mourning system from Mawangdui', Early China 28: 44-8.

Ledderose, L. 200o, Ten Thousand Things: Module and Mass Production in Chinese Art, Princeton: Princeton University Press.

Lewis, M.E. 1990, Sanctioned Violence in Early China, Albany: State University of New York Press.

2006, The Construction of Space in Early China, Albany: State University of New York Press.

Li Haixia 李海霞 2002, Hanyu dongwu mingming yanjiu 漢語動物命 名研究, Chengdu: Ba Shu shushe.

Li Ling 李零 1991, 'Mawangdui Han mu “shenqi tu” ying shu “bibing tu”" 馬王堆漢墓‘神祇圖”應屬 ‘避兵圖’, Kaogu 10, 940-2.

2000 , Zhongguo fangshu kao 中國方術考, Beijing: Dongfang.

Li Xueqin 李学勤 1994, 'Zai lun boshu shi-er shen' 再论帛书十二神, in Jianboyijiyu xueshu shi 简帛佚籍与学术史, Taipei: Shibao, 58-70.

Liu, C.Y., M. Nylan and A. Barbieri-Low (eds) 2005, Recarving China's Past: Art, Archaeology and Architecture of the 'Wu Family Shrines', New Haven/London: Yale University Press.

Liu Keming 刘克明 2003, Zhongguo gongcheng tu xue shi 中国工程 图学史, Wuchang: Huazhong keji daxue.

Liu Pansui 劉盼遂 (ed.) 1990, Lunheng jiaoshi 論衡校釋, Beijing: Zhonghua, 26, 375-6.

Liu Wendian 劉文典 (ed.) 1992, Huainanzihongliejijie 淮南子鴻烈集 解, Taipei: Wenshizhe, 13.432 ('Fan lun').

Liu Yushu 劉玉書 (ed.) 1989, Yinshan zhengyao 饮膳正要, Beijing: Renmin weisheng chubanshe.

Lo, V. 2001, 'Huangdi Hama jing (Yellow Emperor's Toad Canon)', Asia Major, 3rd series 14.2, 61-99.

Loewe M. (ed.) 1988, Early Chinese Texts: A Bibliographical Guide, Berkeley: Institute of East Asian Studies.

Loewe M. and E. Shaughnessy (eds) 1999, The Cambridge History of Ancient China, New York: CUP.

Lu Qinli 逯欽立 (ed.) 1979, Tao Yuanming ji 陶渊明集, Beijing: Zhonghua.

McDermott, J.P. 20o6, A Social History of the Chinese Book. Books and Literati in Late Imperial China, Hong Kong: University of Hong Kong Press.

McLaren, A. 1998, Chinese Popular Culture and Ming Chantefables, Leiden: E. J. Brill. 
Ma Jixing 马继兴 2003, 'Bencao gangmu chuban de kaocha' 本草纲 目出版的考察, in Qian Chaochen 钱超尘 and Wen Changlu 温长 路 (eds), 16-27.

Major, J. and C. Cook (eds) 1999, Defining Chu: Image and Reality in Ancient China, Honolulu: University of Hawai'i Press.

Métailié, G. 1998, 'Un manuscrit en quête d'auteur. Du Plinius Indicus de Johan Schreck au Bencao gangmu de Li Shizhen et au Bencao Pinhui Jingyao de Liu Wentai', Journal Asiatique, 286.1, 211-33.

2007 , 'The Representation of plants: engravings and paintings', in Bray et al. (eds), 487-520.

Métailié, G. and A.G. Haudricourt 1994, 'De l'illustration botanique en Chine', Études chinoises 13.1-2, 381-416.

Nappi, C. 2006, 'The Monkey of the Inkpot: natural history and its transformations in early modern China', PHD diss., University of Princeton.

Needham, J. 1986, Science and Civilisation in China, Cambridge: Cambridge University Press, vol. 6, pt I.

Pei Yaoqing 裴耀卿 (ed.) 2004, Si mu an ji ji yu shi 司牧安焂集語釋, Beijing: Nongye chubanshe.

Powers, M. 1991, Art and Political Expression in Early China, New Haven/ London: Yale University Press.

Qian Chaochen 钱超尘 and Wen Changlu 温长路 (eds) 2003, Li Shizhen yanjiujicheng 李时珍研究集成, Beijing: Zhongyi guji chubanshe.

Reiter, F.C. 1992, 'Some remarks on the Chinese word T'u "chart, plan, design"', Oriens 32, 308-27.

Schmidt-Glintzer, H. (ed.) 1990, Lebenswelt und Weltanschauung im Frühneuzeitlichen China, Stuttgart: Franz Steiner.

Shang Zhijun 尚志钧 (ed.) 1994, Bencao tujing 本草图经, Hefei: Anhui kexue jishu chubanshe.

Sterckx, R. 2002, The Animal and the Daemon in Early China, Albany: State University of New York Press.

2004, 'Attitudes towards wildlife and the hunt in pre-Buddhist China', in Knight (ed.), 15-35.

2005, 'Animal classification in ancient China', East Asian Science, Technology and Medicine 23, 26-53.

in press, 'Zoomorphism and sacrificial religion in early China', in E. Wang (ed.).

Su Kefu 苏克福 1991, Guan Chengxue and Deng Minglu (eds), Su Song yu Bencao tujing yanjiu 苏颂与本草图经研究, Changchun: Changchun chubanshe.

Sun Yirang 孫詣讓 (ed.) 1987, Zhouli zhengyi 周禮正義, Beijing: Zhonghua, 18.689 ('Da situ').

Tang Bingzheng 湯炳正, Li Daming 李大明, Li Cheng 李誠 and Xiong Liangzhi 熊良智 (eds) 1996, Chuci jin zhu 楚辭今注, Shanghai: Shanghai guji.
Unschuld, P. 1986, Medicine in China: A History of Pharmaceutics, Berkeley: University of California Press.

200o, Medicine in China: Historical Artifacts and Images, Munich/London: Prestel.

Wang E. (ed.) in press, Zoomorphic Imagination in Chinese Visual Culture, Periscope and Isabella Stewart Gardner Museum.

Wang Hongbin 王鸿宾 2006, 'Tan “Erya tu” ji qi yuanzuozhe Guo Pu' 谈‘尔雅图'及其原作者郭璞, Wenwu shijie 1, 47-50/70.

Wang Jian 王剑 (ed.) 1996, LiShizhen xueshuyanjiu 李时珍学术研究, Beijing: Zhongyi guji chubanshe.

Wang Liqi 王利器 (ed.) 1993, Yanshijiaxun jijie 顏氏家訓集解, Beijing: Zhonghua, 3.226 ('Mian xue'勉學).

Wang, M.C., E. Wang, R. Sterckx and Guolong Lai 2006, A Bronze Menagerie: Mat Weights of Early China, Boston: Isabella Stewart Gardner Museum and University of Pittsburgh Press.

Wu Hung 1989, The Wu Liang Shrine: The Ideology of Early Chinese Pictorial Art, Stanford: Stanford University Press.

Wuhan daxue jianbo yanjiu zhongxin 武漢大學簡帛研究中心 (eds) 2006, Jianbo 簡帛, vol. 1, Shanghai: Shanghai guji.

Wylie, A. 1901, Notes on Chinese Literature: With Introductory Remarks on the Progressive Advancement of the Art; and a List of Translations from the Chinese into Various European Languages, Shanghai: American Presbyterian Mission Press (Preface 1867).

Xia Henglian 夏亨廉 and Lin Zhengtong 林正同 (eds) 1996, Handai nongye huaxiang zhuanshi 汉代农业画像砖石, Beijing: Zhongguo nongye chubanshe.

Xie Zongwan 谢宗万 1985, 'Bencao gangmu tuban de kaocha' 本草纲 目图版的考察, in Zhongguo yao xuehui yaoxueshi xuehui中国药 学会药学史学会 (ed.), 145-99.

(ed.) 2001, Bencao gangmuyaowu caise tujian 本草綱目藥 物彩色圖鑒, Beijing: Renmin weisheng chubanshe.

Yang Bojun 楊伯峻 1995, Chunqiu Zuozhuanzhu 春秋左傳注, Beijing: Zhonghua, (Lord Xuan, year 3).

Yang Xiaoneng 200o, Reflections of Early China: Decor, Pictographs, and Pictorial Inscriptions, Seattle and London: The Nelson-Atkins Museum of Art and University of Washington Press.

Zhao Zhongyi 趙仲邑 (ed.) 1997, Xinxu xiang zhu 新序詳註, Beijing: Zhonghua.

Zheng Jinsheng 鄭金生 2003, 'Mingdai huajia caise bencao chatu yanjiu' 明代畫家彩色本草插圖研究, Xinshixue 新史學 14.4, 65-120.

Zhongguo yao xuehui yaoxueshi xuehui 中国药学会药学史学会 (ed.) 1985, Li Shizhen yanjiu lunwen ji 李时珍研究论文集, Hubei kexue jishu chubanshe.

Zhou Shirong 周世榮 199o, 'Mawangdui Han mu de "shenqi tu” bohua' 馬王堆漢墓的 ‘神祇圖’ 帛畫, Kaogu 10, 925-8. 


\title{
8 Observational Drawing and Fine Art in Chinese Materia Medica Illustration
}

\author{
ZhengJinsheng 鄭金生
}

There are some 40 illustrated ancient Chinese Bencao (Materia Medica texts or herbals) in existence today, containing a grand total of over 10,00o images (not counting duplicates). Given that these illustrations are intended to facilitate the identification of raw materials and the preparation of medicines, it can be argued that the primary criterion for fitness to purpose must be truth to life. As we shall see, however, Materia Medica illustration fulfilled a multiplicity of functions.

Much of the scholarship evidenced in the earliest Bencao texts, is focused on correctly identifying medicinal substances and establishing their provenance, so as to ensure that they are safe and effective. These concerns fuelled the rise of a distinct genre of Materia Medica illustration.

The term 'bencao tu' - Materia Medica illustration makes its first appearance in Suishu:Jingji zhi 隋書·經 籍志 (The Bibliographic Catalogue of the Sui Dynastic History [581-618]), which lists an illustrated album entitled Lingxiu bencao tu 靈秀本草圖 (Exquisitely Executed Materia Medica Illustrations). ${ }^{1}$ Lidai minghua ji 歷代名畫記 (Record of Famous Paintings Through the Ages) by Zhang Yanyuan 張彥遠 (Tang period, 618-907) cites Shen Nong bencaojing litu 神農本草經例圖 (Exemplary Illustrations from Shen Nong bencaojing), ${ }^{2}$ indicating that an illustrated supplement to Shen Nong bencao jing 神農本草經 (The Divine Farmer's Canon of Materia Medica) was already in circulation prior to the Tang. The first volume of Materia Medica illustrations to be produced under official auspices was Xinxiu bencao: Yao tu 新修本草.藥圖 (Newly Edited Pharmacopoeia: Drug Illustrations), published under the Tang in 659. At all events, owing to the limited diffusion of illustrated manuscripts, none of the hand-drawn Materia Medica illustrations produced up to and including the Tang period have survived. Thus, the story of the genre really begins in the Northern Song period (96o-1127), with Su Song's 蘇頌 Bencao tujing 本草圖經 (Illustrated Classic of Materia Medica).

From the Northern Song onward, all Bencao have illustrations. At first glance, these images seem remarkable for their diversity; they may be black and white or polychrome, they range from rough sketches to exquisitely finished artwork, and they include a subset depicting drug ingredients. But from the point of view of what they set out

\footnotetext{
$1 \quad$ Wei Zheng and Linghu Defen, Suishu:jingji zhi, p. 1,o44.

2 Zhang Yanyuan 1987, pp. 313-14.
}

to represent, they fall readily into two categories: visual records based on actual specimens, drawn from life, and artists' impressions created without direct reference to the originals. By establishing in which areas of the Bencao literature each of these two types of illustration tends to occur, and investigating the identity of the artists, the extent of their knowledge of botany and pharmacy, and the purposes served by the illustrations, we will be better equipped to evaluate these illustrations, and hence to make effective use of them as a resource.

This paper will look separately at observational and artistic Materia Medica illustration.

\section{Observational Materia Medica Illustration}

Bencao tujing 本草圖經 (Illustrated Classic of Materia Medica), published 1061 by the Northern Song writer Su Song 蘇頌 and collaborators (Fig. 1), is China's earliest extant illustrated Bencao. It contains 933 illustrations, all woodblock prints from ink and brush line drawings, based on specimens and pictures culled from all over China.

The compilation of Bencao tujing followed a pattern established by Xinxiu bencao, the state-sponsored pharmacopoeia of the Tang dynasty. A government decree went out to all drug-producing areas, requiring persons with a good knowledge of harmacy to make close observations of the roots, stems, shoots, leaves, flowers and fruit of plants, taking note of their external appearance, colour and size.



Figure 8.1 Pharmaceutical illustrations, from Bencao tujing 本草圖 經 (Illustrated Canon of Materia Medica), 1062 




Figure 8.2 Mineral drugs, from Li Shizhen 李時珍, Bencao Gangmu 本草纲目 (Systematic Materia Medica)

Other items with medicinal uses such as insects, fish, birds, beasts, jade and minerals also had to be observed and recorded (Fig. 2). A drawing was to be made of each item, with notes on flowering, fruiting and harvesting times. In the case of items imported from abroad, foreign merchants were to be questioned, and one or two samples of each item were to be obtained and taken by courier to the capital to be drawn. ${ }^{3}$ Research has shown that the book also makes use of illustrations from earlier pictorial Bencao in the state archives, ${ }^{4}$ as well as drawings obtained from various parts of China during the process of unification under Northern Song rule. Thus Bencao tujing exhibits the following characteristics:

the majority of the illustrations were executed by local experts in pharmacy from the 150 national administrative districts, and almost all of them were drawn from life;

to comply with official guidelines, it was necessary to depict the roots, stems, leaves, flowers and fruit of each plant in its naturally occurring state.

'Tujing bencao zouchi' 圖經本草奏敕, quoted in Tang Shenwei, Chongxiu Zhenghe jingshe zhunyong bencao, p. 548.

$4 \quad$ Zheng Jinsheng 1995 .
Thus many of the illustrations go to great lengths to provide a comprehensive record of the morphology of the source item (i.e. the original animal, plant or mineral), squeezing miniaturised views of entire plants (including large trees such as the clove tree of Guangzhou) into the available space. At the same time, the artists deliberately sought to highlight the parts with medicinal uses, which sometimes led them to distort natural proportions (as in the illustrations of the clove tree and the honey locust [Gleditsia]). The resulting illustrations are somewhat diagrammatic in character.

Already in Bencao tujing, there are instances of illustrations showing pharmaceutical substances, i.e. raw drugs (e.g. dried ginger, euryale seed (jitou shi 雞頭實) or Fuzhou anise). Also, in some entries on larger plants, a part such as a twig is used to stand by synecdoche for a whole tree or vine (see for example the entries on magnolia, privet, arborvitae seed (baishi 柏實) and cork-tree bark in the sections on fruit and trees).

On the whole, the illustrations of Bencao tujing are observational in style. However, in the case of substances originating outside China, or in remote parts of the country, which could not therefore have been viewed in their original form, the illustrations are artists' reconstructions, based on textual descriptions (e.g. Dragon's Blood (qilingjie 麒麟 竭) from Guangzhou; Eaglewood (chenxiang 沉香) from Yazhou 崖州 on Hainan island) or existing collections of illustrations (e.g. borneol [longnao 龍腦]). Because Bencao tujing was of such great practical use, many post-Song Bencao drew on it, or reproduced illustrations from it.

Subsequently, changes in the subject matter and methodology of Bencao studies led to a gradual decline in observational illustration. Starting in the Yuan period (1279-1368), the focus of Bencao scholarship gradually shifted to pharmacology and the clinical applications of drugs. With increasingly professional specialisation, the distance grew between the figure of the apothecary and that of the physician, who had less and less hands-on contact with drugs in their raw form. Thus, following the achievement of Bencao tujing, the tradition of drawing from life saw little further development within the Bencao mainstream. Though illustrated books on materia medica with a realistic or observational character do continue to appear after the Song period, the vast majority of these are personal projects by scholars and artists. The most significant examples are described below.

\section{Lü Chanyan Bencao 履㟴岩本草 (The Cliff Walker's Materia Medica) 1220}

Lü Chanyan bencao is the work of Wang Jie 王介, who flourished around the turn of the 13th century, under the 




Figure 8.3 Shanjianghua 山薑花 (Alpinia japonica), from Lü Chanyan bencao (Cliff Walker's Materia Medica)

Southern Song dynasty. Wang Jie was an artist by profession. According to Tuhui yujian 圖繪寶鑒 (The Precious Mirror of Painting), an art-history text compiled in the Yuan period, he

liked to paint human subjects and landscapes in the manner of Ma Yuan 馬遠 (active 119o-1224) and Xia Gui 夏珪 (active 1180-1230) and was also a skilled painter of plums and orchids. ${ }^{5}$

As his soubriquet Lü Chanyan - the Cliff Walker - implies, ${ }^{6}$ Wang Jie resided in a mountain retreat, where numerous species of medicinal plants grew wild. In his book, he depicts more than 200 plants from first-hand knowledge. On textual evidence, he was based in the western Ciyunling 慈雲嶺 (Cloud of Compassion) hills, outside the Southern Song capital Lin'an 臨安 (modern Hangzhou). This means that Lü Chanyan bencao provides a window on local herbal medicine in the Hangzhou area in the early 13 th century. ${ }^{7}$ This book, which is divided into three juan, or fascicles,

Xia Wenyan, Tuhuiyujian, juan 4, p. 22.

Wang Jie, Lü Chanyan bencao, preface.

Zheng Jinsheng 1980, p. 338. originally had 206 polychrome illustrations, 202 of which still survive today. Drawn from life by a master of flower painting, they are superbly executed, and the paintings of flowering plants are especially fine (Fig. 3). Even though the book contains no descriptions whatever of plant morphology, almost all the plants in it can be identified by species and genus on the strength of the illustrations alone. Wang Jie's paintings have made it possible to identify some two dozen herbs used in Southern Song popular medicine which are not represented in any preceding Bencao, e.g. Datura (mantuoluo 曼陀羅), saxifrage (hu'er cao 虎耳草), Buddleia (zuiyu cao 醉魚草).

From a technical standpoint, where the illustrations in Lü Chanyan bencao improve most notably on their predecessors is in their adherence to natural proportions. In the case of large plants, this is achieved through the technique of using a part to represent the whole, in such a way as to emphasise the plant's distinguishing features. Wang Jie borrowed this technique from the celebrated Southern Song landscape artists Ma Yuan and Xia Jia, already mentioned above, and applied it to botanical illustration. Ma Yuan and Xia Jia specialised in 'partial' (banbian 半邊) or 'single-perspective' ( yi jiao 一角) composition, a highly individual departure from the Northern Song panoramic style exemplified by the famous scroll painting Qingming shanghe tu 清明上河圖 (On the River at Qingming Festival) by Zhang Zeduan 張擇端 (1085-1145). Because of this, they were nicknamed 'Ma yi jiao' 馬一角 and 'Xia banbian' 夏 半邊 (lit. One-angle Ma and One-sided Xia). Influenced as he was by Ma and Xia, Wang Jie made liberal use of their 'partial' techniques to give prominence to the most characteristic parts of each plant. Unfortunately for us, Wang Jie was not an expert in pharmacy. He was however a keen alchemist, involved in experiments to create elixirs of longevity. He therefore depicted several dozen plants used in alchemical processes, but as these are shown only in general outline, without flowers or fruit, they cannot readily be identified.

However, with colour printing technology still in its infancy, the only means of reproducing and disseminating polychrome Materia Medica illustrations like these was hand copying. This inevitably limited their diffusion. Thus although the text of Lü Chanyan bencao is cited in subsequent literature, the illustrations remained largely unknown, and neither the images themselves nor Wang Jie's style and technique influenced later Bencao illustration.

\section{Jiuhuang Bencao 救荒本草 (The Famine Relief Herbal)}

Among woodblock printed Materia Medica illustrations, the most realistic in character are those found in Jiuhuang 




Figure 8.4 Astragalus (Huangqi 黄暮), from Jiuhuang bencao 救荒 本草 (the Famine Relief Herbal) compiled by Prince Zhu $\mathrm{Su}$ 朱橚, 1403-24

bencao (Fig. 4), compiled in the course of $1403^{-24}$ by Prince Zhu Su 朱橚 (1362?-1425), fifth son of the founding emperor of the Ming dynasty, Tai $\mathrm{Zu}$ 太祖 (given name Zhu Yuanzhang 朱元璋, 1162-1227). A vassal prince of the Ming, Zhu Su was posthumously honoured with the title of Prince of Zhou. It is fair to say that Prince Zhu went about the task of compiling a famine herbal in a way that had no precedents and would find few imitators. From mountain farmers, he acquired seedlings of plants that could be used as emergency food in times of famine, and planted them in a garden, where he personally supervised their cultivation. After that he 'waited for them to grow to maturity, and then had drawings made of them by artists. ${ }^{8}$ The herbal has 414 illustrations: 138 of them are taken from earlier publications, but the remaining 276 were all specially drawn.

Owing to the way in which Jiuhuang bencao came into being, it is possible to be confident that the illustrations were drawn from life. Moreover the fact that they were prepared by professional artist-craftspeople is a guarantee of accuracy and stylistic consistency. As artwork, they are admittedly, pedestrian and uninspired, but considered as examples of technical drawing, they are the most useful



Figure 8.5 Pharmaceutical illustrations, from Li Zhongli 李中立, Bencao yuanshi 本草原始 (Origins of Materia Medica), created 1612

for plant identification of any of the woodcut Materia Medica illustrations. It is only to be regretted that the approach to Materia Medica studies pioneered by Jiuhuang bencao, based on observation and recording in the field, did not catch on in China. The book was however highly influential in Japan. Japanese scholars of the Edo period (1603-1868) upheld the tradition of scholarship it initiated, carrying out numerous field studies, and writing up their data in a series of monographs on botany and agronomy. Thus Jiuhuang bencao came to play a crucial role in the development of Japanese botanical science.

Bencao yuanshi 本草原始 (Origins of Materia Medica), created by Li Zhongli 李中立 (Ming period) in 1612, was the first illustrated handbook containing observational drawings of medicinal substances (in their raw state) to be published in China (Fig. 5). Li Zhongli was a native of Yongqiu, in today's Qi 杞 county, Henan province. By his lifetime, there was a clear demarcation between the role of the apothecary and that of the physician. Many doctors were unfamiliar with raw drugs, which led to medication errors. In his 12-juan work, Li Zhongli took as his point of the departure the drugs that contemporary doctors could be expected to have come across, so as to 'fit the names to the substances, verify their thermostatic character and sapors, distinguish their appearance, and establish their 
potencies', setting out to inform through the medium of pictures as well as text. ${ }^{9}$

Bencao yuanshi has 508 entries and 442 illustrations. What is unusual about these illustrations is that Li Zhongli has turned his back on the practice of showing medicinal items in their natural state, focusing instead on the parts with pharmaceutical uses (raw drugs). In this, he recognised that it was unrealistic to suppose that his contemporaries in the medical profession would venture into the mountains to gather simples; but that raw drugs were readily accessible to them. An accomplished artist as well as a writer, Li Zhongli illustrated the book personally, accurately recording the appearance and morphology of each substance, which greatly adds to the scholarly value of the work.

Li Zhongli's book exerted a considerable influence. Illustrations of raw drugs in imitation of Bencao yuanshi are included in various editions of Bencao huiyan 本草匯言 (Collected Remarks on the Materia Medica) by Ni Zhumo 倪朱謨 (Ming period), Bencao hui 本草匯 (Anthology of Materia Medica) by Guo Peilan 郭佩蘭 (Qing), Bencao Gangmu leizuan bidu 本草綱目類纂必讀 (Essential Readings from [Li Shizhen's] Bencao gangmu [Classified Materia Medica]) by He Zhen 何鎮 (Qing), and Bencao qiuzhen 本草求真 (Quest for Truth in the Materia Medica) by Huang Gongxiu 黃宮繡.

Zhiwu mingshitukao 植物名實圖考(Illustrated Survey of Plants and Plant Names) by Wu Qijun 吳其濬 (Qing period) was completed in 1846. In the Qing period, mainstream pharmacological research focused on the clinical applications of drugs, and in consequence no noteworthy examples of observational Materia Medica illustration were produced. However, at the same time, a great upsurge in interest in botany gave rise to botanical drawing of a very high order.

Wu Qijun 吳其濬 (1789-1846), also known as Yuezhai 淪齋 and Yu Lounong 雲婁農, was a native of Gushi 固 始 in Henan province. A career official, he achieved the distinction of coming top in the nationwide imperial examinations in 1817 (22nd year of the Jiaqing reign period). However, his private passion was botany. He took advantage of official postings throughout China to investigate the local flora, and carried out textual research which he published as Zhiwu mingshitukao changbian 植物名實圖 考長編 (Illustrated Compilation of Research on Plants and Plant Names). The 38-juan Zhiwu mingshi tukao brings together drawings and observations that he made in the field.

Although Zhiwu mingshi tukao is not a pharmaceutical text as such, it does include a great many plants with

$9 \quad$ Li Zhongli, Bencao yuanshi, preface. medicinal uses. Of 1,805 illustrations, 1,500 are drawn from life, and only about 300 are reproduced from literary sources, including Bencao tujing, Jiuhuang bencao, Bencao gangmu (Qian Weiqi 錢蔚起 edition, 1640), and Gujin tushu jicheng (Collected Texts and Images from Antiquity to the Present Day); thus the main body of the work consists of original illustrations drawn from life. In the entry on guijiu 鬼臼 (Podophyllum emodi; Dysosma versipellis; Dysosma pleiantha), Wu Qijun records how he came upon the plant:

This plant grows deep in the mountains, and very few northerners have sight of it... on my travels, I chanced to encounter some mountain dwellers who were transporting a plant with huge flowers and leaves on their backs to the city. I made an impromptu sketch of it. ${ }^{10}$

A top-ranking scholar, Wu was a man of uncommon talents. He had a discerning eye for the subtleties of plant identification, and drew with finesse, grace and fluency, which enabled him to bring out the distinguishing features of the plants he depicted from first-hand observation. Furthermore, in comparison with preceding Bencao woodcut illustrations, this book shows a significant advance in attention to scale and proportion. Where it is not feasible to show a tree or creeper in its entirety in a single plate, $\mathrm{Wu}$ instead provides a detailed view, so as to give prominence to characteristic parts of the plant. His lifelike, rigorously accurate drawings, accompanied by meticulous descriptions, achieve a precision that is comparable to modern scientific illustration, and are extremely valuable to scholars.

Zhiwu mingshi tukao belongs to the late Qing period, when Western botanical science was making its way into China. This threw up an issue that has continued to exercise scholars in China and abroad - how to integrate China's vast botanical resources, and the wide range of literary sources relating to plant life, with the advanced taxonomic systems developed in the West. Zhiwu mingshi tukao was able to bridge the gap between ancient Chinese Bencao studies and agronomy, and early modern botanical science thanks to a particular combination of strengths: it brought together an extensive corpus of data, involved field studies, and accurately depicted plant morphology. Many current Chinese terms for botanical families, genera and even species are directly derived from Zhiwu mingshi. At the same time, although it is not itself a Bencao, it is very much an heir to and product of the Bencao tradition.

In addition to the illustrated Bencao described above, some realistic illustrations are also to be found in local Bencao, such as the illustrated versions of Diannan bencao 滇南本草 (South Yunnan Bencao). Diannan bencao is attributed to the Ming author Lan Mao 蘭茂 (1397-1476);

Wu Qijun, Zhiwu mingshi tukao, juan 24, p. 567. 
however it has been transmitted in various versions with discrepancies in both text and illustrations, and it is impossible to establish which of these might represent Lan Mao's original work. Diannan bencao tushuo 南本草圖說 (Illustrated Guide to Diannan bencao) is a Qing work, which survives in a manuscript copy made by Zhu Jingyang in 1773 (38th year of the Qianlong reign period of the Qing dynasty). It contains 221 illustrations, one for each medicinal item. Most of them are realistic in style, but owing to the technical limitations of the artist, they are crude and simple in execution. The Wuben Tang edition of Diannan bencao, engraved in 1887 (23rd year of the Guangxu reign period) has 70 illustrations, which are similarly crude and perfunctory, and it is hard to determine whether they were based on actual specimens.

Caoyao tujing 草藥圖經 (Illustrated Classic of Herbal Medicine, 1827), composed by De Feng 德豐 and edited by Mu Shufan, has 6 o illustrations, one for each drug item. These graceful, naturalistic images do not appear to replicate earlier published illustrations; however it is impossible to tell whether they were actually drawn from life. Of particular interest is Bencao bianfang 本草便方 (Everyday Remedies from the Pharmacopoeia), written and illustrated by Liu Shanshu (Qing period) (Fig. 6). Liu Shanshu died after drafting the work, which was edited for publication by his son Liu Shiji, and appeared in print posthumously in 1870 . It contains 508 illustrations altogether. Though they may strike one as crude and unpolished, they are nonetheless effective in conveying the distinguishing features of medicinal plants, which makes this book an essential source for the study of herbal folk medicine in the Sichuan region.

The brief survey above takes in the main examples of observational Materia Medica illustration. This begs the question of whether the illustrations in Li Shizhen's monumental and immensely influential Bencao gangmu can be regarded as observational drawings.

The earliest edition of Bencao gangmu 本草綱目 (Classified Materia Medica) (the Jingling, i.e. Nanjing, edition of 1593) includes two juan of illustrations, with 1109 images in all. In terms of composition and execution, they strike one as crude and ungainly, a world away from the accomplished productions that adorn the Song Bencao tujing and the MingJiuhuang bencao. In fact, as studies have shown, they are the work of Li Shizhen's sons and grandsons, under pressure to meet a publisher's deadline..11 To judge from various discrepancies between the illustrations and textual descriptions (see, for example, the entries on Gujing cao 穀精草 (Eriocaulon buergerianum Koern)

$11 \quad$ Miyashita 1979.



Figure 8.6 Pharmaceutical illustration: Epimedium (Xianlingpi 仙灵 脾) from Bencao bian fang 本草便方 (Everyday Remedies from the Pharmacopoeia) 1870

and Bihuilei 辟虺雷 (Radix Aristolochiae Cinnabarinae), Li Shizhen himself had no hand in them, and probably did not even supervise their execution. Moreover, Li Shizhen's family members lacked the expertise to produce artwork of a high professional standard. But for all these shortcomings, one should not lose sight of the fact that 91.3\% of the illustrations were specially drawn for Bencao gangmu, ${ }^{12}$ which makes the proportion of reproductions from previous Bencao remarkably low. In view of the close relationship between Li Shizhen and his sons and grandsons, their illustrations are the closest one can get to his original intentions. Though poor execution reduces their scholarly value, they are mostly drawn from life. In later times, the illustrations twice received a thorough aesthetic makeover, which resulted in major differences from the first edition. These later versions cannot be used as evidence in any discussion of the significance of the illustrations in Bencao gangmu.

Liu Wentai's 劉文泰 Bencao pinhui jingyao 本草品彙 精要 (Classified Treasury of Materia Medica, 1505) also deserves mention here (Fig. 7). The polychrome paintings that are a feature of this work are in fact, for the most part, coloured versions of illustrations from Bencao tujing. And while it does contain nearly 400 observational drawings, they are mainly restricted to everyday foodstuffs rather than items commonly used as medicines, which limits their usefulness for the identification of medicinal substances. This book is primarily an example of artistic illustration, and will be dealt with below under that heading.

12 Xie Zongwan 1985, p. 191. 
It should be borne in mind that the Materia Medica illustrations found in Ming and Qing editions of the pharmacological literature do not necessarily belong to the original texts. Because of this they cannot be readily categorised in terms of 'observational' versus 'artistic' illustration, and are probably best described as 'decorative'. They were often added by profit-conscious booksellers and publishers to satisfy an appetite for lavishly illustrated printed books like the illustrated (xiu xiang 繡像) novels of the period. For example, both Bencao mengquan 本草 蒙鉒 (Beginner's Guide to Materia Medica, 1565) by Chen Jiamo 陳嘉謨 (Ming period) and Bencao beiyao 本草備 要 (Ready-Reference Materia Medica, 1683, 1694) by Wang An 汪昂 (Qing period) were originally unillustrated, but were later embellished with illustrations purloined from other Materia Medica texts. This is reflected in sometimes bizarre mismatches between image and text. In the case of Li Shizhen's Bencao gangmu, the book was recognised as a masterpiece, and so the rough-and-ready illustrations of the first edition were repeatedly re-engraved. Successive engravers sought to improve on the original illustrations and make them more aesthetically appealing; and in this process, the illustrations underwent two full-scale makeovers, which changed them almost beyond recognition. These are typical examples of the impact of commercial factors on Materia Medica illustration.

\section{Artistic Materia Medica Illustration}

Pre-Tang Materia Medica illustrations are valued today by collectors as works of art, while many early paintings in the 'flowers, birds, insects and fish' genre were painted from life in a realistic idiom. Thus, no hard and fast distinction can be drawn between Materia Medica illustration and 'fine art' painting. There is an unbroken tradition of visual art with medicinal substances as its subject matter, stretching from antiquity, that did not cease with the rise of woodcut Bencao illustration in the Song. Lüchanyan bencao 履嗳岩 本草 (The Cliff Walker's Materia Medica, 1220) by Wang Jie 王介, discussed above, is a fine example of this.

The expression 'artistic illustration', as used here, does not imply any judgment of artistic merit. It refers to illustrations that are not, in intent, primarily documents of observed reality. 'Artistic Materia Medica illustrations' are artistic in conception and treatment; they are shaped by aesthetic concerns; they make use of symbolism and allusion; and they are not confined to the literal representation of nature. Like today's 'fine art photography', they may creatively transform reality or construct an alternative reality.



Figure 8.7 Chengzhou lily 成州百合, from Bencao pinhui jingyao (Materia Medica Containing Essential and Important Material Arranged in Systematic Order)juan 10. (C) Digital collections, Staatsbibliothek zu Berlin

The germs of artistic Materia Medica illustration can already be seen in the Song Bencao tujing. A very small number of the illustrations in this book showing non-indigenous plants are imaginative creations. However the first truly representative example of this genre is probably the Ming work Bencao pinhui jingyao 本草品彙精要 (1505). The genre would continue to develop and prosper in successive polychrome pictorial Bencao.

Bencao pinhui jingyao contains 1,357 illustrations, executed by Wang Shichang 王世昌 and seven other courtly artists in the meticulous gongbi 工筆 style. Numerous polychrome Materia Medica illustrations were created in the course of the Ming period; however, these beautiful but impractical artefacts were difficult to reproduce and out of step with the evolving requirements of Bencao scholarship. Bencao pinhui jingyao was made possible by particular political circumstances. Under the patronage of the Xuande emperor of the Ming dynasty (r. 1425-35) and his successors down to the Hongzhi emperor (r. 1487- 
1505), the imperial Academy of Art (Huayuan 畫院) had flourished, providing a suitable milieu for the endeavour. The involvement of court artists gives the illustrations their particular artistic character. The fact that Ming polychrome Materia Medica illustrations such as these were circulated and copied by artists long afterwards demonstrates how seriously they were taken as works of art.

Six hundred and ninety-nine of the illustrations in Bencao pinhui jingyao are coloured versions of the ink and brush line drawings that illustrate Bencao tujing. The remaining 668 were especially created for the book. The latter include 384 depictions of common birds, beasts, vegetables, grains, insects and fish, and fruits, all apparently based on preparatory sketches from life. ${ }^{13}$ These exquisite illustrations, the most beautiful and the most precise in the book, have great artistic and scientific value, and constitute the crowning achievement of the illustrators. At all events, observational illustrations make up only $28.3 \%$ of the total. The coloration of monochrome illustrations is in itself an artistic activity, in that it adds a fictive, imaginative dimension to the image, which is no longer 'true to life'.

The 'artistic illustrations' in Bencao pinhuijingyao have a distinctive style and character. They frequently include background scenery, fantastic elements, and scenes of human activity (e.g. collecting medicinal plants, preparing and manufacturing drugs and everyday pursuits). They can be divided into three main types:

1. Ninety-five 'ancillary illustrations', incorporating 147 human figures. These do not depict the actual appearance of the drug, but illustrate its habitat, collection, processing and preparation, method of use, and customs or traditions associated with it, as described in the text.

2. Over 10o illustrations involving fabulous creatures and other fantastic elements - some suggested by the text, some more freely invented. For example the entry on so-called 'dragon's bone' is accompanied by a picture of a dragon.

3. Coloured and retouched versions of monochrome line drawings from Bencao tujing. One of the techniques used by the Ming artists to enhance the originals they were working from was 'grafting' (jiajie 嫁接), whereby drawings of the medicinal parts of an item were inserted into the original image. In general then, the genre of 'artistic Materia Medica illustration' was not restricted to realistic representation. It allowed artists to give free rein to their imagination and expressive skills.

Two Ming works with illustrations modelled on Bencao pinhuijingyao are Shiwu bencao 食物本草 (Materia Dietetica) and BuyiLeiGong paozhibianlan 補遺雷公炮製便覽 (Lei Gong's Guide to Drug Preparation with Addenda). Both

13

Zheng Jinsheng 2003 . these books contain an even larger number of fine art illustrations than their prototype, covering a wider range of subject matter.

Shiwu bencao runs to four juan. The text was written by the Ming author Lu He 盧和, probably during the Zhengde reign period of the Ming dynasty (1506-21), and it was originally unillustrated. Illustrations, in the gongbi zhongcai (meticulous painting with rich colouring) style employed also in Bencao pinhui jingyao, were added by a courtly artist around the Jiajing reign period (1522-66). Of 492 polychrome illustrations, almost half are copied from Bencao pinhui jingyao. The illustrations in the 'Waters' section of Shiwu bencao, as well as the images of foods, fall into the category of artistic illustration. Several dozen of the more imaginative renderings are based on misunderstandings of perfunctory or imprecise textual descriptions. For instance, the initial graph (ling 鯪) of the word linglijia 鯪鯉甲 - pangolin - was mis-written as jiao 鮫, meaning 'shark', and in consequence this scaly anteater has been drawn as a large fish. Similarly, mint (bohe 薄荷) is rendered as its near-homophone the lily (baihe 百合), the finless porpoise (jiangtun 江豚, lit. 'river swine') has been drawn as a pig with a huge head, and the jellyfish (haizhe 海哲) has become a large mollusc, etc.

Buyi Lei Gong paozhi bianlan was created by a courtly artist or artists in 1591. This 14-juan work was based on the Ming text Lei Gong paozhi bianlan 雷公炮製便覽 (Lei Gong's Guide to Drug Preparation) by Yu Ruxi, to which were added mnemonic formulas in verse and polychrome paintings of Materia Medica. No complete exemplar survives. The fragmentary Wanli (1573-1619) copy preserves 1128 illustrations (a supplement dating to the Qing period entitled Jinghui bencao tu 精繪本草圖 (Masterpieces of Materia Medica Illustration) has 34 further illustrations which are not found in the Wanli text). The artists used as their source the original Hongzhi (1488-1505) edition of Bencao pinhui jingyao - so far as one can tell from the surviving images, they produced 302 new illustrations, mostly relating to drug processing and preparation, but all the rest are copies of illustrations in Bencao pinhuijingyao.

Most remarkable are 224 original illustrations of drug preparation, where the artists have taken this subject matter as a point of departure for a series of lively vignettes of people at work (these illustrations contain as many as 910 human figures) (Fig. 8). Notwithstanding, research has revealed that most of these are imaginative recreations based on the text, rather than actual drawings of contemporary pharmaceutical practices. There are also 54 ancillary illustrations, the most striking of which appear in the unprecedented 'Human body' section. These are consummate examples of artistic Materia Medica illus- 




Figure 8.8 Drug preparation, from Buyi Lei Gong paozhi bianlan

tration, full of imaginative and novel conceits. However, due to the artists' limited knowledge of pharmaceutics, the illustrations contain many errors of fact.

Artistic Materia Medica illustration, straddling as it does the fields of fine art and scientific illustration, is a phenomenon unique to early Chinese Materia Medica texts. Because it is not really geared to depicting the morphology of medicinal substances, scholars involved in researching the history of pharmacy need to approach this type of illustration differently from realistic or observational illustrations. On the other hand, artistic Materia Medica illustration adds greatly to the interest of Materia Medica studies by opening windows onto other related areas of life. Because these illustrations generally take their subject matter from contemporary society, they are a rich mine of information about tools, costume and adornment, customs and folkways, etc. They can be enjoyed and appreciated as works of art. 


\section{Concluding Remarks}

Materia Medica illustration includes two distinct genres: observational illustration and artistic illustration. This chapter provides an introduction to the most representative works in each of these genres, and draws attention to differences between them which historians must take account of.

Artistic Materia Medica illustration is a unique phenomenon, peculiar to early Chinese Materia Medica texts. It is a valuable resource for research in other fields beyond Materia Medica and also holds considerable artistic interest.

\section{Bibliography}

\section{Primary Sources}

Li Lian 李濂 (1488-c. 1566), Preface toJiuhuang bencao, in Zhu Su 朱 㙌 (1403-24), Jiuhuang bencao 救荒本草 (Famine Relief Herbal). Modern facsimile edn, Beijing: Zhonghua shuju, 1959.

Li Zhongli 李中立 (Ming period), Bencao yuanshi 本草原始. Edition engraved by Yong Qiuli 雍丘李, 1612, 4oth year of Wanli reign period of Ming dynasty.

Tang Shenwei 唐慎微 (Song period), Chongxiu Zhenghe jingshe zhunyong bencao 重修政和經史備用本草. Modern edn, Beijing: Renmin weisheng chubanshe, 1957

Wang Jie 王介 (Song period), Lü Chanyan bencao 履㟴岩本草 (The Cliff Walker's Materia Medica). Ming manuscript copy.
Wei Zheng 魏征 (58o-643) and Linghu Defen 令狐德荣 (583-666), Suishu:jingjizhi 隋書. 經籍志 (Bibliographic Catalogue of the Sui Dynastic History). Modern edn, Beijing: Zhonghua shuju, 1973.

Wu Qijun 吳其䜭 (Qing), 植物名實圖考 (Illustrated Survey of Plants and Plant Names). Facsimile of edn curated and engraved by Lu Yinggu 陸應谷 (Guangxu period of Qing dynasty, 1875-1908), Shangwu yinshuguan, 1919 .

Xia Wenyan 夏文彥(Yuan period), Tuhuiyujian. Edition engraved in 1366, 26th year of Zhizheng reign period of Yuan dynasty.

\section{Secondary Sources}

Miyashita Saburo 1979, 宮下三郎, ‘本草の図について——本草纲目附 図の解说として’, in Honzô komoku fuzu, Tokyo: Shunyôdô, 9-23. Xie Zongwan 謝宗萬 1985, 'Bencao gangmu tuban de kaocha' 本草綱 目圖版的考察 (An investigation of the plates of Bencao gangmu), in Li Shizhen yanjiu lunwenji 李時珍研究論文集 (Papers in Li Shizhen Studies), Wuhan: Hubei kexue jishu chubanshe, 147-85.

Zhang Yanyuan 張彥遠 1987, Lidai mingyi ji 歷代名畫記 (Records of Famous Physicians throughout History), Shanghai: Shanghai guji chubanshe.

Zheng Jinsheng 鄭金生 1980, 'Lü chanyan bencao chukao' 履㭸岩本 草初考 (A preliminary study of The Cliff Walker's Materia Medica), Zhejiang zhongyizazhi 浙江中醫雜誌 (Zhejiang Journal of Chinese Medicine) 8.8, 338-41.

1995, 'An investigation of the Tianbao danfang drug illustrations', Zhonghua yishizazhi 中華醫史雜誌 (Chinese Journal of Medical History) 23.3, 158-61.

2003, 'Mingdai huajia caise bencao chatu yanjiu' 明代畫家 彩色本草插圖研究 (A study of polychrome Bencao illustrations by Ming artists), Xin shixue 新史學 (New History) 13.4, 79-83. 


\title{
9 Reading Visual Imagery and Written Sources on Acupuncture and Moxibustion
}

\author{
Huang Longxiang 黃龍祥
}

There is a general tendency among scholars of medical history, when recovering and working with primary sources, to focus on the written word at the expense of material artefacts and visual evidence. This has, to a greater or lesser degree, hindered progress across the entire field.

Having spent much of the past 20 years recovering manuscripts, and reconstructing the illustrated scrolls that reveal the development of moxibustion, I have come to feel, more and more strongly, that one picture really is worth the proverbial thousand words. When examining historical data, I find myself wary if significant (or indeed essential) visual evidence is not to hand. This article sets out to show, with the help of some representative examples, the kind of pitfalls that await medical historians when they neglect visual information.

\section{Case 1: The 'Tiansheng Bronze Figure' and Tiansheng Acupuncture Canon}

There are a few acupuncture locations recorded in received literature from the early Han period such as Sima Qian's 司馬遷 Shiji 史記 (Records of the Historian), ${ }^{1}$ which details a treatment at the wai san yang wu hui 外 三陽五會 (Outer Three Yang and Five Meetings, thought to be equivalent to the well known acupoint baihui 百會, One Hundred Meetings) on the crown of the head. Body channels are archaeologically attested in text and artefact from the 2nd century BCE, while the earliest extant set of points for needling can be found in a manuscript from $c$. 1st century CE recovered at the Wuwei 武威 tomb site in modern Gansu, which marks a series of locations sited along the spine, However, those points are not shown on channels. Thus the early evidence suggests a separate development of acupoints and channels. There are also many roughly contemporary acu-moxa points scattered through the Huangdineijing 黃帝內經 recensions, a large corpus of medical writings that were compiled in various recensions over the course of the Han dynasty. However, there was not any systematic coordination of acupoints and channels until Huangfu Mi's 皇甫䍀 (215-82) Zhenjiu jiayijing 針炎甲乙經 (282).

Thereafter, the first standardised national system of acu-moxa locations was established in 1026 (4th year of

1 Shiji 105, edn. Beijing, Zhonghua shuju, 1972, p. 2,792. the Tiansheng reign period of the Northern Song dynasty), with the publication of Tongren shuxue zhenjiu tujing 銅人腧穴針尒圖經 (Illustrated Bronze Man Canon of Acupuncture and Moxibustion), also known as Tiansheng Zhenjing 天聖針經 (Tiansheng Acupuncture Canon). This classic text records 354 acu-moxa points, with methods for finding the points, therapeutic indications, and needling and moxibustion procedures. To reach a wider audience, it was not only published in book form, but also engraved on stone steles. In 1443 (8th year of the Zhengtong reign period of the Ming dynasty), new engravings were made under the auspices of the Imperial Academy of Medicine to replace the time-worn Song steles. Though the Ming stone engravings of the Illustrated Bronze Man Canon no longer survive, three complete rubbings exist in China and Japan.

These national standards were not just set down in writing; they were also given visible and tangible form in the 'Tiansheng Bronze Man' (Tiansheng tongren 天聖 銅人), a standing human figure cast in bronze with the channels and points shown on the surface. Subsequently, no one seems ever to have questioned whether the prevailing interpretation of this canonical text was the correct one, let alone to have considered that it might have been interpreted differently in different epochs, or even by different individuals living in the same epoch. But then came the discovery of a replica of the Bronze Man, created during the Zhengtong era of the Ming (1436-49). All of a sudden, we became aware of a tremendous gulf between our present understanding of the words of the Bronze Man Canon and the intentions of the authors, and we gained a sense of the sheer variety of possible interpretations, both diachronic and synchronic. This was particularly true of the passages on the acu-moxa locations of the head, the shoulders, the abdomen and the back of the thighs. The three locations called hanyan 領厭, xuanlu 懸顱, and xuanli 懸厘 are a case in point (see Table 1). ${ }^{2}$

2 Wang Wei 王惟 (Song period), Tongren shuxue zhenjiu tujing 銅 人腧穴針尒圖經 (Illustrated Bronze Man Canon of Acupuncture and Moxibustion) (3-juan edn). Rubbing of the stone engraving of the Ming Zhengtong era. 




Figure 9.1 Replica of the Song Bronze Man, Ming Zhengtong era



Figure 9.2 Tongren shuxue zhenjiu tujing 銅人腧穴針尒圖經, Ming (1368-1644) woodblock print



Figure 9.3 Tongren shuxue zhenjiu tujing, Song (96o-1279) lithograph



Figure 9.4 Head of the Ming Zhengtong Bronze Man, viewed from the side showing the locations of hanyan 領厭, xuanlu 懸 顱, and xuanli 懸厘 on the back of the head 
Table 9.1 Comparison of Three Acu-Moxa Locations, Hanyan 領厭, Xuanlu 懸覤 and Xuanli 懸厘 as Depicted in the Tongren Tujing 銅人圖經 and in Zhenjiu ZishengJing 針炎資生經

\begin{tabular}{|c|c|}
\hline Tongren tujing 銅人圖經 & Zhenjiu zishengjing 針尒資生經 \\
\hline $\begin{array}{l}\text { Hanyan 領厭, } 2 \text { points, below the temporal hairline } \\
\text { (quzhou, lit. 'curved periphery') at the upper border of } \\
\text { the temple }\end{array}$ & $\begin{array}{l}\text { Hanyan 領厭, } 2 \text { points, below the temporal hairline at the } \\
\text { upper border of naokong 腦空 ('Brain Hollow', above } \\
\text { the occipital protuberance) }\end{array}$ \\
\hline $\begin{array}{l}\text { Xuanlu 懸覤頁, } 2 \text { points, above the temporal hairline in } \\
\text { the middle of the temple }\end{array}$ & $\begin{array}{l}\text { Xuanlu 懸覤頁, } 2 \text { points, above the temporal hairline in the } \\
\text { middle of naokong }\end{array}$ \\
\hline $\begin{array}{l}\text { Xuanli 懸厘, } 2 \text { points, above the temporal hairline at the } \\
\text { lower border of the temple }\end{array}$ & $\begin{array}{l}\text { Xuanli 懸厘, } 2 \text { points, above the temporal hairline at the } \\
\text { lower border of naokong }\end{array}$ \\
\hline
\end{tabular}

In 1992, I was editing the Song text Zhenjiu zisheng jing 針炎資生經 (Classic of Acupuncture and Moxibustion for Nourishing Life), written by Wang Zhizhong 王執中 in 1220, which reproduces passages from the Bronze Man Canon. I noticed that, in describing the locations of hanyan, xuanlu, and xuanli, the term nieru 䫂霣 (temples) of the original had been consistently replaced by 腦空 naokong ('Brain Hollow' - an area at the back of the head). This was evidently not a scribal error, but a deliberate emendation (it is also reflected in the relevant charts). Yet elsewhere, Wang Zhizhong punctiliously follows the text of the Bronze Man Canon.

Where he does take issue with the location of an acumoxa point, he indicates this in a footnote or comment, rather than correcting the original text. For example, describing quepen 缺盆 (Empty Bowl), Wang provides the alternative name, tiangai 天蓋 (Heaven's Cover) and gives its location according to the Bronze Man Canon as under the shoulder, in the hollow of the Collar Bone. Wang notes that a number of texts such as the Suwen 素 問 and the Mingtang 明堂 located a point with the same name above the shoulder in the hollow of the collarbone. Using the strength of combined evidence he suggests that the Bronze Man Canon was wrong. ${ }^{3} \mathrm{He}$ also points out an error with the Bronze Man Canon location of an acu-moxa point below the elbow. The shouyangming 手陽明 (Hand Yangming) point of the channel, it states, lies 3 cun below qu chi 曲池 (Pond at the Crook), whereas other texts such as Mingtang say it is 2 cun below.

So why in the earlier instance did Wang Zhizhong, careful textual scholar that he was, modify his original with such apparent insouciance? With the discovery of the Zhengtong replica of the Bronze Man, the puzzle was solved. Sure enough, on the Zhengtong Bronze Man, the three points hanyan, xuanlu and xuanli are shown on the back of the head. In all probability, when Wang Zhizhong

3 Wang Zhizhong, Zhenjiu zisheng jing 針炎資生經, juan 1; Huang Longxiang 1997, p. $25^{2}$. was writing Zhisheng jing, he had access not only to the text of the Bronze Man Canon, but also to the original Tiansheng Bronze Man itself; otherwise, it is implausible that he would have altered the text in this fashion.

An extreme example of divergent interpretations of the same description of an acu-moxa location is provided by the weiyang 委陽 (Lateral to the Crook) point on the $z u$ taiyang 足太陽 channel, on the lower leg.

Huangdi mingtang jing 黃帝明堂經 states:

Weiyang is anterior to zu taiyang (Greater Yang channel of the leg) and posterior to shaoyang 少陽 (Lesser Yang channel). It emerges between the two tendons at the distal edge of the popliteal fossa, 6 cun below the fucheng 扶承 (Hold and Support) point. This is a divergent collateral (bieluo 別絡) of zu taiyang. ${ }^{4}$

Subsequent texts give much the same account of the location of weiyang. However, acupuncture charts and models display no such unanimity: in fact no two seem to show it in exactly the same position.



Figure 9.5 Variations in the siting of acu-moxa location of weiyang 委陽 (Lateral to the Crook). From left to right: Ming Zhengtong (1436-49) Bronze Man; Japanese Bronze Man, Edo period (1603-1868); Bronze Man from Deoksugung 德壽宮 Palace, Seoul, Korea

Huangdi mingtang jing 黃帝明堂經; Huang Longxiang 1987, p. 235 . 
Were it not for the acu-moxa charts and Bronze Man figures, one wonders how much of the transmitted literature would be understood correctly. There is an old adage: Yuzhi quxue, wu tu mo ke 欲指取穴, 無圖莫可

(roughly: If you want to find acu-moxa points, you cannot do without pictures).

The saying was used in reference to the selection of points for needling but it is equally applicable to the interpretation of texts on the location of points and channels. It explains why charts and models have been a requirement for acupuncture standards from the earliest times to the present day.

There is an important insight to be gained from this: Given the limitations of language and the variability of the human body, any description of an acu-moxa location is bound to involve some ambiguity, and is liable to be understood differently by different people. This is why visual artefacts including Bronze Man figures and acu-moxa charts play a vital and irreplaceable role in the understanding of early texts on acupuncture and moxibustion.

\section{Case 2: Acu-Moxa Charts and Acu-Moxa Prescriptions}

There are two main types of acu-moxa chart:

1. charts showing a full set of acupoints as recorded in the Mingtang jing 明堂經 (Illuminated Hall Canon - a common name for acupuncture texts), known anciently as Mingtang tu 明堂圖 (Illuminated Hall charts). The epithet 'Mingtang' refers to the 'Bright Hall', a ritual structure, reputedly of 12 rooms, through which the emperor would process to coordinate his ceremonies with the passage of the months. The acupuncture body, its channel structure and physiological rhythms are a microcosm of these divisions of time and space. Up until the Song dynasty, these charts normally came in groups of three, comprising a front view, a side view, and a back (or 'prone') view.

2. charts intended to assist in finding the therapeutic locations for specific acupuncture or moxibustion prescriptions. These charts show only the points required for the prescription, with labels identifying their position on the body (see Figs 6 and 7).

Among the texts recovered from Dunhuang is an anonymous illustrated manuscript of moxibustion remedies (now held in the British Library under the two catalogue numbers S.6168 and S.6262, and known by the modern descriptive title Jiufa tu 炎法圖 - The Moxibustion Charts) (See Lo and Tlalim, Chapter 19, Figs 19.4 a and b; Wang Shumin, Chapter 1, Fig. 1.9, in this volume). In it there are two references to a



Figure 9.6 Manuscript recovered from Dunhuang, 1, showing the location of tianchuang 天窗 (Celestial Window). S.6262. ○ The British Library

location called tianchuang 天窗 (Celestial Window). In both cases, it is explicitly stated that this is a single point (yixue 一穴). Over the last few decades, the manuscript has been examined time and time again by medical historians and specialists in Dunhuang studies, from China and elsewhere in the world, and yet they all seem to have turned a blind eye to this signally obvious fact, taking it for granted that tianchuang must refer to the familiar tianchuang location - a pair of points on either side of the neck. But one has only to look at the illustrative charts to see that the point in question is actually located on the midline of the forehead. Numerous further instances are readily to be found in the literature of the period. In Qianjin yaofang 千金要 方 (Essential Prescriptions Worth a Thousand Gold Pieces) and Qianjinyifang 千金翼方 (Supplementary Prescriptions Worth a Thousand Gold Pieces), for example, the term tianchuang turns out almost invariably to refer to a location at the front of the head otherwise known as xinhui 㐫會, or Fontanelle Meeting point. There is ample evidence here to confirm that the xinhui point was habitually known as tianchuang in the Sui (581-617) and Tang (618-907) periods, thereby enabling us to correct a distortion of history. And yet, before looking at the Dunhuang moxibustion charts, I 




Figure 9.7 Manuscript recovered from Dunhuang, 2, S.6168. $\odot$ The British Library

had examined pre-Song moxibustion prescriptions innumerable times without ever noticing this fact, which had been staring me in the face all along.

Uniquely and very strikingly, in the pre-Han literature on acupuncture and moxibustion, the 12 Specific Points (teding wei 特定穴) on the wrists and ankles all bear the same name as the corresponding channel. This can be seen in a prescription for hernia in Wushi'er bingfang 五 十二病方 (52 remedies), a prescription text found in the Mawangdui 馬王堆 Han tomb (sealed in 168 вСE). ${ }^{5}$ The prescription reads: 'Apply moxibustion to Taiyin 太陰; Taiyang 太陽 XX [two illegible characters]; excellent'. Research has established that 'Taiyin' and 'Taiyang' here refer to moxibustion locations, and not to channels.

Because this peculiarity was not understood, terms such as zuyangming 足陽明 and shou yangming 手陽明 (Yang Brightness of the foot or hand) were long erroneously assumed to denote the channels with those names, rather than the acu-moxa points that were originally intended. The location charts in this rare manuscript constitute a vital piece of evidence, which allows a centuries-old enigma to be resolved.

\section{Case 3: The Twelve Waxing and Waning Trigrams of the Months (Xiao-Xi Gua 消息卦)}

Arguably no single text on acupuncture and moxibustion has been misunderstood so thoroughly, or for so long, or by so many people, as the 'Maijie' 脉解 (Explanation of the mai) chapter of Suwen 素問 (Plain Questions - one of the two parts of Huangdineijing 黃帝內經 (The Inner Canon of the Yellow Emperor), the other being Lingshu 靈樞 (The Numinous Pivot). Huangdineijing was written down in the Han period). This chapter discusses the signs and symptoms of diseases of six mai 脉 or channels - three Yang and three Yin. For centuries, it was taken for granted that this was an account of pathologies of the six Foot

For a translation of this recipe, see Harper, 1998, p. 267.



Figure 9.8 Suwen, Song woodblock print, showing a page from the 'Maijie' treatise

channels. With the sole exception of Yang Shangshan 楊上 善 (585-670, early Tang), the physicians and scholars who studied the chapter explained the signs and symptoms it described in terms of the circulation in those channels. The Tang editor Wang Bing 王冰 (c. $710-805)$ had an inkling that Maijie possessed a different frame of reference from the 'Jingmai' 經脉 (Channels) chapter of Lingshu, which gives an account of signs and symptoms in relation to the channels. In a note appended to Maijie he writes:

This chapter is unconnected with the surrounding text, and gives a differing explanation of the aetiology of diseases of the channels. It bears little relation to the account in the Numinous Pivot ${ }^{6}$

Wang Bing's view was rejected by Song writers such as Lin Yi 林億 ( $f l$. 11th century), and failed to spark further interest. In truth, the account of symptoms and signs in Maijie is based on the concept of the Twelve Waxing and Waning Trigrams of the months (xiao-xigua 消息卦), which was current during the Han (Fig. 9); without knowing this, it is unintelligible. The significance of the chapter becomes self-evident when it is read in conjunction with the corresponding set of diagrams, which graphically represent the

\footnotetext{
6 Huangdineijing, suwen 13 (Maijie), Wang Bing (c. 710-805) ed.
} 




Figure 9.9 Twelve Waxing and Waning Trigrams

ascent of Yang Qi and decline of Yin Qi during the first half of the year and the opposite process during the second half.

When one considers the above examples, one cannot help but wonder how much of the enormous mass of available historical data has actually been interpreted accurately - and assuming that it has been accurately interpreted, how often it has been pieced together correctly to build up a historically accurate picture. In my view, any attempt by modern people to understand ancient texts is bound to be inadequate or inappropriate in places, simply because of the limits of written expression. In this regard, visual images can provide vital clues to help us make correct interpretive decisions. But even so, to fully comprehend the technical aspects of ancient texts, one's technical experience and expertise would need to be equal to or greater than the original authors'.

Much of the time, historians are like the blind men in the fable of the elephant. Each of the men touches the elephant in a different place and tries to describe the whole beast, but when they compare notes they cannot agree as to its overall shape and nature. Similarly, we grope at partial truths, while the full story constantly eludes us, and it is a matter of luck if we can make head or tail of what we have in front of us. Where will we find the clues that will lead us out of the shadows into the light?

\section{Bibliography}

\section{Primary Sources}

Huangdi neijing 黃帝內經 (The Inner Canon of the Yellow Emperor), Anon. c. Han dynasty (2O2 BCE-22O CE). Suwen 素問 (Plain Questions), edn Wang Bing 王冰 (c. 710-805); Lingshu 靈樞 (The Numinous Pivot) and Mingtang jing 明堂經 (Illuminated Hall Canon).

Qianjinyaofang 千金要方 (Essential Prescriptions Worth a Thousand Gold Pieces) 652, by Sun Simiao 孫思俘 (581-682).

Qianjinyifang 千金翼方 (Supplementary Prescriptions Worth a Thousand Gold Pieces) 682, by Sun Simiao 孫思絔 (581-682).

Shiji 史記 (Records of the Historian) c. 94 BCE, by Sima Qian 司馬遷 (c. 145-86 BCE) edn. Beijing: Zhonghua shuju, 1972.

Tongren shuxue zhenjiu tujing 銅人腧穴針尒圖經 (Illustrated Bronze Man Canon of Acupuncture and Moxibustion) (3-juan edn), by Wang Weiyi 王惟一, Song (96o-1279) lithograph, Ming (1368-1644) woodblock print.

Wushi'er bingfang 五十二病方 (52 remedies, modern title), Mawangdui 馬王堆 tomb, sealed 168 вСE. Tr. Harper 1998.

Zhenjiu jiayijing 針炎甲乙經 (AB Canon of Acupuncture and Moxibustion) 282, by Huangfu Mi 皇甫䍀 $\left(215^{-282}\right)$.

Zhenjiu zisheng jing 針炎資生經 (Classic of Acupuncture and Moxibustion for Nourishing Life) 1220, by Wang Zhizhong 王執 中 (c.1140-1207).

\section{Secondary Sources}

Harper D. 1998, Early Chinese Medical Literature, Sir Henry Wellcome Asian Series, vol. 2. London: Kegan Paul.

Huang Longxiang 黃龍祥 1987, Huangdi mingtang jing jijiao 黃帝明 堂經輯校 (The Yellow Emperor's Illuminated Hall Canon) edited and collated, Beijing: Zhongguo yiyao keji chubanshe.

1997, Zhenjiu mingzhujicheng 針炎名著集成 (Collection of Famous Texts on Acupuncture and Moxibustion), Beijing: Huaxia chubanshe.

2001, Zhongguo zhenjiu xueshushi dagang 中國針炎學術 史大綱 (The Historical Development of Acupuncture), Beijing: Huaxia chubanshe.

2003, Zhongguo zhenjiushi tujian 中國針炎史圖鑒 (Illustrated History of Chinese Acupuncture and Moxibustion), Qingdao: Qingdao chubanshe. 


\section{The Fine Art of the Tongue}

Nancy Holroyde-Downing
In 1341 during the Yuan Dynasty, a medical text appeared which contained 36 drawn representations of a variety of tongues. Each was accompanied by an explanation of what the individual tongue image depicted in terms of illness, and a directive as to what prescription was necessary to treat that illness. The title of the text was the Aoshi shanghanjinjing lu 敖氏傷寒金鏡錄 (Scholar Ao's Golden Mirror of Cold Damage Disorders), and the provenance of these tongue images weaves a fascinating story.

The inspection of the tongue is now a pervasive aspect of a diagnosis, and it is a fundamental part of the curriculum in most colleges of Chinese medicine worldwide. It is discussed in diagrammatic and theoretical detail in contemporary Chinese medical textbooks and is a feature of the 'signs and symptoms' used in planning or discussing acupuncture and herbal medicine treatment. There are myriad tongue diagnosis teaching texts, most recently with photographic images of tongues in vast quantities showing great varieties of qualities. Tongue diagnosis is usually presented together with pulse diagnosis as systems having comparable antiquity and joint primacy in the clinical encounter. And yet, even a cursory look at classical texts, case histories and formularies from the Han (206 BCE-22O CE) to the Qing (1644-1912) dynasty suggests it has not always enjoyed its current status.

\section{A Brief Textual History}

It is, of course, impossible in this chapter to exhaustively address all the received texts of Chinese medicine that refer to the tongue. However, even a brief glance at a selection of references to the tongue from the Han through the Song Dynasties reveals two interesting facts. These references are neither expressions of a diagnostic system, nor are they conveyed as images.

Texts from the Mawangdui 馬王堆 burial site (closed 168 вCE) are thought to pre-date the classical compilations of Chinese acupuncture theory found in the Huangdineijing 黄帝内經 (Yellow Emperor's Inner Classic), ${ }^{1}$ and the

The Huangdi neijing corpus consists of two treatises: Suwen 素 問 (Fundamental Questions) and Lingshu靈樞 (The Numinous Pivot), written down $c$. 2nd century BCE, plus the $c$. 7 th-century CE Taisu太素 (Great Basis) recension, which overlaps with both. These texts are generally considered to contain the core theory
Nanjing 難經 (Classic of Difficult Issues). ${ }^{2}$ The Mawangdui tomb texts, reflecting the newly emerging physiological medicine of the Warring States and early Han, present early concepts of the body's vessel system. In these texts also, diseased bodies begin to be ascribed to a slow breakdown of a state of internal harmony, rather than to attacks by a displeased ancestor or spirit. Evidence of connection between the tongue and the body's organs is found in vessel descriptions such as: 'attached to the kidney, and presses laterally on the tongue', ${ }^{3}$ or 'emerges at the liver, enters the upper side, and is attached to the tongue'. ${ }^{4}$ The tongue also features in Yin yang mai sihou 陰陽脈死 侯 (Death Signs of the Yin and Yang Vessels), a text from Mawangdui concerned with the prognosis of death, which states: 'when the tongue binds and the testicles curl up, muscle has died first'. 5

By the time of the 'Jingmai' 經脈 treatise of the Lingshu recension of the Huangdi neijing, the anatomical planes of the vessels link clearly with organs and there is an elaborate system of correspondences in place that begins to systematise pulse and complexion diagnosis. Yet the Huangdineijing corpus contains only a handful of references to the tongue within the vessel/organ framework, and only scattered mentions of the tongue's colour or dryness.

The foundations of classical medicine were set out by the end of the Han dynasty. The Nanjing, often considered the work that represents 'the apex, and also the conclusion, of the developmental phase of the conceptual system known as the medicine of systematic correspondence, ${ }^{6}$ only makes passing references to the tongue. In it, we learn that the tongue weighs $283 \mathrm{~g}$, and has a length of $17.8 \mathrm{~cm}$ and a width of $6.4 \mathrm{~cm}$; that when cold and heat have affected the flesh, the lips and the tongue will dry out; that the influences of the heart pass through the tongue; that when the tongue is at ease, one knows the difference between the five tastes; and that when the Foot-Ceasing-Yin vessel is compromised, the testicles draw in and the tongue rolls back. ${ }^{7}$



$\begin{array}{ll}3 & \text { Harper 1997, p. } 210 . \\ 4 & \text { Ibid., p. } 197 . \\ 5 & \text { Ibid., p. } 220 . \\ 6 & \text { Unschuld, 1986, p. } 3 . \\ 7 & \text { Unschuld, } 1986, \text { pp. } 301,387,418,516\end{array}$


Wang Shuhe 王叔和 $(18 \mathrm{O}-c .27 \mathrm{O}$ CE) is the author of the Maijing 脈經 (Pulse Classic), 2nd century CE. In this work, he echoes the tongue presentation in the Yin yang mai sihou, when he writes: 'A sick person with a curled tongue and retracted testicles is bound to die'. ${ }^{8}$ Wang uses the tongue presentation simply as a portent of impending death, indicating nothing about the type of illness contracted or organs involved in the demise. Against the copious writings on information discoverable about the inner body through the palpation of the pulse, we can see that tongue inspection was incidental in scholarly medicine of that time. The presentation of the tongue was noted with certain illnesses, certain vessel trajectories and certain prognosticatory patterns, but not as an indicator of problems within particular organs, and certainly not as a paramount piece of diagnostic information.

The Suwen recension of the Huangdineijing states that the physician who diagnoses using both the pulse and the complexion achieves perfection. ${ }^{9}$ While the physician's gaze was clearly valued during the Han, its focus was on complexion and the tongue hardly receives a mention.

The medical case history is a marvellous window into the actual practice of a physician at a given point in time. It offers the individual records of the diagnosis and treatment of a single patient by a single physician, though the content, context, and application of information may vary. ${ }^{10}$ One of the earliest examples of the case history can be found in the Shiji 史記 (Records of the [Grand] Historian) by Sima Qian 司馬遷, 2nd century BCE. A physician, Chunyu Yi 淳 于意, was summoned by imperial order to give an account of his practice. Offering 25 case histories in defence of his standing as a worthy physician, he writes that 'in every case where your vassal has conducted a medical consultation, he has always made a consultation record zhenji 針劑.'.1 A common turn of phrase is, 'when I examined the mai [pulse]....'2 Nowhere in these records do we find the comparable phrase, 'when I inspected the tongue'. Certainly, to Chunyu Yi, the tongue did not hold vital diagnostic information.

\section{Epidemics and the Search for Effective Treatment}

During the Song Dynasty (96o-1279), an onslaught of epidemics ravaged southern China. Treatments for these



epidemic illnesses were too often unsuccessful, and the Imperial government was anxious to find ways to expand the scope of medical literature in the search for effective remedies.

It established a new bureau, Jiaozheng yishu ju 校正醫 書局 (Bureau for Revising Medical Texts), which it charged with searching out, revising and publishing a selection of ancient and previously neglected classics that might prove useful in combating epidemic illnesses. One of these rehabilitated texts was the Shanghan lun 傷寒論, ${ }^{13}$ written by Zhang Zhongjing 張仲景 (style name Zhang Ji 張機 $f$. late 2nd century $\mathrm{CE}$ ). Zhang is known to have compiled this text after an epidemic swept through his hometown of Changsha (in present-day Hunan province), killing numerous family members and decimating the general population. Over the next two centuries it became one of the most highly regarded and popularly disseminated medical texts. Its particular genius was to pay attention to the individual and evolving condition of each patient during a rapidly changing illness, not just to the fixed disease. ${ }^{14}$

While the Shanghan lun was revolutionary in its strategies for treatment with herbal prescriptions, it offers only scattered mentions of tongue presentation, and no tongue illustrations. One of these few mentions is in line 230 of the text. Under the rubric of 'Yangming illness', it lists a white tongue fur among indications for the use of the prescription Xiao chaihu tang 小柴胡湯 (Minor Bupleurum Decoction). Yet despite the paucity of tongue information in the text, this increased Song focus on treatments for febrile illnesses led to innovations during the following Jin-Yuan period (1115-1368 CE) which had major implications for the development of tongue diagnosis.

\section{Tongue Inspection Emerges as an Illustrated Diagnostic System}

The Jin-Yuan period saw the consolidation of power in the North of China, with concomitant cultural changes, such as the development of neo-Confucianism, in which old ideas were reworked. The current of widespread governmental, cultural and philosophical changes impacted upon medical thinking as well. One of four major medical innovators of the period was Liu Wansu 劉完素 (1110-1209). He put forward the hypothesis that the six pathogenic influences presented in the Suwen all ultimately manifest in a patient as fire. The prescriptions he developed and

13 See Goldschmidt 2009 for a discussion of the Song government's revision and promulgation of earlier medical texts. 
his methods of treatment focused on ensuring that this fire did not accumulate within the body. His approach became known as the Cooling School. Another of the major innovators was Zhu Danxi 朱丹溪, who focused on the movement of 'ministerial fire' in the body. This could pose a risk to the Yin energy in the body, and specifically the Kidney's Yin. He founded the Nourishing Yin School. It becomes possible to imagine that with the reworking of the constraints around Shanghan theories of illness and the many manifestations of fire, there would be increasing interest in developing ways to 'see' and eventually depict pathogenic heat within the body.

Indeed, it is during the Yuan Dynasty, c. 126o CE that we find the first serious evidence of tongue inspection emerging as a system of diagnosis. A book entitled the Jinjing lu 金鏡錄 (Golden Mirror Record), authored by someone known only as Scholar Ao, presented 12 illustrations of tongues, relating them to the Cold Damage Disorders discussed in the Shanghan lun. We know of this lost text through another later text by Du Qingbi 杜凊碧. Du organised and supplemented the Jinjing lu, adding 24 of his own illustrations. The resulting work was published in 1341 as Aoshi shanghan jinjing lu. This text does indeed offer a system of what we would recognise today as tongue diagnosis. Du categorises colours of the body of the tongue, the tongue's shapes, the various peculiarities of surface geography, such as cracks and spots, and the different



Figure 10.1 From 1341 edition of Aoshi shanghan jinjing lu, 敖氏傷寒 金鏡錄 showing Shaoyang presentation with a prescription for Xiao Chai Hu Tang, with dosage possibilities of tongue coatings. Importantly, he connects each illustration with a herbal prescription. Indeed, the Aoshi shanghan jinjing lu records the above-mentioned recipe for Xiao chaihu tang, (Minor Bupleurum Decoction) under Shaoyang 少陽 (Lesser Yang) illness - just as in the Shanghan lun - but additionally provides an illustration of the tongue one would see in a patient with this stage of illness, thereby facilitating diagnosis (Fig. 1).

The Aoshi shanghan jinjing lu appears to have been a much copied text. In the archives of the library of the China Academy of Chinese Medical Sciences (formerly China Academy of Traditional Chinese Medicine) there can be found an array of publications from the 14th century through to the 2oth century containing illustrations of the original 36 tongues presented by Du Qingbi. There are both woodblock prints (Fig. 2) and hand-drawn illustrations (Figs 3 , 4). Many of the woodblock prints are almost exact copies of the early text, but differences also occur. Some differ only slightly with variant titles of the individual illustrations or the sequencing of the drawings. Some compilations give basic black and white illustrations, while others - not necessarily newer ones - offer coloured illustrations. There are replications of the 36 tongues inserted into discussions of Shanghan illness, and compilations of tongue drawings that stand alone as discrete publications. Interestingly, some of the tongue texts include the prescriptions of the Jinjing lu, with and without dosages, and others simply



Figure 10.2 Shanghan zheng zhi zhun cheng (Standards of diagnosis and treatment for Cold Damage) Wang Kentang (Ming 1368-1644). $\odot$ Wellcome Library, London, Loo38014 




Figure 10.3 From a hand-drawn 1445 edition of the Shanghan jinjing lu 傷寒金鏡錄 tongue text. (C) The Library of the Academy of Chinese Medical Sciences

offer the illustrated tongues with a textual description. There are even handwritten texts which dispense with the drawings, having only numbered lists and descriptions of the 36 tongues, suggesting widespread familiarity. The existence of so many of these illustrated tongue texts, published over such a long period of time, highlights the importance they held for physicians, particularly in dealing with febrile illness.

\section{The Tongue's Rise to Prominence}

The taking of the pulse at the radial artery is arguably the most iconic diagnostic image of Chinese traditional medicine, with a great amount of textual history. The mastery of the pulse was an essential skill which traditional doctors achieved through years of practice. For literate doctors, this learning took place while practising as the disciple of another eminent physician, or was learned from the experienced elder physicians within a family network. However, during the Ming dynasty a combination of factors conspired against this usual accumulation of skill and expertise.

The numbers of scholars passing the imperial examinations in this period increased significantly, without an attendant increase in civil service posts. Trade however was on the rise, allowing the expanding and increasingly



Figure 10.4 From Shebian sanshiliu zhong 舌辨三十六種, a 1910 hand-drawn text on tongues. Tongue on right showing the white coat and red tip with a prescription for Xiao Chai Hu Tang without dosage. (c) The Library of the China Academy of Chinese Medical Sciences

wealthy merchant class to finance the education of their sons. Additionally, advances in printing led to an unprecedented availability of texts. Medical texts were among the most desired, and the profession of medicine became an obvious choice for the scholarly gentry. This new rather sideways step from civil service post holder to respected physician had one significant obstacle. There was no sudden increase in scholar physicians available with whom to study. The mastery of medical theory did not always lead to clinical skill, and the new scholar-physicians were in need of assistance. 
Perhaps the tongue's rise to the diagnostic prominence it enjoys today had to do with its particular effectiveness in allowing a clear connection between the visual manifestion of a symptom of illness and the appropriate drug intervention in the treatment of that illness. Essentially, the tongue texts could be seen as a diagnostic shortcut.

The correct diagnosis, based upon the palpation of the pulse, might elude all but the most skilled physician. How different from diagnosis based upon the observation of the tongue, whose qualities are clear and easily categorised. A white, yellow or black coating cannot easily be confused with one other, nor can a pale tongue body be mistaken for a bright red one. Dryness or wetness, the presence or absence of fissures are among diagnostic qualities that can be clearly and objectively determined, whether by novice or skilled physician of long experience.

\section{Artistic Embellishment or Diagnostic Tool}

While discussing the emergence of the tongue images and the development of a system of tongue diagnosis, it is fundamental to note that these drawings were not merely decorative additions to a theoretical process. The drawings were themselves able to function as tools for diagnosis. In the 1341 text attributed to Du Qingbi, the first drawing is accompanied by text stating:

The tongue coat is slippery and white. It indicates the evil has just entered the body. The dantian 丹田 has heat, the chest has cold. This is Shaoyang, half outside, half inside. It is appropriate to use Xiao chaihu tang. ${ }^{15}$

The tongue image here gives the physician a representation of a tongue that could be expected to accompany a particular pattern of heat and cold lodged in the body, and which would impact upon certain channels and organs. In a sense, a cross reference.

Alongside the second drawing, however, we read:

The tongue's red colour indicates heat is amassing inside the body. You don't need to ask which channel. Whatever the channel, it is appropriate to use Touding qingshen san 透頂清神散.

Here is a clear and uncompromised statement that in some instances, the physician's subtle skills of pulse diagnosis, complexion diagnosis, channel and organ diagnostics, and theories of disease progression can all be set aside. All that is needed to treat some illnesses is the observation of a tongue matching the relevant image, and an appropriate herbal formula can be administered without doubt.

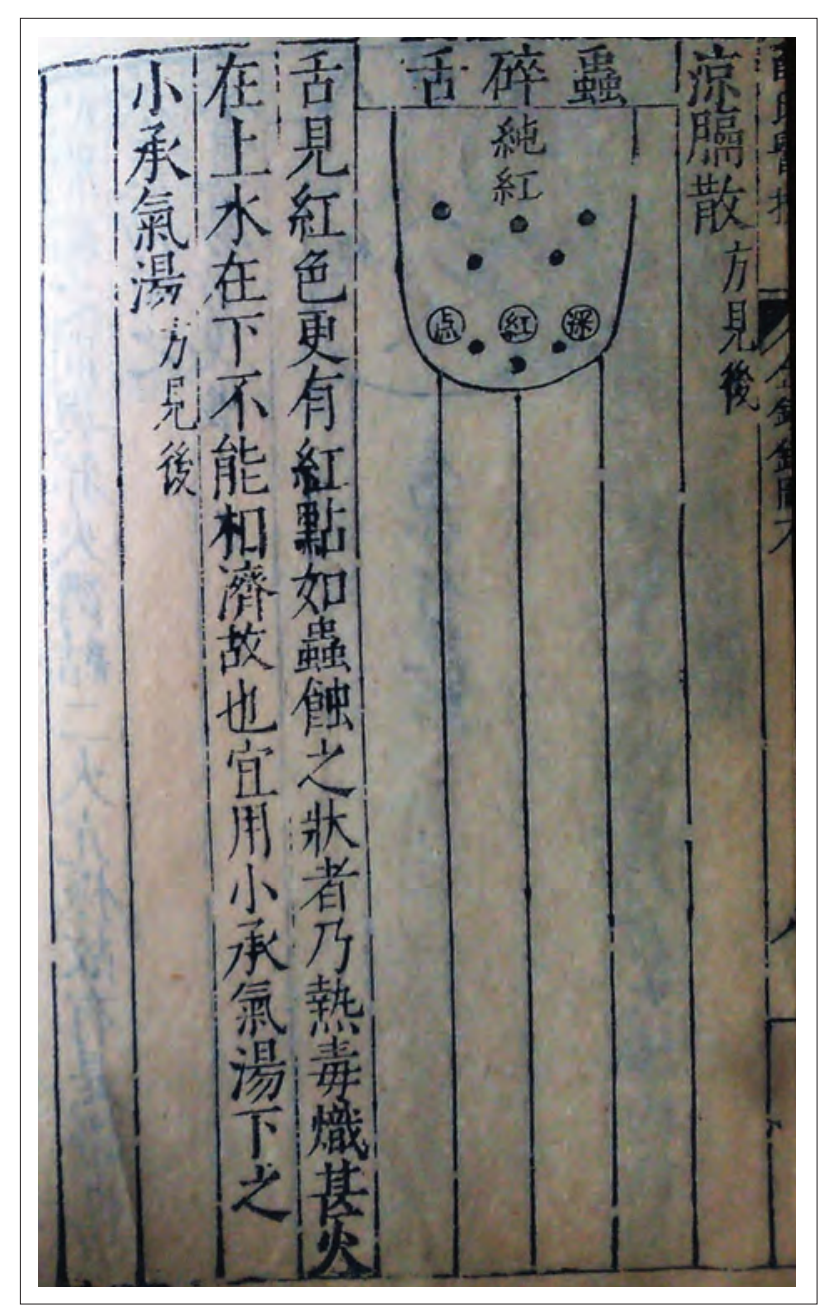

Figure 10.5 From the 1529 edition of Xue Ji's compilation of Waishang jinjing lu 外傷金鏡錄. @ Library of the Academy of Chinese Medical Sciences

\section{Tongue Texts become part of Medical Compilations}

During the Ming Dynasty (1368-1644), Xue Lizhai 薛立 齋 (style name Xue Ji 薛己, (1487-1559) contributed to the recognition and transmission of tongue diagnosis. In 1529 he authored a two-volume, 16-book compilation entitled Xue Shi yi'an 薛氏醫案 (Medical Case Records of Scholar Xue). One of the books in this collection was the Waishang jinjing lu, 外傷金鏡錄 (Golden Mirror Record of External Damage) (Fig. 5). In it he includes the 36 tongue illustrations of the Aoshi shanghan jinjing lu, along with a preface and an endnote extolling the importance of the tongues, and commentaries on them. The publication of this work and the prestige of its author undoubtedly did much to further the transmission of the tongue text.

The Ming was also a time in which case records developed as a distinct genre of medical writing. The advances in printing and the social and economic restructurings of Chinese society during the late Song and Ming facilitated 
the reproduction and dissemination of case records among physicians and the literate population. The compiling of case records became an intrinsic part of literate medical practice. ${ }^{16}$ An as yet cursory look at these records suggests that Ming physicians nearly always palpated the pulse, frequently observed the complexion and listened to the voice of a patient. The inspection of the tongue, however, doesn't yet appear to be a regular feature of diagnosis in the Ming, though it does occur occasionally.

\section{Proliferation of Tongue Texts during the Qing Dynasty}

The tongue diagnosis texts grew out of and at first accompanied the theories and prescriptions of the Shanghan lun, which posited a six-stage progression of illness in the body. Rather than rejecting this paradigm, physicians of the (late) Ming and Qing Dynasty expanded their bases for understanding the progression of disease. There were many innovative physicians, among them Wu Youke 吳又 可 ( $f l$. late 17th century), who wrote the Wenyi lun 温疫 論 (Treatise on Warm Heat). These physicians reworked ideas about the causation of febrile illness, implicating climatic pathogens and developing treatments that differed from the traditional Shanghan theories. Their work led to the development of a school of disease theory known as wenbing 溫病. ${ }^{17}$

During this time, there appeared an ever-increasing number of books discussing the tongue. Shen Douyuan's 申斗垣 ( $f$ l. early 17 th century) Shanghan guanshe xinfa 傷 寒觀舌心法 (Technique for Observing the Tongue in Cold Damage) weaves together existing tongue presentations associated with the six-stage progression of the Shanghan lun and the new wenbing theories. He is also the first to rank the colours of the body of the tongue, and emphasise the importance of the red tongue.

\section{Tongue Diagnosis becomes more Comprehensive}

But the diagnosis of the types and stages of febrile illness is only one aspect of the development of tongue inspection. Along with the proliferation of tongue texts and an increasing range of commentary, by the Qing Dynasty

16 For a discussion of the societal developments and stresses that impacted the practice of medicine in Ming China, see Hymes 1987 and Brokaw and Chow, 2005.

17 See Marta Hanson's investigation of the development of wenbing theory in Hanson 2011.



Figure 10.6 Qing Dynasty text Shejian bian zheng 舌鑑辨正, 1894, showing the mapping of internal organs on to specific areas of the tongue. (C) Library of the Academy of Chinese Medical Sciences

(1644-1911) tongue inspection and the images accompanying its discussion underwent two interesting changes. Firstly, the internal organs of the body were mapped onto the tongue. This allowed the entire framework of Chinese medicine's diagnostic theory to align with the presentation of a patient's tongue (Fig. 6).

As a direct result of this organ mapping, the entire framework of Chinese medicine's diagnostic theory could now align with the presentation of a patient's tongue. A vast range of bodily illnesses could be inferred from changes that were objectively observable on the tongue. The implications of this fact were enormous. Tongue diagnosis could be relevant not only for febrile illness, but for most conditions of 'unwellness'. Fissures on the surface geography of the tongue could not only identify dryness from fevers, but if located in the centre of the tongue could indicate a dearth of fluids or moisture in the stomach and a condition of Yin deficiency. Ulcerations on the edges of the tongue did not only appear with life threatening epidemics, but with types of internal 'heat' or 'fire', and demonstrated their impact on the functioning of the liver. Texts discussing the appearance of the tongue proliferated. 


\section{Western Medicine and the Constraint of Objectivity}

As physicians in China were reworking the diagnostic possibilities of tongue inspection in the Qing Dynasty, Western physicians were arriving in China in ever-increasing numbers and establishing an alternative medical culture and knowledge base. ${ }^{18}$ Put extremely simply, as Western military superiority eroded the power of the Chinese state, Western medicine's superiority benefited by association.

The 1910-11 pneumonic plague outbreak in Manchuria highlighted Western medicine's effective utilisation of isolation, quarantine, and cremation of victims' bodies to stem the contagion. ${ }^{19}$ The North Manchurian Plague Prevention Service was created in its wake, whose remit was to provide Western medical treatment, research and public health measures. For the Chinese government, the Service reflected a desire for international credibility and membership in the scientific community. Bridie Andrews cites a medical periodical in which He Bingyuan 何炳元, a prominent reformer of Chinese medicine, wrote:

Alas! The China of today is a sick nation weakened by injury and oppressed by noxious influences. How can we find someone to treat the nation, to eradicate the roots of the ailment and turn weakness into strength? Although we may wish to strengthen the nation, it is first necessary to strengthen the race. To strengthen the race it is first necessary to pay attention to hygiene. To do this, we must first understand physiology, and if we wish to understand physiology, we must first promote medicine. ${ }^{20}$

All of this suggests a time in which to lack 'modernity' and 'scientific skill and theory' was to be subjugated and inferior. By the end of the 19th and beginning of the 2oth centuries, Western medicine's entire identity was resoundingly modern, scientific, and redolent of superiority. Traditional medicine, on the other hand, was an unappealing art of waning prestige. Additionally, the new medicine arriving from Europe, Japan and America emphasised the importance of anatomical correctness and objectivity. The paradigm in which traditional medicine flourished had relatively little need for exact anatomy, and the primary diagnostic technique, pulse palpation, was an intensively subjective activity. But in traditional medicine's now valued pursuit of scientific respectability, the tongue offered possibilities.

18 There are myriad works on western expansion in China during the igth century. The role of medicine in these encounters is difficult to overstate.

19 For a description of the epidemic's containment, see Wu 2012.

$20 \quad$ Andrews 2014, p.94.
The images of tongues in medical texts that survive from the 14th century onwards display basic similarity. Whether hand-drawn, printed, black-and-white or coloured, they essentially comprise a U-shaped form with relevant colours, coatings, and surface geographies noted - in fact, the same sort of image that is used today by practitioners recording the qualities of a tongue in clinic notes.

But by the end of the 19th century and the beginning of the 2oth, the tongue images in Chinese medicine texts underwent a striking transformation.

The tongue was no longer a discrete site of observation; it was positioned anatomically. As shown below, the tongue was now sited within the skull, the cervical spine clearly defined. Salivary glands and upper-respiratory structures such as the larynx, pharynx and epiglottis figure prominently along with teeth and sinus cavities. In short, European traditions of anatomy had made an impact on Chinese medicine's tongue depictions.

Despite the fact that visualising the tongue with its 'lingual tonsil' or 'foramen caecum' offered no diagnostic advantage, anatomically correct illustrations were adopted in many tongue texts of Chinese medicine during the early 2oth century (Figs 7, 8, 9).

From the original 12 tongues to which Du Qingbi tells us he added an additional 24 , the illustrations in tongue texts have become increasingly numerous and detailed over the past two centuries. It is not uncommon for current texts to list 200 or more variations of tongues.

\section{Tongues and Technology}

Secure in its recent position as an objective and verifiable diagnostic tool (a red tongue is observably not black and a white coating neither yellow nor absent), tongue diagnosis lent itself happily to technological innovation. Beginning in the 1970s the humble photograph was used to make a true and objective record of a patient's tongue. Pictures of tongues have more recently been captured with digital imaging and analysed by computers.

In 2009, researchers in China asserted that an exact analysis of the tongue from digital pictures continues to be difficult. They recommend a further level of precision in which the tongue coating itself would be extracted from the digital photograph and further analysed. Such refinement in electronic management of tongue representations seek to create an objectively unassailable and automatic tongue imaging and diagnostic system.

Current students of Chinese traditional medicine are almost universally familiar with the standardised tongue image above (Fig. 6) of organ correlation and their clinical 



Figures 10.7 and 10.8 Bian she zhinan 辨舌指南, 1920. Figure 7 displays the anatomical placement of the tongue, while Figure 8, a summary of white coloured tongues, suggests a more anatomically correct rendering of the tongue surface, onto which the body's organs are mapped. Fig. 7: ( ) Library of the Academy of Chinese Medical Sciences; Fig. 8 @ Library of Needham Research Institute

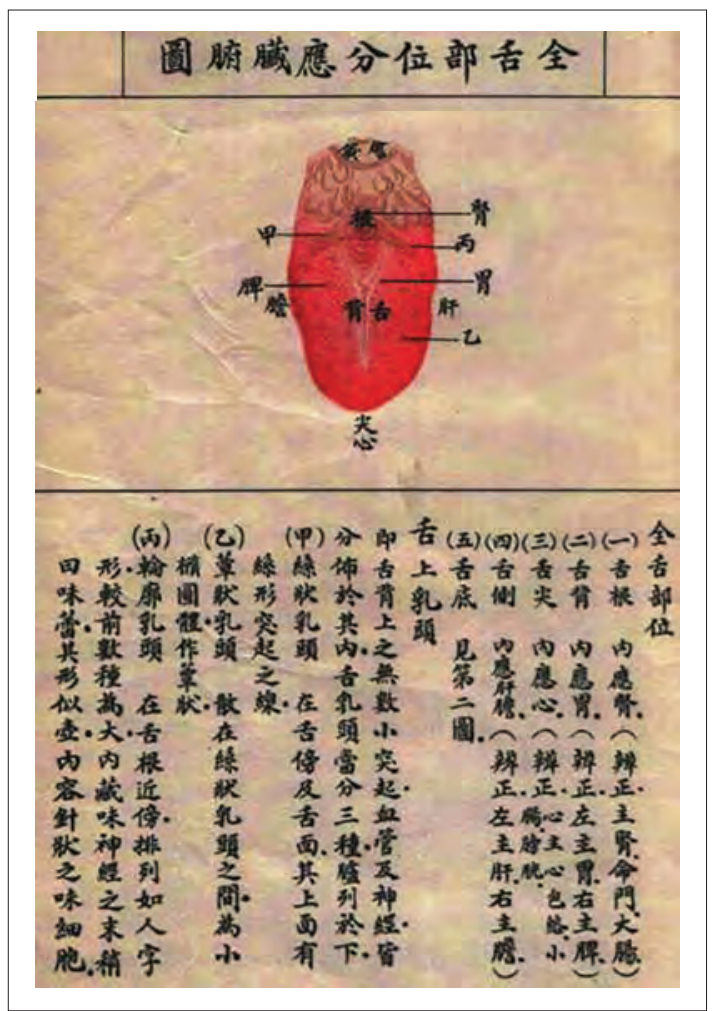

Figure 10.9 Bian she zhinan 辨舌指南, 1920. The body organs mapped onto the tongue. (C) The Library of the Academy of Chinese Medical Sciences notes are more likely than not to have the rudimentary U-shaped drawing with areas of diagnostic significance noted (Fig. 9). For practitioners who have moved from paper notes to computerised records, digital photographs of a patient's tongue are often utilised. In personal communications with various practitioners, I was told that the use of digital tongue photographs is attractive primarily because they are convenient to upload into computerised records, not because they are seen to offer any unique information. In these same conversations, the practitioners nearly always note that tongue diagnosis is easier to master than pulse diagnosis, and often serves as a fall-back technique if the pulse presentation is in doubt. When I described the electronic tongue imaging techniques being researched in China, Western practitioners of Chinese medicine reported finding the research interesting, but not something they were keen to know more about or could imagine using. As technology impacts upon traditional medicine, it is intriguing to consider what role the image of the tongue will play. 


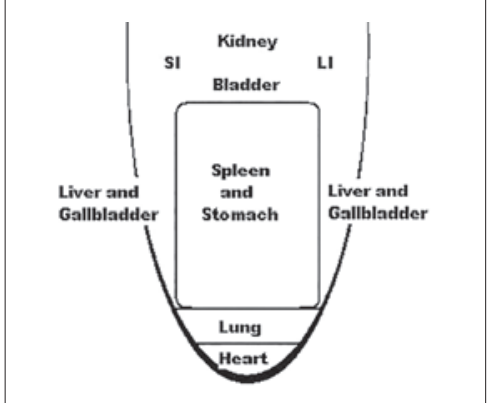

Figure 10.10 Standardised mapping of internal organs onto areas of the tongue, from student handouts. (C) The College of Integrated Chinese Medicine

\section{Conclusion}

Mention of the tongue in Chinese medical writings is seen and referenced as early as the Han Dynasty. A reference, however, is not a system of diagnosis. While a thread of interest in the tongue's appearance winds through the medical writings of early dynasties of China, it was in the Jin-Yuan era (12th-14th centuries) that the first specifically focused book on tongues appeared. It has been postulated that an antecedent to this early Jinjing lu text lies in Cheng Wuji's (1062-1155) Shanghan mingli lun 傷寒明理論, which describes coatings, colours and qualities to be seen on the tongue. ${ }^{21}$ But the marriage of tongue illustration with the required drug treatment was a ground-breaking innovation, which arrived in the form of the 36 tongues illustrated in the Aoshi shanghan jinjing lu.

During the Ming Dynasty, when scholar physician numbers were increasing rapidly, the ability of inexperienced physicians to secure a relatively scarce apprenticeship position was difficult. The tongue text, by then more easily reproduced and circulated, would have been immediately useful. The discourse among physicians focused more intensely on wenbing theories of disease during the Qing dynasty. The tongue's ability to reflect febrile conditions in the body led to increased focus and observation. By the late Ming and through the Qing Dynasty, the original 36 tongues images in the jinjing lu had expanded to include hundreds of distinct images.

The mapping of organs and channels onto the body of the tongue allowed the presentation of the tongue to be relevant to the entire theoretical framework of Chinese medicine. The usefulness of this particular innovation is difficult to underestimate. Students in colleges of Chinese medicine now had a diagnostic tool that was easier to learn

21 I owe the recognition of this precedent on tongue lore to Dr He Wei, who reported on a lecture by Zheng Jinsheng that she attended at the Academy of Chinese medicine in Beijing. than pulse palpation, and it had the additional recommendation of being observable and verifiable.

From the original extant 1341 edition of this visual diagnostic handbook to the automatic tongue image segmentation method now being researched in China, tongue images have played various innovative roles in the practice of Chinese medicine. Not the least of these has been to function as a diagnostic shortcut.

It is now more likely that a physician will observe a tongue to determine the more general state of the Qi, blood, fluids, Yin and Yang as they circulate in the channels and nourish the internal organs of the body rather than to decide specifically upon treatment in the case of febrile disease. Tongue inspection has become a foundation of diagnosis in Chinese medicine. Books with myriad digital photographs of tongues and the association of their appearance with varying symptoms of disease are commonplace. But the hand-drawn image has endured, to be found in the patient notes of generations of practitioners, still conveying relevant and useful clinical information upon which treatments are based.

\section{Bibliography}

\section{Primary Sources}

Aoshishanghan jinjing lu 敖氏傷寒金鏡錄 (Scholar Ao's Golden Mirror Record of Cold Damage) 1341, Du Qingbi 杜清壁; Edition 1529, Library of the China Academy of Chinese Medical Sciences, Beijing. Bian she zhinan 讋舌指南 1920, Cao Bingzhan 曹炳章.

Huangdi neijing lingshu 黃帝内經靈樞.

Huangdi neijing suwen 黃帝内經素問.

Huangdineijing taisu 黃帝内經太素 $c .610$, Yang Shangshan ed. 楊上善. Maijing 脈經, c. 280, Wang Shuhe 王叔和.

Shanghan mingli lun 傷寒明理論, 1156, Cheng Wuji 成無己.

Shanghan zhengzhi zhunshen 傷寒證治準繩, 1589, Wang Kentang, 王肯堂.

Shejian bianzheng 舌鑑辯正, 1897, Liang Yuyu 梁玉瑜. Xue shiyi'an 薛氏醫案, 1529 , Xue Ji 薛己.

\section{Secondary Sources}

Andrews, B. 1996, The Making of Modern Chinese Medicine, 1895-1937, Vancouver/Toronto: University of British Columbia Press.

2013, The Making of Modern Chinese Medicine, 1850-196o, Vancouver: University of British Columbia Press.

Bai, Linda Yunlu 白雲鹿, Shi Yundi 史雲迪, Wu Jia 吳佳, Zhang Yonghong 張永紅, Weng Weiliang 翁維良, Wu Yu 吳显, Bai Jing 白淨, 2009, 'Automatic extraction of tongue coatings from digital images: a traditional Chinese medicine diagnostic tool', Tsinghua Science and Technology 14.2 (April), 170-5.

Brokaw, C. and Chow, Kai Weng (eds), Painting and Book Culture in Late Imperial China, University of California Press, 2005

Cullen, C. 2001, 'Yi'an (case statements): the origins of a genre of Chinese medical literature', in Hsu (ed.), 297-323.

Goldschmidt, A. 2009, The Evolution of Chinese Medicine: Song Dynasty 960-1200, London: Routledge. 
Hanson, M. 2011, Speaking of Epidemics in Chinese Medicine: Disease and the Geographic Imagination in Late Imperial China, London: Routledge.

Harper, D. 1997, Early Chinese Medical Literature: The Mawangdui Medical Manuscripts, London/New York: Kegan Paul International.

Hsu, E. 1999, The Transmission of Chinese Medicine, Cambridge: CUP.

Hsu E. (ed.) 2001, Innovation in Chinese Medicine, Cambridge: CUP.

Hymes, R.P. 1987, 'Not quite gentlemen? Doctors in Sung and Yuan', Chinese Science 8, 9-76.

Kuriyama, Shigehisa, 1999, The Expressiveness of the Body, New York: Zone Books.
Miyashita, Saburo, 1986, A historical analysis of Chinese formularies and prescriptions: three examples, in Ogawa (ed.), 101-16.

Summers, W.C. 2012, The Great Manchurian Plague of 1910-1911: The Geopolitics of an Epidemic Disease, New Haven: Yale University Press.

Taizo Ogawa (ed.) 1986, History of Traditional Medicine, Proceedings of the 1st and 2nd International Symposia on the Comparative History of Medicine - East and West, Tokyo: Taniguchi Foundation.

Unschuld, P.U.1986, Nan-ching: The Classic of Difficult Issues, Berkeley/ Los Angeles: University of California Press.

Wang Shu-He (tr. Yang, Shou-Zhong) 1995, The Pulse Classic: A Translation of the MaiJing, Boulder: Blue Poppy Press.

Wu Liande 1959, Plague Fighter: The Autobiography of a Modern Chinese Physician, Cambridge: Heffer. 


\title{
11 Diagnostic Images of the Tongue: Aetiology and Pathology Made Visible
}

\author{
Liang Rong 梁秌
}

The decisions we make about how to manage a disease are determined by our understanding of the aetiology and pathological changes of the disease. In Chinese medicine, these processes are termed bingyin 病因 (the cause of the disease) and bingji 病因病機 (the mechanism of the disease). Bingyin refers to the direct cause which leads to the onset of disease, such as catching cold, being caught in the rain, getting angry, or eating tainted food. Bingji could be defined as the ways in which the direct cause of the disease gives rise to symptoms. As aetiology and pathology in Chinese medicine share much of the same vocabulary and concepts, such as Wind, Cold, Fire, Heat, Dryness, Dampness, and poisons, the two terms are often found conjoined in the compound bingyin bingji (the cause and mechanism of the disease).

\section{Tactile Diagnosis: Grasping the Causes and} Mechanisms of Disease by the Sense of Touch

Long before tongue observation came to be codified as a diagnostic system, pulse-taking was already a very important method for determining and distinguishing the causes and mechanisms of disease. In the classic text Shanghan lun 傷寒論 (Treatise on Cold Damage, early 3rd century $\mathrm{CE}),{ }^{1}$ each condition is identified by a rubric setting out the disease pattern, treatment indications, and pulse type. The first diagnostic method to be presented systematically in written form, pulse-taking has produced a large body of literature. The earliest known monograph on the subject, Maijing 脈經 (Classic of the Pulse), dates back to the Jin Dynasty $\left(265^{-420}\right)$. It is often said that the pulse is the language oflife, and doctors are its interpreters. ${ }^{2}$ We know, then, that early Chinese medicine relied on touch as a key medium for gathering information about the inside of the human body. However, the diagnosis and treatment of waigan bing 外感病 (or diseases caused by exogenous pathogenic factors) and the resulting accumulation of knowledge posed challenges to the primacy of pulse-taking as a diagnostic technique.

\footnotetext{
$1 \quad$ Shanghan lun sets out a six-stage development of febrile illness caused by cold according to the phases of Yin and Yang (Cold Damage).

$2 \quad$ Cf. Kuriyama 1999, p. 20 et passim.
}

\section{Confusion arising from Haptic Diagnosis in the Differentiation of Cold and Heat Syndromes}

In waigan bing (diseases caused by exogenous factors), the most common causes and mechanisms are Cold (hanxie 寒邪 (malign Cold or Cold evil), hanzheng 寒證 (Cold syndrome), etc.), and Heat (rexie 熱邪 (Heat evil), rezheng 熱證 (Heat syndrome), etc.). Cold and Heat are clearly conceptual opposites, and are easy to contrast in theory. However, both in recorded case histories and in clinical practice, there are situations in which it is extremely difficult to distinguish accurately between Cold and Heat syndromes. The ability to do so hinges on the practitioner's skill in pulse-taking. This dependence upon an individual's haptic sensitivity to the pulse can be seen as a weakness of this diagnostic technique. The following cases illustrate the perils of unskilful pulse-taking.

\section{Case 1}

From Mingyi lei'an: Shanghan 名醫類案 傷寒 (Case Histories Recorded by Famous Doctors: Cold Damage), recorded by Guo Yong 郭雍 (1106-87).

In the 5 th lunar month, a man aged over 50 immersed himself in water after having sex, and became ill with a Cold Damage syndrome. He was inappropriately treated, being prescribed too much Heating medicine, and he developed an oily sweat. He breathed heavily with a thunderous sound. He could not sleep by day or night for several days on end. He had episodes of panic and insane frenzy. He exhaled Qi uncontrollably from his mouth. Many doctors were unsure as to how to treat this.

Doctor Guo felt the patient's pulses and gave his diagnosis: the pulses in the six sectors were deep and lacked strength. This was caused by tiredness due to the patient's inability to sleep. It was a Yang pattern, which should be treated with Huanglian jiedu tang 黃連解毒湯 (Coptis Decoction to Clear Toxins). However, the other doctors considered this treatment to be very dangerous. The patient took one dose, to no effect, so the others suggested using Da qinglong tang 大青龍湯 (Major Blue-green Dragon Decoction). But Doctor Guo said that the patient should take one more dose of Coptis Decoction to Clear Toxins. Thereafter the disease abated, the panting and sweating were relieved, and the patient made a full recovery. ${ }^{3}$

The key feature of this case is that the pulse was weak, and yet the diagnosis revealed not a Yin syndrome but a Yang syndrome.

3 Jiang Guan, Mingyi lei'an (1996 edn), p. 22. 
Case 2

From Mingyi lei'an - Shanghan, recorded by Li

Dongyuan 李東垣 (1180-1251).

A young man named Feng 馮, aged 16 years, had contracted a Cold Damage illness and presented with red eyes, restlessness and thirst. His pulse beat seven to eight times to each breath. His doctor planned to administer Chengqi tang 承氣湯 (Qi Co-ordinating Decoction). But just as the decoction was ready for him to take, Doctor Li came in, whereupon Feng explained the situation to him. Doctor Li took the patient's pulse, and exclaimed: 'You have almost killed this young man'! He explained that the patient was suffering from a febrile disease with a rapid pulse. But if one pressed harder when taking the pulse, no movement could be felt. Such a pulse suggested that the symptoms were caused by Yang Qi being driven out by excessive cold (Han sheng ge yang 寒盛格陽). Thus the disease was no longer a Heat syndrome, but had transmuted into a Yin syndrome. He therefore prescribed ginger and and fuzi (Radix aconiti lateralis preparata). The patient took eight liang $^{4}$ in one dose, began to sweat, and gradually recovered.

The key feature of this case is that the patient had a fast pulse; nonetheless, he was correctly diagnosed as suffering from a Heat syndrome (and not a Cold syndrome).

\section{Case 3}

From Gujin yi'an an -Shanghan 古今醫案按·傷寒

(Medical Case Histories, Past and Present: Cold Damage), recorded by Wang Kentang 王肯堂 (1549-1613).

Wang Kentang treated an officer called Yu Yunwei 余雲佯. Officer Yu had a robust build and abundant Qi; he ate large quantities of meat, and drank copiously. He contracted a sudden febrile disease in June. His limbs were not very hot. Sometimes he moved his hands or feet in a restless fashion. He was unconscious, unaware of what was going on around him. Sometimes he said one or two words, which were barely intelligible. But it was not delirium. The pulse was thin, weak and could hardly be felt. One doctor said that this was a Yin pattern and he should be treated with a warming method (wenfa 溫法). Another said he should be treated by purging the bowels (xiafa 下法). It was up to Doctor Wang to decide. Doctor Wang said that, in theory, if this was a Yang pattern disease with a Yin pattern pulse, it ought to be incurable. However, in this particular case, one should bear in mind the patient's constitution, and the fact that the season was summer, which meant that the temperature was hot, while the meat and alcohol consumed by the patient created inner Heat. All this suggested that there should be wild, burning Heat, and the pulse should be strong, fast and powerful. But the patient's symptoms indicated that the Heat was trapped inside, unable to get out. Moreover, the patient had had no bowel movement for seven days. Therefore, the patient should be treated with Da chaihu tang 大柴胡 湯 (Major Bupleurum Root
Decoction). After taking the medicine, the patient moved his bowels and his pulse could be easily felt. His hands and feet became warm. Later he was then given Coptis Decoction to Clear Toxins. After he had taken a few bags of medicine, the disease was gone. ${ }^{5}$

The key feature of this case is that the patient's pulse was weak, thin, and barely discernible. But the disease pattern was diagnosed as Yang-Heat and not Yin-Cold.

It became apparent that that with some diseases, especially life-threatening ones, a physician's pulse-taking skills might not be sufficient to identify the cause and mechanism of the disease accurately, and could not therefore serve as a guide to treatment. This gave rise to a debate about whether diseases should be treated symptomatically or on the basis of pulse diagnosis (maizheng cong she 脈症從舍).

\section{The Tongue as a Window on the Workings of Fire}

In the Jin-Yuan period (Jurchen and Mongol dynasties, 1115-1368), Liu Yuansu 劉完素 (1110-1209) proposed a new theory of aetiology and pathology whereby the six Qi, or six climatic factors (Wind, Cold, Summer-Heat, Damp, Dryness, and Fire) all could become Fire. He argued that Wind, Cold, Summer-Heat, Dampness, and Dryness are the five direct causes of illness, which invade the human body, but all of them can transmute into a single form of Qi, i.e. pathogenic Heat. This generates febrile disease with a Heat pattern as its core. However, Liu Yuansu merely discussed the mechanism of Fire-Heat as a theory, without offering any new method of observing Fire-Heat inside the body.

In the quest to obtain more accurate evidence for the aetiology and pathology of Fire-Heat, Aoshi shanghanjinjing lu 敖氏傷寒金鏡錄 (Mr Ao's Golden Mirror of Cold Damage Disorders, 1341) was crucial. This text, composed in the Yuan (Mongol) Dynasty (1271-1368), is illustrated with 12 tongue-diagnosis diagrams. The writer adduces fresh evidence for the pathological mechanism of Fire-Heat: the flushed red colour of the tongue (Fig. 1). ${ }^{6}$

According to Shanghan lun, the tongue coating is yellow when there is Heat in the body. From the Song Dynasty (96o-1279) onwards, medical writers state that both a yellow coating and a black coating denote an internal Heat pattern. However, in Chinese medical theory, there is no obvious correlation between these two colours and Fire-Heat. Therefore, Liu Yuansu used a complex process of reasoning based on the theory of the Five Agents (Wood, Fire, Earth, Metal and Water) to explain the relationship 




Figure 11.1 Shanghan diandian jin shu 傷寒點點金書 (Gold-dust Book of Cold Damage), Ao Jiweng (Song period, 96o-1279) and Du Ben (Yuan period, 1206-1368). 1341 (1894) edition, Library of the China Academy of Chinese Medical Sciences, (c) Wellcome Library, London Loo39596 and Loo39595. From left to right:Jueyin 厥陰 tongue - appropriate prescriptions are Lizhong wan 理中丸, Sini tang 四 逆湯. Black colour in the inner area of the tongue - appropriate prescriptions are Tiaowei chengqi tang 調胃承氣湯,Jiedu tang 解 毒湯. Cracked tongue - the appropriate prescription is Liangge san 涼膈散 plus huanglian 黃連. Black colouration on the edges of the tongue - one should take Da chengqi tang 大承氣湯. Black colouration on the tip of the tongue - one should take Zhuye shigao tang 竹葉石膏湯. If there is extreme Heat, one should take Tiaowei chengqi tang 調胃乘氣湯 to help move the bowels. Red spots on the tongue - one should take Fahuang yinchen tang 發黃茵蔯湯, and Yinchen wuling san 茵蔯五苓散. Black spots on the tongue - one should take Xuanshen shengma tang 玄參升麻湯, and Gegen huaban tang 葛根化斑湯.

between the colour black and Fire-Heat. He argued that the extreme degree of Water possessed the characteristics of Fire, and vice versa.

The relationship between Fire-Heat and the colour red was unequivocally clear: in existing medical theories, the colour red represented Fire-Heat. Given that the heart governed Fire, and the orifice into which the heart opened was the tongue, a red tongue was strong evidence of the pathological causation and mechanism of Fire-Heat. In this way, a diagnostic method for the observation of inner Fire-Heat was established.

\section{The Field of Vision - the Six Visible Exogenous Factors}

Once the tongue had become accepted as a medium for observing the operations of internal Heat, discussions arose as to the relationship between tongue presentation and the aetiology and pathology of diseases caused by exogenous pathogenic factors. As the identification of a Cold or Heat pattern was key to the diagnosis of exogenous disorders, a fundamental issue was how and whether Internal cold could be observed via the tongue.
A white tongue coating is mentioned in Shanghan lun, but it is mainly recorded in connection with illnesses such as zangjie 臟結 (a gastro-intestinal disorder), and has no relevance to external disease patterns (biaozheng 表證). However from the Song period onward, Cold Damage disorders were analysed according to a tripartite model comprising external patterns (biaozheng), internal patterns (lizheng 裏證), and combined internal and external patterns. The question that remained to be answered was: how could the presence of Cold be read from the tongue before the six Qi transmuted into Fire - and more particularly, before malign Cold turned into Fire?

The Ming author Shen Douyuan 申斗垣 ( $f l$. early $17^{\text {th }}$ century) describes a tongue presentation indicative of a Cold syndrome, with malign Cold in the external aspect of the body, in a key text entitled Shanghan shebian 傷 寒舌辨 (Tongue Diagnosis for Cold Damage) also named Shanghan bianshe xinfa 傷寒辨舌心法 (Knowledge and Skills of Tongue Diagnosis for Cold Damage). He states that malign Cold first attacks the external aspect and is present in the skin area, which is governed by the Lung. The Lung correlates with metal, which corresponds to the colour white in Five-Agent theory. Therefore, one can first 




Figure 11.2 Tongue graph from Ikedake Zekkan Kuketu 池田家舌函 口訣 (Oral Instructions for Tongue Diagnosis of the Ikeda Lineage, 1835)

observe white foam, then white saliva, white slime, and later a white tongue coating. ${ }^{7}$

A simplified version of this discussion is found in Shanghan shejian: Baishetaizonglun 傷寒舌鑒·白舌苔總論 (Tongue Diagnosis for Cold Damage - General Introduction to White Tongue Coating) by Zhang Deng 張登 (1728). According to that text, when malign Cold attacks the skin area, there will first be white foam on the tongue, then white saliva, white slime, white patches, and then little white blisters. There are differences between the body of the tongue, the tip of the tongue, the middle part and the root of the tongue. ${ }^{8}$

Later on, a moist, slippery white tongue coating came to be seen as the distinguishing sign of Cold syndrome. Figure 2 shows a coloured illustration of slippery white tongue coating from the Japanese text Ikedake Zekkan Kuketu 池田家舌函口訣 (Oral Instructions for Tongue Diagnosis of the Ikeda Lineage, 1835), by Higashiyama Kuniyoshi 東山邦好. The significance of white coating was no longer confined to external Cold. In conjunction with the quantity of jin 津 and ye 液 fluids of the tongue, it could be used to establish whether Cold was located in the external or internal part of the body.

In the Qing period (1644-1911), by observing the tongue during the course of diseases caused by external factors (i.e. waigan bing), moisture was found to be another distinctive attribute of the tongue. Tongue graphs (shexiang 舌象) were developed for acute febrile diseases, and changes in the tongue were regarded as important evidence in the identification of we $i$ 衛, $q i$ 氣, ying 營 and $x u e$ 血patterns

Sheng Menyuan, Shanghan shebian (1995 edn), p. 55.

Zhang Deng, Shanghan shejin (1958 edn), p. 1. (four patterns for differentiating physiological diseases). The observation of Fire by this means gave rise to a new branch of febrile disease, i.e. warm diseases (wenbing 溫病).

\section{From the External to the Internal - Probing the Mechanisms of Endogenous Disorders}

Once it had become possible to establish tongue images indicating Cold, Heat (Fire), Dryness, Dampness (Phlegm), and Blood stagnation patterns of disease caused by external factors, the relationship between tongue images and diseases caused by internal pathological factors came under scrutiny.

Diseases caused by internal pathological factors (neishang bing 內傷病) tend to develop slowly over a long period of time, and the onset and the seriousness of the disease are usually related to emotional factors. Although the causes of neishang bing and waigan bing are different, the theoretical framework for understanding the disease mechanisms and instituting treatment are common to both categories. This led to the realisation that it might be both possible and reasonable to apply tongue diagnosis to neishang bing.

The Qing author Wang Jinghan 王景韓 writes in Shenyang yizong jinjing 神驗醫宗舌鏡 (Spiritually Efficacious Mirror of the Tongue from the Origins of Medicine):

My book on tongue diagnosis focuses primarily on Cold Damage and therefore contains information on the transformations of symptoms and syndrome differentiation according to the channels. Proceeding from a discussion of Cold Damage, almost every syndrome can be covered. This approach can then be applied to other diseases like zabing 雜病 [lit. miscellaneous disorders; internal diseases other than cold-induced diseases and warm diseases] or neishang bing. ${ }^{9}$

Wang Jinghan also states that the breakthrough in the tongue diagnosis of neishang bing came with the establishment of a correlation between areas of the tongue and the zang and fu organs (藏腑), based on similar theories to those involved in pulse-taking. This correlation was gradually codified and developed into the format known today. ${ }^{10}$

One may wonder why the diagnostic model of waigan bing, i.e. the direct observation of Fire from the tongue, was not extended to neigan bing. Wang Jinghan may help us to understand the reasoning behind this. He writes

\footnotetext{
9 Wang Jinghan, Shenyan yizong shejing (1993 edn), p. 10.

$10 \quad$ Liang Yuyu, Shejian bianzhen (1985 edn), p. 9.
} 
that tongue observation cannot provide any evidence for the diagnosis of neishang bing, because such diseases are caused by the seven emotions, which are not represented in the coating on the tongue. ${ }^{11}$ This statement implies two important considerations. First, changes to the tongue are more obvious and occur more rapidly in waigan bing. Second, the seven emotions, which form the aetiology and pathology of neishang bing, are, in essence, states of Qi, and there is little documented experience of the observation of Qi through tongue diagnosis.

However, Wang Jinghan provides a valuable summary, in which he describes characteristic features of the tongue in terms of Cold, Heat, deficiency, and excess. Specifically, if there is Cold, the tongue will be black, or grey; if there is Heat, it will be flushed red or yellow; if there is deficiency, it will be white; if there is excess, it will be a slightly brownish red. ${ }^{12} \mathrm{He}$ also suggests that white tongues can be seen in deficiency patterns. As the consistency of the tongue was not discussed in that period, it is not, however, possible to determine what he understood by white tongue.

Nearer to our own era, Shejian bianzheng: baishe zonglun 舌鑒辨正·白舌總論 (Pattern Differentiation by Tongue Diagnosis: of White Tongue, 1891), by Liang Yuyu, provides detailed descriptions of various types of white tongues (Fig. 3). According to Liang, a white tongue indicates Cold, and can be observed in both biaozheng (external patterns) and lizheng (internal patterns). If the patient is suffering from zabing, the tongue will be white, tender, and slippery. If the tongue is clear after the coating is scraped off, it indicates internal deficiency and Cold..$^{13}$ The white tongue of zabing is usually understood to refer to a change in the colour of the tongue itself, which exhibits a whitish pallor. This abnormal tongue presentation, which was identified after red tongue, has special diagnostic value.

If one accepts that the identification of red tongue as a signifier of internal Heat was a breakthrough moment in the diagnosis of waigan bing, much the same can be said of pallid, white tongue and neishang bing. Though neishang bing are centred in transformations of Qi, they also relate to the mechanisms of Cold, Fire-Heat, Dryness, and Dampness (this is the theory of the five internally originating evils), which are similar to the six malign Qi of waigan bing. If a pale white tongue is evidence of interior deficiency (or Yang deficiency), then it can function as a bridge between the mechanisms of Qi, blood, jin and ye fluids, and those of the five evils originating in the interior. This makes it possible

\footnotetext{
$11 \quad$ Wang Jinghan, Shenyan yizong shejing (1993 edn), p. 10. 12 Ibid

13 Liang Yuyu, Shejian bianzhen (1985 edn), p. 9.
}

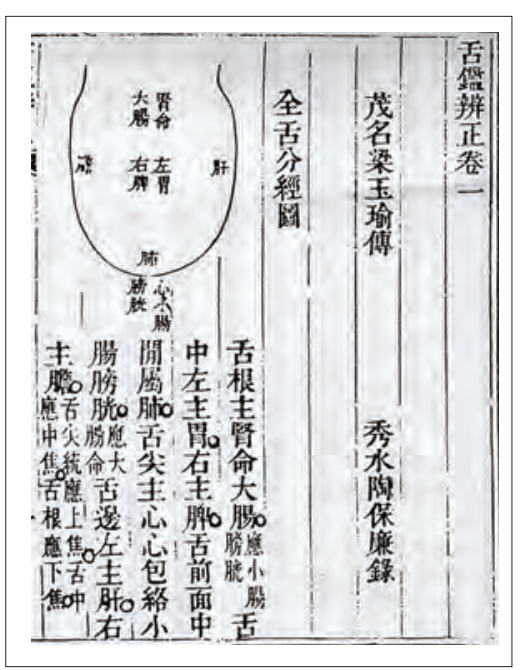

Figure 11.3 Channel distribution on the tongue from Shejian bianzheng 舌鑒辨正 by Liang Yuyu (1891)

The root of the tongue governs the kidney, Mingmen 命 門 [right kidney construed as the Portal of Life], and the large intestine, which is related to the small intestine and the bladder.

The left side of the middle part of the tongue governs the stomach.

The right side of the middle part of the tongue governs the spleen.

The middle part of the anterior region of the tongue corresponds to the lung.

The tip of the tongue governs the heart and pericardium, and connects with the small intestine and bladder, which are related to the large intestine.

The left side of the edge of the tongue governs the liver. The right side of the edge of the tongue governs the gallbladder.

The tip of the tongue is related to the Shang Jiao 上焦 (Upper Burner). The middle part of the tongue is related to the Zhong Jiao 中焦 (Middle Burner). The root of the tongue is related to the Xia Jiao 下焦 (Lower Burner).

to apply the knowledge gained through tongue diagnosis in waigan bing to the investigation of neishang bing.

Through research into tongue diagnosis in neishang bing, tongue graphics for Cold, Heat (Fire), Dryness, Dampness, deficiency, excessiveness, stagnation, and Phlegm were established. This allowed tongue inspection to become a major diagnostic tool for both waigan bing and neishang bing.

In the encounter between Western and Chinese medicine, tongue diagnosis was seized upon as a meeting point between traditions. Grounded as it is in visual observation, Chinese tongue diagnosis seemed to resonate with the anatomical focus and observational style of Western medicine. This gave rise to a genre of 


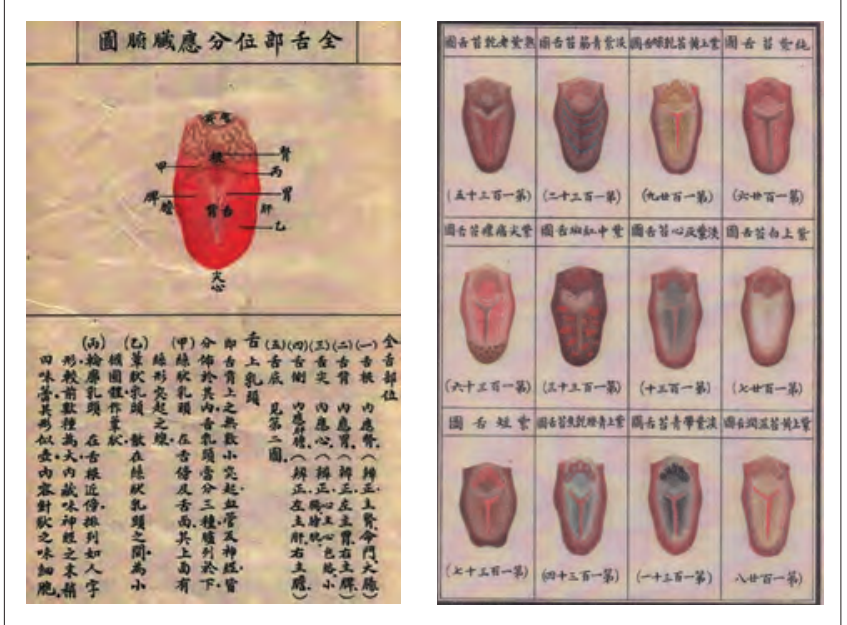

Figure 11.4a (right) Correlations of the zang and fu viscera on the tongue. From right to left, sectors of the tongue: the root of the tongue, related to the kidney (governs the kidney, Mingmen, and the large intestine); the dorsum of the tongue, related to the stomach (the left side governs the stomach, and the right side governs the spleen);

the tip of the tongue, related to the heart (governs the heart, pericardium, small intestine, and bladder); the lateral parts of the tongue, related to the liver and gallbladder (the left side governs the liver, and the right side governs the gallbladder). (See also Holroyde-Downing, Chapter 10 in this volume, Fig. 10.9.)

(C) The Archive of the Library of the Academy of Chinese Medical Sciences, Beijing

Figure 11.4b (left) Bian she zhinan 辨舌指南, 1920. The body organs mapped onto the tongue.

The base of the tongue: lingual papillae. The lingual papillae are many tiny processes on the dorsal surface of the tongue. They contain numerous blood vessels and nerves. They fall into three categories:

filiform papillae, found on the sides and the dorsal part of the tongue. There are filiform processes on the surface; fungiform papillae, located among the filiform papillae and have an oval shape;

circumvallate papillae, located on the root of the tongue and distributed like the Chinese character ren 人. The circumvallate papillae are larger in size than the two types mentioned above. They contain the sensors for taste. The taste bud looks like the Chinese numeral 1 (壹) and it contains the cells for taste, which are needle-shaped. [Translator's note: the characters on the right side of the picture are not clearly legible.]

(C) The Archive of the Library of the Academy of Chinese Medical Sciences, Beijing
While researching the literature on tongue diagnosis, the author has also carried out research and observations in a clinical setting. When we look at the tongue today, can we see the causes and mechanisms of different kinds of diseases in the same ways as the ancient doctors did? Do the ancient theories of aetiology and pathology still have relevance and validity? What scientific propositions can we extract from them? The arena of tongue observation allows us to explore and test possible answers to these questions.

\section{Bibliography}

\section{Primary Sources}

Aoshi shanghan jinjing lu 敖氏傷寒金鏡 (錄 (Scholar Ao's Golden Mirror Record of Cold Damage) 1341, Du Qingbi 杜清壁; edition 1529, Library of the China Academy of Chinese Medical Sciences, Beijing. Bian she zhinan 讋舌指南 1920 , Cao Bingzhan 曹炳章.

Jiang Jin名醫類案 [1503-65] 1996, Mingyi lei'an 名醫類案 (Cases Recorded by Famous Doctors), modern edn, Beijing: Zhongguo zhongyiyao chubanshe.

Higashiyama, Kuniyoshi 東山邦好 1835, Ikedake Zekkan Kuketu 池田 家舌函口訣 (Oral Instructions for Tongue Diagnosis of the Ikeda Lineage, 1835).

Liang Yuyu 梁玉瑜 [1894] 1985, Shejian bianzhen 舌鑒辨證 (Syndrome Differentiation in Tongue Diagnosis), modern edn, Beijing:Zhongyi guji chubanshe.

Shen Douyuan 申鬥垣 [late Ming] 1995, Shanghan shebian 傷寒舌辨 (Tongue Diagnosis for Cold Damage), modern edn, Osaka: Oriento shuppansha.

Tao Hua 陶華 [1369-1450], Shanghan diandian jinshu 傷寒點點金書 (Annotated Golden Book of Cold Damage), Ming period ms copy, Library of Chinese Academy of Chinese Medicine.

Wang Jinghan 王景韓 [Qing period] 1993, Shenyan yizong shejing 神 驗醫宗舌鏡 (Mirror of the Tongue from the Divinely Efficacious Origins of Medicine), Shanghai: Shanghai kexue jishu chubanshe.

Yu Zhen 俞震 [1778] 1998, Gujin yi'an an 古今醫案按 (Medical Case Histories Past and Present), Beijing: Zhongguo zhongyiyao chubanshe.

Zhang Deng 張登 [1667] 1958, Sanghan shejin 傷寒舌鑒 (Mirror of Tongue Diagnosis for Cold Damage), Shanghai: Shanghai weisheng chubanshe.

\section{Secondary Sources}

Cao Bingzhang 曹炳章 1962, Caitu bian she zhinan 彩圖辨舌指南 (Guide to Tongue Diagnosis with Illustrations in Colour), Nanjing: Jiangsu renmin chubanshe.

Kuriyama, Shigehisa 栗山茂久 1999, The Expressiveness of the Body and the Divergence of Greek and Chinese Medicine, New York:Zone Books. Chinese version 2001, Shenti de yuyan 身體的語言, Taiwan:Jiujing chubanshe. tongue graphics based on anatomical criteria (Fig. 4). ${ }^{14}$ However, such images simply depicted the superficial appearance of the tongue rather than truly representing a fusion of medical ideas, and they fell into disuse. 


\section{A Brief Introduction to Illustration in the Literature of Surgery and Traumatology in Chinese Medicine}

\section{HuXiaofeng 胡曉峰}

As we have seen throughout this volume, visual images have been a major vector for the transmission of Chinese medical culture. They figure largely in Chinese medical texts from medieval times onwards, and there are numerous examples in post-Song printed books. This chapter will argue that words and graphics are closely integrated, and most effective, in conveying practical knowledge in the literature of manual therapies such as skin-deep surgery and orthopaedics of various styles; the visual medium therefore assumes a particular importance for Chinese surgical and manual therapies associated with waike 外科, literally 'external medicine', and specifically for shangke 傷科, the treatment of injury and trauma to the body. The very nature of these subjects demands the communication of certain kinds of dexterity and spatial awareness that may be obscured by the textual medium.

There has been a specialised medical literature in China devoted to the science of treating wounds, sores and abscesses, and using mercury to treat skin diseases, with heat sterilisation of surgical instruments, since LiuJuanzi guiwei fang 劉涓子鬼遺方 (Liu Juanzi's Remedies Bequeathed by Ghosts), which dates from the Jin period $\left(265^{-420} \mathrm{CE}\right)$. Early treatises on external medicine and traumatology of this kind were not illustrated, and relied solely on textual description. But as the sum of medical knowledge and the fund of clinical experience grew, this format posed increasing challenges for the student, and so illustrations were introduced as an adjunct to the written text. The earliest surviving illustrated book on surgery and traumatology in China is Weiji baoshu 衛濟寶書 (Wei Ji's Precious Book), in two juan (fascicles), compiled by the hermit Dong Xuan 東 軒 in the Song period (96o-1279). The images illustrate skin diseases as well as therapeutic locations, and demonstrate the kinds of pathologies associated with waike.

These earliest illustrations had an indicative value only, being intended as aids to understanding and mastering difficult points in the text. Later, visual images became an integral part of many medical works, regardless of the degree of difficulty of the text. Lavish productions became ever more numerous and sophisticated. The prime example with the greatest number of surgical illustrations, 260 in all, is the Qing-dynasty book Waike xinfa yaojue 外科心 法要訣 (Knowledge and Skills of External Medicine in Verse), also known as Yizong jinjian waike 醫宗金鑒外科 (External Medicine from the Golden Mirror of Medicine) and Yizongjinjian:waikexinfayaojue 醫宗金鑒外科心法
要訣 (Golden Mirror of Medicine: Knowledge and Skills of External Medicine in Verse). ${ }^{1}$

Images enhanced, and sometimes replaced, textual instructions, but they also had other functions. Thus for example, diagrams of body parts served to identify affected areas and therapeutic locations; illustrations of therapeutic techniques served as an aid to acquiring and carrying out these techniques; and illustrations of medical instruments were important to both the manufacturer and the user. In such cases, illustration played a vital role that words alone could not fulfil. The importance of manual skills and their illustration in the history of medicine in China is often overlooked, particularly as they arose in military medicine, the martial arts, and bone-setting. Historians generally favour researching scholarly medical works, and this bias distorts the record and our appreciation of the range and plurality of medicine in the past in China (for examples from veterinary surgery, see Buell et al., Chapter 22 in this volume). This chapter therefore offers an overview of illustration in the literature of surgery and traumatology in China by way of preliminary redress.

The first edition of the National Union Catalogue of Traditional Chinese Medicine Books (Quanguo zhongyi tushu lianhe mulu 全國中醫圖書聯合目録) lists 12,124 works on medicine published before 1949. They include 448 texts listed as external medicine and 181 listed in the category of traumatology (shangke) - 629 in all, making up a significant $5 \%$ of the total. Weiji baoshu, introduced above, provides a good introduction to the range of interest and expertise in surgery and trauma study. It discusses the causation and pathogenesis of sores and abscesses, the five good practices (wu shan 五善) and seven evil karmas ( $q i$ $e$ 七惡) of Buddhist philosophy, and symptoms of illness. The Buddhist terms wu shan and qi e refer respectively to five or seven prohibitions: no killing, no stealing, no adultery, no lying, and no alcohol; and no killing, stealing, adultery, lying, speaking senselessly, harsh words, and slander. Chinese physicians then brought these Buddhist concepts of morality to bear in describing the state of, for example, the five organs.

\footnotetext{
1 This is a separate edition of the sections on external medicine of Yizong jinjian 醫宗金鑒 (The Golden Mirror of Medicine), a large-scale medical work published under the aegis of the Qing government in 1742 ( 7 th year of the Qianlong reign period of the Qing dynasty).
} 
Weijibaoshu contains illustrations of five categories of skin disease: ai 癌 (cancers), biao 瘭 (whitlows), ju 疽 (carbuncles, abscesses, phlegma), gu 㽽 (chronic, intractable conditions), and yong 痄 (abscesses). Apart from fulfilling the pleasures of professional connoisseurship, differentiating skin diseases presumably served the function of matching symptom with therapeutic procedure. There are also illustrations of instruments and techniques that have obvious practical value: an injection method, the application of poultices to the back, and the moxibustion technique called 'Riding the Bamboo Horse', of which we will see more of in a moment.

The second volume of Weiji baoshu contains $5^{\circ}$ prescriptions for external application, and discusses in detail medications for breast abscesses and soft boils. In addition, the book contains two further diagrams. One of these shows a method for establishing the location on the spine for applying poultices. The other shows six moxibustion points for treating abscesses and ulcers, labelled quchi 曲 池, shou qili 手七里, jianfeng 肩峰, zu sanli 足三里, fengshi 風市, and tuifengguzhong 腿縫骨中.

The 26o illustrations of surgical conditions in Waike xinfa are divided into 16 juan (volumes or fascicles). Juan 1 and 2 form a general introduction. Juan $3^{-11}$ deal with external conditions with a specific location, proceeding from the head to the feet.Juan 12-14 deal with external conditions that do not have a specific location. Juan 15 deals with miscellaneous disorders, and juan 16 is devoted to paediatrics. The entire text is a reworking of Waike dacheng 外科大成 (The Great Compendium of External Medicine), 1665. The entry for each disorder begins with a summary of symptoms and remedies in heptasyllabic verse, for ease of memorisation. This is followed by more detailed explanations. A highly influential text, it was studied and recited from memory by generations of medical students.

The 629 pre-1949 texts on external medicine and traumatology cited above contain a higher-than-average proportion of illustrations. Nonetheless it should be noted that only eight of them actually have the word $t u$ 圖 (illustration, visual representation) in their title, and all of those were published in the Qing period (1644-1911) or later. This is suggestive of an increasing modern attention to establishing this genre of illustrated texts.

Waike and shangke illustrations can be divided into the following 18 categories according to their subject matter.

\section{Affected Parts}

Images showing diseased or injured body parts. This is the largest group of illustrations. They convey information at a glance, in the most readily comprehensible manner. The Ming (1368-1644) work Waike qixuan 外科啓玄 by Shen Gongchen 申拱宸 (1607), contains more than 150 illustrations of this kind. Primarily intended to indicate the location of the disease or injury, they rarely show any

Book Titles Including the Character Tu 圖 (pre-1949)

\begin{tabular}{|c|c|c|c|}
\hline Title & Translation & Date & Author \\
\hline $\begin{array}{l}\text { Zhengcang waike tu } \\
\text { 枕藏外科圖 }\end{array}$ & Surgical Images to be Kept under the Pillow & c. 1767 & Anon. \\
\hline $\begin{array}{l}\text { Waike tuxing maizheng } \\
\text { 外科圖形脉證 }\end{array}$ & $\begin{array}{l}\text { Pulses, Signs and Symptoms of External Medicine } \\
\text { in Pictures }\end{array}$ & 1795 & $\begin{array}{l}\text { Zheng Yutan } \\
\text { 鄭玉壇 }\end{array}$ \\
\hline Waike tushuo 外科圖說 & Pictorial Guide to External Medicine & 1834 & $\begin{array}{l}\text { Gao Wenjin } \\
\text { 高文晉 }\end{array}$ \\
\hline $\begin{array}{l}\text { Waike dazheng xingtu } \\
\text { 外科大症形圖 }\end{array}$ & Major External Disorders in Pictures & $185^{2}$ & $\begin{array}{l}\text { Wang Jinfu } \\
\text { 王晉夫 }\end{array}$ \\
\hline $\begin{array}{l}74 \text { zhong dingchuang tushuo } \\
\text { 七十四种疔瘡圖說 }\end{array}$ & Pictorial Guide to 74 Types of Boils and Sores & 1889 & Mr Ye 葉氏 \\
\hline Ciding tu 刺疔圖 & Illustrations of Lancing Boils & $\begin{array}{l}\text { Qing } \\
(1644-1911)\end{array}$ & Anon \\
\hline $\begin{array}{l}\text { Quanshen gutu kaozheng } \\
\text { 全身骨圖考正 }\end{array}$ & A Study of Illustrations of the Skeleton & c. 1854 & Xu Lian 許槤 \\
\hline $\begin{array}{l}\text { Zhongguo jiegu tushuo } \\
\text { 中國接骨圖說 }\end{array}$ & Pictorial Guide to Chinese Bone-Setting & 1807 & $\begin{array}{l}\text { Ninomiya Hika } \\
\text { 二宮彥可 }\end{array}$ \\
\hline
\end{tabular}


details of the condition itself, which is generally signalled by a dot or circle.

\section{Therapeutic Locations}

These images show the location where treatment is to take place, which does not necessarily coincide with the affected area. As this information could be difficult to convey in words, illustrations were an essential visual aid. The illustration of the moxibustion technique called 'Riding the Bamboo Horse' (Qi zhuma jiutu 騎竹馬炎圖) in Chen Ziming's 陳自明 Song-dynasty work Waike jingyao 外科 精要 (Essentials of External Medicine), 1263, is a case in point (Fig. 1). This is essentially a moxibustion technique, but it demonstrates the manual skill needed to find the locations, and the complexities of collaboration with the patient and the physician's assistants incurred in the treatment of sores and abscesses.

The accompanying text reads:

This is used to treat all kinds of sores and abscesses; it is indicated in all cases. To perform this technique, have the patient lean on the elbow. The wrist must be straight and pointing upward. Extend a string from the horizontal crease at the middle of the wrist, on the left for a man and the right for a woman. Measure along the flesh to the tip of the middle finger, excluding the fingernail, and cut off the string to use as a measure. Then take a bamboo pole, and have the patient undress and sit astride it. Two people should then raise the pole at the back and front, till the patient's feet no longer touch the ground. Two more people should support him/her, ensuring that his/her back is kept straight. Then using the piece of string previously obtained as a measure, measure from the end of the coccyx as the patient sits astride the bamboo pole, up along the back to the end of the piece of string, keeping close to the flesh. Mark this spot with a black ink mark. Note that this is a reference point only; it is not a location for moxibustion. Then using a piece of thin string as a ruler, measure the patient's middle finger joint between the two horizontal creases, on the left for a man and the right for a woman, and cut off the string to use as a measure. Measure this distance to either side of the mark previously made. The outer limits are the locations for moxibustion.

Without reference to the illustration, this explanation is hard to follow, and the therapeutic points could not be reliably identified.

\section{Therapeutic Methods}

Images illustrating particular therapies. A typical example is the illustration of an orthopaedic traction method known as Pansuo diezhuang (Holding ropes and standing on stacks of bricks) in Shangke buyao 傷科補要 (Supplement to traumatology), 1808 (Fig. 2).



Figure 12.1 Riding the Bamboo Horse (moxibustion technique). Chen Ziming 陳自明 1263 , Waike jingyao 外科精要 (Essentials of External Medicine). (c) Library of China Academy of Chinese Medical Sciences

As the accompanying text explains, this is a technique used to treat an injury approximating to the modern slipped disk, with stiffness and pain in the lumbar region. Two lengths of rope are suspended from a height, and a stack of bricks is placed beneath each of them, one on either side. The patient has to stand on the bricks, grasping a rope in each hand. The patient is supported at the small of the back, while the bricks are removed one at a time, obliging him or her to adopt an upright stance. This is done three times, or as long as the patient's feet can still touch the ground. It is supposed to smooth the flow of Qi and disperse stagnations, raise prolapsed vertebrae, and correct curvature. The illustration is both lifelike and aesthetically pleasing.



Figure 12.2 Holding ropes and standing on stacks of bricks. Qian Xiuchang 錢秀昌 1808 , Shangke buyao 傷科補要 (Supplement to Traumatology). $\odot$ Wellcome Library, London Loo38859 




Figure 12.3 Hu Tingguang's 胡廷光 Shangke huizuan 傷科彙纂 (completed 1815), Beijing: Zhongyi guji, 1997, p. 14

Hu Tingguang's 胡廷光 1815 Shangliao huizuan 傷科 彙纂 (Anthology of Traumatology) makes use of vivid illustrations and rhymed couplets to convey information about orthopaedics and bone-setting in an accessible and memorable way. It includes a number of simple techniques especially suited to emergency and first-aid use, such as the two methods for reducing a dislocated shoulder shown in Figure 3.

\section{Bone-Length Measurements}

Illustrations indicating the (relative) lengths of the bones, as discussed in the 'Chapter on Bone-length Measurement' of the Numinous Pivot (Lingshu jing: gudu pian 靈樞經. 骨度篇). Qian Xiuchang's 錢秀昌 1808 Shangke buyao contains bone-length diagrams for the front and back of the body, showing the bones of the head, the thorax and abdomen, the back, the sides, and the limbs (Figs 4a, b).
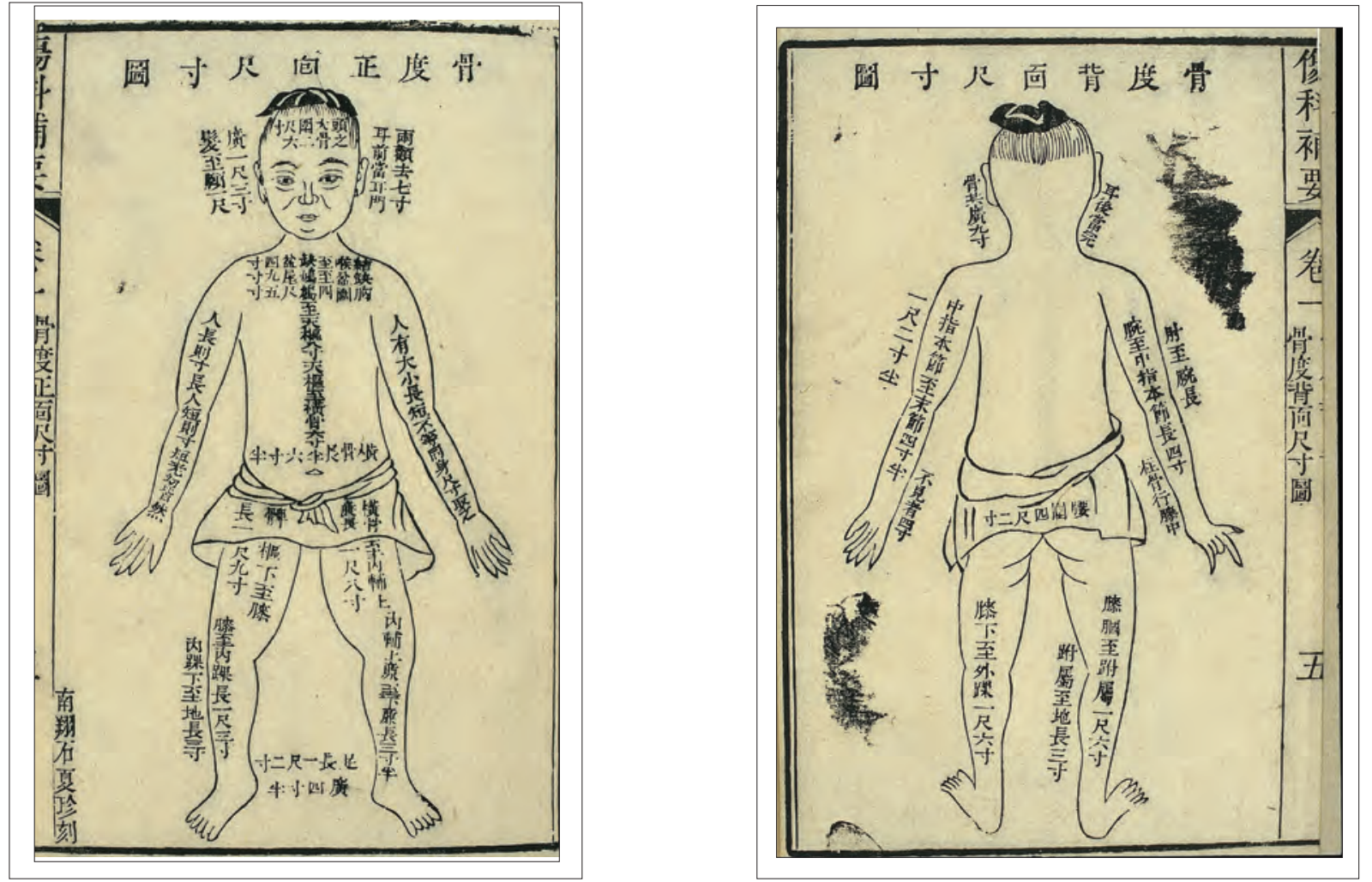

Figures 4a, b Charts of bone-length measurement in chi and cun (anterior and posterior views). Qian Xiuchang 錢秀昌 1808, Shangke buyao 傷科補要 (Supplement to Traumatology), ed. 1976, pp. 9-10. $\odot$ Wellcome Library, London, Loo38855 and Loo38854 


\section{The Skeleton}

Charts showing the number, names, form and structure, and position of the bones of the skeleton. The significance of each element of the skeleton is also explained in words, i.e. whether or not injury to that part would result in death. Shangke huizuan 傷科彙纂 (Traumatology Compilation) has four illustrations of the skeleton: two diagrams of the bones of the skeleton viewed from the front and the back, and two fatality charts, also from the front and back view. The skeletal images in Shangke buyao 傷科補要, when compared with those in the forensic medical literature, for example, Chongkan buzhu xiyuanlujizheng 重刊補注 洗冤錄集證 (1844), do not show any essential difference, but rather indicate an inheritance of illustrations of the earlier genre in the construction of therapeutic rather than legal expertise.

\section{Appearance of Clinical Conditions}

Images were used to show the appearance and signs of specific conditions. For instance, Waike qixuan distinguishes, with the aid of illustrations, 24 types of haemorrhoids, each with a distinct morphology (see table below).
A casual glance through this list impresses the reader with the degree to which visual images are evoked not only through illustration, but also through verbal reference to animals and fruits, blurring categorical boundaries between the human and a distinct non-human world. Over half of the descriptors relate to the common physical morphologies of cow udders or pearls, cockscombs or walnuts etc. From the earliest surviving texts on pathology, we see this kind of animal imagery used to differentiate the sounds emitted during different types of convulsion, or to depict the qualities of the pulse. Waike qixuan also includes the graphically labelled ezhang feng 鵝掌風 (goose-skin palm) as well as other, more plainly named, symptoms such as luo 瘰 (scrofula) and terms containing references to pathogenic causes like Wind-Heat, e.g.xuefeng chuang 血風瘡 (itching eruptions due to Wind-Heat in the blood).

It is interesting also to speculate why it was felt so important to differentiate styles of haemorrhoids in such detail. In Chapter 16 of this volume, by Wan Fang, we will see representations of smallpox. In this case, the list of morphologically distinct varieties of haemorrhoids not only suggests a fascination with the grotesque appearance of ab-

Differentiation of Haemorrhoids in Waike Qixuan

\begin{tabular}{|c|c|}
\hline lingjiao zhi 菱角痔 & Water-chestnut haemorrhoids, multifistular haemorrhoids \\
\hline lianhuazhi 蓮花痔 & Lotus-flower haemorrhoids, hyperplastic haemorrhoids \\
\hline chuanchang zhi 穿腸痔 & Haemorrhoids passing through the intestine, haemorrhoid and anal fistula?) \\
\hline shunai zhi 鼠奶痔 & Rat's breast haemorrhoids \\
\hline lianhuajie 蓮花結 & Lotus clusters \\
\hline fengke zhi 蜂窠痔 & Bee-hive haemorrhoids \\
\hline cixiong zhi 䧳雄痔 & Female and male haemorrhoids \\
\hline zimuzhi 子母痔 & Mother and child haemorrhoids, prolapsed internal haemorrhoids \\
\hline xuanzhuzhi 懸珠痔 & Hanging pearl haemorrhoids, fibropapilloma of the anus \\
\hline gouchang zhi 鈎腸痔 & Haemorrhoids hooked into the intestine \\
\hline hetao zhi 核桃痔 & Walnut haemorrhoids \\
\hline lizizhi 栗子痔 & Chestnut haemorrhoids \\
\hline jiguan zhi 雞冠痔 & Cockscomb haemorrhoids \\
\hline shanhu zhi 珊瑚痔 & Coral haemorrhoids \\
\hline neizhi 内痔 & Internal haemorrhoids \\
\hline danchang zhi 擔腸痔 & Haemorrhoids lifting the intestine \\
\hline chuizhu zhi 垂珠痔 & Drooping pearl haemorrhoids \\
\hline jixin zhi 雞心痔 & Chicken's heart haemorrhoids \\
\hline qizhi 氣痔 & Qi/air haemorrhoids, internal haemorrhoids with prolapse of rectum \\
\hline xuezhi 血痔 & Blood haemorrhoids, internal haemorrhoids with bleeding \\
\hline niunaizhi 牛奶痔 & Cow's udder haemorrhoids \\
\hline yangnai zhi 羊奶痔 & Sheep's udder haemorrhoids \\
\hline chuantun zhi 串殿痔 & Haemorrhoids running through to the buttocks \\
\hline liwaizhi 裏外痔 & Internal and external haemorrhoids \\
\hline
\end{tabular}





Figure 12.5a, b Fixation appliances for fractured kneecap (Baoxi yongfa tu 抱膝用法圖), from Qian Xiuchang's 錢秀昌 Shangke buyao 傷科補要 (juan 1), finalised 1808, printed by Zhuyin tang 竹蔭堂 (Hongkou 虹口, Shanghai) 1818, pp. $49,5^{\circ}$

normal physical conditions, but also would have facilitated the communication of practical procedures. Haemorrhoids like those 'hooked into the intestine' might have required a more dangerous operation, and different surgical instruments from 'cow's udder' or 'hanging pearl' haemorrhoids.

\section{Aetiological Factors}

Pictures showing the causes of medical conditions also evoke the animal kingdom. For instance in Shen Gongchen's 1607 Waike qixuan, pictures of a tiger, a dog, a snake, a scorpion etc. are used as emblems of bites inflicted by these creatures. These animals indicate very direct and easily visualised traumatic causes. Particularly graphic are the 'human bites' as illustrated by a vivid sketch of two men fighting, one of them sinking his teeth into the other's arm. The entry on suicide by cutting the throat shows a general in full armour, in the act of drawing his sword to slit his own throat. More subtle causes such as Wind-Heat or diseases of Qi are not illustrated, probably in consequence of their more abstract nature.

\section{Orthopaedic Instruments}

Illustrations were used to show the appearance and structure of therapeutic instruments, and sometimes also to demonstrate their use.

Shangke buyao contains depictions of a lumbar fixation splint (yaozhu 腰柱), a fixation appliance for fractured kneecaps (baoxi 抱膝) (Fig. 5), and two other devices for setting and fixing broken bones described as shanli 杉篗 (fir wood fence) and muban 木板 (wooden board) (Figs


Figure 12.6 (left) Bamboo curtain, orthopaedic appliance. Hu Tingguang's 胡廷光 Shangke huizuan 傷科彙纂 (completed 1815), Beijing, Zhongyi guji, 1997, p. 12

Figure 12.7 (right) Fir-wood fence, orthopaedic appliance. Xiuchang 錢秀昌 1808 , Shangke buyao 傷科補要. (C) Wellcome Library, London Loo38861

$6,7)$. There are additional illustrations showing these appliances in use.

Ninomiya Hika's 二宮彥可 1807 Waike tushuo 外科圖 說 (Pictorial Guide to External Medicine) contains illustrations of 35 specialised implements used in petty surgery and external medicine. They include:

square-pointed scissors (fangtoujian 方頭剪)

sharp-pointed scissors (jiantou jian 尖頭剪)

claw scissors (zhuajian 爪剪)

tongs or tweezers (jiajian 夾剪)

curved knife (wan dao 彎刀)

hooked knife (gou dao 鈎刀)

willow-leaf knife (liuye dao 柳葉刀)

sharp-pointed knife (jiantou dao 尖頭刀)

razor (bi dao 筆刀)

cooking cylinder, for heating liquid drugs ( pengtong 烹筒)

tube for applying medicaments (yao tong 藥筒)

mercuric oxide tube (shengyao tong 升藥筒)

anal probe (tan gang tong 探肛筒)

needle sheath for anal insertion to treat piles (chuangang zhentao 穿腸針筒)

large forceps (da qian 大鉗)

straight forceps (zhi qian 直鉗)

double hook (shuang gou 雙勾)

silver wire (yinsi 銀絲)

cautery iron (laotie 烙鐵).

It would be hard to obtain an accurate impression of their nature and use from a description alone.

\section{Pharmaceutical Substances}

Illustrations of medicinal plants figure largely in the Materia Medica books (herbals). They are also found 




Figure 12.8 Chart of the healing herb rendongteng 忍冬藤 (Japanese honeysuckle, Lonicera japonica), Waike jingyao 外科精要 1273, modern edn, 2007, p. 21. (C) Library of China Academy of Chinese Medical Sciences

occasionally in works on external medicine and traumatology. For instance, Waike jingyao contains an illustration of rendongteng 忍冬藤 (Japanese honeysuckle, Lonicera japonica) (Fig. 8) with the note:

Liquor made with Japanese honeysuckle is a highly effective treatment for abscesses and ulcers of all kinds. It can also be made into a medicinal plaster with wheatmeal.

In the Materia Medica literature, Japanese honeysuckle is first recorded in Mingyibielu 名醫別録 (Informal Records of Famous Physicians), $c .510$ CE. It is described there as cooling, efficacious in clearing poisons and unblocking the channels, and frequently used to treat external conditions such as abscesses, sores and swellings.

\section{Proportional Somatic Measurements}

Proportional somatic measurement (tongshen chi 同身尺), based on a unit (cun 寸) derived from the patient's own body, was an important topic in early Chinese medicine. It was intended to take account of individual differences in build and stature and to place them within a unified framework. There were various methods for finding the value of the cun unit, based on the fingers, the eye or the mouth; most commonly it was based on the middle finger. Waike jingyao contains a somatic measurement chart (Fig. 9) accompanied by the following text:

The left hand is used for men and the right hand for women. The middle finger is bent, and the middle joint is then measured with a thin string. The horizontal creases at their widest point are the parameters for the proportional cun unit.

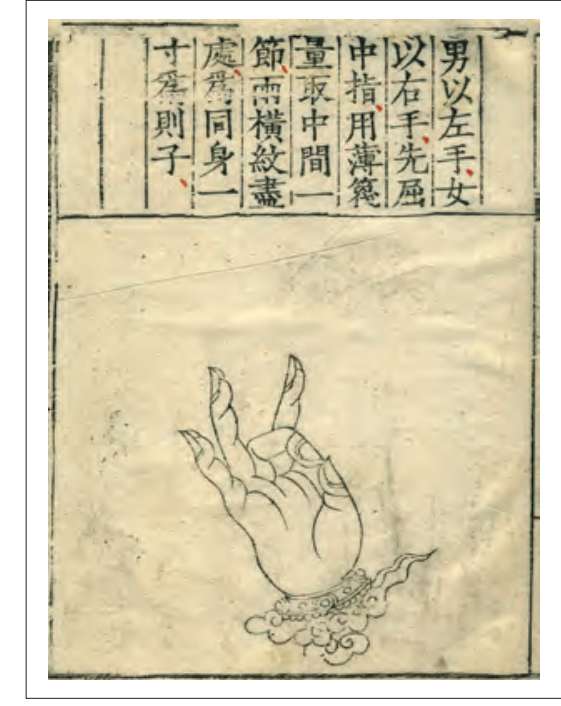

Figure 12.9 Proportional somatic measurements. Chen Ziming 陳自 明 1263, Waike jingyao 外科精要, modern edn, 2007, p. 7 . (c) Library of China Academy of Chinese Medical Sciences

\section{Charts of the Nine Mansions and Travelling Spirit} The phrase 'Nine Mansions and Travelling Spirit' (jiugong kaoshen 九宮尻神) alludes to a theory whereby the human spirit (renshen 神人) processed through the body according to times and seasons; each part of the body became subject to prohibitions on acupuncture and other therapeutic procedures as the spirit passed through it. The current location of the spirit could be calculated by reference to the divinatory systems of the Nine Palaces and Eight Trigrams. Works of external medicine such as Waike zhengzong 外 科正宗 (Orthodox Manual of External Medicine), 1617, contain ready-reference tables of prohibitions enshrining this information, which was a long-standing tradition in determining auspicious times to treat people (Fig. 10). (See Lo and Tlalim, Chapter 19 in this volume, p. 273 et passim.)

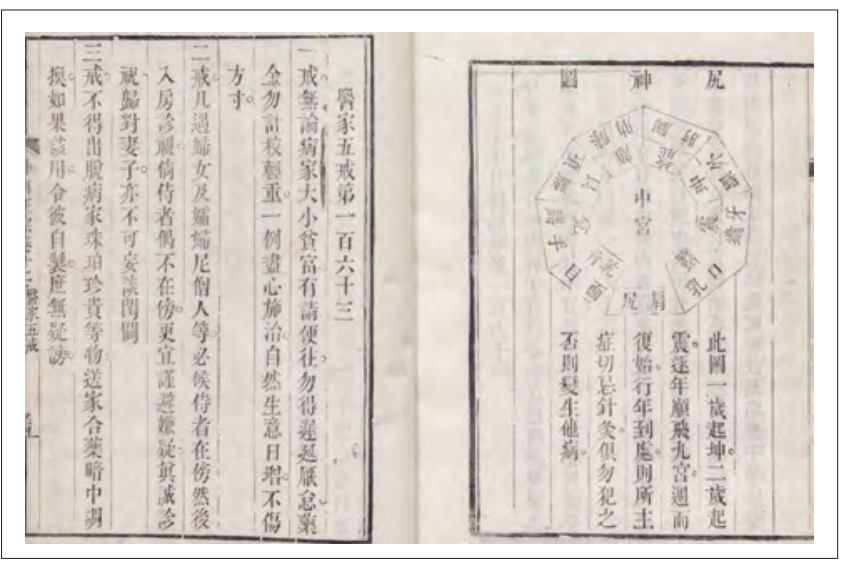

Figure 12.10 Nine Mansions and Travelling Spirit. Chen Shigong陳實 功 1617, Waike zhenzhong 外科正宗, modern edn, 1993, p. 329. (C) Library of China Academy of Chinese Medical Sciences 




Figure 12.11 a, b Charts of the Five Circulatory Phases and Six Climatic Factors; Taiji diagrams. Yangyi daquan 瘍醫大全 176o, modern edn, 1973, ch. 4 pp. 16-18, 21

\section{Charts of the Five Circulatory Phases and Six Climatic Factors; Taiji Diagrams}

The concepts of the Five Circulatory Phases and Six Climatic Factors, and Yin and Yang, so fundamental to Chinese medical theory, were applied in the practice of external medicine. Texts such as Yangyi daquan 瘍醫大全 (Great Compendium of Dermatology), 176o, incorporated tables and charts of the Five Circulatory Phases and Six Climatic Factors and Taiji diagrams to guide the practitioner in differentiating the choice of treatment method for skin-deep disorders, especially as to whether the Qi in the body should be supplemented or dispersed according to changing heat factors (Fig. 11)

\section{Charts of the Channels, Illuminated Hall Charts}

Charts of the channels appear frequently in works of surgery and traumatology. Most of them show 14 channels and the points located on those channels, and are closely modelled on the mainstream literature of acupuncture and moxibustion. For instance, Dongtian aozhi 洞天奧旨 (Profound Teachings of the Heavenly Cavern), 1694, contains 14 channel charts: one each for the 12 regular channels, one for dumai 督脉 (the Governor Vessel) (Fig. 12) and one for renmai 任脉 (the Director Vessel or Conception Vessel). Unusually, Yangyidaquan 瘍醫大全 has separate channel charts for the right and left arm. Yangke huizuan contains an example of the Mingtang tu, or Illuminated Hall chart

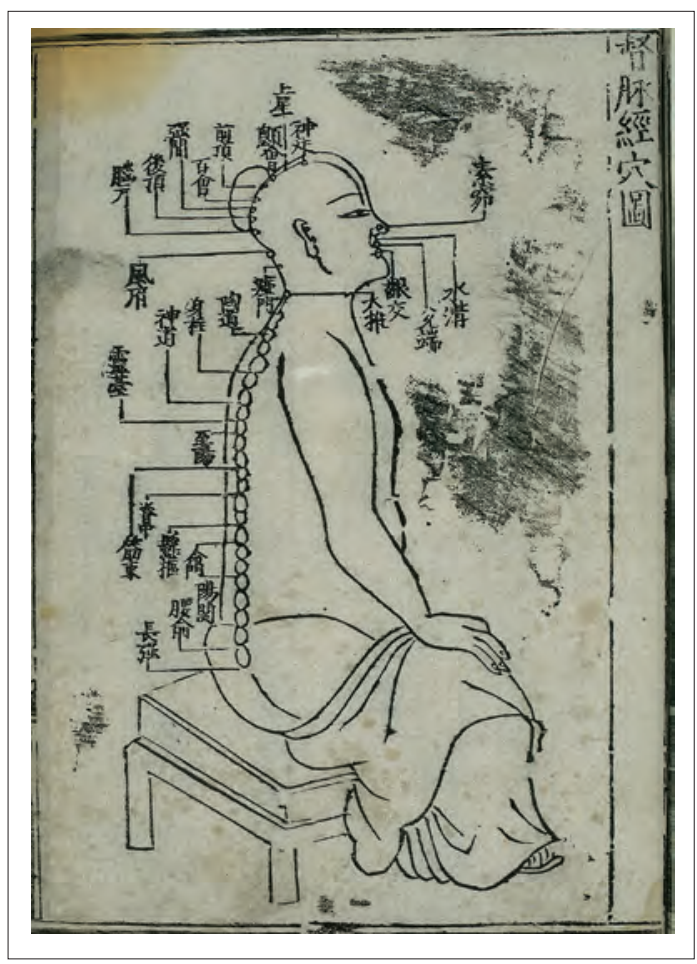

Figure 12.12 The chart of the dumai channel in Chen Shiduo 陈士 铎 1694, Dongtian aozhi 洞天奧旨(Profound Teachings of the Heavenly Cavern) (1992 edn, pp. $5^{-18}$ ) is exactly the same as the version in Gao Wu's 高武 1537 Zhenjiu juying 針炎聚英 (Collected Gems of Acupuncture and Moxibustion). ${ }^{\complement}$ Wellcome Library, London Loo37827

- an atlas of the channel network (See Lo, Introduction, p. 5 ; Wang, Chapter 1; Huang, Chapter 9, in this volume). ${ }^{2}$

The term Mingtang $t u$ 明堂圖 (Illuminated Hall Chart) appeared earlier than Zhenjiu $t u$ 銊炎圖 (Acupuncture and Moxibustion Chart) and Jingluo tu 經絡圖 (Chart of the Channels and Networks). The former term was in use by the Han period, while the latter two terms appeared no later than Tang and Song respectively. But before the Tang period, the term Mingtang tu was not associated with acupuncture and moxa-cautery, but in almost all cases referred to illustrations of a ritual hall in the royal palace, where the emperor dealt with political and official affairs - in an ideal construction of ritual rulership, the emperor moved around the palace according to the season and in concert with astronomical changes. Physicians borrowed the concept of Mingtang 明堂 to denote the human body; where acupuncture or moxa-cautery is involved, the Mingtang tu are, never theless, almost identical to Zhenjiu tu or Jingluo tu. If there is a difference, it might be that the Mingtang tu genre of chart often represents the 'whole' body, and not just its constituent parts or parts under scrutiny

2 Sun Zhenyuan 孫震元 1802 , Yangke huicui 痬科會粹, modern edn 1987, p. 44. 




Figure 12.13 Posterior location chart: Qian Xiuchang 錢秀昌 1808, Shangke buyao 傷科補要, modern edn, 1976, pp. 1-3

in a particular image-text construction. We can often see composite designations (e.g. Mingtang zhenjiu 明堂鍼炎, or Mingtang jingluo 明堂經絡, and Zhenjiu mingtang 鍼 炎明堂 in Song, Ming and Qing texts. There were also the Yuan-period zangfu mingtang tu 藏腑明堂圖, where the term zangfu 藏腑 refers to internal organs. There therefore seems to be no substantial difference between acupuncture charts, and the channel images in traumatology texts, except in terms of the way instructions are given in the surrounding text.

\section{Acu-Moxa Locations for Traumatology}

The charts of acu-moxa locations in monographs on traumatology differ somewhat from the channel and point charts found in the literature of acupuncture and moxibustion. Shangke buyao has three acu-moxa charts: a front view of the body with 19 therapeutic locations marked; a back view with 16 locations; and a side view with 12 locations (Fig. 13). Most of these locations are labelled with names associated with body parts, only a few of which are technical terms from acupuncture. Charts of therapeutic body locations that are labelled primarily according to anatomical terminology, and not the classical acu-moxa locations, are known from medieval moxa-cautery manuscripts (See Lo and Tlalim, Chapter 19 in this volume). In this case, the physical injury suggests that the locations for traumatology belong to a different tradition, which was more closely related to the strategic points in wushu
武術 (martial arts) and perhaps also to forensic medicine, concerned with the establishing of a case history of injury through physical attack to the live or dead body.

\section{Internal Visualisation Images; Illustrations of the Internal Organs}

Images showing the location or form and structure of the five $z a n g$-viscera and six fu-viscera. Chapter three of Yangyi daquan contains internal visualisation images, showing the internal topography of the body, following a chapter devoted to theory, and one on diagnosis. In contrast with internal visualisation charts that represent the inscape of the body as microcosm, which include depictions of the constellations in the form of the sixiang 四像 (the four animals of the constellations) and other spirit and meditational entities such as the chizi 赤字 (the red infant [of immortality]) (See Despeux, Chapter 2 in this volume, pp. 58-65), the chart in Figure 14 concentrates the gaze on mundane anatomy. Apart from the Mingmen 明門, which is a gate of the body and a site of more esoteric anatomy than the viscera, it marks the heart, the lungs, the liver, the kidneys, the stomach, the spleen, the bladder etc. It is followed by versions of the Yanluozi diagrams that we have already seen described in Catherine Despeux' chapter, and then a series of images that isolate the organs themselves. The accompanying text contains excerpts on the viscera from many received medical works, notably recensions of the Yellow Emperor's corpus and the Nanjing. These quotations outline the connections between the organs and their physiological roles. Sun Zhenyuan's 孫震元 1802 Yangke huicui 瘍科會粹 (Treasury of Dermatology) contains an internal visualisation chart with illustrations of the viscera (Fig. 15), but the chart is juxtaposed with other human figures with much more anatomical detail, such as bones and blood vessels, as well as the more common illustrations of the acu-moxa channels. Overall these images serve to focus the attention on knowledge of the physicality of the body and its viscera, in keeping with the manual and practical therapies that follow.

\section{Pulse Diagnosis}

Diagrams and tables showing the locations for pulse diagnosis and the correlations between the pulses and the internal organs. Such charts are most commonly associated with internal medicine. However, the introduction to Yangyi daquan 瘍醫大全 laments the loss of ancient knowledge about the relationship between surgical methods and, for example, the understanding of the superficial and deep pulses as they relate both to the internal organs and to the likelihood of a person surviving a life-threatening situation. Examples are the illustrations of pulse-taking 




Figure 12.14 Internal visualisation images with internal organs: Sun Zhenyuan 孫震元 1802, Yangke huicui 瘍科會粹 (Treasury of Dermatology). (C) Library of China Academy of Chinese Medical Sciences

in Yangyi daquan and the table of correlations between pulses and viscera in the 1742 Waike xinfayaojue 外科心法 要訣 (Knowledge and Skills of External Medicine in Verse).

\section{The Alchemical Furnace}

The alchemical elixir known as danyao 丹藥 was frequently used in external medicine. Both the refining process and the furnace itself were considered crucial to the quality of the finished product. Waike tushuo, 1856, contains illustrations of two types of alchemical furnace. They show implements for creating and refining bagua dajiangdan 八 卦大降丹 (Eight-Trigram Grand Descending Elixir), with special attention given to the internal structure of the alchemical furnace. This elixir is a type of Hydrargyrum chloratum compositum - jiangdan 降丹 or bai jiangdan 白降丹 - obtained through the mixed crystallisation of mercuric chloride $\left(\mathrm{HgCl}_{2}\right)$ and mercurous chloride $\left(\mathrm{Hg}_{2} \mathrm{Cl}_{2}\right)$ by heating mercury together with various minerals in the furnace. It was used to treat ulcers, fistulas, luoli 疼康 (scrofula), xirou 瘜肉 (polyps), wanxuan 頑癬 (chronic itchy skin condition), etc. (Fig. 15).



Figure 12.15 Alchemical Furnace from Waike tushuo 外科圖說 (Pictorial Manual of External Medicine), published in 1856 (6th year of the Xianfeng reign period of the Qing dynasty). (C) Wellcome Library, London, Loo38814

\section{Authors' Portraits}

Some texts were adorned with portraits of the author, like the portrait of the physician Wang Ji 汪機 (1463-1539) in Waike lili 外科理例 (Principles and Examples of External Medicine) (Fig. 16). These images clearly have no practical value in therapy and are apparently inserted as a way of enhancing authorial authority.

\section{Summary}

The extant literature on waike and shangke yields a vast array of visual images, which fall into 18 broad categories as described above. Many of these categories are specific to surgery and traumatology - in particular the illustrations of affected parts, therapeutic locations, orthopaedic methods, clinical presentation, causation, surgical instruments, and the bones and skeleton. Others however, such as the tables of the Five Circulatory Phases and Six Climatic Factors and the channel charts, are drawn from different medical disciplines, indicating that cross-fertilisation occurred between surgery and traumatology, and other branches of learning. The illustrations of acu-moxa locations in particular seem to have more in common with points for combat training than with classic charts of the points and 




Figure 12.16 Wang Ji 汪機 (1463-1539). Waike lili 外科理例 (Principles and Examples of External Medicine). (c) Wellcome Library, London, Loo38739

channels, suggesting a link with martial arts traditions that deserves further research. Above all however, knowledge of the physiology of the body, and the movement of its fluids and spirits according to cosmic and seasonal cycles, and an understanding of when the Qi and the organs and channels were in the most appropriate conditions to respond to intervention, were all essential factors in determining the proper time for treatment.

The function of illustration in writings on external medicine and traumatology was shaped by the nature of these specialist areas and the complexity of the therapeutic methods involved. In carrying out, demonstrating and teaching the variety of therapies illustrated above, we can see that it was necessary to coordinate a community of people for the therapeutic performance: in orthopaedic medicine in particular, the therapist did not work alone, but in conjunction with assistants who pulled, lifted and supported the patient. Considerable skill was also necessary in the production of a wide and inventive range of orthopaedic and surgical instruments. Thus visual images came to play a vital role both in the diagnosis and in the transmission of therapeutic methods, in a way that was a natural and effective supplement to textual instructions.

\section{Bibliography}

\section{Primary Sources}

Anon. (Qing 1644-1911), Ciding tu 刺疔圖 (Illustrations of Lancing Boils). Anon. c. 1767 , Zhengcang waike tu 枕藏外科圖 (Surgical Images to be Kept under the Pillow).

Chen Shiduo 陈士铎 [1694] 1992, Dongtian aozhi 洞天奧旨 (Profound Teachings of the Heavenly Cavern) (Qing), Beijing: Zhongyi guji chubanshe.

Chen Shigong 陳實功 1617, Waike zhengzong 外科正宗 (Orthodox Manual of External Medicine).

Chen Ziming 陳自明 [1263] 2007, Waikejingyao 外科精要 (Essentials of External Medicine), Beijing: Zhongguo zhongyao.

Dong Xuan 東軒 Song period (96o-1279), Weiji baoshu 衛濟寶書 (Wei Ji's Precious Book).

Gao Wenjin 高文晋 1856, Waike tushuo 外科圖說 (Pictorial Guide to External Medicine).

Gu Shicheng 顧世澄 [176o] 1973, Yangyi daquan 瘍醫大全 (Great Compendium of Dermatology), Taibei: Xuanfeng.

Hu Tingguang 胡廷光 [1815] 2006, Shangke huizuan 傷科彙纂 (Anthology of Traumatology), Beijing: Renmin weisheng chubanshe.

Ninomiya Hika二宮彥可 1807 , Zhongguojiegu tushuo 中國接骨圖說 (Pictorial Guide to Chinese Bone-Setting).

Qi Hongyuan 祁宏源 1742, Waike xinfa yaojue 外科心法要訣 (Knowledge and Skills of External Medicine in Verse) [also known as Yizongjinjian waike 醫宗金鑒外科 (External Medicine from the Golden Mirror of Medicine) and Yizong jinjian: waike xinfa yaojue 醫宗金鑒外科心法要訣 (Golden Mirror of Medicine: Knowledge and Skills of External Medicine in Verse)].

Qi Kun 祁坤 1665 , Waike dacheng 外科大成 (The Great Compendium of External Medicine).

Qian Xiuchang 錢秀昌 [1808] 1976 reprint, Shangke buyao 傷科補要 (Supplement to Traumatology), Taibei: Wenguang tushu gongsi.

Shen Gongchen 申拱宸 1607, Waike qixuan 外科啓玄 (Dispelling the mysteries of external ailments).

Sun Zhenyuan 孫震元 [1802] 1987, Yangke huicui 瘍科會粹 (Treasury of Dermatology), Beijing: Renmin weisheng chubanshe.

Wang Ji 汪機 (1463-1539) 1531, Waike lili 外科理例 (Principles and Examples of External Medicine).

Wang Jinfu 王晉夫 $185^{2}$, Waike dazheng xingtu 外科大症形圖 (Major External Disorders in Pictures).

Xu Lian 許梿 c.1854, Quanshen gutu kaozheng 全身骨圖考正 (A study of Illustrations of the Skeleton).

Ye 叶 1889, 74 zhong dingchuang tushuo 七十四種疔瘡圖說 (Pictorial Guide to 74 Types of Boils and Sores).

Zheng Yutan 鄭玉壇 1795 , Waike tuxing maizheng 外科圖形脉證 (Pulses, Signs and Symptoms of External Medicine in Pictures).

\section{Secondary Sources}

Quanguo zhongyitushu lianhe mulu 全國中醫圖書聯合目録 (National Union Catalogue of Traditional Chinese Medicine Books) 1991, Beijing: Zhongyi guji chubanshe. 



\section{Part 3}

\section{Imagining Medical Practice}





\title{
13 Polychrome Illustrations in the Ming Bencao Literature
}

\author{
Cao Hui 曹暉
}

\section{Introduction}

In the aftermath of the Opium Wars, many classics of the Bencao literature were destroyed or dispersed abroad. As a result, Chinese medicine and pharmacology accounts for only about $10 \%$ of classical texts available in China in the modern era. Until recently, China has lagged behind Japan and Korea in research and development, as well as the commercial production and marketing of Chinese pharmaceuticals. Toward the close of the Republican period (1911-49), a number of scholars and lay enthusiasts became interested in compiling a new compendium of Chinese Materia Medica, but due to lack of funding and the sheer quantity of material, this ambitious project was never realised. At the beginning of the 199os, with China embarked on a new phase of stability and economic development, the scene was set for a new golden age of publication. Major editorial projects were set up, which resulted in the publication of Zhonghua dadian: Yiyao dian 中華大典. 醫 藥典 (A Great Dictionary of Chinese Culture: Medical and Pharmaceutical Literature) and Zhonghua bencao 中華本 草 (Chinese Materia Medica). ${ }^{1}$

Soon after the turn of the 21st century, one of the largest non-governmental research groups ever assembled, working independently under the Chinese Cultural Research Institute, completed a monumental cultural project Zhongguo bencao quanshu 中國本草全書 (The Complete Collection of Traditional Texts on Chinese Materia Medica). For an entire generation of scholars of Chinese medicine and culture, seeing this magnum opus finally in print was the fulfilment of a long-cherished dream.

Zhongguo bencao quanshu runs to 410 juan, contains 25,000,000 Chinese characters, and weighs 1.5 tonnes. $^{2}$ It collects together the systematically edited and collated texts of over 800 specialist treatises on materia medica going back as far as the pre-Qin era (before the 3rd century BCE), and over 10,000 other written records from all epochs relating to drugs and the pharmacopoeia. The latter include more than 6,ooo texts on materia medica from medical literature, and more than 8,ooo texts from local annals and gazetteers, pharmacopoeias of various ethnic groups, religious literature and documents, and works by

$1 \quad$ Zhonghua dadian: Yiyao weisheng dian 1999-, Guojia zhongyiyao guanli ju 1998-.

2 Zhongguo wenhua yanjiuhui 1999-.
non-Chinese scholars. It also contains over 10,000 visual images from the whole spectrum of Bencao literature, almost 7,000 of them in colour.

The Bencao literature is not only an important part of China's scientific and cultural heritage; it is also a repository of cultural history. It is a unique resource for both the natural sciences and the social sciences, which cries out to be more fully explored. It is a rich mine of data and theory that encompasses medicine, pharmacology, biology and natural history, and extends beyond these fields into the areas of agronomy, horticulture, handicrafts, metallurgy, disaster and famine relief, and sustainable production, forming a unique, cross-disciplinary intellectual system. It is full of ideas about healthy living and ecology, and cultural and artistic content, embracing folkways, national and local traditions, peripheral cultures and religion. The Bencao tradition is integral to Chinese society and emblematic in many ways of Chinese culture. It is this tradition that has allowed Chinese civilisation to endure through the travails and natural disaster that have marked its long history.

Perhaps more importantly still, in the process of editing and studying this material, one gradually comes to acquire a different perspective on human development from anything afforded by traditional historiography. In the course of history, the Chinese people have performed two unique services for posterity: first, they have accumulated 3,000 years' worth of continuous astronomical observations and research, which are still consulted by the international scientific community; second, they have contributed 2,000 years' worth of clinical records, documenting the interaction between the human body and its natural environment (in the twin aspects of medicine and food), which stretch continuously from antiquity right up to the present day. In the words of the preface to Zhongguo bencao quanshu:

The object of Materia Medica studies is nothing less than life and death itself. And in turn, Materia Medica studies represents the fruit of countless cycles of life and death spanning many thousands of years. It is distilled out of the lives and accumulated wisdom of generation upon generation of human beings. It is like a great river: downstream stand the immeasurable throng of those who have experienced its benefits, and yet very few people have ventured into its upper reaches, or have even considered that such a place might exist. This book will take you on a voyage of discovery to the upper reaches of that river.

Back in the 1980s, when I was working on the state-sponsored Ming Materia Medica texts for my MA thesis, I found 

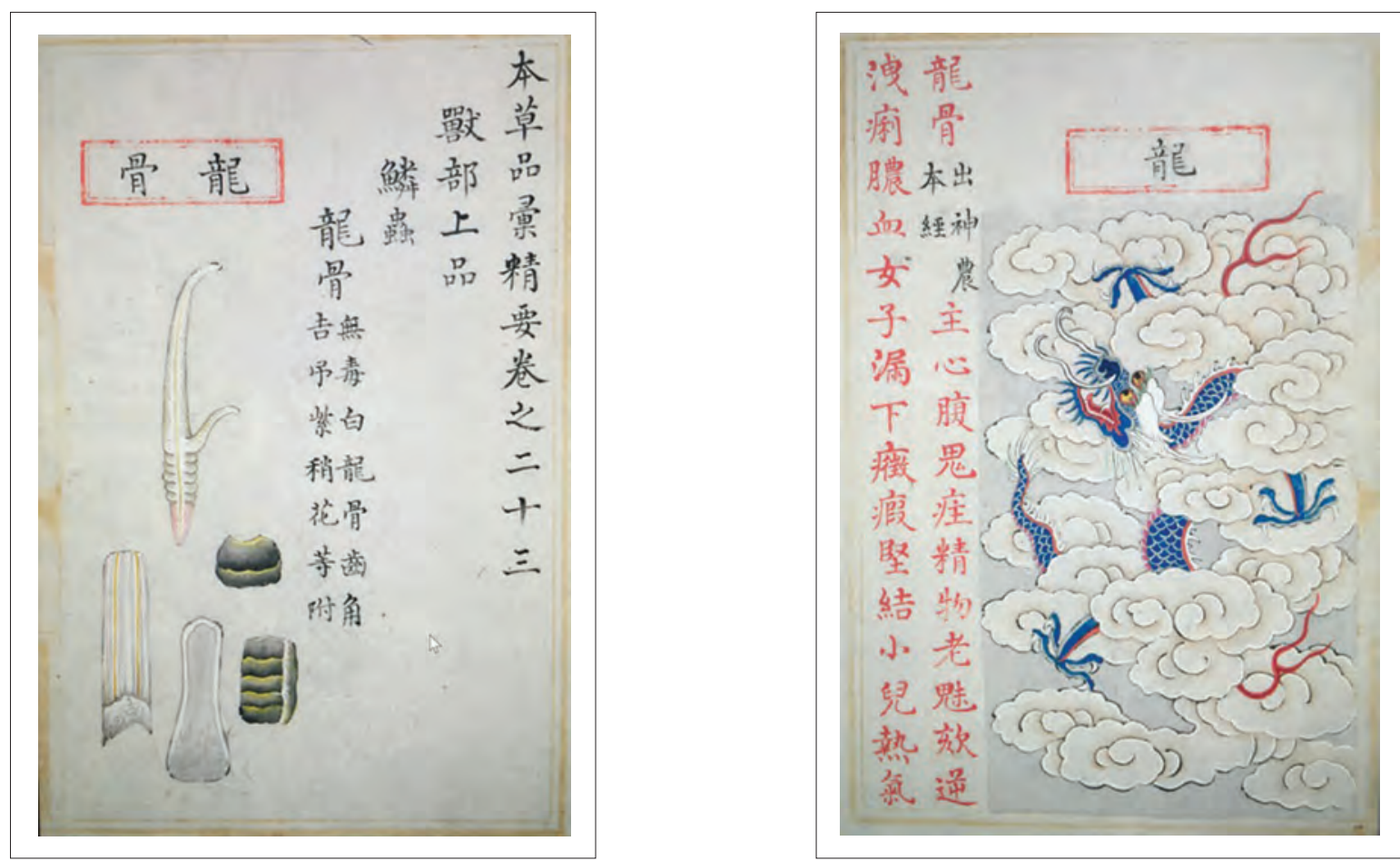

Figure 13.1a 'Dragon Bone' (Longgu 龍骨); 1b) ‘Dragon' (Long 龍), from Bencao pinhui jingyao. Berlin, Staatsbibliothek, Libri sin. Hirth ms. 2, juan 23

myself constantly frustrated by two problems. First, both the original Ming manuscript of the official Bencao pinhui jingyao 本草品彙精要 (Classified Treasury of Materia Medica, begun 1503) and various copies and facsimiles of that manuscript had been dispersed abroad, so that it was hard to gain sight of them (See Chen, Chapter 21 in this volume). But also, those texts that had been preserved in China presented many challenges to the reader and researcher. In the words of the old adage: 'Not even the cleverest housewife can cook without rice'. It was this frustration that set me on a quest that has lasted over 20 years, to seek out, copy and restore this elusive Bencao literature. When I came to publish the annotated critical edition of Bencao pinhui jingyao, I included a selection of over 270 polychrome illustrations that I had collected from texts in China and abroad; but what I failed to realise at that stage was that several versions of Shiwu bencao 食物 本草 (Materia Dietetica, 1505 to 1519) and Buyi Lei Gong paozhi bianlan 補遺雷公炮製便覽 (Lei Gong's Guide to Drug Preparation with Addenda, 1591) contain illustrations copied from originals in Bencao pinhui jingyao. ${ }^{3}$

Of late, a steady stream of illustrated fragments of Bencao pinhui jingyao, Shiwu bencao and Buyi Lei Gong paozhi bianlan have come to light. For instance, Shanghai
Library holds two incomplete juan of an illustrated copy of Bencao pinhui jingyao (juan 24 and 25). In view of the folio size, format, border measurements and sty2le of painting, it seems certain that these are two missing juan from the Berlin manuscript (Figs 1a, 1b). The Kyo-U Library of the Takeda Science Foundation, Osaka, holds three juan of a work entitled Xiuxiang shiwu bencao 繡像食物本草 (Materia Dietetica Illustrated), with illustrations copied from the manuscript of Shiwu bencao in the National Library of China (four-juan version), and eight juan of Jinghui bencao tu 精繪本草圖 (Masterpieces of Materia Medica Illustration), with illustrations copied from the manuscript of Buyi Lei Gong paozhi bianlan in the library of the China Academy of Chinese Medical Sciences (14-juan version). They all belong to a lineage that bears a close relation to the original manuscript of the Hongzhi reign period of the Ming dynasty, 1487-1505.

Since the turn of the 21st century, a dozen editions of Bencao with painted illustrations have been published in China and Japan. The most significant of these as follows.

Xiuxiang shiwu bencao (three juan from the Kyo-U Library collection), colour facsimile, Osaka: Takeda Science Foundation, 2003.

Shiwu bencao (four juan dating from the Ming in the National Library of China), colour facsimile, Huaxia chubanshe, 2000.

Shiwu bencao (four juan dating from the Ming in the National Library of China), Beijing Library Press, 2001. 




Figure 13.2 Guanglangzi 桄榔子 (Fruit of Gomuti sugar palm), Bencao tupu 本草图谱 (Illustrated Register of Materia Medica), Ming Chongzhen era (1628-44)

Bencao pinhui jingyao (Otsuka ms.), colour facsimile, Tokyo: Taniguchi Shoten, 2002.

Bencao pinhuijingyao (Rome ms.), colour facsimile, Kyushu: Kyushu Shuppankai, 2002.

Buyi Lei Gong paozhi bianlan (14juan, China Academy of Chinese Medical Sciences), colour facsimile, Shanghai: Shanghai cishu chubanshe, 2005.

Bencao pinhui jingyao (Forbidden Palace Manuscript), colour facsimile, Shanghai: Shanghai guji chubanshe, 2002.

Bencao tupu 本草圖譜 (Illustrated Register of Materia Medica) (three juan in China Academy of Chinese Medical Sciences and two juan in National Library of China), colour facsimile, Huaxia chubanshe, 2003.

Bencao pinhui jingyao, annotated study edition, Huaxia chubanshe, 2004.

Bencao pinhui jingyao, edited and punctuated, Shanghai: Shanghai kexue jishu chubanshe, 2005.

This chapter will discuss the interrelations between these Bencao texts in light of work by Lu Jun 魯軍, Miyashita Saburo 宮下三郎 and Zheng Jinsheng 鄭金生 on Shiwu bencao and Buyi Lei Gong paozhi bianlan, ${ }^{4}$ with reference to the published editions and facsimiles of Bencao pinhui jingyao listed above, the 12-juan Kangxi (1654-1722) copy

Lu Jun 2000; 2002; Cao Hui 2004; Miyashita Saburo 2003; Zheng Jinsheng 2003; 2005; Bai Hua and Zheng Jinsheng 2003; Zheng Jinsheng and Qiu Jian 2004. of that work held in the National Library of China, and the 26-juan manuscript of 1620 (Taichang era of the Ming dynasty) held in the China Academy of Chinese Medical Sciences, as well as the 12 juan of Jinshi kunchong caomu zhuang 金石昆蟲草木狀 (Description of Minerals, Insects and Plants) of the Wanli Era of the Ming (1573-1621), now in Taiwan. It will also explore the Ming Bencao illustration project constellated around Bencao pinhui jingyao from the perspective of ethno-pharmacology.

\section{Historical Background to the Ming Materia Medica Illustration Project}

\section{Prerequisites}

As a general rule, the publication of a pharmacopoeia is predicated on the following three historical conditions.

\section{An Enlightened Ruler}

The early Tang period witnessed an end to the fragmentation of the country under the Southern and Northern dynasties. The domestic situation was relatively stable, and the economy and culture rapidly revived and developed. With the publication in 659 of Xinxiu bencao 新修本草 (Newly Revised Pharmacopoeia), the Tang established the pattern whereby each successive dynasty would issue an officially sanctioned canon of Materia Medica. Compiled by a team of 23 scholars headed by Su Jing 蘇敬 (599-674), 




Figure 13.3 Jinshi kunchong caomu zhuang 金石昆蟲草木狀

(Descriptions of Minerals, Insects and Plants), Preface by Zhao Lingjun 趙靈鈞, 1617-20 (Ming, 45-48th year of the Wanli era). National Central Library, Taiwan, no. 307.27 06921

Xinxiu bencao was the first pharmacopoeia ever to be published not just in China but in the entire world.

The Song period (96o-1279) brought a spate of state-sponsored Bencao. Shortly after the foundation of the Northern Song (96o-1127) dynasty, and even before the country was fully united under the new regime, the first Song emperor, Taizu (r. 96o-76), ordered and oversaw the compilation of two Bencao: Kaibao xin xiangding bencao 開寶新詳定本草 (Newly Edited Pharmacopoeia of the Kaibao era of the Song Dynasty) 973, and Kaibao chongding bencao 開寶重定本草 (Pharmacopoeia of the Kaibao era of the Song Dynasty, revised edn) 974, for which he personally wrote prefaces.

Three of Taizu's successors followed in his footsteps. The Renzong emperor (r. 1022-63) sponsored Jiayou buzhu Shen Nong bencao jing 嘉佑補注神農本草 (Supplement to Shen Nong's Canon of Materia Medica Compiled in the Jiayou Era), 1061, and Bencao tujing 本草圖經 (Illustrated Canon of Materia Medica), 1062. Huizong (r. 110o-25) sponsored Zhenghe xinxiujingshizhenglei beiyong bencao 政和新修經史證類備用本草 (Newly Revised Classified Pharmacopoeia for Emergency Use, based on Classics and Historical Sources, of the Zhenghe Era), 1116. After the Southern Song transition, Gaozong (r. 1127-62) ordered the publication of Shaoxing jiaoding jingshi zhenglei beiji bencao 紹興校定經史證類備急 (Shaoxing Recension of the Classified Pharmacopoeia for Emergency Use, based on Classics and Historical Sources), 1159. Enlightened rulers such as these helped produce the relatively liberal climate that characterised Tang and Song China.

\section{A Strong, Prosperous Nation}

In the prosperous, outward-looking Tang (618-907), with its highly developed communications and transport systems, interchange between China and the rest of the world intensified, and many medicinal substances and foodstuffs were introduced from regions to the west. Medicine and pharmacy flourished as never before: so much so that this has been termed the golden age of Materia Medica writing. A contrasting example is provided by 15 th-century Korea. Like the Chinese Tang and Song emperors, in 1443 Sejong the Great (r. 1418-50), fourth king of Joseon, sponsored a major medical publishing project: Ui'bang'ryuchui [Yifang leiji] 醫方類聚 (Classified Collection of Medical Prescriptions). However, because of a lack of funds, it took another 43 years - and two more Joseon kings - before the work was ready for publication, and the initial print run was just 30 copies.

An Objective Increase in Actual Need In China throughout the dynasties, life for most ordinary people was a harsh struggle for subsistence under the constant threat of famine, and there are many records of starving people eating roots and tree bark. Ancient agriculture could not deliver food security, and in times of natural disaster such as drought or floods, people consumed wild plants. As a result, they built up a vast body of empirical knowledge about the food value of wild plants. Because, moreover, there was no clear distinction between food and medicine in early China, advances in Materia Medica studies also provided valuable data and practical knowledge about wild plants and their uses as foodstuffs. This is exemplified by the genres of Jiuhuang bencao 救 荒本草 (The Famine Herbal) and Shiwu bencao 食物本草 (Materia Dietetica). The former is devoted to wild plants that can be eaten as famine foods and/or used to combat contagious diseases, while the latter focuses on foodstuffs that also have medicinal properties.

\section{The Historical Background}

Early on in the Ming period - in the Yongle era (1403-25) - three important events took place that fulfilled the three conditions described above. These events also serve as a key to understanding the historical backdrop to the illustrated Bencao industry in the Hongzhi-Wanli period of the mid-Ming (1488-1620). 


\section{The Voyages of Exploration of Zheng He 鄭和}

On ascending the throne as the Yongle emperor in 1403, Chengzu (136o-1424) marked the occasion by sending a mission led by Jiang Binxing 蔣賓興 and Wang Shu 王樞 to South-East Asia, with the intention of strengthening ties with states in the region, and also of impressing them with the power and prestige of the Ming empire. Two years later in 1405 , he sent one of his most senior officials - Zheng He (1371-1433), Director of Court Eunuchs - on the first of the sea voyages that would make him China's most celebrated explorer. Zheng He's first expedition to the 'western oceans' involved more than 27,80o military personnel, coordinated by two commanders-in-chief (tongshuai 統 帥) and 93 commanders (zhihui 指揮), and divided into several battalions (qian hu千戶), companies (baihu 百 戶), units (zongqi 總旗) and divisions (xiaoqi 小旗). The fleet consisted of 208 vessels. The flagship, or Zheng He's treasure ship, was the largest: 44 zhang $(c .145 \mathrm{~m})$ long and 18 zhang (c. 6om) wide, it had 9 masts, a tonnage of 14,000 tonnes, and a cargo-carrying capacity of 7 ,ooo tonnes. The fleet set sail from the great port of Liujia 劉家 at Taicang 太倉, some $50 \mathrm{~km}$ north of Shanghai. Sailing south along the coast via Changle 長樂 in Fujian province, they first made landfall in the main city of the kingdom of Champa. They then proceeded to Surabaya in Java, Pelambang in the south of Sumatra, and Malacca in the south of the Malay peninsula. They continued west to Ceylon (Sri Lanka), then sailed north around the coast of India to Kollam, Cochin and Calicut - the final destination for the main fleet. From here two smaller contingents pressed on: one continued north-west as far as Iran, rounded the Arab peninsula and entered the Red sea, concluding its voyage at Mecca. The other sailed south-west, making directly for east Africa.

In all, Zheng He made seven voyages to the west. On each occasion, he took along no fewer than 18 o medical officials and practitioners, as well as experts in the identification of medicinal herbs, who examined the drugs available for trade in each place they visited. They took with them substances such as ginseng and musk; in return, they brought home medicinals such as rhinoceros and antelope horn, asafoetida, myrrh, cloves, Banksia rose (muxiang 木香), aloes, frankincense, and momordica seed (mubiezi 木鱉 子). Meanwhile, throughout the Hongwu (1368-98) and Yongle (1402-24) eras, several dozen medicinal aromatics, liquors and other prized substances formed part of the tribute regularly sent by the south-east Asian states of Siam (Thailand), Poluo (a part of Borneo), Boni (part of Borneo), Pahang, Srivijay, Sumatra and Java. They included hawksbill, pearls, Acronychia pedunculata, various forms of camphor, roses, sappan wood, aquilaria, gharu wood, ambergris, sandalwood, cloves and nutmeg.
In La Route de la Soie, Aly Mazahéri publishes two official letters from Emperor Chengzu (the Yongle emperor) to the Sultan of Persia, as well as accounts of travels in China written by a Persian emissary and a Persian merchant. From these Persian records, we learn that big cats such as lions, leopards and lynxes were important tribute items in the Ming period. In Chapter 6 of Kețāy-nāma (Records of China), the Persian merchant Ali Akbar Khata'i describes the Ming imperial gardens: 'In the fifth imperial garden, they keep lions, leopards, cheetahs, lynxes and Tibetan dogs'. 5

The voyages of Zheng He confirmed China as the world leader in navigation and ship-building technology of the early $15^{\text {th }}$ century - a century before the age of discovery and exploration dawned in Europe, and just as new sea routes were beginning to open up around the globe. These tremendous feats of navigation propelled China into a golden age unprecedented in nautical history, and helped forge close political, diplomatic and commercial ties with the countries of south-east Asia. They left a lasting legacy of bilateral cultural interchange.

Three of the people who sailed with Zheng He published first-hand accounts of their travels, recording circumstantial details of the voyages as well as personal observations of the countries they visited. These are Yingya shenglan 瀛涯勝覽 (Wondrous Observations of the Ocean's Shores, 1451) by Ma Huan 馬歡, Xing cha sheng lan 星槎勝覽 (Wondrous Observations from the Starry Raft, 1433) by Fei Xin 費信, and Xiyangfanguo zhi 西洋番國志 (Records of Foreign Countries in the Western Ocean) by Gong Zhen 鞏珍. In addition, they and others like them brought home specimens of medicines, foods, and exotic creatures, all of which found their way into the state-sponsored Bencao of the Hongzhi era. Later these would include plants that were native to the Americas, such as maize, ${ }^{6}$ and the peanut, first recorded in Shiwu bencao. Examples of these novel items, such as the lion, Blumea balsamifera (ainaxiang 艾 納香), and numerous drugs and spices are all recorded in Bencao pinhuijingyao.

\section{The Yongle Encyclopaedia and Ming Book Art} Yongle dadian 永樂大典 (the Yongle Encyclopaedia or Yongle Canon), is a monumental work in the leishu genre (combining features of an encyclopaedia, an anthology and a concordance), commissioned by Emperor Chengzu in the seventh month of the first year of his reign (1403). Placed

$5 \quad$ Zheng Jinsheng 2003; Mazahéri 1983.

6 See Xuxiu siku quanshu, 'Zi bu', 'Yijia lei' 續修四庫全書·子部· 醫家類 (Complete Library in Four Sections, continued, 'Masters', 'Physicians'), juan 99o. See also Bencao pinhui jingyao (2002 edn). 




Figure 13.4 Mingjie zenghe qianjia shi zhu 明解增和千家詩注 (A Children's Reader of Poems from One Thousand Authors), Song period. Colour facsimile, Beijing: Wenwu chubanshe and Taibei: Liangyi wenhua, 2015

under the general editorship of imperial preceptor (shaoshi 少師) Yao Guangxiao 姚廣孝 and Xie Jin 解縉, a scholar of the Imperial Academy, the project involved at least 3,000 scholars including Wang Jing 王景 and Zou Ji 鄒輯. The final draft was completed in 1407 after five years of work, and it finally saw the light of day in 1408 , after an additional year spent creating the finished manuscript. The emperor himself gave the book its title and wrote a commemorative preface for it, and it was conserved in the Wenyuan ge 文 淵閣 (Pavilion of Literary Profundity), the library of the National Imperial Academy in Nanjing. The complete work consisted of 22,877 juan (fascicles) of text and 6 o fascicles of indexes, bound into 11,095juan. Altogether it contained 3,700,000,000 Chinese characters. It gathered together material from over 8,ooo texts of all kinds from the pre-Qin period right up until the beginning of the Ming, many of which were not otherwise transmitted. Texts on astrology, geography, human relationships (renlun), government, ethics, institutions, philology, records of the marvellous, topography and meteorology were faithfully transcribed. The Confucian, Buddhist and Daoist classics, histories, drama, both high and popular literature, and works on the arts, craft, agriculture and medicine were all represented. The texts and extracts included were all unabridged, which added greatly to their scholarly value. It was provided with a lexical retrieval system arranged by rhyme and a thematic retrieval system based on key characters, which makes it easy to search. When in 1422 (19th year of the Yongle era) the Forbidden City was completed and the emperor moved the capital to Beijing, Yongle dadian was transferred there also, and permanently installed in the Wenzhao ge library within the Palace complex. 
The rise of the illustrated Bencao should also be seen against the background of the general popularity of illustrated books in the Ming. Novels and books of tales, poetry collections and plays were published in standardised illustrated formats known as 'Picture to the left and text to the right', and 'Picture on top and text below'. As the novelist and essayist Lu Xun 魯迅 put it in 1933, in the preface to Beiping jianpu 北平箋譜序 (Beiping papers):

Song dynasty block-printed books were sometimes illustrated, as can be seen from the extant medical and Buddhist literature, either to help identify objects or to add verisimilitude. This gave rise to the genre of illustrated histories. In the Ming period, illustration was used much more extensively. Every novel and tale had illustrations, some as crude as sand pictures and some as delicate as a hair painting. There were also ravishing albums of polychrome woodblock prints. This was the heyday of the woodcut.

Both the National Library of China and Taiwan National Library possess manuscript copies of Mingjiezenghe qianjia shizhu 明解增和千家詩注 (Ming collection of poems of the Tang and Song Dynasties) with painted illustrations. This book is similar in format to Yongle dadian, and the illustrations are consistent in style and technique with those in Bencao pinghui jingyao, leaving no doubt that it stems from the Ming court milieu. In keeping with the nature of the texts, the illustrations are imaginative and lyrical; paintings and poems complement and illuminate each other. ${ }^{7}$

The creation of Yongle dadian coincided with the beginning of a vogue for illustrated books, and it came to be used as a model for the state-sponsored Bencao editions of the Hongzhi period. Thus for example Bencao pinhui jingyao, Shiwu bencao, and Buyi Lei Gong paozhi bianlan exactly follow the physical format of Yongle dadian. The pages have a double vermilion border; rich, intense mineral pigments are used; drug names are written in gold paint; and the illustrations are executed in a highly-coloured meticulous gongbi style that harks back to the classic academic painting of the Song. Even the paper is the same kind of bamboo folio used for Yongle dadian. This became the standard format for courtly illustrated books in the Ming.

\section{The Publication ofJiuhuang Bencao 救荒本草 (Famine ReliefHerbal)}

At the dawn of the Ming period, the population was still struggling to recover from the oppressive Yuan regime and the hardships of wartime. Life was harder than ever, and famine was rife. In 1389, Zhu Su 朱橚, Duke of Zhou and fifth son of Zhu Yuanzhang, founding emperor of the Ming

$7 \quad$ Mingjie zenghe qianjia shi zhu. dynasty, was exiled to Yunnan in the south-west of China. There, he became keenly aware of the desperate plight of the people, and the critical lack of drugs and medical care. When he was able to return to his fief of Kaifeng, he gathered about him a group of skilled illustrators and specialist scholars including Liu Chun 劉醇, Teng Shuo 滕 碩, Li Heng 李恒 and Qu You 篗佑. They compiled copious visual records, and set up a botanical garden where they cultivated edible wild plants identified from fieldwork among ordinary people. In 1406 (fourth year of the Yongle era), they completed the ground-breaking Jiuhuang bencao. A specialised reference work on edible wild plants, it represents a departure from the traditional Bencao. It is emblematic of the transition of Bencao studies from pharmacology to economic botany.

\section{The Ming 'Illustrated Bencao Project'}

\section{Bencao Pinhui Jingyao 本草品彙精要: the Creation Process}

The Ming was an age of relative political stability, with a highly developed feudal economy, displaying signs of embryonic capitalism in the mid- to late period. Driven by the commodity economy, contacts with the outside world intensified, science, technology and culture developed, and medical standards improved notably. With the steady expansion of foreign trade and interaction came advances in transportation and communications. New drugs were discovered and introduced from abroad, and cross-fertilisation between different branches of learning and technology was encouraged. This fuelled developments in Bencao studies, which in turn added to the sum of agricultural knowledge. On ascending to the throne in 1487 , Ming Xiaozong 孝宗, the Hongzhi emperor, soon showed himself to be a hands-on ruler who involved himself personally in government, cared about the people and exerted himself to make the country prosperous. His reign became known as the 'Hongzhi restoration' (1487-1505).

The phenomenon of the official pharmacopoeia had become well established in the Tang and Song, but it was now three centuries since the last one had been published (none having been issued in the Northern Song, the Jin or the Yuan periods), and in the meantime medicine and pharmacology had moved on. Thus, on the 8th day of the 8th lunar month of 1503 (16th year of the Hongzhi era), Emperor Xiao Zong instructed the Imperial Academy of Medicine (Tai yiyuan 太醫院) to compile a new pharmacopoeia.

According to long established practice, the compilation of the official pharmacopoeia was overseen by the most senior official of the day. In the Tang the actual work was 
carried out by scholars of the Hongwen guan (Academy for the Propagation of Literature) like Kong Zhiyue 孔志約. In the Song, the task generally fell to scholars and literati of the Hanlin Academy like Lu Duoxun 盧多遜, Li Fang 李昉, Wang Guangyou 王光佑, Zhang Yuxi 掌禹錫 and the polymath SuSong 蘇頌. It was not until the Ming that it became the responsibility of the Imperial Academy of Medicine.

The project mobilised the largest specialist team ever to be involved in compiling an official pharmacopoeia, under the overall supervision of the Chief Eunuch of the Directorate for Imperial Regalia (Sishe jian taijian 司設 監太監). The editorial team from the Imperial Academy of Medicine comprised the Commissioner (yuanshi 院 使), five Administrative Assistants (yuanpan 院判), three Court Physicians (yuyi 御醫), and 20 medical practitioners or medical officers. They were joined by the Vice-Commissioner of the Public Pharmacy (Huimin yaoju fushi 惠民藥局副使), and a Chancellor (Zhangyuan 掌院), two Vice-Chancellors (Tong zhangyuan 同掌院) and an Assistant Commissioner (You canyi 右參議) from the Office of Transmission (Tongzheng shisi 通政使司), seven Confucian scholars and scholar-officials from the Central Drafting Office (Zhongshuke rushi 中書科儒士), 49 draughtsmen and other supporting staff, and in the final stages, 14 copyists and eight court artists. Just a year and a half later, the completed pharmacopoeia was presented to Emperor Xiao Zong on behalf of the Imperial Academy of Medicine by senior Administrative Assistants Liu Wentai 劉文泰 and Wang Pan 王盤 and one of the imperial physicians. The emperor granted it the title Yuzhi bencao pinhuijingyao 御制本草品彙精要 (Classified Treasury of Materia Medica by Imperial Appointment), and personally contributed a preface. The finished work, consisting of 42 fascicles plus an additional fascicle of indexes, was bound into 36 juan in emulation of Yongle dadian. Bencao pinhui jingyao contains entries on 1,815 substances, and no fewer than 1,360 exquisite polychrome illustrations, making it the most extensive illustrated Chinese pharmacopoeia still in existence today. ${ }^{8}$

\section{Transmission of the Illustrations of Bencao Pinhui Jingyao}

Copies of the Illustrations from Bencao Pinhui; a Typology

In February 2003, Professor Zheng Jinsheng examined the manuscripts of Xiuxiang shiwu bencao 繡像食物本 草 (Materia Dietetica Illustrated) (three juan) and Jinghui

For further details, see my introduction to Bencao pinhui jingyao. bencao tu 精繪本草圖 (Masterpieces of Materia Medica Illustration) (eightjuan) held in the Kyo-U Library in Osaka. He was able to ascertain that the texts corresponded respectively to the four-juan version of Shiwu bencao食物 本草 (Materia Dietetica) and the 14-juan version of Buyi Lei Gong paozhi bianlan 補遺雷公炮製便覽 (Lei Gong's Guide to Drug Preparation with Addenda) preserved in China, but that the illustrations came from a different source. ${ }^{9}$ The relationship of Bencao pinhui jingyao to the illustrated Shiwu bencao has been studied by myself and Miyashita Saburo, ${ }^{10}$ while Zheng Jinsheng and others have published on its relationship to the illustrated Buyi Lei Gong paozhi bianlan. ${ }^{11}$

The main body of Bencao pinhui jingyao is divided into 1o sections including 'Jade', 'Herbs', 'Trees' and 'Fruit'. It has entries on 1815 substances accompanied by 1367 illustrations (Hongzhi ms.), exquisitely painted in the meticulous court style by eight court artists including Wang Shichang ( $f l$. mid-16th century). In light of similarities to the woodblock illustrations in Bencao tujing, reproduced in Zhenglei bencao, 699 of those illustrations seem to be coloured versions of pre-existing black and white line images, but the remaining 668 are original creations or free adaptations. Circumstances combined to prevent the publication of Bencao pinhui jingyao; not long after the original Hongzhi manuscript of Bencao pinhui jingyao was completed, the chief editor, Liu Wentai and his colleagues were demoted and discredited for political reasons. Moreover, the fine polychrome illustrations did not lend themselves to reproduction in print. However, the illustrations were copied by Ming and Qing artists, who prized them highly as works of art. According to Zheng Jinsheng's typology, the copies fall into three main categories: facsimiles, as in the Rome and Tokyo (Otsuka) manuscripts; modified copies, as in the Taiwan ms. (Wanli era, 1573-1619), Jinshi kunchong caomuzhuang 金石昆蟲草木狀 (Descriptions of Minerals, Insects and Plants) and Beijing ms. (Chongzhen era, 1628-44),Bencao tupu 本草圖譜 (Illustrated Register of Materia Medica); and artistic adaptations like the polychrome illustrations in Shiwu bencao (by court artists of the Zhengde era (1506-21) and Buyi Lei Gong paozhi bianlan (by court artists of the Wanli era). ${ }^{12}$

Elsewhere I have published a detailed study of the two polychrome Bencao containing modified copies of illustra-

\footnotetext{
$9 \quad$ Zheng Jinsheng 2003.

10 Lu Jun 2002 and 2000; Miyashita 2003

11 Bai Hua and Zheng Jinsheng 2003; Zheng Jinsheng and Qiu Jian 2004; Zheng Jinsheng 2005.

12 Zheng Jinsheng 2003.
} 




Figure 13.5 Wanaqi 膃肭臍 (Penis and testes of fur seal), from Bencao pinhui jingyao 本草品彙精要 (Shanghai ms.)

tions in Bencao pinhuijingyao, to which I refer the reader. ${ }^{13}$ With regard to the Bencao containing artistic adaptations, Shiwu bencao is a four-juan work divided into eight sections including 'Water', 'Cereals', 'Vegetables' and 'Fruit', with a total of 386 entries and 492 fine polychrome illustrations (Ming ms. in the National Library of China, Beijing). About $43 \%$ of the illustrations (213 images) are taken from Bencao pinhui jingyao, ${ }^{14}$ and 44 of those images are faithful copies ${ }^{15}$ Buyi Lei Gong paozhi bianlan is divided into 14juan and 10 sections, including 'Metals and minerals', 'Herbs', 'Trees' and 'Human beings'. It contains entries for 957 substances, illustrated with 1162 fine polychrome paintings (the Wanli ms. in the China Academy of Chinese Medical Sciences preserves all but one of the 14juan - the juan on 'Fruits' is missing - with 913 entries and 1,122 illustrations). Approximately three-quarters of the illustrations (880 in total) are derived from Bencao pinhui jingyao, ${ }^{16}$ and 26 of them are exact copies. ${ }^{17}$

\footnotetext{
13 Cao Hui 2004.

14 Zheng Jinsheng 2003.

15 Lu Hui 200o; Miyashita Saburo (2003) calculates the lower figure of 164 images taken from Bencao pinhui jingyao, for both the Beijing ms. and the manuscript in the Kyo-U Library in Osaka.

16 Zheng Jinsheng 2003.

17 Zheng Jinsheng and Qiu Zhen 2004.
}

\section{A Study of the Copies}

As I have discussed elsewhere, nine types of evidence can be adduced to show that the images in Shiwu bencao are directly derived from Bencao pinhui jingyao (principally format, layout, script, artistic style, etc.). ${ }^{18}$ This formal evidence is corroborated by the internal evidence of the subject matter both of the horticultural illustrations and the illustrations of everyday foodstuffs. For example, under the rubric 'Plums' in the section on 'Fruits' in juan two of Shiwu bencao, there are horticultural illustrations of 21 varieties of plum, including green, yellow and purple plums. Eight of these 21 named varieties appear under 'Terminology' under the rubric 'Plums' in juan 34 of Bencao pinhuijingyao. Under the rubric 'Pears' in the same juan of Shiwu bencao, we find illustrations of 11 varieties, including 'milk pear' (ruli, i.e. snow pear, Pyrus nivalis) and 'goose pear' (Pyrus Bretschneideri 'Eli'), 10 of which appear under 'Terminology' in juan 34 of Bencao pinhuijingyao. Similarly for foodstuffs, under the rubric 'Alcoholic beverages' (jiu) in juan 4 of Shiwu bencao, there are illustrations of 16 items, nine of which appear under 'Terminology' under the rubric 'Jiu' in juan 36 of Bencao pinhui jingyao.

Zheng Jinsheng demonstrates with evidence from plant morphology, image titles and artistic technique that the

18

Lu Hui 2000. 
images in Buyi Lei Gong paozhi bianlan are taken from Bencao pinhui jingyao. ${ }^{19}$ The present author has found two further items of supporting evidence.

\section{a. The Preface to Lei Gong Paozhi Bianlan (Lei Gong Paozhi Bianlan Xu 雷公炮䎹論序)}

On the cover page of the Rome manuscript and the Kangxi copy of Bencao pinhuijingyao, in addition to the expected title label, there is a synoptic contents list comprising nine items: tables, compiling officials, prefatory matter $(x u l i$ 序 例), guide to the reader (fanli 凡例), Materia Medica from Shen Nong's Pharmacopoeia, weights and measures, Preface to Lei Gong paozhi lun 雷公炮多論 (Lei Gong's Theory of Drug Preparation), Preface to Lei Gong paozhi 雷公炮制 (Lei Gong's Drug Preparation) and indexes. It turns out that the Preface to Lei Gong paozhi lun reproduces a preface of the same name from the prefatory material in Zhenglei bencao, minus 'Lei Xiao's 14 principles of combining drugs', while the Preface to Lei Gong paozhi was manufactured by the compilers of Bencao pinhui jingyao at the Imperial Academy of Medicine by adding the ' 14 principles' to a short existing commentary on Lei Gong paozhi lun. The two prefaces were later amalgamated to create the Preface to Lei Gong paozhi lun in Buyi Lei Gong paozhi bianlan. This may well have been part of a grand plan to compile a Drug Preparation Bencao, within the overarching Ming Bencao project. As evidence, in reference to 'mouth ulcers and cracked tongue', the preface to Zhenglei bencao uses the character “坼” (zhe) (cracked), while the prefaces to Lei Gong paozhi lun in Bencao pinhui jingyao and Buyi Lei Gong paozhi bianlan both have the character '拆' (zhai).

Lei Gong paozhi lun was the first monograph on the techniques of drug preparation, and was responsible for establishing drug preparation as a separate specialism within the Bencao genre. However, many of the drugs and procedures it describes belong to Daoist alchemy, and thus tend to feature obscure names and elaborate techniques. Therefore the Hongzhi-era editors and artists working on Buyi Lei Gong paozhi bianlan used the earlier texts associated with Lei Gong as a basis, but added seven-syllable mnemonic rhymes and illustrations of processing methods to make the work more appealing and user-friendly. The contemporary Shiwu bencao was similarly based on earlier texts with the same title, with added illustrations. Both Buyi Lei Gong paozhi bianlan and Shiwu bencao took over illustrations from Bencao pinhui jingyao wholesale, adding or substracting elements to fit the images to their new context.

\section{b. The Hongzhi Original and Buyi Lei Gong Bencao} While preparing the critical edition of Bencao pinhui jingyao, I became aware of discrepancies between the text and images in juan 2, 'Jades'. The extant manuscripts of Bencao pinhui jingyao that include juan 2 can be divided into three main systems or families: one comprising the Rome and Otsuka manuscripts, one comprising the Ming Taichang manuscript (held in the library of the China Academy of Chinese Medical Sciences) and the Kangxi facsimile (in the National Library of China, Beijing), and the third represented solely by the Ming Hongzhi copy (also in the National Library of China)..$^{20}$ Among the plethora of copies, two have been shown by Zheng Jinsheng to be directly derived from the Hongzhi original, i.e. the Kangxi facsimile and Buyi paozhibianlan. But in fact there is also a direct copy of the Wanli manuscript:Jinshi kunchong caomu Zhuang (now in Taiwan), which, interestingly lacks any material from juan 2. It is worth considering the reasons for this omission. Could the contents of juan 2 have a bearing on the political struggle that brought about the downfall of Liu Wentai and his colleagues?

\section{The Bencao Illustration Project}

As Bencao pinhuijingyao had been declared a banned book, it stands to reason that the court artists of the Zhengde and Wanli eras could not have used it as source material for illustrated Bencao or drug preparation texts, officially at least. However, all state-sponsored texts had to be produced in duplicate: in addition to the main copy, which was destined to be submitted to the emperor, there was always at least one backup copy in existence, purportedly for editing purposes and to serve as a master copy in case of problems. So despite the official proscription, there was very probably a backup copy of Bencao pinhui jingyao at the Imperial Academy of Medicine or Academy of Art. As time went on, handwritten or traced copies found their way outside the confines of the imperial court. Thus for example the Wanli manuscript of Jinshi kunchong caomu Zhuang in Taiwan contains 1,315 illustrations copied from Bencao pinhui jingyao (but none of the original texts). Coincidentally, the Jiaqing copy of Jinghui bencao tu in the Kyo-U Library in Osaka reproduces 385 images from Buyi Lei Gong paozhi bianlan - also copied without any of the surrounding text - including 34 illustrations from the 'Fruits' section (entirely missing from the manuscript in the library of the China Academy of Chinese Medical

\footnotetext{
$20 \quad$ Cao Hui 2004.
} 
Sciences) and from the 'Metals and Minerals' section (also partially missing).

Thus, we can tentatively conclude that the original grand plan for the compilation of a Ming Bencao, assigned to the Imperial Academy of Medicine by Emperor Xiao Zong in the Hongzhi era of his reign, covered three main areas: the centrepiece was to be a comprehensive pharmacopoeia (Bencao pinhui jingyao), supported by two subsidiary projects: one on Materia Dietetica and famine foods (Shiwu bencao) and one on the preparation and processing of Materia Medica (Buyilei Gong paozhi bianlan). These three elements combined to create the huge and far-reaching 'illustrated Bencao project' of the Ming.

\section{Bibliography}

\section{Primary Sources}

Beiping jianpu 北平䇝譜 (Beiping Papers) 1933, Lu Xun 魯迅.

Bencao pinhui jingyao 本草品彙精要 (Classified Treasury of Materia Medica) 1505, Liu Wentai 劉文泰 (ed.).

Bencao tujing 本草圖經 (Illustrated Canon of Materia Medica) 1062, Su Song 蘇頌 (ed).

Bencao tupu 本草圖譜 (Illustrated Register of Materia Medica) c. 1630, Zhou Shuhu 周淑祜, Zhou Shuxi 周淑禧.

Buyi Lei Gong paozhi bianlan 補遺雷公炮製便覽 (Lei Gong's Guide to Drug Preparation with Addenda) 1591, Anon.

Jiayou buzhu Shen Nong bencao jing 嘉佑補注神農本草經 (Supplemented Revision of the Classified Pharmacopoeia during the Jiayou era of the Song Dynasty) 1061, Zhang Yuxi 掌禹錫 (ed).

Jinshi kunchong caomu zhuang 金石昆蟲草木狀(Description of Minerals, Insects and Plants) 1617-20, Wen Shu 文俶.

Jinghui bencao tu 精繪本草圖 (Masterpieces of Materia Medica Illustration) c. 1590, Anon.

Jiuhuang bencao 救荒本草 (The Famine Herbal) 1406, Zhu Su 朱橚.

Kaibao chongding bencao 開寶重定本草 (Newly Edited Pharmacopoeia of the Kaibao era of the Song Dynasty) 974, Liu Han 劉翰, Ma Zhi 馬志 (ed).

Kaibao xin xiangding bencao 開寶新詳定本草 (Pharmacopoeia of the Kaibao era of the Song Dynasty, revised edn) 973, Liu Han 劉 翰, Ma Zhi 馬志 (ed.).

Lei Gong paozhi lun 雷公炮采論 (Lei Gong's Theory of Drug Preparation) c. 900, Lei Xiao 雷㪒.

Mingjie zenghe qianjiashi zhu 明解增和千家詩注 (Supplemented Notable poems of the Tang and Song Dynasties of the Ming dynasty). c. 1500-160o, Xie Fangde 謝枋得 (ed.).

Shaoxingjiaodingjingshizhenglei beiji bencao 紹興校定經史證類備 急本草 (Revision of the Classified Pharmacopoeia for Emergency Use, based on Classics and Historical Sources in Shaoxing era of Song dynasty) 1159, Wang Jixian 王繼先 (ed.).

Shiwu bencao 食物本草 (Materia dietetica Illustrated) c. 1520, Anon.

Ui'bang'ryuchui 醫方類聚 (Classified Collection of Medical Remedies) 1477, Kim Ye-mong 金禮蒙 (ed.).

Xinxiu bencao 新修本草 (Newly Revised Pharmacopoeia) 659, Su Jing 蘇敬 (ed.).

Xiuxiang shiwu bencao 繡像食物本草 (Materia Dietetica Illustrated) c. 1520 , Anon.

Xing cha sheng lan 星槎勝覽 (Wondrous Observations from the Starry
Raft) 1433, Fei Xin 費信.

Xiyang fanguo zhi 西洋番國志 (Records of Foreign Countries in the Western Ocean) 1434, Gong Zhen 腪珍.

Yingya shenglan 瀛涯勝覽 (Wondrous Observations of the Ocean's Shores) 1451, Ma Huan 馬歡.

Yongle dadian 永樂大典 (the Yongle Encyclopaedia or Yongle Canon) 1403, Yao Guangxiao 姚廣孝, Xie Jin 解縉.

Zhenghe xinxiujingshizhengleibeiyong bencao 政和新修經史證類備 用本草 (Newly Revised Classified Pharmacopoeia for Emergency Use, based on Classics and Historical Sources, of the Zhenghe Era) 1116. Cao Xiaozhong 曹孝忠 (ed).

Zhonghua dadian 中華大典 1999-, Guojia zhongyiyao guanli ju 1998-, Chengdu: Bashu shushe.

Zhongguo bencao quanshu 中國本草全書 (The Complete Collection of Traditional Texts on Chinese Materia Medica) 1999-, Zhongguo wenhua yanjiuhui 中國文化研究會 (ed), Beijing: Huaxia chubanshe.

\section{Secondary Sources}

Bai Hua 白華 and Zheng Jinsheng 鄭金生 2003, 'Buyi Lei Gong paozhi bianla wenzi zuozhe kao' 補遺雷公炮製便覽文字作者考 (Buyi Lei Gong paozhi bianlan: texts and authors), Zhongyao cai 26 (supplement), 57-9.

Liu Wentai 刘文泰 et al. (eds) 2002, Bencao pinhui jingyao 本草品彙 精要, in Xuxiu siku quanshu, 'Zi bu', 'Yijia lei' 續修四庫全書.子部. 醫家類 (Complete Library in Four Sections, continued, 'Masters', 'Physicians'), juan 99o, Shanghai: Shanghai guji chubanshe.

Cao Hui 曹晖 2004, Bencao pinhui jingyao (yanjiu jiaozhu ben) 本草 品彙精要 (研究校注本) (Classified Treasury of Materia Medica [annotated critical edn]), Beijing: Huaxia chubanshe.

Guojia zhongyiyao guanli ju, Zhonghua bencao bianweihui 國家中醫 藥管理局《中華本草》編委會編委會 1998-, Zhonghua bencao 中華本草 (Chinese Materia Medica), Shanghai: Shanghai kexue jishu chubanshe.

Lu Jun 魯軍 2000, Yingyin Shiwu bencao: caihui ben xu 影印食物本草 彩繪本序. Photographic facsimile of Shiwu bencao: preface to the polychrome illustrated edn), Beijing: Huaxia chubanshe.

2002, Yingyin Yuzhi bencao pinhui jingyao $x u$ 影印禦制本 草品彙精要序 (Preface to Classified Treasury of Materia Medica by Imperial Appointment). Photographic facsimile, Beijing:Jiuzhou chubanshe.

Mazahéri, A. 1983, La Route de la soie, Paris: Papyrus. Chinese edn, (tr. Geng Sheng 耿升) 1993, Sichou zhi lu ZhongguoBosi wenhua jiaoliu shi 絲綢之路一中國·波斯文化交流史 (Silk Road: A History of Sino-Persian Cultural Interchange), Beijing: Zhonghua shuju.

Métailié, G. 2015, Science and Civilisation in China: vol. 6, Biology and Biological Technology, Part 4, Traditional Botany: An Ethnobotanical Approach, Cambridge: CuP.

Mingjie zenghe qianjia shi zhu 明解增和千家詩注 1998, (Increased and Noted poems of the Tang and Song Dynasties during Ming dynasty) c. 1500-16oo, Xie Fangde 谢枋得 (ed). Modern facsimile, Beijing: Beijing tushuguan chubanshe.

Miyashita Saburo 宮下三朗 2003, 紼像食物本草.解說一明代的食 材圖鑒, Osaka: Takeda Kagaku Shinkōzaidan.

Unschuld, P.U. 1986, Medicine in China: A History of Pharmaceutics, Berkeley and Los Angeles: University of California Press.

Zheng Jinsheng 鄭金生 2003, 'Mingdai huajia caise bencao chatu yanjiu' 明代畫家彩色本草插圖研究 (A study of Polychrome Bencao Illustrations by Ming Artists), Xin shixue 14.4, 65-120.

(ed.) 2005, Buyi Lei Gong paozhi bianlan 補遺雷公炮製 
便覽·解題 (Lei Gong's Guide to Drug Preparation with Addenda: Problems and Solutions), Shanghai: Shanghai cishu chubanshe. Zheng Jinsheng and Qiu Jian 装儉 2004, 'Xin fuxian Buyi Lei Gong paozhi bianlan yanjiu chubao' 新浮現《補遺雷公炮製便覽》研究 初報 (Newly discovered sources for Buyi Lei Gong paozhibianlan; a preliminary study), Zhongguo yaoxue zazhi 39.5, 389-91. 


\title{
14 Illustrations of Drug Collection and Preparation in Buyi Lei Gong Paozhi Bianlan
} 補遺雷公炮製便覽

\author{
Xiao Yongzhi 肖永芝
}

Buyi Lei Gong paozhi bianlan 補遺雷公炮制便覽 (Lei Gong's Guide to Drug Preparation with Addenda) is a major illustrated Bencao of the Ming period that has only recently come to light. It is dated ' 19 th year of the Wanli reign period of the Ming dynasty', i.e. 1591. Neither the writer nor the artist has been identified. It comprises 14 juan (volumes or fascicles), 13 of which contain the text of the work. It is illustrated with 1,133 polychrome paintings, executed by a master of the meticulous court style (See Zheng, Chapter 8, Chen, Chapter 21 and Cao, Chapter 13 in this volume). The majority of these show the appearance and morphology of herbs and other drugs. However what makes this book unique is a group of 379 paintings illustrating the cultivation, collection and processing of drugs, as well as popular traditions, folkways, beliefs and legends. Most of them are artist's renderings of material in Lei Gong paozhi lun 雷公炮多論 (Lei Gong's Guide to Drug Preparation), attributed to Lei Xiao 雷毁 of the Lei Song period (420-79), depicting human beings at work and a variety of tools and everyday objects. These exquisite vignettes are not only splendid works of art but also a rich mine of visual data on everyday life in the Ming, and traditional processing techniques. In this chapter, I will discuss the scientific and historical significance of this book with regard to portraiture, costume, tools and implements, and drug processing, in light of this set of illustrations.



Figure 14.1 Shoujiao tu 受教圖 (Receiving [Medical] Knowledge). Buyi Lei Gong paozhi bianlan 補遺雷公炮多論 (Supplement to Lei Gong's Guide to the Preparation of Drugs), 1591 ed. Photographic reprint, Shanghai: Shanghai cishu, 2005, preface. @ Wellcome Library, London, Loo39956 




Figure 14.2 Huan kun zhi 浣褌汁 (Laundry Water), showing the typical attire of working people. Buyi Lei Gong paozhi bianlan 補遺雷公炮多論 (Supplement to Lei Gong's Guide to the Preparation of Drugs), 1591 edition. Photographic reprint, Shanghai: Shanghai cishu, 2005, juan 8, p. 1,178. (c) Wellcome Library

\section{Human Figures}

There are 866 human figures in the illustrations of Buyi Lei Gong paozhi bianlan, representing all walks of life. They range all the way down from the highest stratum of legendary emperors and sage kings, to the ministers and generals who ranked just below them, to high-ranking noblewomen, to distinguished scholars, to the craftspeople, foreign visitors and country people who occupied the bottom of the scale. Together, they offer a vivid and nuanced picture of Chinese society in the Ming era.

This is encapsulated in the painting entitled Shoujiao $t u$ 受教圖 (Receiving [Medical] Knowledge) that forms the frontispiece of the book (Fig. 1). The subject - the transmission of medical knowledge from the legendary Yellow Emperor (Huangdi) to Lei Gong - is derived from the dialogue between the Yellow Emperor and Lei Gong in the medical classic Huangdineijing 黃帝內經 (Inner Canon of the Yellow Emperor). Lei Gong is shown prostrating himself



Figure 14.3 Detail of the canopy bed from Renjing tu 人精圖 (Illustration of Human Semen [Essence]) Buyi Lei Gong paozhi bianlan 補遺雷公炮多論 (Supplement to Lei Gong's Guide to the Preparation of Drugs), 1591 edition. Photographic reprint, Shanghai: Shanghai cishu, 2005, juan 8, p. 1,179. ( ) Wellcome Library, London, Loo39984

before the enthroned figure of the Yellow Emperor, who is surrounded by civil and military officials and attendants. The scene is framed by swirling clouds and lofty pines. The golden canopy shading the Yellow Emperor, the splendid furnishings, the incense burner, and the table laden with closed vessels and books create a sense of solemnity and mystery. The protagonists appear to be engaged in conversation; the Yellow Emperor sits upright on his throne, looking down with calm benevolence upon Lei Gong, who kneels on the ground before him in an attitude of humble piety. The civil officials have a refined, cultured air; the general exudes martial vigour; and the attendants are respectful and submissive. The whole composition artfully conveys a web of social relations and social distinctions. Though the subject is drawn from very ancient tradition, the costumes, the binding of the books, and the style of the other artefacts are all typical of the Ming. 


\section{Costume and Personal Adornment}

Most of the paintings of human activity serve to illustrate methods of cultivating, collecting and processing medicines (there are also, as noted previously, some illustrations of popular customs and traditions, and episodes from myth and legend). Because of this the vast majority of human figures are ordinary working people, with a smaller number of emperors, officials, military officers, distinguished personages and aristocratic ladies. In general, men of the gentry classes in the Ming wore a full-length, loose-fitting tunic and a four-cornered, flat-topped hat. Social distinctions were marked by the length of the garments and the width of the sleeves; and so people of high status tend to be depicted with voluminous, flowing robes. Lowlier people wore a short over-shirt with narrow sleeves, and a cloth tied around the head. Most of the working people depicted in this book wear clothing derived from traditional Han costume - albeit with variations in length, and including both jackets and all-in-one tunics. Their garments are fitted for ease of movement, and consist generally of a narrow-sleeved, cross-over shirt or jacket and straight trousers. A cord is generally tied round the waist, and this is used to hitch up the upper garment when working (Fig. 2).

Women generally wear a jacket and skirt; in addition, younger women and servants often wear a short overskirt, a fashionable decorative item that also allowed the wearer to move freely. The jacket generally has a rounded collar and short sleeves. The dress is belted and has a full skirt, and comes in many styles. Colours are significant: gentlewomen are often shown wearing a red robe with voluminous sleeves, while ordinary women wear pink, mauve, green and pale, neutral colours. Ornamentation is used only sparingly.

\section{Implements and Artefacts}

Buyi Lei Gong paozhi bianlan is the first Chinese work on drug processing to be set out as an illustrated guide. As such it provides a wealth of data on a wide range of implements and everyday items, from furniture and household utensils to working tools. According to an initial estimate, the illustrations show more than 2,400 artefacts representing the whole spectrum of daily life in the Ming, including costume and fashion, food and eating, interior design, transport, and labour and production. These items are depicted in precise and exquisite detail, making the book a unique resource for research into traditional furniture design, tools and implements, as well as Chinese folklife studies.

\section{Furniture}

Ming furniture, with its simple, graceful lines and restrained elegance, is regarded as the pinnacle of Chinese furniture design. It is marked by diversity of form and construction within a distinctive style and aesthetic. Classic types of Ming furniture are beds, tables, chairs, cabinets and screens.

In the illustration of 'Human semen' (or human essence, renjing 人精) in juan 8 (Fig. 3), the central element is a classic canopy bed, finished in black lacquer and decorated with gold plant motifs. The bed has a rectangular four-post frame, and is completely enclosed on three sides. The entrance is draped with purple brocade curtains, now let down from their golden hooks to conceal the private space within. Leading up to it is a low, black step, of a type often used as an accessory for canopy beds. The combination of simple, sober forms and fine decoration bespeaks sophisticated craftsmanship. Everything announces that the occupant of the bed is a person of discernment, and probably a nobleman or official. Redolent of a distinctive sensibility, this is a rare and extraordinary visual document for the study of Ming furniture.

The illustrations of drug collection and processing, by their nature, mostly portray the working environment of ordinary labouring people. This is reflected in the style of furniture. Tables are mostly square or long and narrow, and may be of the corner-leg (zhuo 桌) or recessed-leg (an 案) variety. There are few instances of the writing desks, painting tables, wine tables and incense stands so characteristic of the homes of scholars and officials. Drugs and implements are placed on wooden tables of various kinds: oblong corner-leg tables, square corner-leg tables, low corner-leg tables, and narrow oblong tables with recessed legs. Plain and simple, they vary in size, height, and ratio of width to length. Waistless types (with no panel between the the top and apron) and waisted types (with an inset panel between the top and apron) are both found. The former have straight, untapered legs, generally square in section. The latter mostly have legs that curve inwards in a horse-shoe shape, covered along the whole length with intricate carvings. The table-top and base are either unpainted or lacquered in red. In a very few cases, the table-top is adorned with landscape motifs.

Because these are people engaged in manual work, there are few seated figures. Nonetheless, there are a number of depictions of seats and chairs. Sitting on a chair is generally a mark of age or status; junior or youthful figures sit on low compact stools or benches, which are more convenient for physical activity. For example, in Shoujiao tu 受教圖 and in the illustration of 'Tilling the Earth' (Lixia tu 犁下土), the central figure is seated in a typical Ming armchair. 




Figure 14.4 Various receptacles (compilation). Buyi Lei Gong paozhi bianlan 補遺雷公炮多論 (Supplement to Lei Gong's Guide to the Preparation of Drugs), 1591 edition (19th year of the Wanli reign period of the Ming dynasty). Photographic reprint, Shanghai: Shanghai cishu, 2005. (C) Wellcome Library

The curved lines of the backrest and armrests are both aesthetically pleasing and ergonomic, supporting both the lower and upper arm.

There are examples of stools (wudeng 机登, a generic term), barrel stools (zuo dun 坐墩), and benches. Stools can have square, rounded or oblong seats, square-seated stools displaying the greatest variation in style. Waisted and waistless types can be distinguished. Waisted stools are generally made from square stock rather than round stock; they have s-shaped legs terminating in horseshoe-shaped feet, which may curve either outwards or inwards. Waistless stools are made with both square stock and round stock; they have straight legs, which may be either square or rounded, and plain, unadorned feet. All these varieties of stools are well represented in the book.

\section{Everyday Implements and Working Tools}

This includes a variety of items which are integral to daily life but easily overlooked. Over the ages, many thousands of implements have been developed in China for use in everyday life and work, and a wide range of these are represented here. They can be divided into the following broad categories.
Cooking Utensils and Apparatus

Stoves and furnaces - including alchemical furnaces for obtaining the elixir known as dan 丹 (liandan lu 煉丹爐), ordinary cooking stoves and simple furnaces of various kinds. They may be square or conical.

Cooking vessels - flat-bottomed pans or griddles, rounded woks and steep-sided pans.

Steaming and distilling apparatus - rice steamers (zengzi 潧子), tubular steamers (zhengtong 蒸桶), bamboo food steamers (zhenglong 蒸籠) and various bamboo and wooden steaming vessels (longti 籠屨) etc.

Knives and cutting tools - harvesting knives (zhadao 鋇刀), kitchen knives (caidao 菜刀), bamboo blades (zhudao 竹刀), butcher's knives (tuzai dao 屠 宰刀).

Chopping blocks - oblong or round boards, or massive wooden blocks.

\section{Receptacles}

Vats (缸 gang), earthenware urns (weng 瓮), jars ( tan 壇), pots (guan 罐), flasks (ping 瓶), buckets and casks (tong 桶), basins (pen 盆), dippers (tiao 慓), carriers ( $t i z i$ 提子), etc. 
Eating and Drinking Vessels; Tableware Drinking vessels - wine jars (jiu weng 酒器), wine jugs (jiu hu 酒壼), wine cups (jiu bei 酒杯).

Eating vessels- bowls (large and mediumsized food bowls, rice basins, small porcelain bowls); plates, platters and trays (including white porcelain plates, fruit plates, and lacquer plates and trays, seen together with chopsticks and spoons), tea drinking paraphernalia (teapots, tea cups, tea bowls) etc.

Other Items

Wooden artefacts - clothes airers or racks for drying medicines.

Bamboo artefacts - bamboo ladders, baskets, crates and paniers, dustpans and brooms, sieves and strainers, etc.

Metal artefacts - apothecary's scales and steelyards, candlesticks, bronze mirrors, etc.

Textiles

Curtains, hangings and screens.

Tools for Manual Labour

Tools for fragmentation - mallets, grinders, mortars and pestles.

Wood-working tools - axes, saws, hammers, and pliers).

Agricultural implements - hoes, spades, harrows and ploughs.

Other tools, e.g. oil press, apparatus for making alcohol.

These artefacts display variation in material, form and craftsmanship. They reflect the level of development of manufacturing technology in the Ming, and social customs and mores of that period, and they help build up a detailed and nuanced picture of traditional customs and practices.

\section{Methods of Drug Processing}

Though most of the illustrations in Buyi Lei Gong paozhi bianlan are based on originals in Bencao pinhui jingyao 本草品彙精要 (Classified Treasury of Materia Medica, 1505), it also contains 217 unique and (apparently) entirely new illustrations of drug preparation and processing. This sets it apart from Bencao pinhui jingyao itself and other works in the same genre. The artist has mainly followed the entries on drug processing in Lei Gong paozhi lun. The principal methods of drug processing according to that text are set out below.



Figure 14.5 Processing asparagus root. Buyi Lei Gong paozhi bianlan 補遺雷公炮多論 (Supplement to Lei Gong's Guide to the Preparation of Drugs), 1591 edition. Photographic reprint, Shanghai: Shanghai cishu, 2005, juan 3, p. 386. (c) Wellcome Library

\section{Cleansing}

These include: selecting, removing soil and encrustations, removing hard outer layers, removing outgrowths, skimming off foam or scum, wiping, mopping, scraping, paring, peeling and washing in a pan or colander.

\section{Cutting}

These include: cutting, filing, sawing and breaking apart manually.

\section{Pulverisation}

These include: beating or pounding with a hammer or stick (chui 捙), grinding in a mortar (chong 春), pounding with a pestle (dao 搗), grinding (yan 研), grinding with a pestle (chu 杵), milling (mo 磨), grinding with a roller (nian 碾), wet grinding or refining with water (shuifei 水飛) and sifting (guoshai 過篩). 


\section{Drying}

These include: wiping dry (shigan 拭乾), drying in the shade (yingan 陰乾), air-drying (fenggan 風 乾), sun-drying (shaigan 麗乾), drying over fire or parching (beigan 焙乾), drying by stir-cooking with a liquid adjuvant (zhigan 奕乾), and steam-drying zhenggan (蒸乾).

\section{Water and Fire}

This includes: soaking or immersion (jin 浸), blanching and rinsing by frequently refreshing the water (piao 漂), macerating (pao 泡), boiling (zhu 煮), decocting (jian 煎), refining or distilling by fire or alchemical processes (lian 煉), stir-frying (chao 炒), stewing or simmering ( $a \mathrm{o}$ 㥿), roasting ( $k a o$ 烤), baking over a slow fire, by dry heat (bei 焙), roasting or stir-heating at a high temperature, often with hot sand (pao 炮), calcining (duan 煆).

\section{Processing with Adjuvant Substances}

This includes: steeping in bitter rice wine (kujiu jin 苦酒浸), fumigation with vinegar (cu xun 醋 熏), stir-cooking with honey (mitu zhi 蜜塗多), stir-cooking with glutinous rice (nuomi chao 糯米 炒), stir-frying in butter (suchao 酥炒), boiling in sesame oil (mayou zhu 麻油者), soaking in water in which rice has been washed (miganshui jin 米泔水 浸), processing with the urine of prepubescent boys (tongbian zhi 童便制), preparation with distilled medicinal liquid (yaozhizhi 藥汁制).

Nearly all the above methods and techniques of drug processing are illustrated with exquisite paintings. As explained previously, the artist or artists who worked on this book generally followed the text of Lei Gong paozhi lun. The illustrations are shaped by their reading of that text, and their own acquaintance with drug preparation practices, as well as aesthetic and compositional criteria, and involve a certain amount of artistic licence. They were professional artists in the service of the imperial court; they had no specialist expertise in pharmacy, and are scarcely likely to have had any contact with the milieu of drug manufacture. Thus many of the illustrations are imaginative creations based on textual description, and should not be treated as eye-witness records of contemporary tech- niques, practices or artefacts. Inevitably, there are errors or misunderstandings of technical detail. Nonetheless, taken as a whole, the scenarios, methods, substances and instruments depicted in this book are vital source material for research into drug processing.

In summary, Buyi Lei Gong paozhi bianlan is furst and foremost an illustrated Bencao, concerned with explaining and displaying visually the morphological characteristics, cultivation and collection, and pharmaceutical processing of medicinal plants and substances. However the 379 illustrations discussed here are also exceptionally vivid vignettes of life in the Ming at every level of society, full of human interest and telling circumstantial detail. Executed with the finesse and bravura characteristic of Ming court art, they bring to life a host of different characters, whose varied apparel reflects a socially stratified culture of costume and personal adornment. The processing methods and apparatus depicted in the book are mostly taken from the earlier Lei Gong paozhi lun, and a few of them would not have been used in contemporary practice, while others may not be represented with total accuracy. However, with these wonderful paintings, the artists have bequeathed to posterity a treasure house of highly significant data in an accessible visual form.

When viewed as a corpus, the illustrations of drug collection and processing in Buyi Lei Gong paozhi bianlan seem to unfold the world of the Ming like a Chinese handscroll. The quality of the painting, the variety of characters and incident, and the quantity and variety of costumes, artefacts and technologies depicted are virtually unparallelled. They constitute a vital primary source for the history of art, costume, folklife and Chinese medicine.

\section{Bibliography}

\section{Primary sources}

Buyi Lei Gong paozhi bianlan 補遺雷公炮製便覽 (Lei Gong's Guide to Drug Preparation with Addenda), completed spring of 1591 (Xinmao year of the Wanli reign period of the Ming Dynasty), Reprint Shanghai cishu chubanshe, 2005 .

Lei Gong paozhi lun 雷公炮多論 (Lei Gong's Guide to Drug Preparation), attributed to Lei Xiao 雷毁, Lei Song period (420-79). 


\section{The Relationship between Chinese Erotic Art and the Art of the Bedchamber: A Preliminary Survey}

\author{
Sumiyo Umekawa 梅川純代 and David Dear
}

This article will investigate the links between yangsheng 養生 (Nourishing Life) culture dating back to the Western Han dynasty (2O2 BCE-9 CE) and the Ming/Qing era sex illustrations (late 14th to early 2 oth century), as transmitted through the discourse of fangzhong shu 房中術 (the Art of the Bedchamber). From the Ming dynasty (1368-1644) onwards, we see an efflorescence of Chinese erotic art, such as that collected by Robert van Gulik in his 1951 book Erotic Colour Prints of the Ming. Comparisons will be drawn between the Japanese tradition of erotic art known as shunga 春畫 and the Chinese erotic art of chungong hua 春宮畫. ${ }^{1}$ The difference between these two genres highlights the latter's continuing reference to the formulas of the ancient sexology texts, while the former expresses a more directly pornographic ${ }^{2}$ approach to erotic imagery, notwithstanding the availability in Japanese culture of the same philosophy of sexual regimen as transmitted through the Ishimp $\overline{0}$ 醫心方 (Formulas of the Heart of Medicine). ${ }^{3}$

\section{Definitions and Background}

\section{Yangsheng 養生 (Nourishing Life)}

Yangsheng consists of a group of practices and literature aimed at enhancing the well-being of the practitioner. It does so through the operation of Qi 氣 within and without the body of the practitioner(s), and with particular reference to Yin Yang philosophy. In the earliest known texts dating from the Western Han, the discourse of yangsheng merges seamlessly with the then emerging themes of Chinese medicine. The earliest recorded forms of yangsheng physical exercises, called daoyin 導引 (Guiding and Pulling [of Qi]), are the forerunners of modern Qigong and Taiji quan, together with a number of other longstanding exercise practices like the wuqin xi 五禽戲 (The Five Animal

春畫 shunga lit. = 'Spring pictures'. 春宮 chungong (lit. 'Spring palace') in Chinese can refer to a prince's palace - and is also a term for erotica.

2 The term 'pornography' is a problematic one in relation to Asian sexual culture since it is essentially a reflection of Western sexual taboos, or alternatively a way of critiquing the 'morality' of the 'other'. Here it is simply used as shorthand to denote the most directly explicit sexual imagery. See Screech 1999, pp. 13-38.
Mimes). ${ }^{4}$ Besides exercise and breath control practices, yangsheng includes rules and principles for most aspects of daily life with particular concern for food, dietetics and sexology. Yangsheng in a broad sense still exerts a powerful influence on the way ordinary people think about and manage their health to this day. However at the time of its emergence as a distinct philosophy of life in the late Warring States era (4th and 3 rd centuries BCE), it was, in the eyes of many, clearly connected with the search for transcendence and immortality as practised by the xian 仙 cults which flourished in practice until the late Tang (618-907) - and in legend and literature ever since. However from their inception, the philosophies and purposes of yangsheng, medicine and immortality cults were the subject of broad dispute. Works attributed to the philosopher Zhuangzi 莊子 (4th century BCE), have equivocal attitudes to the pursuit of immortality, both speaking of transcendence and mocking those who just stretch and shake their bodies, while the Eastern Han sceptic, Wang Chong 王充 (27 CE-?100 CE), both mocked and criticised the xian immortality cults while acknowledging the value of straightforward yangsheng. ${ }^{5}$

\section{Fangzhong Shu (The Art of the Bedchamber) and Yangsheng (Nourishing Life)}

While the early yangsheng texts concerning sex and sexual relations form a core part of the fangzhong shu, other types of fangzhong shu texts also show the influence of the immortality cults, early Daoist rituals, spiritual alchemy, ${ }^{6}$ and in early modern empire (Song dynasty, 96o-1279 onwards), the impact of works published simply to titillate and entertain as the printing and publishing industries grew and flourished. Thus while yangsheng concerns itself with many things besides sexology, fangzhong shu covers a wider range of sexual practices than those prescribed by yangsheng. The various sexual theories from the realms of medicine, yangsheng, fangzhong shu, Daoism and other religio-spiritual cults all operated over the centuries in a climate of mutual influence around a common vocabulary of Qi and its defining operational philosophies. However, as

\footnotetext{
$4 \quad$ For more on wuqin xi, see Wang and Barrett 2006.

$5 \quad$ Wang Chong (27-97 CE) Lun heng 16.

6 Both waidan 外丹 (External, chemical, alchemy) and neidan 內 丹 (Internal, spiritual, alchemy).
} 
with yangsheng practices and the xian immortality cults, while there is a substantial overlap between yangsheng and fangzhong shu, the two categories are nonetheless separate and distinguishable, the more so as time goes on. ${ }^{7}$

\section{Basic Principles of Yangsheng Sexology}

Yangsheng sexual techniques have a series of interlinked and clearly defined objectives:

to sexually satisfy the female partner by bringing her to orgasm one or more times;

to preserve the physical wellbeing of the male partner; to augment the physical and psychological health of the male partner by absorbing female Yin Qi 陰氣;

to maximise the opportunities for the conception of healthy male heirs;

to allow the male partner to control conception;

to cure female ailments;

to establish and maintain social and domestic harmony between a couple.

The key concept is that the female partner is a potentially inexhaustible ${ }^{8}$ source of Yin Qi. Males by contrast are always in danger of exhausting their supply of Yang Qi 陽 氣 by careless or wanton sexual behaviour, while their vital Yin Qi is constantly under threat of natural depletion. Women are therefore inherently sexually stronger than men. The highest form of female Yin Qi is released by a woman at orgasm. This Yin Qi can be absorbed by the male to benefit his own health because his own inner organs require Yin Qi to nourish them, a process called gubai 固 白 (consolidating white). ${ }^{9}$

The early yangsheng texts place the emphasis on the male partner's ability to bring his companion to orgasm through close observation of her behaviour and an appropriate response in his foreplay to take her to a higher level of excitement. ${ }^{10}$ Once the woman is ready for coitus,

Furth in Gilmartin et al. (eds) 1994, p. 133, notes the increasing criticism in yangsheng texts of 'non yangsheng' fangzhong shu practices in the later empire.

8 Ibid. p. 134. Furth objects to this 'inexhaustible' idea, however inexhaustibility does seem to be consistent with the ideas in the early yangsheng texts, though they do also stress the need not to damage female health and/or Qi through mis-practice.

9 The term gubai 固白 (consolidating white) appears in the fourth and 1oth dialogues of the text excavated from Mawangdui Tomb 3 given the modern title of Shiwen (Ten Questions). Especially, the 1oth dialogue explains the condition indicated by this term in relation to the accumulation of Qi in the five internal organs: Shiwen. p. 147, p. $15^{2}$. and Yang), also from Mawangdui Tomb no. 3 (dated to 68 BCE), and Yang), also from Mawangdui Tomb no. 3 (dated to 168 BCE), is one of the best examples of these techniques. See Harper 1998.

detailed prescriptions are given for the types, sequences and numbers of penetrations the man should make and the various positions in which they can be achieved. Ejaculation control is the key technique that the male needs to apply to his own body.

But as Donald Harper makes clear in a discussion of the aphrodisiac recipes and philtres found at Mawangdui 馬 王堆, Dunhuang 敦煌 and in the Ishimp $\overline{0},{ }^{11}$ the case for a pure tradition of esoteric sexual cultivation, completely divorced from normal human sexual desire and lust, is hard to evidence. In later fangzhong shu works, the idea of augmenting male health becomes a 'battle' in which males seek to garner as much Yin energy from as many partners as possible while not losing any of their own Yang Qi. In this way, men can achieve immortality. This idea is present in the Han dynasty in the legend of Peng Zu 彭祖, the original master of fangzhong shu, who is supposed to have lived to 800 or 900 years through his skill in sexual cultivation. The legend is a clear expression of the influence of the immortality cults. In other legends, and their later corresponding popular stories, females are able to attain immortality through the seduction of multiple male partners. ${ }^{12}$ Similarly in later Daoist practice, the principle of non-ejaculation becomes huanjing bunao 還精補腦 (returning the sperm to nourish the brain) - the idea that not only did the man not ejaculate, but that he was able to conserve his jing 精 (essence) and guide it up to his brain. This was in some cases regarded as a physical practice, in others as a purely meditative one. In both cases, the aim was the achievement of spiritual immortality. Both the idea of 'stealing' Yin and that of an absolute prohibition on ejaculation (as opposed to an enjoinder to restrict it to the minimum possible) can be considered as deviations from yangsheng, and in the eyes of the 5th-century $\mathrm{CE}$ Daoist physician and Patriarch Tao Hongjing 陶弘景, the latter not only offends against true Daoism but also against the Confucian ideal of xiao 孝 (filiality), in that one would thereby be depriving one's parents and ancestors of descendants.

\section{Ishimpō}

During the Tang dynasty (618-907), Japanese scholar monks travelled extensively in China, absorbing and recording all they could of Chinese culture. One product of this was the Ishimpō, compiled by Tamba no Yasuyori 丹波康頼 in 984 CE. It provides us with the most complete record of Chinese

\footnotetext{
11 Harper 2005.

12 See Needham and Lu 1983, pp. 194-5 on the Xi Wang Mu 西王母 (Queen Mother of the West) and Ming novellas like the Zhulin yeshi 株林野史 (The Secret of the Bamboo Grove).
} 
medicine at the time (as opposed to single-authored or single author attributed texts), in addition to giving details of texts recorded in the dynastic histories but subsequently lost or suppressed in the intervening centuries. A number of the Ishimpō texts concern sexology and it was a re-translation of the Ishimpō into Chinese that inspired the late Qing scholar Ye Dehui 葉徳輝 (1864-1927) in his investigations into the ancient sexual arts in general. Ishimpō remained the main source available in this field until the recovery of the Han dynasty yangsheng sexology texts in the tomb finds at Mawangdui and Zhangjiashan 張家山 in the 1970s and 1980s.

\section{Shunga}

Hayakawa Monta 早川聞多 defines Japanese erotic art, shunga, as follows: 'It is the pictures and drawings that illustrate sexual intercourse, and various customs and manners concerning sexual activities.' ${ }^{13}$ It can be taken to correspond to a large extent to the Chinese genre of sexual imagery called chungong hua. These art works would nowadays be classified in English as erotica or pornography, and the category contains images of all varieties of sexual activity, not least homosexual. For the purposes of this chapter, the authors will confine their comments to those images that depict sexual activity between actors of the opposite sex. This is because yangsheng and fangzhong shu texts only direct themselves explicitly to heterosexual relations or solo practices. The authors offer no comment as to whether the ideas and principles there enshrined could apply to same-sex relationships. Interestingly however, Screech in his discussion of Nanshoku 男色 cites the idea in 15th -16 th century Japan of men having sex only with other males as a means of retaining Yang Qi during



\section{The Images}

While there are many talismanic or practical artefacts which use sexual imagery or references from the pre-Song eras, there are none associated directly with yangsheng or fangzhong shu texts. Both van Gulik and the modern Chinese scholar Professor Liu Dalian 劉達臨 posit the existence of ancient sex manuals with illustrations..$^{15}$ As evidence, van Gulik quotes a poem found at Dunhuang,

\footnotetext{
13 Shirakura and Hayakawa (eds) 2003, p. 14.

14 Screech, 1999, p. 84. However from this account, the concern for Yang Q $i$ seems to stem from a sociological rather than physiological perspective.

15 Van Gulik 1974 [1961], pp. 200-6; Liu 2003, p. 242.
}

where erotic imagery also survives amidst the fresco paintings. The poem van Gulik relies upon is the Tiandiyinyang jiaohuan dalefu 天地陰陽交歡大樂賦 (The Heaven and Earth, Yin and Yang Songs of Great Satisfaction in Sexual Pleasure, hereafter referred to as The Songs of Great Satisfaction), which was possibly composed during the Tang dynasty. He refers in particular to the line: 'they read the Canon of the Plain Girl and look at the erotic pictures on the holding screen'. ${ }^{16}$ Van Gulik clearly understands 'the erotic pictures' as depicting the contents of Sunüjing 素 女經 (The Canon of the Plain Girl). However, this reading is open to question. Thus we would hesitate to accept van Gulik's and Liu's suggestion that illustrated sex manuals really did exist from ancient times.

By the Ming Era the situation had changed radically. A flourishing publishing industry produced numerous salacious stories. The Ming period also saw the composition and first publication of the 'secret' masterpiece of Chinese literature, Jinping mei 金瓶梅 (Golden Lotus, or lately in David Roy's modern translation, the more appropriately suggestive Plum in the Golden Vase). ${ }^{17}$ Jinping mei is a savage and shockingly explicit picaresque novel, which satirises contemporary mores in great detail and which itself is seen as prefiguring the accepted pre-eminent masterpiece of classical Chinese literature, Honglou meng 紅樓夢 (Dream of Red Mansions), mid-18th century. It also finds echoes in the mid-17th-century erotic satire Roupu tuan 肉蒲 團 (The Carnal Prayer Mat, or 'Bedclothes of Flesh'), 18 in which the hero, Weiyangsheng 未央生 (Vesperus), torn between spiritual and carnal quests, has his penis enlarged with the insertion of a dog's sexual endowment, the better to pursue the latter path. Both Roupu tuan and Jinping mei make reference to the collections of erotic imagery available at that time. In the former, Vesperus buys a collection of erotic art to give his wife, indicating that such collections of erotic drawings could be freely bought in the marketplace. Two collections are mentioned by name: Sanshi gongchun 三十宮春 (Thirty Palaces of Spring) in both Roupu tuan and Jinping mei, and Ershi si jie 二十四 解 (Twenty-four Descriptions) in Jinping mei. ${ }^{19}$ Shen Defu 沈德符 (1578-1642), a leading intellectual of the Ming era, also reported that erotic items including pictures were sold in many local shops. ${ }^{20}$

\footnotetext{
16 Tiandiyinyang jiaohuan dalefu (Ye Dehui 1995 edn), p. 102.

17 First published in 1610, but circulated in handwritten copies for several decades previously.

18 Roupu tuan, attr. Li Yu 李漁 (1610-80), was composed in 1657 and published in 1693 .

$19 \quad$ Wu 2005, p. 156.

$20 \quad$ Ibid., p. 155.
} 
Two Ming artists in particular, 仇英 (1500-60) and Tang Yin唐寅 (1470-1523), are believed to have created a great deal of erotic material. However, due to the anonymity of their working practice as well as the existence of large number of forgeries, it is quite difficult to provide accurate attributions for each picture. According to Fukuda's 福田 research, most hand-drawn pictures do not have a signature or seal and it is not possible to trace a publisher for most of the printed drawings. ${ }^{21}$ Thus it is very difficult not only to attribute but also to date much of the extant erotic art. Some writers of the Ming and Qing period report the existence of wealthy women fond of producing erotic pictures, ${ }^{22}$ but most of the material would have been the work of anonymous professional artists and artisans living in society at large. The idea of erotic painting as an aristocratic female art form would be consistent with the hedonistic or decadent atmosphere attributed by many to the Ming era. It would also be supported by the evidence of the Jinping mei. However, it should be borne in mind that the Qing historians have a vested interested in portraying the preceding dynasty as degenerate. In fact from the evidence emerging from Liu Dalian's research, erotic and pornographic art proliferated as never before under the outwardly strait-laced Qing. The Qing sources are so extensive that the increase can hardly be attributed simply to the era's relative historical proximity or to improved techniques of production.

In the Ming, artefacts like silk paintings not amenable to mass production would have been costly and exclusive, while on the other hand, the line engravings used to illustrate the aforementioned erotic literature would have enjoyed a mass circulation. One might reasonably assume that those high-production images would have been destined for limited circulation among officials and the wealthy merchant classes. Certainly this is van Gulik's view, but he believed that at the same time these images also had considerable impact on ordinary society. ${ }^{23}$ In the case of the collections mentioned in Roupu tuan and Jinping mei, they could often have formed part of the trousseau of the well-to-do bride as an educational guide in what her husband might expect of her. In the Qing dynasty higher-quality images started to be produced on a systematic basis, notably in the Guangdong area, where several art studios established techniques for the manufacture of large quantities of colour prints and paintings on Chinese

\footnotetext{
$21 \quad$ Fukuda 1981, p. 16.

22 Liu 2003, p. 242.

23 Van Gulik, 1974, p. 332
}

subjects in general for export to the West. These included a considerable amount of erotic material. ${ }^{24}$

It was also from the Ming era onwards that Japanese erotic illustrations, shunga, were imported into China, where they were reportedly much sought after. ${ }^{25}$

\section{Chungong Hua and Fangzhong Shu}

In her paper 'Re-thinking van Gulik', Charlotte Furth says: 'In bedchamber manuals natural philosophy continues to frame the discussion of sex as a microscopic human re-enactment of primary creative processes'. ${ }^{26}$ This is unequivocally true of some of the Han dynasty yangsheng sexology but must be open to question with regard to the broader category of fangzhong shu that had opened up by the Ming era. The purposes of the texts had in many cases moved on, and not necessarily to a more elevated level. The increasing sophistication of the medical discourse in the intervening centuries had stripped the texts of much of their didactic purpose. As we see from Sun Simiao's 孫思激 (581-682) Qianjin yaofang 千金要方 (Essential prescriptions worth 1,0oo gold pieces) and the Ishimpō, sexology for health had become incorporated into a specifically medicalised discourse. On the other hand the Tang dynasty has also been identified as the era in which an unashamedly recreational erotic literature starts to appear. Thus in the Ming era we find erotic illustrations without attached text and un-illustrated Bedchamber texts from previous eras. Can any connection be made between them?

Roel Sterckx in his paper in this volume makes the valuable distinction between performative and illustrative images. $^{27}$

The former type consists of depictions that invite the observer to actively invoke the image as part of a performance (e.g. an incantation, a healing procedure, a mnemonic act). The latter comprises images that complement an existing narrative, mostly texts. While the performative image may be accompanied by explanatory texts, in principle, text remains subsidiary to the image. In the case of the illustrative image, the image offers a type of annotation or commentary to the text.

The classic line illustrations that accompany the text of the Jinping mei are the most obvious example of this latter case. They are simply there to enhance the reader's pleasure - and ensure that the published versions will appeal to the widest possible market, including those who may not be able to appreciate the full literary depth of the novel.

\footnotetext{
24 Nakano 2001, pp. 20, 22.

25 Wu 2005, p. 158.

26 Firth in Gilmartin et al. (eds) 1994, p. 133

27 See Sterckx, Chapter 7 in this volume, p. 136.
} 




Figure 15.1 'Pan Jinlian Enjoys a Midday Battle in the Bathtub' (detail), from Jinping mei, ch. 29. Printed from an original woodblock of the Ming Chongzhen era (1627-44). Source: Jinping mei, Taiping shuju edn, p. 740

However, many of the chungong hua can be said to have some simple performative value by virtue of the fact that most of us share basically recognisable bodily traits and a similar range of movements. The question is to what extent the images can be said or seen to comply with specifically yangsheng values, indicating a performative dimension that can connect them with fangzhong shu. In this regard, the contrast between chungong hua and shunga can be seen to be illuminating.

\section{Shunga and Chungong Hua}

Besides use as pornography ${ }^{28}$ or instruction as to how to enjoy sexual relations, ${ }^{29}$ it is often noted that Japanese erotic art served a humorous function. ${ }^{30}$ While much Chinese erotic art could also have been intended for merriment, the styles of humour in Japanese and Chinese examples are quite different.

One of the key key humorous mechanisms in Japanese shunga is exaggeration. As in Figure 2, male and female sex organs are frequently depicted vividly and realistically, but enlarged to an impossible size. This intense emphasis on the penis and vagina can be related to the Japanese orientation to the 'close-up view'. ${ }^{1}$

In addition shung a reflects the influence of the Japanese dramatic tradition of Kabuki 歌舞伎, which was particularly popular in the Edo period (1603-1867), when much Japanese erotic art was produced. In a Kabuki play, actors

$28 \quad$ Screech 1999, pp. 13-38.

29 See for instance, Shirakura and Hayakawa 2003, p. 16.

$30 \quad$ Ibid., p. 16; Fukuda 2003, p. 9 o.

$31 \quad$ Nakano 2001, p. 7, pp.16-18, p. 36.



Figure 15.2 'Nami Chidori' 浪千鳥 by Katsushika Hokusai 葛飾北斎 (c. 1829). Source: Shirakawa and Hayakawa 2003, p. 162

make up their faces in a unique manner to express the characteristics of the role they are playing and then exaggerate their actions to ensure the audience understands every detail of emotion the character feels. A notable feature of Kabuki is mie 見得, which means to strike an impressive pose. Fukuda suggests that the exaggeration of genital organs comes from this aspect of Kabuki; stage techniques to entertain an audience are effectively lifted to another 'stage', that of erotic art. ${ }^{32} \mathrm{He}$ further points out the fact that male and female figures as well as male and female organs in Japanese erotic art are placed symmetrically or vertically. He calls such composition 'combat style' and regards it as derived from the stage arrangements in Kabuki. ${ }^{33}$

Equally Hayakawa observes that the degree of elaboration in the depictions of faces and genital organs is similar, and that the faces and conjoined genitals are aligned in the composition. ${ }^{34}$ Thus this arrangement of figures and sex organs is one of the special elements in Japanese erotic art and it further suggests the intensive nature of its focus.

This type of exaggeration can also be related to the growing scientific interests of the Japanese in that era. ${ }^{35}$ Erotic pictures as a part of ukiyo-e 浮世絵 (Pictures of the Floating World) were enjoyed mainly by people living in and around Edo, today's Tokyo. Meanwhile parody books called Tengōmonono 転合物 (Joke Goods) became popular in the west of Japan. Such joke books usually imitated didactic, moralistic or medical texts in common circulation. Japan

\footnotetext{
$32 \quad$ Fukuda 2003, p. 92.

33 Ibid., pp. 88, 9 o.

34 Shirakura and Hayakawa 2003, p. 16.

35 Screech 1999, pp. 216-28.
} 
at that time was receiving Dutch medical ideas including anatomical knowledge. Under this Dutch influence, these parody books sometimes discussed the quality of male and female organs, occasionally with detailed illustrations. Sometimes, such books provided quite accurate anatomical descriptions of the organs, female ones being specifically called Ōtsubie 大開絵 (Wide Open Pictures), a not so subtle reference to the anatomical pose necessary for inspection. Thus in shunga, we can identify a series of rationales for the type of imagery from a 'scientific interest' to the attraction to the 'close-up view', the influence of Kabuki style dramatisation and a ribald popular sense of humour based on exaggeration.

In contrast to the shunga images with their essential preoccupation with the moment of penetration, Chinese chungong hua show large numbers of images of foreplay, and rarely, if ever, show penetration in the sort of detail that would have been seen in the imported shunga (as in Figure 2 above). This interest in foreplay can be seen, at least at some level, as carrying a cultural memory of yangsheng, for the Han dynasty texts often expound on the need for the man and woman to achieve emotional harmony before penetration begins - and indeed on the health risks of failing to do so. As mentioned before, the man is specifically enjoined to carefully observe his partner's level of arousal and to act accordingly. Some scholars, from Japan in particular, have noted the lack of passion expressed in the faces portrayed in Chinese chungong hua. This is also in keeping with the yangsheng concept, very much discussed at the time, of the need to avoid extremes; and extreme emotions were seen as being especially damaging to the health. ${ }^{36}$ Unlike shunga, the figures in the Chinese images are often set in natural locations like gardens or spectacular scenery of 'mountains and rivers', ${ }^{37}$ where the whole environment is an expression of the Yin and Yang of the cosmos - and the human actors are therefore merely playing their small part in it. In other cases, the arrangement of details in the image, such as Taihushi 太 湖石 (Lake Tai Stones), expresses the same idea.
However the key to connecting any performative attributes of yangsheng fangzhong shu to chungong hua must come down to a consideration of the positions for coitus enjoyed by the couples. In shunga, realism in this matter is not a necessary requirement. Yangsheng texts however do give detailed sexual positions for the remedy of ailments and the enhancement of the couple's well-being according to specific circumstances. These positions reappear in the Ming illustrations.

\section{Sexual Positioning in Chinese Erotic Art}

In particular it is through a number of texts like Dongxuan $z i$ 洞玄子 (The Master Dongxuan) and the Qisun bayi 七損 八益 (Seven Disadvantages and Eight Advantages) recorded in the Ishimp $\bar{o}^{38}$ that we have details of the use of specific positions to cure or alleviate ailments. The Qisun bayi lists seven sexual positions to cure seven ailments or unhealthy conditions in the male, and eight further sexual positions to enhance male health. ${ }^{39}$ The positions of the Bayi - the Eight Benefits - are set out in Table $1 .{ }^{40}$

Here the sexual satisfaction of the female is secondary to the health benefits available to each partner. The benefits for the female are listed separately without reference to the particular position but with emphasis on the number of penetrations and their required frequency.

In contrast, sexual positioning in erotic pictures did not have such a clear focus on health benefits. Rather, sexual positions are treated as examples of how best to enjoy sexual relations. In spite of this, erotic pictures do still reflect the thinking of the sex manuals, in that the positional variations shown would all have served the primary goal of female orgasm with different positions to be used according to the female's state of arousal and personal preference. Modern Western sexology with its 'discovery' of the G-spot makes this plausible to the modern reader. But let us return to the Qisun bayi and the Seven Disadvantages.
36 Gao Lian 高濂 (c.1527-1603), Zunsheng bajian 遵生八茂 (Eight Discourses on Respecting Life, 1591). See Chen Hsiu-fen 2008, pp. 29-45. traditional forms of Chinese art. 
Table 15.1 Positions, Effects and Number of Insertions of the Eight Benefits

\begin{tabular}{|l|l|l|l|}
\hline Name & $\begin{array}{l}\text { Medicinal } \\
\text { objective }\end{array}$ & Position & $\begin{array}{l}\text { No. of in- } \\
\text { sertions }\end{array}$ \\
\hline $\begin{array}{l}\text { Gujing 固精 } \\
\text { (coagulation of energy) }\end{array}$ & $\begin{array}{l}\text { To congeal male } \\
\text { energy }\end{array}$ & $\begin{array}{l}\text { The female lies on her side and extends her thighs. The male } \\
\text { places himself between her thighs, lying on his side. }\end{array}$ & $\mathbf{1 8}$ \\
\hline Anqi 安氣 (easing Qi) & $\begin{array}{l}\text { To harmonise male } \\
\text { Qi }\end{array}$ & $\begin{array}{l}\text { The female lies on her back, places her head on a high pillow and } \\
\text { extends her legs. The male kneels between her legs. }\end{array}$ & 27 \\
\hline $\begin{array}{l}\text { Lizang 利藏 } \\
\text { (benefiting storage) }\end{array}$ & $\begin{array}{l}\text { To harmonise male } \\
\text { Qi }\end{array}$ & $\begin{array}{l}\text { The female lies on her side and bends her knees. The male lies on } \\
\text { his side behind her. }\end{array}$ & 36 \\
\hline $\begin{array}{l}\text { Qianggu 強骨 } \\
\text { (strengthening bones) }\end{array}$ & $\begin{array}{l}\text { To harmonise male } \\
\text { joints }\end{array}$ & $\begin{array}{l}\text { The female lies on her side with her left knee bent and her right } \\
\text { leg extended. The male lies upon her. }\end{array}$ & 45 \\
\hline $\begin{array}{l}\text { Tiaomai 調脈 (regulat- } \\
\text { ing the vessels) }\end{array}$ & $\begin{array}{l}\text { To smooth male } \\
\text { vessels }\end{array}$ & $\begin{array}{l}\text { The female lies on her side with her right knee bent and her left } \\
\text { leg extended. The male supports himself on the ground. }\end{array}$ & 54 \\
\hline $\begin{array}{l}\text { Xuxue 蓄血 } \\
\text { (depositing blood) }\end{array}$ & $\begin{array}{l}\text { To make the male } \\
\text { more robust }\end{array}$ & $\begin{array}{l}\text { The male lies on his back. The female kneels on him, placing her } \\
\text { buttocks on him to insert the penis deeply. }\end{array}$ & 63 \\
\hline $\begin{array}{l}\text { Yiye 益液 (benefiting } \\
\text { secretion) }\end{array}$ & $\begin{array}{l}\text { To strengthen the } \\
\text { male bones }\end{array}$ & $\begin{array}{l}\text { The female lies on her face and raises her back. The male rides on } \\
\text { her. }\end{array}$ & 72 \\
\hline $\begin{array}{l}\text { Daoti 道體 (body of the } \\
\text { way) }\end{array}$ & $\begin{array}{l}\text { To fill the male } \\
\text { bones }\end{array}$ & $\begin{array}{l}\text { The female lies on her back and bends her legs so that her toes are } \\
\text { placed under her buttocks. The male holds her under the arms. }\end{array}$ & 81 \\
\hline
\end{tabular}


Table 15.2 Therapeutic Sexual Intercourse for the Seven Disadvantages ${ }^{41}$

\begin{tabular}{|c|c|c|c|c|}
\hline Condition & Cause & Symptoms & Position for remedy & $\begin{array}{l}\text { Insertion } \\
\text { instruction }\end{array}$ \\
\hline $\begin{array}{l}\text { Jueqi 絶氣 } \\
\text { Interdicted } \\
\text { Qi }\end{array}$ & Unwilling sex & $\begin{array}{l}\text { Sweat, decrease of Qi, heat in } \\
\text { the heart and dizziness }\end{array}$ & $\begin{array}{l}\text { The female lies on her back } \\
\text { while the male supports her feet } \\
\text { on his shoulders. }\end{array}$ & Deeply \\
\hline $\begin{array}{l}\text { Yijing 益精 } \\
\text { Excessive } \\
\text { ejaculation }\end{array}$ & $\begin{array}{l}\text { Sex before the harmonious } \\
\text { preparation of male and } \\
\text { female, emission of semen in } \\
\text { the middle of intercourse and } \\
\text { sex when drunk }\end{array}$ & $\begin{array}{l}\text { Harm to the lungs which } \\
\text { causes disorders of breathing } \\
\text { and Qi, coughing, extreme } \\
\text { emotional instability, thirst, } \\
\text { and fever }\end{array}$ & $\begin{array}{l}\text { The female lies on her back and } \\
\text { bends her knees making room } \\
\text { for the man between them. }\end{array}$ & $\begin{array}{l}\text { Shallowly, } \\
\text { a half cun. }\end{array}$ \\
\hline $\begin{array}{l}\text { Duomai 奪脈 } \\
\text { Forcing of } \\
\text { the channels }\end{array}$ & $\begin{array}{l}\text { Forced sex without the penis } \\
\text { being sufficiently hard, coer- } \\
\text { cive ejaculation in the middle } \\
\text { of intercourse and sex after } \\
\text { overeating }\end{array}$ & $\begin{array}{l}\text { Exhaustion of Qi, harm to } \\
\text { the spleen, dyspepsia and } \\
\text { impotence }\end{array}$ & $\begin{array}{l}\text { The female lies on her back and } \\
\text { wraps her feet around the male's } \\
\text { buttocks. The male supports } \\
\text { himself with a seat during } \\
\text { penetration. }\end{array}$ & \\
\hline $\begin{array}{l}\text { Qixie 氣泄 } \\
\text { Leakage of } \\
\text { Qi }\end{array}$ & $\begin{array}{l}\text { Sex before the sweat is dry } \\
\text { because of tiredness }\end{array}$ & $\begin{array}{l}\text { Heat in the abdomen and } \\
\text { thirst on the lips }\end{array}$ & $\begin{array}{l}\text { The male lies on his back while } \\
\text { the female rides on him facing } \\
\text { his feet. She supports herself } \\
\text { with a seat during penetration. }\end{array}$ & Shallowly \\
\hline $\begin{array}{l}\text { Jiguan 機關 } \\
\text { (jueshang 厥 } \\
\text { 傷) Fainting } \\
\text { and harm }\end{array}$ & $\begin{array}{l}\text { Sex with those who have } \\
\text { chronic internal ailments or } \\
\text { too soon after defecation/ } \\
\text { urination }\end{array}$ & $\begin{array}{l}\text { Harm to the liver, dim sight, } \\
\text { swellings and impotence }\end{array}$ & $\begin{array}{l}\text { The male lies on his back while } \\
\text { the female rides on him, face to } \\
\text { face. }\end{array}$ & Slowly \\
\hline $\begin{array}{l}\text { Baibi百閉 } \\
\text { Hundred } \\
\text { blockages }\end{array}$ & $\begin{array}{l}\text { Involuntary ejaculation due } \\
\text { to excess of female sexual } \\
\text { desire }\end{array}$ & $\begin{array}{l}\text { No semen at ejaculation } \\
\text { because Qi is exhausted }\end{array}$ & $\begin{array}{l}\text { The male lies on his back while } \\
\text { the female lies upon him sup- } \\
\text { porting herself with a seat. }\end{array}$ & $\begin{array}{l}\text { Let the fe- } \\
\text { male insert } \\
\text { the penis. }\end{array}$ \\
\hline $\begin{array}{l}\text { Xuejie 血竭 } \\
\text { Drying out of } \\
\text { blood }\end{array}$ & $\begin{array}{l}\text { Sex when extremely fatigued } \\
\text { with repeated ejaculation }\end{array}$ & $\begin{array}{l}\text { Drying out of blood, exhaus- } \\
\text { tion of Qi, deterioration of } \\
\text { the skin, pain in the penis, } \\
\text { wetness of the testicles, } \\
\text { emission of blood instead of } \\
\text { semen from the penis }\end{array}$ & $\begin{array}{l}\text { The female lies on her back, } \\
\text { raises her buttocks high and ex- } \\
\text { tends her feet straight. The male } \\
\text { kneels between her knees. }\end{array}$ & Deeply \\
\hline
\end{tabular}






Figure 15.3 'Wang haichao' 望海潮” (Watching the Rising Tide), from Huaying qinzhen 花營錦陣 (Variegated Positions of the Flowery Battle). Printed from an early 17 th-century(?) woodblock. Source: van Gulik [1951] 2004, vol. 2, p. 233

Let us take an example from one of van Gulik's pictorial discoveries, Huaying qinzhen 花營錦陣 (Variegated Positions of the Flowery Battle). Figure 3 is a picture from this collection called Wanghai chao 望海潮 (Watching the Rising Tide). It is identical to the posture Beifeifu 背 飛鳥 (Duck Flying on the Back) (Diagram 1) described in Dongxuan zi 洞玄子 (The Master Dongxuan), and is designated as treatment for the leakage of Qi (see Table 2).

It would appear to be cognate with the posture named 'rabbit sucking fur', from the Xuannü jing玄女經 (The Canon of the Dark Girl), which also involves the woman kneeling astride the male, with her back to him. The description here instructs the man to attack her qinxian 琴弦 (zither strings), which are located at a depth of approximately 1 cun $寸$ from the entrance of the vagina. ${ }^{42}$ Again this appears to correspond to the G-spot. The same location is described in Yufang mijue玉房秘訣 (Secrets of the Jade Chamber) ${ }^{43}$ as a 'spot in which Yin and Yang are harmonised' 44 and the place 'in which Yin falls into



Diagram 15.1 'Beifei fu' 背飛島 (Duck Flying on the Back), based on a description in Dongxuan zi 洞玄子, 'Sanshifa'三十法 (Thirty Sexual Positions). Sketch by Umekawa

trouble'.45 The troubled Yin indicates female orgasm, when the best Yin Qi is produced. Thus we can see how the performative dimension of these images takes shape, where, against the broad background of popular culture, yangsheng and medical knowledge provide the context in which they could be perceived to be effective.

In other examples, a picture named Fenglou chun 鳳 樓春 (Spring in the Phoenix Tower) in The Flowery Battle corresponds to Hai'ouxiang 海鷗翔 (Flying Seagull) in Dongxuan zi, while Queqiao xian 鵲橋仙 (The Immortals of the Magpie Bridge) in The Flowery Battle matches the position called Baibi 百閉 (Hundred Blockages) in Dongxuan $z i$, which heals one of the seven ailments (see Table 2).

In another example of the correspondence between imagery and the texts, Figure 4 appears to depict a position known as Yujie shi 魚唼勢 (Posture of Fishes Kissing) in a sex book called Sunü miaolun 素女妙論 (Precise Theory of the Plain Girl). In the Sunü miaolun, it is described as follows;

Let one of two girls lie on her back, while the other lies on top of her embracing each other as if in intercourse. Where 




Figure 15.4 Fishes Kissing. Anonymous. Source: Bertholet and Yimen [1997] 1998, p. 144

the gates of females press upon each other, they will open naturally like the mouths of fishes. ${ }^{46}$

Of course, superficial correspondence between images and earlier descriptions cannot be taken as a routine proof. Figure 5 is another of the drawings from The Flowery Battle, titled Houting yan 後庭宴 (Celebration in the Backyard). Superficially it might be seen to correspond to Xuannü jing's 'Tiger walking' or the Dongxuan zi 'White Tiger Jumping' (Diagram 2).

White Tiger Jumping: The woman should kneel face downwards. The man should kneel behind her, embrace her waist with his hands, and insert his yujing 玉茾 (Jade Stalk, i.e. the penis) into her zigong 子宮 (Child Palace). ${ }^{47}$

However, Houting yan specifically depicts anal sex. The female's raised leg is a small but probably technically significant difference in the detail of the image, while the name of the posture is extremely close to a sexual position named in Jinping mei as Liting hua 裏庭花 (Flower in the Back Garden) (Fig. 6), in an explicit description of male-female anal sex between two of the novel's key protagonists. This is further re-enforced by the symbolism

46 Sunü miaolun, 1556 text, in van Gulik 1951, vol. II, p. 153.

47 Ishimpō. 28 ('fangnei'), p. 641.



Figure 15.5 Houting yan 後庭宴 (Celebration in the Rear Courtyard), from Huaying qinzhen 花營錦陣 (Variegated Positions of the Flowery Battle). Printed from an early 17 th-century(?) woodblock. Source: van Gulik [1951] 2004, vol. 2, p. 265

of the chrysanthemum in the background of the image, which has been taken as an analogue of the anus. ${ }^{48}$ Like the shunga discussed above, this image was probably designed to induce a humorous as well as erotic reaction. ${ }^{49}$

The Houting yan picture certainly falls into the category of chungong hua, and arguably fangzhong shu, but definitely not yangsheng, which seems to focus exclusively on vaginal penetration. However from our point of view, the most important thing it tells us is that the symbols and details depicted in chungong hua were not arbitrary or random but were indeed composed in relation to a wide range of literary and cultural references. In his study of medicine and literature 'Fictional Medicine: Doctors, Disease and the Curative properties of Chinese Fiction', Andrew Schonebaum observes of the works of this period:

The fact that medical manuals and pamphlets still addressed the supposedly outdated notion of nurturing the essence, even if only to debunk it, shows that the subtext of medical texts was essentially the same as that of erotic literature, and that it was part of the same popular imagination. ${ }^{50}$

From what we have seen above, and many other correspondences, we would suggest that the creators of erotic

See Screech 1999, pp. 151-3.

The story of Aunt Flora in Roupu tuan makes clear that this was indeed a subject of mirth. See Hanan 1996, pp. 264-74.

50 Schonebaum 2004, p. 271. 




Diagram 15.2 'Baihu teng'白虎騰 (White Tiger Jumping), based on a description in Dongxuan zi 洞玄子, 'Sanshifa' 三十法 (Thirty Sexual Positions). Sketch by Umekawa.

illustrations had frequent recourse to the fangzhong shu manuals, as well as to popular stories, for their inspiration. Conversely existing practitioners of the sexual arts would have viewed such pictures as visual confirmation of their knowledge.

\section{Conclusion}

In conclusion, we can say that we do believe, prima facie, that the Chinese chungong hua illustrations represent an extension of fangzhong shu by other means. They were an adaptation to the changed social, technological and commercial situation of their times. Through their focus on realistic positioning and foreplay they show substantial links with fangzhong shu literature and yangsheng sexology texts going back to the Han dynasty, some 1,500 years earlier. The previously mentioned article by Donald Harper on aphrodisiacs and love potions shows that these traditions were multifaceted in that they dealt simultaneously with both sophisticated philosophical concepts and physical desire and that these ideas were transmitted with remarkable consistency over hundreds of years. ${ }^{51}$ Equally, chungong hua can be clearly contrasted with the more explicitly pornographic Japanese shunga of the same era. They are also quite different from the style of literary illustration in which small line figures appear as part of a much wider scene. In Ming China, the discourse of yangsheng to some extent provided the atmosphere which made the large chungong hua illustrations permissible, and certainly it continued to define the way that

$51 \quad$ Harper 2005 .



Figure 15.6 'Liting hua' 裏庭花 (Flower in the Back Garden), scene from Jinping mei, ch. $5^{2}$. Source: Bertholet and Yimen [1997] 1998, p. 108

perceptions of the body and its relationship to the cosmos were conceived. However, the images were competing in a market place with materials whose purpose was simply to stimulate and entertain and thus were subject to a process of commodification ably described elsewhere by both Craig Clunas and Timothy Brook. ${ }^{52}$

Further research needs to be undertaken on the connections between chungong hua and Ming/Qing society and the popular literature of the time and in particular more work is needed on the evolution of fangzhong shu texts themselves which seem to undergo a dramatic transformation during and after the Song dynasty.

\section{Bibliography}

\section{Primary Sources}

Hanshu 漢書, compiled by Ban Gu 班固, c. 100 CE. Modern edn, Beijing: Zhonghua shuju, 1996 .

Ishimpō 醫心方 (Formulas of the Heart of Medicine), compiled by Tamba no Yasuyori 丹波康頼, 984-92. Asakuraya edn, Beijing: Renmin weisheng chubanshe, 1955 .

Jinping Mei 金瓶梅, attributed to Xiaoxiao Sheng 笑笑生. Hong Kong: Sanlian shudian, 1986.

Lun heng 論衡, Wang Chong 王充 (27-97 CE), ed. and tr. M. Kalinowski 2011, Balance des discours: Destin, providence et divination, Paris: Les Belles Lettres.

Shiwen 十問 (Ten Questions). In Mawangdui hanmu boshu zhengli xiaozu 馬王堆漢墓帛書整理小組 (ed.) 1985, Mawangdui Hanmu Boshu 馬王堆漢墓帛書 (The Silk Manuscripts from Mawangdui Han Tombs), Beijing: Wenwu chubanshe, vol. 4.

Sunü miaolun 素女妙論 (Precise Theory of the Plain Girl), attributed to a Daoist of Maoshan sect, with a preface by Zhaihonglou Zhuren 摘紅楼主人 dated 1566. In van Gulik, R.H. 1951, Erotic Colour Prints

$5^{2} \quad$ See Clunas 2004; Brook 1998. 
Tiandi Yinyang Jiaohuan Dalefu 天地陰陽交歓大楽賦, attributed to Bai Xingjian 白行簡. In Ye Dehui 叶德辉 (ed.) 1995, Shuangmei jing'an congshu 双梅影闇从书 (The Collection of Shadows under the Double Plum Trees), Haikou: Hainan guoji xinwen chubanshe.

Zhulin yeshi 株林野史 (The Secret of the Bamboo Grove). Anon. late Ming, early Qing.

Zunsheng bajian 遵生八茂 (Eight Discourses on Respecting Life), 1591, Gao Lian 高濂, 1988, Beijing: Shumu wenxian chubanshe.

\section{Secondary Sources}

Bertholet, F., and Yimen, Rev., 1997, Dreams of Spring Erotic Art in China, Bertholet Collection: Amsterdam, Pepin Press.

Brook, T. 1998, The Confusions of Pleasure: Commerce and Culture in Ming China, Taipei: SMC Publishing Inc.

Clunas, C. 2004, Superfluous Things: Material Culture and Social Status in Early Modern China, Honolulu: University of Hawai'i Press.

Furth, C. 1994, 'Rethinking van Gulik: sexuality and reproduction in traditional Chinese medicine', in Gilmartin et al. (eds), 125-46.

Fukuda Kazuhiko 福田 和彦 1981, Chūgokuno Shunkyūga中国の春宮 画 (Chinese Erotic Art), Tokyo: Haga shoten.

2003, Edo Suhnga no Seiaigaku II - Koushoku no Bi Ishiki 江戸春画の性愛学II一好色の美意識 (Sex and love in Erotic Art during Edo Period II: An Aesthetic Sense in Sexual Desire), Tokyo: KK Besuto Serazu.

Furth, C. 1994, 'Rethinking Van Gulik: sexuality and reproduction in traditional Chinese medicine', in Gilmartin et al. (eds).

Gilmartin, C., G. Hershatter, L. Rofel and T. White (eds) 1994, Engendering China: Women, Culture, and the State, Cambridge, MA: Harvard University Press.

Hanan, P. See Li Yu below.

Harper, D. 2005, 'Chinese recipes for aphrodisiacs and philtres', Asian Medicine: Tradition and Modernity, 1.1, 91-100.

Jin Wenxue 金 文学 2004, Kōshoku to Chūgoku Bunka-Chūgoku no Rekishi ha Yoru Tukurareta 好色と日本文化一中国の歴史は夜作 られた (Love and Sex in Japanese Culture: Chinese Influence on the History of the Night), Saitama: Nihon kyōhōsha.

Kuriyama Shigehisa 栗山茂久 (ed.) 2001, The Imagination of the Body and the History of Bodily Experience (Proceedings of the 15th International Symposium of the International Research Centre for Japanese Studies, Kyoto, 18-22 January, 20oo, Kyoto: International Research Centre for Japanese Studies.

$\mathrm{Li} \mathrm{Yu}$ 李渔, Roupu Tuan 肉蒲団, tr. P. Hanan 1996 as The Carnal Prayer Mat, Honolulu: University of Hawai'i Press.

Liu Dalian 刘达临 2003, Zhongguo xingshi tujian 中国性史图鉴 (Illustrated Handbook of Chinese Sex History), Changchun: Shidai wenyi chubanshe.

Lo V. and C. Cullen (eds) 2005, Medieval Chinese Medicine, London: Routledge Curzon.

Nakano Miyoko 中野 美代 1991, Ryū no Sumu Rando Sukeipu-
Chūgokujin no Kūkan Dezain 龍の住むランドスケープー中国人の 空間デザイン (The Landscape where the Dragon Lives: Chinese Design of Space), Tokyo: Fukutake shoten.

Nakano Miyoko 2001, Rōma Zufu - Chūgoku Shunga Ron Jyosetsu 肉 麻龱譜一中国春画論序説 (Genealogy of Obscene Illustrations: Prolegomena for Chinese Erotic Art), Tokyo: Sakuhin sha.

Needham, J. and Lu Gwei-Djen 1983, Science and Civilisation in China, vol. 5 , pt 5 , Cambridge: CUP.

Okeda Naoto 樋田直人 2001, Chügoku no Nenga-Inori to Kisshō no Hanga 中国の年画一祈りと吉祥の版画 (Lucky Charms in China: Printed Illustrations for Prayer and Luck), Tokyo: Taishūkan shoten.

Sakade Yoshinobu 坂出祥伸 2001, 'Hibikiau shintai' 響きあう身体 (Body in vibration), in Kuriyama Shigehisa 栗山茂久 (ed.), 1-8.

Schonebaum, A.D. 2004, 'Fictional medicine: doctors, disease and the curative properties of Chinese fiction', PHD diss., University of Columbia.

Screech, T., 1999, Sex and Floating World: Erotic images in Japan 1700-1820, London: Reaktion Books.

Shi Chengli 史成礼 et al. (eds) 1999, Dunhuang xing wenhua 敦煌性文化 (Sexual Culture in Dunhuang), Guangzhou: Guangzhou chubanshe.

Shirakura Yoshihiko 白倉敬彦 and Hayakawa Monta 早川聞多 (eds) 2002, Kielletyt Kuvat; Vanhaa Eroottista Taidetta Japanista (Forbidden Images: Erotic art from Japan's Edo period), exhibition catalogue, Helsinki: Helsinki City Art Museum.

Japanese version 2003, Suhnga — Himetaru Waraino Sekai 春画一秘めたる笑いの世界, Tokyo: Yōsensha.

Sterckx, R. 2008, 'The Limits of Illustration: Animalia and Pharmacopeia from Guo Pu to Bencao Gangmu, Asian Medicine, 4. 2, 357-94.

Umekawa, Sumiyo 梅川純代 2004, Sex and immortality - a study of Chinese sexual activities for better-being, PHD diss., School of Oriental and African Studies, University of London.

2005, 'Tiandi Yinyang Jiaohuan and the art of the bedchamber', in Lo and Cullen (eds), 252-77.

Van Gulik, R.H. 1951, Erotic Colour Prints of the Ming, privately published, Tokyo, 1951. Reissued 2004 with introductions by J. Cahill, W.L. Idema and S. Edgren, Leiden: Brill.

1974 [1961], Sexual Life in Ancient China: A Preliminary Survey of Chinese Sex and Society from ca. 1500 BC. till 1644 AD, Leiden: Brill.

Wang Shumin and P. Barrett 2006, 'Profile of a daoyin tradition: the "five animal mimes"', Asian Medicine: Tradition and Modernity $2.2,225-53$.

Wu Cuncun 吴存存 2005, Chügoku Kinseino Seiai-Tanbi to Itsuraku no Oukoku 中国近世の性愛一耽美と逸楽の王国 (Medieval Chinese Eros: Land of Aestheticism and Pleasure), tr. Suzuki Hiroshi 鈴木 博, Tokyo: Seidosha.

Yao Ping 姚平 2009, 'Historicizing Supreme Bliss: the rise of erotica in Tang China', paper presented at The Past, Present and Future of Daoism, Fifth International Daoist Studies Conference, Wudangshan, Hubei, 18-22 June 2009. 


\title{
16 The Vital Role of Illustration in the Literature of Childhood Smallpox
}

\author{
Wan Fang 萬芳
}

In Chinese medicine, diagnosis and treatment are based on the concept of syndrome patterns, ascertained by collating clinical information gained through the four diagnostic methods - looking, listening and smelling, questioning, and feeling the pulses. Paediatrics is no exception to this rule. However, these basic diagnostic principles must be applied in ways that take account of the particular physiological and pathological characteristics of the child. The usefulness of questioning is limited by the child's language skills; and because children do not actively co-operate with the doctor as adults tend to do, it is harder to obtain objective information by taking the pulses. As a result, visual inspection plays the key role in paediatric diagnosis, and is called upon to supplement the other diagnostic methods, where these prove inadequate. This paper seeks to shed light on paediatric diagnosis in Chinese medicine, using examples of the visual diagnosis of childhood smallpox from early illustrated literature. Thanks to the infographic character of this visual material, which is strongly reminiscent of the simple and succinct captions and clear line diagrams of the Tibetan, Chinese and European moxa-cautery charts, it appears to provide a direct window on the ideas and approaches of earlier practitioners, and continues to have reference value for physicians today (See Lo and Tlalim, Chapter 19 in this volume). The diagrams are practical rather than decorative, and can easily be copied and used by non-specialists.

\section{Smallpox}

Paediatric smallpox ${ }^{1}$ has been identified since antiquity as a life-threatening disease, in view of its rapid onset, its virulence, the poor general prognosis, and the very high infant mortality rate in smallpox epidemics. Until the advent of variolation, Chinese medicine was the only available means of combating it. Just how seriously doctors through the ages took this disease is shown by the high percentage of specialised treatises on smallpox found among the extant texts on paediatrics. At the same time,

Xiao'er dou zheng 小兒痘疹. In general the disease categories of Chinese medicine do not correspond exactly to those of Western medicine, however the severe nature of smallpox and the clear visual aspects of the symptoms make this disease a rare exception. this ongoing preoccupation with finding ways of diagnosing and treating smallpox indicates that it was never effectively controlled. Most of the treatises on smallpox draw a typological distinction between cases with a favourable and unfavourable prognosis. If the patient is only mildly affected by pathogenic or malign Qi (xieqi 邪氣), and healthy Qi is present, the course of the disease should run smoothly. Given careful nursing and timely, appropriate medication, the prognosis is favourable. If on the other hand a child who has a naturally weak constitution is infected by virulent malign Qi, it will not be possible to clear this malign Qi, and the patient's condition may become critical at any moment. In cases like this, where a child's life hung in the balance, the doctor bore a heavy burden of responsibility, and rapid and accurate diagnosis was of the essence. As we will see different typologies emerged to differentiate the severity and character of dou zheng 痘疹 (smallpox), and to help in distinguishing it from visually similar conditions. What doctors desperately needed was a well thought-out plan of diagnosis and treatment, and an ever-expanding corpus of medical literature was produced to meet this need. Moreover as the condition of the patient deteriorated, serious complications might appear, leading to organ failure and death. The appearance of the lesions, their colour, their density and distribution pattern, and the presence or absence of vesicular fluid were precious diagnostic clues, which, properly understood held the key to saving lives. Thus the ancient medical writings on smallpox contain numerous illustrations of smallpox lesions, which were of far greater practical use to practitioners than verbal description alone. For these reasons, most of the Ming and Qing Dynasty literature on smallpox is illustrated. The illustrations exemplify the fusion of theory and practice characteristic of Chinese medicine. They represent a crucial point of departure for current research into ancient medical literature, and will amply repay further study. This paper will discuss the significance of medical illustration for the visual diagnosis and typological differentiation of smallpox with special reference to Yulinzhishiyusuizhaiyao 毓麟芝 室玉髓摘要 (Yulinzhishi Chalcedony Digest). 


\section{'Yulinzhishi Chalcedony Digest'}

Yulinzhishi Chalcedony Digest is a partial abridgement of Michuan douzhenyusui 秘傳痘疹玉髓 (Secret Chalcedony Book of Smallpox), attributed to the Yuan physician 黃石 峰. The only known exemplar of the latter is a facsimile copy held in the library of the Chinese Academy of Sciences in Beijing, based on the Ming edition of Yu Xiufen 餘秀峰 published by Jianyi shulin 建邑書林. Secret Chalcedony Book of Smallpox is a monograph in three juan (fascicles). The exact date of composition is unknown. It deals with the aetiology of smallpox, common types of the disease, diagnosis, principles of treatment, and favourable and unfavourable prognoses. It presents 18 medical recipes, making use of 21 separate drugs. It summarises the teachings on smallpox of various eminent physicians, including Zhang Yinbing shi wen 張寅賓十問 (Zhang Yinbing's Ten Questions), Tian Chen lun 錢陳論 (Teachings of Tian Chen), Li Yunyang yongyao shiba bian 李雲陽用藥十八辨 $(\mathrm{Li}$ Yunyang's Eight Criteria for Drug Treatment). The third volume (juan) contains 46 illustrations. The first nine of these show nine types of smallpox that pose particular problems of identification, while the remainder illustrate varieties of smallpox in relation to the channels and the five viscera.

Yulinzhishi Chalcedony Digest itself is is ascribed to the Ming author Peng Duanwu 彭端吾, and was probably first published in 1549. It exists in two- and three-juan formats and circulated both as a self-standing work and in a composite edition called Douzhen quanshu 痘疹全書 (Smallpox Compendium), which also included Douzhen shiyixinfa 痘疹世醫心法 (Knowledge and Skills of Smallpox Culled from the Experience of Hereditary Physicians), a Ming compendium of prescriptions by famous doctors. Numerous examples of both versions survive, so one can assume that it was a well-known text. It is especially important as only a small number of earlier monographs on smallpox are extant.

The last juan bears the self-explanatory heading Yulinzhishi douzhen yusui tuxiang zhujie 毓麟芝室痘疹 玉髓圖像注解 (Annotated Illustrations to the Yulinzhishi Smallpox Chalcedony). Many of the illustrations correspond to those in the facsimile copy of Secret Chalcedony Book of Smallpox, making it a rare and precious source of visual data for pre-Ming times. They show the appearance, location and distribution of the smallpox lesions in diagrammatic form, with explanatory notes and comments on the same page, enabling the reader to take in the relevant information at a glance, and minimising ambiguity. The usefulness of this text as a practical guide speaks for itself. It will be discussed in greater detail below.

\section{Illustrations from the 'Yulinzhishi Chalcedony Digest'}

\section{The Nine Unrecognised Types of Smallpox}

The final volume of the Yulinzhishi Chalcedony Digest contains 76 illustrations, which convey information about various types of smallpox and smallpox treatments through a combination of graphics and text. It begins with 'Nine Unrecognised Types of Smallpox'. Each is given a name according to the location, appearance and course of development of the lesions. On the same page as the diagram, therapeutic principles and the rationale for favourable and unfavourable prognostication are explained in words. The prognosis is also discussed in terms of the channels of the five zang-organs. Graphics and text are closely integrated, so as to aid comprehension. The material on the 'Nine Unrecognised' types of smallpox is extracted from the Mysterious Smallpox Chalcedony; however the expression 'nine unrecognised' (jiu bu shi 九不識) is not found in that work. The author of Yulinzhishi Chalcedony Digest apparently coined the term to highlight the fact that the lesions were easily confused with rashes and other conditions having a superficially similar presentation. He warns the reader strongly of the dangers of misdiagnosis.

The entry on 'Suspended Mirror pox' is typical:

Xuanjing dou 懸鏡痘 (Suspended Mirror pox): These pox run in the opposite direction from the three Yin channels, and are concentrated around the five Transport Points (wushu 五俞). [Symptoms are] agitation, delirium, raging thirst, vomiting, and burning fever. The lesions resemble abscesses on the back. This is unrecognised smallpox no. 1. Chant of Suspended Mirror pox: Suspended Mirror pox presents like abscesses on the back. Do not use the knife or needle, or give drugs haphazardly. If a draught to clear the Liver is given, the danger will resolve of its own accord, the flowers will bloom as of old, and fresh red matter will be vomited.

Note: All too many hereditary doctors are not conversant with the channels. They misdiagnose Suspended Mirror pox, and cause death through error. If acupuncture needles or stone needles are applied, disastrous consequences will immediately be seen. [The patient] will shortly enter the nether world, having perished of smallpox.

The remaining eight 'unrecognised' types are: xiezi dou 蠍 子痘 (Scorpion pox), occurring around the Liver channel; fufu dou (Cauldron-lid pox), on the top of the head where the Yang channels meet; suojing dou 鎖井痘 (Locked Well pox), concentrated on the Spleen channel; panshe dou 盤 蛇痘 (Coiled Snake pox) concealed within the Lung channel; huanhu dou 䅈虎痘 (Tiger-feeding pox), affecting the Spleen and Stomach [channels] and forming a ring around the navel; xuanqiu dou 玄丘痘(Dark Tumulus pox), going 




Figure 16.1 Suspended Mirror pox. Yulinzhishi yusui zhaiyao 毓麟芝 室玉髓摘要 (Yulinzhishi Chalcedony Digest). (C) Library of China Academy of Chinese Medical Sciences

via the Heart to the Kidney [channels], with the poisons concentrated in the penis; shigu dou 師姑痘 (Master and Maid pox), going via the Yangming channel to the armpits, where it is concentrated; and juan'e dou 卷阿痘 (Coiled Mound pox), going via the Spleen channel, with the poisons concentrated on the Yangming channel.

A separate illustration is provided for each type of smallpox. As in the example above, this is accompanied by mnemonic rhymed couplets and explanatory notes. The appearance of the lesions is described through metaphor and simile, and the visible symptoms are briefly and clearly summarised. The writer emphasises that incorrect diagnosis will result in improper treatment, with fatal consequences. In classifying and discussing the 'Nine Unrecognised' types of smallpox in relation to the five zang-viscera, the writer seeks to differentiate them from other conditions with a similar presentation. Thus, he begins by describing the characteristic external appearance of the lesions and their location and distribution so as to assist in identification, and then proceeds to list easily confused conditions: differential diagnosis comes first, and methods of treatment take second place. The same emphasis on differential diagnosis is seen in the illustrations. The author's approach is both detailed and rigorous:

Suspended Mirror pox: this must be carefully distinguished from ulcers on the back (Fig. 1).

Scorpion pox: this must be carefully distinguished from malign abscesses (Fig. 2).

Cauldron-lid pox: this must be carefully distinguished from ringworm (Fig. 2).

Locked Well pox: this must be carefully distinguished from mouth ulcers (Fig. 3).

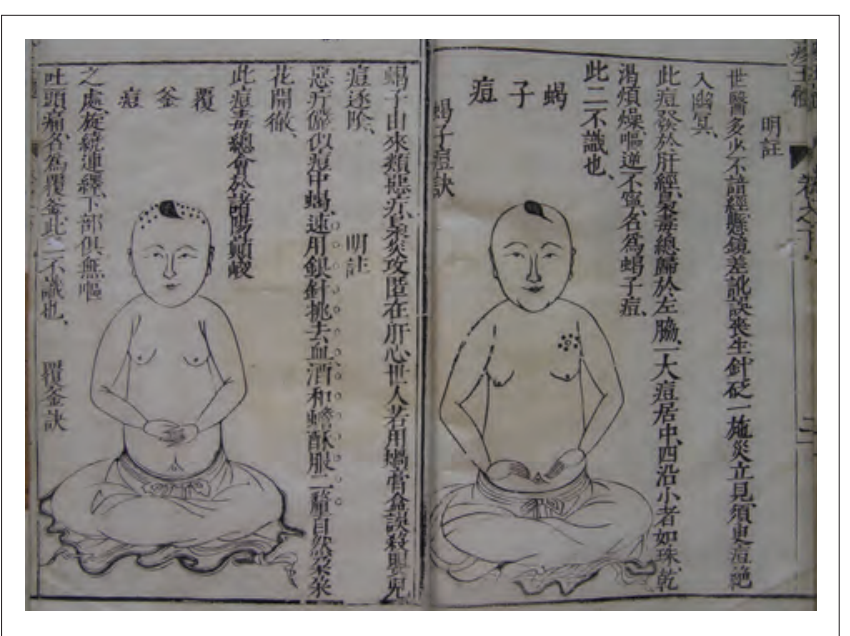

Figure 16.2 Scorpion pox (right-hand page); Cauldron-lid pox (lefthand page). Yulinzhishiyusui zhaiyao 毓麟芝室玉髓摘 要 (Yulinzhishi Chalcedony Digest). (C) Library of China Academy of Chinese Medical Sciences

Coiled Snake pox: This must be carefully distinguished from scrofula (Fig. 3).

Tiger-feeding pox: this must be carefully distinguished from gan syndrome (an infantile condition involving failure to thrive) (Fig. 4).

Dark Tumulus pox: this must be carefully distinguished from syphilitic sores (Fig. 4).

Master and Maid pox: This must be carefully distinguished from banal sores and ulcers.

Coiled Mound pox: This must be carefully distinguished from banal sores and ulcers.

Were it not for the illustrations, one can easily appreciate that practitioners consulting the text would would have been unable to form a clear impression of the appearance,



Figure 16.3 Locked Well pox (right-hand page); Coiled Snake pox (left-hand page). Yulinzhishiyusui zhaiyao 毓麟芝室玉 髓摘要 (Yulinzhishi Chalcedony Digest). ( ) Library of China Academy of Chinese Medical Sciences 




Figure 16.4 Tiger-feeding pox (right-hand page); Dark Tumulus pox (left-hand page). Yulinzhishi yusui zhaiyao 毓麟芝室玉 髓摘要 (Yulinzhishi Chalcedony Digest). (C) Library of China Academy of Chinese Medical Sciences

and distribution patterns of the lesions, or to distinguish them reliably from other conditions mimicking smallpox. They would not have been in a position to make a prompt and accurate diagnosis, and might misinterpret the disease process at work. For the patient, this could mean the difference between life and death. It is evident then that illustration assumed a particular significance in medical texts on smallpox.

\section{Illustrations of Auspicious and Inauspicious Smallpox}

As we have seen, the section on the 'Nine Unrecognised' types of pox focuses on differential diagnosis, and discusses treatment in light of this. The subsequent section deals with favourable and unfavourable prognosis in relation to the five zang viscera. Emphasis is laid on the disease mechanisms and transformative processes involved, which provide the criteria for treatment. Prognostication according to the five viscera is a complex subject involving constantly changing dynamic factors; dangerous symptoms or deterioration in the patient's condition might set in unexpectedly. Accurate and timely observation was the key to a correct treatment plan, and in this, the charts of favourable and unfavourable prognostic signs played a vital guiding role.

In this book, the observation of smallpox cases in relation to the five zang-viscera is discussed in terms of the Keys to Fire, Wood, Metal, Earth and Water (qihuoyao 啟 火錀, qimuyao 啟木錀, qijiinyao 啟金錀, qituyao 啟土錀, qishuiyao 啟水錀). The need for precise, targeted monitoring is strongly emphasised; generic inspection is not sufficient. In keeping with the literary style of the text, each chapter opens with a series of rhyming formulas, setting


Figure 16.5 (left)The Keys to Fire; Figure 16.6 (right) Auspicious smallpox. Yulinzhishiyusui zhaiyao 毓麟芝室玉髓摘 要 (Yulinzhishi Chalcedony Digest). @ Library of China Academy of Chinese Medical Sciences

out the formation, colouration, distribution and location of lesions indicating an auspicious or inauspicious prognosis in relation to one of the viscera. This is followed by an analysis of the implications of auspicious and inauspicious prognosis, and an account of therapeutic principles. For reasons of space, I give just a few examples here.

The Keys to Fire (Fig. 5):

The cheekbones are the location for the Heart channel. If within three days vesicles have formed on the cheekbones, it is a sign of recovery.

Song mountain is the nose; the five [holy] mountains are within it. The smallpox of the five mountains stick out like pomegranate (seeds), whereas smallpox on the hands and feet looks more pleasant (Fig. 6).

Where the area above the cheekbones is covered in vesicles, but vesicles do not form on the lower body, no harm will ensue (Fig. 7, right-hand page).

Where the area from the buttocks downwards is covered in vesicles, but no vesicles form above this, it needs to be treated quickly; delay is fatal (Fig. 7, left-hand page).

Boils known as 'roller blind' develop under the tongue. The tongue is the sprout of the Heart; this is an outbreak of virulent, malign poisons in the Heart channel. Recovery is possible if the Fire in the Heart channel can be subdued; delay in giving treatment will be fatal.

The Keys to Wood:

The eye is the pass of the Liver; if it is purplish black, [this means that] it has been attacked by virulent poisons, and the wood of the Liver has been damaged. This is fatal.

The Keys to Metal:

The forehead is a location belonging to the Lungs. Pox resembling split soybeans are an auspicious sign. The pavilion of the forehead and nose [in physiognomy] has three divisions. It is best of all if the lesions have a pleasant appearance. 


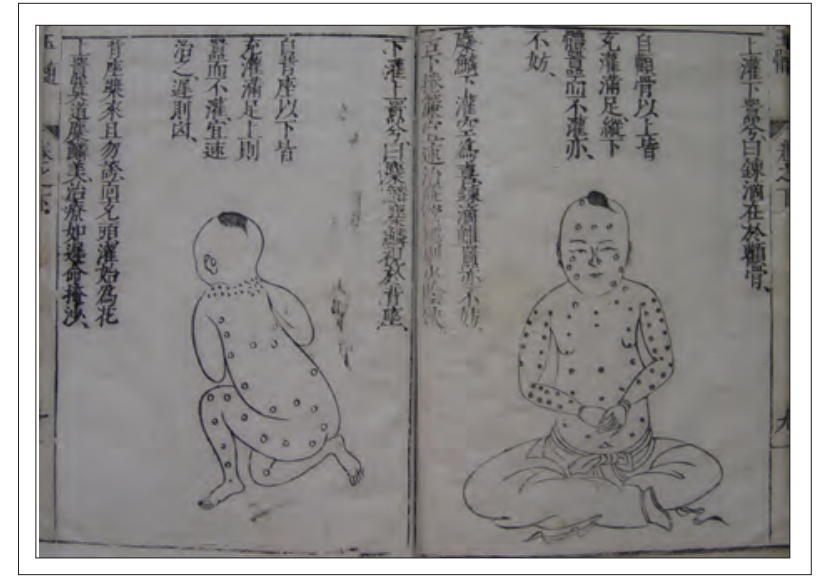

Figure 16.7 Illustration of inauspicious smallpox. Yulinzhishiyusui zhaiyao 毓麟芝室玉髓摘要 (Yulinzhishi Chalcedony Digest). (c) Library of China Academy of Chinese Medical Sciences

The Keys to Earth (Fig. 8, right-hand page):

The upper and lower lips are both restricted; the pox are like a lock, so that the patient cannot feed, leading to death. With emergency treatment, survival is possible.

The Keys to Water (Fig. 9):

The lesions should not appear first on the Pavilion of Earth [i.e. the lower part of the face?], because this is the axis of the kidney. If they first appear here, the pox will be ashen-black and sunken.

As noted at the outset, diagnostics in Chinese medicine is based on a synthesis of four diagnostic methods. With adult patients, practitioners rely primarily on pulse-taking and questioning, complemented by visual inspection, and listening and smelling. But in the literature on childhood smallpox, the emphasis is different. Here, visual diagnosis



Figure 16.8 The Keys to Earth. Yulinzhishi yusui zhaiyao 艈麟芝室 玉髓摘要 (Yulinzhishi Chalcedony Digest). (c) Library of China Academy of Chinese Medical Sciences

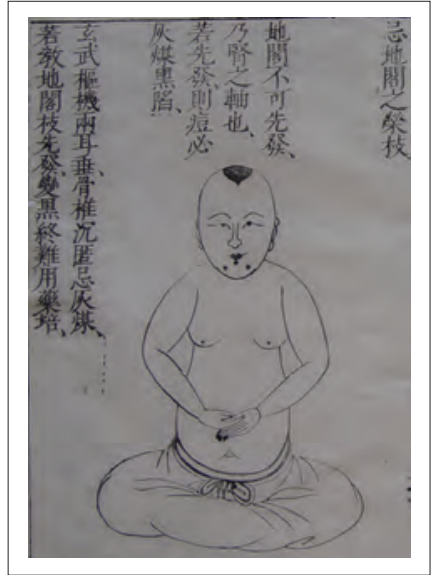

Figure 16.9 The Keys to Water. Yulinzhishi yusui zhaiyao 艈麟芝室 玉髓摘要 (Yulinzhishi Chalcedony Digest). (C) Library of China Academy of Chinese Medical Sciences

is treated in great detail with the aid of copious illustrations, while the remaining three diagnostic methods are relegated to a supporting role, and pulse diagnosis is scarcely discussed at all. This is true of the early literature of smallpox in general. This analysis of childhood smallpox exemplifies wider truths about visual images in medicine: where there are difficulties in communicating verbally (a child, an animal, somebody unconscious), they play a critical role in diagnosis (See Buell et al., Chapter 22 in this volume). Additionally visual keys to typologies take on greater importance where a disorder (here smallpox) is both widespread and dangerously acute, and comes in multiple manifestations that can easily be confused with other, less acute, complaints. From these considerations, it can be deduced that the diagnosis of smallpox was mainly dependent on inspection and questioning, with inspection having pride of place. Almost all the illustrations serve to show the appearance of the lesions, i.e. they are geared towards visual examination, and as such they provided irreplaceable data for diagnosis and prognosis. Because of this, illustrated medical texts on smallpox had a major impact on the diagnosis of the disease. At a time when China was swept by devastating epidemics of smallpox with a very high mortality rate, these illustrations were instrumental in saving many lives.

Today, they are an invaluable resource for research into the history of the prevention, diagnosis and treatment of smallpox.Throughout the Ming and Qing periods, medical texts on smallpox used words and pictures in tandem, and much of the visual material therein was derived from Yulinzhishi Chalcedony Digest. The rise of illustrated monographs reflects the fact that physicians were becoming increasingly conscious of the strengths of the visual medium, and its power to convey information that could 
not be communicated in words. In the literature of smallpox, illustration proved effective in transmitting medical knowledge in a direct and immediately intelligible way.

\section{Bibliography}

\section{Primary Sources}

Douzhen quanshu 痘疹全書 (Smallpox Compendium). Douzhen shiyixinfa 痘疹世醫心法 (Knowledge and Skills of Smallpox Culled from the Experience of Hereditary Physicians) (Ming compendium).

Huang Shifeng 黃石峰(Yuan) Michuan douzhen yusui 秘傳痘疹玉髓 (Secret Chalcedony Book of Smallpox. Edition: Yu Xiufen 余秀峰 ed. (Ming) Jianyi shulin 建邑書林. Facsimile held at Chinese Academy of Sciences.

Li Yunyang yongyao shiba bian 李雲陽用藥十八辨 (Li Yunyang's Eight Criteria for Drug Treatment).

Tian Chen lun 錢陳論 (Teachings of Tian Chen).

Yulinzhishiyusuizhaiyao 毓麟芝室玉髓摘要 (Yulinzhishi Chalcedony Digest).

Zhang Yinbing shiwen 張寅賓十問 (Zhang Yinbing's Ten Questions). 


\title{
17 Picturing Medicine in Daily life: Court and Commoner Perspectives in Song Era Paintings
}

\author{
TJ Hinrichs*
}



Figure 17.1 Hancheng tomb, north wall mural. $\odot$ TJ Hinrichs

Three paintings of medical activity survive from the Song period: ${ }^{1}$

1) a mural depicting a medicinal production workshop from a c. 1070 tomb, recently discovered in Hancheng 韓 城, Shaanxi (Fig. 1);

2) the scroll Qingming shanghe tu 清明上河圖 ('Qingming on the River'), which includes two pharmacies,

* For this chapter, I owe debts of gratitude to Chen Hao 陳吴, for arranging for me to visit the Hancheng tomb; to Li Jugang 李舉 鋼 of the Shaanxi Archaeological Institute for accompanying us to the tomb installation and answering my questions; to An-yi Pan for guidance on Song art history, especially on the Qingming scroll; to Jeehee Hong for introducing me to middle-period art and archaeology, for collaborating with me on an earlier paper on the Hancheng tomb as a whole (see Hong and Hinrichs 2015); to Maria Fernandes, Durba Ghosh, Rachel Prentice, Sara Pritchard, and Marina Welker for detailed comments on an earlier version of the paper; and to an anonymous reviewer. We know the existence of one other painting listed as Village Doctor (Cunyi 村醫), no longer extant, by Chen Tan 陳坦 (Northern Song). See Deng Qiaobin 2005, p. 125. most likely produced at Huizong's 徽宗 court (r. 1101-25), and attributed to Zhang Zeduan 張擇端 (1085-1145) (Fig. 2);

3) another court painting,Jiuaitu炎艾圖 (Moxibustion), showing an itinerant doctor treating a patient in a rural setting, probably produced under Gaozong 高宗 (r. 1127-63), and attributed to Li Tang 李唐 (c.1050-1130) (Fig. 3).

The patrons of each painting - respectively a provincial pharmaceuticals merchant and his family, Huizong's Northern Song court, and Gaozong's Southern Song court - specifically commissioned objects that would visually celebrate the accomplishments of the sponsors. In each case, those achievements entailed the activation of positive social relations and joint communal activities productive of medical healing. This paper explores and compares the ways in which each painting idealises medical practice in social context, and in so doing affirms the efficacy and authority of the Hancheng merchant and of the two Song emperors.

Before examining each painting in turn, it is worth surveying what the three paintings share beyond the theme of medicine - what makes their comparison (as opposed to mere juxtaposition) useful to their analysis. 


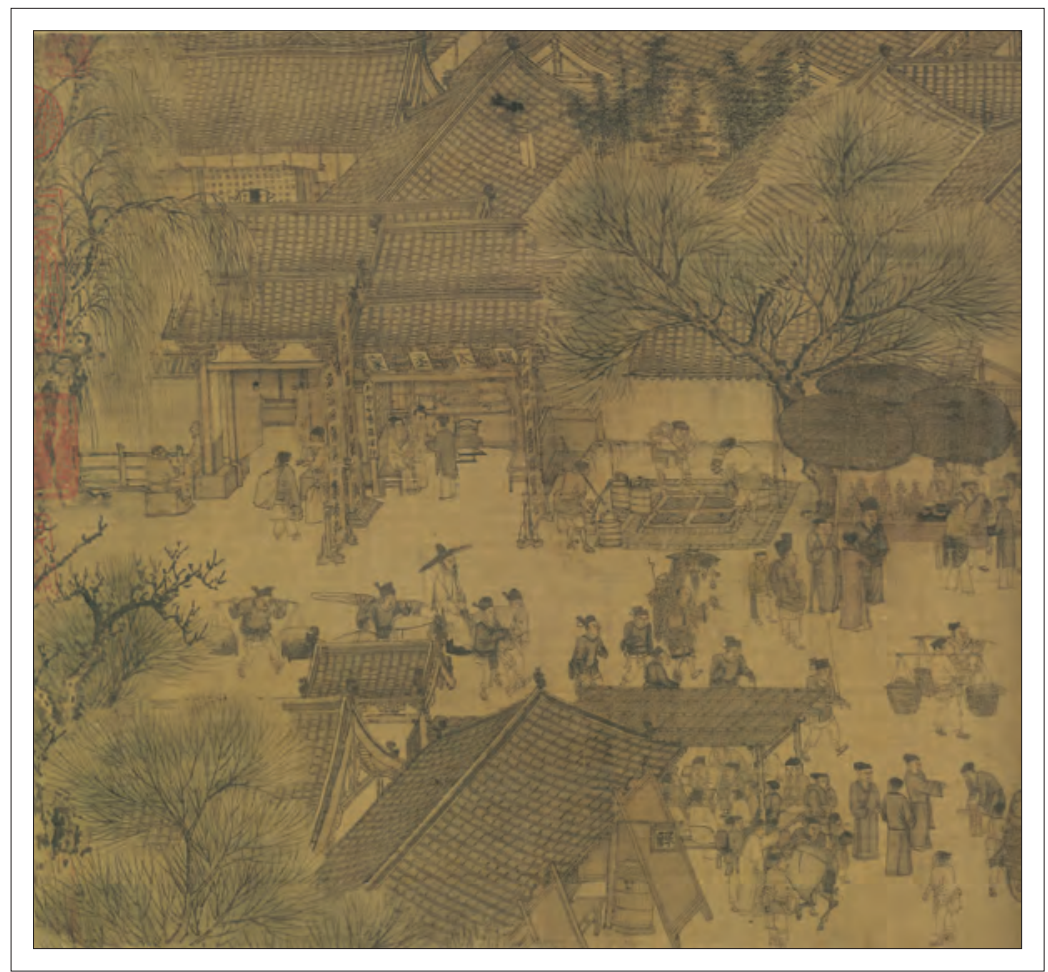

Figure 17.2 Street Scene with Pharmacy from Qingming on the River, attributed to Zhang Zeduan (1085-1145). $\odot$ Courtesy of Palace Museum, Beijing



Figure 17.3 Moxibustion, attributed to Li Tang (c.1050-after 1130). ${ }^{\odot}$ Courtesy of The Collection of the National Palace Museum, Taipei 



First, each painting framed the patron's accomplishments in relation to social ideals of medical production and practice, distilled those achievements into vignettes, then translated key moments of those episodes into a visual mode, and did so in accord with the contemporary styles, respectively, of tomb mural and court 'commoner customs' paintings. While the disparate contexts of merchant burials and courtly displays seem unlikely to share common conventions, in all three the painters portray complex social situations that generate interpersonal vitality. Where, in a landscape painting, our eye might trace flows of Qi through clouds, rocks, and trees, in these paintings we can follow interactions amongst pairs and groups of people: the paintings signal interpersonal engagement not only through involvement in common activities, but through such techniques as portraying affect (most dramatic and vivid in Moxibustion), and eye contact between participants (Fig. 4).

Second, all three paintings are sites for the interpenetration of official and non-official medical activities. In the first painting, we find the state contributing to commercial enterprise with one of its medical publications: the tomb mural prominently features a medical book commissioned by Emperor Taizong 太宗 (r. 976-97), Taiping shenghuifang 太平聖惠方 (the Taiping ['Great Peace' Era] Formulas of Sagely Grace, $992 \mathrm{CE}$ ) (see Fig. 1). The two court paintings, like others of 'commoner customs', pay particular tribute to the court's ability to establish and inculcate good customs and mores amongst the populace - here healing practices productive not only of therapeutic but of social and moral efficacy.
Figure 17.4 Eye contact: a. (top left) detail of Fig. 1. $\odot$ TJ Hinrichs;

b. (below) detail of Fig. 2. $\odot$ Courtesy of Palace Museum, Beijing; c. (bottom right) detail of Fig. $3 . \odot$ Courtesy of National Palace Museum, Taipei



These intersections of state, society, and medicine are not surprising if we consider the era that produced them. Over the long arc of imperial Chinese medical history, the Song (96o-1279 CE) is distinguished by great government projects of medical book production and distribution, including the Taiping Era Formulas; by major augmentations of the medical bureaucracy, of which we see indications in the Qingming scroll; and by innovative campaigns to reform southern healing customs, to bring them into line with norms similar to those portrayed in Moxibustion. ${ }^{2}$ Examining each painting in turn gives us small windows that open onto differently-positioned and shifting ideals of medical, social, and imperial efficacy and authority (Fig. 5).



Figure 17.5 Map of painting production sites in the Song era c. 1070-1100 CE. Map created by Michael Castos

2 For an overview of Song medical policies, with illustrations that include the three paintings discussed in this chapter, see Hinrichs and Barnes 2013, pp. 113-29. 


\section{A Commoner Image of Pharmaceutical Production}

Besides activist state policies, the Song era is notable for a dramatic expansion of commerce, and consequently for the growth of a class of affluent non-literati merchants and landholders. ${ }^{3}$ The additional relaxation of earlier restrictions on commoner burials and death rites led to the emergence of lavishly decorated commoner tombs such as the one found at Hancheng. The Hancheng tomb's centrepiece, the mural on its north wall, also attests to one of the avenues to commoner wealth, pharmaceutical trade, and the contribution of a government publication, the Taiping Era Formulas, to that enterprise. However the occupant came by the book, it was obviously a prized possession, and it was an expensive one: if the occupant possessed the book in its entire 100-chapter edition, it might have cost about as much as the state paid its mid-level military men in three years. ${ }^{4}$

In most ways, the tomb is typical of other north China burials of the time. It was discovered in March 2009 in the course of a Hancheng Municipal Mining Bureau project, and was excavated, encased in concrete, and removed by the Shaanxi Province Archaeological Research Institute. ${ }^{5}$ The tomb is south-facing, $2.45 \mathrm{~m}$ long, $1.8 \mathrm{~m}$ wide, and $2.25 \mathrm{~m}$ tall, the tomb opening just large enough for entry by a single person. Inside the chamber, archaeologists found the skeletons of a $1.8 \mathrm{~m}$-tall man and a $1.65 \mathrm{~m}$-tall woman, heads oriented to the north, laid out side by side on a brick platform topped by a wooden couch carved with peony designs. The woman occupant held coins in her

For a discussion of those historical shifts and their effects on medicine, see Hinrichs and Barnes 2013, pp. 115-18. For an overview of the economic transition, see Shiba Yoshinobu 1970. On commercial markets, a typical book ran 5 o cash/juan. If this edition of the Taiping Era Formulas was typical, it probably sold for around 5,00o cash. For book prices and cost of living figures, see Cheng Minsheng 2008, pp. 372-3, 567, 570.

Nothing in the tomb tells us how the occupant acquired the book; whether he possessed the entire 100 chapters or just a portion, whether he had purchased it outright on the market, whether it had been gifted to him, whether he had stolen it, or whether he had personally copied it, as Taizong intended, at the local prefectural office. An anonymous reader suggested that the occupant may have been a functionary in the medical bureaucracy. Again, nothing in the mural suggests that he currently was or formerly had been a medical official, but some medical officials are known to have set up commercial pharmacy shops both in the capital and in their own hometowns. For examples, see discussion of Qingming Scroll below.

5 Hancheng borders the west side of the Yellow River in the northeast of the Guanzhong plain, roughly $240 \mathrm{~km}$ north-east of Xi'an.

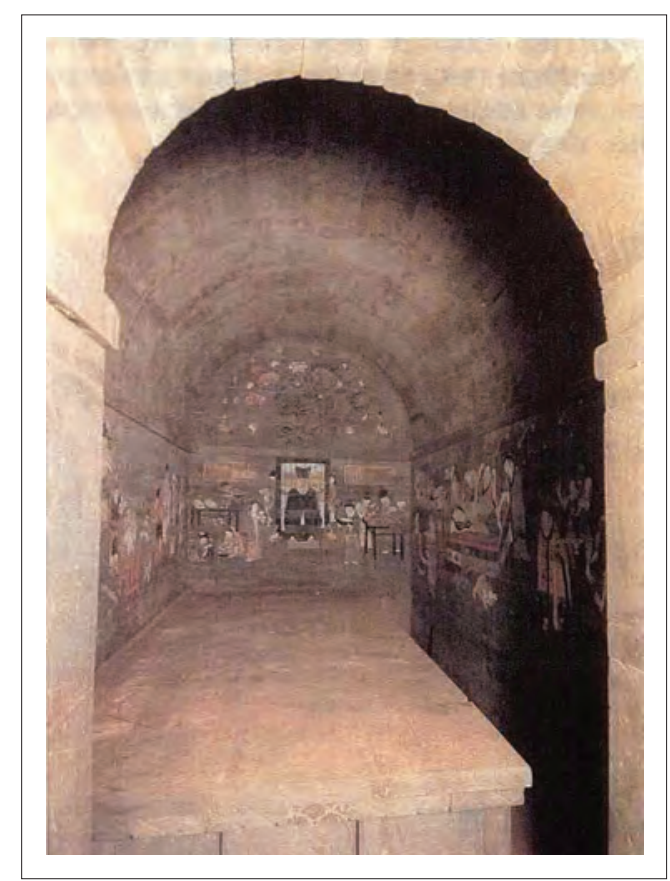

Figure 17.6 Hancheng tomb chamber. ${ }^{\circledR}$ Courtesy of Shaanxi Provincial Institute of Archaeology

hand marked 'Xining yuanbao' 熙寧元寶 (yuanbao was a currency unit), suggesting that she was interred during or not long after the Xining period (1068-77), hence the $c$. 1070 date (Fig. 6).

Two features depart from contemporary Han-Chinese burial practice, causing archaeologists to speculate that the occupants were of west Asian ethnicity. Both wore coarse clothing, and had what reports described as 'red' (hong 紅) or 'yellow' (huang 黃) hair coiled in buns on their heads. Furthermore, other than the coins found in the woman's hand, the tomb, which had remained sealed until its discovery (so the contents should have been intact), contained no grave goods. ${ }^{6}$ Nevertheless, nothing else in the tomb distinguishes it from ethnic-Han practice, and in the absence of other evidence suggesting the relevance of ethnic considerations, the tomb is best read in relation to $c$. 1070 CE north-Chinese burial culture.

As in other tomb murals of the era, many of which show happy domestic scenes and lively entertainments, part of the Hancheng tomb murals' artistry lies in their emotional expressiveness and affective impact. Someone entering the tomb would be confronted first, in ritually central position on the main north wall, with the dynamic and focused activity of the aforementioned pharmaceuticals production workshop. In its midst, they would find the male occupant, seated square and still in an iconic posture of authority. As a man of central importance to the

$6 \quad$ Kang Baocheng and Sun Bingjun 2oog, pp. 79-8o. 


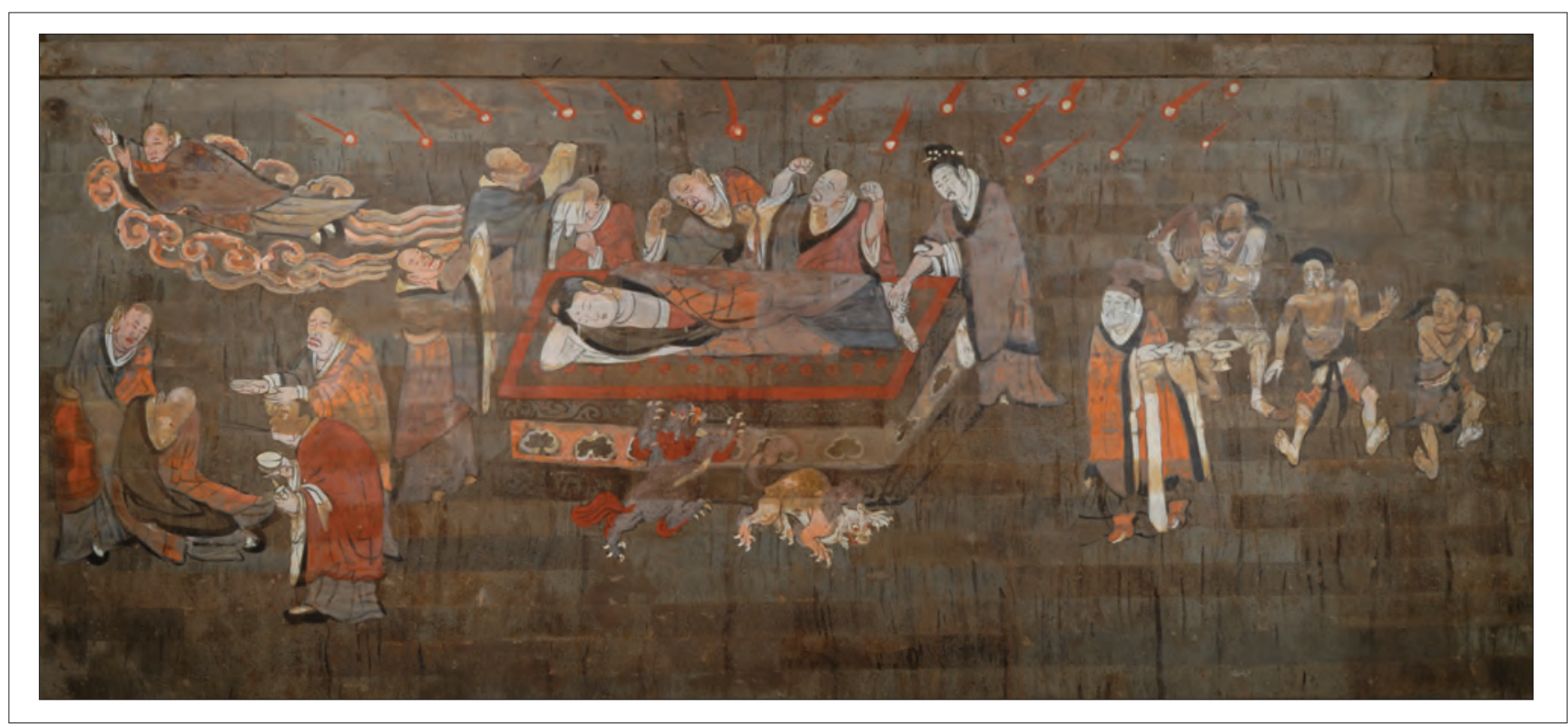

Figure 17.7 Hancheng tomb, east wall mural. Photograph Jeehee Hong

painting and with a status higher than that of the other figures, he is painted larger than the surrounding people. Echoing his calm, an elegant Taihu rock, peony, and crane composition adorns the arch-space above. Turning to the east wall, the viewer is struck by the emotional extremities of mourning. Like the north wall's main figure, the central figure of the reclining, departed Buddha is placid, but his overwhelmed disciples grimace, contort their bodies, beat their chests, flail their arms, and faint in paroxysms of grief. Balancing the lamentations of the eastern wall, the west wall mural of a variety show (zaju 雜劇) evokes raucous mirth, here centring on comic performers surrounded by accompanying musicians (Figs $7-8)^{7}$



Figure 17.8 Hancheng tomb, west wall mural. Photograph Jeehee Hong

$7 \quad$ For a detailed analysis of the spatial orientation of the murals in relation to the occupants and to each other, and of the tomb and its contents in relation to other contemporary tombs, see Hong and Hinrichs 2015. For identifications of the figures in the nirvana mural and of the comic actor types in the theatrical mural, also see Kang Baocheng and Sun Bingjun 20o9, pp. 84-7. 




Figure 17.9 Hancheng Tomb, north wall, left side, detail of Fig. 1. ( ) TJ Hinrichs

In the main mural on the north wall, just to the occupant's right, a young woman offers a tea cup, perhaps containing refreshment, but given the context, possibly an herbal decoction for him to test (Fig. 9). Behind her a pair of young men squat on the floor, one grinding medicines with a mortar and pestle, the other sifting the ground herbs into a larger dish. At their back, a rectangular table bears two thick bookcases, brushes slotted into a brush rack, an ink slab, and bottles, presumably for water to make ink. Behind the table, a young man carries a large dish covered with sprays of herbs. In eye contact with him is a man walking out from behind the screen with a cloth draped over his arm and carrying another dish. From the other side of the screen, a young female servant emerges holding a round fan. ${ }^{8}$

As in many portraits from this period and later, a screen provides a backdrop to the occupant. This screen is decorated with a poem in grass script, the first stanza of which expresses a peaceful vision of meditation in a Buddhist temple:

古寺青松老 The ancient temple's verdant pines, so venerable;

高僧白髮長 The eminent monk's white hair, so long.

夜深禪定後 In the depth of night after meditation is fixed,

8 The image of people emerging from out of doorways is found in Han period tombs. Song tombs saw a revival of this and other Han era motifs, which Wu Hung connects to movements among literati to 'follow ancient models'; in the literati case, this entailed austere tombs, but among 'rich landowners in inland regions', it found expression in complex murals and reliefs. Wu Hung 2010, p. 192.
明 $X \times$ 如霜 Bright $X \times$ like frost,

要 $X X X$ Will $X X X X$.

The banal phrasing of this stanza suggests that the author was probably not a literatus, and was perhaps the occupant himself. Nevertheless, whatever its authorship, the poem shows a reaching for literatus-like sensibilities and literary attainments. The second stanza describes unfettered travel through a divine Daoist landscape, and comes from a poem by Lü Dongbin 呂洞賓 (8th century):

琴劍 \{酒棋龍 $\}$ 鶴虎 Zither and sword, \{wine and chess\}, crane, and tiger.

逍遙\{落\}托永無愁 (憂) Freely roaming, dropping out and begging off, forever without worries.

\{閒\}騎白鹿游三島 $\quad$ \{Idly\} riding a white deer, roaming to the Three Isles [of Transcendants].

悶駕青 $\{$ 牛看\}九州 (十洲) Blithely harnessing blue \{oxen to see\} the Nine Provinces (Ten Continents). ${ }^{9}$

By the Northern Song, Lü Dongbin's poetry was circulating widely. He had also become associated in literature with alchemical pursuits, and was an object of worship popular with drug traders, among others. ${ }^{10}$

And the occupant does seem to have been a drug trader. ${ }^{11}$ To the occupant's immediate left stands a boy presenting

The words inside ' \{\} ' curly brackets are illegible in the mural, and indicate substitutions from an extant version of the poem; the words inside '()' round brackets are the extant versions of the words underlined. Note that "Nine Provinces" refers to Yu the Great's political division of the realm into administrative territories, while 'Ten Continents' (from the extant version of Lü's poem) is another reference to the world of transcendants. Given the instabilities of textual transmission, it is impossible to know to know whether the tomb mural's version is quoting from or modifying then-circulating models.

The transcription of the Hancheng poem is by Chen Yongzheng 陈永正 of Zhongshan University. See Kang Baocheng and Sun Bingjun 2009, p. 80. For an extant version of Lü's poem, see 'Qiyan' 七言, Quan Tang shi 全唐詩 (Beijing: Zhonghua shuju, 1960), juan 857, p. 9,688.

10 See Baldrian-Hussein 1986, 133-69. I thank Jeehee Hong for pointing out these connections. Hong and Hinrichs 2015.

Kang Baocheng and Sun Jianjun inferred from the medical content of the mural that the occupant was a doctor $(2009,81)$. Certainly, other non-literati tombs, if slightly later, included murals and relief carvings portraying scenes from businesses, for example people reading account books. So while the medical content is unique for a tomb mural, portraying scenes from the occupant's vocation and source of wealth are not, although this mural may be an early example. For related discussions and examples, see Wu Hung 2010, pp. 45-7; and Xu Guangji 2012, vol. 2, p. 164 . 




Figure 17.10 Hancheng Tomb, north wall, right side, detail of Fig. 1. (c) TJ Hinrichs

a tray containing three components: out of the top pokes a row of white-stoppered bottles shaped like gourds, long a common form for medicine containers; next the label 'Red Cinnabar Pills' (zhusha wan 朱砂丸); then rows of something white, perhaps paper packaging (Fig. 10).12 Further back is a table covered with a variety of jars and

12 I thank Paul Unschuld for suggesting this identification. 'Perhaps they were formed the same way as their counterparts in Europe. You take a square sheet of paper and fold it from left and right into three identical sections. Then you bend the left and the right sections over the middle section. Now, you fold these three layers again, but this time from the bottom first and then from the top. First, one third from the bottom is folded upwards. This then allows you to take it up, hold it with your left hand and support the lower end with your little finger, keeping the folded lower third in place with your thumb. Then you may pour a powder or pills into the opening on top. Once you have filled the package, you bend the upper third down towards the upward opening of the lower third and insert it into the opening of the lower third that was bent up before. The contents are safely and cheaply stored this way, and they can easily be used by lifting the upper third out of the lower third again.' Email comm., 23 April 2014. gourd-shaped bottles. ${ }^{13}$ Behind the table stand two men, one holding a volume labelled 'Taiping Era Formulas', the other holding white paper packets of a type also common in modern Chinese-style pharmacies, marked with the drug names baizhu 白术 (white Atractylodes) and $d a$ huang 大黃 (rhubarb). ${ }^{14}$ As is common in paintings of human subjects of the period, the people in the painting are shown actively interacting, and these men face each other, apparently consulting about the concoction of a remedy. ${ }^{15}$ This is clearly a workshop for the production of pharmaceutical medicines. Such activity, aimed at the production of medicines to heal others, would have been an appropriately noble activity to portray in a tomb mural (Figs 10-12). ${ }^{16}$

The conjunction of these particular drugs and this book points to the production of a range of disease remedies. In the book, out of a total 16,834 recipes, rhubarb (always specifically Sichuan rhubarb) appears 1,349 times and white Atractylodes appears 941 times, both across a wide range of remedies. ${ }^{17}$ Out of the 941 entries in which the two drugs could potentially appear together, they are combined a total 27 times, just $2.8 \%$ of that total. ${ }^{18}$ The infrequency

13 On the use of the gourd as a symbol for medicine sellers and as a shape for medicine bottles, and for examples, see Unschuld 200o, pp. 48, 79 102, 156, 176, 198.

14 Other roughly contemporary tombs also include labelled books. For example, one of Zhang Shiqing's 張世卿 1116 CE Liao tomb murals include a Buddhist sūtra and the 1oth-century Daoist scripture Changqing jingjing 常清淨經, shown laid out on table in the midst of a domestic tea-making scene. See Wu Hung 2010, p. 232, fig. 227, 228.

15 For further analysis, see Kang Baocheng and Sun Bingjun 20o9, p. 81 .

16 In contrast, the treatment of the occupants' own illnesses would have been a highly inauspicious subject, and therefore one unsuitable for (and unattested in) burial paintings. The instances of bird-bodied Bian Que treating patients as found in Later Han tomb reliefs represent mythistorical episodes, not the occupants' lives.

17 In earlier periods, Atractylodes and rhubarb had been incorporated into some Daoist practices, but in the 11th century, they were much more salient as common therapeutic drugs, both of which wereproduced in Shaanxi, the region of our mural. On the prominence of Atractylodes in Shangqing Daoism, see Stanley-Baker 2013, 175-82. Materia Medica of various periods quote Sun Simiao, and identify the two drugs as being grown in Shaanxi. See Sun Simiao, Qianjin yifang (1959 edn), 2.19, 3.3o.

18 Those combinations appear mainly in juan 11, under Cold Damage or Seasonal Qi (epidemics) complicated by food poisoning or overeating, and under dyspeptic or overeating or drinking conditions, especially those accompanied by constipation or other accumulations (juan 48-51). Although baizhu 白术 (white Atractylodes) and dahuang 大黃 (rhubarb)do not appear to- 




Figure 17.11 'Taiping Era Formulas of Sagely Grace', 'White Atractylodes', 'Rhubarb'. $\odot \mathrm{TJ}$ Hinrichs

of their appearance together is not surprising given that the drugs had contrary effects: Atractylodes treated such conditions as numbness, paralysis, and convulsions; it stopped sweating, fever, violent diarrhoea, and vomiting, and benefited Blood in the lower abdomen. In contrast, far from nourishing abdominal Blood and counteracting diarrhoea and vomiting, rhubarb acted as a purgative that broke up and drained Blood stagnations and blockages along with accumulations and accretions, and cleansed the stomach and intestines. ${ }^{19}$ Marta Hanson has proposed that it is precisely the drugs' incompatibility that explains their function in the mural: as a Yin-Yang pair, standing synecdochically for a full repertoire of drug simples - a reading that agrees with the identification of the mural as representing a workshop that served a sizable clientele. ${ }^{20}$

Reinforcing that interpretation is the inclusion in the mural of the Taiping Era Formulas (Fig. 11). The massive 10o-chapter book collected remedies from physicians throughout the realm, was compiled as a comprehensive reference for medical formulas and disease categories, and was distributed by early Song courts to guide medical relief - i.e., for the treatment of masses of people..$^{21}$ The text was

gether as part of a cinnabar pills remedy, the combination does appear five times in juan 49, which also includes three different versions of cinnabar pills. Chapter 49 focuses on disorders involving accumulations in the abdomen, especially of food that will not disperse. Also see the two drugs combined, for similar conditions, in isolated appearances across juan 6, 9, 14, 16, 38, $43,54,58,71,81-3$.

19 Sun Simiao, Qianjin yifang (1959 edn), 2.19, 3.30.

20 Proposed after joint presentation, with co-author Jeehee Hong, of Hinrichs and Hong 2013.

21 The 100-chapter court-produced formulary was encyclopaedic in scope - the product of Emperor Taizong's call to collect remedies from throughout the realm that would otherwise be trans- so extensive, in fact, that in 1046, Fuzhou 福州 (in Fujian) Prefect Cai Xiang 蔡襄 (1012-67), finding it too unwieldy for everyday use by commoners, commissioned and displayed wood blocks of a much-abridged version, reduced by $6 \circ \%$ to 6,916 entries. ${ }^{22}$ Possession of such an expensive and encyclopaedic work as the Taiping Era Formulas suggests a substantial operation, one that produced standardised remedies for multiple retail outlets, for wholesale markets, for government agencies, or perhaps for some combination of the above.

The division of labour represented in the mural's varied people, activities, and objects also indicates an operation of some scale. All of this points to a functional differentiation of pre-packaged remedies between wholesale production, distribution, and retail (as portrayed in the Qingming scroll), which would in turn have required a certain critical size and level of infrastructure, for example of distribution networks, in commercial pharmaceutical markets. ${ }^{23}$ Around this time, such a division was just appearing in the state's medicinal production and distribution bureaucracy. ${ }^{24}$

mitted only privately within physician lineages, and to organise them in a comprehensive, nosologically indexed reference work. Once it was completed, he ordered two copies distributed to each prefecture, and revived the Tang prefectural post of Medical Erudite (yiboshi 醫博士) specifically to take charge of the text and make it available to commoners for copying. Taizong and some of his successors made concerted efforts to distribute the book. It was compiled under the supervision of Hanlin Medical Officer 翰林醫官使 Wang Huaiyin 王懷隱. Sections on disorders open with analyses drawn from Chao Yuanfang's 巢元 方 Zhubing yuanhou zonglun 諸病源候總論 (Comprehensive Treatise on the Origins and Symptoms of Diseases, 610 CE). See Okanishi Tameto 1969, pp. 713-20. On later efforts to further distribute the work, see Hinrichs in Chia and de Weerdt 2011, pp. 218-19.

22 Okanishi Tameto 1969, pp. 720-21. In contrast to the Taiping Era Formulas, about the time of the tomb or a little later, the state produced a formulary specifically designed for daily application, to guide medical relief by the Imperial Medical Service and the production of pre-packaged drugs by the Pharmacy Service. This book was the Taiyiju fang 太醫局方 (Formulas of the Imperial Medical Service), published in the Yuanfeng Period (1078-85), and was only a modest 1o juan in length. The revised edition of the Taiyiju fang, the Taiping huimin hejiju fang 太平惠民和劑 局方 (Formulas of the Pharmacy Service for Great Peace and for the Benefit of the People, 1107), circulated widely. See Okanishi Tameto 1969, pp. 748, 765-87.

23 There had been a geographically widespread market at least for drug simples for some centuries. See Stanley-Baker 2013; Shiba Yoshinobu 1970, p. 49.

24 There was a separate Office for Compounding Drugs (Heyao suo 合藥所) in operation by 1075, and in 1076 Shenzong's court 




Figure 17.12 'Cinnabar Pills', detail of Fig. 1. ${ }^{\odot}$ TJ Hinrichs

Returning to the mural, the cinnabar pills being offered to the occupant may represent a more complex intersection between the personal and the public (Fig. 12). As an item stocked for sale, cinnabar's popular connotation of powerful life-giving properties would have made 'Cinnabar Pills' a marketable name; beyond that, as in the case of atractylodes and rhubarb, it is impossible to pinpoint a specific application. The term 'Cinnabar Pills' was apparently marketable enough to have named a heterogeneous variety of remedies in the diverse physicians' repertoires that went into the compilation of the Taiping Era Formulas. That book included a total 49 recipes for Cinnabar Pills with 49 different combinations of ingredients, spread fairly evenly throughout. Forty-eight of the 49 formulas treated such ailments as Wind disorders in the Heart Visceral System, various types of epidemic fevers, appetite loss, and demonic intrusions. ${ }^{25}$

The 49th 'Cinnabar Pills' formula, along with the position of the pills in the mural, suggests other layers of meaning. The tray of bottles and packages carrying the label 'Cinnabar Pills' appears, in the setting of the workshop, to be offered to the occupant for his inspection, perhaps to check for quality. In the context of the burial, the pills may also represent a gift to the deceased, not to treat something so unpropitious as illness, but representing descendants' hopes for his long and robust afterlife. ${ }^{26}$ In the Taiping Era

merged it with related facilities and a new Pharmacy Service (Maiyao suo 賣藥所, later Heji ju 和劑局). When Huizong's court expanded the Pharmacy Service, they also instituted separate facilities for drug manufacture and sales. See Li Tao, Xu zizhi tongjian changbian (1986 edn), 271.14A, 275.6B; Goldschmidt 2009, pp. 125-30.

25 I thank Chen Shiau-Yun for double-checking these calculations with Academia Sinica's Hanji dianzi wenxian 中央研究院漢籍 電子文獻 database.

While in some Daoist practice, the mineral could be involved in the alchemical pursuit of transcendence, more commonly and popularly, it seems to have possessed associations with the extension of life. In the 978 collection of tales Taiping guangji 太
Formulas, the 49th Cinnabar Pills entry appeared as the first item in chapter 98, on 'Supplementation and Boosting' (buyi 補益), and that recipe was not a treatment for disease but a tonic for long life:

Cures the hundred [i.e., all] ailments, benefits the Five Visceral [Systems], quiets the Yang-soul (hun 魂) and settles the Yin-soul ( $p o$ 魄), nurtures the Heart and expands Qi, and gives pleasant lustre to the face. Taken over a long time [the pills] will lighten the body, extend the years without aging, lengthen the sinews, supplement the Cinnabar Fields (dantian 丹田), sharpen hearing and sight, and [impart] much skill and strength. ${ }^{27}$

'Cinnabar Fields' refers to loci in the head, chest, and lower abdomen in Daoist bodily landscapes. In general, in Tang and Song medical formularies, distinctively Daoist terminology such as 'Cinnabar Fields' tends to appear in relation to remedies that are either apotropaic, exorcistic, or for 'nurturing life' (yangsheng), at least in the loose sense of 'supplementation and boosting.' ${ }^{28}$

We must remember that such analytic distinctions, between Daoism and medicine, may have been neither salient nor sensible to the occupants and their family. While some Song figures did advocate distinguishing and then removing Daoist elements from medical works, and while, generically, Daoist and medical writings and practice remained discernibly different, the type of boundary work that produced and policed such differences was clearly not of interest to our tomb occupant. ${ }^{29}$ From the point

平廣記, zhusha appears occasionally as a substance ingested for the extension of life, but not for the goal of transcendence. $\mathrm{Li}$ Fang et al., eds [1961] 1981, zhusha: 1.6, 52.321, 237.1824, 437.3896.

27 Wang Huaiyin et al., Taiping shenghui fang ([1958] 1982 edn), juan 98, p. 3,130. Rhubarb pills (大黄丸) appear toward the end of the same chapter, and are identified with more mundane therapeutic actions: 'Harmonising and benefiting the diaphragm, expelling stagnations, pushing what is stale and extending what is fresh, and dispersing Wind and flowing with Qi'. Wang Huaiyin et al., Taiping shenghui fang ([1958] 1982 edn), juan 98, pp. 3,153-4. In this chapter, Atractylodes, so prominent in Shangqing Daoism, only makes the odd appearance in formulas as a subsidiary drug.

28 See, for example, Hinrichs 2003, Appendix 5: 'Prescriptions for Avoiding Warmth and Contagion from Sun Simiao's Beiji qianjin yaofang and Pang Anshi's Shanghan zongbinglun', pp. 251-69.

29 As examples of medical boundary work, Cai Xiang specifically ordered the excision of 'passages on acquiring immortality' from his abridgement of Taiping Era Formulas, for reasons of practicality for commoners. Some decades later, Cheng Jiong (fl. 1163-1176) wrote an entire book calling for the restoration of medicine to canonical foundations, and specifically attacked the use of Daoist physiological terminology. See Hinrichs 2003, pp. 36,215 . 
of view of a modern scholar or of certain Northern Song specialists, just as some parts of the Taiping Era Formulas fold Daoist elements into the field of medicine, so too the mural on the tomb's north wall layers medicine in daily life with medicine in the afterlife. In the same vein, the screen poem combines Daoist with Buddhist elements, and the medical and Daoist content of the northern mural sits between Buddha's nirvana to the east and entertainment to the west. To separate those epistemological, ontological, and salvational realms, though, would have been atypical of the 'eclectic' non-official tomb culture in the Song.

The tomb as a whole and the activity of the north mural in particular, revolve around the male occupant, a structuring of authority in which the patriarch, as object of veneration, occupies a position homologous to that of the ruler. Like the ruler, the occupant sits squarely and centrally, facing south. Just as, in ideal classical formulations, the ruler orders All-Under-Heaven and the Enterprise (ye 業) of state, not through action of his own but through the effects of his potency on others, so too the patriarch orders his family (implicitly present, ritually facing him and organising rituals at the tomb) and his commercial enterprise $(y e) .^{30}$

The Hancheng tomb's north wall mural is a rare primary source, coming as it does from a merchant family's tomb and not a physician's or literatus' brush, on the place of pharmaceutical production in the life of an 11th-century commoner. In official sources regarding the book, we get decrees giving general orders for the Taiping Era Formulas' production and distribution, and for the establishment of positions charged with its 'handling'. In Cai Xiang's preface to his abridged version of the Taiping Era Formulas, we hear that, despite Taizong's order that the book be 'distributed to the prefectures for transmission to the officials and the people', in most prefectures it was just 'strictly locked away, reverently sunned and aired, and that is all. None of the officials or the people gained benefit from it'. ${ }^{31}$

In other sources, we learn that some imprints of the book were riddled with errors, the fault of unruly or lazy woodblock engravers. ${ }^{32}$ In our tomb mural, we can see the

'One who governs through Virtue ( $d e$ 德) is like the Pole Star. It occupies its place while the host of other stars pay homage to it.' 'The virtue of the Noble Man is like the wind, and the virtue of the small people is like grass. When the wind blows over the grass, the grass must bend.' 'To rule through non-action, such was [the sage ruler] Shun. How did he do it? He made himself reverent and exactly faced south, and that is all.' Lunyu 2.1, 12.19, 15.5 .

31 Quoted, with minor alterations, from Hinrichs in Chia and de Weerdt 2011, p. 234.

importance of the text to a merchant's life, as an instrument that contributed to his flourishing pharmaceuticals operation, and as an expensive, luxury object that garnered some of the state's glamour for him and for his family.

In the tomb mural image of the Taiping Era Formulas of Sagely [i.e., Confucian-Imperial] Grace, we see a trace of state medical publishing serving as means of livelihood and as a point of pride in the life of a provincial commoner. For Song courts and civil officials, the book served efforts to care for and educate commoners, and thereby ground the regime's legitimacy. Taizong had ordered the Taiping Era Formulas to be printed and distributed, two copies to each prefecture, both to spread medical knowledge and to guide medical relief. Cai Xiang had commissioned the book's abridged version, whose woodblocks he had posted on the Fuzhou Yamen gates,

in order to guide the Sagely Ruler's inexhaustible grace of morality to penetrate to those below, and also to enlighten people of the error of relying on shamans, and to cause them to return to constant standards. ${ }^{33}$

Taizong's medical publishing, education, and relief policies were just a few of such programmes, which saw unprecedented expansions under the Song. Similarly, Cai's was one of numerous campaigns and imperial decrees aimed at rectifying southern healing practices, often targeting indigenous 'shamanic' healers. ${ }^{34}$ And these programmes of medical governance were part of more general movements among Song courts and civil officials that - like reinterpretations of ancient forms seen in Song tomb mural motifs - reinterpreted Mencian ideals to more deeply root imperial legitimacy in the well-being of commoners, and gave new forms to Xunzian ideals of 'transforming [commoner] customs and mores through education' (jiaohua fengsu 教化風俗). ${ }^{35}$ 34 on the ways in which those views of sovereignty became visualised in paintings, see Powers in Porter 2012, pp. 226-36. On Song policies to 'transform [commoner healing] customs through [medical] education', see Hinrichs in Chia and de Weerdt 2011, pp. $233^{-8 .}$
Hinrichs in Jia and de Weerdt 2011, p. 235.

For a study of these campaigns, see Hinrichs 2003. The term $w u$ 巫, translated here as 'shamanic', appeared in these official documents as an etic term, and referred generally to the shrine officiants and healers indigenous to diverse southern peoples. On Song re-orientations of sovereignty toward commoners and 


\section{Making Commoners Visible at Court}

In the Qingming Scroll and Moxibustion, Zhang Zeduan and Li Tang projected particular inflections of imperial ideology as it related to medicine in commoner lives and livelihoods. The Song state had given qualitatively new, specific, practical, and material forms to medicine-as-ideology, making medicine a more integral and prominent part of governance, and deploying it - as medical relief, medical education, and as healing reform - as a means of actively extending the state's morally transformative power into communities, homes, and bodies. Scholars have shown the ways in which Han ideologies inscribed the imperial bureaucracy on the cosmos, and Han medicine in turn inscribed it on the body. ${ }^{36}$ Han medicine thus held the potential to powerfully naturalise the state at the level of lived experience.

More than any state before it, the Song availed itself of that ideological resource. In addition to imperial formularies such as the Taiping Era Formulas, and the compilation and publication of various Materia Medica, between 1057 and 1069 the Song's Bureau for Editing Medical Treatises (Jiaozheng yishu ju 校正醫書局) canonised and disseminated state-naturalising physiologies and medical treatments through the publication of authoritative editions of the medical classics. ${ }^{37}$ Besides officially recognising and quantitatively expanding the reach of these texts and the ideas they contained, the Song state furthermore tied medicine to the classical Confucian missions of caring for commoners' lives (medical relief) and transforming customs and mores through instruction (reforming southern healing customs and distributing medical texts).

We can see the new visibility of commoners to court and to scholar-officials in a vogue for paintings and poetry that depicted idyllic commoner lives. Recurring themes included village weddings and festival celebrations, and their embodiments of the 'authentic' essence of canonical ritual and human relations; and the classically normative modes of commoner productivity (and contribution, through taxation, to the political economy), agriculture and weaving. ${ }^{38}$ Some court paintings also celebrated livelihoods that, while classically denigrated, had become more economically important to the Song state, i.e., commerce. ${ }^{39}$ And in both

\footnotetext{
36 See, for example, Unschuld 1985, pp. 79-83; Sivin 1995, pp. 5-37; Lewis 2006, pp. 37-47.

$37 \quad$ Hinrichs in Jia and de Weerdt 2011.

38 Hammers 2011; Cheng Wen-chien 2011, pp. 67-106; Cheng Wen-chien 2005, pp. 309-57; Hartman 1995, pp. 1-72; Deng 2005, pp. $125-45$.

the Qingming Scroll and Moxibustion, we find medicine, an area on which Song courts and officials focused great energy and scrutiny.

\section{Qingming on the River}

There is some uncertainty surrounding the dating of Zhang Zeduan's painting of commoner daily life, Qingming on the River, but good evidence points to it having been produced at Huizong's court. ${ }^{40}$ Huizong's was a court that brought new vigour to building institutions aimed at elevating and at the same time unifying literati and technical learning and commoner customs, and caring for commoner welfare. ${ }^{41}$ Huizong connected all of these concerns with the need to improve the quality of medical studies:

We grieve for the blockage of the Great Way, for the [unhealthy] practices entrenched in prevailing customs, for the inveterate bad habits [and chronic ailments] that mire our commoners, and for the reckless doings of mediocre doctors, whose learning lacks profundity and erudition, and whose knowledge lacks insight and analytic comprehension. ${ }^{42}$

In aid of advancing and unifying medical knowledge, the court expanded the Medical School system and extended it to the provinces, and undertook new medical book publishing projects. For commoner welfare, the court also expanded the imperial Poorhouse system and Pharmacy Service, and created state-run Pauper's Cemeteries and Charity Hospitals. ${ }^{43}$ The Qingming Scroll celebrates the desired effects of such policies, even though it only hints at the existence of the programmes themselves.

The name of the scroll, Qingming on the River, refers not to the Qingming festival, but to the month of Qingming, an auspicious season of growth, and simultaneously to the peaceful and prosperous times produced by good governance, the latter a reference to Shijing 詩經 (Classic of Songs, also translated Book of Odes). ${ }^{44}$ Accordingly, the painting exudes prosperity, exuberance, and social vitality. Features such as the painting's several wine shops suggest not just adequate livelihood but plenitude, with space for

On the dating of the scroll, see Murray 1997, pp. 102-5.

41 On Huizong's goals for commoner customs, see for example his 1112 palace examination question, Bol 2006, pp. 195-6.

42 Zhao Ji Shengji zonglu, Preface ([1962 1992 edn] $)$, vol. 1, 3 .

43 For an outline of Huizong's broader set of reforms and institutional innovations, and the place of welfare and medical relief within those, see Chaffee in Ebrey and Bickford 20o6, pp. $37 \mathrm{ff}$. For an overview of Huizong's work in medical education, publication, and medical relief, see Ebrey 2014, pp. 191-4; Goldschmidt 2006, pp. 275-323. de Pee 2010, p. 167 , n. 6 o. 




Figure 17.13 Qingming on the River, detail of Fig. 2. ${ }^{\odot}$ Courtesy of Palace Museum, Beijing

leisurely socialising. ${ }^{45}$ The flow of human motion and activity, from the countryside into the city, teems with intriguing people and happenings, the most dramatic of which is a boat broken from its moorings, which attracts crowds on a bridge and nearby riverbanks to gawk and also to help re-tether the vessel. The scroll is full of vibrant everyday life, excitement, collective work and play, and the occasional manageable and non-life-threatening drama easily resolved through community cooperation.

Following the flow of action from the countryside into the city, one block inside the city wall we find a pharmacy open to the street. (Fig. 13). ${ }^{46}$ The pharmacy's advertisements continue the scroll's larger themes of abundance: one of the shop's placards echoes the theme of liberal drinking with the advertisement: 'Cure the Damage of Wine: Authentic-Recipe Collected Fragrance Pills' (Zhi jiu suo shang zhenfang jixiang wan 治酒所傷真方集香 丸). Another sign refers to disorders of the stomach and intestine (changwei bing 腸胃病), also evoking ample and rich eating. A smaller forward-facing sign advertises remedies for the 'Five Over-exertions and Seven Injuries' (wulao qishang 五勞七傷), ailments mainly of overindulgence and overwork. ${ }^{47}$

45 Zhou Baozhu 1997, pp. 95-104.

46 There is another establishment around the corner, with signs just visible and reading 'Yang Family Clinic' and 'Dr Yang' (yangjia yingzheng 楊家應症; Yang daifu 楊大夫....). For the reading of these and other signs, I follow Zhou Baozhu 1997, p. 88.
In contrast to the themes of these signs, which point to busy lives and boisterous socialising, the human scene in the pharmacy itself is a serene one - an island of tranquillity on a bustling street. Inside we see a pharmacist/doctor quietly examining a small child held on his mother's lap, with a female servant standing to their side. The scene points to the domestic matter of child-rearing, and to a household that could afford a servant. Like the other mini-dramas woven throughout the scroll, this one is non-critical: the child shows no signs of distress, being well enough to be brought to the pharmacy and to sit comfortably upright.

The scroll as a whole in fact reveals no direct signs of official activity, no government offices, no officials parading or inspecting, no apparent policing. As for the greatest preoccupation of Song medical governance, epidemic relief, the small shop advertises no remedies for such ailments. ${ }^{48}$ There are, however, indirect hints at official connections. For example, Tsao Hsingyuan has argued that figures in the scroll hiding their faces with round fans depict incognito

ied between (and even within) medical texts. The Taiping Era Formulas of Sagely Grace followed the nosological work Comprehensive Treatise on the Origins and Symptoms of Diseases (Zhubing yuanhou lun 諸病源候論), with the Five Overexertions as those of will (zhilao 志勞), of thought (silao 思勞), of heart/mind (xinlao 心勞), of worry (youlao 憂勞), and emaciation (shoulao 瘦勞). It includes two sets of 'Seven Injuries', the first having to do with various Yin (male sexual, urogenital, colorectal) problems: Yin (given context, meaning genital) Cold (yinhan 陰寒), Yin atrophy or withering (inability to get an erection) (yinwei 陰萎), painful and urgent diarrhoea (liji 裡 急), frequent seminal emissions (jinglianlian 精連連), paltry semen and damp genitals (jingshao yinxiashi 精少陰下濕), clear, cold semen (jingqing 精清), and painful and frequent urination (xiaobian kushu 小便苦數). The second set of Seven Injuries includes damage to the five Yin visceral systems, skin, and will (zhi 志) from overeating; from strong anger; from the exertion of lifting something heavy; from drinking something cold when already cold; from anxiety and over-thinking; from exposure to wind, rain, and cold; and from immoderate fear. Wang Huaiyin et al., Taiping shenghui fang ([1958] 1982 edn), juan 26, p. 748. Also see Chao Yuanfang Zhubing yuanhou lun jiaozhu 諸病源候 論校注, ed. Ding Guangdi et al. 1991, juan 3, pp. 88-9o.

48 For example, Huizong's 20o-chapter Shengji zonglu 聖濟總錄 (Comprehensive Record of Sagely Beneficence, $1118 \mathrm{CE}$ ) opens with two chapters on the application of Five Circulatory Phases and Six Climatic Influences (wuyun liuqi 五運六氣) theories to the prognostication of 'commoner diseases (minbing 民病)' i.e., epidemics.

Asaf Goldschmidt argues that epidemics were a major driving force behind Song state interest in medicine in general, and epidemic Cold Damage disorders and wuyun liuqi theorisation in particular. See Goldschmidt 2009, pp. 69-102. 
scholar officials. ${ }^{49}$ And while the pharmacy itself does not sport the title of the Pharmacy Service, and is too small to be a government operation, the pharmacy does refer to state medical institutions in two of its signs. The sign that ends with 'stomach and intestine disorders' begins with 'Pills Issued by the Imperial Medical Service' (Taiyi chu wan 太醫出丸). A sign hanging horizontally over the main entryway identifies the place as 'Shop of Imperial [Medical Service] Director Zhao' (Zhao Taicheng jia 趙太丞家). ${ }^{50}$

Just as the Hancheng mural celebrates the occupant's achievements and authority by positioning him as an unmoving nexus of activity, so does the Qingming Scroll pay tribute not to Huizong's acts, to his institutions themselves, but to their desired effects, to happy, simple, and abundant commoner lives. The scroll does, with its self-effacing officials and its former medical officer working commercially, suggest effects overflowing from direct official acts - a pole apart from such remedial governance as poor relief. Along with the vibrant life depicted throughout the rest of the painting, the unruffled child-patient (apparently not urgently sick, but yet seeing a physician), the remedies not for epidemics (the usual object of official medical relief) but for overindulgence - all speak to a measure of bounty beyond sufficiency, and to imperial potency and virtue propagating well beyond the palace and government compounds.

\section{Moxibustion}

In contrast to the Qingming Scroll, and in accord with the tenor of its own times (c.1130-50), Moxibustion eschews any reference to government agency. ${ }^{51}$ By then, Huizong's

49 Tsao Hsingyuan 2003, pp. 166-7.

50 An abbreviation for Taiyiju cheng 太醫局丞, also abbreviated Taiyi cheng 太醫丞, a position in charge of the Imperial Medical Service, established during the Xining period (1068-77) and continued into the Southern Song. Gong Yanming 1997, pp. 284-5. Pharmacy shops opened by former medical officials are attested in 12th-century sources. One of the pharmacy shops (yaopu 藥鋪) listed in the account of the Eastern Capital, Dongjing menghua lu 東京夢華錄, includes such a medical official's title in its name, and other sources describe former medical officials opening pharmacy shops both in their hometowns and in the capital after retirement. See Meng Yuanlao, Dongjing menghua lu (1980 edn), 3.18; Hong Mai Yijianzhi (1981 edn), 夷堅支乙志 7, p. 845 .

$51 \quad$ Li Tang was styled Xigu 晞古 and was a person of Heyang sancheng in Meng District, Henan (孟縣河陽三城). He served in the Painting Academy (Huayuan 畫院) under Huizong. He fled the Jurchen invasion to Lin'an (Hangzhou), and after a period of living in poverty from the sales of his paintings, was re-appointed to the Painting Academy during the Jianyan period $(1127-30)$. His dates are uncertain and sources disagree, but he is

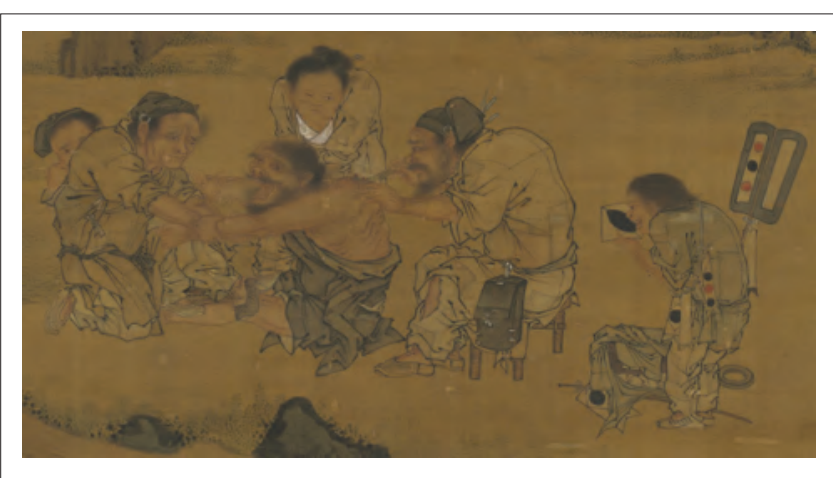

Figure 17.14 Compassion in Moxibustion, detail of Fig. 3. ${ }^{\odot}$ Courtesy of National Palace Museum, Taipei

grandiose ambitions to create a flourishing, unified, and vigorous society through centrally governed institutions had been thwarted, not only by the challenges of bureaucratic implementation, but by catastrophic collapse and disgrace. In 1126, the north-eastern Jin regime 金 (1115-1234) armies invaded Song, prompting Huizong to abdicate. A year later the Jin captured Huizong, the reigning Emperor Qinzong 欽宗 (r. 1126), much of the court, and a large swathe of northern territories. Song refugees regrouped in the south, founding a new 'temporary' capital at Hangzhou, and enthroned one of Huizong's sons as Emperor Gaozong. In the Southern Song (96o-1279), as it came to be called, many blamed the loss of the northern heartland on Huizong and his policies. Thus, in its retrenchment and reorganisation of the government, Gaozong's court eliminated some of Huizong's expensive and government-expanding programmes, including those for medical and poor relief. Where, in the Northern Song, literati of all factions had tended to talk about social transformation in relation to centralised government policies, Southern Song intellectuals advocated reform through extra-official local activism.

Nevertheless, while the Southern Song state scaled back the radical institutional innovations of the pre-invasion era, medical governance, which had become so prominent in the Song's first 16 o years, retained some of its institutional and ideological hold. Despite the crisis of war with the Jin continuing until 1142, and notwithstanding the expense of tribute paid by the Song to the Jin thereafter, medical care for commoners did not drop entirely from official view. In 1136 Gaozong revived the Pharmacy Service, and in 1151 he expanded the network of pharmacies, now called 'Great Peace Bureaus for Benefiting the Common People' (Taiping huimin ju 太平惠民局), to its greatest extent to

generally thought to have died before 105o. On attribution and dating, see Ni Zaiqin 1991, pp. 3-5; Tong Wene 童文娥, 'Jiuai tu' 炎艾圖, in Guoli gugong bowuyuan 2010, p. 389 . 
date, mandating one in each prefecture. ${ }^{52}$ And although official policies to reform southern healing customs and shamans never reached the levels that they had in the Song's first century, Gaozong's court, located in a region formerly targeted in earlier campaigns, made its own forays against southern shamans and their unorthodox approaches to disease. ${ }^{53}$ Not long after Gaozong's time, scholar-official Cheng Jiong 程迥 ( $f l$. 1163-74), railing against shaman healers in the south, commented wistfully: 'The people of the central plains [the lost northern heartland] trust in physicians and do not trust in shamans. ${ }^{54}$

It is just such norms, trust in physicians (and not in shamans), that Moxibustion portrays (Fig. 14). In it, we find a doctor calmly applying a cone of moxa, glowing red and smoking, to a man's bare back. Behind him, a young assistant prepares a poultice to apply to the patient's moxa blister. We can see from the doctor's accoutrements - his stool, his medical kit and bags hanging from his belt and on the ground, and signs with black and red circles advertising his vocation - that he is an itinerant healer. ${ }^{55}$ Besides his kind face, the doctor's coarse, torn, and multiply patched clothing, by suggesting that he does not profit greatly from his treatments, indicates his decency, and distinguishes him from supposedly venal 'shamans' or self-aggrandising charlatans.

The setting and grouping of the figures in the painting also contrasts sharply with literati complaints about southern shamanic healing customs. Scholar-officials singled out for special condemnation the quarantine of the sick in darkened rooms, isolated from family and relegated to the care of 'shamans'. ${ }^{66}$ The issue remained alive in the Southern Song, prompting the aforementioned Cheng Jiong to write an entire book against theories of contagion, which he blamed for helping perpetuate such noxious customs. ${ }^{57}$ The Moxibustion patient, far from sickroom seclusion and shamans' exorcistic intervention, is treated in the sunshine, outside the village wall, surrounded by

52 Xu Song, Song huiyao jigao (1936 edn), zhiguan 職官, 27.66A, $67 \mathrm{~A}$.

See, for example, Gaozong's edicts to control and prohibit shamans who promote 'human-killing and demon-worshipping' in 1142 and 1153, and shamanic healers who prevent commoners from seeking medical care in 1146. In addition, note various campaigns by local officials against shamans under Gaozong. Listed in Wong Cheung Wai 2005, pp. 268, 274-5. For another example of spots being used as a sign for a plaster medicine seller, and for images of plaster medicines, see Unschuld 200o, pp. 45, 47, 79 .

$56 \quad$ See Hinrichs 2003 , ch. 7 .

57 See Hinrichs 2015. and in close physical contact not only with the doctor but with family. As the restorative moxa burns and the patient grimaces and yells, his family express their sympathy with furrowed brows and wincing faces, and help him endure the pain and hold still by tightly gripping his arms and by pinioning his legs with their own feet. Providing some comic relief, on the far left, a child nervously peers around the older woman, and on the far right the doctor's apprentice, inured to such scenes, grins.

As a court painting that celebrates the emperor's ability to establish good commoner customs and mores, Moxibustion spotlights simple, mutually-caring folk, a close-knit family, and a canonically-endorsed mode of healing. In this painting, the state is an assumed, not a marked presence. While Gaozong did continue and even expand the Pharmacy Service, his was not a time for bringing attention to state institution-building or growth. The painting embodies an ideal of imperial efficacy and authority closer to that of classic non-action (wuwei 無爲), a state in which great harmony and stability is achieved with, and precisely because of, minimal state intervention - a restoration of such foundational Confucian ideals as that of the ruler who rectifies himself, faces south, and thereby (and not through centralised institutional apparatuses) transforms the people. ${ }^{58}$

\section{Conclusion}

The three paintings share a vision of patriarchal and imperial authority as a pivot generative of positive human relations. The Hancheng tomb mural situates the merchant in the centre, larger-scale, solidly-sitting, iconically outward-facing. Court paintings assume greater imperial presences: in the Qingming Scroll, hinted at through references to government organs and officials; in Moxibustion, veiled, a pivot operating smoothly-silent and invisible. Despite their stillness and non-action, those

58 'One who governs through Virtue $(d e)$ is like the Pole Star. It occupies its place while the host of other stars pay homage to it.' 'Guide [the people] by means of rules and order them ( $q i$ 齊) [literally, make them uniform] through punishments, and the people will evade [your governance] and will lack any sense of shame. Guide them through virtue and order them through rites, and they will have a sense of shame and will also regulate themselves.' 'The virtue of the Noble Man is like the wind, and the virtue of the small people is like grass. When the wind blows over the grass, the grass must bend.' 'To rule through non-action, such was [the sage ruler] Shun. How did he do it? He made himself reverent and exactly faced south, and that is all.' Lunyu 2.1, $2.3,15 \cdot 4,12.19$ 
patrons' agency, productive of medical production and collaboration, is certain. It is the Hancheng merchant who built the prosperous workshop; it is Huizong who revived and sponsored new medical institutions in his dream of a thriving realm; and it is Gaozong who saved the dynasty and preserved imagined classically-conforming social norms of the Han heartland in the ethnically-mixed south.

There are good reasons why it is the Song period that produced these three paintings. It is in the 1oth and 11th centuries that affluent commoners began building tombs decorated with murals that showed aspects of their daily lives. And the pharmaceuticals trade and government-published medical texts had by then become significant sources of commoner wealth and prestige (at least for our Hancheng merchant). In turn, Song courts invested enough of their legitimacy in the quality of commoner daily life and in medicine to make the two themes and their conjunction fitting subjects for imperial paintings.

As paintings, the three objects celebrate their patrons' social and medical efficacy in ways that texts did perhaps less well, and less strikingly. Where a eulogy might praise the achievements of the departed man in building a pharmaceutical business, a tomb mural can display a conception of him as the stable, authoritative centre of the diverse activities that went into medicinals production..${ }^{59}$ Various edicts proclaimed imperial intent to rectify commoner healing customs, and Huizong's Comprehensive Record of Sagely Beneficence and Gaozong's reinstatement of the 'Great Peace Bureaus for Benefiting the Common People' both proclaimed imperial benevolence through medicine. The Qingming Scroll and Moxibustion, though, reveal visions of imperial benevolence actually being articulated at the community level in compassionate and orthodox forms of medical care, and in tight-knit and positive social relations.

\section{Bibliography}

\section{Primary Sources}

Chao Yuanfang 巢元方 610, Zhubing yuanhou lun jiaozhu 諸病源候 論校注 (Treatise on the Aetiology and Symptoms of Diseases). Modern edn: Ding Guangdi 丁光迪 et al. (eds), Beijing: Renmin weisheng chubanshe, 1991.

Cheng Jiong 程迥 (jinshi 1163) 1176, Yijing zhengben shu 醫經正本書 (Book on the Correct Foundations of the Medical Canon), in Wang Yunwu 王雲五 (ed.) 1939, Congshu jicheng chubian 叢書集成初 編 (Compendium of Collectanea, 1st edn), vol. 1,383, Shanghai:

59 I do not know of any extant eulogies for merchants, and speculate here that such a eulogy would, like literati eulogies, emphasise career and other public accomplishments.
Shangwu yinshuguan.

Li Fang 李昉 et al. (eds) [1961] 1981, Taiping guangji (Extensive Records of the Taiping Era), Beijing: Zhonghua shuju.

Li Tao 李壽 (1115-84) (ed.) Xu zizhi tongjian changbian 續資治通鑑 長編 (Extended Continuation of Comprehensive Mirror to Aid in Government).Modern edn:Shanghai:Shanghai guji chubanshe, 1986.

Lunyuyizhu 論語譯注 (Translation and Commentaries of the Analects). Modern edn: Yang Bojun 楊伯峻 (ed., comm.), Beijing: Zhonghua shuju, 1980 .

Meng Yuanlao 孟元老 (fl.1126-47), Dongjing menghua. Modern edn: Taibei: Dali chubanshe, 1980.

Hong Mai 洪邁 (1123-1202), Yijianzhi 夷堅志 (Records of the Listener). Modern edn: Beijing: Zhonghua shuju, 1981.

Okanishi Tameto 岡西為人 1969 (reprint), Sō izen iki kō 宋以前醫 籍考 (Studies of Medical Books of the Song and Earlier), Taipei: Guting shuwu.

Peng Dingqiu 彭定求 (1645-1719) et al. (comp.) Quan Tang shi 全唐诗 (Complete Tang Poetry). Reprint: Beijing: Zhonghua shuju, 196o.

Porter, D. (ed.) 2012, Comparative Early Modernities, 1100-1800, New York: Palgrave Macmillan.

Quan Tang shi 全唐詩, Peng Dingqiu 彭定求 et al. (eds), Beijing: Zhonghua shuju, 196o.

Sun Simiao 孫思邀 (d. 682?), Qianjin yifang 千金翼方. Modern edn: Taibei: Ziyou chubanshe, 1959.

Wang Huaiyin 王懷隱, et al. 992, Taiping shenghuifang 太平聖惠方. Modern reprint: Beijing: Renmin weisheng chubanshe, [1958] 1982.

Xu Song 徐宋 (1781-1848) (comp.), Song huiyao jigao 宋會要輯稿 (Compilation of Official Documents of the Song). Modern edn: Beijing: Guoli Beiping guoshuguan, 1936.

Zhao Ji 趙佶 (Song Huizong 宋徽宗) $1118 \mathrm{CE}$, Shengjizonglu 聖濟總錄 (Comprehensive Record of Sagely Beneficence), vol. 1. Modern edn: Beijing: Renmin weisheng chubanshe, [1962] 1992.

\section{Secondary Sources}

Baldrian-Hussein, F. 1986, 'Lü Tung-pin in Northern Sung literature', Cahiers d'Extrême-Asie 2, 133-69.

Bol, P.K. 2006, 'Emperors can claim antiquity too: emperorship and autocracy under the new policies', in Ebrey and Bickford (eds), 173-205.

Chaffee, J. 2006, 'Huizong, Cai Jing, and the politics of reform', in Ebrey and Bickford (eds), 31-77.

Cheng Minsheng 程民生 2008, Songdai wujia yanjiu 宋代物價研 究 (Commodity Prices in the Song), Beijing: Renmin chubanshe.

Cheng, Wen-Chien 2005, 'Drunken village elder or scholar-recluse? The ox-rider and its meanings in Song paintings of "Returning home drunk"', Artibus Asiae 65.2, 309-57.

2011, 'Antiquity and rusticity: images of the ordinary in the "Farmers' Wedding" painting', Journal of Song-Yuan Studies 41, 67-106.

Cherniack, S. 1994, 'Book culture and textual transmission in Sung China', Harvard Journal of Asiatic Studies 54.1 (June), 5-125.

Chia L. and H. de Weerdt (eds) 2011, Knowledge and Text Production in an Age of Print: China, 9oo-140o, Leiden: Brill.

De Pee, C. 2010, 'Purchase on power: imperial space and commercial space in Song-dynasty Kaifeng, 960-1127', Journal of the Economic and Social History of the Orient $53,149-84$.

Ebrey, P.B. 2014, Emperor Huizong, Cambridge, MA: Harvard University Press.

Ebrey, P.B. and M. Bickford (eds) 2006, Emperor Huizong and Late Northern Song China: The Politics of Culture and the Culture of Politics, Cambridge, MA: Harvard University Asia Center.

Deng Qiaobin 鄧喬涁 2005, Songdai huihua yanjiu 宋代繪畫研究 (Song Dynasty Painting), Kaifeng: Henan daxue chubanshe. 
Goldschmidt, A.M. 2006, 'Huizong's impact on medicine and on public health', in Ebrey and Bickford (eds), 275-323.

2009, The Evolution of Chinese Medicine: Song Dynasty, 960-1200, London: Routledge.

Gong Yanming 竟延明 1997, Songdai guanzhi cidian 宋代官職辭典 (Dictionary of The Civil Service System in the Song Period), Beijing: Zhonghua shuju.

Guoli gugong bowuyuan 國立故宮博物院 2010, Wenyi shaoxing: Nan Songyishuyuwenhua 文藝紹興: 南宋藝術與文化 (Dynastic Renaissance: Art and Culture of the Southern Song), Taibei: Guoli gugong bowuyuan.

Hammers, R.L. 2011, Pictures of Tilling and Weaving: Art, Labor, and Technology in Song and Yuan China, Hong Kong: Hong Kong University Press.

Hartman, C. 1995, 'Stomping songs: word and image', Chinese Literature: Essays, Articles, Reviews, 17 (December), 1-49.

Hinrichs, TJ 2003, The Medical Transforming of Governance and Southern Customs in Song Dynasty China (96o-1279 CE), PHD diss., Harvard University. 2011, 'Governance through medical texts and the role of print', in Chia and de Weerdt (eds), 217-38.

2015, 'The catchy epidemic: theorization and its limits in Han to Song period medicine', East Asian Science, Technology, and Medicine 41, 19-62.

Hinrichs, TJ and L. Barnes (eds) 2013, Chinese Medicine and Healing: An Illustrated History, Cambridge: Belknap Press of Harvard University Press.

Hinrichs, TJ and Jeehee Hong 2013, 'New perspectives on technical knowledge, funerary rites, and social status in the Northern Song: evidence from a recently discovered tomb' (conference presentation), Templeton International Conference on 'Science and Religion in East Asia, Seoul National University (1 December).

Hong, Jeehee and TJ Hinrichs 2015, 'Unwritten life (and death) of a "pharmacist" in Song China: decoding Hancheng tomb murals', Cahiers d'Extrême-Asie 24, 231-68.

Kang Baocheng 康保成 and Sun Bingjun 孙秉君 2009, 'Shaanxi
Hancheng Songmu bihua kaoshi' 陕西韩城宋墓壁画考释 (Analysis of the Song tomb murals at Hancheng, Shaanxi province), Wenyi yanjiu $11,79-88$.

Lewis, M.E. 2006, The Construction of Space in Early China, Albany: State University Press of New York.

Murray, J.K. 1997, 'Water under a bridge: further thoughts on the "Qingming" scroll', Journal of Song-Yuan Studies 27, 102-5.

Ni Zaiqin 倪再沈 1991, Li Tangji qi shanshui hua zhiyanjiu' 李唐及其 山水畫之研究 (Studies of Li Tang and his Landscape Painting), Taipei: Wenshizhe chubanshe.

Powers, M. 2012, 'Visualizing the state in Early Modern England and China', in Porter (ed.), 217-44.

Shiba Yoshinobu 斯波義信 1970, Commerce and Society in Sung China, tr. M. Elvin, Ann Arbor: University of Michigan.

Sivin, N. 1995, 'State, cosmos, and the body in the last three centuries BC', Harvard Journal of Asiatic Studies 55.1 (June), 5-37.

Stanley-Baker, M. 2013, 'Daoists and doctors: the role of medicine in Six Dynasties Shangqing Daoism', PHD diss., University College London.

Tsao Hsingyuan 2003, 'Unraveling the mystery of the handscroll "Qingming shanghe tu"', Journal of Sung-Yuan Studies 33, 166-7.

Unschuld, P.U. 1985, Medicine in China: A History of Ideas, Berkeley: University of California Press.

2000, Medicine in China: Historical Artifacts and Images, Munich: Prestel.

Wong Cheung Wai 王章偉 2005, Zaiguojiayu shehuizhijian: Songdai wuxi xinyang yanjiu 在國家與社會之間-宋代巫項信仰研究 (Between State and Society: Shamanistic Beliefs in the Song), Hong Kong: Zhonghua shuju.

Wu Hung 2010, The Art of the Yellow Springs: Understanding Chinese Tombs, Honolulu: University of Hawai'i Press.

Xu Guangji 徐光冀 (ed.) 2012, Zhongguo chutu bihua quanji 中國出 土壁畫全集 (Complete Collection of Murals Excavated in China) vol. 2, Beijing: Kexue chubanshe.

Zhou Baozhu 周寶珠 1997, 'Qingming shanghe tu' yu Qingming shanghe xue 〈清明上河圖〉與清明上河學 (The Qingming Scroll and Qingming Scroll Scholarship), Kaifeng: Henan daxue chubanshe. 


\section{PART 4}

\section{Imagining Travelling Medicine}





\section{Images of Healing, Hygiene and the Cultivation of the Body in the Dunhuang Cave Murals}

\author{
WangJinyu 王進玉 ${ }^{*}$ \\ Translated with $\mathrm{Lu} \mathrm{Di}$ 蘆笛
}

Stories of the arrival of Buddhism from India through Central Asia into China recount a succession of delegations of monks who enjoyed audiences at court, beginning with the court of Qin Shi Huangdi 秦始皇帝 (r. 246-210 BCE), first emperor of the Chinese empire. From the mid-1st century $\mathrm{CE}$, members of the royal family apparently began converting to Buddhism, and thereafter the new religion arriving from the West periodically gained imperial sponsorship for the building of monasteries, and official support for the resident communities and their rituals. There were also periods of intense persecution, when Buddhist communities and institutions were destroyed. By the time the building of the Dunhuang cave shrines began, in the mid4th century, Dunhuang was a significant town with a large Chinese population ruled by Turkic nomads. Thereafter, the building and restorations of cave shrines at Dunhuang reached its heyday during the Sui (581-618) and Early Tang (618-907), when the local populace, merchants and the clergy sponsored some 200 excavations and restorations.

Meanwhile the balance of political power in the region fluctuated from the 8th century. While the Tang court was caught up with the Anlushan rebellion (755-63), for example, the Tibetan armies took control of trade nexuses along the routes from the regions of modern Gansu west to modern Tajikistan, Kyrgyzstan and Uzbekistan, and in the winter of 763 , even challenged Chinese power at the Tang capital Chang'an. Nevertheless, mutual knowledge was inevitably gained and religious practices were adopted and adapted during the military contest for the strategic garrison at Dunhuang, which lay at the meeting of the Taklamakan desert and the Hexi corridor leading to central China. We therefore have to assume complex negotiations between the peoples that lived in and moved through the region. In this story, Buddhism and its aesthetics were undoubtedly a cohesive factor in everyday life.

The early impact of Buddhist ideas of medical culture left its mark in China, most famously in Sun Simiao's 孫 思㴞 preface to Qianjin yaofang 千金要方 (Emergency Prescriptions Worth a Thousand Gold Pieces, c. 652) where he made a comment that has been meaningful to many looking for parallels to Hippocratic medical ethics:

Unless otherwise stated all images are provided by the author, Wang Jinyu, with kind permission to publish from the Dunhuang Academy.
Whenever eminent physicians treat an illness, they must quiet the spirit and settle the will, they must be free of wants and desires, and they must first develop a heart full of great compassion and empathy. They must pledge to devote themselves completely to relieving the suffering of all sentient beings. ${ }^{1}$

But there are many other angles from which we can approach the trans-Himalayan history of medicine in this religious context.

In the late 1970s I decided to try a novel approach to analysing the art of Dunhuang, as represented in the grotto murals and painted sculptures, and the images in the manuscripts discovered in the 'Library Cave' (Grotto 17) at the end of the 19th century. ${ }^{2}$ I wanted to see how the Buddhist artistic heritage of the Mogao Grottos could be used as a visual source for the history of science, technology and medicine. In the course of my investigations, much hitherto unknown archaeological evidence for this field came to light (including artwork relating to medicine, health and hygiene), and so this exploratory work pioneered the concept of 'Dunhuang scientific and technical artwork' as a subject of academic enquiry. I first presented my research in a number of articles. ${ }^{3}$ Those were followed by a monograph on the history of science and technology at Dunhuang, which included three chapters with a medical focus: one on Buddhist morality in the provision of medicine, one on the origins of dental hygiene, and one on 'Getting fit, staying in shape, and keeping clean'. ${ }^{4}$ Subsequent studies analysed 23 Dunhuang murals for their illustrations of medieval healing and preventative medicine, as well as hygiene, both personal and environmental. ${ }^{5}$

1 Sun Simiao, Beiji qianjin yaofang 1, 'Preface: Taiyi jingcheng 太 醫精誠, no. 2, p. 1. Tr. Sabine Wilms: https://www.happygoatproductions.com/qianjinfang-ethics (accessed 12/04/2017).

2 'Library Cave' is a common referent for the manuscript depository discovered in 190o. Of course, it is not a library by any modern definition; the term simply refers to the place where the cache of manuscripts was sealed in the early 11th century. There have been many theories proposed about whether the cave was a store for a monastic library or, for example, whether it contained sacred waste.

See Wang Jinyu 1988a and b.

Wang Jinyu 1989, pp. 86-8; 89-91; 92-5.

See Wang Jinyu 2001. This paper falls into three sections: on environmental hygiene and sanitation, cleanliness and preventative healthcare, and the diagnosis and treatment of disease. 




Figure 18.1 Fields of Merit Sūtra painting: regularly offer medical treatment free of charge so as to heal and save from all manner of illnesses. Mogao Grotto no. 296, North Zhou Dynasty (557-81)

When the monk Faxian 法顯 made the perilous journey across the Pamirs in the early $5^{\text {th }}$ century, his mission was to bring home Buddhist scriptures to China. Thanks to his reports, the Indian tradition of sponsoring halls for institutional healthcare also became well known in China, and likewise the traditions of public works, which involved digging wells and planting medicinal herbs. ${ }^{6}$ Personal hygiene had also been a long-standing concern in Indian medicine, since pollutants as 'an unwholesome association of sense and object' $^{7}$ were one of the most basic disease aetiologies in Ayurvedic medicine. While the goal of personal hygiene practices would have been the ritual purity of the body, there is no doubt that many such practices would have been of benefit to the physical health of those who adopted them. So while some of the categories that I have chosen for analysis are certainly modern, like 'environmental hygiene and sanitation', or are derived from modern interpretations and sensibilities, this chapter sets those themes firmly within an transcultural Buddhist context. It provides a window on to the transcultural nature of healing and hygiene through which we see, in breath-taking polychrome, how numerous therapeutic practices entered China in the medieval period. This chapter summarises the aforementioned research under nine headings, and provides essential context for the interested reader in the hope that this will stimulate further research.

$6 \quad$ Wujastyk 20o1, pp. 1-48.

7 Carakasaṃhitā, quoted in Wujastyk 2001, p. 46.

\section{Medicine and Healing}

In the Buddhist art of Dunhuang, the diversity and complexity of everyday life in the medieval period is visualised sometimes directly, sometimes indirectly, but constantly refracted through a religious sensibility. Thus the vignettes of healing and hygiene that are found in the murals function as elements within a larger Buddhist narrative. That narrative has to be understood in light of the many ways in which Buddhist organisations in China rivalled the state in taking responsibility for public works, and created an alternative economic model that was sometimes supported by, and sometimes in conflict with a more centralised authority, particularly when monasteries were allowed to levy their own taxes. Nevertheless, in the many periods when state authority was fractured and fragmented, Buddhist organisation no doubt offered a welcome stability at the local level, building infrastructure and providing essential services. Buddhist authority also functioned as a kind of moral glue, whereby parables and their visual depiction served to instil a system of ethical behaviour transcending the pursuit of personal profit, or allegiances to family or state.

Among the Dunhuang murals there are approximately 30 depictions of medical treatment of various kinds. Interactions between patients and practitioners are portrayed in three Sūtra paintings on the theme of the 'Fields of Merit' (Futian jingbian 福田經變) (Fig. 1), illustrating the meritorious practice of offering medical treatment free of charge, which constitutes one of the seven forms of compassionate service (the remaining six are, in general, 




Figure 18.2 Fields of Merit Sūtra painting: Bone-setting in the fields. Mogao Grotto no. 302, Sui (581-617)

sponsoring images of the Buddha, providing public orchards and washing facilities, building ferries, building new bridges, digging roadside wells, and establishing public toilets). One of these Sūtra paintings is found in Northern Zhou (557-81) Grotto 296, and the other two in Sui $(581-618)$ Grotto 302.

In Figure 1, a male patient lies on his back in the centre of the image, flanked by two family members wearing black, one of whom is spooning medicine into the patient's mouth from a bowl. The patient is naked except for a headdress, which marks his gender. Behind him, the practitioner, his garments now a faded pink, keeps a watchful eye. To the left-hand side, a smaller kneeling figure also in pink, the practitioner's assistant, is preparing medicine with the aid of a pestle. The accompanying Sūtra says: 'Chang shi yiyao liaojiu zhongbing' 常施醫藥療救血病 (Regularly offer medical treatment free of charge so as to heal and save from all manner of illnesses).

In Figure 2, the patient is being tended to in the open air, surrounded by trees and flowers. He is lying on his back with two family members or friends standing by. The practitioner holds his head and neck as if performing some kind of manual bone-setting therapy. Maybe the patient is suffering from a dislocated jaw. This illustration focuses us on the range of practitioners depicted in the murals, and the different types of healing available. It can be compared to the practice of moxa-cautery in the fields. ${ }^{8}$

The Sütra painting on the south wall of Grotto 321, dating from the High Tang (8th century) incorporates a depiction of a patient at home, bent over as if suffering from digestive problems. ${ }^{9}$ Here, the sick man is surrounded by concerned family and friends. The slender figure to the right is identified as a woman by her headdress and skirts. To the left, a group of three people serves to emphasise the involvement of the wider community and their collective responsibility for the care of the sick. This mural was formerly considered to be an illustration of the 'Precious Rain' Sūtra (bayou jingbian 寶雨經變), but in light of research carried out by scholars at the Dunhuang Research Academy (Dunhuang yanjiuyuan 敦煌研究院) in 2004, it has been redesignated as the 'Ten Wheels' Sūtra painting (shilun jingbian 十輪經變) (Fig. 3). The 'Ten Wheels' Sūtra encourages universal responsibility for providing proper care for the sick. High Tang Grotto 74 has a similar mural featuring the same scene. ${ }^{10}$

8 Described by TJ Hinrichs in Chapter 17 in this volume..

9 In the academic history of Dunhuang art, the Tang dynasty is generally divided into four periods: Early Tang (618-704), High Tang (705-780), Middle Tang (781-847), Late Tang (848-9o6).

Wang Huiming 2004. 


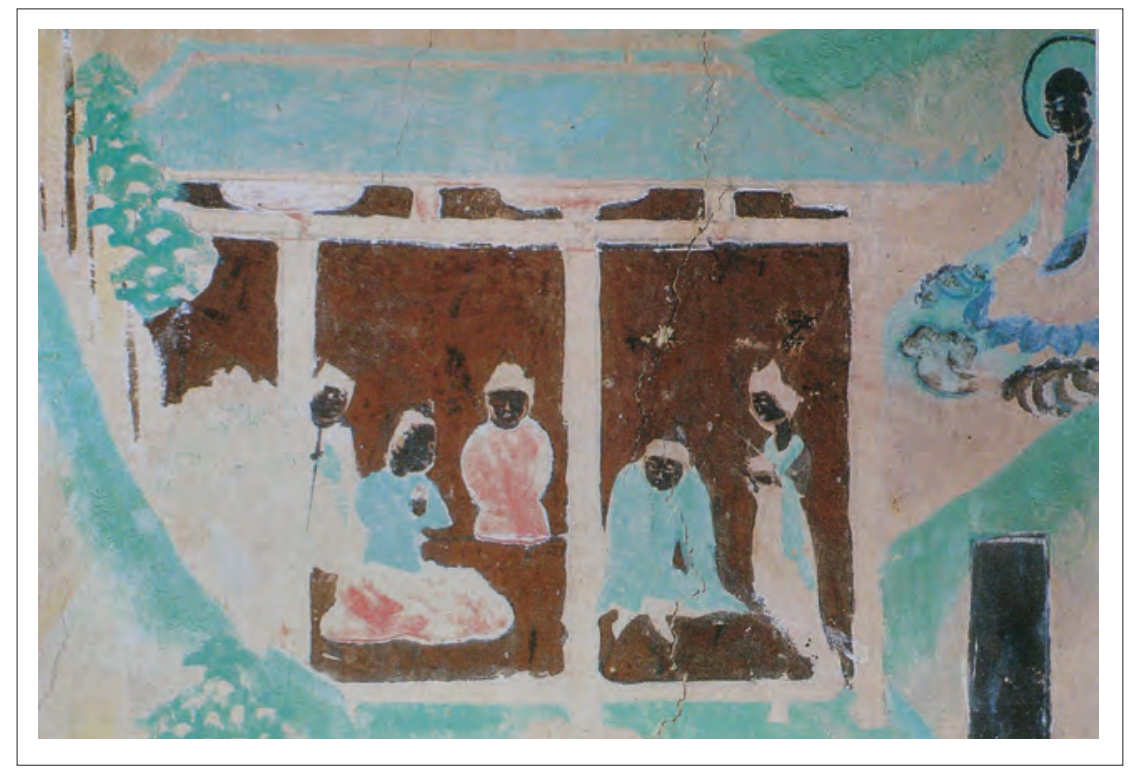

Figure 18.3 'Ten Wheels' Sūtra painting: a universal responsibility to care for the sick. Mogao Grotto no. 321, Early Tang Dynasty (618-712)



Figure 18.4 Lankāvatāra Sūtra: Mogao Grotto no. 61, Five Kingdoms (848-907)

The Dunhuang murals also include 11 allegorical paintings based on the Lañkāvatāra Sūtra (lengjiajingbian 楞伽 經變), one of the first Sūtras to be translated into Chinese. One early rendering is attributed to Zhu Fahu 竺法護 (Sanskrit: Dharmaraksa), who was born in Dunhuang $c$. $233 \mathrm{CE}$, and whose translations were hugely influential in Chang'an and Luoyang. The murals date from the Middle Tang (Grotto 236), the Late Tang (Grottos 9, 85, 138, 156 and 459), the Five Kingdoms period (Grottos 4 and 61), and the Northern Song (Grottos 55, 454 and 456). Most of them show a patient reclining in bed, with one or more attendant figures administering drugs. In the example of Figure 4, from the east ceiling of Grotto 61, we see a patient or possibly a pair of patients lying in bed in an interior, being helped to drink a medical decoction. In each half of the image there is a woman with a high coiffure sitting at the end of a sickbed, with both the surroundings and the patient depicted in a similar manner, except that on the right the practitioner is present and the patient is actually drinking the medicine. The question arises as to whether this is a sequential narrative in strip cartoon fashion with the same patient first becoming sick and then being attended to by the physician. A caption states: 'For example, for the multitude of sick people, a good doctor will go to them and 
give them medicine'. Taken together, these illustrations of the doctor-patient relationship provide a range of contexts within which the medieval doctor administered medicine. The idealised physician was a selfless travelling doctor who might treat a person in the workplace or at home, and perhaps in the company of a number of different assistants. The murals demonstrate the agency of family and community in mediating therapy, and represent the role of the physician in service to his patient.

Themes of medicine and healing also play a major role in the paintings of the Lotus Sūtra (Saddharma PundarikaSūtra), a nother Sūtra apparently first translated by Dharmarakṣa. Yaowang pusa benshi pin 藥王菩薩本事 品 (The acts of the Bodhisattva of Medicine) is the 23rd in a sequence of 28 topoi, which give accessible visual expression to the teachings of Buddhism. Twenty-one examples of this subject survive in the Dunhuang Grottos, and some of them also incorporate the 'Image of finding a doctor' (de yi tu 得醫圖). This image, found in High Tang Grotto 217 (Fig. 5), alludes to a passage in the Lotus Sūtra in which reciting the Sütra is likened to a '... a child finding its mother... / ...a sick person finding a doctor. The Lotus Sütra is just the same as these'.

In the 1950s this painting was reproduced by the distinguished Dunhuang scholar Shi Weixiang 史葦湘, and described in detail for the first time by another distinguished scholar, Chang Shuhong 常書鴻, who noted that the physician is shown conducting a visual examination from outside the chamber where his patient is lying, prior to entering. ${ }^{11}$ In the 198 os the painting was analysed by Wang Daokun 王道坤 and Zhu Yu 朱玉 of Gansu College (now University) of Traditional Chinese Medicine (Gansu zhongyi xueyuan 甘肅中醫學院), who pointed out that the patient is a child. ${ }^{12}$ In this image, the brown-clad doctor, who has a prominent nose and walks purposefully with the aid of a stick, is identified by his large relative size, advanced age and authoritative demeanour; the small white-clad figure of his assistant follows behind carrying a box of medicines. The child sits on her mother's lap, and another, more strongly characterised and portly woman sits cross-legged opposite them. The latter looks like the matron of what is certainly a prosperous household judging by the high quality of the household decoration, and fine architecture of the roof with its elaborately carved eaves (Fig. 5).

The 16th chapter of the Lotus Sūtra, "The lifespan of the Tathagata' (Rulai shouliang pin 如來壽量品), is represented in eight extant murals, which illustrate the text 'What the

$11 \quad$ Chang Shuhong 1956.

12 Wang Daokun and Zhu Yu 1984.



Figure 18.5 1950s reproduction of a Lotus Sūtra painting: Finding a good doctor. Mogao Grotto no. 217, High Tang (705-80)

good physician knows' (Liangyi yu 良醫喻). The southern mural in Grotto 61 portrays a good physician who is prescribing a remedy for a poisoned child, when all hope seems lost. This moving scene first appeared in the Middle Tang. Many illustrations of the chapter of 'The universal gateway of Guanyin Bodhisattva' (Guanyin pumen pin 觀音普門品) contain scenes of people being saved from various calamities by the Bodhisattva. Guanyin was one of the four principal Bodhisattvas in China, and possibly the most popular for her association with childbirth. She links back with the male Bodhisattva Avalokitesvara, who was feminised in China under the Tang.

Grotto 45, and some others dating to the High Tang, contain variants on the same mural, which depicts scenes of people being saved from fire, water, thunder, poison, beasts, snakes and scorpions, the Rāksasa 羅刹 monster, and other disasters such as issues with imperial law. There are also murals illustrating the Sūtra of the Wise and Foolish (Xian yujingbian 賢愚經變). The ‘foolish' may be people who have not yet converted to Buddhism and/or those perceived to be psycho-spiritually abnormal, who are cured after appealing to Guanyin. These murals have a strong religious orientation, and reflect contemporary perceptions of the unenlightened and of disability. Chapter Five, 'Parable of the Medicinal Herbs' (Yaocaoyupin 藥草喻品) is depicted in no fewer than 16 of the Dunhuang murals. ${ }^{13}$ The version in Grotto 23 (Fig.

13 Mogao Grottos 23 (High Tang), 159, 231, 237 (Middle Tang), 12, 85, 
6), dating from the High Tang, shows a rural landscape in the rain, illustrating the text 'Herbs and trees alike grow lush' (Yao shu bing mao 藥木手茂). Farmers are busy ploughing and transporting agricultural produce, and the fields are green and fertile. In the background, rain clouds signify the boundless Dharma, and the ever-present potential for every human being to attain Buddhahood. Texts from a Buddhist Sūtra written beside the image say: 'Yi yun suo yu, cheng qi zhong xing er de shengzhang'一雲所雨, 稱起種性而得 生長 (Rain from a single cloud arouses the essence of the seeds to generate and grow), and 'Gan di pu qia (shi), yaomu bing mao’ 乾地普洽 (濕), 藥木手茂 (Dry land everywhere becomes moist, so herbs and trees both flourish).

Other murals refer indirectly to the healing arts. For example, High Tang Grotto 148 contains a mural about the extraction of Buddha's teeth. The southern mural in Western Wei Grotto 285, entitled Wubai qunzei chengfo 五. 百群賊成佛 (Five Hundred Bandits Convert to Buddhism), portrays the bandits as wailing captives, with their eyes gouged out, but as the Buddha drips fragrant medicinal waters into their eye sockets, they cease to suffer pain and regain their sight.
The painting on the lintel of the western niche in Western Wei Grotto 461 illustrates the Jātaka tale of Śyāma or Shanzi 睒子, who was saved, after an arrow injury, by a celestial being who also administered heavenly drugs to his blind parents so that they could recover their eyesight. The recovery of eyesight in both these cases stands as a metaphor for enlightenment.

Grotto 323 has a mural which illustrates the story of 'Fo Tudeng xi chang' 佛圖澄洗腸故事 (Fo Tudeng washes his intestines) from Gaoseng zhuan高僧傳 (Biography of Eminent Monks), juan 9 (Fig. 7). A man, with a bare upper torso but wearing a cloth around his nether parts, sits cross-legged on a square mat, perhaps in a bamboo grove. He pulls lengths of his intestine from his abdomen and washes them in a nearby brook. Narratives of cleansing the inside of the body have a precedent in Sima Qian's Shiji 史記 (Records of the Historian), where $\mathrm{Yu} \mathrm{Fu}$ 俞跗 is represented as having opened up the flesh of his body and washed out his mai 脉. ${ }^{14}$

In a Buddhist context, 4 th to 5 th century miracle stories of the Bodhisattva Guanyin describe how she would wash out the intestines of devotees while they dreamed in order to heal their sickness. ${ }^{15}$



Figure 18.6 Lotus Sūtra Painting: parable of the medicinal herbs. Mogao Grotto no. 23, High Tang Dynasty (705-80)

138, 156 (Late Tang), 6, 61, 98, 396 (Five Kingdoms), 55, 76, 431, 449 (Northern Song) all contain paintings on the theme of the Parable of the medicinal herbs.
14 Shiji 105, Beijing: Zhonghua shuju p. 2,788.

15 Salguero 2014, p. 131. 


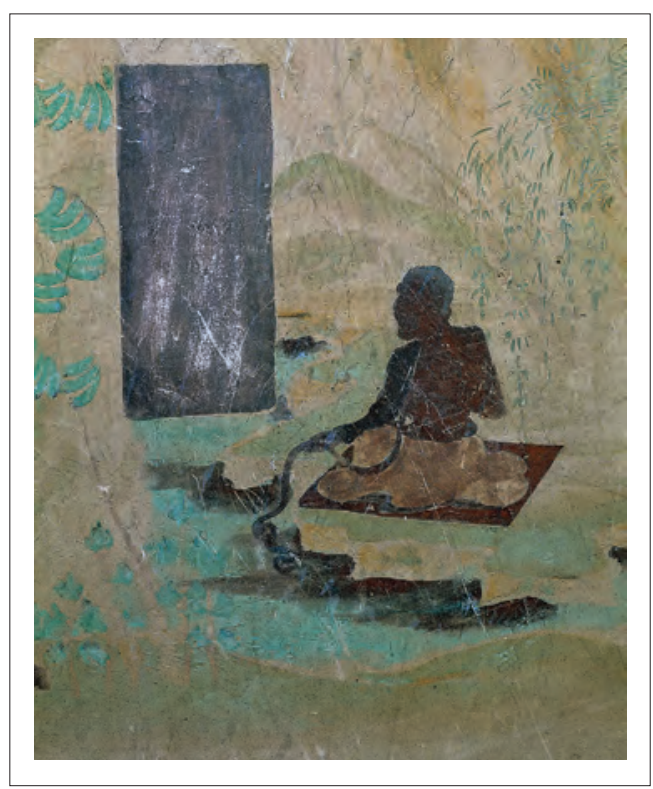

Figure 18.7 Fo Tudeng washes his intestines. Mogao Grotto no. 323, Early Tang Dynasty (618-704)

In the murals, we also see portrayals of people suffering from a variety of medical conditions, for example in the Jātaka tale of King Deer, which is depicted in Northern Wei Grotto 257 (Fig. 8). This well-known story tells of a magical 'nine-coloured' deer who saves a man from drowning. In return, the man promises never to reveal the deer's where- abouts. But when the king of the region wishes to have the pelt of the deer as a trophy, the man assists him in hunting the deer down. The 'nine coloured' deer, awoken from his sleep, recounts the man's betrayal to the king. The king shows mercy to the deer. But as celestial retribution for his betrayal of the deer's kindness, the man contracts a serious case of ulceration, which is graphically illustrated in the murals. We are reminded of the gratitude we should feel to those who perform acts of kindness towards us.

\section{Acupuncture and Moxibustion}

Among the Chinese medical manuscripts preserved at Dunhuang is a work on moxa-cautery which includes diagrams. Assigned the descriptive title Jiujing tu 炎經圖 (Moxa-cautery Charts) by Chinese scholars, it is currently held in the British Library as two separate compilations of fragments, catalogued as S.6168 and S.6262. So far as can be determined, it consists of 18 illustrations with accompanying texts. Five charts are largely intact, but in most cases, only fragments of the illustrations and/or captions survive. All the extant illustrations are full-length diagrams of a human figure, shown either from the front or from the back view (See Wang, Chapter 1, Huang, Chapter 9; Lo and Tlalim, Chapter 19, in this volume).



Figure 18.8 Lotus Sūtra: The betrayal of King Deer. Mogao Grotto no. 257, Northern Wei (386-534) 
Since Professor Ma Jixing 馬繼興 first studied these charts in 1964, they have received attention from numerous Chinese and international scholars. Scholars from Gansu studying medieval acupuncture and moxa-cautery points have not only succeeded in reconstructing 12 of the charts, ${ }^{16}$ but have also replicated their use in a clinical setting. ${ }^{17}$ Lo argues that the Dunhuang moxa-cautery charts were practical in intent: they indicate only those locations to be treated with moxa-cautery, and bear no obvious relation to Qi, the channels, acupuncture techniques or pharmacology. In ignoring more theoretical information they offer first-aid guidance in a simple, accessible format, indicating that the charts were intended for practical use by non-specialists. Together with the large proportion of 'simples' in the Dunhuang remedy literature, they testify to the existence of a distinctive style of 'quick and easy' medicine in the Dunhuang area. ${ }^{18}$

The Tibetan medical manuscripts from Dunhuang, which date from the late Tubo 吐蕃 era (c. gth century), are the earliest extant examples of Tibetan medical literature. They include Tibetan moxibustion fragment P.127, and Tubo Tibetan moxa-cautery charts Pt.1058, both held in Paris at the Bibliothèque nationale de France. The rare books department of the National Library of China possesses three separate photographic reproductions of the charts, but on close inspection, it can be seen that two of the photographs share the same subject matter, and apparently show parts of the same figure. It can therefore be concluded that there are in fact two moxa-cautery charts. The charts consist of line drawings of a standing figure in profile with Tubo traits and distinctive hairstyle. One chart is severely damaged, but the other is preserved intact. The latter has io locations marked in Tubo Tibetan script. Each of location has a line coming from it for a caption, but not all have names (See Lo and Tlalim, Chapter 19 in this volume) .

\section{Imagining Healers}

The grotto art, read together with textual evidence from the Library Cave, gives a sense of the plurality of practitioners and healing practices that were recognised and coexisted in medieval Dunhuang. It is clear that a number of the

\footnotetext{
16 Zhang Nong 1995.

17 Zhang Nong 2001. Lo in Despeux 2010.

18 Xinji beijijiujing 新集備急炎經 (Canon of Emergency Moxa-cautery Remedies, Newly Collected), Jiujing mingtang 炎 經明堂 (Illuminated Hall Moxa-cautery Canon) and Renshen liuzhu人神流注 (The Flow of the Human Spirit) recovered from Dunhuang. See Lo 2005a.
}

medical manuscripts discovered sealed in the Library Cave were in their time available for use by both secular and monastic practitioners. However, it is fair to say that the manuscripts primarily represent literate traditions, whereas the grotto art both represents, and would have been seen by a wider demographic.

According to Tang Liudian 唐六典 (Six Codes of Tang), two doctors of medicine (yixue boshi 醫學博士) and 10 medical students were assigned to serve in each lower prefecture (xiazhou 下州). Brief details of one such scholar-physician are given in Dunhuang manuscript P.2657, Tianbao shizai Dunhuang jun Dunhuang xian chaike bu 天寶十載敦煌郡敦煌縣差科薄 (Book of Official Tasks of the Tenth Year of the Tianbao Reign Period $(742-56)$ in Dunhuang county, Dunhuang prefecture): he was called Linghu Sizhen 令狐思珍, he was then aged 51 , and he was a doctor of medicine and a member of the $Y i$ 翊 (Backup) Guard. He has been identified with a doctor of medicine of that name who served in Shazhou 沙州 (the name of the Dunhuang region in the Tang era) around the Tianbao period. In his official capacity, Linghu Sizhen would have been expected to treat patients in addition to carrying out teaching duties. Dunhuang manuscript P.2005, Shazhou dudufu tujing 沙州都督府圖經, records that in the Tang dynasty, there was a school of medicine in Dunhuang, which was located in the academy of Shazhou, with its own separate faculty offices. According to the Dunhuang manuscripts, there were also Buddhist physicians working within Tang Buddhist temples.

The murals contain lifelike portraits of practitioners including learned physicians of antiquity and folk healers as well as Buddhist monk-physicians, engaged in treating various diseases. Examples are the elderly doctor in the image of 'Finding a doctor' in Grotto 217, and the monk-physician depicted in another version of the same image in High Tang Grotto 31, who is shown sitting on a wooden block cradling a sick child. Images of the monk Suo Falü 索法律 (Dharma Vinaya [Master] Suo), an acknowledged expert in medicine and drugs, also reflect the Buddhist practice of medicine in Dunhuang.

The immortals Po Sou Xianren 婆藪仙人 and Lutou (deer head) Fan Zhi 鹿頭梵志, figures with medical associations, are also frequently to be seen in the Dunhuang murals. Lutou Fan Zhi 鹿頭梵志 a miracle-working doctor often depicted with the eponymous stag's head, appears in murals in 24 grottos dating from the Northern Wei (386-533), Western Wei (535-57), Northern Zhou (557-88), Sui (581-617) and Early Tang periods (Fig. 9). ${ }^{19}$ Lutou Fan

19 The 24 Dunhuang grottos with murals of Lutou Fan Zhi are Northern Wei Grotto 254, Western Wei Grotto 249, Western Wei 
Zhi was an immortal of Indian origin, although not necessarily a Buddhist figure, famed for his wisdom in astrology and medicine. In Grotto 329 he carries a skull with which he performs a divination by knocking and, presumably, reading the sounds in order to tell the physical causes of a person's death. He is depicted as an old brāhmana with curling sideburns and a thick beard. His naked upper body is gaunt, with well-defined ribs, and he wears only a short skirt that hangs from his waist. He looks up at the Buddha, and asks him about the Dharma. This image of ritual healing also attests to the plurality of practitioners that can be detected through close examination of the murals.

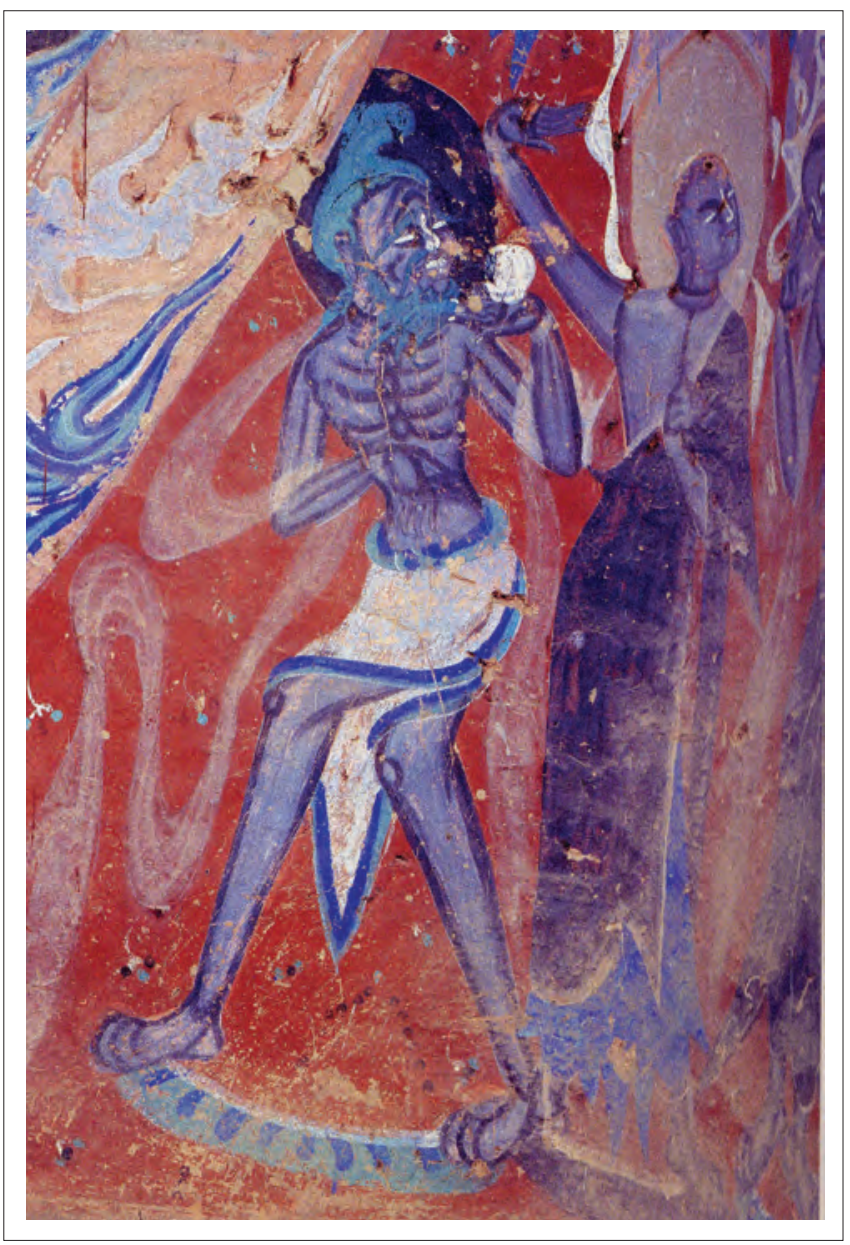

Figure 18.9 Lutou Fan Zhi: the miracle worker with the stag's head. Mogao Grotto no. 329, Early Tang Dynasty (618-712)

Grotto in the Western Thousand-Buddha Cave complex (Xi qian Fo dong 西千佛洞), Northern Zhou Grottos 250, 290, 294, 296, 299 and 438, Sui Grottos 277, 380, 388, 39o, 397, 418, 419, 421, 426, and 427, and Early Tang Grottos 204, 287, 329, 375 and 381. The dear head associates Lutou Fan Zhi with the Deer Head constellation of Orion (Sanskrit: Mrgasirsa).

\section{The Medicine Buddha Paintings}

The paintings of Bhaișajya-guru-vaiḍūrya-rāja Sūtra (Yaoshi jingbian 藥師經變, Sūtra paintings of the Medicine Buddha) are also known as Yaoshi jingtu bian 藥師淨土變 (Illustrated Stories of the Medicine Buddha in the Pure Land) and Dongfang Yaoshibian 東方藥師變 (Illustrated Stories of the Medicine Buddha in the East). For Buddhists, the Sūtra of Bhaișajya-guru - Yaoshi fo 藥 師佛 - the Medicine Buddha, is a potent expression of his arcane divine power, which can bring good fortune and avert disasters, restore the dying to life and health, protect the ruler, and convert any potential misfortune into a good outcome. The incurable or homeless and those who have no access to medical care or relatives to look after them can find comfort and healing by supplicating the Medicine Buddha with appropriate rituals. Their 'Seven Difficulties' can be overcome. The Mogao Grottos now preserve 97 Medicine Buddha mural series, not counting numerous murals on the themes of Jiu heng si 九横死 (Nine Unnatural Deaths) and Shi'er da yuan 十二大願 (Twelve Great Desires) (Fig. 10). ${ }^{20}$

The depictions of a magical healer at work, and the illustrations of the Nine Unnatural Deaths and Twelve Great Desires (of the Medicine Buddha) at either side of the Sūtra paintings of the Medicine Buddha indirectly express the deeply-felt human longing for a safe secure life, free from disease and trauma.

In the High Tang grottos, we begin to see the themes of the Nine Unnatural Deaths and Twelve Great Desires depicted on the left and right-hand panels of murals illustrating the stories of the Medicine Buddha. From Tubo times, this triptych format prevailed. However sometimes the two subjects were treated in separate wall paintings or on folding screens set in niches. The Nine Unnatural Deaths refers to death caused by taking drugs in error; death by imperial sentence; death due to overindulgence in sex, food and alcohol; death caused by fire; death caused by drowning; death caused by fierce beasts; death caused by falling off a cliff; death caused by shigui 尸鬼 'corpse ghosts' and similar beings; and death by thirst or starvation. It is interesting that there was an awareness of the harmful effects of alcoholism, as well as a moral prohibition against excess. The upper caption of the mural of the Nine Unnatural Deaths in Grotto 55 reads: 'Bazhe heng wei duyao qi sishi'八者橫爲毒藥起死時 (The eighth concerns poisonous herbs being the cause of the moment of death)

$20 \quad$ The 97 series can be broken down by period as follows: Sui (4), Early Tang (1), High Tang (3), Middle Tang (21), Late Tang (31), Five Kingdoms (21), Northern Song (9) and Xixia (7). 




Figure 18.10a Sūtra painting of Bhaișajya-guru, the Medicine Buddha. Mogao Grotto no. 85, Later Tang Dynasty (848-907)


Figures 18.1ob-d Sūtra painting of Bhaișajya-guru, the Medicine Buddha. From left to right: b) from Mogao Grotto no. 322, Early Tang Dynasty $(618-712)$; c) from Mogao Grotto no. 310, Northern Song Dynasty (96o-1036); d) illustrating The Nine Abnormal Deaths, here, death by toxic drugs. Mogao Grotto no. 231, Middle Tang Dynasty (781-847)

(cf. Fig. 1od). Paintings on silk from the Library Cave also depict the Nine Unnatural Deaths with captions.

The Medicine Buddha is the great medicine king of the Buddhist Pure Land who cures humankind of ignorance and suffering, and brings comfort to both body and mind. Also known as Dayiwangfo 大醫王佛 (the Great MedicineKing Buddha) and Yiwang shanshi 醫王善逝 (the 'WellGone' Medicine King), he is able to 'save all living creatures from the causes of infirmity, and cure the chronic sickness of benighted ignorance'. Here, the sickness to be treated is not a physical indisposition but an infirmity of nature or character, affecting the sufferer's ability to respond to Buddhist teaching.

The Yin-Yang texts from Dunhuang mention various illnesses and drugs by name, and contain some illustrations. There are seven known exemplars of texts on physiognomy (a diagnostic technique with a very long history in China), all manuscript copies made in the Tang period, three of which are illustrated. ${ }^{21}$

21 Despeux 2005, pp. 176-205. 




Figure 18.10e The Nine Unnatural Deaths: death from consuming poisonous drugs. Mogao Grotto no. 76, Northern Song Dynasty (96o-1036)

\section{Self-Cultivation Practices}

The Dunhuang grottos are also a treasure house of resources for studying ancient forms of physical self-cultivation. In the Chanding 禪定 'Dhyāna' (Chan [Zen]) Grotto, murals, polychrome statues, and epigraphs illustrate some of the same Sūtras that were discovered in the Library Cave. Various forms of self-cultivation are represented in a Buddhist context in the paintings of the Northern dynasties, in the meditation images of various epochs, and in a range of other murals. They comprehend both contemplative practices, which entailed cultivating the breath and static seated meditation, and more physical, muscular and dynamic practices, which involved continuous movement.

Beating and hardening the body was a Buddhist practice. Praying to Gautama Buddha's warrior attendant Vajrapāni, the jingang 金剛 spirit, could make one physically sturdy and capable of magical feats and flying through the air. And beneath the massed ranks of Buddhas and Bodhisattvas on the lower half of at least seven of the Mogao Grottos, there are hundreds of what historians of Buddhist art have called Yao cha 藥叉, a unique form of fighting genie that protected and entertained their spiritual masters. The term Yao cha is derived from the Sanskrit word yaksha, literally the 'wondrous thing', or 'spirit' of non- and pre-Aryan and Vedic ancient Indian religion. These images provide reliable visual sources for the history of internal meditative work on the cultivation of Qi and therapeutic movement in early China, as well as for the martial arts.

On either side of the niche in the western wall of Grotto 272 (Western Liang period, 400-21), there are four rows of gongyang pusa 供養菩薩 (Bodhisattvas of Offerings), ${ }^{22}$

The term gongyang pusa does not originate in Buddhist scriptures. With the exception of the genres of illustration that nar- with five Bodhisattvas in every row. There are two main schools of thought about the significance of these 40 Bodhisattvas: those who believe them to be dancing (performing wudao 舞蹈), versus those who argue that they are practising an early form of Qi cultivation with Buddhist characteristics.

Scholars who hold that the Bodhisattvas are dancing focus on the flowers that some of them carry, and the attitudes in which they sit or kneel before the Lotus Throne. For those scholars, the dance-like attitudes (Figs 11a, 11b) are a rapturous response to the Buddha's sermons. People holding the latter opinion point to the Bodhisattvas' seated poses - with their legs-crossed in padmāsana (Lotus Posture), or angled to one side with a single leg crossed which resemble the kind of static Chinese versions of breath cultivation meditations that are described as 'Brahmanic' in Sun Simiao's Qianjin yaofang, chapter 27, while their hand and arm gestures capture the intention of guiding and directing Qi common to the ancient Chinese practice of therapeutic gymnastics known as daoyin 導引 (guiding and pulling). Some even identify the circles painted on the Bodhisattvas' abdomens as the dantian 丹田 (Cinnabar Field), a vital centre of alchemical transformations in the body. The Dunhuang Buddhist texts also contain records of the human body in upside-down poses, and some scholars link these and related images in the early paintings of Buddhist stories in Dunhuang with modern yoga practice. ${ }^{23}$

rate Buddhist stories or the Buddha's sermons, wherever one sees an individual or group worshipping the Buddha, and Bodhisattvas are involved, they are referred to collectively as gongyang pusa. Such illustrations are scattered throughout the grottos of the Northern Liang (397-46o) and Yuan (1271-1368) periods in Dunhuang.

23 See Mogao Grottos 251 (Northern Wei), 249 (Western Wei), 220 

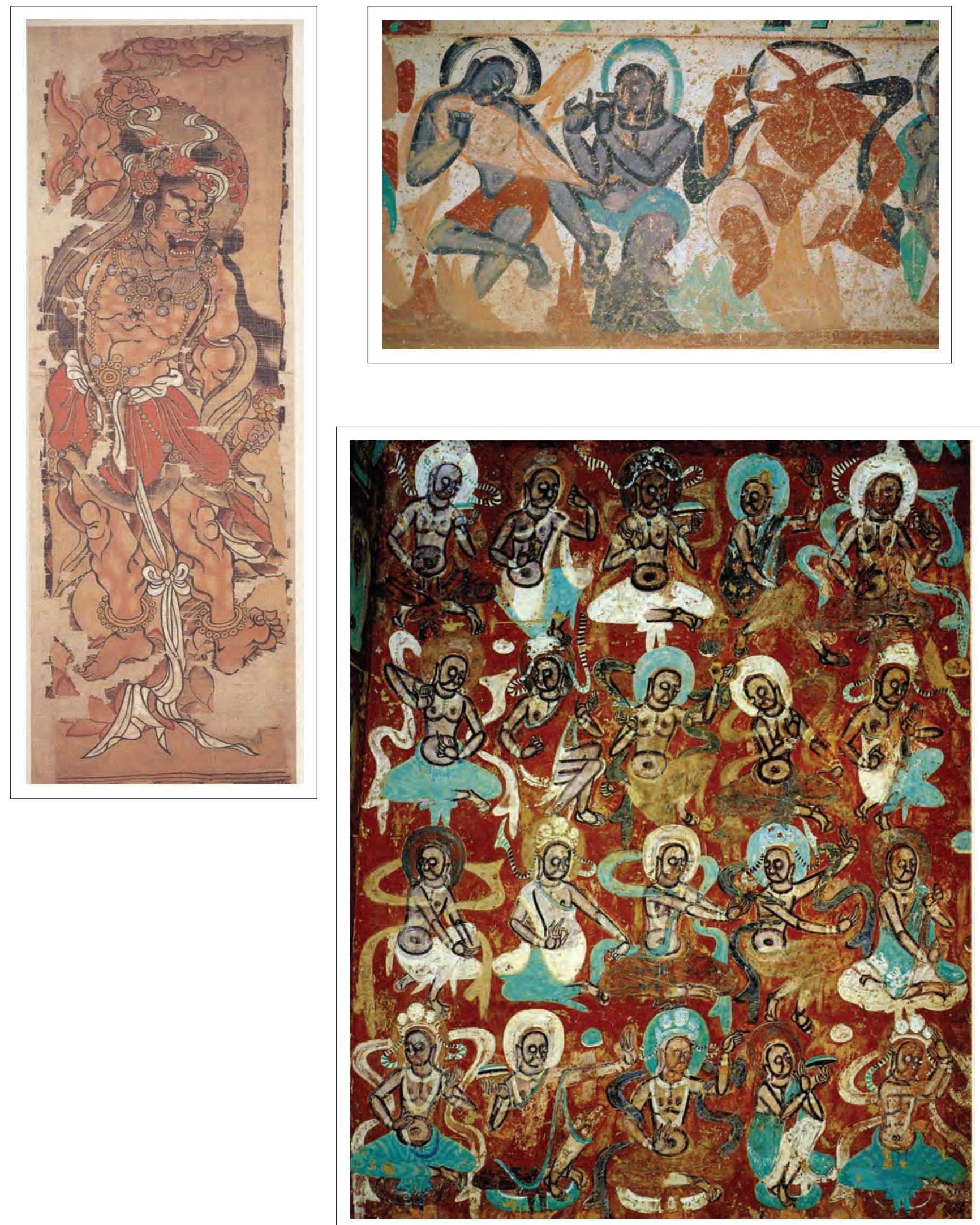

Figure 18.11a (top left) Vajrapāni, wielding a flower-like vajra in a 9th-century painting from Dunhuang. $\odot$ Trustees of the British Museum, BM 1919,0101,0.132

Figure 18.11b (top right) Hundreds of Yao cha (Yakshas) line the lower half of at least seven of the Mogao Grottos. Mogao Grotto no. 249, Western Wei Dynasty $\left(535^{-57}\right)$

Figure 18.11c (below) Worshipping Bodhisattvas: in rapturous dance or self-cultivation? Mogao Grotto no. 272, Northern Liang (397-439) 




Figure 18.12a 'Contest between Sariputra and Raudraksa' (Laoducha dou sheng bian 勞度叉鬥聖變). Mogao Grotto no. 196, Late Tang Dynasty (848-907)

\section{Hygiene, Healthcare and Disease Prevention}

In 1951 an exhibition of cultural artefacts from Dunhuang was held at the Historical Museum of Beijing. It prompted the publication of the first ever historical study of oral hygiene in China. ${ }^{24}$ In this context I identified the illustration of tooth-cleaning in the Amitabha-Sütra paintings (Milejingbian 彌勒經變) in mid-Tang Grotto 159 as China's earliest painting on the subject of oral hygiene. ${ }^{25}$ In the following discussion of various traditions of cleansing the body, we will see that what nowadays counts as a hygienic practice functioned as a key ritual element in choosing a spiritual path in Buddhism, where cleansing the body was tantamount to cleansing the soul.

(Early Tang), 79 (High Tang), 158 and 361 (High Tang), 156 and 196 (Late Tang). Zhou Zongqi 1957

25
An exhibit of particular interest to historians at the 1951 exhibition was the illustration of tooth cleaning from the Sūtra painting of the 'Contest between Sariputra and Raudraksa' (Laoducha dou sheng bian 勞度叉鬥聖變) in Grotto 196 (Fig. 12a). This is one of the most ambitious and successful visual accounts of Buddhist stories. Other murals also contain numerous images of hygiene and body care as they were performed in ritual pursuit of spiritual purity. Of the 18 illustrations of this contest found in the Mogao Grottos and the nearby Yulin 榆林 Grottos at Anxi 安西, dating from the Late Tang, Five Dynasties and Northern Song periods, almost all show Buddhist monks carrying out everyday activities such as bathing, shaving the scalp or face and cleaning the teeth.

This story originates from the 1oth chapter, Xuda qijing she pin 須達起精舍品 (Sudana builds a Vihara) of the Damamūka-nidāna Sūtra (Xianyujing 賢愚經). It narrates Raudraksa's contest with Buddha's student Sariputra: Raudraksa fails several times to overcome his opponent, and finally he and his followers convert to Buddhism. 




Figure 18.12b Teeth cleaning and washing the head. Mogao Grotto no. 196, Late Tang Dynasty (848-907)

Numerous converts to Buddhism are depicted on the lower right side of Sariputra's Lotus Throne, including many who are in the process of initiation into Buddhism and are thus undertaking the inaugural rituals of tonsure, washing their heads and mouths, and cleaning their teeth.

At the bottom of the image there are two men washing their hair and cleaning their teeth (Fig. 12b). Elsewhere, to the lower right-hand side of the Bodhisattva, there is a man gargling and directly to the right a person is having his head shaved.

In these fascinating scenes a tonsured person sits on the ground, one hand in the act of pouring water over his head, the other hand busily washing his head; another tonsured person squats on the ground, holding a flask of water in his left hand, while in his right hand he holds a willow stick with which he brushes his teeth; a tonsured person next to him, clad in a robe, touches his bare head with one hand and clutches his face with the other, his mouth wide open in laughter; another tonsured person, near the Lotus Throne bends over to wash his head, with his legs crossed, his buttocks raised high, and his eyes comically, but also rudely, looking back between his legs; two students of the Buddha, to the right of the Lotus Throne, are tonsuring two ordinands seated on high stools; beside them, a tonsured ordinand sits on the ground, holding a flask of water to his mouth (Fig. 12a).

In addition to a considerable number of partially effaced and faint images of people cleaning their teeth, we can distinguish eight good surviving images of tooth cleaning in the 18 murals that illustrate the battle between Raudraksa and Sariputra. ${ }^{26}$ The vivid image in Grotto 9 also depicts a person cleaning his teeth: he wears a robe, is bare-headed, and has bracelets on his wrists; in his left hand, he holds a

26 The eight images are found in Mogao Grottos 9 and 196 Late Tang), and 98 (Five Kingdoms), and Yulin Grottos 16 and 32 (dates unknown?), and 25, 55 and 454 (Northern Song).



Figure 18.12c Teeth cleaning from Mogao Grotto no. 159, Middle Tang (781-847).

bottle of water, while the thumb and middle finger of his right hand grip a stick which he is using to brush his teeth. The stick being on the right side of his mouth, the right corner of the mouth is open. This stick, made of a willow twig, can be judged the earliest form of toothbrush in China. However, it is unusual. The images of teeth cleaning in murals in other grottos show the instrument as a finger rather than a stick.

There are also several dozen illustrations of the Amitabha-Sūtra dating from the Tang to the Song, most of which include images of novices undergoing tonsure, as well as male and female figures engaged in hygienic and body care activities such as hair cutting, bathing, shaving, rinsing the mouth, and cleaning the teeth. The image of tonsure in the Amitabha-Sūtra painting in Grotto 445 (High Tang) depicts Bhiksus and Bhiksunis: male and female ordained monks and nuns (Fig. 13). It shows the ritual hygiene required of initiates. Shaving the head heralds an important transition. The devotees being tonsured have towels draped around their shoulders; the shorn hair is transferred to a plate, beside which there is a pot filled with holy water and an elaborately decorated basin, used for washing the head after tonsure. ${ }^{27}$

In contrast to more than 80 images of tonsure in Amitabha-Sūtra paintings, there are nine images of cleaning teeth dispersed through the Mogao Grottos. ${ }^{28}$ The portrayal of teeth cleaning and other ritual ablutions in Grotto 159 is especially vivid and dynamic (Fig. 12c). On the left-hand side, we see a man with a bare torso, a towel draped around his neck and dangling down his back. With one hand, he reaches into a big basin, while with the other he washes his head. In the centre top, a man sits on a low

27 Wang Jinyu 1988b. This study was reprinted the following year by Xinhua Wenzhai 新华文摘 (Xinhua Digest), and then received attention from the academic community

28 Grottos 154, 159, 186, 361 (High Tang), 12 (Late Tang), 146 (Five Kingdoms) and 7 (Northern Song). 




Figure 18.13 Tonsure ritual: Mogao Grotto no. 445, High Tang (705-780)

stool, washing his head in a big basin; a person stands beside him, holding his clothes. Towards the right-hand side, another figure squats on the ground; bare-headed and naked to the waist, he also has a towel wrapped round his neck. He holds a flask of water in his left hand, and cleans his teeth with the forefinger of his right hand. The person standing next to him, dressed in a red robe, holds out another towel. These dynamic images of people frozen in a moment of religious transition realistically reflect ritual and also mundane hygiene at that time, and reveal how, in the Middle Tang period not only monks in Buddhist temples, but also land owners, officials, and nobles had developed the good habit of cleaning their teeth.

The image of 'Washing the feet of Sakyamuni' in the illustration of the Vajracchedīkā Sūtra (Jingangjingbian 金剛經 變, Diamond Sūtra painting) on the south wall of Grotto 31 (Fig. 14) alludes to the lines, 'When he had finished eating, he put away his robe and bowl, washed his feet, arranged his seat, and sat down'. Sakyamuni sits on a dais, his right foot in a bowl of water. A middle-aged laywoman is washing his feet. This is the earliest known depiction from China of foot washing as a hygienic devotional practice.

The same image type can be found in nine grottos in conjunction with images of eating rice (Fanshi tu 飯食 圖). ${ }^{29}$ The mural on the theme of washing the feet in Grotto 156 portrays Buddha seated on the Lotus Throne, with his

29 Grottos 112, 147, 150, 198, 359 and 361 (Middle Tang) and 85, 138 and 156 (Late Tang). right leg crossed over the left, and his left foot in a basin; a Bhiksu kneels by the basin and washes the Buddha's feet, and five Bhiksus stand behind the Buddha. In Grotto 85 , the person washing Buddha's feet is a young woman adorned with the robes of a Bodhisattva but without the customary nimbus. A black caption on a red background to the right-hand side of this image explains to the faithful that the Buddha is having his feet washed after his meal.

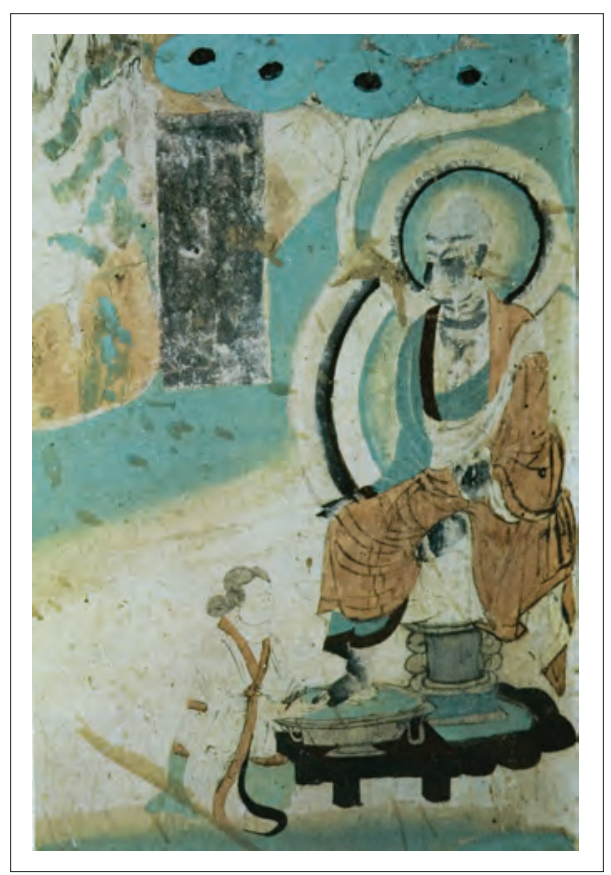

Figure 18.14 Washing the Feet of Sakyamuni: Mogao Grotto no. 31, High Tang Dynasty (705-80) 




Figure 18.15 Boiling milk: Mogao Grotto no. 61, Five Dynasties (9o7-6o)

There are also images of food safety and healthcare in Dunhuang murals. The images of Ananda qiru 阿難乞乳 (Ananda Begging for Milk) that feature in paintings of the Vimalakīrti Nirdeśa Sūtra all portray scenes of milking. ${ }^{30}$ One such image in the murals illustrating stories from the life of the Buddha in Grotto 61 depicts everyday scenes of milking cows and boiling milk (Fig. 15): four dairy cows of various sizes stand on the right-hand side; a woman kneels besides a black cow with a jar in one hand, while with the other hand she milks the cow; in the centre, a woman squats by a three-legged ding 鼎 vessel, which contains steaming hot milk; the black characters against a red background read 'ernüzhuru'二女者乳 (Two women boiling milk); on the left-hand side, a woman kneels on the ground, holding in both hands a bowl of milk, which she offers to Ananda. This rare image of milking cows and boiling milk is a precious resource for research into food safety and food therapy in the Five Kingdoms period. It also indicates that at that time people in northwest China were in the habit of drinking boiled milk.

In later Chinese medical treatises, milk is attributed with various beneficial effects, including restoring the wellbeing of people with deficiencies and weakness, quenching thirst, nourishing the heart and lungs, detoxifying heat poisons, and moistening the skin. The association of milk with treating stomach pain, and various deficiencies, particularly in connection with the syndrome jiaoqi 腳氣 (in modern times, the term for beriberi, a thiamine deficiency disease) is important for the study of early food therapy practices, and is worthy of further research. Chen Cangqi 陳藏器, quoted in Li Shizhen's 李時珍 Bencao Gangmu 本草綱目 (Compendium of Materia Medica, first published 1596),

Mogao Grottos 31 (High Tang), 159 (Middle Tang), 9 (Late Tang), 61 and 146 (Five Kingdoms), and Yulin Grotto 32 (Five Kingdoms). states that the milk from black cows is better than that from yellow/brown cows; milk should be boiled once or twice, and then allowed to cool before drinking, as consuming hot milk will cause yong 癌准 (obstructions) in the body.

\section{Environmental Hygiene and Sanitation}

The murals also portray everyday activities relating to public health and safety and the care of the environment, such as gardening, sweeping, fencing off wells, and constructing latrines. 'Foshuo zhude futian jing' 佛說諸德 福田經' (the Buddha's sermons on various virtues and merits) promotes seven activities; the second consists of irrigating orchards and providing shade by planting trees, and the seventh is building public toilets and making provision for people to relieve their bowels. The mural on the eastern slope of Grotto 290 (Northern Zhou) portrays two persons engaged in sweeping a peaceful courtyard filled with flowers and trees, while another defecates in a toilet with a roof (Fig. 16). The Fields of Merit Sūtra paintings in Grotto 302 (Sui) portray scenes of planting orchards and installing baths, and feature two naked persons washing in an outdoor bath surrounded by trees. Covered wells are depicted in murals illustrating Buddhist stories of merits in Grottos 296 (Northern Zhou) and 302 (Sui), and also in some Buddhist historical paintings of the Tang and Song. The mural on the theme of 'Sudana taizi bensheng' 須達 拿太子本生 (The Story of Prince Sudana) on the eastern slope of the upper part of the main chamber in Grotto 491 (Sui) also portrays a well.



Figure 18.16 Sweeping-up and the latrines: Mogao Grotto no. 29o, Northern Zhou Dynasty (557-81) 




Figure 18.17 Rāksasa (Ye Hua葉華) sweeps the city clean and the Dragon King sends down rain: Anxi Yulin Grotto no. 25, Middle Tang Dynasty (781-847)

Six further Dunhuang caves, and the Buddhist historical murals on the southern wall of Yulin Grotto 38 all contain images of wells. ${ }^{31}$ One mural shows a person wearing a long robe, extending one of his arms towards a square fence around a well, in sign of his inclination to drink water. Most of the wells have high protective fences surrounding them; the lower part of the fence consists of courses of stone or bricks, while the upper part is made from wooden sticks and boards. ${ }^{32}$ Well fences and covers were not only safety features, but also protected against water pollution from debris and dust, especially when the wells were constructed in the vicinity of roads where they would have been vulnerable to both human and animal excrement.

The Sūtra paintings of Maitreya proclaim the transcendent beauty of Maitreya Pure Land, emphasising both its aesthetic and its hygienic qualities. For example, illustrations of the story of Maitreya begging in the city include depictions of the Dragon King who brings showers of rain at night, as well as the Rāksasa called Ye Hua 葉華 who sweeps the city clean. Dunhuang murals depicting the incarnation of Maitreya from the High Tang to Northern Song contain 16 images of the Dragon King, and 18 images of the Rāksasa Ye Hua. ${ }^{33}$ The mural painters have conflated both scenes, which occur in the narrative at different times of the day, into a single image. Typically, the Dragon King gathers the rain in a cloud above the city, sending it down in gentle showers at night to irrigate the land and freshen the air, while outside the city, Ye Hua, with his upper body naked, sweeps the ground perfectly clean with a broom (Fig. 17). Such images were obviously intended to propagandise the Maitreya Pure Land, but they also inevitably reflected the activities of real-life cleaners.

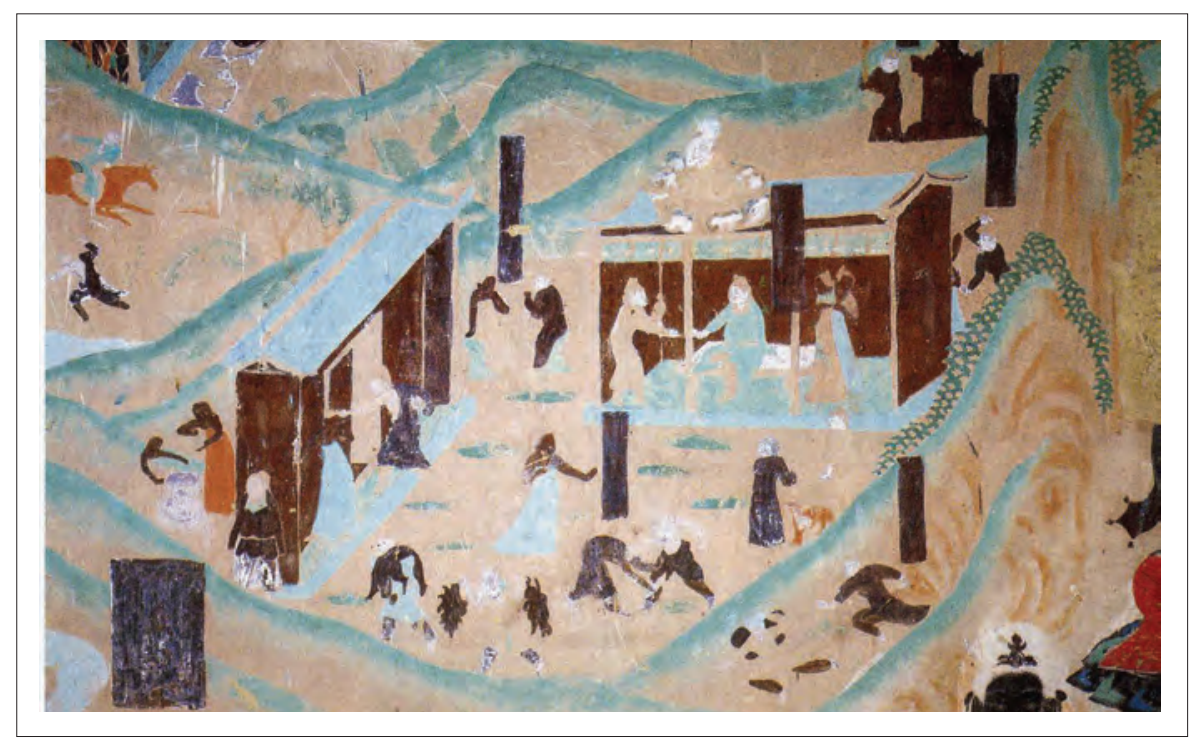

Figure 18.18 Martial arts: Mogao Grotto no. 321. Early Tang Dynasty (618-712)

See the corridors of Grottos 334 and 340 (Early Tang), 39 (High Tang), 98 and 146 (Five Kingdoms), and 454 (Northern Song).
See, for example, Yulin Grotto 38 .

See, for example, Grottos 33 (High Tang; Fig. 17), 445 (High Tang), 202 and 231 (Middle Tang), 9 and 156 (Late Tang), 146 (Five Kingdoms), and 25 and 55 (Northern Song). 




Figure 18.19 Handstand: Mogao Grotto no. 249, Western Wei Grotto $(535-57)$. From his prominent nose and dark skin the figure seems to intend to represent an acrobat from further west.

\section{Sports and Athletics}

In their survey based on an examination of 62 Mogao Grottos and descriptions by scholars of the Dunhuang Research Academy, Zhao Jianxiong 趙健雄 et al. present visual data under three headings - exercise and sport, hygiene and healthcare, and the diagnosis and treatment of disease - and they find only three examples of exercise and sport, from Grotto 272, Western Wei Grotto 285, and Five Dynasties Grotto $98 .{ }^{34}$ I have already suggested in a previous article that this number would have risen to several hundred, if they had not chosen to treat their three categories as mutually exclusive. ${ }^{35}$ In fact the Grottos provide an abundance of compelling visual evidence for research into the history of sports and athletics. (Figs 18, 19).

\section{Tantric Practices}

It used to be thought that early Chinese visual culture did not represent the naked body. Recent scholarship has shown differently, with illustrated manuscripts from the Dunhuang collections, in particular, using nudity to aid the understanding of physiognomy or to exemplify techniques of fangzhong shu 房中術 (arts of the bedchamber) ${ }^{36}$ While

Zhao Jianxiong, Xu Hongda, Wang Daokun et al. 1985.

Wang Jinyu 1988a.

Manuscript P.3589. See Lo and Cullen 2005, p. 232; Harper 2010.



Figure 18.20 Tantric sexual union between deities: Mogao Grotto no. 465. Yuan period (1271-1368)

the latter are associated with health-promoting activities since they were thought to induce an enhanced state of well-being, it is less appropriate to link medieval Tibetan esoteric sexual practice with medicine. Illustrating sexual union between deities and their consorts was a part of the culture of Tibetan rgyud (Tantra) or rdo rje theg pa (Vajrayāna) where the 'divine embrace is a metaphor for the union of eternal bliss and sunyata (the impermanence of phenomena), or wisdom and compassion'. ${ }^{37}$

Mogao Grotto 465 is the only cave in Dunhuang with evidence of Vajrayāna imagery, albeit in a rather different style to other Tibetan paintings on similar themes, which have been reliably dated to a similar period but are located far away in the Ladakh region of Western Tibet. The cave is organised around five groups of colour-coded Dhyani Buddhas of the Five Directions, with a Vairocana Buddha at the centre. The murals themselves are devoted to Anuttara yoga, ${ }^{38}$ representing its focus on the transformation of sensual pleasures to aid spiritual practice, and are located on the second level of the northern side of the cliff in the northern zone.

In particular, each of the southern, western and northern walls of the Grotto contains three sets of mandala murals portraying tutelary deities, male and female vajras,

Dunhuang Research Academy website, Mogao Cave 465 (Yuan period, 1271--1368 CE): http://public.dha.ac.cn/content.aspx?id $=528857548943($ accessed 12/04/2017).

$38 \quad$ Samuel and Johnston 2013, pp. 22-47. 




Figure 18.21 Tantric sexual union between deities: Mogao Grotto no. 465, Yuan period (1271-1368)

referred to in modern Chinese literature as huanxi Fo 歡 喜佛 (happy Buddhas). But the purpose of these unions is not simply to generate pleasure and happiness, or even to perform the kind of bringing together of Heaven and Earth that we know from the Chinese arts of the bedchamber. The sexual union brought about by the coupling of these deities is a ritual generating the immense power and fury deployed in smashing demons. ${ }^{39}$

The three murals on the southern wall depict female and male vajras in sexual union and quelling demons. The mural to the east portrays a male vajra with four faces and four arms, wielding knives, bowls and bows, with one foot subjugating a recumbent demon; the female vajra, Khandroma (Fokong xingmu 佛空行母), has one face but four hands grasping an axe-like knife, a bowl, and the male vajra himself(Fig. 20). Generally temperamentally volatile, this female deity also becomes a muse in more spiritually oriented meditational practice. The middle mural portrays the male Guhyapada vajra (Mijijingang 密跡金岡 $)^{40}$ with his three faces and six arms, carrying an axe-like knife, a bell, a pestle, a flower, a bowl, and a sword; the female

39 One of the scrolls recovered from Dunhuang, P.2702 has an inscription in Tibetan on the verso that reads: 'Spring palace (an allusion to erotic art) paintings of stone lions and birds and beasts.' The first four paintings on the scroll show men and women copulating.

Some scholars consider the figure to be the Kalacakra vajra (shilun jingang 時輪金剛). has one face and six arms, and embraces the male vajra (Fig. 21). The western mural portrays Mahabala vajra (Dali jingang 大力金剛).

On the north wall at the centre there is a portrayal of the Hejavara, a principal yidam deity of the Anuttara yoga sect, an enlightened being, embracing his consort Nairatmyā. Hejavra, with his standard 16 arms, wears a skull crown and is decorated with garlands of some 50 human heads. Here are also, perhaps, links to the Sakya sect who were particularly associated with the cult to Hevajara, one of the two major Tibetan traditions operating at the Mongol court. ${ }^{41}$

The Dunhuang Academy has dated Grotto 465 to the Yuan period, although some scholars believe that the cave itself was excavated much earlier - there being substantial evidence of visitors' inscriptions from the Xixia 西夏 Tangut period of rule at Dunhuang (1032-1227) in neighbouring Grotto 464 . Grottos 464 and 465 share visual motifs such as the Tangut High Lamas' headdresses, which appear to derive from the lotus hats of the Nyingma lineage of Tibetan Buddhism. ${ }^{42}$

\section{Conclusion}

The visual art of Dunhuang is a rich resource for the whole range of human emotion and experience. Figures immersed in everyday life as well as those on a spiritual path, and the deities themselves, embody delight, anger, gloom, longing, sadness or surprise, and throughout, emphasise a recognition of the sacred as it was ever present in life at the Mogao Grottos. Themes of birth, healthcare, aging, illness, and death saturate the imaginary preserved for posterity in the desert caves. The murals, both dynamic and affecting, are the finest of medieval Buddhist artworks. Their illustrations of the surrounding religious landscape weave through successive periods of political rule, in a locality that captures for us Sino-Tibetan-Tangut-Indian, and many other cultural exchanges. That these murals also provide a visual record for themes that are pertinent to how we now think about medicine is somehow incidental; yet at the same time, it is critical to redressing Eurocentric histories of the world. Approaching the murals as a source for the history of disease, public and environmental health and hygiene may verge on the teleological, yet placed within a rich social and cultural context they are, as yet, an unexplored resource for understanding new religious frameworks for innovation in the effective care

\footnotetext{
$41 \quad$ Duan Wujie 1994.

$42 \quad$ Xie Jisheng 2004.
} 
of the body, and strategies for surviving the vicissitudes of life - an appropriate critique of enduring fixations with the supremacy of a European scientific modernity. Much remains to be learned about the history of healing and healthcare from the art of Dunhuang; there is new material still remaining to be excavated, and many new topics still awaiting critical research.

\section{Bibliography}

\section{Primary Sources}

Sima Qian司馬遷, 1st century BCE, Shiji 史記 (Records of the Historian). Modern edn, 1972, Beijing: Zhonghua shuju.

Sun Simiao孫思㴞, c. 652, Beiji qianjin yaofang 備急千金要方 (Emergency Prescriptions Worth a Thousand Gold Pieces). Modern edn, 1995, Shanghai: Shanghai guji chubanshe.

\section{Secondary Sources}

Chang Shuhong常書鴻 1956, 'Dunhuang bihua zhong de lidai renmin shenghuo hua', 敦煌壁畫中的歷代人民生活畫 (Everyday life of people through the ages in the Dunhuang murals), Wenwu cankao ziliao 2, 7-10 (illustrations).

Despeux, C. 2005, 'From prognosis to diagnosis of illness in Tang China', in Lo and Cullen (eds), 176-205.

Despeux, C. (ed.) 2010, Médecine, religion et société dans la Chine médiévale: Étude de manuscrits chinois de Dunhuang et de Turfan (3 vols), Paris: Collège de France, Institut des hautes études chinoises.

Duan Wenjie 段文傑 1994, Dunhuang Art Through the Eyes of Duan Wenjie, edited with introduction by Tan Chung, New Delhi: Indira Gandhi National Centre for the Arts.

Dunhuang Research Academy, website: http://en.dha.ac.cn/ (accessed 26/06/17).

Harper, D. 2010, 'La Littérature sur la sexualité a Dunhuang' in Despeux (ed.), 871-95.

Li Liangsong 李良松 and Liu Jianzhong 劉建忠 (eds) 1995, Foyizongheng - shoujie quanguo fojiao yiyao xueshuyantaohui lunwen huibian 佛 醫縱橫一首届全國佛教醫藥學術研討會論文彙編 (Intersections of Buddhism and Medicine: Proceedings of the First National Symposium on Buddhist Medicine), Xiamen: Lujiang chubanshe.

Lo, V. 2005a, 'Quick and easy Chinese medicine: the Dunhuang moxibustion charts', in Lo and Cullen (eds), 227-51.

2005 b, 'Self-cultivation and the popular medical tradition: Introduction', in Lo and Cullen (eds), 207-25.

2010, 'Manuscripts de Dunhuang et de Khotan sur la moxibustion', in Despeux (ed.), vol. 1, 239-84.

Lo, V. and C. Cullen (eds) 2005, Medieval Chinese Medicine: The Dunhuang medical manuscripts, London; New York: RoutledgeCurzon.

Salguero, C. Pierce 2014, Translating Buddhist medicine in medieval China, Philadelphia: University of Pennsylvania Press.

Samuel, G. 2013, 'The subtle body in India and beyond' in Samuel and Johnson (eds), 22-47.

Samuel, G. and J. Johnston 2013 (eds), Religion and the Subtle Body in
Asia and the West: Between Mind and Body, London and New York: Routledge.

Shi Weixiang 史葦湘 1980, 'Dunhuang Mogaoku zhong de Futian jingbian bihua' 敦煌莫高窟中的《福田經變》壁畫 (Futian Sūtra murals in the Mogao Grottos at Dunhuang), Wenwu 9, 44-8.

Wang Daokun 王道坤 and Zhu Yu 朱玉 1984, 'Dunhuang yixue chutan' 敦煌醫學初探 (A preliminary study of medicine at Dunhuang), Gansu zhongyi xueyuan xuebao 1.1, 36-9.

Wang Huiming 王惠明, 'Dunhuang $321 \mathrm{ku}, 74 \mathrm{ku}$ shilun jingbian kaoshi' 敦煌321窟、74 窟十輪經變考釋 (An examination of the illustrations of the Ten-Wheel Sūtra paintings in Dunhuang Grottos 321 and 74), Yishushiyanjiu 6, 309-36.

Wang Jinyu 王進玉 1988a, 'Dunhuang bihua zhong de kexue jishu' 敦 煌壁畫中的科學技術 (Science and technology in the Dunhuang murals), Ziran zazhi 11, 862-868, 8o8, front cover, inside front cover, inside back cover). 1988b, 'Dunhuang shiku zhong de gudai keji chengjiu' 敦 煌石窟中的古代科技成就 (Achievements of early science in the Dunhuang Grottos), Kexue 4, 307-10, inside back cover, reprinted 1989 in Xinhua Wenzhai 1, 175-9.

1989, Manbu Dunhuangyishu keji hualan 漫步敦煌藝術科 技畫廓 (A Stroll Through the Science and Technology Gallery of Dunhuang Art), 1st edn, Beijing: Kexue puji chubanshe.

1995, 'Dunhuang shiku yiliao weisheng bihua diaoyan' 敦 煌石窟醫療衛生壁畫調研 (An investigation of the Dunhuang medical murals), in Li Liangsong 李良松 and Liu Jianzhong 劉建 忠 (eds), $76-82$.

2001, 'Xingxiang shengdong de yiliao weisheng huamian' 形 象生動的醫療衛生畫面 (Lifelike images of healing and hygiene), in Wang Jinyu (ed.), 247-69.

Wang Jinyu 王進玉 (ed.) 2001, Dunhuang shikuji:Kexue jishu huajuan 敦煌石窟全集 - 科學技術畫卷 (Dunhuang Compendium: The Scientific and Technical Scrolls), Hong Kong: Shangwu yinshuaguan

Wilms, S., Happy Goat Productions, online at: https://www.happygoatproductions.com (accessed 26/o6/17).

Wujastyk, D. [1998] 2001, The Roots of Ayurveda: Selections From Sankskrit Medical Writings, London; New York: Penguin Books.

Xie Jisheng 謝繼勝 2004, 'The Murals of Mogao Cave 465: new evidence for 12 th century Tangut Xia patronage', Orientations 35.5 (June), 38-45.

Zhang Nong 張儂 1995, 'Dunhuang “Jiufa tu” cantu ji guxue de yanjiu’ 敦煌《多經圖》殘圖及古穴的研究 (Study of the Dunhuang 'Moxibustion Chart' fragments and acu-moxa locations in antiquity), Dunhuang yanjiu 2, 146-70.

2001, 'Dunhuang yishu zhong de zhenjiu wenxian', 敦煌遺 書中的針尒文獻 (Literature of acupuncture and moxibustion in the texts recovered from Dunhuang), Dunhuang yanjiu 2, 147-53.

Zhao Jianxiong 趙健雄, Xu Hongda 徐鴻達, Wang Daokun 王道坤 et al. 1985, 'Dunhuang shiku yixue shiliao jiyao' 敦煌石窟醫學史 料輯要 (Summary of data for the history of medicine from the Dunhuang Grottos), Dunhuangxue jikan 2, 115-21.

Zhou Zongqi 周宗岐 1957, 'Kaichi kao - Cong Dunhuang bihua “Kaichi tu” tan dao wo guo lidai de kaichi, shuaya he jiechiji' 揩齒考一從 敦煌壁畫 “指齒圖” 談到我國歷代的指齒、刷牙和潔齒劑 (A study of oral hygiene: tooth cleaning, tooth brushing and dental hygiene preparations in light of the 'Image of tooth cleaning' from the Dunhuang murals), Yixueshiyu baojian zazhi 1.2, 129-32. 


\title{
19 Travelling Light: Sino-Tibetan Moxa-Cautery from Dunhuang
}

\author{
Vivienne Lo 羅維前 and Ronit Yoeli-Tlalim*
}

The practices of blood-letting and cautery, the treatment of wounds and the concept of the Zodiac man all inspired iconographic representations of the body that have become well-known in the history of medicine. ${ }^{1}$ Less commonly known is that a new style of medical imaging associated with cautery techniques seems to have emerged simultaneously in 9th-1oth century Europe, Tibet and China. Common visual characteristics of these manuscript images include simple, sometimes quite roughly executed, outline sketches of the body, aimed to facilitate therapeutic interventions involving the application of burning hot substances to the skin or bloodletting. The medical charts in this new genre were multi-purpose representations of therapeutic know-how. They might be read alone, or together with more scholarly iconography and texts. In some contexts they could function independently as guides to practice; in others, they might require knowledge derived from their respective classical medical milieux, as 'visualisations of the medical word', where the image is subordinated to a dominant corpus of medical texts. ${ }^{2}$ This chapter is primarily concerned with identifying those elements of this medieval medical iconography concerned

Papers which serve the basis of this chapter were presented by the authors in a number of conferences: Vivienne Lo: 'Medicine on the Silk Roads' at the British Library 2005; 'Dunhuang Forum: convergence and innovation', Dunhuang Academy, August 2016; Ronit Yoeli-Tlalim: 'On the notion of channels $(r t s a)$ in the Tibetan medical manuscripts from Dunhuang', The 12th Biennial Conference of Asian Studies in Israel, Haifa University, 25-6 May 2014; "Tibetan medicine from Dunhuang: notes on transmissions of medical knowledge along the Silk Road', Interaction in the Himalayas and Central Asia, Third International SEECHAC Colloquium, Austria Academy of Sciences, Vienna, 25-7 November 2013; 'Tibeto-Chinese/Sino-Tibetan medicine from Dunhuang', 24th International Congress of History of Science, Technology and Medicine, Manchester, 23 July 2013. The proceedings from the British Library conference were published by the authors in a special issue of Asian Medicine: tradition and modernity 2007, vol. 3, issue 2. Many scholars have helped with the research for this chapter, especially Peter Zieme, Geoffrey Samuel and Dieter Maue. We are also very grateful to Jack Hartnell who shared insights which have led directly to the arguments in this chapter. See Hartnell 2017a. Ronit Yoeli-Tlalim's work was supported by the Wellcome Trust.

1 The earliest European version of the Zodiac Man has been identified as BNF ms. Lat. 7028, fol. 154r, dating to the 11th century. Cohen 2014, p. 71. See also Hartnell 2017a and b and Yearl 2011. Hartnell 2017b, p. 331.

with therapeutic know-how that disposed a therapeutic tradition to travelling, to crossing geographic, linguistic, temporal and cultural boundaries.

Fundamental to these diagramatical illustrations are a series of human figures, marked with black dots, and brief textual captions. In the earliest known versions, the dots mark places on the body that are a strategic part of the therapeutic procedure. In later charts from their respective traditions, the dots may also represent a physical axis of correlational knowledge that connects parts of the body to celestial bodies and to physiological notions of the circulation of embodied spirits - mapping forms of astromedical knowledge. But in the earliest European, Chinese and Tibetan charts, the image is unmistakably performative: a series of human figures take centre stage, with captions simply identifying common illnesses, and providing easily understood directions for medical practitioners or householders in search of an instant remedy.

In contrast to the Asian figures that we are soon to meet, the 17 figures that fill the last part of a 9th-century Latin manuscript sketch out scenes where an adult male physician is about to sear the flesh of an apparently unsuspecting patient. In the foreground, a burner, with lively dancing flames, lends a kind of immediacy to the therapeutic action where, in the first image of the sequence, an assistant tends the cautery irons. ${ }^{3}$ The physician's larger scale and sharply drawn eyes embody absolute medical authority. His gaze is trained on the much smaller figure of the patient, perhaps a child. The patients in these images are either prone or supine, their expressions blank, in passive acceptance of what must have been a painful, potentially traumatic, experience. The captions to the images are perhaps the most perfunctory of all we will meet in this chapter. They name symptoms of illness such as 'headache', or [a disorder of the] 'gums' with the cursory directive 'cauterise thus'; (see Fig. 1) Difficult theoretical notions that would have required a formal training in medicine are conspicuously absent. As Hartnell states, 'Instead of linking back to an authoritative text, medical power is invested in the black spots of the image itself', which articulate the essential information for effective action. ${ }^{4}$

3 Plut. 73.41, Biblioteca Medicea Laurenziana.

4 Hartnell 2017b, p. 332. 'Western Manuscript images of this type are presumed to have been around about the same time as the Tibetan and Chinese manuscripts, but are only really known in 


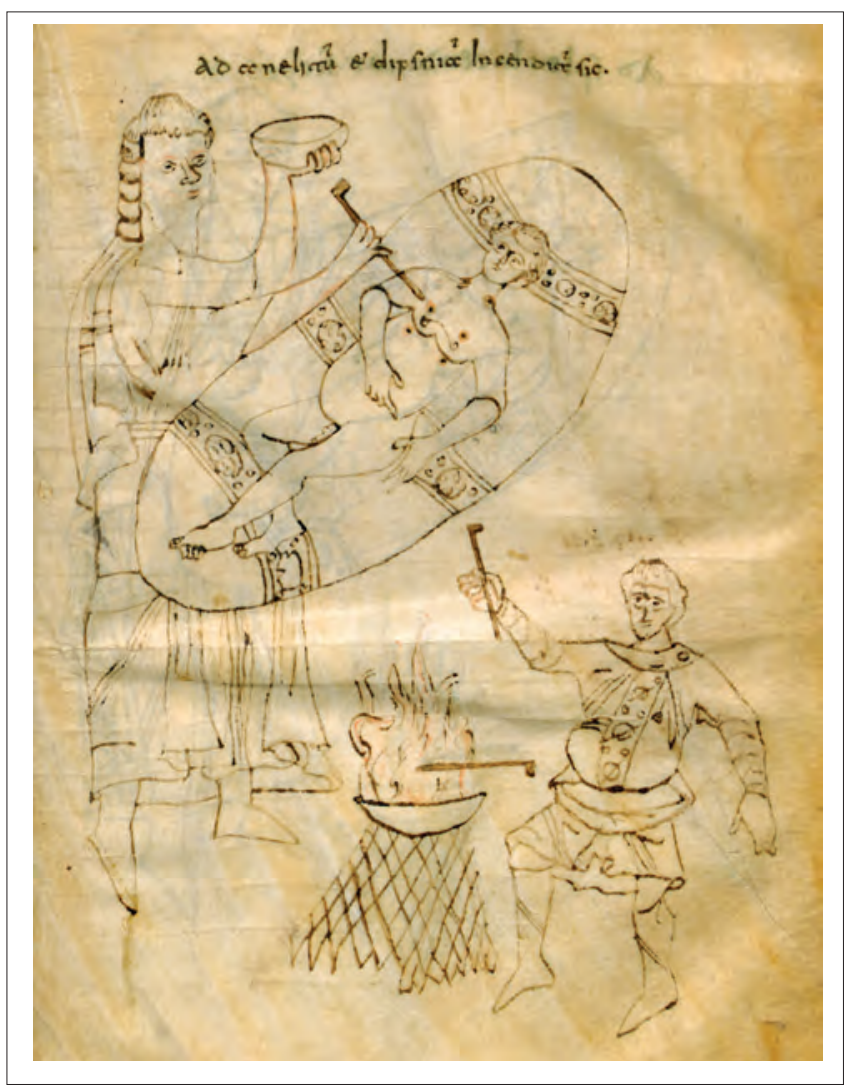

Figure 19.1 Cautery depicted in a 9th-century Latin manuscript, Plut. 73.41, f. 122 r. (C) Florence, The Biblioteca Medicea Laurenziana; reproduced with permission of мівАСт, further reproduction by any means is prohibited

Even though the surviving evidence is, as yet, too slim to make any substantial claims about links in the global medieval world of medicine, such performative images, of which we will hear much more in this chapter, provide intriguing testimony on the basis of which to ask more informed research questions of the period, and imagine new pathways to a history of 'travelling medicine'. They focus us on the following domains for close analysis: the widespread use of cautery and body piercing techniques, the sparse efficient detail of the images, the direct text-image relationship, and the ambiguous relationship with authoritative texts.

For Tibet and China we are fortunate to have $c$. 9th-century charts guiding moxa-cautery therapy, ${ }^{5}$ preserved in

later sources, from the 12 th/13th century. This is partly due to the huge rise in manuscript production and medical practice concurrent with the birth of university culture in the West at that time, so is likely just a bias of what survives rather than the evidence suggesting that practice was only popularised in these later centuries', Hartnell, pers. comm..

5 We have chosen to use the term 'moxa-cautery' rather than the the Dunhuang manuscript collections, and now held at the Bibliothèque nationale de France and the British Library respectively. ${ }^{6}$ These rare witnesses to the practice of medieval medicine not only conjure up a coherent vision of how the body was manipulated in the day-to-day practice of medicine, but also invite an analysis of what facilitated, and indeed facilitates, communication of medical knowledge across cultural boundaries. The connection between the production of the Tibetan and Chinese moxa-cautery charts that we analyse here is fundamental and undisputed. Analysing their continuities, we will ask ourselves: What is the relationship between them? Is one an adaptation of another? We will also identify shared astrocalendrical notions and the discourse of auspicious and inauspicious times for protecting the lunar circulation of bodily spirits. Counter-intuitively, however, after a momentary excitement about similarities, the second and more considered response of the transcultural historian of medicine is key to the methodology of this chapter: what can we tell from the Tibetan and Chinese charts about the nature of the transmission of medical knowledge from the ways that their respective image production differs?

\section{The Images}

Of the tens of thousands of medieval manuscripts discovered in 1900 at the Mogao 莫高 caves near Dunhuang (Fig. 2), the eastern end of the so-called Silk Roads, most are Buddhist scriptures. There are also thousands of secular texts. Some of these secular manuscripts testify to the surprising penetration throughout medieval Chinese society of officially sanctioned texts produced at the Chinese capital. Others reveal a fascinating range of previously unknown literature, in a variety of languages, that were passing through, copied or interpreted at Dunhuang. ${ }^{7}$ More than a hundred of these texts are directly concerned with medicine: most of these are in Chinese. There are also a few medical manuscripts in Tibetan and in Khotanese. In nearby sites, there are also medical manuscripts in Tokharian, Sanskrit, Sogdian, Uighur and Syriac. ${ }^{8}$ Some of

common modern term, 'moxibustion', in order to encompass the range of therapies practised in medieval China and Tibet, some of which were quite invasive.

6 The manuscripts all have a latest date of $c .1035$, the year that scholars generally agree the so-called 'Library Cave' was closed. Many of the manuscripts that it contained were already of some antiquity, and various methods such as script, layout and contents have been used to ascertain their original copying dates.

$7 \quad$ Lo, 2005a, pp. 227-8 and Yoeli-Tlalim (forthcoming).

8 For a discussion of some of these medical interactions see Yoe- 




Figure 19.2 A pile of scrolls, after removal by Stein from Cave 17 in Dunhuang. (C) The British Library, photo 392/27 (587)

the Chinese medical manuscripts from Dunhuang reveal exotic influences and exchanges, hitherto unknown in the Chinese medical literature that has been passed down to us in printed form, and edited countless times since the imperial patronage of medical publication in the Song period $(960-1279){ }^{9}$

In this chapter we are concerned with three medieval illustrated Tibetan and Chinese manuscripts which tell us about the transmission of moxa-cautery and cautery techniques, that is the application of burning materials to the surface of the body at strategic locations for therapeutic reasons: the Tibetan Pt.105 $8^{10}$ and the Chinese S.6168a and $b$, and S.6262. All three manuscripts set out a series of naked or semi-clothed human figures that indicate strategic locations for moxa-cautery. There are also two more Tibetan texts - Pt.127 and Pt.1044 - as well as numerous Chinese texts dealing with moxa-cautery and its prohibitions. ${ }^{11}$ The Tibetan manuscripts have been dated to $c$. 9th

li-Tlalim (forthcoming).

The varying number of manuscripts that contain information related to medicine is sensitive to contested definitions of the term 'medicine', and whether one includes, for example, practices and ideas concerned with gender and sexuality, drugs used in ritual rather than consumed etc. For varying examples see Ma Jixing 1998, list of contents; Despeux 2010, pp. 34-95; Yoeli-Tlalim 2015 .

In Chinese sources appearing as: P.18.o17. See Cong 1994, p. 179. huang, see Arrault 2010, pp. 285-330. Illustrated charts: S.6168, S.6262 (BL); Prohibition Charts: P.2675 (BNF), S.5737 (BL), S.4537 (India Office, BL); Remedy text listing moxa-cautery as an auxiliary technique to a drug recipe: P.2662 $\mathrm{r}^{0}$ and $\mathrm{v}^{0}, \mathrm{P} .3144 \mathrm{v}^{0}, \mathrm{P} .3378$ $\mathrm{v}^{\circ}$, P.3596, S.3395 $r^{\circ}$ (BNF), S.5435 $r^{\circ}$ (BL); Calendar including references to the circulation of the renshen 人神 (human spirit) around the body: P.2591, P.2623, P.2705, P.2765 $r^{\circ}$, P.2973A $r^{\circ}$, P.3247 $v^{o}, \mathrm{P} .3403 r^{\circ}$ and $v^{o}, \mathrm{P} .3492 r^{\circ}$ and $\mathrm{v}^{0}, \mathrm{P} .3555 \mathrm{~B}+\mathrm{P} .3555$ (Piece 9), P.4996, (P.3476) $r^{o}$ (BNF); S.95 $r^{o}$; S.276 $v^{o}$; S.612 $r^{o}$; S.681 $v^{o}$ (BL)

or 1oth centuries. ${ }^{12}$ The script on the Chinese manuscripts has been dated to the Tang period (618-907), but cannot be dated more accurately.

We will also make brief mention of a set of charts, the dating of which is yet to be determined, discovered at Turfan with captions in Uighur, ${ }^{13}$ and some 18th-century Japanese manuscript charts that demonstrate continuities with the illustrated traditions of Sino-Tibetan medicine.

Let us begin with the Tibetan manuscript Pt.1058 (Fig. 3), an illustration of moxa-cautery points, which features two upright, naked, presumably male figures: the one on the left is a static view in half profile, the other, on the right is a side view depicting someone who appears to be in movement. ${ }^{14}$ The figure on the right has six points indicated (foot, knee and arm), and the figure on the left has 13 points indicated (hips, thigh, legs and feet). The manuscript is cut off on the left, where there appears to have been a third image. What has remained of that third image is parts of the legends which were linked to it. Based on these, we can assume this was probably a rear view, since the legends appear to refer primarily to the vertebrae.

Their naked bodies are lean and muscular, with roughly executed striated rib cages, a far cry from the plumper, more opulent Tang dynasty (618-907) ideal of the same period. The Tibetan figures have a high bridge and sharp angle to the nose, full lips and chiselled cheekbones. Unlike later Tibetan medical images made within a Buddhist art tradition, these bear no resemblance to Buddhist artistic conventions. While this entails some raw, almost childlike renderings - such as the fingers of the left image, it also conveys some innovative imaginary, freshness and vitality - such as the dynamic pose of the right image. The limbs of these images are rotated, perhaps to reveal most clearly the strategic therapeutic locations, perhaps in a demonstration of some other kind of individual agency. The hair of each figure is tied loosely in a looping ribbon that gathers up the locks on top of the head in a bow, the ribbons caught by

+ DH.1454 $\nu^{o}$ (Institute of Oriental Manuscripts, St Petersburg); S.1473 $r^{\circ}$; S.24O4 $r^{\circ}$; S.5919; S-P.6 $r^{\circ}$ and $v^{o}(\mathrm{BL})$; BD.15292, WA $37-9$ (NL Beijing).

12

On the dating of the Tibetan Dunhuang manuscripts, see: Uray 1988, pp. 515-28; Takeuchi 2012, pp. 205-16; Dalton, Davis and van Schaik 2007. Dalton and van Schaik 2006.

Cong Chunyu 叢春雨 had previously suggested that the Dunhuang Tibetan medical manuscripts dated between the 7 th and 9th centuries CE. Cong 1994, p. 15 et passim.

13 Müller 1923; Rachmati 1932, pp. 401-48 and Maue, pers. comm.

14 On Pt.1058 see: Luo Bingfen et al.. pp. 34-7, Lalou 1941-2 and Cong 1994, pp. 179-81. The image is accessible on the Bibliothèque nationale de France website: http://gallica.bnf.f/ark:/ 12148/btvib8305595k?rk=21459;2 (accessed, 11/06/2017). 




Figure 19.3 Tibetan moxa-cautery chart from Dunhuang. Image 1 (on our left) marks 13 locations on the lower limbs. To the left-hand side of the text, along with descriptions of three points indicated, there are 12 broken lines of text, apparently relating to another image, now no longer extant. Image 2 has six points marked but the text is damaged. (c) Bibliothèque Nationale, Paris, Oriental ms. Pt.1058

the wind giving the impression of movement. The figures' eyes are intent with dark pupils focussed somewhere in the distance. In contrast to the posed and diagramatic figures of the Chinese moxa-cautery charts we will meet later on, the Tibetan ones are rather individual characters: one with eyelids and lashes lowered; the other with eyes wide open, staring straight ahead (Fig. 3).

The therapeutic points are marked with heavy black dots, with fine black lines stretching to the side where the location of each point is written out with simple anatomical descriptions and directions such as 'measure three fingers from the heart of the calf', or 'in the hollow behind the ankle', 'between the big toe and the second toe..$^{15}$ Unlike comparable Chinese and European charts, the text does not provide any information about the illnesses to be treated or the points indicated for specific ailments. There are no instructions for carrying out the therapeutic procedure. The map is therefore simply concerned with detailing the particular locations for moxa-cautery.

With no information about the nature of moxa-cautery set out systematically on the Tibetan charts, we have to turn

Pt.1058. For a list of all points see Lalou, 1941-2. to related texts in order to understand what kind of therapy is indicated. Two other manuscripts from Dunhuang, Pt.1044 and Pt.127, contain moxa-cautery remedy texts organised according to treatments for groups of symptoms. Generally speaking, Pt.1058 appears to illustrate parts of Pt.127, and discuss the same locations of the body. Pt.1044 and Pt.127 tell us a great deal about the nature and severity of illnesses treated, ideas about anatomy and physiology, moxa-cautery locations and the nature of bodily fluids. Groups of symptoms are listed in relation to the moxa-cautery points and include such problems as digestion, diarrhoea, swelling of the hands and feet, pain in the kidneys, vomiting blood and incessant nose bleed.

The greatest number of references to pathogenic agents in the Tibetan moxa-cautery remedy texts identify strike by wind: wind deviation, wind in the ear, wind causing sudden pain, headache, craziness or loss of motor function. In Pt.127 alone, wind is mentioned as a pathogenic agent no less than 25 times. Wind in Tibetan medicine is one of the three nyes pa, corresponding to the Indian concept of the three dosa: rlung (wind), mkhris pa (bile) and bad kan (phlegm). Wind can be a cause of illness but also 
what enables a practitioner to balance the body through wind-related practices. ${ }^{16}$

The kinds of illnesses treated in Pt.127 and Pt.1044 also include a range of symptoms of a much more severe nature associated with 'chu ser' (Yellow Fluid), a pathology that became common in later Tibetan medical literature. There is a parallel in Chinese pathology related to the term huang bing 黃病 (Yellowing Illness), but the Tibetan concept is quite distinctive. In the Tibetan manuscripts, Yellow Fluid could enter the body, particularly where there was external injury. It could rise to the head, accumulate in the spine, amassing in the joints, the heart or kidneys, or descend to the feet. It could be chronic or acute, associated with febrile illness and both sharp and heavy, swollen pain. Where it accumulated, there could be swelling, and stiffness of the joints and organs, impeding mobility. As it flowed into the head, Yellow Fluid could be observed 'yellowing' the eyes and flesh. These symptoms are often described as being contagious and were clearly febrile in nature. At risk of a teleological observation, the frequent reference to constant bleeding of the nose, swelling and pain of the kidneys and spleen, as well as yellowing of the flesh and eyes is suggestive that Yellow Fluid might cause illnesses that we associate today with typhoid and yellow fever, amongst other serious afflictions.

\section{Moxa-Cautery Texts}

We are accustomed nowadays to imagining a dominant classical tradition of acupuncture, and in that sense the Dunhuang Chinese moxa-cautery texts are quite distinctive. As historians, we should be cautious of a positivist approach, which studies the Chinese medical body as if it had always been a vehicle for needling therapies akin to contemporary acupuncture. Instead, we might heed signs of contestation: early warnings about the dangers of acupuncture, which according to Wang Tao 王濤, writing $c$. $75^{2}$, 'can kill people, and cannot raise the dead'. ${ }^{17}$ Cautery therapy certainly pre-dates acupuncture, and given the widespread availability of substances that could be burnt on the body along with the ease of application, it stands to reason that it was more widely used.

The independent moxa-cautery tradition represented in manuscripts is perhaps better evidence of therapy as it was practised throughout Chinese society than the transmitted scholarly literature which, read alone, might suggest

16 Yoeli-Tlalim 2010.

17 Wang Tao, Waitai miyao, preface. acupuncture was the dominant mode of practice. Not only does moxa-cautery pre-date acupuncture, but it was also much more widespread. Early evidence of moxa-cautery with ai 艾 (Artemisia vulgaris) and other materials, coming from the course and hinterland of the Yangzi valley and its tributaries, can be found in medical texts over one thousand years before the Dunhuang manuscripts, and much earlier in less specialised literature. ${ }^{18}$

Moxa-cautery traditions associated with channel theory, but not with acupoints, have featured in Chinese householder manuals from as far back as the Mawangdui manuscripts of the 2nd century вСЕ and at least as far forward as the 16th-century riyong leishu 日用類書 (encyclopaedias for everyday use), which Chang Che-chia has shown provide household instruction on practically every medical technique except acupuncture. ${ }^{19}$ Representations of Yin and Yang channels of the body, associated with moxa-cautery, and tangentially related to later acupuncture models, survive in texts and in two lacquered figurines excavated from tombs of Han dynasty (206 BCE-220 CE) aristocrats (See Lo, Chapter 3 in this volume). ${ }^{20}$ Confirming the extensive use of acu-moxa during the early empires, Han military records written on bamboo strips, for example, record that moxa-cautery methods were used in emergency medicine by officers in the Chinese garrisons around Dunhuang. ${ }^{21}$

Just before the putative date of the Chinese Dunhuang moxa-cautery literature, the 7 th-century work of Sun Simiao 孫思貌 provides us with a near-contemporary source indicating that illustrated point charts were included in mainstream acupuncture and moxa-cautery manuscript productions at the Chinese court, although they were omitted in later printed editions. ${ }^{22}$ Two hundred years later in 861 the preface to P.2675 sets out the text's intention to abridge the moxa-cautery techniques of a number of schools or teaching lineages in order to provide a practical medicine for those living in outlying regions without access to better quality medicines. P. 2675 is in fact a text more concerned with the proscription and control of what was evidently a popular practice by this time, rather than with prescriptions for guiding treatments.

Since they don't mention acupuncture at all, our extant Chinese Dunhuang charts suggest that there were independent moxa-cautery traditions: on the one hand

\footnotetext{
18 Lo 2002, pp. 99-128; Li Jianmin 2002, pp. 320-31; Yamada 1998.

19 Cong 1994, p.15; Chang Che-chia 2006, p. 183 n. 20.

$20 \quad$ Lo and He 1996, pp. 81-123.

$21 \quad$ Xie Guihua, 2005, p. 97.

22 Sun Simiao, Beiji qianjin yaofang (comp. 650-9): 1995 edn, pp. 508, 513. 王壽, Waitai miyao 外臺秘要 (c. 752), juan 39: 1993 edn, p. 779 .
} 



㞼




we find texts that privileged an understanding of learned concepts of the body which would have required astromedical knowledge, or knowledge of the body's interior, and complex ideas about physiology; on the other we find illustration-based texts which suggest a more practical use in therapy, both needling and moxa-cautery, and in pre- and proscription. But when we consider that charts were also included in scholarly works such as those by Sun Simiao, the independent existence of the exclusively moxa-cautery chart-manuscripts demonstrates that the two genres of medical writings could be read separately, or together, by different groups of readers.

In the Chinese illustration-based manuscripts, black lines lead from the black dots that mark the set places for moxa-cautery, to simple instructions as to how to carry out the treatments, and for what ailments. The simple arrangement of image and text directs the reader's attention to where and how to place the moxa-cautery materials on the patient's body, and with what goal in mind. The sparse information clearly privileges the performative nature of the charts. The combination of image and text included everything that a lay person might need to apply moxa-cautery, so that the charts lent themselves to use in the homestead, as a kind of frontline medical treatment. ${ }^{23}$ Astonishingly, in the Chinese texts moxa-cautery tends to be applied in multiples of 100, and even up to 1,000 in the case of treatment to the yuxingtou 玉茎頭 Head of the Jade Stem, (i.e. the head of the penis) for wulao qishang 五勞七 傷 (five wearinesses and seven injuries), an affliction of men and boys. Here are some excerpts from S.6168 (Fig. 4a):

Moxa for wind floating in the face; as if insects xixi on the face; on rising (they) fall down, whirling...; tian zhuang 天空 (窗) (Heaven's Window); moxa ban mei 板眉 (? Eyebrows); moxa qumei 曲眉 (Bending Eyebrows); moxa two fengfu 風府 (Wind Palaces); ...Shou yangming 足陽 明 (Hand Yangming). In all cases 10 places. 200 times on left and right.

Moxa wulao qishang 五勞七傷 (five wearinesses and seven injuries) in men and boys; losing jing 精 (seminal essence) blood in the urine. You must moxa faji 髪際 (Hair Border); moxa guanyuan 開原 (關元) (Connecting to the Source); moxa liang shou sui kong 兩手髓孔 (Two Hand Bone Marrow Hole); moxa yuxingtou 玉菣頭 (Head of the Jade Stem); moxa liang jiao wuzhou 兩腳五舟 (Two Feet Five Boats); moxa liangjiao bijing 兩腳痹經 (Two Feet Numbness of the Channels); moxa liangjiao zhongfeng 兩[腳中]封 (Central Dike of the Two Feet); not on both sides... 11 places. Each moxa 1,00o zhuang.

Moxa people's various insanities. You must moxa two xuanjiao 玄角 (Dark Corner) moxa bizhu 鼻[柱] (Bridge

$23 \quad$ Lo 2005a, pp. 241-8. of the Nose); moxa two ru tou 乳頭 (Heads of the Breasts); weiguan 胃脘 (管) (Duct of the Stomach), guanyuan, liangshou xiaozhitou 兩手小指頭 (Tip of the Two Little Fingers) zu liang xiao zhitou 足兩小指頭 (the Tip of the Little Toes); 11 places on two sides each. Moxa 500 zhuang. The Bridge of the Nose and the Tip of the Little Fingers, 100 zhuang each; the rest 500 zhuang.

Xiaofu shu 小腹俞 (Connection to the Lesser Abdomen) next to the third vertebra below daji大槌 (椎) (Great Extremity) on both sides 2 cun and 3 fen away. Controls... stiffness/obstruction causing internal haemorrhoids 30 zhuang bao (?). If there is no recovery (?), you can moxa up to 6 o.

Dachang shu 大腸俞 (Connection to the large intestine) at the 16th vertebra on both sides 2 cun 3 fen away. Controls thunderous calling in the belly, large intestine bubbling diarrhoea/dysentery, inability to digest food, small intestine, twistingly hurts, the small of the back and spine are painful and stiff, difficulty in defecating, inability to eat and drink. Moxa 100 zhuang. Excellent.

The Dunhuang Chinese moxa-cautery charts seem to easily fit the bill of household medical manuals, since symptoms treated are mostly non-fatal, non-contagious chronic illnesses predominantly associated with pain and sensory disturbance, gastro-intestinal and locomotive disorders. Many illnesses are associated with zhong feng 中風 (Attack by Wind).

Since the earliest written records in China, untimely winds were known as a cause of sudden disorder in the body of state and the physical body alike. There were good and bad winds and untimely winds always spelled bad fortune. In classical medicine they were 'the origin of 100 illnesses'. ${ }^{24}$ Classical medical treatises depict malevolent demon-like wind spirits, and simultaneously imagine wind as a more abstract cause of physical disorder (Fig. 5).

\section{Moxa-Cautery Images}

There have barely been any analyses that compare the Tibetan medical manuscripts from Dunhuang with their Chinese counterparts, which have been more comprehensibly researched..$^{25}$ The manuscripts were preserved in the

24 Winds were recognised as an independent source of illness from very early times. Kuriyama isolates lack of regularity and sudden change in the winds as the characteristics that qualify it to be the 'origin of 100 diseases', disorders of time and space that could be manipulated by diviner and physician alike. See Kuriyama 1999, pp. 233-70.

25 Lin Meijun and Guo Changqing 2009, pp. 55-7. Complete tran- 


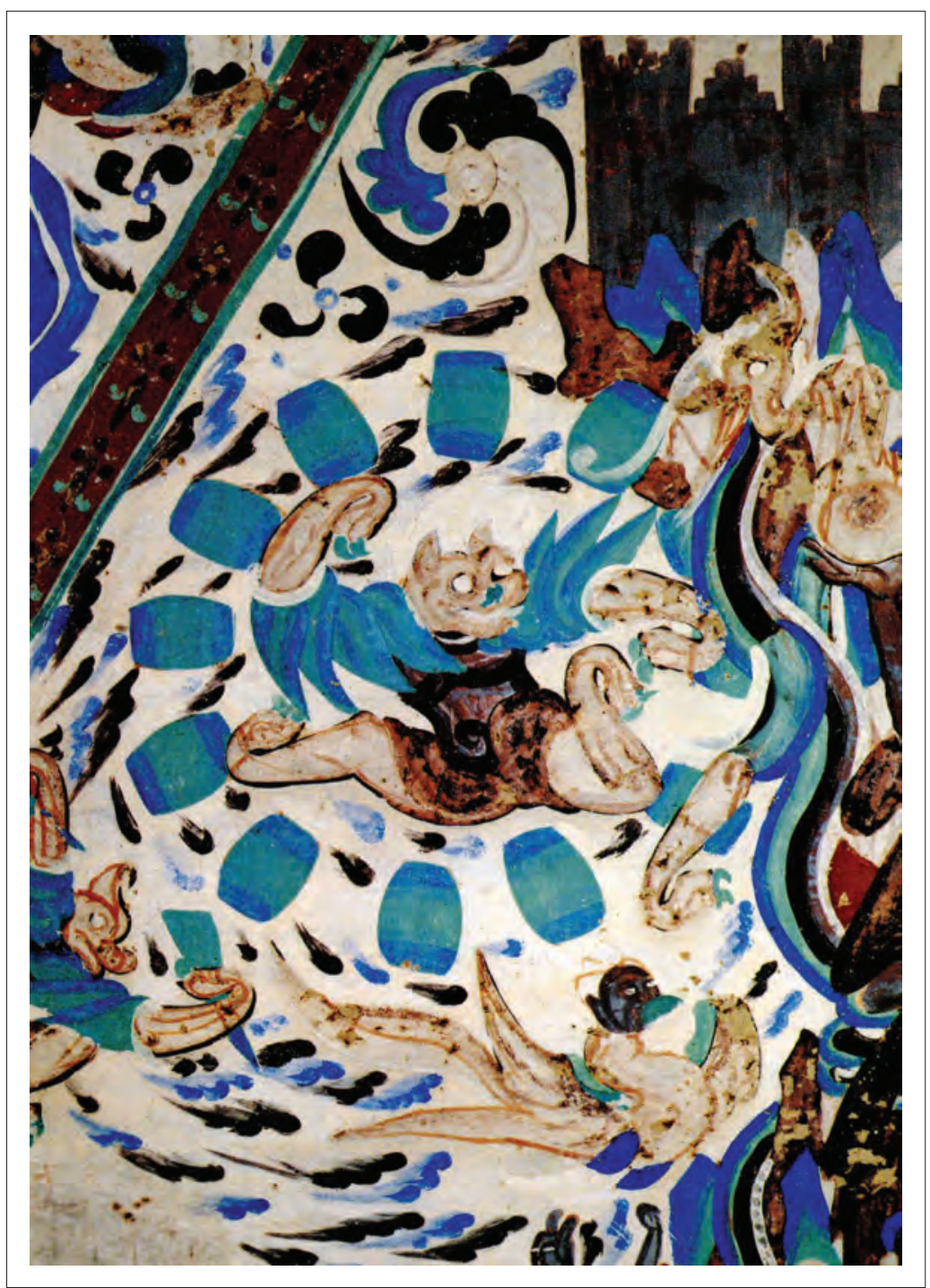

Figure 19.5a and b A ceiling mural from Western Wei $534-556$ Cave 249 at Dunhuang shows Lei Gong 雷 公, the Thunder Duke or the Thunder Spirit, surrounded by drums, together with another image (below right) that some suggest is the Feng Shen 風神 (Wind Spirit), or Feng Bo 風伯 (Wind Uncle) with a bag full of wind on his back. () Wang Jinyu, with kind permission to publish from the Dunhuang Academy

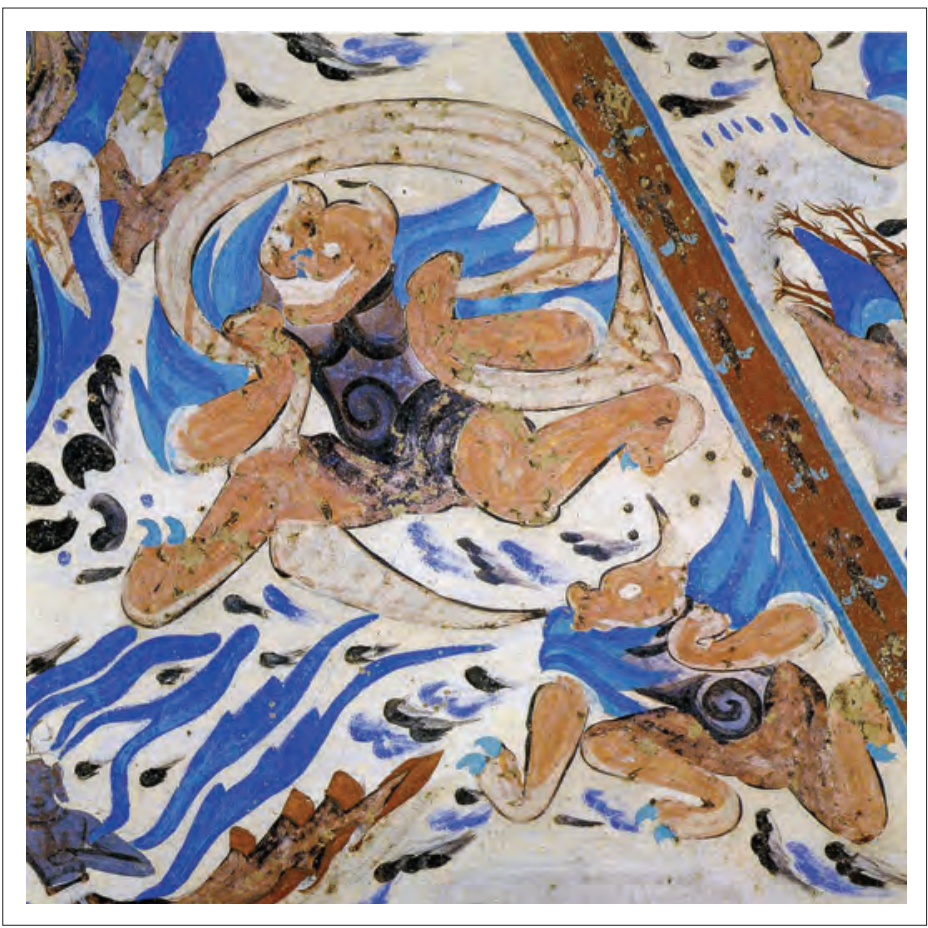


same collection, and therefore their connection in time and space raises the question of connection in content as well. There are, however, a number of factors which indicate the temporal priority of the Chinese moxa-cautery chart tradition. As mentioned above, traditions of Chinese medical illustration survive from one thousand years before the date of either the Tibetan or the Chinese Dunhuang charts, and point charts were included in well-known and widely distributed medical compilations of the 7 th and 8 th centuries, before and contemporary with the probable dating of the Dunhuang texts. The Tibetan versions of the moxa-cautery charts are rough productions that offer only information about the locations for treatment, and do not integrate information about treatment and treatment indications on the chart itself (though that information may be located in moxa-cautery texts Pt.127 and Pt.1044). The Chinese charts offer similar information displayed on many more figures, and integrate greater detail about illnesses and therapeutic technique. Embedded in the Chinese moxa-cautery tradition at Dunhuang, as we will see shortly, are also early Chinese astromedical concepts concerned with care for the physiology of the spirits of the body, concepts which are evidenced in the later Tibetan tradition. The Tibetan Dunhuang manuscripts are the earliest extant Tibetan manuscripts. So while, in the absence of clear earlier Tibetan evidence, none of these factors alone can establish definitively the anteriority of the Chinese tradition, this combined evidence strongly supports the case.

While content-wise the Tibetan and Chinese charts may be similar, visually they are very different. Each of the sets of Chinese images has qualities that point to their production in centres that are far away from the main loci of political power where cultural interaction was a greater feature of everyday life. The Chinese charts, with their articulated rib cages and elongated Buddhist-style earlobes, for example, are not standard central Chinese productions. Perhaps they reflect the growing influence of Buddhism and Buddhist imagery in the Tang court or, as likely, the specific Buddhist culture of Dunhuang as a unique site of intense cultural exchange. The Tibetan charts, as we argue throughout this chapter, demonstrate considerable contact with pre-existing Chinese moxa-cautery traditions. With this in mind the charts provide a unique opportunity to explore the hypothesis that during the gth and 1oth centuries, a Sino-Tibetan medicine was emerging in written form, after which its cross-cultural innovations, to be detailed

scripts of S.6168a and b, and and S.6262 are in Ma Jixing (ed.) 1998, pp. 477-512, and Cong Chunyu (ed.) 19942, pp. 182-20o. See also Lo $2005 \mathrm{a}$ and b, and 2010; Yoeli-Tlalim (forthcoming). in this chapter, were absorbed into the larger Himalayan mélange evident in the canonical texts of Tibetan medicine which were compiled around the 12th century, but not into the Chinese received tradition of a conflated zhenjiu 針尒 'acu-moxa', the category most frequently encountered in post Han medical literature.

Unlike the two Tibetan figures, the 18 male figures of the Chinese charts have been standardised across the sets in all three manuscripts. The two styles of pose, anterior and posterior (and not in profile) are sketched to facilitate inspection, with hands and feet turned out to reveal places marked with black dots. The only clothing is a loin cloth that modestly conceals genitals and buttocks, which would otherwise be exposed in the anterior and posterior views. The hair has been civilised and restrained into two top knots maximising exposure of the face. The faces are standardised to a neat oval shape with curving eyebrows and barely visible eyes, making the expression appear content and passive, altogether pleasing to the eye, if an anodyne blank model on which to inscribe variations of technique. Individuality has been subordinated to functionality.

Both the Tibetan and the Chinese charts feature basic images with a clear practical goal, many of them crudely drawn. Both have emphasised rib cages for ease of positioning abdominal locations. The black lines connecting between black dots on the anatomical figures and the concise hands-on captions may seem almost trivial to our eyes, but in fact they are not: they are some of the earliest - if not the earliest - extant examples of indication lines in anatomical illustrations. ${ }^{26}$

\section{Illnesses and Treatment}

Extending our comparion also to the Tibetan moxa-cautery texts Pt.127 and Pt.1044 reveals striking similarities in form, content and context. ${ }^{27}$ The structure of these moxa-cautery texts is very similar to the text accompanying the Chinese charts S.6168 and S.6262: a list of symptoms, the location on which to apply moxa-cauteries and the number of moxas to be burnt. The descriptions of ailments which are to be treated by moxa-cautery are also very similar. Both the Chinese and Tibetan material deal primarily with many wind (T:rlung, Ch.:feng) related ailments; genito-urinary and reproductive disorders as well as digestive and abdominal illnesses. Some of the descriptions bear almost literal similarities. Such, for example, is the passage found

\footnotetext{
26 For a discussion on indication lines, see Herrlinger 1970, pp. 19 and 54-6o.

27 Yoeli-Tlalim (forthcoming).
} 
in the Chinese ms. S.6168 describing wind in the face like insects on the face, ${ }^{28}$ which is remarkably similar to what we find in Pt.1044: '[If] due to wind (rlung) illness swellings are forming on the face, and there is itching like a walking insect....29 It is interesting also to note here the Tibetan measurement word tshon, a loanword from the Chinese cun, which appears quite frequently in the Tibetan moxa-cautery texts from Dunhuang.

Moxa-cautery in both Tibet and China involved the burning of various qualities of ai 艾 (mugwort or Artemisia vulgaris) on the therapeutic location. There is no specific description for medieval Dunhuang moxa-cautery with ai in either the Tibetan or Chinese charts, but in the Tibetan material there is reference to Turkic style iron cautery. There is also evidence elsewhere of the use of a variety of woods such as elm, orange wood, pine, jujube and mulberry wood in the medieval Chinese tradition. ${ }^{30}$ The moxa-cautery texts Pt.127 and Pt.1044 tell us about combination therapies that recommend the staged use of drugs, dietary therapy, moxa-cautery and bloodletting according to the progression and increasing severity of the illness.

In contrast to the large numbers of moxa placed on the therapeutic locations in the Chinese charts, the Tibetan texts call for the application of rather small numbers.

While most of the remedies preserved associated with the Dunhuang Tibetan medical charts indicate the treatment as forms of moxa-cautery therapy, there is also evidence of fumigation, massage and horn cupping, and bloodletting as well as the use of materia medica.

\section{No Theory}

The moxa-cautery charts and texts from Dunhuang - both the Chinese and the Tibetan - do not discuss theory. Both the Tibetan and Chinese moxibution charts and texts are entirely concerned with practical know-how, rather than formal classical knowledge - no explanations or reasonings are provided relating to Chinese classical physiology of Yin, Yang, Qi. In the Chinese case, the charts could be standalone instructional manuals or, as noted above,

28 See the first sentence of the translation of the excerpts from S.6168 above (Fig. 4a).

29 Pt.1044, lines 26-7.

30 Lo 2001, p. 67. Huangdi hama jing, pp. 55-6. It is not clear whether the wood itself is used therapeutically or simply in the preparation of the cautery material. Variations of this passage are to be found in Waitai miyao, juan 39 and in a section of Ishimpō 2 entitled 'Methods to Prepare Cautery', the latter being attributed to the lost text Xiao pin fang 小品方 (Lesser Grade Remedies); Ishimpō 2, p. $5^{8}$ included in scholarly medical treatises and sit side-byside with learned treatises on medicine. In the Tibetan moxa-cautery texts, although we do have mentions of the two other nyes $p a$ - it is mostly rlung (wind) that the texts are concerned with. There is no dealing with the three: wind, bile and phlegm as a triad and indeed, no mention of the term nyes pa. So while the concept was known in Buddhist contexts, the Tibetan medical manuscripts from Dunhuang reveal a stage where this notion has not yet been integrated into a medical system as we know it from a later stage. In neither the Tibetan nor the Chinese charts is there substantial internal evidence of, or reliance, on theories such as those we know well from the transmitted literature. Within these practical traditions at Dunhuang, it is easy to imagine that therapeutic knowledge transmitted readily, without the encumbrance of theoretical baggage.

\section{Moxa-Cautery, Calendrical Spirits and the Channels of Acupuncture}

Most significantly, neither Chinese nor Tibetan texts from Dunhuang link treatments to a system of channels. This is consistent with how moxa-cautery is later described in the 12th-century Tibetan Gyushi. ${ }^{31}$ In China moxa was undoubtedly more widespread than acupuncture throughout history, being a part of the self-care tradition in the home and evidenced in popular almanacs and manuscript traditions. And so there was a multiplicity of co-existing traditions, some dependent on channel theory and some not. As noted above, the dangerousness of acupuncture treatment meant that the technique went in and out of fashion at various times in imperial history. But in the scholarly written tradition, the notion of points arranged along channels and related to the organ functions was already present in a fairly standardised textual form from the time, in the 3rd century, when Huangfu Mi's 皇甫䍀 Zhenjiujiayijing 針炎甲乙經 (AB Canon of Acupuncture and Moxa-cautery) reordered much of the Yellow Emperor's corpus. And these points related to both acupuncture and moxa-cautery.

The Yellow Emperor corpus is generally thought of as the locus classicus for the distinctive acupuncture and moxa-cautery tradition that coalesced in the centuries around the turn of the previous millennium. Three recensions of this corpus were subsequently edited and printed after the Song period and these form the received

3 For a discussion of moxa-cautery in the Gyushi, see Pasang Yonten Arya 2014. 
tradition. ${ }^{32}$ This received tradition shaped, and continues to shape, the scholarly imagination of medical practice, eclipsing less authoritative writings such as almanacs and the later manuscript tradition which tend to reveal the more extensive use of moxa-cautery rather than needling therapies. The combined evidence therefore suggests a plurality of practice, with moxibustion being more widespread, and in many contexts independent from the acupuncture traditions. In this respect we can say the evidence from Dunhuang reveals a popular moxa-cautery tradition that is more consistent with the classical received tradition of Tibetan medicine - in that it did not integrate with theories of channels or acupuncture - than with the received Chinese tradition, which tends to elide moxa-cautery with acupuncture, as in the catch-all term zhenjiu 針尒 (acu-moxa).

In both the Tibetan and Chinese manuscript charts from Dunhuang, we have a quick and easy type of treatment - a treatment that does not require much advance training. The locations described in the Tibetan and Chinese charts and texts are determined only by surface anatomy, and keyed to illnesses not channels. Acupoints with lyrical Chinese titles such as 'spirit storehouse', 'bubbling spring' or 'celestial pivot' are generally eschewed in favour of terse directions to apply moxa-cautery at, for example, a location liangru shang quepen guxia er leijian 两乳上缺盆骨下二 肋間 (between the two ribs under the clavicle and above the breast) ${ }^{33}$ or zai qixia sancun 在臍下三寸 (3 Chinese inches under the umbilicus). ${ }^{34}$ Of the some 36 o standard acu-moxa locations in Huang Fumi's 3rd-century work. that are well known in the received acupuncture literature, there are only 22 cited among the $c .50$ moxa-cautery locations recorded in the Chinese Dunhuang charts. ${ }^{35}$ These 22 must, in our view, represent the most widely known and popularly used moxa-cautery locations.

\section{What is it that Flows Around the Body?}

One clue to elements of Tibetan medicine that seem to have taken a lead from Chinese precedents can be found

32 The primary medical works attributed to the legendary Yellow Emperor are Huangdi neijing and waijing 黃帝內/外經 (The Inner and Outer Canon of the Yellow Emperor). Over time, the Inner Canon has been rearranged by editors into three sections, forming separate books: Suwen 素問 (The Basic Questions); Lingshu 靈樞 (The Numinous Pivot); and Taisu 太素 (The Grand Basis). P.3378.

P.2662v.

Those that are included are: guanyuan 關元, jianshi 間使, juque 巨䦕, baihui百會, zhongfu 中府 and zuyangming 足陽明. in the descriptions of the Tibetan concepts of bla/ brla..$^{36}$ The notion that there is a cyclical vital force which flows around the body in accordance with the lunar cycle is an important notion in Tibetan medicine, which has various practical implications for the practice of moxa-cautery. This force, termed bla (or: brla) is described as responsible for a person's vitality and well-being. ${ }^{37}$ According to the surviving Tibetan medical tradition, sometimes when a person experiences a great shock, the bla may be lost. Thereafter, the person may show signs of illness. Determining the location of the bla is considered important in Tibetan medical practice, since the use of invasive therapies such as acupuncture, moxa-cautery or blood-letting in an area where the bla resides at the time of treatment is seen to be harmful for the patient. According to Tibetan medicine, these invasive therapies are also to be avoided on days of the new or full moon, when the bla is said to pervade the entire body for a short time.

Pt.1044 is the earliest extant Tibetan source to refer to such a cycle:

As for the method of cauterisation:

The day of the month (tshes grangs) needs to be established and the location of the brla (bla) [needs to be] calculated. Apply accordingly [when] it [the bla] is not descending. ${ }^{38}$

The idea that a vital force flowed around the body in accordance with the lunar cycle, and was vulnerable to damage from invasive therapies, appears in Chinese medical sources both before and after the period of Dunhuang. ${ }^{39}$ In the $c .5^{\circ}$ Chinese calendars found in Dunhuang and dated between the 9 th and 1oth centuries, there are two hemerological methods mentioned: that of the transfer of the daily spirit (riyou 日遊, relevant particularly to childbirth) and that of the location of the human spirit in the body (renshen 人神, relevant particularly to acupuncture and moxa-cautery). According to Harper, the Chinese iatromantic texts in Dunhuang stand apart from other manuscripts on medieval divinatory arts, and have their own distinct place within a larger body of technical literature, which includes medical literature. ${ }^{40}$ While the annotation on renshen found in Dunhuang calendars, however, dates only from the end of the gth century, the medical idea of the spirit flowing around the body emerged much earlier in China.

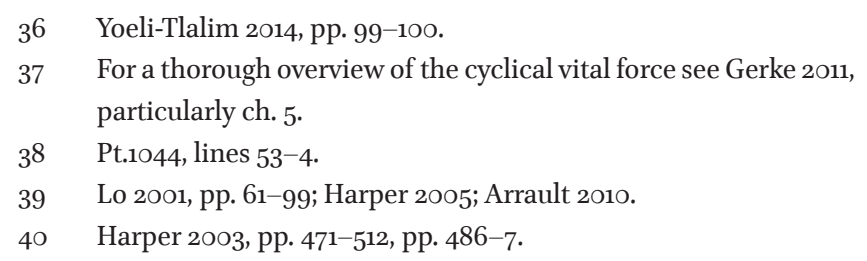


Lo and Harper have shown that the principle of the human spirit moving within the body is found in iatromantic treatises dating to the Han period, and was soon after integrated into the medical practice and theory of classical medical treatises such as those compiled into the Huangdi neijing lingshu 黃帝內經靈樞 (Lingshu for short). ${ }^{41}$ The earliest known exemplar of the notion of the human spirit (renshen) or (renhun 人魂) in circulation has been dated to an iatromantic manuscript found at a 1st-century CE Han dynasty site in Wuwei 武威 (close to Dunhuang). ${ }^{42}$

The concept of a circulating 'human spirit' had thus made up an integral part of the Chinese acu-moxa traditions since the Han period. It closely mirrored ideas about the circulation of renqi 人氣 'human Qi', which in early treatises of the Yellow Emperor corpus was also conceived as a small entity moving around the body, rather than the more fluid-like substance that we imagine today. The Wuwei manuscript also provides the earliest reliably dated evidence of acupuncture at named locations on the body as it records the Huangdi zhibing shenhun ji 黃帝治病神 魂忌 (Yellow Emperor's Soul and Spirit Prohibitions in Treating Illness), which warned of using moxa-cautery at different parts of the body according to a patient's age. ${ }^{43}$ Great caution was taken in order to ensure the safety and free flow of the hun 魂 (soul) and shen 神 (spirit) as it moved predictably according to these cycles. Harper has shown that the Chinese material from Dunhuang provides examples of various types of renshen, but he has suggested that those based on days of the lunar month may have been the most widely disseminated and influential, to the point of being general knowledge in medieval society. ${ }^{44}$ Classical texts such as Huangdineijing lingshu had already repeated the notion that the Qi and the shen 'spirit' travel in and out of the body, and identified 365 locations suggesting an annual cycle. By the 7 th and 8th centuries, many Chinese medical books record cycles of the human spirit and related treatment prohibitions as a staple part of the acupuncture and moxa-cautery traditions, including three manuscripts in the Dunhuang collections (P.2675, S.5737 and P.3247) and the numerous calendars which refer to the renshen. 45

The notion that a spirit travelled around the body on lunar and diurnal cycles, taking up lodging at knowable locations, was part of a shared Sino-Tibetan medical culture that was emerging in the cultural interactions around



Dunhuang. In the Chinese case, there is a textual lineage of iatromancy from antiquity to the medieval period, and evidence of this tradition survives in illustrated manuscript form in Japan, as we shall see, and as prohibitions which are integrated into the received Chinese medical sources. But the concept of the circulating renshen lost its central position in the scholarly Chinese medical tradition. In contrast, in the Tibetan case, this tradition has remained very much alive as an integrated part of the written and practical tradition.

A graphical reflection of the Sino-Tibetan nature of the bla/renshen 人神 (human spirit) notion can be seen in manuscripts which have both Chinese and Tibetan inscriptions. ${ }^{46}$ Both the Chinese and Tibetan moxa-cautery texts reflect links with time reckoning and divination. The Tibetan scroll Pt.127 is in fact a compilation of several different texts: the moxa-cautery text discussed above (recto, lines $78-184$ ), along with a number of divination and calendric texts on the rest of the recto as well as the verso. These different texts appear to have been written by the same hand. We also find such juxtapositions of medical and divinatory texts among the Chinese texts. Indeed, common to the Tibetan and Chinese cultures of the prohibition texts and charts was this circulation of the spirit, conceived of astromedically as a vulnerable orb or planet circulating around the system in need of careful protection.

\section{Origins of Moxa-Cautery Method: the Land of the Indian King?}

As we dig beneath the surface of the Dunhuang manuscript traditions that describe the transmission of moxa-cautery techniques, many strata of medical practice are revealed. These may help us to identify aspects of medical technology that were most mobile, and amenable to moving from one linguistic and cultural sphere to another.

At the close of Pt.127 a final statement tells us that the preceding moxa-cautery procedures have wide-ranging cultural origins:

This text (yig) on medical practice (dpyad) is not even [to be found] at the archives (phyag sbal). It is a compilation of all traditions of medical practice (dpyad yig thams $c a d$ ), in addition to being compiled according to the indigenous (?phugs pa) medical practice (dpyad phugs) of Zhang Zhung. ${ }^{47}$

\footnotetext{
$46 \quad$ Kalinowski 2003, p. 149.

47 Pt.127, lines 183-4. See Yoeli-Tlalim 2013.
} 
Even more intriguing is the colophon of Pt.1044, saying: 'This type of method comes from a land of the/an Indian king'. ${ }^{48}$ The moxa-cautery practices which are described in Pt.1044 - as in the other Tibetan moxa-cautery chart and text - are not known to have been used in classical Indian medicine. Indian cauterisation practices are delineated, for example, in the chapter devoted to cauterisation in the Aștāingahrdaya samhitā. Although the Tibetan term for moxa-cautery - me bsta' - was used in the 11th century by the translators of the Aștāingahṛdaya samphitā to render the Sanskrit term for cauterisation (agnikarman) in Tibetan, ${ }^{49}$ we know that the Indian cauterisation practices are fundamentally different from the moxa-cautery practices found in Pt.1044.

Indian cauterisation practices are discussed in the chapter devoted to the subject in the Aștāingahrdaya sam hitā (ch. 30 of the Sütrasthāna part $)^{50}$ where there is a brief discussion on two types of burning: one by placing an alkaline paste (for treating conditions like haemorrhoids or diseases of the eyelids) and the second is thermal cautery (S: agni karma) - used on the skin, muscles, veins, tendons, joints and bones, for treating 'diseases like black mole, weakness of body parts, headache, adhimañtha (a disease of the eye), warts, cysts etc. Burning of the skin should be done either with a lighted wick, tooth of a cow, rock crystal, arrowhead or others (such as pippalī, excreta of a goat, iron-rod, piece of bangles)'. ${ }^{51}$ Burning is also used for treating haemorrhoids, rectal fistula, tumors, various types of ulcers, illness of the eyelids, bleeding, blue mole or surgical wounds. There is also a chapter devoted to cauterisation (agnikarman) in the Suśruta (ch. 12).

How then should we read this reference to a land of the/an Indian King?'

The next line in the colophon provides us with an intriguing clue. It explains that this technique derives from Ha-ta-na-bye, a Tibetan transliteration of the old Khotanese name hvatana for Khotan. ${ }^{52}$

Khotan, an oasis kingdom on the southern branch of the Silk Road, was a major centre of Buddhist learning in the ist millennium. Chinese and Tibetan accounts on the

48 Pt.1044, l. 52: rgya gar gi rgyal po'I yul nas byung bai dpyad rnam gchIg las; Luo Bingfen et al. 2002, p. 238.

49 This point was made by Fernand Meyer. Meyer 2002. RYT wishes to thank Fernand Meyer for sharing his paper with her.

$50 \quad$ Murty 1991, vol. 1, pp. 343-53.

51 Ibid., vol. 1, p. 35 o.

$5^{2}$ We are grateful for the help of Peter Zieme for the suggestion that Ha-ta-na-bye is a Tibetan transliteration of the Old Khotanese word hvatana, the geographic designation being hvata$n a-k s ̦ i r a$, and the language hvatanau. Encyclopaedia Iranica 2016, online entry for Khotan (accessed 10/10/2016). foundation of Khotan associate it with the son and ministers of Emperor Aśoka - hence 'the Indian king'. The view of Khotan as an Indian colony endured, even until the 1oth century when its rulers continued to bear Indian names. ${ }^{53}$

The population of Khotan was ethnically mixed, as was its culture, bringing together Indian, Chinese and Iranian elements. ${ }^{54}$ We can also trace Tibetan cultural input, primarily as a result of Tibetan rule of Khotan from the late 8th century till mid-9th century. During the 1oth century the contacts between the Chinese, Khotanese, and Uighurs intensified as a result of marriage alliances. ${ }^{55}$ An instructive description in this regard appears in the Tibetan 9 th or 1oth century History of Khotan (Li yul lung bstan pa):

Li (Khotan) being a country where Indians and Chinese met, the common language agrees with neither Indian nor China. The letters agree one by one with India. The customs of the people agree for the most part with China. The religious customs and the religious language agree for the most part with India. ${ }^{56}$

Khotanese manuscripts were found by Stein in Dunhuang and in the Khotan area. The language, an Iranian language contemporary with Middle-Persian and Sogdian, written in Indian Brāhmī script, with an extensive vocabulary borrowed from Sanskrit, was unknown by the 2 oth century. Following Stein's discoveries, Khotanese was deciphered by Hoernle.

The Khotanese manuscripts and fragments date from the


Khotanese Buddhist texts include both translations from known texts (mostly from Sanskrit) as well as some local compositions. ${ }^{57}$ With a Buddhist culture based mostly on Sanskrit sources, it is not surprising that Khotanese medical texts predominantly reflect links with Ayurveda: these include a Khotanese version of Ravigupta's Siddhasāra ${ }^{58}$ and the so-called 'Jivaka Pustaka', the title given by Bailey to a 73-folios long, Khotanese and Sanskrit bilingual, medical text. ${ }^{59}$ The text is incomplete and contains no colophon. ${ }^{60}$

Beyond this level of learned medicine, we also find a host of texts which can be viewed as belonging to a more popular strand. Some of these correspond to the testimo-

\footnotetext{
53 Emmerick [1992] 1997, pp. 1-3.

54 Emmerick 1979 .

55 Rong Xinjiang 2004, pp. 57-62 and pp. 6o-1.

56 Quoted in Emmerick 1979b, p. 169 (from Emmerick's edn, pp. 2O-1). For the proposed dating, see Thomas 1935, I, p. 42. Thomas suggested that the text was composed in the Dunhuang area.

57 See Maggi 20o9; Emmerick [1992] 1997.

58 Emmerick 1980.

59 India Office Library ms. Ch ii oо3 - IOL кнот, pp. 87-110.

6o For an English translation, see Konow 1941. See also, Hoernle 1917, pp. 415-32; Emmerick 1980; Emmerick 1979a.
} 
ny of the Tibetan History of Khotan text quoted above, stating that the 'customs of the people agree for the most part with China'. These are yet to be studied properly, but some of these popular medical texts can be pointed out. They include, for example, a number of fragments from the Crosby Collection $(78+79 ; 104+105 ; 184+185 ; 190+191)$, which mention needles and cauterisation. ${ }^{61}$

There are also a number of Khotanese texts within the sphere of popular ritual medicine, such as omen texts and divination texts based on the 12 year cycle, which appear to bear resemblance to Chinese and Tibetan texts, some also including Chinese parallel text. ${ }^{62}$ It is thus wholly understandable how Pt.1044 could be a Tibetan adaptation of a Khotanese medical text which preserved Chinese notions - or at least that this is how its author could have perceived it.

\section{A Uighur Parallel}

The process of transcreation did not stop there. Elements of the illustrated Dunhuang medical traditions continued to have traction around the Taklamakan Desert. One Uighur manuscript fragment testifies to the longevity of the local tradition. A Uighur chart preserved on the northern route around the desert survives in the Turfan collection at the Berlin-Brandenburg Academy of Sciences and Humanities (Fig. 6). ${ }^{63} \mathrm{~A}$ preliminary dating by Müller has placed the texts on this scroll in the 11th-12th centuries, but according to Dieter Maue, this dating needs to be revised. ${ }^{64}$
61 Emmerick 1993.

62 Maggi 2009. A text combined with drawings where demons causing children's illnesses are depicted together with a collection of formulas against demons. Khotanese with Chinese parallel text (Ms. Ch oo217 a-c; Maggi 1996, pp. 123-37. Reproductions in Whitfield et al. 1990, pp. 90-1 (no. 69).

63 Mainz o725, p. 2.

64 Dieter Maue, pers. comm.
Once again, we see the basic elements of the early moxa-cautery charts: the simple outline anterior view of figures with striated rib cages, 11 black dots marked on the upper body wherein the medical significance of the texts resides, the lines linked to terse captions, for example, bašta the 'head', köküztä the 'chest', talta 'the spleen'. Other lines point to captions indicating places on the forearm and the knee. Where the lines on the first figure indicate spots on the head, and the upper body, the blocked text next to it describes symptoms also located in the head: 'heat in the head', 'curling tongue' and 'unclear speech'. In the Uighur case there is also a new feature integrated into the chart. The treatments themselves seem to include medical recipes to be combined with moxa-cautery. The recipes include instructions on how to prepare complex remedies with animal substances, horn, fat and a form of pepper, measured according to standardised weights.

The Uighur figures reveal a shared Buddhist quality to medical figure drawing - the central figure sits in padmāsana (Lotus Posture), with hands positioned in a Buddhist gesture, possibly dhyāna-mudra. ${ }^{65}$ The rough execution and the slightly angled view of the central and right-hand side figures associate the Uighur chart with the Tibetan moxa-cautery chart. The figures are also less standardised than the Chinese versions, and the hairdos a rather elaborate curly coiffure, neatly restrained, perhaps a version of the way the Tibetan figures have their hair swept back from the face in a ribbon. More modesty is evident in the substantial underwear of the figure on the right, although clearly outlined breasts in the naked upper body of the figure on the left identify one of the figures as a nude female. Their empty pupil-less eyes lack the lively intentionality of the Tibetan figures, but in contrast to the Chinese versions they are wide open, which serves to convey a certain individual expressiveness, if yet a passive receptivity.

65 Müller 1923, pp. 21-6. 


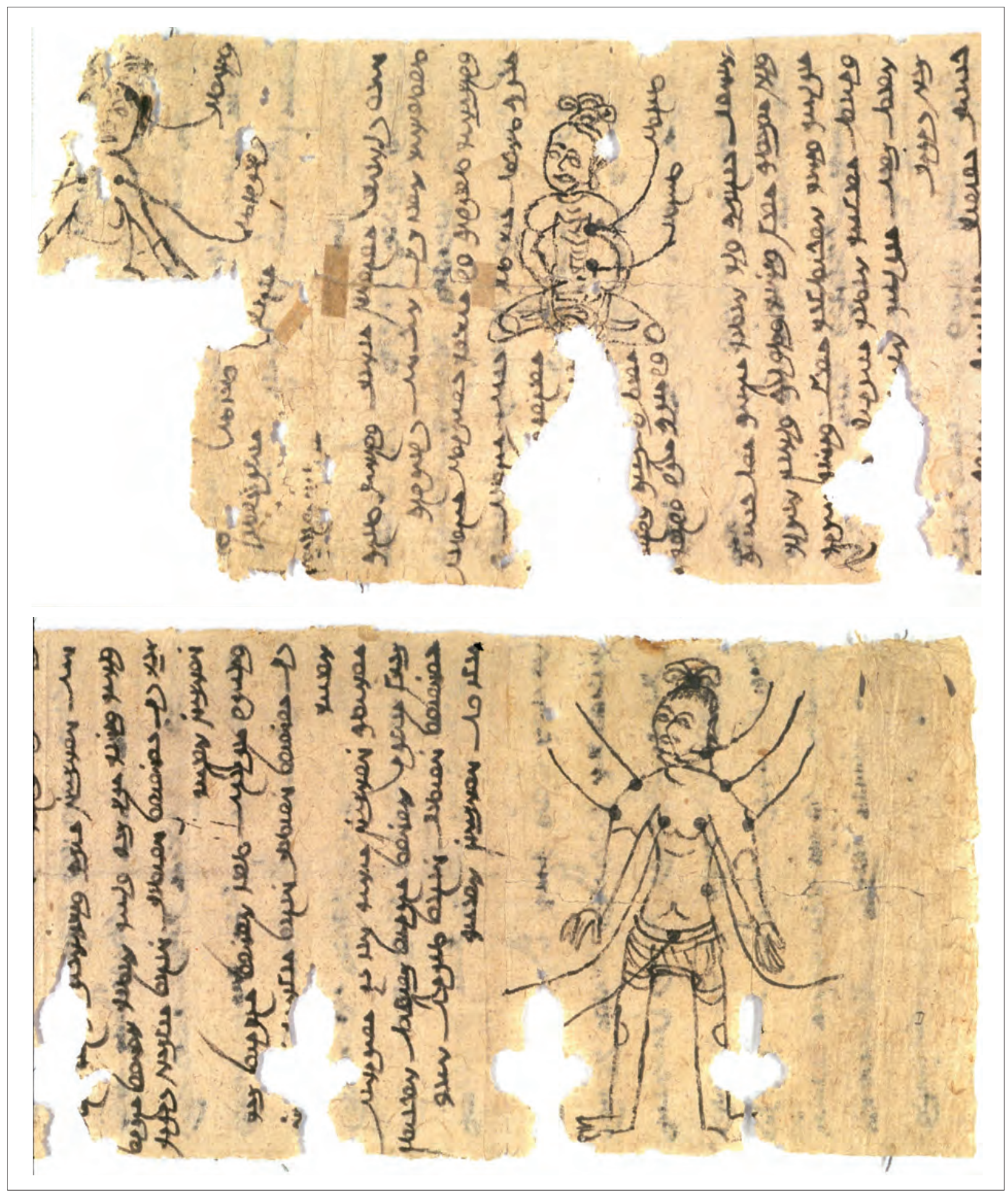

Figure 19.6 A Uighur moxa-cautery chart with remedies. Mainz o725, p. 2. @ Berlin-Brandenburg Academy of Sciences and Humanities

\section{Continunities Beyond the Medieval World}

Fortunately for us, the Chinese prohibitions protecting the flow of the human spirit were transmitted to Japan from the ioth century onwards, where many lost Chinese medical traditions are preserved in text and manuscript.
As a result, while the illustrated tradition is otherwise lost in the transmitted Chinese literature, it is preserved in prohibition texts in Japan which demonstrate direct continuities with the Tibetan and Chinese medieval moxa-cautery charts. 




Figure 19.7 The circulation of bla in the body: the different locations are marked by syllables. Tibetan Medical Painting no. 12 (Ulan Ude set)

In Tibet we also find a later visual depiction of the flow of the bla, represented in the Tibetan Medical Paintings, which illustrate Sangye Gyatso's (1653-1705) Blue Beryl. Medical Painting no. 12 has a visual depiction of flow of the $b l a$, as it was synthesised with Indic tantric notions found in the Kälacakra Tantra, where each day of the lunar month (and the flow of the bla) is associated with a syllable (Fig. 7) ${ }^{66}$

Meanwhile in Japan, we find the Hama jing 蝦蟆經 (Toad Canon), extant in several 18th-century illustrated

$66 \quad$ Gerke 2011, ch. 5 .
Japanese manuscripts, testifying to further continuities in this pan-Asian tradition. The pairing of a practical tradition of moxa-cautery and acupuncture practice with calendrical prohibitions survives therein over some eight centuries from north-west China (modern Gansu) from the time of the Han dynasty Wuwei manuscripts to the Dunhuang cave library manuscript collection, and then on another millennium to the Hama jing ${ }^{67}$ Once again we see the black

67 Tamba no Yasuyori 984, Ishimpō 2. The Hama jing survives in an 1823 woodblock edition known as the Weisheng huibian 衛 生彙編. Five 19th-century illustrated Japanese manuscripts at- 




Figure 19.8 The Toad and the Hare in the Moon. Acu-moxa prohibitions on the full moon and the day before the full moon, to protect the passage of the human spirit. Hama jing, Zhongyi guji, Beijing, 1984, pp. 16-17

and white outline images of the body with lines indicating strategic black dots on the body; in this case the dots mark the place where the Qi or the 'human spirit' resides on a particular day of the lunar cycle, and where treatment is contra-indicated. The toad in the manuscript title alludes to the toad and hare that traditionally reside in the moon, much as in Europe we might see a pareidolic man in the moon. Each of the 30 figures depicted in the Hamajing illustrates where the human spirit resides on one day in the lunar cycle, with a sketch of the moon itself marking the progression, the dark sides of the moon's phases gradually obscuring and then revealing the two animals until they are both visible at the full moon (Fig. 8).

Comparatively long textual explanations in the Japanese editions of the Hama jing list the perils of damaging the human spirit - from minor reactions like redness of the eyes, to violent death, and many instances of sexual and

test to the illustrated tradition. Kokkai Toshokan 国会圖書館 (National Library); Kyōdai Fujikawa Bunko 京大富士川文庫 (Fujikawa Library at Kyoto University) [edn with commentaries by Mori Yakushi 森約之 in 186o]; the private Tokyo Mukyukai kan-narai Library 無窮会神習文庫 [edn with commentaries by Mori Risshi 森立之 $c$. 1830, privately owned by Ota Shojirō 太田晶二郎]; of unknown location formerly in the possession of Tomioka Tessai 富岡鉄斎 (1836-1924). Both editions with commentaries refer to a further lost manuscript owned by $I s$ $s h \bar{u} d \bar{o}$ 聿修堂. The $5^{\text {th }}$ edition, in hanging scroll form, dating to 1831, was rediscovered by Nagano Hitoshi in the archives of the Naitō Kinen Kusuri Hakubutsukan 内藤記念薬博物館 (Naitō Memorial Museum for Medicine). This may be a copy of Tamba no Motoyasu's original text since it attempts to copy his original seal (i.e. ‘廉夫’ and ‘丹波元簡'). The 'rediscovery' and naming of this copy is described in Nagano 2001, p. 414 et passim. reproductive disorder. Just as in the surviving Tibetan tradition which imagines the bla flooding the body once a month at full moons, it was deemed particularly inauspicous to treat at the full moon when it is not fitting to harmonise Yin and Yang [engage in sexual intercourse]; women will be struck by Wind illness. Greatly prohibited, not insignificant. On the sixth day of the lunar month sex will bring on carbuncles and ulcers' ${ }^{68}$

The Hamajing concludes with a short and unusual treatise that captures the plural nature of the moxa-cautery traditions. ${ }^{69}$ The moxa-cautery materials were to be prepared under an effigy of Bian Que 扁鵲, a well-known patron of the acupuncture traditions, and the treatment was conducted in a ritual space sanctified by the Celestial Physician and the Celestial Master, deities in the oldest recorded institution of Daoism. The incantation, which is fully transcribed, reflects the bureaucratic character of Celestial Master Daoism. It exorcises malevolent spirits, and poisons contracted from ghosts, wind, cold, and impurities of the diet, and calls on the assistance of Daoist deities and the animals of the constellations:

To the left, the empress, Mother Queen of the West. To the front, the Vermilion Bird. To the rear, the Black Warrior 玄 武, (the Herdboy?) and Weaving Girl cause me to moxa you.

It then concludes:

The needle does not encounter the spirit. In piercing I do not cause harm or injury. Sickness and illness quickly be

68 Huangdi hamajing 1984 reprint, p. 164.

69 Partially reproduced in Zhi zhubing xiang bei zhou 治諸病向背 咒 (Incantation used with directional orientation for treating various medical disorders), Ishimpō 2, p. 59. 
gone. Urgent, urgent, in accordance with the statutes and orders, ${ }^{70}$

and the final instructions: 'In all cases when treating illness recite the incantation thrice, and afterwards moxa and needle them'.

This passage provides us with a unique social and religious context for the preceding thirty illustrations of the flow of the human spirit in the Hamajing, and so brings this chapter to a neat conclusion with evidence of the survival of both content and illustrative genre in medical literature transmitted into modern times. The practitioner, most probably a Celestial Master Daoist, has created a ritual Chinese environment to optimise cautery and acupuncture treatment, and as a part of his preparations he must know and avoid the exact location of the human spirit as it flows on its lunar cycle around the body, for fear of injuring it and causing terrible damage to the patient.

\section{Conclusion}

The structure of the moxa-cautery charts, the simple black and white figures with their brief, instructive, captions provided a seemingly infallible short-cut to cross-cultural therapy, a quick and easy visual code available to all those with basic literacy. The simple diagrammatic representations could override barriers of language and education, obviating the need to know complex medical theories, and give the impression of facilitating the transmission of medical knowledge through focusing on the essentials. The Tibetan and Chinese moxa-cautery texts represent very similar but not identical styles of practice. We have to imagine that at some point during the 9 th-1oth centuries in the Dunhuang region medical writers and copyists fused concepts of the Tibetan brla spirit with Chinese lunar cycles of the circulation of Qi and the renshen 'human spirit'.

Sensitised to these nuances we begin to perceive the charts as an empty vessel into which could be poured new and culturally specific concepts of the body and its internal workings; each time moving from one context to another the charts become evidence of a new collective endeavour, stimulating changing outputs and interpretations. The Tibetan chart is not simply a copy of the Chinese charts; it is not a passive acceptance of the priority of Chinese

70 The use of the term, jïji ru lü ling 急急如律令 (Urgent, urgent, in accordance with the statutes and orders) often implies that the practitioner is speaking with the authority of an ordained priest of the Celestial Master; $c f$. the use of this formula throughout Qianjin yifang ch. 29-30. See Sivin 1995, ch. VII, n. 18. Our italicisation knowledge, but represents a way of adapting foreign techniques to enhance local know-how - the two colophons of the related Tibetan moxa-cautery texts are forms of legitimating the new fusion with a new trans-cultural imperial and religious identity.

Collectively, the moxa-cautery and prohibition charts offer direct witness to a medieval visual exegesis that seamlessly reconciled divergent meanings. They are vivid testimony to the success of the simple medieval line diagram in facilitating practical therapies - not just spontaneously in isolated places as distant as medieval Europe and Japan, but in the execution of 'travelling medicine', 'travelling light' as it passed through the scribes and medical practitioners in and around Dunhuang.

\section{Bibliography}

\section{Primary Sources}

\section{Manuscripts}

Mainz 0725, Berlin-Brandenburg Academy of Sciences and Humanities Melothesia Astromedical text Lat. 7028, fol. $154 r^{\circ}$ (BNF)

Pelliot tibétain 1058 (BNF)

Pelliot tibétain 127 (BNF)

Pelliot tibétain 1044 (BNF)

Plut. 73.41, Biblioteca Medicea Laurenziana, Florence

Stein Or.8210/S.6168 (BL)

Stein Or.8210/S.6262 (BL)

Wuwei 武威 Hantanpo 旱灘坡 medical manuscripts (Eastern Han). Modern edn: Wuwei Handaiyijianyanjiu 武威漢代醫簡研究, Zhang Yanchang 張延昌 and Zhu Jianping 朱建平 (eds) 1996, Beijing: Yuanzineng chubanshe

\section{Printed Sources}

Beiji qianjinyaofang 備急千金要方 (Recipes Worth A Thousand Gold for Emergencies) (comp. 650-9), Sun Simiao 孫思貌 (d. 682). Reprint, Shanghai: Shanghai guji chubanshe, 1995.

Huangdi hama jing 黃帝蛤蟆經. 1823 woodblock edn known as the Weisheng huibian 衛生彙編. Kokkai Toshokan国会圖書 館 (National Library); Kyōdai Fujikawa Bunko 京大富士川文庫 (Fujikawa Library at Kyoto University) [edn with commentaries by Mori Yakushi 森約之 in 186o]; the private Tokyo Mukyukai kan-narai Library 無穹会神習文庫 [edn with commentaries by Mori Risshi 森立之 $c .1830$, privately owned by Ota Shojirō 太 田晶二郎]; of unknown location formerly in the possession of Tomioka Tessai 富岡鉄斎 (1836-1924). Reprint: Beijing: Zhongyi guji chubanshe, 1984.

Huangdineijing suwen 黃帝內經素問. Edn, 762, Wang Bing 王冰 (ed.). Beijing: Zhongyi guji, 1994.

Huangfu Mi 皇甫䍀 Zhenjiu jiayi jing 針炎甲乙經 (AB Canon of Acupuncture and Moxa-cautery) 3rd century. Tr. Unschuld, P.U.U. and H. Tessenow 2011, Huang Di nei jing su wen, Berkeley/ Los Angeles: University of California Press.

Ishimpō, Tamba no Yasuyori 丹波康賴 984. Reprint, 1993. Beijing: Huaxia.

Qianjin yifang千金翼方 (Supplement to Recipes Worth A Thousand 
Gold) (comp. c. 682) Sun Simiao 孫思邀 (d. 682). Reprint, 1955. Beijing: Renmin weisheng chubanshe.

Rgyud bzhi (Four Tantras) (bdudrtsi snying po yan lag brgyad pa gsang ba man ngag gi rgyud), 1982. Modern edn: Bod ljongs mi dmangs dpe skrun khang, Lhasa, 2000.

Waitai miyao 外臺秘要 (c. 752), Wang Tao 王壽. Reprint, 1993, Gao Wenzhu 高文鑄 (ed.). Beijing: Huaxia chubanshe.

\section{Secondary Sources}

Akasoy, A., C. Burnett and R. Yoeli-Tlalim (eds) 2008, Astro-Medicine: Astrology and Medicine, East and West, Florence: Sismel.

Arrault, A. 2010, 'Esprit humain (renshen) et esprit du jour (riyou)', in Despeux (ed.), 285-332.

Arya, Pasang Yonten 2014, 'External therapies in Tibetan medicine: The Four Tantras, contemporary practice, and a preliminary history of surgery', in Hofer (ed.), 64-89.

Chang Che-chia 張哲嘉 2006, 'Riyong leishu [yixue men] yu chuantong shehui shumin yixue jiaoyu' 日用類書「醫學門」與傳統社會庶民 醫學教育 (The medical sections of everyday encyclopaedias and the education of ordinary people in traditional society), in Mei Jialing (ed.), $167-85$.

Cohen, S. 2014, Transformations of Time and Temporality in Medieval Art, Leiden: Brill.

Cong Chunyu 叢春雨 1994, Dunhuang Zhong yiyao quanshu 敦煌中 醫藥全書, Beijing: Zhongyi guji chubanshe.

Dalton, J. and S. van Schaik 2006, Tibetan Tantric Manuscripts from Dunhuang: A Descriptive Catalogue of the Stein Collection at the British Library, Leiden: Brill.

Dalton, J., T. Davis and S. van Schaik 2007, 'Beyond anonymity: paleographic analyses of the Dunhuang manuscripts', Journal of the International Association of Tibetan Studies 3, 1-23 (available online).

Despeux, C. (ed.) 2010, Médecine, Religion et Société dans la Chine Médiévale, Paris: Collège de France.

Dotson, B., Kazushi Iwao and Tsuguhito Takeuchi (eds) 2013, Scribes, Texts, and Rituals in Early Tibet and Dunhuang, Wiesbaden: Reichert-Verlag.

Emmerick, R.E. 1979a, 'Contributions to the study of the Jivaka-pustaka', BSOAS XLII 2, 235-43.

1979b, 'The historical importance of the Khotanese manuscripts', in Harmatta (ed.), 167-77.

1980, The Siddhasāra of Ravigupta, Wiesbaden: Franz Steiner Verlag (2 Vols.)

[1992] 1997, A Guide to the Literature of Khotan, (2nd edn), Tokyo: International Institute of Buddhist Studies.

1993, 'Notes on the Crosby collection', in Skalmowski and van Tongerloo (eds), 57-64.

Emmerick, R.E. and M. Macuch (eds) 2009, Persian Literature. Companion, vol. 1: The Literature of Pre-Islamic Iran, London: Tauris.

Gerke, B. 2011, Long Lives and Untimely Deaths: Life-span Concepts and Longevity Practices among Tibetans in the Darjeeling Hills, India, Leiden/Boston: Brill.

Glaze, F.E. and B.K. Nance (eds) 2011, Between Text and Patient: The Medical Enterprise in Medieval and Early Modern Europe, Micrologus Library 39, Florence: SISMEL/Edizioni del Galluzzo.

Harmatta J. (ed.) 1979, Prolegomena to the Sources on the History of Pre-Islamic Central Asia, Budapest: Akadémiai Kiadó.

Harper, D. 2003, 'Iatromancie', in Kalinowski (ed.), 471-512.

2005, 'Dunhuang iatromantic manuscripts P.2856 ro and P. $2675 \mathrm{v}^{\mathrm{o}}$ ', in Lo and Cullen (eds), 134-64.

Hartnell, J. forthcoming 2017a, 'Bloodlines. Medicine and cosmology in France, China, and Mexico', in Essays on Manuscript Illustration,
Brepols.

- forthcoming 2017b, 'Medicine's image', in Hourihane (ed.), $326-43$.

Herrlinger, R. 1970. History of Medical Illustration: from Antiquity to AD 16oo, Munich: Pitman Medical \& Scientific Publishing.

Hoernle, R.A.F., 1917, 'An ancient medical manuscript from eastern Turkistan', in Commemorative essays presented to Sir Ramkrishna GopalBhandarkar, Poone: Bhandarkar Oriental Research Institute, 415-32.

Hofer, T. (ed.) 2014, Bodies in Balance: the Art of Tibetan Medicine, New York: Rubin Museum of Art.

Hourihane, C. (ed.) forthcoming 2017, The Routledge Companion to Medieval Iconography, New York: Routledge, 326-43.

Kalinowski, M. (ed.) 2003, Divination et société dans la Chine médiévale: Étude des manuscrits de Dunhuang de la Bibliothèque nationale de France et de la British Library, Paris: Bibliothèque nationale de France.

2005, 'Mantic texts in their cultural contexts', in Lo and Cullen (eds), 109-33.

Konow, S. 1941, A Medical Text in Khotanese: ch. II. oo3 of the India Office Library, Oslo: Dybwad.

Kuriyama, S. 1999, The Expressiveness of the Body, New York: Zone.

Lalou, M. 1941-2, 'Texte médical tibétain', Journal asiatique 233, 209-11.

Landau A.S. (ed.), 2015, Pearls on String: Artists, Patrons, and Poets at the Great Islamic Courts, Baltimore: Walters Art Museum and University of Washington Press.

Li Jianmin 李建民 2002, 'Aihuo yu tianhuo - jiu liaofa yanshenzhi mi 艾火與天火炎療法延伸之密' (Moxa fire and celestial fire: the riddle of the evolution of cautery treatment), Ziran kexue shi yanjiu 21.4, 320-31.

Lo, V. 2001, 'Huangdi hamajing (Yellow Emperor's Toad Canon)', Asia Major 14.2, 61-99.

2002, 'Spirit of stone: technical considerations in the treatment of the jade body', BSOAS 65.1, 99-128.

2005a, 'Quick and easy Chinese medicine', in Lo and Cullen (eds), 227-51.

2005b, 'Self-cultivation and the popular medical traditions', in Lo and Cullen (eds), 207-225.

2010, 'Manuscrits de Dunhuang et de Khotan sur la moxibustion', in Despeux (ed.), 239-84.

Lo, V. and C. Cullen (eds), 2005, Medieval Chinese Medicine, London: Routledge.

Lo V. and He Zhiguo 何志國 1996, 'The channels: A preliminary examination of a lacquered figurine from the Western Han period', Early China 21, 81-123.

Lo, V. and Y.D. Wang 2013, 'Blood or Qi circulation? On the nature of authority in Rashīd al-Dīn's Tānksūqnāma [The Treasure Book of Ilkhān on Chinese Science and Techniques]', in Akasoy, Burnett and Yoeli-Tlalim (eds), 127-72.

Lo, V. and R. Yoeli-Tlalim (eds) 2007, Asian Medicine: Tradition and Modernity 3.2, Special Silk Roads Issue [whole issue].

Lo, V., Berlekamp, P. and Y. Wang 2015, 'Administering art, history, and science in the Mongol Empire', in Landau (ed.), 53-85.

Luo Bingfen et al., (eds) 2002, Dunhuang ben: Tufan yixue wenxian jingyao 敦煌本: 吐蕃醫學文獻精要 (The Dunhuang Manuscripts: Essentials of Tibetan Medical Literature), Beijing: Minzu chubanshe [Tun hong nas thon pa'i bod kyi gso rig yig cha gces bsdus. Pe cin: Mi rigs dpe skrun khang].

Ma Jixing (ed.) 1998, Dunhuangyiyao wenxian jijiao 敦煌醫藥文獻輯 校 (The Dunhuang Medical Texts Edited and Collated), Jiangsu: Jiangsu guji chubanshe.

Maggi, M. 1996, 'A Chinese-Khotanese excerpt from the Mahāsāha- 
srapramardan̄̄', in La Persia e l'Asia centrale da Alessandro al X secolo, Rome: Accademia nazionale dei Lincei, 123-37.

2009 'Khotanese literature', in Emmerick and Macuch (eds), $330-417$.

Meyer, F. 2002, 'Quelques traces parmi les sources de la médecine tibétaine: Les manuscrits médicaux tibétains de Dunhuang', paper delivered at the Colloque Franco-Japonais: Interactions et translations culturelles en Eurasie (EPHE et Université de Tokyo), Paris, 12-13 December 2002 (unpublished).

Mei Chia-Ling (Mei Jialing) 梅家玲 (ed.), Shibian zhong de qimeng: wenhua chongjian yu jiaoyu zhuanxing (1895-1949) 世變中的啟蒙: 文化重建與教育轉型 (1895-1949), (Out of Turmoil Comes Enlightenment: Cultural Reconstruction and Educational Transformation 1895-1949), Taibei: Maitian chubanshe.

Müller, R. 1923, 'Ein Beitrag zur ärztlichen Graphik aus Zentralasien (Turfan)', Archiv für Geschichte der Medizin, 15 November, (7oth birthday Festschrift for Karl Sudhoff), 21-6.

Murty, K.R. Srikantha (tr.) 1991, Vāgbhața's Așțāñga Hrdayam: Text, English Translation, Notes, Appendix and Indices, Varanasi: Krishnadas Academy.

Nagano, Hitoshi 2001, “Koutei Gamakyō” Rinmo Insha Kyū Shohon no Shutsugen 黄帝蝦蟇経” 臨模影写旧鈔本の出現' (Emergence of the Rinmo copy of Hama jing), Shinkyū Osaka 鍼炎大阪 (Osaka Journal of Acupuncture and Moxibustion) 61, 5-9.

Rachmati, G.R. 1932 'Zur Heilkunde der Uiguren II', Sitzungsberichte der Preußischen Akademie der Wissenschaften, 401-48.

Ramble, C. and U. Roesler (eds) 2015, Tibetan and Himalayan Healing: an Anthology for Anthony Aris, Kathmandu: Vajra Publications.

Rong Xinjiang 2004, 'Official life at Dunhuang in the tenth century: The case of Cao Yuanzhong,' in Whitfield (ed.), 57-62.

Scherrer-Schaub, C. (ed.) 2012, Old Tibetan Studies dedicated to the Memory of R.E. Emmerick: Proceedings of the 1oth Seminar of the IATS 2003, Leiden: Brill.

Sivin, N. 1995, 'Taoism and science', in Sivin, Medicine, Philosophy and Religion in Ancient China, Aldershot: Variorum, ch. viI.

Skalmowski, W. and A. van Tongerloo (eds) 1993, Medioiranica: Proceedings of the International Colloquium Organised by the Katholieke Universiteit Leuven 21-23 May199o, Leuven: Peeters.

Smith, P. (ed.) forthcoming, Entangled Itineraries of Materials, Practices, and Knowledge: Eurasian Nodes of Convergence and Transformation, Pittsburgh: University of Pittsburgh Press.
Thomas, F.W. 1935, Tibetan Literary Texts and Documents Concerning Chinese Turkestan, London: Royal Asiatic Society.

Takeuchi, Tsuguhito 2012, 'Old Tibetan Buddhist texts from the post-Tibetan imperial period (mid-9th cent. to late 1oth cent.)', in Scherrer-Schaub (ed.), 205-16.

Uebach H. and J.L. Panglug 1988, Tibetan Studies: Proceedings of the Fourth Seminar of the International Association for Tibetan Studies, Munich: Bayerische Akademie der Wissenshaften.

Uray, G. 1988, 'New contributions to Tibetan documents from the post-Tibetan Tun-huang', in Uebach and Panglug (eds), 515-28.

Whitfield, R. et al. 1990, Caves of the Thousand Buddhas: Chinese Art from the Silk Route, London: British Museum Press.

Whitfield, S. (ed.) 2004, The Silk Road: Trade, Travel, War and Faith, London: British Library.

Xie Guihua 谢桂华 2005, 'Han bamboo and wooden medical records discovered in military sites from the north-western frontier regions', in Lo and Cullen (eds), 77-105.

Yamada, Keiji 1998, The Origins of Acupuncture, Moxibustion and Decoction, Kyoto: International Research Centre for Japanese Studies.

Yearl, M.K.K. 2011, 'Bloodletting as recreation in the monasteries of medieval Europe', in Glaze and Nance (eds), 217-43.

Yoeli-Tlalim, R. 2010, 'Tibetan 'wind' and 'wind' illnesses: towards a multicultural approach to health and illness', Studies in History and Philosophy of Biological and Biomedical Sciences 41.4, 318-24. 2013, 'Central Asian mélange: early Tibetan medicine from Dunhuang,' in Dotson, Iwao and Takeuchi (eds), 53-6o.

2014, 'Medicine, astrology, and divination' in Hofer (ed.), 90-109.

2015, 'Between medicine and ritual: Tibetan 'medical rituals' from Dunhuang,', in Ramble and Roesler (eds), 739-46.

forthcoming, 'The Silk-Roads as a model for exploring Eurasian transmissions of medical knowledge: views from the Tibetan medical manuscripts of Dunhuang', in Smith (ed.).

Yonten, Pasang 2014, 'External therapies in Tibetan medicine: the Four Tantras, contemporary practice, and the preliminary history of surgery', in Hofer (ed.), 64-89.

Online Resources

Encyclopaedia Iranica: www.iranicaonline.org. 


\section{Chasing the Vermilion Bird: Late-Medieval Alchemical Transformations in The Treasure Book of Ilkhan on Chinese Science and Techniques}

\author{
Vivienne Lo 羅維前 and Wang Yidan 王一丹
}

Edward Schafer's 1967 title, The Vermilion Bird, was partially a trope to convey Tang (618-907) Chinese images of the tropical south as a mysterious, exotic, sensual, and therefore dangerous, realm. One of its many manifestations was the red planet Mars emerging from the velvet black of the tropical night. To the medieval Chinese it was the spirit 'Anger of Red Sparks' or the 'Dazzling Deluder'. His place is in the 'Southern Quarter'... The Fire red God is embodied in a Vermilion Bird... . The northward drift of the sun, inhabited by a red crow (a cousin of the Vermilion Bird), to its ultimate goal, the Tropic of Cancer... . ${ }^{1}$

The Vermilion Bird was custodian of the night sky, emblem of the southern constellations. Like the other si xiang 四 象, the animals of the four directions that we know from Han astronomical/astrological traditions, it also lived within the viscera of the medico-religious body in China, determining human health and well-being. Our Vermilion Bird for, example was a nervous, flighty, creature who lived in the Heart and determined a person's essential stability. This chapter draws on others in this volume to highlight the overlapping registers of medicine, religion and astronomy that made these multiple animal transformations possible (See Despeux, Chapter 2; Zhang, Chapter 27, in this volume). It will then analyse what happened as the Vermilion Bird tried to fly West, outside of its Chinese domains, and encountered conflicting Persian beliefs about the landscape of the inner body.

The rise of the Mongolian empire and its four Mongol khanates - the Yuan in China (1271-1368), the Golden Horde in Russia (1240s-1502), the Chagatai in Central Asia (1225-16o7), and the Ilkhans in Iran (1245-1343) precipitated the largest scale movement of peoples, ideas and practices in the pre-modern world. In the diverse political, religious and commercial exchanges that followed there was a tension between the pragmatic need to develop commensurable knowledge that could facilitate integration of local cultures to the new world order, and the desire to maintain local identity and difference. This tension will be illustrated with a study of the reception of Chinese medical imagery in the translation projects of the eminent Jewish court physician and minister Rashīd al-Dīn Tabīb (1247-1318) of Hamadān.

Among the prolific works of this polymath the most famous is Jāmi al-Tawārīkh (Compendium of Chronicles) completed 1307 to 1316 . Even though this is a work dedicated mainly to Mongolian history it is also the earliest history of the world to aspire to cultural inclusivity and includes the stories of Adam, the Buddah, Moses, as well as Mohammed, and lists Chinese rulers from Pangu 盤古, the first mythic king, to the Song and Jin emperors. This inclusive world history seems to be an intellectual reflection of the political ambitions, administrative techniques and the cosmopolitan tastes of Rashīd al-Dīn's Mongol leaders, tempered with his own experience of the diverse worlds of the Ilkhanate.

Rashīd al-Dīn also masterminded the earliest extant monograph about Chinese medicine to be produced to the West of China, the Tansūqnäma-i İlkhān dar funūn-i 'ulüm-i Khatā' $\bar{\imath}$ (The Treasure Book of the Ilkhan on Chinese Science and Techniques, hereafter Tansūqnāma); dated anno Hegirae 713 (1313). Tansūqnāma contains substantial tracts of translation that provide a rich resource for analysing the process of interpretation through which remote 'ethnic' medical ideas were assimilated to the knowledge of an ever-expanding Islamic universe.

Rashīd al-Dīn faced many challenges as he set about reconciling Chinese and Persian religious realities as they related to medicine. Some of these he resolved creatively, others he decided not to take on. The esoteric traditions of Chinese internal alchemy (neidan 內), as opposed to waidan 外丹, external alchemy, which was concerned with drug compounding) were steeped in beliefs about the way spirits and spirit animals inhabited the body and were active in refining bodily elixirs in the pursuit of long life and transcendent states. The imagery associated with these spirit animals, and particularly the four directional spirits of antiquity (the Vermilion Bird in the South, White Tiger in the West, Blue-Green Dragon of the East, Black Turtle in the North), in their association with specific organs of the body, features in charts directing Daoist meditation and healing, as well as more mainstream medical texts (Fig. 1) (See Zhang, Chapter 27; Shin, Chapter 23, Fig. 23.1, in this volume). While Rashīd al-Dīn accepted and copied many medical charts from Chinese printed books, he seems not to have been keen on the spirit animals. We will suggest some reasons for his hesitation in embracing and copying the complete range of Chinese medico-alchemical illustrations into Tansūqnàma, despite the probability that he was familiar with them. 


\section{Background}

In 1264 Genghis Khan's grandson Qubilai Khan (r. 126o-94) consolidated Mongol rule in the Middle Kingdom, controversially establishing their winter capital in the east at Beijing. In 1271 he formally inaugurated China's Yuan dynasty. Geographically speaking, the Mongol empires were reaching their zenith, and facing all the inevitable tensions that come with rapid imperial expansion. In the administration of this new world Qubilai learnt well the lessons of a millennium and more of Chinese imperial bureaucratic practice, its attention to empire-wide standardisation of coinage, writing and vehicles, in order to ensure smooth operation of the economy, taxation, and military movements. Accordingly, he commissioned a new script intended to facilitate communication across an ever more linguistically diverse people.

Under the influence of a powerful Tibetan Buddhist, aPhags-pa (1235-died 1280), Qubilai Khan announced Buddhism as the official religion of a multi ethnic, multi religious empire. ${ }^{2}$ Of the many religions that he had been exposed to from across Asia, Tibetan Buddhism was probably closest in its ritual and practice to his native Mongolian shamanism and the religion of the last great empire in central Asia. ${ }^{3}$

Within 30 years, towards the west of the Yeke Mongol Ulus (the great Mongol patrimony) at the Ilkhanid capital of Azarbaijan, Rashīd al-Dīn, following in the spirit of the times, converted to Islam. Islam was the faith of the majority of the non-Mongol local population and that of his patron, Ghazan Khan (1271-1304), whose own conversion from Christianity on 16 June 1295 had gained him the support of the strategic, but more radical, Muslim General, Nawrūz. ${ }^{4}$ Despite Ghazan Khan's expedient decision to have all his subjects convert to the Islamic faith, and episodic suppression of competing religions under his rule, he apparently also practised Mongolian shamanism, worshipped their celestial deity, Tengri, and kept the Mongolian law and traditions. He had a Chinese Mongolian wife and spoke Chinese, Arabic and 'Frank'.

In the tension between a belief in the efficacy of political, administrative and religious unity and the enduring affinities of birth and ethnic identity peculiar to the Mongolian empires, we find the background dynamic to our story of medico-religious resistance, assimilation and transformation - at one end of the tale in a 13th and 14th century
Daoist alchemical tradition in China and at the other in the transformations that took place as Chinese illustrations and text were adapted to a 1313 Persian treatise on blood circulation and physiology in late medieval Tabriz. In the enterprise of one particular individual vested in building bridges for personal survival and academic excellence, we find a world that appreciated the power of universal truth yet understood the creative potential that lies in the flexibility of meaning - a world uniquely receptive to the kind of adaptations that would facilitate knowledge in transit. We will also discover the limits of that creativity.

\section{a) Travelling Knowledge and Practice}

Much of our interest in Jāmi al-Tawārīkh lies in the information about Chinese history that Rashīd al-Dīn assimilated from Buddhist monks, travellers and scholars. Two Chinese described in the introduction apparently brought to Tabriz all kinds of historical and other texts, attributed to the work of three monks. They had come themselves across the breadth of the well-ridden routes of the empire from China and Tibet, through Dunhuang, Kuche, Khotan and Samarkand to Rashīd al-Dīn's kitabkhāna, the translation workshop and scriptorium that he established in the suburb of Tabriz. This centre in the Rab'-i Rashīdī, with its hospital and medical school became what Allsen claims was one of the 'leading cultural clearing houses of medieval Eurasia'.

The deep entanglement of medicine and religion in the transcultural history of Central Asia is well known. Military campaigns had laid the earliest routes through central Asia, but it was trading and religious networks that really forged enduring links between Persia and China. Large translation projects such as those evidenced by the Mogao cave library, and the copying and printing of votive and other Buddhist texts were an integral feature of programmes of salvation in the medieval Chinese world, and the production of texts concerned with medicine and healing was a part of this enterprise. ${ }^{7}$ Medical knowledge and practice moved in both directions, east and west, in the form of books, manuscripts, practices and materia medica. In the 1st and 2nd centuries CE, for example, Sogdian trading families with Persian names were active in bringing Buddhism from the Indo-Parthian kingdoms of western India to China. Familes with Persian names dominated the trade in fragrant herbs and their presence in medieval China stimulated the Tang dynasty vogue for exotic commodities. ${ }^{8}$ Some of those Persians were Christian

\footnotetext{
$6 \quad$ Allsen 2001, p. 144. Lo and Cullen, 2005

$7 \quad$ Barrett 2007, pp. 42-55.

8 Chen 2007, pp. 240-63.
} 
Nestorians, well known for their skills in opthamology, which was used, perhaps, as a form of proselytising. ${ }^{9}$

Chinese medical culture also moved in the opposite direction. Seventeen drugs associated with China are recorded in Ibn Sīnā's (Avicenna. d. 1037 CE) Kitāb al-Qānūn fi al Tibb (Canon of Medicine), demonstrating their value in the Muslim world. ${ }^{10}$ The Mongol courts apparently exchanged consignments of medicine. Rashīd al-Dīn himself mentions cubebs, cinnamon, white pepper, and a prepared headache medicine from China. ${ }^{11}$ By his lifetime the combined socio-economic institutions of tribute, trade and religion, through which the transmission of the material culture of medicine, of medical knowledge and practice, had been operating across the trade routes of Central Asia, along the so-called Silk Roads, for at least 6 oo years, if not much longer.

\section{b) Tansūqnāma}

In the 78 page-long introduction to Tansūqnāma Rashīd al-Dīn praises the superiority of Chinese technology and script and credits a Chinese scholar and two young Persians for assisting him in translation: one was Safī al-Daula va al-Dīn and another was an interpreter who was born in China and knew Chinese very well. ${ }^{12}$

There has been some controversy over the main sources for this first book on sphygmology, but it has been established that the attribution to Wānk Shükhü 王叔和 (Wang Shuhe, 265-317) author of the Maijing 脈經 (Pulse Canon) is anachronistic. The main source for the text after page 202 was a very popular 12th-century sphygmology text in rhyme titled Maijue 脈訣 (Chants of the Pulse) attributed to Gao Yangsheng 高陽生 (Song 96o-1279).

After the introduction a list of contents refers to the four sections of the work and some of the principle sources: one section is on Chinese medical principles and sphygmology, a skill much admired in the Mongolian world; the second section purports to be a translation of Wang Weiyi's 王 惟一 (c. 987-1067 CE) Tongren shuxue zhenjiu tujing 銅 人腧穴針尒圖經 (Bronze Man Illustrated Chart of the Transporting Points for Acupuncture and Moxibustion) and describes different forms of acupuncture (See Huang, Chapter 9 in this volume), the inner body, the 12 channels

\footnotetext{
$9 \quad$ Allsen 2001, pp. 146-7.

10 Akasoy and Yoeli-Tlalim 2007, pp. 217-40.

11 Allsen 2001, pp. 152-3; Klein, Zhu and Dai 2001, pp. 559-65.

12 Rashīd al-Dīn Fażl Allāh 1313, ed. M. Mīnuvī 1971, pp. 23-4, 31. There is a Turkish translation of the introduction in Süheyl Ünver 1939; see also Süheyl 1944, as quoted in Needham 1954, vol. 1, pp. 218-21. See also Franke 1951, p. 22.
}

and the circulation of the blood. ${ }^{13}$ The third section is translated from a work in the Chinese Bencao 本草 (Materia Medica) tradition and contains treatises on drug qualities and a remedy collection; the fourth and last section is a treatise on how the realm of the body and its physiology is analogous to the structure and workings of empire. ${ }^{14}$

After page $2 \mathrm{O} 2$ of Tansūqnāma the text is dedicated to the Maijue. After page 220 the short sections of text dedicated to each of the chants are frequently divided into three, beginning with a Persian phonemic transliteration of the Chinese chants about the pulse, followed sometimes by a direct one-to-one translation, and finishing with teaching notes about difficult issues that might arise for a Persian audience. From this structure it is evident that the intention of the compiler was to aid Persian medical students to recite the Chinese poems in Chinese, with a teacher then explaining the meanings of the original Chinese text and elucidating difficult details.

Scholars have been unable to identify a single text as the source for the translation prior to page 201, which is a compilation of medical and alchemical texts dating to the early empires, including excerpts from the Huangdineijing 黃帝内經素問/靈樞 corpus (Yellow Emperor's Inner Canon (c. 1st-3rd century), and Huangfu Mi's 皇甫䍀 (215-82) Zhenjiu jiayi jing 針炎甲乙經 (AB of Acupuncture, 3 rd century) that were deemed of interest to a Persian audience. Judging by its content Rashīd al-Dīn was clearly interested in blood circulation according to diurnal rhythms, and the physiology of the organs.

It seems likely that Rashīd al-Dīn's three monks were working with printed books and manuscripts together, the latter being more amenable to reorganisation and compilation into new works, but less easy to identify retrospectively.

Earlier analysis of Rashīd al-Dīn's work has had a tendency to concentrate on 'misunderstandings and imagination' in translation. The truly creative solutions embedded in his responses to intercultural difference have proved difficult not to notice. ${ }^{15}$ As Klein-Franke and Zhu note, to avoid a 'cultural clash', Rashīd al-Dīn reshaped the Tansūqnāma in the spirit of Islam. In excerpted translations of the Persian scholar's deliberations on the inconstant nature of heat, Kitāb Bayān al-Haqā'iq (The Explanation of Truths), for example, they introduce us to an important debate about whether the drug characteristics of inducing hot or cold in the body could have universal applicability. Both

\footnotetext{
13 Lo and Wang 2013, pp. 127-72.

14 Rashīd al-Dīn Fażl Allāh, ed. Mīnuvī 1971, pp. 79-81. Lo and Wang 2013, p. 127-72.

$15 \quad$ Klein-Franke and Zhu 1996, 400.
} 




Figure 20.1 Han dynasty stone ceiling relief from Qilingang 麒麟崗 in present-day Henan province with Taiyi 太乙 (Grand Unity) between the emblems of the constellations of the four directions. The Vermilion Bird is at the centre top, flanked by the White Tiger and the Blue-Green Dragon.

bodies and appropriate drugs changed, it was observed, as people entered different ages in their lives, or according to environments in which they lived. Universal prescriptions might be dangerous. As any good physician knows, Rashīd al-Dīn's opined, the mixture of heat and body fluids is not the same in Persians, Indians and Chinese, or even in the young and old. ${ }^{16}$ Whether these syntheses were unsatisfactory cross-cultural compromises, or creative innovations in the pursuit of deeper truths is open for consideration.

\section{c) Internal and External Chinese Alchemy}

The illustrative tradition of internal alchemy was a major source for the Tansūqnāma. The classic objective of early Chinese alchemy was to investigate and understand the universe through examining its material nature. By combining and heating the alchemical ingredients in a hermetically closed vessel a substance could be studied as it moved from its original state through different stages of transformation. The alchemist and scholar of what came to be known as waidan 外丹 'external alchemy' would then draw analogies with sequences of time, from the very origin to the end of time, followed by a return to the beginning and the source. Then the alchemist would try to speed up time by carefully regulated and repeated heating and cooling in order to turn an imperfect substance into a perfected one - base metal into 'gold', equivalent in the compounding of drugs to producing the elixir of long life. 'External alchemy' had its heyday from the 3 rd to the 9th centuries CE. ${ }^{17}$

$16 \quad$ Klein-Franke and Zhu 2003, pp. 977- 82.

17 Pregadio 200o, pp. 165-95.
The alchemists' mastery of the material world naturally extended their authority into the realm of drug taking. ${ }^{18}$ The fact that mercury, derived from the highly symbolic ore, cinnabar, plays an important role in the refining of gold would have reinforced the connection between refining precious metals and compounding elixirs. While the widespread search for immortality through drug taking came to be associated with certain strands of Daoism, it was not an exclusively Daoist practice. It is simply that by virtue of their knowledge of alchemy and materia medica significant Daoist masters like Ge Hong 葛洪 (c. 283; d. 343/363) and Tao Hongjing 陶弘景 (456-536) led others, particularly those in powerful political parties, to demand the elixir of immortality of them. ${ }^{19}$

The practice of alchemy was also an important feature of cross-cultural trade across Central Asia. There is evidence that, five centuries before Rashīd al-Dīn lived, Persians were involved in the practice of alchemy in China. In the 8th/9th century the family of Li Xun 李珣, author of the Haiyao bencao 海藥本草 (Overseas Pharmacopaeia), for example, were immigrants from Persia. Li Xun's religious background reflects the kind of eclecticism exemplified by the later Mongol rulers. Most Persians at that time were Zoroastrian. But it seems that Li Tingyi 李廷儀, Li Xun's elder brother, was interested in Daoism and, given the Daoist preoccupation with compounding the elixir of

18 Traces of lead and mercury were found in the corpse of the near perfectly preserved countess of Dai, buried in the Mawangdui burial mound in 168 BCE. She had taken all of these minerals for a long period during her lifetime. Her clothes were also soaked in cinnabar. Lo 2002, pp. 99-128.

19 Wilms 2007, vol. 2, pp. 543-4. and vol. 5, pp. 1,219-2. 




Figure 20.2 Li Jiong's Neijing tu 内景圖 (Chart of the Inner Landscape). Huangdi bashiyi nanjing zuan tu jujie in Zhengtong Daozang 1436-49, Hanfen Lou, Shanghai. (The Ming edition of the Daoist Canon). Wellcome Library, London, Loo34715

life, is likely to have been familiar with external alchemy. Haiyao bencao records many alchemical drugs in use at the time. ${ }^{20}$ Given the well-known Persian involvement in trade in fragrant aromatics, spices and medicinals it is likely that there was also some awareness of radical changes that came about in the Chinese alchemical tradition during the Tang period.

Early alchemical preparations had contained mercury, and arsenic, which, when taken over a long period of time, had the effect of nerve poisoning, the symptoms being lapses in consciousness, weakness and heart paralysis, whole body numbness, delusions, and diarrhoea. ${ }^{21}$ But the gradual nature of the pathology, coupled with bouts of ecstatic hallucination, might have deceived the user as to the nature and outcome of their habit. Eventually, it is said, over the course of some three centuries innumerable literati and some of the emperors themselves died of elixir poisoning, of arsenic and mercury.

The mistake was also made 5 oo years later in Persia. Arghun, Hülegü's grandson, was also partial to East Asian medicinals and in 1291 probably hastened his death by taking a course of cinnabar (the raw material used for making mercury), one of the drugs used extensively in Chinese alchemy. Although the physicians said to have poisoned Arghun were variously recorded as Uighur or Indians the alchemical practice also associates this tradition with
China. ${ }^{22}$ It is probable that a growing recognition of the dangers of poisoning saw the decline of external alchemy and a concentration on the safer practice of neidan 内丹 'internal alchemy' as exemplified in Figure 2, the Neijing $t u$ 內景圖 (hereafter, Chart of the Neijing tu).

\section{d) Cultivating Perfection: Chinese Medico- Alchemical Illustration}

The intertwined traditions of formal medical illustration and the illustration of internal alchemy were overlapping registers of body mapping that have already been elegantly introduced in Catherine Despeux's article in this volume (See Despeux, Chapter 2 in this volume), but are worth reiterating here. Based on analogies between macrocosm and microcosm, medicine and alchemy shared common terminology and a common illustrative history.

Internal alchemy was a uniquely Chinese practice that generated extraordinarily beautiful charts of the inner body concerned with xiuzhen 修真 (Cultivating Perfection), the practice of breath and visualisation meditations. It used a language that bears marked similarities to that of external alchemy, but the focus of its practices were, in part, the essences of the human person, which explains the shared medical history. ${ }^{23}$ Adepts worked on the ingredients of the inner elixir i.e. their own prime physiological constituents: Qi 氣, jing 精 (Essence) and shen 神 (Spirit).

They also trained their practice on perfecting an immortal being within the physical body, as illustrated in the Chart of the Internal Landscape (see Fig. 2 and note the child of immortality below the navel). At least from the $5^{\text {th }}$ century CE, if not much earlier, there had been a complex of many spirits or gods which were actively invited by Daoist adepts to take up residence within the body. ${ }^{24}$ The Huangting jing 黃庭經 (Canon of the Yellow Court), a meditation manual used in the Shangqing 上清 (Highest Clarity) Daoist tradition posited visualising 24 deities resident in both the inner and outer body. Adepts might also seek to project perfected replicas of themselves outward to meet the deities in the astral realms where they dwelled (See Fava, Chapter 25 in this volume). ${ }^{25}$

\footnotetext{
22 Allsen 2001, p. 143.

23 Skar and Pregadio 200o, pp. 464-97.

24 Records of this practice also exist in the Taiping jing of the later Han Dynasty, but the dating of that work is problematic.

25 Cultivating Perfection xiu zhen refers to a synthesis of the texts and practices of the Tianshi (Heavenly Masters) Daoist sect and the traditions of the immortality seekers that date to the Han, involving directing ritual religious practice into the interior landscape of the body. Despeux 1994, p. 52; Pregadio 2008, vol. 1, pp. 76-80: 'The Body in Inner Alchemy' 'and 'The Body as residence of Gods and Spirits'.
} 
One part of this practice involved cultivating the animal spirits within the body, initially those associated with the four quadrants and their constellations, Vermilion Bird, Black Warrior (a turtle), Blue-green Dragon, White Tiger, as well as a less familiar character Gouchen 勾陳 (Angular Arranger) representing the centre. ${ }^{26}$ (In Figure 1 the Bluegreen Dragon is on the upper left of the image, the White Tiger to the right of the Infant of Immortality, the Black Warrior (a turtle) to the left of the figure's neck and the Vermilion Bird flies away above, and to the right-hand side of his head. Note also the Deer at the base of the spine that we will encounter later.)

Not all the animals that took up residence in the body were as benign as these. Even at birth the body contained the seeds of its own destruction - initially the mother's diet of grains could corrupt its purity, but then the adept's own lifestyle was called to account. On the physiological level the body nourished three worms - also called the three cadavers or three sisters that gnawed away at the body and lodged inside it (see Fig. 2, above the Blue-green Dragon). On a moral level these integral parasites acted on the three spheres of desire, wealth, food and lust travelling to heaven to expose the sins of the individual that nourished them. Then there were were seven $p o$ 魄 'bodily souls' that performed the same function: Gluttonous Thief, Flying Fish, Filth, Stinking Lung, Dog Cadaver, Flabby Piss, Bird's Sex (see Fig. 2, to the top left). These spirits had only one desire, to kill their host and bring her/his body back to the earth to which they belonged. Many of these characters, and a host of others, feature in the genre of Cultivating Perfection diagrams, along with images of the body's mountains, starscapes, and seas in a tradition that took shape in the 13th and 14th centuries during Mongol rule.

The earliest extant Chinese version of the Chart of the Internal Landscape, is part of a set of illustrations that form a part of a medical work known as the Classic of 81 Difficult Issues (in the Inner Canon of the Yellow Lord) (Huangdi bashiyi nanjing 黃帝八十一難經), edited by Li Jiong 李駰 in 1269 CE. ${ }^{27}$ It is first seen in the Ming Daoist Canon of 1445 .

Li Jiong's set of images is one of the earliest Chinese witnesses to the Cultivating Perfection tradition, as is the Xiuzhen shishu 修真十書 (Ten Books of Cultivating Perfection), also attributed a Song/Yuan date and preserved in the Daoist Canon. ${ }^{28}$

Tr. Major 1993, p. 81. Gouchen are six stars close to the five stars of beiji 北極, the north pole asterism located in Ursa Minor.

Pregadio and Skar 200o, pp. 464-97.
Apart from the Chart of the Internal Landscape Li Jiong's text includes illustrations that have a much more anatomical flavour, in a tradition that began with depicting the organs of the executed rebel fighter Ou Xifan 歐希範. Ou Xifan was executed in $1041 \mathrm{CE}$ and the diagrams were printed under the title of Images of the Five Viscera of Ou Xifan (OuXifan wuzang tu 歐希範五臟圖). The tradition of anatomical drawings on the execution ground continued with a set known as the Yanluozi 煙蘿子, lit. 'Master of the Smoke Curtain' - diagrams. Yanluozi was a figure thought to have flourished between 936 and $941 \mathrm{CE}$. (See Despeux, Chapter 2 in this volume, pp. $5^{8-9}$ and 65). The Yanluozi diagrams feature as a part of Li Jiong's set in Classic of 81 Difficult Issues, as do a series of diagrams concerned with the Yin and Yang measurements and movements of the body, and with the manner of taking the pulse in diagnosis.

\section{Rashīd al-Dīn and the Reception of Chinese Medical Illustration}

Tansūqnāma's value is immeasurably enhanced through its inclusion of what have become some of the earliest extant copies of Chinese divinatory, medical and alchemical illustrations: these include a versions of the Hetu 河圖 (River Diagram) (See Yoeli-Talim, Chapter 30, p. 436-8, Fig. 3o.6; Despeux, Chapter 2, p. 64, in this volume.), the Taijitu 太極 圖 (Diagram of the Grand Ultimate), and the Yanluozi 煙蘿 子 anatomical diagrams, described above. ${ }^{29}$

The 23 illustrations of the Tansūqnāma are ranged across 14 sections of the second chapter of Book 1 . Most of the illustrations are concerned with explicating aspects of the Chinese concepts of the physiology of the inner organs, of Yin and Yang and the mai 脈 (that concept that is so difficult to translate and that equates with aspects of the pulse, the blood vessels and muscular contours of the body), as seen through Persian eyes; they are an important resource for understanding the intercultural exchange of ideas about the movement of blood and the pulse..$^{30}$ Of the first 14 illustrations 13 are reproduced after a fashion from, and virtually in the same order as, images collected in Li Jiong's Classic of 81 Difficult Issues and include anatomical diagrams in the Ou Xifan and Yanluozi tradition (Fig. 3).

29 In the Persian text the Yanluozi images seem unrelated to the surrounding text which describe the correspondences with the Five Agents 五行, Water, Wood, Fire, Earth or Metal in the, by then, long established ritual and technical tradition. 

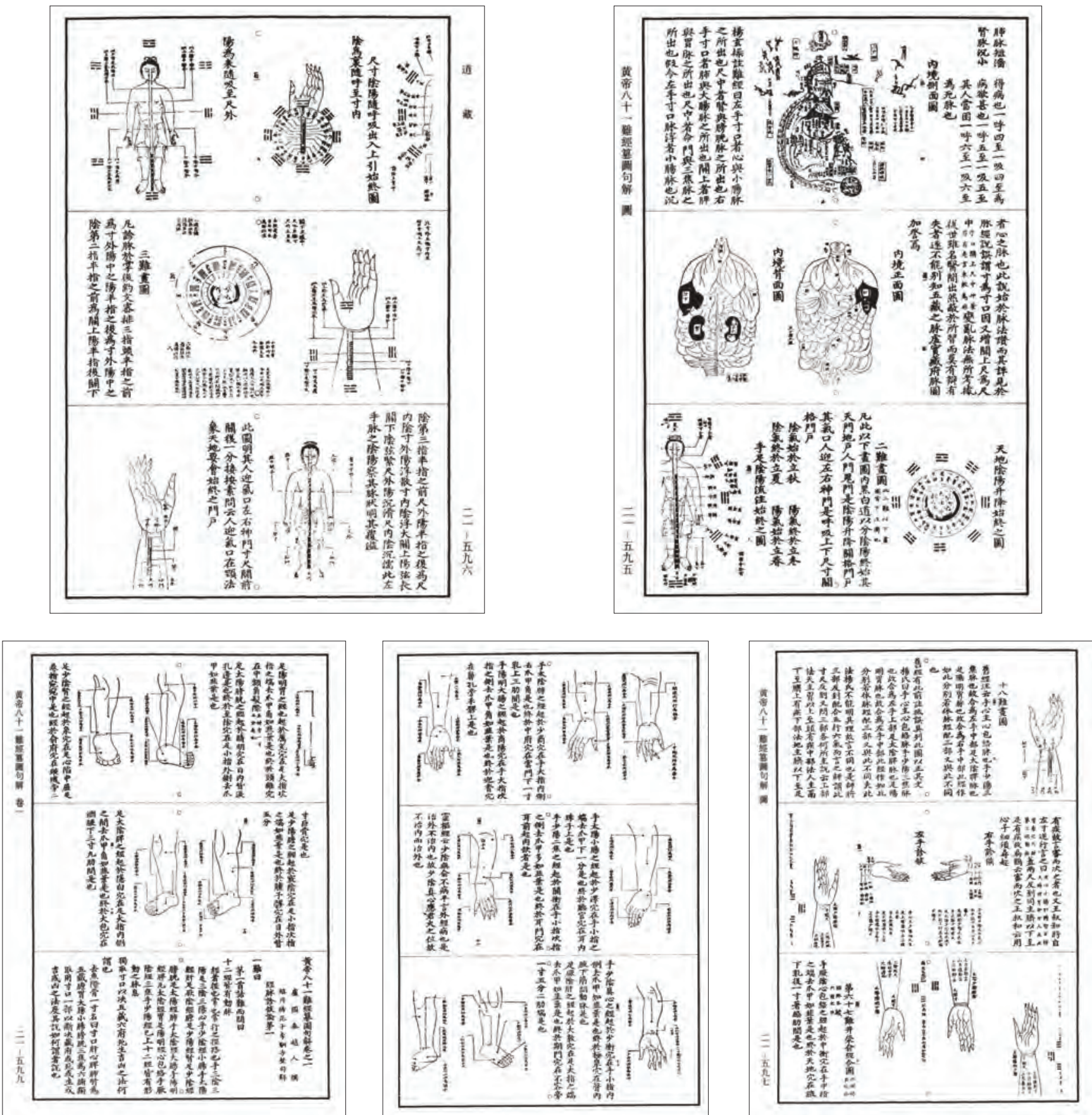

Figure 20.3 A selection of images from Huangdi bashiyi nanjing zuan tu jujie in Zhengtong Daozang 1436-49, Hanfen Lou, Shanghai, (The Ming edition of the Daoist Canon), including the Chart of the Inner Landscape with the Yanluozi images of the internal organs in the top right-hand corner. (c) Library of China Academy of Chinese Medical Sciences

This is powerful evidence that Li Jiong's text is in fact the source of Rashīd al-Dīn's illustrations (Fig. 4). Only the first of Li Jiong's images, our Chart of the Internal Landscape (Fig. 2), and the last set of five depicting the acupuncture points of the extremities, are missing from the Persian text - omissions that require explanation.

The introduction to the second chapter of Book 1 states that the illustrations are taken from Wang Shuhe's second chapter, chosen in relation to Neijing tu (Chart of Internal
Landscape). While it has already been established that the allusion to Wang Shuhe is anachronistic, the reference to the Chart of the Internal Landscape places the source material firmly within the alchemical tradition. The explicit reference to the 'Internal Landscape' is to the vision of the body and the inner organs as a microcosm of the astrological, social and spiritual worlds used in Daoist meditation described above. 




Figure 20.4 Rashīd al-Dīn's Tansūqnāma version of $\mathrm{Li}$ Jiong's images in the Yanluozi tradition Tabriz, 1313, fols 63b-64a. (c) Süleymaniye Library, Aya Sofya Collection. Türkiye Yazma Eserler Kurumu Başkanlı̆̆

Tansūqnàma illustrations three through seven are derived from a collection of images in another medical text, the Yuannmen maijue neizhao tu 元門脈訣內罩圖 (Primordial Portal, Secret Art of the Pulse, also known by the alternative title of Xuanmen maijue neizhao tu 玄門脈 訣內罩圖 Portal to the Mysteries, Secret Art of the Pulse) which, although containing materials that may date to the Sui/Tang period, is only extant in a Ming Jiaqing period edition (1522-66 CE). As early testimony to this tradition the Tansūqnāma images are therefore of extraordinary value. ${ }^{31}$ Figures $5 \mathrm{a}$ and $\mathrm{b}$ show the Persian interpretation of the 'sea of Qi', a place where Qi converged above at the diaphragm. These works are anatomical in flavour, and not explicitly concerned with meditation, but the epithet xuanmen 玄門 Portal to 'the Mysteries' links the text across the overlapping medical/alchemical fields to Daoist traditions, the mystery in question being the most profound spiritual goal of Daoist attainment as described in the classics - an oft-quoted trope in Daoist maps of the body and the Heavens.

An explanation for the missing acupuncture point illustrations may well be that the practice of pulse-taking was more attractive to Persian physicians than the practice of acupuncture, and that this led to the two Chinese


Figure 20.5a (left) A Qing period, manuscript illustration of the Yuanmen maijue neizhao tu (Internal Visualisation Charts from the 'Primordial Portal' Secret Art of the Pulse), attributed to Hua Tuo, a renowned physician of the 3 rd century BCE. This chart shows the relative position of qihai (the Sea of Qi), the diaphragm and the five zang viscera. Library of China Academy of Chinese Medical Sciences. (c) Wellcome Library, London, Loo38697

Figure 20.5b(right) Rashīd al-Dīn's version of the qihai (Sea of Qi). The Tansūqnāma, Rashīd al-Dīn, Tabriz, 1313, fol 66b. (c) Süleymaniye Library, Aya Sofya Collection. Türkiye Yazma Eserler Kurumu Başkanlığı 




Figure 2o.6 Chart of the Heart Connections, The Tansūqnāma, Rashīd al-Dīn, Tabriz, 1313, fol. 65b. (C) Süleymaniye Library, Aya Sofya Collection. Türkiye Yazma Eserler Kurumu Başkanlığı

techniques bifurcating in Persian practice. Pulse-taking was a familiar practice in the Persian medical world, but acupuncture would have been seen as strange and illogical. In 1241, for example, a local solution to this conundrum was devised. Physicians tending to Ögödei, Genghis Khan's third son, had diagnosed an irregularity in his pulse, Chinese style, but he did not receive acupuncture treatment for his illness. Instead, the moral solution of an amnesty was declared. ${ }^{32}$

The selection of illustrations in Tansūqnāma demonstrates that Rashīd al-Dīn's religious sensibilities were challenged by the medieval Chinese Daoist adoption of anatomical texts for meditation purposes, and by the image of the human body as embraced within the influence of, and inhabited by, the planets, stars and their spirits. ${ }^{33}$ Besides Rashīd al-Dīn was trained in other competing traditions through which he would filter Chinese ideas about the movement of animal spirits in the body. An explanation of the omission of the Cultivating Perfection image is the main focus of the rest of this chapter.

\section{The 'Chart of the Heart Connections'}

Tansūqnāma book 1, chapter 2, section 2 states that the accompanying image comes from Wang Shuhe and bears the legend Xinxitu 心系圖' (Chart of the Heart Connections) (Fig. 6). We know that the Wang Shuhe attribution is anachronistic and that this image is exant in medical collections such as the Xuanmen maijue neizhao tu. The ideas alluded to by the text in both the Chinese Xuanmen maijue neizhao tu and the Persian interpretation have some similarities if we

\footnotetext{
$32 \quad$ Allsen 2001, p. 146.

33 Levi 1989, pp. $105^{-27}$.
}

loosely equate the passage of clear air with the movement of Qi described in the Chinese text. Both texts deal with the physiology of the Lungs, Heart, Liver and Kidneys and Bladder and its relationship to the bone structure. Where the Chinese text describes Qi moving from the Lungs to the Heart, the Persian text has clear air moving in the opposite direction. It then moves through three vessels back to the Lungs, to each of the Kidneys, finally connecting to the Bladder. Both texts describe a scattering of fluids/ Qi at the level of the Bladder. The Persian account tells us how animal spirits (soul) travel downwards with the Yin from the Heart to the lower body and it is this aspect of the text that particularly concerns us here.

Tansūqnāma Book 1: Chapter 2. Section 2: On the explanation of the heart and its arteries, including all of its vessels, known as the 'Chart of the Heart Connections'.

From the Heart there is a vessel that connects to all the vessel passageways of the organs. Now we will narrate how the animal soul is born, and also how it pours into the organs. According to what they say, the blood fluids are born in the Liver and, moreover, when it all ripens, there is a kind of vapour that flows into the Heart, and is nurtured there, changing into the animal soul. When one breathes the clear air into the Lung, then from the Lung the clear air arrives at the Heart. When the clear air from the Lungs arrives at the Heart, it fills it with this animal soul through the vessels to all the organs and to the extremeties, and all the bones are filled with this animal soul. In this way when the animal soul goes quietly it is without illness and its nature does not change, and maintains that equilibrium, the organs are also healthy without illness; whenever this animal soul changes for the weaker or its nature changes, all the organs will be harmed by this. First, from the Heart there are three branches of the vessels that go to the Lung: one branch ascends; each one of the other two connects to a blood vessel branch, one branch goes right, and the other branch flows towards the door of the Lungs... straight to the Kidney organ. When it arrives behind the left and 
right Kidneys, then from the left and right Kidney it goes forward to the Bladder, and as it comes to the organ of the Bladder it begins to scatter. ${ }^{34}$

We find here in the juxtaposition of text and illustration early testimony to the rich mix of medical and inner alchemical traditions of physiology, east and west. Many of the ideas contained in Tansūqnāma, such as the production of blood in the liver, are not contained in the original Chinese text. The introduction of the idea that the production of blood at the liver stimulates a ripening process which in turn generates vapour to the lungs has a decidedly Greco-Roman-Arabic ring to it. For Galen the liver was central in haematopoiesis and the origin of a venous system which transported nourishment around the body. ${ }^{35}$ Our text stops short of differentiating the ventricles of the heart, and the passage of the blood through the pulmonary artery, or air through the venous artery (as it was understood by Galen). ${ }^{36}$

Islamic physicians tended to accept the Galenic theory of the movement of blood between the organs and although Ibn al Nafis (d.1288) added detail about the blood flow from the right ventricle to the heart and back into the left ventricle it didn't catch on. It is most likely, given the overall coherence of the text to a Persian physician, that Rashīd al-Dīn and his translators took the original Chinese explanation of the Chart of the Heart Connections and interpreted it through the lens of familiar GalenicArabic-Persian physiological paradigms. The text tells us that all the passageways and tributary branches that come together around the lung and intersect at the heart organ bring clear air to the heart organ, and nurture an animal soul that grows there - and that the organs are all dependent on the quiet strength and the constant nature of this entity. But how are we to understand this animal soul?

\section{On the Animal Spirits and The Vermilion Bird}

The choice to ignore the animals of the four directions as illustrated on the Chinese Chart of the Internal Landscape in Tansūqnàma focuses our attention on the selective reception of Chinese ideas in late medieval Persia, and on an environment increasingly dominated by Islam and the assimilations of Greco-Roman and Arabic medicine in the Islamic world. The text that accompanies the Chart of the

Also compare with the text accompanying the Chart of the Heart Connections in Hua Tuo neizhao tu.

Temkin, p. 154

Poorman and Savage-Smith 2007, pp. 45-7.
Heart Connections, translated above, describes a physiology that fuses Chinese and Galenic-Arabic-Persian ideas. Given Chinese familiarity with the idea of in-dwelling animals in the body, the Persian interpretation of an 'animal soul' moving together with the clear air may not have seemed too much of a departure from the original meaning to the Chinese involved in translation. But it would not have meant the same thing to the Persians themselves for the following reasons.

In late antiquity Galen wrote a great deal about psychikon


most spirituous part of the three types of pneuma best exemplified in the human body - as part of his discourse on the soul. This term had been translated into Latin as spiritus animalis, the adjective animalis referring to anima (soul) and not to animals at all. The two Latin words do however have a common root, and there may well have been misinterpretations, or creative interpretations, as they were translated across medical cultures. The other kind of spirit important in Galenic physiology is the Vital



The key physiological terms concerned with spirits as they translated through Greek and Latin into late-medieval Persian were the rūh nafasānī (Vital Spirit), or rūh hayawānī (lit. Animal Spirit), and sometimes rūh tabì̄ (Natural Spirit), the latter of which was often also the term used to translate pneuma $\pi v \varepsilon \hat{\mu} \mu \alpha$ (lit. 'wind' or 'breath'). These entities formed the physiological counterpart of the tripartite soul. ${ }^{37}$ Galen quoted Erasistratus saying that 'the Psychic Spirit proceeds from the brain, the Vital Spirit


described the generative potential of pneuma somewhat differently. ${ }^{38}$

For Galen the liver created venous blood that nourished the body. Some blood ascended to the right ventricle of the heart. Crossing into the warm environment of the left ventricle the blood vitalised by mixing with air. The resulting Vital Spirits ascended to a place called the rete mirabile where, after a further refinement, they transformed into the Psychic or Animal Spirit. Galen speaks of the Vital Spirits which moved through the moving vessels (the arteries) and animated the sensory body. These were perhaps the same moving vessels that we find in the opening sentences of the explanation of the Chart of the Heart Connections in Tansūqnàma. In the Persian translation of the Chinese text, we find a kind of air flowing into the heart and nurturing the rūh hayawāni (Animal Spirit) that forms there and then moves around the body through the blood vessels.

$\begin{array}{ll}37 & \text { Temkin 1977, p. } 154 . \\ 38 & \text { Ibid. }\end{array}$


By late antiquity Galen's ideas crystallised into three different systems that had their own kinds of spirits. Arabs of late medieval times, such as Ibn Sīnā (Avicenna, c. 980), also imagined three spirits in the body. Galen wasn't talking about real animals inhabiting the body such as we find in the Chinese alchemical landscape, his Animal Spirit was a sort of physiological vitality. Tansūqnāma uses the term rūh hayawānī in what therefore emerges as a very Galenic, Persian, Chinese medical text.

Unlike the received Chinese tradition of Li Jiong's set of illustrations in the Daoist Canon, the Persian edition lacks the illustration featured in Figure 2, and therefore does not represent the animals of the four directions, and their constellations - a common feature of astronomy, astrology and medical designs from very early times. (See Zhang, Chapter 27 in this volume). As we know, in the Chinese alchemical tradition, the inner body was inhabited by planets, stars and spirit animals. But the concept of spirit animals residing in the body was not limited to the alchemical traditions.

The power and presence of animals must be understood within larger paradigms of change and transformation that shaped Chinese views of the cosmos. ${ }^{39}$ As Wang and Barrett state:

If Western cosmologies have tended to present contingent physical phenomena as a mere (partial or distorted) reflection of a transcendent or universal reality, in Chinese models of the world, all things partook of the same reality, 'through a system of correspondence which meshed the manifold phenomena of the world into a single fabric' 40 The boundaries between animals and human beings were at once less perilous and more permeable. ${ }^{41}$

Animals were capable of metamorphosis and spirit-like transformations across boundaries of form or species. By visualising animals within the body human beings could assimilate themselves to the creative and transformative forces of nature. The animals were also immanent within the microcosm of the body and resident in its organs. ${ }^{42}$

The following is an excerpt from the extant Hamajing 蝦蟆經 (Toad Canon), ${ }^{43}$ an 1823 Japanese woodblock

39 The essential text is Sterckx 2002.

$40 \quad$ Harper 1985, p. 467.

$41 \quad$ Huangdi hamajing 1984, pp. $43-4$.

42 See Sterckx 2002, especially ch. 6 'Changing animals' for numerous examples. The concept of spirits resident in the organs exists in Taiping jing 太平經 (Canon of Heavenly Peace), which may be datable in part to the later Han Dynasty. Lo 2001, citing Taipingjing hejiao, p. 24, n. 67 . 黃帝蝦蟆圖隨月生毀避炎判法 (Yellow Emperor's Toad Chart: method for avoiding cautery and pan treatment according to the

edition of much earlier Chinese writings that prohibited acupuncture or sexual relations on certain days in the calendar. One passage gives a day related to each of the spirits/constellations of the viscera according to the ancient sixty-day ganzhi cycle that had structured the Chinese calendar since Shang times:

The Liver is the Qing long 青龍 (Blue-green Dragon); spirit is at dingmao. The Heart is the zhu que 朱雀 (Vermilion Bird); spirit is at gengwu. The Spleen is the gouchen 勾陳; spirit is in the centre. The Lung is the White Tiger; spirit is at guiyou. The Kidney is the xuan wu 玄武 (Black Warrior) constellation; spirit is at jiazi. ${ }^{44}$

The inner dimension of the body was an image of the Heavens and our Vermilion Bird resided in the Heart. These are either five different spirits belonging to the five different viscera flourishing in sequence according to the ganzhi cycle; or one spirit moving around the body according to the time or position of the person and animating its visceral residence. All of the terms that identify the five viscera refer in some way to groups of star formations through the rubric of the sixiang, the animals of the four directions. Four of the directional animals then may form a group of constellations that constitute seven of the 28 xiu 宿 (lodges). ${ }^{45}$

Compare then the illustrations in the Daoist compendium Yunji qiqian 雲笈七籤 (Seven Tablets from a Cloudy Basket) collected for the Emperor Zhangzong (r. 997-1022). In the 54 sections that describe the ritual, contemplative and medical practices expected of a Daoist priest there are illustrations collated from three editions of the Huangting jing corpus. They focus on the outward appearances of the creatures dwelling in the body used in visualisation and clearly draw on the earlier medical traditions. Together they help us make sense of Figure 2. In these three texts only the correspondence of the Heart with the Vermilion Bird and the Liver with the Dragon are the same as that traditionally expressed in the medical correspondences, but these variations in practice are only to be expected. For the rest there is a white beast (a lion) in the Lungs, a phoenix

waxing and waning of the moon).

44

45
Huangdi hama jing 1984, pp. 43-4.

The Black Warrior is the turtle depicted with the other three animals in Han motifs. The turtle is evidently elevated to heavenly status through carapace divination, and associated with war through the image of its armour. The fifth direction, the six stars of gouchen, is needed to correlate to the power of five governed by the five agents, Earth, Water, Metal, Wood and Fire, and manifest here in the five viscera. It refers to the centre, and is part of a conglomerate of eleven stars including the five of the beiji 北極 (north pole asterism) in Shi Shi's 石氏 star catalogue. Sun and Kistemaker 1997, pp. 42-52, p. 5o n.3, and pp. 113-46. 




Figure 20.7 Shangqing huangting neijing wuzang liufu zhenren yuzhou jing 上清黄庭五藏六腑真人玉軸經 (Precious Scroll of the Zhenren on the Six Receptacles and Five Viscera of the Yellow Court of Shangqing) DZ 1402. Library of China Academy of Chinese Medical Sciences

in the Spleen and a deer in the Kidney, as well as a gui-she (turtle/serpent) - all metaphors for the distinctive spirit or character of each organ (Fig. 7). These are counterparts to the Vermilion Bird, the Blue-Green Dragon, the White Tiger, the Deer and the Turtle that we have already seen in Figures 1 and 2. It may be that the animals were simply representative of the character of the organ, and in the case of the Vermilion Bird, the fluttering feelings of the Heart, but for the purposes of the argument we are concerned with them as visual representation. Representations of the intertransformability of beings and objects were powerful and enduring in both religion and medicine in China.

Here is a translation of the text that accompanies the Vermilion Bird from Yunji qiqian:

The personal name of the spirit is Dan Yuan 丹元 (Cinnabar Origin), its scholarly name is Shou Ling 收靈 (Guarding the Spirit); the appearance of the Heart is like a Vermilion Bird; the spirit is like a lotus flower hanging (upside down) with the colour of pale white silk on deep red growing. It is located between the Lungs and the Liver opposite the place one cun below the jiuwei 鳩尾 (Dove's Tail). The channel comes out at the centre of zhongchong 中衝 (Middle Rushing). Middle Rushing is in the hollow at the end of the left-hand finger two fen and more away from the nail. ${ }^{46}$ (See Zhang, Chapter 27 in this volume, p. 391)

Medical literature would have the Vermilion Bird restless and unpredictable in nature, a heart spirit prone to flighty, unsettled changes of whim. ${ }^{47}$ We can chase the figure of the bird (in this case Schafer's Red Crow, who he terms the

46 Yunji qiqian 14 (preface 1028 or 1029 edn 1992), pp. 116-20. $47 \quad$ Zhang 2008.



Figure 20.8 A number of cosmic birds represent the stars and planets. This mural from Dunhuang depicts the ancient myth that there is a crow resident in the Sun held in the hand on the right-hand side of the figure. On the left one can see the toad in the moon together with the hare who is compounding the elixir of immortality. Mogao Grotto no. 35. Image (c) Wang Jinyu, by kind permission to publish from the Dunhuang Academy

cousin of the Vermilion Bird) in its migration to the northwest and towards the eastern end of the Silk Roads where it is incorporated into designs on the Dunhuang cave murals and, as the phoenix, was integrated seamlessly into the Islamic, art-historical tradition. But as a motif associated with the Chinese astromedical traditions, its flight west does not cross the Taklamahan desert to reach Tabriz (Fig. 8). ${ }^{48}$ It is true that the probable Chinese source of the Chart of the Heart Connections, the Xuanmen maijue neizhao tu, does not depict the Vermilion Bird, or any illustration from the traditions of internal alchemy such as the Chart of the Internal Landscape. But Li Jiong's collection did (see Fig. 2.1). And with these two collections as the major sources for Tansūqnāma illustrations our question about the nature of the 'animal soul' remains pertinent to understanding the kind of difficulties Rashīd al-Dīn's resident scholars experienced at the point of translation.

Might there also have been a cultural factor that prevented Rashīd al-Dīn copying all of Li Jiong's illustrations? It is well known that there was no formalised prohibition against figurative art based on doctrine derived directly from the Koran, and that the comparative lack of imagery in early Islamic art reflected the limited formal vocabulary available to Muslim artists. ${ }^{49}$ The denunciation of traditions of representation of the life of the prophet was, however, interpreted differently at different times and in different places. Initially, in the early part of the 8th-century, iconoclasm did not have a widespread effect

Berlekamp, Lo and Wang, 2015, p. 55.

Grabar 1987, pp. 105-33. Berlekamp 2010, pp. 251-76. 
in Byzantium, even if it strengthened its hold in the later part of the century. ${ }^{50}$ Nevertheless, with the growing influence of the 'hadith', that body of interpretation of Islamic law, came a very clear statement that 'the maker of images or pictures is the enemy of God', that the painter is the competitor of God. ${ }^{51}$ The message was strengthened in various campaigns so that by the time of Rashīd al-Dīn Islam was very clearly opposed to the representation of living beings on theological grounds.

The rich traditions of Islamic art, not the least as manifest in the illustrations in Rashīd al-Dīn's own Jāmi“ al-Tawārīkh (Compendium of Chronicles), however, testify that any Islamic prohibition against figurative art in the mosque and madrasahs did not extend into the secular world. Thus, the depictions of the inner organs that we have seen in a medical text, and the maps that measure the body in terms of its Yin and Yang, were apparently easily embraced within Rashīd al-Dīn's medical discourse. But what about the sacred space of the inner body, and the suggestion of a multiplicity of animals resident as gods and spirits?

Ghazan Khan's conversion to Islam in 1295 heralded a tightening of control over writing and representation. Chronicles became more clearly orthodox in character. Non-Muslim monuments were destroyed and the physical evidence of Buddhism in the Ilkhanate was eradicated. Most of the Muslim community in the Persian lands were Sunni, and the Sunni community were less permissive than the Shi'ites. ${ }^{52}$ With the conversions of the military and nobility all around, Rashīd al-Dīn must surely have been keen to keep faith with Islamic orthodoxy. In this politically sensitive environment Li Jiong's contemplative Chart of the Internal Landscape with all its lurid worms, White Tigers and Vermilion Birds would certainly have stretched Rashīd al-Dīn's Mongolian masters' eclecticism a step too far.

\section{Conclusion}

Rashīd al-Dīn's Treasure Book of Ilqān on Chinese Science and Techniques contains the earliest witnesses to a 13th- and 14th-century Chinese alchemical tradition of illustration. Not a startling discovery, but nevertheless strange and important. It stands as enduring testimony to the culture of valuing other people's knowledge that was nurtured by Rashīd al-Dīn and others in Tabriz.

\footnotetext{
$50 \quad$ Flood 2002, pp. 641-55.

$5^{1} \quad$ Grabar 1987 , pp. 82-7.

$5^{2} \quad$ Blair 2002, pp. 105-10.
}

It was within an eclectic religious context that these illustrations of the body were reinvented for the Persian world of the Ilkhanate. The Yanluozi diagrams were secular in origin, but had already been adapted to the contemplative techniques of inner alchemy, and were preserved in China within a Daoist context. Rashīd al-Dīn ascribed the Chinese texts that he received to three monks. Given the predominance of the institutions of Buddhism along the route from China, these were most likely Buddhist monks who had compiled the particular set of books and manuscripts that arrived at his door. This should be no surprise as there is already evidence from the 8th century Dunhuang (Thousand Buddha) Caves store of Daoist medico-religious works being copied and transmitted within a Buddhist context. 53

With his personal multi-faith religious history Rashīd al-Dīn would have had no trouble accommodating other medico-religious realities into his world view. Our detailed study of the Heart Connections Chart and its text serves as a model for understanding what pressures might have come to bear upon him during that process. It has hypothesised about some of the compromises and creative solutions that he and his scribes and scholars arrived at. Drawing on the complex strands of Greco-Roman, Arabic and Chinese physiology that are interwoven into the explanation of the generation of the animal soul in Tansūqnāma we have teased out some examples of the intellectual and aesthetic difficulties he faced. In translating Chinese physiology for a Persian audience, the Vermilion Bird transmutated into the animal soul and Vital Spirits of Galenic medicine, losing both its cosmological significance and its corporal Chinese form on the way. People are generally more comfortable with ideas that have a well-used familiarity and, however we might choose to respect other traditions, the familiar persists in informing our reception of knowledge arriving from afar.

Mongol appreciation of and assimilation of the ideas, technologies and things local, to the enrichment of their universal project, provided a fertile environment for Rashīd al-Dīn's eclectic intellectual innovations. Like Joseph Needham, perhaps Rashīd al-Dīn believed that ethnic knowledge would ultimately offer up its treasures to the common universal pool of knowledge and, 'like all other ethnic cultural rivers ... flow into the sea of [modern/ Islamic] science'. ${ }^{54}$ And so it did. Rashīd al-Dīn's complex

53 See for example Bibliothèque nationale de France, Paris: cat nos. P.3810 and P.4038. There is a great deal of scope for future research comparing the texts in the Tansūqnāma compilation with the Dunhuang manuscripts in the British Library and BNF. 
religious and intellectual history gave him a head start in appreciating the value of cross-cultural knowledge. Whether his bricolage of traditions, his late-medieval multi-culturalism, had any enduring impact on the development of medical knowledge in Europe remains to be seen. His methods, however, are arresting and controversial enough to deserve serious attention across many modern academic disciplines.

\section{Bibliography}

\section{Primary Sources}

Hamajing 黃帝蛤蟆經. Edn cited: 1984, Beijing: Zhongyi guji.

Huangdi bashiyi nanjing zuan tu jujie 黃帝八十一難經纂圖句解 in Zhengtong Daozang 1436-49 (The Ming edn of the Daoist Canon, fasc 668-70), Shanghai: Hanfen Lou.

Tansūqnāma-i İlkhān dar funūn-i 'ulūm-i Khatā'̄ (The Treasure Book of the Ilkhan on Chinese Science and Techniques, hereafter Tansuqnama), Rashīd al-Dīn Fażl Allāh AH 713 (1313), Süleymaniye Library, Aya Sofya.

Yuanmen maijue neizhao tu 元門脈訣內罩圖 (Internal Visualisation Charts from the 'Primordial Portal' Secret Art of the Pulse), attributed to Hua Tuo, of the 3 rd century вCE. Library of Zhongguo zhongyi yanjiu yuan (China Academy of Traditional Chinese Medicine).

Yunji qiqian 雲笈七籤 (Seven tablets from a Cloudy Basket) c. 1029. Preface 1028/1029, comp. Zhang Junfang 張君房, fl. 1oth and 11th centuries.

\section{Secondary Sources}

Akasoy, A. and Yoeli-Tlalim, R. 2007, 'Along the musk routes: exchanges between Tibet and the Islamic world', Asian Medicine 3.2, 217-40.

Akasoy, A., Burnett, C., Yoeli-Tlalim, R. (eds) 2013, Rashīd al-Dìn as an Agent and Mediator of Cultural Exchanges in Ilkhanid Iran, London: Warburg Institute,

Allsen, T. 2001, Culture and Conquest in MongolEurasia, Cambridge: CuP.

Berlekamp, P. 2010, 'The limits of artistic exchange in fourteenth-century Tabriz: the paradox of Rashīd al-Dīn's book on Chinese medicine', Muqarnas 27, 251-76.

Berlekamp, P., V. Lo and Wang Yidan 2015, 'Administering art, history, and science in the Mongol empire', in Pearls on a String: Artists, Patrons, and Poets at the Great Islamic Courts, Seattle: Walters Art Museum/University of Washington Press.

Blair, S. 2002, 'Religious Art of the Ilkhanids', in Komaroff and Carboni (eds), 104-33.

Boyle, J. 1971a, 'The significance of the Jāmi' al-Tawārīkh as a source on Mongol history', in Proceedings of the Colloquium on Rashìd al-Dìn Fadlallāh, Tehran/Tabriz, 2-7 November 1969, 1-8.

— 1971b, 'Rashīd al-Dīn: The first world historian', Iran 9, 19-26.

Chen Ming 2007, 'The transmission of foreign medicine via the Silk Roads in medieval China: a case study of Haiyao Bencao', Asian Medicine 3.2, 241-64.

Despeux, C. 2005, 'Medicine and pharmacology', in Schipper and Verellen (eds), 772-4.

Feher. M., R. Naddaff and N. Tazi (eds) 1989, Fragments for a History of the Human Body, Part 1, New York: Zone.

Flood, F.B. 2002, 'Between cult and culture: Bamiyan, Islamic iconoclasm and the museum', Art Bulletin 84.4, 641-59.
Franke, H. 1951 'Some sinological remarks on Rašīd ad-Dīn's History of China', Oriens 4, 21-6.

Grabar, O. and R. Ettinghausen 1987, The Art and Architecture of Islam 650-1250, London: Pelican.

Harper, D. 1985, 'A Chinese demonography of the third century BC', Harvard Journal of Asiatic Studies 45, 459-98.

Jackson, P. 2005 The Mongols and the West 1221-1410, London/New York: Routledge.

Kistemaker J. and Sun Xiaochun 1997, Chinese Sky: Constellating Stars and Society, Leiden: Brill.

Klein-Franke, F. and Zhu Ming 1996, 'Rashīd al-Dīn as a transmitter of Chinese medicine to the West', Le Muséon 109, 395-404.

2003, 'The Book of Heat From Rashīd ad-Dīn's Kitāb Bayān al-Ḥaqā’iq', The American Journal of Chinese Medicine 31:06, 977-82.

Kohn, L. (ed.) 200o, Daoism Handbook, Leiden/Boston: Brill.

Komaroff L. and S. Carboni (eds) 2002, The Legacy of Genghis Khan: Courtly Art and Culture in Western Asia, 1256-1353, New York: Metropolitan Museum of Art.

Levi , J. 1989, 'The Daoist coat of arms', in Feher, Naddaff and Tazi (eds), 104-27.

Lo, V. 2002, 'Spirit of stone: technical considerations in the treatment of the jade body', BSOAS 65, 99-128.

2001, 'Huangdi Hamajing (Yellow Emperor's Toad Canon)', Asia Major 14 (2), 61-99.

Lo, V., and Wang Yidan 2013, 'Blood or Qi circulation? On the nature


[The Treasure Book of Ilqān on Chinese Science and Techniques]', in Akasoy, Burnett and Yoeli-Tlalim (eds), 127-72.

Ma Jixing 馬繼興 1990, Zhongyi wenxianxue 中醫文獻學 (The Study of Chinese Medical Literature), Shanghai: Shanghai kexue jishu.

Major, J. 1993, Heaven and Earth in Early Chinese Thought, Albany: Suny.

Mīnuvī M. ed. 1971, Tansūqnāma-i İlkhān dar funūn-i 'ulūm-i Khatā'̄, Tehran: Intishārāt-i Dānishkada-i Adabiyāt va 'Ulūm-i Insānī-i Dānishgāh-i.

Needham, J. 1970, 'Elixir poisoning in medieval China' in Needham, Clerks and Craftsmen in China in the West, Cambridge: CuP, 316-19.

Needham, J. [1964] 2013, 'Science and society in East and West', in Needham, The Grand Titration: Science and Society in East and West, London: Routledge, 190-217.

Pormann P. and E. Savage-Smith 2007, Medieval Islamic Medicine, Edinburgh: Edinburgh University Press.

Pregadio, F. (ed.) 2008, The Encyclopedia of Taoism, London/New York: Routledge.

Schafer, E. 1967, The Vermilion Bird, Berkeley and Los Angeles: University of California Press.

Schipper, K. and F. Verellen (eds) 2005, The Taoist Canon, Chicago: University of Chicago.

Skar. L. and F. Pregadio 200o, 'Inner alchemy (Neidan)', in Kohn (ed.) 464-97.

Sterckx, R. 2002, The Animal and the Daemon in Early China, Albany: sunY Press.

Temkin, O. 1977, The Double Face of Janus, Baltimore/London: John Hopkins.

Wang Shumin and P. Barrett 2006, 'Profile of a daoyin tradition: the 'five animal mimes' Asian Medicine 2.2, 225-53.

Wang Shumin and V. Lo (eds) 2007, Xingxiang Zhongyi 形象中醫, Beijing: Renmin weisheng.

Wilms, S. 2007, 'Zhong shiji de Zhongguo gudai yangtai fa' 中世紀的 中國古代養胎法' in Wang Shumin and Lo (eds), 144-5.

Zhang Qicheng 2007, 'Wuzang, liufu, buixie tu jieshuo' 五藏六腑補 瀉圖解說, in Wang Shumin and Lo (eds), 175-81. 


\title{
21 Fanciful Images from Abroad: Picturing the Other in Bencao pinhui jingyao
}

\author{
本草品彙精要
}

\section{Chen Ming 陳明}

Bencao pinhui jingyao 本草品彙精要 (Materia Medica Containing Essential and Important Material Arranged in Systematic Order) was the last great pharmacopoeia to be produced under official auspices in pre-modern China. It belongs to the high Ming period. Begun in 1503 (16th year of the Hongzhi 弘治 reign period of the Xiaozong 孝宗 emperor of the Ming) the entire project was completed within the space of one and a half years, by a large team of subject specialists and artists headed by Eunuch Zhang Yu 張瑜 and Imperial Physician Liu Wentai 劉文 泰 ( $f l$.1488-1505 CE). Following the format established by its Song-dynasty predecessor Zhenglei bencao 證類本草 (Categorised Pharmacopoeia), composed by Tang Shenwei 唐慎微 in the late 11th century, the work is divided into 1o sections covering jade and minerals, fruits and trees etc., subdivided into 42 juan or fascicles, and all the drugs are classified as 'upper' (shang 上), 'middle' (zhong 中) or 'lower' (xia 下). There are 1,815 drug entries, written in red and black ink. It is a manuscript - a deluxe handmade artefact - not a printed book: the editors chose not to avail themselves of the sophisticated woodblock engraving and printing technology available to them. Instead, they selected 14 artist-craftspeople to copy the texts in coloured ink, and eight courtly painters, among them the renowned artist Wang Shichang 王世昌, to provide the exquisite polychrome illustrations. As a result, Bencao pinhuijingyao constitutes the largest collection of coloured pharmaceutical illustrations surviving from pre-modern China, including 667 pictures that were specially created or freely adapted for the book, in addition to over 700 coloured versions of monochrome line drawings taken from Zhenglei bencao.

The Emperor Xiaozong himself added a preface to the completed work, which was then bound in 36 volumes in emulation of Yongle dadian 永樂大典 (the Yongle Encyclopaedia, 1408, commissioned by the Ming Yongle emperor), and placed in a protective camphorwood box as befitted an official imperial manuscript. However, just two months later, Xiaozong died under mysterious circumstances. Of the 49 editors of the book, 12 were arrested and investigated on suspicion of conspiracy to murder the emperor. Bencao pinhui jingyao was locked away in a restricted archive, never to be published. Nonetheless, some copies were made, and during subsequent periods of war and upheaval, some of them were dispersed abroad.
One copy found its way to Italy, and was acquired by the National Central Library of Rome in $1877 .^{1}$

\section{The Seven Pictures of Non-Chinese in Bencao PinhuiJingyao}

In addition to pharmaceutical illustrations of plants, animals and minerals, the illustrations in Bencao pinhui jingyao include vivid and detailed vignettes of everyday life, notably scenes of collecting herbs and manufacturing drugs. They are thus of enormous value not only for the history of pharmacology, but also for social history, as well as the history of art. $^{2}$

Seven of the illustrations have always particularly intrigued me - the so-called Huren tu 胡人圖 (Pictures of foreigners/non-Chinese/non-Han people), i.e. the illustrations for Aina xiang 艾納香 (blumea) in juan 12; Zhihan 質汗 (citragandha, a composite drug) ${ }^{3}$ in juan 15; Jiangzhen xiang 降真香 (acronychia), Suhe xiang 蘇合香 (storax), Longnaoxiang 龍腦香 (borneol), and Anxixiang 安息香 benzoin) in juan 17; and Diyejia 底野迦, theriac, a kind of antidote) in juan $23 .{ }^{4}$ In comparison with the other human figures portrayed in the book, the figures in these illustrations are all clearly connoted as non-Han and/or foreign: they have curly hair, Roman noses, deep-set eyes and characteristic moustaches, and wear distinctly un-Chinese apparel (hats, robes, turbans and belts), mostly accessorised with tall boots.

The Tang and Song Bencao sources of Bencao pinhui jingyao also contain numerous items of foreign provenance. But only in these seven entries from Bencao pinhui jingyao do images of non-Chinese people feature as signifiers for exotic substances.

The detailed content of the seven illustrations is set out below.

\section{Aina xiang (blumea): Collecting drugs}

A Chinese figure (a doctor) is picking herbs beneath a blumea tree; beside him stands a non-Chinese figure with a collecting bag on his back. The latter

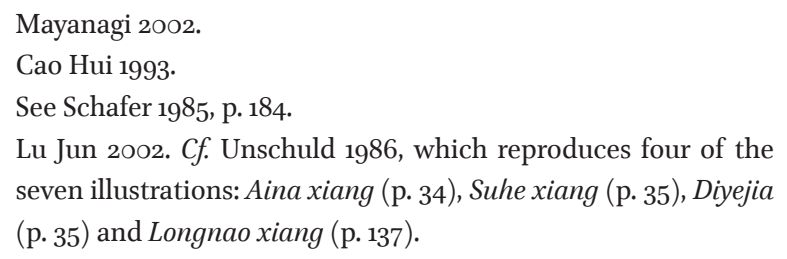





Figures 21.1-3 Bencao pinhui jingyao: 1 (left) Citragandha (Zhihan 質汗, a composite drug); 2 (middle) Acronychia pedunculata (Jiangzhen xiang 降真香); 3 (right) Borneol (Longnao xiang 龍腦). ( ) Biblioteca Nazionale Centrale di Roma, Oriental Collections

seems to be showing a handful of herbs to the Chinese doctor and saying something to him.

2. Zhihan (Citragandha): Decocting drugs (Fig. 1) A non-Chinese figure (a servant) with a bulbous head, wearing a yellowish robe and short boots, squats down to tend a brazier, on which stands a tripod cauldron (a ding 鼎). A second, standing non-Chinese figure, wearing a long belted gown, a close-fitting, front-fastened upper garment with lapels, and tall black boots, stirs a decoction in the cauldron. On a table beside them are four dishes heaped with various drugs, and two bowls containing black and red liquids. The cauldron resembles those used by Chinese Daoist alchemists refining the elixir of immortality.

3. Jiangzhen xiang (acronychia): Carrying drugs on the shoulders (Fig. 2)

This illustration depicts a lone, robed, non-Chinese male figure with tall boots and headband, carrying a length of acronychia wood.

4. Suhe xiang 蘇合香 (storax): Transporting drugs There are three non-Chinese figures in this picture. Two of them are carrying a jug of drugs between them; the third, who follows behind, is apparently their master, a foreign merchant. He wears a long mantle and a turban surmounted by a tall hat. The whole picture could be interpreted as representing the introduction of foreign aromatic drugs to China, or the role of drugs in the imperial tribute system. In any case, it undoubtedly relates to the trade in imported drugs.
5. Longnao xiang (borneol): Selecting Drugs (Fig. 3) Beneath a Borneo Camphor tree, a non-Chinese figure squats on the ground in front of a big bamboo basket full of white aromatic drugs. He is using hand-held tools to sift through the drugs in the basket, depositing the waste matter in a heap beside it.

6. Anxixiang (benzoin): Collecting drugs

On the right, a non-Chinese man in a green robe with a belt, holding a drug spatula, is slicing open the bark of a styrax benzoin tree to obtain the resin. On the left, a non-Chinese man in a purple robe (with an unidentified object in his right hand) holds out a small white bowl in his left hand to catch the resin. Under the tree, there is also a small keg for collecting drugs.

7. Diyejia (theriac): Drugs as tribute A non-Chinese figure in a turban genuflects before a seated Chinese figure (the emperor? a physician?). With both hands, he offers him in tribute a bowl of red and black theriac pills.

The implements and apparatus depicted in the Seven Pictures of Non-Chinese are: a bag for collecting drugs; a tripod cauldron (ding); a long spoon; a table; drug containers including bowls, dishes, an earthenware jar, a large basket and a small keg; a drug spatula. All of these resemble artefacts in use in the central plain area of China, or rather, they are domesticating depictions of indigenous artefacts from the Chinese heartland.

The relation between these seven illustrations and the corresponding texts is relatively tenuous, except in the cases of Ainaxiang (blumea) and Anxixiang (benzoin). This 



Figures 21.4-6 Buyi Lei Gong paozhi bianlan: 4 (left) Citragandha (Zhihan 質汗, a composite drug); 5 (middle) Acronychia pedunculata (Jiangzhen xiang 降真香); 6 (right) Borneol (Longnao xiang 龍腦香). Photographic reprint, Shanghai: Shanghai cishu, 2005, juan 5, p. 884, juan 6, p. 971 and p. 997. (C) Wellcome Library, London

limits their usefulness from a pharmacological perspective; however, they are highly significant for the history of cultural interchange.

\section{The Eight Pictures of Non-Chinese in Buyi Lei Gong Paozhi Bianlan 補遺雷公炮製便覽}

Buyi Lei Gong paozhi bianlan (Lei Gong's Guide to Drug Preparation with Addenda), hereafter Buyi Lei Gong, was completed in the spring of 1591 (Xinmao year of the Wanli reign period of the Ming Dynasty). Like Bencao pinhui jingyao, it is a manuscript work, richly illustrated in the courtly meticulous style. It contains 1153 polychrome illustrations, 302 of which are original creations, including exquisitely painted vignettes of drug manufacture in the Ming period. This book survives in a single exemplar, which is now conserved in the library of the China Academy of Chinese Medical Sciences 中國中醫科學院 (formerly known as 中國中醫研究院 China Academy of Traditional Chinese Medicine). A full-colour facsimile edition was published in 2005 by Shanghai cishu chubanshe 上海辭 書出版社 (Shanghai Lexicographical Publishing House), a division of Shanghai shiji chuban jituan 上海世紀出版 集團 (Shanghai Century Publishing Group).

While visiting Beijing in September 2005 for the conference on 'Chinese Medicine: A Visual History', ${ }^{5}$ I was

$5 \quad$ Jointly sponsored by the China Institute for the History of Medicine (China Academy of Traditional Chinese Medicine) and the lucky enough to be able to examine this book at first hand. As I then ascertained, it contains eight 'Pictures of NonChinese': the same seven as in Bencao pinhui jingyao plus an additional illustration for Tuode hua 陀得花 (hops). This picture shows a scene of drug collection: one non-Chinese figure is picking hops, while a second, a curly-haired boy, is dropping the flowers into a vat. A side-by-side comparison with the corresponding images in Bencao pinhui jingyao confirms that the non-Chinese illustrations in Buyi Lei Gong are derived from the earlier work, but it remains to be established which copy of Bencao pinhuijingyao constituted the immediate source. The differences between the pictures of non-Chinese in the two works are listed below:

1. Aina xiang: there are differences in the shape of the tree branches and the twigs in the knapsack, and the colour of the figure's clothing is different; moreover, in Buyi Lei Gong, he is holding a single twig in his hand in his hand, rather than a handful of the herb.

2. Zhihan (Fig. 4): the shape of the table and the arrangement of the drug bowls differ. The squatting figure has been changed into a standing servant-boy, and the seated figure is now standing.

3. Jiangzhen xiang (Fig. 5): a second non-Chinese figure has been added, also carrying a length of acronychia wood.

4. Longnao xiang (Fig. 6): a second, seated, non-Chinese figure has been added.

Wellcome Trust Centre for the History of Medicine at UCL. 
5. Suhe xiang: there are some differences in the colour and shape of the figures' garments and headgear.

6. Anxixiang: almost identical save for minor differences in the tree branches and the colour of the figures' clothing.

7. Diyejia: almost identical.

\section{Images of Foreigners and Foreign Medicine: Sources of Visual Evidence}

In the period spanned by the Han and Tang dynasties, cultural exchange between China and other countries intensified. Images of non-Chinese people are found in a variety of sources dating back to this period, including famous paintings transmitted by copyists, paintings on silk and paper discovered in archaeological excavations, murals in cave-temples along the Silk Roads, and screens, murals and reliefs from tombs. Art historians have written extensively on all these subjects; I will therefore confine myself to a few pertinent examples.

A celebrated example is Zhigong $t u$ 職貢圖 (Image of tribute bringers), originally created by Emperor Yuan of the Liang dynasty (personal name Xiao Yi 蕭繹 508-55), a gifted artist. The original work no longer exists, but a copy made in the Song period (96o-1279) is conserved in Nanjing Museum. It depicts emissaries from 12 countries, including Persia, Huaguo 滑國 (Uar, Hephthalites), and Baiji 百濟 (Baekje), each with distinctive garb and physical appearance. A slightly later and more elaborate Zhigong $t u$ from the Tang period, painted by Yan Liben 閻立本 6o1-73, is likewise known from a Song copy, now held in the Imperial Palace Museum of Taibei. It features lively, detailed depictions of 27 emissaries from countries including Poliguo 婆利國 (Borneo[?]) Luochaguo 羅刹國 (Sri Lanka) and Linyiguo 林邑國 (Champa, present-day Vietnam), as well as a rich variety of tribute items, visually representing the bold assertion that 'ten thousand countries come to honour the empire; hundreds of alien nations come to pay tribute'. ${ }^{6}$ The stone cave temples along the Silk Road, and especially the murals showing foreign merchants in the Mogao 莫高 Grottos at Dunhuang (present-day Xinjiang), provide a corpus of images of non-Chinese people. In addition, many of the donors portrayed or documented in Buddhist paintings and religion texts unearthed on the Silk Road, are not Chinese. In recent years, a series of Sogdian mausolea have been excavated in central China, shedding new light on the social fabric of China in the early Middle Ages - notably the tombs of An Jia 安伽 (d. 579), Kang Ye 康

$6 \quad$ Zhang Yanyuan 1964, p.17o.
業 (d. 571) and Shijun 史君, Sogdian name Wirkak (d. 580) in Xi'an; and the tomb of Yu Hong 虞弘 (d.592) in Taiyuan, Shanxi province). An Jia, whose tomb was excavated in 200o, held the title of sabao 薩保 (sartapao), i.e. leader of the local Sogdian community. His tomb has murals by the entrance and coloured paintings on the panels surrounding the coffin-platform - 12 paintings in all - depicting religious activities and scenes from everyday life. ${ }^{7}$

Many of the murals in Dunhuang include subject matter that is highly relevant for the history of medicine. Dunhuang shiku quanji: kexue jishu huajuan 敦煌石窟全 集.科學技術畫卷 (Dunhuang Caves, Complete Edition: Science and Technology Paintings Volume), edited by Wang Jinyu 王進玉, reproduces 249 paintings, chosen from the many thousands of murals for their scientific (including medical) interest. The majority of these are Sūtra paintings (jingbian 經變), and were not created with any medical intent. Nonetheless, many of them document healing activities and their social context in the Sui, Tang and the Five Dynasties period, or have a clear connection with medicine and medication. ${ }^{8}$ These visual documents of medical activity have been described by scholars such as Cong Chunyu 敢春雨 as 'visual iatrology'. Examples are the painting of 'Diagnosing disease and administering drugs' from the Sūtra of the Fields of Merit in northern Zhou (557-81) cave no. 296, and the painting of 'Finding a doctor according to the disease' in High Tang (705-81) cave no. 217, which illustrates a passage in juan 22 of the Lotus Sūtra. ${ }^{9}$ The Library Cave at Dunhuang has yielded a number of specifically medical images, such as Moxibustion Charts S.6168 and S.6262, ${ }^{10}$ now held in the British Library, as well as various images in manuscripts on augury and physiognomy that are also relevant to Chinese medicine.

Detailed catalogues published over the past couple of decades provide a sense of the wealth of medical imagery generated in China from earliest times to the Ming. ${ }^{11}$ For instance, a series of stone reliefs from the Eastern Han period (25-220 CE), which show the legendary bird-headed doctor Bian Que treating a patient with needles, testify eloquently to the antiquity of acupuncture in China. ${ }^{12}$ However, the vast majority of images in pre-modern

\footnotetext{
7 Anon 2001; Shaanxi sheng kaogu yanjiusuo 2003. For more studies, see: Rong Xinjiang 2001; Jiang Boqin 2004.

$8 \quad$ Wang Jinyu (ed.) 2001, pp. 247-69.

$9 \quad$ Fu Weikang (ed.) 200o, pp. 70; 9 .

10 Lo in Lo and Cullen (eds) 2005, pp. 211-22.

11 See Li Jingwei (ed.) 1992; Fu Weikang (ed.) 2000; Zhong Jun and Wu Hongzhou (eds) 2001.

12 Unearthed at Liangcheng 兩城, Weishan 微山, Shandong Province
} 
medical texts relate to the medicine of the Han Chinese, and overt representations of foreign people and drugs are extremely rare. Even in the Sūtra paintings from multicultural Dunhuang, mentioned above, the human figures are, to all appearances, Han Chinese.

Although there seems to be very little direct visual evidence of non-Han or non-Chinese medical activity in Chinese art from the Silk Roads, the three examples below all appear to relate indirectly to non-Han medical culture:

\section{Mural Depicting a Sacrifice on a Zoroastrian Fire} Altar, Tomb of AnJia, Xi'an (see above)

On the altar, we see the herb haoma. Cognate with the ancient Indian longevity elixir Soma, ${ }^{13}$ it has close connections with medico-religious beliefs in ancient Persia.

\section{Mural of Mourners at the Buddha's Nirvana, North Wall, Dunhuang Cave no. 158}

This painting shows the rulers of many countries, including 13 figures identifiable by their costume as potentates from central Asia or the western regions of China, grieving for the death of the Buddha (his passage into Nirvana). Some of them express their grief through violent acts of self-mutilation: one mourner is in the act of severing his left ear with a knife held in his right hand; to his left, another hacks at his breast with twin daggers; in front of them, a mourner grasps his nose in his left hand so that he can cut it off with the knife in his right hand. Further to the left, a man, naked from the waist up, stabs himself in the heart with a sword. According to Lei Wen 雷聞, this scene of violent grief has associations with Zoroastrian ritual magic, which enjoyed popularity in Tang China. ${ }^{14}$ Although the subject matter of the picture has no direct connection with medicine, the haemostatic procedures and first aid treatment required in the aftermath of these violent actions fall into the category of surgery. The scene calls to mind the famous episode, recounted in the Tang dynastic histories, of the ethnic Sogdian An Jinzang 安金 藏, who attempted suicide by disembowelment, and was saved by doctors who sutured his intestines with white mulberry bark and applied external medicines to the wound. Similar specialised surgical procedures to treat protruding or lacerated intestines are recorded in the Ishimp $\bar{o}$ 醫心方 (Formulas of the Heart of Medicine), a medical compendium compiled by the Japanese physician Tamba Yasuyori 丹波康賴 (912-95) in 984 on the basis of

13 Rao Zongyi 2002.

14 Lei Wen 2003. earlier Chinese texts. ${ }^{15}$ This mural undoubtedly offers us imaginative space for non-Chinese surgery.

\section{Two 8th-Century Prints Unearthed in Dandan- Uiliq, Khotan (Present-day Xinjiang)}

Each print shows three deities, the one in the middle being a female deity holding a child. She is generally identified as the mother demon-goddess Graha, or Bala Graha, in the guise of protector of children. Belief in Bala Graha (spirits who seize children and cause convulsions) was widespread in the Khotan area under the influence of Indian culture. Indian scholar Lokesh Chandra has studied images of Graha in Khotan in his thesis 'The Khotanese Mural of Hàrãtã in Shrine D II at Dandan-Uiliq.16 This identification is corroborated by a series of drawings on paper from a fragmentary bilingual (Khotanese and Chinese) manuscript from Dunhuang, 'Images of the goddess protecting children' (Ch.oo217a-c, now held in the British Library. ${ }^{17}$

\section{Foreign Drugs and Drug illustrations in the Bencao Literature from the Tang to the Ming (7th-17th Centuries)}

By the Sui-Tang period, medical cultural exchange between China and foreign countries was flourishing. Numerous imported drugs were accepted into the mainstream pharmaceutical repertoire, and were gradually included in the Bencao literature. Specialist works recording foreign drugs also began to be published. The state-sponsored Tang bencao 唐本草 or Xinxiu bencao 新修本草 (Newly Revised Pharmacopoeia) (659) is one of the earliest known illustrated pharmaceutical texts, although it is not the very earliest, as is sometimes assumed. ${ }^{18}$ Its story begins in 657 , the second year of the Xianqing era, when court counsellor Su Jing 蘇敬 petitioned Emperor Gaozong (r. 649-83) to publish an updated version of Tao Hongjing's 陶弘景Bencaojingjizhui 本草集注. The emperor agreed and assigned a team including the high official Xu Jingzong 許敬宗 to undertake the task. Volume 82 of Tang huiyao 唐會要 (the institutional history of the Tang dynasty) (961) recounts:

It was decreed that drugs should be collected from all over the country, and recorded in pictures. Sikong Liji 司 空李勣 was put in charge of the work. The whole book

\footnotetext{
15 Okano 2000.

16 Chandra 2003.

17 Bм ms. OA 1919.1-1.0177[1-3]). Whitfield and Farrer 199o, pl. 69, p. 88 and pp. 90-1; Chen Ming 2005, pp. 91-100.

18 Zheng Jinsheng 198ob, 1989 and 1999.
} 
with illustrations [the author's italics] ran to 55 volumes. It was finished on the 17 th day of the first lunar month of the fourth year.

Xinxiu bencao was made up of three parts: the Bencao ( $m a-$ teria medica),Yaotu 藥圖 (drug pictures) and Tujing 圖經 (illustrated descriptions). Volume 25 contained polychrome drug illustrations 'with magnificent use of colour, beyond anything that can be imagined. 19

The Tang also saw the publication of the first Bencao dedicated to describing foreign and non-Han drugs (now sadly lost): Hu bencao 胡本草 (Barbarian Pharmacopoeia), compiled around 740 by the multi-talented, mid-Tang scholar Zheng Qian 鄭虔 (705-64). The famous poet Du $\mathrm{Fu}$ 杜甫 (712-70), a friend of Zheng Qian, celebrated the latter's achievements in a long poem entitled Gu zhu zuo lang bian Taizhou sihu Xingyang Zhen Gong Qian 故著 作郎貶台州司戶焱陽鄭公虔 (Zheng Qian of Xingyang, Former Secretary in The Imperial Library, Demoted to the Finance Bureau at Taizhou):

In Shen Nung's work there are omissions;

Yellow Stone would have been embarrassed by his mastery. His handbook of medicines covered plants from the Far West;

Military strategy was at his fingertips...

'The three perfections', wrote the emperor himself,

And people throughout the land admired him even more....$^{20}$

The great 17th-century naturalist and pharmacologist Li Shizhen 李時珍 wrote: 'In these seven volumes are contained all the foreign drugs, which are not [all] known today'. Unfortunately, however, nothing now remains of this long-lost work save a few snippets preserved in the 9th-century Beihu lu 北戶錄 (Records of the Northern Gateway) by Duan Gonglu 段公路. ${ }^{21}$

During the Five Dynasties period (907-6o), Han Baosheng 韓寶升 from the state of Shu (Shuguo 蜀國, corresponding to today's Sichuan region) compiled a regional Bencao entitled Chongguang yinggong bencao 重廣英公本草 (Expanded Pharmacopoeia of Yinggong) (commonly known as Shu Bencao 蜀本草), which was illustrated after the manner of Xinxiu bencao. Another Bencao work from the Five Dynasties period, specifically

19 Su Jing ed., Shang Zhijun 1981, pp. 1-2.

20 Translation from Chou, E. Shan 1984, pp. 40-2. The emperor was Xuanzong 玄宗 of Tang (r. 712-56), and the 'Three Perfections' refers to Zheng Qian's exceptional skills in poetry, painting and calligraphy. It is not known whether Hu bencao actually contained any pictures by Zheng Qian.

$21 \quad$ Unschuld 1986, p. 258 describing foreign drugs, was Haiyao bencao 海藥本草 (Overseas Pharmacopoeia) by Li Xun 李珣. The book itself is no longer extant, but some of its contents are preserved in later Bencao including Daguan Bencao 大觀本 草, Zhenglei bencao 證類本草 and Bencao gangmu 本草 綱目..$^{22}$ It appears from the surviving sources that Haiyao bencao was not illustrated.

The Song (96o-1279) was a significant period for illustrated Bencao.Kaibao bencao 開寶本草 (Pharmacopoeia of the Kaibao era), compiled c. 963 just after the establishment of the Northern Song (96o-1127), did not, however, have any illustrations. ${ }^{23}$ In 1057 (2nd year of the Jiayou era) during the reign of Emperor Renzong of Song 仁宗, a team headed by Zhang Yuxi 掌禹錫 and Su Song 蘇頌 began work on Jiayou buzhu Shen Nong bencao 嘉祐補注神農本草 (Expanded and Annotated Edition of Shen Nong's Materia Medica, Commissioned in the Jiayou Era), which combined text and images after the pattern of Xinxiu bencao. The same team also produced a companion work - Bencao tujing 本草圖經 or Tujing bencao 圖經本草 (The Pictorial Pharmacopoeia) - which set out to 'use pictures to convey the forms and colours of the drugs'. It was completed in 1061 (6th year of Jiayou), and issued together with Jiayou buzhu Shen Nong bencao. It contained 'over 800 drug entries, 642 of which are illustrated, with a total of 933 images, and it is China's first block-printed illustrated Bencao'. ${ }^{24}$ Most of the illustrations were realistic. Composed towards the end of the 11th century, Zhenglei bencao 證類本草 (Categorised Pharmacopoeia) contains 1764 drug items, with more than 3,00o prescriptions, plus illustrations and details of preparation methods for the main drugs. Originally a personal project of scholar-physician Tang Shenwei 唐慎 微 (active 11th-12th century), it was subsequently re-edited and printed under official auspices. There were three main families of official editions:Jingshizhenglei daguan bencao 經史證類大觀本草 (Categorised Pharmacopoeia of the Daguan Era, Based on Classics and Histories) 1108; Chongxiu Zhenghejingshizhengleibeiyong bencao 重修政和 經史證類備用本草 (Revised Categorised Pharmacopoeia of the Zhenghe Era, Based on Classics and Histories, and Organised For Practical Use) 1116; and Shaoxing jiaoding jingshizhengleibeiyong bencao 紹興校定經史證類備用本 草 (Revised Categorised Pharmacopoeia of the Shaoxing Era, Based on Classics and Histories, and Organised For Practical Use) 1159. The drug illustrations are, generally speaking, reproduced with great consistency across all three families. 
In 1220 (4th year of the Xingding era), in the Southern Song (1127-79), the artist Wang Jie 王介 created the three-volume Lü Chanyan bencao 履㟴岩本草 (Cliff Walker's Materia Medica), illustrated with naturalistic paintings in the meticulous courtly gongbi style. Of the original 206 illustrations, 202 survive. A Ming facsimile copy of this work is preserved in Beijing Library. ${ }^{25}$ In the early 14th century under the Yuan (Mongol) dynasty (1271-1368), the imperial dietetic physician Hu Sihui 忽斯 慧 wrote the three-volume Yinshan zhengyao 飲膳正要 (Principles of Correct Diet), a nutritional manual for the Mongol empire. ${ }^{26}$ The library of the China Academy of Chinese Medical Sciences holds a block-printed edition of Yinshan zhengyao from the Ming period, published by the Ming Jingchang 經廠 (the Palace Treasury printing house), which is illustrated. However, it can be deduced that the illustrations are a late accretion, especially as the people and scenes depicted all appear to be Han Chinese. The version of Yinshan zhengyao in Sibu congkan xupian 四部叢刊續編 (Supplement to the Sibu congkan anthology of the classics) reproduces the illustrations from the Ming edition. The most direct reflection of the spread of foreign, and more particularly Islamic medical knowledge in this period is Huihui yaofang 回回藥方 (Huihui [Muslim] Medicinal Recipes). Beijing Library holds four volumes of an incomplete Ming hand-copy which has no illustrations, and it is quite possible that the original work was unillustrated. ${ }^{27}$

Li Shizhen's encyclopaedic Bencao gangmu (Compendium of Materia Medica), the best known Bencao from the Ming period (1368-1644), contains entries on 1,892 drugs with more than 1,10o illustrations. Various other illustrated works in the Bencao genre have been transmitted from the Ming and Qing dynasties. According to Zheng Jinsheng's 鄭金生 calculations, there are five major Ming illustrated Bencao with polychrome painted artwork, which between them account for a grand total of 10,709 illustrations (either the originals or facsimile copies). Of these, Bencao pinhui jingyao is the earliest, and it also contains the most illustrations. ${ }^{28}$ The most beautiful Ming illustrated Bencao is surely the magnificent Buyi Lei Gong paozhi bianlan, discussed in detail above. Besides the Ming exemplar in the library of the Chinese Medical Academy, the Kyo-U Library (杏雨書 屋) of the Takeda Science Foundation in Osaka conserves an illustrated copy of Buyi Lei Gong paozhi bianlan made in or after the Jiaqing 嘉慶 reign period of the Qing dynasty

\footnotetext{
25 Li Jingwei (ed.) 1992, pp. 52-3; Zheng Jinsheng 198oa, pp. 338.

26 Buell and Anderson 2000.

27 Jiang Runxiang 1996; Song Xian 2000.

28 Zheng Jinsheng 2003.
}

(1796-1820). The Jinshi kunchong caomu zhuang 金石昆 蟲草木狀 (Description of Minerals, Insects and Plants) compiled by Wenshu 文淑 in 1620 (48thyear of the Wanli era of the Ming), which is now held in the National Central Library of Taiwan, contains ' 1,316 coloured illustrations, accounting for over $96 \%$ of the 1,358 original Hongzhi-era illustrations in Bencao pinhui jingyao'.29 Beijing Library holds a four-volume illustrated version of Shiwu bencao, which is textually similar to the Ming-dynasty edition by Xueyi 薛已, but additionally contains 492 polychrome paintings in meticulous gongbi style. ${ }^{30}$ Around the end of the Ming and the beginning of Qing Dynasty, the artist sisters Zhou Hu 周祜 and Zhou Xi 周禧 provided 75 painted illustrations for the five-volume Bencao tuhui 本草圖繪 or Bencao tupu 本草圖譜 (Illustrated Register of Materia Medica), all based on originals in Bencao pinhuijingyao. ${ }^{31} \mathrm{As}$ Zheng Jinsheng has pointed out, the majority of the Ming illustrations listed above are imaginative works by creative artists who were not specialists in materia medica. Their pharmaceutical significance should not be exaggerated. ${ }^{32}$

To the best of my knowledge, there are no images of non-Chinese people like those in Bencao pinhui jingyao and Buyi Lei Gong paozhi bianlan in any of the other Bencao books listed above, or indeed in Yinshan zhengyao and Huihui yaofang. Uniquely, the eight pictures in Bencao pinhui jingyao and Buyi Lei Gong represent, with vivid immediacy, the presence of foreign medical culture in China.

\section{The Bedrock of Imagination: the Trade in Aromatic Drugs and the Diffusion of Huihui Drugs in the Song, Yuan and Ming Dynasties ${ }^{33}$}

As we have seen, the seven pictures from Bencao pinhui jingyao discussed in this chapter almost all relate to aromatic drugs. From a social perspective, the genesis of these images is bound up with the trade in aromatics and fashions for exotic drugs, which form the bedrock of the painters' imagination. The historical background to their creation includes the importation of Huihui aromatic drugs in the Song and Yuan periods, the establishment of Huihui medical institutions in the Yuan, the publication

\footnotetext{
29 Cao Hui 1992.

30 Or 467 , according to Li Jingwei (ed.) 1992, p.73. Zheng Jinsheng (2003) says that it contains 492 pictures, 279 of which are original creations.

$31 \quad$ Li Jingwei (ed.) 1992, p. 76; Zheng Jinsheng1989.

32 Zheng Jinsheng2005, pp. 219-51.

33 'Huihui' in this period referred primarily but not exclusively to Muslim people.
} 
of Yinshan zhengyao and Huihuiyaofang, etc. The pictures of non-Chinese in Bencao pinhui jingyao must thus be seen in the wider context of an influx of Huihui medicine and medicinals. In a sense, they present a microcosm of medical cultural exchange between China and the rest of the world from the Six Dynasties period through to the Yuan and Ming Dynasties, and they reflect the historical fact that Huihui medical culture was widespread in China from the Song and Yuan periods onward.

Medical interchange between China and Da Qin 大秦 (i.e. the Roman Empire), Persia and the Arabian regions began with 'tribute' and diplomacy. In the Tang, knowledge of ophthalmology from Daqin and India spread to central China (especially jinbi shu 金篦術, a technique for operating on cataracts using a golden needle). Imported remedies such as 'Persian medicinal decoction' (Bosan tang 餑散 湯) were recorded in classic medical texts. The trade in Arabian aromatics peaked in the Song, when Guangzhou 廣州, Quanzhou 泉州 and other coastal cities became famous trading ports. ${ }^{34}$ Song huiyao 宋會要 (the Song institutional history), records an imperial proclamation issued by Taizong in 920 ( 7 th year of Taiping Xingguo), naming 37 imported drugs that were approved for general use. Xiangyao, Jiangzhen xiang, Longnao xiang and Anxi xiang were among the aromatics on the list. Song huiyao also reports data for the trade in crude drugs for the years 1133 and 1141 ( 3 rd and 11th years of the Zhaoxing era), showing that an extremely rigorous and detailed classificatory system for aromatics was in place by the 12 th century. For borneol/camphor drugs, there were nine categories: Shunao 熟腦, Meihua Nao 梅花腦 (borneol), Minao 米腦, Baicang nao 白蒼腦, Younao 油腦 (camphor), Chicang 赤 蒼, Naoni 腦泥, Lusu nao 鹿速腦 and Muzha nao 木答腦. It is not difficult to imagine the grand scale of trade in that period. The documentary evidence is further corroborated by archaeological evidence of aromatic drugs from the 13th-century Quanzhou shipwreck.

In the Yuan period, Huihui physicians enjoyed great success. An outstanding example is Aixue or Ngai-Sie 愛 薛 (1227-1308), who held office in the Beijing Medicine Department and Guanghui Si 廣惠司 (the Office of Ample Benevolence) in 1263 and 1273. Guanghui Si, the institution to which most Huihui doctors were subject, played an important role in the administration of medicine in the Yuan. In 1292, the Yuan government set up special Huihui Drug Departments (Huihui yaowuyuan 回回藥物院) in the two capital cities - Dadu 大都 (Great Capital), near present-day Beijing and Shangdu 上都 (Supreme Capital) in what is now Inner Mongolia. Shanjuxinyu 山居新語 (New
Writings from a Mountain Dwelling) (vol.1) by Yang Yu 楊 㻦 (14th century), and Chuogeng lu 輟耕錄 (Notes Made While Resting from the Plough) (vol. 22 'Thaumaturgy from the Western Regions' 西域奇術) by Tao Zongyi 陶宗儀 ( $f l$. 136o-8) also extol the marvellous skills of Huihui doctors. Yinshan zhengyao and Huihuiyaofang, the Yuan (Mongolera) texts referred to above, are historical records of Huihui medical expertise. Ruizhutang jingyan fang 瑞竹堂經 驗方 (Empirical Recipes from Auspicious Bamboo Hall) (15 volumes in total), by the Uyghur physician Sademishi (Shatumusu.Saqianzhai 沙圖穆蘇·薩謙齋), probably published in 1326, contains many prescriptions requiring the use of aromatic drugs imported from central Asia. Much has already been written on the subject of imported drugs in the Yuan and early Ming, and I will therefore not discuss it further here. ${ }^{35}$

\section{Conclusion}

In light of the preceding discussion, I would argue that the seven Pictures of Non-Chinese in Bencao pinhuijingyao are intended as representations of 'Huihui' people from the Persian and Arabian regions; through the representation of exotic people and drugs, they create an imaginative space to envision life outside the boundaries of Chinese culture. In my reading of the seven pictures, I have focused on tracing the trajectories of transmission of foreign medical culture. But also, it is important to note that the pictures embody a socio-cultural perspective in which Chinese medicine occupies a position of centrality. This is especially obvious in the illustrations of Diyejia and Aina xiang. This sense of medicine as a marker of identity and difference is integral to the Sinocentric world view of pre-modern Chinese doctors and scholars.

\section{Bibliography}

\section{Primary Sources}

Jiang Runxiang 江润祥 (ed.) 1996, Huihui yaofang 回回药方 [c.14th century], Hong Kong: Xianggang Zhongguo bianyi yinwu youxian gongsi.

Lu Duoxun 卢多逊, Li Fang 李昉 et al. [Song], ed. Shang Zhijun 1998, Kaibao bencao 开宝本草 (compiled and restored edn), Hefei: Anhui kexue jishu chubanshe.

Lu Jun 鲁军 (ed.) 2002, Yuzhi bencao pinhui jingyao 御制本草品彙精 要 (Imperially Commissioned Materia Medica Containing Essential and Important Material Arranged in Systematic Order) (new, printed edn), Beijing: Juzhou chubanshe.

Shang Zhijun 尚志钧 (ed.) 1997, Haiyao bencao 海药本草 (compiled

Li Jingwei (ed.) 1998, pp. 98-157; Ma Jianchun 2003, pp. 284-301. 
and corrected edn), Beijing: Renmin weisheng chubanshe.

Su Jing 苏敬 (ed.) [Tang period], ed. by Shang Zhijun 尚志钧 1981, Xinxiu bencao 新修本草 (Newly Revised Bencao) (2nd edn), Hefei: Anhui kexue jishu chubanshe.

Su Song 苏颂 (ed.) [Song period], ed. Shang Zhijun 尚志钧 1994, Bencao Tujing 本草图经 (compiled and restored edn), 'Foreword', Hefei: Anhui kexue jishu chubanshe.

Zhang Yanyuan 張彥遠, ed. with notes Yu Jianghua 俞劍華, 1964, Lidai minghuaji歷代名畫記 (A Record of Famous Paintings through the Ages), Shanghai: Shanghai Renmin meishu chubanshe.

\section{Secondary Sources}

Buell, P.D. and E.N. Anderson 200o, A Soup for the Qan: Chinese Dietary Medicine of the MongolEra as seen in Hu Szu-Hui's Yin-Shan ChengYao, London and New York: Kegan Paul International.

Cao Hui 曹晖 1992, 'Bencao tupu zai kaobian' 本草图谱再考辨 (Bencao tupu revisited), Zhongguo yaoxue zazhi, 27 (Special for Medical History and Study on Bencao, supplement), 32-35.

1993, 'Bencao pinhui jingyao zhiyaotuji qi tese kaocha' 本草 品汇精要之药图及其特色考察 (Review of the drug illustrations in Bencao pinhui jingyao and their features), Xinyuan Zhongyi wenxian zazhi $1,5^{-8}$.

Chandra, L. 2003, 'The Khotanese mural of Hārītī in shrine D II at Dandan-Uiliq', Archiv Orientální, quarterly journal of Asian and African studies 71.3, 293-302.

Chen Ming 陈明 2005, Shufang yiyao: Chutu wenshu yu xiyu yixue 殊方异药: 出土文书与西域医学 (Foreign Medicine in Medieval China: Medical Manuscripts Discovered in Dunhuang and Western Regions), Beijing: Beijing daxue chubanshe.

Chou, E. Shan 1984, Tu Fu's 'Eight Laments': allusion and imagery as modes of poetry. Diss. Harvard University.

Fan Xingzhun 范行准 1952, 'Zhōngguó yǔ Yalabo yīxué de jiāoliú shǐshí' 中国与亚拉伯医学的交流史实 (Historical facts on the exchange between Chinese and Arabian iatrology), Yisi zazhi 4.2, 83-110.

Fu Weikang 傅维康 et al. (eds) 200o, Zhongguo yixue tongshi 中国医 学 (A General History of Chinese Medicine), Volume of Illustrative Plates of Cultural Relics, Beijing: Renmin weisheng chubanshe.

He Zhongjun 和中浚 and Wu Hongzhou 吴鸿州 et al. (eds) 2001, Zhonghua yixue wenwu tuji 中华医学文物图集 (Illustrated Collection of Chinese Medical Cultural Relics, Chengdu: Sichuan renmin chubanshe.

Jiang Boqin 姜伯勤 2004, Zhongguo Xianjiaoyishu shi 中国祅教艺术 史 (A History of Chinese Zoroastrian Art), Beijing: Sanlian shudian.

Lei Wen 雷闻 2003, 'Ge er li mian yu ci xin poufu - cong Dunhuang 158 ku beibi Niepan bian wangzi ju'ai tu shuoqi' 割耳第面与刺心 剖腹一从敦煌158窟北壁涅槃变王子举哀图说起 (Severing the ears, cutting the face, stabbing the heart, disembowelment: a study of the painting of princes mourning for the Buddha's Nirvana in Dunhuang Cave no. 158), Zhongguo dianji yu wenhua 4, 95-104.

Li Jingwei 李经纬 (ed.), 1992, Zhongguo gudaiyishitulu 中国古代医史 图录 (Illustrated Catalogue of Ancient Chinese Medical History), Beijing: Renmin weisheng chubanshe.

Li Jingwei 李经纬 et al. (eds) 1998, Zhongwai yixue jiaoliu shi 中外 医学交流史 (History of Medical Interchange between China and Abroad), Changsha: Hunan jiaoyu chubanshe.
Lo, V. 2005, 'Quick and easy Chinese medicine: the Dunhuang moxibustion charts', in Lo and Cullen (eds), 211-51.

Lo, V. and C. Cullen (eds) 2005, Medieval Chinese Medicine: The Dunhuang Medical Manuscripts, London/New York: Routledge Curzon.

Ma Jianchun 马建春 2003 , Yuandai dongqian xiyuren ji qi wenhua yanjiu 元代东迁西域人及其文化研究 (Eastward Migration of the Peoples of the Western Region in the Yuan Period: a Cultural Study), Beijing: Minzu chubanshe.

Mayanagi, M. 真柳誠 2002, 「『本草品彙精要』ローマ本・大塚本・ベ ルリン本の成立関係」, 『漢方の臨床』49.9, 1130-1133; 1207-1220.

Okano Makoto 冈野诚 2000, ‘唐の安金藏の割腹', published in Japanese《法史学研究会会报》5 (July), 33-7.

Rao Zongyi 饶宗䝠 2002, 'Soma yu busi yao' Soma 与不死药 (Soma and the elixir of immortality), Zhongguo xueshu 4, 1-12.

Rong Xinjiang 荣新江 2001, Zhonggu Zhongguoyu wailai wenming 中 古中国与外来文明 (Medieval China and Foreign Civilizations), Beijing: Sanlian shudian.

Shanxi sheng kaogu yanjiusuo 陕西省考古研究所 2001, 'Xi'an Beizhou An Jia mu fajue baogao' 西安北郊北周安伽墓发掘简报 (Report on the excavation of the tomb of An Jia of the Northern Zhou dynasty in Xi'an), Wenwu 1. 28-35.

Shaanxi sheng kaogu yanjiusuo 陕西省考古研究所 2003, Xi'an Beizhou An Jia mu 西安北周安伽墓 (Tomb of An Jia at Xi'an of the Northern Zhou Dynasty), Beijing: Wenwu chubanshe.

Schafer, E.H. [1963] 1985, The Golden Peaches of Samarkand:A Study of T'ang Exotics, Berkeley, Los Angeles: University of California Press.

Song Xian 宋岘 2000, Huíhuiyàofāng kăoshi 回回药方考释 (Study and Exegesis of Huihuiyaofang), Beijing: Zhonghua shuju.

Unschuld, P.U. 1986, Medicine in China: A History of Pharmaceutics, Berkeley/Los Angeles/London: University of California Press.

Wang Jinyu 王進玉 (ed.) 2001, Dunhuang shiku quanji: kexue jishu huajuan 敦煌石窟全集・科學技術畫卷 (Dunhuang Caves, Complete Edition: Science and Technology Paintings Volume), Hongkong: The Commercial Press (Hong Kong) Ltd.

Whitfield, R. and A. Farrer 199o, Caves of the Thousand Buddhas: Chinese Art from the Silk Route, London: British Museum Publications.

Zheng Jinsheng 郑金生 1980a, 'Lü Chanyan bencao chukao' 履㭸岩 本草初考 (A preliminary study of Lü Chanyan bencao), Zhejiang Zhongyi zazhi 8,338 .

1980b, 'Tang bencao yiqian de bencaotu' 唐本草以前的本 草图 (Bencao illustrations before Tang bencao), Zhonghua yishi zazhi 2,82 .

1989, 'Zhongguo gudai caihui yaotu xiaoshi' 中国古代彩 绘药图小史 (A brief history of early Chinese polychrome drug illustrations), Zhejiang Zhongyi zazhi 9, 422-424.

1999, '清以前本草図の作风と价值' (Style and value of pre-Qing bencao illustrations), 日本医史学杂志Japanese Medical Historiography 45.2, 296-7.

2003, 'Mingdai huajia bencao chatu' 明代画家本草插图研 究 (A study of Bencao illustrations by artists of the Ming period),Xin shixue 14.4, 65-120.

2007, Yaolin wai shi 药林外史 (Unofficial History of Medicine), Guilin: Guangxi shifan daxue chubanshe. 



\title{
22 Chinese Horse Medicine: Texts and Illustrations
}

\author{
Paul D. Buell, Timothy May and David Ramey*
}

The domesticated horse appeared early in China. It was used to draw chariots no later than Shang times (late 2nd millennium), and for riding during the mid-Zhou (after the 6th century BCE), in imitation of China's pastoral enemies, then pressing upon the Middle Kingdom. ${ }^{1}$ Nonetheless, in spite of its importance for maintaining mounted armies and other uses of the horse, China was late to develop a specialised horse medicine. Veterinarians are among those medical officials mentioned in the Zhouli 周禮 (Rituals of the Zhou). This is a work of early Han 漢 times. ${ }^{2}$ There are also hints of specialised knowledge of horses in other sources (e.g., the Bo Le 伯 樂 tradition found in the Zhuangzi 莊子), ${ }^{3}$ but there are no actual texts until much later. The most important Chinese works date only from the Ming 明 dynasty (1368-1644).

\section{Texts}

The earliest surviving veterinary texts of any sort in China are a few stray horse recipes found among Dunhuang 敦煌 documents. These are largely from Qin 秦 $\left(255^{-206}\right.$ BCE $)$ and Han 漢 (206 BCE-220 CE) times. ${ }^{4}$ In them, treatment is overwhelmingly herbal. This parallels the main focus of Chinese medicine for humans down to the present. Later, the popular sixth-century manual Qimin yaoshu 齊民要 術 (Essential Arts to Assist the People) (hereafter QMYs), included its own selection of veterinary recipes. ${ }^{5}$ They were not just for horses, but for a variety of other animals as well.

In these early texts, clear development is evident. The QMYs, for example, calls not just for herbal treatment but also for a specialised bleeding and cauterisation. This is a first for Chinese veterinary medicine although not, apparently, for humans. ${ }^{6}$ Be that as it may, bleeding and



$6 \quad$ All this depends upon when the texts currently making up the Huangdineijing 黃帝內經 (Yellow Thearch's Inner Canon) were written. A Han date is claimed but existing versions go back no farther than Song or Tang. On bleeding in these texts, see Epler 1980.

cauterisation are clearly not treatments of choice in the QMYS. These remained herbal. Already the QMYS shows the influence of the theoretical system developed earlier for human patients, including the key concept of Qi 氣, the resident Yang 陽 energy and motive force pervading the body according to the Chinese medical system, although references to Qi remain a very minor tradition in the QMYS.

The QMYS was written during the Toba Wei 魏 dynasty (386-535), a period not only of foreign rule in China, but also of great foreign influence, especially through the medium of Buddhism. This may be the source of some of the new ideas in the QMYS. Its bleeding and cauterisation, for example, are differently applied compared to what we know of the human medicine of the time. ${ }^{7}$ In fact the closest equivalents of the bleeding and cauterisation of the QMYS must be sought in Greece and Rome, where such treatments had a far longer history than in China. ${ }^{8}$

Shortly after the time of the QMYs (early 6th century), references to specialised veterinary texts begin to appear in our sources. Several are mentioned in early bibliographies, including that accompanying the Suishu 隋書, the official history of the Sui 隋 dynasty (580-618). It was written in Tang 唐 times. Unfortunately, none of these early texts appear to have survived, except perhaps as fragments in later documents. ${ }^{9}$ Veterinary information does continue to occur in passing in other sources that do survive, including a Northern Song 宋 (96o-1125) military manual,10 but information in them is limited. A Northern Song date is claimed for a treatise on camel medicine, but this text only survives in a late, extensively edited form, ${ }^{11}$ and as such is an unreliable guide to Song practice. By contrast, a late 14th-century compilation does contain genuine works from earlier times. This is the Simu anji ji 司牧安驥集 (Collections for Pacifying Stallions when Administering Flocks) (hereafter SMAJJ), published in 1384. Judging from internal evidence, most of its components are apparently

The cauterisation called for in Qimin yaoshu recipes, for example, had little or nothing to do with moxa-cautery, the indigenous tradition of cauterisation already established in China. It seems to represent something entirely novel, and probably an introduction from somewhere else.

8 See Buell, May and Ramey 2010.

9 See the discussion in Zhou Jiezheng 196o.

10 See von den Driesch and Peters 2003, p. 6o.

11 Discussion in Franke 1997. We do not share Franke's views on the early dating of this text. 
from the 11th and 12th centuries, but some may be later. In particular, some show the signs of the developed correspondence theory that first came to fruition in human medicine during late Song, Jin 金 (1125-1234) and Yuan 元 (126o-1369) times. Most SMAJJ texts are probably northern in origin (China was disunited at the time) although some, probably those added last, may have been Southern Song $\left(1125^{-1279)} \cdot{ }^{12}\right.$

From roughly the same period comes a more significant work, the first surviving monograph devoted exclusively to horse medicine, the Xinbian jicheng mayifang 新編集 成馬醫方, (Newly Printed and Collected Recipes for Horse Medicine), which, although written in Chinese, is actually a Korean work. It was first published in $1399 .{ }^{13}$

Despite these important early efforts, the most significant works in the genera of horse medicine date only from the Ming 明 dynasty (1368-1644). Among them is the Yuan Heng liaoma ji 元亨療馬集 (Yuan and Heng's Collection for Treating Horses) (hereafter YHLMJ), first published under that title in 1608. The existing version is supposedly the work of two brothers, horse specialists with long experience. In fact, this is not likely to have been the case. As the Korean scholar Kang Myun Hee 姜冕熙 has shown, the YHLMJ is actually a new edition of an earlier text, the Liaoma ji 療馬集 (Collection for Treating Horses), now lost. It lacked any reference to two brothers Yuan and Heng. ${ }^{14}$ Although emphasised in the preface to the $16 \circ 8$ edition, this fraternal tradition is also suspect because the two characters Yuan 元 and Heng 亨 can also be taken as a reference to the Qian 乾 chapter of the Yijing 易經 (Book of Changes). ${ }^{15}$ In any case, the existing YHLMJ has clearly been compiled from a variety of existing sources. In this respect it is like the older SMAJJ, although editors have made the material in the YHLMJ fit more seamlessly than is the case with the heterogeneous material in the earlier collection.

Spurious attribution notwithstanding, the YHLMJ is an extraordinarily rich work. It is in six books and includes

12 For further analysis and discussion, see Buell, May and Ramey 2010 .

The text was later republished in China, in 1634, and subsequently in Japan, showing its popularity. The authors are given as Quan Zhonghe 權仲和 and Han Shangjing 韓尚敬 (reading their names in Chinese).

14 Kang Myun Hee 1988.

15 The Qian chapter, the first in the book, begins: Yuanheng li chen 元亨利真, 'the originating benefits the propitious'. In this case the 'originating' refers to the originating of the 10,00o things, particularly those comprising part of the biological world. Despite the preface to the 1608 edition, it is unlikely that any literate Chinese would have failed to note the reference. detailed discussion of a variety of topics: horse physiognomy; horse whorl lore, a kind of divination based upon close examination of the configurations assumed by a horse's hair that is also found outside of China; ${ }^{16}$ horse diagnosis and physiology; horse pathology, including a consideration of the eye diseases of the horse; taboo and propitious days for treatment; correspondences; and a huge section, comprising all of books 3 and 4 , on specific conditions and the treatments preferred for them. At the end of the book, there is also a detailed listing of herbal medicines and preparations and their specific applications.

The Ming edition apparently became rare, the case today too, and in the 18th century, an entirely new edition was made of the YHLMJ. This edition is still in general use. Editions based on this new edition have continued to be made down to the present, each, as a rule, with changes to suit different times, making the use of the most modern editions of the text problematical at best. The 18th-century reworking was by Guo Huaixi 郭懷西 and was published in 1732 in his collection Xinke zhushi ma niu tuo jing daquan 新刻注釋馬牛駝經大全 (Newly Printed and Annotated Horse, Ox, and Camel Classics).

Another major horse text of the Ming period is the Xinke ma shu 新刻馬書, (Newly Printed Horse Book), from 1594. It is by the Ming literatus Yang Shiqiao 楊時喬 (d. 16o9), who is also the author of a treatise on Ming horse management, the Mazheng ji 馬政記 (Record of the Horse Administration). The latter work is mostly devoted to official pronouncements. This is not the case with Yang's 'Horse Book'. It is in 13 books and does not come down to us complete. The text is highly literary and is replete with quotations from classical sources and copies out whole documents in some cases. Topics discussed include physiognomy and general aspects of judging horses, general methods for treatment, correspondence theory (the fullest discussion of this in the traditional veterinary literature), physiology, pathology, diagnosis, and bleeding and cauterisation, with some consideration of specific pathologies. Book 12 is lost and book 13 is in fragments only; the latter deals with the treatment of donkeys. One chapter reproduces a treatise on 'difficult issues' already known from the SMAJJ. ${ }^{17}$ Throughout the book, where treatment is called for, the emphasis is herbal. Bleeding and cauterisation is more prominent than in the Korean Xinbian jicheng mayi fang of 1399, for example, possibly indicating that this form of treatment, which is also important in the YHLMJ, had gained in acceptance.

16 See, for example, von den Driesch and Peters 2003, p. 68.

17 This is the Zaofu bashiyi nanjing 造父八十一難經, (Father Zao's Classic of 81 Difficult Issues) in the form of poetic couplets. 




Figure 22.1 'Map of Indentations'. Source: SMAJJ, p. 28

These texts, including major revisions, as in the case of the YHLMJ, have set the tenor of Chinese horse medicine down to the present. Their style is also closely imitated by a rich manuscript tradition, including texts from various periods collected by David Ramey. In fact, little changed in China in the basic treatment of horses until the introduction of modern veterinary practices under the Nationalist and Communist regimes. This included, from the 196os, the first veterinary acupuncture with fine needles practised in China. This has since been taken up not only in China but throughout the world including the United States and Europe, where it has achieved some popularity. ${ }^{18}$

\section{Illustrations}

One major feature of the recent, printed tradition of Chinese veterinary medicine is that nearly all the texts, starting, in the case of horse medicine, with components of the sMAJJ, are illustrated, some very well illustrated. This is true of many of the major manuscripts as well, some in

18 Actually, as we will show in a separate paper, the use of acupuncture on animals appeared in Europe before it appeared in China, in the early 19th century and veterinary acupuncture, like human acupuncture, has been heavily influenced by French physicians and their ideas. the Ramey collection, for example. One is even beautifully illustrated in colour, although most sketches in them are black and white.

Typical of the printed tradition is the short Bo Le zhenjing 伯樂鍼經 (BLZJ) (Bole's Needling Classic), reproduced in the SMAJJ of 1384 , but which clearly circulated separately before that date, although how much before that date is uncertain. The current BLZJ, along with a parallel text, the Bo Le hualuo tu ge jue 伯樂畫烙圖歌訣（The Song Secrets of the Diagram of Bo Le's Branding) (BLHLTGJ), to be discussed next, are most likely works of the Yuan dynasty (126o-1368). Both texts appear to show Western influences, and the Mongol Yuan period was a maximal period for such influences in China, particularly those coming from the Islamic world, including its veterinary literature and methods of practice. ${ }^{19}$

In its present form, BLZJ is in two parts. Part One, and this may be the oldest component of the text, is a 'Map of Indentations' (xueming tu 穴名圖), here reproduced from a modern print of an 18th-century edition, the earliest available, showing 78 places on a horse. Each is named, for therapeutic intervention (Fig. 1).

19 See the detailed discussion with examples of the medical convergences involved in Buell, May and Ramey 2010. See also Buell 2008. 




Figure 22.2 Figure from Künstlicher Bericht und allerzierlichste Beschreybung. Source: Stork 1971, p. 681

Part B is the BLZJ proper. The first part is a general theoretical consideration of 'needling'. The language is very much influenced by the theory of human acupuncture, although the actual interventions, which follow, have nothing whatever to do with it. ${ }^{20}$ Not one calls for

2O SMAJJ, pp. 29-34. The following are examples of the relevant sections. Numbers are the order that the discussions appear in the text:

[1.] Eye Pulse Indentation (Yanmo xue 眼脉穴): [This is] four fingers behind the eye. One needle 2 fen 分 [1oth of Chinese inch] deep at this indentation. Bleed. It cures liver and organ heat, eye swelling, and crying disease.

[18.] Liver Main Conduit Indentation (Ganchu xue 肝腧穴): [It is in] the left inside kernel bank [zuo lijenpan 左裡仁畔], the inside of rib number 5 from the rear, 1 chi 尺 [Chinese foot], 5 cun 寸 from the spine. One fire needles at this indentation 1 cun. It treats all diseases associated with the liver.

[21.] Great Wind Gate Indentations (Da fengmen xue 大風門 穴): [They are located] one finger behind the bases of the two ears. One cauterises at this indentation with a branding iron, and a round brand. The depth is $3 f e n$. One applies oil. It treats needling perse. All insertions are for purposes of bleeding, cauterisation ('hot needling'), branding, or to achieve minor surgery (using scalpels, 'white needles'), making the whole tradition, including points identified, quite parallel to equivalent material from the West. The West had its own diagrams of insertion points, nearly as old as those from China, and the Islamic World. ${ }^{21}$ Figure 2 shows a German example, based upon many earlier precedents reaching back to the Middle Ages. The close similarity of the 'map'

a sudden seizure, destroying wound wind, or various wind diseases.

[33.] Heart Main Conduit Indentation (Xinchu xue 心腧穴): [This is] on the breast bone. In the case of this indentation, if there is suffering from heart jaundice disease, one uses white needle [scalpel] barbs, 10 or more needles, to needle and produce yellow water and blood. Take a qian 錢 [Chinese ounce] of salt and rub into the needle wound. Draw out the yellow water and poison Qi. If there is no treatment, an ulcer will form that will penetrate to the heart and lung.

21 See the discussion and sample translations in Buell, May and Ramey 2010. 




Figure 22.3 Bo Le Branding Diagram. Source: SMAJJ, pp. 48-9. See complete translation in Buell, May and Ramey 2010.

The 12 places in the top illustration are (from top, clockwise): 1. 'The ink stone'; 2. 'The foreleg bone'; 3. 'The adverse wind [bone]'; 4. 'The shoulder dragon [bone]'; 5. 'The kneecap bone'; 6. 'The transfer bone'; 7. 'The fish net hoof bone'; 8. 'The fathering sinew bone'; 9. 'The fox bone'; 10. 'The bird sinew bone'; 11. 'The seizing grass bone'; 12. 'The great thigh bone'.

Below, the first two items are: Song for branding the transfer bone. [forked three]: pain of the transfer bone makes the feet drag. There can be no harmonisation due to twisting breaking. If one brands a forked three at this bone joint, at that time the [feet] will be light and strong, and they will accurately sharpen [adjust] themselves; Song for branding the ink stone bone. [seal script character for $z h i$ 柧, 'gardenia nut']: The ink stone bone is very much a cause [of disease], and there is thus a great deal of swelling as a result of wounds from blows. One uses a fire branding iron and brands a zhi character. One will thereby avoid causing neglect, and one will be able to attain a proper arrangement.

shown in Figure 1 to this German illustration is striking. Both provide information on cauterisation and bleeding.

The BLHLTGJ is similar to the BLZJ, but showing fewer places on the body of a horse for actual intervention, in this case therapeutic branding. This is a special kind of cauterisation, either with specially shaped brands (the likely interpretation) or with 'fire irons' intended to write out and build up shapes. The purpose is to produce a brand at determined points responding to associated conditions. Interestingly, such branding was already well known in the European and Islamic West. Also interestingly, some of the symbols called for are quite similar to the Chinese: ${ }^{22}$

22 See Buell, May and Ramey 2010 for a detailed comparison with symbols from the horse book of Abu Bekr (Egypt, first half of the 14th century) 




Figure 22.4 Colic from the Xinbian jicheng mayifang (56a). The text reads: Number 24: Disease origin [is] in a stiff intestine and excrement that does not move. The song: When there is a stiff intestine and excrement that does not move it is not difficult to treat. [The animal] lies down and gets up frequently. The spleen and belly are high. [Textual note in smaller characters:] (The Yellow Thearch asked [Ma] Shi Huang [馬] 師黃 [皇] [Horse-Master Huang?] saying: as for the condition where the intestine is stiff and the excrement does not move, what is it? He replied saying: when the intestine is stiff and the excrement does not move it is a matter of the horse eating grass excessively on account of difficulty, and there is a lot of excrement. It hardens for a long time. The horse drinks and does not rest. Also it sometimes eats to satiation and sometimes harbours chill and heat and this is not in accord with the brightness of the intestines. It is primarily this illness. It is also said: when the Qi 氣 goes up and down and does not go through, the belly swells. On account of difficulty [the horse] eats grass and [this] forms knots. Inside the intestines the knots are a reason for chill or heat. It does not accord with what is in the stomach. It is a matter of this.) If there is no improvement for five mornings and seven days, the Qi ways follow this and run to the rear loins. [Textual note:] (What is to be considered is that the illness first is transmitted to the stomach. The stomach transmits into the kidneys. The kidneys [are ruled by] water. What is to be considered is that the earth [?] Qi does not accord with what is dominant in the loins. The Qi goes up and down and mutually conflicts.) Take grass ash, rat dung, and pig oil [lard]. Heat [each] and in succession make an oily powder. Combine into an ointment. The five flavours [spices] will subsequently be combined [with it]. Make sure [the material] is abundant. A hard iron will also dissipate it.



Figure 22.5 Hoof Problems from the Xinbian jicheng mayi fang (58a). The text states: Number 28: disease origin situated in cramping manifestations, and in five gathering pain when lying down and getting up. The song [says:] at the time of cramping manifestations, the four hooves [ $\mathrm{ti}$ 啼 $=$ 蹄] are gathered because of the fact that the blood pulse does not go through and is caught up. [Textual note:] (The Yellow Thearch asked [Ma] Shihuang saying: when there are cramping manifestations of the blood pulse what reply is there? Cramping manifestation blood pulse is because of long term heat and wounding and [because the horse] eats to repletion going beyond [what it should] to be greatly careful. Also spring and autumn, if there is at the time pulling of the six pulses, then they will then not accord with the four seasons and there is pouring and chewing and cramping manifestations. The blood gathers together. Most of the blood is obstructed and congeals and does not accord. A lesser Yin 陰 [condition] dominates the disease. It is also said that heat goes up and attacks. Blood and chill descend. This is puddle Qi. The blood goes up and attacks up into the brain, thus the blood is chilled and flows into the four hooves. This is gathering cramping.) If in five days and seven mornings there is no sign of healing, if the loins [of the horse] are crooked and the head is held low, and the gait and steps are difficult, then (also it says if the heart's blood is a sea. Blood is greatly wounded in the heart. The heart is harmed in the spirit. If the head is held down it is wounded Qi. The Qi wounds all the main arteries of the lung.) in the case of the herbs in the Fire Care Recipe, see that the medicinal cake draws out its six flavours. [The condition] will be calmed naturally. 
Although many of the early illustrations in the printed tradition are of this sort, self-contained, and probably separately circulated as independent publications, connected sets of illustrated texts also appear. These are the single largest component of illustrated material in the YHLMJ, for example.

Opposite are two examples (Figs 4 and 5) of the early illustrations from the Korean Xinbian jicheng mayi fang. The first shows a horse suffering from a form of colic. The illustration clearly shows the horse in a position typical of colic (non-specific pain originating from the abdomen), looking at its abdomen, and in obvious discomfort. The text identifies the condition, with help from a quotation mentioning the Yellow Thearch himself, an originator and patron saint in veterinary as well as human medicine. The clinical description, in terms of the lack of excrement, failure to rest, and persistent drinking, is accurate. It then provides treatment options. The second shows another unfortunate horse, this one suffering from a hoof problem (laminitis). Again, the illustration is remarkable, as it shows the horse unwilling to bear weight on its fore feet, as is typical of laminitis, a condition in which the connections

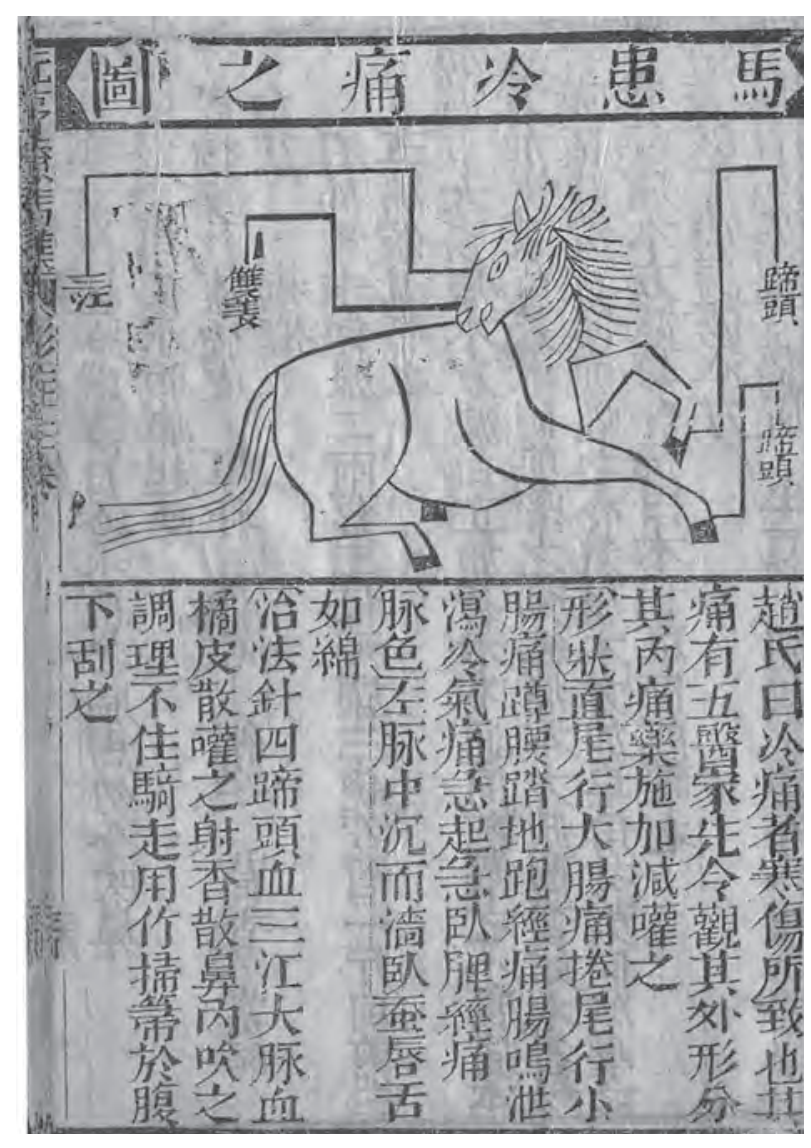

Figure 22.6 Horse suffering from Chill Pain (2) (continued) holding the hoof to the underlying structures are compromised, and which causes considerable pain. The format is the same, except that treatment is more generalised and the implication is that the horse will recover on its own if allowed to. Again, this is a remarkably accurate observation, as it is well-known that in many cases of laminitis it will resolve spontaneously.

In the YHLMJ, the basic format remains the same but symptoms and treatment are described in far more detail, and text and its illustration are not confined to a single printed sheet but take as much space as needed. In addition, the YHLMJ tends to describe horse diseases in terms not of pragmatic symptoms - largely (but not exclusively) the case for the Korean text above - but as they relate to the abstract qualities of human medicine as applied to animals. Figure 6 and its companion Figures 7, 8 and 9 are devoted to a horse suffering from 'chill pain'. The illustration is from a very late version of the YHLMJ, a copy of which is included in the Ramey collection. ${ }^{23}$ Significantly, in spite of its late publication date, this edition clearly reflects the original, Ming edition and not later re-workings.



Figure 22.7 Horse suffering from Chill Pain (1)

23 Yuan heng quan tu niu ma tuo jing ji, 3, 23b-25a. 




Figure 22.8 Horse suffering from chill pain (4) (continued)

The text passage begins with the title of the condition discussed, number 18 in the series, and a text source for the basic information (giving some indication of the multiple sources of the YHLMJ). Next is a general discussion of the symptom described and its causes, followed by a rhymed summary. This is perhaps the oldest material in the section and is one of the few things that remain unchanged from edition to edition. There follow quotations from authorities including another summary poem, a description of the behaviour of a horse with the condition, pulsing considerations and treatment methods, including bleeding, drenching and herbal application through the nostrils followed by post-treatment options. This section is followed by herbal formulas, varied to suit symptoms, and preparation instructions. The discussion is quite complete and traditional veterinary medicine could add little to what is said here, although some material is rearranged in different editions. ${ }^{24}$

The pattern of the YHLMJ as here became pervasive after Ming times, both in printed texts and in the rich veterinary manuscript tradition, including those manuscripts in the

24 An example of the YHLMJ text of this type, in this case from Guo Huaixi's revised edition is translated in Buell, Ramey and May 2010. It pertains to a surgical operation.



Figure 22.9 Horse suffering from chill pain (3) (continued)

Ramey collection, for example. Figure 10 shows a horse suffering from what seems to be prolapse of the anus, a serious condition, albeit rarely occurring. On the right is a description of symptoms, again, remarkably accurate, and easily recognised by equine veterinary practitioners, followed by a suggested treatment: surgical intervention and herbal treatment. A recipe for the proposed herbal remedy, for irrigation, follows with details about how to prepare and apply the remedy. The only difference between this manuscript, which is possibly late 19th-century, and the YHLMJ is that treatment is more pragmatic and less theory is discussed, although other pages (not this one, however) do include quotations from authorities.

\section{Physiognomy}

In addition to illustrations used to set forth theoretical summaries (e.g., points for cauterisation and what they are used for), and relating to specific diagnostic categories, a popular subject, for horses in particular, was physiognomy. In fact, a large part of manuals such as the YHLMJ is devoted exclusively to horse physiognomy and even texts without extensive illustration go into physiognomy in some detail. 




The concern is an ancient one for many reasons. First of all, fine horses are beautiful and owners rejoice in them, in their looks and pedigrees, these sometimes going back many centuries in the Arabian world. Indeed, one study of Bedouin vocabulary listed over a thousand words related to the horse. ${ }^{25}$ These terms include not only ones related to physiognomy, but also epithets and terms used in the commercial exchange of horses. The epithets also provide some insight into traits valued by the Bedouin as well as features that, while not necessarily a negative, were somewhat unusual. One such example is the appearance of a sa'din, which is a double jibhe or shield-like protrusion on a wide-forehead. It is regarded, as indicated by the root of the word, as being auspicious. ${ }^{26}$ These terms can also lead to qualities of a horse deemed favourable such as a mare who can run long distances without becoming winded. Such a mare is known as $q \bar{a} z \bar{a} t$.

And because owners appreciate their horses, they would prefer to predict their qualities and confirm their investments. Secondly, there is the - also age-old - issue of how to choose a good horse, camel, or ox. Above all, physiognomy texts seek to provide a guide to judging horses and other large animals prior to their purchase. This includes determining animal ages. Chinese sources, for example, include teeth diagrams for this purpose. Such

25 Raswan 1945.

26 Raswan 1945, p. 126
Figure 22.10 Prolapse of the Anus (Ramey A, p. 67). The full text is as follows: [Condition:] Picture of a horse suffering from prolapse of the anus [tuo gang 脫肛]: Whenever a horse unseasonably has an overexertion [crossbow, $n u$ 驽 = 努] throat, raises up its tail, the haunches are curved, the head is lowered, the ears are rolled up, and the grass fine hairs [of the coat] are scorched, then this is called the pathocondition [zheng 症] of chill Qi 氣 of the entrails, and overexertion wound of apparent force. Whenever treating it, one cuts the lotus hole and drenches with Debris Powder Putting-Through-the-Pass and washes with fangfeng [Ledebouriella seseloides] Debris Power decocted into a soup.

It is prohibited to ride the horse energetically for seven days.

Debris Power Putting-Through-the Pass: plum seed, peach seed, Chinese angelica [Angelica sinensis], fangfeng, qianghuo [Notopterygium incisum root], rhubarb [rhizome], Chinese honey-locust [Gleditsia sinensis]. Powder. Each time take 1 qian 錢 [Chinese ounce], 5 fen 分. Produce an oil from the dose. Drench evenly mixed with half a cup of water before [the horse eats] grass.

Fangfeng Debris Powder: fangfeng, jingjie [Schizonepeta tenuifolia herb], Chinese flower pepper, common alum, cangshu [Atracylodes spp.], mugwort [leaf].

Masticate these herbs together. Take two cups of water and decoct together. Bring to a boil three to five times. Remove sediment. Wait until the herbs are cooked. Clean away the pus and blood [from the prolapsed anus]. First use a pair of scissors and remove all the 'wind' membrane skin, Remove so as to be able to insert a finger into the anus and remove the hard covering [?] powder. Wash again and remove the blood. Send [the herbs] into the anus head. When dry, cauterise below the shoe and when ready, apply an iron.

diagrams are a comparative rarity in illustrated veterinary literature found elsewhere. This concern manifests in the Islamic world as well. The concern there was not simple appreciation for the beauty of the horse, but also practical necessity. Although works on furūsiyya or horsemanship existed through medieval Islamic history, a particular emphasis on furussiyya arose during the period of the Mamluk Sultanate of Egypt and Syria (126o-1517). As a result several of these manuals compiled many ideas and drills for horsemanship as part of the military training of the Mamluks, slaves who were purchased to be trained as soldiers. In addition to listing drills and techniques that were practised in a hippodrome, the furūsiyya manuals also included sections on veterinary care and the qualities of a good horse. This was literature not just for the literati and intellectuals, but for the military. As the Mamluks fought from horseback, the care and knowledge of the horse was crucial to their success. One of the most important furūsiyya manuals is the Kitāb al-siyāsah fì 'ilm 


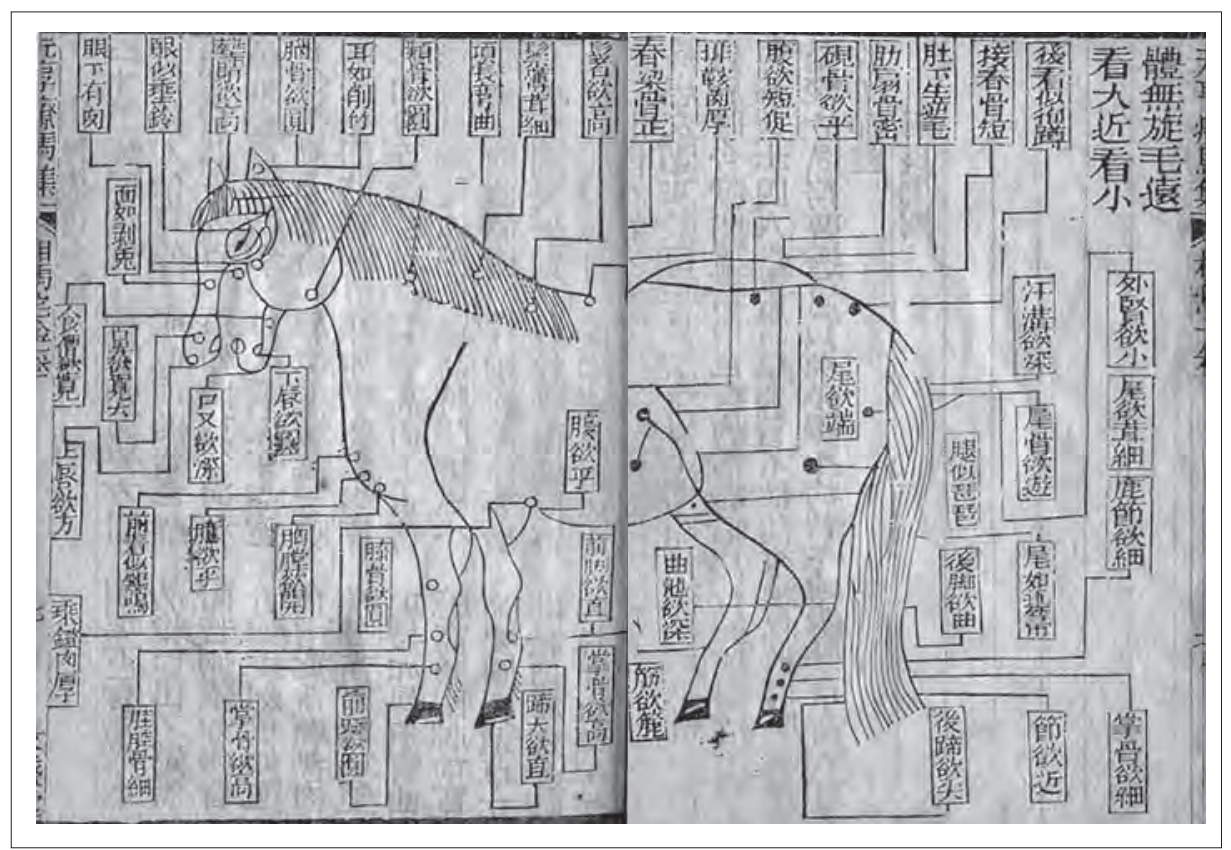

Figure 22.11a, b Physiognomy, detail from the Yuan Heng liaoma ji 元亨療馬集 (YHLMJ), 1608.1, pp. 6b-7a

al-farāsah wa-ashā'ir al-khayl wa amā'irhā (The Book on the Art of Horsemanship and the Knowledge of First-rate Horses and Their Characteristics and Features). Although the text lists the fourth Caliph, 'Alī ibn Abī Tālib (d. 661) as the author, this seems unlikely. Indeed, the copy that exists in the National Library of Medicine was produced in 1830 . As a result, it is uncertain at what point the text came into existence or from where. Nonetheless, it does reinforce the importance of certain qualities such as length and texture of the mane and tail, the shape of the horse's back, neck, and head. The horse should be strong and deep in the shoulder. A curling of hair, such as in the sa'din, anywhere on the body of the horse, except above the nostrils, was deemed a good omen, which could cause a prospective buyer to overlook another fault. Of course, there were also inauspicious marks as well, such as a white mark on the left foreleg accompanied by a white mark on the right hind leg.

Indeed, due to the horse's importance to the Mamluk military, knowledge of horse care and veterinary science was deemed important. This is demonstrated in the Aqrābādhin fí 'ilm tibb al-khayl (Medical Formulary for Horses) which entered the Mamluk sultanate as part of booty taken from a Mamluk assault into the region of Cilicia or Little Armenia in 1266. Sultan Baybars (r. 126o-77) had the work translated from Armenian into Arabic. ${ }^{27}$ In the work, there are sections devoted to the good qualities of a horse, medical care for specific symptoms, and even breeding. An example of a desirable quality according to the Aqräbādhin is a horse with a thin nose or snout as well as determining the strength or quality of a horse's leg bones. Although it is easy to assume that the ideal animal was an Arabian horse, the Mamluks procured horses from a variety of areas such as Cyrenaica Libya, which produced an entirely different type of horse. Thus while references such as a thin snout may sound similar to an Arabian horse, the Mamluks also sought horses such as the Cyrenican horse, which possessed sturdier legs and was stouter - thus better able to handle rough terrain as well as the weight of the Mamluk in full armour and accoutrements. In short, the contents of these works vary.

In China references to horse physiognomy actually predate any real indications of hippiatrics. Bo Le, for example, was known for his physiognomy before there was any attempt to associate him with medical treatment of horses. ${ }^{28}$ Also predating the now surviving medical literature are the many paintings of famous horses, those attributed to Han Gan 韓幹 (706-83), in particular, although most of the existing paintings attributed to Han are late copies. Under the Mongols, and much of our existing materials seems to date back to the Yuan, China's foreign rulers took a particular interest in the horse, and the Mongolian language, like Arabic is meticulous in its horse descriptions to the point that Mongolian sources of the time spend more time describing what a hero rode than the hero himself, or even his actions. How much Mongolian ideas of horse

28 On Bo Le, see Buell, May and Ramey 2010. See also the relevant sections in Sterckx 2000. 
physiognomy, as expressed in its later manuals, influenced Chinese remains uninvestigated.

Figures 11a and b, part of the same diagram from the YHLMJ (1, 6b-7a), details some principal points on a horse of physiognomic significance with short explanations (e.g., 'one wants the tail bone to be short', 'the ears like sliced bamboo', etc.). ${ }^{29}$ This set of illustrations is then followed by another set of proper characteristics, not supported by an illustration, and then the illustrated detailed guide to horse teeth. Elsewhere in the text there are other illustrations and discussions of more specialised topics related to physiognomy. A connected topic is the determination of the proper days and hours for such activities as bleeding, and cauterisation. This tradition is of great importance for the entire older Chinese veterinary tradition, although mostly forgotten today, particularly by Western practitioners who prefer to see Chinese veterinary medicine as more scientific than it actually is.

\section{Conclusion}

China's rich tradition of veterinary medicine, which becomes a flood during Ming times, is early associated with textual illustrations, including some that clearly once circulated separately. Illustrations, in fact, even when accompanied by explanatory texts, usually have a standalone quality, although some later texts clearly integrate illustrations more or less completely into general discussions of given conditions.

In their essence, traditional Chinese veterinary illustrations are more diagrams than scientifically based technical presentations (compared with medical illustration in late Renaissance texts). They seek to summarise lore and serve as aids to memory rather than seriously present scientific information, but then, half magic, half pragmatic experience, science was never the goal of Chinese horse medicine. Nevertheless, while the treatment options may have little relevance in terms of modern veterinary medicine, the accurate clinical observations indicate that the Chinese were keen observers of the horse.

What is most interesting about them is that the illustrations seem to have roots in early printing (although we have no truly early examples), similarly to Chinese religious broadsides for example; and before such texts were printed, in an illustrated manuscript tradition well

29 For a fuller physiognomy compare, for example, the relevant sections in Guo Huaixi's reworking of the YHLMJ (Guo Huaixi 1732). For a Middle Eastern example of such material, see the horse chart illustrated in Dum-Tragut 2005, p. 101. known from Silk Road finds. The important consideration is that such texts travel easily, witness elements of Chinese popular art that found their way to Iran before and after the Mongol period. ${ }^{30}$ Could this fact explain, given that there were Western equivalents, the apparent easy exchange of veterinary ideas in Eurasia? The traditions become almost identical at both ends of the Silk Road, although, as we demonstrate elsewhere, ${ }^{31}$ the most important ideas went from West to East, and not the reverse.

\section{Bibliography}

\section{Primary Sources}

Bo Le hualuo tugejue 伯樂畫烙圖歌訣 (The Song Secrets of the Diagram of Bo Le's Branding) (BLHLTGJ).

Bo Le zhenjing 伯 樂鍼經 (BLZJ) (Bole's Needling Classic).

Guo Huaixi 郭懷西 1732, Xinke zhushima niu tuojing daquan 新刻注釋 馬牛駝經大全 (Newly Printed and Annotated Horse, Ox, and Camel Classics), reprinted in Zhongguo nongye congkan xumu shouyizhi bu 中國農業叢刊畜牧獸醫之部, Beijing: Nongye chubanshe, 1988, 3-4. Qimin yaoshu 齊民要術 (QMYs), 6th century.

Simu anji ji 司牧安驥集 (SMAJJ) (Collections for Pacifying Stallions when Administering Flocks), 1384.

Xinbian jicheng mayi fang 新編集成馬醫方, (Newly Printed and Collected Recipes for Horse Medicine), 1399.

Yang Shiqiao 楊時喬 (d.16o9), Xinke ma shu 新刻馬書, (Newly Printed Horse Book), 1594.

Yuan Heng liaomaji 元亨療馬集 (YHLMJ) (Yuan and Heng's Collection for Treating Horses), $16 \circ 8$.

Yuan Heng quan tu niu ma tuo jing ji 元亨全圖療牛馬駝集 (The Yuan and Heng Completely Illustrated Collection for Treating Oxen, Horses and Camels), Sanyi tang zang 三義堂藏, Guangxu 光緒 period, jichou 己丑 year (1889).

\section{Secondary Sources}

Buell, P.D., T. May and D. Ramey 2010, 'Greek and Chinese horse medicine: déjà vu all over again', Sudhoffs Archiv 94.1, 31-56.

2008, 'How did Persian and other western medical knowledge move east, and Chinese west? A look at the role of Rashīd al-Dīn and others', Asian Medicine, Tradition and Modernity 3.2, 278-94.

Cooke, B. (ed.) 200o, Imperial China, The Art of the Horse in Chinese History, Exhibition catalogue, Lexington: Kentucky Horse Park.

Dum-Tragut, J. 2005, Kilikische Heilkunst für Pferde - Das Vermächtnis der Armenier, Hildesheim: Editorial oLMs Verlag.

Epler, D.C. 1980, 'Blood-letting in early Chinese medicine and its relation to the origin of acupuncture', Bulletin of the History of Medicine 54, 337-7.

Franke, H. 1997, 'Zur traditionellen Kamelheilkunde in China', Sudhoffs Archiv 81.1, 84-98.

Kang Myun Hee 姜冕熙 1988, 'Kankoku jūigaku genshi to Kanpo jūigaku no eikyō‘ 韓國原始獸醫學と漢方獸醫學の影響, Nihon Jüishigaku Zasshi 日本獸醫學雜誌 (23 March), 34-45.

This topic is vast, see as an introduction Komaroff and Carboni (eds) 2002.

$31 \quad$ See Buell, Ramey and May 2010. 
Komaroff, L. and S. Carboni (eds) 2002, The Legacy of Cinggis-qan, Courtly Art and Culture in Western Asia, 1256-1353, New York: The Metropolitan Museum of Ar/New Haven/London: Yale University Press.

Lo, V. and C. Cullen (eds) 2005, Medieval Chinese Medicine: The Dunhuang Medical Manuscripts, London/New York: RoutledgeCurzon (Needham Research Institute Series).

Ramey, D. and P. Buell 2001, 'Equine medicine in sixth century China: Qimin yaoshu', in Rossdale and Green (eds), 154-61.

Raswan, C.R. 1945, 'Vocabulary of Bedouin words concerning horses', Journal of Near Eastern Studies 4.2, 97-129.

Rossdale, P. and R. Green 2001 (eds), Guardians of the Horse, II, Newmarket: Romney Publications.

Sterckx, R. 2000, The Animal and the Daemon in Early China, Albany:
SUNY Press.

Stork, M. 1971, 'Lehrschemata in europäischen Tierheilkundebüchern des 16. bis 18. Jahrhunderts: Lassrösslein - Krankheitsnamenpferd - Fehlerpferd', Zentralblatt für Veterinärmedizin, Reihe A, vol. 18:8, 661-707.

von den Driesch, A. and J. Peters 2003, Geschichte der Tiermedizin, 50ooJahre Tierheilkunde, 2nd edn, Stuttgart/New York: Schattauer.

Wang Shishun 王世舜 et al. (eds) 1983, Zhuangziyizhu 莊子譯注 (Jinan: Shandong jiaoyu chubanshe.

Xie Guihua (tr. V. Lo) 2005, 'Han bamboo and wooden medical records discovered in military sites from the northwestern frontier regions', in Lo and Cullen (eds), 78-106.

Zhou Jiezheng 鄒介正 1960, 'Tangdai di zhenlao shu' 唐代的針烙術, Nongshiyanjiujikan 3, 159-74. 


\title{
23 Korean Anatomical Charts in the Context of the East Asian Medical Tradition
}

\author{
Shin Dongwon 신동 원 \\ Translated by Kim Yuseok 김유석
}

To the best of current knowledge, traditional Korean medical literature contains only three sets of images showing the internal organs of the human body. All three are highly significant in various ways for our understanding of East Asian traditions of anatomical representation.

The earliest set, in Ui'bang'ryuchui 醫方類聚 (Classified Collection of Medical Remedies) 1477 CE, reflects a Daoist vision of the internal landscape of the human body. It comprises six separate images illustrating the five zang organs (heart, spleen, liver, lungs and kidney) and the gallbladder, one of the six fu organs (the others are the stomach, large intestine, small intestine, urinary bladder and 'Triple Burner'). They are in fact integral reproductions of the images from Hu Yin's 胡愔 Huangting neijing wuzang-liufu tu 黃 庭內景五臟六腑圖 (Yellow Court Atlas of the Inner View of the Five Zang Organs and the Six Fu Organs), a Chinese Daoist work dating from the Tang dynasty c. 848 CE (Fig.



Figure 23.1 P'yejangdo 肺藏圖, Image of the lung. Kim Yemong et al. 1477, Ui'bang'ryuchui 醫方類聚, vol. 5, p. 51, (original edition in Kyoto Palace, Japan; micro-film copy in National Library, Korea)
1). These images are no longer extant in China, although the text that originally accompanied them is included in the Ming Daoist Canon of 1445 (Zhengtong Daozang 正統 道藏). By transmitting the images together with the text, the Classified Collection of Medical Remedies sheds a unique light on 9th-century East Asian anatomy.

The second set, in Chi'mkuyokyel 鍼炎要訣 (Essential Formulas for Acupuncture and Moxibustion) 16oo CE, by the Confucian scholar-physician Yu Seongnyong 柳成龍, consists of one illustration of the zang and fu organs, and two channel charts (Fig. 2). They are reproductions of the identically named illustrations in Yixue rumen 醫學入門 (An Introduction to Medicine) 1575 CE, by the Ming dynasty physician Li Chan 李梴.

The third set, in Heo Jun's Dong'ui'bo'gam (Treasured Collections of an Eastern Physician, hereafter Treasured Collections) 1613 CE, comprises Sinhyeng jangbudo 身形

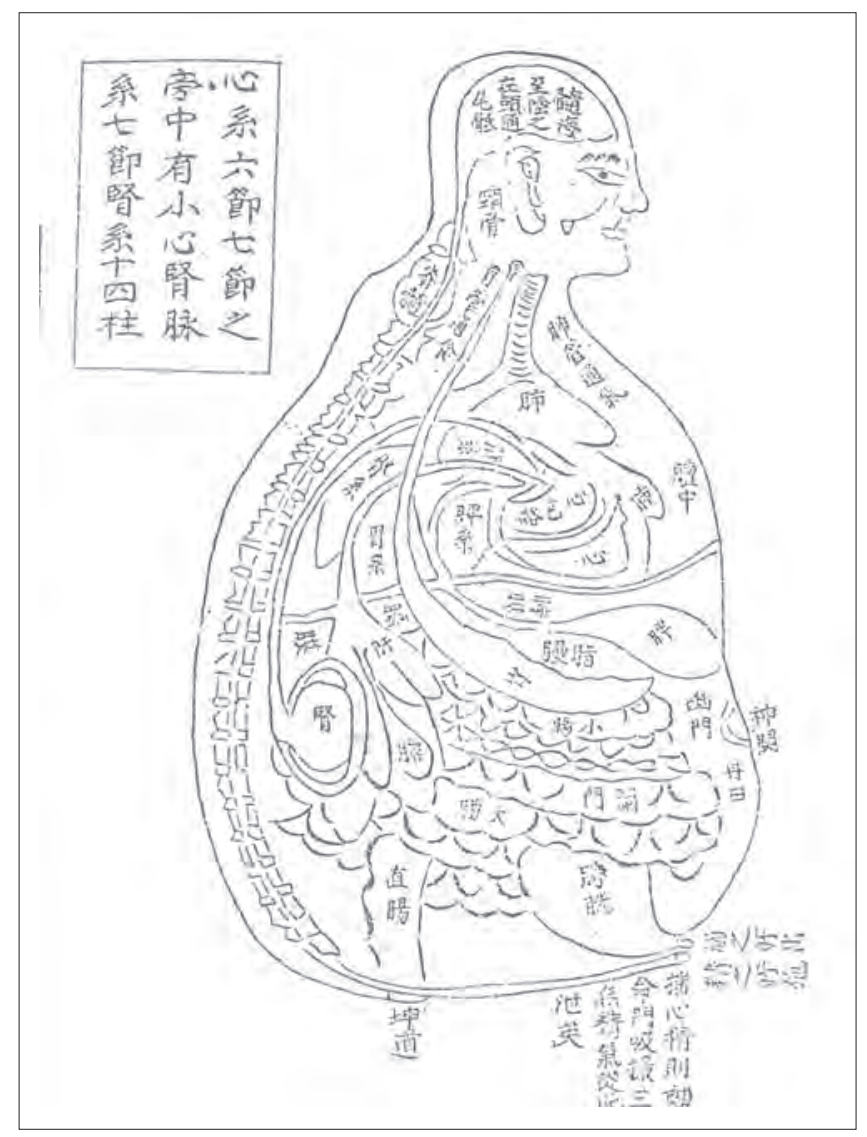

Figure 23.2 Changbu naegwando 藏腑內觀圖, Image of the organs in the body. Yu Seongnyong 1600 p. 12, ed. 1994, p. 340 




Figure 23.3 Sinhyŏng changbudo 身形藏腑圖, Image of the form of the body and the zang and fu organs. From Heo Jun 1630, ed. 1994, p. 227

臟腑圖 'Image of the form of the body and the zang and $f u$ organs', as well as illustrations of five of the internal organs' (Fig. 3). At first sight, 'Image of the form of the body and the zang and fu organs' appears almost identical to an image of the body in Wanbing huichun 萬病回 春 (Recovery from Ten Thousand Diseases) 1585 CE, by the Ming-dynasty Chinese physician Gong Tingxian 驡 廷賢, while the illustrations of the organs seem to differ but little from Chinese illustrations of the Song and Ming Dynasties (Fig. 4). On closer scrutiny however, significant differences become apparent.

The illustrations in Treasured Collections are the sole pictorial representations we possess that reflect the approach and sensibility specific to Korean physicians of the Joseon dynasty (1392-1910 CE). Studying the text over the years, I have been constantly intrigued by these images. ${ }^{1}$ Similar to anatomical charts in early Ming-dynasty Chinese medical texts, yet in some ways strikingly different from them, what do they actually represent? And what function do they perform within the medical text Treasured Collections? Where do they come from? What place do they occupy in the East Asian tradition of anatomical charts? The present

\footnotetext{
$1 \quad$ Shin 2001, pp. 172-9o.
}



Figure 23.4 Chuúksinindo 側身人圖, Lateral view of the body. Gong Tingxian (Ming), ed. 1999, p. 43

paper is an attempt to answer these questions. In my view, in the process it also provides clues to understanding the unique character of traditional Korean medicine in the $17^{\text {th }}$ century and thereafter.

\section{A Short Biography of Heo Jun}

Heo Jun remains the most famous physician in Korean history. A royal doctor by his early 3 os, he served the Royal Hospital for more than 40 years and wrote at least seven medical treatises, including the internationally-known Treasured Collections. After its first publication in 1613, the book saw more than 10 reprints in Korea, more than 30 in China, and at least two in Japan.

Heo Jun's mother was a concubine, and his father was legally barred from taking office in the civil service or military. Thus the only professions open to a boy of Heo Jun's birth were astronomy, the law, the interpretation of Chinese and Japanese texts, and medicine.

Nothing is known about Heo Jun's medical training. In 1581, while working at the Royal Hospital, he was ordered by King Seonjo 宣祖 (r. 1567-16o8) to revise an important 
book on diagnosis, which medical students had been using as a basic text for at least a century.

Heo Jun became famous in 159 o for curing thousands of smallpox patients, among them the Crown Prince, Goang'haegun 光海君. At that time he was the only royal physician to ignore the religious prohibition against treating people for the disease. He refused to accept the contemporary belief that the god who brought smallpox would kill any patient who sought medical treatment. This success encouraged him to write a medical text aimed at putting an end to the practice of not treating smallpox patients, Oenhaedu'chang'jip'yo 諺解痘瘡集要 (Essentials of Smallpox with Korean Translation).

Treasured Collections, completed in 1610, brought Heo Jun long-lasting fame. After the first Japanese invasion of Korea in 1592, King Seonjo ordered him to compile a medical book to expose the effect of war upon medicine. Work began with a team of six doctors, five of them Royal doctors, but after the second Japanese invasion in 1597 , his colleagues scattered and he had to finish the treatise alone. It took Heo Jun 10 years to complete this 25 -volume work. Meanwhile, as the first Royal doctor, he was impeached for King Seonjo's death and exiled between $16 \circ 8$ and $16 \circ 9$ to Uiju, at the northern frontier of Korea. Although the exile was painful, it gave Heo Jun the time that he needed to complete most of the book, which was finally published in 1613 .

Treasured Collections consists of five sections: inner body physiology and symptoms, outer body symptoms, common diseases, pharmacology, and acupuncture and moxibustion. This book had three distinctive features. First, it attached more importance to cultivation of the mind and body than to external medical treatment. The popularity of Neo-Confucian and Daoist self-cultivation techniques among the ruling yangban 兩班 class in Korea was influential in this trend. Secondly, it made an attempt to resolve apparent contradictions in traditional East Asian medical discourse such as the tension between knowledge and practice highlighted by the four great doctors of the Jin and Yuan. Between the Han and Ming dynasties Chinese medical texts and remedy collections were widely disseminated throughout the territory equivalent to modern day Korea. Thirdly, it emphasised the benefits of using locally produced drugs, according to Korean tradition.

During 16o1, while working on Treasured Collections, Heo Jun also wrote manuals on obstetrics, emergency treatments, and smallpox. All of these books, originally written in Chinese script, as was conventional in Korean literature, were also translated into vernacular Korean to allow easy access for a popular audience. Heo Jun spent his last days studying the prevention of typhus and scar- let fever. As the leading doctor in Korea he championed preventive health, writing two short books on infectious diseases. His book on scarlet fever, Byuk'yeok'sinbang 辟 疫神方 (Divine Remedies for Treating Contagious Illness) attracted serious attention from medical historians for his unique and original observations unprecedented either in China or Korea. The Japanese medical historian Sakae Miki insists that Heo Jun was the first person to study the disease in East Asian countries. His descriptions of the symptomatology including fever, headache, sore throat, dropsy, rash, and desquamation seem so accurate that they have been compared to Daniel Sennert's 1627 European treatise on fever.

Heo Jun died in 1615. In an honour unprecedented in Korean history the king conferred upon him posthumously the highest court rank.

\section{The Nature of 'Form' in 'Image of the Form of the Body and the Zang and Fu Organs'}

To return to Treasured Collections, under the rubric 'Image of the form of the body and the zang and fu organs', we find the anatomical chart in question and two passages of textual commentary relating to it. To understand the nature of the image, we need to begin with the material data afforded by the text: the title; the chart itself; the commentary; and the physical position of the chart within the text.

To begin with, the title itself brings together two conceptually distinct categories: the outward form of the body (形, Chinese xing) and its inward substance (臟腑, Chinese zangfu). It announces the author's intention to represent the body both externally and internally; to convey both observational knowledge of the body and the experience and imagination of its inner workings.

The image itself consists of an outline of the body, shown laterally from the right, with the internal organs as well as various external features drawn and labelled. The external contour of the body is confined to the head and the trunk, with the limbs entirely omitted. The eyebrows, eyes, ears, nose, mouth and chin are drawn on the head; the neck, chest, waist and abdomen are indicated on the trunk; and the navel is drawn protruding. The names of external bodily landmarks all appear outside the outline.

As regards the internal organs: the brain and the spine are shown running continuously down the full length of the body; the pharynx and the larynx begin at the same height as the mouth; the larynx connects with the lungs, directly below which sits the heart. The diaphragm - the frontier between the organs of the upper and lower body - is clearly marked and labelled. The downward passage 
from the pharynx divides into three. One branch leads to the diaphragm, the second branch leads to the kidneys, and the middle branch continues on, to bifurcate further down with one path leading to the spleen and stomach, and the other to the liver and the gallbladder. Underneath the stomach is the small intestine, and underneath that is the large intestine, shown with many folds. Depicted below the small and large intestines is the urinary bladder. Below the gallbladder and to the left of the intestines, we see the rectum and urethra, the paths through which food waste and urine make their way to the exterior. Although there is no textual explanation of the relations between the internal organs, the image compellingly suggests how they are organically connected with one another.

The organs drawn inside the body all have distinctive and evocative visual forms. The brain resembles the bough of a tree, the spine is a long chain of interlinked bones, the pharynx and the larynx look like holes, the lungs are depicted like many layers of leaves, the spleen and the stomach resemble drooping sacks, the liver looks like a long flower bud, the kidneys resemble beans, and the urinary bladder is like an inverted heart.

\section{Heo Jun's Explanation of 'Image of the Form of the Body and the Zang and Fu Organs'}

The text accompanying this image is particularly detailed and provides a clear guide to the way in which the author, Heo Jun, intended the image to be read. It consists of two parts, which begin, 'Sun Zhenren 孫眞人) ${ }^{2}$ says...' and 'Zhu Danxi 朱丹溪 ${ }^{3}$ says...' respectively. The first part is as follows:

Sun Zhenren states: the human head is round in emulation of the roundness of heaven, and the human foot is square in emulation of the square shape of the earth. Just as heaven has the four seasons, man possesses the four limbs. Just as heaven has the five agents [also translated as elements or phases], man possesses the five organs; just as heaven has the six directions, man possesses the six viscera. Because there are winds from the eight directions in heaven, man possesses the eight joints; and just as there are the nine stars in heaven, humans have the nine orifices. The 12 human channels emulate the 12 hours of heaven; and the 24 human points emulate the 24 solar terms of heaven. In addition, because there are 365 degrees in heaven, man likewise possesses 365 joints. Just as there are the sun and the moon in heaven, man possesses the eyes and the ears. Just as there are day and night in heaven, man sleeps and wakes. Just as there are thunder and lightning

Sun Zhenren, or Sun Simiao, 孫思絈 (581-682 CE). Zhu Danxi, or Zhu Zhenheng 朱震亨 (1281-1358 CE). in heaven, man feels joy and anger. Just as there are rain and dew in heaven, man possesses tears and nasal mucus. Just as there are Yin and Yang in heaven, man possesses cold and body heat. Just as there are fountains on earth, man possesses blood vessels; and just as trees and grass grow on earth, man possesses hair both on the body and on the head. Just as there are metals and rocks in earth, man possesses teeth. Everything takes form through the four great agents and the five agents. ${ }^{4}$

This passage gives us a sense of the range of constituents (body parts, physiological phenomena, affects) that are regarded as making up human bodily form. With detailed examples, it presents the human body as a homologue of heaven and earth, or the cosmos and natural phenomena, structured according to universal numerological schemes.

It asserts the belief that the various parts and aspects of the body are formed not coincidentally and independently of one another but in accordance with cosmic patterns. In essence, all parts of the body are formed according to the order of the four great agents of earth, water, fire and wind, a Buddhist concept, and the five agents, an idea from Chinese natural philosophy. The second part states:

Zhu Danxi says: when human form is examined, the tallness and shortness of height are unequal, the largeness and smallness of build are unequal, and the fatness and thinness of flesh are unequal. The fairness and darkness of skin tone are unequal, lightness and bluishness are unequal, and thinness and thickness are unequal. Those who are fat abound in the wet vital breath, those who are thin abound in the fiery vital breath, those who are fair are deficient in the vital breath in the lungs, and those who are dark are sufficient in vital breath in the kidneys. Because shapes, colors, the organs, and the viscera thus differ, treatment methods vastly differ even when the outward symptoms are similar. ${ }^{5}$

Whereas the first passage, from Sun Simiao, describes characteristics universal to human bodies, the quotation from Zhu Danxi discusses individual differences in height, build and skin tone. These differences in bodily form are the outward manifestation of disparities in vital breath in the internal organs. In light of this, Zhu Danxi establishes the principle that even where the presenting symptoms are identical, medical treatment must be differentiated and personalised on the basis of the disparities among individual bodies. By citing this passage, Heo Jun sought to demonstrate that a discussion of bodily form is germane to the correct treatment of diseases.

\footnotetext{
$4 \quad$ Heo 1994, p. 278.

5 Ibid., p. 278.
} 


\section{The View of the Human Body of 'Treasured Collections of an Eastern Physician'}

\section{What 'Image of the Form of the Body and the Zang and Fu Organs' Represents}

'Image of the form of the body and the zang and fu organs' is placed close to the beginning of Treasured Collections. Because this position in the material text is important in relation to our overall understanding of the image, it needs to be examined more closely:

Preface;

General catalogue (2 fascicles);

Chapter on internal view (4 fascicles): 'collection of cases'; 'successive medical formula examinations'; 'image of the form of the body and the zang and fu organs'; 26 categories including 'bodily form';

Chapter on outward form (4 fascicles): 26 categories including 'head';

Chapter on miscellaneous diseases (11 fascicles): 38 categories including 'celestially and terrestrially determined fate';

Chapter on brews and decoctions ( 3 fascicles): 17 categories including 'prefatory material to brews and decoctions';

Chapter on acupuncture and moxibustion (1 fascicle): 'acupuncture and moxibustion'.

Although 'Image of the form of the body and the zang and $f u$ organs' is included in 'Chapter on the internal view', on examination it differs from the other individual categories in this chapter, with their discrete contents. Apparently, it belongs to the level of general summaries like 'Collection of cases' and 'Examination of successive medical formulas'. 'Collection of cases' is the section in which HeoJun discusses the structure, organisation, noteworthy features, and title of the book, while in 'examination of successive medical formulas' he enumerates works deemed to have made an important contribution to the medical tradition in both China and Korea during successive eras. What then is the role of the illustration? In conjunction with 'Examination of successive medical formulas', which provides a diachronic dimension, this section gives a general account of the body, the object of medicine.

The diverse elements that we have examined so far reveal the view of the body held by the author of Treasured Collections. The contents of 'Image of the form of the body and the zang and fu organs' correspond, point to point, to the principles set out in 'collection of cases':

As I respectfully observe, the body has the five organs and the six viscera inside and muscles and bones, blood vessels, and skin outside, thus completing its form.

In addition, essential matter (jing 精), vital breath (qi 氣),


body parts. Consequently, the three joys of Daoism and the four great agents of Shakyamuni Buddha all indicate this. The Classic of the Yellow Court includes the section 'internal view', and the medical text also includes mirror images. The basis of Daoism lies in clear and peaceful selfcultivation, and medicine cures with medication, food, acupuncture, and moxibustion, which is how Daoism has acquired its finesse and medicine, has acquired its roughness. Now, this book first presents 'Inner Chapter', including in it essential matter, vital breath, spirit, organs, and viscera in the internal view, then presents 'Outer Chapter', including in it the head, face, hands, feet, muscles, pulse, bones, and flesh of the external realm... . ${ }^{66}$

As can be seen in the above passage, Treasured Collection of an Eastern Physician distinguishes three aspects of the body - the organs inside the body; the outward form of the body; and essential matter, vital breath and spirit, which animate the body. 'Image of the form of the body and the $z$ ang and $f u$ organs' is an attempt to incorporate all three of these aspects into an anatomical chart.

\section{The Addition of the Concept of 'Bodily Form' to Anatomical Charts}

The concept of 'bodily form' that emerges elsewhere in Treasured Collections is more inclusive than this image shows us. The 'Chapter on outward form' includes the locations and lengths of the head, face, eyes, mouth and lips, teeth, neck, back, chest, breasts, abdomen, waist, sides, armpits, and legs (which are not separately indicated on the drawing). This chapter also discusses the characteristics of superficial and deeper structures of the body with reference to the skin, flesh, pulse, muscles, and bones. At the same organisational level, there is a separate account of hair.

The ordering of the categories in 'Chapter on outward form', or 'bodily form' broadly follows three criteria. The first criterion hinges on the concept that 'heaven is round and earth is square'. By starting out with the statement that: 'the human head is round in emulation of the roundness of heaven, and the human foot is square in emulation of the square shape of the earth', the author sets up a primary distinction between the head and the trunk. Based on this distinction, 'Chapter on outward form' begins with the head and the face and then proceeds to the trunk, concluding with the waist and sides. The second criterion is one of depth. Starting with the skin, it moves deeper, progressing to the flesh, pulse, muscles, and bones. Thus, a three-dimensional understanding of the outward form becomes possible. The third criterion is the concept of centre and periphery. The limbs, genitalia, anus, and head, all of which are attached to the trunk, together with the hair on the

$6 \quad$ Heo 1994, pp. 265-6. 
head, the moustache and beard, and the bodily hair, all of which protrude from the skin, are classified as peripheral.

Never before Heo Jun, in the history of East Asian medicine, has the surface of the body been so systematically organised. This is because other medical texts organised their material with regard to the symptoms or nature of diseases. For instance, it is instructive to compare Treasured Collections with Li Chan's Yixue rumen 醫學入門 (An Introduction to Medicine), which exercised a considerable influence on Heo Jun's work. ${ }^{7}$ In that text, material corresponding to 'Chapter on outward form' in Treasured Collections is partially included in 'external medicine', and is classified into the categories of 'neck', 'hands', 'chest and abdomen', 'hips', 'legs', and 'girth of the body' only. Gong Tingxian's 龔廷賢 Wanbing huichun 萬病回春 (Recovery from Ten Thousand Diseases, $1585 \mathrm{CE}$ ), which is more detailed, conflates body parts with diseases under such categories as 'stomach-ache', 'lumbago', 'waist pain', 'side pain', 'hip pain', 'shoulder pain', 'headache', 'hair on the head', 'face diseases', 'ear diseases', 'nose diseases', 'mouth and tongue', 'lips', 'teeth', 'eyes', and 'pharynx and larynx'. ${ }^{8}$ Unlike other, earlier medical texts, Treasured Collections does not combine sections on the body with sections on diseases but, instead, brings the former together under the overarching category of 'bodily form'. In other words, the concept of disease is consistently subordinated to that of the body.

\section{An Emphasis on Daoist Physiology for the Preservation of Health}

'Image of the form of the body and the zang and fu organs' indicates specific bodily organs and parts by means of both images and captions. From top to bottom, these named sites are: Mud Pill Palace, suihainao, Jade Pillow Gate, larynx, pharynx, lungs, heart, diaphragm, Well Pulley gate, spleen, stomach, liver, gallbladder, kidneys, small intestine, large intestine, navel, urinary bladder, Tailbone Gate, large intestine and anus, and urethra. The fact that only these parts were selected from a plethora of possibilities demonstrates that Heo Jun, attached a particular significance to them.

However, 'Image of the form of the body and the zang and fu organs' includes no explanation of why it depicts the human body in the way it does or why it draws attention to certain parts. The relevant categories in 'Chapter on the internal view' and 'Chapter on outward form' provide detailed accounts of the locations or shapes of each of the organs or body parts, and it is illuminating to read 'Image of the form of the body and the zang and fu organs' against these accounts. In particular, the separate 'Image of the

Shin 2001, p. 213.

Gong 1999, pp. 236-7. five zang organs' explicates in greater detail the forms of the liver, heart, spleen, lungs, and kidneys, as well as the gallbladder and the stomach (two of the six fu organs).

Simple and economical in its visual language, 'Image of the form of the body and the zang and fu organs' neither attempts to represent the entire compass of Treasured Collections, nor reflects the detail of the text in a straightforward manner. Let us examine the location of the liver as an example. According to the main text of 'diagram of the liver', the liver 'is attached from below the diaphragm to the right ribs, and penetrates the diaphragm upward, to enter the lungs and connect with the diaphragm'. However, in 'Image of the form of the body and the zang and fu organs', the liver, though shown below the diaphragm, is not depicted as penetrating the latter or as being connected to the lungs. There is no suggestion of a connection with the right ribs, which are not represented at all. Likewise, there are notable omissions in the depiction of the heart. The main text of 'A diagram of the heart' states explicitly that

the systems of the five organs are linked to the heart, and the heart is linked to the systems of the five organs so that the systems of the heart and the five organs are linked to one another.

It goes on to explain in detail:

The system of the heart is linked upward to the lungs. The other system that branches out begins in the middle of the two leaves on either side of the lungs, penetrates the spine toward the back, and reaches the kidneys. It then proceeds from the kidneys to the urinary bladder and, together with the content of the urinary bladder, reaches to where urine is voided outside. This is the lowest place.

However, 'Image of the form of the body and the zang and fu organs', does not depict the heart as being connected to the systems of the five organs. Nor, for that matter, does it depict the systems leading to the lungs and the kidneys. Moreover, this incongruity between explanations and diagrams applies to all the remaining organs.

In fact, this textual information on the positions of the organs inside the body is derived from Li Chan's 李梴 $A n$ Introduction to Medicine, a Ming medical text published in 1575, 28 years earlier than Treasured Collections. An Introduction to Medicine also includes an anatomical chart with the title 'Image of the zang and fu organs', in which the various internal organs are represented in accord with the text. But 'Image of the zang and fu organs' was not an original creation of Li Chan. In fact, it goes back to Jinlan xunjing 金蘭循經 (Book Beside the Golden Orchid) by Hut Bilic 忽泰必烈, a master of acupuncture and moxibustion of the Yuan (Mongol) dynasty. Because Hut Bilic's original chart was extremely large, it was subsequently simplified and reduced. Reduced versions are found in Wu Kun's 吳 
崑 Zhenjiu liu ji 鍼炎六集 (Six Volumes of Acupuncture and Moxibustion) and Lou Ying's 樓英 Yixue gangmu 醫學綱目 (Outline of Medicine), both from the early Ming dynasty, and it is to this tradition that Li Chan's $A n$ Introduction to Medicine belongs. Currently, the oldest known extant exemplar is the one in Lou Ying's (undated) Outline of Medicine. ${ }^{9}$ While Treasured Collections cites An Outline of Medicine as a source, it is uncertain whether the version of the image that Heo Jun consulted was one of those accompanied by 'Image of the Illuminated Hall and the zang and fu organs' as an addendum.

Hut Bilic's anatomical chart aimed at demonstrating that man's five organs and six viscera, entire body, nine orifices, and meridians and collaterals, penetrate the body and are connected to every nook and cranny without discontinuity.

The 'Illuminated Hall' images in Lou Ying's work consist of three drawings: 'Illuminated Hall image', showing the internal organs, and two channel charts, showing front and back views of the body. ${ }^{10}$ Through these three diagrams, Hut Bilic sought to represent visually the connections between the inside and outside of the human body, the internal organs, and various body parts. Where the internal organs are concerned, noteworthy here is his endeavour to establish an anatomical basis for the connections among the five zang organs and six fu organs. Because traditional Chinese medicine is characterised by a physiology of the zang and fu organs and the six viscera according to the mutual generation and restriction of the five agents (also translated the five phases or elements: wood, fire, earth, metal and water), it was necessary to demonstrate the anatomical interconnectedness of the internal organs. The 'Illuminated Hall image' and 'Image of the zang and $f u$ organs' elaborate and represent visually four organic systems with the heart at the centre, and thereby lend support to the idea that 'the heart is the sovereign organ'.

'Image of the form of the body and the zang and fu organs' both adds to and subtracts from the content of the earlier 'Illuminated Hall image' and 'Image of the zang and $f u$ organs', revealing very different priorities. It does not portray the systematic connections of the five zang and six $f u$-viscera. Instead, it names and thus emphasises a series of places between the brain and the base of the spine, i.e. Suihainao, Mud Pill Palace, Jade Pillow Gate, Well Pulley gate, and Tailbone Gate. Drawn directly from the vocabulary of Daoism, these terms reflect a Daoist understanding of the human body. Suihainao, which the Hermit Sütra (仙經) identifies as the 'upper elixir field', is

$9 \quad$ Huang 2003, pp. 67-8.

10 Ibid., p. 70 here described as the 'storehouse of vital breath'. Mud Pill Palace (also known as the Yellow Court, Kunlun Mountains, and Heavenly Valley) is, according to Daoism, one of the nine palaces of the brain, where the Original Spirit resides. In addition, it is through this orifice that the soul and vital force enter and exit. According to the Hermit Sūtra there are three gates at the back of the body: the Jade Pillow Gate at the rear of the brain; the Well Pulley Gate on the back; and the Tailbone Gate at the base of the spine. In Heo Jun's 'Image of the form of the body and the zang and fu organs', these three gates are identified as pathways for essential matter and vital breath: 'If the three gates circulate well like the pivotal part of the Big Dipper, essential matter will circulate up and down just as the Milky Way flows and circulates'.11 By focusing on these parts in this way, Heo Jun presumably wishes to emphasise the activities of essential matter, vital breath, and spirit, which are the three constituent elements of the human body.

In 'Collection of cases' in Treasured Collections, Heo Jun sets out the principle that:

The basis of Daoism lies in clear and peaceful self-cultivation, and medicine cures with medication, food, acupuncture, and moxibustion, which is how Daoism has acquired its finesse and medicine has acquired its roughness.

The same principle is reflected, as a matter of course, in the anatomical chart called 'Image of the form of the body and the zang and fu organs'. By making the Daoist context explicit and suppressing the medical context, which centres on the intrinsic and extrinsic relationships among the $z$ ang and $f u$ organs, Heo Jun reveals the nature of his work, which foregrounds the Daoist principle of the preservation of health.

\section{The Images of the Five Organs in 'Treasured Collections of an Eastern Physician'}

'Chapter on the internal view' in Treasured Collections includes five images of the five zang organs: 'Image of the heart', 'Image of the spleen', 'Image of the lungs', 'Image of the liver' and 'Image of the kidneys' (Fig. 5). Judging from the fact that only these five drawings were created or included, we can conclude that Heo Jun regarded these five organs as the most important of all the bodily organs. The liver, heart, spleen, lungs, and kidneys each occupy one of the 108 categories in Treasured Collections, and the images are placed at the beginning of their respective categories. Because each image is provided with an explanatory text, what Heo Jun sought to communicate through the images

$11 \quad$ Heo 1994, pp. 285-6. 




Figure 23.5 Five images of the five zang organs五臟圖. Source: Heo Jun's Dong'ui'bo'gam (Treasured Collections of an Eastern Physician). Shin Dongwon 2001, pp. 184-5

is unmistakable. For example, his explanation of the image of the liver goes as follows:

The liver is shaped like two large leaves and one small leaf, like gaping bark. At the centre of each, channels and collaterals emit harmonious Yang vital breath (Neijing zhu 內經注 (Annotations on the [Yellow Emperor's] Inner Canon)). The liver is shaped like two large leaves and one small leaf. It is divided into three leaves on the left and four leaves on the right, and is like bark split and gaping in many pieces (An Introduction to Medicine). The liver weighs 4 catties and 4 taels. There are seven leaves altogether, three on the left and four on the right and it holds the soul (Classic of Difficult Issues). ${ }^{12}$

It can be seen from this that the subjects that Heo Jun found it necessary to discuss in conjunction with 'Image of the liver' were the form, weight, and function of this organ. Following 'Image of the liver', the category 'Liver' treats the location of the liver, pulse diagnosis of liver diseases, liver diseases themselves, and the treatment of liver diseases. Thus 'Image of the liver' serves to provide the anatomical grounds for the various considerations that follow. In other words, the liver is not an abstract entity but a physical thing with form, weight, and specific functions. The same concreteness and physicality hold true of other organs.

These diagrams of the five organs differ considerably from earlier drawings of the $z$ ang and $f u$ organs. In Chinese medical history, there are two traditions of separate representations of the internal organs. ${ }^{13}$ One of these is inspired by Daoist ideas and techniques of internal visualization. This tradition is exemplified by the drawings of the five zang and six fu organs by Hu Yin of the Tang dynasty, the oldest form of which has been transmitted through $A$ Collection of Medical Formulas, a Korean medical text. As outlined earlier, this work contains six illustrations showing the liver, heart, spleen, lungs, kidneys (the five zang organs), and the gallbladder (one of the fu organs). Each

\footnotetext{
$12 \quad$ Heo 1994, p. 541.

13 Huang 2003, p. 30.
}

illustration presents the shape of the organ in question as well as the hexagrams corresponding to it in the Yijing 易 經 (The Book of Changes) and the gods and animals associated with it in Daoism. These six images seek to express the cosmic symbolism of the organs as well as the marvel of each organ's function.

The other tradition is represented by Zhenjiu juying 鍼炎聚英 (A Glorious Anthology of Acupuncture and Moxibustion), an early Ming medical text. The illustrations in this work depict 10 of the 11 zang and fu organs (excluding the Triple Burner due to its ambiguous location). The hexagrams from the Classic of Changes and the corresponding animals are omitted, with only the organs represented pictorially. This subsequently became the most widespread model in East Asian medical texts. Treasured Collections depicts the five organs only, without the deities and symbolic creatures found in A Collection of Medical Formulas. Judging from this, it is possible to surmise that the author of Treasured Collections confined himself to the Daoist preservation of health and did not accept Daoist religious symbolism.

Treasured Collections differs from both the traditions described above. Its images of the five organs evidently follow neither 'Image of the zang and $f u$ organs' nor $A$ Glorious Anthology of Acupuncture and Moxibustion.

This becomes more obvious when these anatomical charts are examined in detail. In the case of 'Image of the liver', Treasured Collections shows two large leaves on either side and one large leaf in the centre. In total, there are three leaves on the left side (hidden in the centre is the gallbladder) and four on the right side. As for A Collection of Medical Formulas, the liver resembles boughs in clusters, whereas in A Glorious Anthology of Acupuncture and Moxibustion, there are patterns on an image that likewise resembles boughs in clusters.

In the case of 'Image of the heart', in Treasured Collections the heart is shaped like a flower bud, with seven holes and three strands of hair at the top, surrounded by the pericardium. In contrast, A Collection of Medical Formulas presents a simple image of an upside-down lotus blossom. In A Glorious Anthology of Acupuncture and Moxibustion, a tube at the top connects the heart with the lungs, and three tubes connect the heart with the liver, spleen, and kidneys.

In the case of 'Image of the spleen', in Treasured Collections the spleen is shaped like a horseshoe, whereas A Collection of Medical Formulas depicts this organ like an upside-down flower pot, and A Glorious Anthology of Acupuncture and Moxibustion presents a knife-like image.

In the case of 'Image of the lungs', Treasured Collections depicts the lungs like two broad-shouldered ears of grain, with six leaves underneath and 24 points in eight rows 
and three columns at the bottom. In contrast, the lungs in A Collection of Medical Formulas have three leaves on the left side and four leaves on the right side, with all the leaves displaying a pattern. The lungs in A Glorious Anthology of Acupuncture and Moxibustion have six leaves, with each leaf showing a pattern (the muscles).

In the case of 'Image of the kidneys', Treasured Collections depicts the kidneys like two red beans touching each other, their surface lined with scale-like markings. In contrast, the kidneys in A Collection of Medical Formulas resemble two beans touching each other while those in A Glorious Anthology of Acupuncture and Moxibustion look like two beans separated by the spine in the centre, and neither of the latter two drawings shows scale-like lines.

Of these illustrations, those closest to actual dissections are the ones in A Glorious Anthology of Acupuncture and Moxibustion. The images in A Collection of Medical Formulas and Treasured Collections are the furthest removed from anatomy. In fact, the images of the five organs in Treasured Collections are not anatomical illustrations in a literal sense at all. Rather, they are pictorial representations of the contents of older texts such as the Neijing 內經 (Inner Canon), Wang Bing's 王冰 Neijing zhu 内經注 (Annotations on the Inner Canon), and Nanjing 難經 (Classic of Difficult Issues), attributed to Bian Que, as well as more recent ones like the Yixue zhengchuan 醫學正傳 (True Lineage of Medicine) and Yixue rumen 醫學入門 (An Introduction to Medicine). ${ }^{14}$ 'Image of the liver' pictorially represents the contents of An Introduction to Medicine, which in turn combines the teaching of Annotations on the Inner Canon that 'it [the liver] is shaped like two large leaves and one small leaf, like gaping bark', with that of the Classic of Difficult Issues that 'there are seven leaves altogether, three on the left and four on the right'. 'Image of the heart' represents the teachings of Annotations on the Inner Canon that 'it [the heart] is shaped like an as yet unopened lotus blossom and has nine holes', of An Introduction to Medicine that 'there are seven holes and three strands of hair in the centre', and of the True Lineage of Medicine that 'the membrane surrounding the heart is the pericardium'. The holes and the hairs of the heart are renderings of the following passage in An Introduction to Medicine, citing from the old Daoist compilation, Yunji qiquan 雲笈七籤 (Seven Sticks from the Cloudy Basket).

The heart resembles a lotus flower that is yet to bloom; it has seven holes in the middle and three strands of hair. The wisest ones have nine holes and three strands of hair; wise ones have seven holes and two strands of hair; less wise ones have five holes and one strand of hair; ordinary people have two holes

$14 \quad$ Yi 1957, p. 263. and no hair; and unwise ones have nothing but one hole. These holes and strands of hair are the channels of communication with Qi from Mother Nature 본성 (本性). ${ }^{15}$

'Image of the lungs' illustrates the statements in Annotations on the Inner Canon that 'they [the lungs] are like broad shoulders, and there are 24 points in the middle', and in the Classic of Difficult Issues that 'there are a total of eight leaves, which consist of six leaves and two ears'. 'Image of the spleen' illustrates the opinion of Annotations on the Inner Canon that 'it [the spleen] resembles a horseshoe and embraces within it the gastric cavity'. 'Image of the kidneys' illustrates the view of the Inner Canon that 'there are two kidneys, and their shape is like that of two red beans facing each other, and they are attached diagonally to the muscles of the back. They are coated with oil on the surface, and they are white inside and black outside, and their role is to hold essential matter'.

The Ming Glorious Anthology of Acupuncture and Moxibustion and later diagrams of the five organs tend to represent the organs in a way that echoes the actual experience of dissection; in contrast, the anatomical images in Treasured Collections hark back to more ancient traditions. The same approach runs through those sections of Treasured Collections that explicate body parts other than the six viscera. In the section on the stomach, the Numinous Pivot, Plain Questions, and An Introduction to Medicine are cited as authorities for the length, shape, and diameter of this organ. In the case of the small intestine, the Numinous Pivot is cited with reference to its length, circumference, diameter, weight, and number of folds and capacity. In the case of the large intestine, the Classic of Difficult Issues is cited on its length, circumference, weight, number of folds, and capacity. In the case of the urinary bladder, the Classic of Difficult Issues is cited on the circumference of the upper orifice of the bladder, the circumference of the centre of the organ, and the capacity and weight of the entire bladder. This emphasis on older texts coincides with the author's declared motive for composing Treasured Collections: 'because recent years have seen far too many heterodox opinions and divergent formulas, which has led to the loss of the spirit of the Numinous Pivot of old, it [the composition of the text] is to correct this'. The argument that the orifices and tiny hairs of the heart are indicators of a person's degree of wisdom likewise reveals that Treasured Collections sought to pay homage to ancient knowledge rather than moving toward anatomical empiricism, as does the explanation that, because the heart corresponds to the Three Terrace Stars 삼태성 (三台星), the utmost sincerity

15 Heo 1994, p. 549 
can move even heaven. This is why Heo Jun felt no need for dissection, and devised 'imaginary' anatomical charts that correspond to statements in older medical texts.

\section{Conclusion}

In sum, the anatomical images in Treasured Collections differ from earlier East Asian anatomical charts in three important ways. First, they embody the view that Daoist practices for preserving health and vitality (yangsheng) are closer to the essence of life than is medicine. Consequently, while based on anatomical charts from the medical tradition, they supplement their sources with key content for Daoist concepts of the preservation of life. In particular, the three gates of the spine, which are the channels for essential matter, vital breath, and spirit, and the Mud Pill Palace (brain) have been introduced into 'Image of the form of the body and the zang and fu organs'.

Second, unlike preceding medical texts, which mainly focused on the organs inside the body and the channels on the surface of the body, Treasured Collections seeks to incorporate other elements of the body into 'bodily form'. Bodily constituents other than zang and fu organs and the channels, including the head, face, eyes, mouth and lips, teeth, neck, back, chest, breasts, abdomen, waist, sides, armpits, arms, legs, skin, flesh, pulse, muscles, and bones are seen in this work as main components of 'bodily form'. However, rather than representing all of these explicitly by visual means, the author alludes to his intention through the title 'image of the form of the body and the zang and fu organs'.

Third, Treasured Collections aims to establish a medical standard faithful to the spirit of older texts, an enterprise that is especially noticeable in the diagrams of the five organs. In doing so, this work pays more regard to the anatomical content of the earlier Inner Canon and the Classic of Difficult Issues than to the contributions of positivistic anatomy from and after the Song and Yuan Dynasties, and the diagrams of the five zang organs are devised in accord with such a view.

This approach and set of priorities subsequently became a salient characteristic of Korean medicine. Indeed, medicine in Korea during the late Joseon dynasty was strongly characterised by Daoist ideas of the preservation of health, which in turn resonated with the spirit of Confucian scholar-officials, who attached great importance to the pursuit of physical and mental cultivation. In addition, Heo Jun's successors repeatedly condensed and rearranged Treasured Collections, and used it as a model; and in this process, the section of the text entitled 'Treatise on bodily form' acquired the status of a standard for classifying the body and diseases.

Above all, dissection was subject to powerful taboos in Joseon society. This was not so much a consequence of the publication of Treasured Collections, as part of the general ethos of which Heo Jun's text partook. In fact, there exists only one record of an actual dissection conducted in Korea before the introduction of Western ideas and techniques, and it dates from the same period as Treasured Collections. According to the record, the medical skills of one Jeon Yuhyeong 全有亨 became even more refined following his dissection of three corpses during the Japanese Invasions of 1592-1598. However, this record is found in the context of emphasising the taboo against dissecting human bodies: precisely because he conducted this dissection, Jeon Yuhyeong was unjustly charged with treason and later put to death. ${ }^{16}$

The passage below illustrates the attitude towards dissection of Korean physicians after Heo Jun. It consists of a dialogue between Nam Dumin 南斗旻, a physician who visited Japan in 1763 as a member of a Korean delegation, and a Japanese Confucian scholar-physician.

Kitayama Arawatsu 北山彰: So here is my question. In my country, it was heard that a doctor who likes strange things (Miwaki Toyo) dissected the dead body of an executed prisoner, drew the shapes and locations of the internal organs in different colours and published a book entitled 'Theory of Organs'. He said that while Neijing claimed there were 12 organs, he dissected the body, discovered there were only nine of them, and could see the large intestine but not the small one. He tried to prove this quoting Shang Shu (Book of Documents), Zhouli (Rites of Zhou) and other books from miscellaneous schools. If the so-called arrangement of the human organs and the theory of Five Agent correspondences, described in the Inner Canon, were to be dismissed because of this, all of us in the medical profession are destined to fail miserably, it is said. Is there any such theory in your country, too? What is your opinion about this?

Nam Doo-min 南斗旻: Scholars in your country like to present strange theories; I am not sure if it is because they have a predilection for this. In my country, we all follow the principles of Huangdi 黃帝 (the Yellow Emperor) and his teacher, Qi Bo 岐伯, and we do not seek any new theories. One who learns only after doing dissection is unwise, while knowing without doing dissection is the ability of a sage. Please do not be carried away [by such theories]. ${ }^{17}$

16 An, Jeong-bok, Seongho saseol yuseon 星湖僿說類選, 1929, p. 445 .

17 Kitayama Shō 北山彰 1764 , Keydan waumei 鷄壇嚶鳴 (http:// jisik.kiom.re.kr/, 鷄壇嚶鳴, 席上賦一律呈) (accessed 22/06/17). 
The general attitude of Korean physicians including Heo Jun was that one should remain faithful to the medicine bequeathed by sages instead of developing new medicine through dissection, and this holds true also of their views on anatomy.

\section{Bibliography}

\section{Primary Sources}

Gong Tingxian 竟廷賢 (Ming), Wanbing huichun 萬病回春. Modern edn: Gong Tingxian yixue quanshu 竟廷賢醫學全書, Beijing: Zhongguo zhongyiyao chubanshe, 1999.

Heo Jun 許浚 1613, Dong'ui'bo'gam 東醫寶鑑 (Treasured Collections of an Eastern Physician). Edited:Seoul:Yeokang Chu'lpansa, 1994, vol.1.

Kim Yemong 金禮蒙 et al.1477, Ui'bang'ryuchui 醫方類聚 (Classified Collection of Medical Formulas). Modern edn: Tokyo: Kunaichō shoryōbu (Archives and Mausolea Department, The Imperial Household Agency), 1998, vol. 5. Copy held by the National Library, Korea.

Kitayama Shō 北山彰 1764 , Keydan waumei 鷄壇嚶鳴 (Twittering on the Roost) vol. 1 (http://jisik.kiom.re.kr/, Keydan waumei 鷄壇嚶
鳴, Sekijō fu ichiritsu tei 席上賦一律呈 (Writing a Poem in the Seat) (accessed 16/04/2014).

Li Chan 李梴 1575, Yixue Rumen 醫學入門 (An Introduction to Medicine). Edited Li Chan 編註醫學入門 (An Introduction to Medicine) Seoul: Namsandang, 2002.

An Jeongbok 1929, Seongho saseol yuseon 星湖僿說類選 (Selected Discourses of Seongho), 'Ojangdo' 五臟圖 (Illustration of the Five Viscera), Seoul: Mun'gwanseorim.

Yu Seongnyong 柳成龍 16oo, Chi'mkuyokyel 鍼炎要訣 (Essential Formulas for Acupuncture and Moxibustion). Edited:Seoul:Yeogang ch'ulpansa, 1994.

\section{Secondary Sources}

Huang Longxiang 黃龍祥 2003, Zhongguo zhenjiushi tujian 中國針 炎史圖鑑 (Illustrated history of Acupuncture and Moxibustion in China), vol. 1, Qingdao: Qingdao chubanshe.

Shin Dongwon 신동원 2001, Chosǒn Saram Heo, Jun 조선사람 허준 (A Biography of Heo Jun), Seoul: Hankyere chulpan.

Yi Yeongtaek 이영택 1957, 'Uri nara esǒ silyong doiǒon inche' haeputo' (The Charts of Human Anatomy Which Have Been in Practical Use in Korea), Nonmunchip Chayen kwahak, 5 , Seoul National University. 



\title{
24 Imagining Acupuncture: Images and the Early Westernisation of Asian Medical Expertise
}

\author{
Roberta Bivins
}

Chinese medicine draws upon a rich iconographic tradition; through this tradition (as well as careful observation, and the translation of medical texts), European medical practitioners and patients first became aware of the medical expertise of Asia. As a therapeutic modality defined by and practised in conjunction with a unique set of body maps, acupuncture's Western history in particular can be traced in the images through which it was represented. From Wilhelm Ten Rhijne's 1683 Mantissa Schematica:De Acupunctura to contemporary medical textbooks, changes in the European visual culture of acupuncture record different attempts to incorporate the technique into Western theory and practice - and expose, too, areas of cultural, medical, and scientific intransigence. Thus such images offer us primary evidence of the processes by which Chinese medical knowledge and culture has been globalised: how it has been perceived, translated, transmitted, received, and practised in other medical settings and cultures.

What we summarise under the heading of 'transmission' is, of course, a complex and multi-faceted process even within a single culture. As the 17 th-century French, natural philosopher Blaise Pascal recognised, the communication of knowledge involves many interactive elements (parsed by scholars of his work as content, medium, receiver and context). Perhaps more crucially, Pascal noted that successful communication depended not just on the value or truthfulness of the knowledge to be conveyed, but on knowing the tastes, pleasures and accepted truths of its would-be recipient:

We must pay attention to the person whom we are addressing: we must know their mind and heart, what principles they grant, what things they like... the art of persuasion consists in pleasing as much as in convincing - humans being, as they are, governed so much more by whim than by reason. ${ }^{1}$

Rather more recently, Elisabeth Hsu (among others) has explored the complex processes whereby Chinese medical knowledge is transmitted from one generation to another within China. The pathways Hsu isolated - 'secret', 'person-

1 For the intracultural transmission of (expert, scientific) knowledge, see the essays in Kusukawa and Maclean (eds) 2006, which also examine the role of images in stabilising polysemic knowledge. For Pascal, see specifically Scholar 2006, p. 3, where this quotation from Blaise Pascal, De l'Esprit Géométrique, c. 1655 , can be found. al' and 'standardised' - operate to a greater or lesser extent in any medical culture. ${ }^{2}$ One or another of these pathways may dominate the transmission process for any particular kind of medical knowledge, but will rarely if ever operate exclusively. For example, while medical education in the West may well be dominated by 'standardised' knowledge transmission, both 'personal' and 'secret' pathways certainly still operate, as illustrated by Julie Anderson and John Pickstone's work on the Charnely artificial hip, and by the persistence of the culture of surgical 'firms'. ${ }^{3}$

As Volker Scheid has demonstrated in relation to contemporary Chinese medicine, the specific content to be transmitted also affects the process and pace of transmission. ${ }^{4}$ In the sphere of medicine, three broad genres of knowledge are involved. Medical theory, comprising cosmologies, models of the body, and aetiologies of health and illness, is perhaps most readily transmissible across physical barriers, as it can take an exclusively textual form. ${ }^{5}$ But cultural barriers are another matter. Medical systems, as many scholars have argued, are highly culturally specific - and the availability of another culture's medical texts is no guarantee of their assimilability or persuasiveness. Medical practice, meanwhile, entails methods of diagnosis, prognosis and treatment; the content and style of the doctor-patient encounter; and the management of illness and health; these are perhaps best transmitted through personal contact and direct experience. And the technologies of medicine - its materia medica, instruments, devices, body maps, diagnostic charts, etc. - may be more or less transportable, but are rarely complete without both textual and experiential glosses. Consider, for example, the stethoscope - or more properly, the use of mediate auscultation (the technique of diagnosing chest conditions through listening indirectly to the sounds of respiration,


straightforward process, in which meaning in the origin culture and meaning in the destination culture bear a one-to-one relation to each other. See for example the studies (focused primarily on the transmission of scientific rather than specifically medical knowledge) in Ragep and Ragep, with Livesey (eds) 1996. Moreover, as Pascal's wry quotation suggests, the movement of knowledge from one location to another does not ensure the successful communication of that knowledge. 
etc.). Three components had to be transmitted for the practice to be successful and stable: the tool itself, the method of using it effectively, and the knowledge required to differentiate between newly audible sounds, and to associate those sounds with specific pathologies. Detailed knowledge of anatomy and personal experience using the technique under the close supervision of an expert proponent - in this case, the stethoscope's inventor, René Laënnec - were vital to its successful adoption, as were accessible and excellent translations of relevant treatises. ${ }^{6}$ Images too played a role in the eventual adoption of the stethoscope. In his first treatise on mediate auscultation, Laënnec presented detailed images and a cross-section of his novel (but fairly simple) instrument. These images allowed doctors remote from Paris to experiment with the tool themselves, and to test and assert its utility through direct experience. ${ }^{7}$

\section{Images and Acts of Transmission}

The study of images is increasingly recognised as essential to understanding the processes whereby expertise diffuses within, or passes from one culture to another. ${ }^{8}$ As the case of stethoscopy demonstrated, images can connect audiences and contexts otherwise separated by time, space, language or intellectual bent. Even within a single expert culture, images can operate as more than just illustrations: the philosopher Michael Ruse has offered compelling evidence that pictures - in particular, the portrayal of genetic processes in terms of a topographical landscape - were crucial to the communication of mathematical models of evolutionary genetics to mathematically disinclined evolutionary biologists, who only then could use them to produce key experiments. ${ }^{9}$ The use of images can also extend scarce resources and offer up surrogate experiences, as in the case of anatomical dissection and anatomical atlases. ${ }^{10}$ But images do not always play such straightforward roles, and certainly do not reliably speed the transmission process. Crucially, images can create unintended meanings; William Hunter, the famous 18th-century anatomist,

6 See Reiser 1978, and in more detail, Smith 1998. For a slightly different perspective, see Nicholson, 1993. On the importance of textual glosses for tools and images, see also Kusukawa and Maclean (eds) 2006.

7 See Smith 1998, pp. 183-216.

8 See for examples Gilman 1995, ch. 1; Kemp 1993, pp. 85-121. For more on the 'visual turn' in history, see Jordanova 2012.

Ruse 1996, pp. 303-10.

10 See Kemp 1996, esp. pp. 43-59; and Kemp, 1993; see also Berkowitz, 2011, pp. 259-62. noted that, while art could present information 'so plain that the unlearned as well as the learned understand it at first sight', it could also be dangerously misrepresentative. ${ }^{11}$

An additional complexity in the iconography of Western medicine and science since the Renaissance is that the images were intentionally directive as well as descriptive: they showed surgeons and artists 'routes' around and through the body, suggested 'appropriate' comparisons and valuations for the observed structures, and so constrained as well as communicated knowledge of the body's inner spaces. ${ }^{12}$ Mark Jenner's recent assessment of English physician Sir John Floyer's early 18th-century response to Chinese haptic diagnostics illustrates the operation of such constraints, even in Floyer's very positive response to the images of Chinese 'pulse-taking' in Andreas Cleyer's Specimen medicinae Sinicae. ${ }^{13}$ Moreover, images were routinely separated from the texts that glossed them, and re-used, derailing the intended transmission process and sometimes replacing their original meanings with entirely new ones..$^{14}$ As we will see, this has been common in cross-cultural transmission.

\section{Portraying Technique, Mapping Transmission?}

The case of acupuncture neatly exemplifies the difficulties of transmission and their effect on the globalisation of Chinese (or indeed any other) medical culture. Here, I will focus primarily on images created or copied by Westerners, and particularly medical professionals, whether intended for lay or medical audiences. Many of those images, particularly in the first modern wave of acupuncture's transmission to the West (roughly 1670 os to 173os), consisted of maps of the jingluo 經絡 and xue 穴 - the dense network of channels through which energy flows around the body and the specific points at which particular channels and flows could be stimulated by surface interventions, including the insertion of needles or the burning of moxa. Standing between the poles of text and technology, the original maps, charts and models of a subtle body were certainly tools in Chinese and Japanese medical practice and education. Foreign observers noted these uses. In Japan, interested (if faintly disapproving) Europeans also

\footnotetext{
11 Quoted in Kemp, 1993, p. 87. For unintended meanings, see also Luthy 2006.

12 Kemp 1993, p. 94 and Berkowitz 2011 hint at this, as do many primary source accounts. See Schiebinger 1989 for a detailed study of anatomical images and gender in science and medicine.

13 Jenner 2010, pp. 663-7.

14 Kusukawa and Maclean (eds) 2oo6, pp. 92-6.
} 
described the use of these medical images by an enthusiastically self-medicating laity to self-puncture - as one Dutch physician-observer put it, 'the needles are among the portable treasures of the capsule-loving nation'.15 This apparent transparency and directness in medical practice was much admired by Westerners more accustomed to the rhetoric and obscurantism - the 'verbal globs of honey' and 'contrived and controversial nonsense'- of elite European physick. ${ }^{16}$ And so they intended to emulate it in transmitting knowledge about acupuncture. One early proponent of the technique remarked: '

Since this method is so very different from the practice of Westerners, and since a thorough demonstration of it would involve a huge amount of labour and since this would not be suited to the flat surface of a book page, I thought it best to present illustrations clarifying separate dissertations. ${ }^{17}$

This physician clearly expected his maps to be attractive and useful to his intended Western audience: European medical professionals. His charts were to be virtual demonstrations, as well as maps for needle placement. He had, after all, seen similar documents working in exactly that way in Japan, as tools and guides of users' hands. But the maps and models also embodied an entirely different medical cosmology, and were imbued with considerable sophisticated medical theory. It was precisely these elements of the images that were obscured or erased when the role of images in Chinese and Japanese medical practice was unquestioningly equated with the purely illustrative and corroborative role that images were coming to have (at least in theory) in Western medicine.

Thus although often presented by their authors as direct copies of Asian originals, the maps that were actually transmitted to early modern Europe were in fact translations, and as such, were subject to exactly the same kind of errors that have plagued translations of Asian medical texts. Like Chinese medical terminology, the images were variously exoticised, domesticated, over-simplified, and embellished. Moreover, in seeking to understand the process of transmission, it is essential to remember that none of these images existed in a vacuum: they were 'read' in conjunction with an array of material and especially textual sources. Just as the images inflected (and reflected) Western perceptions of acupuncture, so responses to them were enduringly influenced by Western perceptions of China; similarly, internal debates and trends in Western

15 Engelbert Kaempfer, 'Acupuncture, A Japanese Cure for Colic', tr. in Bowers and Carrubba 1970, p. 304.

16 Ten Rhijne, 'De Acupunctura' and 'Mantissa Schematica', tr.in Carrubba and Bowers 1974, p. 375 .

17 Tr. in Carrubba and Bowers 1974, p. 376.

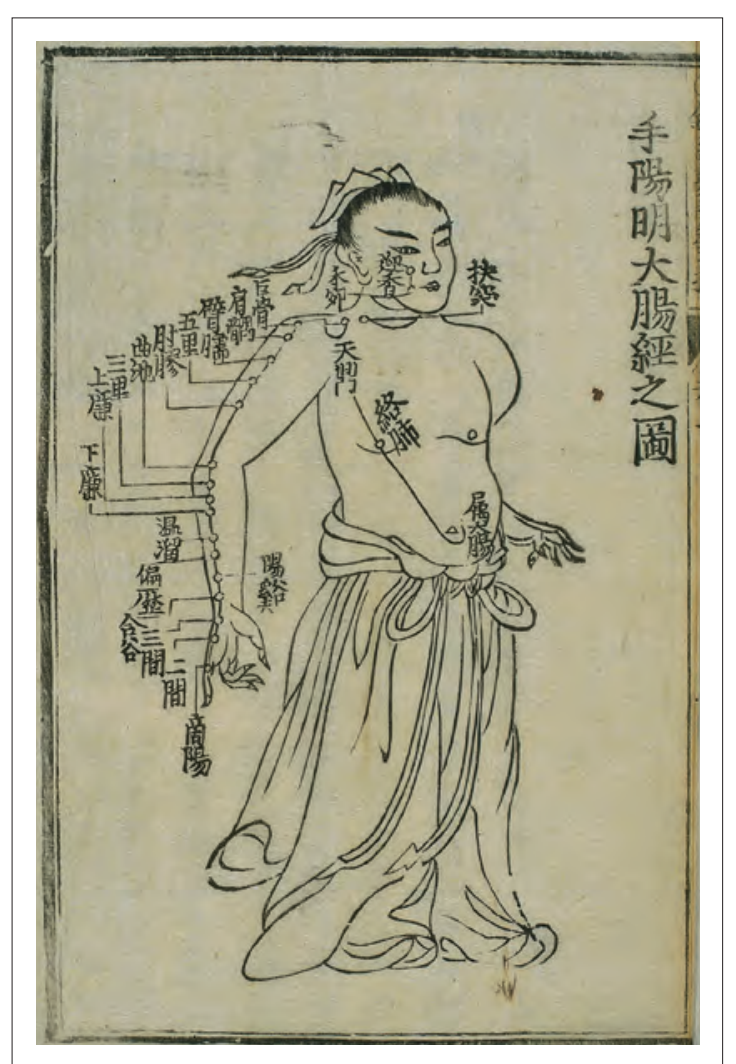

Figure 24.1 The Large Intestine Channel of Hand Yangming, from Gao Wu (Ming period, 1368-1644), Zhenjiu jiuying fahui (Explanations of 'Collected Gems of Acupuncture and Moxibustion), engraved and published in Japan. Library of Zhongguo zhongyi yanjiu yuan (China Academy of Traditional Chinese Medicine). (c) Wellcome Library, London, Loo37831

medicine played a profound role in both the iconography of acupuncture, and its reception. ${ }^{18}$

The earliest European medical treatise on acupuncture, Wilhelm Ten Rhijne's 1683 Dissertatio de Arthritide: Mantissa Schematica:De Acupunctura:Et Orationes Tres..., was based on Ten Rhijne's own observations of medical practice in Japan and his interpretations of rather painfully translated Chinese medical texts. Ten Rhijne confided to his readers:

I gathered and translated these into Latin, with the assistance of Iwanaga Zoko, a Japanese physician who knows Chinese, and with the assistance of Monttongi Sodaio, our interpreter, who speaks faltering Dutch in half words and fragmentary expressions. ${ }^{19}$

Indeed, Ten Rhijne grumbled, his interpreters' 'inexperience and limited vocabulary in Dutch' forced him to 'omit

For examples of interactions between internal medical debates and responses to acupuncture in the 18 th and 19th centuries, see Bivins 2000, esp. chs 2 and 3.

19 Tr. in Carrubba and Bowers 1974, pp. 377-8. 


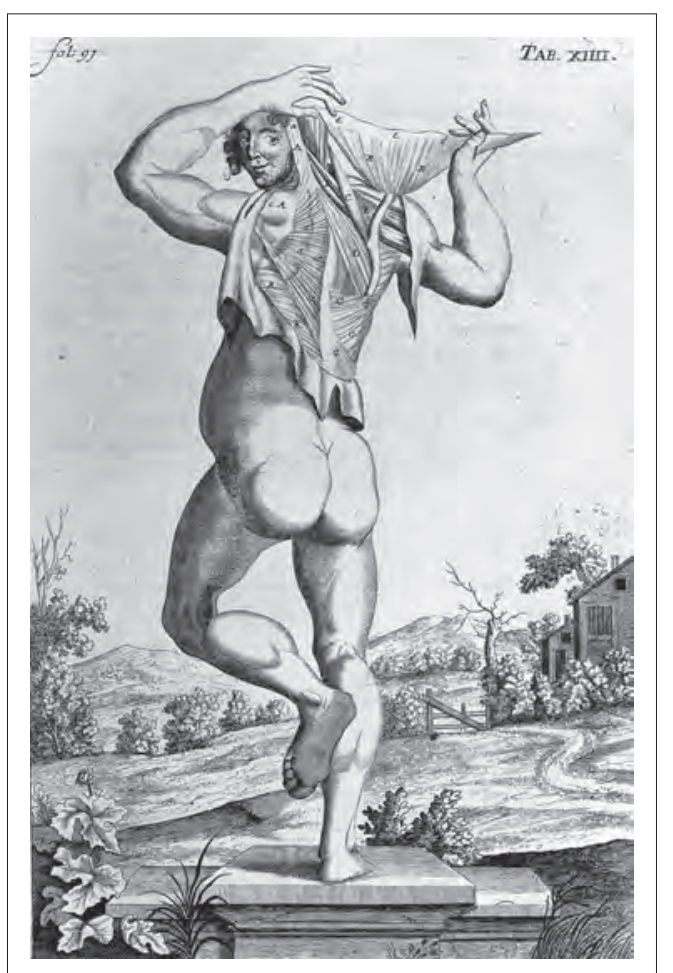

Figure 24.2 Anatomy of the upper back, from John Browne, A Compleat Treatise of the Muscles: as they appear in humane body, and arise in dissection..., London 1681. Note the similarities with Ten Rhijne's 'Chinese' image below, Fig. 5. (C) Wellcome Library, London, Loo28419



Figure 24.3 Dissected hand and arm, from Govard Bidloo, Anatomia Humani Corporis: centum \& quinque tabulis, engraved G. de Lairesse, Amsterdam 1685. () Wellcome Library, London, Looo6365



Figure 24.4 'Anatomical Figure’ from Andreas Cleyer, Specimen Medicinae Sinicae, sive opuscula medica ad mentem Sinensium, Frankfurt, 1682. (c) Wellcome Library, London, Looo4105



Figure 24.5 'Effigies Sinica' from Willem Ten Rhijne, Dissertatio de Arthritide: mantissa schematica: de acupunctura, London 1683. Compare John Browne's partially dissected Fig. 2. (c) Wellcome Library, London, Loo22387 
much that was written in Chinese in the original documents'. ${ }^{20}$ Just as Ten Rhijne's source texts were, in general, first translated from Chinese into Japanese, from Japanese into Dutch, and finally from Dutch into medical Latin, so too were his images multiply 'translated' - from Chinese originals to Japanese copies (see Fig. 1) and finally to images modified to suit European iconographic conventions.

In medical publishing, those conventions were rooted in rapidly changing models of the human body drawn from contemporary anatomical studies (see Figs 2, 3). Clearly, there is a striking contrast between the Asian and European styles of representing the body. From a Western perspective, the Chinese image shows a body mapped and by implication, known - primarily on the surface, and with little anatomical detail or specificity. Contemporary Chinese images of the internal organs did little to improve Western medical opinion, since they flatly contradicted the anatomical knowledge of the day. Indeed, as an example of the role of selection in the transmission pathway, it is worth noting that Ten Rhijne - medically trained and exhorting emulation, or at least experimentation in a medical audience - did not duplicate these images; his non-medical contemporary, the missionary Andreas Cleyer, did (see Fig. 4 ). By contrast, Western images from the same period as the Mantissa Schematica portrayed ever-greater levels of fine detail, and ever-deeper explorations beneath the body's surface. ${ }^{21}$ As a part of the pursuit of naturalism and the culture of 'virtual witnessing', a natomical art conventionally displayed the sheets of flesh which dissection peeled away. ${ }^{22}$ This convention is respected in the adaptations of Chinese body maps published with Ten Rhijne's account of acupuncture (Fig. 5), where similar flaps have been added. Additionally, the pose of one of Ten Rhijne's 'Chinese' figures shares many features with a Western anatomical image published in London only two years earlier (Fig. 2). Ten Rhijne himself would have been familiar with an earlier version of this same figure, which appeared in an anatomical treatise he presented to the Japanese official charged with controlling foreign trade in $1765 .^{23}$

Although rendered visually more familiar to European eyes by the addition of 'dissected' flaps of skin, Ten Rhijne's versions preserved the most distinctive aspect of acupuncture maps: the jingluo. Ten Rhijne also discussed them in his text. He acknowledged, 'a person especially skillful in

\footnotetext{
20 Ibid.

21 On 'surfacing the body interior', see Taylor 2005, pp. 746-8.

22 The classic discussion of 'virtual witnessing' is Shapin and Shaffer 1985; for more on anatomical art in the West, see Carlino 1999 .

23 Cook 2004, pp. 25-6.
}

the art of anatomy will belittle the lines and the precise points of insertion'. ${ }^{24}$ He knew that a Western audience used to the increasingly sophisticated heuristics of contemporary anatomical atlases might have other criticisms as well, that they would

censure the awkward presentation of the short notes on the diagrams, when these should be more closely identified with the walls of the blood vessels... ${ }^{25}$

Nonetheless, he told his readers,

[t]he various movements of the blood must be learned through the precepts and rules as laid down by the Chinese... if the cure is to be undertaken according to their regimen. ${ }^{26}$

In other words, he asserted that theory and practice were inseparable. Ten Rhijne's successor, Kaempfer, would disagree, erasing the jingluo - fundamental to any understanding of acupuncture's modus operandi-from his own visual and textual accounts of the technique.

Engelbert Kaempfer, like Ten Rhijne, served as medical officer to the Dutch community on Deshima. Also like his predecessor, he was a close observer, deeply interested in the medicine of Japan. He was considerably less sympathetic, however, to its different model of the body. Where Ten Rhijne urged his European counterparts to experiment with acupuncture as it was used in China and Japan - as a compound entity combining practice and theory via needles, maps, and body-model - Kaempfer divorced the former element from the latter. Discussing both moxibustion and acupuncture, Kaempfer described in minute detail the exact methods practised in relation to burning, needle-insertion and movement. He stressed the care taken in choosing the location of insertion or the placement of moxa, but he dismissed the jingluo altogether: they were not anatomical and therefore not 'reasonable'.

The Main art lies in the knowledge of the parts which it is proper to burn [or puncture] in particular distempers. ... [O]ne would reasonably imagine that place to be the most proper which is nearest to the affected part, yet the operators frequently choose such others, as are not only very remote from it, but would be found, upon an Anatomical inquiry, to have scarce any communication with it, no more than by the common integuments. ... the most skillful Anatomist would be at a loss to find out any particular correspondence of these remote and differing parts with one another. ${ }^{27}$

In an earlier version of his account, Kaempfer added a further comment on his Japanese counterparts:






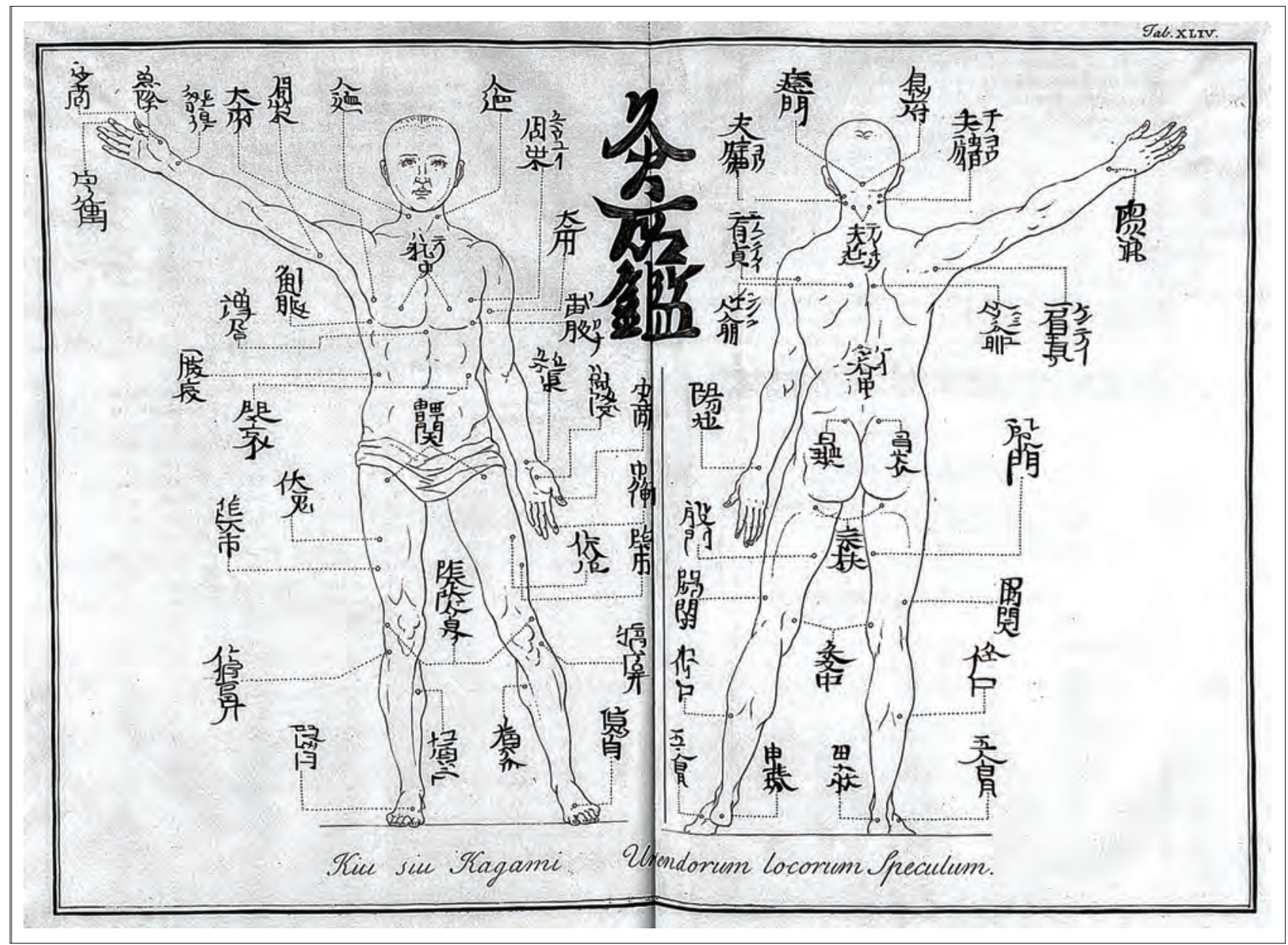

Figure 24.6 'Kiu siu Kagami Urendorum locorum Speculum' from Engelbert Kaempfer, The History ofJapan, London 1728. Note the absence of jingluo. (c) Wellcome Library, London, Loo23384

The results do not allow us to accuse them all of deception, yet sound reasoning does not permit us to testify in defence of all of them. ${ }^{28}$

The images Kaempfer included reflected and emphasised his materialist bias and scepticism of Chinese understandings of the body (see Fig. 6). ${ }^{29}$ They carefully charted the points, the empirical efficacy of which Kaempfer had witnessed. But they erased the structures underpinning them as anatomically disproven. With these images, the theoretical rationale of acupuncture's efficacy disappeared.

Images and texts addressing Chinese (and Japanese) medicine were examined by Western audiences over the course of the 18th and 19th centuries, though not always in conjunction with each other. The results, despite their authors' best efforts, were mixed. For some, the images served to confirm what (often exoticised) translations

\footnotetext{
28 Tr. in Bowers and Carrubba 1970, pp. 270-310.

29 For an expanded treatment of these two authors and texts, see Bivins 2000, ch. 2.
}

of Chinese medical texts had already suggested: that the medical skill of the East was negligible. English polemicist William Wotton, for example, wrote scathingly of Cleyer's illustrated translations:

The anatomical figures annexed to the tracts, which also were sent out of China, are so very whimsical, that a man would almost believe the whole to be a banter, if these theories were not agreeable to the occasional hints that may be found in the travels of the missionaries..$^{30}$

While Wotton included a short extract of the translated text on the grounds that, 'few will in all probability have patience to go through them, since they are not very pleasant to read', he deleted the images altogether. ${ }^{31}$ On the other hand, almost a century later, Dujardin, in his compendious and influential Histoire de la Chirurgie reproduced Ten Rhijne's images exactly. ${ }^{32}$ Other scholars, including the French Encyclopédistes, mentioned the existence of

\footnotetext{
$30 \quad$ Wotton 1694, p. 152.

31 Ibid., p. 147.

$32 \quad$ Dujardin 1774, pp. $75^{-98}$.
} 
maps and figures depicting acupuncture or moxibustion points, sometimes referring to Ten Rhijne, Kaempfer, or Dujardin - but did not reproduce the images themselves. Images were expensive to reproduce, and these 'curious anatomies' were clearly not considered important enough to justify their additional cost.

Although maps of the body's surface were among the most dramatic and controversial Western representations of acupuncture, they did not provide the only images of the technique. The technology of acupuncture - needles, needle cases, striking implements - and, in the 19th century, three-dimensional figures too, were well-studied and frequently portrayed, particularly in medical texts and instrument catalogues. Even authors who ignored Ten Rhijne and Kaempfer's body-maps often included images of the implements of acupuncture. ${ }^{33}$ The drawback to their visual prevalence, and indeed the (again) orientalised beauty of many of these images, was that they tacitly reinforced Western perceptions of acupuncture as a standalone technology, to be applied purely empirically and in accordance with Western anatomical understandings of the body. Later, as in the 19th-century British study of the technique authored by James Morss Churchill, the deliberate Westernisation of therapeutic needling was reflected in a very Westernised representation of the needle itself. (Figs 7, 8). These images, however, were nonetheless important in widening access to the technique, much as they had been in the nearly contemporaneous case of the stethoscope. ${ }^{34}$ Doctors looking at Churchill's pictures could make their own acupuncture needles, and could test in person the miraculous, incomprehensible and therefore almost unbelievable efficacy of therapeutic needling in certain conditions.

The late 19th and 2oth centuries brought significant changes in the pathways by which Westerners, whether of the laity or the medical profession, accessed information about acupuncture. Immigration brought whole communities, with their medical practitioners and practices, to Western Europe and North America, where the latter swiftly gained a following among locals. ${ }^{35}$ In 1887 , for example, a US commentator noted: 'Many of the Chinese stores in our American cities keep a supply of Chinese drugs, and all of them sell Chinese proprietary medicines'. Alongside these over-the-counter remedies, such stores often hosted a Chinese practitioner able to diagnose and treat patients individually. ${ }^{36}$ New and more accurate translations of

33 See for example Heister 1743, p. 314.

34 See above, and Smith 1998.

35 Liu 1998; Marcus and Chen 2011.

36 Culin 1887. See also Liu, 1998; Shah 2001, pp. 55-6, 213 et passim.

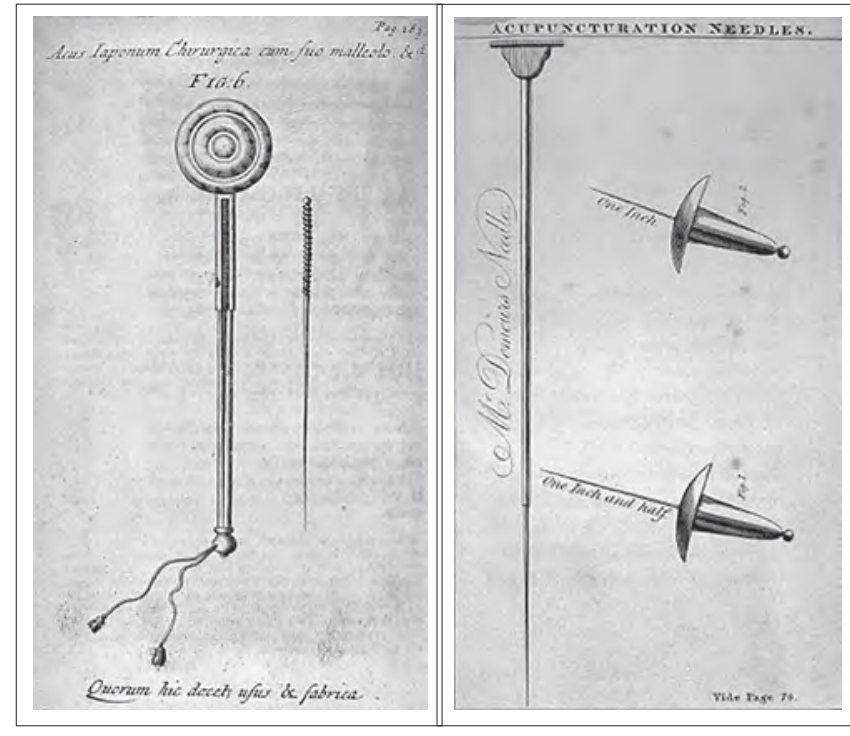

Figure 24.7 Acupuncture implements, as illustrated in Willem Ten Rhijne, Dissertatio de Arthritide: mantissa schematica: de acupunctura, London 1683

Figure 24.8 Mr Demours' Needles', from James M. Churchill, $A$ Treatise on Acu-puncturation; being a description of a surgical operation originally peculiar to the Japonese [sic] and Chinese, and by them denominated Zin-King, now introduced into European practice, with directions for its performance, and cases illustrating its success, London, 1821

Chinese medical, cosmological and philosophical texts likewise became more readily available, and Chinese language originals were mass-produced for the migrants; in Philadelphia, for example, complete copies of the Golden Mirror sold for $\$ 2.25$ in $1887 .{ }^{37}$ Chinese culture and its productions more generally became the subject of popular interest and enquiry, sometimes in ways that encouraged appreciation, rather than orientalism or racial stereotyping. ${ }^{38}$ And more Westerners were able to travel to East Asia to observe or study acupuncture in situ.

Nonetheless, even with these new avenues of transmission, images and models have remained central to understandings of acupuncture in the West. Like their predecessors, they reflect syntheses of different models of the body. Anatomical carcasses, stripped of skin but covered in $x u e$, dominate educational texts, while popular accounts and introductions - for example Ted Kaptchuck's Web That Has No Weaver - carries diagrams which could have been taken directly from Ten Rhijne's 'Japanese' images of three centuries before. Western commercial representations of acupuncture, meanwhile, heavily emphasise the exotic, the

37 For an interesting account of the creation of the Golden Mirror itself, and the ways in which this too was a process of selection, translation, and sometimes erasure, see Hanson 2003.

$38 \quad$ Zboray and Zboray 2004. 
unusual, and the historic qualities of 'Traditional Chinese Medicine' in images as well as text. ${ }^{39}$ Kaempfer's images remain popular. Their persistence may offer testimony to the accuracy with which he estimated Western tolerances for non-Western medical expertise - or to the ease with which its simpler lines, unlike those of Ten Rhijne's images, could be copied and reproduced. Visual distinctiveness here re-emphasises the now-attractive qualities of theoretical and practical divergence from a Western medical culture, which can be as mystifying, as obscure, and as alienating to its patients as Chinese medicine and culture once was to Western eyes.

So what, more broadly, do acupuncture's images, understood in their historical context, tell us about the processes by which medical knowledge and expertise become globalised? Clearly, whether philosophical, anthropological or historical, any model of transmission of expertise must include the following processes: the observation and collection of information, specimens, and technologies (and inevitably, therefore both accidental and deliberate selection); the translation, interpretation and description of the observed and collected materials; the publication and/or display of such materials in the destination culture; and the reception, re-interpretation, and response to them by the members of that culture. Both culture and chance inflect each stage of the process. A closed origin community, a higher market value for secret knowledge, cultural traditions about bodily privacy or sanctity, the mandates of governments, shipwreck, bookworm, sudden death, and of course, the personal tastes, ambitions and intellectual hobbyhorses of observers and observed: all can act as drivers of or as limits to comprehensive and persuasive cross-cultural transmission. Images cannot evade the exigencies of selection or of translation - nor can they escape culturally specific interpretation; indeed, as Western responses to Ten Rhijne and Kaempfer's images suggest, they may be particularly susceptible to interpretive drift and redefinition. Yet, as demonstrated by their persistence in the face of rapidly shifting technologies both of pedagogy and of diagnosis, images remain essential to the teaching and practice of medicine. Ten Rhijne himself acknowledged both the limitations and the value of imagery in communicating knowledge at a distance in another treatise, on leprosy in Asia:

It is not enough, for one who has never seen the disease, to send a description of it, consisting of a few hypotheses

39 For more on 2oth-century perceptions of acupuncture, see Bivins 2002, pp. 84-105; and on the use of tradition and exoticism in the commercial spread of Chinese (and Ayurvedic) medicine, see Bivins 2007, pp. 117-99. formed from guesswork rather than experience ... embellished, according to present day dash custom [sic], with much true-seeming chop-logic; making the nature and particularities of the disease like the paintings which have been made of it more from imagination than from real life. ${ }^{40}$

Such flawed and partial attempts, he acknowledged, had some merit: 'if everyone adds a line, then the picture will gradually become like nature, and after thorough observation, be at last completed'. They were, in short, better than nothing. Crucially, however, Ten Rhijne exhorted his readers not to 'stare at the manikin... always keep the living object in view'. Scholars, practitioners, and patients today continue to debate whether contemporary Western images (and imaginings) of acupuncture - biomedical, popular, or scholarly - are ossified, orientalised, or like nature', and with what experimental or experiential tools each additional line should be drawn. I have suggested here that such questions are of more than theoretical interest. Remarkable visual representations of acupuncture played a central role in acupuncture's early westward transmission and its initial reception in Europe. ${ }^{41}$ But with observers captivated or repelled by these 'manikins' and largely cut-off from the 'living object', acupuncture as it was originally understood, practised, and experienced (as an entity comprised of theories, practices, and technologies) was first transfigured, and then erased from view until the mid-2oth century.

\section{Bibliography}

\section{Primary Sources}

Kaempfer, E. 1728, The History ofJapan, London.

Dujardin, M.F. 1774, Histoire de la Chirurgie, depuis son origine jusqu'à nos jours, vol. 1, Paris: L'Imprimerie Royal.

Heister, L. 1743, A General System of Surgery in Three Parts, London: W. Innys.

Ten Rhijne, W. [1687] 1937, Treatise on the Asiatic Leprosy, Amsterdam: Abraham Van Someren, tr. D. Schoute, in Opuscula Selecta Neerlandicorum de Arte Medica, fasc. 14, Amsterdam: 'Sumptibus Societatis', 36-113.

Wotton, W.B.D. 1694, Reflections upon Ancient and Modern Learning, London: Peter Buck.

\section{Secondary Sources}

Anderson, J. 2006, 'Greenhouses and body suits: The challenge to knowledge in early hip-replacement surgery, 196o-1982', in Timmermann and Anderson (eds), 175-92.

Baigrie, B. 1996, Picturing Knowledge: Historical and Philosophical Problems Concerning the Use of Art in Science, Toronto: University

40 Ten Rhijne 1687, pp. 37-40.

41 For a detailed account of this process, see Bivins 2000 . 
of Toronto Press.

Berkowitz, C. 2011, 'The beauty of anatomy: visual displays and surgical education in early nineteenth-century London', Bulletin of the History of Medicine 85.2 (Summer), 248-78.

Bivins, R. 2000, Acupuncture, Expertise and Cross-cultural Medicine, Basingstoke: Palgrave.

2002, 'Acupuncture and innovation: 'New Age' medicine in the NHS', in Stanton (ed.), 84-105. 2007, Alternative Medicine? A History, Oxford: oup.

Bowers, J. and R. Carrubba 1970, 'The doctoral thesis of Engelbert Kaempfer: "On tropical diseases, oriental medicine and exotic natural phenomenon", Journal of the History of Medicine and Allied Sciences 25, 270-310.

Bynum, W.F. and R. Porter (eds) 1993, Medicine and the Five Senses, Cambridge: CUP, 134-53.

Carlino, A. 1999, Books of the Body: Anatomical Ritual and Renaissance Learning, Chicago: University of Chicago Press.

Carrubba, R. and J. Bowers 1974, 'The western world's first detailed treatise on acupuncture: Willem Ten Rhijne's "de Acupunctura”, Journal of the History of Medicine and Allied Sciences, 29, 371-98.

Cook, H. 2004, 'medical communication in the first global age:Willem ten Rhijne in Japan, 1674-1676', Academia Sinica 11, 16-36.

Culin, S. 1887, 'The Practice of medicine by the Chinese in America', reprinted from Medical and Surgical Reporter, 19 March, available online at http://babel.hathitrust.org/cgi/pt?id=uc2. ark\%3 A\%2F1396o\%2Ft22b8w48v;page=root;view=image;size=100;seq=6;num=2 (accessed 2/7/2013).

Gilman, S. 1995, Picturing Health and Illness: Images of Identity and Difference, Baltimore: Johns Hopkins University Press.

Hanson, M. 2003, 'The Golden Mirror in the imperial court of the Qianlong Emperor, 1739-1742', Early Science and Medicine 8.2, 111-47.

Hsu, E. 1999, The Transmission of Chinese Medicine, Cambridge: CuP.

Jenner, M. 2010, 'Tasting Lichfield, touching China: Sir John Floyer's senses', The Historical Journal 53: 647-70.

Jordanova, L. 2012, The Look of the Past: Visual and Material Evidence in Historical Practice, Cambridge: cup.

Kemp, M. 1993, “"The Mark of Truth”: Looking and learning in some anatomical illustrations from the Renaissance and eighteenth century', in Bynum and Porter (eds), 85-121.

1996, 'Temples of the body and temples of the cosmos: vision and visualisation in the vesalian and copernican revolutions', in Baigrie, $40-85$.

Kusukawa, S. and I. Maclean (eds) 2006, Transmitting Knowledge:
Words, Images and Instruments in Early Modern Europe, Oxford: OUP.

Liu Haiming 1998, 'The resilience of ethnic culture: Chinese herbalists in the American medical profession', Journal of Asian American Studies 1.2, 173-91.

Luthy, C. 2006, 'Where logical necessity becomes visual persuasion: Descartes' clear and distinct illustrations', in Transmitting Knowledge: Words, Images and Instruments in Early Modern Europe, Oxford: oup, 97-133.

Marcus, K. and Chen Yong 2011, 'Inside and outside Chinatown: Chinese elites in exclusion era California', Pacific Historical Review $80.3,369-400$.

Nicholson, M.1993, 'The introduction of percussion and stethoscopy to nineteenth century Edinburgh', in Bynum and Porter (eds), 134-53.

Pickstone, J. 2006, 'Bones in Lancashire: towards long-term contextual analysis of medical technology', in Timmermann and Anderson (eds), $17-36$.

Ragep, F.J. and S.P. Ragep, with S. Livesey (eds) 1996, Tradition, Transmission, Transformation: Proceedings of two conferences on pre-modern science, held at the University of Oklahoma, Leiden: Brill.

Reiser, S.J. 1978, Medicine and the Reign of Technology, Cambridge: CuP. Ruse, M.1996, 'Are pictures really necessary? The case of Sewall Wright's “Adaptive Landscapes"', in Baigrie, 303-38.

Scheid, V. 2002, Chinese Medicine in Contemporary China: Plurality and Synthesis, Durham, NC.: Duke University Press.

Schiebinger, L. 1989, The Mind Has No Sex: Women in the Origins of Modern Science, Cambridge MA: Harvard University Press.

Scholar, R. 2006, 'Introduction', in Kusukawa and Maclean (eds), 1-9. Shah, N. 2001, Contagious Divides: Epidemics and Race in San Francisco's Chinatown, Berkeley:University of California Press.

Shapin, S. and S. Shaffer 1985, Leviathan and the Air-Pump: Hobbes, Boyle, and the Experimental Life, Princeton: Princeton University Press.

Smith, H. 1998, 'The transmission of medical knowledge: the introduction and acceptance of mediate auscultation in Great Britain, 1816-1843', PHD diss., University of Minnesota.

Stanton, J. 2002 (ed.), Innovations in Health and Medicine, London: Routledge, $84-105$

Taylor, J. 2005, 'Surfacing the body interior', Annual Review of Anthropology 34, 741-56.

Timmermann, C. and J. Anderson (eds) 2006, Devices and Designs: Medical Technologies in Historical Perspective, Basingstoke: Palgrave.

Zboray, R. and M.S. Zboray 2004, 'Between "Crockery-dom and Barnum: Boston's Chinese Museum, 1845-47', American Quarterly $56.2,271-307$. 



\section{PART 5}

Esoteric Contexts and Knowledge Transmission 



\title{
25 The Body of Laozi and the Course of a Taoist Journey Through the Heavens
}

\author{
Patrice Fava*
}

At the border of Hunan and Jiangxi, where Taoist (Daoist) rituals and the Nuo tradition of masked theatre have undergone a large scale revival in recent decades, a Taoist master of the Orthodox Zhengyi 正一 sect, Master Yi Songyao 易 松堯, has preserved a number of rare ancient paintings and manuscripts that have been transmitted to him as the liturgical texts of his lineage; these include a map of the body of Laozi and a chart of the course of a Taoist journey through the Heavens. The following brief introduction to these two documents serves mainly as an example of how an intimate knowledge of Taoist ritual can provide a key to the performative nature of the charts and indicates the rich scope for future research.

The fact that many of the rituals involved in Taoist practice have the aim of healing the body at their core provides one reason to include an a chapter on what amounts to an out-of-body shamanic journey in an edited volume on medicine. After all, Taoists were responsible for a very large proportion of healthcare in pre-modern China. Their iconography and topographies of the body also overlap in with medical maps and an analysis of chart and textual itinerary is an invaluable resource for understanding the intersections with more mainstream medical illustration.

Laojun gulou 老君骷髏 (Fig. 1), or Laojun tu 老君圖, is a religious topography of the body of Laozi 老子, the foundational deity of Taoism and putative author of its sacred text, Daode jing 道德經 (Scripture of the Way and its Power). ${ }^{1}$ It is derived from a Taoist manuscript entitled

All images in this chapter are copyright of the author.

Early accounts describe the scribe Laozi, or Lao Dan 老聑, as the 6th-century BCE author of the Daode jing, a quietist philosophical text propounding detachment, simplicity and removal from worldly affairs. During the early unification of China, he became worshipped by the imperial house, and he also appeared in a vision as Taishang Laojun 太上老君 (Lord Lao of Grand Supreme) to the founder of the Celestial Masters (Tianshi 天師) Daoist sect. Records of the early Tang describe Laojun, under the title Celestial Worthy of the Way and its Power (Daode Tianzun 道德天尊), as one of the three Celestial Worthies (Tianzun 天 尊), a trinity of anthropomorphic manifestations of the Dao who are coterminous with the Three Purities, celestial realms associated with the teachings of different Taoist sects. Daode Tianzun is associated with the Supreme Purity (Taiqing 太清) heaven. The reason why Laozi was associated with this specific Pure One is, of course, that the title of the book attributed to his authorship contains the same collocation, 'daode' 道德, the
Lingbao taiji lianmi 靈寶太極鍕秘 (Alchemical Secrets of the Supreme Ultimate Tradition of the Sacred Jewel) in the collection of Hunan Taoist Master Yi Songyao 易松 堯 of the Orthodox Zhengyi 正一 sect. According to an attribution on the cover, it had been in the possession of Master He Huaide 何懷德 whose ordination name (luming 録名) was Yuanzhen 元真. He copied it in the middle of the 19th century from a manuscript by Zhang Fazheng 張法正, who received the text himself in the 36 th year of the Kangxi reign period (1697). The entire manuscript, which includes some 70 double sheets, and contains many texts, illustrations and diagrams related to Taoist ritual, is stitched together using the traditional xianzhuang 線 裝 string binding.

This chapter discusses the Chart of the Skeleton of Lord Lao in relation to another chart in Master Yi's collection entitled Xian zhang feixing sanjie zhi tu 獻章飛行三界之 圖 (Chart of a Flying Journey through the Three Worlds to Present a Petition) (Fig. 2). While the latter chart is not a map of the body, but a representation of Heaven, both charts relate to Taoist ritual, and particularly to the liturgy of Thunder Magic (leifa 雷法). ${ }^{2}$ Both are also unique inasmuch as they have no close match in either Taoist or medical archives. ${ }^{3}$ It is outside the scope of this chapter to provide a full historical contextualisation of every detail of the complex pantheons and places listed on the two

\section{Way and its Power.}

Thunder Magic was an exorcistic class of ritual which gained prevalence during the Song dynasty. It had, as its core, methods designed to appropriate the powers of thunder for vitalising the body and punishing enemies using judicial, bureaucratic and meditative rituals. The foremost collection of these rites is in the Daofa huiyuan 道法會元 (Taoist Method, United in Principle). This is the largest compendium of the Taoist Canon, in 268juan, and represents the rites ( $f a$ 法) of 39 different sects from the Song and Yuan dynasties. See van der Loon 1979, pp. 401-5. For the historical background to these rituals and a résumé of the whole collection, see Schipper and Verellen 2004, pp. 1,056-7, 1081-3, 1105-15. Boltz 1987 pp. 47-9 mentions 6o different sects in her discussion of this corpus of Taoist ritual. See also Skar 1996-7, pp. 159-202. Davis 2001 bases his description of Song society on the Daofa huiyuan.

3 I have seen only one other witness to this kind of text in a collection belonging to John Lagerwey. However, the well-known chart of the body called Neijing $t u$ 內經圖 and specially the Xiuzhen $t u$ 修真圖, share common features with Laojun gulou. See Despeux 1994, pp. 12-29, 46-65. 


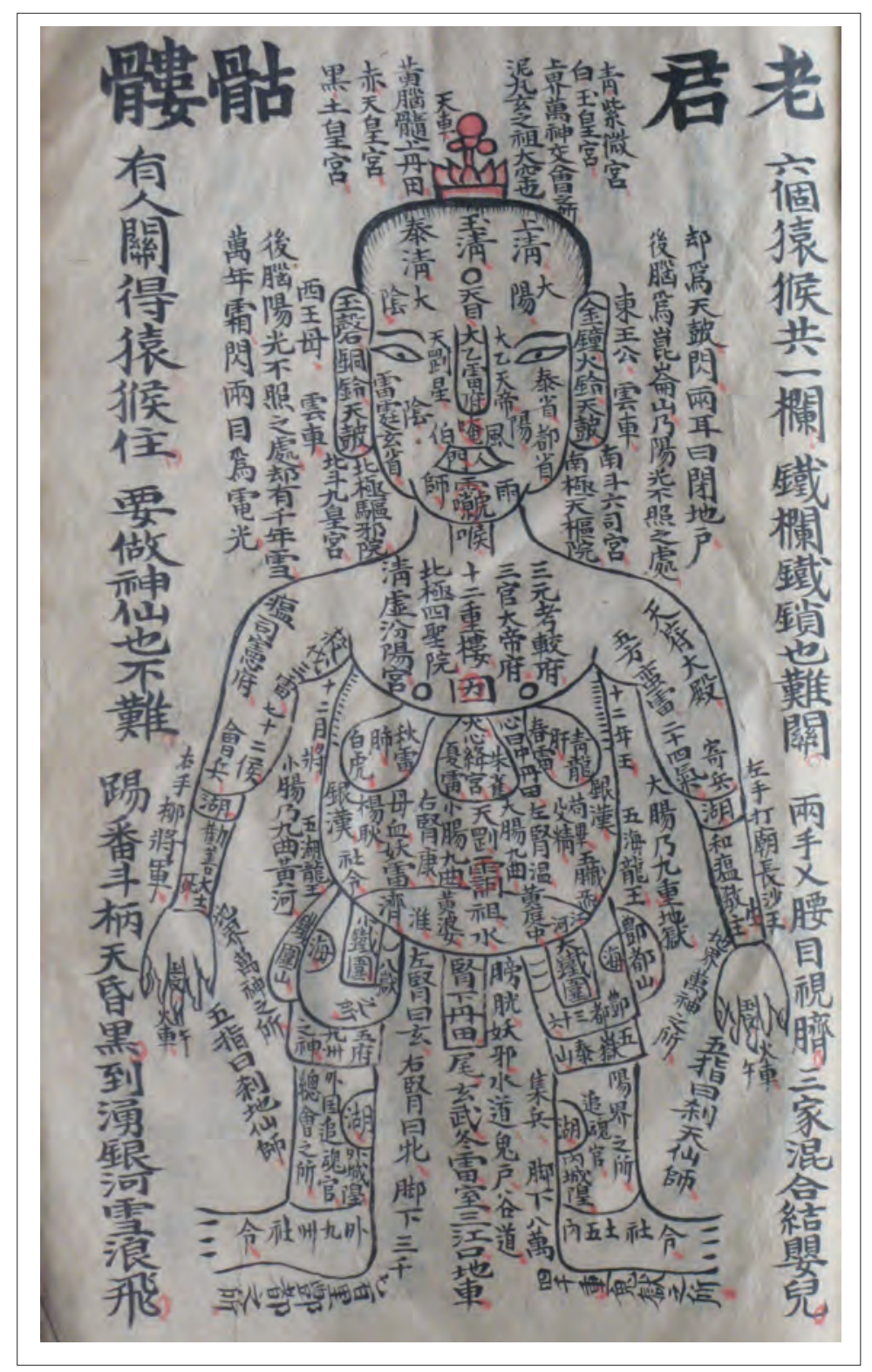

Figure 25.1 Laojun gulou 老君骷髏 (Chart of the Skeleton of Lord Lao)

charts; however my argument here is that, while they focus on different aspects of the Taoist ritual, the two charts are actually interdependent. The journey to present the petition was enacted via the ancient ritual of chuguan 出 官 (the exteriorisation of the gods that inhabit the adept's body) and required a working knowledge of the celestial domain and the names and locations of a host of different deities within the body.

Generally speaking, a Taoist master inherits his manuscripts from his father and will only transmit them to his eldest son, but he will also let his disciples copy his books during their apprenticeship. At the time of ordination, the initiate must be in possession of all the books he will use for his rituals. The Taoist texts, which are mostly in the form of manuscripts, are not transmitted to outsiders, and to this day, this is considered a very serious matter by many Taoist masters. Even when copying a manuscript for a Western researcher, the Master will sometimes write:

This secret book must not be transmitted, even to one's own son if he is without virtue; only people with karmic affınities can receive it.

符法不傳無義子 天機當度有緣分.

Despite a steady increase in published Taoist texts, due to the development of Taoist studies, practitioners only use the books transmitted by their masters and generally ignore the immense corpus of Taoist texts available. Among the great amount of liturgical texts included in the Taoist canon, there are many instructions for meditation and visualisation to accompany the performance of rituals, but each Taoist has his own way of delivering the memorials to the gods he addresses. In keeping with tradition, the 


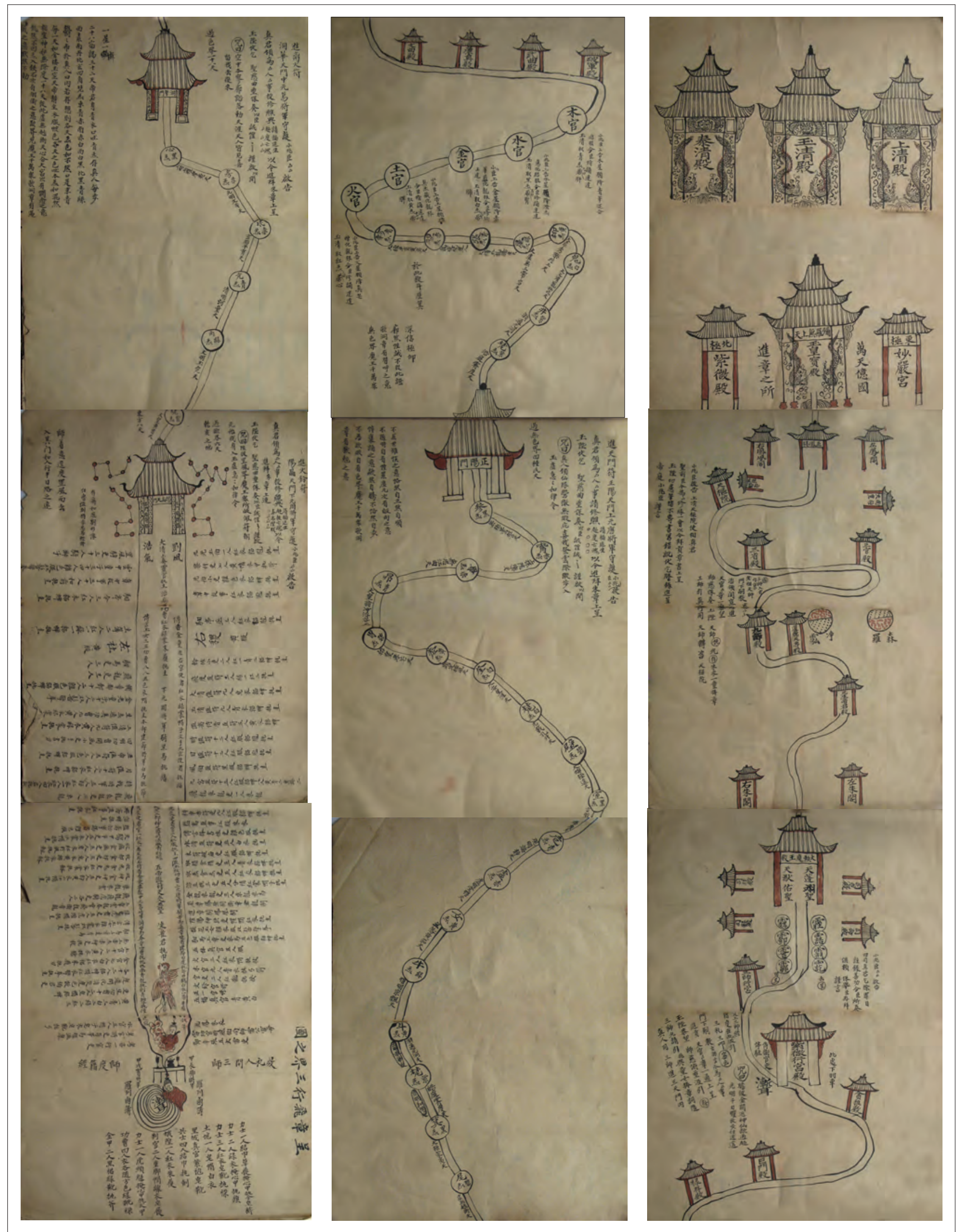

Figure 25.2 Xian zhang feixing sanjie zhi tu 獻章飛行三界之圖 (Chart of a Flying Journey through the Three Worlds to Present a Petition) 


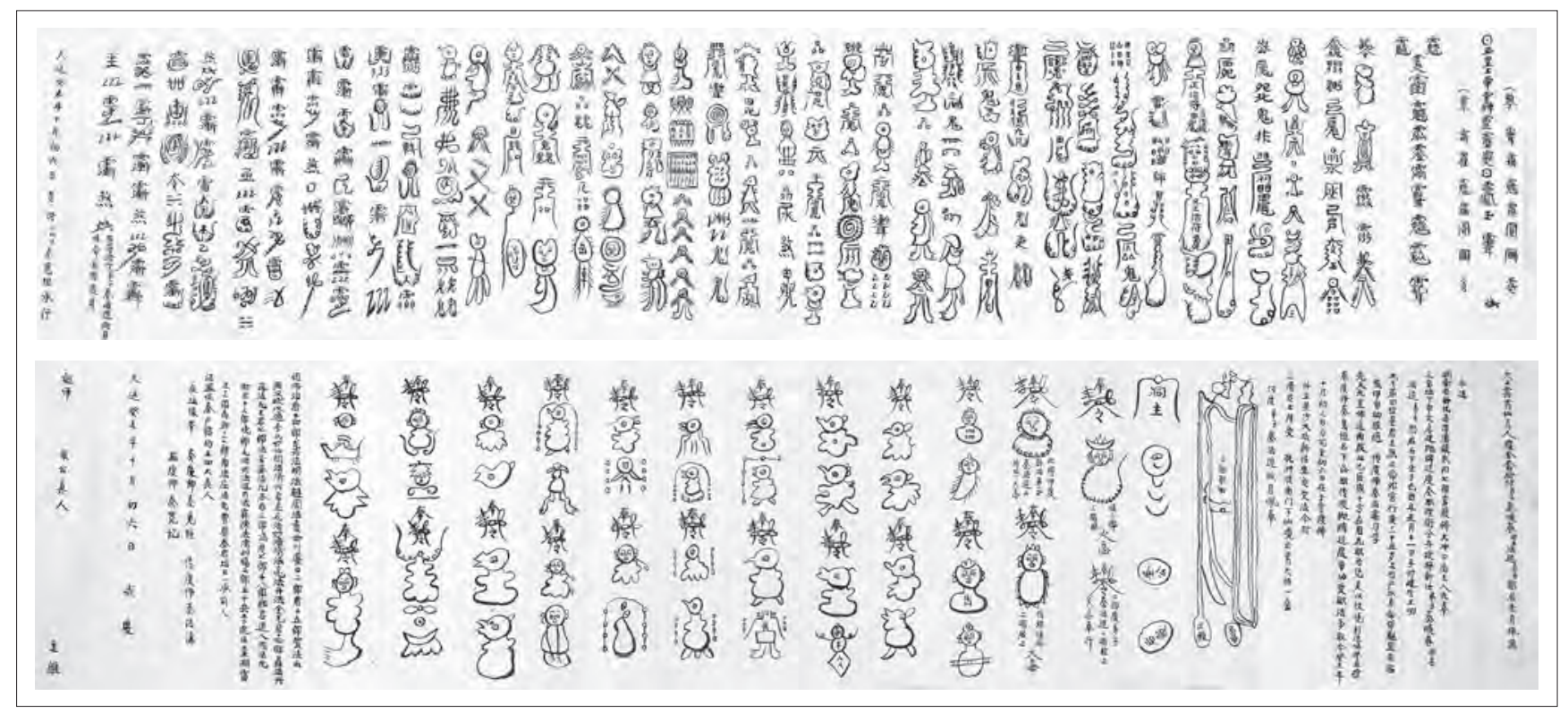

Figure 25.3 Two Ordination documents prepared by Master Qin Guorong 秦國榮 for his disciple. 'All these soldiers and generals will accompany the newly ordained ritual master and will protect him'. Original size: $245 \times 28 \mathrm{~cm}$ and $160 \times 28 \mathrm{~cm}$

manuscripts in Master Yi's possession were kept secret until he let us photograph them for the purposes of our research.

Editing The Taoist Canon: A Historical Companion to the Daozang, Kristofer Schipper separated the texts into two categories: those for restricted distribution and those for general transmission. As he points out in the general introduction:

The vast majority of the books in the Daozang are those that in principle should be transmitted only within the framework of initiation and ordination... . Before the commercial production of the Ming Canon of $1926,{ }^{4}$ these texts were normally not available in general libraries. Not all works contained in the canon were, however, subject to ritually prescribed transmission. Most writings by philosophers, doctors, historians, and the like, were intended for everybody and thus circulated freely. These texts can therefore also be found outside the canon. The distinction between books in general circulation and those whose distribution was ritually restricted is of great importance for the study of Taoist literature. ${ }^{5}$

I visited Master Yi together with Professor Liu Jinfeng 劉 勁峰 while researching the relationship between Nuo theatre (nuoxi 儺戲) and Taoism. In 30 years of participant observation of Taoist ritual, this was the first time I had come across documentary and illustrative evidence of the secret knowledge and practice that is at the heart of the Taoist Liturgy. Master Yi is actively involved in the local Taoist community where he is the Gaogong 高功,

\footnotetext{
4 I.e. the first reprint of the Zhengtong Daozang by the Commercial Press (Hanfenlou 涵芬樓) in Shanghai.

5 Schipper and Verellen, 2004, p. 4.
}

the highest officiant in the Taoist ritual known as the jiao 醮, or sacrifice. ${ }^{6}$ Despite excellent studies of Taoist body illustration, no published research has previously linked these two charts by considering together the relationship between the internal body structures and a spiritual journey through the celestial realm, as two aspects of the same practice. ${ }^{7}$ The title, Laojun gulou 老君骷髅 (Chart of the Skeleton of Lord Lao), (Fig. 1) employs the term 'skeleton' in the metaphorical sense evoked by Schipper when he wrote: One must have 'the bones', the skeleton of an Immortal, to qualify for the dignity of being the representative of heaven and the master of the gods. This genetic quality is not exactly a question of physiology or of physiognomy (although a tao-shih must be of sound mind and body); 'the skeleton' is a hidden quality, an inner quality that expresses itself, perhaps unexpectedly but nonetheless significantly, in the special privilege of being the keeper of the sacred writings. 8

6 The jiao is a community sacrificial ritual, usually performed to affirm the contractual association between a community and its tutelary deity, which can last up to a week. While some elements are open for public interaction (e.g. offerings of sacrificial food, juggling displays and other performances), the central rites take place in a closed temple to which only the primary priests of the rite and certain members of the community have access. Jiao rituals can be performed on a regular basis, once every three or more years, or they can be offered to prevent calamities of a specific kind.

The only European-language study of these images of the body to date is Despeux 1994.

8 Schipper 1983, p. 58 . 
To expand upon Schipper's insight, one might add that the cosmic body of Laozi, as it is represented here with its deities and celestial bodies, does not set out to represent a mundane physiology, but the Taoist body as it is cultivated over several years under the formal instruction sanctioned through the ordination ceremonies variously known as $z o u z h i$ 奏職 (bestowal of office), shoulu 受録 (receiving the register), shoujie 受戒 (receiving precepts), chuanjie 傳戒 (transmitting precepts) or shoudao 受道 (receiving the Tao). During this time, the Taoist initiate (daoshi 道 士) receives the liturgical instruments of his priesthood and the register of those gods whom he can command to intervene in his rituals.

While this image of the body of Laozi is a replica of the macrocosm, it also recalls the so-called 'registers' ( $l u$ 等录) which, in the form of cosmic diagrams or lists of deities, constitute a vital part of the alliance between the initiate and the transcendent powers that is established during ordination. In Hunan province, during ordination, ritual masters (fashi 法師) receive a long 'register' including hundreds of national and local gods (difang shen 地方神) gods of the Thunder Department (Leibu 雷部), soldiers and Celestial Marshals, some wielding fierce exorcistic powers, who will, subsequent to the ritual, constitute the specific group that are under the command of the Taoist initiate. ${ }^{9}$ These gods must be familiar to him. Although there are many register charts in the Daozang, until the recent discovery in Hunan of ordination registers for fashi ritual masters, we have had no evidence of contemporary Taoist registers. That is why Schipper has opined that the ordination register $(l u)$ is a 'fictive document'. ${ }^{10}$

The registers must be 'inspected' (yue 閲) on certain dates during meditation sessions to ensure that all the deities are present and correct. The process involves recitation of specific sūtras and breathing techniques that enhance the purification process. Writing about the register Dongxuan lingbao ke zhong fa 洞玄靈寶課中法 (Method for Determining [the Dominant Qi] in each Class [of register] DZ 1246), from the Early Tang (618-907), Schipper opines:

The human body at birth is like the hundun 混沌 [ hundun (lit. chaos) is a central concept in Taoism that describes the moment before creation when nothing is differentiated], but thanks to the revelation of the Zhengyi mengwei lu 正一盟威籙 (Register of the Orthodox Covenant with the Powers), the Qi differentiates and manifests in form: 'the correct spirits of the Three Pneumas $(Q i)$ of the three Cinnabar Fields can change themselves into myriad forms.

$9 \quad$ There are many references to these spirits in Reiter 2005.

10 For a reproduction of a Register from the Daozang, see, for example, Pregadio (ed.) 2oo8, pp. 39-42.
To do this, one must know how to visualise on the inside as well as on the outside (內外兩存)'.11

The Cinnabar Fields are the three alchemical furnaces in which the adept refines the movement and quality of Qi 氣, originally undifferentiated, into the elixir of immortality. Although locations vary within limits, they most usually reside in the lower abdomen, the chest and the forehead, and correlate with the successive transmutation of jing (精, seminal essence) into Qi, and Qi into shen 神 (spirit[s]), the most common transformative process in inner alchemy. The Zhengyi (Orthodox Covenant) sect, to which Master Yi Songyao belongs, is steeped in the culture of inner alchemy and its traditions of visualisation of the Cinnabar Fields.

The guan 冠 (crown) is the headdress par excellence worn by the Taoist master at the major ritual ceremonies. Once rolled into a chignon on top of the head, the master's long hair is fastened with a flame-like cap (or a three-headed flower hua 華). This marks the place where the gods ascend and leave the body, moving in harmony with the rhythm of the breaths of the master. From the crown, we can identify that Laojun gulou is an illustration of the body of a Taoist master as linked to its liturgical functions. Beginning at the top of the body, marked at the summit of the skull, are the Three Purities, or Three Pure Ones: Jade Purity (Yuqing 玉清), Highest Purity (Shangqing 上清), and Supreme Purity (Taiqing 泰清). The Three Purities are the highest deities of Taoism; they represent the Tao, but they are also representations of the gods and the palaces where they live - the ultimate destination of the Taoist master ascending to heaven with his petition (chengci 呈詞).

Figures 4 to 6 are modern representations of these palaces from the Daqiantu shuo 大千圖說 (Explanations of One Thousand Illustrations), published in 1916.

The two eyes of Lord Lao are identified with the sun and the moon (taiyang 太陽 and taiyin 太陰), and the Celestial Eye (tianmu 天目) in between. The nose is the location of the Grand Unity's Prefecture of Thunder (Taiyi leifu 大乙雷 府). Flanking this on both sides are the Celestial Emperor,

11 Schipper 1973. The Zhengyimengwei lu (DZ 1208) is a Song dynasty (96o-1279) text on the 24 registers that the initiate can receive during his life. The first is intended for children from seven to eight years, and comprises one general, while the last is reserved for couples and includes 150 generals. These 24 registers are interpreted as an image of 24 dioceses of the Celestial Masters and the deities who inhabit them. Incorporating them into his own body through the alchemical process of refinement (liandu 煉 度), the initiate can then appeal to the deities and encourage them/coerce them to intervene. Schipper and Verellen 2004, pp. 971-2. For the Dongxuan lingbao ke zhong, and particularly the Zhengyi registers, see Schipper and Verellen 2004, p. 472. 


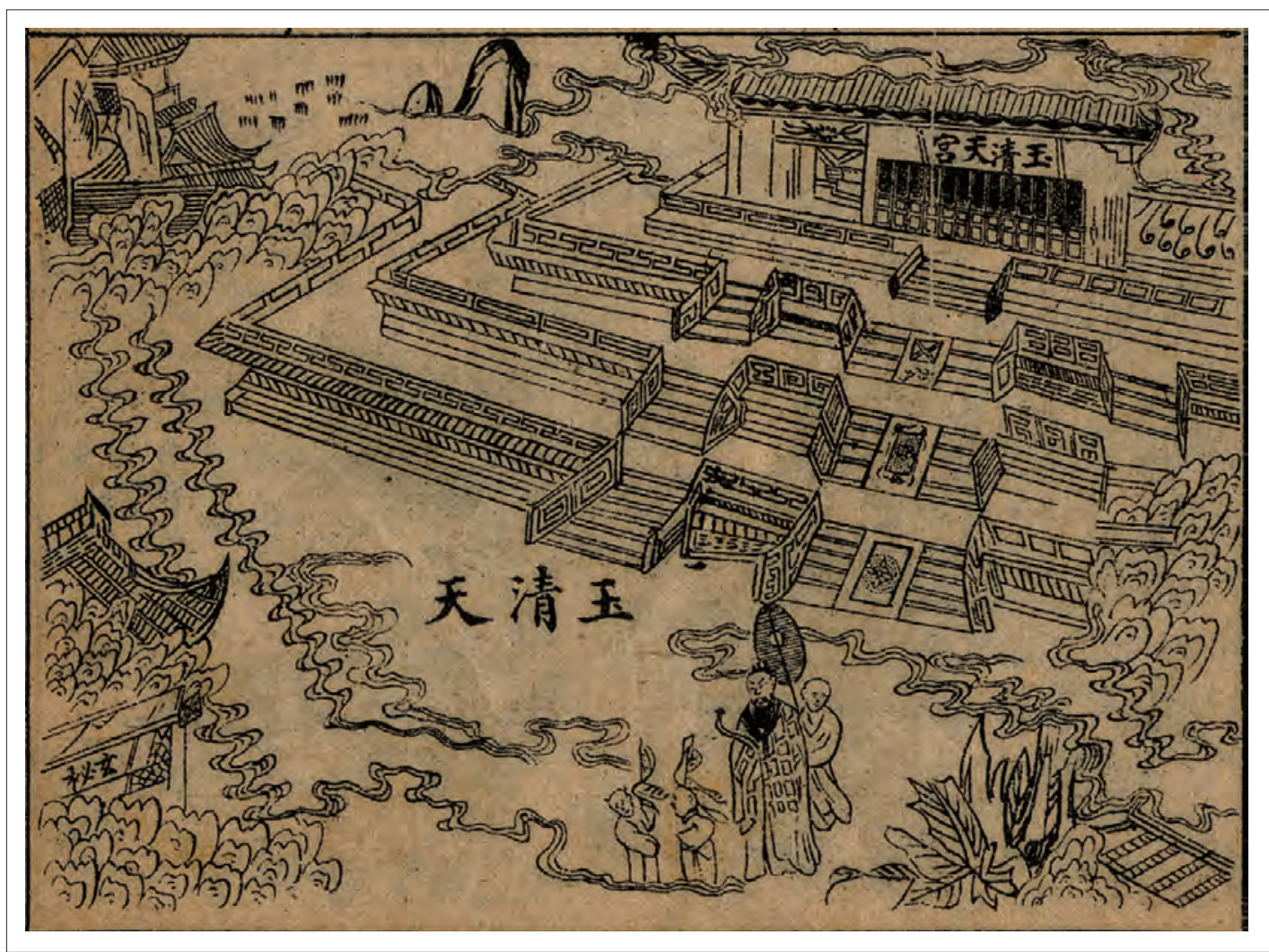

Figure 25.4 Heaven of Jade Purity (Yuqing 玉清), residence of the Celestial Worthy of the Primordial Beginning (Yuanshi Tianzun 元始天尊)

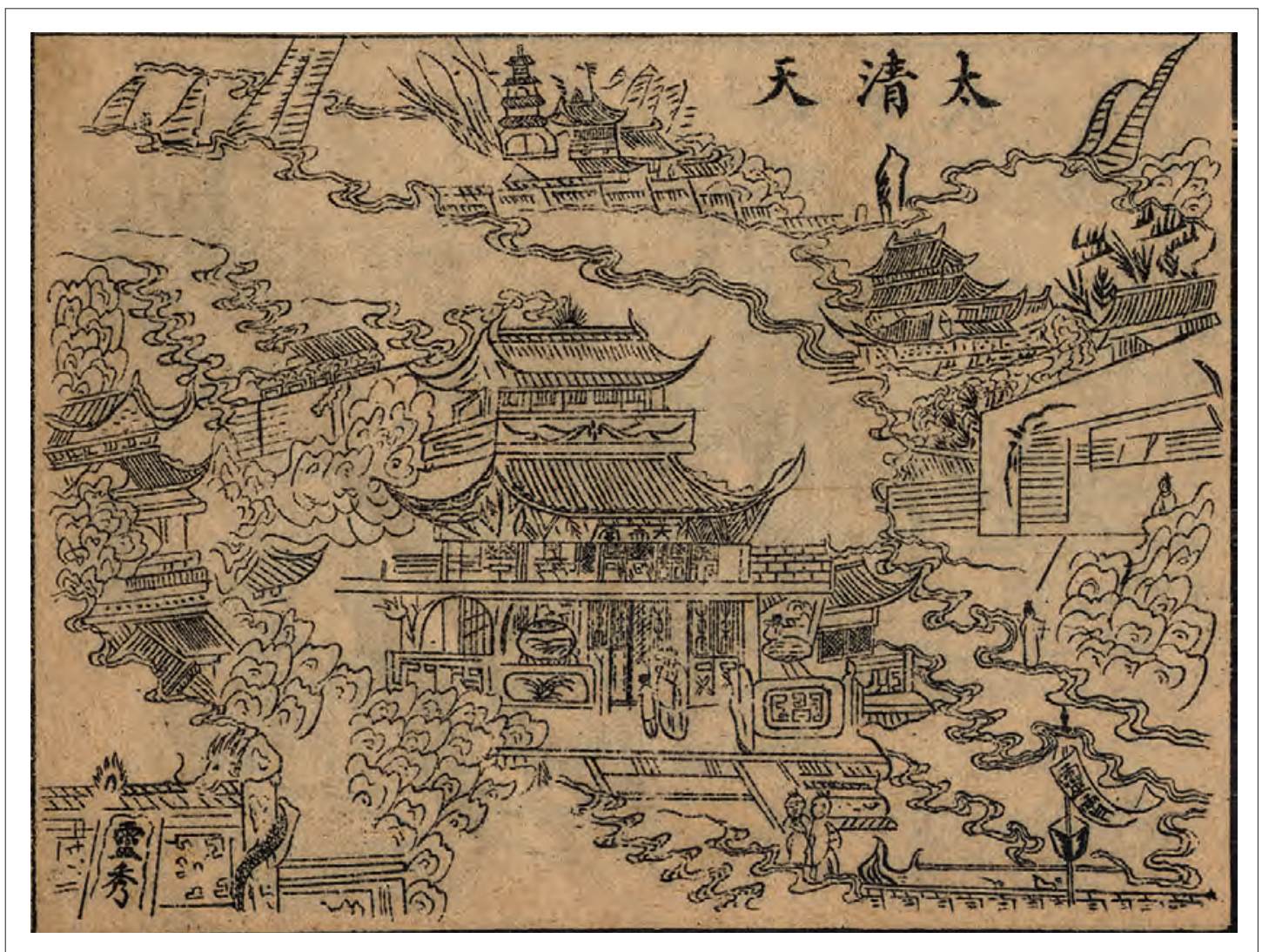

Figure 25.5 Heaven of the Supreme Purity (Taiqing 太清), residence of the Celestial Worthy of the Sacred Jewel (Lingbao Tianzun 靈寶天尊) 


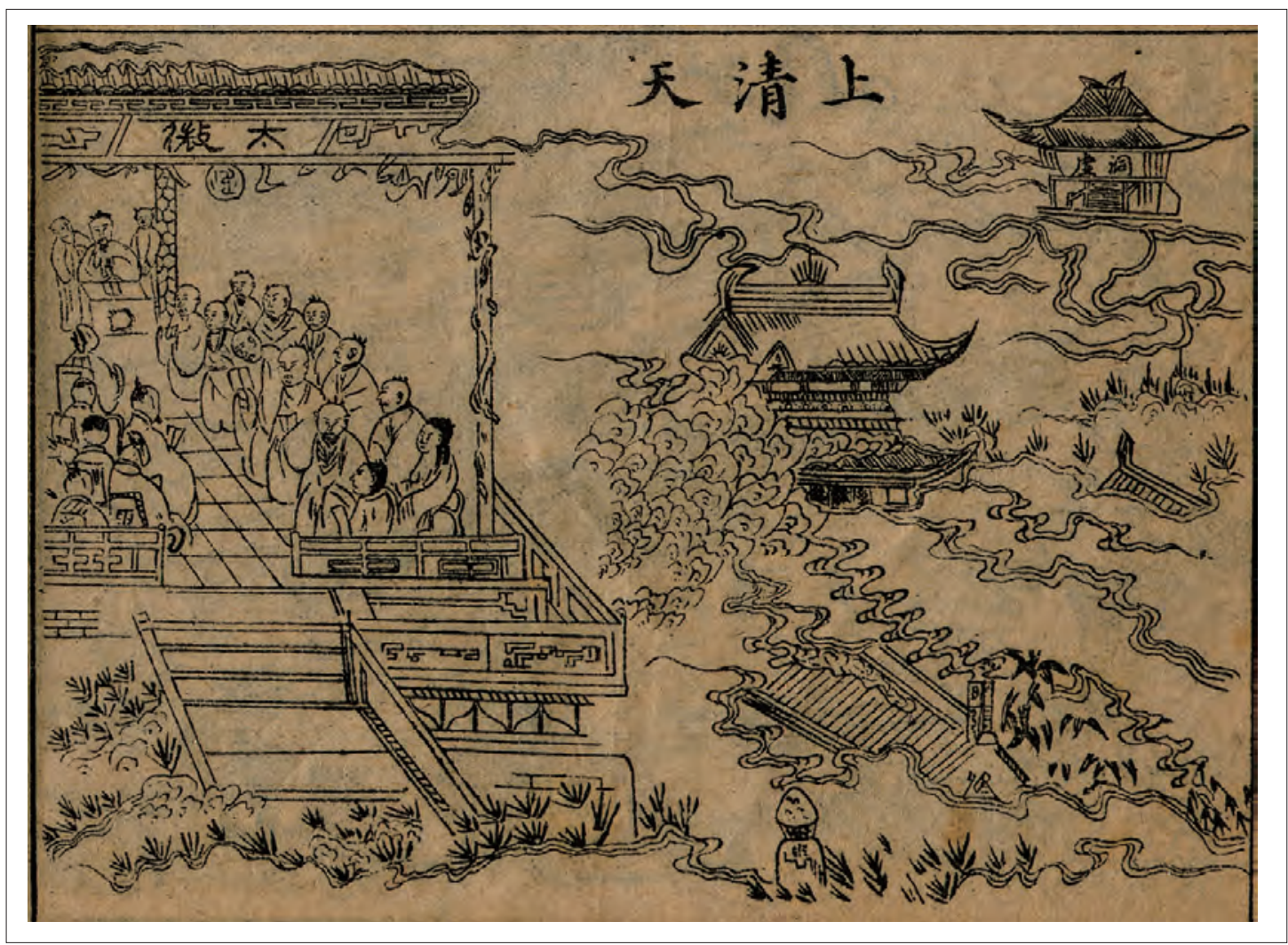

Figure 25.6 Heaven of the Supreme Purity residence of the Celestial Worthy of the Tao and its Potency (Daode Tianzun 道德天尊)

Great Unity (Taiyi tiandi 大乙天帝) and the Celestial Net (tiangang 天罡), which includes both the star and Ursa Major. The right side of the face is designated as Yin and the left as Yang. The mouth is called the Gate of Man (renmen人 門). The titles Fengbo 風伯 (Lord of the Wind) and Yushi 雨 師 (Rain Master) stand as metaphors for breath and saliva. On the left and right cheek are two major centres of Taoist administration, the Mysterious Province of Light (leiting xuansheng 雷霆玄省) and the Capital of Great Mystery (taixuan dusheng 泰玄都省).

The ears are interpreted as musical receptors: on the left is written 'Large Golden Bell' (jinzhong 金鐘), 'Small Fire Bell' (huoling 火零) and 'Sky Drum' (tiangu 天鼓); and on the right ear, 'Jade Chime' (yuqing 玉磬), 'Small Bronze Bell' (tongling 銅鈴) and 'Sky Drum'. In Taoist cultivation, these terms refer to 'knocking the teeth', an ancient practice which occurs in a variety of contexts, including yangsheng 養生 (nurturing life) practices for health, exorcistic ritual, and entering or departing a ritual space. In hygiene practices it is associated with the production of elixir in the form of saliva from the jade pool under the tongue, which was thought to have rejuvenative qualities. These liturgical instruments are used in ritual and produce a celestial music that invites the gods to descend and participate in the dramatic performance of Daoist ritual. While the drum, cymbals, gong and bell are all percussion instruments, there are also wind instruments (flutes or suona 嗩呐) and string instruments (nanhu 南胡 and erhu二胡).

The throat is the channel of communication between the two alchemical furnaces, the Upper Cinnabar Field (shang dantian 上丹田) and the Middle Cinnabar Fields (zhong dantian 中丹田). Under the character 'hou' 喉 (throat) is written Twelve-Storey Building (shi'er chong lou 十二重 樓), sometimes represented in the form of a pagoda and referring to the layers formed by the cartilage of the throat that one can palpate and see outlined at the front of the neck. On both sides, we find the Administrative Offices of the Three Origins (sanyuan kaojiao fu 三元考較府) and the Prefectural Offices for the Three Officers (sanguan dadifu三官大帝府), the Palace of Pure Emptiness (qingxu fenyang gong 清虛紛陽宮) and the Court of the Four Saints of the Far North (beiji sisheng yuan 北極四聖院). Two other administrations are referred to at the left and right shoulders: the Great Hall of the Celestial Talisman (tianfu dadian 天符大殿) and the Censorate of the Commander of Pestilences (wensi xianfu 瘟司憲府). The name of the seventh office of the celestial administration, the Crimson Palace of the Fiery Heart (huoxin jianggong 火心絳宮), is inscribed on the central axis and is associated with the Vermilion Bird (zhu que 朱雀) and Summer Thunder (xialei 夏雷). The Vermilion Bird is one of the animals that represent the constellations of the South. References to 
Summer Thunder, and other terms that I will come to later, associate this chart with the liturgy of the Five Thunders (wuleifa 五雷法), with which the Daoist initiate will light his internal fires. The fires then ascend into the Fire Palace of the Heart, here located in the lower chest. Visualising the fire ball ascending through the heart and entering the niwan (mud ball), at the brain in modern-day terms, the adept's ultimate aim is to nurture the infant of immortality.

Common to the three charts at the top is mention of the Three Pures (sanqing 三清), their residences on the one hand and on the other the names of the deities imprinted on Laozi's forehead. There are also Tianpeng 天蓬, Tianyou 天猶, Yisheng 翌聖 (aka Heisha 黑殺, the Black Killer) and Zhenwu 真武, martial deities and commanders of their own spirit generals, who became associated as the Four Saints (sisheng 四聖) in the Song dynasty and were called upon to assist in exorcistic rites. They are the subject of, for example, the Song text Taishang jiutian yanxiang di'e sisheng miaojing 太上九天延祥滌厄四聖妙經 (DZ 26). Mention of the Four Saints links the Laozi body chart specifically to the exorcistic traditions within the liturgy of the Five Thunders that dates to Song times. ${ }^{12}$ Further details of our 17 th-century charts will testify to a direct association with the Song liturgy, introducing radical changes in Taoist organisation and ritual. Scattered around the chest, stomach and arms, there are multiple references to the Five Thunder Rites: the seasons, the calendrical spirits, the 24 divisions of the year, the five viscera, and the heraldic animals, i.e. the Green Dragon, White Tiger, Vermilion Bird and Black Tortoise.

Expressions such as huibing 會兵 (assembled troops) and jibing 寄兵 (despatched troops), together with the Four Saints, and in particular Heisha, associate the exorcisms in our chart with the exorcistic rituals of the Thunder liturgy or Thunder Magic (leifa 雷法) as they are described in the 14th-century text Daofa huiyuan 道法會元 (Taoist Method, United in Principle), where the same expressions are also found. These are all troops in the celestial militia in the service of the master during the ritual. They are the souls of the deceased, recruited from the 84,ooo gui 鬼 (ghosts) that reside under the sole of the left foot and the 3,700 under the right foot. The body thus depicted is both an alchemical crucible and a world in miniature in the image of the cosmos, populated by a host of spirits and deities.

As a representation of the sites for refinement and circulation of Qi, the body is a dynamic system interacting with the cosmos. The interactive quality of the Taoist body is a manifestation of the resonance between state, cosmos and

See, for example, all the references to these Celestial Marshals (yuanshuai 元帥) in Davis 2001. body so eloquently described for medicine in the classical period by Nathan Sivin. Qi, or shenqi 神氣 'numinous Qi', permeates the universe and body alike. Chinese medicine, Sivin remarks, remained largely holistic: 'the resonance of the body with natural process is an ideal toward which man must strive; ignoring it brings on disease'.13 Lord Lao's body contains elements that link canonical medicine, structural anatomy, alchemy and meditation techniques. But above all it is distinguished by its lists of deities and ritual spaces, which uniquely chart the body according to Taoist liturgy - that is to say it is the body of the Taoist master as he works through the prescribed ritual activities and transforms himself and those around him.

If we focus on the performative function of the text, there are two terms written on the two middle fingers, yuwen 玉文 (the Jade Wrinkles) at the middle phalanges and $w u$ 午 at the tip of the finger, which have a specific function. ${ }^{14}$ They simultaneously correspond to constellations, calendrical data and/or internal Qi. By pointing with his thumb to yuwen or $w u$, the master links his own body to the external world, remotely exerting control over external phenomena, and mobilising the gods and deities. Listed at the index finger, the Chariot of Fire (huoche 火車) is the divine vehicle pulled by dragons in which the gods travel between worlds. Another reference to the liturgy of Thunder is the inscription on the left forearm of the name of the King of Changsha who Destroys Temples (damiao Changsha wang 打廟長沙王) and on the right forearm that of General Liu (Liu jiangjun 柳將軍), both major exorcists in the Ministry of Thunder.

As in almost all maps of the Taoist body, the Court referred to is the Yellow Court (Huangting 黃庭), a term that first emerges in the 2nd century and reminds us of the antiquity of notions of inner landscape, the gods of the body and transmutation. Huangting jing nei/waijing 黃庭 經內/外景 (Canon of the Yellow Court: 'Inner Landcape' and 'Outer Landscape'), two meditational manuals used in the Shangqing 上清 (Supreme Purity) tradition of Taoism, contain the earliest writings to link the microcosm of the body with a list of its resident gods. This was later absorbed into xiuzhen 修真 (cultivating perfection) practice. ${ }^{15}$ Since then, the image of the body-microcosm has evolved differently in different contexts, but the fundamental concept of the human body as a replica of the macrocosm is shared by medical and religious cultures. With this map of the skeleton of Taishang Laojun, Taoists show clearly how they

\footnotetext{
13 Sivin 1995.

14 The Jade Wrinkles are the horizontal wrinkles of the middle phalangeal joints of the hand.

15 See the introduction to Schipper et al. 1975, p. ii.
} 
have abolished the boundaries between the cosmos and the physical body. ${ }^{16}$

Progressing downwards, we leave the territory of the myriad gods of the Earth (dijie wanshen zhi suo 地界萬 神之所) to enter the underworld, which is located in the lower body. Here we find Mount Fengdu 豐都山 and its 36 courts of Justice (Fengdu sanshiliu 豐都三十六), ${ }^{17}$ Iron Encircled Mountain (tieweishan 鐵圍山), Mount Taishan, one of the Five Sacred Peaks (wuyue taishan 五获泰山), the Small and Large Iron Courts (xiao tieyuan 小鐵院, da tieyuan 大鐵院) - all symbols of a formidable penal system - together with the City Thearchs (chenghuang 城隍), who represent the underworld above ground, and the gods of the Five Prefectures and Nine Provinces (wufu jiuzhou zhi shen 五府九州之神). Here too is Winter Thunder (donglei 冬雷), coupled with the True Warrior (Zhenwu 真 武), regarded as a powerful deity and exorcist, who reigns over the darkness of the netherworld and is symbolised by the tortoise and snake, and the Dragon Kings (longwang 龍 王), who are water gods and also reside in the lower body.

The inscriptions around the periphery of Laozi's body provide us with more information. Proceeding from head to toe, at the top and corresponding to Heaven and the Upper Cinnabar Field, are the Palaces of the Five Emperors, the couple Xi Wangmu 西王母 (Mother Queen of the West) and Dong Wanggong (Duke of the East 東王公) as well as the names of several constellations. In the ritual transformation of body and space (bianshen biantan 變身 變壇), which is enacted during the creation of the sacred internal space, the Taoist master visualises a whole army of celestial soldiers leaving his body through his four limbs. Most of the deities, generals and officers mentioned in the body chart of Lord Lao are the deities that the adept

16 Despeux 1994, p. 52; Pregadio 2008, 'Neidan', pp. 762-6.

17 The numbers of the courts of justice vary in Taoist tradition. Here there are 36 . At the Taishan or Dongyue temples there are 70 or 76 . In the Jin 晉 dynasty Zhengao 真誥, which contains extensive descriptions of Fengdu, there are only six outer and six inner palaces. The locus classicus for 36 heavens of Shangqing occurs in a later Shangqing text, the Waiguo fangpin qingtong neiwen 外國放品青童內文, DZ 1373. This was later codified in the Tang into a system linking three and nine heaven groupings, which itself produced other variants. One frequently mentioned is found in the Yunji qiqian 雲篖七籤 (Seven Tablets from the Cloudy Satchel), a Song Daoist encyclopaedia, where there is one Daluo Tian 大羅天, three Purities (sanqing 三清), four heavens for the Seed-People (zhongmin 種民), four realms of Formlessness (wuse jie 無色界), 18 realms of form (sejie 色 界) and six realms of desire (yujie 欲界). There is also an early reference in Weishu 魏書 114.3052 to 36 heavens, linking it to a genealogy of the Celestial Masters sect. has received during his ordination. As the ritual evolves, he lets them intervene at strategic moments, and as the ordination draws to a conclusion, they return to his body. In acknowledgement of the work done, both master and deities receive a promotion. As Schipper has often pointed out, rituals denoted as $k e$ 科 refer to a kind of 'upgrading'. The practice of internal alchemy (neidan 內丹) shared techniques and goals with Taoist ordination ritual, before autonomous individual practices were absorbed into the more widespread yangsheng practice of lay people, neiguan 內觀 (inner observation or inner contemplation), and shouyi 守一 (preserving unity).

Min Zhiting 閩智亭, once the best known representative of modern Taoist organisations at international ecumenical meetings, and author of Xuanmen risong zaowankejingzhu 玄門日誦早晚功課經注 (A Commentary on Morning and Evening Rituals), has described one of the eight secret formulas for purification of the mouth (jingkou shenzhou 淨 口神咒), a technique which is accompanied by alchemical techniques (xiulian 修練). Invoking the gods of the teeth, throat, Qi, heart and thought, the adept recites the formula, and the gods of the mouth (all mentioned in the Book of the Yellow Court) return to their respective places in the body. Maintaining the gods in their designated locations, the adept can be united with the Tao (yu dao hezhen 與 道和真). ${ }^{18}$

The sending of petitions and memorials to Heaven has been part of the Taoist liturgy since the foundation of the Celestial Masters Sect 天師道 (Tianshi dao) in the 2nd century, especially during the healing rituals of the three major Taoist assemblies of the year that are held in honour of the agents of Heaven, Earth and Water. Several scroll paintings illustrate the ascent to Heaven undertaken by Taoist masters with their documents in different ways. Our second chart from Master Yi's collection is one of these illustrations, and a study of the performance of the ceremony reveals how aspects of the ritual body are connected with the Chart of the Skeleton of Lord Lao. The rituals to submit the petitions take different forms, according to particular Taoist schools and local traditions. Taoists from White Cloud Temple 白雲觀 (Baiyun guan) in Shanghai use paper cranes as their intermediaries. In Beijing, the Gaogong 高功 (Grand Master of the Ritual) mimes the master's ascent to Heaven by pacing the patterns of the constellations (bugang 步罡) on a brocade carpet, which is embroidered with the Taiji 太極 symbol surrounded by the Eight Trigrams. He presents the official envelope containing the petition (fanghan 方函). On his dragon robe, a ritual dress which appropriates the power of the

18 Min 200o, p. 20. 

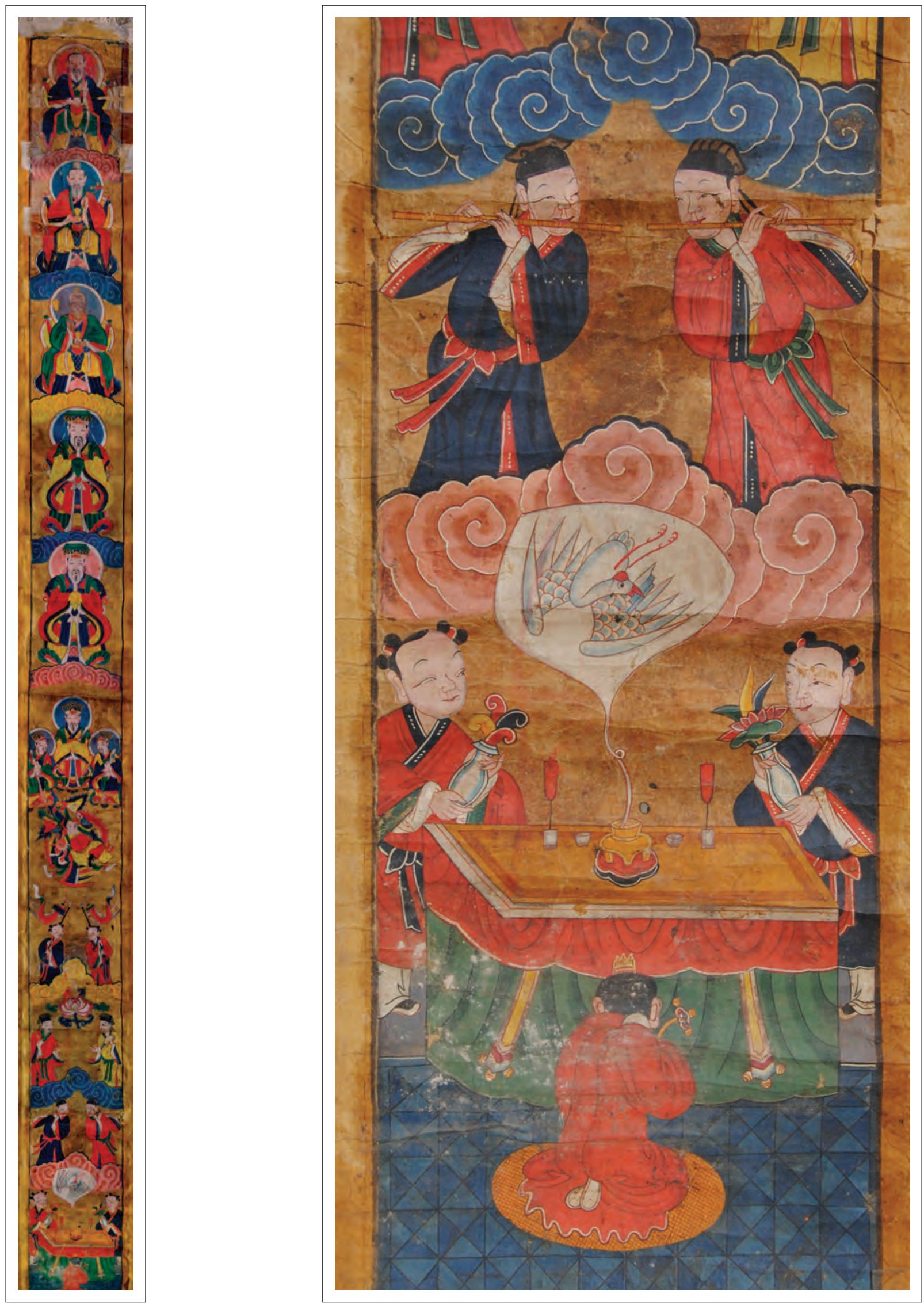

Figure 25.7 Hunan painting describing the submitting of a petition to Heaven (350 x 33cm). Fava: Private collection

Figure 25.7.1 Detail: The Master prostrates himself before the altar. A crane flying out of the incense burner represents his ascentto the domain of the Immortals. 


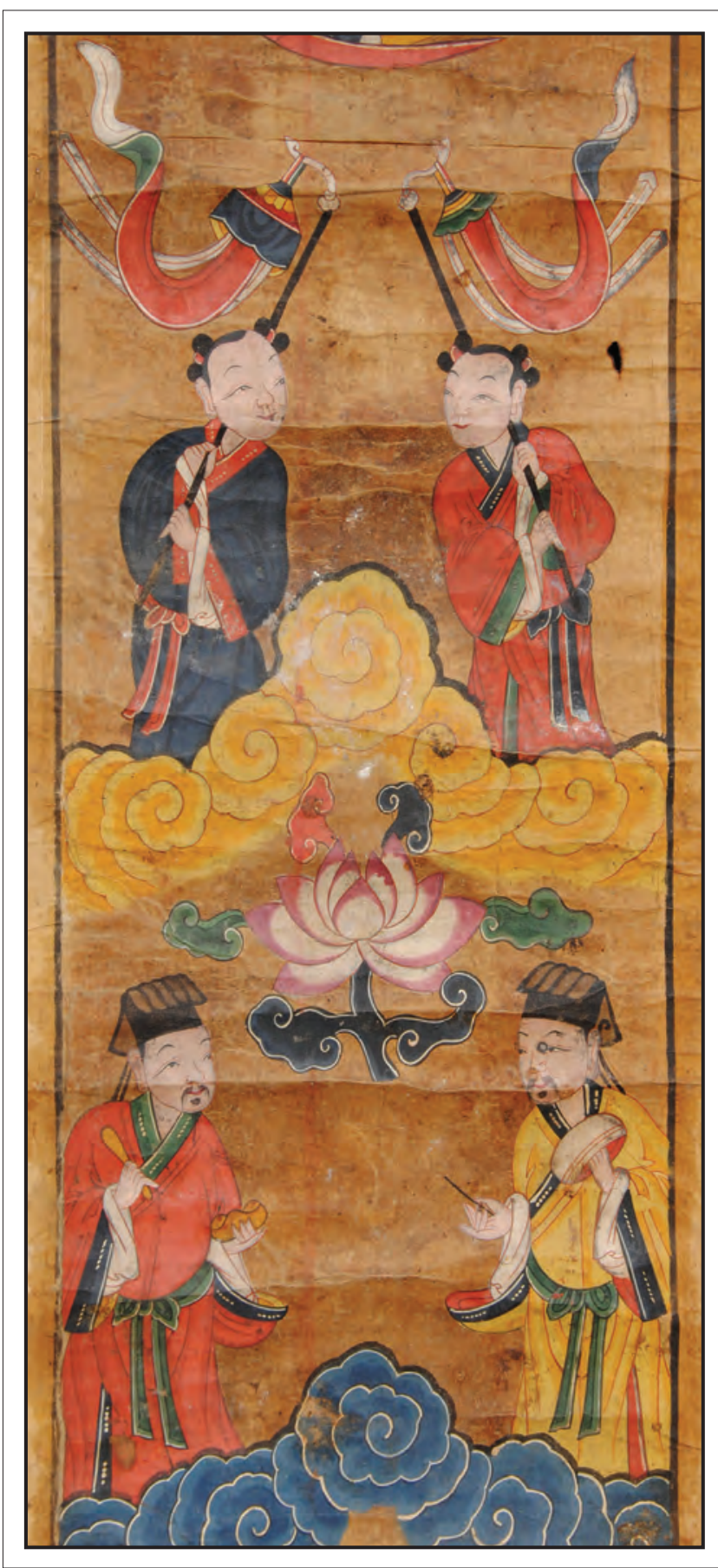

Figure 25.7.2 Detail: Two Taoist musicians and two Immortals

emperor, are hung talismans to protect him and to open the doors of paradise.

Figure 7 is an example of the sort of scroll that I have frequently seen hung by Hunan Taoists outside the entrance to their altar. This scroll probably dates to the Republican period (1912-49), and, at least as a decorative object imbued with ritual power, has a performative function in the ceremonies, where its presence augments the numinous power of the ritual ground. The question of whether it was originally intended as a pedagogical tool

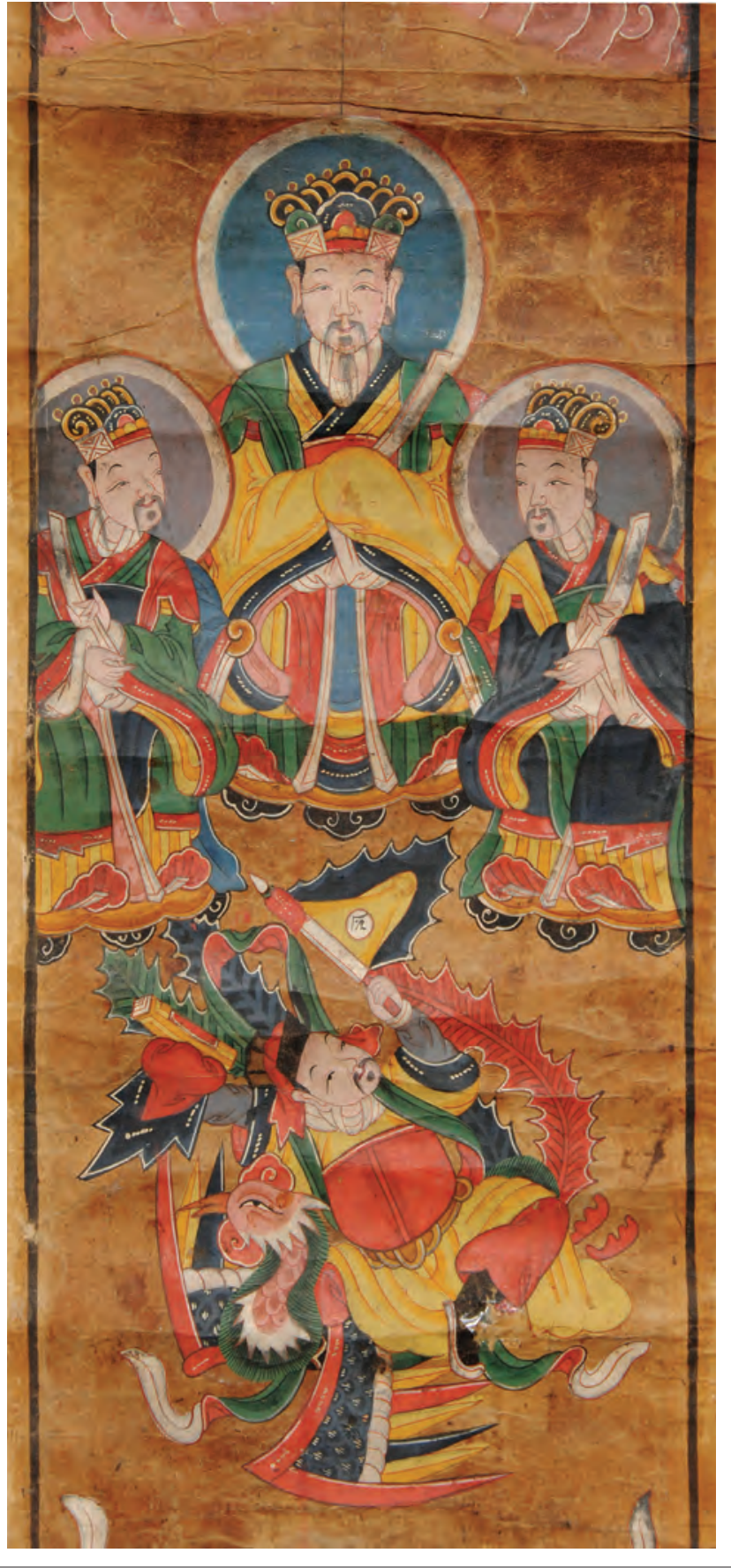

Figure 25.7.3 Detail: The Celestial Emissary (gongcao) and the Three Officers (sanguan)

in the illustration of the ritual process through which this kind of petition should be submitted is a subject for more lengthy analysis, but it certainly serves as evidence for the historical reconstruction of the meaning of Master $\mathrm{Yi}$ Songdao's second chart.

In the Hunan painting, the master or main officiant (gongcao 功曹) respectfully prostrates himself in front of the altar. A crane flies from the mouth of the incense burner, representing the ascent to the domain of the Immortals. The master visualises the journey through the clouds. On 


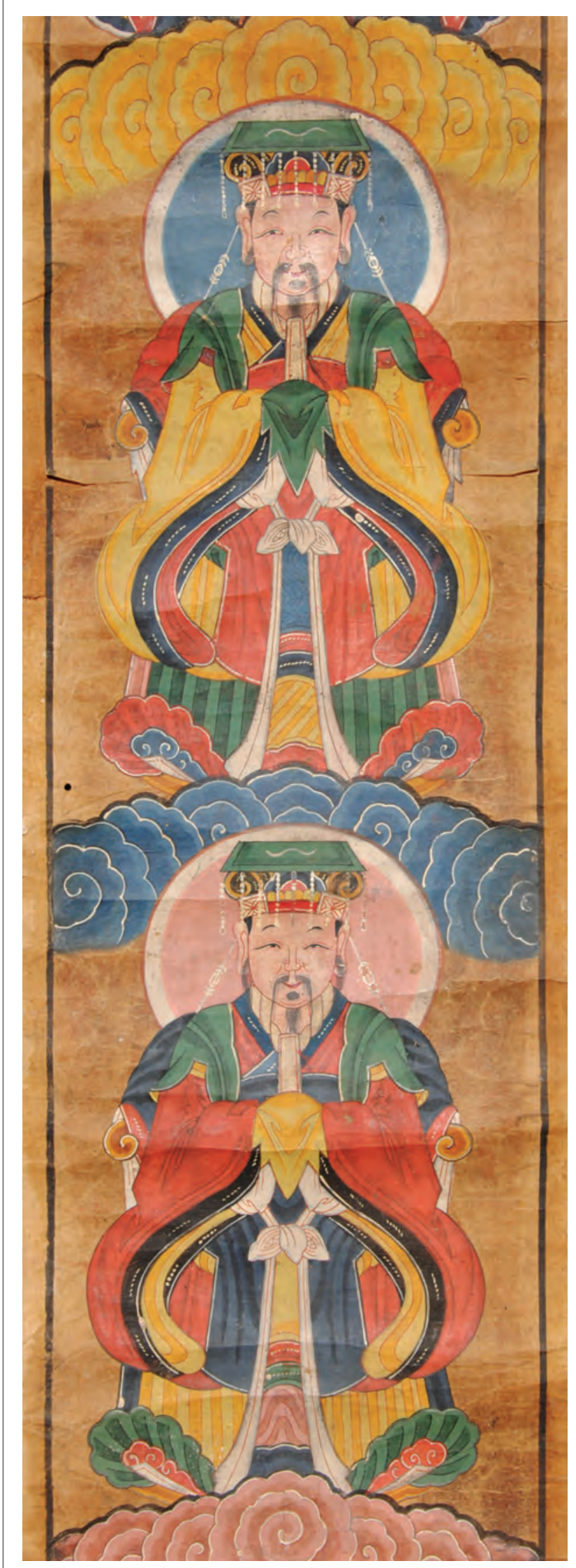

Figure 25.7.4 Detail: The Two Emperors (erhuang 二皇)

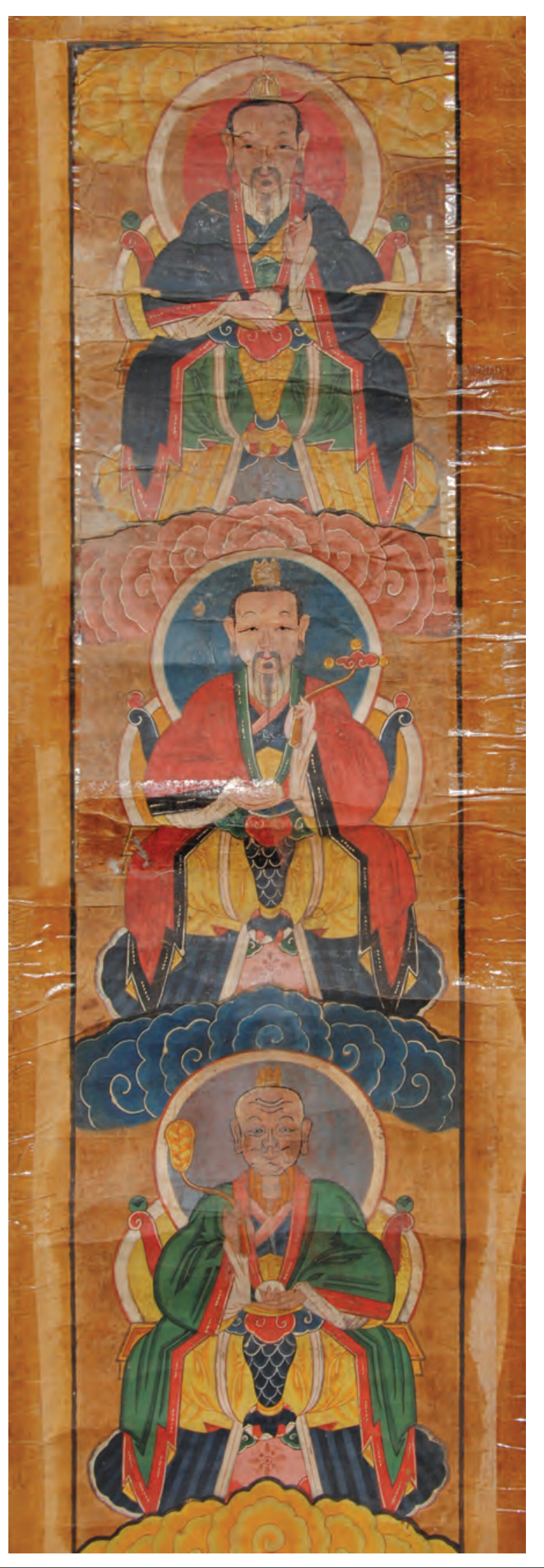

Figure 25.7.5. Detail: The Three Pure Ones (sanqing 三清) 


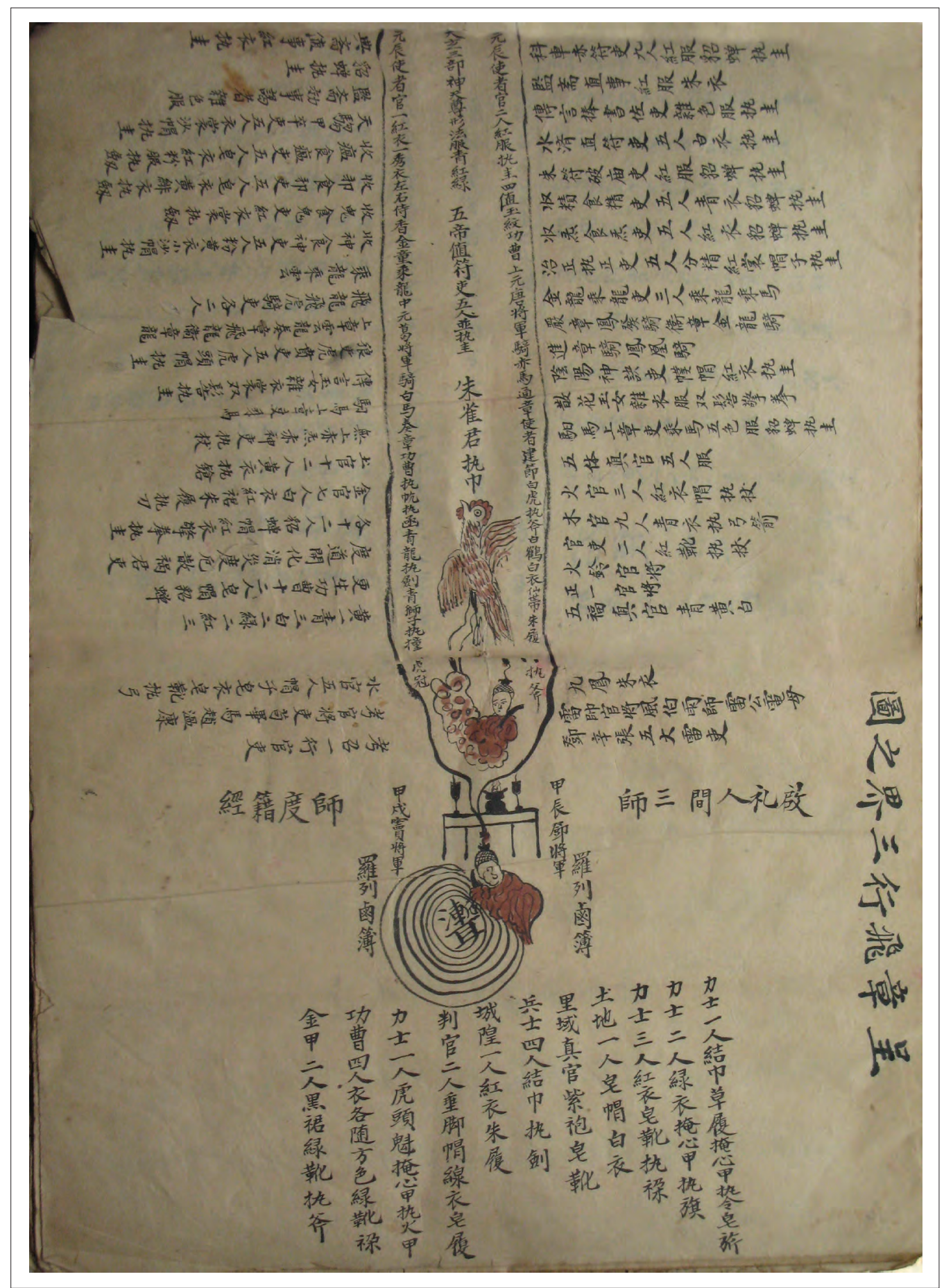

Figure 25.8 Map of the Journey to Heaven of a Taoist master presenting a petition to the Palace of the Three Pures, dating to 1843 Figure 25.8.1 The master kneels in front of the altar. The Vermilion Bird leads the way through the clouds

each side of the altar, two assistants have prepared offerings. After rising through several stages, the master, holding an envelope in which a petition is contained, arrives at the gates of the Palace of the Three Officers 三官 (sanguan), where stand two guardians with their banners. Beyond the gates, he will submit the petition to the Emperor of Heaven 天皇 and the Jade Emperor 玉皇, and then on to the Three Pure Ones 三清, whose names we have also seen inscribed on Lord Lao's forehead.

The petitioner's journey through the Heavens (zhang mi yunlu 章秘雲路) is charted in the second manuscript under consideration, entitled Xian zhang feixing sanjie zhi tu 獻 章飛行三界之圖 (Chart of a Flying Journey through the Three Worlds to Present a Petition) (Fig. 8), also transmitted to Master Yi Songyao by Master He Huaide. It depicts 


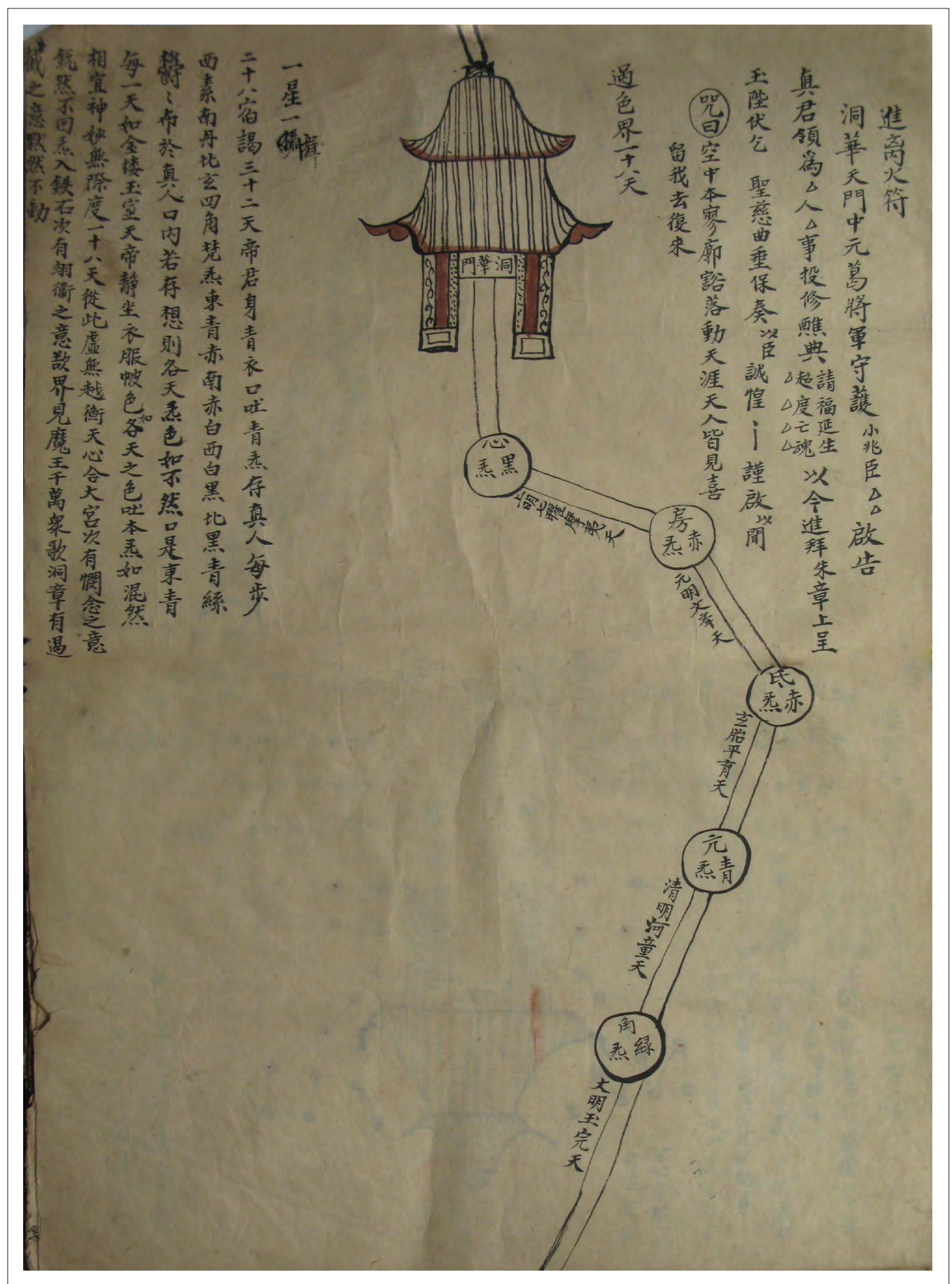

Figure 25.8.2 At the entrance of Heaven, the Crystal Gate

the same journey, but in a much more detailed manner. The chart is in nine pages and dates to the 23rd year of the Daoguang period (1843). The date is written on the inside of the back cover. Master He Huaide indicates that he was ordained under the 6oth Celestial Master Zhang Peiyuan 張培源 (1829-59) and his ordination lasted three days. He mentions where it took place, the name of his master of initiation, Wang Chunhua 王春華, and the title he received.

To our knowledge, there is no other document that records so much information about the celestial journey and the major agents as the Chart of the Heavens manuscript transmitted to Master Yi. It belongs to that genre of books and manuscripts that circulated only within closed lineages. Representing the organisation of the celestial administration and therefore the internal process of the master sending a memorial to Heaven, it reveals the invisible and intimate part of the ritual. This illustration is inserted into a folio of $5^{2}$ pages. The introductory statement explains the journey and all the deities and institutions that the Daoist initiate will encounter on his journey, and the appropriate 


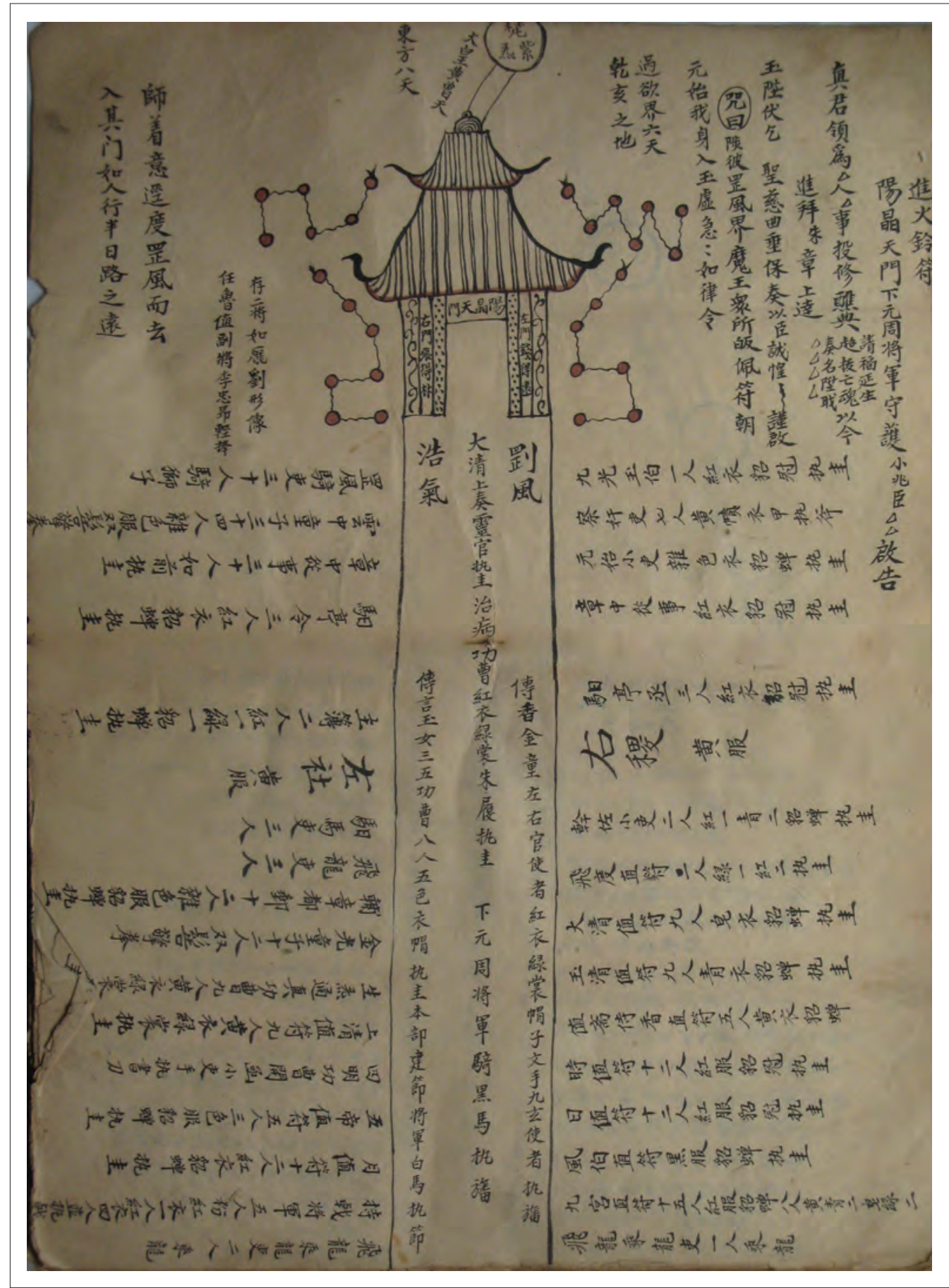

Figure 25.8.3 Beginning of the ascent through the 32 heavens. The master arrives before Donghua Gate, the second gate of heaven.

rituals to perform at each stage. In describing the elements in the illustration, I have relied on these explanations. In this chart, the petitioner is represented as transcending his body through his flame-like cap (which, in contrast, in the scroll illustration, takes the form of a flower on his crown). A little further on, we find that his court tablet (often carved from ivory or peachwood and held high so as not to pollute the face of one's superiors with mortal breath) has shifted onto his shoulder for ease of travel. The master will carry it in front of him when granted a hearing before the Emperor. The bird here is not a crane, but clearly identified and illustrated as the Vermilion Bird, one of four heraldic animals.

Flanking the route chart are the names of a host of emissaries, gods, celestial bureaucrats and different departments and ministries. Continuing the ascent, we traverse three celestial portals: the Crystal Courtyard, the Grotto of the Belles, and Upright Yang - residence of Tang 唐, Ge 葛, and Zhou 周, the generals of the Three Origins. Onwards and upwards we arrive at the Palaces of the Five Agents, 


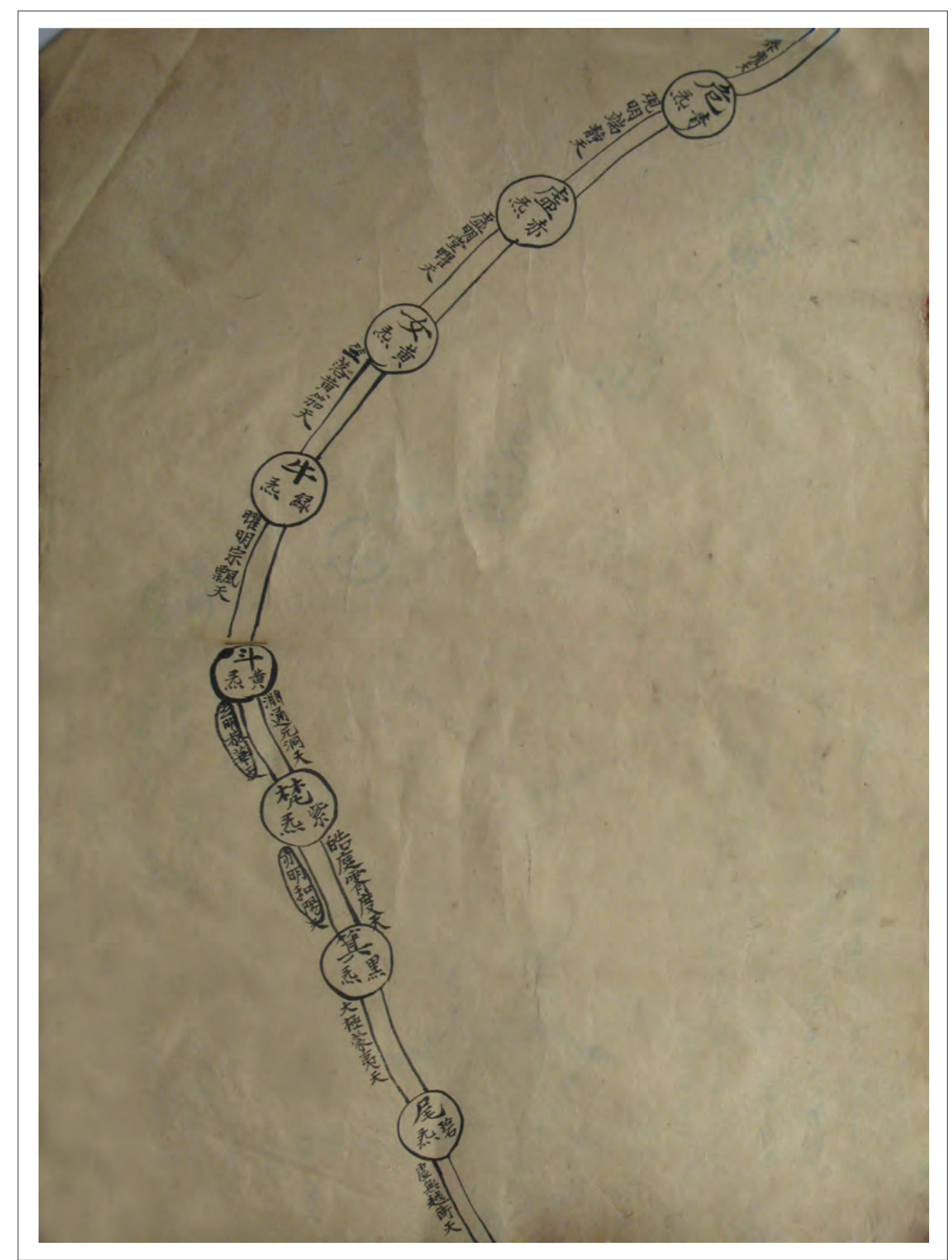

Figure 25.8.4 Across the 28 mansions and the four Brahma heavens

residences of the officers of Fire, Earth, Metal, Water and Wood. The ascent to Heaven continues through the constellations and the 28 lodges, each marked with Qi of a different colour, at the summit of which presides the Pole Star. To the 28 celestial lodges are added the four Brahma Heavens (四梵), familiar from the Lingbao 靈寶 Daoist traditions, and therefore the 32 heavens of the journey through the Empyrean. Proceeding from one position to the next, the master passes 32 named heavens.

Beyond these 32 heavens, the master enters the domain of the Five Officers (guan 官) who represent Fire, Earth,
Metal, Water and Wood. Going up, he passes through the seven stars of the Big Dipper and the crowning Pole Star, Purple Tenuity (ziwei 紫微). Arriving at the constellation of the Three Masters (sanshi 三師), he passes close by the four Directors among the Perfected Lords (sisizhenjun 四 司真君): Tianpeng, Tianyou, Yisheng and Yousheng. Ahead in the centre, he reaches the Palace of the Four Saints listed in Lord Lao's Body chart and pays homage to each of the dignitaries. In the margin, a note indicates that he has entered the three Heavens (santian mennei 三天門內, where he prostrates himself in front of the lineage masters 


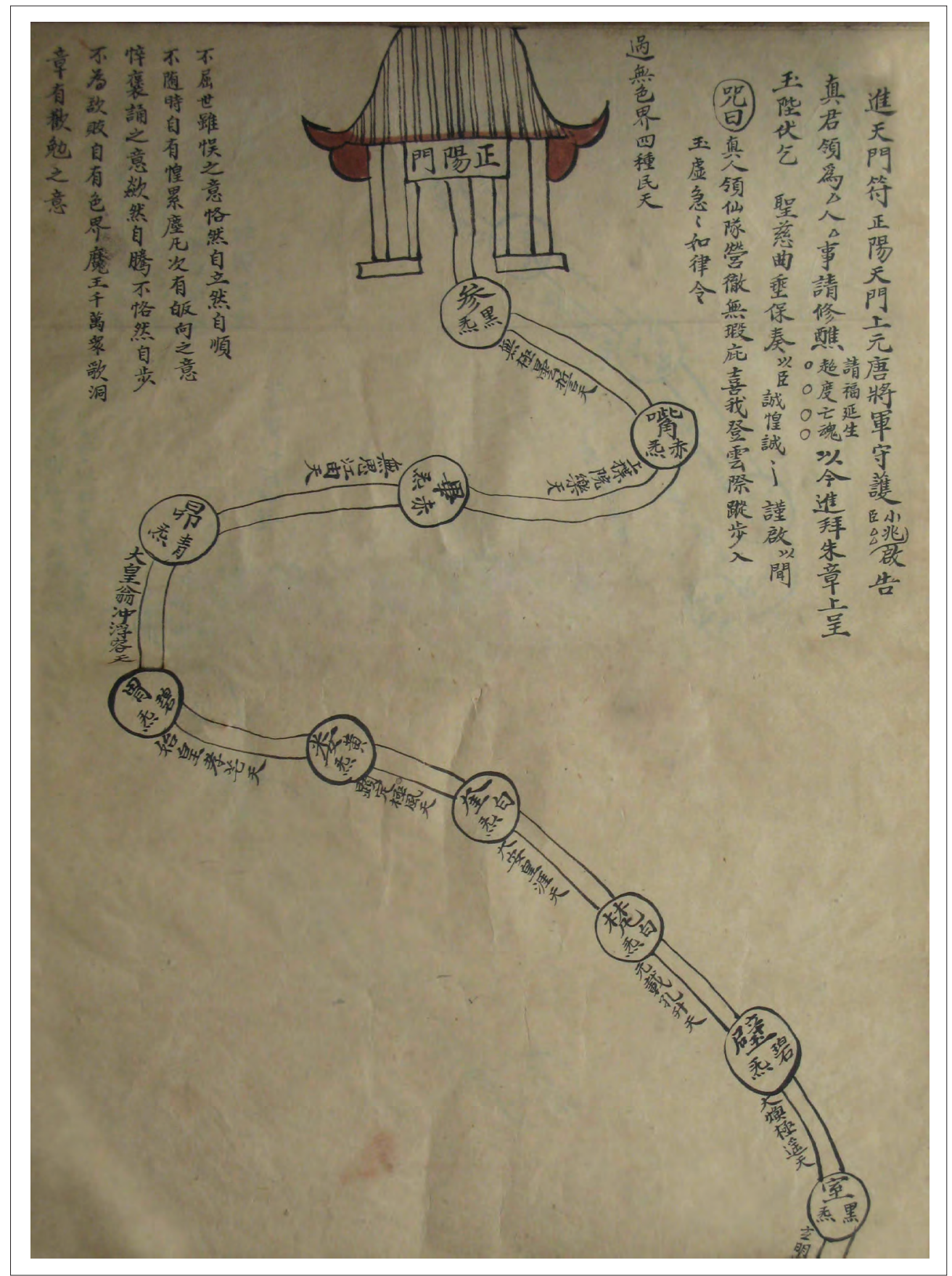

Figure 25.8.5 Arrival at the third Gate of Heaven

of the three previous generations, listed in descending seniority (jing 經, $j i$ 籍, $d u$ 度), and introduces himself and the purpose of his mission to the officiant.

Thereafter he salutes the Lord of the Origins (Yuansheng dijun 元生帝君), the Celestial Worthy of the Dao and its Efficacy (Daode Tianzun 道德天尊), the nine masters ( $j i$ ushi 九師), and the Three Officers (sanguan 三官), before entering the Hall of the Three Mysteries (sanxing dian 三 省殿) and the Court of the Celestial Pivot (tianshuyuan 天 樞院). From that point, he visits the Emperor of the North Pole who is in charge of the Office of Exorcisms (beiji quxie
北極驅邪) and the Emperor of the South Pole, who resides opposite. Now he approaches his ultimate destination and the zenith of the Heavens. After the Gate of the Five Phoenixes (wufeng lou 五風樓), he can see the residence of the Jade Emperor flanked by the Palace of the Pole Star of the Extreme North and the Marvellous and Majestic Palace of the Extreme East (miaoyan gong 妙嚴宮). The manuscript mentions that when the master arrives at the Gate of the Five Phoenixes, he kneels and gazes upon the Jade Emperor with his Celestial Eye (tianmu天目). He also visualises the Celestial Master, who transmits his petition 


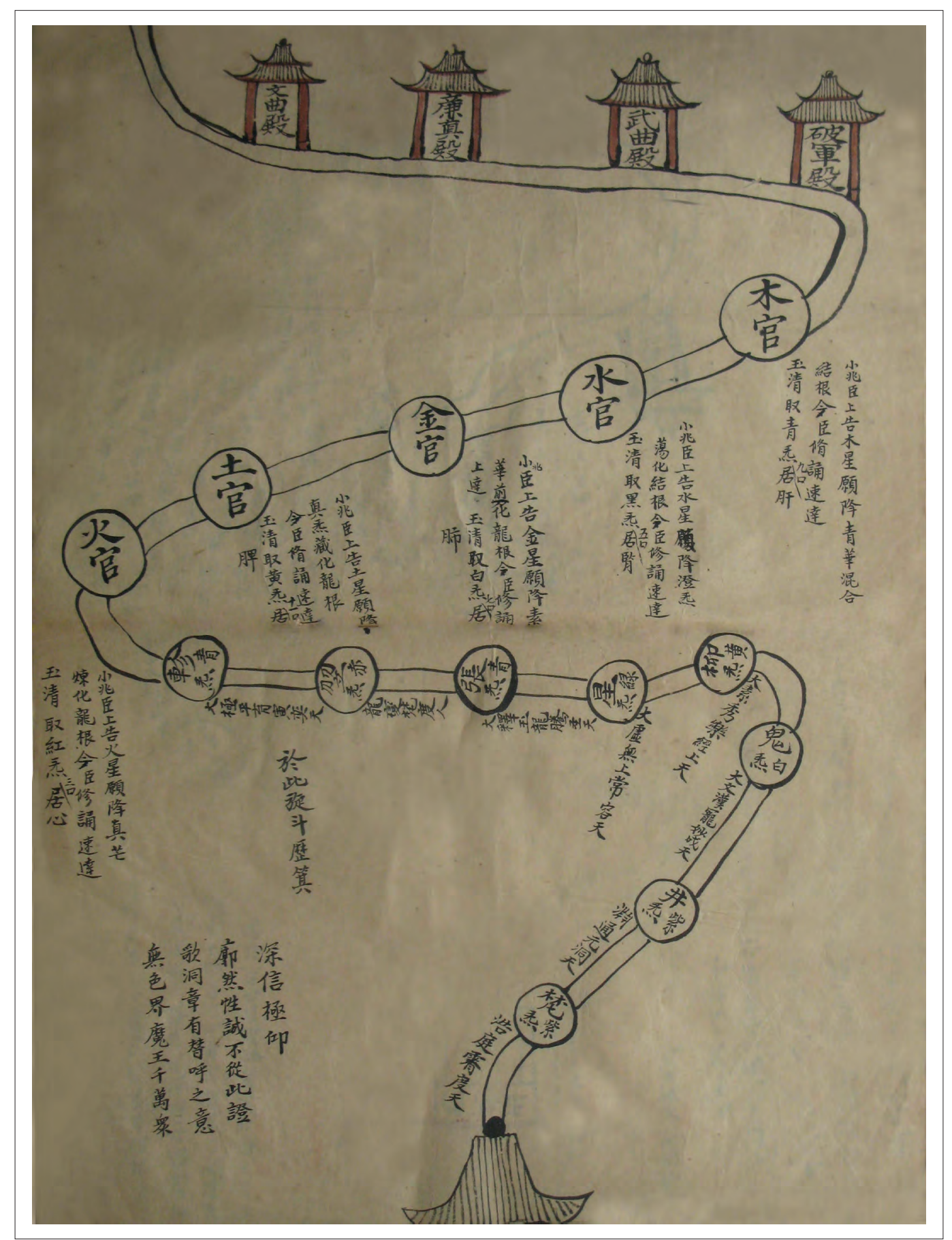

Figure 25.8.6 Beyond the 28 mansions are the constellations of the Five Agents and the stars of Ursa Major

to a young maiden and follows her to the Palace of Supreme Purity (Taiqing gong 泰清宮), which is the residence of the Celestial Worthy of the Tao and its Efficacy (Daode Tianzun), the third of the Three Pure Ones, identified with Laozi himself.

Schipper, in the fifth chapter of The Taoist Body, described this trip as follows:

Curled up in the fetal position, the Great Master then makes a spiritual journey within his own body. First he visualizes a newborn child in the area of his abdomen, there at the site of the Cinnabar Field (tan-t'ien) [dantian] below his navel. Then he concentrates on his heart, where he rediscovers the newborn child, who is now called the True Person. He is dressed in red and two guards in military uniform stand beside him. They are 'generals'. He visualizes all three, standing on a terrace bathed in the light of dawn. Then, with an escort of other generals, they ascend a twelve-storey tower (which corresponds to the trachea) and present themselves before the Pass of the Tiger and the Leopard (throat). They enter the mouth, then the nose and continue their path all the way to the Gates of 


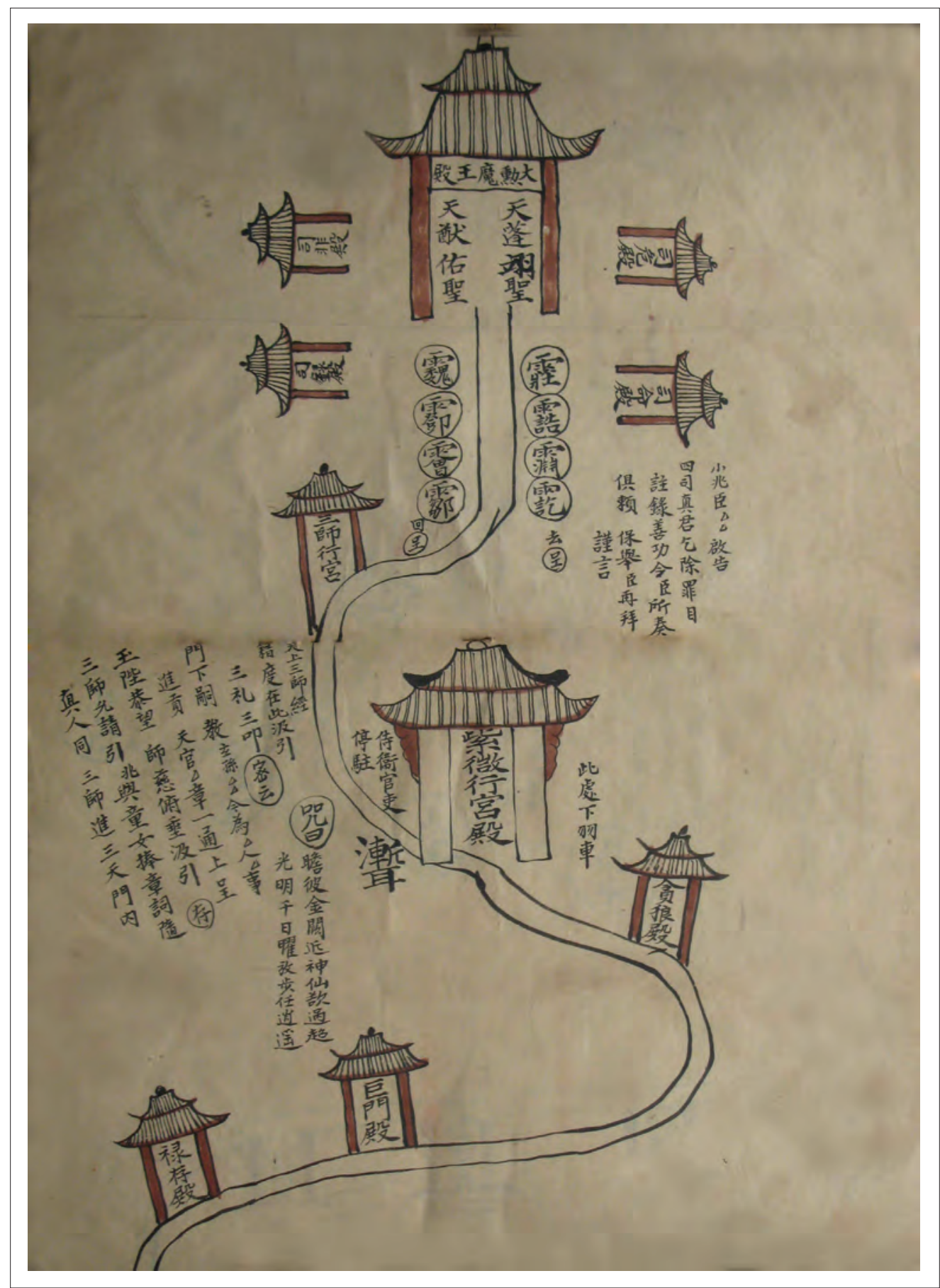

Figure 25.8.7 The seven stars of Ursa Major have the Pole star at their top (ziwei). Beyond are the Palace of the Three Masters, the Four Offices of the Stellar Lords and the Palace of the Four Saints

the Sun and Moon (the eyes), which open into the point between the brows. Finally, they arrive at the top of the head and from there enter the cavity inside the crown. There stands the Golden Gate. On his knees, facing the flaming pearl - a white light emanating from the original energy - the True Person presents the memorial on which he has placed several $f u$ or passes. Once the memorial has been accepted by the officers of the Heavenly Chancellery, the True Person turns back. But the journey to the heavenly regions has transformed him. Now he appears as a venerable old man, the Great Lord of Long Life, the stellar deity of Immortality, who slowly descends to reintegrate himself with the point of origin, The Cinnabar Field. ${ }^{19}$

There are many descriptions of the submission of Taoist petitions and the process through which the Taoist master externalises the officers of his body. In Chisongzizhangli 赤 松子章曆章曆 (Master Red Pine's Almanac), the officiant, prostrate in front of the altar, visualises a red Qi emanating

$19 \quad$ Schipper 1993, pp. 98-9. 


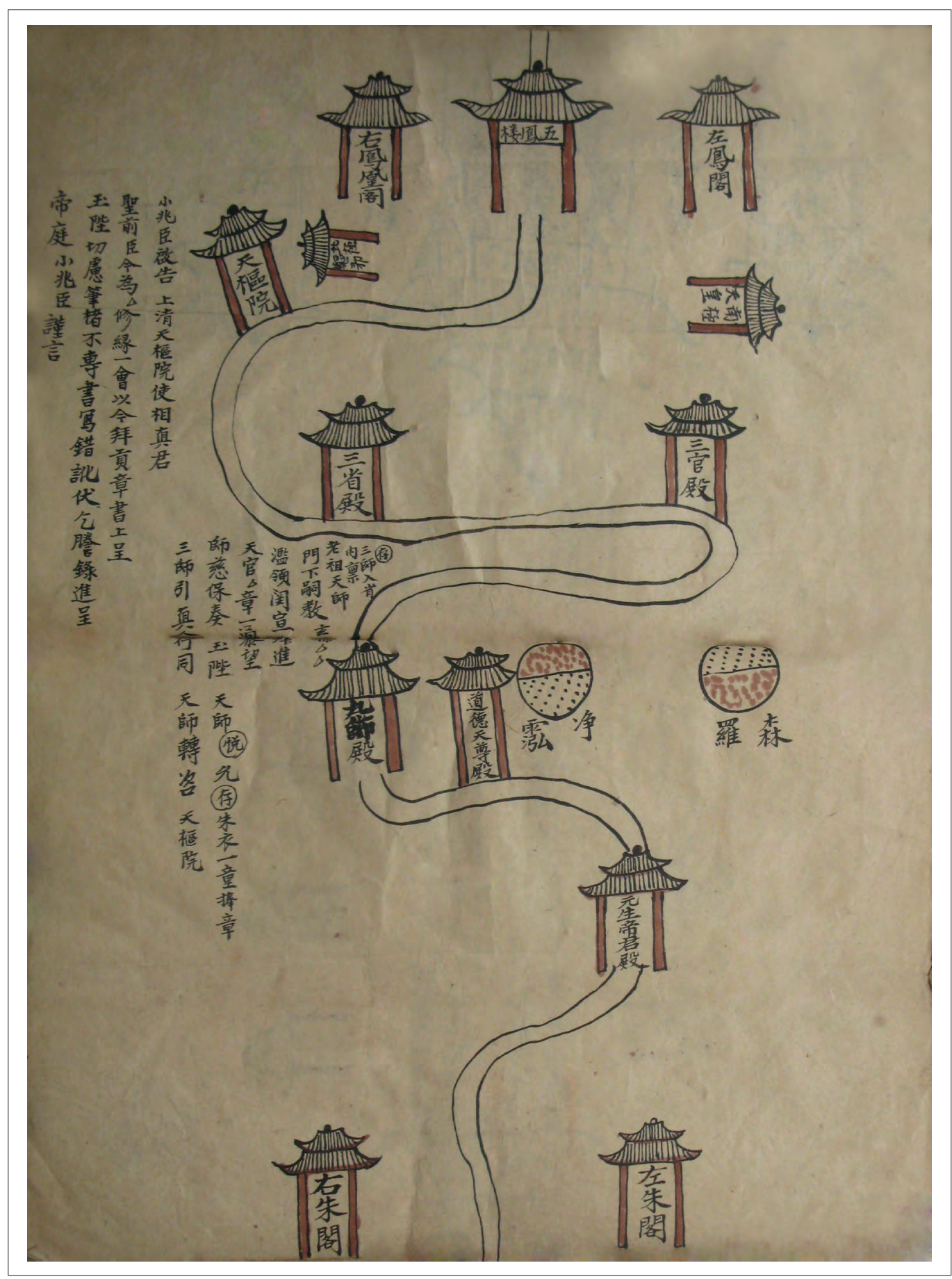

Figure 25.8.8. Traversing the last palaces among which are those of the Three Officers of Water, Earth and Heaven

from his heart, passing along the course of the huangdao 黃道 (solar ecliptic) and arriving at the Gate of Heaven, where he is hosted by Zhang Daoling 張道陵, the founder of the Celestial Masters sect. Once there, an Immortal approaches to receive his petition. The Taoist master is then brought before the Palace of the Three Pure Ones who read the document. He withdraws respectfully, and then returns to the altar while they examine the petition, and finally he performs the rites of thanks. ${ }^{20}$ Chisongzizhangli is a collection of zhang 章 (petitions) dating to late Tang times, which probably contains documents used by Taoist masters in late Han.

Another very complete account of the submission of a petition is that of the great Taoist master and prominent liturgist Lu Xiujing 陸修靜 (406-77). Lu wrote a manual for the ordination rites in which hundreds, if not thousands, of 


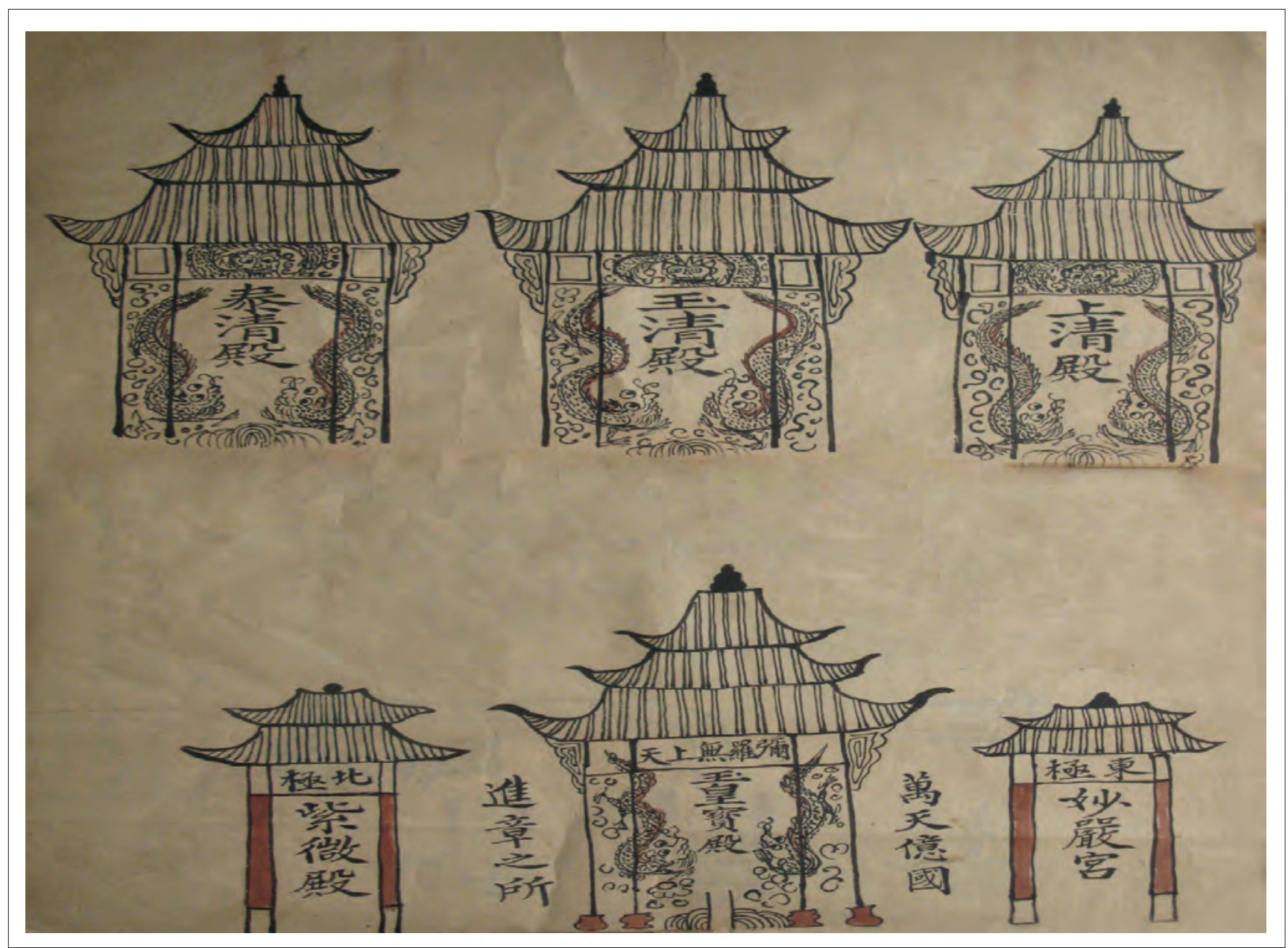

Figure 25.8.9. Arrival at the court of the Jade Emperor and the Palaces of the Three Purities

gods are dispatched to Heaven during the chuguan ritual, and then re-integrated (fuguan 復官) at the end of the ritual in readiness for their next mission. ${ }^{21}$ During such ordination ceremonies, which sanction a lengthy training in Taoist ritual, the initiate becomes the alter ego of the gods who enter into his service and to whom he will give orders, like a general to his troops. But lest the military metaphor be over-read, we must remember that the Taoist initiate is also an alchemist. During the long retreat that precedes ordination, the applicant prepares a bowl of sacred water into which he mixes the ashes of $f u$ 符 (talismans) that record the secret (hui 諱) names of his divinities. He will drink this water, mixed with cinnabar, on the day of his inauguration so that the gods can pass into his body.

$21 \quad$ A very detailed summary of this work by Lu Xiujing, entitled 'Taishang dongxuan lingbao shou du yi' 太上洞玄灵宝授度仪 (DZ 522), is in Benn 1991, pp. 121-36. For an account based primarily on the ritual of Master Chen Yung-sheng Tainan, see also Lagerwey 1987, pp. 126ff.
As was succinctly observed by Kenneth Dean:

The medieval liturgical manuals reveal that the talismanic-orders were not only burned and sent to the Gods or to the souls. Some were burned and then the ashes were consumed by the priest. Descending into his body, they could affect the summoning of the gods within his body, whom the priest would then send forth to merge with their counterparts in the macrocosm. ${ }^{22}$

Master Yi Songdao's Chart of the Skeleton of Lord Lao and Chart of a Flying Journey through the Three Worlds to Present a Petition have focused us on connections between the inner and outer geography of the Taoist ritual. Together the charts preserve knowledge of an ancient tradition belonging to the shamanic sub-strata in Taoism, as Piet van der Loon has described ${ }^{23}$. Simultaneously alchemists, exorcists, official dignitaries of the Tao and masters of the gods, Taoists are also the inheritors of ancient shamanism, of which this journey into the celestial realm is the best illustration.

\footnotetext{
22 Dean 1993, pp. 48-9.

23 Van der Loon 1979.
} 


\section{Bibliography}

\section{Primary Sources}

Daofa huiyuan 道法會元 (Taoist Method, United in Principle) Daozang no. 1220.

\section{Secondary Sources}

Andersen, P. and F.C. Reiter (eds) 2005, Scriptures, Schools and Forms of Practice in Daoism: A Berlin Symposium, Wiesbaden: Harrasovitz Verlag.

Bauer, W. (ed.) 1979, Studia Sino-Mongolica, Festschrift für Herbert Franke, Wiesbaden: Franz Steiner

Benn, C.D. 1991, The Cavern-Mystery Transmission: A Taoist Ordination Rite of $A D$ 711, Honolulu: University of Hawai'i Press.

Boltz, J. 1987, A Survey of Taoist Literature, Berkeley: University of California Press.

Davis, E. 2001, Society and the Supernatural in Song China, Honolulu: University of Hawai'i Press.

Dean, K. 1993, Taoist Ritual and Popular Cults of South-East China, Princeton: Princeton University Press.

Despeux, C. 1994, Taoïsme et corps humain, Paris: Guy Trédaniel. 2005, 'Visual representations of the body in Chinese medical and Daoist texts from the Song to the Qing period (tenth to nineteenth century)', Asian Medicine: Tradition and Modernity $1.1,10-52$.

Fava, P. 2005, The Revenge of Han Xin, A Taoist Mystery, DVD 90 min., EFEO-CNRS Images (A film about Taoist ritual in Hunan province), distributed by CNRS Images, France.

2013, Aux portes du ciel, la statuaire taoïste du Hunan: Art et anthropologie de la Chine, Paris: Les belles lettres.

Lagerwey, J. 1987, Taoist Ritual in Chinese Society and History, New York: MacMillan Publishing Company.

Min Zhiting 閔智亭 2000, Xuanmen risong zaowan gongke jingzhu 玄門日誦早晚功課經注, Beijing: Zongjiao wenhua chubanshe.

Pregadio, F. (ed.) 2008, The Encyclopedia of Taoism, London: Routledge. Reiter, F. 2005, 'The name of the nameless and Thunder Magic', in Andersen and Reiter (eds), 97-116.

Schipper, K. 1973, 'Some remarks on the function of the Inspector of Merits' (unpublished study).

\section{2, Le corps taoiste, Paris: Fayard.}

(tr. K. Duval) 1993, The Taoist Body, Berkeley: University of California Press.

Schipper, K. et al. 1975, Concordance du Tao-Tsang:titres des ouvrages, Paris: École française d'Extrême-Orient.

Schipper, K. and F. Verellen 2004, The Taoist Canon: A Historical Companion to the Daozang, 3 vols, Chicago: University of Chicago Press.

Sivin, N. 1995, 'State, cosmos, and body in the last three centuries BC', Harvard Journal of Asiatic Studies 55.1, 5-37.

Skar, L. 1996-7, 'Administrating Thunder: a thirteenth-century memorial deliberating the Thunder Rites', Cahiers d'Extrême-Asie 9, 159-202.

Van der Loon, P. 1979, 'A Taoist collection of the fourteenth century', in Bauer (ed.), 401-5.

Verellen, F. 2004, 'The Heavenly Master liturgical agenda, according to Chisong zi's petition almanach', Cahiers d'Extrême-Asie 14, 291-343. 


\title{
26 Clinical Medicine Texts: The Earliest Stone Medical Inscription
}

\author{
Zhang Ruixian 張瑞賢, WangJiakui 王家葵, Michael Stanley-Baker 徐源 *
}

South of the ancient capital of Luoyang lie the world-renowned Longmen 龍門 Caves, a beautiful sacred site carved into the cliff faces of two mountains facing the River Yi 伊. From the turn of the 6th century the caves have attracted hosts of pilgrims from near and far, not merely for their beauty and sacred power, but as a place to make conspicuous displays of devotion and wealth, and it is now recognised as a UNESCO World Heritage Site. Royalty, aristocrats, poets, wealthy officials and guilds all came to Longmen to make votive offerings and pray for blessings. Amidst these visual manifestations of devotion, compassion, and monumental art lies the 'Medical Recipes Cavern', a carved cave (no. 1387) on the stone walls of which are engraved a hundred or so ancient medical formulas, commonly known as the Longmen Drug Recipes. The cave lies near the main thoroughfare, not $20 \mathrm{~m}$ from the Fengxian monastery 奉 先寺 and less than $15 \mathrm{~m}$ from Guyang cavern 古陽洞, thus occupying a central location within easy access of all who passed through the region.

Simple to use, and composed of easily available ingredients, the Longmen formulas are not terribly complex, and appear accessible to general use by common people without any specialised medical training. Yet when placed in their location at a sacred site dominated by monumental statuary, dedicatory and scriptural inscriptions with exclusively Buddhist overtones, the presence of medical formulas strikes a distinctive chord. How did the medical recipes come to be carved here? What messages are carried by the medium of cave inscription?

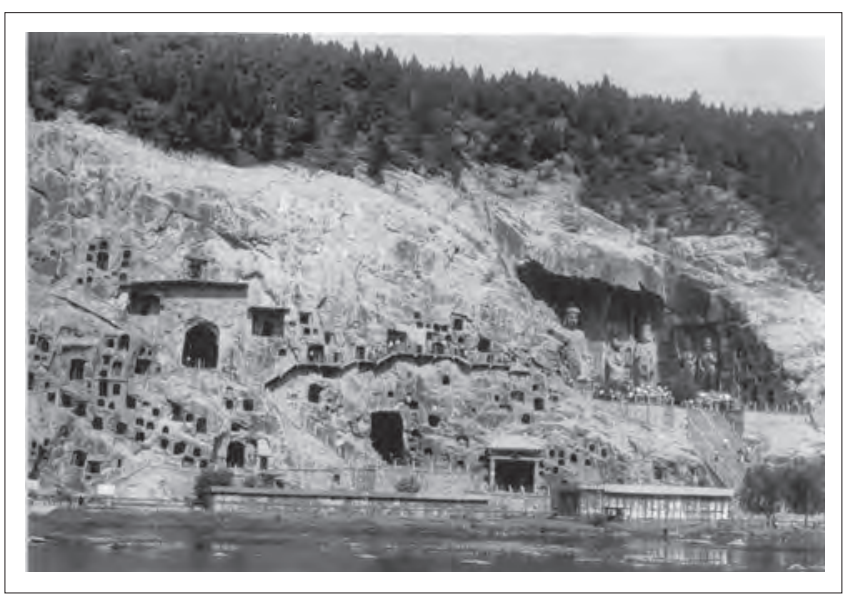

Figure 26.1 Panoramic view of the Longmen Caves in present-day Henan
The Longmen recipes have been of interest to numerous scholars, no matter whether in the field of medical history or archaeology. Fragmentary studies began in the Qing dynasty (1644-1911), and the recipes appear in several important epigraphic collections, such as Gu Yanwu's 顧炎武 (1613-82) Jinshi wenziji 金石文字記 (Records of Bronze and Stone Inscriptions) and Wang Chang's 王昶 (1742-1806) Jinshi cuipian 金石萃篇 (Collected Chapters of Stone and Bronze Inscriptions). Since the 1980s there has been abundant research both within and outside of China, from which new insights and research results are constantly being developed. ${ }^{1}$ Section 1 of this chapter describes the contemporary patterns of medical and monumental display on which these inscriptions drew as they communicated distinct messages about Buddhist medicine at the time. Section 2 presents orthographic and stylistic analysis and data to argue for a narrower dating of the inscriptions to between $65^{\circ}$ and $653 \mathrm{CE}$. Section 3 describes common features among the Longmen formulas and three Dunhuang scrolls, and thus argues that they share common origins. Altogether this chapter will demonstrate that the carving of these medical recipes within Chinese Buddhist monumental contexts creates a divine spectacle which serves to enhance the recipes' religious significance, and to imply their associated material and spiritual rewards.

\section{The Content and Structure of the Recipes}

The inscriptions across the east and north walls of the cave contain 154 recipes in total, treating 41 different diseases. ${ }^{2}$ The recipes are presented in a uniform, organised man-

This paper has benefited from comments and insights of Jonathan Pettit and Pierce Salguero.

$1 \quad$ Most significantly, see the extensive study Zhang Ruixian (ed.) 1999, the earlier article on dating the inscriptions by Chang Qing 1989, and the contextualising work by Liu Shufen 劉淑芬 2005 . For more recent work on the political, economic and religious motivations involved in the creation of the Longmen caves, see also McNair 2007. The introduction provides an extensive review of scholarship on Longmen since Okakura Kazuko's trip in 1894 up to 2007. On folk production of medieval Buddhist imagery in general, see Abe 2002.

2 The recipes are not numbered, and because different transcriptions of the recipes vary, leading scholars to disagree on the number of recipes. The numbers here represent a comparison and translation of various modern editions in Stanley-Baker and Yang 2017. 


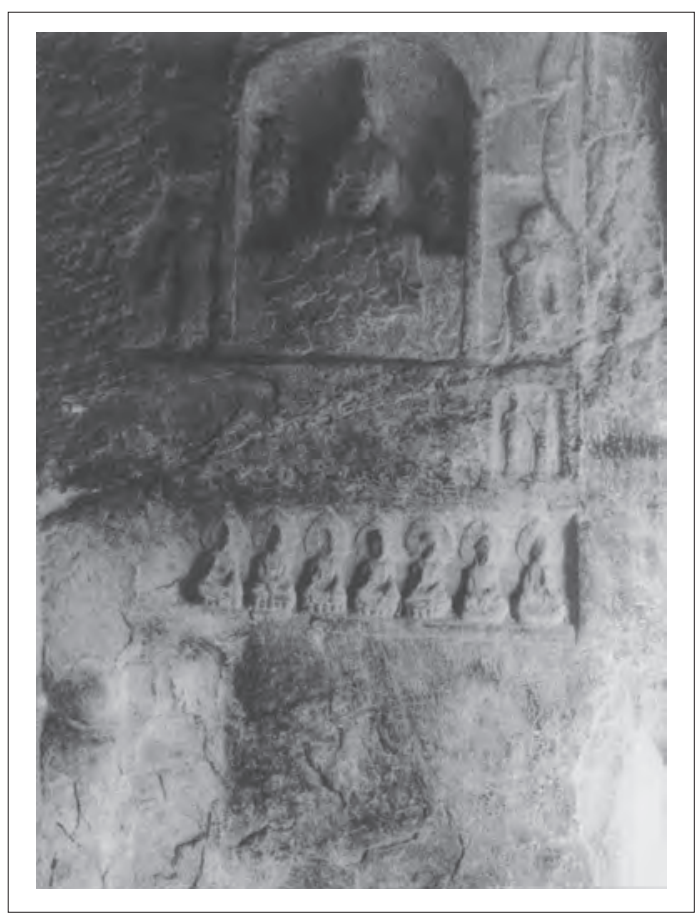

Figure 26.2 Seven Buddhas shrine, stele with medical formulas below, Medical Recipes Cavern, Longmen

ner, grouped according to specific diseases. Each disease group begins with the name of the problem and an initial formula, followed by one or several alternative recipes, as in the example below.

1 療瘧方: 燕翼一合, 末。取方寸七, 候發日平旦, 和 酒半碗攪, 令患人兩手捧碗, 當鼻下取氣, 勿飲。神良。

2 又, 蜀漆, 末, 方寸七, 發前和酒服。

3 又, 黃連搗末, 發前, 三指撮和酒服, 並驗。

4 療卒狂言鬼語方: 針刺足大拇趾甲下二分, 即止。

5 又方, 以甑 帶急縛兩手, 又指炎左右脅, 握肘頭, 兩火俱起, 各七狀。須舆, 鬼自道姓名, 乞去。徐徐解。 驗。

1. Recipe for curing maleria-like disease: 1 ge ( $\sim 103 \mathrm{ml})$ of swallow dung. Powder it. Take 1 square-inch spoonful, and wait until dawn of a fever day. Combine with half a bowl of wine and mix it. Cause the patient to raise it up with both hands in front of his or her nose and inhale the vapours. Do not drink it. (Divinely effective.)

2. Another: Grind lacquer from Shu 蜀. [using] 1 squareinch spoonful. Before the fever mix it with wine and drink.

3. Another: Grind Coptis chinensis (huanglian) into a powder. Before fever, mix a pinch with wine and drink. Also proven. ${ }^{3}$

Transcribed from Shao Dianwen 1993. In comparison with Liu Jinglong and Li Yukun 1998, Shao interpolates corrupted characters from Jia Zhihong and Ren Bangding 1989, and Mizuno Seiichi et al. 1941. These are marked with square boxes 字.

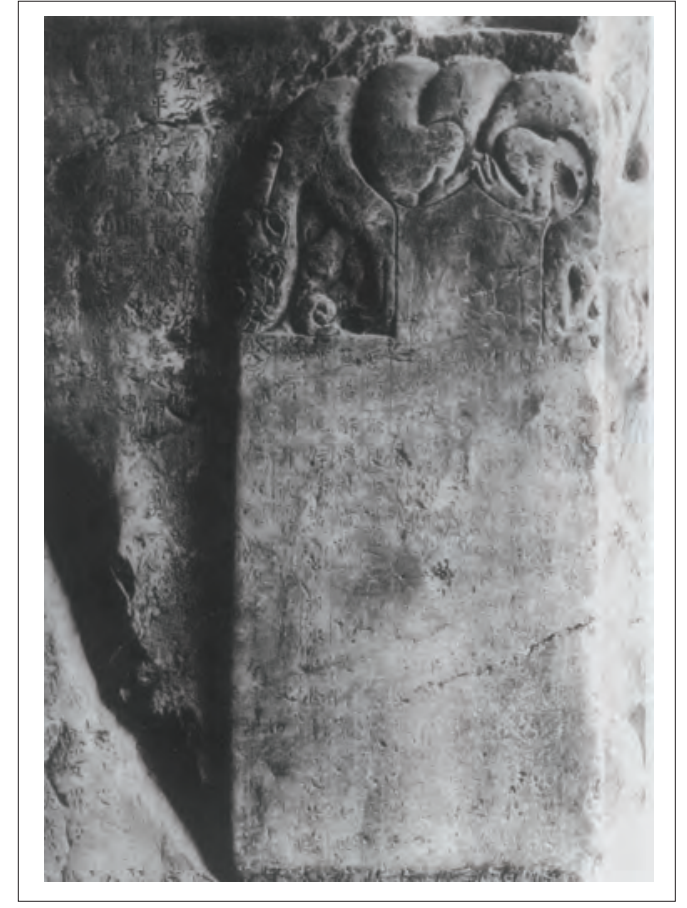

Figure 26.3 Votive statue and opening inscription on north wall

4. Recipe to treat rapid crazy speech in the language of ghosts: Pierce with a needle two fen below the large toenail, then it will stop.

5. Another recipe: Use a steamer-belt ${ }^{4}$ to tightly bind the two thumbs, then cauterise the flanks to the right and left while controlling the elbows. The heat should be equal on both sides, seven times each. Shortly thereafter the ghost will speak his surname and given name of its own accord and beg to leave. Slowly release the patient. Proven.

The diseases can be grouped into larger categories. Although the different diseases in these larger groups are scattered throughout the general inscription, aggregating them is useful to show the predominant medical foci of the inscription. The table below shows the disease categories we have grouped together, the total recipes per category, and the number of recipes per disease. The largest general category is skin lesions, boils and swellings, indicating repeated interest in these types of problems. However, when considering the number of recipes for single diseases, issues of internal medicine and feverish diseases rank the highest, with the most recipes for heart pain, and for accumulations in the chest, closely followed by varieties of jaundice and wasting and thirsting disorder.

The treatments used do not require specialised medical knowledge and can be applied by anyone. Methods

A strap used to tie a steamer basket to its water supply. Later these became used in decoctions as pharmaca in their own right. Bencao gangmu 本草綱目, Li Shizhen 李时珍, p. 2, 209. 
List of Recipes Grouped According to Disease Treated

\begin{tabular}{|c|c|}
\hline $\begin{array}{l}\text { Skin lesions, swellings, boils } \\
\text { Total recipes: } 46\end{array}$ & $\begin{array}{l}\text { 20 Sores, wounds or lesions 瘡, of the following types: } \\
3 \text { boils 疔 } \\
3 \text { inverted flower 反花 } \\
3 \text { wounds from metal [weapons] 金 } \\
5 \text { oozing 㾫 } \\
3 \text { lacquer 漆 } \\
\text { 3 Sores and boils on someone on the verge of death due to wind invasion [i.e. leprosy] 瘡腫 } \\
\text { 風入垂死 } \\
8 \text { Noxious swellings 惡腫 } \\
6 \text { Massive swelling over the whole the body 遍身洪腫 } \\
4 \text { Sudden eruption of boils on the whole body 卒遍身生皰 } \\
3 \text { Rising Qi causing the spitting of blood and pus 上氣唾膿血. } \\
3 \text { Five types of hæmorrhoids } \\
2 \text { Sores on the back 發背 }\end{array}$ \\
\hline $\begin{array}{l}\text { Feverish, wasting diseases } \\
\text { Total recipes: } 25\end{array}$ & $\begin{array}{l}5 \text { Noxious infixation entering the heart-mind and causing one to wish to die 惡㾏入心欲死 } \\
4 \text { Acute jaundice, Heat jaundice, Interior jaundice type 急黃疸黃內黃等 } \\
4 \text { Sudden disruption disorder 霍亂 } \\
4 \text { Rising Qi, cough, abdominal fullness and oedema 上氣咳嗽腹滿體腫 } \\
3 \text { Malaria-like diseases 瘧 } \\
3 \text { Wasting and thirsting 消渴 } \\
2 \text { Feverish epidemics 瘟疫 }\end{array}$ \\
\hline $\begin{array}{l}\text { Various internal problems } \\
\text { Total recipes: } 39\end{array}$ & $\begin{array}{l}11 \text { Red and white diarrhoea (i.e. with blood and pus) 赤白利 } \\
8 \text { Heart pain 心痛 } \\
7 \text { Urinary dificulty, 小便不通, 五淋 } \\
4 \text { Infantile malnutrition 療急蚶方 } \\
\text { 4 Constipation 大便不通 } \\
3 \text { Thoracic masses 癖 } \\
1 \text { Abdominal fullness and stone-hard accumulations, without decrease over years. 腹滿堅如 } \\
\quad \text { 石積, 年不損 } \\
1 \text { Sudden impediment, stumbling and lack of mobility in the legs 脚忽痹蹶不隨 }\end{array}$ \\
\hline $\begin{array}{l}\text { Throat, breathing } \\
\text { Total recipes: } 13\end{array}$ & $\begin{array}{l}\text { 4 Choking噎 } \\
\text { 4 Difficult breathing 咻 } \\
3 \text { Fish bones in the throat 魚骨鯁 } \\
2 \text { Bi-obstruction in the throat 喉痹 }\end{array}$ \\
\hline $\begin{array}{l}\text { Traumatic injury } \\
\text { Total recipes: } 14\end{array}$ & $\begin{array}{l}5 \text { Cramps extending from the feet up to the abdomen 腳轉筋及入腹 } \\
4 \text { Vicious stab wounds 惡刺 } \\
3 \text { Insect poisoning 蟲毒 } \\
2 \text { Unending bleeding from wounds due to metal 金瘡血出不止 } \\
\end{array}$ \\
\hline $\begin{array}{l}\text { Epilepsy, fits, spasms, crazy } \\
\text { speech } \\
\text { Total recipes: } 11\end{array}$ & $\begin{array}{l}4 \text { Falling fits 顛狂 } \\
3 \text { Loss of speech 失音不語 } \\
2 \text { Opisthotonus (lit. arching backwards like a bow) due to wind entering the body, and women } \\
\text { struck by wind 被風入身, 角弓反張, 及婦人中風 } \\
2 \text { Acute onset of mad speech and ghostly gibbering 卒狂言鬼語 }\end{array}$ \\
\hline $\begin{array}{l}\text { Vomiting, acid reflux } \\
\text { Total recipes: } 6\end{array}$ & $\begin{array}{l}3 \text { Stomach reflux 反胃 } \\
3 \text { Vomiting and heaving 嘔噦 }\end{array}$ \\
\hline
\end{tabular}

used include needling and moxibustion at clearly defined locations, with no use of medical names for the points, ingestion of substances (often mixed with water or wine), and binding the patient in various ways. The ingredients are fairly common, easy to get hold of, and the recipes are simple to prepare, requiring no more than two or three ingredients. Decocted or mixed substances include common ingredients found around the household, such as willow (liu 柳), wild rice (gucao 菰草), Glauber's salt (mangxiao
芒硝), cocklebur fruit (cang'er 蒼耳), pork, chives (jiu非), mungbeans (lüdou 綠豆) and cloves (dingxiang 丁香). Materials from medical pharmacopoeias include angelica (danggui 當歸), evodia fruit (wuzhuyu 吳茱实), cinnamon bark (rougui 肉桂), white mulberry root bark (sanggen baipi 桑根白皮), apricot seed (xingren 杏仁) and others. Less commonly seen materia medica include: human hair; human, cow, horse, swallow and earthworm dung; roof tiles; and hedgehog skin. 


\section{Cultural Context of the Recipes}

\section{Merit-Making among the Elite Classes}

In order to get a clearer idea of the cultural context in which the recipes appeared, it is helpful to look more closely at the surrounding inscriptions. Although the inscription bordering the recipes on the eastern wall was inscribed 100 years prior, it provides useful clues to the social, spiritual and genealogical properties of Buddhist engraved texts in general, which bear on the recipes by virtue of their shared context.

夫 $x \times$ 沖眇, $x$ 尋光影, 可以依悕, 是以太中大夫 $x \times$ 將 軍 $\times \times$ 天都/督(清)水鄉開國公李長壽妻陳暈, 宿因莫 遇, 嫡為李室, 敬善內敷, / 口口超悟。割舍家財, 造釋 迦像一堪 (龕), 愿夫亡高 $X$, 帝王龍念. (又) 愿已身亡 息, 征 $\times \times$ 任, 所生者使 $\times$ 子平康。聖賢祐助, 家眷安 寧, 命齊天 / 算, 行深 $X$ 正。一切從愿。永安三年六月 十二日X 餝銘記。 5

When $X X$ was but little, he sought luminous glory, and had enduring longing. He was the Superior Grand Master of the Palace, the $X X$ General, and $X X$-Heaven Commanderin-Chief, Founding Duke of Qingshui (清) 水 province. Li lived to a great age, marrying Chen Yun 陳量, but never saw his karma come to fruition. His descendant Li Shi 李 室 respected virtue which he practised internally, and [achieved] $X X$ transcendental awakening. Severing myself from family wealth through giving donations, I have made a shrine to Śakyamuni, with the aspiration that my husband might attain $X$ heights in death, and that the monarch bestow his affection. I also pray that when I have died and am at rest, I may go to $X$ and serve as $X$, and that those who live on cause $X$ children to be peaceful and healthy. May the sages and worthies bestow their divine aid, that the family descendants may be peaceful, their lives be as long as the measure of heaven, and that they may be deeply righteous and $X$ in their comportment. May all accord with this aspiration. The 23 day of the sixth month of the third year of the Yong'an 永安 reign [530 CE].

Commissioned by the relative of a high-ranking official, the inscription describes the glory of their family history, and expresses the aspiration to receive blessings both for the deceased husband, for the donor, and for their descendants. The wishes are for royal favour (diwang chongnian 帝王寵念), for a good death, for peace and health, and for virtuous descendants.

As will be discussed later, the shrine and its inscription transform capital and social influence into spiritual power. The very clear denotation of name, family, rank (and in other cases, place of birth) ensures the correct transfer of spiritual power and is ubiquitous in Chinese religions. These inscriptions are found in Daoist, Buddhist and common religious rituals, public and private, even to

$5 \quad$ Chang Qing 1989, p. 38 the present day. ${ }^{6}$ In Longmen, however, this public identification of donors takes on another dimension, because elites from very high strata of society down to the levels of lower officials and collective guilds were embroiled in the habitus of competitive, conspicuous display, forming a context wherein individuals jostled for social positions and even public offices. ${ }^{7}$ The clamour to outdo one another in mortuary display became so strident and widespread through the Wei dynasty, that families began to destitute themselves in potlatch fashion, so that in 472 , an imperial edict came down, calling for a halt to the expenditures. ${ }^{8}$ Stakes were high, and much was thought to be gained by establishing a presence among elite mortuary monuments.

We can tentatively say that the merit generated by monumental art like that in Longmen thus worked in two, possibly three vectors: the ancestral, the social and the personal. Firstly, the merit from these monuments was transmitted along family lines via the dedications, which generated good fortune for them in the future. Secondly, the production of monumental art in a location like Longmen located the donor and their associates and relatives in elite circles, providing direct material or relational benefits for the living. Thirdly, the act of 'selfless' compassion (shanxin 善心) also cultivated the individual's own merit and virtue, which went towards improving one's own karmic merit and brought one a step closer to cessation of rebirth. ${ }^{9}$

The recipes are not in themselves merit-making inscriptions (apart from any merit earned by those directly responsible for their creation); they do not profess to bring ancestral merit to anyone, nor magical power to bestow blessings. In the context of the Buddha images at Longmen, however, they take on the visual language of the inscriptions around them, and are tacitly imbued with these powers. By bringing broad benefits to people at large through disseminating medical knowledge, they follow the general

6 It is common practice in temples of any denomination in Taiwan to announce one's name, family and birthplace when offering incense to deities. More formal rituals such as Daoist petitioning rites (shang zhang 上章 or qi biao 起表) also state very clearly the donor's name, position, address, family lineage and place of birth, as well as that of the intended recipient of the ritual's power.

7 Zhang Ruoyu argues, for example, that Prince Li Tai's efforts to finish the Binyang 賓陽 South Grotto in honour of his mother, were motivated by his desire to impress his father with his filial piety in order for a chance at the throne. See Zhang Ruoyu 1980. On the social and spiritual functions of the Binyang Grotto, see McNair 2007, ch. 2.

8 History of the Wei Dynasty, Weishu 魏書, Wei Shou 魏收, p. 114. 3038, tr. in Gernet 1995, p. 234, cited in McNair 2007, p. 52.

9 The idea of triple merit is introduced in Hsieh Shu-wei 2010. 
subtext of merit-making. Although they do not articulate a bureaucratic process of spirit-communication like that imagined in Daoist talismans, these writings dispelled diseases commonly thought to be produced by demons and ghosts. Their placement amidst elite monuments lent them status by association, separating them out from common recipe handbooks.

\section{Charisma of Buddhist Textual Engravings}

Engravings of Buddhist scriptures were also regarded as sacred objects in their own right, and were initially associated with mortuary monuments as well as more general public sites. One of the early forms of mortuary monuments was the stūpa, developed initially as a reliquary for the śarira or cremated remains of the Buddha. They later came to house the remains of high-level Buddhist practitioners, whose knowledge of Buddhist teachings caused them to receive an equivalent reverence to the Buddha, and whose bodies came to be revered as having the same spiritual and healing powers accorded to the Buddha and the stūpas which housed his remains. During the Northern Qi (550-77) dynasty, the sites of stūpa-caves also came to be decorated with scriptural wall-engravings, but were also carved on cliff-faces and boulders, 'blurring the boundaries between relic and text, image and ideology'. ${ }^{10}$ Strongly promoted by Sengchou 僧稠 (481-56o), the accomplished Confucian scholar who then became a Buddhist meditation master, this activity may have been inspired by engravings of Confucian classics in stone, but went on to become widespread through various caves in the Northern Qi. ${ }^{11}$

As Katherine Tsiang argues, the engravings may have been motivated by 'various concerns, such as reverence for texts and learning, preservation, the generation of merit and the beneficial protective effects of copying and keeping the texts', but they also were bound up with notions that worship of texts themselves was superior to the worship of relics, as it was equal to seeing the whole body of the Buddha, the Thus-come-one or Tathägata. This idea was grounded in the Mahayana Buddhist trikāya (sanshen 三身) or 'triple body' theory, which distinguishes three modes of existence of all Buddhas. ${ }^{12}$ The created or transformative

$10 \quad$ Tsiang 2005, pp. 55-6.

11 Tsiang 2005, p. 56 . Tsiang cites an inscription in the central cave at Xiaonanhai 小南海, Baoshan 寶山, near Anyang, which records that these were carved at Sengchou's suggestion in 555 .

The trikāya philosophy has a much more complex history than we have space to describe here, as different schools of thought, such as Tantric Buddhism, Huayan, or Kegon Buddhism incorporated different Buddhas, deities and cosmic principles within body (Skt. nirmānakāya, Ch. huashen 化身) refers to the physical incarnation of saviours such as the Śakyamuni Buddha; the bliss body (Skt. sambhogakāya, Ch. baoshen 報身), or body of communal reward, is a subtle body of infinite form; the truth body (Skt. dharmakāya, Ch. fashen 法身) is the ultimate reality from which all Buddhas emerge and to which they return. In ritual practice, the nirmānakāya came to be materially represented by śarira or relics, the sambhogakāya by sculpted images, and the dharmakāya by scriptural texts. Dharmakāya was also translated in some early Chinese scriptures as Fojing shen 佛經身 (Corpus, or body, of Buddhist scripture), while others used collective terms such as deng 等 (class), and zang 藏 (storehouse). ${ }^{13}$ While the terms deng and zang are otherwise used in medieval Chinese to refer to textual collections, the embodied metaphor of shen is not - this rendering denotes a particular and deliberate equivalence with the body of the Buddha. Scriptures, as a physical form of the Buddha, were intimately bound up in metaphors of the Buddha's body, and in the Northern Qi, also came to be associated with the power of relics and public display in mortuary monuments.

Scriptures were considered to have numinous power in and of themselves as physical objects, carrying the power of the deities invoked therein, or of attendant spirit-guardians. They were considered to bestow benefits, protection and healing power on those who possessed them and treated them with reverence. ${ }^{14}$ Although, again, the Longmen Recipes are not Buddhist scriptures and thus do not pertain directly to ideas of dharmakāya, as a coherent collection of knowledge sharing the same context and medium of scriptures that would have been associated with numinous power, the Drug Recipes draw on that charismatic status for their own authority and efficacious potential. Their placement among forms of the Buddha lends them the aura of expressions of grace, of munificent knowledge, and thus positions them as part of the extended knowledge of the Thus-come-one, and ultimately, of his compassion.

Other numinous texts with healing power also circulated in China, particularly talismans ( $f u$ 符), arcane writings which were used, among other things, to entreat spirits to dispel ghost-borne disease from the bodies of their recipients. ${ }^{15}$ Insofar as the Longmen Recipes are a form of performative writing which results in ghostly diseases

these three terms.

13 Tsiang 2005, pp. 57-6o.

14 On Buddhist notions of the numinous power in scriptures and their reception in Chinese religion, see Hsieh Shu-wei 2005, Bumbacher 2012, pp. 134-76.

15 Most recently, Bumbacher 2012, pp. 57-81. 
being banished from the body, there is, at the very fringes of association, some connection between the two forms of writing, but this quickly fades on closer examination. ${ }^{16}$ Talismans were considered to communicate with the spirit realm, and the intended readership of the Longmen recipes is very clearly human. The network of numinous power on which Buddhist monumental display operated was a karmic one, whereas talismans operated through the spiritual bureaucracy. These distinctions would have been very clear to 7 th-century readers.

From the above comparisons with other monumental texts in the Longmen caves and beyond, we conclude that the adoption of the medium of cave engraving gives the recipes resonant associations with Buddhist salvific intentions and textual practice. They invite the reader to identify the recipes and their content as a distinctly Buddhist type of knowledge, and moreover, one deserving the attention of the elite classes. As we explore later in this chapter, the recipes have no precedent in Chinese medical documents, and it is likely that their provenance was west of China. However, for all the social and religious undertones that pertain to the medium of the Longmen cave inscriptions, it would be unwise to imagine that the recipes served a directly salvific function. So how then can we better interpret the uses and implications of the recipes in the context of the broader medical history of the period?

\section{Buddhist Medicine ${ }^{17}$}

Buddhist medicine had been making its entry into China through a variety of means over the previous five to six centuries, initially with mixed reception. This excerpt from a 6th-century apocryphal work describes monks practising acupuncture, moxibustion and dispensing drugs:

何故未來世中一切俗人輕賤三寶。正以比丘比丘尼不 如法故。身被法服輕理俗緣。或誦咒術以治他病。或 復修禪不能自一心。以邪定法占睹吉凶。或行針尒種 種湯藥以求衣食。

What could lead all of the commoners to belittle and slight the Three Treasures in their future lives? It is precisely when monks and nuns do not accord with the dharma,

16 From early on, drugs were considered to have apotropaic powers which enabled them to dispel ghost-borne disease. See Unschuld 1985, p. 114, et passim.; Stanley-Baker 2013, pp. 157-61, 205-8; Stanley-Baker 2014; Harper 1998.

17 Buddhist medicine is gradually becoming an object of more focused scholarly attention. See the seminal paper, Demiéville and Tatz 1985, "The broad eclectic selection of esoteric practices' in Strickmann 2002, and the most recent review of the field, bibliography and analysis of Sino-Indic medical transmissions, Salguero 2010. On Buddhist medicine and government medical policy in the Tang and Song, see Liu Shufen 2005. when, wearing the cloth, they slight reason and engage in common practices [such as...] using the art of chanting and invocations to dispel other diseases,... or practising acupuncture, moxibustion and various decoctions for a living. ${ }^{18}$

Although the tone of this passage is critical, describing ways in which false monks distract the laity from the dharma and lead them astray through practising common arts, including medicine, it is clear that medicine was becoming widespread enough in Buddhist communities to warrant this censure. The practice of Buddhist medicine became increasingly accepted as time went on, as in the legendary figure of the transmission and imagination of Buddhist medicine, doctor Jīvaka (Qi Po 耆婆), also known as the Medicine King (Yaowang 藥王). Texts and arts attributed to him circulated in China from at least the 4th century, and constituted an important part of the mystery and allure of Buddhist healing methods. ${ }^{19}$

During the Northern and Southern Dynasties (420-589), there were increasing numbers of monasteries through which common folk could get medical care, as well as local charitable Buddhist associations (fahui 法會, yiyi 義 邑, yihui 義會), which operated at the village level. Both stored up food in times of famine, and dispensed medicines during times of epidemics. ${ }^{20}$ Monks however, provided other forms of medicine that the laity could not, such as sūtra-recitations, talismans, merit-making rituals, dhārāni spells, confession rites and esoteric rituals, to name but a few, as well as practising, as mentioned above, acupuncture, moxibustion and pharmaceutics. ${ }^{21}$ Monks provided treatment to the sick who arrived at monastery doors, giving care in far-off regions away from urban centres, places where government supplied care would have been less easily accessible.

\section{Buddhism and State Medicine}

At the political level, ethical models of charity and benevolence, including state provision of medical supplies, reached the ears of heads of state through at least two channels. One was the ideal of the Cakravartin king, a noble benefactor who acted in accord with the dharma, idealised

18 Xiangfa jueyijing 像法決疑經 (Sūtra for Resolving Doubts during the Age of the Semblance Dharma), T85n2870.1337b28-co6. On date of this text, and for examples of famous monk doctors, see Liu Shufen 2005, pp. 151-2.

19 Salguero 2009.

$20 \quad$ Salguero 2010, p. 5 o.

21 For an in-depth survey of Buddhist esoteric healing techniques, see Strickmann 2002. 
in the narratives of the Indian King Aśoka. ${ }^{22}$ Travellers' tales reported by merchants and high-profile monks also described hospitals, treatments and the public dispensing of drugs that they had observed in India. These narratives appealed to some Buddhist monarchs of China, such as Emperor Xuanwu 宣武 (500-16) of the Northern Wei.

In the summer of 510 , there was an epidemic in Pingyang commandery 平陽郡 23 in which roughly 3,ooo people died. ${ }^{24}$ Later that year, Xuanwu ordered widespread public health provisions, building the first hospital in China, public distribution of medical care, drugs, and, significantly for our case, the posting of 3 o or more volumes of medical recipes in and outside cities and towns across the country: 可敕太常於閑敞之處, 別立一館, 使京畿內外疾病之 徒, 咸令居處。嚴敕醫署, 分師療治, 考其能否, 而行賞 罰。雖齡數有期, 修短分定, 然三疾不同: 或賴針石, 庶 秦扁之言, 理驗今日。又經方浩博, 流傳處廣, 應病投 藥, 卒難窮究。更令有司, 集諸醫工, 尋篇推簡, 務存 精要, 取三十餘卷, 以班九服。郡縣備寫, 布下鄉邑, 使知救患之術耳。

We thus command the Chamberlain for Ceremonials to establish one hostel in a spacious, fenced-off area, for the service of the sick in the capital and surrounding area, with my full command that it be a residential space. I sternly command the medical bureau 醫署 to distinguish between the preceptors' curative abilities, examine whether or not they are able, and distribute rewards and punishments accordingly. Although age has its limits, and its length is apportioned and fixed, the three illnesses are not the same: ${ }^{25}$ whether it is relying on needle-stones, or the many sayings of Bian [Que] 扁[鵲] from Qin, [the bureau should] organise and verify them for today. Also, masters of classical recipes jingfang 經方 with broad knowledge should spread out into far regions, respond to diseases by dispensing medicine, and thoroughly research acute and difficult [disorders]. I further command that there should be an officer si 司 who will gather all of the [texts of] medical workers yigong 醫工, seeking their chapters and offering up summaries. He should work at preserving their essentials, and extract 30 or more volumes to be published throughout the nine regions. The commanderies and counties shall thoroughly copy these, and display them in the counties and cities, solely in order to cause the arts of curing disease to be known. ${ }^{26}$

Salguero 2010, p. 52. On the reception of the Chakravartin King concept in China, see Salguero 2009, Zhang Ruoyu 1980, pp. 226-33.

23 Near modern-day Yaodu 堯都 area of Linfen 臨汾 city in Shanxi 山西 Province, roughly around 36.o775, 111.5247. (http://authority.ddbc.edu.tw/place (accessed July, 2010).

24 Weishu, p. 154.2433 The figure is described as precisely 2,730 in Li Wenbo 李文波 (ed.) 2004, p. 72.

25 For a discussion of what Xuan Wu meant by the term 'three illnesses' sanji 三疾 see the Appendix.

26 Weishu, p. 8.210 partially cited in Liu Shufen 2005, p. 168.
This edict is worthy of note on many counts, not least because it legitimates the first government-sponsored residential medical clinic in China, and thus one of the earliest hospitals. ${ }^{27}$ Additionally it calls for an early governmental review of the medical classics, about which we have little detail, but which may have significantly influenced the trajectory of later editions of medical texts circulating in northern China. Furthermore, it indicates the wide dispersal of government-employed doctors and, where their influence could not reach, the further spread of medical knowledge through posting on official notice boards.

It is likely that the posting of the recipes followed the Han precedent for public proclamations, which were posted by writing them either on the walls of provincial and county offices, or onto wooden boards which were hung there. ${ }^{28}$ Whether written on wooden boards or directly onto the walls, the recipes could not have stayed there in that form for very long. No doubt they were copied down by individuals, and were circulated from that point as paper or bamboo texts, in whole or in part. At that point they would have stopped being directly accessible to the general public, and entered the private text market, like many other medical texts of the time, such as the Longmen recipes. We can assume that, through this mechanism, the formulas posted spread beyond the geographic and temporal limits of the government-sponsored postings and circulated through networks of various types, including medical workers, families and local elites.

\section{Official Medicine in the Tang}

Government intervention in medical practice became more sophisticated over time. During the Tang dynasty, when the Longmen recipes were carved, there were three levels of medical bureaucracy which included:1) the Palace Medical Bureau (shangyao ju 尚藥局), which served the emperor directly; 2) the Pharmaceutical Bureau (yaocang $j u$ 藥藏局), which served the princes, and 3) the Imperial Medical Office (taiyi shu 太醫署), which was responsible for medical education and the provision of medical care at the national level. ${ }^{29}$ The Imperial Medical Office had 200 medical staff; they did not treat the general people, but served favoured individuals and officials and military

27 This followed the precedent of Xiao Ziliang 蕭子良 (r. 483-94), a Buddhist king of the Southern Qi dynasty, who ordered the first permanent hospice with a dispensary in 491 (Needham and Lu Gwei-Djen 200o, p. 54).

28 Liu Shufen 2005, p. 168. On the Han model, see Zhu Guihai 1999, pp. 153-9.

29 Li Jingwei and Lin Zhaogeng (eds) 200o, pp. 230-1, cited in Liu Shufen 2005, p. 163 . 
staff. They were also responsible for preparing drugs to use for Cold Damage (shanghan 傷寒), seasonal illness (shiqi 時氣), malaria (nüe 虐), diarrhoea (li 痢), internal injury (shangzhong 傷中), and wounds from metal objects (jinchuang 金瘡), and distributing them to various regions or in times of epidemics. ${ }^{30}$ At the provincial level there was one Medical Professor (yixue boshi 醫學博士) at each of the Governor's Palaces ( $d u d u f u$ 都督府), under whom were 10 to 12 medical students, who specialised in internal medicine (tiliao 體療), sores, wounds and swellings (chuangzhong 瘡腫), paediatrics (shaoxiao 少小), eyes, ears mouth and teeth (ermu kouchi 耳目口齒), and cupping (jiaofa 角法). ${ }^{31}$ Yet, as Liu Shu-fen cogently argues, although studies of government medical policy clearly describe the bureaucratic structures in place at different times, they often fail to show the insufficiencies of official medicine. ${ }^{32}$ She points out that, during their training, these students were unable to treat patients apart from under direct supervision of their medical Professor, and after they finished there were no official posts for them - they would most likely have gone off into the 'alleys and lanes' (lüyan 閤閻) to practise their trade, and it is at that point that government medicine ended, and private practitioners became dispensers of medical care.

The transmission of medicine in medieval China, when not through government bureaus, was most frequently through family and master-disciple lines, as well as through lineages of transcendents. ${ }^{33}$ Shrouded in esotericism from early doctors such as Chunyu Yi 淳于意 (205-150 BCE), medical knowledge was not circulated widely or easily. Xuanwu's policy thus marked a quite distinct brand of medical transmission. The Longmen recipes follow suit, in a time when we can be quite confident that Xuanwu's recipes were no longer available to the common public, and Tang medical policy had not yet enabled medical care to reach far out beyond urban areas. ${ }^{34}$ Serving as a gesture

Tang Liudian 唐六典 Compendium of Administrative Law of the Six Divisions of Tang Bureaucracy, Tang Liudian 唐六典, Zhang Jiuling 張九齡 and Zhang Shui 張說, p. 10.409, cited in Liu Shufen 2005, p. 163 .

31 Tang Liudian, p. 10.410, cited in Liu Shufen 2005, p. 163.

32 Liu Shufen 2005, p. 146.

33 On transmissions of transcendent pharmaca, see Pettit 2009; and 2013 .

34 Two Tang emperors did later follow the Xuanwu's policy example by publicly posting their own recipe collections, Xuanzong 玄宗 in 723, and Dezong 德宗 in 796. When Dezong made his edict, he recognised the need for broader distribution, as he stipulates they should 'Post notice boards in the counties, villages and on main roads, in order to inform and show the people' 散 片旁鄉村要路, 曉示百姓. Liu Shufen 2005, p. 169. of compassionate care, alleviating illness both in the local area and in farther reaches (through travellers who copied the recipes and took them with them), the Longmen recipes would have helped to portray Buddhism as a provider of the medicine of the people, in concert with other Buddhist healing practices and in contrast to more sophisticated, but less accessible government-sponsored medicine.

\section{Periodisation of the Longmen Inscriptions}

The earliest inscriptions in the cave in which the Longmen Drug Recipes are inscribed date to $5^{20-5}$, during the Zhengguang 正光 reign of Emperor Xiaoming 孝明 (r. 516-28) of the Northern Wei dynasty. ${ }^{35}$ The name 'Longmen drug recipes' refers to the drug formulas carved on either side of the entrance, and a few entries carved deeper inside the cave itself. The cave shows signs of an interruption between two stages of development, which is most likely due to the stoppage of works at Longmen because of the Heyin Incident (Heyin zhi shi 河陰之事).

During the regime change in 528 after Emperor Xiaoming was poisoned at the command of his mother, the Empress Dowager Hu 胡, Xiaoming's ally General Erzhu Rong 爾朱榮 (493-530 stylename Tianbao 天寶) held a coup d'état outside the capital Luoyang. On the pretence of sacrificing to the new emperor, Erzhu invited over 2,ooo palace officials to his camp along the river at Heyin 河陰, outside Luoyang, where he slaughtered them en masse before installing his own candidate for the throne. In anticipation of this, the Empress Dowager ordered all of Xiaoming's consorts to take Buddhist vows, and also had her own head shaved, although she never took vows before she died. ${ }^{36}$ This resulted in a general disturbance of Buddhist practice, so that carvings were stopped and altars were burned at Longmen, and the development of local policies on sacrifice and religious devotions in the region for some time thereafter. Rites were banned in the area until the Northern Qi dynasty (550-77), from which time new caves at Luoyang continued to be carved through the Sui and Tang dynasties.

These interruptions resulted in two distinct periods of work on the Drug Recipe Cave, which can be observed through various structural and stylistic analyses. The cave faces west, but the images on the north, south and east walls are not placed in a coherent relationship to one another, unlike other well-known caves whose north and south walls have central niches with carving that surround

\footnotetext{
35 Chang Qing 常青, pp. 38-9.
}

36 Weishu, p. 10.255ff. 


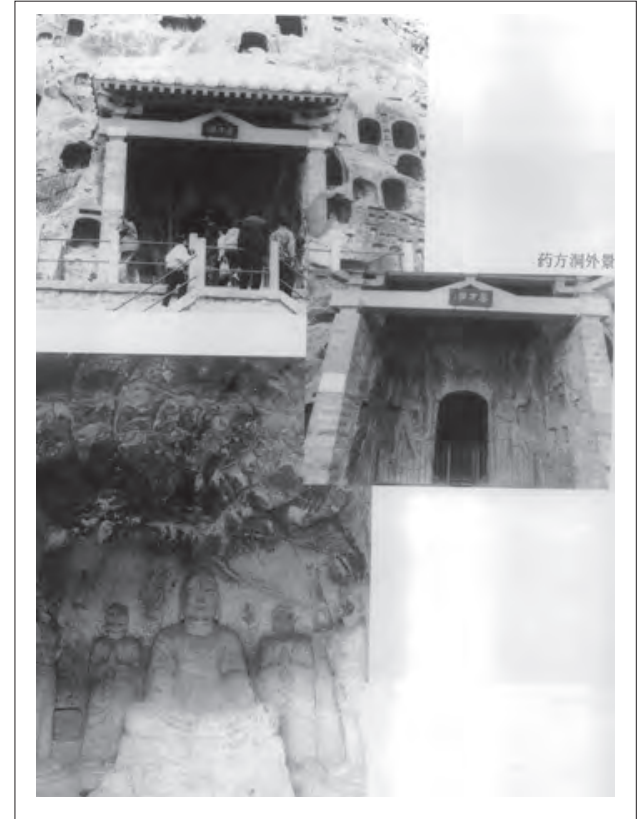

Figure 26.4 Buddhist statuary, Medical Recipes Cavern, Longmen

them. The earliest dedicatory inscription lies on the upper portion of the western face of the south wall amidst two others. Therein lies a sharply arched alcove $0.66 \mathrm{~m}$ wide by $0.46 \mathrm{~m}$, containing one Buddha, two disciples and two Bodhisattvas, with one stalwart on either side of the alcove. The stalwart on the western side has been damaged, presumably during the carving of the right side of the Bodhisattva. Also depicted are two men and six women performing worship (see Fig. 2). Outside of the niche is an inscription dated to $53 \mathrm{O} \mathrm{CE}$ (the 23 day of the sixth month of the third year of the Yong'an 永安 reign 永安三年六月 十二日). This date, combined with stylistic features that match other engravings from late Northern Wei in Longmen and elsewhere, suggests that the cave was opened some time before $530 .^{37}$

On the north portion of the eastern wall, there are two other niches, which are dated to 532 and 534. Other undated niches in the cave share the same drop-shaped apex of the alcove. The main alcove in the southern wall also has the same shape and engraving style as the other smaller ones mentioned above.

The Medical Recipes and the Votive Images in the Cave Scholars disagree about the date of the recipe inscriptions: Some think Northern Qi, some think Sui-Tang. So far there has been no definitive thesis, and therefore we have made the following study to date the engravings.

The recipes are in three areas of the cave: there are three sections on the north wall, one section on the south wall,

$37 \quad$ Chang Qing 1989, p. 39. and two sections on the interior east wall. The sections on the north wall are placed to the upper left, lower left, and directly below a votive statue (Fig. 3). The dedication, thought to predate 530, is on the upper portion of the western side of the south wall.

The recipes do not include an inscription date nor name of the donor, and are thus distinct from the nearby statue which states:

北齊武平六年歲次乙未六月甲申朔

Sixth year of the Wuping 武平 reign of Northern Qi 齊 (575), to the new moon on 14 July of the following year. ${ }^{38}$

This statue's dedication refers to medicinal drugs:

自非傾珍建像,焉可熾彼遺光; 若不勤栽藥樹,無以療 茲襲韾.

Without using up one's treasures to erect images, how can one illuminate others and bestow one's glory? If one is not diligent in planting medicinal shrubs, one cannot heal the deaf and the blind.

The author of this dedication argues that the practice of commissioning Buddhist statuary is a means to disseminate the wealth and social status accrued in one's lifetime for the greater good. Disseminating wealth is compared to planting medicinal shrubs to provide drugs in order to treat illnesses, an image that would not have been lost on its readers, as by this time the dispensing of medicine was already well-known as a major Buddhist merit-making activity. ${ }^{39}$ The diseases mentioned here are metaphoric, and refer to symbolic diseases of spiritual impediment, rather than the boils, trauma and feverish diseases that the recipes treat. In both cases, the statues and the drugs act as mediators of compassion between the donor and the broader public, converting one's diligence or wealth of treasures into herbs or statues, which in turn bring broad benefit to those at large.

The function of the shrine combined with the dedication is grounded in the principle of karmic merit, wherein good actions are repaid with good fortune and bad with bad in this life or the next. The selfless wish of an individual for the good of others, combined with the donation (Skt. dāna; tanna 檀那, tuona 陀那), of funds towards the making of sacred objects, is an action which generates spiritual power. These monumental displays thus function as karmic convertors, as it were, which transform capital and social influence into spiritual power, to be directed at the discretion of the donor, in most cases towards other members of the family.

38 Date for new moon from Xue Zhongsan and Ouyang Yi (eds) 1961.

39 Demiéville and Tatz 1985, pp. 43-5o, Salguero 2010, pp. 49-50. 
From the Qing dynasty on, collectors of epigraphy have included the statue, its inscription and the drug recipes as a single unit. ${ }^{40}$ These various ink rubbings and other associated articles suggest four strong reasons to indicate that the image and the medical recipes are unrelated:

1. The writing of the drug recipe which surrounds the statue's dedication is consistent in meaning from top to bottom, without any lacunae, and it is clear that no text from the drug recipe was erased in the carving of the statue's dedication.

2. The dedication is $3 \mathrm{~cm}$ higher than the recipes, and the dedicatory stele is set at a slanting angle, so that some of the recipe text abuts the dedication at a slanted angle. This indicates that there may have been text or images surrounding the statue which were scraped away by carvers in order to inscribe the formulas. This occurred because of differences among the carvers and their uses of the stone wall.

3. The image inscription states: 'if one is not diligent in planting medicinal shrubs, one cannot heal the deaf and the blind'. This argument encourages readers to exert themselves so that positive benefits can be bestowed on others. It also trades on the dispensing of medicine as a core image which, in the context of the statue inscription, functions as an explanation for the act of commissioning votive carvings, and a defence for this expenditure. We argue that it is not a literal argument for dispensing drugs, or for planting.

4. It should be admitted that the calligraphic style of the image inscription and the medical recipe indeed have similarities in certain areas. However, with detailed analysis, one can still make out differences between the two. The characters of the image inscription are slightly smaller, and the dots and strokes are slender and vigorous, already foreshadowing the kaishu 楷書 script of the early Tang. On occasion, however, the corner strokes show angularity, indicating that the stone carvers had not yet cast off the style of Northern Dynasties calligraphy. The characters of the medical recipe are larger, and while the weight of the brush seems heavier, it doesn't lose its lively movement or floating elegance, and the curves are natural.

40 See, for example: Wang Chang's王昶 Jinshi cuipian 金石萃編; Bi Yuan's 畢沅 (1730-97) Zhongzhou jinshi ji 中州金石記 (Records of Epigraphy from the Central Provinces); Hong Yixuan's 洪頣煊 (1765-1833/7?) Pingjin dubeiji 平津讀碑記 (Records of Beijing and Tianjin Stelæ Readings); the Yilutang jinshi ji 宜錄 堂金石記 (Records of Stone and Bronze Epigraphy from the Hall of Notable [Steles]) by Zhu Shiduan 朱士端, a provincial graduate (juren 舉人) of 1821, and Lu Zengxiang's 陸增祥 (181682) Baqiongshi jinshi buzheng 八瓊室金石補正 (Amendments to Stone and Bronze Epigraphy from the Eight-Jade Chamber).
It is truly an example of 'elegant and refined calligraphy, like the formal (zheng 正) script of the Tang', as described in Zhonguo shufa dacidian 中國書法大辭典.41

\section{Date for the Carving of the Medical Recipes}

Although the recipes are engraved in three separate sections in the cave, they share the same calligraphy. From the unity of style across the steles, the calligraphic style itself, the taboo words and the borders of the image inscription, we can make an estimate of the date of carving.

\section{Calligraphic Style}

When comparing the inscriptions of the medical recipes and the votive images, the characters nian 年 and wen 文 show clear differences, and although the structure of the other characters in the inscriptions is the same, the hand of the votive inscription uses square corners, whereas the medical recipes have round turns. The two hands are clearly quite distinct.

Comparison of the body of the medical recipe characters against classic examples of Northern Qi style from the Jiangzuan zaoxiang 姜纂造像 (Jiang's Edited Collection of Sculptural Inscriptions, 565), and early Tang works such as Ouyang Xun's 歐陽詢 (557-641) Huadu sibei 化度寺 碑 (Temple Conversion Steles), and Yu Shinan's 虞世南 (558-638) Kongzi miaotang bei 孔子廟堂碑 (Steles from Confucian Temples and Shrines) reveals that the medical recipes differ widely from Northern Qi steles in engraving style, but that they match up with characteristics of early Tang kaishu script, and are especially close to Ouyang and Yu's styles.

\section{Characteristics of Variant Characters}

The medical recipe engravings use a large proportion of variant characters. One useful description of character variants in early medieval China is chapter 19 of the Yanshi jiaxun 顏氏家訓 (Yan Family Admonitions) the author of which describes changes in frequency and type of character variants over various periods. ${ }^{42}$ Regarding writing and pronunciation standards in the Northern Dynasties, the author makes the following comment:

\footnotetext{
$41 \quad$ Liang Piyun 1986.

42 A successful official who managed to transition through various dynastic administrations leading up to and including the Sui dynasty, Yan Zhitui 顏之推 (531-91), saw first-hand a variety of cultural, literary and political changes during this period, which he recorded in the Yan Family Admonitions Yanshi jiashun 顏氏 家訓 in 589 .
} 
略不可看北朝喪亂之餘書迹。鄙陃加以専輙造字, 猥 拙甚於江南。乃以百念為憂, 言反為變, 不用為罷, 追 來為歸, 更生為蘇, 先人為老。

Generally, one cannot read traces of writing that remain from the chaos and strife of the Northern Dynasties. Their base vulgarity is compounded by careless errors in writing, which is far more cluttered and clumsy than in the south. So they use '1oo thoughts' 百念 for 'worry' 憂; 'speak contrary' 言反 for 'change' 變; 'stop using' 不用 for 'cease' 罷; 'chase here' 追來for 'return' 歸; 'live again' 更 生 for 'revive' 蘓 (equivalent to modern 蘇); 'person from before' 先人 for 'aged' 老.43

These are representative of local dialectical variances. Some are ambiguous, and still others are more likely graphic equivalences, such as xian 先 and lao 老. None of these substitutions can be found in the Longmen recipes.

However, there are many books, especially in the Tang, such as Yan Yuansun's 顏元孫 (d.714) collection, the Ganlu zishu 干祿字書 (A Writing Book for the Upwardly Mobile), in which the graphic variants for the words tong 通 and $s u$ 俗 are quite close to those in the medical recipes. This leads us to think that the orthography of the drug recipes is closer to that used in the Tang.

\section{The Relationship to the Borders of the Images}

The medical recipes on the south wall of the entrance to the cave are divided into two records: the record on the top half has 24 lines, the bottom half has 26 . The bottom half is severely weathered - only the lower halves of the two lines along the edge of the doorway still survive. At the place where the walls of the cave's archway meet the outside cliff face, there are waisted octagonal lotus pillars on the left and right. The area on the south face of the cave-wall where the medical recipe meets the octagonal pillar has five lines of text, which have been broken in the middle. Ding Mingde 丁明德 believes that this was broken when the pillar was being carved, and that the pillars were constructed at a later date than the medical recipes. ${ }^{44}$ According to Li Wensheng's 李文生 study, there are engravings outside the cave dating to 653 (永徽 Yonghui year 4), 656 (顯慶 Xianqing year 1) and 664 (麟德 Linde year 1). ${ }^{45}$ These indicate that the waisted octagonal lotus pillar was carved before 653 , and after 575 when the votive inscription inside the cave was carved.

On the north side of the interior east wall there is a shrine to the Seven Buddhas [of past kalpas] (qi shi Fo 七 世佛), ${ }^{46}$ with medical formulas to the right and below.

\footnotetext{
43 Yan Family Admonitions, p. 1,367.

44 Ding Mingde 1979.

$45 \quad$ Li Wensheng 1981.

46 The seven past Buddhas are: Vipaśyin, Śikhin, Visvabhū (of the
}

The medical recipes in this area of cave are for curing accumulated masses (liao pi 療癖), and are related to the formulas on the north face of the cave. This can be seen from the fact that once the space for the drug recipe below the northern dedication was used up, the formula was then continued on the eastern face of the cave. In total there are three lines of text; the left is near the north of the opening to the cave, the second is near the two-Bodhi (puti 菩提) shrine above the Seven Buddha shrine. The remaining portion of the recipes, which are for loss of voice, (liao shiyin buyu 療失音不語) continue on to the area below the Seven Buddha Shrine, clearly showing that the two-Bodhi shrine predates the medical recipes. According to Li Wensheng's study of their special formal characteristics, refined bearing, spiritual harmony and sculptural style, the two figures carved in the two-Bodhi shrine are very similar to the shrines on the cave's northern wall, carved by Yang Junya 楊君雅 in 649, and Gong Shi'an 宮士安 in 657 , indicating that they are all from the same period. The Seven Buddha shrine, the single example of such in the Longmen caves, was begun in the Tang.

\section{Taboo Characters}

\section{Sui Dynasty Taboo Characters}

The herbal recipes don't follow the dynastic character taboos of the Sui, which prohibit the use of specific characters found in the names of Sui rulers. For example, the recipe title Fuman jian rushi 腹滿堅如石積 (abdominal fullness and stone-hard accumulations), uses the character jian 堅, in direct contravention of the taboo on the name of the founder of the Sui, Yang Jian 楊堅 (541-6o4). The Sui also adopted taboos on 'words similar to royal names' (xianming 嫌名), such as that of Yang Jian's father, Zhong 忠. This rule entailed banning the character zhong 中, so that throughout the Sui, all instances of the word zhong 中 should have been changed to nei 內. For example, the title Zhongshu 中書 (Director of the Secretariat) was changed to neishi 內史 (Inner Secretary), and the name for Zhongmou 中牟 county was changed to Neimou 內 牟. ${ }^{47}$ However, the character zhong 中 occurs frequently in the medical recipes, such as luzhong pingdan 露中平 旦 (the dew arrives at dawn), nayao kongzhong 納藥孔中 (draw the medicine into the cavity), fanchuang zhongfeng

previous zhuangyan 莊嚴 kalpa), and Krakucchanda, Kanakamuni, Kāśyapa, and Śākyamuni (of the xian 賢 or present kalpa). Soothill 1937 .

47 Translations of most official titles in this chapter rely on Hucker 1985 . 
凡瘡中風 (all wounds struck by wind). These are in direct contravention of the 'similar name' taboo on Yang Jian's father's name, making it clear that the recipes were not carved during the Sui.

\section{Tang Dynasty Taboo Characters in the Recipes}

\section{Missing Strokes in the Character 世}

According to the Shihuijuli 史諱舉例 (Examples of Taboo Terms from History) taboos during the Tang were much more strict, forbidding not only the base character, but also similar names, and even the components of that character. In the early Tang, the primary forbidden characters were yuan 淵, hu 虎, bing 昞, shi 世, min 民 among others. To cope with this, the missing stroke method emerged within Tang calligraphy, which enabled copyists and writers to continue without excessive interruption. Chen Yuan states:

During the Tang, there were special ways to write taboo characters, such as leaving stroke(s) out, a method which began at the beginning of the dynasty. Once this precedent was set, then there was far less changing of old texts and writings. Original copies of the classics were relied on and preserved. ${ }^{48}$

Although we cannot see any of the characters mentioned above in the medical recipes, close examination of the character $z e$ 池 in the phrase 'wu shi qichi' 勿使氣池 (do not use the qichi point), ${ }^{49}$ reveals strokes that have been excised. Similarly, the 世 radical in the character ye 葉 in the phrase 'qing cong ye, gan huang' ye 青葱葉, 趕黃葉, (leaves of green scallion and of Penthorum chinense Pursh) is also missing strokes. This is clearly due to the taboo on the Tang emperor Taizong's 太宗 name, Li Shimin 李世 民 $(627-50)$.

The writing style of the characters chi 池 and ye 葉 deserves attention. The Youhuan jiwen 遊宦紀聞 states: ${ }^{50}$

As for the character 世, since Taizong tabooed the Shi 世 clan name, the characters die 牒, ye 葉 and $q i$ 棄 all have had the radical 世 removed from them.

According to the first chapter of Emperor Gaozong's 高 宗 (650-84) biography in the Old Tang Histories (Jiu Tang shu 舊唐書), it says: ‘十二月... 庚午, 改「昌」「葉」字’;

48 Chen Yuan $195^{8}$.

49 Although not one of the standard acupoints from Huangdi neijing 黃帝內經 and derivative literature, this point appears in tuina 推拿 massage manuals, such as Youke tuina mishu 幼科推 拿秘書, Luo Rulong 駱如龍, and Xiao'er tuina guangyi 小兒推 拿廣意, Xiong Yingxiong 熊應雄.
'On Jan... 26, 657, the characters hun 昌 and ye 葉 were changed. 51

Thus one knows that the writing of $y e$ 葉 as began in the second year of Gaozong's xuanqing 顯慶 reign (657), and that, according to juan 9 of Youhuan jiwen, “漏泄縲 紲, 又去世而從曳'. In the phrases 漏池 and 縲絏 [they] removed 世, and used 曳.

Thus 池 was written 洩, as in the Shijiazhaiyangxin lu 十駕齊養新錄, 52 which says:

A Tang stele of the Classic of Odes (Maoshi 毛詩) has 洩 洩其羽 for 泄泄其羽 (Ode 33), 桑者洩洩兮 for 桑者泄泄 兮 (Ode 111), 無然洩洩 for 無然泄泄 (Ode 254), 是縄祥也 for 是紲祥也 (Ode 47), 俾民憂洩 for 俾民憂泄 (Ode 253), all of which are taboos on 世.

Regarding the moment when the 'binomial exception' (pian hui 偏諱) ${ }^{53}$ began to be introduced,

In the Old [Tang] History, when Taizong became the Prince, he commanded saying: 'According to the Book of Rites one should not taboo both names, but in current times the tabooing of both characters in a name has already resulted in many losses. Classics with tabooed characters, official titles and personal names, public and private letters and documents which have the two characters 世 and 民 do not need to be banned when they are not written alongside one another'.54

Now although in the recipes the characters 池 and 葉 show missing stroke marks for taboo purposes, they have not been transformed to 洩 or 菜. Because of this, they must have been engraved between 65 o, the first year of Taizong's reign and 658 when the above variant characters came into use.

\section{療 Standing in for 治}

Emperor Gaozong's birth name was Zhi 治, so all instances of 治 (also a common verb meaning 'treat, cure') were changed to 療 or 主. In the Xinxiu bencao 新修本草 55 and in fragmentary documents from Dunhuang, this kind of evidence is often seen in the fairly numerous formulas

$5^{1} \quad$ History of the Former Tang, Jiu tangshu 舊唐書, Liu Xu 劉昫 (887-946), p. 4.77. Dates from Xue Zhongsan and Ouyang Yi (eds) 1961.

52 Shijiazhaiyangxin lu十駕齋養新錄, Qian Daxin 錢大昕 (17281804).

53 The binomial exception follows the 'Dianli' 典禮 chapter in Liji 禮記 (the Book of Rites), which states: 'Er ming bu pian hui' 二 名不偏諱, 'When someone has two given names, they should not both be tabooed'.

54 Discussions of the 17 Histories, Shiqishi shangque 十七史商榷, Wang Mingsheng 王鳴盛 (1722-98), juan 70.

55 Newly Revised Materia Medica, Xinxiu bencao 新修本草, Su Jing 蘇敬. 
which also use the character 寮. Throughout the whole text not one 治 character shows up, strong evidence that the recipes were inscribed during Gaozong's reign or the one following.

\section{Characters after Emperor Zhongzong 中宗} After Gaozong came the brief reigns of Zhongzong (684) and Ruizong 睿宗 (684-9o), who were both deposed by their mother Wu Zetian 武則天, but were re-established on the throne in 705 and 710 respectively. The characters 顯 and 旦 were added into the Tang taboo characters, and although we have not come across any instances of the character 顯, the term 平旦 (dawn) comes up frequently in the medical recipes. In addition, similar characters like 疸 (jaundice) were also banned. Because of this, we can verify that the medical recipes are not later than Zhongzong.

Based on the observation that the recipes do not use the new forms of the characters 年 and 日 established during Wu Zetian's 武則天 (684-705) reign, in combination with the date of the octagonal lotus pillar, we propose that the recipes were carved between 650 and 653 .

\section{The Same Source for the Longmen Herbal Recipes and the Dunhuang Scrolls}

The source of the Longmen herbal recipes has long been a mystery. Medical texts from Chinese history do not contain these formulæ. The Japanese Honzo wamyō 本草和名56 (918) lists a title called Longmen baiba 龍門百八 (Longmen 108), which is the earliest record of any document related to the recipes. The next surviving text, the Ishimpo 醫 心方 (Formulas of the Heart of Medicine) ${ }^{57}$ includes 102 entries titled Longmen yaofang 龍門藥方, but this does not match up with the number of recipes in the Longmen caves. However, our research has revealed a clue hidden amidst the Dunhuang scrolls.

\section{Clues in the Dunhuang Scrolls P.3596 and S.3347}

The best volume of Dunhuang medical manuscripts at present is Ma Jixing's 馬純興 edited collection Dunhuang guyaoji kaoyi 敦煌古藥籍考釋.58 Comparison of the untitled medical formulas Type 9 (P.3596) and Type 13 (S.3347) revealed interesting data. According to the critical study based on taboo characters by Ma Jixing et al., these two

56 Pharmacopoeia with Japanese names, Honzō wamyō 本草和名, Fukane Sukehito 深根輔仁 (898?-922?). Core Medical Formulas, Ishimpō 醫心方, Tamba no Yasuyori 丹 波康賴 (912-95).

$58 \quad$ Ma Jixing 1988. manuscripts were written in the early Tang. Since their taboos happened to be from exactly the same period as the Longmen recipes, the inscription and the manuscript dates are very close in time. Entry 213 in the formulas on P.3596 is exactly the same as one from the Longmen inscription, and there are up to 54 entries that are basically the same across both copies. (Because the text of the entries is divided into sections differently, many entries are largely the same as each other, while others are not the same at all. In total there are 58 entries in Type 9 which are either exactly or almost completely the same). In this kind of textual comparison, which relies on fragile decaying scrolls and dilapidated steles from a period before extensive use of printing, miscopied and variant characters ought to be very common. However, the high level of orthographic similarity between the two old texts is rarely seen elsewhere. From this we can see that the Longmen recipes and the Type 9 untitled medical formulæ must have been derived from the same source material. Similarly, S.3347 has 77 entries that are entirely identical with the Longmen recipes and 25 which are fundamentally similar. This demonstrates that the Type 13 untitled recipes are also derived from the same source material.

These two scrolls clearly show that they are derived from the same source as the Longmen recipes. But what relationship do the two scrolls bear to each other? It appears that they may also come from the same source, as they each include 40 entries which are either identical or very similar. Clearly these two scrolls are two different hand-copies of the same medical text, which served as the same source for the Longmen recipes. But we know nothing of the source material itself, its provenance, circulation or even any text titles name, and this remains a mystery.

\section{The Dunhuang Copy of Beiji Danyan YaofangJuan} 備急單驗藥方卷 S.9987

Wang Beiqing 王冀青 reported in 1991 that the East Asian section of the British Library discovered two document fragments from S.9987, whose contents, paper, calligraphy, character shape, style and pigmentation are the same as S.3347 and S.3395, and that all three come from the same manuscript. ${ }^{59}$ This means that S.3347, S.3395 and S.9987 were part of the same volume. Of further interest is the mention of the title of the said manuscript: Beiji danyan yaofang juan 備急單驗藥方卷 (Individually Verified Recipes Prepared for Crises). On the front of S.9987 there are nine entries similar to the Longmen recipes. Could it be that the Longmen recipes and the Beiji danyan yaofang

$59 \quad$ Wang Jiqing 1991. 
juan share the same origins? We think this is a possibility, for the following reasons:

1. There are a high proportion of similar entries.

2. In S.9987 verso it states:

求刊之岢石,傳以救病,庶往來君子錄之備急用。

I sought the carved stone inscriptions, and spread them in order to relieve illness, and many travelling gentlemen record them for use in all manner of maladies.

3. The following statement from S.9987 verso 2 is identical to one in the Honzō wamyō 本草和名, compiled in 918 by Sukehito Fukane 深江輔任 (898?-922?), who cites it as appearing in the Longmen babai 龍門八百 (Longmen 108):

服之立效一百八方、以人有一百八煩惱、合成此 $\square$ 。

Taking this [formula] is effective like the 'recipes for 108'. When a person has 108 vexations, combine and complete this $\square$.

The expression '108 Vexations', also called the 108 karmic bonds (jieye 結業), is a typical Buddhist term and refers to the multitudes of bad karma which are produced from a vexed mind. The Beiji danyanyaofang shows Buddhist influence, in that it refers to illnesses as the 108 vexations. The Longmen caves are sacred Buddhist ground, and carving the recipes there gave them clear Buddhist associations, which are borne out in the manuscripts described above. It could be that the formulas were those used at the Fengxian monastery at Longmen, but this is impossible to verify.

4. S. 9987 verso states:

$\square \square / \square$ 止、取對目前、豈得輕其賤穢、棄而不服者哉? 依用自取 $\square$ / $\square$ 鳩集單驗、始晤天地所生、還為天地所 用、觸目能療而 $\square / \square$ 救急易得、服之立此效。

This is like pig urine (猪零) and human faeces being able to cure crises of heat illness (熱病急) $\square \square$ Stop and hold it up in front of your eyes. How is that, slighting it as lowly and filthy, one discards it without ingesting it? Relying on $\square \square$ which you have acquired yourself, successively accumulating individual verifications begins to awaken you to [those things] which are produced by Heaven and Earth, and return [them] to the service of Heaven and Earth. Pressing it to the eyes can cure them and $\square \square$. Curing of crises can be easily achieved; ingesting it produces its effects.

This formula is very similar in content, metaphor and tone to one Longmen recipe in particular, but other uses of excrement can be found throughout the other Longmen recipes. ${ }^{60}$

Since the time of writing this chapter, more textual evidence has come to our attention, in the form of P.2666 $v^{o}$, which contains a number of similar or identical recipe and merits closer comparison. Furthermore, readers can

6o Zhang Ruixian et al. 1998, p. 117 . also direct their attention to the complete table of received literature which cites the Longmen Recipes by Catherine Despeux. ${ }^{61}$ It bears considering that despite the strong similarity across different manuscript and received sources, in many the order of recipes and sometimes their content varies. This leaves it ambiguous as to whether these records are testimony to a singular original text, or a common set or genre of recipes.

For these reasons, namely: the similarity between entries; the reference to a stone inscription; the use of Buddhist numerology; and the use of excrement, we can say that the Longmen recipes and the Beiji danyanyaofang juan share the same source material. Furthermore, from internal evidence in the inscription, and in comparison with the surrounding dedications in the cave itself and with the Dunhuang manuscripts, we can say that the inscription was commissioned by lay Buddhists or monks, and that they can be dated between $65^{\circ}$ and 653 .

Having dated the inscriptions with greater precision to the early Tang dynasty enables us to more accurately understand the religious, political and medical contexts which would have shaped the recipes' reception at the time of inscription. ${ }^{62}$ The display of the recipes communicated subtle but quite clear messages, regardless of their producers' tacit or conscious intentions. Their position among the votive carvings of the Longmen caves marked them as Buddhist at a time when Buddhist medicine had reached the apex of its influence in China. ${ }^{63}$ Through sharing the same location and medium as the votive offerings in the caves, the recipes - and metonymically, Buddhist medicine in general - accrued layers of association as convertors and transmitters of merit, and expressions of Buddhist compassion. The numinous power and elite patronage of Longmen lent the recipes power and authority, hinting that they were suitable not just for recipients of Buddhist charity, but for elite consumption - factors which likely influenced the copyists who saw fit to circulate them to regions as far as Dunhuang. The public display of the recipes in a high traffic area echoed earlier national medical policies; what effects this had on the public perception of Buddhist medicine, we cannot be sure. ${ }^{64}$

61 Thanks to Catherine Despeux for pointing this out: Despeux, 2010, pp. 382-6.

62 On the historical as an emerging, if not now fully established, critical approach in the history of medicine, see Lloyd and Sivin 2002, pp. xi-xlii, 3, as well as various papers from XxiI International Congress of History of Science, Beijing 2005.

63 Salguero 2010, pp. 54-7.

64 It is worth remarking that in the final year of the period in which the recipes were carved, 653 , the government issued a decree, which banned healing by the Buddhist community. 


\section{Appendix to Note 26}

What Xuan Wu meant by the term 'three illnesses' sanji 三疾 invites pause. The Chinese locus classicus for the term is Confucius' reference to three categories of moral illness in the Analects: kuang 狂 (madness), jin 矜 (conceit), and $y u$ 愚 (stupidity) (Lunyu 論語 17.6). However, by the Tang and Song, encyclopaedic works describe them as three categories of varying degrees of debility: can 殘 (debilitating), fei 廢 (crippling) and $d u$ 篤 (incapacitating) (Baikong liutie 白孔六帖 33.8a, Siku quanshu edn). During the Tang, the three illnesses took a central role in legal considerations of appropriate severity of corporal punishment (Tang Liudian 唐六典 30.23a and Tanglü shuyi 唐律疏義 $21.4 \mathrm{ff}$, Siku quanshu edns).

In Buddhist literature, the semantically and orthographically cognate sanbing 三病 represents a host of categories. Texts attributed to the Eastern Han translator from Parthia, An Shigao 安世高 ( $f$. $148 \mathrm{CE}$ ), use sanbing 三病 to refer to moral illnesses of the Sanskrit tri-ryādhi, listed as tan 貪 (avarice), chen 瞋 (anger or hate), and chi 癡 (stupidity). (Foshuo chuchu jing 佛說處處經 T730, 17.524b1). Closer to Xuanwu's time, specific meditations on filth (bujing 不淨), compassion (cixin 慈 心) and the wisdom derived from comprehension of causes and conditions (yinyuanzhi 因緣智) were prescribed as 'medicines' for these three illnesses in Dharmakșema's (385-433) translation of the Mahāparinirvāna-sūtra. (Daban niepan jing 大般涅槃經 T374, 12.593c11-13).

However, texts attributed to An Shigao also used sanbing to translate the Indian nosological theory of tridosha, that is, vata, pitta and kapha or śleșman. (Qichu sanguan jing 七處三觀經 T150A, 2.882a1417). This use of 三病, with varying translations for the doshas, is found throughout the Buddhist Canon. (Salguero 2010, 123-33). By the 6th century, alternative nosologies also began to appear. Zhi Yi 智顗 (53897), the Tiantai 天臺 founder, refers to three origins of disease as: $y e$ 業 (karma), gui 鬼 (ghosts) and sida 四大 (the four elements) (Shi chan boluomi cidifamen 釋禪波羅蜜次第法門 T1916, 46.505c23-27).

Furthermore, sanbing was used to distinguish three levels of prognosis. The Northern Liang 北涼 Mahāparinirvāna-sūtra defines the three illnesses as yi zhi 易治 (easy to treat), nan zhi 難治 (difficult to treat), and buke zhi 不可治 (impossible to treat) (T374 12.56oc14-15). This typology was extended to refer to three character flaws which could prevent someone from converting to Buddhism, 'treatment' for the disease of worldly existence.

What can Xuanwu have been referring to? The edict concerns medical issues, and is grounded in a revision of classical and other received medical lore. It is thus somewhat removed from Confucius' and An Shigao's moral deliberations. His reference to indigenous medicine (stone needles and Bian Que), together with the general lack of integration of court medicine with Buddhist theory, and the lack of Buddhist language in the edict, makes a tridosha reference seem incongruous, although not impossible. Although Xuanwu was not discussing corporal punishment, and many of the debilities mentioned in later legal texts are either congenital or the result of injury, it does not seem out of place to consider that he is referring to unequal distributions of debilitating disease.

\section{Bibliography}

\section{Primary Sources}

Baqiongshi jinshi buzheng xubian 八瓊室金石補正 899-901, by Lu Jihui 陸繼煇 and Lu Zengxiang 陸增祥 2002, Baqiongshi jinshi buzhengxubian 八瓊室金石補正續編, vol. 3, Shanghai:Shanghai guji chubanshe.

Bencao gangmu 本草綱目 1578, by Li Shizhen 李时珍 1975, Bencao gangmu: jiaodian ben 本草綱目 : (校点本), Beijing: Renmin weisheng chubanshe.

Ganlu zi shu 干祿字書 (A Writing Book for the Upwardly Mobile), by Yan Yuansun 顔元孫 and Zhang Shen 張參. In Congshu jicheng chubian 叢書集成初編, Wang Yunwu 王云五 (ed.) 1936, Shanghai: Shangwu yinshu guan.

Gu Yanwu quanji 顧炎武全集, Gu Yanwu 顧炎武 (2011), Shanghai: Shanghai guji chubanshe.

Hong Yixuan 洪頣煊 1966, Pingjin dubeiji平津讀碑記 (Records of Beijing and Tianjin Stelae Readings), 藝文印書館, Taibei: Yiwen yinshuguan.

Honzō wamyō 本草和名 918, by Fukane Sukehito 深根輔仁, in Taki Motohiro 多紀元簡 (ed.) 1796, Edo: Izumiya Shojiro.

Ishimpō 醫心方 984, by Tamba no Yasuyori 丹波康賴 1973 , vol. 30, Tokyo: Nihon Koigaku Shiryo Senta.

Jinshi cuibian 金石萃編 (Collected Chapters of Stone and Bronze Inscriptions) by Wang Chang 王昶 in Luo Ergang 羅爾綱 (ed.) 2003, Jinshi cuibianjiaobu 金石萃編校補, Beijing: Zhonghua shuju.

Jinshiwenziji 金石文字記 (Records of Bronze and Stone Inscriptions), by Gu Yanwu 顧炎武. In Gu Yanwu quanji 顧炎武全集, 2011, Shanghai: Shanghai guji chubanshe.

Jiu Tangshu 舊唐書 by Liu Xu 劉昫 1975, Beijing: Zhonghua shuju. Shi jiazhai yangxin lu 十駕齋養新錄 by Qian Daxin 錢大昕 1965 , in Sibu beiyao, Zibu Taipei: Taiwan Zhonghua shuju.

Shiqishi shangque 十七史商榷 by Wang Mingsheng 王鳴盛 in Shuhui Huang 黃曙輝 (ed.) 2005, Shiqi shangque 十七史商榷, Shanghai: Shanghai shudian.

Tang liudian 唐六典 (Compendium of Administrative Law of the Six Divisions of Tang Bureaucracy) by Zhang Jiuling 張九齡 and Zhang Shui 張說 2005, Beijing: Zhonghua shuju.

Weishu 魏書 by Wei Shou 魏收 1974, vol. 8, Beijing: Zhonghua shuju. Xinxiu bencao 新修本草 by Su Jing 蘇敬 ( $f l$. 7 th c.), Shanghai: Shanghai guji chubanshe.

Xiao'er tuina guangyi 小兒推拿廣意 by Xiong Yingxiong 熊應雄 1888, Chen Shikai 陳世凱 pub.

Yanshijiashun 顏氏家訓 (Yan Family Admonitions) by Zhou Yiliang 周一良 1992, Zhongguo dabaike quanshu 中国大百科全书, Beijing and Shanghai: Zhongguo dabaike quanshu chubanshe.

Youhuan jiwen 游宦紀聞 in Zhang Shinan 張世南 et al. (eds) 1981, Tang-Song shiliao biji congkan 唐宋史料筆記叢刊, Beijing: Zhonghua shuju.

Youke tuina mishu 幼科推拿秘書 by Luo Rulong 駱如龍 1784, Jinling: Sijiao tang.

Zhongzhoujinshiji 中州金石記 (Records of Epigraphy from the Central Provinces) by Bi Yuan 畢沅, in Wu Yunwang 王雲五 (ed.) 1966, Congshujichengjianbian 叢書集成簡編, Taipei: Taiwan shangwu yinshuguan.

\section{Secondary Sources}

Abe, S.K. 2002, Ordinary Images, Chicago: University of Chicago Press. Bumbacher, S.P. 2012, Empowered Writing: Exorcistic and Apotropaic 
Rituals in Medieval China, St Petersburg, FL: Three Pines.

Chang Qing 常青 1989, 'Longmen yaofangdong de chuchuang he xuzao niandai' 龍門藥方洞的初創和續鏊年代, Dunhuang yanjiu, $1,38-46$.

Chen Yuan 陳垣 1958, Shihuijuli 史諱舉例 (Examples of Taboo Terms from History), Beijing: Kexue chubanshe.

Demiéville, P. and M. Tatz 1985, Buddhism and Healing: Demiéville's Article 'Byo' from Hobogirin, Lanham, MD: University Press of America.

Despeux, C. (ed.) 2010, Médecine, religion et société dans la Chine médiévale : étude de manuscrits chinois de Dunhuang et de Turfan, Paris: Collège de France - Institut des hautes études chinoises.

Ding Mingde 丁明德 1979, 'Luoyang Longmen yaofangdong de shike yaofang' 洛陽龍門藥方洞的石刻藥方, Henan wenbo tongxun 2, 27-33.

Gernet, J. 1995, Buddhism in Chinese Society: an Economic History from the Fifth to the Tenth Centuries, New York: Columbia University Press.

Harper, D. 1998, Early Chinese Medical Literature: The Mawangdui Medical Manuscripts, London/New York: Kegan Paul Intl.

Hsieh Shu-wei (Xie Shiwei) 謝世維 2005, 'Writing from heaven: celestial writing in Six Dynasties Daoism', PHD diss., Bloomington: Indiana University.

2010, 'Shouguo yu chanhui: Zhonggu shiqi zuigan wenhua zhitantao' 首過與懺悔: 中古時期罪感文化之探討 (Repentance and confession: a study of the concept of guilt in medieval China), Qinghua xuebao 40.4 (Dec.), 75-104.

Hucker, C.O 1985, A Dictionary of Official Titles in Imperial China, Stanford, Calif.: Stanford University Press.

Jia Zhihong 贾志宏 and Ren Bangding 任邦定 1989, Longmen shiku yaofang 龙门石窟药方, Zhengzhou: Henan kexue jishu chubanshe.

Li Jianmin 李建民 (ed.) 2005, Cong yiliao kan zhongguo shi 從醫療看 中國史, Taipei: Lianjing chuban shiye gongsi.

Li Jingwei 李經緯 and Lin Zhaogeng 林昭庚 (eds) 2000, Zhonggu yixue tongshi:gudaijuan 中國醫學通史一古代卷, Beijing: Renmin weisheng chubanshe.

Li Wenbo 李文波 (ed.) 2004, Zhongguo chuanran bing shiliao 中国 传染病史料 (Historical Records of Infectious Diseases in China), Beijing: Huaxue gongye chubanshe.

Li Wensheng 李文生 1981, 'Longmen shiku yaofangdong kao' 龍門石 庫藥方洞考 (An examination of the Medical Recipe Cave in the Longmen Cave), Zhongyuan wenwu $3,63-5$.

Liang Piyun 梁披雲 1986, Zhongguo shufa dacidian 中國書法大辭典 (Dictionary of Chinese Calligraphy), Hong Kong: Xianggang shupu.

Liu Jinglong 劉景龍 and Li Yukun 李玉昆 1998, Longmen shiku beike tiji huilu 龍門石窟碑刻題記彙錄, Beijing: Zhongguo da baike quanshu chubanshe.

Liu Shufen 劉淑芬 2005, 'Cong yaofangdong dao huiminju - Sengren, guojia he yiliao de guanxi, cong yiliao kan Zhongguo shi' 從藥方 洞到惠民局一僧人、國家和醫療的關係, in Li Jianmin 李建民 (ed.), $145^{-202 .}$

Lloyd, G. and N. Sivin 2002, The Way and the Word:Science and Medicine in Early China and Greece, New Haven: Yale University Press.

Ma Jixing 馬繼興 1988, Dunhuang guyiji kaoshi 敦煌古医籍考釋 (Ancient Medical Manuscripts of Dunhuang, examined and explained), Nanchang: Jiangxi kexue jishu.

McNair, A. 2007, Donors of Longmen: Faith, Politics, and Patronage in Medieval Chinese Buddhist Sculpture, Honolulu: University of Hawai'i Press.

Mizuno Seiichi 水野清一 et al.1941, Kanan Rakuyō Ryūmon sekkutsu no kenkyū 河南洛陽龍門石窟の研究, Tokyo: Zauho Press.
Needham, J. and Lu Gwei-Djen 2000, Science and Civilisation in China, vol. 6., pt. 6, Biology and Biological Technology: Medicine, Cambridge: CuP.

Pettit, J. 2009, 'Barbarian hemp and the propagation of holy plants in China', conference paper, given at American Academy of Religion, Montreal.

Salguero, P. 2009, 'The Buddhist medicine king in literary context: reconsidering an early medieval example of Indian influence on Chinese medicine and surgery', History of Religions 48.3, 183-210. 2010, 'Buddhist medicine in medieval China: disease, healing, and the body in crosscultural translation (2nd to 8th Centuries CE)', PHD diss., Baltimore: John Hopkins.

2014, A study of the Chinese Reception of Indian Medicine, as Brought to China via Buddhist Texts, Philadelphia: University of Pennsylvania Press.

(ed.) 2017, Buddhism \& Healing in East Asia, New York: Columbia University Press.

Shao Dianwen 邵殿文 1993, 'Yaofang dong shike yaofang kao' 药方洞 石刻药方考 Zhonghua yishi zazhi, 23.4, 242-9.

Soothill, W.E. and L. Hodous 1937, A Dictionary of Chinese Buddhist Terms, London: K. Paul, Trench, Trubner \& Co.

Stanley-Baker, M. 2013, 'Daoists and doctors: the role of medicine in Six Dynasties Shangqing Daoism', PHD diss., London: University College London.

2014, 'Drugs, destiny, and disease in medieval China: situating knowledge in context', Daoism: Religion, History and Society $6,113-56$.

Stanley-Baker, M. and Yang, D. 2017, 'Dung, hair and mungbeans: household remedies in the Longmen recipes', in Salguero (ed.), 456-77.

Strickmann, M. 2002, Chinese Magical Medicine, Stanford, Calif.: Stanford University Press.

Unschuld, P.U. 1985, Medicine in China: a History of Ideas, Berkeley: University of California Press.

Tsiang, K.R. 2005, 'Embodiments of Buddhist texts in early medieval Chinese visual culture', in Wu Hung and Tsiang (eds), 49-78.

Wang Jiqing 王冀青 1991, 'Yingguo tushuguan cang “Beiji danyang yaofang juan” S.9987 de zhengli fuyuan’ 英国图书馆藏《备急单 验药方卷》 (S.9987) 的整理复, Dunhuang yanjiu 4, 103-6.

Wu Hung and K.R. Tsiang (eds) 2005, Body and Face in Chinese Visual Culture, Cambridge, MA: Harvard University Press.

Xue Zhongsan 薛仲三 and Ouyang Yi 歐陽頣 (eds) 1961, Liangqian nian Zhong-Xi li duizhao biao 兩千年中西曆對照表 (A Sino-Western calendar for two thousand years, 1-2000 AD), Hong Kong: Shangwu yinshuguan.

Zhang Ruixian 张瑞贤, et al. 1998, 'Luoyang longmen shiku yaofang yu Dunhuang juanzi “Beiji danyang yaofangjuan” tongyuan' 洛阳 龙门石窟药方与敦煌卷子《备急单验药方卷》同源, Zhonghua yishi zazhi 28.2, 113-17.

(ed.) 1999, Textual criticism of the Longmen Prescriptions, 龍門藥方釋疑, Zhengzhou: Henan yiliao daxue.

Zhang Ruoyu 張若遇 1980, 'Yinque fokan zhi bei he Qianxisi, Binyang dong' 伊關佛弇之碑和潛溪寺, 賓陽洞 (The Yinque shrine stele in relation to the Yinxi Monastery and Binyang Cave), Wenwu 1, 63-71.

Zhu Guihai 注桂海 1999, Handai guan wenshu zhidu 漢代官文書制 度 (Official Document Systems of the Han), Nanning: Guangxi jiaoyu chubanshe.

Zhu Shiduan 朱士端 1921, Yilu tang shoucang jinshiji 宜錄堂收藏 金石記 (Records of Stone and Bronze Epigraphy from the Hall of Notable [Steles]), Shanghai: Xiling yinshe. 


\title{
27 Embodying Animal Spirits in the Vital Organs: Daoist Alchemy in Chinese Medicine
}

\author{
Zhang Qicheng 張其成 \\ Translated and Edited by WangJing 王晶 and David Dear
}

The Tang and Song Dynasties saw the production of three of the most important books in the Daoist Canon in relation to medicine. These were:

Huangting dunjia yuanshen jing 黃庭遁甲緣身經 (Book of the Hidden Period and the Causal [Karma] Body of the Yellow Court, hereafter YSJ). DZ o873;

Shangqing ${ }^{1}$ huangting wuzang liufu zhenren yuzhou jing 上清黃庭五藏六腑真人玉軸經 (Precious Scroll of the Zhenren on the Six Receptacles and Five Viscera of the Yellow Court of Shangqing, hereafter YZJ). DZ 1402;

Huangting neijing wuzang liufu buxie tu 黄庭內景五藏 六腑補瀉圖 (Chart on the Procedures for Filling and Emptying the Six Receptacles and Five Viscera according to the Inner Landscape of the Yellow Court, hereafter BXT). DZ 0432.

The importance of these three books is that they are all based on the zangfu 藏腑 2 theory, which was comprehensively set out in different treatises of Huangdineijing 黃帝內 經 (Yellow Emperor's Inner Canon) and the Huangting jing 黃庭經 (Canon of the Yellow Court), compilations which date respectively to $c$. 1st century CE and 2 nd -3 rd centuries CE. This theory divides the body's organs into six zang 臟 inner (Yin 陰) organs and six fu 腑 outer (Yang 陽) organs. In the versions of the texts incorporated into the Daoist Canon, a real or mythical beast was selected to represent each one of the five zang organs and one $f u$ organ, i.e. the Gallbladder. This marks a major development in zangfu theory made by Daoist doctors and medical thinkers in the era between the Northern and Southern Dynasties, $420-589 \mathrm{CE})$ and the Song (96o-1279 CE). The Lungs were represented by the lion, the Heart by the red (vermilion) bird, the Liver by the dragon, the Spleen by the phoenix, the Kidneys by the (two-headed) deer and the Gallbladder by the gui-she (a tortoise or turtle and snake entwined). These conceptions had an important influence on the practices of both medicine and Daoism. This chapter will examine and compare the six beasts based on their descriptions in the three books.

1 Shangqing 上清 (Highest Purity) Daoism developed from the 3rd century CE onwards. It had a highly literate and scholarly focus, and as the name suggests, was much concerned with the expurgation of the vulgar, popular practices (especially sexual) of earlier 'Daoisms'.

2 Unschuld, 1985, pp. 77-83.
The discourse of medieval Chinese medicine was open to the incorporation of alternative and contemporaneous authorities about the body. One significant element was to be found in the writings and institutions of Daoist alchemy. Daoist visualisation of animal and other spirits as resident in the vital organs became a part of classical Chinese medicine. This chapter shows how esoteric ideas contributed to an ongoing search to articulate physiology. The individual functions of the organs were characterised by animal spirits, which through their natures expressed a delicate relationship between organic function and states of emotion. By looking at the way in which the text-image dynamic constituted a homology, we understand how emotional excesses could disrupt organic function and, potentially, vice versa. Thus the emotions were rooted in organic function and could, conversely, be regulated through correct visualisation.

The beasts were selected to represent their respective organs according to the principles of one or more different theories:

\section{Xingzhuang ZhiXiang 形狀之象 (Physical Resemblance)}

The organ in question simply bears an imagined resemblance in some of its characteristics to the beast which represents it, or possibly they are associated through analogy with another object which they are both believed to resemble. These 'intermediary objects' are things like xuanqing 懸馨 (hanging 'bells'), lianhua 蓮花 (lotus flowers), xuanpao 懸歇 (hanging gourds), fupen 覆盆 (blackberries),yuanshi 圓石 (round stones). This is a particular feature of the YZJ, where both the beasts and the organs themselves are illustrated.

\section{Fangwei Zhi Xiang 方位之象 (Direction Corre- spondence with the Wuxing 五行 (Five Agents))}

In the wuxing correspondence system, the Five Agents (Wood, Metal, Earth, Water and Fire), also themselves correspond to points of the compass (East, West, Centre, North and South), and again in turn to organs of the body. So the Lungs represent duijin 兌金 (Metal) and correspond 
to West; the Heart represents li huo 離火 (Fire) and the South; the Liver represents zhen mu 震木 (Wood) and East; the Spleen represents kun tu 坤土 (Earth) and the Centre; the Kidneys and the Gallbladder represent kan shui 坎水 (Water) and North. However, in these three texts, only the correspondences of the Heart, Vermilion bird and South, and of the Liver, Dragon and East, are entirely consistent with the regular directional correspondences of the Five Agents.

\section{Texing Zhi Xiang 特性之象 (Character of the Image)}

According to the descriptions given, each of the zangfu has its own xiang 象 (image) and shen 神 (spirit). The descriptions of the xiang vary in length but are of a similar type. Once again the intermediary objects feature as described above under Xingzhuang zhixiang. However the descriptions of the shen are quite different. They appear to use the animal imagery as metaphors for the distinctive spirit or character of each organ. This is an idea derived from Daoism of the Tang and Song. The Daoists were themselves developing the ideas of Shenzang sixiang 神藏思想 (spirit-organ theory) found in the Huangdineijing. The latter mentions only briefly the physical form of each organ but the 3rd-century CE Huangting jing gives detailed descriptions of the shen (spirit) of each of the zangfu according to the characteristics of each of the associated animal spirits.

\section{Fei Shen 肺神/Lung Spirit (zang)}

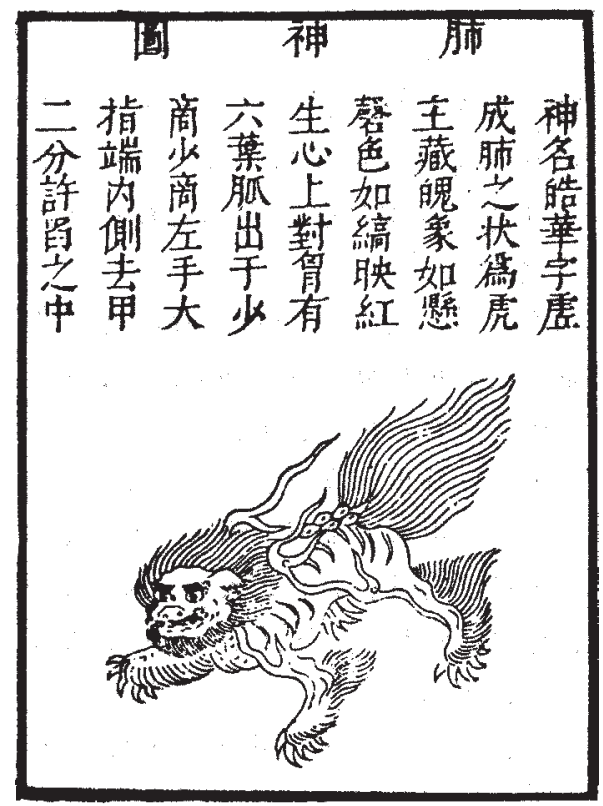

Figure 27.1 Lion/White Beast of the Lung, DZ 1402.

\begin{tabular}{|c|c|}
\hline Spirit Image/ Designation & $\begin{array}{l}\text { Bai Shou 白獸: The White Beast/Lion } \\
\text { The YZJ says 'Its spirit is like the white beast' (qi shen ru bai shou 其神如白獸). The YSJ has the } \\
\text { same description except that the final shou character is not 獸 (beast) but the homophone } \\
\text { 狩 (hunting). There is no such clear description in the BXT, but the picture, a lion in profile, is } \\
\text { nearly identical to that in the YSJ. In the YZJ the lion is viewed from head on. On the evidence } \\
\text { of Shuowen 說文 (Explanation of Characters) and the Shijing 詩經 poem 'Che gong' 車攻 (Our } \\
\text { Chariots Were Strong), the characters shou 獸 (beast) and shou 狩 hunt) were graphic variants, } \\
\text { so it can be assumed that the Lung Spirit was called the White Beast (bai shou 獸). }\end{array}$ \\
\hline Types of Shen & $\begin{array}{l}\text { All three books describe the spirit of the Lungs (fei shen 肺神) as a po 魄 (earthly soul), but in } \\
\text { differing ways. The YSJ says the Lungs are the master of the soul (fei zhu po 肺主魄). The YZJ } \\
\text { says the Lungs give birth to the soul (fei sheng po 肺生魄). This slight difference in the zhu/ } \\
\text { sheng 主/生 formulation is repeated throughout these two books. } \\
\text { The largely un-annotated BXT says: 'the Gate of Soul (po men 魄門) is above the Jade Hall (yu } \\
\text { tang 玉堂)'. }\end{array}$ \\
\hline $\begin{array}{l}\text { Character } \\
\text { (not addressed in вXт) }\end{array}$ & $\begin{array}{l}\text { The YSJ says the key characteristic of the fei shen is irascibility. (qi shen duo nu 其神多怒). The } \\
\text { YZJ says that anger originates in the Lungs. }\end{array}$ \\
\hline $\begin{array}{l}\text { Manifestation } \\
\text { (Not addressed in BXT) }\end{array}$ & $\begin{array}{l}\text { According to both the YSJ and YZJ the spirit takes the form of a young boy, } 7 \text { cun 寸 } \\
\text { (approximately } 23 \mathrm{~cm} \text { ) in height, who resides in the Lungs. }\end{array}$ \\
\hline Principles (for soothing) & $\begin{array}{l}\text { The YSJ and YZJ both prescribe controlling desires, being kind-hearted and just. Anger should be } \\
\text { avoided and one should not allow oneself to be stirred up by others. Through control, harmony } \\
\text { will be created within the body. } \\
\text { BXT does not specifically describe a principle of soothing the temperament of the Lungs, but it } \\
\text { prescribes personal cultivation (xiuyang fa 修養法), drug prescriptions, and the six Qi/breaths } \\
\text { method (liu qifa 六氣法), along with various intra-mensual dietary prohibitions (yue shi jinjifa } \\
\text { 月食禁忌法) and daoyin - all in great detail compared to the other two books. }\end{array}$ \\
\hline
\end{tabular}


Summary

The spirit of the Lung organ (feizang 肺臟) is a po (earthly spirit) and is characterised by being easily roused to anger. The lion is chosen to represent this force because it is associated with a similar fierceness of spirit, and is a beast that is regarded as dominating others.

Xin Shen 心神/ Heart Spirit (zang)

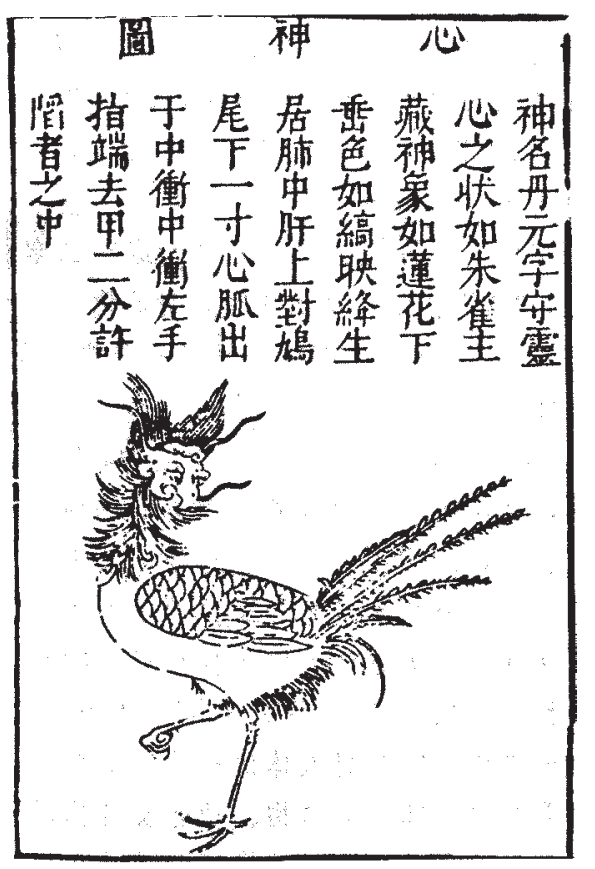

Figure 27.2 Vermilion Bird of the Heart, DZ 1402.

\begin{tabular}{|l|l|}
\hline Spirit Image/ Designation & $\begin{array}{l}\text { Zhu Que } \text { 朱 雀: The Vermilion Bird } \\
\text { Both YSJ and YZJ tell us 'its spirit is that of the vermilion bird (zhu que). } \\
\text { In BXT the image is unnamed but is very similar to the image in YSJ, and in both cases the bird is } \\
\text { depicted in profile. In the YZJ, as with the bai shou, it is seen head on. }\end{array}$ \\
\hline Types of Shen & $\begin{array}{l}\text { The YSJ says that the Heart dominates the spirit. The YZJ says that the Heart gives birth to the } \\
\text { spirit. The BXT says that the Heart is the location of life's Root Spirit (ben shen 本神). }\end{array}$ \\
\hline $\begin{array}{l}\text { Character } \\
\text { (not addressed in BXT) }\end{array}$ & $\begin{array}{l}\text { The YSJ and the YZJ both say that the spirit of the vermilion bird is restless and unpredictable } \\
\text { (qi shen zao er wu zhun 其神燥而無准), 'flighty' we might say in English. The YZJ also warns } \\
\text { that all sudden unexpected 'attacks' on one's health are due to its action in the Heart. }\end{array}$ \\
\hline $\begin{array}{l}\text { Manifestation } \\
\text { (Not addressed in BXT) }\end{array}$ & $\begin{array}{l}\text { The YSJ and YZJ say that the spirit appears as a young girl with a height of } 8 \text { cun (c. 27cm); she } \\
\text { carries a priceless jade and moves in and out of the Heart. }\end{array}$ \\
\hline Principles (for soothing) & $\begin{array}{l}\text { The YSJ and the YZJ say that to achieve physical and mental peace and calm one should be filial, } \\
\text { loyal, just and kind-hearted. Avoid a quick temper, settle the body and the spirit, and your Heart } \\
\text { will be at peace. } \\
\text { The BXT recommends the same practices as for the Lung Spirit above, but with no specific } \\
\text { reference to any theory behind it. }\end{array}$ \\
\hline Summary & $\begin{array}{l}\text { The Heart Spirit is the Root Spirit. It is restless, unpredictable and liable to violent attacks. It } \\
\text { corresponds to Fire and the South and is symbolised by the vermilion bird, the } z h u \text { que. }\end{array}$ \\
\hline
\end{tabular}


Gan Shen 肝神/ Liver Spirit (zang)

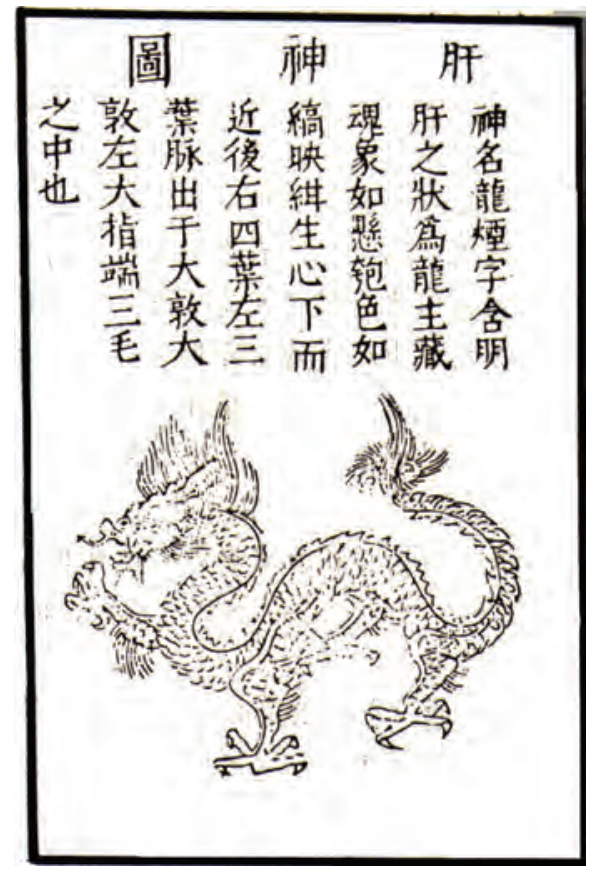

Figure 27.3 Dragon of the Liver, DZ 1402.

\begin{tabular}{|l|l|}
\hline Spirit Image/ Designation & $\begin{array}{l}\text { Long } \text { 龍: The Dragon } \\
\text { YSJ and the YZJ both say the spirit of the Liver (gan 肝) is like a dragon. There is an image of a } \\
\text { dragon which appears in each. A similar image appears in the BXT but with no descriptive text. }\end{array}$ \\
\hline Types of Shen & $\begin{array}{l}\text { The YSJ says that the Liver 'rules' ( } z \text { hu主) the hun 魂, the heavenly or eternal soul (as opposed } \\
\text { to the po 嵬, the earthly one, which decays after death). The YZJ says that the Liver 'generates' } \\
\text { (sheng 生) hun. } \\
\text { BXT says that the Liver is the main organ where the hun is dominant. }\end{array}$ \\
\hline $\begin{array}{l}\text { Character } \\
\text { (not addressed in BXT) }\end{array}$ & $\begin{array}{l}\text { The YSJ and YZJ describe the essence of the spirit as benevolence and kind-heartedness. Benev- } \\
\text { olent persons get their motivation from this organ. }\end{array}$ \\
\hline $\begin{array}{l}\text { Manifestation } \\
\text { not addressed in BXT) }\end{array}$ & $\begin{array}{l}\text { The YsJ it says that this spirit manifests as a young girl and a young boy together, one black and } \\
\text { the other yellow. Each is } 7 \text { cun (c. 23cm) tall, one is accompanied by a dragon and the other } \\
\text { holds a bottle of elixir. They move in and out of the Liver. } \\
\text { There is a similar description in the YZJ, but there their heights are given as } 1 \text { cun (c. } 3 \mathrm{~cm}) \text { each. }\end{array}$ \\
\hline Principles (for soothing) & $\begin{array}{l}\text { The YsJ and YZJ both say that to calm the Liver and live a long life, one should live frugally in } \\
\text { poor surroundings, avoiding all luxuries. This will create a higher harmony in the body. } \\
\text { BXT as above. }\end{array}$ \\
\hline Summary & $\begin{array}{l}\text { The Liver Spirit is a hun, or heavenly soul. Its character is benevolence. Its image is the dragon } \\
\text { (long). The dragon is associated with rain, beneficial to all living creatures. Its traditional corre- } \\
\text { spondent direction is the East }\end{array}$ \\
\hline
\end{tabular}




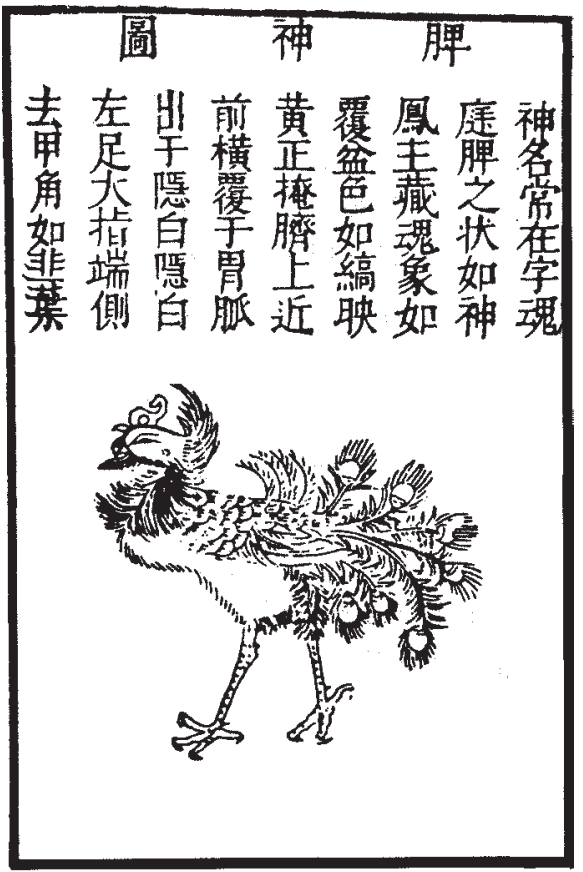

Figure 27.4 Phoenix of the Spleen, DZ 1402.

\begin{tabular}{|l|l|}
\hline Spirit Image/ Designation & $\begin{array}{l}\text { Feng } \text { 風: The Phoenix } \\
\text { The YSJ and YZJ both say 'its spirit is like a phoenix' (qi shen ru feng 其神如風). } \\
\text { The BXT does not name the creature but the image is very similar to that in the other books } \\
\text { with only a few differences in artistic technique }\end{array}$ \\
\hline Types of Shen & $\begin{array}{l}\text { The YSJ and the YZJ say that the Spleen is the master of one's intentions (pi zhu yi 脾主意). } \\
\text { According to BXT, the Spleen is the pivot of the five zang-organs, and the seat of all intentions. }\end{array}$ \\
\hline $\begin{array}{l}\text { Character } \\
\text { (not addressed in BXT) }\end{array}$ & $\begin{array}{l}\text { The YSJ and the YZJ say the character of the spirit is jealousy and that this feeling grows from the } \\
\text { Spleen. }\end{array}$ \\
\hline $\begin{array}{l}\text { Manifestation } \\
\text { (Not addressed in BXT) }\end{array}$ & $\begin{array}{l}\text { The YSJ and the YZJ describe the spirit as a young girl with the height of } 6 \text { cun }(c .20 \mathrm{~cm} \text { ), who } \\
\text { circulates within the Spleen }\end{array}$ \\
\hline Principles (for soothing) & $\begin{array}{l}\text { The YSJ says to calm this spirit one should control sexual desire. The YZJ concurs and adds } \\
\text { that one should not think too much. This will prolong one's life in harmony with Taiyin 太陰, } \\
\text { cosmic Yin. } \\
\text { BXT as above. }\end{array}$ \\
\hline Summary & $\begin{array}{l}\text { Intention (yi 意) is founded in the Spleen organ (pi zang 脾藏). The main characteristic of the } \\
\text { spirit is jealousy. Both the YSJ and the YZJ state that jealousy in women is caused by excessive } \\
\text { Yin. } \\
\text { The phoenix is selected to represent this as it is the counterpart of the dragon, which is Yang. }\end{array}$ \\
\hline
\end{tabular}




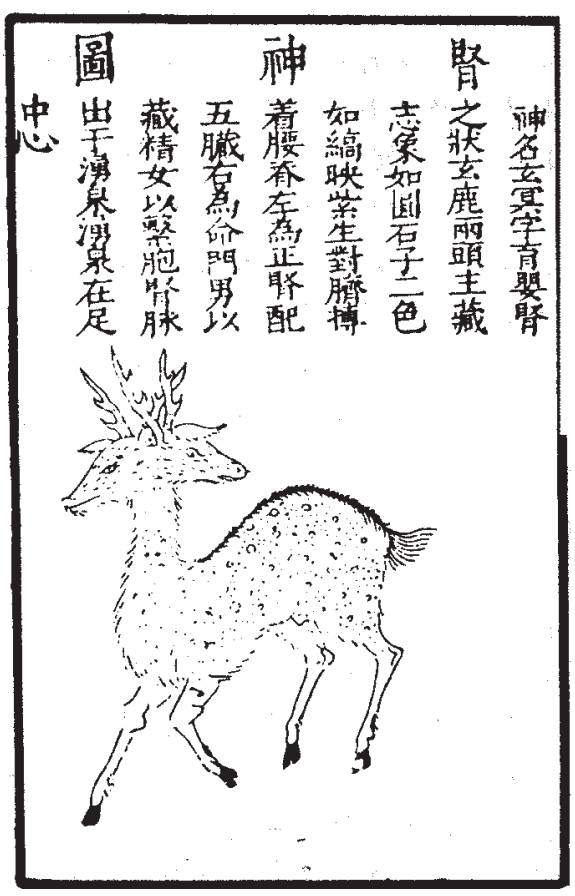

Figure 27.5 Deer of the Kidney, DZ 1402.

\begin{tabular}{|l|l|}
\hline Spirit Image/ Designation & $\begin{array}{l}\text { Lu } \text { 鹿: The Deer } \\
\text { The YSJ and the YZJ say 'the spirit is like a white deer with two heads'. There is no written de- } \\
\text { scription of this in the BXT but the illustration is of a two-headed deer in a standing position, } \\
\text { similar to that in the YSJ. In the YZJ, the beast is shown lying down. }\end{array}$ \\
\hline Types of Shen & $\begin{array}{l}\text { Both the YSJ and the YZJ are vague about the spirit of the shen zang 腎藏, the Kidney Spirit, oth- } \\
\text { er than saying it is the essence of Yin. The BXT says that the Kidneys are the origin and storage } \\
\text { point of all the essences. }\end{array}$ \\
\hline $\begin{array}{l}\text { (not addressed in BXT) } \\
\text { Manifestation }\end{array}$ & $\begin{array}{l}\text { The YSJ and the YZJ say the nature of the spirit is harmoniousness. Obedience in a person starts } \\
\text { in their Kidneys. }\end{array}$ \\
\hline Principles (for soothing) & $\begin{array}{l}\text { The YSJ and the YZJ say the spirit appears as a young boy with a height of } 1 \text { chi } \text { 尺 (1 Chinese } \\
\text { foot, c. 33cm), who moves in and out of the Kidneys. }\end{array}$ \\
\hline $\begin{array}{l}\text { According to the YSJ and the YZJ, to rest this spirit, one should be kind-hearted, calm and gentle. } \\
\text { By nourishing all the things in accordance with Tai Qing 太清 (supreme purity), one increases } \\
\text { one's essence and puts one's will in order, then one's physical form becomes whole. } \\
\text { BXT as above. }\end{array}$ \\
\hline $\begin{array}{l}\text { Summary } \\
\text { Jing (essence) is the key attribute of the Kidney. This is different from medical theory of the } \\
\text { time, which believes } z h i \text { 志 (will) is its key attribute. Daoism prioritised cultivating essence } \\
\text { (jingqi } \text { 精氣). Its character is described as soft and obedient, virtues embodied by the deer. The } \\
\text { two heads of the deer relate to the physical form of the kidneys. Deer also represent long life in } \\
\text { traditional beliefs. As the Kidneys are the prenatal root of life (Kidney essence is also cognate } \\
\text { with male sexual essence), the image of the deer expresses the aspiration for longevity. }\end{array}$ \\
\hline
\end{tabular}


Dan Shen 胆神/ Gallbladder Spirit $(f u)$

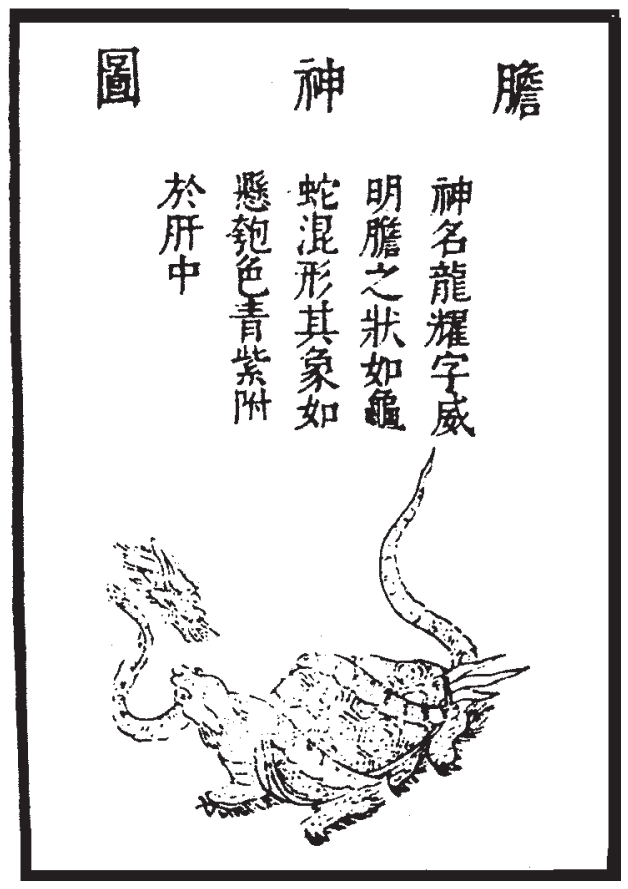

Figure 27.6 Turtle-Snake of the Gallbladder, DZ 1402.

\begin{tabular}{|l|l|}
\hline Spirit Image/ Designation & $\begin{array}{l}\text { Gui-she } \text { 龜蛇: The Turtle and Snake } \\
\text { The YSJ says 'the spirit is a turtle and a serpent'. The YZJ says 'the spirit is like a turtle } \\
\text { and a serpent'. No clear description is given in BXT and there are differences in artistic } \\
\text { technique as well as in the illustration itself from the other two books, but it is none- } \\
\text { theless clearly a combination of a turtle and a serpent. }\end{array}$ \\
\hline Types of Shen & $\begin{array}{l}\text { Both the YSJ and the YZJ describe it as the essence of gold (jin zhi jing 金之精). This is } \\
\text { not mentioned in BXT. }\end{array}$ \\
\hline $\begin{array}{l}\text { Character } \\
\text { (not addressed in BXT) }\end{array}$ & $\begin{array}{l}\text { The YSJ and the YZJ say that the spirit's characteristic nature is courage. Courage is } \\
\text { rooted in the Gallbladder. }\end{array}$ \\
\hline $\begin{array}{l}\text { Manifestation } \\
\text { (not addressed in BXT) }\end{array}$ & $\begin{array}{l}\text { The YSJ and the YZJ describe the spirit as a young boy with the height of } 1 \text { chi, who } \\
\text { runs carrying a halberd in his hand. }\end{array}$ \\
\hline Principles (for soothing) & $\begin{array}{l}\text { The YsJ says that, to rest this spirit, one should avoid arguing or debating with others. } \\
\text { Be kind-hearted and just, and then you can live a long life. The YZJ uses the same } \\
\text { phrasing bar one character difference - the meaning is the same. } \\
\text { BXT recommends personal cultivation methods (xiuyang fa 修養法), the guiding and } \\
\text { pulling (daoyin) traditions of therapeutic movement, and Qi manipulation, (qifa 氣 } \\
\text { 法). }\end{array}$ \\
\hline Summary & $\begin{array}{l}\text { There is no clear single designation for the Gallbladder Spirit. The two annotated } \\
\text { texts (YSJ, YZJ) say it is the essence of gold (jin zhi jing). This differs from traditional } \\
\text { medical theory and may be related to Daoist practices. As with the deer, gui-she repre- } \\
\text { sents long life. }\end{array}$ \\
\hline
\end{tabular}




\section{Conclusion}

The three books all share the same structure with identical chapter titles and chapter orders, with each of the zangfu described in terms of its symbolic moving spirit. The illustrations in the three books are also very similar and their correspondence to the organs identical.

There are, however, some differences between the three books. In the Yuanshen jing and the Yuzhoujing, there are clear explanations for the naming of the images, and these in themselves are similar, except for some minor differences in relation to the Lung Spirit. However in the Buxie tu, there are no clear names or descriptions. Though the type of beast is the same in each book, there are differences in the style and technique of the illustrations as well as in the postures and the points of view from which they are shown. In the Yuanshen jing and Buxie tu, only the animal spirit itself is illustrated. In the Yuzhoujing, there is in addition a simple illustration of the actual organ. Kind-heartedness and tranquillity seem to be the common factor for soothing most of the spirits in question. This idea was to be carried through to the yangsheng 養生 self-cultivation tradition of future centuries.

All told, the three books represent a continuation of earlier traditions extant in the medical classics Huangdi neijing and the more esoteric Huangting jing writings, but expressed in imagery derived directly from Daoist meditation practices. Text is simply used as an adjunct to supplement the power of the animal images. The variations from earlier wuxing and directional correspondence theories can be seen as confirmation of the influence of developments in Daoist thought and practice.

\section{Bibliography}

\section{Primary Sources}

Huangting dunjiayuanshenjing 黃庭遁甲緣身經 (Book of the Hidden Period and the Causal [Karma] Body of the Yellow Court) (YSJ) DZ o873.

Huangting neijing wuzang liufu buxie tu 黃庭內景五臟六腑補瀉 圖 (Chart on the Procedures for Filling and Emptying the Six Receptacles and Five Viscera according to the Inner Landscape of the Yellow Court) (вХт) DZ 0432.

Shangqing huangting wuzang liufu zhenren yuzhou jing 上清黃庭 五藏六腑真人玉軸經 (Precious Scroll of the Zhenren on the Six Receptacles and Five Viscera of the Yellow Court of Shangqing). (YZJ) DZ 1402.

\section{Secondary Sources}

Unschuld, P.U. 1985, Medicine in China: A History of Ideas, London: University of California Press. 


\section{A Phoenix Amid the Flames: Mount Emei Big Dipper Finger-Point Method, Daoyin and Qigong}

\section{Liao Yuqun 廖育群}

\section{Introduction}

The images presented here are taken from the Emei shan tiangang zhixue fa 峨眉山天罡指穴法 (Mt Emei's Big Dipper ${ }^{1}$ Finger-point Method) by Zhou Qianchuan 周 潜川 (1905-71), from Sichuan province. Zhou was a renowned physician operating in an arcane and mystical tradition. ${ }^{2}$ The book describes 28 different hand positions for manipulating Qi 氣 in others. However the book has had a troubled history and does not exist in its complete original form. Though first published in 1962, the original text with any accompanying images was destroyed. The book's author, Zhou Qianchuan, came to be regarded as a miscreant for his work in regard to Qigong and religious medicine, subjects that came to be regarded as mixin 迷 信 (outlawed superstition). This chapter offers a window on to the 2oth-century history of a secret medico-religious tradition through the biography of a major protagonist and the reconstruction of one of his key works by his disciples and others who were close to him.

\section{My Family Involvement}

More than 5 o years ago, I used to accompany my father to Zhou's house in Beijing where my father had become one of Zhou's students. I would then see my father, a marine engineer by profession, go home and make drawings of the techniques Zhou had taught him (Fig. 2).

My father and his family had a longstanding interest in Chinese medicine and herbal medicine in particular. My great-uncle had received a secret transmission of analgesic herbs from a doctor who posed for a while as a vagrant, in order to establish my great-uncle's suitability to receive the recipe. When my father had fallen ill in 1957, he had cured himself with herbal medicine. Then Zhou arrived out of the blue from Shanghai and my father was introduced to him. My father would study with Zhou several times

1 Emei mountain is a famous Daoist temple site in Sichuan, while 'Tiangang' is the constellation Ursa Major, also know as the Big Dipper, an object of veneration in Daoist ritual.

Zhou Qianchuan's death as 1962. Here I have corrected this in line with Zhou Huaijiang's 1985 article. Zhou Huajiang is Zhou Qianchuan's grandson.

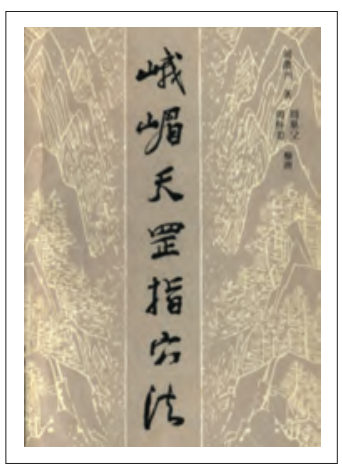

Figure 28.1 The cover of Emei shan tiangang zhixue fa 峨眉山天罡指 穴法 (Mt Emei's Big Dipper Finger-point Method) 1985 edn

a week and when in the early 6os Zhou was invited to Shanxi to work, he specified that any remaining patients in Beijing should be referred to my father. After a while my father gave up his job in Beijing to follow Zhou to Shanxi. However their relationship was brought to a stop by the political movements of the mid-6os and thereafter my father had to return to Beijing. Zhou was persecuted and imprisoned. After a period of unemployment, my father had some great good fortune in the form of a backdated pension allowance. It was the fulfilment of a prediction that Zhou had had made that my father would enjoy a financial windfall in his early forties. Zhou himself had no such good fortune and died for want of adequate medical treatment while still in prison in 1971.
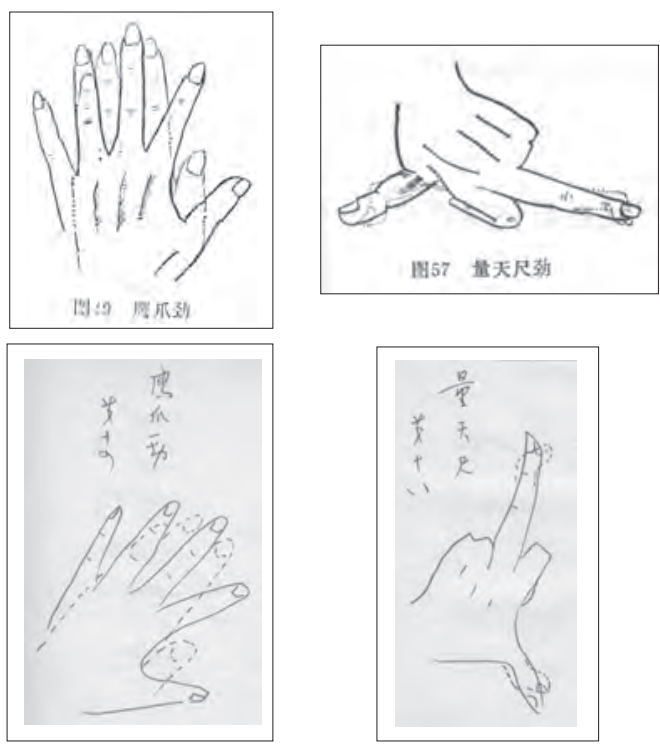

Figure 28.2 Emei Tiangang Zhixue Fa: (above) two of my father's illustrations; (below) from the 1985 publication 


\section{Zhou Qianchuan's Career and History of his Book}

Zhou Qianchuan was from Sichuan Province. He began his career as an army medical orderly. Subsequently, with financial support from his well-to-do father-in-law, he was able first to attend Wuhan University, and then to travel to Great Britain to further his studies in military engineering. After suffering an injury while practising martial arts, he was successfully treated using Mongol medicine. This aroused his interest in Chinese medicine in general. He went to the Emei and Wudang mountains, where he studied a wide range of subjects from many different traditions, including divination and the martial arts. However his core interest was in Chinese medical theories and techniques. His research in this field enabled him to develop his own unique form of practice.

After the civil war Zhou began to practise medicine in Shanghai. Then in the 1950s he was invited to treat patients in Beijing by some of the celebrated figures there. Thanks to his exceptional therapeutic results, he received a recommendation to the central government, and a vice minister in the Ministry of Health arranged for him to move from Shanghai and practise at Beijing's Sanshi ${ }^{3} x u e h u i$ 三 時學會 (The Three Era Study Association), an influential lay Buddhist research centre in Beijing. In the evenings he gave lectures on medicine, which my father would attend. In those days Zhou's treatments cost five yuan; equivalent to a month's salary. From that we can tell that he enjoyed considerable status and respect. In the early 1960 s, he was invited to work at the Shanxi TCM Research Institution after successfully treating some officials from that province. Zhou accepted the offer on condition that he would be able to continue to research, teach and publish on Chinese medicine and neidan 內丹 alchemy, a form of inner meditation aimed at refining the elixirs of life (See Despeux, Chapter 2 in this volume, pp. 63-4). In Shanxi, Zhou regularly treated members of the political and military elites. He published a number of books during this time, including the Qigong yao'er liaofa 氣功藥䬣療法 (Qigong Tonics and Remedies) and the Emei shi'er zhuang shimi 峨眉十二莊釋密 (Secret Explanation of the Emei Shi'er Zhuang), and he also gave a series of unpublished lectures which his students would record and circulate amongst themselves.

Sanshi (tri-kāla), a Buddhist term, refers to ' $\mathrm{t}]$ he three divisions of the day, i.e. dawn, daylight, and sunset, or morning, noon, and evening; also the three periods, after his nirvāna, of every Buddha's teaching, viz., 正 correct, or the period of orthodoxy and vigour; 像 semblance, or the period of scholasticism; and 末 end, the period of decline and termination' (Soothill and Hodous 1937, p. 536).
After the end of the Cultural Revolution, Zhou's case was reassessed and he was exonerated. Interest in both Qigong and Daoist medicine then started to grow again, as it had in the 195os. With the appetite for information about such techniques reaching a nationwide fever pitch, ${ }^{4}$ in 1985 Zhou's son had his father's book republished (Fig. 1). It was the survival of the unpublished lectures collected by his students that made the re-publication of the text possible. However some of the original images were missing and had instead been to be replaced by brief descriptions.

It is by comparing the illustrations in my possession that were made by father in the 196 os with the ones re-published in 1985 that I can verify the accuracy of the latter.

\section{Analysis of the Text}

\section{Mt Emei's Big Dipper Finger-Point Method List of 28 Hand Positions}

- Hezui jin ${ }^{5}$ 鶴咀勁 (The Crane's Beak)

- Fengchaijin 風釷勁 (The Phoenix and Hairpin)

- Yingzui jin 鷹嘴勁 (The Eagle's Beak)

- Shetou jin 蛇頭勁 (The Snake's Head)

- Yazuijin 鴨嘴勁 (The Duck's Beak)

- Riyue koujin 日月扣勁 (Fastening Sun and Moon)

- Chongtian chu jin 沖天杵勁 (Soaring Pestle)

- Yizhichanjin 一指禪勁 (Single Finger Meditation)

- Jingou jin 金鈎勁 (Golden Hook)

- Wuding kai shan jin 五丁開山勁 (Five Fingertips Open the Mountain)

- Huzhaojin 虎爪勁 (Tiger Claw)

- Long tan zhua jin 龍探爪勁 (The Dragon's Claw)

- Dingtoujin 丁頭勁 (Head of Strong Man)

- Yingzhua jin 鷹爪勁 (The Eagle's Claw)

- Long xian zhu jin 龍銜珠勁 (Dragon with Pearl in its Mouth)

- Pingzhijin 平指勁 (Even Fingers)

- Fuyufanyunjin 複雨翻雲勁 (Recurrent Rain and Rolling Clouds)

- Tongtian jin 通天勁 (To Reach the Sky)

- Liangtianchijin 量天尺勁 (Ruler to Measure the sky)

- Jianjuekaiqijin 劍决開氣勁 (Sword to Open Qi)

· Lijing jin 離經勁 (Separating the Channels)

- Luoyan jin 落雁勁 (Wild Goose Landing)

- Pengshajin 捧沙勁 (Cupping Sand)

· Taiji mo yun jin 太極摩雲勁 (Taiji Rubbing Clouds)

· Shaoyang zuqijin 少陽祖氣勁 (Shaoyang Ancestral Qi)

4 Editor's note: on Qigong fever (氣功熱) see D.A. Palmer, Qigong Fever, New York, Columbia University Press, 2007.

5 Jin 勁 at the end of each name simply means 'force'. It can be taken as an indication of their distinct, dynamic and transformative natures in the eyes of the practitioner. 


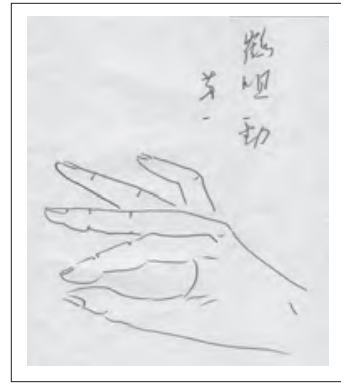

Figure 28.3 Hezui jin (The Crane's Beak)

- Bao pujin 豹撲勁 (Leopard Pounce)

- Xiudijin 袖底勁 (Sleeve Ends)

- Tuotian jiedi jin 托天截地勁 (Support the Sky and Press the Earth)

Each hand position has some descriptive verses dedicated to it, followed by a further explanation. The verses are somewhat antique in style and content. They include both the traditional master/disciple teaching that Zhou received and his own, subsequently developed, personal understanding and interpretation of the subject matter. The first verse on each gesture usually describes the shape and the action of the hand. For example, the first verse of the 'Hezui jin' 鶴咀勁 (The Crane's Beak) reads: ${ }^{6}$

The Crane's beak swallows snakes and pecks fish. When it pecks the pine tree, its neck is agile and its wings are open. When doing this, the movement of the wings and the beak are coordinated. Repeat it, using the eight methods.[Bafa 八法 - see item 3 below.]

The main points of the author's explanation are summarised below.

1. The five fingers form the beak and wings of the crane while the wrist forms the neck.

2. The 'beak' can be opened (to swallow a snake) or closed (to peck fish). The strength of the 'peck' can be adjusted by opening or closing the 'wings'.

3. The bafa 八法 (Eight methods) are: han 含 (keep in the mouth), tun 吞 (swallow), tu 吐(spit), rou 揉 (rub/ knead), $p i$ 繴 (split/crack open), tan 彈 (flick), zhen 震 (shake/vibrate), tui 推 (push). These are all essentially simple massage techniques, related to the type of hand movement involved.

The two or three verses that follow are mostly about the clinical usage of the technique, including the treatment of common diseases, how to use them to treat problems arising from Qigong practice and their use in the diagnosis of disease. The last verse for each technique is about its use in martial arts. For example, the last verse of Chongtian chu jin 沖天杵勁 (The Soaring Pestle) goes as follows:

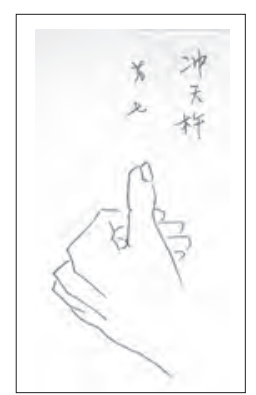

Figure 28.4 Chongtian chujin (Soaring Pestle)

In Chongtian chujin, the Qi rushes up to the heavens. It is only taught to girls for self defence against attackers. If a master teaches its use on the sixue 死穴 (death point), the result will be fatal. This must not be taken lightly.

\section{Chongtian Chu Jin (Soaring Pestle) (Fig. 4)}

This belongs to the field of advanced martial arts, known as yijing zhi dong 以靜制動 (stillness overcoming movement). An adept looks to strike his opponent's death points thus putting an end to matters. Similarly the last verse in yizhi chan jin 一指禪勁 (Single Finger Meditation) describes how you use the technique to locate the LR-14 Liver 14 qimen acupoint on an assailant's chest beneath the nipple. Striking there will lead to the other party vomiting blood with death following within seven days. Unsurprisingly it is regarded as extremely dangerous and the use of this technique is strictly proscribed under the code of Wude 武德 (martial arts ethics). The problematic question of death points I will come back to in my discussion below.

Zhou Qianchuan describes the techniques in four aspects: properties, provenance, purpose and pre-requisites.

\section{Properties}

In an author's preface, dated 15 July, 1962, it says that $M t$ Emei's Big Dipper Finger-point Method is the daoyin dianxue $s h u$ 導引點穴術 ('leading and guiding’ therapeutic exercise, acupoint technique) of the Emei yangsheng xuepai 峨嵋養 生學派 (Mt Emei School of Nurturing Life). ${ }^{7}$ This daoyin dianxue shu has two types of technique: neijing 内景 and waijing 外景 (inward and outward looking). The hand gestures we have listed all belong to the waijing daoyin shu (outer daoyin technique). In previous generations these techniques were highly secret, only transmitted orally from master to disciple and memorised by heart.

The neijing techniques are differentiated from the waijing in the book's first chapter with the following passage: 
Regulate your breath by tuna fa 吐納法 (breathe in and out with one's mind). Focus your conscious by guanxiang $f a$ 觀想法 (observation and meditation) in order to rectify imbalances of Yin and Yang, Qi and blood. Refine jin 津 (liquid) into jing 精 (essence), and then refine jing 精 into Qi 氣, then refine Qi 氣 to shen 神 (spirit), finally refine shen back into $x u$ 虛 (emptiness).

Both neijing and waijing were used to regulate Yin, Yang, Qi, and blood through out the body. Using the techniques while expressing one's neigong zhenqi 內功真氣 (internal arts for true Qi $)^{8}$ to stimulate the channel and acupoints on the exterior of a patient was to be in accordance with the systematisation of traditional diagnosis that was developing in the 1950s movement to modernise and standardise Chinese medicine, bianzheng lunzhi 辨證論治 (condition-based diagnosis and treatment determination). ${ }^{9}$ This unique synthesis was classified as waijing daoyin anqiao shu 外景導引按蹺術 (outer daoyin and massage technique).

\section{Provenance}

The history of these techniques is described in the first chapter of Mt Emei's Big Dipper Finger-point Method. It is claimed they date back to the latter part of the Southern Song Dynasty (13th century CE) and were created by Baiyun Chanshi 白雲禪師 (White Cloud Zen Master), a monk living on Emei Mountain. According to the legend, he combined the theories of Buddhism, Daoism, and related elements of popular practices. His work covered a series of methods including liangong 練功 (martial arts and other esoteric body training), danyao 丹藥 (alchemical pills and medicine), daoyin ('leading and guiding' therapeutic exercise) and anqiao 按蹺 (massage). Techniques attributed to him, the Emei shi'er zhuang 峨眉十二莊 (The Twelve Statements of Emei) include donggong 動功 (movement exercises), sancheng gongfa 三乘功法 (the three levels exercise method), jingong 靜功 (exercises without movement), and xuanmen daxiao danyao 玄門大小丹藥 (Dark Gate large and small elixirs), as well as MtEmei's Big Dipper Finger-point Method.

The Twelve Statements of Mt Emei throws considerable light on the source of the Big Dipper Finger-point Method. A number of the latter's hand positions can be found directly described in the former, albeit sometimes with different names. These include Hezui jin, Tuotian jiedi jin, Chongtian chu jin, Wuding kaishan jin, and Huzhao jin. However all

8 Neigong 內功 refers to martial arts exercises which benefit the inner organs. Zhenqi 真氣 refers to 'true Qi, one's inborn vitality'.

9 For a discussion of bianzheng lunzhi see Scheid 2002, pp. 200-4. the published evidence available in China today points to Zhou himself being the pre-eminent transcriber, perhaps even re-inventor of this Emei shi'er zhuang tradition. ${ }^{10}$

\section{Purpose and Pre-Requisites}

The purpose of Emei tiangang zhixue gestures can be summarised as follows:

- to correct Qigong piancha 氣功偏差 (Qigong deviation $)^{11}$ - in this case, problems arising from errors in practice; to treat disease generally;

- to aid diagnosis, assessing the Qi and blood in a channel while taking the pulse.

The requirements to be able to practise the Emeitiangang zhixue are described as:

- a basic knowledge of TCM theory, channels and acupoints; the practitioner must regularly practise both donggong and jingong (see above). This will allow the emission of zhenqi towards the patient, creating a genuine neigong treatment instead of simple body contact.

\section{Discussion}

All the above, and particularly the biography of Zhou Qianchuan, raises many further questions about the different domains of medico-religious and martial arts techniques. In his story we see how, during the 2oth century, overlapping religious and medical traditions were variously subject to cross-cultural and international knowledge transfer, state intervention, and both the opening up and censure of the esoteric traditions. Rapid change in the nature of acceptable knowledge, and in the structure and role of education, had serious consequences for the survival of the individuals involved and the traditions alike. And, specific to the aims of this volume, we have examined the history of illustration in the survival of Mt Emei's Big Dipper Finger-point Method.

For example, what is the significance of 'death points' and how do they relate to dianxue 點穴, or acupoint

Online searches reveal five books relating to the Emei shier zhuang tradition. In each case, the prefaces would seem to indicate Zhou Qianchuan as the origin of the material.

11 'Qigong Deviation' has become a politically loaded term in the post-1949 era, but there is widespread recognition both in current and historical sources that Qigong practised without proper safeguards can put the practitioner at serious risk. It can be equated with the psychological syndrome identified in classical texts as zouhuo rumo 走火入魔 - possession by an evil spirit. For a historical discussion of the subject, see Eskildsen 2008, pp. 259-91. 
pressing techniquest? How do they relate to medicine? Yi-Li Wu has recently published on the shared technologies of 'apertures' of the body in the realms of 'acupoints, bones, and mortal spots - to discuss points on the body that were more or less vulnerable to injury'. ${ }^{12}$ Roman Sieler has explored vital spots in the martial and medical arts of Kalari in Tamil Nadu. ${ }^{13}$ It is clear that besides position and timing, enormous force makes the difference between therapeutic and lethal applications. ${ }^{14}$ From his recorded sayings and his own writing, we can tell that Zhou Qianchuan accepted the existence of the death point technique. However as a medical doctor, whether for an ambivalence born of his cross-cultural training, or a doubt that his more esoteric views about the body might not prove acceptable, he generally avoided the subject. Perhaps the technique only existed in legend since, in the run of everyday life, one does not have much opportunity to apply it. In a military context practitioners might easily think failure to apply the death points was a consequence of an inadequate level of ability. In the same way, while there is no reliable evidence for yogic flying, the highest level of practice supposedly attainable in that art, this does not prevent many yoga practitioners from believing that it is still an achievable goal. If, on the other hand, the death point technique has some efficacy, it can only be as a feature of the grander claims for the Asian martial arts, which would see the ability to cause internal damage at places which would otherwise seem biologically invulnerable as an aspect of the supra-normal practice of Qigong. Such skills maintain their power and prestige, especially in specialist and secret training. One can point to the Qigong practices demonstrated by the Security Forces, where policemen famously break cobble stones with their bare hands. The techniques are still kept secret and nothing is published about them in the mass media. Audiences may applaud related performances at the New Year festival, but no one really takes it seriously enough to fund research in this area.

But in this respect, the martial arts maintain their prestige through secrecy in contrast with developments in the field of medicine. The martial arts focus on the achieving

\footnotetext{
$12 \quad$ Wu Yi-Li 2015, pp. 64-6.

13 Sieler 2012.

14 In Celestial Lancets, Lu Gwei-Djen and Joseph Needham refer to the example of the Indian mahout or elephant handler. The mahouts are supposed to use a similar death point technique, which could be taken as evidence that such points exist in all animals. But Needham is unable to tell us how the mahouts apply such techniques, or whether they have or require any relationship with Qigong or similar exercises. Lu and Needham [1980] 2002, pp. 69, 316-17.
}

of certain levels of ability and efficacy in an individual's training and practice. Generally speaking, the biomedical field, as it developed in 2oth-century China, promotes extensive public discussion, which in turn allows people access to styles of knowledge that privilege the combination of theory and technique in the pursuit of new forms of treatment. To pursue an analysis of master-disciple styles of knowledge transmission in religious medicine and their efficacy would lead deep into the subject of religious medicine and efficacy, a topic too large to be addressed here. So I will simply make a few internalist observations about the use of these techniques to correct the problems brought about by 'errors' in practising Qigong.

It is well known that xingqi 行氣 and daoyin therapeutic breathing and exercises have 2 millennia of history in China. If we include Fojiao zuochan 佛教坐禪 and Mizong xiulian 密宗修煉 (Buddhist meditational training) then the potential for zouhuo rumo 走火入魔 (lit. passing in to the fire and entering demonic possession - nowadays thought of as obsession brought about by mispractice) through inappropriate practice was huge. Zhang Lu 張璐 (1617-99) wrote a book entitled Zhang shi yitong - rumo zouhuo 張氏醫通・入魔走火 (Dr Zhang's Observations of Passing into the Fire and Entering Demonic Possession, i.e. mistakes in Qi practice). It records cases not otherwise mentioned by doctors, such as those where monks or practitioners experienced problems (zouhuo rumo) during Qi practice, and ancient texts offered no solutions. Apparently they had no option but to rely on stock remedies such as Tianwang buxin dan 天王補心丹 (The Heavenly King's Supplementing the Heart pills) or Liuwei dihuang wan 六 味地黃丸 (Six Flavour dihuang pills) to treat themselves.

From 1950s onwards China set up three Qigong institutions. Under their aegis, many Qigong therapists were trained and Qigong practice became widely disseminated. However after the founding of the Tangshan Institute, the Shanghai Institute was set up in 1957 with part of its remit specifically to deal with Qigong piancha 氣功偏差 (Qigong deviation). Since the techniques studied revolved around the jingong and donggong exercises taken from ancient texts, their approach to correcting errors in practice was simply to try to explain to the practitioner his or her mistakes, while in extreme cases referral to mental health experts was required. ${ }^{15}$ This marks a significant contrast with Zhou's method of using massage and daoyin to treat Qigong deviation.

Unfortunately for us the photographs of Zhou treating piancha patients were lost in the Cultural Revolution. But why were Zhou's methods not more widely adopted by his

$15 \quad$ Ma Jiren 1983, p. 267. 
friends and followers in the field ${ }^{16}$ The answer is simply because in this area the practice of every individual is different, and requires specialist attention. Zhou himself practised two types of daoyin; namely the big and small daoyin (da daoyin 大導引 and xiao daoyin 小導引). ${ }^{17} \mathrm{Da}$ daoyin is a very particular exercise. The practitioner needs both a deep inner power and to be very familiar with the neijing jingluo 內景經絡 (inner channel) syndrome differentiation principles in order to practise successfully.

Thus even though we still have Zhou's theories, it is not possible to recreate the particularity of his abilities and practice. In such a system every practitioner is the unique engine of his or her own development. Furthermore Wang Songling 王松齡, in his research into Qigong history, has said that although the major schools, like Wudang or Emei, can be broadly traced back over hundreds or thousands of years, the nature of their traditional system of oral transmission makes detailed study almost impossible. ${ }^{18}$ However my personal experience of Zhou Qianchuan has given me at least some small insight into his methods.

\section{Conclusion}

In the course of this story we have seen the Qi traditions of exercise, massage, acupuncture and the martial arts survive and transition from secret arts allied to divination and ritual practice into school and university education. This now is a domain that, in the aftermath of the Cultural Revolution, remains wary of superstition and religion and is keen to align itself with the authority of the new sciences. Quite a lot was lost or endangered in the process, including Zhou Qianchuan's illustrated text, not to speak of the one-to-one method of training in medico-religious arts which focuses on the quality and skill of the individual and unique practitioner. The secret knowledge that was once only passed on by a doctor who might 'pose for a while as a vagrant' (in order to make sure of the skill and worthiness of his disciple) is now lost in a system dominated by textbooks and college education. Nevertheless, we are

16 For example, Liu Guizhen, who established the first Qigong practice institute (in Tangshan city) in 1951, was expelled from the CCP, forced to resign as President of the Institute and sent to the countryside for re-education as a result of the case against Zhou Qianchuan in 1965. Liu was made to suffer further cruel treatment during the Cultural Revolution. See Wang Songling 1989, pp. 344-55.

17 In xiao daoyin the commonly seen problems are a heavy sensation in the head, shaking of the head, drowsiness, and an accumulated but static Qi (qiju busan 氣聚不散). grateful to those students of Zhou, who in opening up the semi-closed teaching lineage that surrounded their master, shared their notes and made multiple copies of his illustrations, so that we can now compare and contrast them in the re-creation of the master's knowledge. Together, text and image, with the vivid figurative titles so evocative of images of movement themselves - animals, clouds, the heavenly bodies - permit a sharing of information in multiple and interdisciplinary forms, no longer restricted to a small group of elite practitioners, available now to both massage and TCM schools, and increasingly accessible on the internet as part of the more public, global life of Chinese medicine and the martial arts.

\section{Bibliography}

\section{Primary Sources}

Zhou Huaijiang 周懷䔬, (son of Zhou Chaofu 周巢父, grandson of Zhou Qianchuan 周潜川) 1985, 'Emei tiangang zhixue fa' 峨眉 天罡指穴法, in Qigong jingxuan xubian 氣功精選續編 (Further Selected Qigong Classics), Beijing: Renmin tiyu chubanshe, 2-70. Zhou Qianchuan 周潜川, Zhou Chaofu周巢父, Zhou Huanjiang 周 懷輜 (eds) 1985, Emei Tiangang zhixue fa 峨眉天罡指穴法 (Mt Emei Tiangang Finger-point Method), Taiyuan: Shanxi renmin chubanshe.

\section{Secondary Sources}

Brownell S. (ed.) 2011, From Athens to Beijing: West Meets East in the Olympic Games, vol. I: Sport, the Body, and Humanism in Ancient Greece and China, New York: Greekworks.

Dear, D. 2012, 'Chinese Yangsheng: self-help and self-image', Asian Medicine 7.1, 1-33.

Eskildsen, S. 2008, 'Some trouble and perils of Taoist meditation', Monumenta Serica 56, 259-91.

Liao Yuqun 廖育群 2001, 'Zhou Qianchuan, Liao Houze yu Gumaifa' 周潜川、廖厚澤與《古脉法》(Zhou Qianchuan, Liao Houze and Ancient Pulse-taking Methods), Zhongguo keji shiliao 4, 9-25.

Lo, V. 2011, 'Training the senses through animating the body in ancient China', in Brownell (ed.), 67-85.

Lu Gwei-djen and J. Needham [1980] 2002, Celestial Lancets: A History and Rationale of Acupuncture and Moxa, London: RoutledgeCurzon.

Ma Jiren 馬濟人 1983, Zhongguo qigongxue 中國氣功學 (Chinese Qigong), Xi'an: Shaanxi keji chubanshe.

Scheid, V. 2002, Chinese Medicine in Contemporary China, Durham/ London: Duke University Press.

Sieler, R. 2012, 'Kạ̣ari and vaittiyacālai: medicine and martial arts intertwined', Asian Medicine 7.1, 164-95.

Soothill, W.E. and L. Hodous 1937, A Dictionary of Chinese Buddhist Terms, London: K. Paul, Trench, Trubner \& Co.

Wang Songling 王松齡 1989, Zhongguo qigong de shi lifa 中國氣功 的史・理・法 (History, Theory, and Principles of Chinese Qigong History), Beijing: Huaxia chubanshe.

Wu Yi-Li 2015, 'Between the living and the dead: trauma medicine and forensic medicine in the mid-Qing', Front. Hist. China 10 (1), 38-73. 


\section{Moving towards Perfection: Physical Culture in Dzogchen as Revealed in Tibet's Lukhang Murals}

Ian A. Baker

A set of murals created in Lhasa at the end of the 17 th century reveal mind-body practices within Tibetan Buddhism that have traditionally been kept hidden from non-initiates, in part because of the practices' perceived threat to monastic and clerical conventions. The bodybased disciplines depicted on the walls of what was once a private meditation chamber for Tibet's Sixth Dalai Lama are part of the Dzogchen (rdzogs chen), or 'Great Perfection' teachings that developed within Tibet's earliest transmission of tantric Buddhism. Commissioned by Tibet's then ruling political regent Desi Sangye Gyatso (Sde srid sangs rgyas rgya mtsho) (1653-1705), the Lukhang murals' artistic virtuosity reflects intellectual, doctrinal, and political concerns during one of Tibet's greatest periods of cultural production and innovation.

This chapter explores the diverse motivations that informed the creation of the Lukhang murals, but focuses more specifically on the murals' enduring didactic content: the means by which the human body can be cultivated, according to the 'Great Perfection' teachings of the Nyingma, or 'ancient' order of Tibetan Buddhism, as an agency of release from suffering and discontent, culminating in realisation of the mind and body's inherent 'Buddha Nature' (de gshegs snying po, Skt: tathāgatagarbha).

\section{The Yoga of Non-Duality}

The 'Great Perfection' or Dzogchen teachings of Tibet are upheld as revealing the ultimate unconditioned nature of human consciousness without recourse to the theological rites and practices that characterise the tantric, or Vajrayāna, form of Buddhism from which Dzogchen arose. ${ }^{1}$ Described as both the essence and culmination of

1 Dzogchen, is also known as Ati, or 'utmost' Yoga (shin tu rnal 'byor), and is presented in Nyingma tradition as the culmination of nine processual vehicles for actualising enlightenment - union with all-encompassing awareness transcendent of all afflictive fluctuations of consciousness. The first two vehicles refer to the Hīnayāna stages of Śrāvakayāna and Pratyekayāna that lead to the solitary realisation of the arhat, as represented today by Theravāda Buddhism. The third vehicle, Bodhisattvayā$n a$, introduces the Mahāyāna or greater vehicle, and cultivates enlightenment not just for oneself, but for all beings. The fourth, fifth, and sixth vehicles are the so-called Outer Tantras of Kriyātantra, Caryātantra, and Yogatantra, all of which are part of the Vajrayāna ${ }^{2}$ Buddhist path to 'pure and total presence' (byang chub kyi sems), Dzogchen is held to liberate consciousness from latent discontent through ever-present awareness (rig pa) of the ultimately non-dual (gnyis med) nature of mental experience. As explicated in the 'Six Vajra Verses' (Rig pa'i khu byug), one of Dzogchen's earliest known literary sources:

Recognising that everything is self-perfected from the very beginning and beyond the constraints of conceptualising mind, the malady of striving is spontaneously relinquished. One remains immaculately at ease in innate perfection. ${ }^{3}$

the Vajrayāna, or third turning of the wheel of doctrine, but are still dualistic in their orientation. The three Inner Tantras were transmitted to those deemed of higher capacity and consist of Mahā Yoga, which emphasises the development stage (bskyed rim) of creative perception, Anu Yoga, which cultivates co-emergent bliss and emptiness through completion stage (rdzogs rim) practices based on an imaginal body of channels, winds and essences, and Ati Yoga, the resultant non-duality of Dzogchen with its liberating praxis of primordial, self-existing perfection. The three Inner Tantras of the Nyingma further correlate with the Unsurpassed Yoga Tantras (Anuttarayogatantra) of the Kagyu, Sakya, and Geluk lineages, all of which culminate in the non-dual (Advaitayoga) view of reality as expressed in Essence Mahāmudra (ngo bo'i phyag rgya chen po) which is identical, in outlook, to Dzogchen, the 'Great Perfection', but differs in method and presentation.

2 Founded by Indian mahāsiddha, or realised adepts, Vajrayāna, is a multifaceted and complex system of Buddhist thought and practice, which evolved over many centuries. Also known as Tantric Buddhism, Tantrayāna, Mantrayāna, Secret Mantra, Esoteric Buddhism and the Diamond Way or Thunderbolt Way, Vajrayāna, refers to one of three Buddhist vehicles or routes to enlightenment, the other two being Theravāda and Mahāyāna. For discussion of the phases of introduction of Vajrayāna Buddhism to Tibet, see Kapstein 20oo; Snellgrove 1987.

3 Also known as 'The Cuckoo's Song of Total Presence', this seminal text is considered the basis of Dzogchen practices emphasising the nature of consciousness (sems sde). A version of this text was discovered among materials sealed into a chamber at the oasis of Dunhuang in the 1oth century, but the text is held by tradition to date originally to the 8th century when Tibet's first Dzogchen master, Vairocana, allegedly received it from Śrī Siṃha in the legendary land of Uḍdiyāna. 


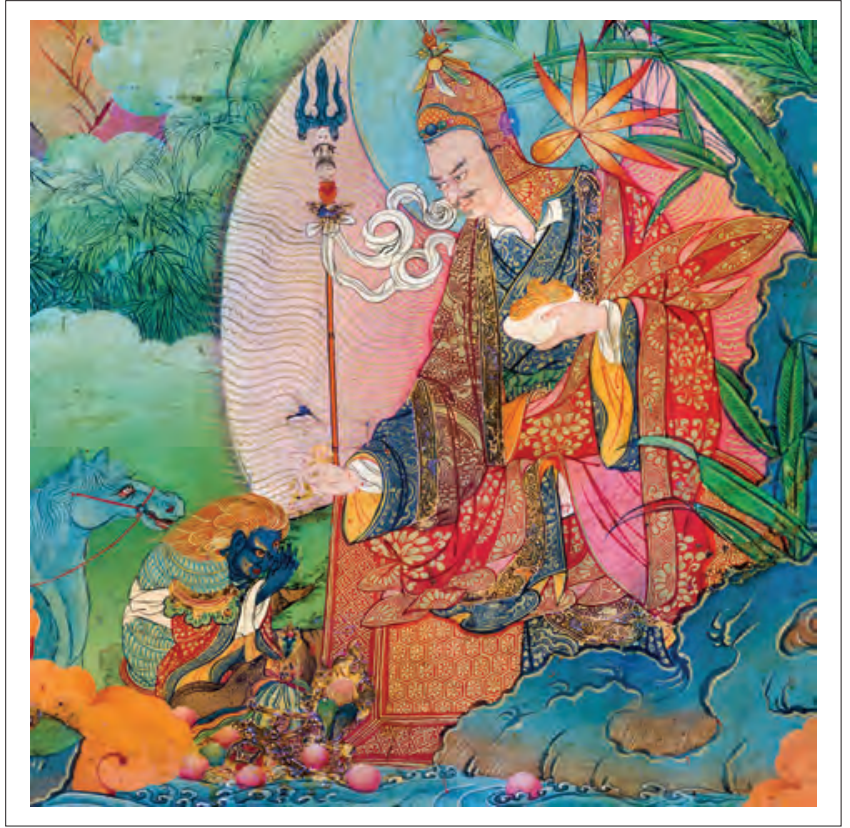

Figure 29.1 Padmasambhava accepting obeisance from an elemental nature spirit called a Lu (Skt: naga), as shown on a mural in Tibet's Lukhang Temple. ${ }^{\odot}$ Ian Baker

Similarly, Padmasambhava, ${ }^{4}$ the 8 th-century figure most commonly identified as having established the Dzogchen teachings in Tibet, reputedly proclaimed that:

In its true state the mind is naked, immaculate, clear, without duality, transparent, empty, timeless, uncreated, unimpeded; not realisable as a separate entity, but as the unity of all things... . To know whether or not this is true, look into the nature of your own mind. ${ }^{5}$

Padmasambhava, the 'Lotus Born', is a quasi-supernatural personality in Tibetan Buddhist hagiography, who is credited with eight archetypal manifestations and innumerable emanations. He is said to have originated in Uḍdiyāna, a region known for its syncretic traditions, currently encompassing parts of northern Pakistan, Afghanistan, and Kashmir. Asked who he was, he allegedly replied; 'My father is wisdom. My mother is selflessness. My country is Reality, just as it is. I take thoughts as food and have come here to destroy sorrow'. There are clear parallels between the Dzogchen tradition attributed to Padmasambhava and non-dual Kashmiri Śaivism that includes the practice of 'Sambhava Yoga', referring to the radiance of intrinsic consciousness when freed from all ideation. Similarly, Uḍ̣iyāna, from where Padmasambhava is said to have originated, refers to a specific hațha yoga practice, Uḍdiyāna, or 'flying upward', $m u d r a \bar{a}$, used for directing energy and awareness into the body's central meridian (rtsa dbuma). For evidence of the influence of Kashmiri Śaivism on the development of Vajrayāna Buddhism, see Alexis Sanderson's extensive publications in the field (www. alexissanderson.com). To compare relevant passages of the śaiva āgama with Dzogchen pith instructions (man ngag), see Singh 1979; Odier 2005.

$5 \quad$ Evans-Wentz (ed.) 1954, p. 183.
While Dzogchen is commonly perceived, and presented, as pertaining to the innate 'self-liberating' potential of human consciousness and thus considered superior to transformation-based tantric disciplines, its practice is traditionally infused by physical exercises that push the body - and thereby the mind - beyond conventional limits and constraints. These disciplines range from physically and imaginatively demanding 'preliminary practices' (sngon'gro) to supportive Qigong and hațha-yoga-related exercises (rtsa rlung 'khrul 'khor) 6 that amplify innate somatic processes preparatory to Dzogchen's more widely known and transmitted contemplative techniques of 'cutting through' (khregs chod) and 'leaping over' (thod rgal), the latter involving precise body postures, breathing techniques, and visual focus. Auxiliary Dzogchen practices involve condensed renditions of the so-called Six Yogas, ${ }^{7}$ geared towards recognising the Natural State (gnas lugs) of non-dual awareness (gnyis med) at all stages of waking, dreaming, sleeping, and posited post-mortem states of consciousness. In all aspects of Dzogchen practice, the body is cultivated and its natural processes enhanced and ultimately transcended, in order to facilitate effortless abiding in the self-liberated radiance of intrinsic awareness, equated in Dzogchen with the 'Buddha Nature' (bde bar gshegs pa'i snying po, Skt: sugatagarbha) held to be latently present within all beings. As stated in the Secret Nucleus:

6 rtsa rlung breathing practices that alter the flow of somatic energy are typically enhanced by the dynamic yogic movements of 'khrul 'khor to both improve health and expand the body's capacity for inner experience. Geoffrey Samuel has pointed out that the Tibetan Buddhist Resource Center (TBRC) alone includes 26o 'khrul 'khor-related texts, although little research has been devoted to the subject. He further notes that some of these texts are included in the Tengyur and thus held to be translations of original Indian texts.

7 Associated with the 11th-century Indian mahāsiddha Nāropā (956-1041), the 'Six Yogas' ( a ro chos drug) derive from the 'Yoginī Tantras' of Anuttarayogatantra and refer in reality to a larger and shifting set of processual yogic practices directed towards awakening habitually dormant human capacities during the recurring phases of waking, dreaming, and sleeping as well as at the time of death and its aftermath. The inclusion of the Six Yogas in 'rediscovered' Dzogchen treasure texts (gter ma) suggests the influence on the Nyingma tradition of teachings brought from India to Tibet by Marpa Lotsawa in the 11th century and propagated within the Kagyu lineage of 'whispered secrets'. For an overview of the Six Yogas and their diffusion in Tibet in connection with the development of the Kagyu, Sakya, and Geluk orders, see Mullin 20o6; Dudjom Rinpoche 1991, pp. 284-9; Kongtrol 2005, pp. 330-7. 
The Perfect Buddha is not found in any of the 10 directions or four times. Other than the perfect Buddha which is mind-as-such, do not seek the Buddha elsewhere. ${ }^{8}$

As Dzogchen treatises repeatedly point out, the human body is an unsurpassable vehicle for the realisation of the radiant and selfless expanse of enlightened awareness that dualistic subject-object mentation habitually obscures. ${ }^{9}$

Dzogchen teachings in Tibet were traditionally closely guarded as their uncompromising and doctrinally challenging elucidations of the nature of mind were often perceived as being in conflict with monastic traditions based on ceremonial magic and scriptural study. Dzogchen methods for awakening to the posited innate mind of enlightenment were transmitted primarily through Tibet's Nyingma, or 'Ancient' order, which traces its origins to the 8th-century Buddhist masters Padmasambhava and Vairocana, who are credited with having brought the teachings of 'Great Perfection' from Uḍ̣iyāna, a legendary realm at the northwest frontiers of the Indian sub-continent. The Dzogchen, or Ati Yoga, lineage is held to have spread beyond Uḍdiyāna with the 'Three Incisive Precepts' (Tshig gsum gnad brdegs) transmitted by the quasi-historical Prahevajra (Tib: Dga' rab rdo rje) to his Brahman disciple, Mañjuśrīmitra ('Jam dpal bshes gnyen). ${ }^{10}$ Dzogchen evolved in Tibet and neighbouring Himalayan countries from the 8th century onward and, beginning in the 11th century, through a tradition of rediscovered 'treasure texts' (gter $m a)^{11}$ revealed

8 Guhyagarbha Tantra, Tib: Rgyud gsang ba'i snying po. This Tantra predates the appearance in Tibet of specifically Dzogchen works and is upheld in Nyingma tradition as authenticating Dzogchen's deconstructionist presentation of experiential reality. The 'Reverberation of Sound' (Sgra thal 'gyur), the root tantra for the 17 Dzogchen Tantras of the secret instruction series, states that 'the union of body and mind connects primordial space with pristine awareness ... realising that, all beings are naturally buddhas!'

10 Prahevajra's final testament summarises Dzogchen's existential view, meditative practice, and ubiquitous application. These are encapsulated in outer, inner, and secret precepts: recognising the nature of mind (sems sde), remaining in non-dual 'spaciousness' (klong sde), and applying that realisation in all circumstances (man ngag). These three aspects of Dzogchen are further associated with the interrelated experiences of clarity, open presence, and the unity of emptiness and appearance.

11 Revealed treasure texts (gter $m a$ ) are invariably attributed to Padmasambhava and typically consist of meditative instructions (khrid), empowerments (dbang), and root tantras (rgyud), with miscellaneous ritual treatises often appended at the end. They can vary in length from a single text to more than a hundred. Treasure texts reinforce tradition by attributing literary and conceptual innovation to the founding figure of Padmasambhava while including new and sophisticated techniques of yo- by Padmasambhava's lineal successors. While faithful to Dzogchen's original ethos of the primordial purity $(k a$ dag) of selfless unitary consciousness and non-reliance on external rites, the Dzogchen treasure texts nonetheless increasingly incorporated hațha-yoga-like techniques that were perceived as supportive and complementary to Dzogchen's core contemplative practices by which the physical body could ultimately be dematerialised into an immortal body of rainbow light ('ja'lus). ${ }^{12}$

gic practice that entered Tibet from the late 1oth century onward in a subsequent wave of transmission from Indian mahāsiddhas, or realised adepts. Devotees of Tibet's original Nyingma order maintain that comparable teachings had been transmitted centuries earlier by Padmasambhava to his primary Tibetan consort, Yeshe Tsogyal, who encoded them on 'yellow scrolls' (shog gser) and hid them in rock walls, temple pillars, and other locations to be discovered in future generations by designated 'treasure finders' or terton (gter ston). This practice allowed renewal of Nyingma doctrine to occur within the stability of orthodox tradition. But, as was the case throughout Tibet's history, the ambiguity of the texts' historical sources raised concerns of authenticity while the overtly creative and non-conventional lives of the tertons themselves allowed for the possibility, at least to their detractors, of their subverting the practice to support elaborate agendas of self-advancement. The lack of any traceable Indic source for the revealed treasure texts attributed to Padmasambhava continues to vex Tibetan Buddhist tradition. While certain schools of Tibetan Buddhism view texts such as Pema Lingpa's 'Compendium of Enlightened Spontaneity' (Rdzogs chen kun bzang dgongs' dus) as indisputable expressions of Padmasambhava's enlightened mind and, by extension, the essence of the transcendental Buddha Amitābha that Padmasambhava is held to embody, other schools view the texts with suspicion and at times with outright hostility. Matthew Kapstein describes this historical rift between the idealism of living revelation and a more critical historical orientation as having led, over time, to 'purely political considerations whose perverse alchemy transformed the gold of religious vision into the base metal of violent sectarianism' (Kapstein 2000, p. 135). As Kapstein points out, the great Tibetan scholar and tantric master Tāranātha (1575-1634) had articulated the possibility of a middle ground 'by affirming that while at least some of the treasures were genuinely based upon early materials, they were at the same time, nevertheless, elaborated by their "discoverers". In this formulation alone do we find the possibility of an alternative to the aporia of unimpeachable authenticity versus willful fraud'. (Kapstein 2000, p. 135). See also Kapstein 1989, pp. 217-44 and Gyatso 1993, pp. 97-210. For further discussion of the terma (gter ma) tradition, see Gyatso 1998, as well as Thondup 1986.

12 Tibet's pre-Buddhist Bon tradition also includes hața-yoga-like practices but, rather than ascribing them an Indic source, maintains that they originated in Tibet through the lineage of the Shang Shung Nyengyud. For the Bon presentation of Dzogchen, see Namdak and Myrdhin Reynolds 2006; Namdak and Dixey 2002. 


\section{The Lukhang Murals}

In the $15^{\text {th }}$ century, the Nyingma treasure master Terton Orgyen Pema Lingpa (Orgyan padma gling pa) (1450-1521) revealed a highly influential Dzogchen treasure text entitled 'Compendium of Enlightened Spontaneity' (Rdzogs chen kun bzang dgongs'dus) ${ }^{13}$ from a stūpa above Samye Monastery in Central Tibet. Ascribed to Padmasambhava, the treasure text emphasises Dzogchen pith instructions (man $n g a g$ ) for realising the exalted state of 'Great Perfection' through body-oriented techniques ranging from methods of hațha and kuṇdalini yoga ${ }^{14}$ to specific

'Great Perfection: Compendium of Enlightened Spontaneity' (Rdzogs chen kun bzang dgongs' dus) is one of three texts revealed by Pema Lingpa that elucidates the Great Perfection (rdzogs chen). Alternative translations of Rdzogs chen kun bzang dgongs' dus include Sarah Harding's 'Great Completion: Union of Samantabhadra's Intentions'. Kun bzang is a contraction of kun tu bzang po meaning 'All Good' or 'Ever Good', a name referring to the primordial Buddha and the principle of enlightenment. $d$ Gongs pa is often translated as 'intention', but as Buddha activity is spontaneous and unpremeditated, I prefer the alternative translation 'wisdom' which retains more of the spontaneity expressed in Pema Lingpa's presentation of the Dzogchen teachings. Although I have chosen to render Pema Lingpa's revealed text as 'Great Perfection: Compendium of Enlightened Spontaneity', it could also be 'Great Perfection: Compendium of Spontaneous Wisdom'. For a full inventory of Pema Lingpa's revealed treasures, see Harding 2003, pp. 142-4. A set of Pema Lingpa's original manuscripts is preserved at Gangteng (or Gangtey) Monastery in Bhutan. Reproductions were sponsored by Her Majesty the Royal Grandmother of Bhutan, edited by the great Nyingma master Dudjom Rinpoche, and published in 21 volumes by the National Library of Bhutan in Thimphu. Pema Lingpa's treasure corpus is also available as digital scans at Tibetan Buddhist Resource Center (www.tbrc.org) through the efforts of Gene Smith and the generous support of Chris Tomlinson. For more on the life and activities of Pema Lingpa, see Harding 2003; Aris 1988; Tshewang et al. 1995 .

14 Hatha, literally 'forceful', yoga was expounded in a 15th-century Sanskrit treatise by Svāmi Svātmārāma entitled Hațha Yoga Pradīpikā. Widely available in modern English translations, the text was previously considered to be one of the earliest presentations of hațha yoga, although recent research by Birch (2011), Mallinson (2014), Singleton (2015), and others has revealed far earlier textual origins. Like the two other well-known medieval classics of hațha yoga, Gheranda Samhitā and Siva Samhitā, the Hațha Yoga Pradīpikā offers a detailed overview of yogic postures (āsana) and breathing techniques (prānāayām) as well as the metaphysical anatomy of channels (nädi) and cakra and psychophysical energy of kuṇdalinī, or candāalī (Tib: gtum mo) that underlie both Hindu and Buddhist Tantra. Physical yogic practices in Tibet are generally held to have begun in the 11th century during the second diffusion of Vajrayāna, in particular body postures associated with Dzogchen's ultimate goal of allowing the body's psychophysical constituents to decompose into their intrinsic luminosity. Pema Lingpa's treasure text has had an enduring influence throughout the Himalayan world and elucidates the role of physical cultivation in teachings dedicated to attaining what Vajrayāna Buddhist tradition upholds as humankind's highest potential, the literal photonic enlightenment of mind and body.

In the late $17^{\text {th }}$ century, at what is often considered the height of Tibet's political consolidation and cultural efflorescence, ${ }^{15}$ the reincarnation of the Fifth Dalai Lama (Ngag dbang blo bzang rgya mtsho) (1617-82) was identified by Tibet's reigning chief minister as Rinchen Tsangyang Gyatso (Tshang dbyangs rgya mtsho) (1683-1705), a direct descendent of the Bhutanese Treasure Revealer Pema Lingpa, discoverer of the 'Compendium of Enlightened Spontaneity'. ${ }^{16}$ As Tibet's interregnum ruler, Desi Sangye Gyatso (Sde srid sangs rgyas rgya mtsho) (1653-1705) was furthermore a lineage-holder of the practices described in Pema Lingpa's treasure text, leading him to oversee the creation of a set of murals illustrating the contents of Pema Lingpa's revealed treatise on the walls of a meditation chamber intended exclusively for the Dalai Lamas and their closest preceptors and attendants. ${ }^{17}$ Representing

in association with the Kagyu order's transmission of 'whispered precepts'. The inclusion of hatha-yoga-like practices in a text ascribed to Padmasambhava supports the contention that the Nyingma tradition of revealed treasures was, at least in part, a way of including in the Nyingma canon advanced tantric methods of internal transformation such as those described in the Six Yogas of Nāropā as well as the similarly formulated Six Yogas of Niguma that date from the same period and continue to be passed down within the Shangpa Kagyu sub-order. Like the Hața Yoga Pradīpikā, Pema Lingpa's 'treasure text' makes clear its intent to illuminate the essence of tantric methods that, within monastic settings, were often burdened with liturgical rites and coded language that concealed more than they revealed.

15 For details of events at this time, see Aris 1988; Baker 2011.

16 The Sixth Dalai Lama was a direct descendent of Pema Lingpa through his father, the Nyingma master Rigdzin Tashi Tendzin (Rig 'dzin Bkra shis bstan 'dzin) (1651-97), who hailed from the mountainous regions east of Bhutan and disseminated Pema Lingpa's lineage of non-celibate lay ordination to others of his Nyo clan, the progenitors of Bhutan's nobility and eventual royal family.

17 As Pema Lingpa's teachings spread within Tibet, 'Compendium of Enlightened Spontaneity' became central to the curriculum at Orgyen Mindroling, a prominent Nyingma monastery founded between 1670 and 1676 by Minling Terchen Gyurme Dorje (Smin gling gter chen gyur med rdo rje) (1646-1714), known also as Rigdzin Terdak Lingpa (Rig 'dzin gter bdag gling pa). Towards 
spiritual practices and insights beyond Tibet's monastic conventions, the murals continue to serve as visual guides to the psychophysical practices that infuse the Dzogchen tradition in which the human body is perceived and cultivated as a medium of spiritual illumination. ${ }^{18}$

The sequestered murals line the walls in the uppermost chamber of the Lukhang (klu khang), the 'Temple to the Serpent Spirits' situated on a willow-fringed lake beneath the southern escarpment of the Dalai Lamas' Potala Palace in Lhasa. ${ }^{19}$ Flowing across the walls within a surreal bluegreen landscape influenced by the painting styles of Qing dynasty China and Mughal India, the murals cover three sides of the room, as well as the corners, and illustrate

the end of the 17th century, Terdak Lingpa wrote an extensive commentary on Pema Lingpa's 'Compendium of Enlightened Spontaneity' entitled 'Total Illumination of the Essence: The Teaching Manual for the Union of Samantabhadra's Intentions' which continues to be used today at teaching centres associated with H.E. Gangteng Tulku Rinpoche, one of Pema Lingpa's three contemporary emanations. Summarising the contents of the Lukhang murals, the commentary begins with teachings on ngon dro (the common preliminary practices of Refuge, Bodhicitta, Vajrasattva, Mandala Offering, and Guru Yoga) followed by the $r d z o g$ chen extraordinary preliminaries (Inner, Outer, Secret, and Ultra Secret 'khor 'das ru shan, 'distinguishing samsāra and nirvāna'). The text then directly introduces intrinsic awareness (rig pa) and the associated practices of ka dag khreg chod, thod rgal, the four visions, arriving at direct experience, and transferring consciousness at the moment of death. Terdak Lingpa transmitted these teachings to Ngawang Lobsang Gyatso ( Ngag dbang blo bzang rgya mtsho), the Fifth Dalai Lama (1617-82) as well as to Rinchen Tsangyang Gyatso, the Sixth Dalai Lama (1683-1706/46), who, after his enthronement in 1697, rejected monastic ordination in favour of esoteric yogic practices such as those disclosed in Pema Lingpa's treasure text.

18 The link between Pema Lingpa's treasure text and the Lukhang murals was identified in 2002 by Jakob Winkler. See Winkler 2002, pp. 321-43.

19 Lukhang means 'Temple to the Serpent Spirits' and refers to its origins in a vision of the Fifth Dalai Lama during the construction of the Potala Palace. A serpent-like water deity called a $l u$ appeared to him during his meditations and warned that excavations behind the palace were disturbing the subterranean realm of the $l u$. In an act of reconciliation, the Fifth Dalai Lama vowed to build a temple to appease the lu once the Potala Palace was completed. This promise was fulfilled during the lifetime of the Sixth Dalai Lama (1683-1706) who made the resulting island temple his primary residence, in keeping with his controversial preference for romantic trysts and poetic composition over affairs of state. Over succeeding centuries the Lukhang continued to serve Tibet's Dalai Lamas as a place of spiritual inspiration and contemplative retreat. For details concerning the contents of the murals on the two lower floors of the Lukhang, see Luczantis 2011. the principal contents of Pema Lingpa's treasure text 'Compendium of Enlightened Spontaneity'. 20

Apart from Padmasambhava's 25 Tibetan disciples who are shown grouped around the 8th-century Tibetan emperor Trisong Deutsen (Khri srong lde'u btsan), and images of Pema Lingpa, Pema Lingpa's previous incarnation Longchen Rabjampa (1308-64), and his descendant, Rigdzin Tashi Tendzin - father of the Sixth Dalai Lama - almost all other figures in the murals are shown as being of Indian origin. These include an entire mural depicting 42 of the 84 canonised Indian mahāsiddha (grub thob chen po), progenitors of Tibet's tantric lineages, as well as anonymous Dzogchen practitioners portrayed on the north and west walls, whose distinguishing hairstyles, skull bowls, and trident staffs connect them with the Kāpālika Śakta Saiva tantrism which shaped both the iconography and ritual practices of Vajrayāna Buddhism's most esoteric traditions. ${ }^{21}$

Although Pema Lingpa's treasure text directly associates the Dzogchen teachings with the practices of the $r s ̦ i$, or

20 According to the current Fourteenth Dalai Lama, the paintings that inscribe the private meditation chapel were executed under the combined direction of Desi Sangye Gyatso and Terdak Lingpa's younger brother, the accomplished artist Minling Lochen Dharma Shri (1654-1717). Prior to Desi Sangye Gyatso's production of the Lukhang murals, he masterminded the creation of a series of 79 scroll paintings depicting the Tibetan medical system as it was taught at Lhasa's Chakpori Medical College, which he founded in 1696. The artistic and didactic paintings were based on the Blue Beryl (Vaidūrya sngon po), the Desi's extensive commentary on the foundational Four Medical Tantras (Rgyud bzhi) that had been compiled in the 12th century and which continue to form the basis of Tibetan medical practice today. The medical paintings generated by the Desi, in collaboration with Tibet's top artists and physicians, continue to be reproduced throughout the world, although less now for instructional purposes than for their artistic merit and the insight they provide into Tibetan civilisation at its height of artistic and cultural production. See Gyatso 2012 for an overview of the various copies that have been produced of the 79 medical thangkas. Also see Parfionovitch, Dorje and Meyer 1992 and Baker 1997, the latter of which presents a late 2oth-century Nepalese rendition.

21 Vajrayāna's Yoginī tantras, in particular, draw on a corpus of Śaiva tantras classified as the Vidyāpitha, or 'locus of knowledge', of the Bhairava component of esoteric Kashmiri Śaivism. The Buddhist Yoginī tantras adapted pre-existing Śaiva texts while assimilating Śaiva deities, mantras, ritual procedures, mandalas, Kāpālika accoutrements, specialised terminology, and secret gestures into a system of yogic practice infused with the core Mahāyāna principles of benevolence, compassion, joy, and equanimity. Most tellingly, Pema Lingpa's revealed treasure text, as transcribed on the wall, begins with the words, 'Thus follow the teachings of the rși, the Vedic seers of ancient India. 


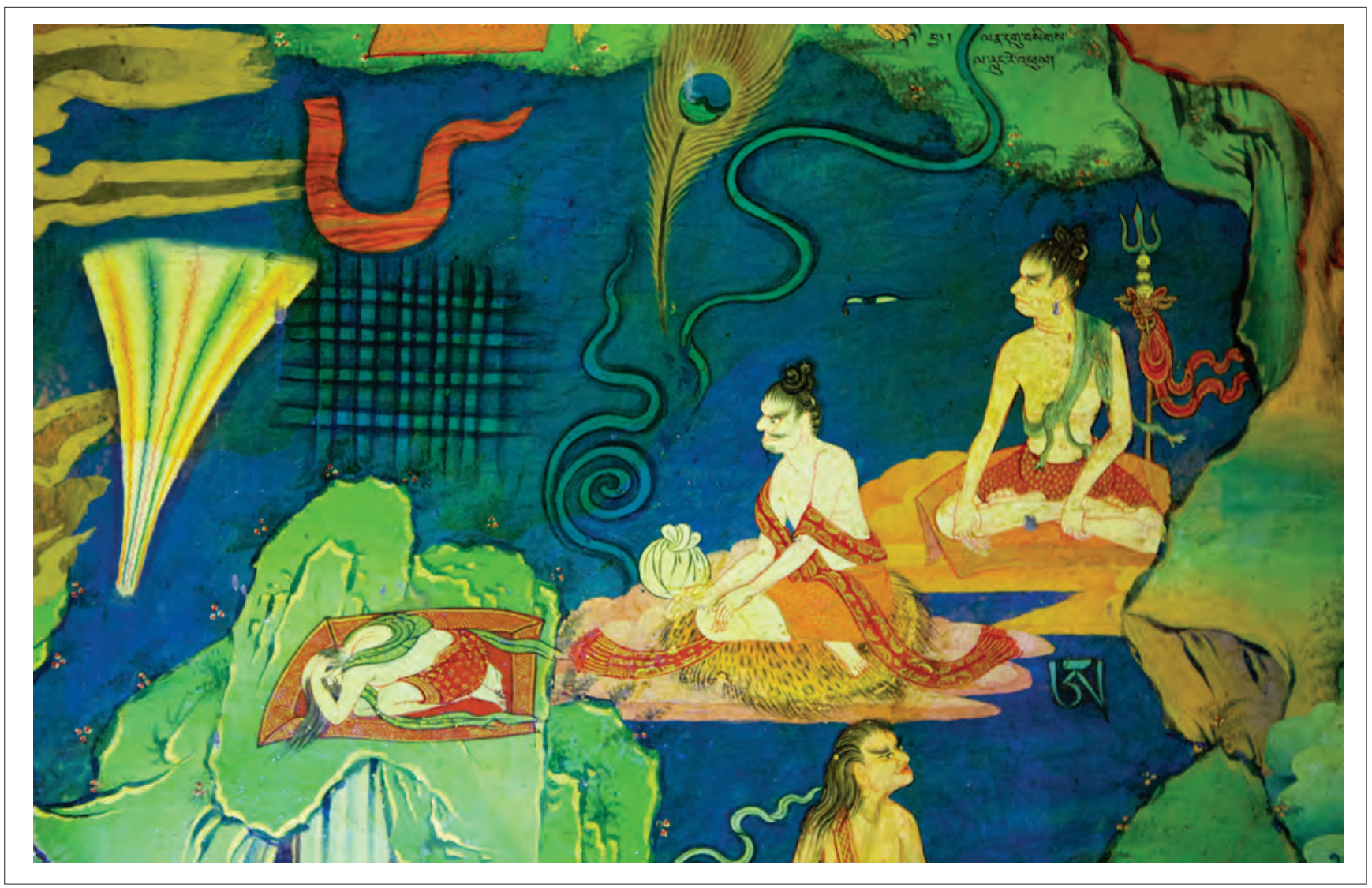

Figure 29.2 Practitioners of 'Great Perfection' (Dzogchen) on the walls of the Lukhang are typically shown as Indian ascetics. Accoutrements such as the three-pronged katvanga staff - visible at the right - associate them with the Kāpālika school of tantric Śaivism which deeply influenced the development of Vajrayāna Buddhism in India prior to its diffusion in Tibet. The abstract geometrical visions depicted in the sky anticipate 21st century abstract art which, like the practice of Dzogchen, sought to illuminate deeper levels of perceptual reality. ${ }^{\odot}$ Ian Baker

Vedic seers of ancient India, it was entirely the Desi's choice to present the generic practitioners of Dzogchen depicted in the murals as Indian ascetics rather than as Tibetans (Fig. 2). Beyond their instructional content, the Lukhang murals thus promote an ideological Indian genesis for the Dzogchen teachings, in defence against rival Tibetan claims that Dzogchen lacks legitimising Sanskrit sources. ${ }^{22}$

\section{Visual Transmission}

The Lukhang murals were created during a vibrant period of Tibetan history when cultural knowledge was on the one hand subjected to increased intellectual scrutiny and, on the other, to comprehensive, state-sponsored documentation. These initiatives began with the Fifth

22 The presence of the rși also establishes a clear case against rival Bonpo claims that Dzogchen originated in the pre-Buddhist civilisation of Shang Shung in Western Tibet. For the Bon presentation of Dzogchen, see Namdak and Myrdhin Reynolds 20o6; Namdak and Dixey 2002.
Dalai Lama, Ngawang Lobsang Gyatso (1617-82) - the first leader of a fully united Tibet - who commissioned a lavishly illustrated version of a section of his Sealed and Secret Biography (gSang ba'i rnam thar rgya can) entitled the Gold Manuscript ${ }^{23}$ which, in turn, contains a chapter entitled 'Visions and their Significance'24 that documents the Fifth Dalai Lama's own accounts of his life-long paranormal and visionary experiences. ${ }^{25}$ The Fifth Dalai Lama recounts

23 The Gold Manuscript contains 16 texts, consisting of 184 folios and five groups of illustrations with a total of 886 separate drawings on 61 illuminated folios, mostly consisting of ritual instruments and diagrams but also mandalas, cakras, and lingams, painted largely in gold on a charcoal background. The folios measure $29 \times 6 \mathrm{~cm}$.

24 The section of the Gold Manuscript entitled 'Visions and their Significance, being the elucidation of the visions recorded in the sealed volume' (rGya can gyi 'khrul snang rnams gsal bar bkod pa mThongba don (dan) contains 886 separate drawings on 55 illuminated folios.

25 The Fifth Dalai Lama refers to his paranormal visions as 'the flowing melody of divine beings, secret spells and wisdom at play, echoing freely through my body, speech, and mind'. See 
in his biography that he documented his visions with the 'belief that they might be beneficial to myself and others' and, especially, that they might 'guide the unititiated' and serve 'those who wish to do drawings and paintings of the heavens... and record their roamings in the Pure Lands'. ${ }^{26}$ The paintings in the Gold Manuscript were made under the Fifth Dalai Lama's direct supervision by a lay tantrika named Guru rTa mgrin between the years 1674 and 1681, the year before the Fifth Dalai Lama's death.

The Gold Manuscript of the Sealed and Secret Biography extended the scope of the written word by illustrating in minute detail the complex diagrams, ritual implements, mantras and methodological procedures of compassionate, if coercive, sorcery that the Fifth Dalai Lama had undertaken in order to solidify Tibet's political power and, in part, to repel invaders from its borderlands. Artistically, the Gold Manuscript was unique in being the earliest example of Tibetan painting in which a charcoal black background is illuminated with figures executed in silver and gold with subtle malachite, azurite and vermilion shading. ${ }^{27}$ It is also the first known instance of illustrating tantric ritual. Although not performative as a textbook of ritual magic, the Gold Manuscript nonetheless revealed the ways in which visual illustration could enhance understanding of a written text and extend its applicability.

After the death of the Fifth Dalai Lama in 1682, Desi Sangye Gyatso (1653-1705), the chief minister and de facto ruler of Tibet since 1679, continued the Great Fifth's initiatives in consolidating cultural knowledge and increasing access to Buddhist scriptures and instructional treatises through unprecedented forms of artistic production. ${ }^{28}$ In 1686 and 1688, the Desi expanded on the Gold Manuscript by commissioning two sets of 23 scroll paintings (thangka) depicting the Fifth Dalai Lama's life and visions.

He simultaneously embarked on a more ambitious and public project, collaborating from 1687 with artists and medical practitioners to transform his own four-volume

Karmay 1988, p. 28.

$26 \quad$ See Karmay 1988, p. 28.

27 See Karmay 1988. The original manuscript - painted in gold, silver and mineral pigments on charcoal blackened paper - is in the Musée Guimet in Paris. The manuscript served as a prototype for the later 'black thangka' genre of Tibetan painting, the origins of which can be discerned in 11-12th century Nepalese manuscripts.

28 In order to ensure completion of the imperial projects that 'The Great Fifth' had initiated, the Desi concealed the Fifth Dalai Lama's death for 14 years, during which time he completed the construction of the colossal Potala Palace and secretly prepared Rinchen Tsangyang Gaytso for his later installation as the Great Fifth's successor. treatise on Tibetan medicine, The Blue Beryl (Vaidūrya sngon po), into a series of 79 scroll paintings which the Desi himself described as being 'without any equivalent in the past' in their aim of providing information as lucidly as 'fruit [seen] in the palm of one's hand'. ${ }^{29}$ Following the Desi's Blue Beryl commentary on the 12th century Four Medical Tantras (rGyud bzhi), the 79 medical paintings depict human anatomy, physiology, pathology, diagnostic techniques, therapeutic methods, and pharmacological substances used in Tibet's syncretic 'science of healing' (Gso ba rig $p a$ ), which drew historically from both Traditional Chinese Medicine and Indian Ayurveda. The paintings are extraordinary, however, not only for their pedagogical illustrations of Tibetan medical science, but also for their poignant vignettes of everyday life, including a wide range of vocational pursuits, dress styles, and facial expressions. ${ }^{30}$

In a political gesture intended to establish the teenage reincarnation of the Fifth Dalai Lama as a patron of Tibet's newly systemised medical tradition, the Desi formally offered the 14-year-old Sixth Dalai Lama, Rinchen Tsangyang Gyatso, the first 62 finished paintings at the time of his enthronement in 1697. The full set of 79 medical scrolls was completed in 1703 and subsequently used as a teaching tool at Chakpori (Chags po ri) Medical College, which the Desi had established in 1696 on a hill overlooking the Potala Palace. ${ }^{31}$ Apart from representing cultural knowledge in visual form, the medical paintings were a conscious departure from prior conventions of Tibetan art, characterised by Janet Gyatso as an 'unprecedented sidelining of Buddhist elements in favor of a larger picture of the world in which religion is but one part'.32

The encyclopedic range of the secularly oriented and publically displayed medical paintings contrasted with Desi Sangye Gyatso's concurrent project of representing in artistic form Tibetan Buddhism's most esoteric meditational practices. The murals that the Desi commissioned for the Sixth Dalai Lama's private meditation chamber on the top floor of the Lukhang temple share common features with

\footnotetext{
$29 \quad$ Gyatso 2015, p. 62.

$30 \quad$ For an in-depth and illuminating analysis of the Desi's 79 medical paintings as they pertain to Tibetan society at the turn of the 17th century see Gyatso 2015 .

$31 \quad$ Chakpori Medical College - along with the original set of thangkas - was destroyed during the Chinese crackdown that followed the 1959 Tibetan uprising against Chinese occupation and was reopened in Darjeeling, India, in 1992.

32 The medical paintings controversially present religious practice as a potential cause of disease and distress in the form of disputes, infighting, and ineffective magical rituals. See Gyatso 2015 , p. 68 .
} 


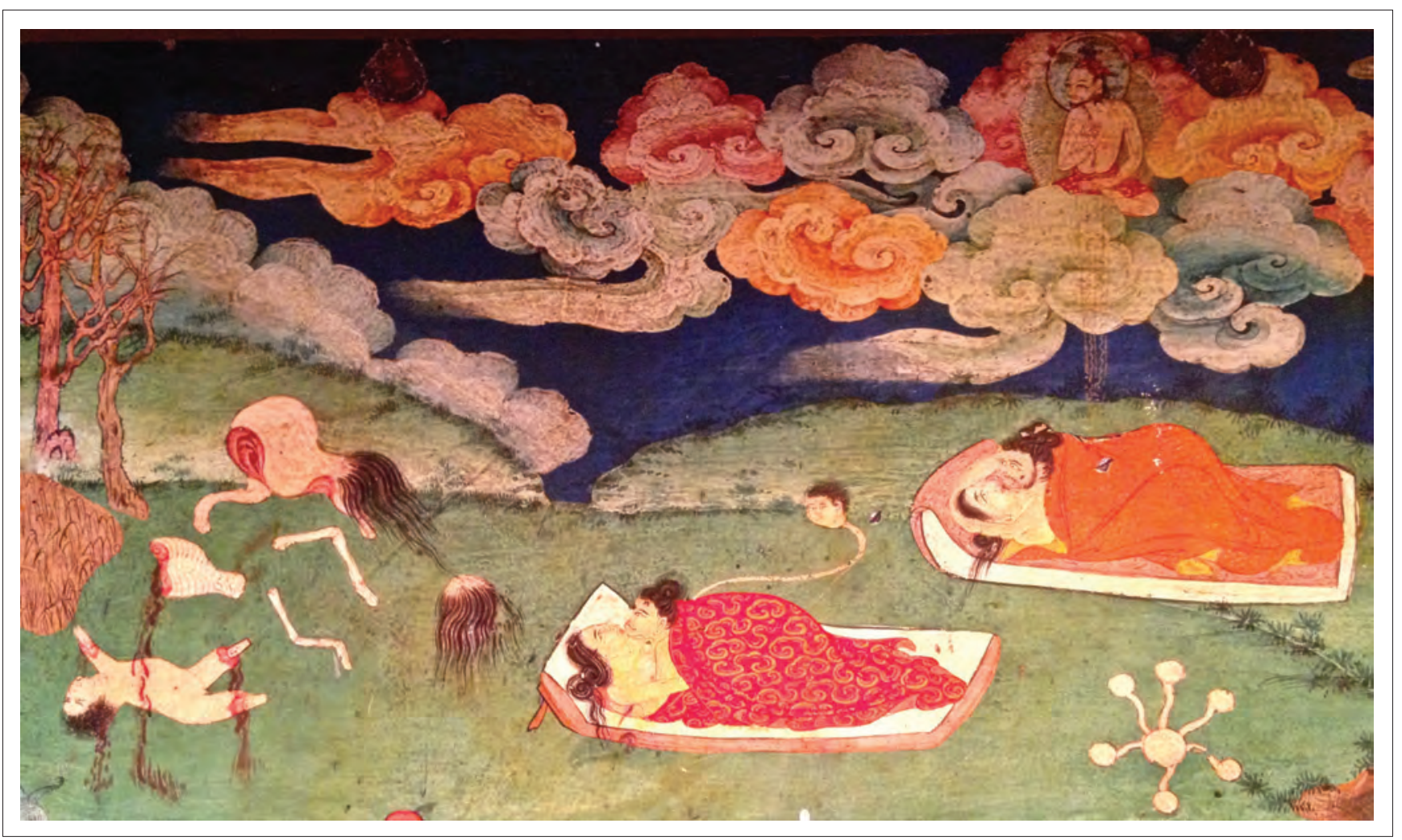

Figure 29.3 The Lukhang's northern mural includes a deconstruction of the Buddhist Wheel of Life and Rebirth. The opening vignette shows a disembodied 'soul' in the process of incarnating in a woman's womb - a choice leading to continuing cycles of birth and death, signified by the dismembered body parts at the left. The haloed figure at the upper right has opted out, as indicated by the gesture of his right hand, symbolising the Buddhist path to liberation. The image in the lower right-hand corner signifies a spermatozoa and the six realms of existence into which one may be born and is thus a reference to, and departure from, the more conventional and elaborated ways in which these realms are typically represented. ๑Ian Baker

the 79 medical thangkas, including depictions of conception, embryology, birth and death, as well as representations of the network of subtle energy channels within the human body that chart mind-body interactions and serve as a basis for both Tibetan medical theory and advanced tantric Buddhist practice. In this sense, the murals partly represent a 'medicalisation' and rationalisation of tantric practice, contextualising it within natural processes of the human body.

As with the medical paintings, the wall paintings in the Lukhang include descriptive captions, drawn from the murals' source text - Pema Lingpa's 'Compendium of Enlightened Spontaneity'. Also akin to the medical paintings, the murals feature generic representations of everyday life that serve a subliminal didactic purpose. Such images in the Lukhang murals include a miser buried under the weight of his jewel-encumbered home as well as scenes of an impassioned butcher and couples making love within a charnel ground of severed body parts (see Fig. 3). Other symbolic elements such as crystals, mirrors, and spheres of light recur throughout the murals, emphasising the encompassing Dzogchen context of self-illuminating non-dual awareness.

Whereas the medical paintings present their contents in sequential horizontal rows - read from left to right - the Lukhang murals represent the practices of Dzogchen in sinuous unbounded vignettes within surreal, and often inverted, landscapes that must be visually deciphered in order to relate them to the specific chapters of Pema Lingpa's treasure text from which the illustrations largely derive.

The mural on the north wall is particularly notable in its deconstructed depictions of the Buddhist cosmos and the 'wheel of rebirth' (srid pa'i 'khor lo) that, by convention, typically frame the entryways of Tibetan Buddhist monasteries. In the case of the Lukhang, the primal elements of the cosmos are shown as issuing from a naturalistically rendered vagina with a depiction of the mythical Mount Meru shown, without accompanying inscription, as an almost purely ornamental feature, representing a description of the universe that the current Dalai Lama has himself dismissed in favor of more scientific accounts of the cosmos. The Buddhist wheel of rebirth depicting the causes of cyclic existence is similarly deconstructed: 


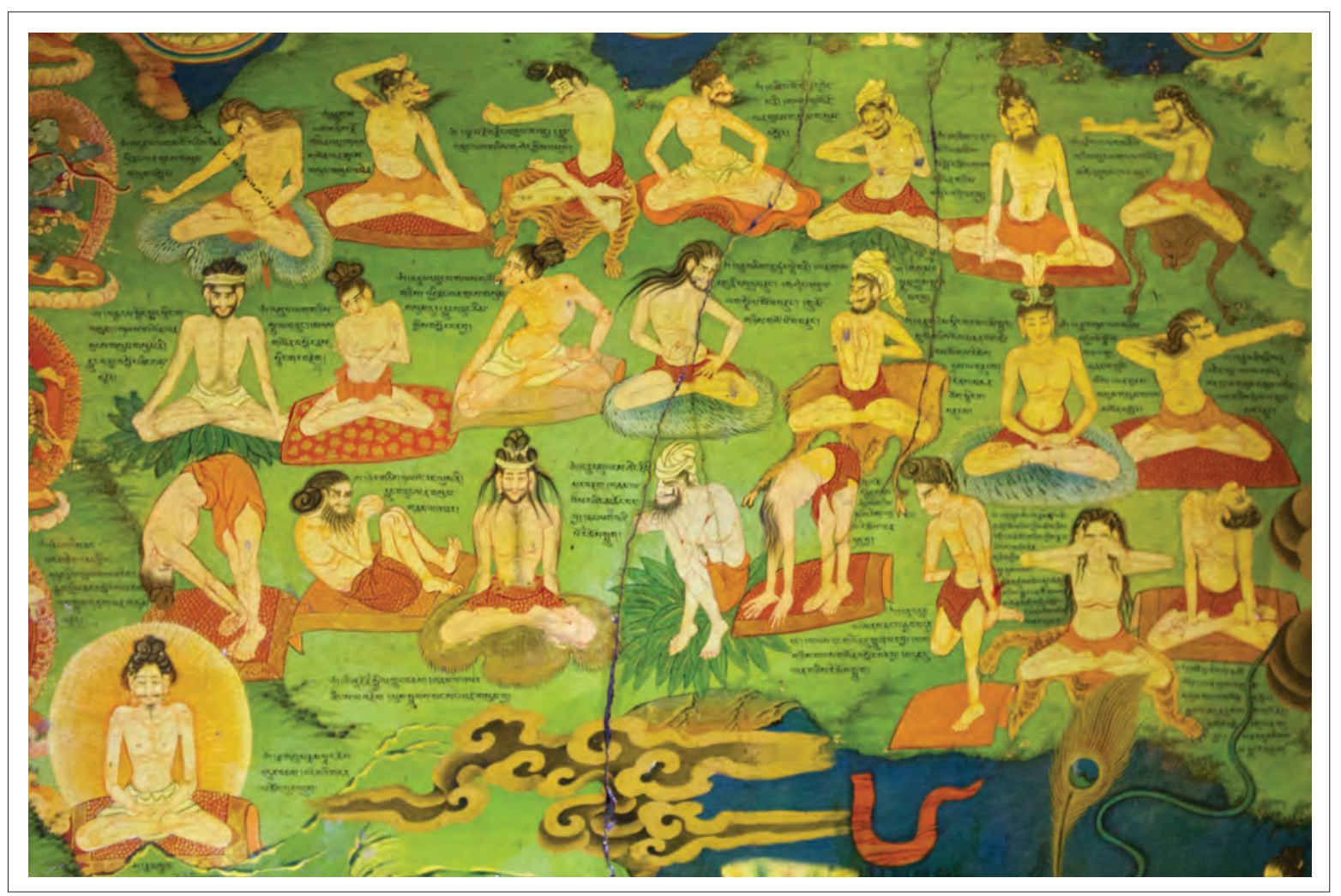

Figure 29.4 A section of the Lukhang murals illustrates Pema Lingpa's 'Secret Key to the Channels and Winds' (Rtsa rlung gsang ba'i lde mig) and includes depictions of 23 yogic exercises ('khrul 'khor) that prepare body and mind for visionary gazing practices called thod rgal ('leaping over the skull'), visions of which are pictured at the lower right. $\odot$ Ian Baker

The pig, rooster, and snake that symbolise the mental afflictions of ignorance, greed, and aggression are shown in the Lukhang murals as circling within the breast of an ordinary human being, rather than at the hub of a wheel in the hands of Mara, the Buddhist characterisation of egocentric existence. As in more traditional renderings of the wheel of life, a Buddha figure is shown nearby offering an alternate source of identification. This cycle of birth, death, and reincarnation, as well as its transcendence, is recapitulated in the opening vignette of couples making love (see Fig. 3), a reference to the 12 stages of interdependent origination that are normally depicted on the outer ring of the Buddhist wheel of life.

Throughout the Lukhang murals, generic human figures are paired with often humanistically rendered Buddhas and Bodhisattvas that emphasise the murals' overall iconographic and intellectual programme: a visual guide to the realisation of one's innate indwelling Buddha Nature. In this sense, the Lukhang murals graphically illuminate the original intent of Pema Lingpa's revealed treatise, while detailed representations of intra-ocular visionary phenomena exceed the scope of the written word. The murals were nonetheless not performative, in the sense that simply looking at them would not allow one to put them into practice without personal guidance by a realised master of the tradition. This same argument can also be made for the Desi's medical paintings that serve even today as a mnemonic course outline rather than as a comprehensive how-to guide to medical practice.

The chapter entitled 'Secret Key to the Channels and Winds' (a translation of which is appended to this chapter) from Pema Lingpa's 'Compendium' clearly reveals the limits of both words and visual representations for transmitting Dzogchen's core practices in absence of a qualified human preceptor. The chapter describes 23 sequential yogic movements, but the Lukhang murals illustrate only one position within each of the numbered exercises, rather than representing each movement's multiple stages. Although inscriptions drawn from Pema Lingpa's text accompany each of the images, a viewer would not be able to follow the practice without additional instruction any more than one could play a musical instrument simply by deciphering a sequence of musical notations. Both Pema Lingpa's source text and the illustrations on the walls of the Lukhang are, in this sense, mnemonic reminders, rather than comprehensive guides, to actual practice. Yet, as with the Desi's other highly innovative projects, no prior attempts had ever been made to illustrate esoteric practices connected with the subtle body on the walls of a temple. 
Despite Desi Sangye Gyatso's commitment to detailed realistic illustration, the encrypted nature of the images of yantra yoga ('khrul 'khor) on the walls of the Lukhang are fully in keeping with tantric Buddhism's traditional emphasis on one-to-one oral transmission. This may account for the complete omission of textual inscriptions in the section of the mural below the labelled sequence of 23 yogic movements. The images of practice illustrated in this section of the northern mural - such as a seated figure with flame erupting from his head (Fig. 8) and another gazing at a disembodied eye suspended in space (Fig. 2) - are some of the most compelling in all of Tibetan art history, but their function cannot be fully deduced even from reading Pema Lingpa's source text. Nonetheless, the effective rendering of the subjective phenomenology of these practices clearly shows the degree to which the creation of the Lukhang murals involved active collaboration with accomplished Dzogchen lineage holders. ${ }^{33}$

The convention of secrecy regarding tantric yogic practice was one reason that, unlike the medical paintings, the murals in the Lukhang remained sequestered, concealed to all but the Sixth Dalai Lama and his closest attendants. In this sense, the Lukhang murals remained a hidden treasure whose time for public revelation had not yet arrived. Even if the Desi's ultimate intention had been to confer further prestige to the Tibetan state through the revelation of an illuminated encyclopaedia of Vajrayāna Buddhism's most esoteric practices, he did not live long enough to see this materialise, as he was beheaded by Lhasa's Mongol overlord Lhasang Khan soon after the murals' completion. ${ }^{34}$ Later the same year, 1705, Lhasang Khan deposed the rebellious Sixth Dalai Lama and dispatched him, as a prisoner, to Beijing. The exiled Sixth Dalai Lama is alternately said to have died on the way to China or to have gone on to live a secret peripatetic life outside Tibet until 1746 .

Tibet's complex political circumstances at the turn of the 17 th century offer further insight into the genesis and subject matter of the Lukhang murals. Tibet's Mongolbacked and ecclesiastically disposed Gelukpa order was openly hostile to Tibet's earlier Nyingma tradition and its non-monastic teachings of 'Great Perfection'. As both secular and spiritual leaders of the Tibetan people, the Dalai Lamas nonetheless secretly practised Dzogchen, and the

Desi Sangye Gyatso was himself a Dzogchen initiate who received the transmission of Pema Lingpa's 'Compendium of Enlightened Spontaneity', as well as other Dzogchen practices, from Terdak Lingpa, the abbot of Oryen Mindroling, central Tibet's most important Nyingma monastery.

34 By some accounts, the Desi was murdered by Lhasang Khan's wife with whom he allegedly had an adulterous affair.

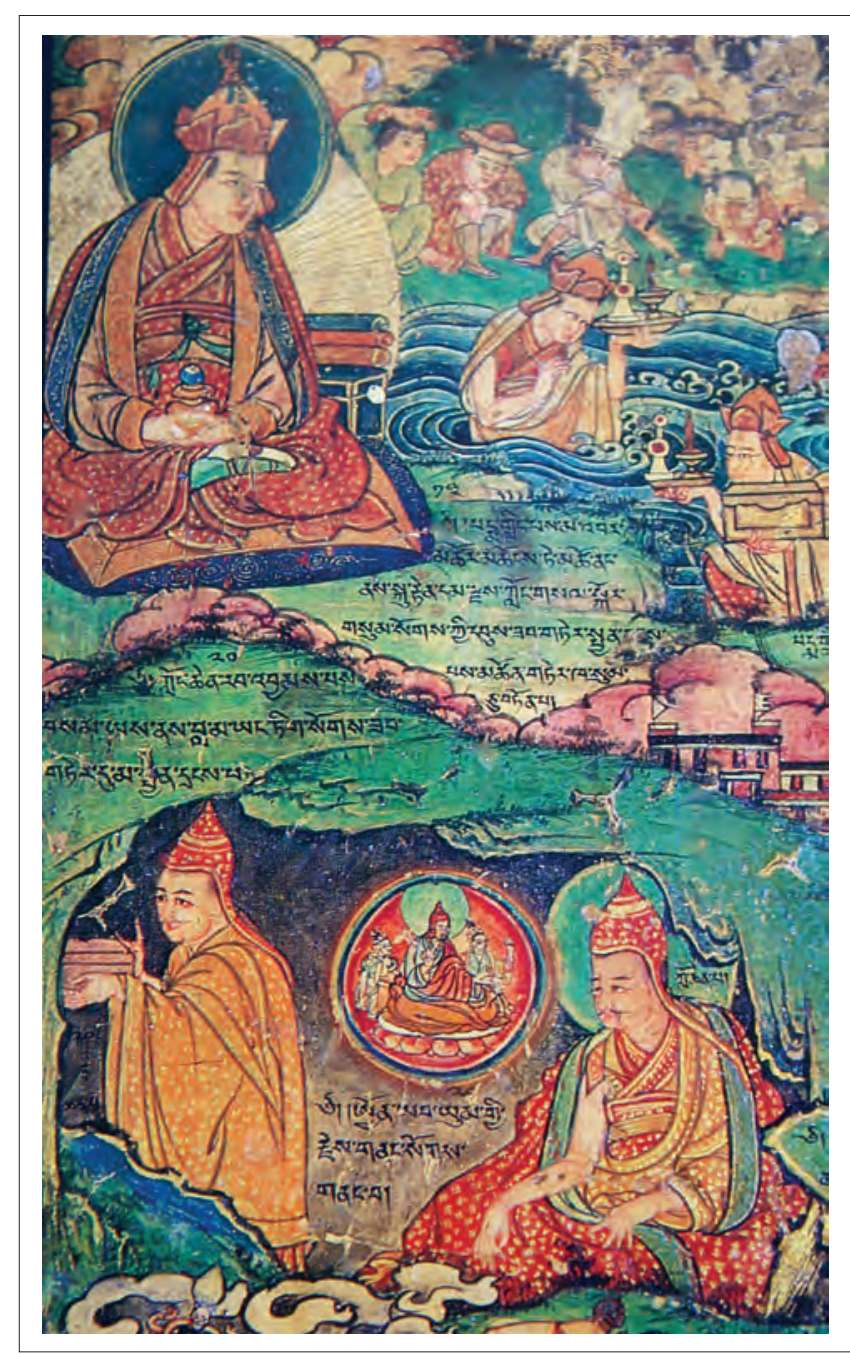

Figure 29.5 Pema Linga publicly extracting treasure texts (gter) from 'Burning Lake'. A detail from the Lukhang murals. ${ }^{\circledR}$ Ian Baker

vivid murals created by Desi Sangye Gyatso within the private precincts of the Lukhang helped ensure that successive reincarnations of the Dalai Lama remained fully aware of the 'Great Perfection' practices, even if Tibet's sectarian political climate remained hostile to their dissemination.

Beyond preserving endangered esoteric knowledge in artistic form, the subject matter of the Lukhang murals also served a political function in helping to justify Desi Sangye Gyatso's clandestine and unilateral selection of Tsangyang Gyatso as the Sixth Dalai Lama, without recourse to the established protocol for identifying reincarnate Dalai Lamas. The south-east mural on the top floor of the Lukhang illustrates Tsangyang Gyatso's hereditary descent from Pema Lingpa, who is shown at multiple points in the mural extracting ter, or religious treasures, from earth and water (see Fig. 5). ${ }^{35}$

This detail from the Lukhang murals illustrates a scene in which a sceptical local governor challenged Pema Lingpa to prove his 
This panel also celebrates Pema Lingpa's previous incarnation as the revered Dzogchen master Longchen Rabjam (1308-63), who is depicted performing a tantric rite of subjugation. The dissemination of Pema Lingpa's revealed treasure teachings by Terdak Lingpa Gyurme Dorje (1646-1714) at Orgyen Mindroling, the most important monastery in central Tibet for the transmission of Dzogchen, is also vividly illustrated on the mural, as is the figure of the Sixth Dalai Lama's father, Rigdzin Tashi Tendzin, from whom Terdak Lingpa received the full transmission of Pema Lingpa's revealed teachings. Pictured next to Rigdzin Tashi Tendzin is an idealised, yet unlabelled, rendering of his son Tsangyang Gyatso, the Sixth Dalai Lama, as a temporal and religious monarch surrounded by devoted subjects (see Fig. 6). This image is entirely unprecedented - as well as politically expedient - in showing a figure of Amitābha, the Buddha of Infinite Light, emerging from the Sixth Dalai Lama's head turban, linking him iconographically to Tibet's first emperor and 'dharma king' Songtsen Gampo (617-49). Such a bold and compelling visual account of Rinchen Tsangyang Gyatso's spiritual and hereditary origins would have helped protect both him and the Desi from potential detractors, establishing the Sixth Dalai Lama as a legitimate embodiment of Avalokiteśvara, the Bodhisattva of Supreme Compassion. ${ }^{36}$

All of Desi Sangye Gyatso's works reveal an unusually creative and interpretive mind using art to convey cultural, medical, and religious practices beyond the limits of both written expression and iconographical conventions. Although the Lukhang murals represent an unprecedented moment in Tibetan intellectual history- portraying knowledge at the cutting edge of innovation and creative imagination and revealing complex cultural and socio-political agendas - the primary intent of this chapter is to clarify the nature of the practices and body-based spirituality that the Lukhang murals depict.

authenticity by retrieving a treasure text from an underwater grotto in front of a large audience. Pema Lingpa declared that if he was genuine, he would return with the text before his butter lamp extinguished. If he were a fraud, he would die in the water. He dived in and returned with a small box and a sculpture, his butter lamp still burning.

This idealised portrait of the Sixth Dalai Lama is still more striking in its inclusion of ritual objects linking him to the other two principle Bodhisattvas widely represented in Tibetan art. A religious text and a sword are pictured at his left, connecting him to the Bodhisattva of transcendental wisdom Manjusri, while a vajra (dor je) to his right links him with Vajrapani, the Bodhisattva of wrathful, yet compassionate, power.

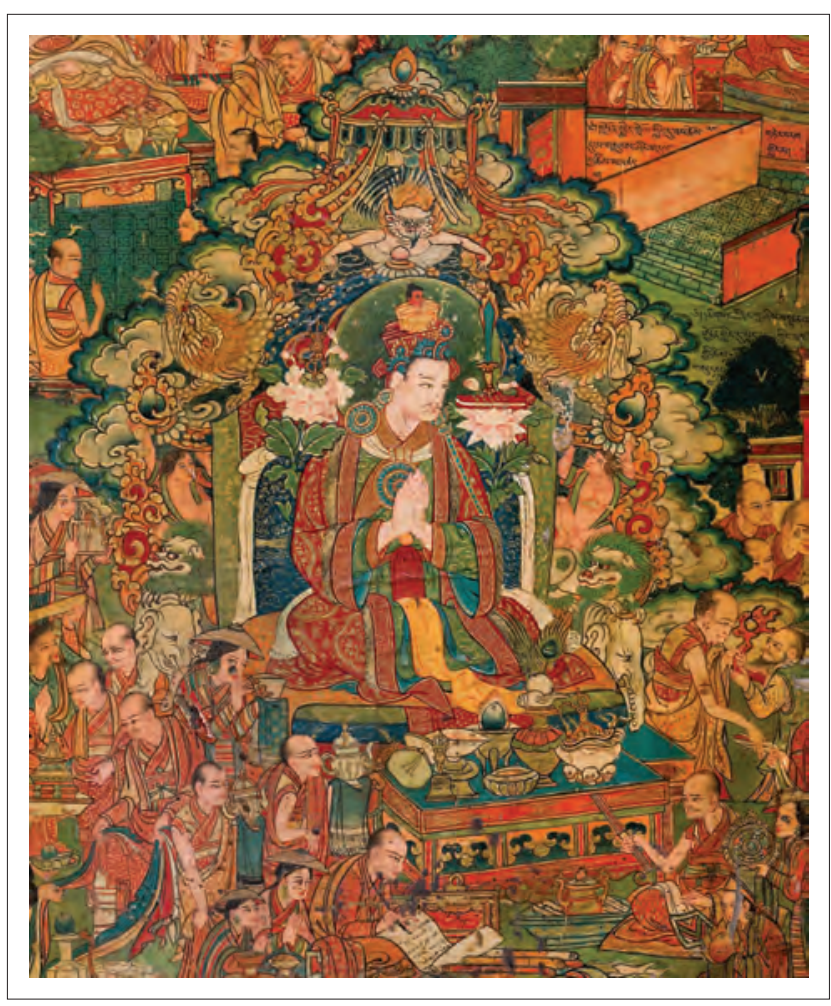

Figure 29.6 This detail from the south-east corner mural of the Lukhang temple presumably shows Tsangyang Gyatso, the Sixth Dalai Lama, as a 'Bodhisattva king', modelled on the iconography of Avalokiteśvara holding the wishfulfilling gem of supreme compassion in his joined palms, and with the accoutrements of wisdom and spiritual power emerging from lotuses at his shoulders. $\odot$ Ian Baker

\section{Preludes to Perfection}

Advanced practice in Vajrayāna, or Tantric Buddhism, is customarily preceded by foundational practices called Ngondro (sngon 'gro), designed to transform the adept's subjective experience of reality. These generally consist of development phase (bskyed rim) ${ }^{37}$ methods of visualisation and mantra recitation that reorient consciousness and develop concentration and insight. These are customarily followed by completion stage ( $\mathrm{rdzogs}$ rim) practices based on a metaphysical anatomy of channels, winds, and subtle essences within the human body. These methods lead, in turn, to the enlightening realisation of the inseparability of outer and inner experience. Dzogchen differs from tantra in maintaining that enlightenment is spontaneously present as humankind's innermost nature and needs only to be recognised. The foundational practices in Dzogchen thus forgo graduated liturgies and elaborate rites of trans-

37 For a concise presentation of the development stage (bskyed rim) of Mahā Yoga associated with sngon 'gro, see Dudjom Rinpoche 1991, pp. 275-83. See also ch. 18 in Kongtrul 2005. 


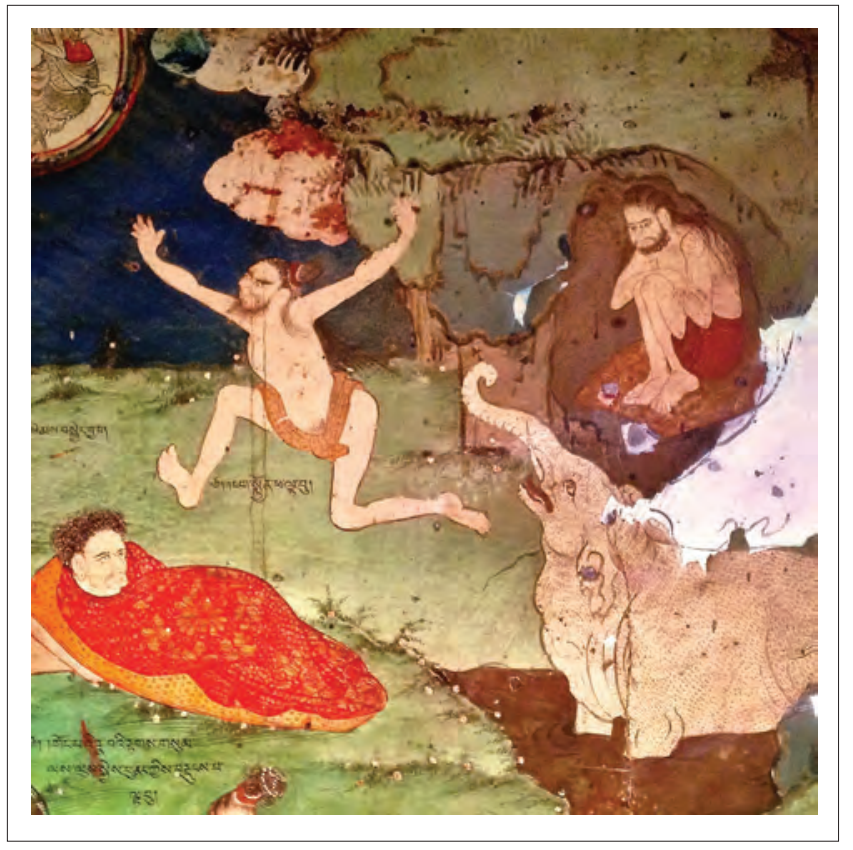

Figure 29.7 This detail from the Lukhang's western mural shows a Dzogchen yogin acting out interior states of consciousness in a process called Korde Rushen ('khor 'das ru shan), 'distinguishing Samsāra from Nirvāṇa'. The elephant emerging from a quagmire symbolises the freedom of consciousness that the practice facilitates. ${ }^{(C)}$ Ian Baker

formation and focus instead on embodying primordial awareness ( $r i g ~ p a)$ in every conceivable circumstance.

Divided into Outer, Inner, Secret, and Ultimately Secret methods for distinguishing the spontaneous self-liberating awareness (sem nyid) of Nirvāṇa (T: mya ngan 'das) from the bounded mental habits of Samsāra (T: 'khor $b a$ ), Dzogchen's foundational practices are called Korde Rushen ('khor'das ru shan) and are normally undertaken in wilderness settings. Although their purpose is to induce a radical reorientation of consciousness - distinguishing the contents of conscious from consciousness itself - the methods by which this is achieved involve pushing both body and mind beyond all habitual limits. In the practices of Outer Rushen, the adept is instructed to physically act out conjured existences from heavens to hells and thus to recognise the self-created nature of conditioned existence. These practices are illuminated on the western wall of the Lukhang murals in images such as that of a yogin shown leaping like a rogue elephant through a shape-shifting landscape of blues and greens (see Fig. 7). As a Dzogchen treatise entitled 'Flight of the Garuda', contemporary with the Lukhang murals, instructs:

With the conviction that Samsāra and Nirvāna are of one taste... walk, sit, run and jump, talk and laugh, cry and sing. Alternately subdued and agitated, act like a madman. Finally abide in a state of ease and contentment... .
Practising in this way your realisation becomes as vast as the sky and your meditation naturally radiant.... Without reference points, prejudice or attachment, your actions become spontaneous and saintly, making no distinction between self and others. Detached from your utterances, your speech is like a melodious echo. Without desire for anything at all you are like a celestial eagle soaring through space, or like a fearless and intrepid lion... free from the very beginning, like bright clouds in the sky. ${ }^{38}$

When body and mind have exhausted their capacity for consciously directed dramatic expression, one rests in a state of lucid relaxation ( rnal du ba), corresponding to the śavāsana, or 'corpse' pose, of hațha yoga.

By alternating unrestrained enactment of the imaginative projections of the mind with pristine objectless awareness, the practitioner of Outer Rushen discovers how Samsāra, the state of dualistic distraction, and Nirvāna, open selfless presence, emerge from the same 'primal purity' ( $k a \mathrm{dag}$ ) beyond being and non-being. As Padmasambhava explicates in a treasure text entitled 'Liberation Through Seeing with Naked Awareness' (Rig pango sprod gcer mthong rang grol):

Saṃsāra and Nirvāṇa have no difference other than being unaware or aware [of the intrinsically undivided nature of reality]... . Since we are not deluded by perception but by fixation, Liberation naturally occurs when we recognise that fixated thoughts are only mind grasping at its own empty manifestations.

Through the cathartic improvisations ${ }^{39}$ and physical actions of Outer Rushen, the practitioner releases all inhibitions and attachments and realises a freer dimension of being, based on the mind's essential and abiding nature rather than its transient expressions.

The practices of Inner Rushen ${ }^{40}$ focus attention on six areas along the central axis of the physical body and soles

38 See Dowman 1984. For a detailed account of the relation of Pema Lingpa's texts to the different sections of the Lukhang wall paintings, see Winkler 2002, pp. 321-43.

39 The methods of Outer Rushen recall the creative and empowering dramatisations of psychic contents used in therapeutic modalities such as Psychodrama, defined by its founder, Dr J.L. Monroe, as 'the science which explores the truth by dramatic methods'. Monroe was the author of an influential book, The Theatre of Spontaneity (1924), which addresses the cathartic effects of uninhibited self-expression in therapy and personal growth.

40 The precise instructions for Korde Rushen practices vary between different Dzogchen lineages. In some, the 'Purification of the Six Lokas' as described here is performed prior to the Outer Rushen described above. As with all aspects of Dzogchen, the key point is never technique but the end result: integrating awareness of the mind's innermost non-dual nature within all 
of the feet correlated with inhibiting psychological potentialities and the karmic traces of past actions. Envisioning associated 'seed-syllables' (bija mantra) ${ }^{41}$ at each of the six points, one clears them of all subconscious residue with the intonation and visualisation of blazing white, red, and blue light resounding from the seed mantras $\mathrm{Om}, \mathrm{Ah}$, Hum, representing the triune totality of pristine awareness. Consciousness thus shifts from identification with the body's materiality to its underlying luminescence (nang gsal). ${ }^{42}$ As with the practice of Outer Rushen, sessions focused on the physical body are alternated with motionless periods of concept-free awareness in which thoughts dissolve within the encompassing space of primordial awareness (rig pa).

Inner Rushen's clearing of subconscious somatic obscurations prepares the body for the practices of Secret Rushen, which commence with adopting a highly strenuous isometric posture - the 'position of the vajra' ( $r d o$ rje'i 'dug stang) - which, supported by associated mental imagery, pushes body and mind into a state of positive eustress, leading to the emergence of adaptive mental and physical capacities. Standing tensed with heels together, knees stretched out to the sides, chin to larynx, and the hands palm to palm above the crown of the head while visualising oneself as an indestructible blazing blue vajra ( $r$ do rje), the practitioner observes the flow of energy and sensation within the body while pushing through barriers of pain and exhaustion. The position is held until the body physically collapses and then is continued on the ground to whatever degree is possible. Then, with the resounding of the seed-syllable Phat, one lies down in a state of unconditioned concept-free awareness. When thoughts arise, one repeats the process in continuing cycles of effort and repose. More than any other Dzogchen practice, the 'vajra posture' engages the physical body in ways designed to alter

circumstances and experience.

41 The syllables $\mathrm{Ah}, \mathrm{Su}, \mathrm{Nri}$, Tri, Pre, and $\mathrm{Du}$ correlate with the forehead, throat, heart, navel, base of the trunk, and soles of the feet. The upper five centres are also associated with seed-syllables representing the Five Elements. Early reference to practices associated with the recitation of the panca bhüta (five element) bija mantras (Ham, Yam, Ram, Vam, and Lam) can be found in the ShriJabala Darsana Upanishad, composed between 200 вСЕ and $200 \mathrm{CE}$.

Photon emission associated with bioluminescence within humans and other species has been identified as a side-effect of metabolic reactions, the result of highly reactive free radicals produced through cell respiration interacting with free-floating lipids and proteins. The 'excited' molecules that result can react with chemicals called fluorophores to emit photons (The Guardian, 17 July 2009). the flow of psychosomatic energies and to embody experientially the mind's capacity to transcend and gain from physiological stress as it identifies ever increasingly with the essence of consciousness, rather than its adventitious forms including the illusory notion of an abiding self. ${ }^{43}$

The body practices of Secret Rushen are followed by practices of sound and vibration in which a blue seed-syllable Hum is intoned and visualised as multiplying until small Hums fill the entire universe. The syllables and sounds of Hum then fill one's entire body propelling it imaginatively through space. At the end of the session one again lies down as in the practice of the body and rests in open presence. When thoughts arise, one begins the practice anew, using the primal energy represented by the seed-syllable Hum to alter habitual perceptions and conceptions of reality. According to the texts, signs of accomplishment include physical bliss, mental lucidity, and non-conceptual awareness.

The subsequent mind practice of Secret Rushen uses the seed-syllable and sound of $A h$ to enter into direct experience of the pure potentiality, or emptiness, that underlies all appearances. Dissolving all perceptions into effulgent light, one remains in a unified state of luminous cognition, beyond conceptions of time and space. The quiescent culmination of the mind component of Secret Rushen forms the basis for Ultimately Secret Rushen in which the energetic currents of one's inner being are expressed dynamically 'in order to free what has been stabilised'. Spontaneously manifesting as a wrathful tantric deity representing the creative volatility of sensation, thought, and emotion, one stands with hands formed into horned mudrās, pivoting from left to right on heels driven into the earth. With eyes rolling in the sky, one emits loud thought-subduing laughter from the deepest core of one's being, filling all space with the syllables and sounds of $\mathrm{Ha}$ and $\mathrm{Hi}$. As in all Rushen practices, the body is used to its fullest potential to bring the mind to an innate state of lucid, all-pervading awareness, the foundation for all subsequent Dzogchen practices based on the existential ideal of discovering ultimate freedom within the flow of ordinary experience.

43 The development of increased mental and physical capacities through pushing through habitual limits is known in biology as hormesis, a process whereby beneficial effects such as increased strength and resilience, growth, and longevity can result from deliberate and systematic exposure to therapeutic stress. 


\section{Flowing Wholeness}

A seminal chapter of Pema Linga's treasure text 'Compendium of Enlightened Spontaneity' is illustrated on the north wall of the Lukhang temple, further illuminating the role of physical practices in Dzogchen. Entitled 'The Secret Key to the Channels and Winds' (Rtsa rlung gsang ba'i lde mig), ${ }^{44}$ the chapter focuses on methods of self-cultivation that view the body as a vehicle for realising a liberated condition beyond all physical and conceptual limits. (See the Appendix to this chapter for an illustrated translation of Pema Lingpa's 'Secret Key'.) Based on the Dzogchen formula of 'Four Sessions for Realising Buddha Nature' (thun bzhi sangs rgyas ro mnyam pa nyid), the interconnected practices are undertaken at dawn, daytime, dusk, and night and reframe the Six Yogas of tantric tradition in the context of Dzogchen's radical view of non-duality. 45

Unusually for a Dzogchen treasure text, the chapter begins with a description of 23 yogic exercises ('khrul'khor) that, combined with specific breathing techniques, ${ }^{46}$

44 Rdzogs chen kun bzang dgongs'dus, vol. 2, pp. 33-40. 'Winds and channels' (rtsa rlung. Skt: nādī vāyu) refer to tantric representations of the body as a flow of subtle energies that, harnessed and controlled through yogic practice, lead to illuminating states of awareness. The principal energy channels represented in the Lukhang murals are the body's central channel (rtsa dbu ma, Skt: avadhüti) and the paralleling 'solar' and 'lunar' side channels (rtsa ro ma and rtsa rkyang ma) (see Figs 11 and 15). A translation of 'The Secret Key to the Channels and Winds' is appended to this chapter, illuminating the fundamental dynamics of mind and body at the heart of the Dzogchen tradition and the ways in which primordial unitary awareness (rig pa) arises vibrantly and unconditionally in response to physiology and perception pushed beyond their accustomed limits, whether in states of waking, sleeping, dreaming, or near-death experiences. In contrast with Kagyu, Sakya, and Geluk presentations of the Six Yogas, Pema Lingpa's 'Secret Key' emphasises the Dzogchen approach of 'self-liberation' rather than that of transformation (see also n. 7). The order of the 'four sessions' varies between $\mathrm{Dz}$ ogchen lineages. The Yeshe Lama, for example, describes them as follows: 'Before sunrise, one focuses on a white $A h$ emerging in the sky before one from the white $A h$ at the heart. During the daytime, one meditates on the natural visions of thod rgal. At dusk, one practises gtum mo. During the night, one withdraws the senses into a luminous $A h$ in the vase of the heart (she bya bum 'jug)'.

46 The breath or vital energy (rlung, Skt: präna) of the body is often likened to a horse and the mind to the rider of the horse. The movements of 'khrul 'khor, sometimes called Yantra Yoga, are normally performed with the breath held in the lower abdomen 'like a vase' (bum can, Skt: kumbhaka) in order to direct the prānic winds into the body's central meridian and alter the are held to clear the pathways of the channels $(r t s a)$ and activate the flow of awareness through the body's central meridian (rtsa dbuma) (see Fig. 4). The exercises culminate in the seven-point meditation posture (rnam snang chos bdun $)^{47}$ that forms the basis for the subsequently described evening practice of 'fierce heat' (gtum mo) in which the physical senses are drawn inward towards the experience of 'four joys' (dga'ba bzhi, Skt: caturānanda) at specific points along the body's central axis, giving rise to the self-transcending rapture of co-emergent bliss and emptiness (bde ba'i stong pa nyid). ${ }^{48}$

The text goes on to describe practices of lucid sleep and dreaming - 'retaining wakefulness in the vase' - whereby the habitual divisions between conscious and subconscious states are gradually overcome as the inner winds (rlung, Skt: prāna) resolve at the heart into all-pervasive Luminosity ('od gsal). ${ }^{49}$ Pema Lingpa's text next describes 'reviving natural awareness at dawn', a method involving the propulsion of the heart-syllable Hum through the crown of the head to a bow's length in front and above until it naturally dissolves and becomes co-extensive with infinite space, the ultimate posited nature of mind and body. The text then introduces the daytime practice of thod rgal, or 'leaping over', by which the mind, through attending to

flow of consciousness. When combined with the 'Four Sessions for Realising Buddha Nature', the practices of 'khrul 'khor are normally undertaken before the evening practice of gtum mo or in the early morning before the practice of thod rgal. In thod rgal, however, the practitioner refrains from any manipulation of the prānic winds so as to directly engage primary non-dual consciousness. This is accomplished through an alternative network of 'light channels' ('od rtsa).

47 The seven points consist of the legs in full lotus position, the spine erect, the shoulders broadened, the neck bent slightly forward, the hands in the gesture of equanimity (with the right hand resting on the left palm), the tip of the tongue touching the palate, and the gaze directed beyond the tip of the nose.

The bliss that is cultivated through the practice of gtum mo represents the transformation of dualistically oriented desire into innate rapture beyond subject or object (bde ba chen po, Skt: mahāsukha, sahajananda). The process is described through the experience of four progressive states of bliss, each associated with a different energy locus along the body's central axis, and culminating in open enstatic awareness beyond divisions of pleasure and pain.

49 The practices of inner yoga described in the chapter 'The Secret Key to the Channels and Winds' are elaborated in other chapters of 'Compendium of Enlightened Spontaneity' such as one entitled 'The Secret Practice of Noetic Fire (gtum mo)' and another on 'taking desire as the path' entitled 'Churning the Depths of Samsāra'. For a detailed description of the practice of gtum mo, see Yeshe 1998 . 


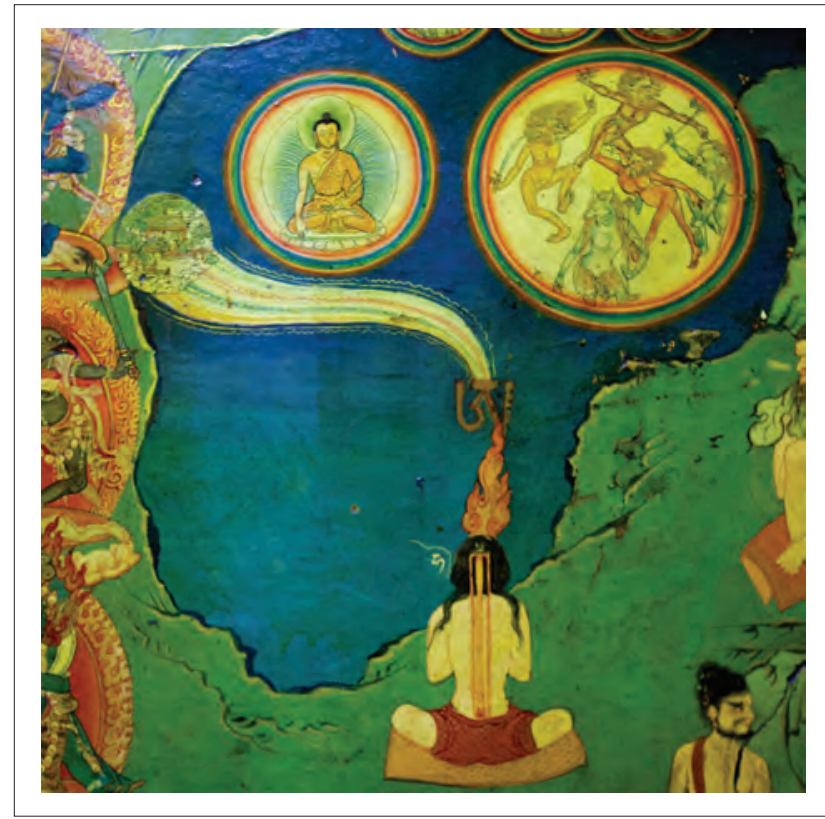

Figure 29.8 This detail from the Lukhang's northern mural appears beneath the section of the wall that illustrates 23 yogic exercises that prepare the mind and body for meditation and interior yogic practices (see Fig. 4). This image illustrates the actualisation of Buddha Nature (de gshegs snying po, Skt: tathāgatagarbha) through the practice of 'fierce heat' (gtum mo) and the ejection of consciousness ( $p h o b a$ ) to a paradisiacal Buddha Realm. $\odot$ Ian Baker

spontaneous displays of numinous photonic spheres (thig le), ${ }^{50}$ becomes coextensive with the expanse of reality (chos kyidbyings, Skt: dharmadhātu) and the body, at the time of death, dissolves into rainbow light, the subtlest expression of the five elemental energies held to comprise the manifest universe (see Figs 2 and 12).

While all of the meditative practices described in Pema Lingpa's text involve the support of the physical body, the sequence of 23 hatha-yoga-related movements illustrated on the northern wall of the Lukhang are the most revealing. While analogous exercises are described in commentaries to the Hevajra Tantra and other early Vajrayāna Buddhist works for facilitating the transformative flow of kundalini

The Tibetan word thig le (Skt: bindu) has multiple meanings depending on context. Within thod rgal practice, thig le refers to spheres of multicoloured light that mutate into an infinite variety of intermediary forms held to represent the inherent dynamics of primordial awareness (rig pa). As entoptic phenomena (visual effects whose source is within the eye itself as images cast upon the retina), thig le also bear comparison with muscae volitantes-'floaters' that can spontaneously arise within the visual field as luminous chains or drifting orbs - as well as with phosphenes, subjective perceptions of light in the absence of light actually entering the eye, such as when one presses on one's eyeballs.

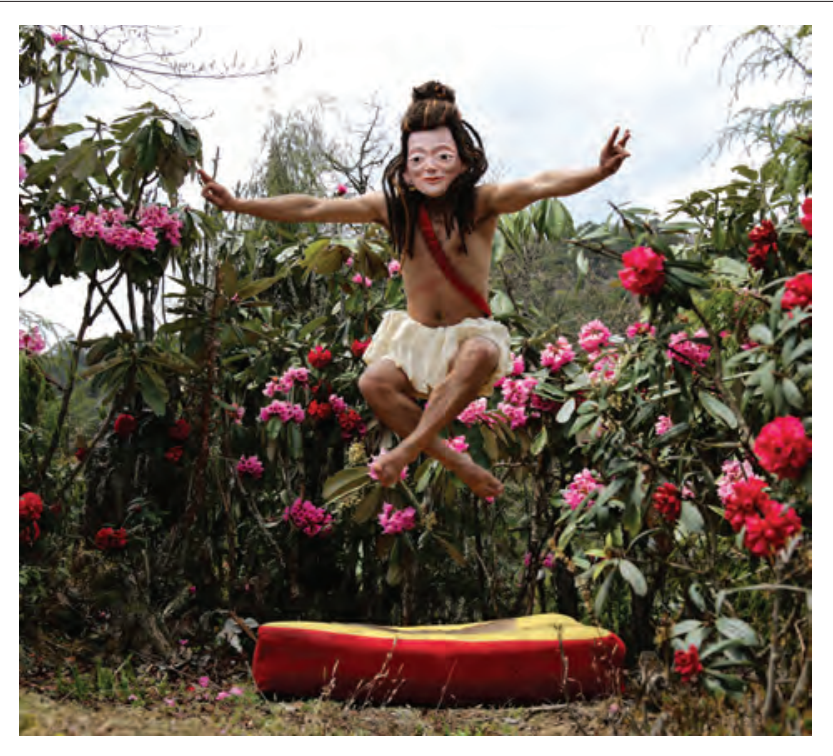

Figure 29.9 The practices illustrated on the walls of the Lukhang remain a living tradition in Bhutan, where a Nyingma yogin - concealing his identity behind a mask - demonstrates the sequence of 23 yogic exercises ('khrul'khor) central to Pema Lingpa's 'Secret Key to the Channels and Winds'. The movement shown in this contemporary photograph is a controlled fall (bep) that concentrates energy in the body's central channel (susumnā) to facilitate the practice of 'fierce heat' (gtum mo). ${ }^{\odot}$ Ian Baker

(gtum mo, Skt: cand̄ālī) through the body's central channel (rtsa dbuma, Skt: avadhüti), no definitive records exist of the inclusion of such practices in early Dzogchen (see Fig. 9).

Nonetheless, rtsa rlung 'khrul 'khor forms a major part of the Dzogchen gter ma tradition from the 11th century onwards, when practices based on the body's 'inner mandala' (nang pa'i dkyil 'khor) of subtle energy channels were introduced in Tibet through the Kagyu and Sakya lineages. The Nyingma tradition of inspired revelation continues to this day, however, in the appearance of works such as the 'Union of Sun and Moon' ('khrul 'khor nyi zla kha sbyor gyi dgongs 'grel dri med nor bu'i me long), credited by the Tibetan scholar and meditation master Chogyal Namkhai Norbu to Vairocana, a Tibetan contemporary of Padmasambhava, who lived during the 8th century. The text's detailed presentation of 108 sequenced movements, linked by precise breathing methods for directing the flow of internal energy, includes postures (asana) that are otherwise known only in works of hatha yoga that appeared in India from the $15^{\text {th }}$ century onward. A verifiable historical source for the text translated and published by Namkhai Norbu as Yantra Yoga: The Tibetan Yoga of Movement ${ }^{51}$ sion of Yantra Yoga ('khrul 'khor) from Padmasambhava who, in 


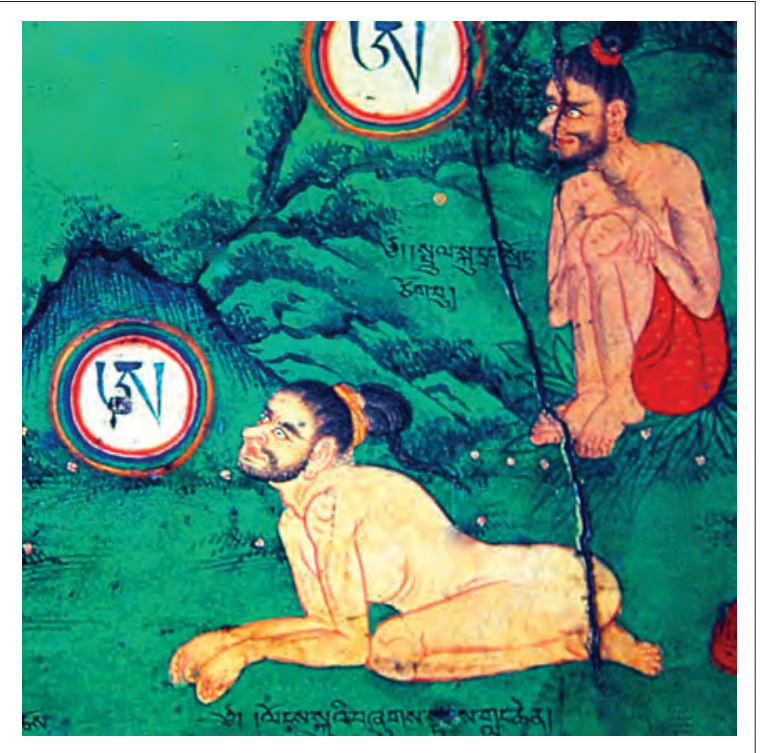

Figure 29.10 “The Dzogchen 'leaping over the skull' (thod rgal) postures of elephant and sage, as illustrated on the western mural of the Lukhang temple in Lhasa, Tibet, $c .1700$. The seed-syllable $A h$ in the rainbow-encircled nimbuses symbolises the mind's primordial 'alpha' nature. ${ }^{\odot}$ Ian Baker

would change not only current understanding of the history of postural yoga, but would also clearly identify the existence of $r$ tsa rlung and 'khrul 'khor practices in Tibet prior to their introduction through the Kagyu and Sakya lineages during the second diffusion of Vajrayāna that began in the 11th century. In any event, the complexity and diversity of Yantra Yoga ('khrul'khor) in Tibetan Buddhism suggests that, like Tibetan medicine, it was an eclectic and syncretic tradition drawing on methods and practices derived from multiple cultural sources. ${ }^{52}$

Pema Lingpa's 'Secret Key to the Winds and Channels' includes many of the same movements described in Vairocana's 'Union of Sun and Moon'. It also bears strong similarities to the 'khrul 'khor practices promulgated by Pema Lingpa's preceding incarnation, Longchen Rabjampa (Klong chen rab 'byams pa) (1308-64), as well as those

turn, had received it from a Nepalese mahāsiddha named Humkara who had himself learned it from the great adept Shri Simha, an early Dzogchen lineage-holder who had spent considerable time at Mount Wutai in China. The esoteric movements of 'khrul 'khor as practised in Dzogchen resemble not only Indian hatha yoga but also Taoist-Chan Buddhist methods of Qigong that were current at Mount Wutai within The East Mountain Teachings. Further research may thus indicate syncretic origins for Tibet's rtsa rlung and 'khrul 'khor practices. For elucidation of 'khrul 'khor within Tibet's Bon tradition, see Wangyal 2011; Chaoul-Reich 2006.

See Baker 2017 and the forthcoming Tibetan Yoga: Secrets from the Source. codified in the 'Heart-essence of Infinite Expanse' (Klong chen snying thig), a mind treasure revealed by the scholar and adept Jigme Lingpa (1730-98). ${ }^{53}$ As all traditions of Dzogchen maintain, the practice of rtsa rlung and 'khrul 'khor can result in improved health and well-being and prepare the body for inner tantric practices such as gtum mo, but their primary function within Dzogchen is to coordinate the body's psychosomatic winds (rlung, Skt: prāna) so that the nature of awareness becomes ever more manifest and embodied through the Dzogchen contemplative techniques of thod rgal, or 'leaping over the skull' (Figs 2, 10 and 12).

\section{The Body of Light}

'The Secret Key to the Channels and Winds' presents the 'khrul'khor exercises as preparations for the contemplative practices of lhun grub thod rgal, or 'leaping over the skull into spontaneous presence, ${ }^{54}$ which include prescribed body postures, breathing techniques, and associated gazes (Skt: $d r s ̦ t i$ ) for shifting consciousness from left- to right-brain activity, correlated with unitary awareness and the dissolution of distinctions between subject and object. Using the support of sunrays and the sky, awareness expands from restricted egocentric consciousness to a self-transcendent totality and the spontaneous display of what is held to be intrinsic non-dual reality (see Figs 2 and 12). The physical body is upheld as the vehicle by which awareness eventually expands to encompass all dimensions of being, described in Vajrayāna as the triune 'bodies' (sku gsum, Skt: trikāya) of Dharmakāya, Sambhogakāya, and Nirmānakāya, referring respectively to infinite undifferentiated totality, intermediate visionary experience, and manifest temporal reality.

Specific body postures and gazes are associated with each of the three kāyas, and the practitioner may freely shift between them during a single session of contemplation while maintaining 'three-fold motionlessness of body, eyes, and consciousness'. As further described in Pema Lingpa's treasure text, the Dharmakayya posture of a seated lion involves squatting on the ground with the soles of the feet together and the hands resting on the ground in front in vajra fists (with the tips of the thumbs touching the base of the ring finger). The upper body is extended upwards with the chin tucked slightly in and the spine and back of

53 For an updated history of 'khrul 'khor in Vajrayāna and Tibetan history, see Baker 2017.

54 See Namdak and Dixey 2002; Chagme 200o; Baker 2011, pp. 11621. 

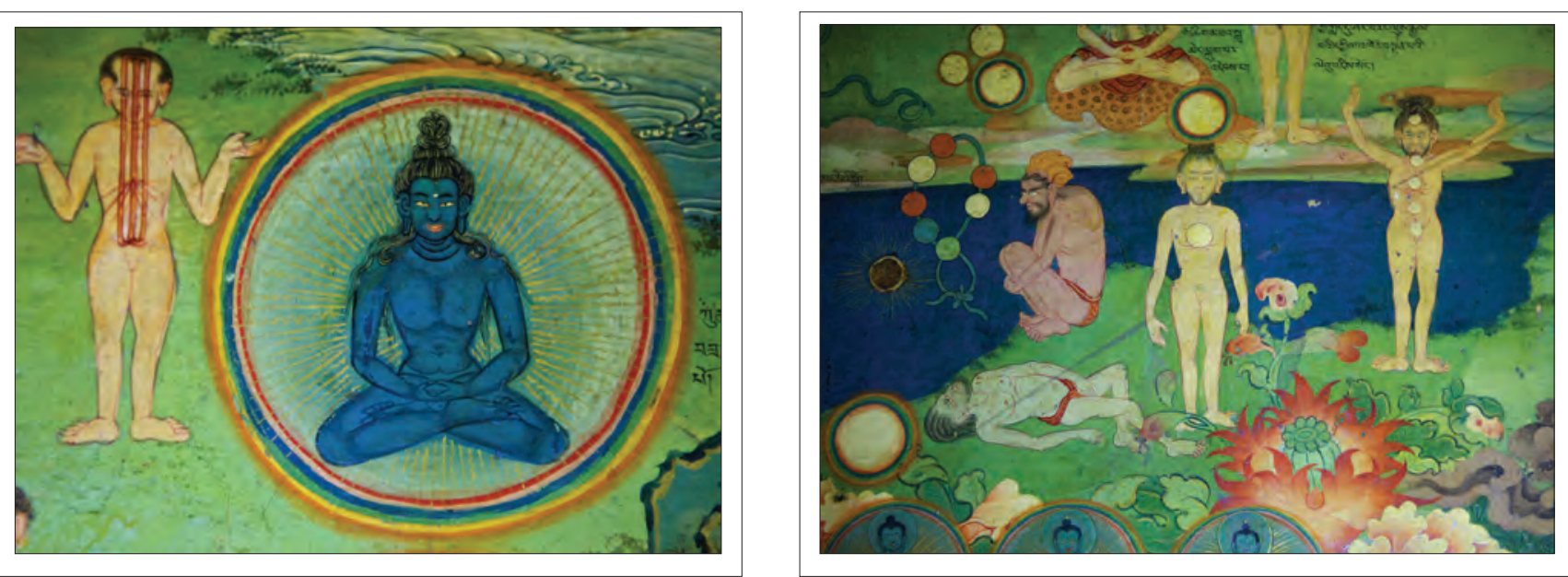

Figures 29.11-12 The images in the Lukhang murals represent processes of physical and psychological illumination in which the practitioner of Dzogchen awakens to his or her indwelling Buddha Nature, experienced as a transpersonal dimension of bliss, clarity, and compassion. The paintings, completed at the turn of the 17th century, preserved a deeply embodied and assessable form of spiritual practice, independent of Tibet's monastic and ecclesiastical traditions. ${ }^{\circ}$ Ian Baker

the neck straightened so as to allow the free flow of vital energies through the cranial arteries and associated light channels' connecting the heart and eyes. With the breath extended outward through gently parted teeth and lips, the eyes are rolled upwards past an imagined protuberance at the crown of the head into the limitless expanse of sky.55

In the Sambhogakayya posture of a reposing elephant (Fig. 10), one lies down with the knees tucked beneath one's chest, toes extended behind, elbows on the ground and hands placed either in front as vajra fists or cradling the chin as the spine elongates and the eyes gaze to the sides into pure visions reflecting the innate activity of consciousness and perception. In the Nirmānakāya posture of the sage (rși) (see Figs 10 and 12), one squats with one's ankles together, knees pressed against the chest, and one's arms crossed in front, either with hands resting in the armpits or hugging the knees. The gaze is directed slightly downward through half-closed eyes to control the body's vital energies and bring the mind to stillness. As Pema Lingpa's text maintains, pristine awareness 'dwells like a lamp in a vase at the heart' of the physical body but only manifests as direct experience by maintaining the key points of posture, 'just as the limbs of a snake only become apparent when it is squeezed'.

The physical postures, suspended breath, and raised, lowered and sidelong gazes used in the Dzogchen practice of thod rgal serve as 'doors' to the direct experience of the multi-dimensionality of being beyond the single dualistic dimension to which human beings are habitually con-

This gaze correlates with śāmbhavimudrā in hațha yoga, which is said to synchronise the two hemispheres of the brain and lead directly to samādhi. See Birch 2011. strained. The indwelling pristine awareness that liberates consciousness is posited as the innermost essence of the heart (snying thig), and all methods of Dzogchen are directed towards making this a lived reality (see Fig. 11). The innate radiance (lhun gyis grub pa) of the heart-centre is held to manifest first as flickering and darting gossamer strands and later as spontaneously present spheres of light through a subtle channel that, like a white silken thread and 'far-ranging noose' connects the heart and eyes. The thig le, or subtle essences, appear in the empty mirror of the sky as reflections of innate reality and develop through four sequential visions. ${ }^{56}$ The halos of light ultimately divide and transform into five concentric circles, the central one symbolising pure unmanifest potentiality. As the circle widens through steady gaze, it eventually pervades immeasurable space as distinctions between subject and object, mind and body, vanish in the luminosity of enlightenment. ${ }^{57}$ As Pema Lingpa's revealed treasure text illustrated on the walls of the Lukhang proclaims:

Through the unmediated perception of reality, conceptual analysis naturally subsides... . Free of conflicting views, all things arise as intrinsic Luminosity... . As tainted aggregates dissolve, the body itself is naturally Enlightened (illuminated)!

$5^{6}$ The 'Four Visions' of thod rgal are described as the Direct Perception of the Ultimate Nature, the Vision of Increasing Experience, the Perfection of Intrinsic Awareness, and the Dissolution of [phenomena into] the Ultimate Nature. The visions are said to arise from the heart as reflections of the body's purified channels, energies and subtle essences. For further details, see Baker 2011 and Baker 2017.

57 This process echoes Matthew 6:22: 'Therefore when thy eye be single, thy whole body will be full of light'. 


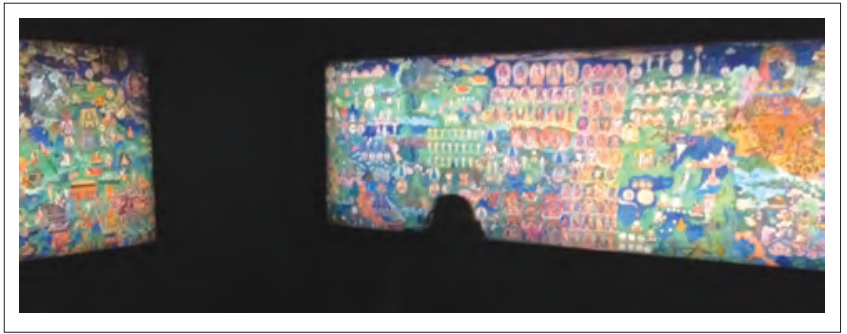

Figure 29.13 A 2015-16 exhibition at London's Wellcome Collection entitled 'Tibet's Secret Temple: Body, Mind and Meditation in Tantric Buddhism' featured life-sized facsimiles of the Lukhang murals, bringing to public view images that were once meant only for the eyes of Tibet's Dalai Lamas. Merging contemporary light technology with the murals' avant-garde depictions of human potential, the exhibition offered visitors both an educational and contemplative experience. $\odot$ Ian Baker

\section{Conclusion}

The Lukhang murals illustrating Rigdzin Pema Lingpa's treasure text 'Compendium of Enlightened Spontaneity' vividly reveal the essential role of the physical body in cultivating the integral states of awareness associated with Dzogchen, or Ati Yoga. From foundational practices that push body and mind to unaccustomed and instructive extremes to hatha yoga exercises for awakening the body's 'inner mandala' (nang pa'i dkyil 'khor) of subtle channels and vital energies, to the prescribed body postures for experiencing the visionary display of the body-mind's innermost essence (snying thig), Dzogchen is permeated by pro-somatic practices that lead to integrated, self-transcendent dimensions of being, held to represent the highest capacities of the human mind and body. Pema Lingpa's 'Secret Key', as illuminated on the walls of the Lukhang, offers an invaluable visual guide and inspiring artistic presentation of yogic practices formerly kept secret within initiatic lineages of Tibetan Buddhism. Transcending their historical context, the murals invite continued reflection on the intersection of physical and psychological disciplines in promoting human flourishing, and reveal the diverse ways that the human condition can be optimised through systematic physical cultivation concurrent with liberating reorientations of consciousness.

As visual culture, the Lukhang murals' fluent depictions of mind-body practices at the heart of the Tibetan Buddhist tradition reflect the words of Wassily Kandinsky, who wrote in 1911 in 'Concerning the Spiritual in Art' that:

Each period of culture produces an art of its own that can never be repeated... directed to the improvement and refinement of the human spirit. ${ }^{58}$
Analogously, Desi Sangye Gyatso, Tibet's visionary ruler at the turn of the 17th century who conceived of 'translating' Pema Lingpa's revealed treasure text into wall paintings in the private quarters of the Sixth Dalai Lama, achieved the 'unprecedented' by both preserving and interpreting an endangered body of cultural knowledge in artistic form. He simultaneously legitimised his choice of Tsangyang Gyatso as the Sixth Dalai Lama through a visual record of the transmission of teachings that Pema Lingpa revealed and to which Tsangyang Gyatso was ancestrally connected.

Beyond the cultural, doctrinal, and political concerns that contributed to their original production, the wall paintings in the Lukhang have been re-envisioned in the 21st century in ways that expand on the murals' innovative means of representing knowledge. In 2015, life-size digital facsimiles of the murals formed the core of an exhibition at London's Wellcome Collection entitled 'Tibet's Secret Temple: Body, Mind and Meditation in Tantric Buddhism' that transformed the murals' once highly esoteric subject matter into a public educational resource. Back-lit by panels of LED lights, the digitalised and illuminated facsimiles of the murals revealed not only an alternate vision of the human condition and its possibilities, as imagined in $17^{\text {th }}$ century Tibet, but the ways in which science, art, and technology can dynamically combine in the 21st century towards a renewed aesthetics of transcendent experience. The exhibition also consciously reflected on its own breaching of norms in popularising 'secret images' connected with a living esoteric tradition. This decision was guided by the published words of the current Fourteenth Dalai Lama who, in specific reference to the Lukhang murals, stated that 'the time of secrecy is over'. 59

Beyond their original initiatory context, the images nonetheless maintained an analogous function in challenging unexamined habits of perception and, as religiously inspired art, inviting transcultural reflections on the sources of human meaning and wellbeing. What was gained and what was lost in moving the murals from the domain of the sacred to that of the resolutely secular is perhaps best understood within the Great Perfection's own internal commitment to reconciling false dichotomies. As Padmasambhava, the reputed source of the teachings that the murals illustrate, is said to have stated: 'Samsāra and Nirvāna have no difference other than being unaware or aware' of the intrinsically undivided nature of reality. ${ }^{60} \mathrm{In}$ other words, from the non-dual perspective of Dzogchen, enlightenment consists in recognising the unitary na-

See H.H. the Dalai Lama's 'Introduction' to The Dalai Lamas Secret Temple: Tantric Wall Paintings from Tibet (Baker 2011).

6o See p. 414 above, for the full quotation. 
ture of all experience, beyond the dualising tendencies of thought and perception. It is surely such coalescent insight that inspired Desi Sangye Gyatso to envision and commission the Lukhang murals. It may also have been this view, applied too personally and out of context, that led to the Desi's beheading - a reminder that, as in its Western sense a century later, the movement towards perfection, or Enlightenment, however variously enacted, is not without hazard. ${ }^{61}$

\section{Appendix}

\section{'The Secret Key to the Channels and Winds' (Rtsa Rlung Gsang Ba'i Lde Mig)}

A Chapter from Pema Lingpa's Compendium of Enlightened Spontaneity (Rdzogs chen kun bzang dgongs'dus). ${ }^{62}$

Homage to Innate Enlightenment, Samantabhadra (kun tu bzang po)!63

I, the Powerful Skull-Garlanded Lotus (Rdo rje thod phreng rtsal) ${ }^{64}$ have composed this 'Secret Key' to elucidate the [nature of the] Inner Winds (rlung, Skt: prāna) to help practitioners to open their subtle channels, balance the three humours of wind, bile and phlegm, and bring wholeness to body and mind.

In an isolated place free from disturbances, abide in the non-conceptual space of 'leaping over the skull' (thod rgal) and generate boundless love and compassion for all beings. ${ }^{65}$

61 Enlightenment, in its Western sense, connotes the cultural ascendency of reason and intellect over faith and tradition. See $n$. 34 concerning the circumstances of Desi Sangye Gyato's murder.

$62 \quad$ KZGD, vol. 2, pp. 453-6o.

63 The primordial purity and self-liberated nature of human consciousness is symbolised in Dzogchen as the Buddha Samantabhadra, literally 'All Pervading Goodness'. The male polarity signifies supreme compassion while the female polarity represents all-encompassing wisdom.

64 A title of Padmasambhava, the 8th-century Buddhist master credited with having established Vajrayāna, or Tantric Buddhism in Tibet, and the reputed author of the 'Secret Key'.

65 Pema Lingpa presents the yogic exercises of channels and winds (rtsa rlung 'phrul 'khor) as preparations for the Dzogchen practices of ka dag khregs chod and lhun grub thod rgal, 'cutting through to primordial purity' and 'leaping over the skull', the latter of which relies upon subtle channels connecting the heart and eyes. Although khregs chod commonly precedes the practice of thod rgal, early Nyingma expositions of Nyingtik, or 'Heart Essence, often present thog rgal first. As with all practices of Vajrayāna Buddhism they are framed by the altruistic Mahāyāna intent to achieve enlightenment on behalf of all beings.

\section{The Yogic Movements [for Clearing Hindrances $]^{66}$}

First, expel the stale winds three times [from the two side channels]. ${ }^{67}$ Then rub the palms of your hands together and massage the left and right sides of the body.

Second, turn the feet outward and alternate turning them to the left and right.

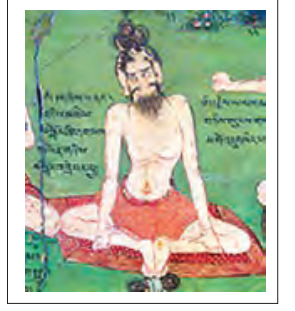

Third, apply [sesame] oil [to the body] and shake.

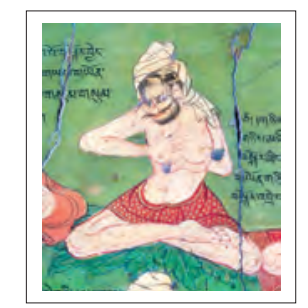

Fourth, churn the abdomen three times to the left and three times to the right. ${ }^{68}$

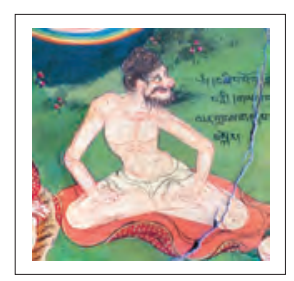

66 Pema Lingpa's text is illustrated below with relevant images from the paintings on the northern wall of the Lukhang temple. See Baker 2017 for a detailed history of 'magical movements' within the Tibetan Buddhist tradition. For a description of an elaborate system of 'phrul 'khor attributed to Vairocana, a contemporary of Padmasambhava, see Norbu 2008. See also Wangyal 2011 and publications by Chaoul-Reich for further explication of 'khrul 'khor within the Tibetan Bon tradition. The word 'magical' refers to the unusual psychological effects that the movements have on the mind.

67 Similar to methods of nādi shodana in hatha yoga, the stagnant winds are cleared through forceful exhalation through alternate nostrils while the other nostril is held closed with the thumb or ring finger. Emptying the two lateral channels concentrates energy and awareness in the body's central meridian.

68 Unlike uddiyāna bandha and nauli in hatha yoga, the abdomen is not pulled in towards the spine. 
Fifth, as if pushing a vajra, hold the breath [in the lower abdomen] and firmly press out both arms.

Sixth, like throwing a rock, fling the left and right arms outwards three times. ${ }^{69}$
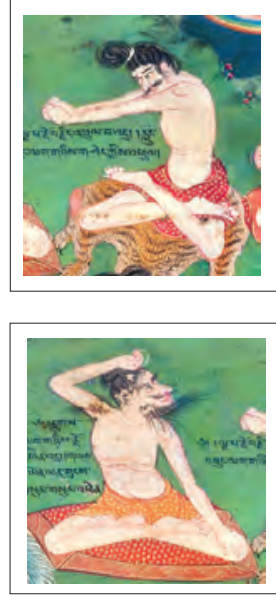

Seventh, rotate the wrists inwards and outwards three times each.
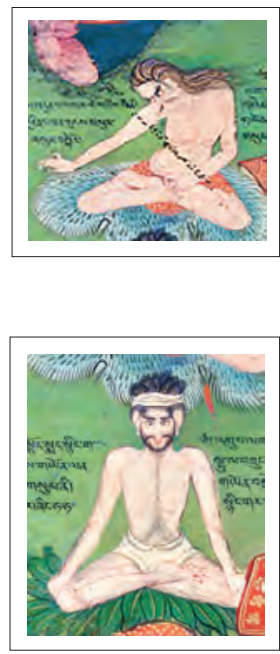

Eighth, while holding the breath [in the lower abdomen], rotate the entire body and the heart-centre three times to the left and right and exclaim $\mathrm{Ha} \mathrm{Ha}$.

Ninth, with hands [crossed in front and] holding the upper arms rotate to the left and right and then strike the heart-centre.

Tenth, while holding the breath [in the lower abdomen], rotate the left and right shoulders in and out three times each [with the arms straight and the fists pressing against the thighs].

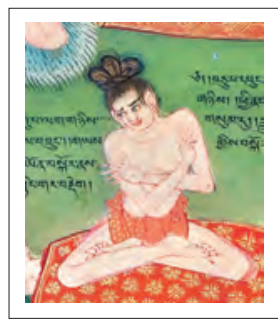

Eleventh, strongly strike your fists nine times against your navel.

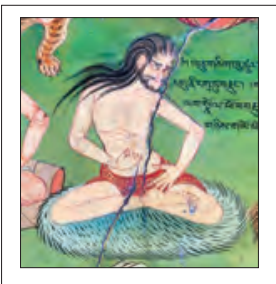

Twelfth, join the palms at the heart-centre and alternate striking your elbows against the sides of the ribs and then bang both [elbows against the body] simultaneously.

Thirteenth, interlacing your fingers, push back against the back of the neck and rotate [the head] three times to the left and three times to the right.

Fourteenth, like drawing a bow, pull three times to the left and three times to the right and, while crossing the arms, strike the point of the shoulder. ${ }^{70}$

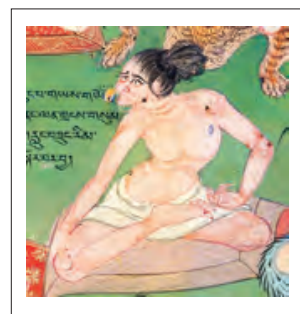

Fifteenth, rotate the head and eyes three times to the right and three times to the left. Then stretch the head nine times to the back and front, and nine times from side to side. Then shaking [the head] nine times, expel
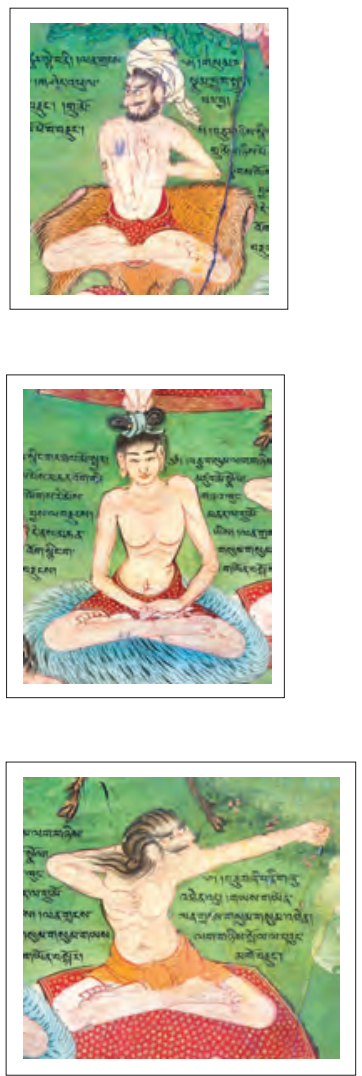
all stagnant air.

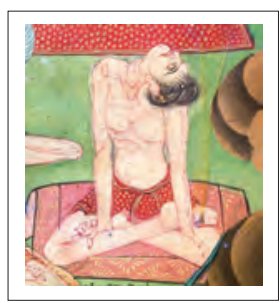

69 In traditional Chinese medicine this movement has the reputed benefit of opening the lung and heart meridians.
This movement is said to unblock and open the heart-centre, freeing it from stagnant 'wind' (rlung) and unproductive mental states. 
Sixteenth, the 'Lion's Frolic': Seal the eyes with the two index fingers, block the ears with the two thumbs, block the nostrils with the middle fingers and, with the small and ring fingers, seal the lips. ${ }^{71}$ Rotate the head three times to the left and three times to the right. Then shake [the upper body] three times [while exhaling].

Seventeenth, while standing up, shake the legs to the back and front and from left to right. Turn the arms to the left and right and shake them to the front.

Eighteenth, bend forward and touch the hands to the ground. Standing up, repeat nine times.

Nineteenth, while standing up strike the heels [on the ground] then, rising up, jump. Then alternate shaking the arms and legs. ${ }^{72}$

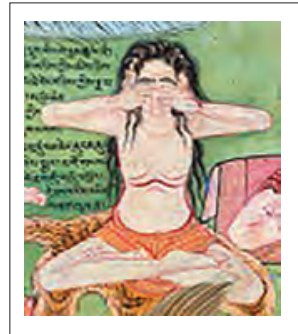

Twentieth, sit in [full lotus] vajra posture and lift up the body [with the hands pressing against the ground], then drop the body against the floor. Do this three times, shaking the body into wakefulness. ${ }^{73}$

Twenty-first, while lying back, hold the breath [in the lower abdomen] and lift your body up three times.
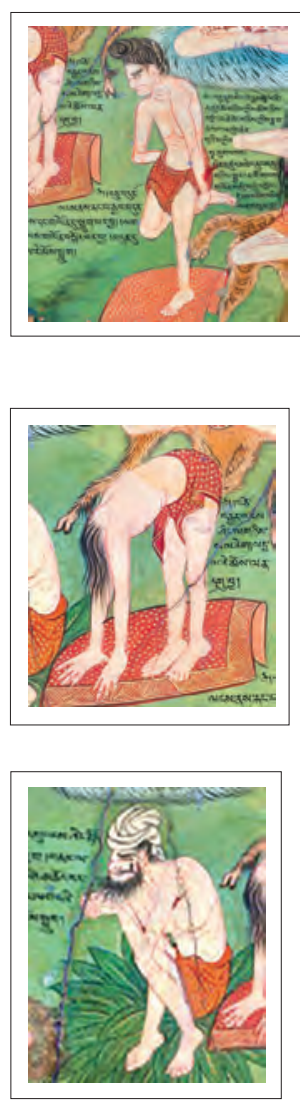

Twenty-third, sit comfortably in the seven-point posture of Vairocana. ${ }^{74}$

Twenty-second, bend forward and touch the forehead to the ground. Then rolling back, touch the toes to the ground. Shake the body and utter a strong Ha three times.
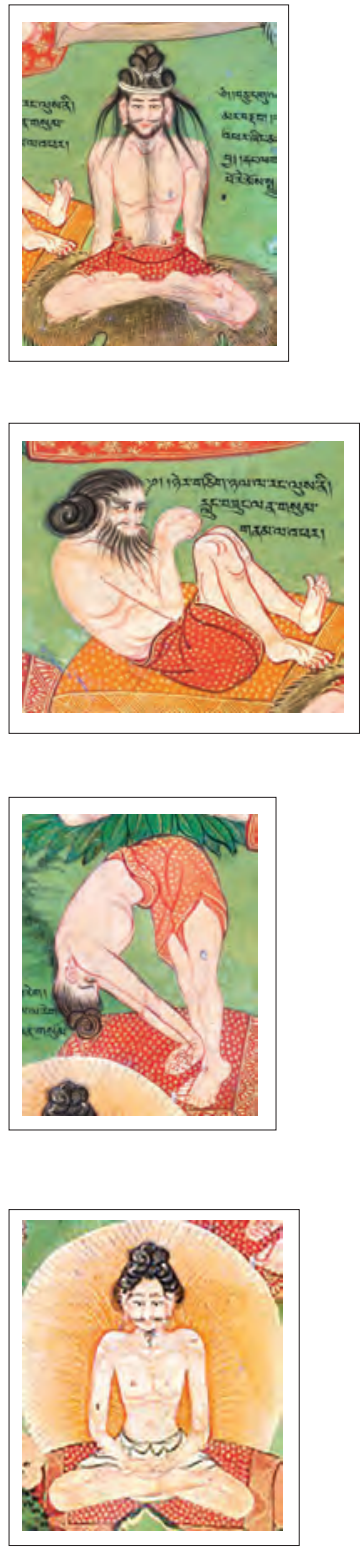

I, Pema [Padmasambhava], wrote down the [descriptions of these] 23 secret yogic movements. I pray to my heart son [Pema Lingpa] that they will benefit all beings.

This completes the first section, the stage of 'khrul 'khor [the yogic movements].

Samaya. Sealed! Sealed! Sealed!
This method of closing the doors to the senses is described in Patañjali's Yoga Sūtras as pratyahara (so sor sdud pa), signifying the inward withdrawal of the senses towards progressively deeper states of unitary awareness. Following an alternate spelling, the exercise is sometimes alternatively translated as 'Lion's Liberation'.

This description appears to be shorthand for the controlled falls (bep) used in Yantra yoga ('khrul 'khor) to concentrate vital energy in the central channel and to stimulate the awakening of gtum mo, or 'fierce heat' (see Figs 9 and 14).
73 This is a version of mahāvedha, a hața yoga exercise described in the corpus of 11th- to 12th-century texts entitled Amrtasiddhi, or 'Attainment of Immortality' ( $:$ : bDud rtsi grub pa). See Mallinson 2012 and Baker 2017.

74 The seven points consist of the legs in full lotus position, the spine erect, the shoulders broadened, the neck bent slightly forward, the hands in the gesture of equanimity (with the right hand resting on the left palm), the tip of the tongue touching the palate, and the gaze directed beyond the tip of the nose. 


\section{The Channels of the Human Body ${ }^{75}$}

In the crown chakra of great bliss

There are 360 channel petals;

In the throat chakra of gathering taste

There are 16 channel petals;

In the heart-centre, the dharma chakra

There are eight channel petals;

In the navel chakra of emanation;

There are 64 channel petals;

In the secret centre, the chakra of preserving bliss ${ }^{76}$

There are 28 channel petals.

The entire body contains 21,00o channel petals.

The three principal channels are:

The uma, roma, and kyangma.77

The presentation of the subtle energy channels ( $r t s a$, Skt: $n \bar{a} d i)$ in Tibetan Buddhism derives from Indian sources and varies in its enumeration of the number and location of specific channels and energy plexuses ('khorlo, Skt: cakra). The Tantras and Tibetan medical systems generally describe 72 ,0oo such channels within the human body that allow for the flow of awareness throughout the physical organism. The hollow, tubular ducts are described as symbolising the unlimited space through which consciousness can express itself, with the thig le, or psychophysical essences, representing the base for all appearance and manifestation. Pema Lingpa's overview of the subtle body focuses on three principal energy channels that parallel the spine and which, along with five associated energy plexuses (cakra), form the basis for the completion stage practices of Anu Yoga (rjes su rnal'byor) (see Figs 11 and 15).

This refers to the root cakra in the genital region that serves as a reservoir of bliss so long as the relative thig le, or sexual fluids, are retained and not lost through ejaculation, especially within the context of dual-cultivation with a consort (las kyi phyag rgya, Skt: karmamudrā).

The three fundamental channels rtsa dbu ma, ro ma, and rkyang ma differ in fundamental ways from the sushumna, ida, and pingala nādi described in hațha yoga (see Figs 11 and 15). In the Dzogchen view of embryology, the navel cakra develops first, followed by the crown cakra as a result of a connecting life-channel' (srog rtsa) that further differentiates into the spinal cord and spine, yet remains as the unifying energy of the rtsa dbu ma (Skt: avadhūti) central meridian at the core of tantric yoga. Four finger-widths below the navel, the dbuma intersects with two ascending lateral channels called ro ma and rkyang ma. The ro $m a$ is visualised as white in colour and as corresponding to lunar energy. It is said to lie on the right side of the body in men and on the left side in women. Ro, in Tibetan, means 'flavour', and the principal function of the ro $m a$ is to provide the sensation of pleasure. The ro $m a$ is thus further correlated with the tantric principle of thabs lam, or method. The rkyang ma is connected with the principle of ' $\mathrm{grol}$ lam, or liberation and is visualised as red and as corresponding to solar energy. It lies on the left side of the body in men and on the right side in women. Rkyang means 'sole', and unlike the ro ma, the rkyang ma is not connected with any secondary channels. Control of the energies that flow through the rkyang ma is considered fundamental for develop-
Their inner space is erect like pillars.

Their upper ends appear like the horns of the bamen ['wild ox']. Their lower ends are curved like the Tibetan letter cha.

Uma, the channel of the wisdom flow,

Has three different names: ${ }^{78}$

Outwardly it is known as Uma [the 'central'], inwardly as Kundarma [the 'all-encompassing'], and secretly as Shel Bugchen, [the 'great crystalline tube']. ${ }^{79}$

It is empty and transparent and free of blood and fluids. Along its length are four principal channel wheels [cakra] through which Awareness Wisdom (rig pa'i ye she) ${ }^{80}$ flows unimpeded. Primordial Awareness (rig pa) is supported, in turn, by a luminous energy sphere [located at the heart cakra]. The 21,0oo channel petals [of the subtle body] connect to 21,00o internal winds that differentiate the pure and impure essences (thig le) of the body.

This completes the section on the subtle channels $(r t s a)$ of the physical body.

Samaya. Sealed! Sealed! Sealed!

\section{Igniting Blissful Heat to Enhance Awareness ${ }^{81}$}

Expel the stale airs three times [from each nostril] and inhale in an instant the essence of the wisdom präna from the tip of

ing insight into emptiness as the energetic basis for all manifestation. The goal of all practices connected to the channels and winds (rtsa rlung) can be generalised as the unification of the energies of the body's two lateral channels into the wisdom flow of the central meridian (rtsa dbu ma) that runs from the base of the spine to the crown of the head, an energetic current possibly related in human anatomy to the prenatal notochord.

78 The three principle channels further correlate with Dzogchen's presentation of the triune reality of the ground of being (kun gzhi). The Essence or Pure Potentiality (ngo stong pa) relates to the avadhüti itself, the Luminous Nature (rang bzhin gsal ba) to the ro $m a$, and the unceasing Energy of Manifestation (thugs rje kun khyab) to the rkyang ma.

79 This microscopic and translucent channel is described in Dzogchen as originating in the innermost centre of the heart cakra and rising up through the central channel into the brain where it bifurcates and arcs forward to terminate at the centre of the pupils of both eyes. In distinction to the channels that circulate prāna, or vital energy, the crystalline kati channel is described in oral tradition as a 'light channel' that connects the Clear Light wisdom located in the heart cakra with the Clear Light awareness 'seated' in the brain. As with all practices of Dzogchen, it remains 'secret' until manifested through direct experience.

$80 \quad$ The Tibetan word rig pa generally means 'awareness' or 'knowledge'. In Dzogchen, it further refers to abiding in the mind's essential nature as distinct from ordinary mental processes. The word ye shes generally translates as 'primordial wisdom' and is an intrinsic feature of enlightened consciousness.

81 This section describes the technique of gtum mo, literally 'fierce female energy', which corresponds with the Indian system of 
the nose and swallow the saliva. Pull up the lower winds [by gently contracting the pelvic floor] and [while pressing down with the upper winds] hold the energy at the navel centre [by applying the 'Vase Breath' (bum chen, Skt: kumbhaka]. Then gradually perform the 'khrul'khor [the 23 yogic movements].

[Again] expel the stale winds three times [from the side channels]. Then gently unite the lower and upper winds [at a point four finger widths below the navel] by applying jam rlung [exhaling and inhaling softly and completely with a gentle breath retention]. Next, visualise the central channel (rtsa dbuma) as ascending from the secret centre [genitals] to the crown [of the head], empty and unimpeded, the size of a bamboo arrow, bluish white and free of blood, lymph, or any fluids. ${ }^{82}$ At the navel cakra, within the central channel, is a red four-petalled lotus. In its centre is the syllable Yam and, on top of it, a red Ram. ${ }^{83}$ Wind stirs from the Yam and strikes the Ram, igniting a fire that rises [within the central channel]. When the flame touches the white [inverted] Ham at the crown cakra, it causes the red and white essences (thig le) to descend. When they reach the Ram at the navel cakra the inner fire blazes and incinerates all habitual [and limiting] tendencies [of the mind]. While meditating on the expanding bliss, imagine that you are receiving the third empowerment ${ }^{84}$ that [activates the channels and winds and] awakens the innate heat and wisdom [of the umbilical centre].

canḍāầ or kuṇdalinī yoga. In the context of Vajrayāna Buddhism the incandescent bliss that rises through the practice is not an end in itself but 'expedient means' (thabs lam, Skt: upāya) for recognising the true nature of the mind and thus achieving liberation from conditioned states, including attachment to bliss itself. All physical methods in Dzogchen can be divided into either bogs dun or bgegs sel, practices that either enhance the realisation of non-dual wakefulness (such as gtum mo) or dispel hindrances (such as 'khrul'khor).

82 See n. 78 for discussion of the role of the central channel in tantra and Dzogchen. For the practice of gtum mo there are two main bodily positions. In the first, the practitioner sits crosslegged with the arms held along the sides of the body with the hands turned upward and, with thumbs connected to the roots of the ring fingers, pressed against the insides of the thighs as 'vajra fists'. In the second position, one sits with the legs raised in front with arms crossed and the elbows resting on the tops of the knees to simulate a 'stove', often supported by a meditation belt.

$83 \quad$ Yam and Ram are seed-syllables (yi ge) signifying air and fire. Ham, visualised in Pema Lingpa's presentation of gtum mo as located at the crown of the head, represents water, or the dimension of fluidity. In other contexts, this practice is also performed by visualising a short blazing $A h$ four finger-widths' beneath the navel that, in turn, causes the 'white element' at the crown of the head to melt and drip down through the central channel.

84 Vajrayāna practice is normally connected to receiving four successive tantric empowerments (dbang bzhi) that prepare the mind and body for the experiences that arise through the practices of the Inner Tantras. The third empowerment of 'knowledge-wisdom' (shes rab ye shes kyi dbang) authorises the practitioner to meditate on the union of bliss and emptiness aroused by the Anu Yoga practices of gtum mo, rtsa rlung, and
This completes the third section of meditating on Fierce Heat (gtum mo) in the evening. ${ }^{85}$

Samaya. Sealed! Sealed! Sealed!

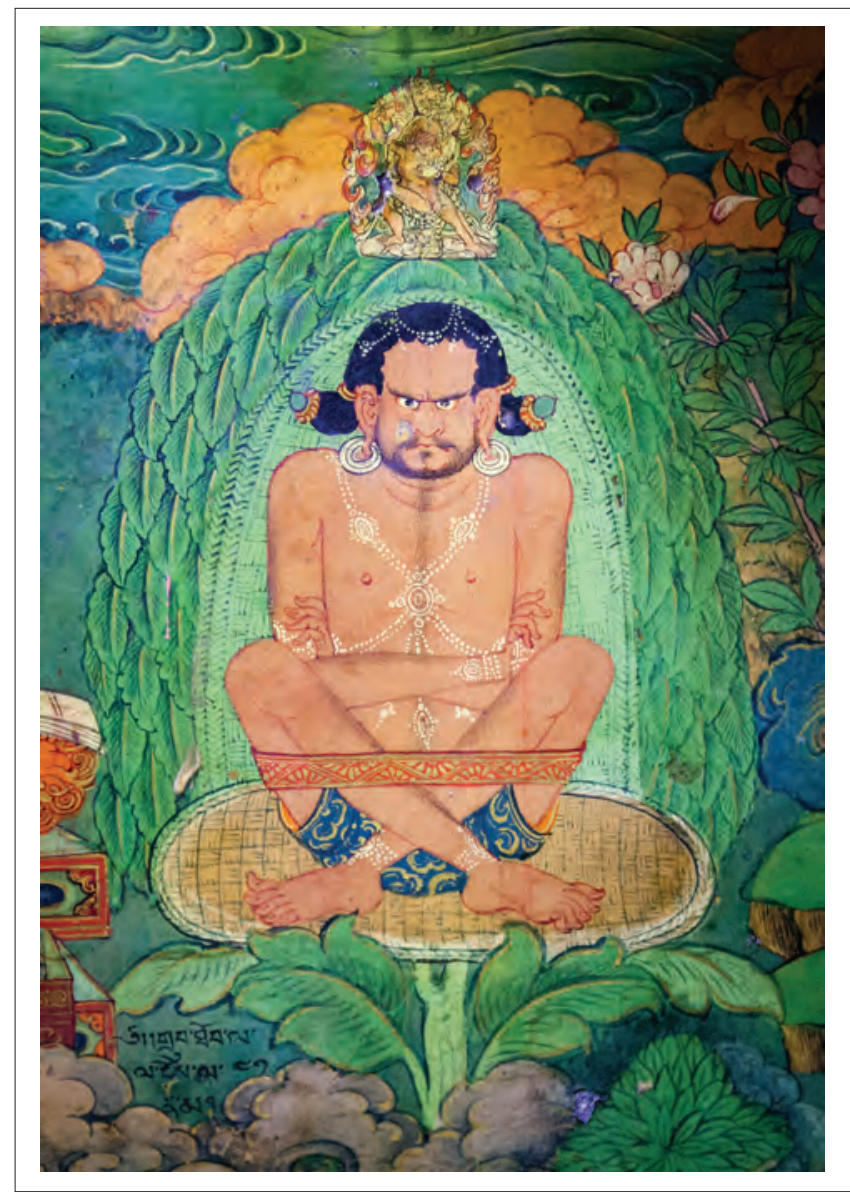

Figure 29.14 A mahāsiddha, or realised adept, pictured on the Lukhang murals sits in a posture associated with the practice of Fierce Heat (gtum mo), as described in Pema Lingpa's revealed treatise, 'Secret Key to the Channels and Winds'. 이 Ian Baker

\section{Entering Consciousness into the Vase at Night $^{86}$}

[For the practice of sleep] lie on your right side and meditate that the central channel (dbuma) is the size of an arrow and empty and transparent and free of blood and fluids. Primordial Awareness (rig pa) abides as a white $\mathrm{Hum}^{87}$ at

\section{karmamudrā.}

85 When gtum mo is combined with other Anu Yoga practices, it is conventionally performed in the evening; otherwise it may be practised at any time of day or night.

86 'Vase' refers, in this context, to the containing of the senses as one falls asleep. The vase into which the sensory functions are withdrawn can refer to either the heart cakra or the navel cakra.

87 The seed-syllable Hum refers in Vajrayāna Buddhism to the innate wisdom mind while, in Dzogchen, the seed-syllable $A h$ represents the mind's primordial nature (Logos). While the syllable Hum is illustrated on the western mural in the context of 
the navel cakra, in the centre of a four-petalled lotus. The Hum is unmoving, clear, and brilliant. Contemplate that all the coarse and subtle thoughts that ride on the vehicle of the breath dissolve into the upward-facing Hum. When thoughts arise, concentrate on the Hum at the navel cakra.

Following this, at the point of sleep, imagine your Primordial Awareness as a radiant white Hum blossoming in your heart cakra at the centre of a four-petalled lotus. Meditate that all thoughts [and sensations] dissolve into this luminous Hum. Join the lower and upper winds at your heart cakra. The Hum shines within the heart like a lamp inside a vase. Meditate on clarity and emptiness free of grasping and your sleep will naturally arise as unobstructed Luminosity. Meditate in this way again and again and the movement of outer and inner thoughts will dissolve into [all-pervasive] Clear Light. This completes the fourth section of entering the objects of consciousness into the vase.

Samaya. Sealed! Sealed! Sealed!

\section{Resting Awareness in its Natural State in the Early Morning}

At the moment of waking, sit in the rși posture ${ }^{88}$ and expel the stale winds three times [from both nostrils]. Then, release the two winds [that were joined] at your heart-centre and [while shouting $\mathrm{Ha}$ into the sky] project the Hum through the crown of your head to a bow's length above and in front. Focus your Awareness and internal energy (rlung, Skt: prāna) on the Hum [in the sky above you] while holding a gentle 'vase breath'. ${ }^{89}$ Meditate undistractedly in a state of naturalness, giving rise to non-conceptual wisdom. Allow the Hum to naturally dissolve [into infinite space] and remain free of all conceptual activity.

When the sun is shining focus [your attention] on [the naturally arising visions of ] thod rgal. ${ }^{90}$

the preliminary Dzogchen practices, $A h$ is pictured far more frequently and often substitutes for instances in which Pema Lingpa's text specifies the visualisation of a Hum. In any case, the practice described here, 'Retaining Wakefulness in the Vase', is often done while visualising an $A h$ at the centre of the heart which lights up the interior of the body and radiates beyond it, leading to 'capturing Luminosity during deep sleep'.

88 One of three positions emphasised for the practice of thod rgal, the rssi posture consists of sitting with an erect spine with the legs pulled up in front and the arms either around or resting on top of the knees. This practice is also commonly done while crouched in the 'lion's posture' (see Figs 10 and 12).

89 In the practice of thod rgal, breathing occurs almost imperceptibly through the mouth without any manipulation of the prāna. The out-breath is naturally longer with a gentle holding of the empty breath before inhaling. In Anu Yoga, prāna is controlled so as to control the mind. In Ati Yoga (Dzogchen) the mind is controlled so as to bring prāna naturally into balance.

The visions of thod rgal can be compared with so-called blue field entoptic phenomena identified in 1924 by the German ophthalmologist Richard Scheerer and described as the appearance
Spend your days and nights united with ultimate reality.

An instruction of this type cannot be found [elsewhere] in Tibet or India.

This sacred heart blood of me, Pema [Padmasambhava], may it meet with my destined heart son [Pema Lingpa].

Samaya, Sealed! Sealed! Sealed!

The light rays of Orgyen Pema's [Padmasambhava's] compassion

Opened the lotus heart of me, Pema [Lingpa and revealed]

The supreme path for accomplishing stainless Buddha [Nature].

For the benefit of qualified practitioners

The light rays of the white conch scrolls shone forth,

And were written down from the yellow parchment [shog gser].

May these teachings spread throughout the world!

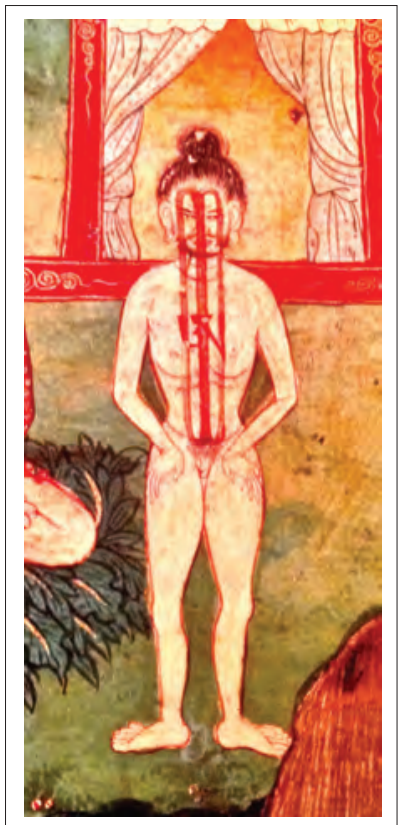

Figure 29.15 This detail from the Lukhang murals shows the three principal energy channels cultivated in Tibetan yoga. The syllable $A h$ at the figure's heart centre signifies the state of primordial awareness (rig pa. Skt. vidya). ${ }^{\odot}$ Ian Baker

of bright dots flowing along wave-like lines in the visual field when looking into bright blue light, such as the sky. Ophthalmologically, the dots appear as a result of leukocytes, or white blood cells, flowing through the macular, retinal capillaries of the eyes in synchrony with the cardiac cycle between the beginning of one heartbeat and the onset of the next. In a convergence of science and traditional accounts, the luminous orbs that appear before one's eyes are manifestations of the cellular structure of one's own blood, or heart essence (nying thig). The blue field entoptic phenomena are distinct from other entoptic phenomena such as 'floaters' (muscae volitantes) which result from debris floating on the eye's vitreous humour. In the context of thod rgal, subjective entoptic phenomena and associated hypnogogic imagery serve as affective entry points into liberative non-dual states of consciousness. 


\section{Bibliography}

\section{Primary Sources}

Ngag dbang blo bzang rgya mtsho'irnam thar'phro 'thus (Autobiography of the Fifth Dalai Lama Ngawang Lobzang Gyatso, 1617-82). Completed by Desi Sangge Gyatso. 3 vols. Block print. 'Bras spung, Lhasa. Dga' ldan pho brang. Available at твRC W8239.

Padma gling pa, Rdzogs chen kun bzang dgongs'dus, in 'The Rediscovered Teachings of the Great Padma-glin-pa', 21 vols. Thimphu: Kunsang Tobgay 1975-76. Also available at твRC (resource ID T1851).

gSang ba'irnam thar rgya can (A Record of the Visionary Experiences of the Fifth Dalai Lama Ngag dban blo bzang rgya mtsho, Sman rtsis Shesrig Spendzod, vol. 42), Leh 1972.

Sde srid gSangs rgyas rgya mtsho, Dr'in can rtsa ba'i bla ma ngag dbang blo bzang rgya mtsho'i thun mong phyi'i rnam thar du kula'igas bzang glegs bam gsum pa'i 'phros, vols IV-VI, Zhol edn.

- Thams cad mkhyen pa drug pa blo bzang rin chen tshangs dbyangs rgya mtsho'i thun mong phyi'i rnam par thar pa du kü la'i 'phro'thud rab gsal gser gyi snye ma. Block print. 'Bras spungs, Lhasa: Dga' ldan pho brang. Available at твRC $\mathrm{W}_{2} \mathrm{CZ}_{7} 844$.

Ngag dbang blo bzang rgya mtsho'i rnam thar 'phro 'thus, 3 vols. Block print. 'Bras spung, Lhasa. Dga'ldan pho brang. Available at TBRC W8239.

1973. Bai dūr snon po: Being the text of Gso ba rig pa'i bstan bcos sman bla'i gsal byed bai dur sngon po'i ma Iii ka. 4 vols. From a Lcags po ri block print carved 1888-92. Leh: T.Y. Tashigangpa.

1994. gSo ba rig pa'ibstan bcos sman bla'idgongs rgyan rgyud bzhi'i gsal byed bai dūr ya sngon po'i malli ka, 2 vols., Dharamsala: Bod gzhung sman rtsis khang.

\section{Secondary Sources}

Aris, M. 1979, Bhutan: The Early History of a Himalayan Kingdom, Warminster: Aris \& Phillips.

1980, 'Analysis of the biographies of Padmasambhava according to Tibetan tradition', in Aris and Aung San Suu Kyi (eds) $45^{-52}$.

1988, Hidden Treasures and Secret Lives: A Study of Pemalingpa (1450-1521) and the Sixth Dalai Lama (1683-1706), Delhi: Motilal Banarsidass.

Aris M. and Aung San Suu Kyi (eds), 1980, Tibetan Studies in Honour of Hugh Richardson, Warminster: Aris \& Phillips.

Baker, I. 1997, The Tibetan Art of Healing, London: Thames \& Hudson. 2017, 'Tibetan yoga: somatic practice in Vajrayāna and Dzogchen', in Maas et al. (eds).

- Forthcoming 2018, Tibetan Yoga: Secrets from the Source, London: Thames \& Hudson.

Baker, I. and T. Laird 2011 [2000], The Dalai Lama's Secret Temple: Tantric Wall Paintings from Tibet, London: Thames \& Hudson.

Birch, J. 2011, 'The Meaning of Hatha in Early Hathayoga', Journal of American Oriental Studies 131.4, 527-54.

Blezer H. et al. (eds) 2002, Religion and Secular Culture in Tibet: Tibetan Studies II: Proceedings of the Ninth Seminar of the International Association of Tibetan Studies, Leiden 200o, Leiden: Brill.

Cabezón J. and Jackson R. (eds) 1996, Tibetan Literature: Studies in Genre, Ithaca, NY: Snow Lion Publications.

Chagme, K. (tr. B.A. Wallace) 2000, Naked Awareness: Practical Instructions on the Union of Mahamudra and Dzogchen, Ithaca NY: Snow Lion Publications.

Chaoul-Reich, A. 2006, 'Magical movements ('phrul'khor): ancient yogic practices in the Bon Religion and contemporary medical perspectives', PHD diss., Houston: Rice University.

Dargyay, E. 1977, The Rise of Esoteric Buddhism in Tibet, Delhi: Motilal Banarsidass.

Davidson R. and Goodman S. (eds) 1992, Tibetan Buddhism: Reason and Revelation, Albany: State University of New York Press.

Dorji, C.T. 1995, A Political and Religious History of Bhutan, Delhi: Prominent Publishers.

Douglas, K., and G. Bays (tr.) 1978, The Life and Liberation of Padmasambhava, 2 vols, Berkeley: Dharma Publishing.

Dowman, K. (tr.) 1984, Sky Dancer, London: Routledge and Kegan Paul.

Dudjom Rinpoche, J.Y.D. (tr. Gyurme Dorje and M. Kapstein), 1991, The Nyingma School of Tibetan Buddhism: Its Fundamentals and History, 2 vols, Boston: Wisdom Publications.

Evans-Wentz, W.Y. (ed.) 1954, The Tibetan Book of the Great Liberation, London: OuP.

Gangteng, Literary Committee (2008), The Rosary ofJewels: Biographies of the Successive Throne Holders of Gangteng, Bhutan: Gangteng Monastery.

Guenther, H. 1987, The Symbolic Recreation of the World According to the Tibetan Buddhist Tradition of Tantric Visualisation Otherwise Known as The Developing Phase, Lotsawa House.

Gyatso, J. 1992, 'Genre, authorship, and transmission in visionary Buddhism: the literary traditions of Thang-stong rGyal-po', in Davidson and Goodman (eds), 95-106.

- 1996, 'Drawn from the Tibetan treasury: the gTer ma Literature', in Cabezón and Jackson (eds), 147-69.

1998. Apparitions of the Self: The Secret Autobiographies of a Tibetan Visionary. Princeton: Princeton University Press.

2012, 'Looking for gender in the medical paintings of Desi Sangye Gyatso, Regent of the Tibetan Buddhist state', Asian Medicine: Tradition and Modernity 6, 217-92.

2014. 'Buddhist Practice and Ideals in Desi Sangye Gyatso's Medical Paintings', in Hofer (ed.), 198-220.

2015, Being Human in a Buddhist World: An Intellectual History of Medicine in Early Modern Tibet, New York: Columbia University Press.

Dorje G. 1994, Total Illumination of the Essence: The Teaching Manual for The Union of Samantabhadra's Intentions, Boulder, co: Yeshe Korlo Publications.

Harding, S. 2003, The Life and Revelations of Pema Lingpa, Ithaca, NY: Snow Lion Publications.

Hofer T. (ed.) 2014, Bodies in Balance: The Art of Tibetan Medicine, Seattle: University of Washington Press.

Kapstein, M. 1989, 'The Purificatory Gem and its cleansing: a late Tibetan polemical discussion of apocryphal texts', History of Religions 28(3), 217-44.

2000, The Tibetan Assimilation of Buddhism: Conversion, Contestation, and Memory, Oxford: oup.

2006, The Tibetans, Oxford: Blackwell Publishing.

Kandinsky, W. 1977, Concerning the Spiritual in Art, New York: Dover Publications.

Karmay, S. 1988, Secret Visions of the Fifth Dalai Lama. The Gold Manuscript in the Fournier Collection, London: Serindia Publications.

Kongtrul, J. (tr. E. Guarisco and I. McLeod) 2005, The Treasury of Knowledge, Book Six, Part Four: Systems of Buddhist Tantra, , Ithaca, NY: Snow Lion Publications.

Kvaerne, P. 1990, 'Review of Hidden Treasures and Secret Lives', Acta Orientalia 51, 302-6.

Lipman, K. 1987, 'The dynamic yoga of Tibet: combining asanas, breathing exercises, and flowing movements, Yantra Yoga aims to return us to our "natural state"', cited in Yoga Journal, May 1987, no. 74, Active Interest Media. ISSN 0191-0965. 
Luczantis, C. 2011, 'Locating Great Perfection: the murals of the Lhasa Lukhang', Orientations 42(2), 102-11.

Maas P. et al. (eds) 2017, Yoga in Transformation: Historical and Contemporary Perspectives on a Global Phenomenon. Vienna: Vienna University Press.

Mallinson, J. and M. Singleton. 2017, The Roots of Yoga: A Sourcebook from the Indic Traditions, London: Penguin Classics.

Mayer, R. 1992, 'Review of Hidden Treasures and Secret Lives', Religion $22(2), 187$.

Meyer, F. 1992. 'Introduction: the medical paintings of Tibet', in Parfionovitch, Dorje and Meyer (eds), 2-13.

Mullin, G. 2006, The Practice of the Six Yogas of Naropa: Tsongkhapa's Commentary, Ithaca, NY: Snow Lion Publications.

Namdak, T. and R. Dixey 2002, Heart Drops of Dharmakaya, Boston: Wisdom Publications.

Namdak, T. and J. Myrdhin Reynolds 20o6, Bonpo Dzogchen Teachings, Boston: Wisdom Publications.

Norbu, N. (tr. A. Clemente) 20o8, Yantra Yoga: The Tibetan Yoga of Movement, Ithaca, NY: Snow Lion Publications.

Odier, D. 2005, Yoga Spandakarika: The Sacred Texts at the Origin of Tantra, Rochester, VT: Inner Traditions.

Parfionovitch, Y., G. Dorje and F. Meyer (eds) 1992, Tibetan Medical Paintings: Illustrations to the Blue Beryl Treatise of Sangye Gyamtso (1653-1705), 2 vols, New York: Abrams.

Robinson, J. 1996, 'The lives of Indian Buddhist saints: biography, hagiography and myth', in Cabezón and Jackson (eds), 57-69.

Rossi D. and C. Oliphant of Rossie (eds) 2016, Sharro - Festschrift for Chögyal Namkhai Norbu. Rudolfstetten: Garuda Verlag:

Samuel, G. 1993, Civilized Shamans: Buddhism in Tibetan Societies, Washington, DC: Smithsonian Institution Press.

2008, Origins of Yoga and Tantra: Indic Religions to the Thirteenth Century, Cambridge: cup.
Singh, J. 1979, Siva Sūtras: The Yoga of Supreme Identity, Delhi: Motilal Benarsidas.

Singleton, M. 2010, Yoga Body: The Origins of Modern Postural Practice, Oxford: OUP.

Snellgrove, D. 1987, Indo-Tibetan Buddhism:Indian Buddhists and their Tibetan Successors, Boston: Shambhala Publications.

Thondup, T. 1986, Hidden Teachings of Tibet: An Explanation of the Terma Tradition of Tibetan Buddhism, Boston: Wisdom Publications. 1990, 'The Terma Tradition of the Nyingmapa School', Tibet Journal 15(4), 149-58.

Thurman, R. 1991, 'Review of Hidden Treasures and Secret Lives', The Journal of Asian Studies 5o(2), 375-7.

Tshewang, P. 1995, 'The Biography of Pemalingpa', in Tshewang et al., 23-98.

Tshewang, P., K. Phuntshok Tashi, C. Butters and S. Saetreng 1995, The Treasure Revealer of Bhutan: Pemalingpa, the Terma Tradition and Its Critics, Bibliotheca Himalayica, series III, vol. 8, Kathmandu, Nepal: EMr Publishing House.

Van Schaik, S.J. 2004, Approaching the Great Perfection: Simultaneous and Gradual Approaches to Dzogchen Practice in the Longchen Nyingtig, Boston: Wisdom Publications.

2004b, 'The Early Days of the Great Perfection', Journal of the International Association of Buddhist Studies 27(1):165-206.

Wangyal, T. 2011, Awakening the Sacred Body: Tibetan Yogas of Breath and Movement, London: Hay House, Inc.

Winkler, J. 2002, 'The rDzogs Chen Murals of the Lhasa Klu-Khang', in Blezer et al. (eds), 321-43.

2016. 'The Literary Sources of the Klu-Khang Murals', in Rossi and Oliphant of Rossie (eds), 319-39.

Yeshe, L.T. 1998, The Bliss of Inner Fire: Heart Practice of the Six Yogas of Naropa, Boston: Wisdom Publications.

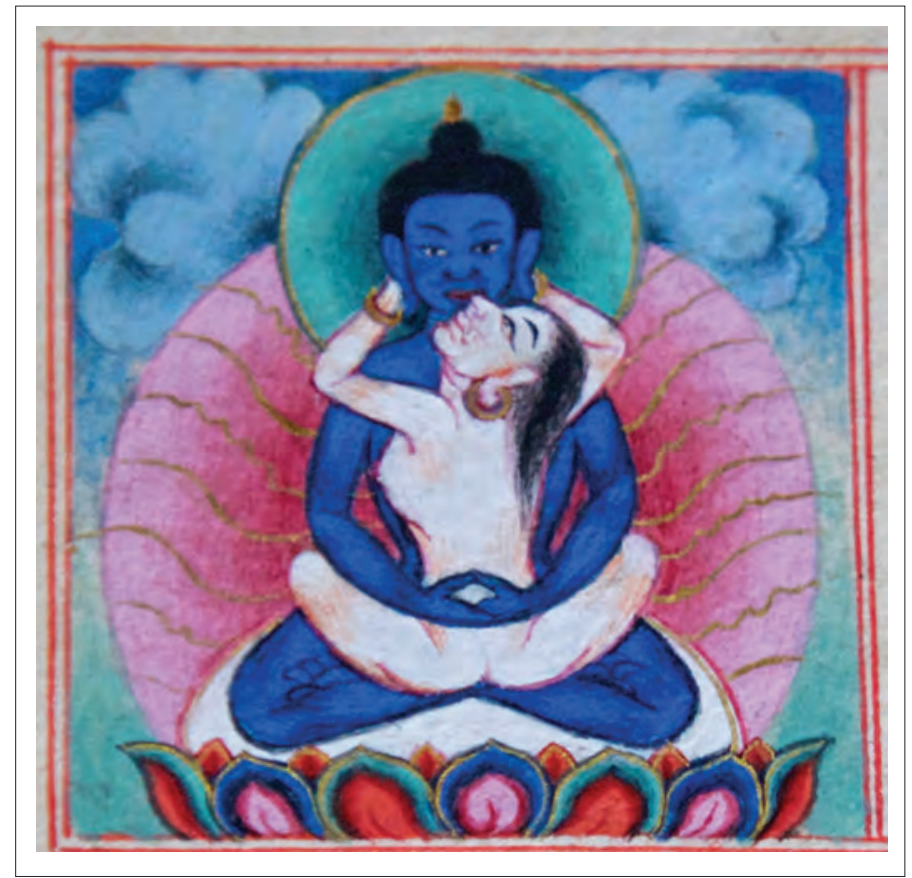

Figure 29.16 This image of Buddha Samantabhadra, 'All Pervading Goodness', appears on the cover folio of vol. 1 of a two-volume 17th-century copy of Pema Lingpa's 'Compendium of Enlightened Spontaneity' that served as a source text for the Lukhang murals. The original manuscript is kept at Gangtey Monastery in Bhutan (see also note 13). $\odot$ Ian Baker 


\title{
30 A Tibetan Image of Divination: Some Contextual Remarks
}

\author{
Ronit Yoeli-Tlalim
}

This paper will focus on a Tibetan image of divination found in the Wellcome Images collection (no. Loo35124, Fig. 1), ${ }^{1}$ illustrating the close association between divination and medicine as taught and practised in the Tibetan medical tradition.

The present contribution will link some of the visual imagery found in this illustration with some of its corresponding theoretical considerations and practical applications. I will focus here on two main motifs in the Wellcome image: the turtle and the magic square. I shall look at some similarities between the Tibetan and Chinese notions illustrated in this Wellcome image, as well as a parallel from the Ilkhanid court in Iran.

The material under discussion provides us with an intriguing case study of how ideas of medicine and divination change as they move between different cultural contexts. This material allows also to reflect on some of the connections between images and knowledge on-themove, and contributes another angle to one of the grand questions which this volume addresses as a whole, namely the role of images in contributing to our understanding of transmissions of medical knowledge.

When looking at the links between medicine and divination in a cross-cultural perspective, one finds many striking similarities in a variety of Euro-asian cultures, in spite of their adoption into very different cultural contexts. ${ }^{2}$ Recent research, primarily of a collaborative nature, has revealed that many of these similarities are due to multi-faceted networks of transmission of knowledge. ${ }^{3}$ Research into these networks is slow and complex. Analysing visual representations of some of these transmitted notions is one prism which can allow glimpses into cultural traces, traces which might otherwise be easily ignored or marginalised. ${ }^{4}$

Most of the theoretical concepts depicted in the central figure of this image are derived from Chinese notions. This specific visual compilation, however, like the very similar

1 Research for this chapter was supported by the Islam and Tibet Project (2005-7, funded by the AHRC) and the Wellcome Trust (2007-10, Tibetan Medicine: A Himalyan Melange, grant no. o76159).

2 See Akasoy, Burnett and Yoeli-Tlalim (eds) 2008. For an overview of similarities, see the editors' Preface, pp. ix-xii.

On these networks in the ancient world, see Mair 2006.

For Edward Said's discussion of Gramsci's notion of traces, see Said 2003 [1978], p. 25.

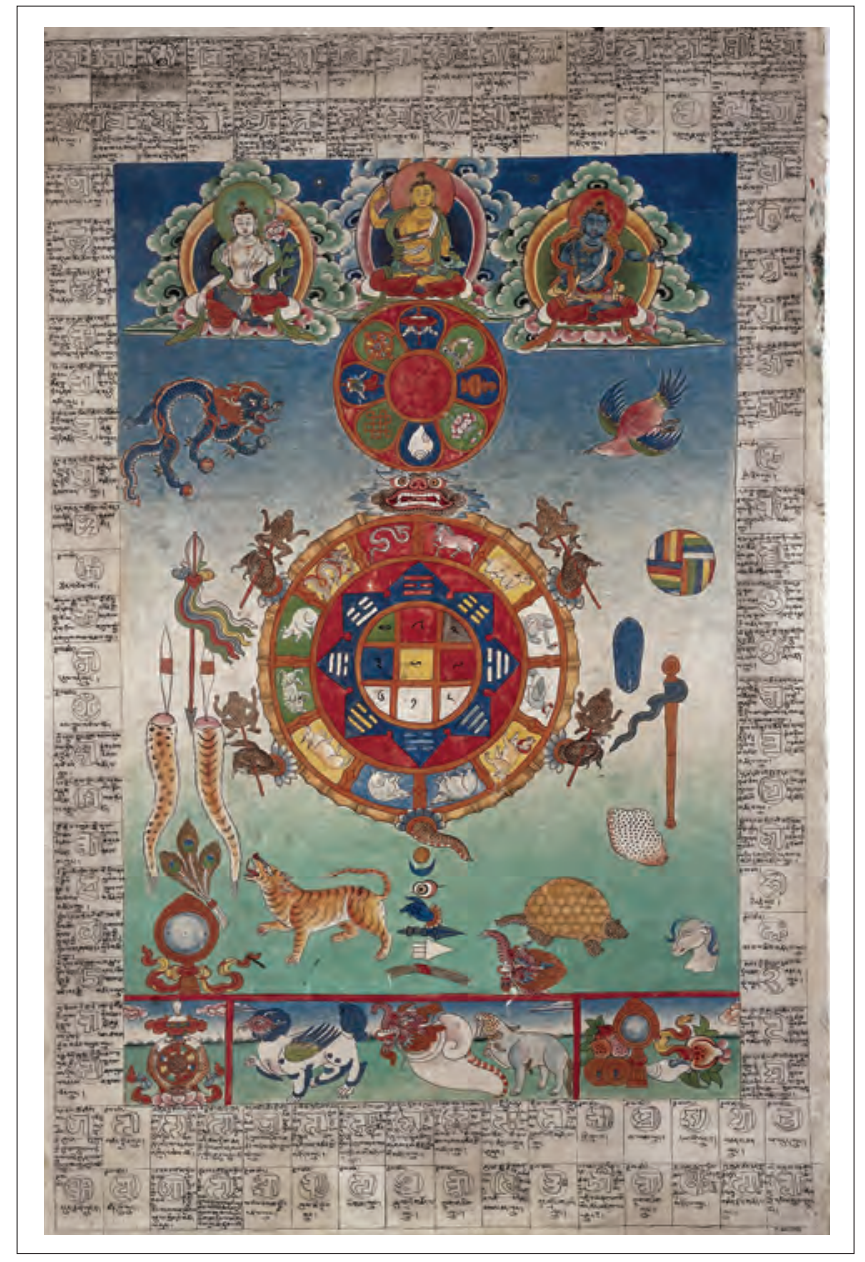

Figure 30.1 A Tibetan Protection painting containing the main elements of Tibetan divination. Tibet, 19th century. (C) Wellcome Library, London, Oriental Tibetan 114, Loo35124

and hugely popular Tibetan charts known as sridpa ho, is specifically Tibetan.

The central motif of this image is of a large turtle, with a magic square of nine (sme ba dgu) in its midst. Surrounding it are the eight trigrams (spar kha brgyad). These are surrounded by a depiction of the 12-year cycle (lo skor bcu nyis). Below the turtle, we find a depiction of the seven-day planet week. The entire image is presided over by Buddhist deities, while Buddhist auspicious symbols are depicted around the main image. Surrounding the image are 104 squares containing instructions for making amulets, which are to be written on a piece of cloth. The image as a whole can be seen as a kind of visual summary of the key elements which stand at the heart of Tibetan medical divination. The overall composition of this image 
is arranged like a mandala, one of the most common visual images in Vajrayāna Buddhism.

\section{Tibetan Medical Divination - General Contextualisation $^{5}$}

Astrology, time computation and divination (rtsis) have been part of Tibetan medicine throughout its history. ${ }^{6}$ In Tibetan, the term rtsis, which is usually translated as 'astrology', refers to astronomy, time computation and divination. The astral sciences are further divided into what is usually translated as 'elemental astrology' ('byung rtsis) and astronomy/astrology (skar rtsis). 'Byung rtsis, also known as 'Chinese divination' (nagrtsis), refers to the system of divination based on concepts found in Chinese divination: the relationships formed between the five agents (Wood, Fire, Earth, Metal and Water) and their various representations, the 12 animal signs, the trigrams (sparkha) and numeric squares (sme ba).

In his explanation of why the science of the stars (skar rtsis) is so central to the healing arts, Sangye Gyatso (Sangs rgyas rya mtsho, 1653-1705), Regent of the Fifth Dalai Lama, to whom a number of important books on medicine and on divination are accredited, explains:

Using it one calculates the times, directions, and dates when the collecting, preparation, and taking of medicines becomes effective and auspicious. There is a great difference between these benefits and the disastrous consequences that come from not knowing this astrology (skar rtsis).

Black [or, elemental] calculation is so called because it spread from the country of black clothes (i.e. China). It describes pulse evaluation - calculating mother, son, enemy, and friend pulses - and urine analysis in conjunction with turtle diagrams and so on, topics that are of prime interest for medical literature. It also covers subjects such as karma calculation, which can predict lifespans and foretell whether one will survive an illness without medical intervention. It deals with the examination of the year, month, day, and hour to divine whether various therapies are appropriate and to calculate the auspicious and inauspicious times for compounding medicines, for performing various medical practices, and so on. It also covers the consulting of land guardians to divine auspicious and inauspicious directions. In particular, using

This overview is based on Yoeli-Tlalim 2014.

For an overview on Tibetan sources on astrology and time computation, see Schuh 1973, pp. 22-46; Dorje 2001. For an illuminating work on Kālacakra time computation, see Henning 2007. On Tibetan divination, see Stein 1939; Jhampa Kalsang 1999; Chime Radha Rinpoche 1981; Jampa Dagthon et al 1998; Cornu 1997; Laufer 1914. only illness divination and time divination, you can make prognoses and learn which demon is causing the illness and what rituals are to be performed, all without resorting to guesswork or relying on other forms of divination, oracles, and the like. In this way elemental calculation has a very strong connection with medical science. ${ }^{7}$

Medicine and astrology have been closely interlinked in Tibet, theoretically, practically and institutionally. Both medicine and astrology have been taught together at medical colleges. To this day, Tibetan doctors are required to study astrology as part of their training. Indeed the two main Tibetan medical colleges, in Lhasa and in Dharamsala, are both called sMan-rtsis-khang (Institute of Medicine and Astrology).

Divination has played - and still plays - a very significant role in all levels of Tibetan society. ${ }^{8}$ Calculations of the relationships formed by the five elements are frequently applied in all parts of life such as birth, marriage, detecting obstacles, analysing disease, analysing spiritual progress and foretelling death. There are various types of auspicious and inauspicious dates, which are marked on every Tibetan calendar. The waxing half of Tibetan lunar months is considered in general more auspicious than the waning half. Therefore, most Tibetans will begin constructive, positive practices during the first half of the lunar month.

Astrological divination of illness constitutes a major part of divination practices in general. These practices, assigned to an astrologer rather than a doctor, are discussed especially in relation to cases where the patient is not seen to respond to medical treatment. Some of the topics examined within this context include: the degree to which the life-spirit or lifespan has been dissipated; the degree to which one is pursued or exploited by malevolent forces; the degree to which the 'heavenly life-line' has been cut, and others. ${ }^{9}$

Divination is also used in the medical context in order to determine the existence of what is termed 'spirit-inflicted illnesses'. These are illnesses that are otherwise unexplained, which are attributed to nine types of spirits. Various divination methods for diagnosing such illnesses are described in the medical writings. It is said that in order to treat the illness, it is necessary to identify which type of spirit is causing the affliction. These divination

\footnotetext{
7 Sangs rgyas rgya mtsho, tr. Kilty 2010, pp. 453-4.

8 For an account of current uses of Tibetan astrology and divination in medical contexts, see Vasstveit 2014.

9 Divination of illness is discussed in Sangs rgyas rgya mtsho, Phug lugs rtsis kyi legs bshad Vaidūra dkar po, 1972 edn, I, fols 556-6o7 (original pagination: fols $274 \mathrm{r}-299 \mathrm{v}$ ). For a translation of these chapters, see Dorje 2001, pp. 294-305.
} 
methods include urine divination and observing seasonal influences on pulse diagnosis..$^{10}$

According to Tibetan medicine, since the seasons have an effect on the pulse and since each season has its activity pattern, a doctor needs to know the exact time of the season in order to be able to read the pulse correctly. Of a more divinatory nature are the 'seven astonishing pulses', which are based on the mother-son and friend-enemy relationship between the five agents and the patient's organs, as delineated in Chinese divination. Based on those, a doctor is described as being able to read from the pulse information regarding the patient's family, guests, enemies, finances, relationship with spirits, prospects and procreative fate. ${ }^{11}$ Other topics of astro-medicine include the collection of medicinal herbs and the preparation of medicines, which need to be performed in accordance with certain astrological events, as well as the association between the days of the week and the planets and the medical implications that they entail. As with medicine more generally, Tibetan astro-medicine is derived from several cultural contexts: predominantly Indian, Chinese and indigenous, codified within an overall Buddhist context. ${ }^{12}$

\section{China as the Land of Divination in Tibetan Sources}

Tibetan sources often refer to China as the land of divination'. A popular saying in Tibet maintains this connection: 'cho (religious doctrine) came from India and $t s i$ (astrology) from China'.13

According to some Tibetan accounts, Songtsen Gampo brought to Tibet knowledge from the four great countries surrounding it. Pawo tsuglag (dpa'bo gtsug lag, 1504-66), for example, tells us:

In the east, from China and Minyak, he took books of technology and of divinatory calculation (others say: medicine and calculations) of the five elements. ${ }^{14}$

The section dealing with pulse diagnosis appears in the rGyud bzhi, Phyi ma rgyud (subsequent Tantra), ch. 1. bDud rtsi snying po yan lag brgyad pa gsang man ngag gi rgyud, Lhasa, 2000 edn, pp. 557-67. For an English translation, see Yuthok Yonten Gonpo, tr. Thokmay Paljor et al. 2011. See also Parfionovitch, Dorje and Meyer 1992, pls, pp. 123-39.

11 Parfionovitch, Dorje and Meyer 1992, pls, pl. 55, pp. 125-6.

12 See Yang Ga 2014.

13 Chime Radha Rinpoche 1981, pp. 6-7.

14 Quoted in Stein 1972, p. 52, n. 2. See also Chime Radha Rinpoche 1981, pp. 6-7.
Tibetan sources distinguish between two strands of Chinese divination teachings: an older strand of Chinese divination (rgya rtsis rnying ma) and a later one (rgya rtsis gsar ma), which was introduced from the 17 th century onwards. ${ }^{15}$

While the later strand has received scholarly attention - primarily through Gyurme Dorje's monumental work on Sangs rgyas rgya mtsho's treatise on divination, the White Beryl (Vaidurya dkar po), ${ }^{16}$ not much was known about the older strand. Thanks to recent studies into Chinese and Tibetan divination material from Dunhuang and nearby sites, conducted by Strickmann, Kalinowski, Nishida and others, our understanding of the older strand is slowly evolving. ${ }^{17}$

Looking at the Chinese and Tibetan material from Dunhuang, we can see that Tibetan and Chinese calendrical and astrological systems co-existed and interacted in Dunhuang in various ways. Beyond the calendrical and astrological texts themselves, we also have a literary source which attests to this interaction in a Chinese Dunhuang manuscript, which recalls a discussion between the Grand Astrologer and Wu Zixu 伍子胥 about the differences between the calendrical cycles of the Chinese (Han 漢), the Barbarians ( $\mathrm{Hu}$ 胡), the Tibetans (Fan 蕃), the Tuyuhun (Hun 渾) and the Xianbei 鮮卑..$^{18}$

The Tibetan connection to Chinese divination is also revealed in Tibetan divination texts from Dunhuang which mention Confucius, rendered as Kong tse, as their author. In the Dunhuang manuscript ITJ.742, for example, Kong tse is mentioned as the author of a divination text. ${ }^{19}$ Among the manuscripts from Dunhuang we also find Tibetan translations of Confucian maxims (Pt.987 and Pt.988). ${ }^{20}$ According to Chinese sources from Dunhuang, we know that Confucian doctrines were taught in the prefectural Dunhuang school, and that special rites for his worship

\footnotetext{
15 Dorje 2001, pp. 16-17.

16 Dorje 2001.

17 The Chinese sources have been studied by Kalinowski and his team. See Kalinowski (ed.) 2003. The Tibetan divination material from Dunhuang has recently been studied by Ai Nishida. For a list of manuscripts and previous studies, see Nishida 2011. See also Nishida's PHD diss. (Nishida 2012). Strickmann's breathtaking study (Strickmann 2005) was published posthumously by Bernard Faure.

18 P.3288 (cols 229-38), see Deng Wenkuan and Liu Lexian in Kalinowski (ed.) 2003, p. 68.

19 Nishida 2011. See also MacDonald 1971, particularly pp. 282-3. Other Tibetan manuscripts from Dunhuang, which mention Kong tse (in its variant spellings) are Pt.987, Pt.988, Pt.992, Pt.1284. For a discussion of these, see Lin Shen-yu 2007.

$20 \quad$ Stein in McKeown (ed. and tr.) 2010.
} 
were conducted twice a year, at the time of the equinoxes, in which the entire body of doctors participated. ${ }^{21}$ Chinese diviners sought to legitimise their texts and techniques by referring to the Yijing and to Confucius. ${ }^{22}$

\section{Buddhisation of Divination}

Particular to the adaptation into a Tibetan context is the process of 'buddhisation' which concepts of divination, as well as their visual representations, have undergone. Tibetan Buddhist accounts maintain that medicine as well as astrology was taught by Buddha Śākyamuni himself. Both medicine and astrology have been categorised as Buddhist classical sciences (rig gnas). ${ }^{23}$ Amongst the Buddhist classical sciences, medicine is traditionally categorised under the five major sciences (rig gnas che $b a),{ }^{24}$ and astrology and divination under the five minor sciences (riggnas chung ba). ${ }^{25}$ It is worth noting here that the Tibetan term 'rig gnas', which is usually translated as 'science', also means 'art', 'culture' or 'field of knowledge'.

In Mahāyānic monastic learning in India, astronomy and astrology were taught as ancillary to medicine. Xuanzang 玄牀, the renowned 7 th-century Chinese pilgrim to India, informs us that both medicine and divination were part of the curriculum at Nālanda:

They studied Mahayana teachings and the doctrines of the 18 schools, as well as worldly books such as the Vedas. They also learned about works on logic, grammar, medicine, and divination. ${ }^{26}$

From a Buddhist-Mahāyānic point of view, the study of these 10 'fields of knowledge' has been described as essential in the path of the bodhisattva's striving towards omniscience. This omniscience is considered in Buddhist

21 Kalinowski in Lo and Cullen (eds) 2005, pp. 120-1.

22 Kalinowski and other contributions in Kalinowski (ed.) 2003.

23 The link between the sciences (rig gnas) and Buddhism is established in Tibetan medical literature and Tibetan astral sciences literature, as well as Buddhist literature. For an overview of the 10 Buddhist sciences in Tibetan Buddhist literature, see Ruegg 1995, pp. 93-147, 'Science religieuse et sciences séculières en Inde et au Tibet: vidyāsthāna indo-bouddhiques et rig gnas indo-tibétains'; see also Wallace, 2001, ch. 4, and Gold 2007, esp. ch. 1 .

The other four are inner science (i.e., Buddhism, nang rig pa), epistemology and logic (gtan tshigs rig pa), grammar (sgra rig pa), and arts (bzo rig pa).

The other four are poetry (snyan ngag), metrics (sdeb sbyor), lexicography (mngon brjod) and drama (zlos gar).

26 literature both as a means of helping others and as a way of knowing oneself. ${ }^{27}$

The Gyushi (rGyud bzhi, Four Tantras), thought by scholars to have been composed in the 12th century, and still regarded as the locus classicus of Tibetan medicine, begins with an account of how the Buddha, manifesting as the Medicine Buddha, gave the teaching encompassed in the text. ${ }^{28}$ Other Tibetan accounts ascribe the source of the knowledge of divination to Mañjuśrī (or Mañjughoșa) - seen in Figure 1 at the top centre. The presiding position of this Buddhist deity of knowledge here is a visual representation of the Tibetan systemisation of medicine and astrology within a Buddhist framework.

A significant Indic influence on Tibetan astro-medicine was also derived from the Kālacakra, an Indian Buddhist tantra composed in the 1oth century, which reached Tibet in the 11th century. The Kălacakra describes the association between the human body and the external world. In the Kālacakra tradition, the integral application of astronomical and medical knowledge is perceived as facilitating the flourishing of human potential and attainment of well-being. It focuses on the ways in which celestial bodies correlate with and influence the human body. This is based on two fundamental premises. The first is that both the human body and the cosmos partake of the nature of time. The second is that both the human body and the cosmos are composed of the same particles that make up the elements of earth, fire, water, wind, space and gnosis. ${ }^{29}$

The first two chapters of the Kälacakra, called respectively 'The Universe' and 'The Individual', demonstrate the Buddhist tantric view of the universe as macrocosm and the individual as its microcosm. The Kälacakra inquiry into the nature of the external world and the individual applies various disciplines such as Buddhist cosmology, astronomy, chronometry, embryology, physiology, botany, psychology and pharmacology. The aim is to provide an analysis of the natural world, which is viewed conventionally as an object of purification and ultimately as a manifestation of the Buddha's mind. One of the goals of the Kälacakra is to demonstrate the correspondence of the universe to

\footnotetext{
27 See Schaeffer 2003.

28 See rGyud bzhi (bDud rtsi snying po yan lag brgyad pa gsang ba man ngag girgyud), Lhasa 2000 edn, p. 1. Similarly, the Kälacakra Tantra, a main source for much of Tibetan astral sciences, has been presented as the words of the Buddha himself: see for example Bu-ston 1965, vol. nga (4), fol. 12b, l. 3-6.

For an analysis of how Tibetan medicine constructed itself to be part of Tibetan Buddhism, see Garrett 2006. 
the individual by identifying the properties of the external physical universe in the body of the individual. ${ }^{30}$

Buddhism also stands at the heart of the reasoning behind explanations provided in related divination manuals. Predictions are not considered to be deterministic, but cautionary and prescriptive. If the outcome is auspicious, no action needs to be taken. If the outcome is negative, the subject is advised to undertake the appropriate counteracting rites, such as the recitation of some Buddhist scriptures or mantras, the offering of purifying smoke (bsangs), the making of thread crosses ( $m$ dos) for ritual exorcism, alongside the carrying of amulets or talismans (such as the srid pa ho) or the offering of torma (gtor ma) cakes.

\section{The Turtle}

The common Tibetan depictions of the turtle in the context of divination are derived from the Chinese association of the shell of a sacred turtle and divination. Indeed in China the association between divination and turtle is inherent in the word $b u$ 个, which originally meant 'to divine by the turtle' and later became the general term for all forms of divination. ${ }^{31}$ The turtle also played an important role in Indian cosmological myths, as well as Western and Arabic ones. $^{32}$

Tibetan sources which present legendary Chinese accounts on the origins of divination reflect the link between the turtle and divination. According to these accounts as they are redacted in Tibetan sources, the mythical Chinese emperor Fu Xi 伏羲 (spa huh shi dhi) saw a gold-coloured turtle (rus sbal) which was offered to him by a subject from the coastal region. Upon his inspecting it, the patterns of the eight trigrams (T: spar kha, Ch: bagua 八卦) first arose in his mind. Consequently, the divination system based on the elemental relationships formed by the eight trigrams, the nine numeric squares (sme ba $d g u$ ) and the 12-year cycle (lo skor bcu gnyis) were devised and treatises were gradually composed by kings, ministers and learned scholars who had mastered these systems. ${ }^{33}$

The earliest known Sino-Tibetan turtle divination manuscript comes from Dunhuang and was brought to light by Sam van Schaik. ${ }^{34}$ This manuscript, Or.8210/S.6878

This principle also appears in earlier Ayurveda texts such as the Caraka Compendium and the Suśruta Compendium. See Wallace 1995, p. 31.

$31 \quad$ Kalinowski in Nylan and Loewe (eds) 2010, p. 339.

32 See Patyal 1995.

33 Dorje 2001, p. 16; Kelényi in Ardussi and Blezer (eds) 2002.

34 Van Schaik, 'The Golden Turtle: A Sino-Tibetan Divination Man-

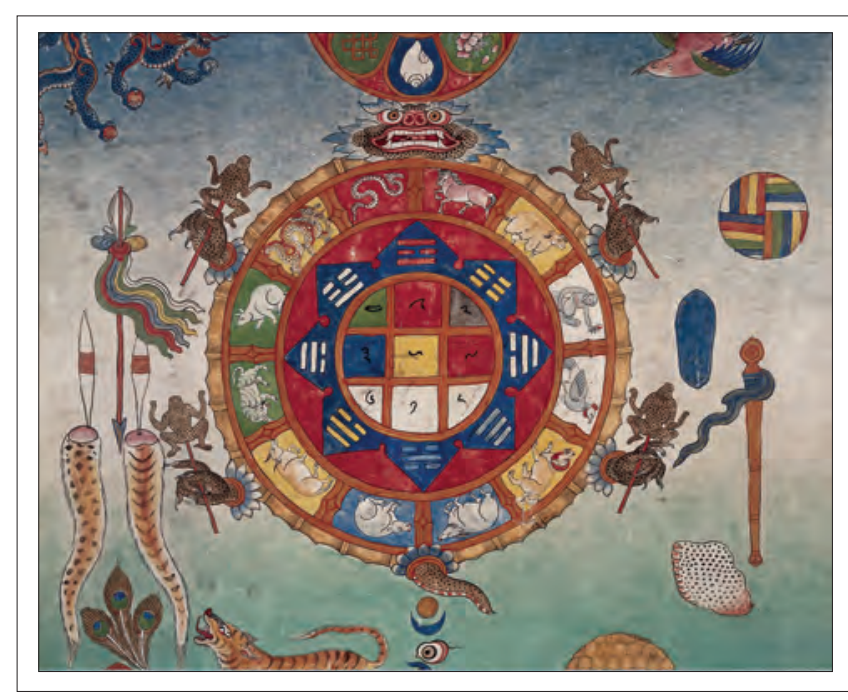

Figure 30.2 Detail of the tortoise, from Figure 30.1. (c) Wellcome Library, London, Loo35124

(Text 50), includes an image of a turtle and a number of divination texts (Fig. 3). It reflects the earliest extant evidence of Sino-Tibetan transmission of visual images related to divination. The manuscript includes sections on 13 methods of astrological divination, each illustrated with a diagram. Visually, these diagrams are precursors of some of the visual depictions we see in the Wellcome image (Fig. 2): divisions of a circle into nine sections, similar to later depictions of the magic square in a circle; divisions into 12 sections of the 12-year cycle; and division into eight sections, reminiscent of the later association with the eight trigrams.

The turtle is inscribed on its back with the words 'Golden Turtle' (gser gyi ru sbal). It has eight locations marked on its back: head (mgo), two ears (rna ba), two arms (lag pa), two sides of the body (mchan khung), two feet (rkang pa) and tail (mjug ma). The image of the turtle is followed by a mo divination text, using the eight types of days to find objects which have been lost.

The name for a turtle as depicted in this drawing, rubel ( rus sbal) literally means 'frog of bones' ( $r u s=b o n e s ; ~ s b a l=-$ frog). Indeed, the rubel from Dunhuang looks something like a cross between a frog and a turtle. In later depictions, the name rubel remains, but the image looks more like a turtle. An interesting Naxi variation, which maintained the link with a frog, was reported by J.F. Rock. ${ }^{35}$ The link with frogs might explain the presence of frogs surmounting the poles and held by the turtle in its four paws in the Wellcome image. The frogs depicted here represent spirits

uscript', available online at: earlytibet.com (accessed 10/o1/2014). See also Iwao, van Schaik and Takeuchi 2012. The image of the tortoise appears in text 5 o (S.6878), pls p. 83 .

35 See Hummel 1969. 


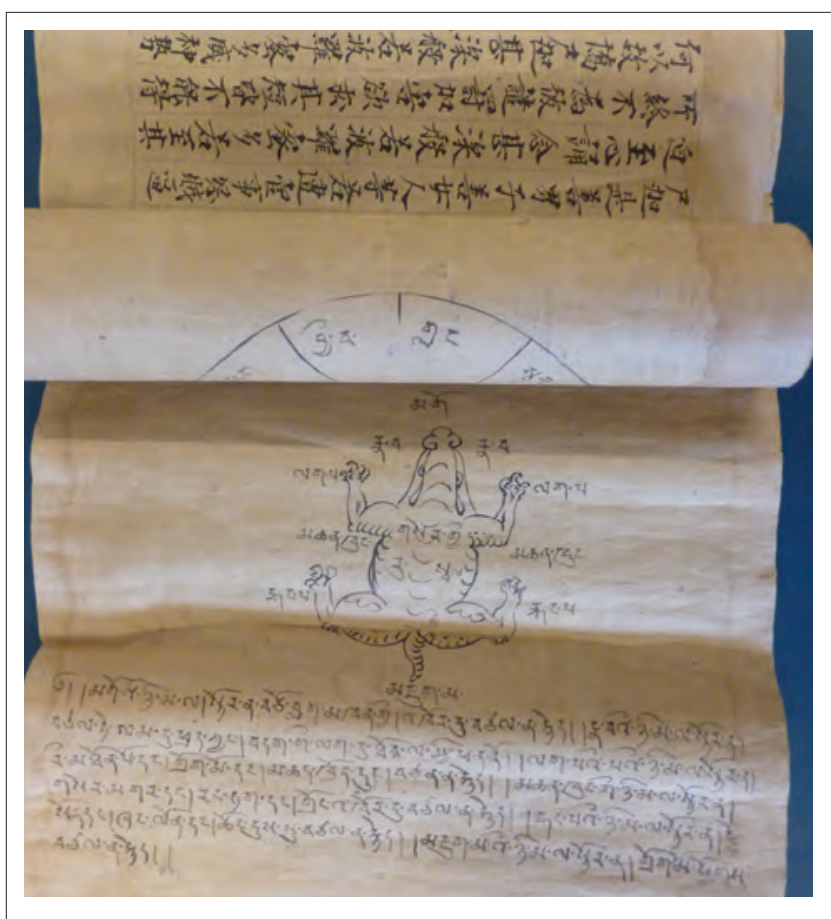

Figure 30.3 A Tibetan Frog/Turtle divination chart for the purpose of finding lost objects. Dunhuang; 9th-1oth century. This chart, along with other Tibetan divination charts, is found at the back of a Prajnaparamita Sütra written in Chinese. (c) British Library ms. Or.8210/S.6878

of the earth. Pictured nailed onto a stick, they symbolise 'pinning down' into stability (Fig. 2).

The eight locations on the Dunhuang turtle-frog appear to foreshadow the eight turtle locations on which we find the eight trigrams in later formulations, such as in the Wellcome illustration. One such later description is found in the 17 th-century White Beryl (Vaidurya dkar po):

Then the eight trigrams assembled separately, each with his or her own wealth, on the golden turtle of sentient existence. The father (Khen) was entwined with the left leg of the turtle, and the mother (Khon) with the left arm, while the younger daughter $(\mathrm{Li})$ was placed at its head, the second son (Dva) at its heart, the first incestuous offspring (Kham) at the tail, and the second (Zin) at the right armpit, the eldest daughter (Zon) at the first tier of Mount Sumeru and thence the right arm, and the eldest son (Gin), who became the general of the spirit lords of the soil (sa-bdag dmag-dpon), at the right leg. Following this separation, conflict then arose between Gin and Zon, Dva and Zin, Khon and Khen, and between Kham and Li, in consequence of which the eight trigrams (kha-brgyad) became known as the 'eight blazing trigrams' (spar-kha brgyad). ${ }^{36}$

It is interesting to note here that in the White Beryl the golden turtle is presented as a manifestation of Mañjuśrī.

$36 \quad$ Dorje 20o1, p. 46.

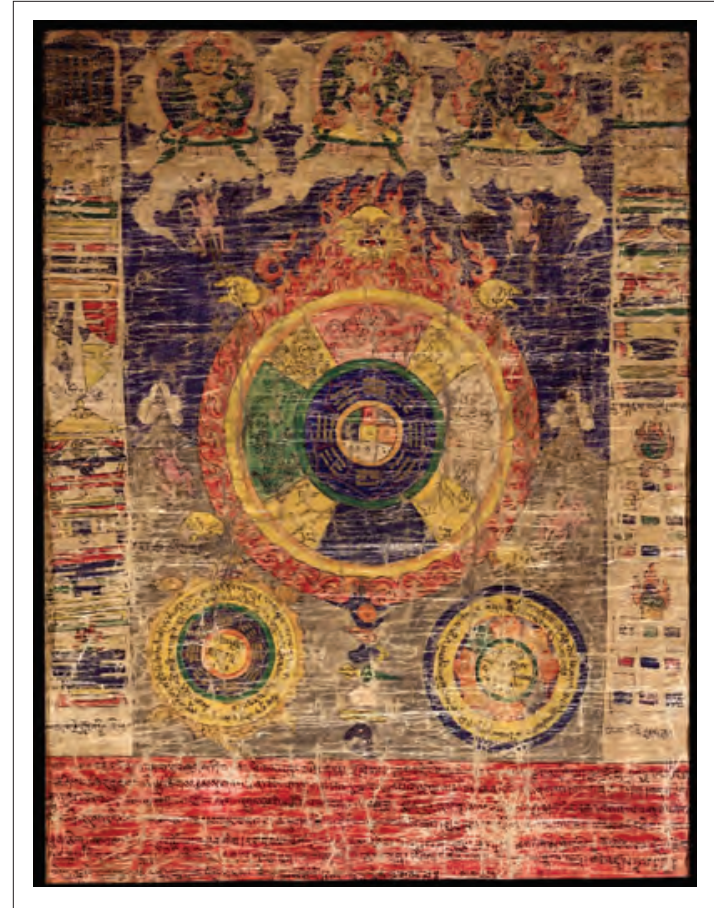

Figure 30.5 A sipaho (srid pa ho) protective chart. (c) Shelley \& Donald Rubin Foundation

The White Beryl discusses a number of different turtles representing various Buddhist notions. ${ }^{37}$

Associating a turtle chart with nine sections for divination purposes also became integrated into practices of Tibetan urine divination. In this technique, a fresh sample of urine is placed in a shallow container of round or oblong shape. A turtle divination chart is placed on top of the container. Changes in the urine specimen are then observed in the nine different sections. According to the changes in the urine, the doctor may identify which type of negative spirit is affecting the patient (Fig. 4). ${ }^{38}$

The image of a divination turtle became central in Tibetan images of divination and good luck. The Great Golden Turtle (mahā gser gyi rus sbal) became a common figure in Tibetan folk religion, with the role of preventing trouble and bad luck. The turtle has remained a popular Tibetan image, found as part of the very common srid pa ho image (Fig. 5).

The srid pa ho charts are very common amongst Tibetans to this day. They are hung on doors in order to prevent evil spirits from entering the home. They are also used as amulets for protection from bad luck, and are displayed on auspicious or inauspicious occasions, such as the time of marriage, important transitions, the time of death etc.

$37 \quad$ See Dorje 2001.

38 Parfionovitch, Dorje and Meyer 1992, pls, pp. 64-6. 


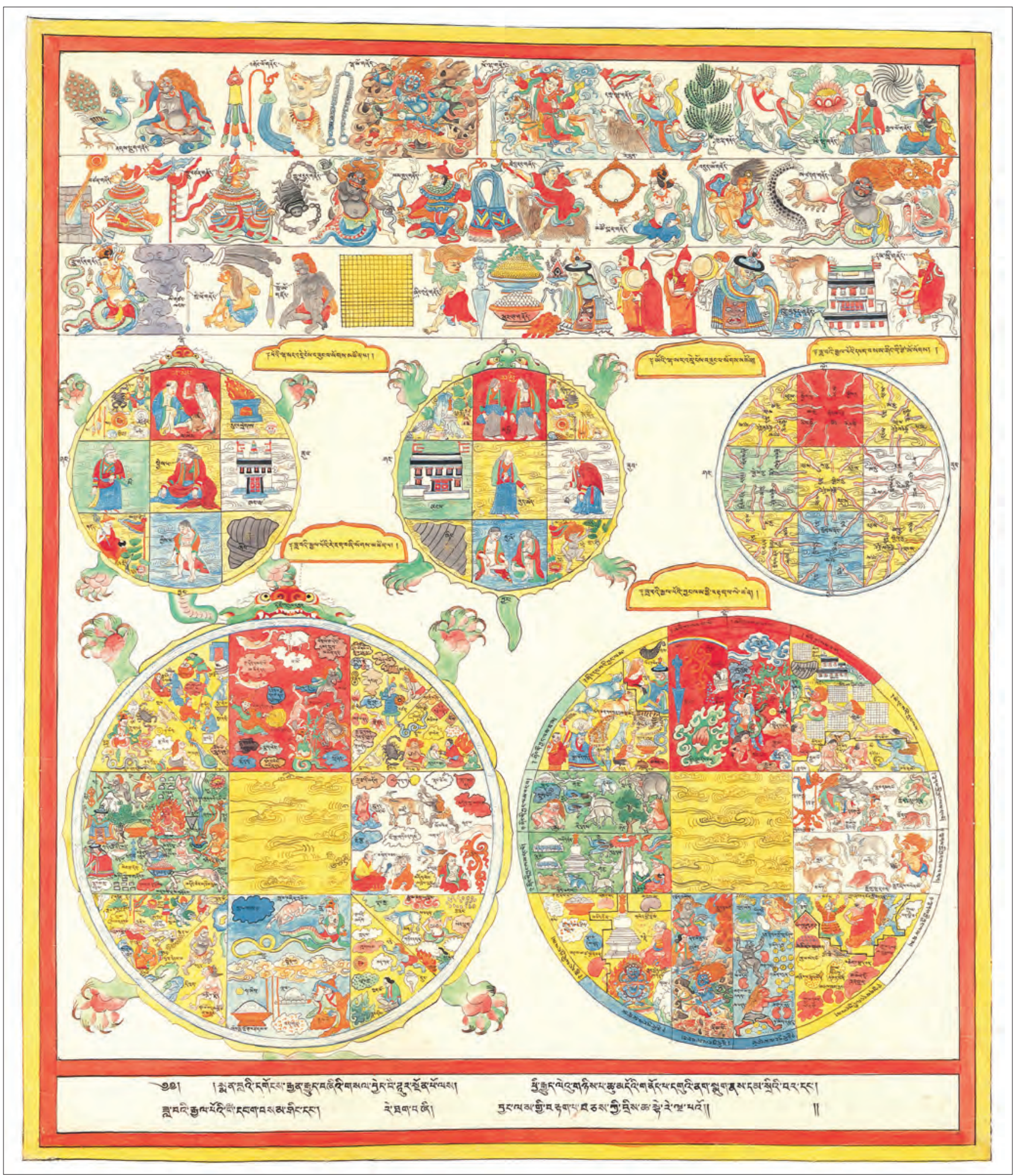

Figure 30.4 Tibetan medical painting no. 65: Urine divination using Turtle charts. Source, copyright? 


\section{The Magic Square}

At the centre of the turtle of the Wellcome image we find a magic square of order three. In a magic square the sum of all the rows, columns and diagonals is the same. In our case here, a magic square of order three, the sum is 15 . The magic square represented in the Wellcome image is:

\begin{tabular}{|c|c|c|}
\hline $\begin{array}{c}4 \\
\text { Green }\end{array}$ & $\begin{array}{c}9 \\
\text { Red }\end{array}$ & $\begin{array}{c}2 \\
\text { Black }\end{array}$ \\
\hline $\begin{array}{c}3 \\
\text { Blue }\end{array}$ & $\begin{array}{c}5 \\
\text { Yellow }\end{array}$ & $\begin{array}{c}7 \\
\text { Red }\end{array}$ \\
\hline $\begin{array}{c}6 \\
\text { White }\end{array}$ & $\begin{array}{c}1 \\
\text { White }\end{array}$ & $\begin{array}{c}8 \\
\text { White }\end{array}$ \\
\hline
\end{tabular}

We can see that the positions of the 6 and the 8 have been switched and that this magic square should look like this:

\begin{tabular}{|c|c|c|}
\hline $\begin{array}{c}4 \\
\text { Green }\end{array}$ & $\begin{array}{c}9 \\
\text { Red }\end{array}$ & $\begin{array}{c}2 \\
\text { Black }\end{array}$ \\
\hline $\begin{array}{c}3 \\
\text { Blue }\end{array}$ & $\begin{array}{c}5 \\
\text { Yellow }\end{array}$ & $\begin{array}{c}7 \\
\text { Red }\end{array}$ \\
\hline $\begin{array}{c}8 \\
\text { White }\end{array}$ & $\begin{array}{c}1 \\
\text { White }\end{array}$ & $\begin{array}{c}6 \\
\text { White }\end{array}$ \\
\hline
\end{tabular}

In Tibetan, the arrangement of the nine numbers is known as sme ba dgu, or the 'nine naevi' (nine moles). Sme ba literally refers to the white or black moles or spots found on the skin. The reference to moles or spots probably refers to their representation in the Luo shu diagram of white and black spots (Fig. 6).

As mentioned above, Chinese sources describe a sacred turtle with a magical square on its shell which appeared to the mythical King Yu 禹 from the waters of the Luo 雒 river at the time of taming the floods. ${ }^{39}$ It was called $L u o$ shu 洛書, or 'Document of the Luo (Lo) River', and was generally represented by a pseudo-archaic arrangement of black and white knots or beads on short strings of cord. Cammann has traced the depiction of the Luo shu in dotted form to the 1oth century $\mathrm{CE}{ }^{40}$

Tibetan sources too refer to these nine numbers as birthmarks on the turtle. Tibetan sources reflect the Chinese associations linking the numbers with colours, directions and the five agents. There are different variations for these associations. One set that is often found in Tibetan sources is: ${ }^{41}$

39 The turtle episode is also associated with Huangdi, the Yellow Emperor, in some Daoist writings. See Cammann 196o, p. 118.

40 Cammann 1961.

41 See Henning 2007, p. 168. For Chinese variations, see Major 1984.

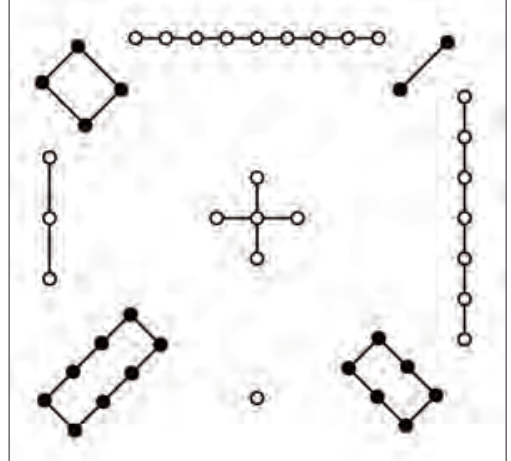

Figure 3 o.6 Luo shu: Chinese magic square of three

$\begin{array}{llll}1 & \text { White } & \text { Iron } & \text { north } \\ 2 & \text { Black } & \text { Water } & \text { south-west } \\ 3 & \text { Blue } & \text { Water } & \text { east } \\ 4 & \text { Green } & \text { Wood } & \text { south-east } \\ 5 & \text { Yellow } & \text { Earth } & \text { centre } \\ 6 & \text { White } & \text { Iron } & \text { north-west } \\ 7 & \text { Red } & \text { Fire } & \text { west } \\ 8 & \text { White } & \text { Iron } & \text { north-east } \\ 9 & \text { Red } & \text { Fire } & \text { south }\end{array}$

Nine Palace diagrams (jiugong tu 九宮圖), which form the basis for magic squares, have been found in China in texts dating to the 2nd century всЕ. ${ }^{42}$ The first of these, Xingde B (Xingde yipian 刑德乙篇), a diagram which has been found in the Mawangdui tombs, is the earliest known representation of the Nine Palaces diagram. Unlike later representations in the form of a square divided into nine equal cells, this diagram consists of a central circle from which radiate the eight sectors of the world, represented by squares (Fig. 7).

A similar star shaped representation of the Nine Palaces was also found on a Taiyi 太乙 diviner's board from Fuyang 阜陽 in present-day Anhui province. A date inscribed at the back of the instrument dates it to the 7 th year of the Emperor Wen of the Han dynasty, i.e. 173 BCE. ${ }^{43}$ Kalinowski has shown that by the Han period, the different components of magic squares were already linked together: the directions (four cardinal directions, four intermediary directions and the centre), numbers (1-9) and the eight trigrams. ${ }^{44}$

The grid of the magic square provided a visual image of the way in which mathematical structures stand at the basis of spatial order. ${ }^{45}$ As argued by Lewis, the grid constituted the most important mechanism for correlating earthly events to astral phenomena. The 'controlled space'

\footnotetext{
$42 \quad$ Kalinowski 1998-1999.

43 Ibid., p. 138, n. 36 and pp. 198-9.

44 Kalinowski 1985.

45 See Lewis 2006, pp. 245-305.
} 


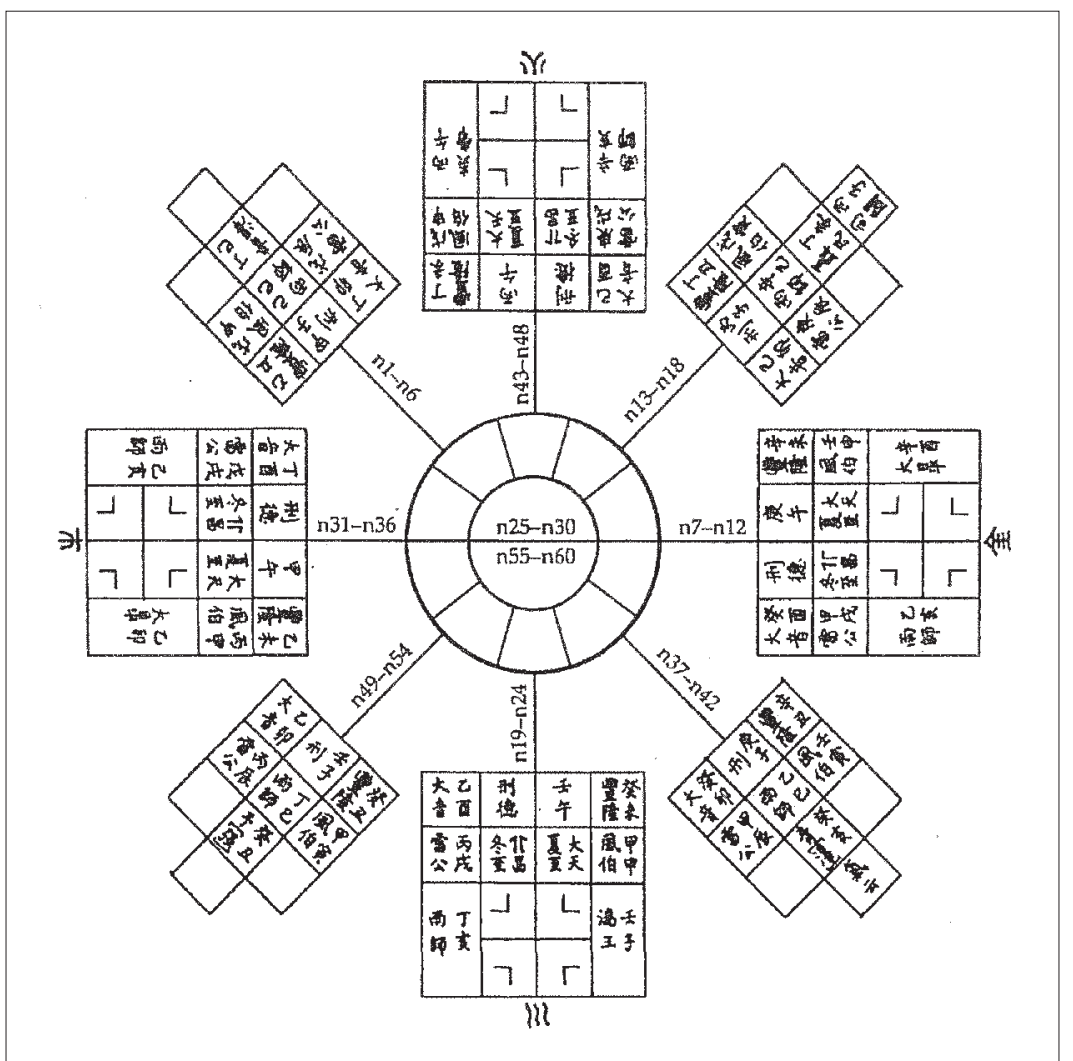

Figure 30.7 Representation of the Nine Palace diagram in Xingde B, Mawangdui tombs (2nd century BCE) (Rendering by Kalinowski). From Kalinowski 1998-9, p. 178, fig. 13

of the grid also formed a frame for linking cosmological models and ritual actions. The nine-square type of grid, formed by the intersection of two horizontal lines and two vertical lines at right angles, was the most common type. The earliest literary occurrence of this type of grid was in Mencius's model of the 'well-field' system. In this system, a piece of land was divided into nine sectors in the shape of the Chinese graph 'well' ( jing 井): the outer eight were cultivated by individual households, while the middle one, which had the well in it, was owned by the landlord and was cultivated collectively. As magic squares evolved in China, a schematic cosmography of the earth's nine regions became linked with magic squares and notions of the five agents, creating an integrated whole. ${ }^{46}$

\section{A Persian Parallel}

An image which is based on the same ideas at the basis of the Tibetan image under discussion, but which took a very different visual shape, is found in a Persian text from 14th-century Ilkhanid Iran. The text, Tansūqnāma-i

Major 1984.
İlkhān dar funūn-i 'ulūm-i Khatā' (The Treasure Book of the Ilkhan on Chinese Science and Techniques), known as the Tansūqnäma, was composed in the court of Rashìd al-Dīn and and includes a number of intriguing illustrations. Rashīd al-Dīn (1274-1318), the court physician and a powerful minister in the Ilkhanid court in Tabriz, assembled Chinese, Tibetans, Indians, Kashmiris and others at the Ilkhanid court and systematically translated and promoted the collection and dissemination of the different medical traditions of the cultures under Mongol rule at the time. ${ }^{47} \mathrm{His}$ monumental multi-cultural oeuvre - which is yet to receive the place it deserves in global intellectual history - was formed through noteworthy political levels of support. ${ }^{48}$

The Tansūqnāma, completed in 1313 , is divided into two; the first part is an introduction by Rashīd al-Dīn. The second part is a collection of Chinese classical medical writings. As far as is presently known, this is the first indication of the transmission of classical Chinese medicine into the west. ${ }^{49}$

47 On Rashīd al-Dīn, see Akasoy, Burnett and Yoeli-Tlalim (eds) 2013. .

48 Prazniak 2014.

49 See Lo and Wang 2013; Berlekamp 2010; Klein-Franke and Zhu 


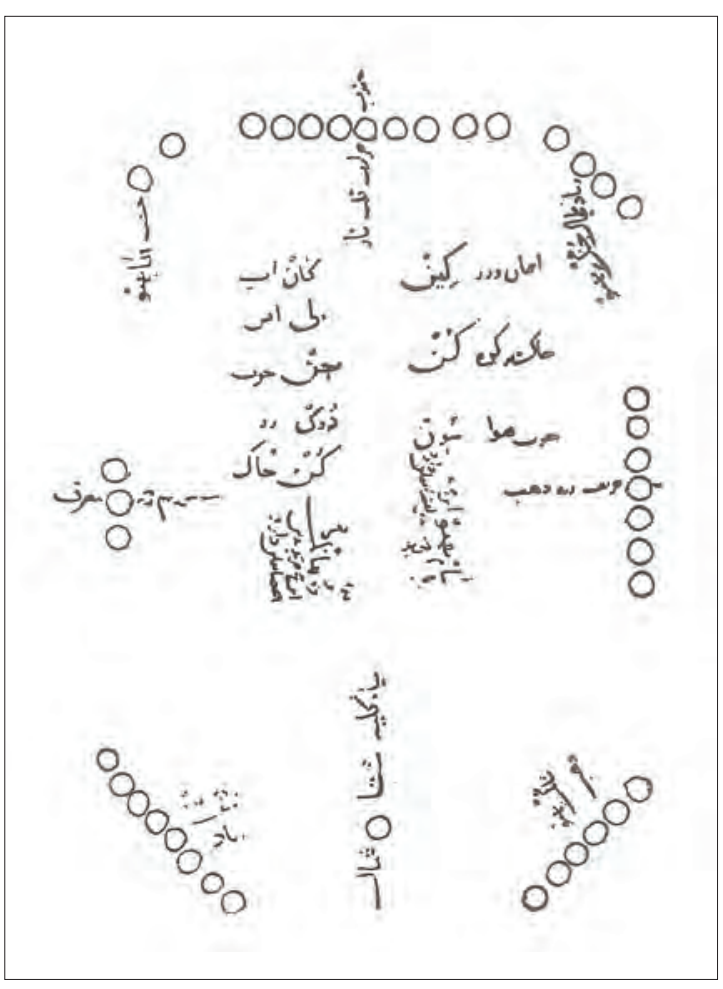

Figure 30.8 The Tansūqnāma of Rashid al-Din. Tabriz, 1313, ms. 3596, fol. 55b. (c) Süleymaniye Library, Aya Sofya Collection. Türkiye Yazma Eserler Kurumu Başkanlığı

Figure 8 from Tansūqnāma shows a visual representation of a magic square in the Chinese style. The magic square depicted here, clearly based on the Luo shu depictions, is set out thus:

\begin{tabular}{|l|l|l|}
\hline 2 & 9 & 4 \\
\hline 3 & & 7 \\
\hline 8 & 1 & 6 \\
\hline
\end{tabular}

Like the Tibetan image, it inverts the position of two numbers: here, the 2 and the 4 . The place of the central 5 is taken up by the names of the trigrams, the five agents and the relevant associations with body parts and the seasons.

Magic squares are documented in the Islamic world since the 9th-1oth centuries, and by Rashīd al-Dīn's time were well known in the Islamic world. A set of magic squares was presented in the Rasāil of the Ikhwān as-Ṣafā (Brethren of Purity), an encyclopaedia of sciences composed in Basra during the gth and oth centuries. As Baffioni points out, the Rasā'il was an Arabic compendium of foreign sciences, reworking Babylonian, Indian and Persian knowledge. ${ }^{50}$ Cammann has suggested that the section of magic squares

Ming, 1996. See also Lo and Yoeli-Talim, Chapter 20 in this volume.

Baffioni 2012. in the Rasāil incorporated Chinese notions, possibly via Indian mediation. ${ }^{51}$ Indeed, the Rasā'il includes many references to India and the Buddha. ${ }^{52}$ Early Islamic magic squares are also attributed to Jābir ibn Ḥayyān, known in Europe as Geber. ${ }^{53}$

Magic squares became an important part of the vocabulary of talisman-makers and compilers of magical manuals in Islamic cultures, particularly after the 12 th century. ${ }^{54}$ The $3 \times 3$ magic square became so important in Islamic cultures that the name itself of this type of magic square, budūh, acquired its own occult potency. It was known as a charm for easing childbirth. Magic squares also became known and popular in Hebrew and European languages.

Analysing the transmission of magic squares and amulets - many of them using magic squares - between the Chinese and the Muslim worlds, Johan Elverskog has shown that the Ilkhanid court played a key role in transmission. ${ }^{55}$ Although trigrams and their associations might have seemed alien to the compilers of the Tansūqnāma, the notion of the magic square probably fell on receptive ground.

\section{Magic Squares, Borders of Medicine, Borders of Cultures}

When somebody eventually sits down to write a global history of magic squares, he or she is bound to find a treasure-trove of unexplored stories of transmission. For the time being, what I have shown here is that this popular visual depiction, used in Tibetan medical and other divination practices to this day, has its roots in China and many parallels in what we may term 'popular medicine' among Muslims and Jews, and in Europe. ${ }^{56}$

In the long history of magic squares in China, they gained and lost meanings pertaining to various links between a human being and the world. The formula of the magic square was an effective way of distilling in a concise schematic structure a multitude of linkages between the micro-cosmos and the macro-cosmos. Its simple visual representation made it suitable both as a mnemonic device and for cross-cultural transmission.

\footnotetext{
$51 \quad$ Cammann 1969.

$5^{2}$ Walker, 'ekwwān al-ṣafā'.

53 Savage-Smith 1997, p. 106.

54 Savage-Smith 2004, "Introduction", pp. xiii-xliv.

55 Elverskog 2010, ch. 2, 'Understanding', especially pp. 104-14.

56

Cammann 1960. Cammann refutes a number of other exaggerated claims on the origins of magic squares. See also Cammann 1961 .
} 
From studies on the transmission of diagrams such as magical squares across cultures and religions, we know that these were useful vessels not only for transmission but also for adaptation. In the Tibetan case, the visual representations went hand in hand with the transmission of Chinese divination theory and practice. In other cultural contexts, magic squares gained numerous new symbolic meanings that were part of their new cultural contexts.

\section{Bibliography}

\section{Primary Sources}

Bu-ston 1965, 'History of the Kālacakra' (Dus 'khor chos 'byung rgyud sde'izab don sgo 'byed rin chen gces pa'i lde mig), in Collected Works, New Delhi: International Academy of Indian Culture, vol. nga (4). rGyud bzhi (bDud rtsi snying po yan lag brgyad pa gsang ba man ngag gi rgyud), Lhasa: Bod ljongs mi dmangs dpe skrun khang, 2000.

Sangs rgyas rgya mtsho, Vaidurya dkar po [Phug lugs rtsis kyi legs bshad Vaidūra dkar po. Reproduced as The Vaidūrya dkar po of sde-srid Sans-rgyas-rgya-mtsho], New Delhi: T. Tsepal Taikhang, 1972.

\section{Secondary Sources}

Akasoy, A., C. Burnett and R. Yoeli-Tlalim (eds) 2008, Astro-Medicine: Astrology and Medicine, East and West (Micrologus' Library, 25), Florence: SISMEL/Edizioni del Galluzzo.

C. Burnett and R. Yoeli-Tlalim (eds) 2013, Rashid al-Din as an Agent and Mediator of Cultural Exchanges in Ilkhanid Iran, London: Warburg Institute.

Ardussi, J. and H. Blezer (eds) 2002, Impressions of Bhutan and Tibetan Art: Tibetan Studies III, Proceedings of the Ninth Seminar of the IATS 200o, Leiden: Brill.

Baffioni, C. 'Ikhwān al-Safā", The Stanford Encyclopedia of Philosophy (Summer 2012 edn), ed. E.N. Zalta: http://plato.stanford.edu/ archives/sum2012/entries/ikhwan-al-safa (accessed 27/o6/17).

Berlekamp, P., 2010, 'The limits of artistic exchange in fourteenth-century Tabriz: the paradox of Rashid al-Din's Book on Chinese Medicine, Part I', Muqarnas 27, 209-50.

Cammann, S. 1960, 'The Evolution of Magic Squares in China', Journal of the American Oriental Society 8.2 (April-June), 116-24.

Cammann, S. 1961, 'The magic square of three in old Chinese philosophy and religion', History of Religions 1.1 (Summer), 37-80.

1969, 'Islamic and Indian magic squares. Part I', History of Religions, 8.3 (February), 181-209.

Chime Radha Rinpoche 1981, 'Tibet', in Loewe and Blacker (eds), 3-37. Cornu, P. 1997, Tibetan Astrology, Boston: Shambala.

Deng Wenkuan 鄧文寬 and Liu Lexian 劉樂賢 2003, 'Uranomancie', in Kalinowski (ed.), 35-83.

Dorje, Gyurme (comm. and tr.), 2001, Tibetan Elemental Divination Paintings: Illuminated Manuscripts from The White Beryl of Sangs-rgyas rGya-mtsho: with the Moonbeams Treatise of Lo-chen Dharmaśri, London: John Eskenazi, 2001.

Elverskog, J. 2010, Buddhism and Islam on the Silk Road, Philadelphia: University of Pennsylvania Press.

Gold, J. 2007, The Dharma's Gatekeepers: Sakya Pandita on Buddhist Scholarship in Tibet, Albany, NY.: SUNY Press.

Garrett, F. 2006, 'Buddhism and the Historicising of Medicine', Asian Medicine: Tradition and Modernity 2, 204-24.
Henning, E. 2007, Kälacakra and the Tibetan Calendar, New York: Columbia University Press.

Hofer, T. (ed.) 2014, Bodies in Balance: The Art of Tibetan Medicine, New York: Rubin Museum of Art and Washington University Press.

Hummel, S. 1969, 'The sMe-ba-dgu, the Magic Square of the Tibetans', East and West 19, 139-46.

Imaeda, Yoshiro, M. Kapstein and Tsuguhito Takeuchi (eds) 2011, New Studies of the Old Tibetan Documents: Philology, History and Religion, Tokyo: Research Institute for Languages and Cultures of Asia and Africa.

Iwao, Kazushi, S. van Schaik and Tsuguhito Takeuchi 2012, Old Tibetan Texts in the Stein Collection Or. 8210, Tokyo: Toyo Bunko.

Jampa Dagthon et al. 1998, Tibetan Astronomy and Astrology: a Brief Introduction, Dharamsala: Men Tsee Khang, 1998.

Jhampa Kalsang 1999, Tibet Astro Science, Rome: Tibet Domani.

Kalinowski, M. 1985, 'La transmission du dispositif des neuf palais sous les six-dynasties', in Strickmann (ed.), 773-811.

1998-1999, 'The Xingde texts from Mawangdui', Early China $23^{-4}, 125^{-202 .}$

(ed.) 2003, Divination et société dans la Chine médiévale: Étude des manuscrits de Dunhuang de la Bibliothèque nationale de France et de la British Library, Paris: Bibliothèque nationale de France.

2005, 'Mantic texts in their cultural context', in Lo and Cullen (eds), 109-33.

2010, 'Divination and astrology: received texts and excavated manuscripts', in Nylan and Loewe (eds), 339-66.

Kelényi, B. 2002, 'The myth of the cosmic turtle according to the late astrological tradition', in Ardussi and Blezer (eds), 69-9o.

Klein-Franke, F. and Zhu Ming 1996, 'Rashīd al-Dīn as a transmitter of Chinese medicine to the West', Le Muséon 109, 395-404.

Laufer, B. 1914, 'Bird divination among the Tibetans', T'oung Pao 15, 1-110.

Lewis, M.E. 2006, The Construction of Space in Early China, Albany, NY: SUNY.

Li Rongxi 李荣熙 (tr.) 1995, A Biography of the Tripitaka Master of the Great Ci'en Monastery of the Great Tang Dynasty, Berkeley, CA: Numata Center for Buddhist Translation and Research.

Lin Shen-yu 林純瑜 2007, 'The Tibetan image of Confucius', Revue d'Études Tibétaines 12, 105-29.

Lo, V. and C. Cullen (eds) 2005, Medieval Chinese Medicine: The Dunhuang Medical Manuscripts, London: RoutledgeCurzon.

Lo, V. and Wang Yidan 王一丹 2013, 'Blood or Qi circulation? On the nature of authority in Rashīd al-Dīn's Tānksūqnāma ('The Treasure Book of the Ilkhan on Chinese Science and Techniques')', in Akasoy, Burnett and Yoeli-Tlalim, (eds), 127-72.

Loewe M. and C. Blacker (eds) 1981, Divination and Oracles, London: George Unwin.

MacDonald, A. 1971, 'Une lecture des Pelliot tibétain 1286, 1287, 1038, 1047, et 129o', in MacDonald (ed.), 19o-391.

- (ed.) 1971a, Études tibétaines dédiées à la mémoire de Marcelle Lalou, Paris: Adrien Maisonneuve.

Maddison, F. and E. Savage-Smith 1997, Science, Tools and Magic (Pt 1: Body and Spirit, Mapping the Universe), London: Nour Foundation.

Mair, V. 2006, 'Kinesis versus stasis, interaction versus independent invention', in Mair (ed.), 1-16.

(ed.) 2006a, Contact and Exchange in the Ancient World, Honolulu: University of Hawai'i Press.

Major, J. 1984, 'The Five Phases, magic squares, and schematic cosmography', in Rosemont Jr. (ed.), 133-66.

Nishida, Ai 2011, 'An old Tibetan divination with coins: IOL Tib J 742', in Yoshiro Imaeda, Kapstein and Tsuguhito Takeuchi (eds), New Studies of the Old Tibetan Documents: Philology, History and 
Religion, Tokyo: Research Institute for Languages and Cultures of Asia and Africa, $315^{-27}$.

2012, 'Ko-Tibet-go uranaimonjyo no kenkyū 古チベット語占

い文書の研究’ (A study on old Tibetan divination manuscripts), PHD diss., Kobe City University of Foreign Studies.

Nylan, M. and M. Loewe (eds) 2010, China's Early Empires: A ReAppraisal, Cambridge: CuP.

Parfionovitch, Y., G. Dorje and F. Meyer 1992, Tibetan Medical Paintings: Illustrations of the Blue Beryl of Sangya Gyatso, London: Serindia Publications.

Patyal, H.C. 1995, 'Tortoise in mythology and ritual', East \& West 45, 97-108.

Prazniak, R. 2014, 'Ilkhanid Buddhism: traces of a passage in Eurasian history', Comparative Studies in Society and History, 56.3, 650-8o.

Rosemont, H. Jr. (ed.) 1984, Explorations in Early Chinese Cosmology, JAAR Thematic Studies 50.2, Chico, CA: Scholars Press

Ruegg, D.S., 1995, Ordre spirituel et ordre temporel dans la pensée bouddhique de l'Inde et du Tibet: quatre conférences au Collège de France, Paris: Collège de France.

Said, E.W. 2003 [1978], Orientalism, London: Penguin Books.

Sangs rgyas rgya mtsho, tr. G. Kilty 2010, Mirror of Beryl: A Historical Introduction to Tibetan Medicine, Boston: Wisdom.

Savage-Smith, E. 1997, 'Talismanic charts', in Maddison and SavageSmith, 106-23.

(ed.) 2004, Magic and Divination in Early Islam, Aldershot: Ashgate-Variorum.

Schaeffer, K. 2003, 'Textual scholarship, medical tradition and Mahāyāna Buddhist ideals in Tibet', Journal of Indian Philosophy 31, 621-41.

Schuh, D. 1973, Untersuchungen zur Geschichte der Tibetischen Kalenderrechnung, Wiesbaden: Steiner Verlag.

Stein, R.A. 1939, 'Trente-trois fiches de divination tibétaines', Harvard Journal of Asiatic Studies 4, 297-371. 1972, Tibetan Civilization, London: Faber and Faber. huang manuscripts, in Stein 2010a, 273-335.

2010a, RolfStein's Tibetica Antiqua with Additional Materials, ed. and tr. A. McKeown, Leiden: Brill.

Strickmann, M. (ed.) 1985, Tantric and Taoist Studies in Honour of R.A. Stein, vol. 3 (Mélanges chinois et bouddhiques, vol. 22), Brussels: Institut des Hautes Études Chinoises.

(ed. B. Faure) 2005, Chinese Poetry and Prophecy: The Written Oracle in East Asia, Stanford: Stanford University Press.

Van Schaik, S. 'The Golden Turtle: a Sino-Tibetan divination manuscript', available online at: http://earlytibet.com/20o8/11/28/ the-golden-turtle (accessed 27/o6/17).

Vasstveit, I.K. 2014, 'A day at the astrology department of the Men-TseeKhang in Dharamsala, India', in Hofer (ed.), 105-10.

Walker, P.E., 'Ekֵwān al-Șafā”, Encyclopaedia Iranica, available online at: http://www.iranicaonline.org/articles/ekwan-al-safa (accessed 27/06/17).

Wallace, V.1995, 'The Buddhist tantric medicine in the Kālacakratantra', The Pacific World:Journal of the Institute of Buddhist Studies, New Series 10-11, 155-74.

2001, The Inner Kälacakratantra: a Buddhist Tantric View of the Individual, New York/Oxford: OuP.

2008, 'A convergence of medical and astro-sciences in Indian Tantric Buddhism: a case of the Kālacakratantra', in Akasoy, Burnett and Yoeli-Tlalim (eds), 2o9-22.

Yang Ga 2014, 'The Origins of the Four Tantras and an account of its author, Yuthog Yonten Gonpo', in Hofer (ed.), 154-77.

Yoeli-Tlalim, R. 2014, 'Medicine, astrology and divination', in Hofer (ed.), 90-104.

Yuthok Yonten Gonpo, tr. Thokmay Paljor et al. 2011, The Subsequent Tantra: From the Four Tantras of Tibetan Medicine, Dharamsala: Men-Tsee-Khang Publications. 


\section{Part 6}

Imagining Modern Medicine 



\title{
31 Places and Traces: Selections from Professor Ma Kanwen's 馬堪溫 Ethnography of 1955
}

\author{
Abridged by Penelope Barrett with an Introduction by Vivienne Lo 羅維前*
}

This chapter presents the ethnographic journey in 1954 of Professor Ma Kanwen, one of the 'Two Mas' who were members of the Institute for the History of Chinese Medicine and Medical Literature at the China Academy of Traditional Chinese Medicine (Zhongguo zhongyi yanjiuyuan 國中醫研究院, now China Academy of Chinese Medical Sciences (Zhongguo zhongyi kexueyuan 中國中 醫科學院), hereafter 'the Academy', during its founding years. The other Ma is, of course, Professor Ma Jixing, to whom this book is dedicated. Professor Ma Kanwen has also been a long-term colleague of ours since the days of the Wellcome Trust Centre for the History of Medicine, where he worked for 23 years. It is fitting that we should begin this section on 'Imagining Modern Chinese Medicine' in celebration of the man who bridges the two institutions that came together in the creation of the book.

His illustrious 6o-year career began at the prestigious Yenching University (now Peking University), one of the first modern universities in China, when he wrote his doctoral dissertation on the subject of famous Chinese physicians. He had been influenced by Li Tao 李濤, the father of a college friend. Li Tao was a famous historian of science, and director of Peking Medical College (Beiyi Xueyuan 北醫學院). After graduation, as a promising young researcher, Ma Kanwen was sent out into the field to create a photographic record of the historical sites of some 30 eminent physicians from 3 millennia spanning the pre-Qin era to the Qing period (1644-1911).

His project formed part of a new historical enterprise that came at time when there was a concerted official endorsement of Chinese medicine as a valid medical system. We can identify histories of Chinese medicine and medical figures throughout the imperial period, and notably in biographies of eminent physicians - so the programme was not entirely without precedent. But what was so extraordinary was that the new post-liberation' 1950s discipline of the History of Chinese Medicine meant that the past was being used (and in many senses re-created and re-configured) to serve the present and the future. Whereas the norm elsewhere in constructing 'modern' scientific medical systems was to discard the 'pre-scientific' past, the new revolutionary government intended to incorporate and scientise it. Professor Ma's account, which

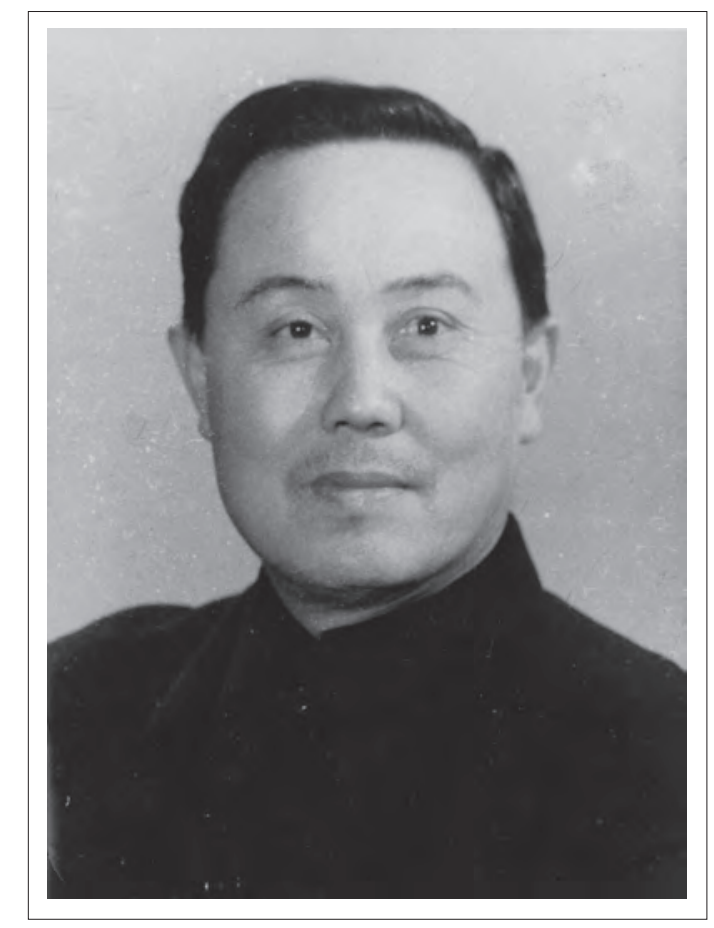

Figure 31.1 Professor Ma Kanwen 1927-2016. ㄷ Ma Kanwen

follows in part, is redolent with the political tensions that ensued, especially as Chinese medicine encountered the Cultural Revolution (1966-76), with its ruthless re-definition of tradition and drive to purge Chinese society of its superstitious and religious elements.

In 1953-4, the years leading up to the inauguration of the first modern institutions to teach Chinese medicine, in Shanghai, Guangzhou, Chengdu, Nanjing and Beijing, the identity and future of traditional medicine were caught up in the intense ideological debates that heralded the radical economic and social campaigns of the Great Leap Forward (1958-61). The Red $v s$ Expert controversies of early Communist China pitted numerous scientists against those committed to the ideals of the Communist revolution. Many Chinese physicians had trained abroad, and the Chairman of the Communist Party, Mao Zedong, turned against all those who, in his view, harboured untrustworthy political allegiances to hostile imperialist and capitalist regimes and their hierarchies of knowledge. He was also dealing with an impending split with the Soviet Union, and all that this would mean for the withdrawal of 


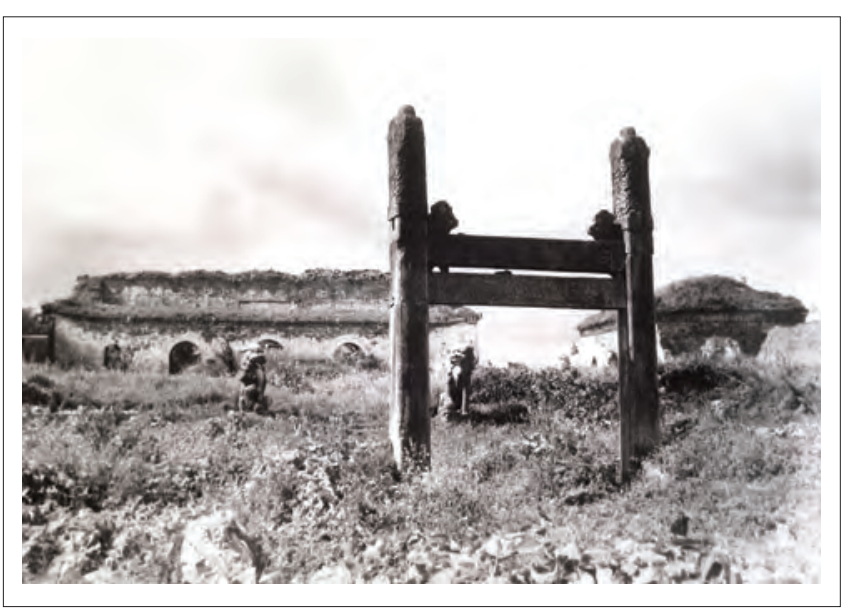

Figure 31.2 Ruins of Yuchen Daoist temple, Maoshan, on the site of Tao Hongjing's mountain retreat, Maoshan, Jiangsu province. () Ma Kanwen

Soviet support for medical infrastructure. Self-sufficiency was firmly on his agenda. ${ }^{1}$

In the revolutionary years, seeking support for the revolution, Mao had made historical pacts with practitioners of Chinese medicine, promising to support them following any CPC success. All this resulted, in the 1950s, in a sudden investment in traditional medicine in order to bring it to a par with modern medicine. In a classic Mao Zedong move, he turned the status quo on its head, decreeing that practitioners of Western medicine, who constituted a large section of the educated elite, should learn from China's 'national treasure' and that 'Western medicines and Chinese medicines should unite' (zhong-xiyi 中西醫). Like everything else, however, Chinese medicine would be subject to continuing revolution. It would be required to professionalise and adopt all the structures of a modern discipline: institutional education, hospitals, and clinical evaluation, and ultimately, to integrate its home-grown cultural genius with a modern, forward-looking scientific approach. $^{2}$

As famous practitioners of Chinese medicine congregated in Beijing to teach the doctors of Western medicine, Professor Ma Kanwen was also participating in this Chinese medical revolution aimed at renewing the tradition, by undertaking a parallel journey out of town to conduct fieldwork on ancient Chinese physicians, looking for temples, statuary and steles. During this personal voyage of discovery, he not only recorded what the local gazetteers said about the physicians, but personally visited many places in the north, south and west of China including Jiangsu, Hebei, Shaanxi and Zhejiang, where he talked to local residents and wrote down what he saw and heard,

\footnotetext{
1 Taylor 2005, p. 43 et passim.

$2 \quad$ Taylor 2005, pp. 3-39.
}

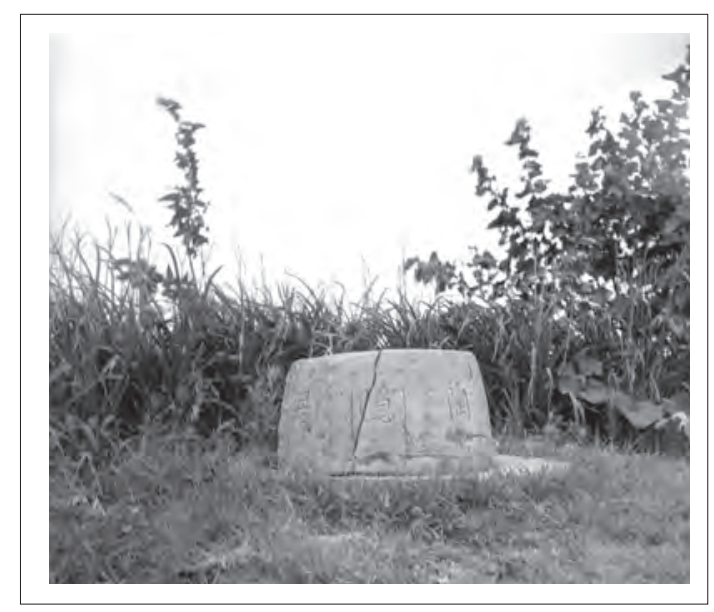

Figure 31.3 'Cinnabar Well' of Tao Hongjing, in front of Dongxu Daoist temple, near Tao village, Jintan county, Jiangsu province. (C) Ma Kanwen

as well as taking photographs. His record is an intimate account of his own physical encounter with the material culture of healing and healers. His declared purpose was to create a visual, photographic record, but his account is also a sensory history that goes beyond the visual. It documents physical sensations, sounds, qualities of light, and the daily rhythms of the journey.

In such a way, the historians at the Academy set out to create their new discipline not only from written documents, modern histories, and scattered textual references in local and family records, but also by reclaiming and finding ways to reconnect with this material culture: through conserving what was preserved at that time in places associated with ancient physicians, and at religious sites associated with festivals and cults. It was clearly important to build up a rich history that could legitimate the new enterprise, but as the following account testifies, the process of making that history was also an intensely personal experience. Professor Ma's aesthetic encounter embodied many of the conflicts inherent in the processes through which practitioners and historians of Chinese medicine were constituting a new collective identity.

By far the largest part of Professor Ma's account concerns the curious figure of Bian Que, a patron of the Chinese medical arts whose story and legacy is redolent of myth and magic. Ironically, in modern times Bian Que has been hailed as a founding father of scientific Chinese medicine, and is placed in a lineage of practice that distinguished itself from the practitioners of ritual medicine - the $w u$ 巫 (variously translated as 'witches', 'shamans' 'spirit mediums' 'ritualists'). Penelope Barrett has translated, abridged and edited extensive sections of this work below. Sadly, the account of the 30 physicians is too long to translate in its entirety here, but we append some photographs of sites associated with the other physicians and those who 


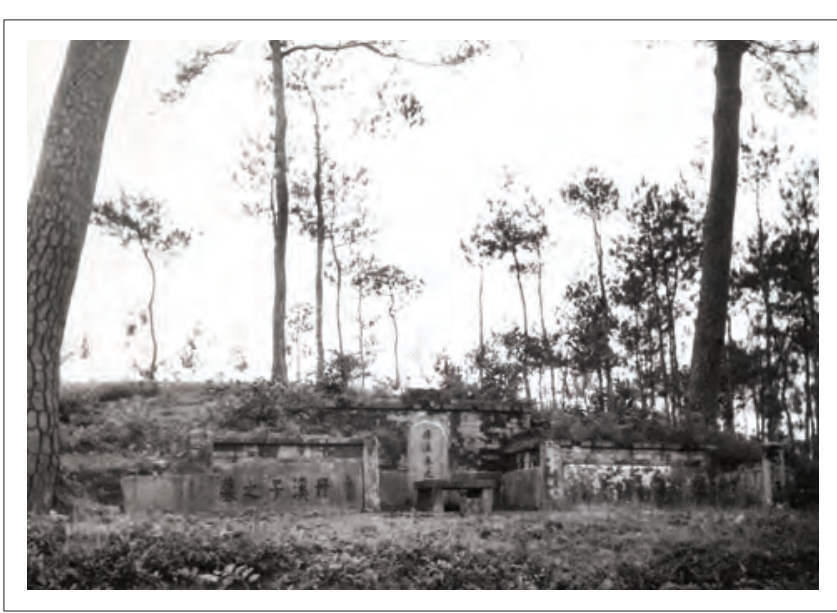

Figure 31.4 Tomb of Zhu Zhenhong in Dongzhu village, Yiwu, Zhejiang province. ( ) Ma Kanwen

contributed to medical traditions, to give a flavour of the full range of his work: from Tao Hongjing 陶弘景 $\left(45^{6}-536\right)$ to Zhu Zhenheng 朱震亨 (1281-1358).

A full record is archived at the Wellcome Library.

Against the background of all the political turmoil of the 195os, Professor Ma's travels were a rare opportunity to leave Beijing, and the following excerpts offer fellow scholars a glimpse of the challenges and rewards of his journey. Before his departure, Professor Ma had to make contact with the local authorities and other official bodies, to obtain assistance and accommodation in situ. By 1955 his preliminary work was nearing completion. However, for various reasons, including notably a succession of political movements, it was not then possible to pursue the project further to publication. Professor Ma and his fellow historians of Chinese medicine had clearly hoped to preserve a core part of China's cultural heritage through this field trip, but their enterprise was short-lived. Later campaigns against religious practice and superstition destroyed some of the precious remains that Professor Ma and his companions photographed. In the fuller draft of the chapter, the narrative is punctuated by recurrent elegiac descriptions of broken ruins, which acquire additional poignancy when read against the destruction of material culture, and of many people's hopes for new China, that would ensue during the subsequent Cultural Revolution period (1966-76).

Professor Ma retained a nostalgia for the project, so it gives us great satisfaction to reproduce a part of it here, in the hope that others will take advantage of his work and perhaps revive his ambitions to complete the record. His sustained interest in the continuities with the past, in this case in archaeological remains, represents a broader testimony to the survival of many traditional forms in Chinese medicine, which are emerging more widely in contempo- rary scholarship. ${ }^{3}$ The radical break with the past, once imagined as a feature of China's communist modernity, did not wholly rupture many aspects of lineage teaching, of religious healing, and oral transmission, especially in communities far from urban centres. ${ }^{4}$

As his postscript shows, in the last decade there has been a significant relaxation of constraints on constructing the historical identity of Chinese medicine, at least in the academic world, and efforts are being made to understand the contributions of religious organisations to the formation of medical practice in China's past. Professor Ma's textual history of Bian Que is as erudite as we would expect from a great scholar. His diary and reflections, but particularly the visual record, bring his journey alive and preserve data from a brief, but crucial moment for Chinese medicine in the mid-2oth century.

Professor Ma had worked for Li Tao as an Assistant Editor for China Bulletin of New Medicine (Xin yixue bao 新醫學 報), based at Peking Medical College. In 1954, he became a premier researcher in the department for the History of Medicine at the Central Academy of Health (Zhongyang weisheng yanjiuyuan 中央衛生研究院), before transferring to the new Academy in 1955.

After moving to Britain in 1987 , he worked as a Senior Research Fellow at the Wellcome Institute for the History of Medicine, Senior Research Fellow of the Joint Department of Human Sciences and Medical Ethics at the London Hospital Medical College, and Guest Professor of the Institute for the History of Medicine, Vienna University. He served as a council member for several professional organisations, including the International Society of Medical History, and the Standing Committee for the Translation and Publication of Joseph Needham's Works, and as a member of editorial committees for several journals, including the Chinese Journal of Medical History (Zhonghua yishi zazhi 中華醫史雜誌) and Acupuncture in Medicine.

He lectured worldwide and at the time of writing he was Honorary Senior Adviser to the Association of Traditional Chinese Medicine (UK), Senior Adviser to the World Federation of Chinese Medicine Societies, and Adviser to the China National Committee for the Standardisation of English Translation of Chinese Medical Terms. As an author, co-author or co-compiler, he published 12 books and dictionaries and 160 articles on Chinese medicine and the history of Chinese medicine. This list of achievements needs no further elaboration.

\section{Vivienne Lo 羅維前 2016}

\footnotetext{
$3 \quad C f$. the work of Volker Scheid, notably Scheid 2007.

4 See also Liao, Chapter 28 in this volume.
} 


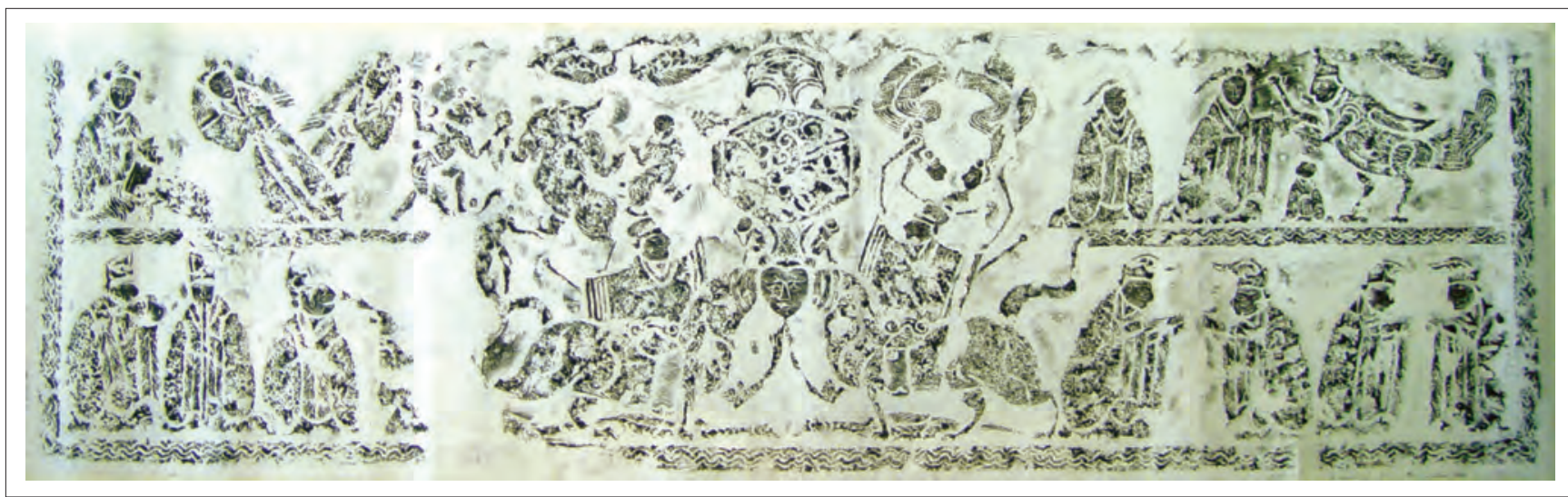

Figure 31.5 Tomb relief showing, in the top right-hand corner, Bian Que, the human-headed bird, administering acupuncture to waiting patients, with a child looking on from below. Tomb relief, Eastern Han $\left(25^{-220} \mathrm{CE}\right)$ dynasty, from Liangcheng 兩城, Weishan 微山, Shandong province. National Museum of China, image courtesy of Huang Longxiang 黃龍祥

\section{Excerpts from Ma Kanwen's Account}

\section{Bian Que 扁鵲 (6th-5th Century BcE)}

The main biographical source for Bian Que is Shiji 史記 (Records of the Historian), the earliest extant general history of China, compiled by Sima Qian 司馬遷 (traditional dates $145-86$ BCE; born 145 or 135 BCE, death unknown). ${ }^{5} \mathrm{He}$ is among the earliest and most renowned of the medical figures documented in that work, a distinction he shares with the Han physician Chunyu Yi 淳于意 (3rd century BCE). According to Shiji, Bian Que was a native of Bohai 勃海 in the ancient county of Mao 鄚, now Renqiu 任丘 county in Hebei province. He originally bore the name Qin Yueren 秦越人. As a young man, he worked at an inn in the local township, where he was initiated into the healing arts by a guest named Zhang Sangjun 長桑君, an expert healer, who passed on to him his secret remedies. Thereafter, he became an itinerant physician, travelling widely in the States of Qi 齊 (present-day Shandong), Zhao 趙 (which included areas in modern Inner Mongolia, Hebei, Shanxi and Shaanxi provinces) and Yan 燕 (present-day north Hebei and west Liaoning provinces) performing miraculous cures and gathering fame wherever he went.

He is supposed to have become known as Bian Que while practising as an itinerant physician in the state of Zhao. Eventually, this soubriquet eclipsed his personal name. 'Bian Que' is composed of two distinct semantic elements. The graph bian 扁 Chinese functioned in early China as a variant of bian 遍 (everywhere, ubiquitous), and presumably refers to his itinerant lifestyle. Que means magpie (also xique, lit. 'lucky magpie'). A lucky portent in China

Sima Qian, Shiji, 'Bian Que Can Gong liezhuan’ 扁鵲倉公列傳 (Biography of Bian Que and Cang Gong), juan 105. since antiquity, the magpie is described as an auspicious bird in Qin jing 禽經 (Classic of Birds), China's earliest ornithological treatise, ${ }^{6}$ and figures as a symbol of good fortune in tales and poetry. It was supposed, traditionally, to fly from place to place announcing good tidings with its song. Clearly, the fact that Qin Yueren's medical ministry was equated with the flight of the bird of good omen reflects the high esteem in which he was held. But also, it is certainly no coincidence that the name is linked with the people of Zhao, who revered birds as totems.

Among a collection of Han dynasty stone reliefs excavated at Liangchengshan 兩城山 in Weishan 微山 county, Shandong province, is a carving of a figure with a human head and bird's body (Fig. 5). Its mouth resembles a bird's beak, and in its hands it holds needle-like implements, with which it is apparently treating a patient. This figure recalls the assertion in the 'Zhaoshijia' 趙世家 (Biographies of the House of Zhao) chapter of Shiji that the first ancestor of the Zhao lineage had a 'human head and bird's beak'. ${ }^{7}$ After examining the evidence, scholars have concluded that the relief shows Bian Que practising medicine. ${ }^{8}$

Bian Que is said to have learnt and applied a range of medical techniques including acupuncture and moxibustion, drug preparation, and therapeutic massage. He is also credited with special expertise in pulse diagnosis. Shiji calls him China's first pulse-diagnostician, and presents him as a key figure in the early differentiation of a Chinese medicine, as groups of practitioners distanced themselves from the practice of magic in favour of treatments based on the emerging theories of the Yin and Yang channels and of Qi. In later times, the magical healer Bian Que

\footnotetext{
6 Qinjing, attr. Shi Kuang, 6th century вСE, juan 1.

7 Sima Qian, Shiji, juan 43.

8 See, for example, Lo in Lu and Needham 2002, p. xxxiii.
} 
would be re-imagined as a master of the classical medical arts, far removed from the worlds of spirit mediums. As Medicine King and tutelary hero of classical medicine, he would be particularly celebrated for his opposition to ritual medicine. Again according to Shiji, there were six types of illness that he would not treat, one of these being cases where the patient 'trusts in the spirit mediums and ritualists instead of medicine'. 9

Over the centuries, Bian Que's name recurs constantly in the medical literature and historical records, and successive generations have erected temples, steles and shrines in his honour in many parts of China. His status is illustrated by the story of the Ren Zong 仁宗 emperor of the Song dynasty (r. 1022-63), and the famous acupuncturist

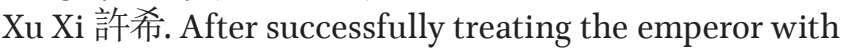
acupuncture when he fell ill in 1034 (1st year of the Jing You 景祐 era, 1034-8), Xu Xi had rewards and honours lavished upon him, whereupon he kowtowed to the west. When asked by the emperor why he did this, he replied that he was giving thanks to the ancient sage Bian Que, for it was only due to the teachings of Bian Que that he had been able to cure his sovereign's illness. Moreover he entreated the emperor to use the money he had been awarded to build a temple to Bian Que. The emperor granted his request and founded a temple to the west of Kaifeng, the Northern Song capital. This is the earliest known temple to Bian Que to have been established by imperial decree. Around 1057 (towards the beginning of the Jia You 嘉祐 era, 1056-63), Emperor Ren Zong fell ill once more, and drug treatment was ineffective. The emperor worshipped at the temple of Bian Que and subsequently recovered; thereupon he issued a decree bestowing upon Bian Que the title of 'Shen Ying Hou' 神應侯 (Prince to whom the Spirits Pay Heed), and in 1069 the temple was rebuilt by his order. ${ }^{10}$

\section{Fieldwork in Renqiu 任丘 County}

According to the Annals of Renqiu county, ${ }^{11}$ relics of Bian Que in the county once included an ancestral shrine, a temple, his former home, his tomb, and a temple dedicated to his teacher, Zhang Sangjun. During fieldwork based in the Maozhou 鄚州 locality in April 1954, the author discovered the existence of a temple to the 'Medicine King', about $2 \mathrm{~km}$ north of the East Gate of the old county town, and $20 \mathrm{~km}$ north of present-day Renqiu county. By that

‘信巫不信醫. Sima Qian, Shiji, 'Bian Que Can Gong liezhuan', juan 105 .

10 Toqtohan et al. (eds) Song shi, juan 462, 'Fangji' 方技 (Recipes and techniques).

11 Compiled by Liu Tong 劉統 (Qing period), edition engraved in 1762 (27th year of the Qianlong era of the Qing dynasty).

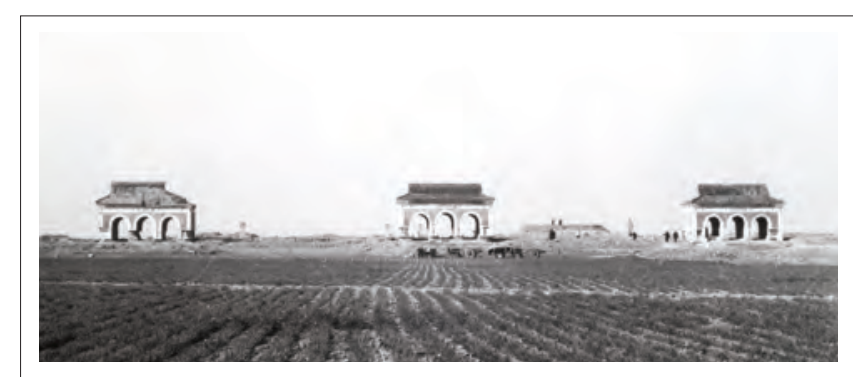

Figure 31.6 Entrance gates ('Mountain Gates') of the Three Kings temple complex, Renqiu 任丘 county, Hebei province. On the right, the gateway to the temple of Bian Que. (c) Ma Kanwen

time, all that remained of the temple was a monumental archway, constructed mostly of white stone, bearing the five-character inscription 'Chijian Yaowang miao' 敕建藥 王廟 (Temple of the Medicine King, erected by imperial order). To the left of the gateway stood the ruins of two further temples, on a similar scale: an inscription over the gateway of one read 'Chijian San Wang dian' 敕建三皇殿 (Hall of the Three Kings, erected by imperial order) and the other, 'Chi jian Wen Chang ge' 敕建文昌閣 (Pavilion of the Wen Chang, erected by imperial order) (Fig 6). The 'Three Kings' generally referred to Fuxi 伏羲, Suiren 燧 人 and Shen Nong 神農, three legendary ancient rulers and ancestral deities of the Chinese people, who were the object of popular cults. Wenchang is Wenchang gong 文昌 宮 or Wenqu xing 文曲星 ( $\delta$ Ursa Majoris), a constellation associated with fame and good fortune. This juxtaposition testifies to Bian Que's significance as a tutelary hero of medicine, and the reverence in which he was held.

On the date of construction of the temple, Wang Taoyun 王兆雲 (Song period) writes in Hui chen xin tan 揮塵新談 (New Discourses on Whirling Dust) that a temple dedicated to Bian Que was in existence at Maozhou prior to the Yuan period. ${ }^{12}$ However, there are no surviving records of the date or the precise circumstances of its foundation.

The Qing Annals of Renqiu county state that the ancestral temple ( $c i$ 祠) of Bian Que is located to the north of the town of Mao, and record several reconstructions. They also tell us that an annual temple fair was held there in the 4 th lunar month. ${ }^{13}$

A stele commemorating the reconstruction of the temple (now described as 'Ying Wang' 應王 Temple) in 1513 (8th year of the Zhengde era of the Ming dynasty) reads:

The old temple of 'Shen ying Wang' 神應王 (the King to Whom the Spirits Pay Heed) was located to the north of the town of Mao, more than $100 l i[c .50 \mathrm{~km}]$ from the

12 See Lü Chaoru 1948.

13 Annals of Renqiu county, juan 2, 'Jianzhi' 建置. 
seat of the prefectural government. The inscription no longer survives, so it is not known when it was originally constructed.

From the above we learn that by the Ming period, Bian Que had become known in Renqiu as 'the King to Whom the Spirits Pay Heed' (presumably a development of the name 'Shen ying hou' 神應侯 - the Prince to Whom the Spirits Pay Heed - which was conferred on him in the Song), and that he was worshipped in a temple situated to the north of the old town of Mao. The change of name from Bian Que miao 扁鵲廟 (Temple of Bian Que) to Yaowang miao 藥王廟 (Temple of the Medicine King) is explained by the fact that, in the Zhizheng 至正 era of the Yuan dynasty (1264-1294), the government had a temple to the Medicine King erected in every county of China. This is borne out by another stele commemorating the refurbishment of the Temple to the Medicine King at Maozhou by official order in 1584 (12th year of the Wanli era of the Ming dynasty). That stele also states that there has been a temple of the Medicine King at Maozhou for many years; according to tradition it was once a temple for the cult of Bian Que; when the local people fell ill they would go there to pray to him, and their prayers would quickly be answered; however the building collapsed because the foundations were insubstantial, whereupon the Wanli emperor gave orders for it to be rebuilt.

The reconstruction, possibly the most significant that this temple underwent in the Ming period, began in the third month of that year, and was completed in five months. According to the Annals of Renqiu county, the reconstructed temple was sited three $l i(c .1 .5 \mathrm{~km})$ north of the town of Maozhou. It was approached via a ceremonial gateway with three arches, beyond which a path flanked by incense burners and steles led to the entrance of the temple itself. Bian Que was worshipped in the imposing main hall; to east and west of this stood side halls, containing devotional statues of the 10 illustrious physicians of past ages - Wang Shuhe 王叔和, Zhang Zhongjing 張 仲景, Taiyi Lei Gong 太乙雷公 (Lei Gong the Great, the Thunder Duke), Chunyu Yi 淳于意 and Hua Tuo 華伦 in the east hall; and Sun Simiao 孫思邀, Huangfu Mi 皇甫 䍀, Han Kang 韓康, Ge Hong 葛洪 and Liu Shouzhen 劉 守真 in the West Hall.

A second phase of rebuilding took place from 1591 to 1593 (19th to 21st years of the Wanli era of the Ming dynasty). The occasion for this was a severe bout of illness suffered by the Wanli emperor, during which the empress dowager had incense lit and prayers said for his recovery in Medicine King temples throughout the land. By then, the temple buildings at Maozhou had grown dilapidated, although the foundations still stood firm. In view of this, orders were issued for a temple hall dedicated to the 'Three Kings' to be added to the west of the temple of the Medicine King itself, and at the same time the existing temple halls and statues were renovated and beautified. On this occasion, the building work took two years, being completed in 1593 (21st year of the Wanli era of the Ming dynasty). A stele engraved at that time states that the Three Kings worshipped in the new temple hall were Pao Xi 庖 羲 (i.e. Fu Xi 伏羲), Shen Nong 神農 - the Divine Farmer, and Huangdi 黃帝 - the Yellow Emperor. The temple was refurbished once more in the Qing period (there is a stele commemorating this, dated 1861 [11th year of the Xian Feng era of the Qing dynasty]).

According to the Annals of Renqiu county, corroborated by local informants, the anniversary of Bian Que's birth fell on the 28th day of the fourth lunar month. During the whole of that month, pilgrims flocked from far and wide to the Temple of the Medicine King at Maozhou to worship and offer incense. At its height, the Temple Fair with its constant stream of pilgrims was a famous sight, and one of the most important events of the traditional year in the area. The temple itself was said to be one of the 'great temples under heaven'; a drawing in juan 1 of the Annals gives some idea of its size and magnificence in its heyday. Very sadly, the temple was completely destroyed by fire in the Japanese invasion of 1937. By the time of the author's visit, nothing remained to be seen but some ruined arches, amid a blank expanse of open fields.

The original home of Bian Que was located, according to local informants, some $20 \mathrm{~km}$ north of the county town, in a village called Yaowang zhuang 藥王莊 (Medicine King hamlet or mansion), outside the eastern gate of Maozhou town. ${ }^{14}$ The county Annals ${ }^{15}$ preserve poems on the home and burial place of Bian Que by writers including the famous Ming scholar Yang Shiqi 楊士奇 (1364-1444) and other literati, demonstrating that both sites had become significant destinations for literati by the early modern period. When the author went to Yaowang zhuang to investigate, the eponymous village was still in existence; however any trace of Bian Que's dwelling had long since vanished. Local people also reported that the village had been named in honour of Bian Que after his lifetime. As to Bian Que's burial place, according to the Annals of Renqiu county, it was located approximately $3 l i(c .1 .5 \mathrm{~km})$ northeast of Maozhou town, or 3 li north of Yaowang zhuang. ${ }^{16}$

\footnotetext{
14 See also Annals of Renqiu county, juan 1, 'Zhai' 宅.

15 Annals of Renqiu county, juan 11, 'Yiwen xia' 藝文下.

16 Annals of Renqiu county, juan 1, 'Yu di muzhong' 與地墓冢.
} 
The author duly went in search of it, but could find no vestige of that either.

Da Qing yitongzhi 大清一統志 (Comprehensive Geography of the Great Qing Dynasty) states:

The ancestral temple ( $c i$ 祠) of Zhang Sangjun [Bian Que's teacher] lies three li north of Maozhou town. To the west of the temple is the Temple of the Medicine King, where Bian Que is worshipped. ${ }^{17}$

However, according to the Qing Annals of Renqiu county, though there was at one time a shrine to Zhang Sangjun to the north-east of Maozhou town, it had ceased to exist by the Qianlong era $(1736-95){ }^{18}$

It should be said that temples and mausolea of Bian Que are or have been alleged to exist in many parts of China, tombs being particularly numerous. For instance, the Annals of Xianyang 咸陽 county in Shaanxi place the tomb of Bian Que in Xianyang. A mausoleum to Bian Que is found in Fudaocun 伏道村, in Tangyin 湯陰 county, Henan province; and according to the local gazetteer, it was there in Fudaocun (a village $20 \mathrm{li}[\mathrm{c} .1 \mathrm{okm}]$ north of the county town) that Bian Que was assassinated by an envious imperial physician. Zhenjiu dacheng 針尒大成 (The Great Compendium of Acupuncture and Moxibustion) explains that the village is called fu dao (lit. prostrate on the road) because he died by the roadside. ${ }^{19}$ In Bian Que mucitang ji 扁鵲墓祠堂記 (Notes on the Mausoleum of Bian Que), published in 1248 on the cusp of the Jin and Yuan dynasties, Zhang Dou 張都 writes that the tomb of Bian Que at Fudao was destroyed in a violent uprising in the Zhenyou 貞佑 era of the Jin dynasty (1213-16). There was also a tomb of Bian Que, known as 'Lu yi zhong' 盧 醫塚 (grave of physician Lu), in Lilicun 孝裡村 village in Qing 請 county, Shandong province. In the northern outskirts of Jinan city, there was one called Lu yi muzang 盧醫墓葬. Both of those were tombs only, without any temple buildings. Other putative tomb sites were found in the village of Nanchencun 南陳村 in Ma'e zhen 馬額鎮 township, 30 li (c. $15 \mathrm{~km})$ north of Lintong 臨潼 county, ${ }^{20}$ and east of the county town of Jixian 濟縣 in Shandong province. There is little evidence to support any of these claims, and it is probable that many so-called tombs were actually memorials. It goes without saying that it would be very hard to establish which of them, if any, is the true burial place of Bian Que. The one thing that can be affirmed with certainty is that all these traditions reflect a widespread cult of the figure of Bian Que.

17 Da Qing yitongzhi, juan 16.

18 Annals of Renqiu county, juan 2, 'Jianzhi' 建置.

19 Yang Jizhou, Zhenjiu dacheng, juan 11.

$20 \quad$ See the Annals of Lintong county.
Fieldwork in Neiqiu 内丘

According to the county Annals, ${ }^{21}$ the village of Shentoucun 神頭村, at the foot of Queshan 鵲山 mountain in Neiqiu county, contained many relics associated with the figure of Bian Que, including a temple dedicated to him. The writer therefore undertook a field trip to the village, in search of traces of Bian Que. ${ }^{22}$ Neiqiu is an area in the south-west of Henan province, near to Bian Que's birthplace in Renqiu county in the adjoining province of Hebei. In the Warring States period (475-221 BCE), it was known as Xing 邢, and formed part of the Zhao kingdom. It acquired its present name during the Sui period (581-618). The village itself nestles among mountains. Looking westward from the county town, one sees an unbroken chain of lofty peaks: this is Pengshan 蓬山 mountain, a continuation of the Taixingshan 太行山 range. Tradition has it that Bian Que practised in these mountains as an itinerant physician, and one of the peaks bears the name Queshan 鵲山 (Bian Que or Magpie mountain). Another peak has been renamed Taizi yan 太子岩 (Prince's crag) in accordance with a tradition that the Prince of Guo 虢 retired to Pengshan after being revived from a death-like coma by Bian Que. ${ }^{23}$

Shentoucun is located some $63 l i(c .31 .5 \mathrm{~km})$ from the county town of Neiqiu. On 10 November 1954, with the assistance of the head of Neiqiu Health Department, the author and his colleague Ding Jiantang 丁鑑塘 set out on an eight-day bicycle journey up winding mountain paths to the village. Because of the steepness of the mountains and the rugged terrain, we often had to dismount and push and drag our bicycles, and we finally arrived in Shentoucun after several hours on foot. The village was completely surrounded by mountains, which formed a natural fortification with only one way in. On entering, one saw a flowing brook with dry-stone constructions on both banks, made from many-coloured mountain rocks. Interspersed with clumps of persimmon trees weighed down with vermilion fruit, they gave the village an extremely picturesque air. At the far end of the village, we came upon the temple to Bian Que mentioned in the Annals. Built on a hillside connected with Queshan, it rose against the backdrop of Pengshan mountain and the adjoining peaks of Queshan and Taizi yan. If one stood on the mountain slope to the south of the village and looked north, one saw tier upon tier of temple buildings amid the dense green of ancient

$21 \quad$ Neiqiu xian zhi 內丘縣志 (Annals of Neiqiu county), compiled by Qiao Zhonghe 喬中和, edition engraved in 1642 (15th year of the Chongzhen era of the Ming dynasty).

$22 \quad$ 10-17 November 1954.

23 Zhongguo lishi ditu ji 中國歷史地圖集 The Historical Atlas of China), Tan Qixiang 譚其驤 (ed.) 1982, vol. 4. 


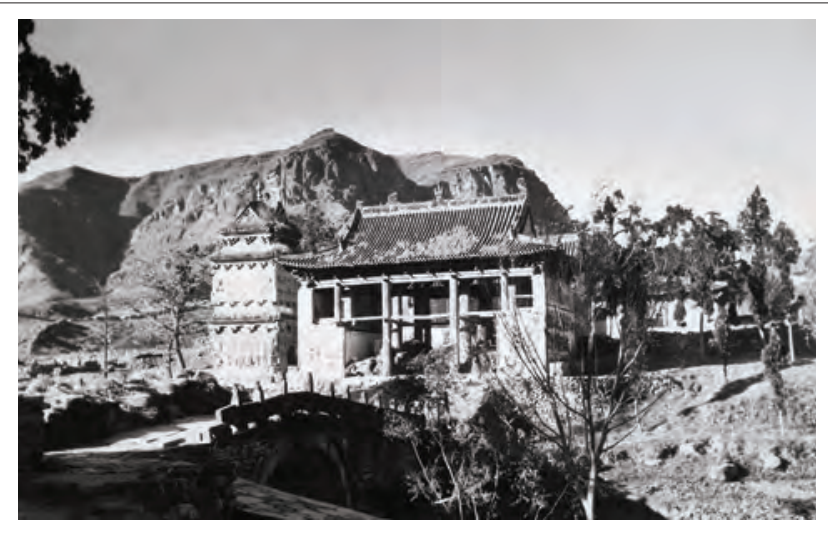

Figure 31.7 Temple of Bian Que, Shentou village 神頭村, Neiqiu 內 丘 county, Hebei province. (C) Ma Kanwen

conifers, and towering in the distance beyond them, the mighty mountain-range of Pengshan, creating a vista of solemn, primordial beauty (Fig. 7).

In front of the temple was an imposing stone bridge. According to an inscribed stele in the temple, dating from 1543 (gui-mao year of the Jiajing era of the Ming dynasty), this bridge was constructed to commemorate Bian Que's transcendent medical skill. Originally called Huisheng qiao 回生橋 (Bridge of Reanimation), it was twice renamed, first as Longdeng qiao 龍登橋 (Soaring Dragon bridge), and then in 1539 (ji-hai year of the Jiajing era), as Jiulong qiao 九龍橋, (Nine Dragon bridge). The latter name alluded to the nine ravines of Queshan, which were transformed into torrents and waterfalls when it rained in the mountains. The bridge was rebuilt and extended in 1543 using dressed blocks of locally quarried white jade: it had a single arch, and was 1 zhang 7 chi high, 2 zhang 6 chi wide, and 2 zhang 8 chi long (approximately $5.6 \mathrm{~m}$ high x $8.6 \mathrm{~m}$ wide $\mathrm{x} 9.2 \mathrm{~m}$ long according to today's equivalents). The external faces of the bridge were clad with square slabs carved in relief and the apex of each of the 16 piers was ornamented with carvings of lion cubs. Most of the reliefs depicted motifs from Chinese popular history, including Grand Duke Jiang 姜 catching fish, Su Wu 蘇武 herding sheep, and Wu Song 蘇武 killing the tiger, as well as auspicious creatures and rare birds and flowers, in a lively, realistic style. Beneath it rushed the babbling brook, so clear and limpid that you could see right to the bottom.

To the south of the bridge rose a hillside; to the east there stood a huge rock with the two characters yao shi 藥石 (Medicine Stone) engraved on it). The inscription was dated 'Wanli era, gui-mo year', i.e. 1583 . The Medicine Stone was flanked by two steles. One, dated 1355 ( 15 th year of the Zhizheng era of the Yuan dynasty) was inscribed 'Record of prayers for rain answered at Queshan'. The text was worn away and only partially legible, but the gist was clear: In the summer of that year there had been a severe drought, and for three months on end, there were no fish to be had and the crops dried up and withered in the fields. Officials from Shunde lu 順德路 set off to the temple of Bian Que to pray to him for rain, but when they were only halfway there, they saw dark clouds billowing up from Queshan mountain, and then without warning the rain fell in torrents. The stele was erected as a record and thank-offering. The second stele was inscribed: 'Record of the prince heeded by the spirits at Queshan'. Sadly the surface of the stele had crumbled away making the rest of the inscription illegible. However, the style and dimensions suggested that it formed a pair with the former stele, and it could also be dated to the Yuan period.

On the hillside beyond the southern end of the bridge stood nine, mighty, century-old cypresses. Their trunks were so thick that two men could not join hands around them, their colossal roots reached deep into the cracks and crevices in the stone, and their tall, spreading branches formed a dense green canopy. Also on the hillside stood another large rock with five characters 'Jiu long qiao shi bai' (Nine dragon bridge stone cypress[es]) engraved on it. It was too thickly overgrown with moss to make out the date of the inscription. At all events it appeared that the planting and naming of the nine cypresses had a close connection with the Nine Dragon bridge.

Up on the slope to the north of the 'Nine Dragon bridge' stood Que lo - Bian Que tower. The tower, which was approximately 5 zhang tall (about $16.5 \mathrm{~m}$ ), contained various stone steles dating from the Qing period. Built into two of the walls were small stone tablets from the Yanyou era of the Yuan dynasty (1314-20), mostly containing poems celebrating the feats of Bian Que or praising the beauties of the temple and its surroundings. Beyond the tower was a ceremonial gateway, only slightly less tall than the pagoda. In front of the gate, on the right-hand side, were two steles; one of them, erected in 1069 (2nd year of the Xining era of the Song dynasty), commemorated the restoration of 'Shenying hou miao' (the Temple of the Prince to Whom the Spirits Pay Heed); the other, erected in 1283 (gui-mo year of the Yuan dynasty), recorded the restoration of 'Shen ying miao' (the Temple of [the One] to Whom the Spirits Pay $\mathrm{He})$ at Queshan. Both steles were over 8 chi $(c .2 .6 \mathrm{~m})$ tall.

On the left-hand side stood the Hall of Steles, a three-storey building which was even taller than Que lo. Each storey had carved reliefs on all four walls; those on the middle storey appeared to represent the Four Heavenly Guardians of Buddhist iconography. The storeys got progressively narrower from bottom to top, like a pagoda. The upper part of the building was covered in yellow and green glazed tiles. The overall effect was tasteful and artistic. Even 
though it had become dilapidated over time, and the tiles had fallen away in many places, while wilting grass and weeds sprouted from it here and there, the building still retained a unique style.

The hall contained a stele dated 1268 ( $5^{\text {th }}$ year of the Zhiyuan era), which commemorated the restoration of 'Que wang shen ying wang miao' (The temple of King Que, Prince to Whom the Spirits Pay Heed). The stele, which was made from bluish-green stone, was $6 \mathrm{~m}$ tall, o.94m wide and $0.3 \mathrm{~m}$ thick. The top of the stele had an arched shape and was ornamented with sinuous dragons, and the base was in the shape of a turtle. The older inhabitants recalled the scenes at the annual temple fair held in the third lunar month, when a constant stream of pilgrims and other visitors would flock here, jostling one another to touch the stele - which was said to cure illness - with their hands or with a coin. In places, the surface of the stele had been rubbed as smooth as a mirror, and the inscription was all but erased. From the traces of lettering that could still be made out, it was possible to gather that the text had been composed by Wang E 王鶚 (119o-1273), a high-ranking scholar in the imperial Hanlin academy. It was written out in kaishu regular script by Liu Bingzhong 劉秉忠 from Xingtai 邢臺 (in present-day Hebei province), a senior official at the Yuan court, who held the post of imperial tutor. The heading at the top of the stele, written in seal characters, was contributed by Liu Yu 劉鬱, former office administrator at the Imperial Metropolitan Secretariat (Zhong shusheng dushi 省中書省都事.

The stele summarised the life history of Bian Que as recounted in Shiji and gave dates for construction and restoration work at the temple of Bian Que up until the Yuan period. It stated that a temple to Bian Que had existed here from the Han (206 BCE-220 CE) to the Tang dynasty (618-907), but was destroyed in fighting during the turbulent Five Dynasties period (907-6o). When peace was restored in the Xiande era (954-9) of the Later Zhou, the last of the Five Dynasties, the military governor Chen Sirang 陳思讓 had the temple rebuilt. By this time, there was already a stone tablet in place describing Bian Que as 'Wang' (King), but it was not known when that appellation first became current. The stele also recounted that at the beginning of the Jiaoyou era (1056-63), the Emperor Renzong fell ill, and all attempts to cure him were in vain, until he went to the temple of Bian Que to pray for recovery and his prayers were answered. This was the origin of the title 'Shen ying hou' (Prince to whom the Spirits Pay Heed). Also, when crops failed in 1069 (the 2 nd year of the Xining era of the Northern Song dynasty), the district magistrate of Xingzhou 邢州, Li Guanglu 李光祿, prayed at the temple of Bian Que, whereupon the harvest became abundant. In gratitude, physicians and the general public contributed money to have the temple refurbished. It was renovated again in 1190 (1st year of the Mingchang era of the Jin dynasty), under the auspices of the Neiqiu county administration, but it subsequently burnt down, and was not rebuilt until the Yuan.

Passing through the gateway, one came to the sacrificial hall of 'King Que' (Que wang xiandian) 鵲王獻殿. In front of the hall was an open courtyard, with tall, ancient cypress trees growing in it. The whole area was overgrown with weeds, now dry and withered, and littered with remnants of steles. Some of them lay toppled on the ground, some of them had been smashed to pieces so that the top of the stele with the epigraph was disconnected from the body of the inscription, and of others only the base remained. The scene was one of utter chaos. Among the best preserved was a row of four steles, over a metre tall, still upright and relatively intact. One, erected in 1543 (gui-mao year of the Jiajing era of the Ming dynasty) was headed Queshan dingjian Jiulongqiao ji 鵲山鼎建九龍橋記 (Record of the construction by the state of the Nine Dragon bridge at Queshan). The remaining three, erected in 1570 (4th year of the Longqing era), contained texts relating to the consecration of the temple. One of the latter was inscribed in the hand of Gui Youguang 歸有光 (1507-71), a famous essayist of the Ming period and graduate of the highest metropolitan jinshi examination. At this time, Gui Youguang was a leading literary figure in the classicising 'Tang and Song movement', so the fact that he could have been induced to write a dedication indicates that the temple of Bian Que at Neiqiu enjoyed a high profile.

Behind the Sacrificial Hall was Que wang dian 鵲王獻 殿 (the Hall of King Que). This was the main hall of the temple, and the largest building in the complex. With its roof of dazzling yellow and green glazed tiles, glistening in the sun, it was a magnificent and arresting sight. To its right stood an enormous cypress, even taller than the trees to the south of the Nine Dragon bridge, its trunk more than three arm-spans in circumference. According to the village elders, it was planted in the Tang, a claim which is plausible in light of the evidence for the construction date of the temple seen by the author. Behind the Hall of King Que was Que wang houdian 鵲王後殿 (the Rear Hall of King Que) and various other buildings that bore no direct relevance to Bian Que, including Qian Nainai dian 前奶 奶殿 (the Front Hall of the Temple of the Grandmother Goddess), Xilou 戲樓 (a traditional opera theatre), Shuifo dian 睡佛殿 (Hall of the Reclining Buddha) and Yuhuang ge 玉皇閣 (Pavilion of the Jade Emperor). At the time of our visit, those buildings were in use by the local primary school as classrooms and offices, and nothing remained 
of the devotional statuary or sacrificial altars. They had vanished into the past, along with the pomp and festivities of the annual temple fair, which once drew worshippers and tourists from as far afield as 1,00o li (50okm).

A curious tale about the construction of the temple at Bian Que still survived in local tradition: the project was superintended by Yuchi Jingde 尉遲敬德, a high-ranking general of the early Tang period. On being invited by the local authorities to view the finished temple, the stern general was so impressed that he could not forbear from smiling with delight. However, rumour had it that the general only ever smiled when he was killing his enemies. That smile struck such terror into the county official that he hanged himself the very next day. Yuchi Jingde, who had intended to reward the official, and was quite unaware of the reason for his suicide, had a small temple erected in his memory alongside the main temple building. Whether or not this tale has any basis in fact, it suggests the former glory of the temple of Bian Que. The Annals of Neiqiu refer to a stele which read:

Constructed for the third time by Jingde of the great Tang empire in the first year of the Zhenguan era of Emperor Taizong of the Tang dynasty (627).

However, this stele no longer exists.

It should be particularly noted here that, in addition to the steles described above, the writer saw many other stone inscriptions during his fieldwork in the area. Unfortunately however, most of them were damaged and fragmentary. According to local informants, the interior and exterior of the temple of Bian Que had once been a veritable forest of steles. However, the area fell victim to successive waves of fighting and violence, and each time, large numbers of steles were knocked down and smashed. Many of them were removed by local people and used in walls and foundations, as stairs and table-tops. In all the sources identified by the author, there is no record of any further reconstruction work after the close of the Qing period. Of the steles that I saw during fieldwork, about 3 o were relatively intact or partially legible, most of them dating from the Yuan to the Qing periods.

Having heard local people talk of the numerous engraved stones once to be found in and around the temple, I carried out a search in the vicinity for any inscribed records that might still exist. To my excitement, among the stones on the riverbank close to the Nine Dragons bridge, I found a fragment of a stele, used at one time by the villagers for treading their laundry. When I took it down to the water's edge and rinsed it, I was able to ascertain, to my surprise and delight, that it dated from the Xiande era (954-9) of the later Zhou dynasty, in the Five Dynasties period. The fragment consisted of the very top of the stele and one of

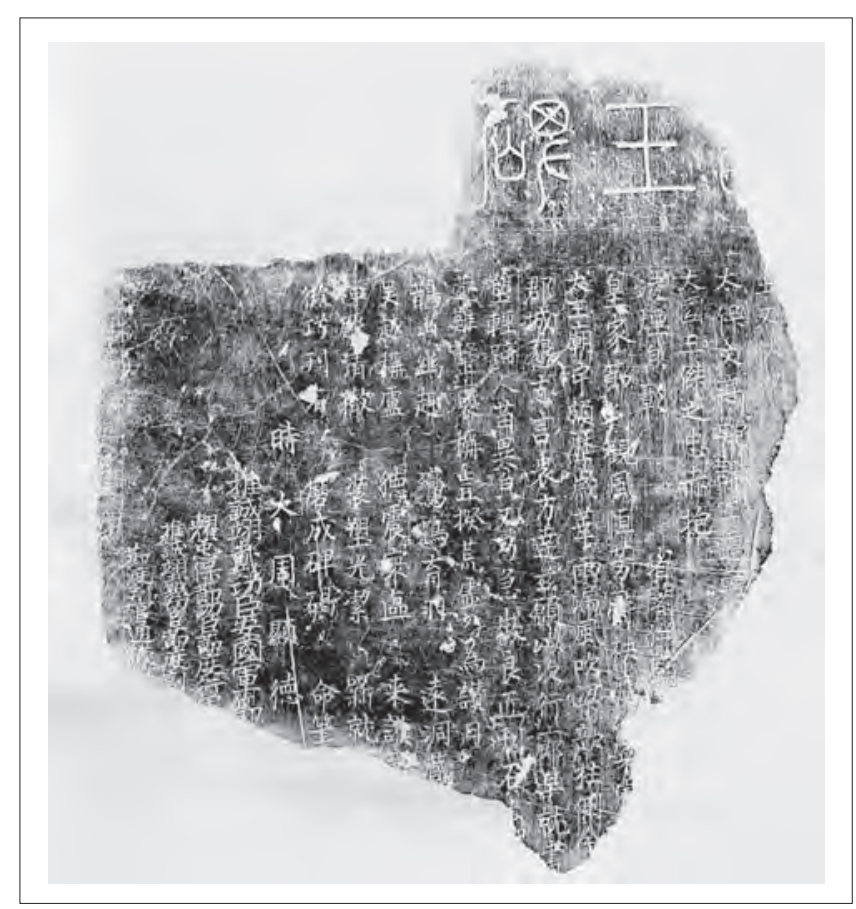

Figure 31.8 Fragment of a stele of the Xiande era of the Later Zhou dynasty (954-6o), at the temple of Bian Que, Shentou village 神頭村, Neiqiu 内丘 county, Hebei province. (C) Ma Kanwen

the upper corners, and measured about 1 chi (c. o.3m) in height and a little more than $1 / 2$ chi in breadth. From the epigraph, one edge of the characters 'Wang bei' 王碑 (King stele) and Que 鵲 ([Bian] Que) was preserved (see Fig. 8). What remained of the body of the inscription was largely illegible, but it evidently recorded a major rebuilding of the temple. One of the better preserved passages read:

The temple of the Great King, much dilapidated by the passage of time, the rain leaking into it and the wind blowing through it, the rafters... the columns collapsed... Mount Queshan is a place of delightful seclusion, where the mandarin duck and crane abound, and deep caverns... In the time of the Xiande era of the great Zhou dynasty.

From the Xiande stele, it can be gathered that the temple was founded in the Tang dynasty at the latest, and that it was a splendid building. We also learn that Pengshan had changed its name to Queshan by the time the inscription was composed, and that there were statues and inscribed tablets in the temple. This information was corroborated by another stele in the Hall of the Steles to the left of the temple gateway, which also confirmed the date of the restoration and gave the full name of the official responsible for it.

The latter stele carried the epigraph 'Guochao chongxiu Que wang shen ying wang miao bei' 國朝重修鵲王神應王 廟碑 (Stele on the imperial reconstruction of the temple of King Que, the King to Whom the Spirits Pay Heed). The inscription was written by Wang E 王鶚 in 1268 ( 5 th year 
of the Zhiyuan era of the Yuan dynasty). It contained the words:

Since the Han and Tang dynasties, people have made offerings to the statues of old. In the Xiande era of the [later] Zhou, when peace was brought to the country, the military governor Chen Sirang 陳恩讓 had it rebuilt. At that time, there was already a stele calling [Bian Que] 'King', but it is not known in what period this name was conferred.

In addition to providing supporting evidence for the Xiande stele fragment, this passage attests to the antiquity of the temple foundation.

The Xiande stele was the most ancient artefact associated with Bian Que and his temples that was observed during this fieldwork exercise, and it was one of the most significant finds.

Another early record that came to light was 'Record of the rebuilding of the temple of the Prince to Whom the Spirits Pay Heed' (Congxiu shen ying hou miao ji 重修神 應侯廟記), dated 1069 (2nd year of the Xining era of the Song). It recounted the exploits of Bian Que, the renaming of mount Pengshan as Queshan in memory of Bian Que, and the story of how, at the beginning of the Jiayou era of the Song, the Emperor Renzong contracted a disease that would not respond to medication, and was miraculously cured by praying to Bian Que, upon which Bian Que was granted the title of 'Shen ying hou' - the prince to whom the spirits pay heed.

According to the Annals of Neiqiu county, and the author's own research and observations during fieldwork, besides the stele inscriptions, a large body of poetry was composed from the Tang to the Qing (1644-1911) celebrating Bian Que, the Great Temple, and the scenery of Pengshan and Queshan. The earliest known example is a poem by $\mathrm{Yu} \mathrm{Gu}$ 於鵠 ( $f l$. Dali-Zhenyuan eras of the Tang dynasty, 766-8o5) on the mountain cave where Bian Que is supposed to have made his home: 'Qin Yueren dongzhong yin' 秦越 人洞中吟 (Qin Yueren chants in the cavern). This provides further confirmation that, by the Tang, the temple of Bian Que already existed and had moreover become a famous landmark in its own right.

As to the date of the original foundation of the temple, the Annals of Neiqiu county state: 'It already existed in the Han and Tang, but the original date of construction is unknown'. ${ }^{24}$ The inscriptions described above and other written sources support the idea that it is a foundation of great antiquity.

“漢唐已有之,始建不詳”.

\section{Ma Kanwen - Conclusions and Epilogue}

Field research in sites associated with famous Chinese physicians of antiquity can deepen and round out our understanding of their lives and times, and provide new source material for medical historians, offering insights not available from the written records. Many findings are of ethnographic and archaeological interest, and shed light on the lingering resonances of famous physicians from Bian Que onward. The figures discussed above were all aligned, to a greater or lesser extent, with the three major belief systems of China - Confucianism, Buddhism and Daoism - and thus reflect the diverse and complex cultural background of Chinese medicine. Some of them were deified and became the subject of institutionalised cults, although the intentions of the ruling classes in supporting these cults no doubt diverged from the concerns of ordinary worshippers, rooted in folk religion.

The fieldwork described here was conducted more than half a century ago, and it has proved impossible to ascertain the current state of many of the sites mentioned. Some monuments, like the Temple of Bian Que in Neiqiu and the Temple of the Medicine King in Yaoxian 耀縣, with its inscribed steles and stone caves, have been restored, but only after suffering further damage during the 10 years of the 'Cultural Revolution'. It should be noted that a number of local authorities have recently been active in restoring monuments with medical associations. Thus for example extensive restoration work has been carried out on the Temple and other historic relics in Neiqiu.

From 24-25 October 2005, I was invited to Shentoucun, Hebei province, to advise on the restoration of the Temple of Bian Que. It was a delight to return to my old haunts of half a century ago, and to see for myself the transformation of both temple and village. According to those in charge, the restoration was actually based on the photographs and fieldwork reports from my visit in 1954. Sadly the original Nine Dragon bridge had been swept away by floods, and it had not been feasible to replace it with an exact replica. Moreover, the Yuan (Mongol) period (1271-1368) steles that once stood to the south of the bridge, on either side of the 'Medicine Stone', had both disappeared. However, with those exceptions, the temple area had by and large been faithfully restored to its former appearance. Some dozen stone steles dating from the Song period onward had been preserved inside the temple, and most of these were now inset into a new eastern wall to ensure their continued survival. To my disappointment, however, the mid-1oth-century-stele from the Xiande era of the Later Zhou dynasty (954-6o) that I discovered in the village in 1954 seemed to have vanished without a trace. By the time 
the restoration work got underway, the Bian Que Tower that had stood beside the temple was in a state of ruin and had been despoiled of its polychrome glass tiles, so that it proved impossible to salvage any of the original materials to use in restoration.

On 29 October of the same year, I attended the 'Bian Que Cultural Festival and Temple Fair', which was organised by the county government and presided over by city- and county-level officials. As of old, it attracted visitors from far and wide, as well as thousands of local residents, who lined the entrance to the temple and thronged the hillsides round about, dressed in their holiday best. The entire village was lit up by lanterns and festooned with colourful decorations. In the limpid light of a bright, clear northern autumn, the newly restored buildings, set amid tall pines and cypresses, looked especially magnificent against the backdrop of the thickly wooded Queshan and Pengshan mountains, resplendent in their autumnal colours.

On 30 October, I chaired a colloquium on 'Bian Que and his culture', convened by the local authorities, which brought together more than 100 participants, including scholars from the fields of cultural studies, history, ethnography and history of medicine from across the country, as well as city and county officials from the Xingtai area, for in-depth discussions of the figure of Bian Que, his significance, the events of his life, and sites and artefacts associated with Bian Que in Neiqiu county. This must surely have been the most remarkable commemorative event that the temple has witnessed in its long history. It was very gratifying to hear from local officials that the temple buildings and artefacts in Shentoucun had been accorded protected status as national cultural heritage. I was also informed that the great temple at Maozhou was under reconstruction under the auspices of Renqiu county government.

Besides these projects, according to the modern redaction of the Annals of Yiwu 義烏 county, ${ }^{25}$ the local Bureau of Health, Bureau of Culture, and Science and Technology Commission had jointly funded the reconstruction of the Temple of Zhu Zhenheng 朱震亨, with a dedicatory inscription by the famous calligrapher Sha Menghai 沙 孟海 (1900-92). This last restoration project entailed one major change in layout: the tombs of Zhu Zhenheng's two sons and heirs to his medical lineage were relocated from their former position on either side of their father's tomb to a new spot behind it.

To the best of this author's knowledge, no other place on earth possesses such a rich, extensive and ancient heritage of historic sites related to medical culture as does China.

25 Yiwu xian zhi bianzuan weiyuanhui (eds) 1987, pp. 644-5.
When I embarked on the fieldwork described here, I was a young man with my life before me. The conviction that I was achieving something concrete with my work was enough to sustain me and spur me on, during the long, hard treks on foot through the mountains and remote rural areas, exposed to the elements and extreme weather. Today, as a much older man with the wisdom of hindsight, I look back on that time with mixed emotions. I only hope that from now on China will protect and cherish her medical sites and other historic monuments so that they will never again suffer wanton destruction, and this precious inheritance and the cultural continuity they represent can be carried forward into the future.

\section{Bibliography}

\section{Primary Sources}

Anon., Da Qing yitongzhi 大清一統志 (Comprehensive Geography of the Great Qing Dynasty), first published 1743.

Feng Zhao 馮照 et al., Jintan xian zhi 金壇縣志 (Annals of Jintan county), Chengwen chubanshe, 1896 (bing-shen year of the Guangxu era of the Qing dynasty); revised edn, 1921/2 (xin-you year of the Republic of China).

Gong Jiajun 龔嘉俊 and Li Ke 李揢, Hangzhou fu zhi 杭州府志 (Annals of Hangzhou Prefecture), stereotype print, 1922 (11th year of the Republic of China); photographic facsimile, Taibei: Chengwen chubanshe, 1974.

Li Hongzhang 李鴻章 et al., Guangdong tongzhi 廣東通志 (Annals of Guangdong), photographic facsimile, Shangwu yinshuaguan (Commercial Press), 1934 (23rd year of the Republic of China).

Liu Tong 劉統修 (ed.), Renqiu xian zhi 任丘縣志 (Annals of Renqiu County), 1762 (27th year of the Qianlong era of the Qing dynasty).

Qiao Zhonghe 喬中和, Neiqiu xian zhi 內丘縣志 (Annals of Neiqiu County), edn engraved in 1642 (15th year of the Chongzhen era of the Ming dynasty).

Ruan Yuan 阮元 and Li Hongbin 李鴻賓, Guangdong tongzhi 廣東通 志 (Annals of Guangdong), 1864 (3rd year of the Tongzhi era of the Qing dynasty), reprinted 1914; photographic facsimile, Shangwu yinshuaguan (Commercial Press), 1st edn, 1934 (23rd year of the Republic of China.

Shi Kuang 師曠 (6th century BCE) (attr.), Qinjing 禽經 (The Classic of Birds). Modern edn: Shanghai: Shanghai guji chubanshe, 1989.

Sima Qian 司馬遷, Shiji 史記 (Records of the Historian), 'Bian Que Can Gong liezhuan’ 扁鵲倉公列傳 (Biography of Bian Que and Cang Gong), juan 105. Modern edn, Beijing: Zhonghua shuju 1973(?). Toqtohan (Tuo Tuo 脫脫) (c. 1343) et al. (eds), Songshi 宋史 (Song Dynastic History). Modern edn: Zhonghua shuju, 1977.

Yang Jizhou 楊繼洲 (1522-1620), Zhenjiu dacheng 針炎大成 (The Great Compendium of Acupuncture and Moxibustion), 1601.

Yiwu xian zhi bianzuan weiyuanhui 義烏縣志編纂委員會 (eds) 1987, Yiwu xian zhi 義烏縣志 (Annals of Yiwu County), modern edn, Hangzhou: Zhejiang renmin chubanshe.

Yu Shuyun 於書雲 and Zhao Xifan 趙錫蕃, Peixian zhi 沛縣志 (Annals of Pei County), stereotype print, 1920 (9th year of the Republic of China).

Zhang Dou 張都, Bian Que mucitang ji 扁鵲墓祠堂記 (Notes on the Mausoleum of Bian Que), 1248. 
Zhang Shaotang 張紹棠 and Xiao Mu 蕭穆, Xuzuan Jurong xian zhi 續纂句容縣志 (Annals of Jurong County, continued), 1904 (3oth year of the Guangxu era of the Qing dynasty).

Zhu Xin 朱忻 et al., Xuzhou fu zhi 徐州府志 (Annals of Xuzhou prefecture), edn engraved in 1874 (13th year of the Tongzhi era of the Qing dynasty).

Zhu Zigu 諸自穀 et al., Yiwuxian zhi 義烏縣志 (Annals of Yiwu county), engraved 1802 ( 7 th year of the Jiaqing era of the Qing dynasty).

\section{Secondary Sources}

Lei, S. Hsiang-Lin 2014, Neither Donkey nor Horse: Medicine in the Struggle over China's Modernity, Chicago: University of Chicago Press.
Lo V. 2002, 'Introduction', in Lu Gwei-Djen and Needham, xxv-li. Lu Gwei-Djen and J. Needham 2002, Celestial Lancets: A History and Rationale of Acupunture and Moxa, London: Routledge Curzon.

Lü Chaoru 呂超如 1948, Yaowang yu Mozhou Yaowang miao kao 藥王 與鄚州藥王廟考 (A Study of the Medicine King and the Temple of the Medicine King at Maozhou), Guohua yinshua chang, (37th year of the Republic of China).

Scheid, V. 2007, Currents of Tradition in Chinese Medicine, 1626-2006, Seattle: Eastland Press.

Tan Qixiang 譚其驤 (ed.) 1982-7, Zhongguo lishi ditu ji 中國歷史地 圖集 (The Historical Atlas of China), vol. 4, Beijing: Zhongguo ditu chubanshe.

Taylor, K. 2005, Chinese Medicine in Early Communist China, 1945-63: A Medicine of Revolution, London; New York: RoutledgeCurzon. 



\section{Visualisation in Parasitological Research: Patrick Manson and his Chinese Assistants}

\author{
Shang-Jen $L i$ 李尚仁
}

Patrick Manson, the 'father of tropical medicine', played an important role in the transformation of the study of tropical diseases into a specialty. Manson was the medical advisor to the Colonial Office and exerted crucial leverage in the foundation of the London School of Tropical Medicine, which trained medical men serving in the colonies. ${ }^{1}$ Manson spent his early career in China, where he made some of his most important scientific breakthroughs. In 1877, at the Chinese treaty port of Amoy, Manson conducted a series of feeding experiments on the human filarial worm, and demonstrated that the mosquito was the intermediate host of the parasite. This was the first discovery to identify the role of the insect in the transmission of human disease. It provided a new orientation for research about tropical medicine with a focus on the insect-host role in the transmission of parasitic diseases. ${ }^{2}$

When we read Manson's filarial research reports, one feature that immediately attracts our attention is the parasite drawings published along with these reports. Some of the pictures are more detailed than others. For example, the drawings of canine filarial worms, such as Filaria immitis and Filaria sanguinolenta (Figs 1, 2), show greater detail in the anatomical structures of reproductive organs and mouths than those of the human filarial worm Filaria hominis sanguinis (Figs 3, 4). This was not because Manson paid more attention to the former. The latter, in fact, was the real focus of his research. This phenomenon was simply caused by the fact that the adult canine filarial worms were substantially larger than the human filarial embryos. Manson, moreover, observed the worms with the aid of a microscope. As Ian Hacking points out, microscopic observation is not 'passive looking'; it involves 'learning by doing.' ${ }^{3}$ Seeing with a microscope is a skill that requires substantial training and which involves active manipulation of both the instrument and the specimen. What Manson saw was mediated by a microscope and was, to a large extent, conditioned by the resolution of the lens, the preparation of the slides and his skill operating the instrument. These factors, in turn, contributed to the completion of Manson's drawings, which were representations of what he had observed through the microscope. This paper examines Patrick Manson's visual-

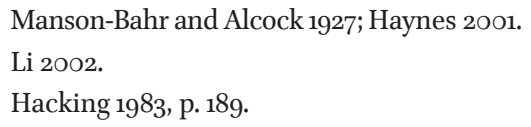

isation skills with regard to filarial worms, analysing the techniques he developed and deployed during the course of his research. Instead of conducting an iconographic or semiotic study of the printed images of the parasites, this chapter focuses on the scientific practice that rendered the production of the images possible. Finally, I also discuss the role of Manson's Chinese assistants in the visualisation process.

\section{The Microscope}

During the period in which Manson conducted his research, carrying out a microscopic examination of filarial worms was not a simple task. Manson encountered patients suffering from elephantiasis and lymph scrotum and began treating these patients in 1871 after he was transferred to Amoy. Elephantiasis and lymph scrotum were disfiguring diseases (Fig. 5). Although at the time, Manson believed the diseases were caused by inflammation resulting from repeated malarial infections and did not suspect that they were caused by parasitic infections, he still conducted microscopic examinations of the blood of some of the patients. He was not, however, able to observe parasites in either patients' urine or in their blood.

In fact, I kept a man for three or four years for the purpose of watching the progress of his lymph scrotum, and though I am convinced now that this man's blood contained filariae, yet, on account of my examining probably only a small part of one slide and with a high power, I always missed them. ${ }^{4}$

Successful microscopic observation depends on skills learned through repeated practice. Microscopic observation of different objects, such as bacteria, parasitic worms, protozoon, animal and plant tissues, often requires different levels of skill and nuanced variation in technique. When encountering a new research subject, even an experienced microscope observer may struggle for a period of time to learn a new technique. Even though Manson had received solid training in microscopy when he was an undergraduate student at Aberdeen University, he still had to work diligently to hone his filarial worm observation skills. Manson discovered that two species of filarial worms, Filaria immitis and Filaria sanguinolenta,

$4 \quad$ Manson 1887a, p. 37. 


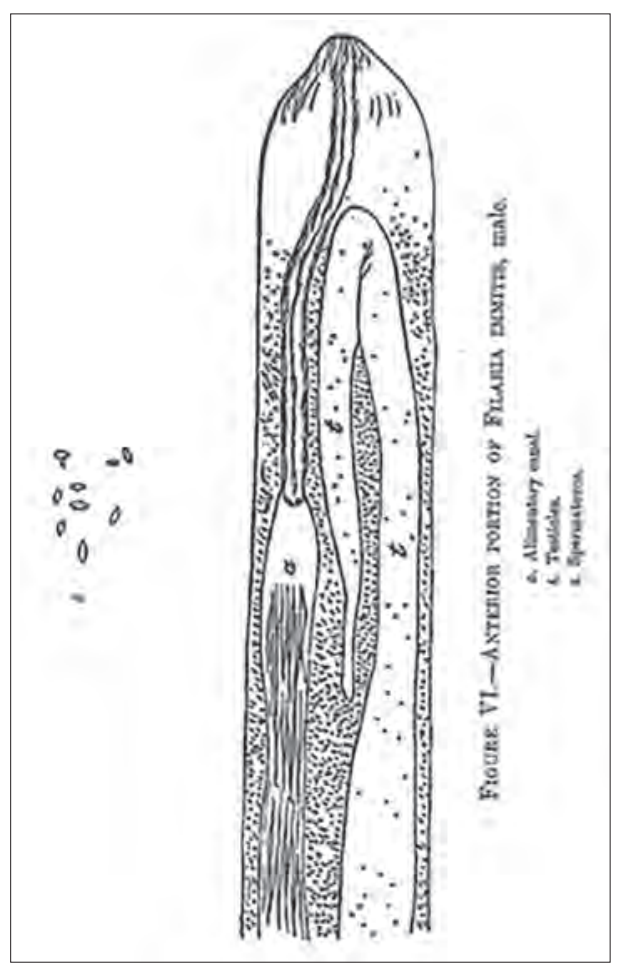

Figure 32.1 Filaria immitis. From Manson, 'Report on Haematozoa', Medical Reports, Customs Gazette, no. 13 (1877), p. 22

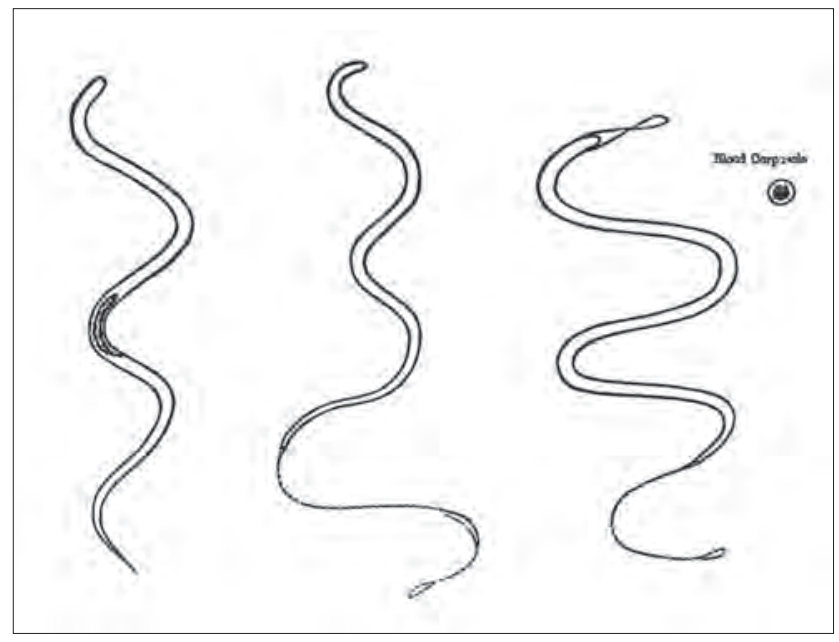

Figure 32.3 Filaria hominis sanguinis. From Manson, 'Report on Haematozoa', Medical Reports, Customs Gazette, no. 13 (1877), p. $3^{2}$

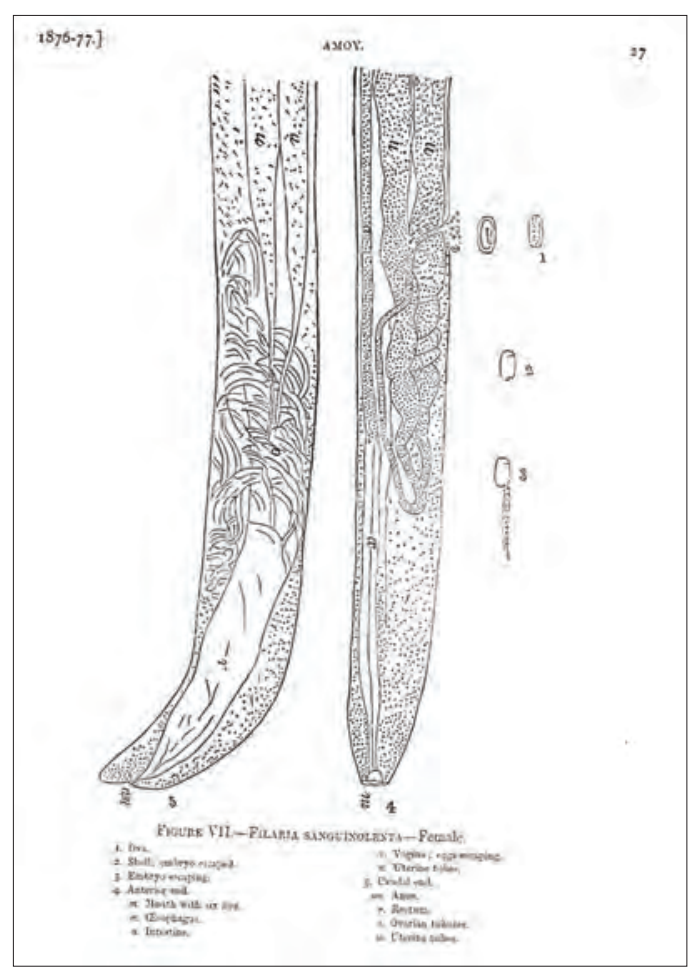

Figure 32.2 Filaria sanguinolenta. From Manson, 'Report on Haematozoa', Medical Reports, Customs Gazette, no. 13 (1877), p. 27

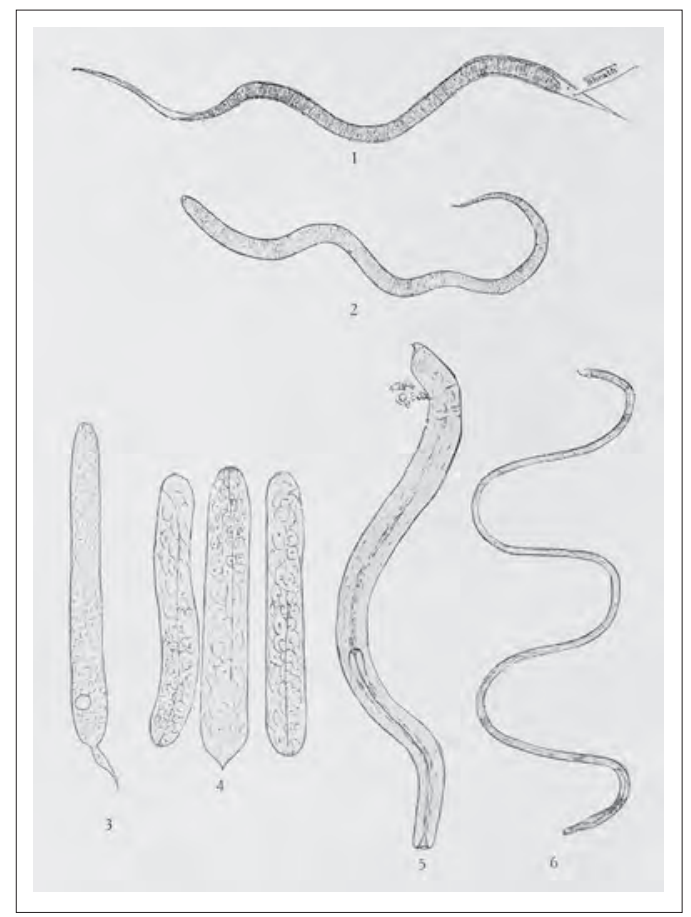

Figure 32.4 Filaria hominis sanguinis. Selection of Manson's drawings of the metamorphosis of Filaria bancrofti in the mosquito. 1. Few hours after ingestion; 2. Embryo released from sheath; 3 . About three days later; 4 . About five days later; 5 . About six days later; 6. Final stage in mosquito. From Manson-Bahr and Alcock, 1920, pl. viI. (C) Wellcome Library, London, Moo126oo 
infected dogs in China. He proceeded to conduct numerous post-mortem examinations of the infected dogs and undertook microscopic observations of the two species of filarial parasites. Canine filarial worms are substantially larger than human filariae. As a result, Manson could investigate the morphology, development and life history of canine filariae with greater ease. In the meantime, he also practised his filarial parasite observation skills. Manson called his approach 'comparative pathology'.

Manson's biographers, Alcock and Manson-Bahr, mention that Manson bought a 'more powerful' microscope during his furlough in England. ${ }^{6}$ They do not explain what they mean by the term 'more powerful', however. In this case, this term does not necessarily indicate a microscope with higher magnification power. ${ }^{7}$ In fact, Manson warned against using a lens with too great a capability for magnification.

The power employed as a searcher should not be a high one, a quarter or half-inch is sufficient, but power lower than these will do, provided they will distinctly define a blood corpuscle. ${ }^{8}$

Selecting a suitable lens and developing lens technique appropriate to the microscopic organism under investigation is of crucial importance for the success of observation. With regard to these issues, Manson is likely to have gained great insight from Timothy Richard Lewis, whose work he consulted during his furlough. Lewis provided technical instruction for microscopic observation of human filarial worms, which probably proved helpful to Manson. Lewis cautioned the observer never to assume 'that the Filariae are to be detected by taking a mere peep through the microscope' and that the slides should 'be carefully and systemically examined; a lateral and horizontal stage-movement being very useful for this purpose' because it ensured that every part of the slide was examined. This was time-consuming work that demanded patience. The examination of a slide took approximately 15 minutes. Moreover, 'any one who imagines that they can be detected with the same ease as a white-blood corpuscle had better not make the

5 Manson, 1887a, p. 31. For more on Manson's comparative pathology, see Li 2002, pp. 216-18; Haynes 2001, pp. 49-5o.

$6 \quad$ Manson-Bahr and Alcock 1927, p. 43.

7 For an analysis of 19th-century discussions of microscope selection for various purposes, see Gooday in Lightman (ed.) 1997, pp. 425-32.

8 Manson 1877 a, p. 37 . It is possible that Manson bought a microscope with better optic qualities, such as higher resolution, less achromatic and spherical aberration, etc. A technique invented by Joseph Lister in the mid-19th century to reduce achromatic and spherical aberration brought about a major advance in microscopy. See Bracegirdle in Bynum and Porter (eds) 1993, p. 112. attempt'. Sometime observers had to examine several slides in order to detect the parasites. ${ }^{9}$ Lewis argued that the selection of a suitable lens was of great importance to the observation process.

The search should not be undertaken with too high a magnifying power, but it should be sufficiently high to define the outline of a red-blood corpuscle quite distinctly. I have found that a good two-third of an inch objective answers the purposes of a searcher admirably; it embraces a tolerable large area so that the preparation can be examined in a comparatively short time; but care should be taken to keep the fine adjustment screw constantly moving, so as to examine the deeper as well as the superficial layer of fluid in each field as it passes under observation.

If anything unusual was detected, Lewis advised, the observer should replace the low-power objective with a one-fourth inch or one-eighth inch objective. ${ }^{10}$

Manson must have been impressed by the significance of Lewis' instruction on this microscopic technique as he quoted from the above passage in the first report on filariasis he penned after his reading in the British Museum. ${ }^{11}$ His technical advice was almost an exact copy from Lewis. For example, he also warned 'others against a hasty examination of the blood, and against concluding that, because no filariae are found, none exist'. At least six slides were to be prepared for one patient. Each slide had to be carefully examined. ${ }^{12}$ Manson was also aware of the importance of light for the microscopic study of filarial worms. He pointed out that strong light rendered observation of 'the very transparent body of the haematozoon' difficult and caused eye fatigue in the observer. ${ }^{13}$

From Manson's and Lewis' reports, it is clear that microscopic observation differs greatly from observation carried

\footnotetext{
$9 \quad$ Lewis 1872, pp. 18-19.

10 Ibid., pp. 19-2o. The one-third inch or one-fourth inch in Lewis' and Manson's texts refers to the focal length of the objective. An objective with shorter focal length has greater magnifying power. After 1875 the objectives produced by Carl Zeiss included one inch, two-third inch, one-third inch, one-fourth inch and onesixth inch. See Carpenter 1891, p. 34. Today's microscopes specify the magnifying power of their objectives. In the 19th century, objectives of the same focal length would differ in magnifying power if they were made by different manufacturers. The observer had to use micrometers to calibrate the magnifying power of his lens. On the use of micrometers, see Beale 188o, pp. 41-5. Neither Manson nor Lewis mentioned either the brands or the magnifying powers of their microscopes.

$11 \quad$ Manson 1875 , p. 8.

$12 \quad$ Manson 1877a, p. 37.

13 Ibid. p. 37. For more on the use of light in 19th-century microscopy see Gooday 1997, pp. 425-32.
} 
out using the naked eye. They are different ways of seeing. ${ }^{14}$ To see with a microscope involves not only the eyes, but also the fingers. The observer needs to move the slide and the adjustment screw of the microscope carefully, and it is a desideratum that the movement be coordinated with the act of seeing. To borrow a phrase from Bruno Latour, 'visualization and cognition' is 'thinking with eyes and hands'.15

\section{The Slide}

Besides a familiarity with the morphological features of the parasite and the possession of microscopic observation skills, it was equally important that the observer learn techniques for blood drawing and slide preparation. These techniques were crucial for successful detection of parasites under the microscope. When discussing techniques for drawing blood, Lewis suggested the following method:

A piece of narrow tape is coiled tightly round the end of one of the fingers or toes, so as to produce a little temporary local congestion of the part, but not to such an extent as to cause the slightest pain; and with a clean, finely-pointed needle the finger is gently pricked... I find it a good plan to squeeze out only a very small drop, and transfer it altogether to one slide by drawing the edge of the covering glass along the tip of the finger so as to scrape off the 'droplet'. The glass is then carefully pressed on to the slide by a gliding motion, in order to produce as thin a layer of fluid as possible, and to ensure that all the fluid removed is retained beneath the cover, because there is a tendency on the part of the fluid to carry the Hæmatozoa towards the edge of the slide....

The ways in which investigators drew blood from patients could determine the outcome of microscopic examination. Lewis, for example, found that he occasionally had to prick more than one finger of the same patient because he sometimes failed to find any filarial worms in several slides made from the blood of one of the patient's fingers, but observed numerous worms in blood from other fingers. He suspected that the tiny hole made by the puncture was 'plugged by fat', and, as a result, the parasites, which were significantly larger than red blood cells, were prevented from coming out. ${ }^{16}$

Manson emphasised that if blood was not drawn out after pricking a patient's finger, it was important not to press the finger too forcefully. The fact that blood failed to be released might simply be due to the small size of the

\footnotetext{
14 Berger 1972.

15 Latour 1986.

16 Lewis 1872 , pp. 18-19.
}

puncture. As a result, even if blood could be forced out by pressing the finger, the filariae in the blood might not come out along with it. Instead, another finger should be pricked. Placing 'too much blood under the cover glass', moreover, should be assiduously avoided. The observer should simply scrape off 'the finger with the edge of glass... and then slide along till the blood has almost reached the circumference of the covering glass'. This was because most of the time the filarial worms were 'found quite close to the edge of the patch of blood, and, should this escape from below the covering glass, the chance of finding them' would be extremely slim. ${ }^{17}$ Manson later improved Lewis' method of slide preparation. He no longer divided a drop of blood into six slides. He also gave up the use of covering glass. Instead he put 'the entire drop between two slides, using one as covering glass for the other'. Manson claimed that this would greatly reduce the chance of the parasites slipping out 'from under the covering glass' and could also save time during slide preparation. ${ }^{18}$ This improved slide-making technique enabled Manson to procure research materials with greater efficiency.

The examination of parasites in patients' blood constituted only one part of Manson's filarial research. To investigate the life cycle of the parasite, it was necessary to observe its metamorphosis in its intermediate host, the mosquito. This task required additional observational skills which Manson developed during the course of his research. He devised the following procedures. First, mosquitoes were killed with chloroform.

The legs and wings and head were torn off. The thorax was then separated from the abdomen, each being placed on a separate slide. In the case of the abdomen the contents were expressed by rolling a penholder from the free to the severed thoracic end; and a cover-glass was then placed on the expressed blood. If necessary, a little water or sulphate of soda solution... was used to soften the blood and allow of the easy removal of the two large ovisacs, which, when crushed, obscure the slide very much. The thorax is best treated by being broken up, and teased out with a couple of sharp needles in a droplet of water, before the cover-glass is applied.

Manson pointed out that with slides prepared in this way using 'an inch objective' could easily detect 'the parasites among the tissue of the insect'. After finishing an examination, if a filarial specimen were to be preserved, the observer should carefully lift up the cover-glass and use 'a fine forceps' to remove

the large pieces of mosquito debris... . After drying for a day, or better, two, it may be stained with an aniline dye

\footnotetext{
17 Manson 1877a, pp. 37-8.

18 Manson 1877b, p. 1.
} 
(gentian violet answers very well), and after washing and redrying mounted in Canada balsam.

If the filariae were from the abdomen of the mosquito, it was not necessary to stain the specimen as the 'red colour of the blood' would provide 'sufficient contrast to display, at all events, the outline of the parasites'. However, Manson emphasised that the observer should also examine 'fresh and unstained preparations', because although 'stained specimen' could well reveal an 'outline', they could not show 'the details of internal structure'. ${ }^{9}$

Michael Lynch has correctly argued that we should 'replace a preoccupation with images on the retina (or, alternatively 'mental images' or 'pictorial ideas') with a focus on the 'externalised retina' of the graphic and instrumental fields upon which the scientific image is impressed and circulated'. ${ }^{20}$ It should be pointed out that the 'instrumental fields' of Manson's parasitological research included not merely the microscope but also techniques for drawing blood, preparing slides, and staining. ${ }^{21}$

\section{Chinese Assistants}

Manson argued that, as most of the Chinese infected by filariae did not develop symptoms or afflictions, such as chyluria or swelling of the scrotum and lower limbs, even if they did come to the hospital, physicians might not suspect that they harboured the parasites. And those patients who suffered from mild 'varicose of lymphatic gland' or 'lymph scrotum' would not seek medical assistance in order to specifically treat these afflictions. In order to develop a better statistical estimate of the prevalence of filariasis, Manson decided to examine the blood of patients who came to the hospital even if they were seeking medical attention with relation to other conditions. ${ }^{22}$ In 1877 , Manson reported that he had trained two Chinese assistants to help him with excessively tedious and laborious work. Any Chinese person who came to the hospital - regardless of whether they had any symptoms of filarial infection or not - was examined by a Chinese assistant, as long as they were willing to have blood drawn from their fingers. At least six slides of blood were made for each patient, and these

\footnotetext{
19 Manson 1884, p. 370.

$20 \quad$ Lynch in Lynch and Woolgar (eds) 1990, p. 154.

21 Hacking claims that 'many of the chief advances in microscopy have had nothing to do with optics'. They were, instead, the result of improved techniques for staining, specimen preparation and the use of light; Hacking 1981, p. 192.

22 Manson 1877b, p. 1.
}

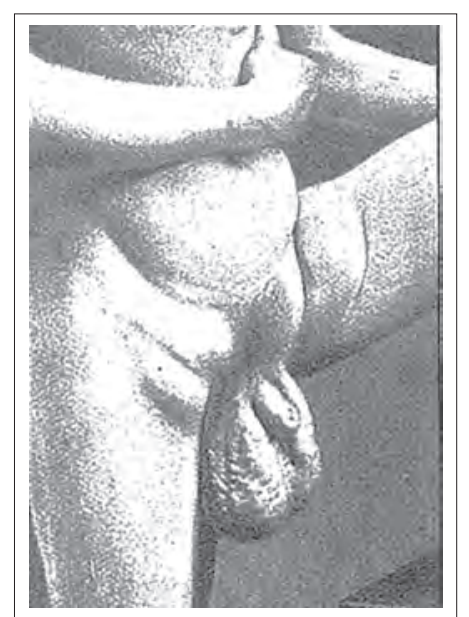

Figure 32.5 A Chinese patient suffering from Elephantiasis. From Manson, 1898, p. 475

slides were then carefully examined. The slides for 190 patients were examined, and Manson and his assistants were surprised to find that 15 of the patients were infected with the filarial worm. As a result, Manson was able to derive a rough statistical estimate of the prevalence of the disease in areas around Amoy. ${ }^{23}$ Moreover, Manson was also able to collect numerous clinical materials for his filarial research. This was an area in which Manson succeeded where Lewis had failed. Lewis could not persuade his patients to stay in the hospital for a long enough period to allow him to conduct his research, and he found it difficult to follow up his patients because he usually did not possess information as to their whereabouts. ${ }^{24}$ Manson, on the other hand, was able to gain access to a virtually limitless supply of research materials by using his Chinese assistants effectively.

In his study of the history of experimental genetics, Robert Kohler argues that enlargement of the scale of research was the key to success for scientists using fruit flies as experimental organisms. The repeated occurrence of some mutations, which could easily be overlooked if the research scale was not big enough, caught the attention of researching scientists and enabled them to design experiments that subsequently led to important discoveries. ${ }^{25}$ Similarly, the enlargement of Manson's scale of observation by means of his assistants enabled him to discover 'filarial periodicity'.

When Manson announced that the mosquito was the intermediate host of the filarial worm in 1878 , he had not yet discovered the 'filarial periodicity' phenomenon. Two years later he noticed that while one of his assistants often

\footnotetext{
23 Manson 1877a, p. 31.

24 Lewis 1872 , pp. 8-10.

$25 \quad$ Kohler 1994, pp. 46-9.
} 


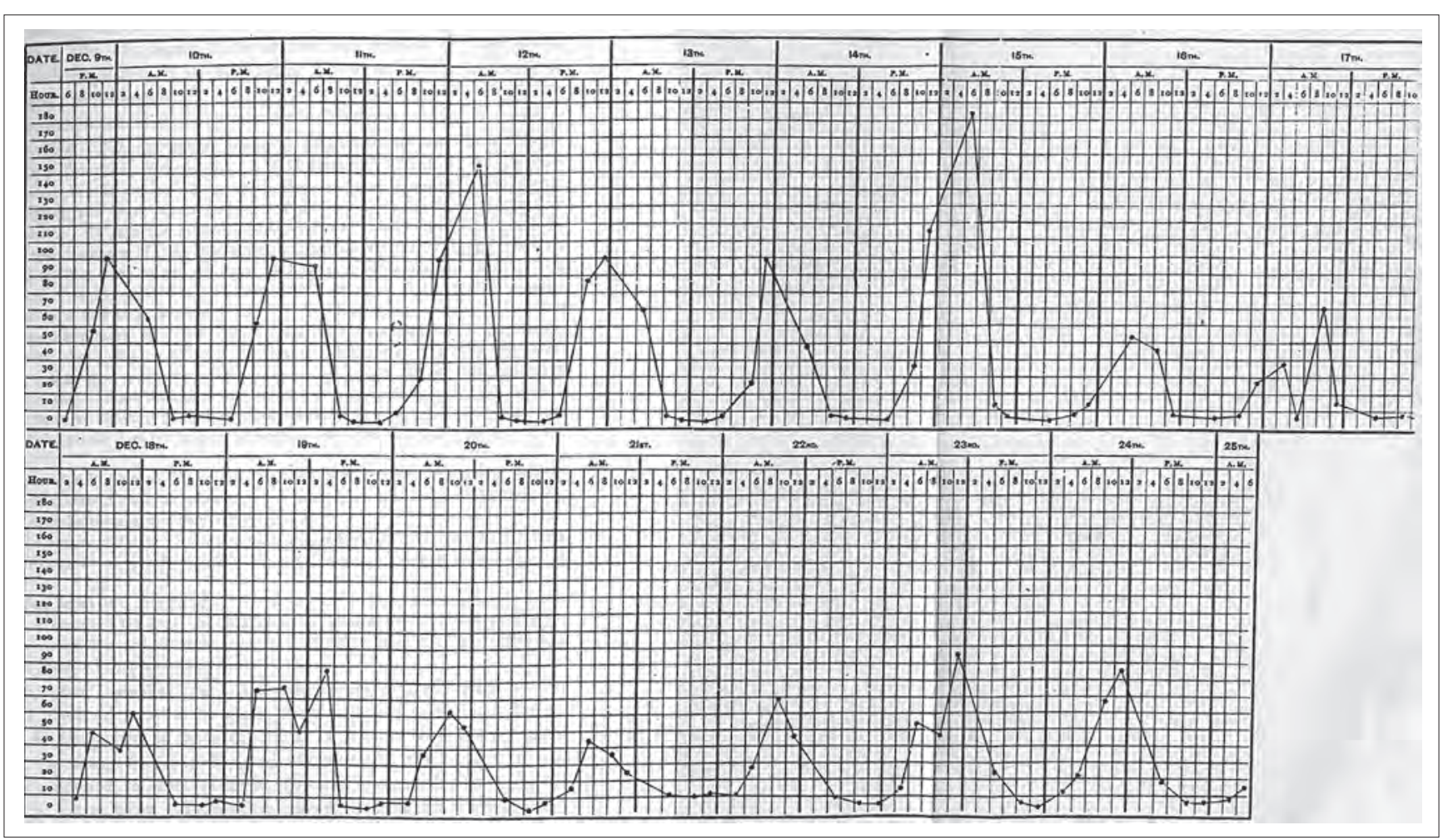

Figure 32.6 Manson's Chart of Filarial Periodicity. From Manson, 'Notes on Filaria Disease', Medical Reports, China Imperial Customs, 23rd Issue, (1882): 1-16, Chart I

observed filariae in patients' blood samples, the other usually did not. At first he thought this was an accident, but later he noticed that the assistant who seldom observed filariae took patients' blood during the day, while the assistant who often succeeded in finding the worms performed examinations in the evening. Manson and his assistants later conducted systematic examinations of persons infected by filariae and found that during the day the worms seldom presented themselves in the peripheral blood circulation. This situation was reversed after sunset. Manson immediately explained this phenomenon as another piece of evidence which could be used to support his intermediate host theory, arguing that the nocturnal habits of filariae

are adapted to the nocturnal habits of the mosquito, its intermediary host, and [this] is only another of the many wonderful instances of adaptation so constantly met with in nature. ${ }^{26}$

With the aid of a graph, another visualisation technique Manson often used, he was able to present his findings by means of a dramatic and persuasive image (Fig. 6).

It is significant that when Manson noticed the inconsistency in the observations of his assistants, he did not suspect one of them of cheating, but considered the inconsistency a clue worthy of further investigation. As historians

26 Manson 188o, see especially pp. 36-7. of science have argued, establishing trust is extremely important within the scientific community. Manson's trust in his assistants was not based on their social status, but on the regime of discipline and surveillance he had established. 'To prevent mistakes or imposition', Manson claimed, 'when the parasite is found by my assistants, I take care to verify the observation for myself from a fresh specimen of blood, which I see drawn'. On four occasions, the results of Manson's examinations were inconsistent with those of his assistants. While the assistants reported the occurrence of parasites in the patients' blood, Manson could not find the parasites. Manson, however, believed that the 'assistants' observations were correct' because 'they were confirmed by persons who supplied the blood'. He claimed that the patients' 'horror at the snake-like animal they had given birth to was conclusive'. ${ }^{27}$ From the above description, we know that Manson not only checked his assistants' work regularly but also asked his assistants to show their examination results to patients, having patients observe their own blood samples under the microscope. In a sense, Manson enlisted patients as participants in the verification of his assistants' observations, and thus, these patients became part of Manson's regime of surveillance and discipline.

$27 \quad$ Manson 1877 a, p. 37. 


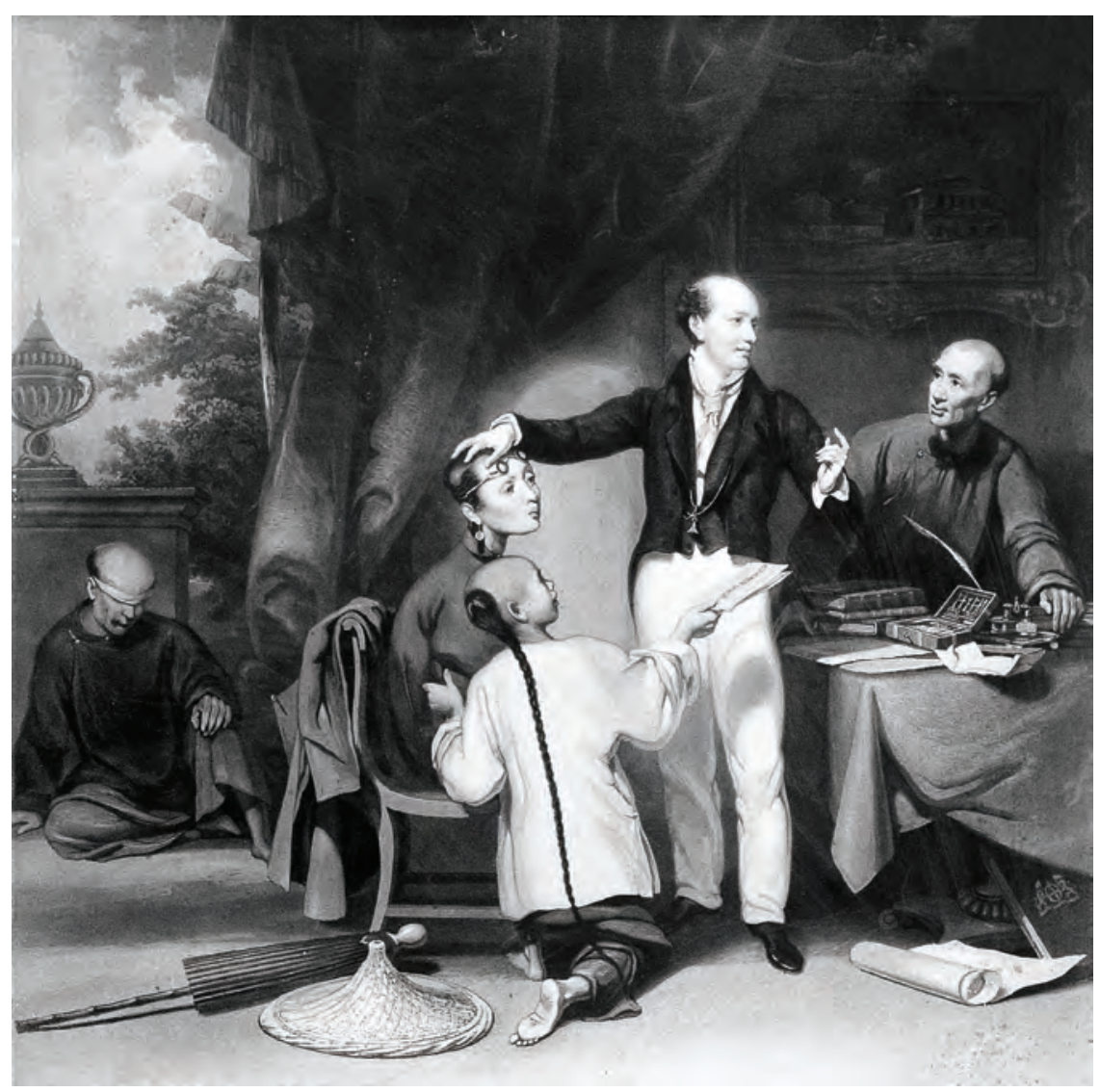

Figure 32.7 Portrait of Thomas Colledge, a surgeon working for the English East Indian Company at Macao in the early 19 th century, and his Chinese assistant. Mezzotint, 1834, by William Daniell after George Chinnery. Wellcome Library, London, Loo17163

Historians and sociologists of science, such as Chris Lawrence, Harry Collins and Trevor Pinch, have shown that skills are crucial to scientific research and clinical practice. ${ }^{28}$ Although they were neither responsible for drafting the reports nor for drawing the parasites, the skill and labour of Manson's Chinese assistants contributed significantly to Manson's ground-breaking discovery. It should also be pointed out that the tasks performed by the assistants required skills that could only be acquired through rigorous training. However, within the colonial context, the skills of native assistants, just like those of technicians in early modern natural philosophy, were not always highly valued. ${ }^{29}$ In contemporary portraits of European medical men and their Chinese assistants, the assistants are usually relegated to the background (Figs 7, 8). The painting commissioned by Sir Henry Wellcome, which depicts Manson's discovery of the mode of transmission of filariasis, follows this convention. The humbleness and subordinate position of the Chinese assistant is

Pinch, Collins and Carbone 1996; Lawrence 1985.

Shapin 1994, pp. 378-83; Schaffer 1988. emphasised by his short stature and slight hunchback in the painting (Fig. 8).

In his famous study of 'invisible technicians' in early modern experimental natural philosophy, Steven Shapin claims that, in scientific literatures, technicians and assistants have generally remained anonymous. Technicians, according to Shapin, are 'triply invisible'. First, they have been ignored by historians of science.

Second, they have been largely, if not entirely, invisible in the formal documentary record produced by scientific practitioners... it is extremely difficult to retrieve information about who they were and what they did.

Finally,

technicians have arguably been invisible as relevant actors to those persons in control of the workplaces in which scientific knowledge is produced. ${ }^{30}$

Although we do not know many details about Manson's Chinese assistants, they are not completely anonymous. In his 1877 report, Manson did not mention who his assistants were, but in his reports published in 1883 he mentioned that

Shapin 1994, 359-6o; see also pp. 355-497. 


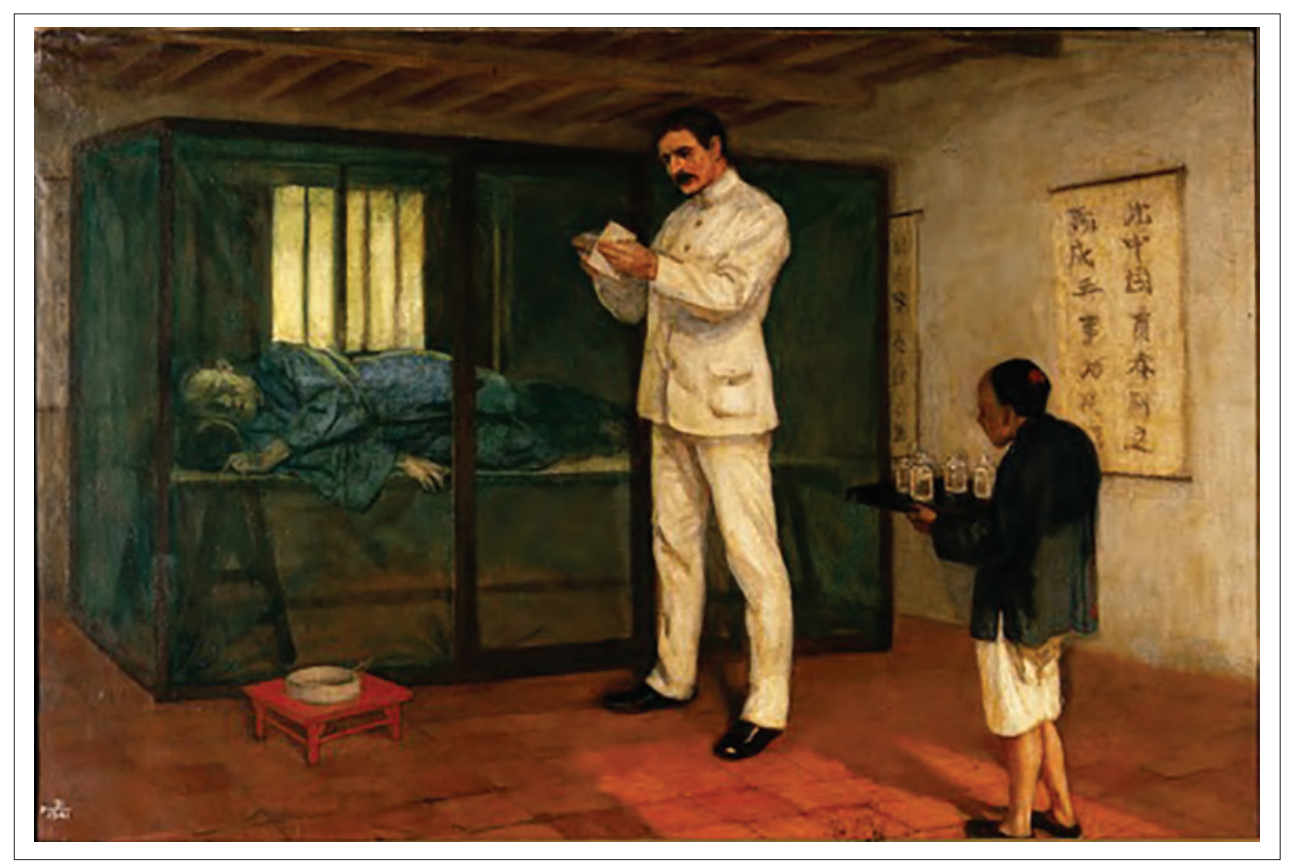

Figure 32.8 Portrait of Patrick Manson experimenting with filaria sanguinis hominis on a human subject in China. Painting by Ernest Board, c. 1912. Wellcome Library, London, Voo18137

he had hired two sufficiently intelligent assistants named Li Kha and Tiong Seng in 188o. From Manson's report, we learn that both of his assistants came from Hooihoah, an area about a three days' journey from the north of Amoy. Both were 21 years old. There are even records documenting the health conditions of these assistants. For example, Li Kha 'gave no history of fever, lymphangeitis, or of any serious disease whatever...'. For Manson, there was one more advantage to hiring the two young men:

As they themselves are filarious, and the subject of their own observations, the work could be prosecuted easily, with little fear of interruption, and with the sympathies of the observers entirely on the side of accuracy and truth. ${ }^{31}$

Nevertheless, Manson still checked the work of his assistants regularly. The trust he bestowed upon them was based upon three-fold supervision conducted by Manson, his patients, and by the assistants themselves. Compared with other Chinese assistants working for Western doctors during this period, we know relatively more about Manson's assistants. Due to their special status not merely as technicians, but also as providers of research materials and as subjects of Manson's experiments, these Chinese assistants did not become completely invisible.

That Chinese assistants were regarded as mainly responsible for manual work is often highlighted by contemporary portraiture. Shapin argues that in the early modern period, it was commonly assumed that technicians possessed merely manual skills. Moreover, a natural philosopher,

$31 \quad$ Manson 1882, p. 2. such as Robert Boyle, would hold that the 'assistants' hands were subsumed in his head'. ${ }^{22}$ In a colonial context, even if a native assistant's hands were subsumed in a European scientist's head, his thought was probably not. Manson had received solid training in natural history and microscopy when he studied medicine at the University of Aberdeen. After his reading in the British Museum during his furlough, he was also well versed in medical literature about elephantiasis and lymph scrotum, and some of these writings, such as Lewis' report, also contained important information about the relationship between these diseases and filarial parasites. ${ }^{33}$ Manson's Chinese assistants possessed none of his medical knowledge and came from a very different intellectual background. After a period of training, however, they successfully acquired blood drawing, slide preparation and microscopic observation skills. Another interesting example from Maryinez Lyons' study of the history of sleeping sickness illustrates this point well. Early 2oth-century field research on sleeping sickness involved microscopic examination of spinal fluid. Detecting the appearance of trypanosome in the fluid was a demanding task. It took about 20 minutes to scrutinise each slide. However, in a 1905 letter to his family, British investigator John L. Todd stated that microscopic examination entailed 'no necessity to think of the work - it is done reflexly [sic] - and my mind is left free to all sorts of curious thoughts.... After some training, moreover,

$\begin{array}{ll}32 & \text { Shapin, p. } 363 . \\ 33 & \text { Li 2002. }\end{array}$


African assistants were perfectly able to perform such microscope examination. 'The African technicians were skilful at detecting trypanosomes under the microscope', Lyons points out,

a difficult task even in good laboratory conditions... . They understood perfectly well that sleeping sickness was caused by tsetses. But they also knew perfectly well that it was due to witchcraft that the trypanosomes were present in certain individuals and not others. ${ }^{34}$

All of these examples demonstrate that the skill of microscopic observation could be acquired by peoples whose ideas and understandings of disease differed greatly from those of people who had developed their knowledge through a study of modern Western medicine. Skill could exist independently of a conceptual framework or theoretical knowledge. 'Observation is a skill', as Hacking points out, and skill can be acquired and improved 'by training and practice'. ${ }^{35}$ This study of Manson's Chinese assistants also has further historiographical significance. The philosopher Michael Polanyi claims that skills are 'an art which cannot be specified in detail and which cannot be transmitted by prescription, since no prescription for it exists'. Skill, Polanyi argues, 'can be passed on only by example from master to apprentice. This restricts the range of diffusion to that of personal contacts..... He further claims that for those countries which have become richer than European countries,

$[w]$ ithout the opportunity offered to young scientists to serve an apprenticeship in Europe, and without the migration of European scientists to the new countries, research centres overseas could hardly ever have made much headway. ${ }^{36}$

The situation of Manson's Chinese assistants or of the African technicians in sleeping sickness research teams was the other way around: they had acquired skills, but they did not possess scientific knowledge, or rather their knowledge of the disease that they were observing was very different from knowledge or understanding that would be gained through Western medical training.

Following the opening of Chinese treaty ports after the Opium War, an increasing number of European medical practitioners, who served as medical missionaries or as medical officers to the Chinese Maritime Customs, arrived

Lyons 1992, pp. 80, 182. See also Hacking's discussion of the experimental study of the positron using photographic plates. 'An assistant,' Hacking claims, 'can be trained to recognise those tracks without having a clue about the theory'. Hacking 1981, p. 179 . in China. These practitioners established hospitals and dispensaries and practised medicine among the local people. Faced with an increasing number of patients seeking medical assistance - sometimes more than 100 per working day - many of these Western medics trained and employed Chinese assistants to ease their burden. These Chinese assistants helped European medics prepare medicines, served as interpreters during clinical encounters with Chinese patients, and worked as dressers during surgical operations. They also dispensed medicine and performed minor surgery independently. Most of the first generation of Chinese practitioners of Western medicine served as assistants to Europeans practitioners before they started their own independent practices. In the early stages of the dissemination of Western medicine to non-Western countries, knowledge transferred to local practitioners probably consisted mainly of skill-related knowledge and not of articulate contents. The significance and implication of this form of transmission still awaits further historical exploration.

\section{Bibliography}

Beale, Lionel S., 1880, How to Work with the Microscope, London: Harrison.

Berger, J. 1972, Ways of Seeing, Harmondsworth: Penguin.

Bracegirdle, B. 1993, 'The microscopical tradition', in Bynum and Porter (eds), 102-19.

Bynum, W.F. and R. Porter (eds) 1993, Companion Encyclopedia of the History of Medicine, London: Routledge.

Carpenter, W.B.1891, The Microscope and Its Revelations, Philadelphia: P. Blakiston, Son \& Co.

Gooday, G.J.N. 1997, 'Instrumentation and interpretation: managing and representing the working environments of Victorian experimental science', in Lightman (ed.), 409-37.

Hacking, I. 1981, Representing and Intervening: Introductory Topics in the Philosophy of Science, Cambridge: Cambridge University Press.

Haynes, D.M. 2001, Imperial Medicine:Patrick Manson and the Conquest of Tropical Disease, Philadelphia: University of Pennsylvania.

Kohler, R.E. 1994, Lords of the Fly: Drosophila Genetics and the Experimental Life, Chicago: University of Chicago Press.

Latour, B. 1986, 'Visualization and cognition: thinking with eyes and hands', Knowledge and Society, 6, 1-40; reprinted as 'Drawing things together', in Lynch and Woolgar (eds), 19-68.

Lawrence, C. 1985, "Incommunicable knowledge”: science, technology and the clinical "art" in Britain, 1850-1910', Journal of Contemporary History 20, 503-20.

Lewis, T.R. 1872, On a Hcematozoon Inhabiting Human Blood:Its Relation to Chyluria and other Diseases, Calcutta: Office of the Superintendent of Government Printing.

Li, Shang-Jen 2002, 'Natural history of parasitic disease: Patrick Manson's philosophical method', Isis 93, 206-28.

Lightman, B. (ed.) 1997, Victorian Science in Context, Chicago: University of Chicago Press.

Lynch, M. 199o, 'The externalised retina: selection and mathematization in the visual documentation of objects in the Life Sciences', in 
Lynch and Woolgar (eds), 153-86.

Lynch, M. and S. Woolgar (eds) 199o, Representation in Scientific Practice, Cambridge, MA.: The MIT Press.

Lyons, M. 1992, The Colonial Disease: A Social History of Sleeping Sickness in Northern Zaire, 1900-1940, Cambridge: CuP.

Manson, P. 1875, 'Remarks on lymph scrotum, elephantiasis and chyluria', Medical Report of the Chinese Imperial Maritime Customs 27 (1875), 1-14.

1877a, 'Report on hæmatozoa', Medical Report of the Chinese Imperial Maritime Customs 13, 13-38.

1877 b, 'Further observations on Filaria sanguinis hominis', Medical Report of the Chinese Imperial Maritime Customs, 14, 1-26. 1880, 'Additional notes on Filaria sanguinis hominis and Filaria disease', Medical Report of the Chinese Imperial Maritime Customs 18, 31-51.

1882, 'Notes on Filaria disease', Medical Report of the Chinese
Imperial Maritime Customs 23, 1-16.

1884, 'The metamorphosis of Filaria sanguinis hominis in the mosquito', Transactions of the Linnean Society of London, 2nd Series, Zoology 2.10, 367-88.

1898, Tropical Diseases. A Manual of the Diseases of Warm Climates, London: Cassell \& Co.

Manson-Bahr, P.H. and A. Alcock 1927, The Life and Work of Sir Patrick Manson, London: Cassell.

Pinch, T., H.M. Collins and L. Carbone 1996, 'Inside knowledge: second order measures of skill', Sociological Review 44, 163-86.

Polanyi, M. 1983, Personal Knowledge: Towards a Post-Critical Philosophy, London: Routledge and Kegan Paul.

Schaffer, S. 1988, 'Astronomers mark time: discipline and the personal equation', Science in Context 2.1, 115-45.

Shapin, S. 1994, A Social History of Truth: Civility and Science in Seventeenth-Century England, Chicago: University of Chicago Press. 


\title{
33 Marketing Medicine to Koreans
}

\author{
Soyoung Suh 서소영
}

On 1 September 1911, a full-page advertisement for Jintan 仁丹 (Humane Elixir) was placed in Maeil sinbo 每日申 報 (Daily News), targeting Korean consumers. A lady in Western costume (Fig. 1) testifies to the merits of the elixir. The text is multilingual; the English copy reads:

Whenever, however, and wherever you may be, you'd surely enjoy excellent health in taking Jintan as a task. Jintan is a wonderful tonic and the best mouth refresher. ${ }^{1}$

First manufactured by Morishita Hiroshi (1869-1943) in 1900, Humane Elixir became one of the most notable Japanese marketing successes in Korea. ${ }^{2}$ Not only Humane Elixir, but medicine in general was extensively advertised in Korean-language newspapers. Medicine-related advertisements occupied $30 \%$ of advertising space in Tonga ilbo 東亞日報 (East Asia Daily) in 1923, rising to more than half by $1938 .^{3}$

Korean newspapers began to carry advertisements as early as 1886 . However, of the six newspapers then distributed in Seoul, only Taehan maeil sinbo 大韓每日 申報 (Korean Daily News) continued to publish after the Japanese annexation in 1910, albeit with the word Taehan (Korea) removed from the title. Thus until the East Asia Daily and Chosŏn ilbo 朝鮮日報 (Korean Daily) appeared in 1920 , advertisements in Korean found few media outlets. ${ }^{4}$

At the same time, Japanese-language newspapers, published and owned by the Japanese in Korea, flourished with the growth of Japanese colonial governance. According to statistics from the Government-General, in 1913 there were 25Japanese-language newspapers in national circulation, most of which had been launched between 1905 and 1909. These Japanese newspapers were designed to appeal not only to Japanese residents of Korea but to indigenous

$1 \quad$ Maeil sinbo, 1 September, 1911.

2 For the success of Humane Elixir in Japan, see Burns 2009, pp. 194-8. In China, an attempt was made to imitate Humane Elixir by the Shanghai businessman, Huang Chujiu 黄楚九, who successfully popularised Chinese-made, Western-style patent medicine in early 2oth-century China. The Chinese version of Humane Elixir was slightly modified, with a trivial change of the brand name - the original character ren (仁), which means 'benevolence', was replaced by ren (人), a homophone with the same tone meaning 'human', i.e. 'Humane Elixir' became ' $\mathrm{Hu}$ man Elixir' (人丹). Cochran 2006, pp. 46-7.

3 Han'guk Kwanggo Yŏn'guwŏn 1996, p. 50. Shin and Sǒ 2011, p. 110.

4 Shin 1980, pp. 18-19. For an English version, see Shin and Shin 2004 .
Koreans as well. ${ }^{5}$ Furthermore, over half the advertisements placed in Korean newspapers during the 1920 s were sponsored by Japanese companies. Recognising the significance of the Korean market, Japanese entrepreneurs set out to attract more Korean consumers. For instance, by 1923 Japanese advertisements made up 36\% of the total in the East Asia Daily, increasing to $40 \%$ by 1925 and $61 \%$ by 1938. Japanese enterprises were major sponsors of Korean newspapers, publicising Japanese commodities to a Korean market. 6

Humane Elixir was one of the two Japanese products most frequently advertised in the Daily News in the 1910 s and $1920 .^{7}$ As its popularity grew, profits were ploughed back into increasingly sophisticated advertising. In addition to the iconic trademark (the Humane Elixir man with his

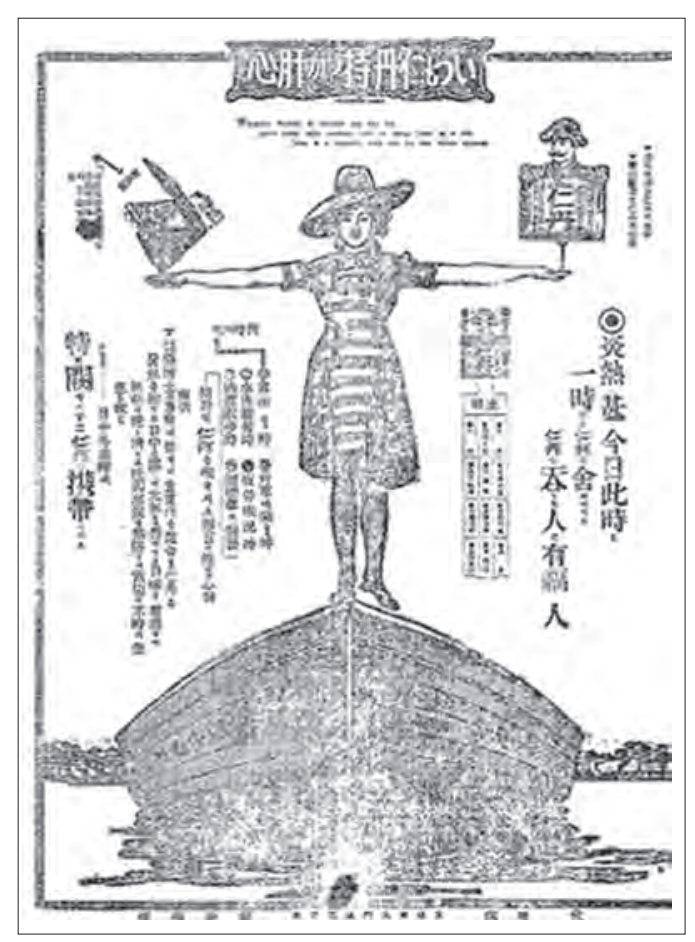

Figure 33.1 Advertising 'Jintan’ 仁丹 (Humane Elixir) in Maeil Sinbo 每日申報 (the Daily News), 1 September 1911

5 Han'guk Kwanggo Yŏn'guwŏn 1996, p. 65.

6 Ibid., pp. 49-5o.

7 The other item was Ajinomoto 味の素, a Japanese-made seasoning for every kind of food, now well known as monosodium glutamate (MSG). It was successfully marketed in Korea by portraying it as indispensable to the modern household. Shin 1980, p. 53; Han'guk Kwanggo Yŏn'guwŏn 1996, pp. 238-43. 


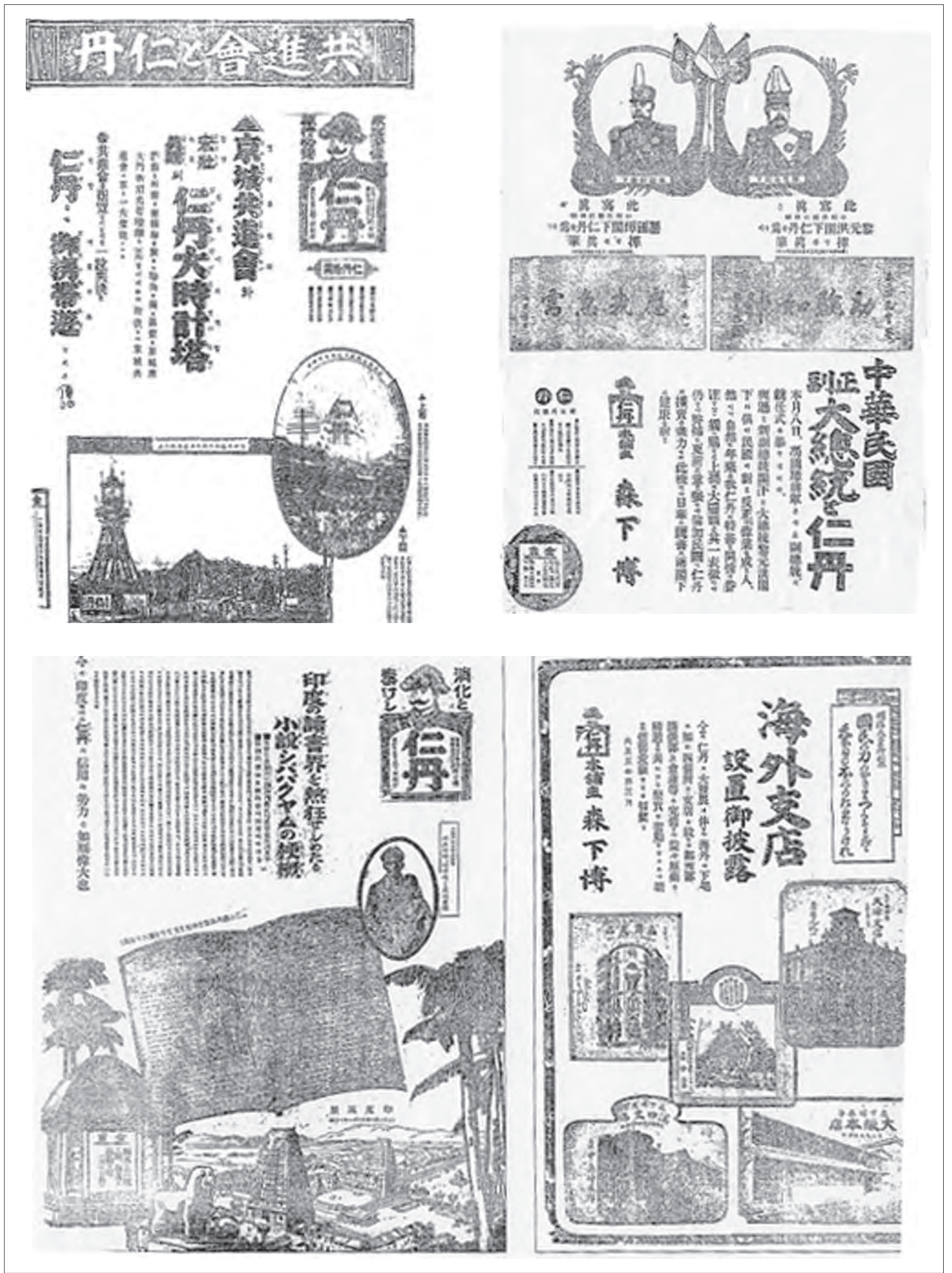

Figure 33.2 Various advertisements for 'Humane Elixir' placed in the Daily News in the 1910s 
admiral's hat and distinctive moustache), print advertisements utilised a variety of illustrations and catchphrases.

Special events and presentations aimed to create a festive atmosphere reminiscent of New Year celebrations. The elixir was advertised as having important health benefits for children. In 1914 much was made of the fact that 6oo,ooo doses of Humane Elixir had been sent to China in to aid flood survivors. When Kyŏngsŏng Kongjinhoe 京 城共進會 (a competitive trade fair) was held in Seoul in 1915, a billboard for Humane Elixir was erected on the clock tower, signalling its leading position, and this event was also exploited for newspaper advertisements. Its popularity abroad was highlighted: Humane Elixir figured in a popular Indian novel; four overseas sales branches were touted as evidence of its international fame. When Li Yuanhong 黎 元洪, (1864-1928) became Generalissimo of the Chinese Republic in 1916, portraits of him and Vice-Generalissimo Feng Guozhang 馮國璋 (1859-1919) featured in advertisements for Humane Elixir with product endorsements in their own handwriting (Fig. 2). ${ }^{8}$ Humane Elixir, with its sophisticated advertising techniques and financial clout, dominated the newspaper's medical advertisements, not to mention the Korean market, during the first half of the 2oth century.

Humane Elixir well exemplifies the Japanese pre-eminence in marketing medicine in Korea. How, then, did Koreans respond to Japan's domination of the market? Did Korean drug sellers or pharmacists try to emulate Japanese initiatives? Or did Koreans develop their own marketing strategies? As Figure 1 shows, medical advertisements in Korean newspapers often displayed hybrid features. This example incorporates texts in Korean, Japanese, English and Chinese alongside an image of a modern lady. Invoking scientific modernity, the advertisement points out that Humane Elixir is specifically manufactured under the direction of two famous Japanese researchers. Yet although the advertisement employs the imagery and rhetoric of modernity, the text does not mention biomedical principles at all. Instead, concepts from traditional medicine are deployed to explain bodily processes.

Given this situation, what role did indigenous features play in fashioning medical advertisements aimed at Koreans? Did Korean advertisers focus on local specificities or did they ignore indigenous attributes in order to emulate the modern approaches adopted by the Japanese? By examining early 2oth-century medicine advertisements, this chapter considers the ways in which the concepts of

8 Maeil sinbo, 1 January, 1916; 1 January, 1915; 21 July, 1914; 6 October, 1915; 8 March, 1916; 31 March, 1916; 22 November, 1916; 1 August, 1934. 'medicine for Koreans' was utilised and/or disregarded in the process of creating opportunities for Korean drug sellers.

\section{Japan's Domination of the Korean Market}

Japan had a significant impact on restructuring the pharmaceutical market in Korea, primarily by enhancing legal regulation. In March 1912, the Japanese GovernmentGeneral of Korea (Chosŏn Ch'ongdokpu 朝鮮 總督府) promulgated the Regulations for the Enforcement of Laws for Medicinal Products and the Medicinal Product Businesses (Yakuhin oyobiyakuhin eigyō torishimarishikō kisoku 藥品 及藥品營業取締令). This law specified four categories of pharmaceutical professionals, differentiating their range of knowledge and degree of commercial engagement: a pharmacist (cheyaksa 劑藥士) should 'combine chemicals according to doctors' prescriptions'; a manufacturer (cheyakja 劑藥者) should 'prepare and sell medicinal products'; a drug seller (yakchongsang 藥種商) should 'sell chemicals'; and finally, merchants of patent medicines (maeyagŏpcha 賣藥者) should 'sell patent medicines by manufacturing, introducing, or importing.' ${ }^{9}$ Although the boundaries between the categories were often blurred, those concerned gradually reoriented their professional careers in line with the official licensing system, and the newly imposed regulation remained in force until 1945 .

Even before the 1910 annexation of Korea, Japan had begun to establish modern biomedical hospitals in Korea, thereby paving the way for the introduction of biomedically trained personnel and medical commodities. Clinics run by Japanese army doctors were first set up in Pusan in 1877, Wŏnsan in 1880, and Seoul and Inch'ŏn in 1883. Named Government-Sponsored Hospitals (Kwallip pyŏngwŏn 官 立病院), these institutions aimed to 'relieve (Japanese) settlers' diseases and to provide hygiene'. ${ }^{10}$ Compared with the Government-Sponsored Hospitals, the Seoul Hospital (Hansŏng pyŏngwŏn 漢城病院), established by Japan in 1895 , was more explicitly intended to display imperial benevolence toward Koreans in need. Upon annexation, the Government-General Hospital of Korea (Chosŏn ch'ongdokpu ǔiwŏn 朝鮮總督府醫院) replaced the Hospital of Great Han (Taehan ǔiwŏn 大韓醫院), which had been established by the Residency-General (T'onggambu 統監府) in Seoul in 1907. Paralleling the initiative in Seoul, regional branches of charity hospitals (Chahye pyŏngwŏn 慈惠病院) began to be built nationwide beginning in 19o9; after 1925 these regional

\footnotetext{
9 Park 2005, pp. 322-3o, Burns 2012, pp. 8-10.

10 Ibid., pp. 57-68.
} 
hospitals were redesignated as provincial hospitals (torip pyŏngwŏn 道立病院). Overall, 41 provincial hospitals had been established in Korea by $1938 .^{11}$ Through these national facilities, biomedically trained Japanese pharmacists were also introduced into Korea. After a period of service, they frequently opened apothecary shops, and made a variety of Japanese commodities available for sale to the public, including cosmetics and miscellaneous consumer goods as well as medicinal items. Around the turn of the 2oth century, a few Japanese wholesale drug sellers established themselves in major cities in Korea. Arai Kotarō 新井虎太 郎 (b. 1867) and Yamagishi Yūtarō 山岸祐太郎 (1868-1927), for instance, set up businesses in Seoul in the early 1900 . When the two railway links opened between Seoul and Pusan in 1905 and Seoul and Sinŭiju in 19o6, traversing the Korean peninsula, Japanese apothecaries gradually expanded and came to dominate the Korean market. ${ }^{12}$

Yamagishi, one of the most famous Japanese-run apothecary businesses in Seoul, first set up a wholesale outlet in 1906, and prospered by supplying medicine and medical equipment to the charity hospitals. In 1913 the Yamagishi enterprise employed a staff of 30 , with sales of $¥ 500$,oo per month rising to ¥1 million per month in 1935. During the 1920s major Japanese pharmacies and pharmaceutical companies established branch offices in Seoul. As the Japanese imperial vision for Manchuria took concrete form with the establishment of Manchukuo 滿洲 國 in 1932, drug manufacturers opened new area offices in Korea and increased their marketing budgets. Korean branches were regarded as a significant bridgehead to the Chinese continental market. Having seen business boom during the 1920s and 193os, Japanese companies began to construct factories in Korea during the early 1940s to produce agricultural chemicals, tonic soups, and medicines using Korean raw materials and special products. ${ }^{13}$

The Japanese initiative in Korea was underpinned in a variety of ways, primarily using the associations of Japanese pharmacists and drug merchants to consolidate the Japanese network in the country. The Association of Pharmacists of Korea (Han'gukyakchesahoe 韓國藥劑師 會) was first established in 1909 under the leadership of Japanese pharmacists working for the Residency-General (the Japanese Government-General in Korea after 1910) and its affiliate hospital, the Government-General Hospital of Korea. Of 28 regular members, only one was Korean.

Ibid., pp. 247-67.

Hong 1972, pp. 158-9.

Ibid., pp. 155-76, pp. 191-4. By 1941, Seoul had approximately 300,000 Japanese residents, out of a total population of about 1,000,00o.
Originally, membership was limited to those holding a licence in biomedical pharmacy. ${ }^{14}$ However in 1913, the Association was renamed the Pharmaceutical Society of Korea (Chosŏn yakhakhoe 朝鮮藥學會), in an attempt to recruit anyone with connections to the pharmaceutical field. It held lectures to publicise newly promulgated qualifications for pharmacists, provided information about the regulation of toxic chemicals, and specified biomedical principles for the preparation of medicines. A short course managed by the society developed into a training school, which later became the School of Pharmacy of Korea (Chosŏn yakhakkyo 朝鮮藥學校). ${ }^{15}$

Several major newsletters fortified the Japanocentric network. The first of these, Kyŏngsŏng hakpo 京城藥報 (Newsletter of Pharmacy of Seoul), was launched in 1915, sponsored by the Arai and Yamagishi apothecaries. As trade prospered, Arai also funded Mansŏnji hwa jangp'um sangbo 滿鮮之化粧品商報) (Commercial News of Cosmetics in Manchuria and Korea) and Mansen no ikai (Mansŏnji ŭigye 滿鮮之醫界, Medical World of Manchuria and Korea). Stimulated by the positive response to these new ventures and the successful impact of the Newsletter of Pharmacy of Seoul, drug manufacturers in Pusan published Chosŏn yakpo 朝鮮藥報 (Newsletter of Pharmacy of Korea) in 1927. All these periodicals were published in Japanese. ${ }^{16}$

Japanese influence also made itself felt in the aggressive strategies and advanced techniques employed for advertising medicine in Korea, as exemplified by the Humane Elixir publicity campaigns discussed above. Introduced to Korea via Japanese traders in the early 2 oth century, Humane Elixir was soon circulating and being consumed nationwide. A Korean copy of the elixir appeared, but as in China, the original Japanese brand retained its dominant position.

In sum, the Japanese dominated the early zoth-century Korean medicine market by regulating the modern licensing system, introducing biomedically oriented training, organising nationwide associations, and employing a variety of marketing strategies. Anti-Japanese sentiment was not a major issue in the market in that period. Although nationalism grew during the 1910 s and 1920s, it was difficult and unnecessary for Korean drug-sellers to contest Japanese supremacy overtly while under colonial rule.

\footnotetext{
14 The licensing system for medical professions in Korea was not fully operational until 1913 .

Hong 1972, pp. 5-9.

16 Ibid., pp. 184-5.
} 


\section{Latecomers' Strategies}

\section{Patent Medicines $^{17}$}

Under Japanese domination, Koreans developed independent commercial power primarily by manufacturing and marketing patent medicines. Advertisements for patent medicines from the late 19th and early 2oth centuries generally juxtapose traditional herbs familiar to Korean practitioners and traders with Western imagery, sometimes in conjunction with ingredients associated with biomedical practitioners. Korean drug sellers involved in trading herbs with Qing China were among the first to notice a flow of new commodities from China and Japan. A few entrepreneurial spirits took the opportunity to manufacture their own unique brands by turning their knowledge of native herbs to new combinations.

Two notable examples well illustrate the opportunistic and eclectic aspects of patent medicines at the turn of the last century. Whal Myung Su (Hwalmyŏngsu 活命水, 'Lifesaving Water'), an oral liquid digestive, was one of the most popular medicinal products ever to be sold in Korea. It was first manufactured in 1897 by Min Pyŏng-ho 閔並浩, who came from a family belonging to the yangban (兩班) ruling class, and had himself gained an official position by passing the state examination. He was known to be wellversed in the medical classics and the secret prescriptions that circulated among Chosŏn court doctors, but what inspired Min was the perceived superiority of 'Western' medicine. As a Christian, Min had a general interest in Western culture, and he set about acquiring knowledge of biomedicine through a friend who worked at a newly-founded Western-style hospital managed by Western missionaries. Based on his experience and knowledge, Min first extracted a few herbs including dried orange peel and silver magnolia, then added recently-imported catechu, menthol and alcohol. ${ }^{18}$ The result was Whal Myung Su, and the news of its miraculous efficacy quickly spread beyond his own locality. His apothecary shop, Tonghwa yakpang 同和藥房, prospered, and he registered 98 patent medicines in 1908. ${ }^{19}$ Although Min was neither a trained

17 The practice of patenting medicine involves some kind of institutional legitimisation. Hereafter I define patent medicines as medicines registered with their own brand names, even though the ingredients and efficacy may be similar to other ready-made or pre-compounded medicines. The Japanese government officially controlled patent medicines with detailed regulations from 1912 onward, although the patenting of medicines had already been practised since 1907 .

18 Hong 1972, pp. 3-4, Ye 2009, pp. 16-17.

19 Tonghwa yakpang opened in 1897 , developed into Dong Wha (Tonghwa) Pharmaceutical Industrial Company Limited in 1962, pharmacist nor a merchant, he found a way into the market by combining his knowledge of traditional medicine with elements of Western medicine.

Another example is the Pill for Clearing the Heart and Guarding Life (Ch'ŏngsim pomyŏngdan 清心保命丹, hereafter the Pill for Clearing the Heart), manufactured by Yi Kyŏng-bong 李庚鳳, who, following in a family tradition, was engaged in the herb trade with China in the years around the turn of the 19th century. Spurred on by the success of Humane Elixir, Yi was determined to manufacture his own patent medicine. The Pill for Clearing the Heart reflects his knowledge of herbs and sensitivity to market changes. It was, like Whal Myung Su, a digestive preparation. Made from ingredients like borneol and peppermint, it tasted sweet and came in the form of small red granules. Being a domestic product, the pill was sold for 5 chŏn, ${ }^{20}$ half the price of Humane Elixir. Yi's handsome and Westernised appearance was in line with his eloquent and flamboyant style of public speech, which conferred a modern image on this medicine. Frequent street performances with music and colourful banners were used to promote the medicine, providing a form of public entertainment (Fig. 3). On the strength of the success of the Pill for Clearing the Heart, Yi opened an apothecary shop, Chesaengdang yakpang 濟生 堂藥房, first in Chemulp'o, then in Seoul. ${ }^{21}$ By recognising the commercial features of Japanese patent medicines and appealing to Korean aspirations for the visual culture of modernity, Yi was able to create a new market.

The successful practices of Min, Yi, and other drug sellers shared the following characteristics. They were all versed in herbs and familiar with the herb trade. They were inspired to produce their own commodities by patent medicines from China and Japan. With the exception of Min, whose major market was in P'yŏngyang, they all began their business in treaty ports and then moved into the main streets in the centre of Seoul. Min was interested in biomedical practices, which presumably influenced his patent medicine. However, neither he nor the others were trained in biomedicine, and hardly any biomedical principles or ingredients were involved in their products. They stepped into a gap in the market, using materia medica that were familiar to them, and imparting a veneer of modernity to the finished product. During the heyday of these traditional patent medicines, the claim that this was 'a medicine of our own' was not made explicit. But the herb traders did indirectly appeal to Korean nationalism, claiming to

and continues to be one of the leading pharmaceutical companies in contemporary Korea. Ye 20o9, p. 31.

20 One chŏn was the equivalent of 1 US cent at that time.

21 Hong 1972, pp. 12-13. 


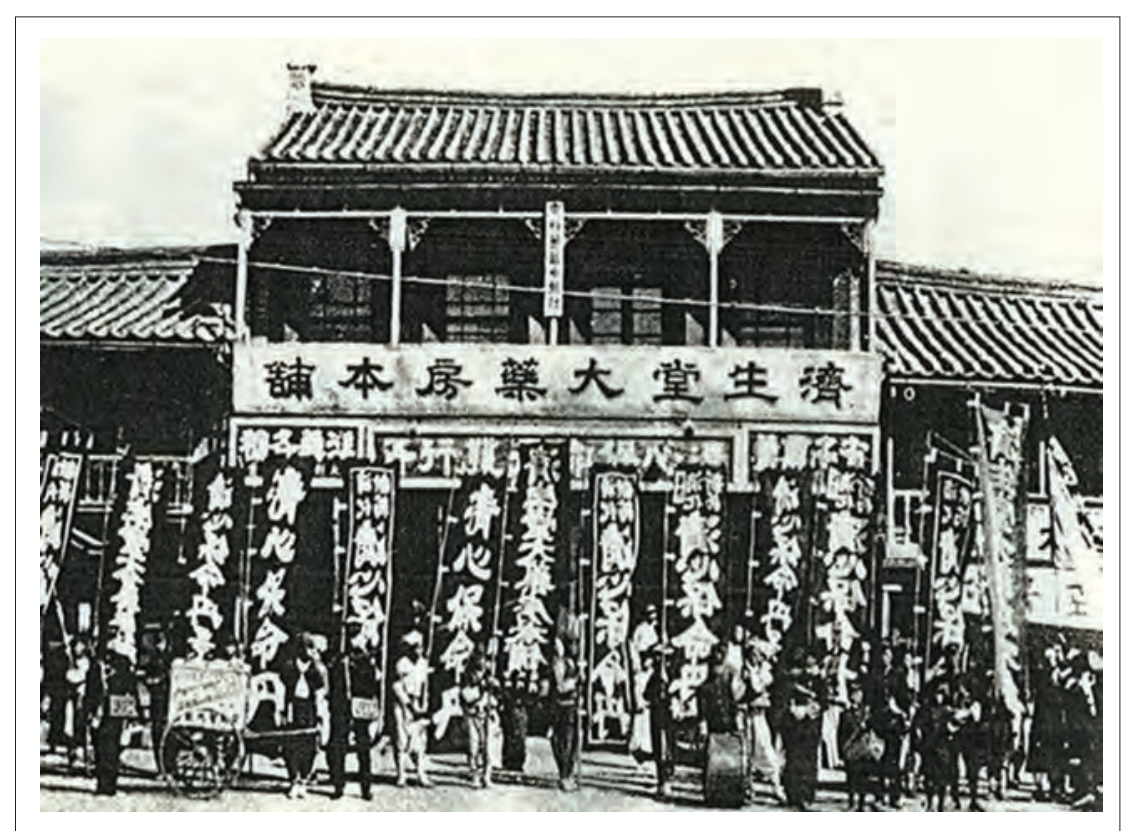

Figure 33.3 Advertising ‘Ch’ŏngsim pomyŏngdan’ 清心保命丹 (Pill for Clearing the Heart and Guarding Life) in front of Chesaengdang yakpang 濟生堂藥房

produce patent medicines that were both convenient and familiar to Koreans, or to utilise 'something Korean'. ${ }^{22}$ In some cases, growing nationalism provided a new motivation to establish a Korean pharmacy that might compete with Japanese domination. For instance, Min's son, Min Kang 閔疆 (1883-1931), who expanded and modernised his father's apothecary business, first supported and then actively participated in the Korean independence movement. Min Kang's nationalist consciousness, however, put his business in jeopardy. As a result of his anti-Japanese activism, 60 of his 86 medical patents were cancelled by the Japanese colonial government. ${ }^{23}$ Under Japanese colonialism, it was unwise for traders to explicitly claim Korean traits for their products.

Under these circumstances, Korean specificity was expressed, not in any explicit catchphrases or images, but through merchants' social networks. For instance, the successes of wholesale herb dealers called for brokers with the capacity to establish local branches and extend the market into every corner of the Korean peninsula. Ch'onnil yakpang 天一藥房, a wholesale pharmacy, attracted local dealers by offering free gifts or trips, and became known for its varied programme of high-profile public events. As discussed earlier, the market for Korean patent medicine had surged by the 1920s. Most Korean apothecaries registered more than 40 or 50 medicines with their own brand names, yet many of these were similar in terms of ingredients and therefore medical efficacy. In the 1920 over

22 For this quotation, see ibid., p. 14.

23 Ibid., p. 22. Ye 20o9, pp. 62-3.
1500 patent medicines were on sale in Korea. Newspapers criticised their low quality and the excessive competition among apothecaries. While the retail shops usually took a $40-50 \%$ profit margin, latecomers who attempted to market knock-offs had to offer bargain prices, allowing a profit of up to $60-70 \% .{ }^{24}$

Most Korean apothecaries, then, attempted to seize the advantage, not by improving the quality of the medicines they sold, but by commissioning ever more impressive advertising and publicity stunts. To appeal to the public, Ch'ŏnil yakpang sponsored a variety of spectacular events including a parade of Ford cars. A free cinema ticket was given away with each purchase of a large-size tonic. A chamber pot was among the most popular free gifts on offer! Additionally, Ch'ŏnil yakpang published its own monthly newsletter, Ch’ǒnil yakpo 天一藥報 (Medical Newletter of Ch'ŏnil), which circulated among traders nationwide. Special events for middlemen or local sales forces were frequently held. Ch'ŏnil yakpang spent substantial sums on treating local traders, wooing them with calendars printed in Japan and a variety of entertaining free gifts such as Korean playing cards or the Four-Stick Game. Frequently, traders were invited to one of the most lavish and flamboyant restaurants in Seoul, which usually provided scores of singing girls (kisaeng 妓生). Famous singers, a comic storyteller and a dancing master were enlisted to put on local performances. ${ }^{25}$

\footnotetext{
24 Hong 1972, pp. 47-8.

25 Ibid., pp, 112-13.
} 
The highlight of Ch'ŏnil yakpang's publicity events in Seoul was an aeroplane ride. Though it lasted only five minutes, more than 8 o traders gathered in the city for their turn. All travel and accommodation expenses were borne by Ch'ŏnil yakpang. Another popular event was a trip to north-eastern China. Eight days in duration, it took in the major cities of northern and southern Manchuria in addition to Beijing and Tianjin. These types of sales promotions continued until 1939. Though costly, they did ultimately pay off: the extraordinary sales strategies were reported in newspapers, doubling their effect. In addition, the initial cost for manufacturing Ch'ŏnil yakpang's flagship item, Chogoyak 趙膏藥, a medicinal plaster originating from the Cho family, was less than 2 chŏn, but it was then sold for 10 chŏn at retail, and 5 chŏn at wholesale. ${ }^{26}$ Despite the financial cost, special sales promotions continued because they consolidated sales networks by retaining local dealers. To be popular and profitable, medical efficacy was not enough. Patent medicines needed to be packaged with free gifts, lavish entertainment, and spectacular events.

Korean drug sellers at the turn of the 19th century recognised the commercial possibilities presented by patent medicines. Herbs grown in Korean soil or familiar to Korean tastes were lent a sense of novelty by the terminology and imagery of 'Western' biomedicine, sensational advertising, and local salesmanship. While indigenous medicine did not lose favour, drug sellers under Japanese domination tactically managed whether local, familiar medical ways should be recognised as 'Korean' medicine or not.

\section{Combining Nationalism with Biomedicine}

Manufacturing biomedicines gave Koreans another opportunity to strategically establish their own commercial networks. Without relying on Japanese brokerage, Koreans founded pharmaceutical businesses by first importing biomedicines, then ultimately manufacturing indigenous branded items. The Yuhan Corporation (Yuhanyanghaeng 柳韓洋行) is a case of successful marketing of biomedicine which evoked nationalist sentiment while challenging aspects of ethnic identity. A significant example of a modern Korean pharmaceutical company in Korea that utilised purely Korean capital and management, while competing with Japanese companies, the Yuhan Corporation is instinct with the distinctive career and nationalist vision of Yu Ir-han (1895-1971). ${ }^{27}$

26 Ibid., pp, 113-14.

27 For detailed stories about Yu Ir-han and the Yuhan Corporation, see Cho 2005, Yu Ir-han Chŏn'gi P'yŏnjip Wiwŏnhoe 1995, and
Yu was the first-born son of a local merchant of P'yŏngyang. His father, Yu Ki-yŏn, a Christian convert, was impressed by Western missionaries' medical services and educational efforts. Concerned at the advance of Japanese colonialism, he decided to educate his eldest son abroad. Thus at the age of nine, Yu Ir-han was sent to San Francisco, then to high school in Nebraska. After majoring in business at the University of Michigan, Yu pursued a variety of business careers, establishing the highly successful La Choy Food Products Inc., producing canned food, in the US in 1922. Within four years the company was grossing $\$ 500,000$ p.a., and expanding its commercial network throughout the major US cities. ${ }^{28}$ Imbued with American capitalism, Yu envisioned a trading firm that would manufacture medical supplies and miscellaneous goods to improve the quality of Korean lives. Accordingly in 1926, he established the Yuhan Corporation, which introduced insecticides, anti-tuberculosis drugs, plasters for skin diseases, etc. to Korea under its own brand name. It also imported sundry goods like farming implements, paints, dyes, toilet paper, cosmetics, chewing gum, and chocolates, all of which were wonderful novelties for many Koreans.

Every biography of Yu cites nationalism and Christianity as the two most significant factors shaping his entrepreneurship, and relates anecdotes revealing his nationalist activism. ${ }^{29}$ But although nationalism was undoubtedly embedded in the Yuhan Corporation's management, the company did not openly display ethnic traits or employ nationalist catchphrases; rather its advertisements relied on a variety of messages encompassing enlightenment and modernity, which sometimes ran counter to indigenous sentiments.

Prontosil and Neotone well exemplify this point. Determined to manufacture and not merely import biomedicines during the 193os, Yu endeavoured to keep abreast with the latest achievements in biomedicine that might be applicable to his modern manufacturing facilities. A PHD chemist from Vienna was invited to act as a consultant in the development of new medicines; a Korean who had gained a degree in Japan followed, and finally Yu and his company were able to exploit the commercial possibilities of the recent discovery of Prontosil. ${ }^{30}$

Prontosil, a sulphanilamide derivative ( $\mathrm{p}$-aminobenzene sulphonamide) synthesised by a Viennese chemist in 1908, was discovered to have antibacterial use by Gerhard

Kim 1984.

28 Cho 2005, pp. 158-66.

29 For instance, Yu Ir-han Chŏn'gi P'yŏnjip Wiwŏnhoe 1995, pp. 193-4, p. 215 .

$30 \quad$ Hong 1972 , pp. $67-71$. 


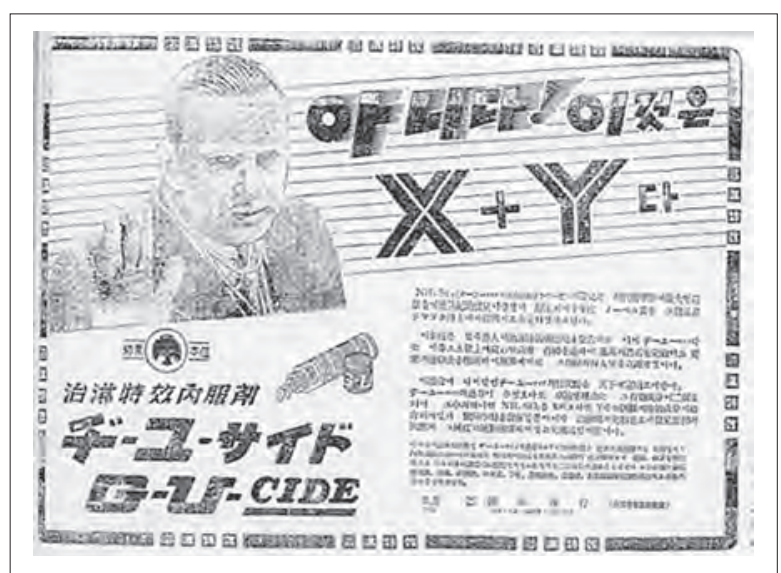

Figure 33.4 Advertising 'G-U-CIDE' in 1939

Domagk in 1934, for which he was awarded a Nobel Prize in 1939. The discovery of Prontosil opened up new ways of synthesising and commercialising the antibacterials which would become known as sulfa drugs. For instance, Satsutarimu サツタリム, a Japanese brand of Prontosilbased antibiotics, was developed for for treating gonorrhea. ${ }^{31}$ Not long after it was first introduced to Japan via Germany in 1937, the Yuhan Corporation manufactured a variety of branded items containing Prontosil, which it sold in domestic and overseas markets. G-U-CIDE, the Korean brand of Prontosil, was Yuhan's most financially successful item (Fig. 4).

Interestingly, the success of G-U-CIDE is often ascribed to a marketing catchphrase. ${ }^{32}$ The advertising placed much emphasis on 'difference': G-U-CIDE was presented as quite different from any previous medicine, and readers were urged by implication to completely disconnect and distance themselves from the inadequacies of old-fashioned medicine and pre-modern inefficiency. In a sense, this language clearly reflects the desire of the Yuhan Corporation to cure Korea's ills through the introduction of biomedicine, but also through the use of attendant modern methods, knowledge and even marketing strategies. Just as the Yuhan Corporation's new medicine destroyed pathogenic bacteria, so too would the modernity of biomedicine cure the deep-seated germs of Korean backwardness and inferiority.

Another of the Yuhan Corporation's brands was Neotone, a tonic that claimed to promote health by adding nutrition and strengthening users' immunity to disease. Lack of nutrition and resistance was described as not only a problem of individual bodies, but also the problem of the

$31 \quad$ Advertisements for Satsutarimu frequently appeared in the Daily News of Manchuria and Korea between 1940 and 1943. For a case study of the Japanese pharmaceutical company and Japanese research on Salvarsan, see Nihon Keieishi Kenkyūjo 2001. national body as a whole. Alluding to the deterioration of the nation's health in general, advertisements for Neotone encouraged people to consume the medicine to be healthy members of the Korean nation. ${ }^{33}$

An analogy between the individual and the nation is more explicitly expressed in Yuhan's corporate advertisements. While appealing to people to contribute to the 'Korean fund' for Korean business, advertisements for the Yuhan Corporation from 1926 emphasise the link between prosperity and national health:

By business, the nation-state ( $k u k k a$ 國家) becomes saved, and so does the individual. The nation-state that fails to develop business falls to the level of an uncivilised country and those who do not understand business become poor. Acknowledging this principle, we should be interested in business and work hard to find a means of survival. This is why we founded our company. We aim to show our reserves of strength and do our best in order to establish a Korean business that is solid and perfect. ${ }^{34}$

When the company offered investment stock in the same year, it argued that the company should be owned and managed by Koreans, using racial categories to underline the need for Koreans to take the initiative in investing in their own enterprises:

If we offer stocks for subscription to Caucasians (paegin 白人), we are sure to easily collect a huge fund in a short time. Yet, that would result in transferring our company to Caucasians, not Koreans. ${ }^{35}$

These brief examples demonstrate that manufacturers of biomedicine as a commodity utilised nationalist ideas, but often projected indigenous attributes negatively. The swift marketing of Prontosil by the Yuhan Corporation was motivated and sustained by Yu's and his colleagues' nationalistic ardour. From the beginning the owners of the corporation set out to establish a Korean pharmaceutical company with its own skill sets and markets, aiming to cure Korean diseases, and improve their fellow countrymen's health. ${ }^{36}$ The biomedically enhanced Korean body was going to be a radically different thing from bodies constructed and managed through indigenous ways of living. Hence the Yuhan Firm's nationalist passion looked for its fulfilment, to a great extent, through enlightenment and hygiene rather than through Korean culture. Even when marketing its vitamins, nutrients, tonics, and antibacterials in Manchuria and Vietnam, the Yuhan Corporation did not showcase 'Korean' attributes in its advertisements.

\footnotetext{
33 Manson ilbo, 3 December, 1940.

34 Yu Ir-han Chŏn'gi P'yŏnjip Wiwŏnhoe 1995, p. 518.

35 Ibid., pp. 519-20.

36 See, for instance, Hong 1972, pp. 53-61.
} 
But while the Yuhan Corporation's advertising rhetoric remained indifferent to any native 'Korean' characteristics, it still appealed to Koreans to support 'Korean business for Korea's own sake'.

\section{Seeking Markets in Manchuria ${ }^{37}$}

Manchuria, during the first half of the 2oth century, provides an intriguing lens through which to view the eclectic attributes of 'Koreanness', because it engaged the nationalist imagination of Korea's pure and primitive antiquity within the multi-ethnic circumstances that Koreans experienced outside Korea under Japanese colonialism. Koreans moved into Manchuria beginning in the 18th century for a variety of reasons: freedom from bondage, avoidance of severe taxes and forced labour, escape from criminal punishment or harsh creditors, and the quest for more fertile soil for farming. Whatever the reasons, during the late 18th and 19th centuries Korean settlers were willing to risk their lives by going against the Qing xenophobic policy of 'seal and prohibit' and the Chosŏn prohibition on emigration to Manchuria. It is those 'illegal' immigrants who are now remembered as the first generation of Korean immigrants in China.

Japan gained international approval for colonising the Korean peninsula after it won the Sino-Japanese war in 1895 and the Russo-Japanese war in 1905. It became obvious after 1905 that Korea, once a tributary kingdom belonging to the Sinocentric world order, was now compelled to fit herself into a Japanocentric political world. China, the Middle Kingdom, the millennia-long source of political, intellectual and cultural authority, was rapidly being marginalised in every sphere, including diplomatic ceremonial and education, and in public consciousness. ${ }^{38}$ The appeal of the new, combined with Japanese colonisation, rapidly displaced the centrifugal force of the Middle Kingdom. Given the changing contours of world politics around the Korean peninsula, it is not difficult to imagine that Koreans in Manchuria encountered even more complicated political and cultural issues than did Koreans in Korea: questions of land ownership, the extent of jurisdiction, and extraterritoriality, which mainly resulted from the ambivalent status of citizenship of Korean settlers in Manchuria, often

37 Regarding the term Manchu, see Duara 2003, pp. 41-2. Koreans have called Manchuria Manju (滿洲, Manzhou in Chinese), Yŏnbyŏn (延邊, Yanbian in Chinese) or Kando (間島, Jiandao in Chinese), the last of which literally means an island in-between. triggered disputes and even provoked physical conflicts among Koreans, Chinese, and Japanese. ${ }^{39}$

Japan argued for extraterritoriality. The Japanese claim over Korean citizenship made most Chinese uncomfortable. Koreans in Manchuria, particularly when in dispute with local Chinese farmers or officials, were thought to be agents of Japanese imperial penetration into China. The more Chinese nationalism grew, the more strongly Korean ties to Japanese rule were criticised. By way of compromise, a few Korean leaders in Manchuria at the end of the 1920 s persuaded their communities to become naturalised Chinese. ${ }^{40}$ However, when Korean settlers felt they were being unfairly treated, bullied or plundered by Chinese petty officials, landlords and local gangsters, they looked to Japanese consular jurisdiction as a resource. When Manchukuo (1932-45) was established, Koreans in Manchuria were considered to belong to the state of Manchukuo, and were classified as one of the ethnic groups of that state. But the general Chinese view was that Koreans were closer to the Japanese empire than other ethnic groups, including Manchurian and Mongols as well as Han Chinese. In other words, even when Koreans were defined as people of Manchukuo, they were still represented as the second people of Japan, or as Korean Japanese, with more intimate cultural, linguistic, and social connections to Japan than to China. ${ }^{41}$

Given this milieu, Korean in-betweenness in Manchuria was experienced in every corner of people's lives. Ethnically, culturally, and politically, Koreans were situated in between Chinese and Japanese officials; between Han Chinese and Manchurians; and between the lost motherland and occupied foreign lands. Caught in the middle of these intersecting ethnic groups and therefore needing to be open to political negotiation, Koreans in Manchuria cooperated or resisted, and more often compromised merely to survive or to recover their losses. ${ }^{42}$ As a reflection of this weakened identity, Korean immigrants' plight was described in metaphors of sickness, and Korean writers in Manchuria underlined the way in which harsh circumstances created psychosomatic wounds that were accumulated and exacerbated into more serious chronic diseases.

Medical advertisements conveyed their messages with a vocabulary and visual register that set the agenda whereby unhealthy conditions were defined and proper treatment was claimed. After the establishment of Manchukuo, both

\footnotetext{
39 Kwŏn 1990.

$40 \quad$ Shin 1999, p. 184.

41 Kwŏn 199o, pp. 276-7. K. Kim 2004, pp. 16-25.

42 For the in-betweenness expressed in literary works by Koreans in Manchuria, see, J. Kim 2004.
} 
Japanese and Korean traders actively expanded their markets in Manchuria. Little research exists charting the early 2oth-century rise of the medical trade between Korea, Japan, and Manchuria. However, the advertisements in Mansŏn ilbo 滿鮮日報 (Daily News of Manchuria and Korea) and Mansen no ikai 滿鮮之醫界 (Medical World of Manchuria and Korea $)^{43}$ suggest that major Japanese drugstores and pharmaceutical companies with Korean branches in Seoul established additional headquarters in Changchun (長春), the capital of Manchukuo. Their Korean counterparts responded similarly. The Yuhan Corporation established the Yuhan Pharmaceutical Company of Manchuria (Yuhan manju cheyak 柳韓滿 洲製藥) in Fengtian (奉天) in 1938, aiming to trade its own-brand items throughout Manchuria. ${ }^{44}$ Kŭmgang Pharmaceutical Company (Kümgang cheyak 金剛製藥), which synthesised Salvarsan and anaesthetics containing morphine or cocaine, also sold its branded medicines in Manchuria. ${ }^{45}$ Not only these major pharmaceutical companies but also successful apothecary stores aimed to trade their patent medicines in Manchuria in the 193os. The number of Korean immigrants exceeded that of Japanese residents in Manchuria, so although the Japanese held administrative and political authority, Koreans were able to maintain economic rights, not to mention their ethnic identity.

The ways in which medicines were marketed to Koreans in Manchuria did not explicitly articulate the liminal politics of Korean 'in-betweenness'. However, the advertisements do show how Korean ethnic identity was deliberately fashioned as a reflection of the physical and cultural circumstances that Korean immigrants faced in Manchuria. Needless to say, most advertisements by the Japanese pharmaceutical companies and the Yuhan Corporation are copies of the domestic versions. Yet advertisements for medicines like Paekpohwan 百補丸 (the Pill

43 Mansŏn ilbo was first published in 1937, combining two previous local newspapers, Kando ilbo間島日報 (The Daily News of Kando), which appeared in the 1920s, and Mangmong ilbo滿蒙日報 (The Daily News of Manchuria and Mongolia), first printed in 1933 and published in Korean. Mansŏn ilbo is one of the most significant sources of information on the daily lives of Korean immigrants in Manchuria during the first half of the 2oth century. In 1936, approximately 875,908 Koreans resided in Manchuria. K. Kim 2004, p. 34.

44 Hong 1972, pp. 69-71. Yu Ir-han Chŏn'gi P'yŏnjip Wiwŏnhoe 1995, p. 585. In addition to the Fengtian branch, the Yuhan Firm also established branch shops in Dalien 大連, Tianjin 天津, Shanghai 上海, and Taibei 臺北, and branch offices in Los Angeles and Tokyo, between 1933 and 1943 . of Nourishment with One Hundred Ingredients, hereafter the Pill of Nourishment) and Mansu paekpohwan 萬壽百補 丸 (the Longevity Pill of Nourishment with One Hundred Ingredients, hereafter the Longevity Pill) include content specifically tailored to appeal to Korean immigrants.

First and foremost, they highlight the Chinese origin of traditional medicine. An advertisement for the Longevity Pill explains that traditional medicine originated in Manchuria (i.e. a part of China) and not Korea or Japan, and suggests that the pill is based on a traditional secret formula that guarantees greater efficacy than newer products manufactured in Korea or Japan. Although the drugstore selling the Longevity Pill - Kŭmgang Pharmaceutical Company (Kŭmgang cheyak 金剛製藥) - was not exclusively based in Manchuria, it emphasised geographical difference as a way to establish its authentic traditional credentials, thus differentiating its brand from other nourishing pills. ${ }^{46}$

Furthermore, the advertisement uses the harsh environmental conditions peculiar to Manchuria - drought and extremes of hot and cold weather that sapped water, energy and primal vitality (wŏn'gi 元氣) from both plants and human beings - to point to the origins of a perceived decline in immunity suffered by Koreans living there. Hence, even those who have never previously taken the 'Pill of Nourishment' are urged to consider doing so without delay (Fig. 5). ${ }^{47}$

Sickness among Koreans in Manchuria was often ascribed to the strong and hazardous winds of the region. A series of articles based on interviews with Korean women in Manchuria revealed that Korean settlers were apt to be troubled by severe cold, muscular fatigue, and mental exhaustion, symptoms that were not curable by one-dose medicines. The women interviewed ascribed their bodily discomfort to the merciless wind of Manchuria, which in their expression, 'broke through' every joint of their limbs. ${ }^{48}$

A corollary of the portrayal of Manchuria as the cause of chronic illness was the portrayal of Korea as the best place for healing herbs. The Pill of Nourishment was apparently the best in the world because its ingredients were rooted in Korean soil. Unlike medicines imported from the West or even from other places in East Asia, the pill was made from mysterious grass roots from the homeland and the fruit of trees free from all kinds of chemical adulterants and foreign contamination. Korean soil was an indispensable element of health, even for those who resided elsewhere. ${ }^{49}$

\footnotetext{
46 Mansŏn ilbo, 5 December, 1939.

47 Ibid., 13 January, 1940.

48 See the series of interviews published in Mansŏn ilbo, 1 January, 1940.

49 Mansŏn ilbo, 7 December, 1939.
} 


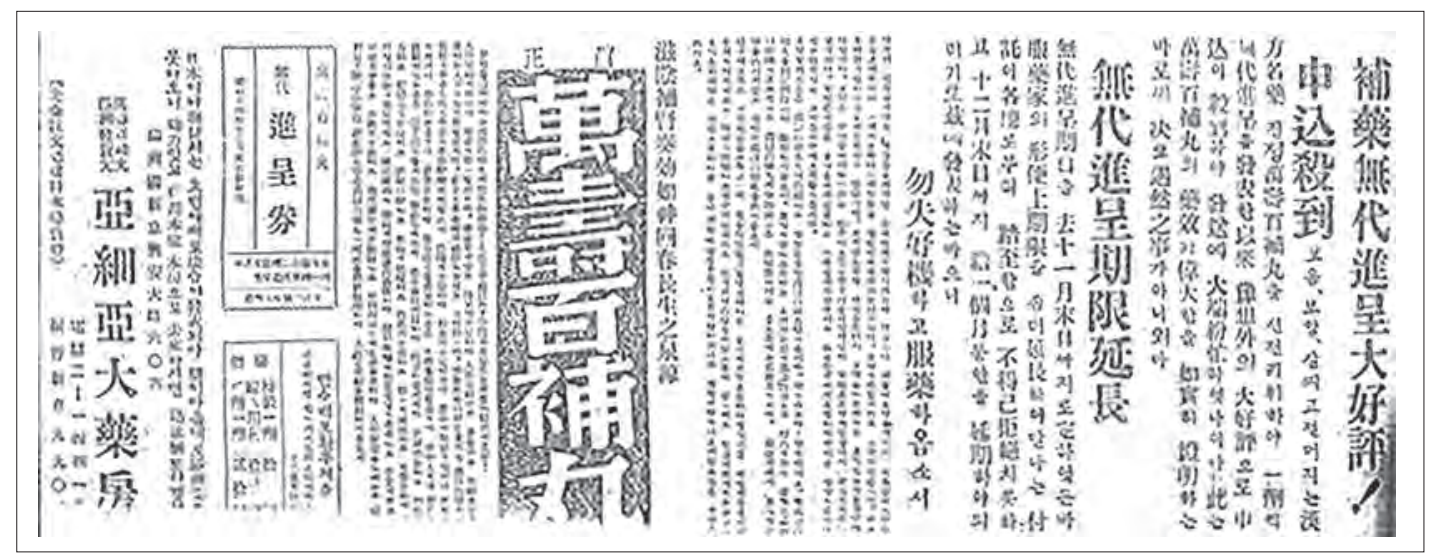

Figure 33.5 Advertising 'Mansu paekpohwan' 萬壽百補丸 (Longevity Pill of Nourishment with One Hundred Ingredients) in Mansŏn ilbo 滿鮮 日報 (Daily News of Manchuria and Korea) December 1940

Furthermore, the authority of the Government-General was invoked to highlight the connection between health and the native Korean condition. The Imperial University, with the support of the Government-General, encouraged research into Korean indigenous medicine and botanicals native to Korea..$^{50}$ To target Korean immigrants in Manchuria, some medical advertisements described how medicine from Manchuria (China) guaranteed superior efficacy, while others emphasised that Koreans needed purely Korean medicine whose ingredients were exclusively grown in Korea.

In addition, the Longevity Pill deployed biomedical terminology as part of its advertising arsenal. Interpreting knowledge drawn from traditional medicine in the new language of endocrinology, the advertisements explain sexual apathy, nervous breakdown, and general symptoms of sensitivity.

The eclectic and hybrid features of medicine advertisements should be understood in the context of an adjustment of both manufacturing and marketing to the changing political and cultural contours of the region. At times, an appeal to the local and indigenous qualities of the medicines proved an effective marketing strategy. However, there existed no purely indigenous medicine. Rather, there were various medical commodities with multi-regional ingredients and various methods of advertising, all of which claimed to serve the best health of Koreans.

\section{Conclusion}

Medical advertisements published in the early 2 oth century provide a lens to view the contingent and eclectic aspects

$5^{\circ}$

See, for instance, Japanese research published in Mansen no ikai 68 (1926) to 89 (1928). of Korean medicine. The multifarious elements that constituted Korean medicine need to be considered, as the purity of indigenous medicine was often asserted in conjuring up traditional medicine as a national heritage. With a critical perspective on a contemporary framing of the uniquely Korean medicine, it is possible to avoid essentialising a Korean medicine as if it were a self-determining unit of analysis. Traditional medicine in Korea shares its textual heritage with China and has often looked to China for medical innovations. Novel texts from China were constantly in demand; the Korean physicians who accompanied the official envoys to China during the 16th century sought to learn from Chinese doctors; the theories of the Four Masters of the Jin (1115-1234) and Yuan (1271-1368) period ${ }^{51}$ were carefully read and interpreted by Korean scholars. At the end of the 19th century a Korean doctor practising in Seoul would proudly legitimise his own medical writings by claiming an unbroken line of descent from the Yellow Emperor and the Four Masters.

But while Koreans valued the Chinese origins of their medical tradition, they also emphasised the local qualities of Korean medicine. Publications on local botanicals increased during the 15 th century; in the 17 th century, Hŏ Chun 許浚 (1539-1615) acknowledged that Korea had her own indigenous medical tradition - Tongǔi 東醫 (Eastern Medicine) - while at the same time, he synthesised Chinese Song-Yuan-Ming medicine (medicine from the mid-1oth

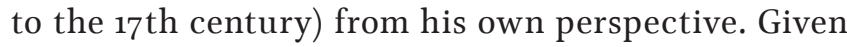
Korea's dual position as both an importer of medicine from China and a producer of its own medicine, the traditions of Korean medicine should be viewed as an outcome of tension and compromise between the claim to the local

\footnotetext{
51 Liu Wansu 劉完素 (1120-1200?), Zhang Congzheng 張從正 (1156-1228), Li Gao 李杲 (1180-1251) and Zhu Zhenheng 朱震亨 (1281-1358).
} 
and an aspiration toward the universal. The changing relationship between the indigenous and the foreign, or the local and the universal, enables us to view the ways in which the identity of Korean medicine has been created and modified over time.

The verbal and graphic language of medical advertisements in the major newspapers provides an example of this changing mode of styling indigenous products. Against a colonial backdrop, and enthused with a growing sense of nationalism, drug sellers, as we have seen, were among those who shaped indigenous medicine to their own ends. They exhibited a marked sensitivity to the changing relationship between local and universal legitimacy for their products as they negotiated the demands of their target audience. The Japanese and Western agents attempting to expand their markets in Korea were sources of new manufacturing skills and advertising strategies. Navigating the overwhelming flow of medical commodities from abroad, Korean drug sellers gradually established markets for their own goods. In that process, new strategies were developed, yet there was also continuity in the methods of both manufacturing and selling medicinal products.

Consistent representation of the indigenous was not an issue in the competitive drug trade. However, to maximise profits under Japanese domination, Korean drug sellers had to develop local networks to attract retailers in outlying areas, and they utilised Korean sociocultural references to promote sales. Early 2oth-century advertisements for medicine show that the indigenous features of Korean medicine were both utilised and ignored as Korean drug sellers sought to create and exploit new opportunities for themselves. 'Medicine for the Koreans' was undoubtedly emphasised. Yet advertising tactics from Japan, new theories from the West, and the ancient authority of Chinese medicine were selectively employed to style the imagery and meanings of the 'Korean' attributes of Korean medicine.

\section{Bibliography}

\section{Primary Sources}

Maeil sinbo 매일신보 (Daily News).

Mansŏn ilbo 滿鮮日報 (Daily News of Manchuria and Korea).

Mansen no ikai (Mansŏn ji ŭigye) 滿鮮之醫界 (Medical World of Manchuria and Korea).

\section{Secondary Sources}

Brooks, T. and A. Schmid (eds) 2000, Nation Work: Asian Elites and National Identities, Ann Arbor, MI: University of Michigan Press.

Burns, S. 2009, 'Marketing health and the modern body: patent medicine advertisements in Meiji-TaishōJapan', in Purtle and Thomsen (eds), 179-202.

2012, 'The Japanese patent drug trade in East Asia: consumer culture, colonial medicine, and imperial modernity'. Paper presented at 'Medicine, Politics, and Culture in the Japanese Empire', University of Chicago, 11-12 May.

Cho, Sŏnggi 조성기 2005, Yu Ir-han p'yŏngjŏn 유일한평전 (A Critical Biography of Yu Ir-han), Seoul: Chagŭn ssiat.

Cochran, S. 2006, Chinese Medicine Men: Consumer Culture in China and Southeast Asia, Cambridge, MA: Harvard University Press.

Duara, P. 2003, Sovereignty and Authenticity: Manchukuo and the East Asian Modern, Lanham, MD: Rowan \& Littlefield Publishers Inc.

Han'guk kwanggo yŏn'guwŏn 한국광고연구원 1996, Han'guk kwanggo paengnyŏn 한국광고백년 (Korean Advertising: 100 Years), Seoul: Han'guk kwanggo tanch'e yŏnhaphoe.

Hong, Hyŏn-o 홍현오 1972, Han'gukyagŏpsa 한국약업사 (A History of Pharmacy in Korea), Seoul: Handok Yagŏp Chusikhoesa.

Kim, Jae-young et al. 김재용 외 2004, Chaeilbon mitchaemanju ch'inil munhak üinolli 재일본및재만주친일문학의논리 (The Rationale of Pro-Japanese Literature Published in Japan and Manchuria), Seoul: Yŏngnak.

Kim, Kyo-sik 김교식 1984, Yu Ir-han, Yuhan Yanghaeng gŭrup 유일 한, 유한양행그룹 (Yu Ir-han, and the Yuhan Corporation), Seoul: Kyesŏng ch'ulp'ansa.

Kim, Kyŏng-il et al. 김경일 외 2004, Tongasia ŭiminjok isan kwa tosi 동 아시아의민족이산과도시 (Korean Diaspora in Manchurian Cities in the Early 2oth Century), Seoul: Yŏksa Pip'yŏngsa.

Kwŏn, Rip 권립 1990, "Historical survey of the legal status of Korean Chinese', Chosŏn hakyŏn'gu 朝鮮學研究 (Korean Studies) 2, 266-85.

Nihon Keieishi Kenkyūjo 日本經營史研究所 (Japan Business History Institute) (ed.) 2001, Medicine for the People: the First 85 Years of Banyu Pharmaceutical 1915-2000, Tokyo: Banyu Pharmaceutical Co. Ltd.

Park, Yun-jae 박윤재 2005, Han'gukkŭndae ǔihakŭikiwŏn 한국근대의 학의기원 (The Origins of Modern Medicine in Korea), Seoul: Hyean.

Purtle, J. and H. Thomsen (eds), Looking Modern: East Asian Culture from Treaty Ports to World War II, Chicago IL: Art Media Resources.

Schmid, A. 200o, 'Decentering the "Middle Kingdom": the problem

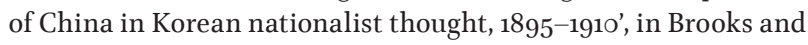
Schmid (eds), 83-107.

Shin, Chu-baek 신주백 1999, Manchu chiyŏk hanin ŭiminjok undongsa 만주지역한인의민족운 동사 (A History of the Korean Nationalist Movement in Manchuria), Seoul: Asea munhwasa.

Shin, In-sup 신인섭 1980, Han'guk kwanggo paltalsa 한국광고발달 사 (A History of Advertising and its Development in Korea), Seoul: Ilchogak.

Shin, In-sup 신인섭 and Shin, Kie-hyuk 2004, Advertising in Korea, Seoul: Communication Books.

Shin, In-sup 신인섭 and Sŏ, Pŏm-sŏk 서범석 2011, Han'gukkwanggosa 한국 광고사 (A History of Advertising in Korea), Seoul: Nanam.

Ye, Chong-sŏk 예종석 2009, Hwalmyŏngsu 10o nyŏn sŏngjang ŭi pimil 활명수100년성장의비밀 (Hwalmyŏngsu, the Secret of 10o Years of Development), Seoul: Lidŏsŭ buk.

Yu Ir-han Chŏn'gi P'yŏnjip Wiwŏnhoe 유일한전기편집위원회 1995, Nara sarang ŭi ch'am kiŏbin, Yu Ir-han 나라사랑의참기업인유일 한 (Yu Ir-han, Patriot and Businessman of Integrity). Seoul: Yuhan yanghaeng. 


\title{
34 The Visual Language of Medicine Advertisements in The Ladies'Journal
}

\author{
Chang Che-chia 張哲嘉
}

The Ladies'Journal (Funü zazhi 婦女雜誌) was the most highly regarded, the longest running, and the most widely circulated magazine for women in early Republican China. ${ }^{1}$ During the 17 years of its publication (1915-31), it built up a loyal readership. It was among the top 10 most popular magazines as voted by Chinese secondary school students. ${ }^{2}$ Published in Shanghai, it had subscribers all over China and in Chinese communities overseas, and can be considered one of the most influential periodicals of its time. Its broad appeal makes it an excellent case study for patent medicine advertisements in this period.

It is over 20 years since Huang Ko-wu 黄克武 pioneered the study of medicine advertisements in a seminal article that examines ideas of the body and illness as reflected in the Chinese advertisements in Shenbao 申報 (a popular and authoritative daily newspaper, also published in Shanghai), thereby providing a general overview of the medico-social life of ordinary Chinese people in the early Republican period. ${ }^{3}$ This chapter adopts a different approach, focusing on the visual language and strategies of the medical advertisements that appeared in The Ladies' Journal. ${ }^{4}$

Every issue of The Ladies' Journal includes medical advertisements. Over its run of 17 years, it carried over 900 advertisements for patent medicines, making this the second most frequent type of advertising. ${ }^{5}$ Furthermore, the content of the journal itself reveals the pervasive influence of advertising. From 1925 to 1931, it ran a 'Medical Advisory Column' - a doctor's answers to readers' letters - in response to persistent enquiries about the patent medicines advertised in its pages. Even though the column repeatedly exhorted readers not to believe all the advertisers' claims, and not to equate patent medicines with 'Western medicine' as recognised by the medical profession, they continued undeterred to write in, requesting instructions on the most effective ways to take the advertised drugs. Nothing, apparently, could stem their faith. ${ }^{6}$ It seems that medical authority could not compete with

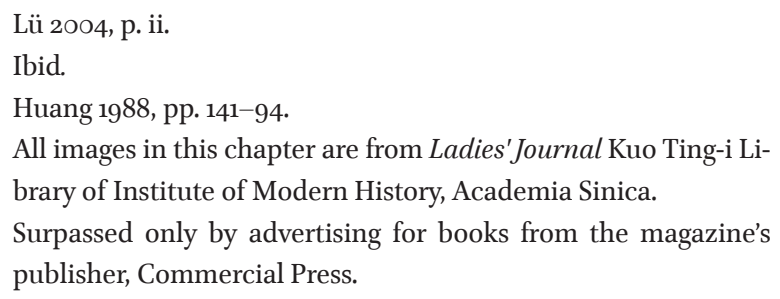

6 Chang 2004, pp. 145-68. the power of advertisements, however far-fetched their claims. Moreover, due to a lack of regulation, pharmaceutical businesses could exaggerate as much as they pleased without legal consequences. This chapter will explore the visual language that these advertisements used to convey their messages, and the kinds of marketing strategies they deployed in order to convince Chinese readers.

Most of the pharmaceutical products advertised in The Ladies'Journal are of foreign origin. The small minority of advertisements placed by Chinese manufacturers are by and large plain, simple and unsophisticated, in comparison with the more visually inventive and deliberately targeted work of foreign advertisers, so although they continued to be published throughout the entire run of the magazine, they are unlikely to have made much impact on consumers.

To highlight the theme of visual language, I will begin with a brief review of visual advertising techniques in pre-modern China and Japan, and then consider three particularly striking cases of foreign drugs marketed in China: Chūshōtō 中將湯 (Chūshō's Decoction) from Japan, aspirin from Germany, and DrWilliams' Pink Pill for Pale People from Canada, in comparison with domestic Chinese products. I will examine how the advertisements for these foreign drugs were localised so as to appeal to Chinese tastes, and look at the techniques used to stimulate consumers' desire to purchase the drugs.

\section{Print Media and Drug Advertising in Pre-Modern East Asia}

Merchants in pre-modern China utilised various kinds of visual material to call attention to their wares. Their main tools were signboards and banners, which relied heavily on text to communicate their message. Occasionally, purely visual signs are found, generally trademarks or emblems of the type of merchandise on sale. For example, sellers of plasters and poultices would hang out a string of model plasters, while oculists displayed a big image of an eye at the entrance to their premises. ${ }^{7}$ Sometimes, merchants would use visually appealing small objects, such as decorative containers or packages, to impress customers and catch the eye of passers-by. ${ }^{8}$ These simple and rudimentary

$7 \quad$ Yomeda 1931, p. 106; Qu 200o, pp. 73-81.

$8 \quad$ Xu 2006, pp. 112-13. 
visual signals served to announce the presence of a store of a particular type in the area, but can scarcely have generated additional business.

In comparison with China, Japan has the reputation of being more highly sensitive to visual presentation. It is interesting therefore to see how print advertisements for traditional drugs compare with those published in China. As historians of advertising have shown, by the end of the Edo Period (16o3-1867), Edo (Tokyo) had become the largest metropolis on earth, and commercial activity in the city had reached an unprecedented level, providing fertile ground for new developments in advertising. ${ }^{9}$ As early as 1683, the Japanese store Echigoya 越後屋 created a genre of advertising leaflets known as insatsu 引札, which were handed out in the street. Impressively, Echigoya also utilised these leaflets to evaluate its marketing strategy. In 1762, Echigoya distributed leaflets in 12 Japanese cities, inviting merchants to bring their wares to a large-scale fair (similar to a modern trade fair). This marketing method proved effective and was soon adopted by many other businesses, including drug merchants, as is evidenced by the considerable numbers of promotional leaflets for drugs that still survive. ${ }^{10}$ Early 19 th-century Japan surpassed Europe and America in the scope and quantity of advertising activities, creating the prototypes of modern advertising methods as taught in the fields of mass communications and mass media. ${ }^{11}$

Edo drug-sellers also invented a genre of artistic prints called eshi 絵紙, i.e. 'painted paper', offered to customers as promotional free gifts. The subject matter of eshi included landscapes, portraits of beautiful women, and popular theatre plays of the day. Though hardly any of the eshithemes were related to the drugs on offer, the pictures attracted customers. The innovators of this advertising method, which is reported to have produced excellent marketing results, were travelling drug sellers from Toyama 富山 fiefdom. ${ }^{12}$ However, while Edo Japanese drug merchants certainly exploited the power of illustration in their advertising material, they do not seem to have used images to generate demand by creating a concept of the effectiveness of a drug. This strategy would develop later.

\section{Chinese and Japanese Medicine Advertisements in The Ladies'Journal}

In the mid-19th century, the Western powers began to exert their influence in East Asia, bringing new consumer goods and new means of circulating information. One of the few Chinese businesses advertising in The Ladies' Journal was Yong'an Tang 永安堂 (Wing On Tong; Hall of Everlasting Peace), which focused on marketing two signature products: Wanjin you 萬金油 (Tiger Balm; lit. Oil of Ten Thousand Gold Pieces) and Bagua dan 八卦丹 (Pat Kwa Tan; lit. The Pill of Eight Trigrams), a refreshing medicinal compound that is still popular in Taiwan, Hong Kong, and Southeast Asia to this day. Another major provider of advertisements was Guangdong Zhongde Yuan 廣東 種德園 (Garden of Planting Virtues in Guangdong), which offered a wider product line including gynaecological and paediatric patent medicines. Overall, the style of illustration in these advertisements is simple and straightforward. Almost half of a full-page advertisement for Yong'an Tang is taken up by the firm's trademark, a tiger. Guangdong Zhongde Yuan's advertisements generally include pictures of women and children, the target users. However, the advertising designers seem to have made little attempt to entice customers. They employ no specific persuasive techniques, and do not highlight the indigenous character of the product as a selling point.

The Japanese drug Chūshōtō, a compound of over a dozen crude medicinal substances, was often assumed to be a traditional Chinese medicine, although it was in fact formulated in the 19th century by Dr Saeki Riichirō 佐伯 理一郎 (1862-1953). The name of the drug is derived from a historical figure of the 8th century, called Chūshōhime 中將姬 (747-75), whose story resembles a Japanese version of Snow White. The great-granddaughter of Fujiwara no Kamatari 藤原鐮足 (614-69), an important figure in the Taika Reforms (645) and founder of the powerful Fujiwara clan, Chūshōhime was a devout Buddhist. When Chūshōhime reached the age of 14, her stepmother, jealous of her beauty and wisdom, ordered a family retainer to take the young girl into the forest and kill her. However, the servant, touched by her virtues and piety, let her live, and she was taken in and cared for by a countryman named Fujimura 藤村. When Chūshōhime's father found her, after years of searching (by which time she had attained an advanced Buddhist state and decided to become a nun), he rewarded Fujimura with a secret gynaecological formula handed down from earlier generations.

By the 19th century, this tale was still a popular subject in the Japanese theatre, but the recipe for the drug was long lost. In 1893, however, the merchant Tsumura Jūsha 津村重
Ōbushi 1999, p. $5^{2}$.

Takakuwa 1994, pp. 66-7.

Ibid., pp. 69-71.

Netsuka 1979, pp. ${ }^{148-56 .}$ 
舍, a member of the Fujimura family on his mother's side, acquired Dr Saeki's gynaecological formula. In marketing the drug, he drew on his family's associations with the tale of Chūshōhime, which inspired the brand name. Exhibiting a remarkable talent for marketing, Tsumura linked his product with an episode rooted in Japanese tradition to create an instant sense of familiarity. In addition, Tsumura actively used the new media - newspapers - to launch Chūshōtō, taking the unprecedented step of buying up three-quarters of the front page of a newspaper for his advertising campaign, so as to maximise brand recognition. Tsumura was also the first person in Japan to use neon signs. This coordinated strategy made Chūshōtō an overnight success, and a textbook case in the history of Japanese advertising. ${ }^{13}$

In Japanese newspapers, Chūshōtō was marketed through a two-pronged approach, targeting two contrasting groups. Advertisements for the conservative customer show a senior businessman in the centre of the page, paying homage to the customer for purchasing his product, following a time-honoured advertising convention of the Edo period. Advertisements aimed at the younger generation feature smiling, fashionably dressed young ladies, hinting that Chūshōtō will confer beauty and satisfaction on the consumer.

However, neither of these themes was adopted in the pages of The Ladies'Journal. Culture-specific Japanese conventions and associations could not be directly transplanted to China, where moreover images of European - not Japanese - women were high-status symbols of fashion. In China, Chūshōtō employed a completely different visual strategy. In The Ladies'Journal, two patterns are particularly worth noticing. One type of advertisement describes the manifold sufferings of women, such as sickness, debility, and brief life expectancy, suggesting that the reason for all these sufferings is that women do not take Chūshōtō. The other type appeals to Chinese women's desire for progeny. Figure 1 shows a middle-aged couple gazing dotingly at a baby held aloft in the husband's arms. The text says:

Even if one possesses many-storeyed mansions or 10,000 hectares of fine rice paddies, what treasure does a family own if it has no children?

And:

For women who are childless, the effects of taking Chūshōtō promptly will surpass those of praying to Buddha. ${ }^{14}$

The images and texts exploit the cultural imperative to carry on the family line and the social reality that a wom-

\footnotetext{
$13 \quad$ Kaga 2003, pp. 139-42.

14 The Ladies'Journal, 1.1 (1915), front page.
}

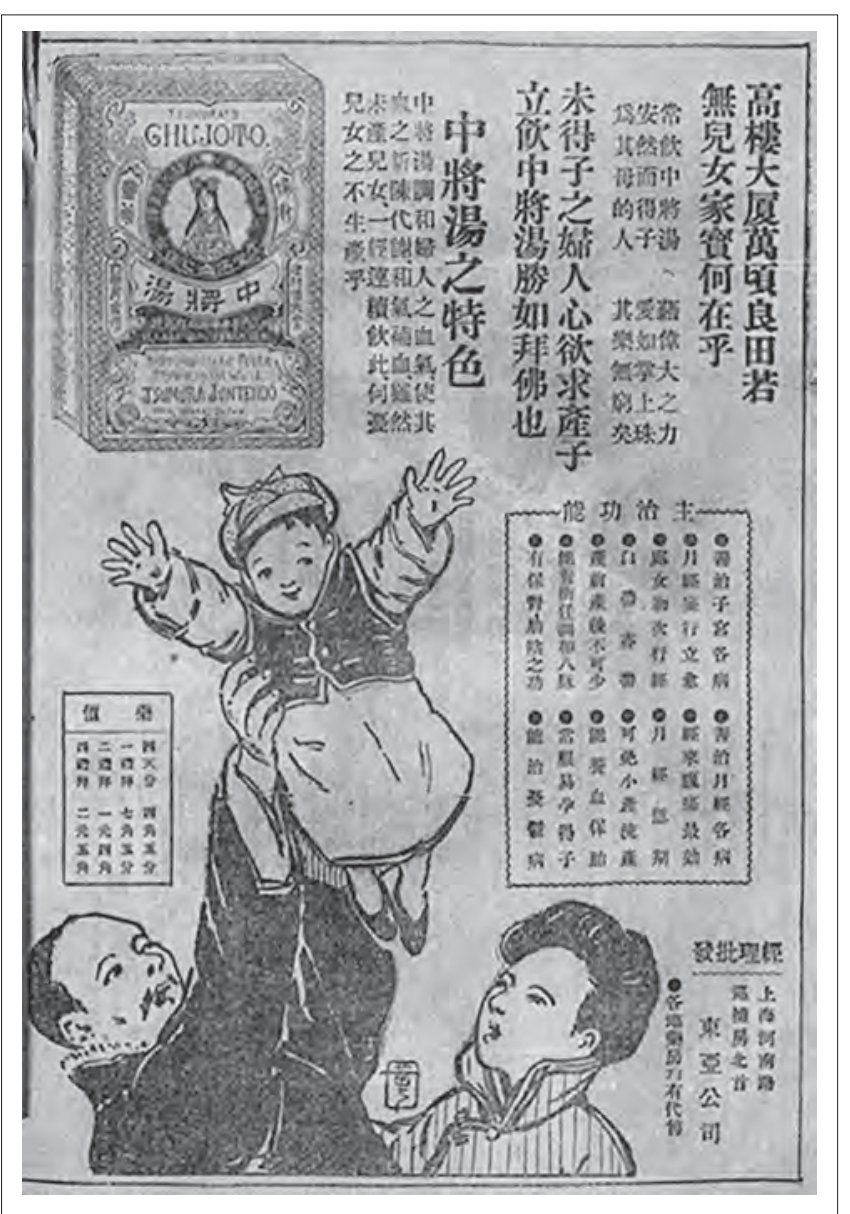

Figure 34.1 The Ladies'Journal: 中將湯 Chūshōtō advertisement

an without a son had no status, in an attempt to win the hearts and minds of female customers.

Other Japanese medicine manufacturers, such as Rixin Dayaofang 日信大藥房 (Nisshin Dispensary) and Shanghai Jishengtang 上海濟生堂 (Sai Sei Do, The Japanese Pharmacy; lit. Hall of Rescuing Lives in Shanghai) also advertised in The Ladies' Journal when it was first launched in 1915. However, not long afterwards, all these Japanese businesses, including Chūshōtō, withdrew from advertising in the magazine, probably due to anti-Japanese feeling in China at the time. Thereafter, almost all the medical advertisements were sponsored by European or American companies.

\section{European and American Drug Advertisements}

In early Republican China, European and American businesses were the major players in the pharmaceutical market. In the context of The Ladies' Journal, objects, topics and products are often presented as coming from the 'West', and are thereby connoted as being progressive, up-to-the-minute and therefore a good thing. However, some advertisements also use Chinese linguistic devic- 


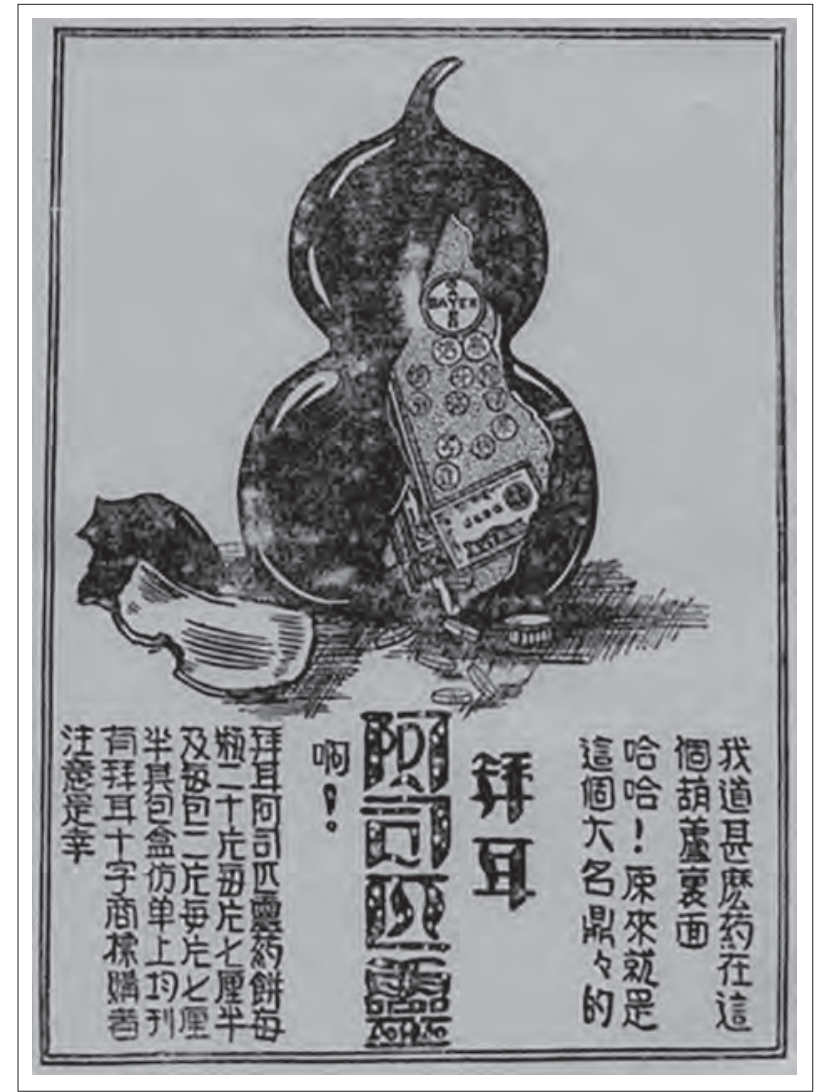

Figure 34.2 The Ladies'Journal: Bayer 拜耳 Aspirin Advertisement 1

es and local associations to appeal to readers. A classic example of advertising for a Western product that has been localised for the Chinese market is that of aspirin, marketed by Friedrich Bayer \& Co from Germany. Aspirin was invented in 1897 by the scientist Dr Felix Hoffman, and became available commercially from $1899 \cdot{ }^{15}$

Bayer excelled not only in developing drugs, but also in devising marketing strategies. A key feature of Bayer's drug advertisements was the use of Chinese linguistic and historical elements as a vehicle for the advertising message. Figures 2 and 3 ('The broken gourd' and 'Agreement without prior consultation') are characteristic examples. Figure 2 includes the idiomatic expression 'What drugs are hidden in the gourd waiting to be sold?', thus linking aspirin with the legendary panacea contained in the gourd of the Immortals. ${ }^{16}$ The central motif in Figure 3 consists of two men wearing Chinese gowns, showing each other the palms of their hands, on which are written the Chinese characters for 'Bayer Aspirin', and smiling at each other as though in perfect agreement. ${ }^{17}$ This image would have reminded readers, in a highly effective and economical fashion, of a story known to almost every Chinese person

15 Mann and Plummer 1991, pp. 23-7.

16 The Ladies'Journal, 16.7 (1930), front page.

17 Ibid., 16.1(1930), front page.

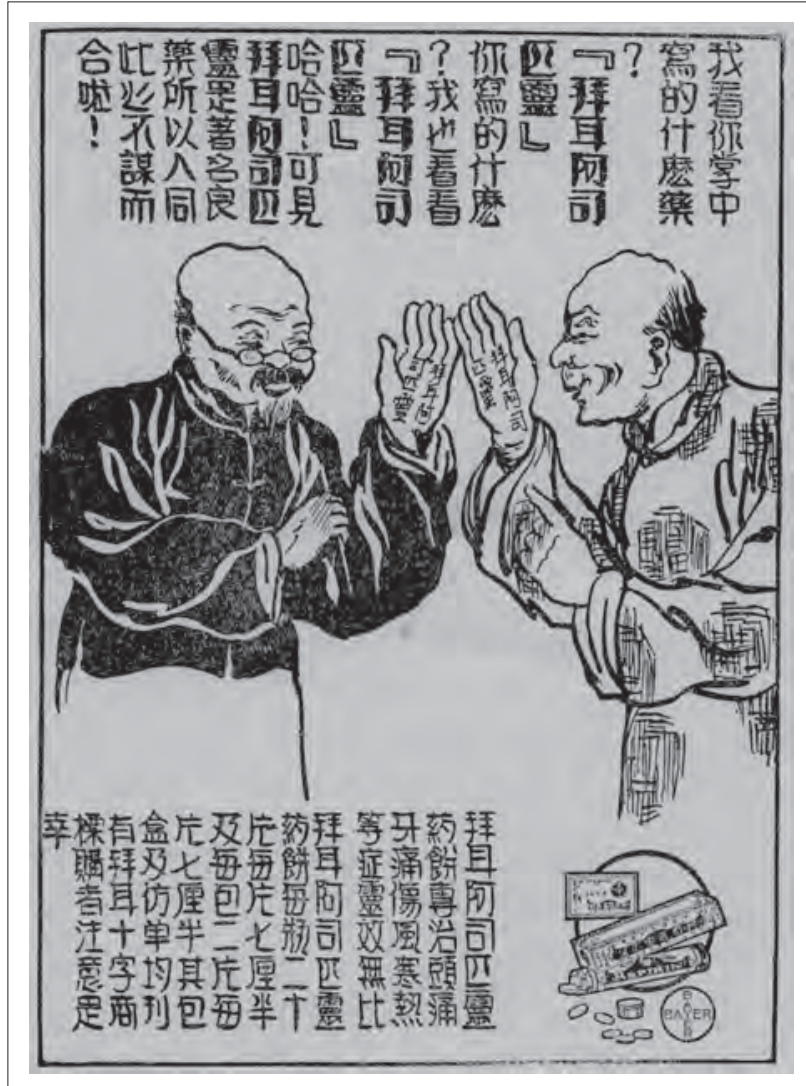

Figure 34.3 The Ladies'Journal: Bayer 拜耳 Aspirin Advertisement 2

- the decisive Battle of Chibi in the Romance of the Three Kingdoms. The novel recounts how, on the eve of that battle, the wise statesman Zhuge Liang and the famous general Zhou Yu discuss strategies for the defeat of a great army led by Cao Cao. Lest their conversation be overheard, they decide to write down their stratagems on their palms. Both Zhuge and Zhou write the character 'fire'. They simultaneously realise that they have both had the same idea, and exchange a smile of complicity. Through this image, the advertisement suggests that taking aspirin will bring about decisive victory in the battle against illness, at the same time hinting that those who buy aspirin are as wise as Zhuge Liang and Zhou Yu.

Most pharmaceutical companies were less innovative, and instead made use of the prevailing local tradition of personal recommendation to promote their products. In the absence of public institutions to certify the quality of physicians and drugs, the main social mechanism to avoid being duped by a charlatan or sold a fake nostrum was word of mouth, and patients canvassed friends and relatives for reliable recommendations. Thus it seemed only natural that drug companies should provide personal testimonials in their advertisements. As Huang Ko-wu points out, the most frequent form of medical advertising in the popular daily newspaper Shenbao was the "Testimonial of 


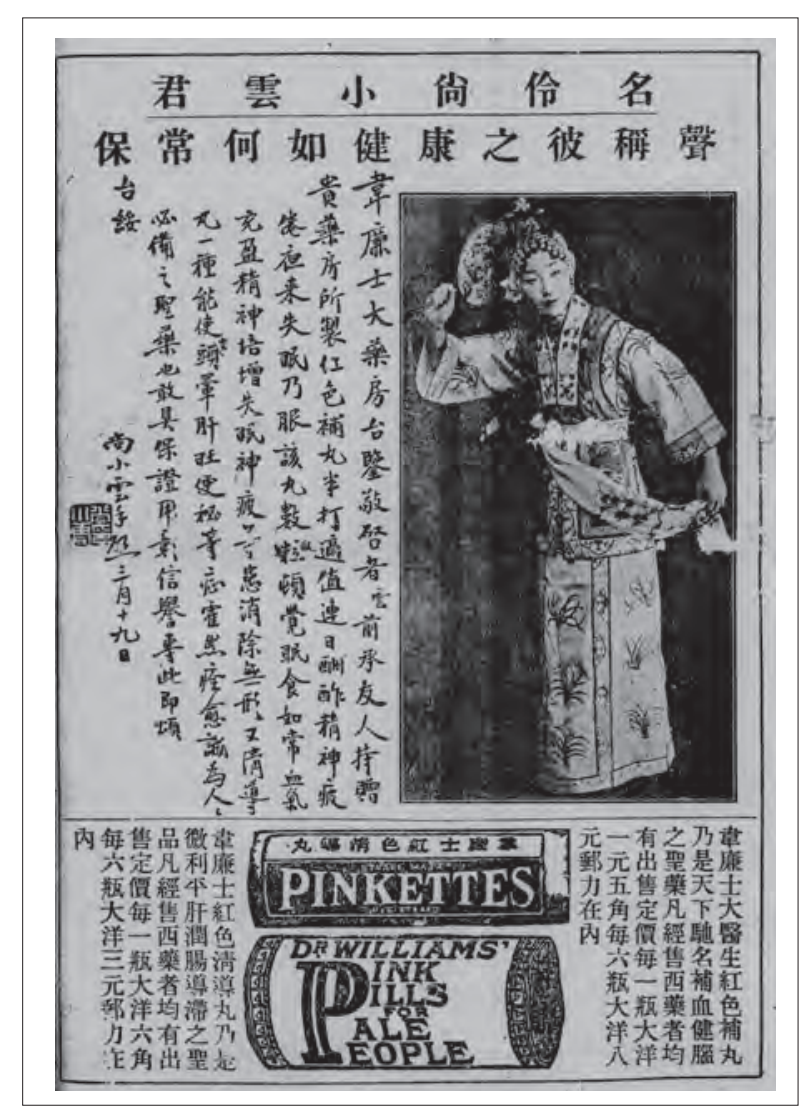

Figure 34.4 The Ladies'Journal: Handwritten testimonial by actor Shang Xiaoyun 尚小雲

Successful Healing'. ${ }^{18}$ This is also the case in The Ladies' Journal, where a considerable proportion of drug advertisements include testimonials describing how the patient has recovered from a problematic or serious disease after taking the advertised medicine. These testimonials were generally referred to as zhengshu 證書 (lit. holograph or letter of certification). In such advertisements, the image, trademark and testimonial function as complementary forms of persuasion.

Figure 4 is a typical example of this strategy. It takes the form of an autograph letter by the famous actor Shang Xiaoyun 尚小雲 $(1900-72)$, expressing his gratitude for the effects of Dr Williams' Pink Pills for Pale People. At the upper right is a photo of Shang Xiaoyun, magnificently attired in traditional Peking Opera costume. On the left is a facsimile of a letter in which Shang lists his various ailments prior to taking the wonder drug. The trademark and packaging of Dr Williams' Pink Pill are clearly displayed below, to enable the customer to identify the correct product. ${ }^{19}$

This advertisement exemplifies the time-honoured commercial strategy of endorsement by authorities and celebrities, which was common in East Asia long before

$18 \quad$ Huang 1988, p. 154.

19 The Ladies'Journal, 15.5 (1929), back cover.

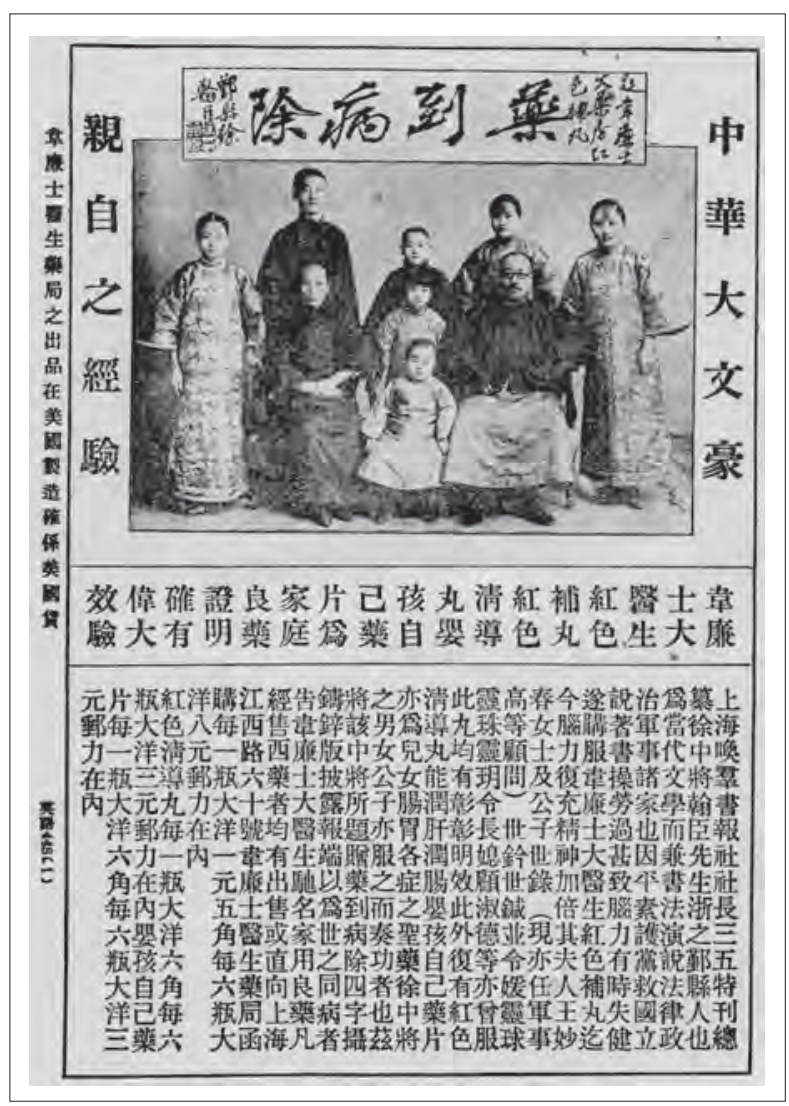

Figure 34.5 The Ladies'Journal: Dr Williams' Pink Pill for Pale People

the introduction of Western marketing methods. Japan's earliest known example of using famous actors to promote merchandise is an advertisement for the cosmetic Senjo Kaori 仙女香 (Fairy Fragrance), printed in 1821. The main image shows a female actor in the leading role of the popular theatre drama Ekyōdai chūshinzō 絵兄弟 忠臣蔵 (The Treasury of Loyal Retainers), making herself up with Senjo Kaori before a mirror. Inset in the upper right-hand corner of the page is a depiction of one of the superstar's well-known stage performances. This format must have attracted considerable attention at the time. ${ }^{20}$ The same strategy was used in China prior to the adoption of Western ideas of advertising. In addition to Shang Xiaoyun, The Ladies'Journal carried drug advertisements endorsed by the still more famous actor Mei Lanfang 梅蘭 芳 (1894-1961). However, instantly recognisable names, like these do not seem to have been a requirement in Chinese advertising, as most of the authors of testimonials are impossible to identify.

Figure 5 incorporates a testimonial from Xu Hanchen 徐翰臣, who is described in the advertisement as a 'Great writer of China', but is scarcely known to historians nowadays. Though he may well have enjoyed some measure

$20 \quad$ Nakada 1999, pl. on p. 22. 
of local renown, he was certainly no national celebrity. Nonetheless, this example is important, because it is emblematic of the ways in which testimonials were used to impress consumers with the efficacy of patent medicines. At the top of the advertisement is what looks like a commemorative tablet inscribed with four large hand-written characters, Yao dao bing chu 藥到病除 (As soon as the medicine is taken, the disease will be removed), and several lines of smaller characters. However, the message is delivered not merely by the means of text, but also through the spatial arrangement of the entire advertisement. With the tablet placed like a signboard across the top of the advertisement, and below it a multi-generational family posed as though for a formal photograph, and framed by two halves of a Chinese couplet, the composition carries very specific connotations. Anyone familiar with Chinese social conventions would have known that this kind of tablet was commonly presented as a gift of thanks for successful treatment by a patient to a doctor, who would display it prominently in his clinic to publicise his skills and merits. Although a self-promoting physician could easily fabricate a story of successful healing and purchase his own tablet, such a tablet would nevertheless serve to impress a potential patient walking into an unfamiliar clinic, and inspire a degree of confidence in the doctor. With the aid of advertisements in newspapers or magazines, pharmaceutical merchants could construct a virtual space where visual images served to invoke authority and confidence in the same way as a gratitude tablet. ${ }^{21}$

Xu Hanchen, who endorses the product in this advertisement, was at least well known within his home province, if we are to believe the accompanying text. But most of the names attached to testimonials are those of ordinary people, of whom nothing else is known. According to the advertisements, they were of good social standing, but their existence was impossible to prove or disprove.

The most active provider of drug advertisements was Dr Williams' Medicine Company, the trading arm of G.T. Fulford \& Company, formed in 1887 by Canadian national George Fulford (1852-1905). In 189o, Fulford acquired the rights to Dr William's Pink Pills for Pale People, and went on to make his fortune. Although the company was founded in Canada, the advertisements constantly claim that it is British.

An advertisement for Dr Williams' Pink Pills for Pale People appeared in almost every issue of The Ladies' Journal, with only a very few exceptions. Most of them centred on a testimonial - frequently a letter of gratitude from one of those obscure figures described above, an 'Old

$21 \quad$ The Ladies'Journal, 14.4 (1928), back cover.
Madame Cheng' (Cheng lao furen 程老夫人) 22 or 'Mr Chen' (Chenjun 陳君). ${ }^{23}$ Another favourite method was to play on the reader's concerns about a specific disease, and then present the Pills as a cure for it. Some advertisements whet the reader's curiosity with a tale full of suspense, which culminates in a demonstration of the prodigious efficacy of the Pills. There is always a lengthy passage in advertorial style boasting of a range of miraculous effects; and no matter which strategy is adopted, the advertisements are always vividly illustrated and full of colourful description. With these impressive productions appearing on the back cover of each monthly issue of The Ladies'Journal, it was highly unlikely that any reader could remain ignorant of Dr Williams' Pink Pills for Pale People.

The main ingredients of Dr Williams' Pink Pills were iron oxide and Epsom salts. The pills were originally marketed to veterans of the American Civil War suffering from digestive problems, malaria, wounds, and post-traumatic stress disorders. Later, the marketing strategy was redirected to a much broader pool of potential customers: women. As the colour of the pills might suggest, the advertisements emphasised their efficacy in diseases related to the blood, and claimed that they were a remedy for many female ailments, able to restore the blood and nerves. In 1899, the pills were advertised as a cure for all forms of weakness in men as well as in women, and diseases resulting from vitiated humours in the blood. Like Tsumura Jūsha, George Fulford used newspapers and magazines to keep the name of his product before the public eye, and achieved impressive commercial success. Within a mere five years, Fulford's company had expanded throughout North America, Europe and the British Empire and was advertising in 82 countries. $^{24}$

In the English-speaking world, advertisements for Dr Williams' Pink Pills were strong on visual presentation. In an advertisement published in New Zealand, the figure of a doleful-looking girl, holding a laundry basket, appears in an inset on the left. The accompanying text, headed 'Doing a Day's Washing', explains the importance of the Pink Pills to a housewife. In a testimonial attributed to 'Mrs Mary Brown' we are told how much happier her life has become since she discovered the amazing effects of the pills. ${ }^{25}$

The New Zealand advertisement is similar in format to those advertisements in The Ladies'Journal that seek to draw attention to a particular health need. One can
Ibid., 1.12 (1915), back cover.
Ibid., 10.6 (1924), back cover.
Loeb 1999, pp. 126-45.
See: http://www.soyonlineservice.co.nz/articles/pink\%2opills. htm (accessed 13/12/2008). 
therefore rule out the possibility that this format was specifically designed to appeal to a local Chinese audience, but neither did it form part of a global communication strategy. As in the New Zealand case, the Chinese advertisements combine images and personal testimonials to persuade customers. However, Dr Williams' Pink Pills for Pale People did make some adjustments to suit the Chinese market. For example, some advertisements for the Pink Pills emphasise their efficacy as a fertility treatment, in the same way as Chūshōtō. Moreover, similarly to the strategy adopted for aspirin, the advertising concept is often based on a Chinese idiom.

In one advertisement, a figure dressed in a traditional official's robe, adorned with a depiction of a Pink Pills package, has just prevented a man, in the nick of time, from severing a piece of flesh from his thigh. ${ }^{26}$ The caption reads: 'Why is it necessary to cut your own thigh to cure your parents?' 'Cutting one's thigh to cure one's parents' is a traditional Chinese form of self-mutilation, founded on the belief that if filial sons or daughters-in-law sacrifice their own flesh, Heaven will heal their ailing parents. This advertisement suggests that a son can fulfil his filial duty while preserving the integrity of the body given to him by his parents, which is in itself an act of filial piety. ${ }^{27}$ In return for a small financial outlay, a son can be filial without physical harm to himself.

The persuasive approaches of the purveyors of Pink Pills discussed above directly address the medical concerns of people who are already suffering from poor health. But the Pink Pills were also targeted at those who were not, as yet, sick. Dr Williams' Medicine Company also wanted to impress this sector of the market with the miraculous effects of the Pink Pills, so that they would think of the pills when the need arose.

A strategy frequently adopted to address this particular audience was to pose a question that seems at first glance to be totally unrelated to medicine, so as to induce the potential customer to read on. For example, one advertisement bears the caption 'How did Major General Yi defeat his enemies?' and is illustrated with a portrait of the General and pictures of his troops in training. The interested reader will find that General Yi's military exploits were made possible by Dr Williams' Pink Pills, which helped him to recover his strength, and gave him the energy to lead his troops to victory. ${ }^{28}$

Another example is illustrated with two pictures, showing a small boat in a stormy sea and a family reunion, with

\footnotetext{
26 The Ladies'Journal, 14.12 (1928), back cover.

27 For details, see Qiu 1995.

28 The Ladies'Journal, 2.7 (1916), back cover.
}

the captions: 'Strong winds assailed the little boat and struck terror into the woman on board' and 'If you want to know what happened next, please read the text below'. The design even obscures the ubiquitous brand-name and packaging of the Pink Pills. Only after reading to the end will the reader realise that the story was merely an exemplum of the wondrous effects of the Pink Pills. ${ }^{29}$

\section{Conclusion}

With the exception of pedlar's shows, the main visual method used to advertise proprietary drugs in pre-modern China was the signboard. This technique is like telling potential customers, 'I am here'. In contrast, modern advertisements go one step further and announce, 'You need me'! They aim to position the image of the product in the consciousness of the consumer before the desire to purchase arises. By the time a demand has been created, the customer will already have been primed to think of the product as a favoured option.

As Sherman Cochran has pointed out, some Chinese drug merchants purveying the so-called 'new medicine' in the Republican period used images associated with the West to suggest that their merchandise was modern and more effective than traditional medicines against the illnesses that afflicted Chinese people. ${ }^{30}$ For their part, advertisements for bona fide overseas drugs did not hesitate to claim that they offered the best solutions to health problems conceptualised as specifically Chinese, such as spermatorrhoea. ${ }^{31}$ To impart an aura of familiarity, foreign businesses flexibly adopted Chinese conventions and other cultural resources to make their advertisements more memorable and acceptable to Chinese customers. The use of familiar, localised images served chiefly to suggest that international drugs would cater to the needs of Chinese customers, implying that they were as well suited to the Chinese bodily constitution as to the constitution of patients from elsewhere. ${ }^{32}$ In fact, advertisements for domestic drug companies - or at least the cases in The Ladies'Journal - never attempt to exploit the argument that Chinese products are best suited to the Chinese body. In a sense, these advertisements naturalise rather than nationalise the drugs consumed in China.

\footnotetext{
29 Ibid., 16.9 (1930), back cover.

30 Cochran 2000.

$31 \quad$ Hugh Shapiro's excellent article shows that there are many other such cases, see Shapiro 1998.

$3^{2}$ Sometimes the advertisements illustrate their amazing effectiveness with foreign patients, especially Pink Pills for Pale People.
} 
The introduction of modern print media redefined the drug market in China. Since periodicals carrying advertisements could be delivered throughout the country by mail, drug merchants were able to penetrate many new markets. Furthermore, since many periodicals had specific themes and audiences, it was possible to gauge the common interests and characteristics of their readership, so as to design targeted advertising campaigns. Besides, because the magazines were delivered regularly to subscribers' homes, the advertisements could exert a stronger and more sustained effect on the minds of readers. From the strategies reviewed in the medicine advertisements in The Ladies' Journal, it can be seen that patterns of competition and marketing techniques in the Chinese drug market underwent a radical transformation in the early Republican period.

\section{Bibliography}

\section{Primary Sources}

Funü zazhi 婦女雜誌 (The Ladies' Journal), issued by Shangwu yinshuguan 商務印書館 (Commercial Press), 1915-31.

\section{Secondary Sources}

Chang Che-chia 張哲嘉 2004, 'Funü zazhi zhong de “yishi weisheng guwen” 《婦女雜誌》中的「醫事衛生顧問」(The 'Medical Advisory' Column' in The Ladies' Journal), Jindai zhongguo funü shiyanjiu 近代中國婦女史研究 (Research on Women in Modern Chinese History 12, Special issue on The Ladies'Journal, 145-68.

Cochran, S. 2000, 'Marketing medicine and advertising dreams in China', in Yeh Wen-hsin (ed.), 62-97.

Go, S. 2003, Hong Kong Apothecary: A Visual History of Chinese Medicine Packaging, Hong Kong: MCCM Creations.

Hanson, M. and J. Farquhar (eds) 1998, Empires of Hygiene, Durham NC: Duke University Press.

Huang Ko-wu 黃克武 1988, 'Cong Shenbao guanggao kan Minchu Shanghai de yiliao wenhua yu shehui shenghuo, 1912-1926' 從申 報廣告看民初上海的醫療文化與社會生活, 1912-26 (The medical culture and social life of early republican Shanghai reflected in the advertisements of Shenbao, 1912-26), Bulletin of the Institute of Modern History, Academia Sinica 17. 2, 141-94.

Kaga Sansenjun 加賀三千尋 2003, Meiyaku tanhō 名薬探訪 (Reports on famous patent medicines), Tokyo: Dojidaisha.

Loeb, L. 1999, 'George Fulford and Victorian patent medicines: quack mercenaries or Smilesian enterepreneurs?', Canadian Bulletin of Medical History $16,125-45$.

Lü Fang-shang 吕芳上 2004, 'Daoyan' 導言 (Introduction), Jindai zhongguofunü shiyanjiu近代中國婦女史研究 (Studies of Modern Chinese Women) 12, Special issue on The Ladies'Journal, i-x.

Mann, C.C. and M.L. Plummer 1991, The Aspirin Wars: Money, Medicine, and 100 Years of Rampant Competition, New York: Alfred A. Knopf.

Nakata Setsuko 中田節子 1999, Kōkoku de miru Edo jidai 広告で見る 江戸時代 (The Edo Period seen through Advertisements), Tokyo: Kakukawa Shoten.

Netsuka Isomatsu 根塚伊三松, 1979, Baiyaku hanga-Omake eshino miryoku 売薬版画一おまけ絵紙の鬼力 (Patent Medicine Prints: The Appeal of Pictures as Free Gifts), Toyama: Kogen shuppan.

Obushi Hajime 大伏肇 1999, Zusetsu Nihon kōkoku sennenshi: Kodai chūsei kinseihen 図説日本広告千年史: 古代、中世、近世編 (Illustrated History of One Thousand Years of Japanese Advertising: Ancient, Medieval, and Modern), Tokyo: Nihon zusho senta.

Qiu Zhonglin 邱仲麟 1995, 'Bu xiao zhi xiao - Tang yilai ge gu liao qin xianxiang de shehuishi chutan' 不孝之孝 - 唐以來割股療親現 象的社會史初探 (A Socio-historical Study of the Phenomenon of 'Cutting Flesh to Heal Parents' from the Tang Dynasty to Modern China), New History 6.1, 49-94.

Qu Yanbin 曲彦斌 200o, Zhongguo zhaohuangyu zhaohuang shisheng -Chuantong guanggaoyishu shilüe 中国招幌与招幌市声一传统广 告艺术史略 (Shop Signs and Street Cries of China: a brief History of the Traditional Arts of Advertising), Shenyang: Liaoning renmin chubanshe.

Shapiro, H. 1998 'The puzzle of spermatorrhea in Republican China', in Hanson and Farquhar (eds), 551-96.

Takakuwa Suehide 高桑末秀 1994, Kōkoku no sekaishi 広告の世界史 (World History of Advertising), Tokyo: Nikkei Kokoku kenkyusha.

$\mathrm{XuJunji}$ 許俊基 2006, Zhongguo guanggao shi 中國廣告史 (A History of Advertising in China), Beijing: Zhongguo chuanmei daxue chubanshe.

Yeh Wen-hsin (ed.) 200o, Becoming Chinese: Passages to Modernity and Beyond, Berkeley/Los Angeles/London: University of California Press.

Yomeda Yutaro 米田祐太郎 1941, Shina Kokoku sendenno gijutsu 支那広 告宣伝の技術 (Advertising Techniques of China), Tokyo: Kyozaisha. 


\title{
35 Beauty and Health: Images of Health and Illness from 2oth-Century China
}

\author{
ZhouXun 周遜
}

\section{Introduction}

As Peter Burkes has rightly argued, 'images can bear witness to what is not put into words.' ${ }^{1}$ Visual documents provide invaluable evidence that both bolsters and supplements other forms of information available to historians. In the early 20 th century, the visual arts featured prominently in the everyday life of ordinary Chinese, not only in cosmopolitan urban centres but also in rural areas. They became an expression of 'modernity'.2 For a huge number of Chinese at the time, especially the urban elites, being 'modern' (modeng 摩登) had multiple meanings and appeals: it was often interchangeable with the concept of 'hygiene'. ${ }^{3}$ In turn, the term 'hygiene' (weisheng 衛生) could imply being 'beautiful' (mei 美), 'healthy' (jian 健) or 'fashionable' (shishang 時尚). Images of healthy, beautiful, stylish women, and bonny babies, as well as muscular men, penetrated into people's homes and consciousness through advertisements, calendars, popular magazines, posters

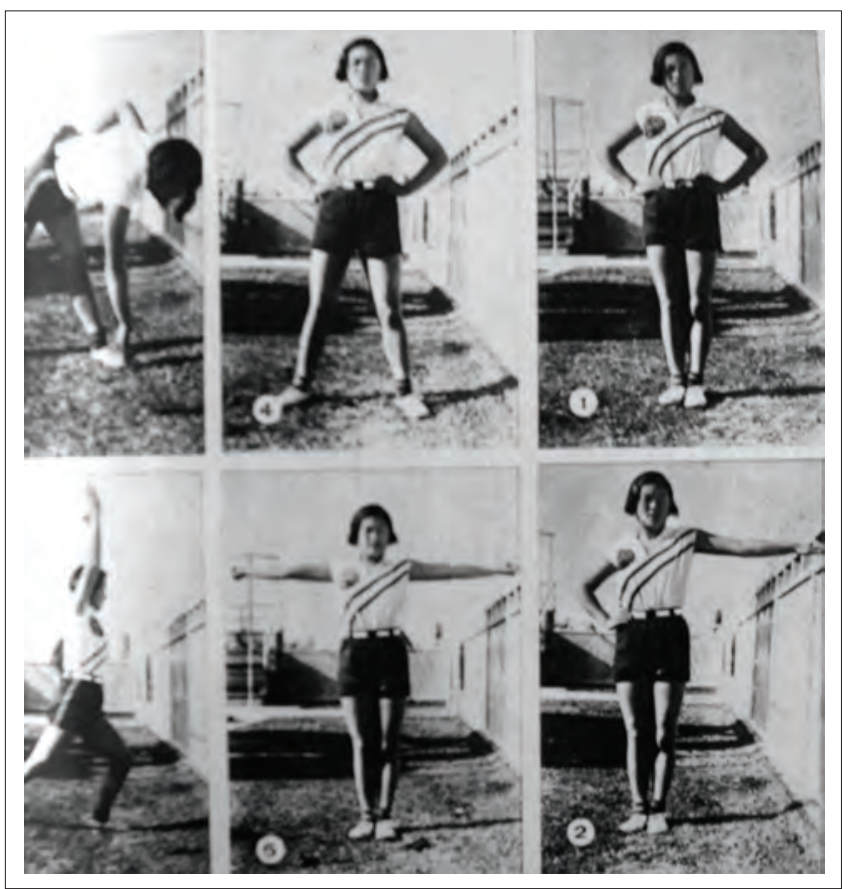

Figure 35.1 Take home gymnastics for women 良友畫報 (Young Companion Pictorial), 1930s

Burkes 2001.

For further reading, see Laing 2004

Ruth Rogaski argues that in the 1920 s and ' 30 , in Chinese treaty ports such as Tianjin, hygiene (weisheng) was intertwined with the desire for modernity. See Rogaski 2004, p. 226. and photographs. On the one hand, medical and hygiene products were more than just a means to cure and prevent illnesses, or to preserve health; they were also beautiful objects of consumption and desire. On the other hand, many everyday items, from toilet paper to soap to men's vests, as well as various kinds of manufactured foodstuffs, were advertised as hygienic or as hygiene products, serving to promote health.

The present chapter shows that the use of visual images was an integral part of the discourse on 'medicine', 'health', 'illness' and 'modernity' in early 2 oth-century China. In particular, the advent of new technologies such as photography played an important role in the proliferation of images of health in China. The camera appealed to individuals of very different social backgrounds, transforming the ways in which ordinary people experienced seeing the world around them. Photographs and coloured posters were regarded as 'mirrors of truth'. Popular magazines were lavishly illustrated with photographs that most readers

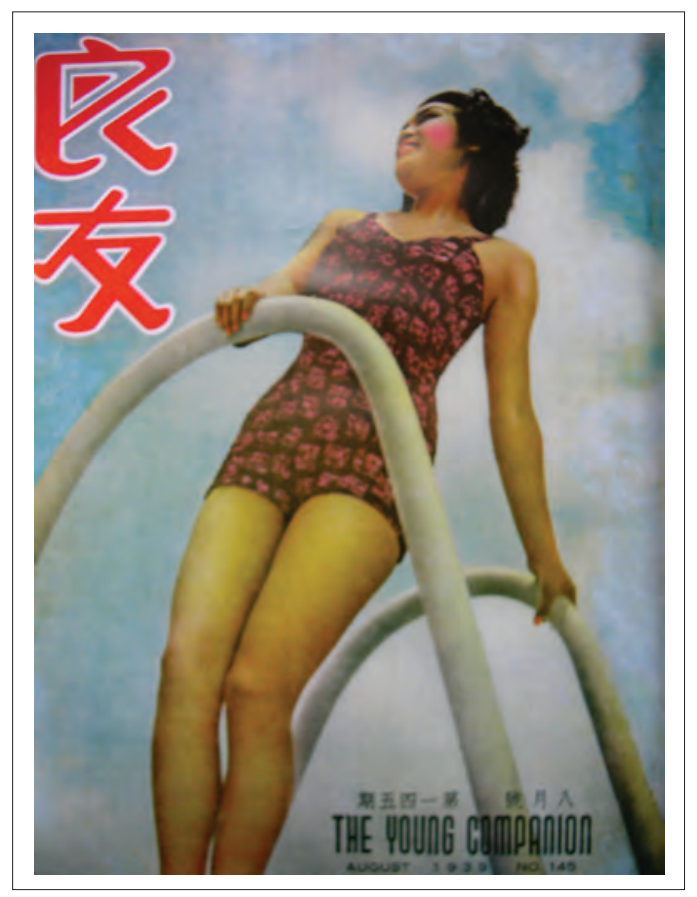

Figure 35.2 Swimming Pool Beauty 良友畫報 (Young Companion Pictorial), front cover, no. 145, August 1939 


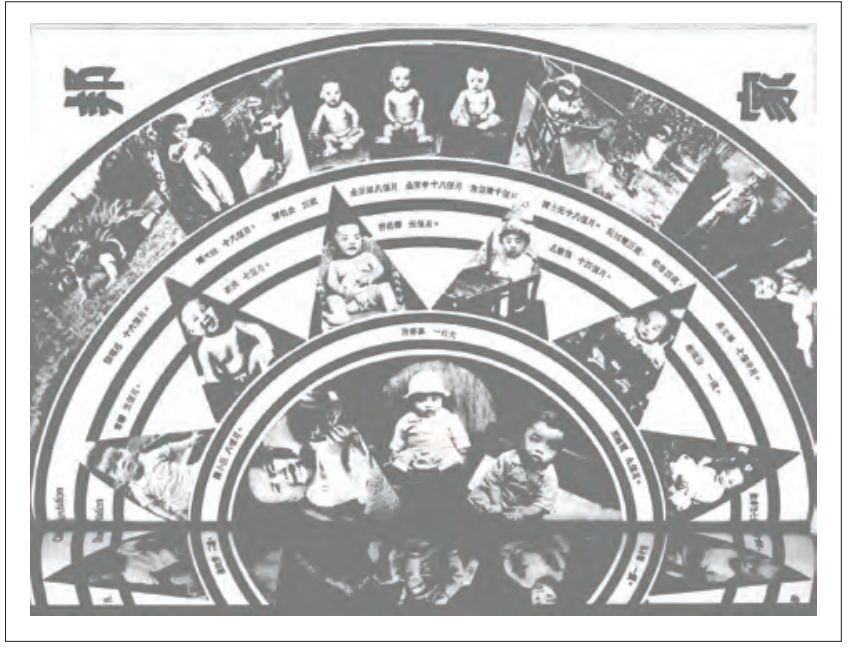

Figure 35.3 Healthy baby photos 禮拜六, Saturday Magazine, 1930s

could easily relate to, especially shots of babies, children and women. ${ }^{4}$ For instance, photos of Healthy Baby Contests (jiankang ertong bisai 健康兒童比賽), and pictorial manuals of bodily exercises (jianmeiyundong 健美運動), as well as the swimming pool photos of the 193os, were used by modernising elites to spread their message of nationalism, emphasising that healthy and beautiful individual bodies were the necessary basis of a healthy nation (Figs 1-3). Such images gradually changed ordinary people's ideas of the body, health and beauty: the traditional concept of longevity was reconfigured into images of a modern, fit masculinity, of full-breasted females and chubby babies.

\section{Bodies as Commodities}

In Critique of Commodity Aesthetics, Haug argues that there is an intense and special connection between sensuality, the body, and the power of modern commercial discourse. ${ }^{5}$ By the turn of the 2oth century, hygiene and cleanliness, as associated with the body, had become commodified, and were assigned a significant consumable value, which was promoted through pictorial advertisements. The early visual culture of modern consumption in the West is conspicuously replete with bodily images: advertisements featuring hygiene and toiletries, first in Europe and America, and then in urban China, played an integral role in refiguring the body.

New hygiene practices that developed in Victorian Britain, as exemplified by popular writers such as the dermatologist Erasmus Wilson (1809-84), continued to dominate questions of health and beauty until well into

\footnotetext{
4 Dikötter 2007.

$5 \quad$ Haug 1986, p. 83 .
}

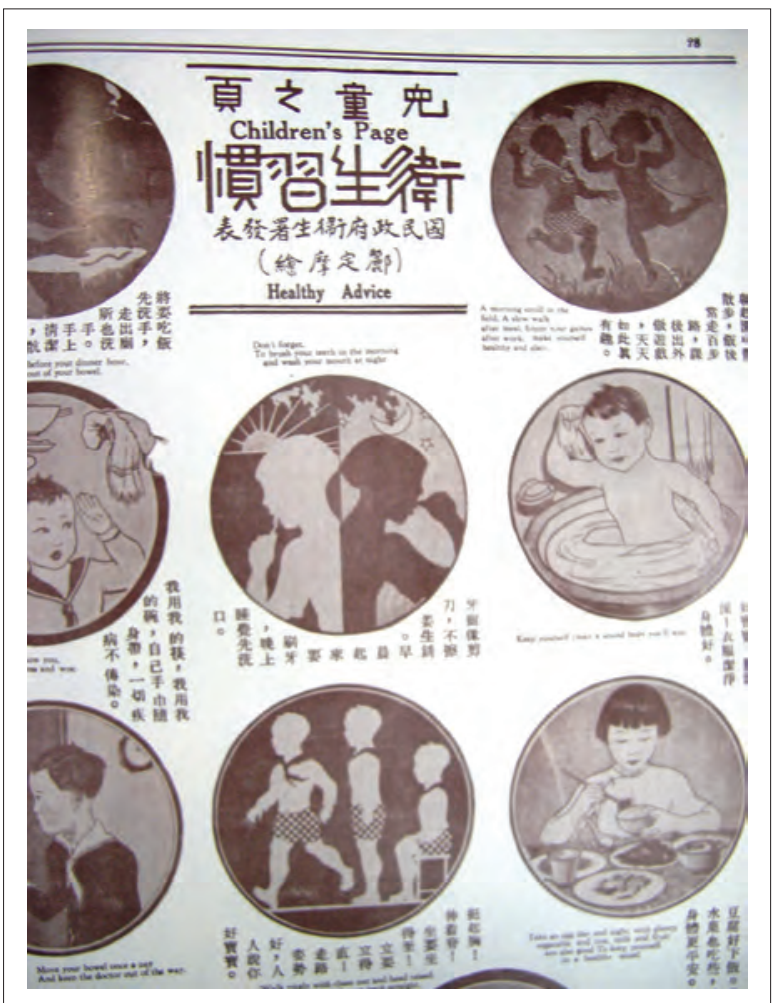

Figure 35.4 Public health poster for the Republican Health Bureau 國民政府衛生署: ‘Hygienic habits for children', 1931

the early 20 th century, propelled by the evolving 'germ theory of disease', which displaced perceptions of dirt as 'an essence that oozed immorality and degeneracy and contravened the law of God'. ${ }^{\text {The }}$ 19th-century transformation of sanitation, personal cleanliness, and collective hygiene, while exercising a positive impact on general health, was also a part of the medico-moral politics of the time. ${ }^{7}$ As a result, pictorial and textual representations of cleanliness, personal appearance and beauty were increasingly used to define social hierarchy and difference in 19th-century western Europe. These representations helped to shape what was socially acceptable. Personal and social hygiene were seen as key attributes of feminine domesticity and beauty, and were given social power through a range of new institutions and official practices that took shape throughout western Europe and in the United States at the turn of the 2oth century. The English working class, for example, were portrayed as 'unwashed' or physically malformed, and colonial subjects were likewise seen as having dirty or undesirable bodies. ${ }^{8}$

In pre-modern China, body decoration and hygiene were already an important component of bodily vocabularies

\footnotetext{
6 Burke 1996, p. 18.

$7 \quad$ For further reading, see Mort 1987.

8 For further reading, see Stepan 1982, and Stocking 1987.
} 


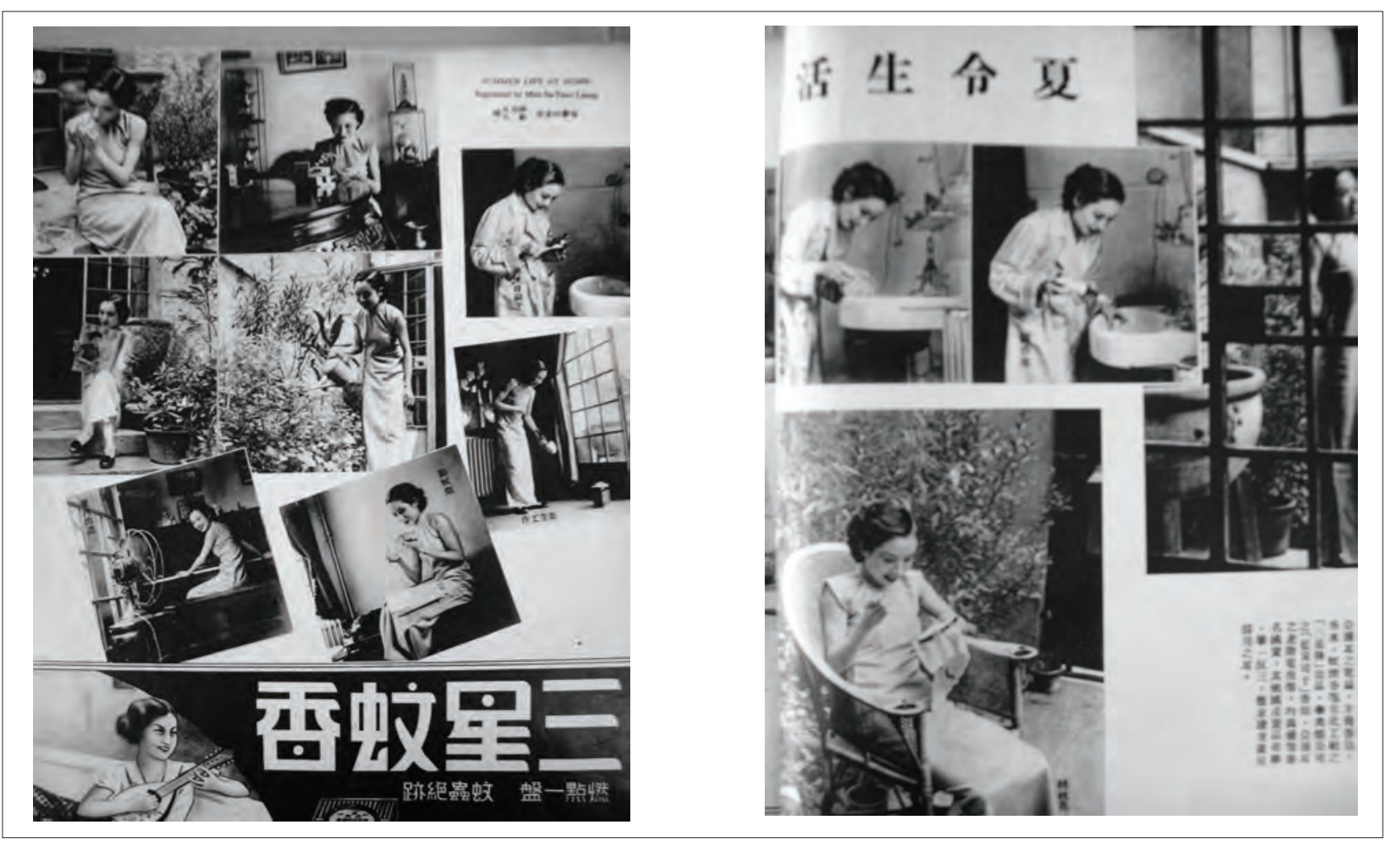

Figures 35.5 and 35.6 'Summer Life at Home’ 良友畫報 (Young Companion Pictorial)

marking various social distinctions, including status, gender and age. However practices denoted as 'weisheng' (the root meaning of which is 'guarding the body') were not restricted to keeping the body clean. The term covered a wide range of regimes for promoting health and longevity, encompassing diet, exercise and the arts of the bedchamber. Weisheng practices recommended for a member of the nobility differed greatly from those considered appropriate for a peasant. Nonetheless, an understanding of gender and age was also vital in day-to-day weisheng practices for ordinary Chinese people.

In the first decade of the 2oth century, the tone of teachings about cleanliness, the (modern) body, domesticity, and 'civilisation' began to change. As in many places throughout the world, European-inspired practices of cleanliness were generally accepted in China along with other 'common-sense' attitudes to the body, health and manners. This shift was due to several factors. Professionalised advertising and the growth of toiletry manufacturing had come to exercise an increasing impact on idealised images of bodily appearance and health. This had begun in Victorian England with the rise of illustrated advertising, using sexualised celebrities such as Lillie Langtry to sell Pear's Soap. Also the weight and persistence of discourses about hygiene and domesticity had to some degree established European models of bodily behaviour as a form of hegemonic 'common knowledge' for many educated Chinese in urban settings. State propaganda was more specific and to some extent less didactic, often involving daily activities ranging from eating to personal grooming. For example, children, as representatives of the future of the Chinese race, were given detailed instructions by the Ministry of Health and Hygiene (weishengshu 衛生署) on how to brush their teeth, how to bathe and how to eat (Fig. 4).

This official propaganda as well as the popular print media also presumed that women had an innate aptitude for the task of superintending the nation's hygiene; and modern women were understood as the caretakers of the future of China. ${ }^{9}$ As shown in this photo essay, published in a Shanghai's popular women's magazine in the 1930s, discourses of science, modernity and cleanliness were embedded in daily female domestic norms and practices among a small but significant section of the population

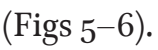

Traditionally in China, health and longevity were, in large part, achieved through specific exercises and diet regimes. From the early 2 oth century, however, thanks to the power of marketing, cleanliness and hygiene could now be purchased; in other words, a modern healthy body could be bought instead of being constructed through

$9 \quad$ For further reading on hygiene and government, hygiene and modernity, hygiene as a female responsibility, see Farquhar and Hanson (eds) 1999; Rogaski 2004; Leung and Furth (eds) 2011. 


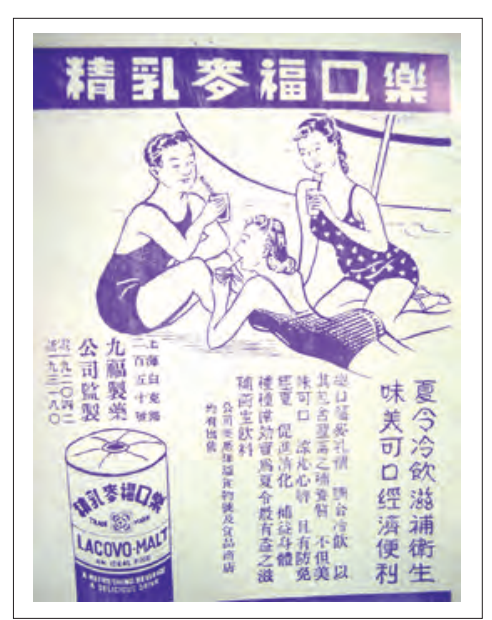

Figure 35.7 Lacovo Malt drink advertisement

bodily reform and control. Whereas health was previously associated with the inner self - with styles of being - a healthy lifestyle was now expressed by keeping clean and consuming 'hygiene' commodities, including toiletries, canned and manufactured foods, bottled beverages and undergarments. Canned malt drinks, for example, were promoted as not only delicious and nutritious but also hygienic, and particularly healthful for the summer season (Fig. 7).

In this context, the more expensive a product was, the cleaner and more hygienic, and hence the healthier it was felt to be. Disease became definitively linked with being 'dirty', with the lack of sanitation and overcrowding of the urban slums as well as unhygienic rural conditions. Thus the new constellation of concepts of health, cleanliness and hygiene endorsed social segregation. Rural life, traditional culture, parents and elders, the urban poor, and coarse food were all defined at various moments as dirty, repellent, unhygienic, disease-ridden, embarrassing, primitive, or inadequate. Being clean and hygienic became synonymous with being modern and fashionable, and was hence desirable. Hygiene products were marketed for the 'young', 'modern' and 'sophisticated', the antithesis of the 'old', 'traditional' and 'backward'. Advertisements regularly endowed hygienic products with the ability to provide 'health'. They also began to replace traditional forms of medical practices for modern consumers.

The concept of the aspirational and status-conscious professional was also a favourite with advertisers. The cost of importation meant that imported toiletries, tonics and food such as milk powder were often positioned as prestige products. The commodification of cleanliness and domesticity, reflected in new practices of using toiletries - soap, creams and lotions, skin lighteners, cosmetics, perfumes, toothpaste, deodorants, and shampoos - was instrumental in producing 'modern' Chinese bodies.

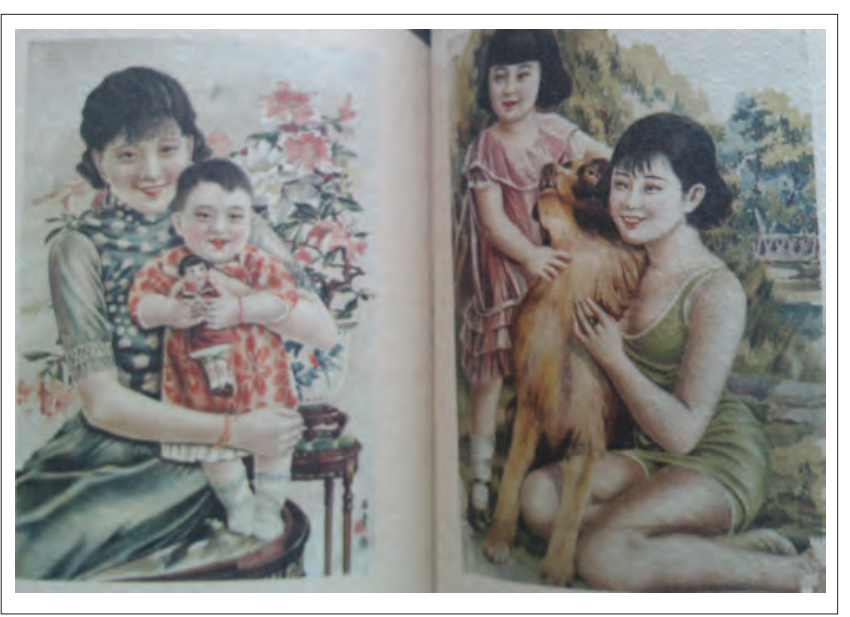

Figure 35.8 Popular advertisement for luxury fabric (1930s)

As in the more recent case of Zimbabwe, new goods did not only present new needs, and new challenges; the process of acquiring them also presented a bewildering experience, a new surface for cultural inscription, a new opportunity for imagination and creativity. ${ }^{10}$ Soaps and skin care products, as well as other hygiene products, and the interlocking of their cultural meanings, played a vital part in generating the modern Chinese body and in that body's role in the presentation of self in everyday life. As marketers sought to give public expression to the anxieties that characterised daily life in urban China, hygiene products were promoted as 'modern' Chinese products, enabling social aspiration.

Just as educators and public campaigners had held women responsible for transforming the social content of Chinese life, marketers targeted women by assigning them the crucial role in organising the 'modern' Chinese home. ${ }^{11}$ Women were depicted as 'mothers of the citizens' (Minguo zhi mu 民國之母), and their capacity to satisfy male demands and organise their households - to be good wives and loving mothers - featured regularly in advertisements (Fig. 8).

Women also became 'sufferers' from headaches, menstrual pains, stomach pains, tiredness, sadness and 'neurasthenia'. 'Neurasthenia', according to its creator George Miller Beard in the 187 os was the 'American disease. ${ }^{12}$ From the early 20 th century, however, it became the most fashionable illness in China, as avant-garde intellectual elites sought to construct a modern Chinese identity. Western medications were advertised as having the power to give the modern women 'new blood and new health', and thus 'new happiness' (Fig. 9). An 'unhealthy'

\footnotetext{
$10 \quad$ Burke 1996, p. 202.

$11 \quad$ For further reading, see Dikötter 1995. See also Phillips 2006.

12 See Beard 1881.
} 


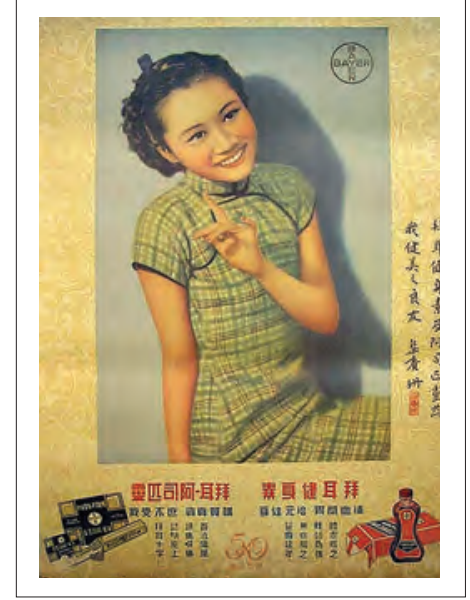

Figure 35.9 'Bayer medicines keep me healthy and beautiful'. Advertisement for Bayer company's Western medicine

woman was thought to have lost the ability to fulfil her duties, namely reproduction, maintaining a healthy and hygienic household, satisfying her husband, and raising healthy children.

Furthermore, hygiene products were marketed in terms of glamour, smartness and beauty. Thus being hygienic became a marker of feminine beauty and virtue. While women bore the burden of ensuring their own purity and the purity of their households through the use of soap and other hygiene products, the Pinkettes laxative pills claimed to help women maintain a healthy, beautiful body, invoking the worldwide popularity of Lydia Pinkham's women's tonic (Fig. 10). The beauty of women's bodies and the modernity of their manners became a major topic of domestic and hygienic education.

\section{The 'Modern' ‘Chinese' Body}

In comparing medical traditions in China and the West, Shigehisa Kuriyama noted that, while a muscular body was representative of health in Greek tradition, there was

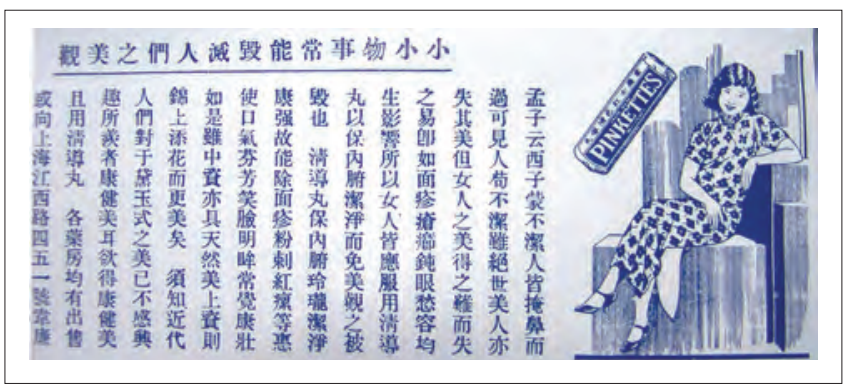

Figure 35.10 193os advertisement for Pinkettes

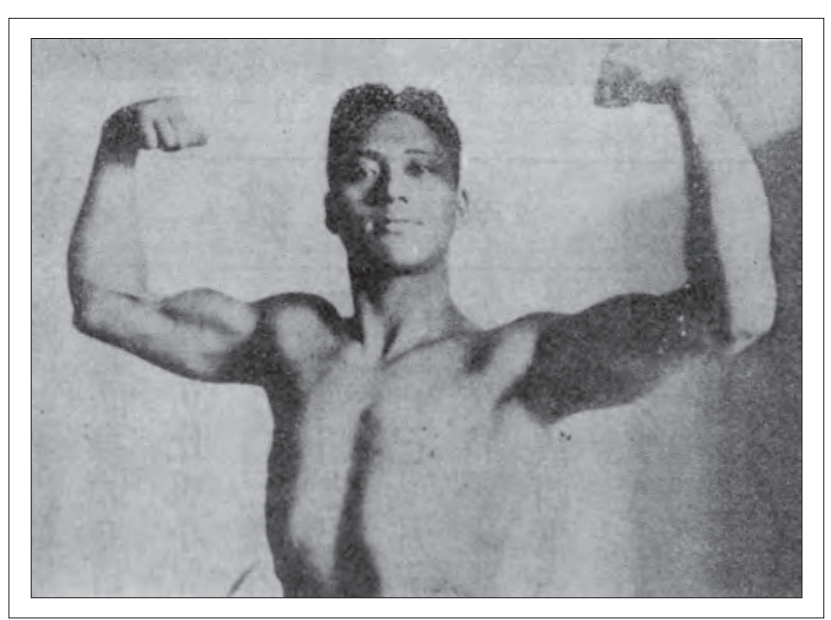

Figure 35.11 Body builder

a 'neglect' of musculature in Chinese medical tradition. ${ }^{13}$ This was certainly no longer the case by the early part of the 2oth century. Spurred by new knowledge about human anatomy, the mechanical metaphor featured prominently in modern medical discourse of the body. ${ }^{14}$ The modern body was viewed as a finely-tuned machine, with intricate mechanisms that could be adjusted and regulated. Placed within an industrial landscape, the human body became a factory. Muscles were the representations of a strength and power that would guarantee the future of China. ${ }^{15}$ The body of an opium addict on the other hand was emblematic of China's weakness. If China was to emerge as a modern nation and to survive on the world stage, the 'Chinese man' must attain a modern body: a healthy virile body - the body of a strong and powerful production force.

In 1934, Earle Liederman's Muscular Development was translated into Chinese by Zhao Zhuguang 趙竹光 (190991), the 'Father of body building' in China. Liederman was an American pioneer of strength training. From 1910, for eight years, he toured the vaudeville circuits of the United States demonstrating his skills in weight lifting, acrobatics, and physique display. He subsequently turned his hand to writing, and published his exercise regimen as a homestudy course, which he sold widely through mail order. It became a great success. His Muscular Development, an illustrated booklet explaining his techniques and philosophy, enjoyed great popularity and went through at least 20 editions.

Zhao Zhuguang, a Cantonese student deeply concerned about the survival of China and the Chinese race, took Liederman's mail-order course while studying in Shanghai

\footnotetext{
13 Kuriyama 1999, pp. 111-12.

14 Rabinbach 1990.

15 Shen 1946, p. 629. Cf. Dikötter 1995, pp. 20-2.
} 
in the late 1920s. In 1932, he founded the Far East's first body-building society in Shanghai, and began his career as the 'Liederman of China'. Believing that a strong China needed muscular men, from the late 1930s to late 4os, Zhao devoted his energies to developing body-building culture in China, and published numerous books, pamphlets and journals on the subject. One of these was Jian li mei 健 力美 (Strength, Power and Beauty), a popular Shanghai magazine of the 1940s, which was distributed in many other Chinese cities. The body-building movement and Zhao's many publications helped to spread the message that the modern men of China should be strong and muscular. Photographic images of muscular men filled the pages of popular magazines and newspapers, and became the representation of modern Chinese manhood par excellence (Fig. 11).

The body of a modern Chinese woman was however believed to be inherently different from that of a modern Chinese man: gender differences were visibly expressed throughout the body. While a muscular man was the builder of a modern and industrialised China, a woman's health was bound up with her capacity to reproduce healthy future generations, and her ability to raise healthy children and maintain a healthy home, attributes seen as fundamental to the survival of the Chinese race. Besides having a healthy body, it was believed that a modern Chinese woman should possess virtue, beauty, knowledge, and an adventurous spirit, as well as power and wealth. Photos of healthy brides or idealised 'model' females became a regular feature in women's magazines. These female role models included Madame Chiang Kai-shek (Soong May-ling 宋美齡, representing power and virtue), Madame Chiang's mother $\mathrm{Ni}$ Kwei-tseng (Ni Guizhen 倪桂珍, longevity and prosperity), the writer Ding Ling 丁玲 (intellect), China's swimming star Yang Xiuqiong 楊秀瓊 (health), the famous actress Hu Die 蝴蝶 (beauty), and Jewish tycoon Silas Hardoon's Buddhist wife Luo Jialing 羅迦陵 (wealth).

Medicalised discourse deployed the language of modern science to describe the processes of bodily degeneration. A degenerate female body had shrivelled, shrunken breasts and suffered from thin blood. An unhealthy body, in both men and women, was said to store up fatty tissues, which would eventually lead to a pathological state of obesity ( feipangbing 肥胖病). While the modern medicine Lipolysin promised to help one to reduce body fat, exercise was also considered essential for men and women alike in order to keep their bodies shapely, youthful and full of vitality. Western-style forms of physical exercise were recommended by medical journals and cultural elites, including the leaders of the New Culture Movement (1915-21), who were profoundly influenced by Social Darwinism. In his essay

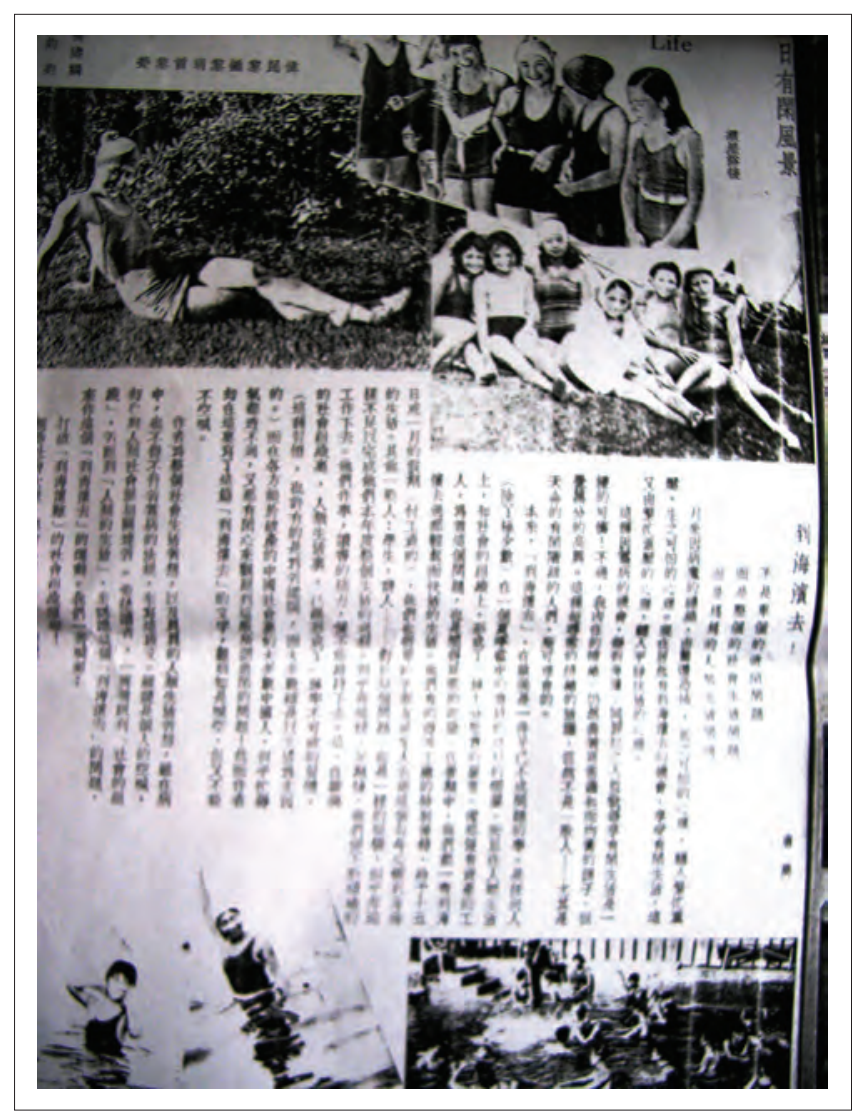

Figure 35.12 Go to the Seaside', Arts and Life, 5 (August 1934)

on modern education, Chen Duxiu 陳獨秀, founder of New Youth (Xin qingnian 新靑年) magazine, the flagship publication of the New Culture Movement, promoted physical education as a means to save the nation. According to him, the contemporary youth of China did not even have enough strength to catch a chicken and lacked the mental vigour to think on a grand scale:

Their skin looks pale and their bodies look fragile. They look as though they were not fully grown. They are afraid of cold and cannot bear the hot weather. They appear extremely weak, like sick people. Full of citizens with feeble minds and bodies, how can our country develop any further? ... The new youth of the 2oth century must also be physically fit. ${ }^{16}$

Echoing Chen, in 1917, the young and as yet little-known Mao Zedong produced a comprehensive study of physical education with detailed procedures for exercising the body including the arms, the legs, the trunk, the chest, the shoulders, the abdomen and the buttocks. 'Strength comes from drill', opined Mao, although Mao himself showed no taste for drill. ${ }^{17}$

\footnotetext{
16 Chen Duxiu 1915.

17 Mao Zedong (April 1917) in Shram (ed.) 1992.
} 


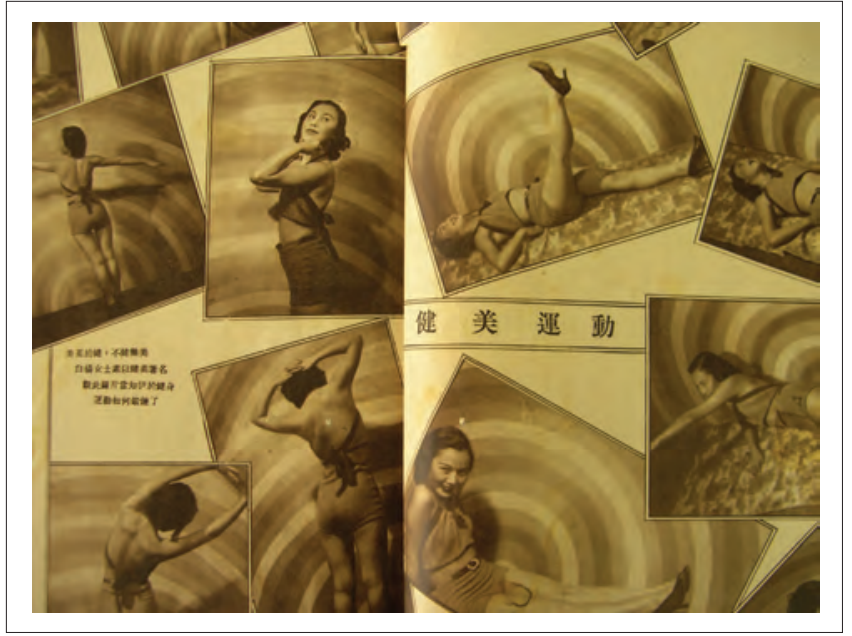

Figure 35.13 Film star Bai Yang (1920-96) doing calisthenics

Sports clubs sprung up in big cities such as Shanghai, Tianjin, Canton, Beijing, Chengdu and Wuhan. The YMCA in Shanghai boasted one of the first modern fitness centres in China with a heated indoor swimming pool that attracted a great number of devotees. Swimming became a popular pastime for fashionable urban youth, and swimming pools and the beach became some of the most captured photographic subjects. The well-known writer and art historian Tang Jun 唐隽 declared in a photo essay that he wrote in 1935 for Meishu shenghuo 美術生活 (Arts and Life), one of the most popular pictorial magazines of the 1930s, which catered to an audience interested in the lives of the rich and the famous: 'Go to the beach; it is not merely a leisure activity, but has implications for the whole of society and one's entire life'. Seaside holidays, according to Tang, were the best cure for any disease as well as the stresses of modern life. By going to the beach, each individual could become healthy and happy, and could thus help to produce a better and happier society. The article was illustrated with images of healthy, good-looking young women, as well as children, posing at swimming pools or at the seaside (Fig. 12). ${ }^{18}$

The appeal of images of bathing belles clearly went beyond the aspiration to be healthy; health and beauty or 'jian' and 'mei' had gradually merged into one. For a modern Chinese woman, being healthy was tantamount to being beautiful and glamorous, and equally a beautiful woman was a healthy woman. Famous actresses such as Bai Yang 白楊 and others became the ultimate role models for modern Chinese women (Fig. 13).

\section{Healthy Babies}

'Better Baby Contests' were an American phenomenon that emerged in the early 20 th century. ${ }^{19}$ Conceived as a eugenic and public health programme to teach parents about childcare, hygiene and sanitation, with the aim of improving children's health and preventing racial degeneration, the Better Baby Contests gradually evolved after World War I into the Fitter Family campaign.

Modelled on the American Better Baby Contests, 'Healthy Baby Contests' (jiankang ertong jingsai 健康 兒童競賽) became widespread in China in the 1920 s and 3 os, and by the end of the 1920s, were incorporated into the country's eugenic programme..$^{20}$ Beginning in 1922, Libailiu 禮拜六 (Saturday), the best selling weekly magazine in Shanghai in the 1910s and 2os, held a series of competitions for photos of babies and children. The entries were published in the magazine each week. In fact, some of the children in the photographs look far from healthy. Well dressed in modern clothing, and portrayed with modern toys, they were evidently selected because they came from rich or well-known families. While the focus of these photos is clearly on physical appearance, they also reflect the social and material transformations taking place in China at the time. Photographs of the upcoming generation functioned as a visual display of the prestige of the emerging middle class and their desire for things modern. The trend continued into the 193os. Photos of healthy babies and children appeared regularly in Liangyou 良友 (Young Companion pictorial), one of the most popular pictorial magazines in Shanghai at the time. According to the founder of the publication, Wu Liande 伍 聯德, photos of healthy infants, as well as photos of screen actresses, society ladies, political leaders, famous artists and wealthy industrialists, helped to sell copies.

In 1926, Wu Liande decided to hold the first Healthy Baby Contest to further promote his new magazine. ${ }^{21}$ The competition was sponsored by American-owned Baohua 寶華 Biscuits Company. A couple of months earlier, the magazine published a pictorial advertisement for Baohua's milk powder. According to this advertisement, healthy citizens were the basis of a healthy nation, and healthy citizens began as healthy babies. Baohua milk powder, imported from America, was a modern and hygienic product essential for raising healthy children

\footnotetext{
$19 \quad$ For further reading, see Dorey 1999.

20 For further reading on the Eugenic movement in Republican China, see Dikötter 1997.

$21 \quad$ For further reading on Healthy Baby Contests and the Young Companion, see Wang Zhenzhu 2011.
} 


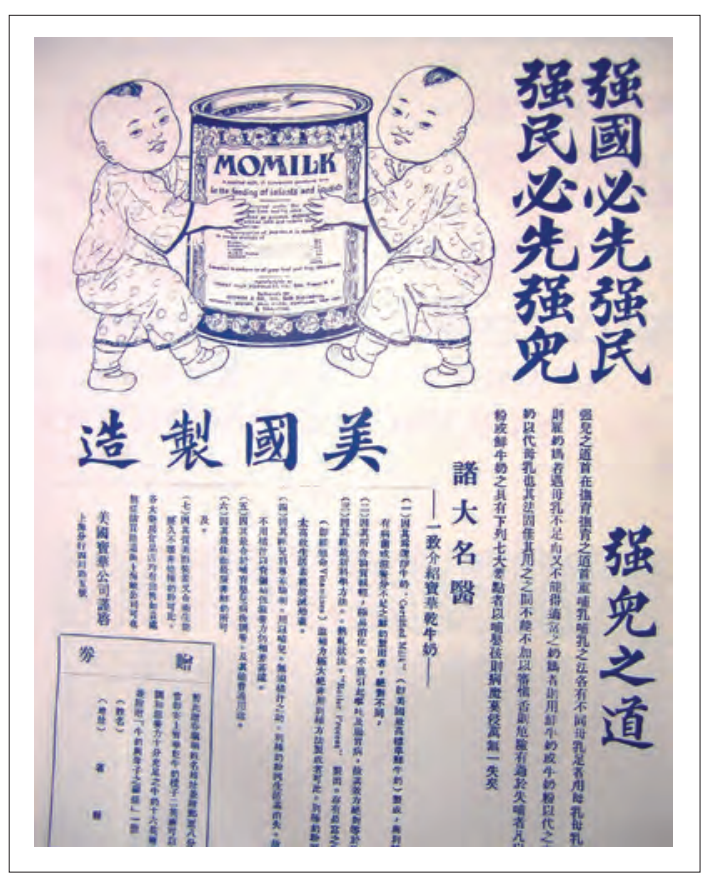

Figure 35.14 'To strengthen the nation you must first strengthen its people. To strengthen the people you must first make your children strong'. Advertisement for baby milk from the USA, $c .1930$

(Fig. 14). ${ }^{22}$ Soon afterwards, Healthy Baby Contests, as an integral part of the discourse on the future of China and the survival of the Chinese race, spread through Chinese cities. In the same year, Baohua Company also sponsored a Healthy Baby Contest organised by Suzhou Institute of Child Hygiene. The winners were depicted as the future of China. Gradually the Healthy Baby Contests became more and more medicalised through the effort of the state and medical professionals.

After the Nationalist party consolidated its power in 1927, concern for national revival became one of the main political and social priorities of central government. Public health campaigns and sanitation programmes were seen as an integral part of the nation's regeneration. 'Positive' eugenics (in Chinese, youshengxue 優生學, literally 'science of superior birth') was promoted throughout China by public health campaigners. Child health became an important issue. In 1928, the Ministry of Health proposed the introduction of a nationwide health service for pregnant women, and the First Midwifery School was founded in Beijing in 1929. Many maternal and child welfare initiatives were established in big cities. In the 1930s, a Child Health Institute was established in Beijing (Peiping) and Yang Chongrui 楊崇瑞 was appointed its director. Yang was a graduate of the Union Medical University for Women and John Hopkins School of Hygiene and Public Health.

22 Liangyou, no. 9, October 1926, p. 2.
Prior to her appointment, she had already helped to build the child health programme for the Peiping Social Health Area. The programme encompassed Western-style modern prenatal care, obstetric care, infant clinics and well-baby care, pre-school clinics and midwifery training. Besides antenatal clinics and 24-hour delivery assistance, public health nurses also visited homes regularly. After taking up the directorship of the First Midwifery School, Yang was also put in charge of the newly organised Department of Maternity and Child Health in the Central Field Health Station, which oversaw a number of pilot programmes in Beijing, Tianjin and Nanjing. One of the programmes entailed setting up an annual Healthy Baby Contest.

The inaugural state-sponsored Healthy Baby Contest took place in Tianjin. The elites of Tianjin saw the contest as an integral part of the re-construction of China and the production of healthy Chinese citizens. They argued that China had been ridiculed by the world as the 'Sick man of the East'. To transform this image, it was necessary to change the physique of Chinese people; to make them healthy. Children's health was seen as essential to this process. To promote children's health, it was proposed to hold a week-long Healthy Baby Contest. The contest took place in October 1930, and doctors and health personnel were invited to give lectures on the subject. Parents were encouraged to follow health advice and to bring up their children in a proper, scientific way:

This is not about competing for competition's sake, but through competing, parents will learn to pay attention to their children's physical health and personal development, so that the child will grow up both mentally and physically fit. ${ }^{23}$

After the Tianjin event, the Healthy Baby Contest became a nationwide movement, and April 4 was declared National Children's Day. ${ }^{24}$

The 1933 Changsha Fine (healthy) Baby Contest was a major event in its time. Four hundred babies entered the competition, which was sponsored by major international and national companies, including Nestlé Anglo-Swiss Milk Co., the Bank of China, the Agriculture Bank and Yu Shin Photographic Co. All the competing babies were weighed and measured by nurses; doctors and senior medical students then listened to their chests with stethoscopes and examined their noses, eyes and throats in a most thorough

23 See Li Xianzhe, 'April 4th Children's Day in Republican China': www.cnlu.net/disp.asp?id=51702 (accessed 23/o8/2013).

24 In December 1949, the Maoist government decided to change the date of National Children's Day to 1 June, following the agreement a month early to adopt International Children's Day by the Soviet Union and other Communist countries. 

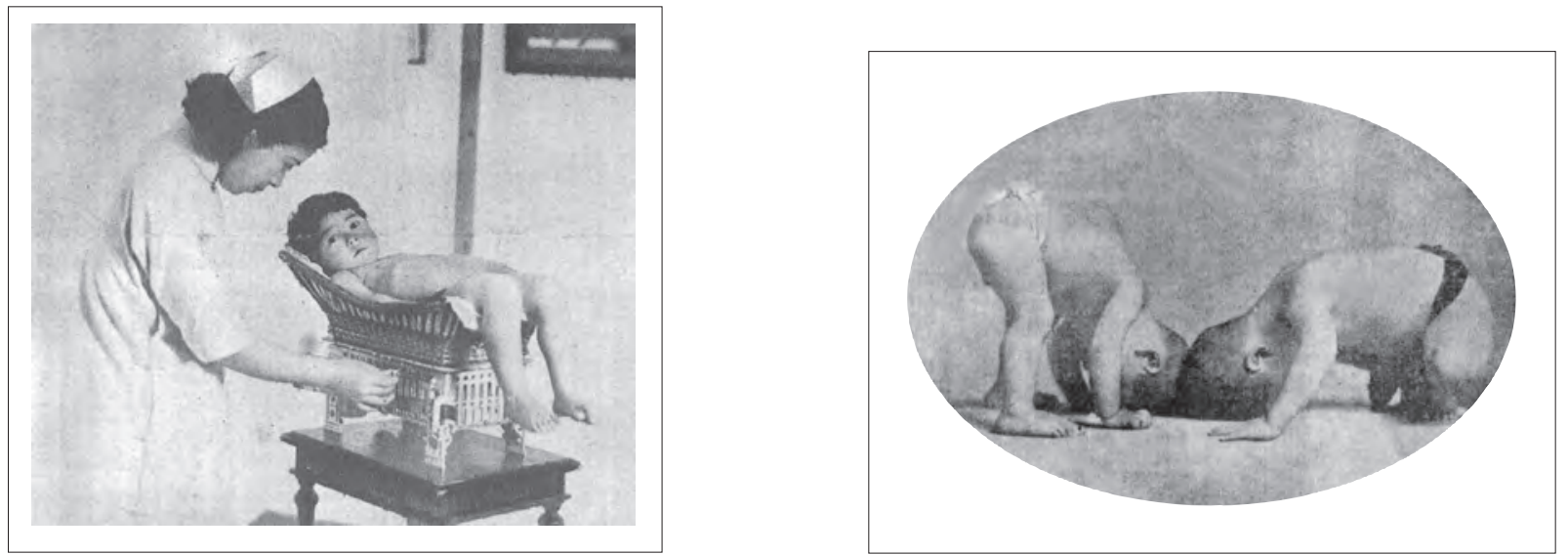

Figures 35.15 and 35.16 Healthy Baby Contest from Republican period (from Second Historical Archive of Nanjing)

fashion. The prize-winning 'fine babies' were required to have sound limbs, ears, nose, eyes and throat, and their weight and height had to conform to criteria set for their age, but points were also given for beauty and charm. ${ }^{25}$

Encouraged by medicalised notions of physical perfection, emphasis was placed on abstract mensuration, including the correlation between weight and height. Measurements and quantification boosted claims to scientific exactitude (Fig. 15). Traditional links between health, balance and appearance were reconfigured into sets of figures and statistics. Thus for instance, 'small ears', 'primitive shape of the ears' and 'low-set ears' became markers of congenital abnormality. While standards were set for weight and height, even the size of the penis was precisely stipulated. The shape of the skull, on the other hand, was believed to be an indication of the psychological propensities or character of the child. By these standards, the health of the baby was judged.

Photographs were taken of each session, to be used as medical evidence, for propaganda purposes and as prizes for the parents of winning babies. Ironically, the babies were reported not have enjoyed the competition. According to the North China Herald, most babies seemed to consider it as adding insult to injury to be photographed sitting in a weighing machine, and 'they lifted up their voices and howled'.26

To be thoroughly fit, both physically and mentally, was also a prerequisite for success. At the majority of baby contests, mental and physical tests were also carried out. Survival of the fittest was the watchword: the stronger the baby, the better, as shown in this photo taken at one of the contests (Fig. 16).

\footnotetext{
25 'Fine Baby Show in Changsha', North China Herald, 31 May, 1933, p. 334. I am grateful to Valentina Boretti for first alerting me to this material. Ibid.
}

Fine, healthy babies were the future of Chinese race, and as Yan Fuqing 顏福慶 (F.C. Yen), war-time director of the Chinese National Health Administration, put it, the task of national reconstruction 'must fall on the shoulders of the children of China today and of future generations'. ${ }^{27}$ The message of this photo poster, commissioned by the Nationalist government, resounds loud and clear (Fig. 17).

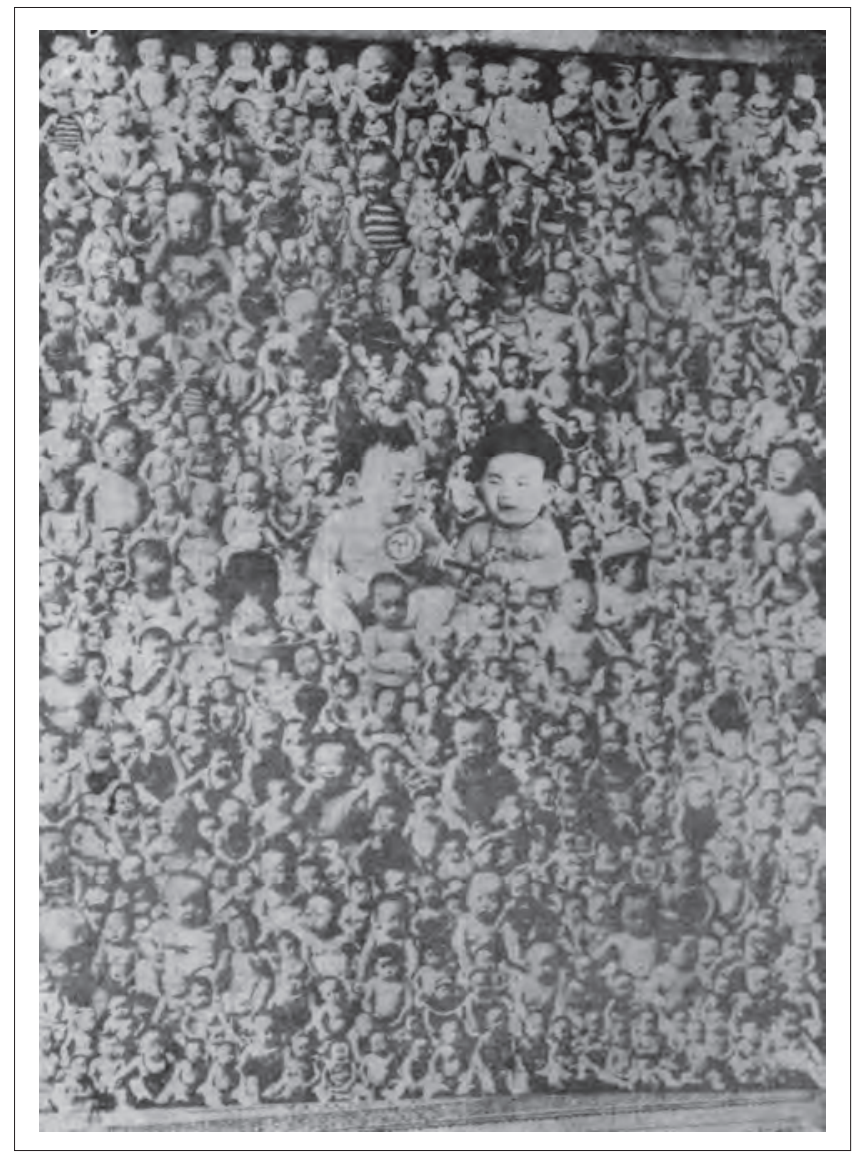

Figure 35.17 'Healthy Babies are China's Future', Republican government poster (from Second Historical Archive of Nanjing)

$27 \quad$ Yen 1938, p. 117. 
For ordinary people in China, pictorial posters and photographs of better/healthy baby contests served as talismans to invoke chubby, healthy offspring. They became a popular decorative item on people's walls and doors from big cities to the countryside. In this respect, pictorial images of health spread further, wider and faster than any written discourse.

\section{Conclusion}

In early 2oth-century China, visual images, in particularly photographs, played an important role in the proliferation of images of 'health'. While the modernising elites used them to spread their message of nationalism, emphasising that the individual healthy and beautiful body was the necessary basis of a healthy nation, pictorial advertisements, calendars, popular magazines and posters helped to transform the ways in which ordinary people experienced 'health' on an everyday level, and gradually changed popular ideas of the body, health and beauty. As elsewhere in the world, 'health' and 'hygiene' became interlinked, and formed an integral part of a discourse of modernity. With the advent of a new urban economy, imported medical and hygiene products also became beautiful objects to be desired and consumed. Not only did they promise to cure one and make one healthy; they also had the power to make one beautiful - all for the cost of a bar of soap or a tonic.

\section{Bibliography}

Beard, G.M. 1881, American Nervousness: Its Causes and Consequences: a Supplement to Nervous Exhaustion (Neurasthenia), New York: G. P. Putnam's Sons.

Burke, T. 1996, Lifebuoy Men, Lux Women: Commodification, Consumption, \& Cleanliness in Modern Zimbabwe, Durham: Duke University Press.

Burkes, P. 2001, Eyewitnessing: The Uses of Images as Historical Evidence, Ithaca, NY: Cornell University Press.

Chen Duxiu 陳獨秀 1915, 'Jinri zhi jiaoyu fangzhen' 今日教育方針 (Today's education policies), Xin qingnian 新青年 (New Youth) 1.2. Dikötter, F. 1995, Sex, Culture and Modernity in China, London: Hurst. 1998, Imperfect Conceptions: Medical Knowledge, Birth Defects, and Eugenics in China, London: Hurst. in China, London: Hurst.

Dorey, A.K. Vance 1999, Better Baby Contests: The Scientific Quest for Perfect Childhood Health in the Early Twentieth Century, Jefferson, NC: McFarland.

Farquhar, J. and M. Hanson (eds) 1999, Empires of Hygiene, special issue of Positions: Asia Critique 6.3.

'Fine Baby Show in Changsha', North China Herald, 31 May, 1933, 334.

Haug, W.F. 1986, Critique of Commodity Aesthetics: Appearance, Sexuality, and Advertising in Capitalist Society, Minneapolis: University of Minnesota Press.

Kuriyama, Shigehisa 栗山茂久 1999, The Expressiveness of the Body, New York: Zone Books.

Laing, E. Johnston 2004, Selling Happiness: Calendar Posters and Visual Culture in Early Twentieth-Century Shanghai, Honolulu: University of Hawai'i Press.

Leung, A. Ki-che and C. Furth (eds) 2011, Health and Hygiene in Chinese East Asia: Policies and Publics in the Long Twentieth Century, Chapel Hill: Duke University Press.

Li Xianzhe 李賢哲 ‘Minguo shiqi de “4-4” ertong jie’ 民國時期的 “四 四” 兒童節 (4 April Children's Day in Republican China). Online at www.cnlu.net/disp.asp?id=51702 (accessed 23/o8/2013).

Lo, V. (ed.) 2012, Perfect Bodies: Sports, Medicine and Immortality, London: British Museum Research Publications no. 188.

Mao Zedong 毛澤東 1917 (April), 'A study of physical education', in Shram (ed.), 113-27.

Mort, F. 1987, Dangerous Sexualities: Medico-Moral Politics in England since 1830, London: Routledge and Kegan Paul.

Phillips, T. 2006, 'Building the nation through women's health: modern midwifery in early twentieth-century China', PHD diss., University of Pittsburgh.

Rabinbach, A. 199o, The Human Motor: Energy, Fatigue, and the Origins of Modernity, New York: Basic Books.

Rogaski, R. 2004, Hygienic Modernity: Meanings of Health and Disease in Treaty-Port China: Berkeley: University of California Press.

Shen Juanqi 沈焦淇 1946, Jixie rensheng 機械人生 (Mechanical Life), Shanghai: Wentong shuju.

Shram, S.R. (ed.) 1992, Mao's Road to Power: Revolutionary Writings 1912-1949, vol. 1: The Pre-Marxist Period, 1912-1920, New York: M.E. Sharpe.

Stepan, N. 1982, The Idea of Race in Science: Great Britain 1800-1960, Hamden, Conn.: Archon Books.

Stocking, G.W.Jr. 1987, Victorian Anthropology, New York: The Free Press.

Tang Jun 唐隽 1935, 'Dao haibian qu' 到海濱去 (Go to the Seaside), Meishu shenghuo 美術生活 (Arts and Life) no. 5 (August).

Wang Zhenzhu 汪珍珠 2011, 'Popular magazines and the making of a nation: the healthy baby contest organized by the Young Companion in 1926-27', Frontiers of History in China 6.4, 525-37.

Yen, F.C. [Yan Fuqing 顏福慶] 1938, 'Problems of public health', People's Tribune new series 23, 113-19.

Zhou Xun 周遜 2012, 'Fitness and modernity in late nineteenth and early twentieth century China', in Lo (ed.), $143^{-56}$. 


\title{
36 Sketching the Dao: Chinese Medicine in Modern Cartoons
}

\author{
Judith Farquhar and Lai Lili 賴立里
}

The way that can be realised is not the lasting Way, the names that can be uttered are not the lasting Name 道可道非常道, 名可名非常名.

These famous first lines from Laozi's 老子 Daode jing 道 德经 incorporate a whole theory of representation, one that has been immensely influential throughout Chinese history. This originary formula, contemporary with the beginning of the written history of Chinese medicine, teaches us that all efforts to understand ultimate realities through any form of representation will fail. The lasting Way, the permanent names, may be approached, but they cannot be captured by mere human devices. Language and all forms of representation necessarily fall short of ultimate realities, and Laozi teaches us to forego all ambitions of taming reality by naming it.

At the same time, though, this ancient formula paradoxically invites us to think about representation in a different way. The idealism of the Laozi text is rigorous, no doubt: if the goal is to achieve harmony with elusive cosmic patterns, to become one with the Dao, human ethical systems and the names on which they rely can only fall short. In the text as a whole (as is well known), the ethical principles taught by Confucian thinkers are depicted as especially misleading, substituting hypocrisy, arrogance, and ungenerous calculation for directness, surrender, and spontaneity. Mere mortals armed only with a familiar morality and conventional classifications (names), will never become one with the lasting Dao; in fact our ethical practice leads us ever further from it. Most of us, however, are not world-renouncing Daoists. Under these circumstances, we might as well abandon any more properly 'Daoist' aims of converging with cosmic process. The way that can be realised is not the lasting way. Shouldn't we, then, concern ourselves with the realisable? On the question of names, in what follows I want to trace in modern forms of representation a more properly 'ethical' (or Confucian?) question: what can human language achieve? How should names be improved to encourage virtue or wisdom in mere mortals? What are the forms of representation that can be both uttered and realised? If the great names known to metaphysics necessarily miss their always-transforming cosmic referents, then we must ask what a more concrete and particular naming process can achieve.

For present purposes I want to add a line to the first chapter of the Daode jing: 'Hua kehua fei chang hua' 画可
画非常画 - the picture that can be drawn is not the lasting picture. Pictures have their own ways of assigning names and limiting reference, their own generic shortcomings in efforts to reach the Dao. A picture may be worth a thousand words, as the English proverb has it, but if words fundamentally fail to tell us the truth, if they fail to name the 'lasting Way', then pictures also fail. The philosophical point holds for all forms of representation. At the same time, pictures, words, and conventional morality do something. It is this 'something', this humble functionality of words and images, which can be approached through a reading of popular graphic versions of Chinese medicine and philosophy. The comic books I consider in what follows are examples of a popular genre of publishing devoted to the classics of Chinese thought, as I will describe below. I will ultimately argue that these humble and unpretentious cartoons are effective in surprising ways. This effectiveness is limited, but interesting for an ethnography of reading and for a study of visual rhetorics. So I argue there that cartoons are as successful as many other genres in capturing and conveying the meanings - and perhaps especially the practical implications - of ancient Chinese medical and metaphysical works; and where they fail it is for similar reasons. In other words, I will conclude with a return to Laozi's scepticism about the powers oflanguage and representation, but I want to suggest that in practice there may be syntheses a philosopher cannot dream of.

In what follows, I will maintain a focus on both the successes and failures of visual representation in medical materials published for popular consumption. Successes and failures are here conceived in relation to the goal of representing - making present again - a reality. As often as not, this might be conceived of as producing a full and accurate idea of something for a knower, making a sign that fully evokes its referent. I adopt this definition in an effort to remain true to the representational goals of the language of the Daode jing rather than in relation to contemporary semiotics or literary realism. The concerns of linguistic semiotics have informed this chapter to some extent, but I have avoided technicalities while attending more centrally to indigenous Chinese approaches to language and representation. After some historical preliminaries, I will provide a reading of a few examples from medical classic comics from the point of view of content analysis. I will then turn to some popular appropriations of these comics drawn from field research in Beijing in 2003, 
linking texts to practice and thus back to the question of 'realising the Dao'.

Medical classic comics constitute a relatively ephemeral genre of medical illustration: widely available cartoon versions of classical writings from the history of Chinese medicine. Examples are several versions under the title (Huangdi neijing yangsheng tudian 黄帝内经养生图典 (Classic Comic of Life Nurturance in the Yellow Emperor's Inner Canon), ${ }^{1}$ Manhua xuedao anmo yangsheng shu 漫画穴道 按摩养生术 (Arts of Nurturing Life Through Acupressure Massage in Comic Form), ${ }^{2}$ and Zhongyi yaoshi tudian 中 医药食图典 (Classic Comic of Chinese Medicinal Foods). ${ }^{3}$

We begin by placing illustrated books like these in historical context, presenting some other cartoon and illustration genres to which they can be compared, especially with reference to the effectiveness of images and words. These comparative materials include cartoons that developed in mid-2oth-century Chinese print media, Maoist propaganda comics, and the illustrations used in relatively 'serious' publications about Chinese medicine. Each of these genres forges its own relationships between image, word, and referent; none is as popular as 'classic cartoons'. We then turn to a close reading of the typical representational strategies of a few cartoon versions of Chinese medical knowledge, and close by commenting on how books like this are being used in contemporary Beijing. Reading practice returns us in the conclusion to some of the concerns of the Daode jing.

\section{2oth-Century Illustrated Genres}

As materials presented in this volume make abundantly clear, the history of conjoined text and image in China is as long as the history of the nation itself. In fact a Chinese picture that incorporates no words at all is rare, as we are reminded by genres as diverse as technical illustrations and Song-dynasty landscape painting. Indeed, one could argue that any theoretical divide between words and pictures for the Chinese linguistic tradition would be illegitimate. For many Chinese thinkers, words are pictures. Modern publishing, with its standard machine fonts and separation of calligraphic dedications or illustrations from the text itself, have made the arbitrariness of the sign a more plausible linguistic theory for the 2oth and 21st centuries. But it might still be unproductive to explore 'the text-image

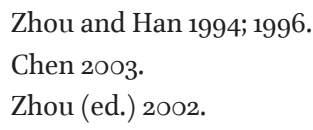

relationship' in China as if the two modes of representation were really distinct. ${ }^{4}$

The modern popular genre of cartoons (漫画 manhua, 连环画 (ianhuanhua) has a particular and rather lengthy pedigree in East Asia, but in China as in the United States the history of modern cartoons proper - published in the mass media and made for general consumption - is not long. The precursors of the modern mass market comics in China who produced New Year woodblock prints and illustrated fiction were influential in the development and styles of manhua, ${ }^{5}$ but most historians link the rise of the genre to the availability of lithography and the rapid development of newspapers in the late 19th century. ${ }^{6}$ The modern terms for comics, manhua and lianhuanhua, are recent, having been coined in the 1920 os. $^{7}$ The 2 oth century saw a vast expansion in cartoon genres and venues, some included in newspapers as a few frames and others published as little books' (xiaoshu 小书 orxiaorenshu 小人书) or broadsides. In Shanghai in the late 193os, there were at least 17 magazines devoted almost entirely to the genre. ${ }^{8}$ By the 1930s it had become possible to theorise, and at the same time politicise, the efficacies of words and images in the popular genre of the cartoon. ${ }^{9}$ Nebiolo quotes the influential left-wing writer Lu Xun 鲁迅, for instance, on the propaganda potential of the comics:

Not long ago in the review Modern Age, I read an article by Su Wen, the art critic, condemning the comics from an aesthetic point of view... . In the art histories to which

4 Mitchell has extensively explored and critiqued Western modernist criticism, which has given the appearance of a 'battle of text and image' (Mitchell 1986, p. 154). In his own interpretive project he occasionally mentions both 'Chinese calligraphic landscape' (Mitchell 1994, p. 98) and cartoons as modes of suturing word and image (ibid., pp. 91-4). The approach he advocates explores 'the problem of the image/text', allowing genres and works to be seen as historically and formally particular in the relations they materialise between images and texts. Such a critical project would not, perhaps, be needed in relation to classical Chinese linguistics and literary theory.

5 Bai et al. (eds) 1993.

6 See Hung 1994, pp. 28-9; Isao 2001, p. 137; Nebiolo 1973, pp. viixvi; Schodt 1983; Shandong Illustrated Press Editorial Group (ed.) 2002; Wong 2002, pp. 11-16; Zhang 2001.

$7 \quad$ See Hung 1994; Shen 2001; Xiao 2007.The first free-standing 'comic' with narrative in the United States appears to have been 'The Yellow Kid' in 1895 (Blackbeard 1995).

8 Wong 2002, pp. 14-15.

9 Ibid., pp. 13-15, argues that a genre of political cartoon critical of Qing policies had already begun to flourish in Hong Kong in the late 19th century. Until 1949 there was a great deal of exchange among artists, intellectuals, and publishers including Hong Kong and all the coastal cities of the mainland. 


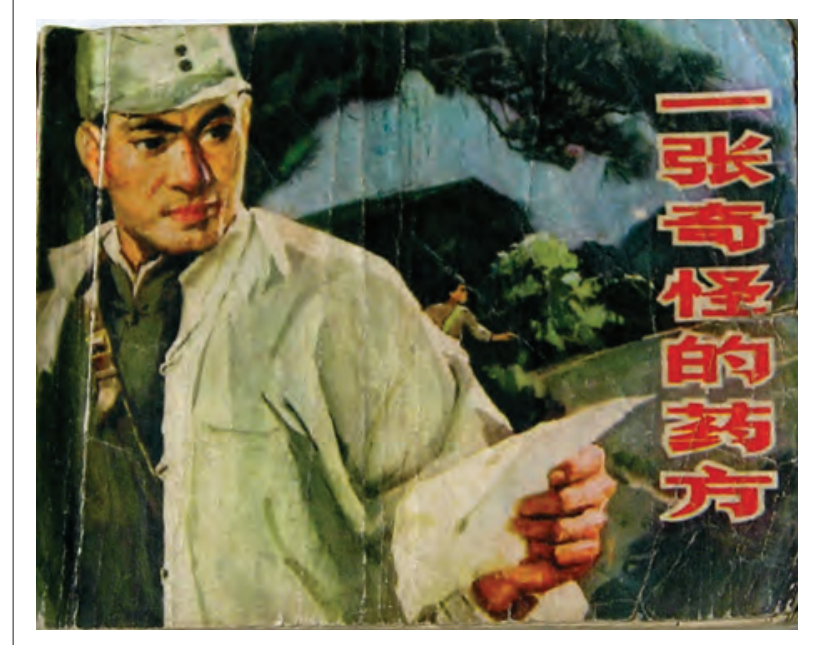

Figure 36.1 Cover of Xiaorenshu 'A Strange Herbal Prescription' 一张奇怪的药方

we are accustomed we find no reproductions from comic books, and in exhibitions we see only such things as 'Rome at Twilight' or 'Western Lake at Dusk'. Obviously, comic books are considered too low-brow to belong to such respectable company. But if you visit the Vatican... you will find that all the marvellous frescoes fundamentally tell stories from the Old Testament and the Acts of the Apostles. When an art historian reproduces one of them under the title 'The Creation of Man' or 'The Last Supper', no one considers it vulgar or propagandistic. And yet the originals are fundamentally of a propagandistic nature. The same thing holds true in the East... . Both the life of the Buddha and the [Analects] of Confucius are obviously propagandistic publications, in which pictures are meant to stimulate the reader's interest. ${ }^{10}$

This is not a surprising interest on Lu Xun's part, given his simultaneous involvement in reform of both visual and linguistic genres of art and his concern with the politics of representation practices. Folklorist Qu Qiubai 篗秋白 also argued for the revolutionary potential of cartoons (along with 'operas and spoken dramas, songs and ditties') 'because the numbers of the literate are so extremely small'.11 As the Japanese mobilised to occupy Chinese cities in the 1930s, a number of publishers and artists involved in comics production openly cast their mission as political and committed their work to public education and mobilisation. ${ }^{12}$ As Japanese control over Chinese cities tightened, mass media artists moved their work and publications ahead of the front. Controversies surrounding particular

$10 \quad$ Nebiolo 1973, p. xiii. See also Hung 1994, p. 31; Xiao 2007.

11 Wen and Ye (eds), 1997, p. 124, cited in Bohnenkamp 2005. A recent study of 193 os woodcut narratives (lianhuanhua) by Tie Xiao is particularly illuminating about the political climate of the time among artistic activists (Xiao 2007).

12 See Hung 1994, pp. 31-5, 93-150; Shen 2001; Zhang 2001, p. 135.
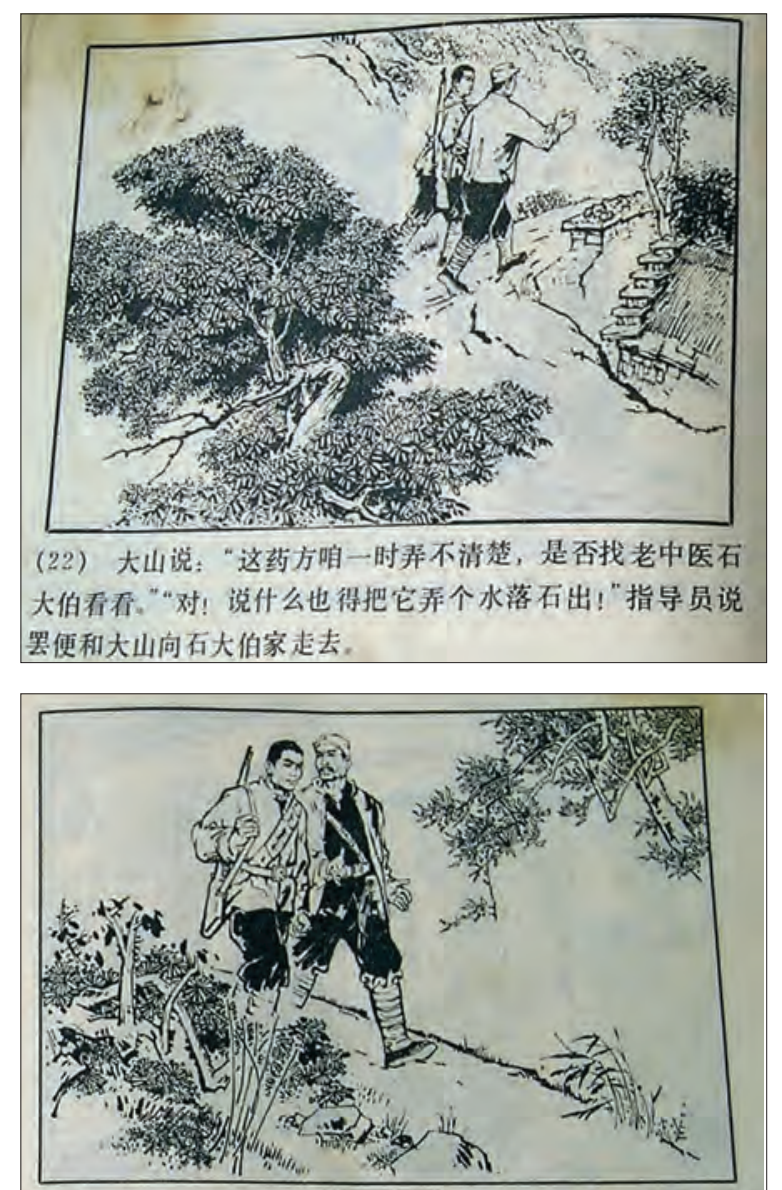

（23）路上, 指导员说: “地主山羊胡被斗以后，他弟弟三 角眼逃跑到县城当了蒋韮军官。现在山羊胡又和独眼㖪鬼混 在一起, 因此值得我们特别繁惕。”大山点了点头。

Figure 36.2 Two pages from 'A Strange Herbal Prescription'

cartoonists and individual works attest to the power of these combinations of images and words.

After the founding of the PRC the new propaganda apparatus took up Lu Xun's advice to exploit the educational and populist potential of images: the cartoon story, often in the form of books small enough to be carried in a pocket, became an important propaganda genre directed at people of all ages (Fig. 1).

If the high artistic quality of the drawing in these books is any indication, the 'little book' was meant to educate and entertain, but not to amuse in a comic sense. The informal and irreverent styles of drawing that had been popular in cartoon genres before 1949 were replaced by a more traditional aesthetic incorporated into the 'revolutionary romanticism' propagated by the state. ${ }^{13}$

An experience reported by Gino Nebiolo, an Italian visitor to China in the 1960 s, attests to the popularity and appeal of these xiaorenshu, despite or even because of their

13 See Anderson 1990. 
ponderousness. On an overnight train in the late 196os, all passengers received a little cartoon book when their tea was delivered by the train staff. Passengers traded books as they finished them, and most stayed awake all night reading. Nebiolo reports:

All through the night we exchanged comic books, without ever speaking... . The comic books did not tell heroic tales or episodes from history alone; some of them reflected everyday life, particularly in the country. One that fell into my hands was entitled 'A Pailful of Manure', whose peasant heroine, Qien Er-xiao, was, to be sure, a conscientious worker, but was corroded by the subtle poison of bourgeois selfishness. From her personal latrine she removed a pailful of potential manure for use in her own vegetable garden. Fortunately her husband was a good citizen, ready to give up the manure for the good of the community and, indeed, to scatter it over the collective field.... Around the smelly pail the entire village took part in a doctrinal dispute, and social considerations, quite rightly, won. Qien Er-xiao understood what she was supposed to do, happily gave in to her altruistic husband, and thanked the villagers for opening her eyes. ${ }^{14}$

In the reform period the amount of this kind of state-produced cartoon material has diminished drastically, at least in the domain of adult reading. The 'little book' format, with its elegant drawing style, persists as boxed sets illustrating classics of pre-modern Chinese literature. But more 'commercial' and - once again, 'comic' - uses of cartoons for adults started to appear in the publishing boom of the late 1980 s and 199os. Nowadays, of course, unlike the era of the xiaorenshu, little of what's published could be classed as state propaganda. Even works of serious scholarship and political thought have difficulty finding a publisher, as most presses need to turn a profit, so they seek genres that sell. In fact, bookstore stocks reflect publishers' market research rather closely, as any books that don't move are sent back at least bi-weekly to the presses. The new entrepreneurial publishing field in China tries not to make the same mistake twice about an author or a genre. Cartoons appear to sell. The bigger Xinhua Bookstores in Beijing now have whole departments devoted to cartoon books, stocked with works ranging from facsimile reproductions of Qing dynasty illustrated manuals to imported and translated black humour and dirty jokes. Among these are the very popular 'classic comics' to which I now turn.

\section{Classic Comics}

The materials I present here belong to a thoroughly contemporary genre. Unlike the propaganda materials discussed above, and unlike some other kinds of illustrated popular books on Chinese medicine, they make few pretensions to including 'good art'.

Cartoon versions of classic philosophical works, originally produced by the well-known Taiwan animator Cai Zhizhong 蔡志忠 (Tsai Chih Chung), began to appear in the late 198 os and 199 os as a vehicle for popularising 'traditional Chinese culture'. Cai published cartoon versions of the classic philosophical works of Zhuangzi 庄子 and Laozi 老子, the Confucian four books, Shijing (The Book of Odes), the Han Feizi 韩非子, and even Song poetry. The genre is called “classic comics' or 'pictorial classics' (jing dian manhua 经典漫画, tudian 图典), and following the immediate popular success of Cai's first publications the genre as a whole caught on in the book market throughout sinophone East and Southeast Asia. ${ }^{15}$ Cai's books and those of his mainland imitators have been translated into Japanese, Korean, and English, and they are printed in runs of up to 20,000. Most recently Cai has produced hard-bound books with colour illustrations which include both a DVD and a CD-Rom. ${ }^{16}$

Once the Chinese philosophical and poetic classics had been represented as comics, Chinese medicine presented itself as a natural body of literature to be handled in this way. ${ }^{17}$ An artist named Zhou Chuncai 周春才 began to produce Chinese medicine 'pictorial classics' as early as the mid-199os, but the Chinese medicine branch of this publishing field became really active around 1998. Publishers clearly thought that many readers who might be intimidated by the classic works and medical textbooks would both want to know more about classical Chinese medicine and would prefer to read a cartoon version of its famously abstruse canonical works. This expectation is made clear by the following description from the back cover of Zhou Chuncai's 1994 Huangdi neijing yangsheng tudian (Classic Comic of Life Nurturance in the Yellow Emperor's Inner Canon):

The Yellow Emperor's Inner Canon is a wellspring of our fatherland's medicine and an exotic flower of human wisdom. Its theoretical system of 'resonance of man and heaven', and 'Yin-Yang and Five Phases' is not only a cen-

\footnotetext{
$15 \quad$ Wei 2001.

16 For example, Cai 2003 .

17 The presentation of science in cartoon form was not entirely unprecedented. In 1952 palaeo-anthropologist Jia Lanpo published a serial picture book, 'Our Ancestors 500,00o Years Ago' (see Schmalzer 2008).
} 
tral model (宗 zong) in medicine, it is also an important approach to people's daily habits of eating and drinking, rising and resting. For example, how to 'accord with the seasons and adjust to winter and summer'; how to 'nurture Yang' in the spring and summer, how to 'nurture Yin' in the fall and winter; what the relationship is between human emotions (情志 qingzhi) and health; how the five flavours nourish the body; how the 12 organs (器官 qiguan) are connected; etc. etc. There is nothing in it that is not of intimate interest to every individual.

In addition to all of this, the Yellow Emperor's Inner Canon not only nurtures life, it also explains and interprets people's behaviour and thinking. So this book purposely adopts the comic form that everyone finds so enjoyable to read, and provides lively image commentaries (形象生动 的诠释) for this archaic and abstruse canon, in the hope that more people can derive inspiration from it.

Here the authority and importance of these works (perhaps especially to cultural nationalists) is juxtaposed to their mundane usefulness and their ready accessibility in comic form. 'Image commentaries' are seen as lively and helpful, even 'inspiring', suggesting that the book's publicists recognised that images might have an added value in conveying significance. There is a Chinese medical subtext here, perhaps: many leaders in Chinese medical education lament that classical Chinese medicine is 'too abstract', and thus difficult to convey to young medical students who have been trained in the very concrete natural sciences. Echoing Lu Xun's enthusiasm for the 'realistic' power of cartoon images ${ }^{18}$, this volume presumes that 'the masses' are more responsive to the mundane and the concrete than to the elevated metaphysical language of the medical classics.

When I turn to these books in preference to the many scholarly editions of classics on my own bookshelves, it is for speed and convenience. Other habitual readers of Chinese medical works with whom I've spoken noted the same usefulness for these little 'classics'. If one is looking for the exact wording of a famous quote, or needs to be reminded of the correspondences of colours to viscera or the symptoms associated with wind, these fragmentary kinds of knowledge can be found very quickly in a comic. This scholarly short-cut is not imagined as the primary function of these books, of course. Recall that the publishers believe that these medical cartoon books are of 'intimate interest' to everyone. Some readers pick up these books as their primary access to the insights of Chinese medicine about the life of the body and the nurturance of life - their own body, their own life. Can the comics be relied on for teaching Chinese medicine to the masses?

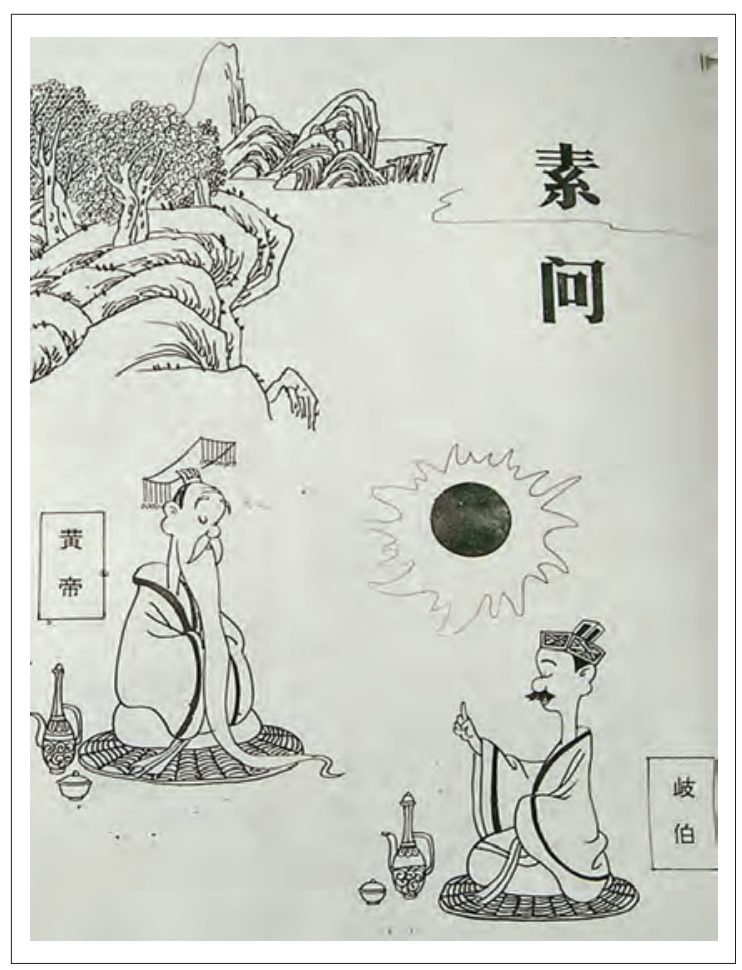

Figure 36.3 The Yellow Emperor and his teacher Qi Bo

In fact, do ordinary non-specialist readers actually find the comic form of medical classics 'so enjoyable to read'? A closer reading of the content of these books, as well as a consideration of how they are actually received, can begin to answer these questions.

\section{Images}

I noted above that these cartoon classics make no pretensions to offering beautiful art. The cartoon images differ somewhat depending on the artist, but all are sketchy and unpretentious. The drawings themselves have a 'comic' character. Though actual jokes are relatively rare in the classic comics genre, the mere act of depicting something so ancient and dignified as classical medicine and metaphysics through sketches of ordinary people doing ordinary things is amusing enough. In these pages we have perfectly recognisable modern individuals discussing cosmic processes in words that sometimes depart relatively little from 2,00o-year-old classical language. Most of these classic comics include the original text (usually as a fragment), some commentary or modern Chinese translation, and not quite reverent remarks from the cartoon 'characters', often in speech balloons.

Though Huangdi 黄帝 and Qi Bo 岐伯, the chief interlocutors of the Yellow Emperor's Inner Canon (Fig. 3), are in dressed in cartoon versions of Han Dynasty clothing, 


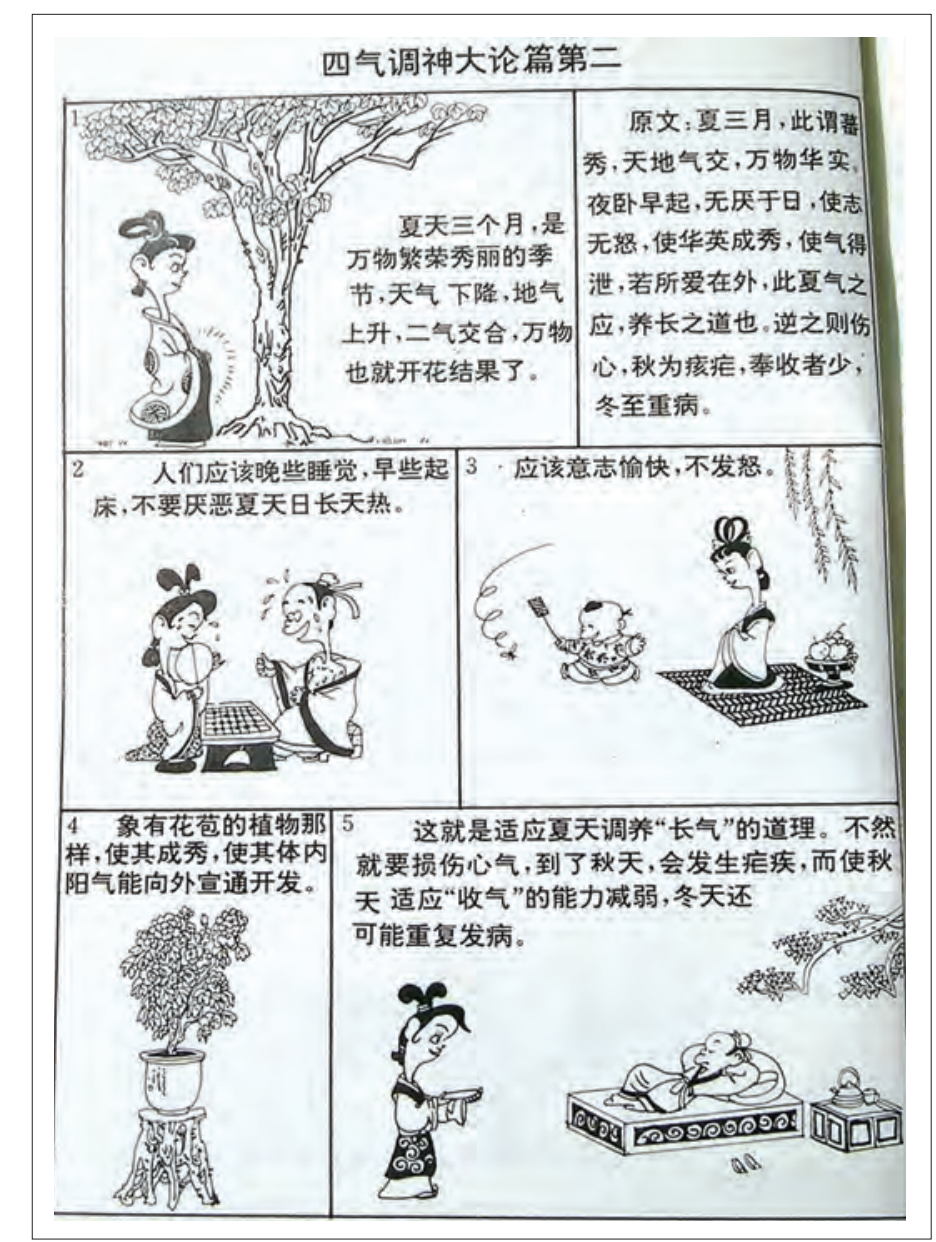

Figure 36.4 Adjusting to Summer weather is both an ancient and a modern problem

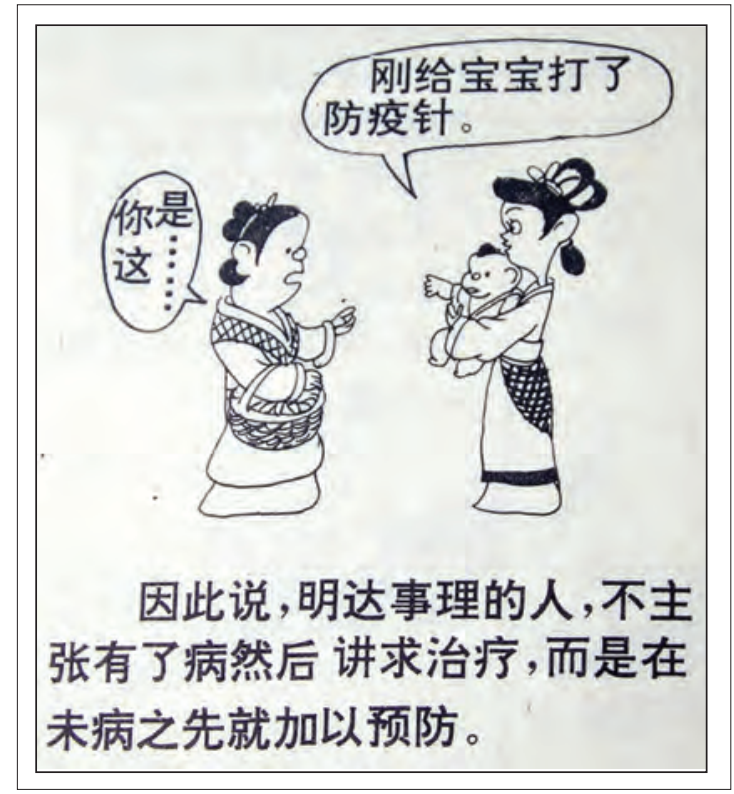

Figure 36.5 'I just took the baby for his shot' and display a certain ancientness in their serenely seated postures (but isn't Qi Bo's moustache rather suspiciously 2oth century?), the many ancillary characters are aggressively modern.

The gestures, facial expressions, and spontaneous comments of these ordinary men and women, workers and farmers, are purposely anachronistic (Fig. 4); they certainly lack the gravitas of actors one would think of as 2000 years in the past. This temporal dissonance, the source of the comic sense of the genre as a whole, is clear in a frame where a Han dynasty mother, committed to the ancient idea of preventive medicine, takes her baby for a vaccination shot ('The highest doctor treats the not-yet-ill' 上工治未病) (Fig. 5). 19

Then there's the one of the person afflicted with stomach troubles hogging the neighbourhood 'w.c.. ${ }^{20}$ The overall impression given by the cartoon style is similar to that of the many Han and Tang dynasty soap operas on Chinese 


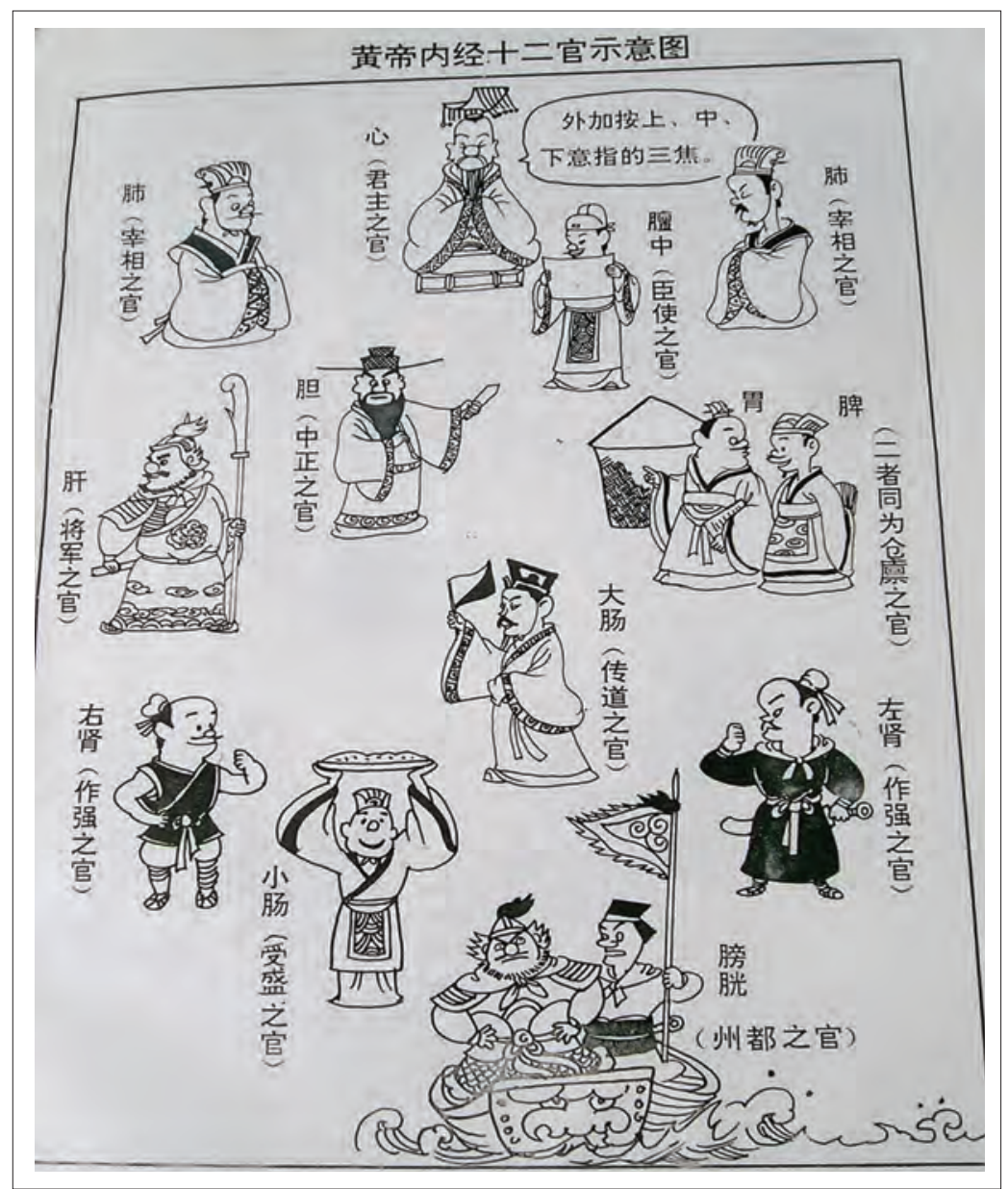

Figure 36.6 The 'Twelve Officials' of the visceral systems (Zhou 1994, p. 3)

television. The message is, 'except for their clothes and hairstyles, those ancients were just like us'.

Perhaps this visual depiction ironically persuades modern readers to take the science of the Chinese medical classics more seriously than they otherwise would. If Qi Bo is just like us - even if he is a little more educated about the ways of the universe and more practised at living in accord with the Dao - then his advice should be directly relevant to the ways we live our lives today. After all, knowing the Dao of health and long life should not go out of style; even if words and images cannot grasp it, the most lasting Dao toward which these representations are oriented should never get relegated to the dead past. Looking through these comics with Beijing friends, I've noticed that they find a comic quality in the casual everyday language that is juxtaposed with the formally intoned correspondences of the original text. When a mother with wet hair gets struck by wind pathogen, and her bathing baby cries out, ‘妈妈, 你怎么了?' (Ma, what's the matter?), this is occasion for a chuckle. ${ }^{21}$ When a cartoon time-keeper urges the sluggish

$21 \quad$ Zhou and Han 1994, p. 94. drugs tianzhuhuang 天竹黄 and huomaren 火麻仁, to wake up and get to work, and the soldiers representing these drugs rub their eyes and say, 'I'm sleepy,' this brings a comfortable and pleasant recognisability to the vast technical field of herbal pharmacy. ${ }^{22}$ In these images the authority of the past is brought at least halfway down from the Olympian heights of the sage kings and their interpreters, with their difficult classical language, to a level where consumers of medical knowledge can connect each fragment in a straightforward way to some aspect of their own (possibly just as fragmented) modern lives.

The kind of immediate bodily relevance sought by these books is consistent with the pedagogical-popular aims of the genre. But the images provide a different kind of mnemonic technique than the classic texts themselves ever could, even in the modern translated and annotated versions most specialists read. An interesting example of a divergence in message between classic and comic forms is clear in a page depicting the 'twelve officials' corresponding to the visceral systems (wuzang liufu 五脏六腑) (Fig. 6).

22 Ibid., p. 174. 
In this cartoon depiction of the metaphorical 'ministers' who classically divide responsibility for the body's visceral bureaucracy, the various officials are arranged in a way that corresponds to the spatiality of a modern Westernmedical or anatomical body. This depiction hardly follows a classical Neijing (Inner Canon) understanding of the hierarchy of the visceral systems, the five zang and six $f u$. There are two little officials for the lungs and two for the kidneys, for example, though these visceral systems are not usually spoken of as dual in the classics; the heart is given the highest position, though many would accord priority to other viscera; the physiological importance of the kidney system, the spleen, and the liver system are curiously minimised; the Triple Burner - one of the six fu - is forgotten altogether; and the urinary bladder, depicted as a relatively large and fierce navy, looks a lot more important than classical medicine would normally claim. Modern organs known through an anatomical medicine are a dominant, if unstated, referent for this cartoon spatialisation of the functional body. Moreover, with the exception of the spleen and stomach, who seem to collaborate a bit, these bureaucrats all appear as free-standing individuals rather than interrelated functional systems. One might also note that the arithmetic doesn't even work: there is no way to count these officials so that they add up to 12! If lungs and kidneys are counted as one, and spleen and stomach are (properly) kept separate, there are 11 officials; if lungs and kidney are counted as two, and even if spleen and stomach are combined, there are 13 ! A reader who recalls the spatial and functional relationships of the viscera from this illustration, as she reads about the zangfu in later parts of this book, will continue to see the ancient material through a certain anatomical lens, and will strive to turn profoundly interrelated systems into discrete individuals with distinct domains of responsibility. This cartoon, then, is a potent (mis)representation of a central form of knowledge found in the classical sources.

I am not here simply arguing that comic books over-simplify and distort the correct meaning of the medical classics. A moment of reflection would persuade us that any pictorial representation of the bureaucratic metaphor for the zangfu as used by the Neijing would reduce the allusiveness of the original text and necessarily miss the dynamic functionality of the visceral systems. Any picture that could be designed would occlude some aspects of the body and cosmos found in the Inner Canon even as it presented - perhaps very persuasively - those aspects deemed to be most central. There's another way of saying this: every picture is about the same as a caricature (or a cartoon?) when it is compared with the richness of the medical concepts and realities, and even the medical classical language, at

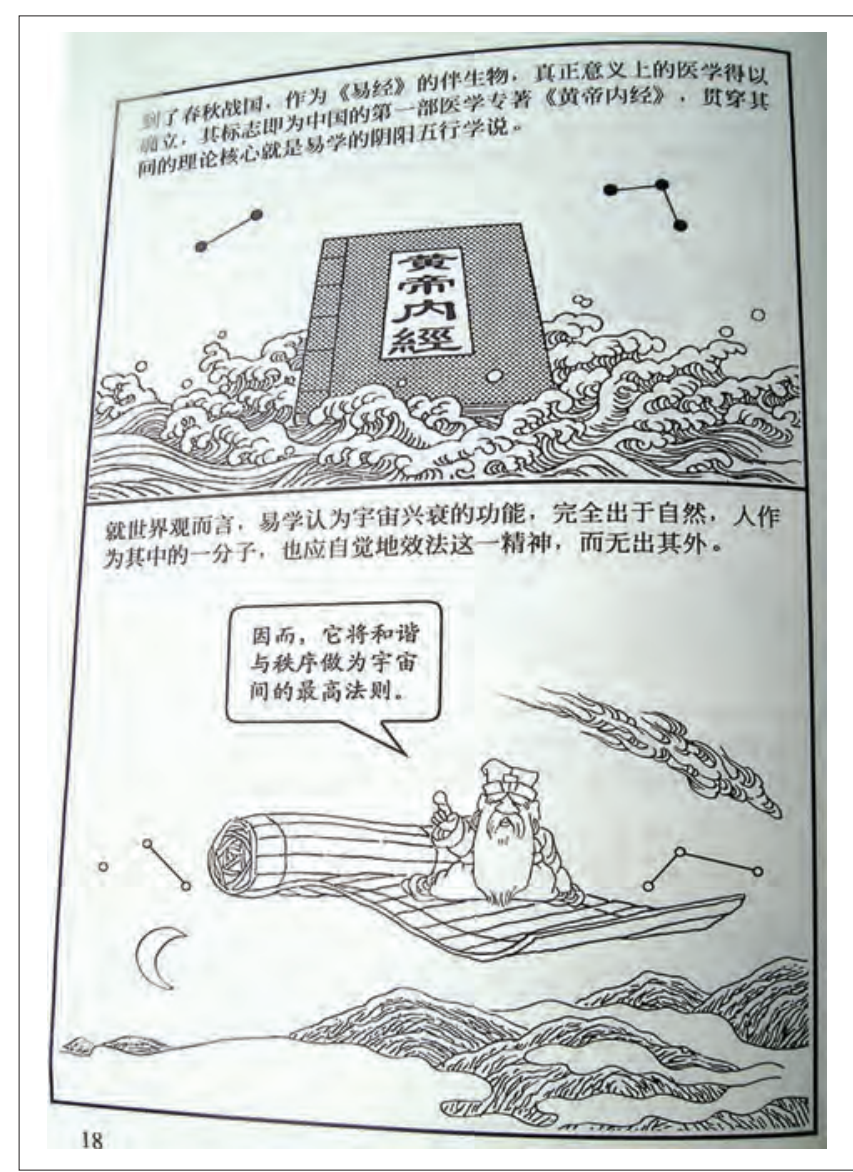

Figure 36.7 The Comic Yellow Emperor's Inner Canon (Zhou 2002, p.

18)

which it aims. If the hierarchy of visceral functions in the Chinese medical body is a dynamic and recursive one (and it is, of course), then a list of bureaucrats who 'rule' various functions is a very remote metaphor indeed. Even if a tacit understanding of the interactivity of the bureaucratic process is presumed (as it is by the original texts), these 'twelve' officials are at best only mnemonic devices that can place the zangfu in relation to each other, spatially and functionally. This sort of picture falls very far short of the 'lasting Way'.

But perhaps, as concepts become more abstract, art can be more successful, more comprehensive? Is a casual sketch an especially good medium for a broad abstract notion? Oddly enough, in my reading of the comics this seems to be the case. A sequence from Zhou Chuncai's Zhongyi yaoshi tudian can leave the purist feeling a bit more positive about the power of images. This book is a bit unusual in that it provides no textual extracts from classic books. Instead it explains the lessons about food and medicines, or medicinal foods that can be derived from Chinese medicine's classic sources. With a cheerful, bearded, and very old Chinese doctor - perhaps meant to be Shen Nong 神农, the Divine Farmer, though he is nowhere identified - as our guide, 


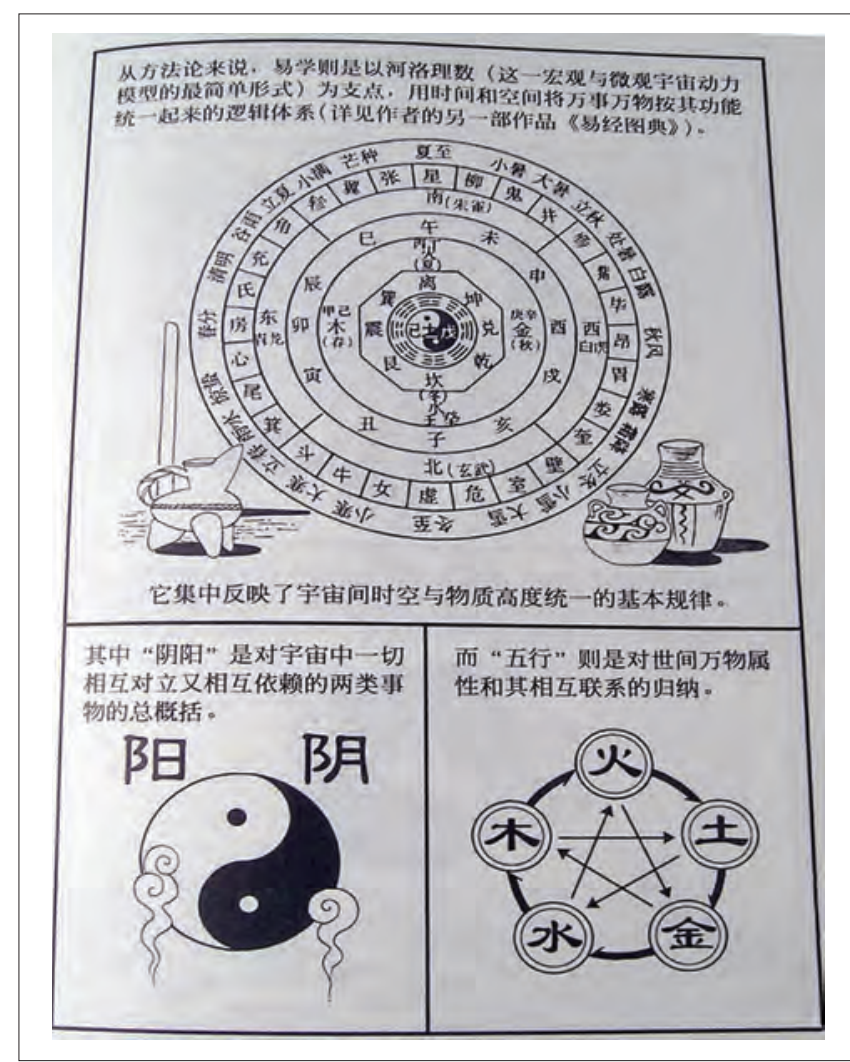

Figure 36.8 Systematic method (Zhou 2002, p. 19)

we mainly find here a catalogue of medicinal foods. The book is organised according to medical principles (rather than, for example, botanical principles), much as a textbook would be organised. Most of it is very concrete. But in a few introductory pages on 'Origins', a strong statement is made that this kind of information relies on the theoretical system of Yin-Yang and Five Phases derived (it is claimed) from the Book of Changes (Fig. 7).

The cosmic scope and totalising ambitions of this theory are represented by asterisms in the sky; its dynamism and limitlessness are emphasised by ocean waves; and the intimate connection of both knowledge and knowers to these cosmic processes is represented by the string-bound Huangdineijing floating on the ocean as well as by the sageking riding his flying bamboo mat high above mountains and clouds. This depiction of 'theory' is both witty and effective. It opens a window on to the cosmos, leading the reader imaginatively far from the cloistered study or the dusty herbal pharmacy. It urges her to see the relatively humble question of what to eat for health as linked to ultimate questions of order and harmony in the universe.

The next few frames (Fig. 8) draw the eye back down to some well-known diagrams that graphically put Yin-Yang and the Five Phases, time and space, into conventional relationship with each other, but the section concludes

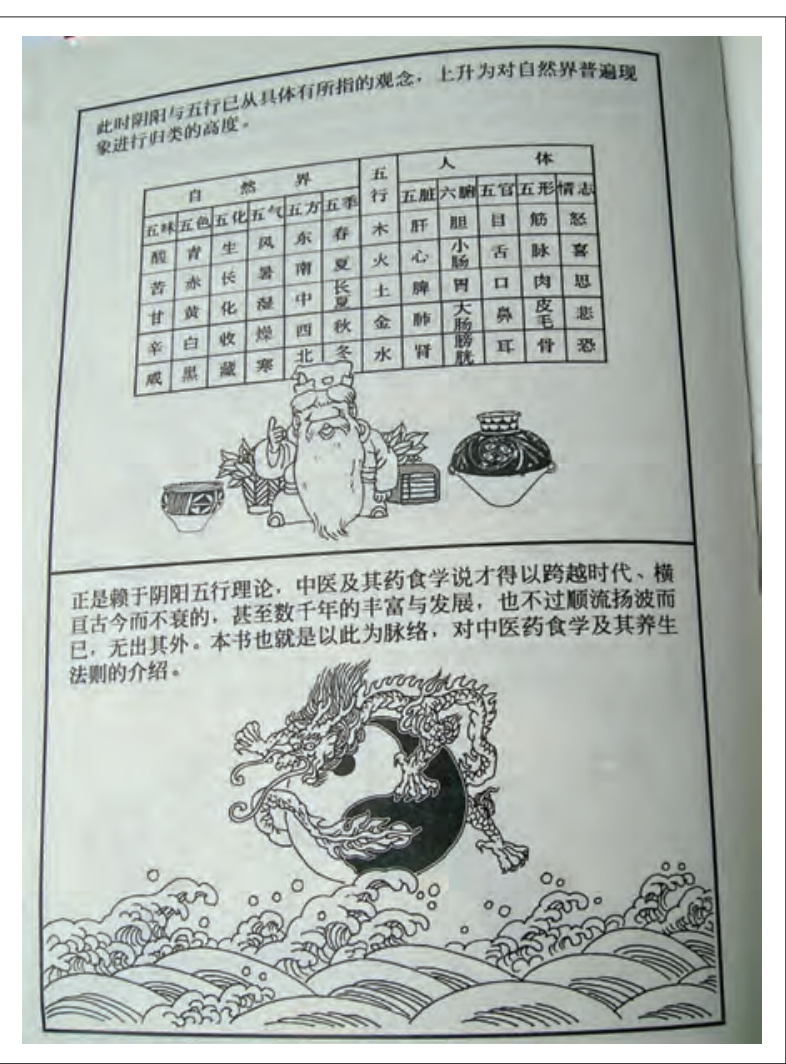

Figure 36.9 Systematic method followed by the ineffable dragon

(Zhou 2002, p. 20)

cosmically again with a lovely image and an ambitious thought (Fig. 9).

Like a dragon, the theoretical system of Yin-Yang and the Five Phases, as well as ancient knowledge of medicinal foods, leaps across the millennia. These philosophies have lost none of their cosmic connection and none of their relevance for us today. In fact, as the twice-repeated formula 无出其外 'there is nothing beyond it' insists, these principles, and the dragon of ancient Chinese wisdom, will always be with us, and they are everywhere meaningful.

It doesn't really matter, for present purposes, whether such images of origins and the cosmos are historically accurate (they are almost surely not what a historian would find in the literature); nor need we be concerned with any limitations in the ideas about China, the heritage, and Chinese medicine that might be encouraged by these images. Rather what interests me is the success of a cartoon in representing something like metaphysical theory and something like the flow of cosmic processes. Though this is not great art, it aims in these few frames to open our horizons; though this is not detailed information, it offers a background against which the herb and food information to follow can be found significant. These drawings and their accompanying text interrupt the ordinary instrumentalism of daily food consumption and rituals of illness prevention 
to give us a vast sea of dynamic process, a look back up at the heavens, a glimpse of a dragon.

\section{Reading}

The question thus far has been, how do cartoon images capture (or not) a concept, an intellectual aim, the deeper or more complex meanings of a source text? Now I turn to a different sort of relationship between sign and referent, asking: how do cartoons reach out to the body and life of the reader? How do they achieve saliency for readers? These questions are generally classified under the heading of 'reception studies', and they are not easy to answer. When we ask how an image or a piece of text is incorporated into the life or opinions of a reader, we venture into a generally unspoken region of everyday experience. Reading takes place, but its particular efficacy in making meaning and influencing readers is difficult to track in ordinary conversation. So here I can only speculate a bit about how Chinese medical comics are received by readers, based on field research I have been doing over the last decade in collaboration with Prof. Zhang Qicheng 张其成 of the Beijing University of Chinese Medicine. We have conducted interviews with a number of Beijingers who practise some form of yangsheng, or the arts of nurturing life. These interviews, and our less formal interactions with city-dwellers interested in yangsheng, provide insights into how Chinese medical knowledge is received and understood by non-experts in contemporary China.

There are many popular media representations of Chinese medicine, ranging from drug advertising to formal lectures in neighbourhood committee offices, from $\mathrm{TV}$ documentaries to magazine articles, and including a wide range of inexpensive books that turn Chinese medical expertise into easily-understood public health advice. The cartoon books that are the subject of this chapter are prominent in this media mix, and publishers specialising in health and medicine are enthusiastic about the genre. They say people do buy these books and the publishers are anxious to keep producing them. In general, looking for them in bookstores, I found them less often displayed with other comic books and more often in sections devoted to personal healthcare or traditional medicine. This supports the suggestion made above, that the comic version of the Yellow Emperor's Inner Canon is seen more as pedagogy than entertainment.

Sharing Chinese medicine cartoon books with ordinary city-dwellers I know, I can get them to say that they are 'interesting', or 'a good idea'. They often see books as full of information that they should probably take seriously. I haven't been able to elicit commentary on precisely how the combination of classical language, informal modern commentaries, and the pictures themselves actually speak to the lives and values of these acquaintances, however. The most interesting responses I have got from non-specialists have singled out isolated practical facts - such as the uses of a particular drug for common ailments - and taken issue with it. Teachers of Chinese medicine, on the other hand, are very enthusiastic about comic classics in the field. They seem to view them as a sort of Cliff's Notes that will no doubt help their struggling students to prepare for exams.

Readers of this Chinese medical knowledge take up particular facts or principles according to needs, experiences, and assessments of their own. In our interviews and more casual conversations, we have noted a certain scepticism about information that can be found in books or advertisements. Asked about where they learn the most reliable health information, Beijingers usually tell us they find their friends' advice the most useful. This kind of freely shared common sense about bodies, food, medicine, costs, and reliable expertise is more important, for example, than newspaper articles, lectures organised by the street committee, or commercials on TV. Further, when we asked about the management of diet or the desiderata of exercise regimes, people told us over and over, 'you should do what feels good'. These two orientations - a reliance on informal lore and a tendency to listen to one's own experience - impress me as very important for understanding the reception of authoritative health advice and even classical knowledge about bodies, nature, and the cosmos. ${ }^{23}$ Both of these preferences take embodied experience to be more important than formal knowledge. Surely, people will say, my friend who has had to control her diabetes with dietary changes, and whose life is so much like my own, really knows more that is useful than any handbook that can be picked up in the clinic. Surely the pleasure I derive from eating oily griddle cakes and twice-cooked fatty pork is as good for my health as any unsatisfying bland diet the doctors might recommend. Though curiosity about good techniques for health maintenance runs fairly high, and Beijingers are always willing to hear advice from any quarter about how to be 'healthy, wealthy and wise', the people we talked with

23 Even the comic book versions of the central theoretical classic, The Yellow Emperor's Inner Canon, are dominated by abstract cosmological explanations and a pre-modern science of correspondences. I cannot believe that very many of my 'life nurturing' acquaintances and interviewees in Beijing really care about the fine points of ancient medical metaphysics. Perhaps it is for this reason that more concrete everyday life advice is scattered through the comic Inner Canon. 
nevertheless preferred common-sense to science, present experience to authoritative traditional wisdom. The matrix within which Chinese medical information takes on significance for ordinary city-dwellers is a complex mix of health experience, friendly conversations, rumours and urban legends, and ways of being embodied.

This sort of experience could be thought of as a taken-for-granted world toward which the text quite self-consciously gestures. Unlike the tight and beautifully illustrated xiaoren shu narratives shared by train-riders in the 1970s, which were read with absorption, classic medical comics (and most other public health publishing) are designed to be nibbled rather randomly. Readers flip through looking for titbits they can use or share with friends. Short snatches of text, engaging subheadings, and - of course pictures catch the eye but do not demand a continuous reading. So much miscellany could make for a bad book, one supposes. But it is precisely this parcellisation that allows the cartoon genre to articulate so well with people's everyday lives. Readers pick and choose bits of lore that accord with their own embodied and untheorised experience; they don't expect a comprehensive account of everything that can be known, or that they should know, about medicine and bodily life. They wouldn't trust such a vision if they got it.

On the other hand, there is something about the comic sketch that has a particular efficacy. It draws attention to itself, generically, as a rapid approximation, not a ponderous realistic representation. A few lines, a gesture, are enough to point toward that outside world of experience and embodiment, that excess over the text, that informs the reading process. There is no need to fill out the frame with more detail; this would only limit the usefulness of the parcels of information that are made livelier through illustration. The very down-to-earth cartoon people who romp through the pages of the Huangdineijing yangsheng tudian or the Zhongyiyaoshitudian, despite their pre-modern clothing, may be more reminiscent of a Beijing reader's friends and neighbours than the figures of Huangdi, Qi Bo, and Shen Nong the same reader might imagine as she scans a non-illustrated text. These cartoon figures may bring the Dao of the medical classics a little closer to the apartment complexes and hutongs of the modern city. But everyone knows that their own dimensional, changing, stubbornly material life is not a cartoon. And they know that habits of 'daily eating and drinking, rising and resting' have an impact on the degree of satisfaction they can find in life. So for these readers, too, both words and images fall short of the lasting Way.

\section{Conclusion}

Thus it can be argued from two points of view that the picture that can be drawn is not the lasting picture. The first is the point of view of content analysis, which has shown us how much of the rich body of Chinese medical understanding is missed or - at best - rendered quite abstract by the cartoon form. The second is the scepticism of the people who actively assemble their understanding of health, medicine, and ways of forging a wholesome bodily life from experiences that exceed all forms of representation. Readers understand the failings of representations. At least in the comic genre, books are treated more as resources and diversions than as authorities; yet they offer images of everyday life and ordinary problems resolved by the theory and techniques of Chinese medicine, and so they can always provide grist for the experiential mill. For some readers, Shen Nong and Qi Bo - only allusively sketched - might become just as much companions in the nurturance of life as one's neighbours, family, and friends.

Comparing medical classic comics with the more systematic, even 'scientific', texts of Chinese medicine, as well as with the beautifully drawn propaganda illustrations of the xiaoren shu, it is tempting to feel that much has been lost in contemporary graphic publishing. But these other forms of representation have their shortcomings too. Scholars of formal Chinese medicine lament that the classic textual materials are 'too abstract' and alienate today's scientifically-trained youth. No ordinary reader turns to the official editions of the Huangdineijing or the Bencao gangmu for everyday health advice. In fact, fewer and fewer medical student actually read these classics; indeed I know a teacher in a Shanghan lun 伤寒论 (Treatise on Cold Damage) department at a college of Chinese medicine who was delighted when I gave her a comic book version. She intended sharing it with her students immediately, feeling it would make the material so much more accessible for them. As for the 'better' pictures we have considered, the beauties and warriors, model workers and conscientious cadres of both old and new 'little books' may seem quite remote to readers, subjects of an imaginary if sometimes pleasurable other world. It is important to recall, then, that the inadequacy of images and words to capture or guide experience is not specific to just a few, relatively 'popular' or 'vulgar' genres. Every form of representation enables some readings while preventing others. There is no total picture, and the way that can be realised is not the lasting Way. 


\section{Bibliography}

Anderson, M. 1990, The Limits of Realism: Chinese Fiction in the Revolutionary Period, Berkeley: University of California Press.

Bai Chunxi 白纯熙, Liu Yuheng 刘玉衡 and Zhang Jinlu 张金路 (eds) 1993, Zhongguo lianhuanhua fazhan tushi 中国连环画发展 图史 (A Pictorial History of Chinese Comics), Beijing: Zhongguo lianhuanhua chubanshe.

Blackbeard, B. (ed.) 1995, R.F. Outcault's The Yellow Kid: A Centennial Celebration of the Kid who Started the Comics, Northampton mA: Kitchen Sink Press.

Bohnenkamp, M. 2005, 'The Chinese folklore movement, Qu Qiubai's revolutionary mass culture, and the contradictions of Modernity'. Unpublished ms.

Cai Zhizhong 蔡志忠 2003, Laozi Shuo 老子说 (Laozi Says), Beijing: Xiandai chubanshe.

Chen Xuguang 陈旭光 2003, Manhua xuedao anmoyangsheng shu 漫 画穴道按摩养生术 (Arts of Nurturing Life Through Acupressure Massage in Comic Form), Beijing: Nongcun duwu chubanshe.

Hung, Chang-tai 1994, War and Popular Culture: Resistance in Modern China, 1937-1945, Berkeley: University of California Press.

Isao, Shimizu 2001, 'Red Comic Books: The origins of modern Japanese manga', in Lent (ed.), $137-5$ o.

Lent, J.A. (ed.) 2001, Illustrating Asia: Comics, Humor Magazines, and Picture Books, Honolulu: University of Hawai'i Press.

Liu Yanchi (tr. Fang Tingyu and Chen Laidi) 1988, The Essential Book of Traditional Chinese Medicine, illustrated by Cheng Duoduo, New York: Columbia University Press.

Mitchell, W.J.T. 1986, Iconology: Image, Text, Ideology, Chicago: University of Chicago Press.

1994, Picture Theory: Essays on Verbal and Visual Representation, Chicago: University of Chicago Press.

Nanjing College of Chinese Medicine (ed.) 1979, Nanjing jiaoshi 难经 校释, Beijing: Renmin weisheng chubanshe.

Nebiolo, G. 1973, 'Introduction', in Wilkinson (tr.), vii-xvi.

Schmalzer, S. 2008, The People's Peking Man: Popular Science and Human Identity in Twentieth-century China, Chicago: University of Chicago Press.
Schodt, F.L. 1983, Manga! Manga! The World ofJapanese Comics, New York: Kodansha International and Harper and Row.

Shandong Illustrated Press Editorial Group (ed.) 2002, Manhuazhongde Lishi 漫画中的历史 (History in Cartoons), Jinan: Shandong Illustrated Press.

Shen, Kuiyi 2001, 'Lianhuanhua and Manhua - Picture Books and Comics in Old Shanghai', in Lent (ed.), 100-20.

Shen Xiazhu 沈夏珠 and Chen Cong 陈聪 2002, Manhua Huangdi neijing suwen, 'zhenduan' juan 漫画皇帝内径素文诊断卷 (The Comic-book Plain Questions of the Yellow Emperor's Inner Canon, Diagnosis Volume), Shanghai: Wenhui Press..

Wei, Shu-chu 2001, 'Redrawing the past: modern presentation of ancient Chinese philosophy in the cartoons of Tsai Chih-chung', in Lent (ed.), 153-70.

Wen Mu 文木 and Yu Hua 郁华 (eds) 1997, Qu Qiubai sanwen 篗秋白 散文 (Essays of Qu Qiubai), Beijing: Zhongguo guangbo dianshi chubanshe.

Wilkinson, E. (tr.) 1973, The People's Comic Book, Garden City NY: Anchor Books, Doubleday and Company.

Wong, W. Siuyi 2002, Hong Kong Comics: A History of Manhua, New York: Princeton Architectural Press.

Xiao, Tie 2007, 'From "His Suffering" to "Her Awakening": A transnational/ translational visual encounter - Wen Tao's wood-engraving lianhuanhua and its Belgian connection'. Unpublished ms.

Zhang, Yingjin 2001, 'The corporeality of erotic imagination: a study of pictorials and cartoons in republican China', in Lent (ed.), 121-36.

Zhou Chuncai 周春才(ed.) 200o, Zhongyi yangsheng tudian 中医养 生图典 (Classic Comic of Life Nurturance in Chinese Medicine), Beijing: Zhongguo wenlian chubanshe.

Zhou Chuncai 周春才(ed.) 2002, Zhongyi yaoshi tudian 中医药食图 典 (Classic Comic of Chinese Medicinal Foods), Beijing: Zhongguo wenlian chubanshe.

Zhou Chuncai 周春才 and Han Yazhou. 韩亚洲 1994 and 1996, Huangdi neijing yangsheng tudian 黄帝内经养生图典 (Classic Comic of Life Nurturance in the Yellow Emperor's Inner Canon), Beijing: Zhongguo wenlian chubanshe.

Zhou Chuncai 周春才 et al. (eds) 1999, The Yellow Emperor's Medicine Classic: Treatise on Health and Long Life, Singapore: Asiapac. 


\section{Periodisation of Chinese History - Principal Dynasties}

$$
\begin{aligned}
& \text { c. 21st-16th century BCE } \\
& \text { c. 1600-1046 ВСЕ } \\
& \text { 1046-256 ВСЕ } \\
& 1046-771 \text { ВСЕ } \\
& 77 \text { О-256 ВСЕ } \\
& 722-476 \text { ВСЕ } \\
& 475^{-221 ~ В С Е ~}
\end{aligned}
$$

221-206 вСE

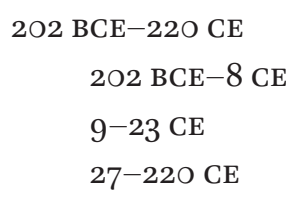

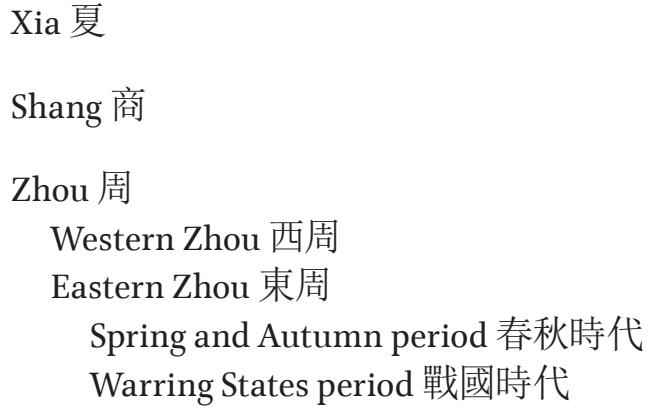

Qin 秦

Han 漢

Western (Former) Han 西漢

Xin 新 (Wang Mang 王莽 interregnum)

Eastern (Later) Han 東漢

Age of Division 大分裂時代

Sui 隋

Tang 唐

Five Dynasties (North China) and Ten Kingdoms (South China) 五代十國

Liao 遼 (Khitan)

Song 宋

Northern Song 北宋

Southern Song 南宋

Western Xia 西夏 (Tangut)

Jin 金 (Jurchen)

Yuan 元 (Mongol)

Ming 明

Qing 清 (Manchu)

Republic of China 中華民國

People‘s Republic of China 中華人民共和國 


\section{Author Biographies}

Ian A. BAKER, cultural historian, anthropologist and art curator, studied art history, literature, and comparative religion at Oxford University and Columbia University, and Medical Anthropology at University College London(UCL). His publications on Himalayan and Tibetan culture include The Heart of the World: A Journey to the Last Secret Place (2007), The Tibetan Art of Healing (2015) and Tibetan Yoga: Secrets from the Source (forthcoming). He curated the 2015-16 Wellcome Collection exhibition Tibet's Secret Temple: Body, Mind and Meditation in Tantric Buddhism. He is currently conducting doctoral research at Strathclyde University on the heterodox and esoteric use of toxins and intoxicants during the British colonial period in South and East Asia.

Penelope BARRETT is a researcher and translator based in the China Centre for Health and Humanity (CCHH) at UCL. She has a background in medieval European languages and literatures as well as Chinese studies. She is currently pursuing a PHD on 'Bernard Read: Translating medical worlds'.

Roberta BIVINS is a historian of medicine at the University of Warwick. Her first two books examined the cross-cultural transmission of medical expertise, particularly in relation to global and alternative medicine (Acupuncture, Expertise and Cross-CulturalMedicine, 2000 and Alternative Medicine? A History, 2007). Since 2004, funded by the Wellcome Trust, she has studied the impacts of immigration and ethnicity on post-war British health, medical research and practice. In 2015 she published findings from this work as a book, Contagious Communities: Medicine, Migration and the NHs in Post War Britain. She is now exploring the culture and effects of Britain's National Health Service in the UK and internationally since 1948.

Paul D. BUELL, PHD, MA, independent scholar, is a Mongolist, Turkologist and Sinologist. He is widely published, including in comparative medical history. His books include A Soup for the Qan: Chinese Dietary Medicine of the Mongol Era as Seen in Hu Sihui's Yinshan Zhengyuan, with Eugene N. Anderson and Charles Perry, second revised and expanded edition (Leiden and Boston: Brill, 2010), and Historical Dictionary of the Mongol World Empire and its Successor States, second revised and expanded edition (Lanham, MD and Oxford: Scarecrow Press, 2018). His forthcoming projects include Crossroads of Cuisine: The Eurasian Heartland, the Silk Roads and Food, with Eugene N. Anderson and Montserrat de Pablo (London: Reaktion Press, forthcoming, 2019).

CAO Hui 曹暉 is Director of the National Engineering Research Centre for Modernisation of Traditional Chinese Medicine, academic adviser to The Hong Kong Society of Chinese Medicines, Professor of Jinan University, and a member of the Chinese Pharmacopoeia Committee. His research interests span research and development of TCM, quality control of TCM, Bencao and Chinese medical history.
CHANG Che-chia 張哲嘉 is an Associate Research Fellow of Institute of Modern History, Academia Sinica. He received his PHD degree in 1998 with a dissertation on the physician-patient relationship in the Late Qing palace, 1874-19o8. Since then he has worked on a variety of topics in the history of medicine in China and Japan, including drugs, medical education, astrological medicine, forensic medicine, and ideas of the human body. He has been a Visiting Fellow of the International Research Center for Japanese Studies, Wellcome Trust Centre for History of Medicine, Harvard-Yenching Institute and IKGF, Universität Erlangen-Nürnberg. In 2008 he won the Zhu Kezhen Award of the International Society for History of East Asian Science Technology and Medicine with the paper 'The Myth of Rhubarb: The Strategic Rationale and Cultural Implications of China's Prohibitions on the Export of Rhubarb to Britain and Russia in the Qing Period. He is now conducting a project on the history of translation of anatomy into China, and another on pharmaceutical exhibitions in Edo Japan.

CHEN Ming 陳明 is a Professor in the Department of South Asian Studies at Beijing University (PKU). He researches the history of cultural communication between China and central and south Asia in the medieval period, mainly but not exclusively in matters related to medicine.

David DEAR is a PHD candidate at UCL and Script Editor for CCTV9 Documentary Channel in Beijing. His area of research involves the history, construction and re-construction of Yangsheng (養生) culture in Chinese society.

Catherine DESPEUX is Professor Emeritus at the National Institute of Oriental Languages and Civilisations (Institut National des Langues et Civilisations Orientales [INALCo], France) and associate member of the East Asian Civilizations Research Centre (Centre de recherches sur les civilisations d'Asie orientale, CRCAO). She has studied representations of the body in Daoism and Chinese medicine, the history of Daoist women and the great classics of Chan Buddhism.

Judith FARQUHAR is Max Palevsky Professor Emerita of Anthropology at the University of Chicago. Her research focuses on the development and practice of traditional Chinese medicine in modern China, and on cultures of health and embodiment in both rural and urban China. She is the author of Knowing Practice: The clinical encounter of Chinese medicine (1994), Appetites: Food and Sex in Post-Socialist China (2002), and Ten Thousand Things: Nurturing Life in Contemporary Beijing (2012) co-authored with Zhang Qicheng 張其成 of Beijing University of Chinese Medicine. Her current research on ethnic traditional medicines in China is collaborative with Lai Lili 賴立裡 of Peking University Medical Humanities.

Patrice FAVA, sinologist and anthropologist, is an associate researcher of the Ecole française d'Extrême-Orient (Beijing Centre). He is the author of Aux portes du ciel, la statuaire taoïste du Hunan, Art et anthropologie de la Chine, preface by Kristofer Schipper (Paris: Les Belles Lettres/EFEO, 2014).

Gabriel FUENTES received his Masters of Oriental Medicine in the United States in 2004. In 2005 he moved to Taiwan to 
pursue further studies in Chinese medicine and he has remained there ever since. His research focus has been on the development and education of Chinese medicine in Taiwan and the de-contextualization of Chinese medical education. His other interests lie in exploring folk medical traditions and non-academic Chinese medical traditions which are prevalent in Taiwan. Gabriel is a full-time lecturer at China Medical University, where he has been teaching for over seven years.

TJ HINRICHS is an Associate Professor in the Department of History at Cornell University. She co-edited Chinese Medicine and Healing: An Illustrated History with Linda Barnes (Belknap Press of Harvard University Press, 2013), and wrote Shamans, Witchcraft, and Quarantine: The Medical Transformation of Governance and Southern Customs in MidImperial China (Harvard East Asia Series, forthcoming).

Nancy HOLROYDE-DOWNING gained her PHD in 2017 in history of medicine in China. Her interest lies in the diagnostics of Chinese medicine, their transformations and changing primacies. Her publications include articles for Asian Medicine and the Journal of Chinese Medicine, and she was a contributor to Disease, Religion and Healing in Asia. She has practised Chinese traditional medicine for the past 32 years.

Elisabeth HSU is Professor in Anthropology at the University of Oxford. She has done extensive textual research on the Historical Records (Shiji), chapter 105, first in 1986-7, then again in 1999-2001, in recognition of which she obtained a Habilitation in Sinology from the University of Heidelberg in 2002 (the elaborated version from $2003^{-7}$ was published in 2010). Her current textual project is a longitudinal study of the qinghao in the Bencao genre (published in 2010) and the fangji literature.

HU Xiaofeng 胡曉峰 obtained his PHD in Chinese medical history and literature from the China Academy of Traditional Chinese Medicine. He is currently Director of the Institute for History of Medicine and Medical Literature, China Academy of Chinese Medical Sciences. His main publications include A Compilation of Images in Ancient Chinese Medical Books [I CAN'T FIND ORIGINAL TITLE]. His research interests and projects centre on the history of medicine in the period of the Republic of China, the history of herbal medicine, and images in Ancient Chinese medical books.

HUANG Longxiang 黃龍祥 is a Principal Researcher at the China Academy of Chinese Medical Sciences. His research interests span the theory, history and literature of acupuncture and moxibustion. His publications include work on the academic history of acupuncture, historical illustrations of Chinese acupuncture, and evidence-based surface anatomy for acupuncture, as well as critical editions of early texts.

LAI Lili 賴立裡 received her PHD in Anthropology from UNC-Chapel Hill in 2009. After her two-year postdoc at the Department of Sociology at Peking University (PKU), she joined the Institute of Medical Humanities of PKU in 2011. She is now Associate Professor of Anthropology. Lai's research interests focus on the body, everyday life and medicine.
Shang-Jen LI 李尚仁 is a research fellow at the Institute of History and Philology, Academia Sinica. He did his PHD at Imperial College, London and was a Wellcome post-doctoral research fellow at UCL (1999-200o) and a Harvard-Yenching Society Visiting Scholar (2006-7). Shang-Jen Li works on the history of British tropical medicine and Western medicine in 19th-century China. He has published articles in Isis, and Journal of the History of Biology and Social History of Medicine. He has also published a biography of Patrick Manson in Chinese.

LIANG Rong 梁嶸, academic and physician, is Professor of Diagnostics at Beijing University of Chinese Medicine. She serves on national committees for Traditional Chinese Diagnostics, Integrative Medcine, and Health Management. Her interests span the intellectual history and contemporary clinical applications of pulse diagnosis and tongue diagnosis. Her recent research has focused on digital tongue diagnosis, including methods, criteria and implications for health information management. She has led many major research projects, won national and regional awards, and published numerous books and articles. Recent publications include Tongue Diagnosis and Management of Internal Heat 上火的 舌診與調理 (2013).

LIAO Yuqun 廖育群, historian of science and medicine, is former director of the Institute of Natural History, Chinese Academy of Sciences, and director of the Chinese Society for the History of Science and Technology. His numerous publications include Reconstructing Medical Images of the Qin and Han Periods 重構秦漢醫學圖像 (2012), and Traditional Chinese Medicine中國傳統醫藥 ([2006] 2010), translated into English, French and Spanish).

Vivienne LO 羅維前 is the director of the UCL China Centre for Health and Humanity (CCHH). Her research focuses on the social and cultural origins of acupuncture and therapeutic exercise. She translates and analyses manuscript material from early and medieval China and studies the transmission of scientific knowledge along the so-called Silk Roads through to the modern Chinese medical diaspora.

LU Di 蘆笛 holds a PHD in History from UCL. He carried out his doctoral research on the making of modern Chinese materia medica at the China Centre for Health and Humanity (CCHH), and the Department of History, UCL. His research interests include transnational history of medicines and cultures of natural history.

MA Kanwen 馬堪溫 (1927-2016) was a distinguished authority on the history of Chinese medicine. After graduating from the former Yenching University, Professor Ma worked as an Assistant Editor for China Bulletin of New Medicine 新醫學報. In 1954, he became a premier researcher in the department for the History of Medicine at the Central Academy of Health before transferring to the new China Academy of Traditional Chinese Medicine in 1955. After moving to the UK in 1987, he worked as a Senior Research Fellow at the Wellcome Institute for the History of Medicine, Senior Research Fellow of the Joint Department of Human Sciences and Medical Ethics at the 
London Hospital Medical College, and Guest Professor of the Institute for the History of Medicine, Vienna University. He was a council member for several professional organisations, including the International Society of Medical History and the Standing Committee for the Translation and Publication of Joseph Needham's Works, and a member of editorial committees for several journals including the Chinese Journal of Medical History 中華醫史雜誌) and Acupuncture in Medicine. He lectured worldwide, and served as Honorary Senior Adviser to the Association of Traditional Chinese Medicine (UK), Senior Adviser to the World Federation of Chinese Medicine Societies, and Adviser to the China National Committee for the Standardisation of English Translation of Chinese Medical Terms. As an author, co-author or co-compiler, he published 12 books and dictionaries and 160 articles on Chinese medicine and the history of Chinese medicine.

Timothy MAY (History, U. Wisconsin-Madison PHD) is Professor of Central Eurasian History at the University of North Georgia, where he currently serves as the Associate Dean of Arts \& Letters. He is the author of The Mongol Conquests in World History (2012) and The Mongol Art of War (2007), and the forthcoming Mongol Empire (Edinburgh University Press) and The Encyclopedia of the Mongol Empire (ABC-Clio).

David RAMEY DVM is a 1983 graduate of Colorado State University School of Veterinary Medicine, and a practising equine veterinarian. He has published on horse medicine, horse history, and horse-human interactions. His collection of Chinese veterinary manuscripts pertaining to horses is thought to be the largest private collection in the world.

SHIN Dongwon 신동원 is a Professor at the Korea Advanced Institute of Science and Technology (KAIST). His research focuses on the history of Korean medicine and Korean science and technology, especially on issues of culture, power and modernity. He has published numerous articles on Korean medicine and medical discourse including . His publications include Hohwan, Mama, Cheondu (A History of Concept of Disease, 2013), Heyeolja, Joseoneul Seupgyeokhada: Momgwa Uiha-ui Hanguksa (A Korean History of the Body and Medicine, 2004) and Hankuk Keundai Pogeon Uiryosa (History of Modern Healthcare in Korea, 1997).

Michael STANLEY-BAKER 徐源 is an historian of Chinese medicine and religions. He writes on the interrelation of medicine and religion in early imperial China, as well as topics in Chinese medicine and Daoist studies more broadly. He has published a critical translation of the Longmen recipes, and various papers on Daoist health and healing practices, and is currently working on a monograph concerning the emergent distinctions between medicine and religion in early imperial China. He is an associate professor teaching medical humanities at Nanyang Technical University, Singapore, and previously held research positions at the Max Planck Institute for the History of Science in Berlin, the Needham Research Institute, Cambridge, and Academia Sinica in Taipei. He studied for his PHD in medical history at the Wellcome Trust Centre for the History of Medicine at UCL. He currently serves as vice-president of the International Association for the Study of Traditional Asian Medicines.

In addition to close text reading and philology, Dr StanleyBaker develops and employs Digital Humanities tools for the history of medicine and of religion. In his 'Drugs Across Asia' project, he has developed tools for tracing and analysing the distribution of materia medica and other material practices across Buddhist, Daoist and medical corpora, and across time and space.

Roel STERCKX is Joseph Needham Professor of Chinese History, Science and Civilization and head of the Department of East Asian Studies at Cambridge University. His research interests lie in classical and literary Chinese language and philology; the cultural history, religion and thought of pre-imperial and early imperial China; text and manuscript studies (Warring States, Qin, and Han periods). His work has focused on forms of knowledge about the natural world in pre-modern China (cultural ecology, agricultural thought, natural history); food and dietary culture in Chinese philosophy and religion; and the interplay between moral and material values in Chinese thought.

Soyoung SUH 서소영 is an Associate Professor of History at Dartmouth College. She wrote Naming the Local: Medicine, Language, and Identity in Korea since the Fifteenth Century (Cambridge, MA: Harvard University Asia Center, 2017). She is now interested in the transnational history of breast cancer, which will explore the origins of gendered medical culture in post-World War II Korea.

Sumiyo UMEKAWA 梅川純代 is a part-time Lecturer at Nihon University, Meiji Pharmaceutical University. After graduating from Tokyo University of Foreign Languages, Sumiyo gained an MA in Literature of East Asia (1997) and a PHD in History (2003) at SOAs, University of London. Her research focuses on the history of Chinese Art of the Bedchamber, Japanese evaluations of Chinese Sexual Art, and Japanese ideology as observed in the Tachikawa sect. Her publications include Daoist Art of the Bedchamber and the Idea of Qi: An Ancient Chinese Sexual Art for Longevity which is Still Active Today「気」の思想から見る道教の房中術—いまに生きる 古代中國の性愛長壽法, with Yoshinobu Sakade 阪出祥伸 (Tokyo: Goyo Shobo, 2003).

Wan FAN 萬芳 obtained a Master's degree in Chinese medical history and literature from the China Academy of Traditional Chinese Medicine. He is currently a senior research fellow in the Institute for History of Medicine and Medical Literature, China Academy of Chinese Medical Sciences. His main publications include Methodological Thinking on the Development of the Theories and Technologies of Traditional Chinese Medicine 中醫藥理論技術發展的方法學思考 (2011). His research interests and current projects lie in the literature of herbal medicine literature and its history in China.

WANG Jiakui 王家葵 is a professor in the School of Pharmaceutical Science at Chengdu University of Traditional Chinese Medicine. Since receiving his PHD from Chengdu Chinese Medical Institute in 1998, he has written an ongoing 
column for the Southern Metropolis Daily, and published or co-edited works including Researches into the Shen Nong Materia Medica 神農本草經研究, Resolving Uncertainties around the Longmen Medical Recipes 龍門葯方釋疑), Critical Edition of Tao Hongjing's works 陶弘景叢考, Critical Commentary and Researches on the Jiuhuang bencao 救荒本 草校釋與研究, and The Development and Regional Character ofChinese Materia Medica 中藥材品種沿革及道地性. He has worked as medical editor of the National Project on the History of Chinese Daoist Science and Technology, and published extensively on Materia Medica studies, pharmacology and Daoist studies. In recent years he has focused on art history, early modern printing and calligraphy.

WANG Jing 王晶 (Crystal WANG) is a graduate of Beijing University of Chinese Medicine and a practitioner and researcher in acupuncture at Beijing тсм Hospital, Capital Medical University.

WANG Jinyu 王進玉 studied Chemistry at Lanzhou University before pursuing a Master's degree in conservation at Fudan University. Now a researcher of the Conservation Institute of the Dunhuang Academy, he has been engaged since 1978 in research on the conservation of the Dunhuang grotto relics, and the history of science and technology in China. He has participated in some 6o Chinese and international scientific research projects including joint projects with the China-Getty Conservation Institute and the Tokyo National Research Institute of Cultural Properties for the conservation of the Mogao Grottos. His numerous publications include Dunhuangology and the History of Science and Technology 敦煌學和科技史 (2011).

WANG Shumin 王淑民 is Emeritus Professor of the History of Chinese Medicine at the China Academy of Chinese Medical Sciences. Her publications include Medical Formulae Found Hidden in the Dunhuang Grottoes 敦 煌石窟秘藏醫 方 and Medical Manuscripts in British Collections: Facsimiles, Transcripts and Commentaries 英藏敦煌醫學文獻圖影與注 疏. She has been extensively involved in producing transcripts and editions of early Chinese medical texts, including the Dunhuang and Mawangdui manuscripts. Professor Wang is the chief editor of the Chinese version of Imagining Chinese Medicine (形象中醫, Beijing: Renmin weisheng chubanshe, 2007).

WANG Yidan 王一丹 is Professor of Persian literature and philology and Director of the Institute of Iranian Culture Studies at PKU. Her historical research focuses on Persian documents concerning China, especially the works of Rashīd al-Dīn (1247-1318). She has also published translations of the poetry of Rumi and Omar Khayyam.

Sabine WILMS studied Chinese and Japanese in Germany and Taiwan, before moving to the us for graduate studies in Asian Studies and Medical Anthropology. While her academic background has given her a solid foundation in early Chinese philosophy, science, and cosmology, she also enjoys approaching Chinese medicine as a living, clinically effective response to any cultural environment, and she is happiest when engaged in dialogue with practitioners Some of her favourite topics are gynaecology and reproduction, paediatrics, medical ethics, and 'nurturing life'. Besides lecturing, mentoring, writing, and publishing books on Chinese medicine through her company Happy Goat Productions, Sabine loves raising happy goats. Her publications include Bèi JíQiān Jìn Yào Fāng 備急千金要方, vols 2-4 on Gynaecology (bilingual edition, 2007), Divine Farmer's Classic of Materia Medica (translation, 2016), and Concise Introduction to Chinese Medicine (co-authored with Nigel Wiseman, forthcoming).

XIAO Yongzhi 肖永芝 obtained her PHD in Chinese medical history \& literature from the China Academy of Traditional Chinese Medicine in 1996. She is currently a senior research fellow at the Institute for History of Medicine and Medical Literature, China Academy of Chinese Medical Sciences. Her main publications include Selected Overseas Chinese Ancient Medical Books Series 珍本海外回歸中醫古籍叢書 (editor). Her research interests lie in medical literature and its communication history in China, Japan, Korea and Vietnam. Future projects include further editions of ancient medical books, and research on Japanese herbal literature and the historical material on medicine in The True Record of the Joseon Dynasty, The Daily Records of the Royal Secretariat, and The Records of the Korean Envoys to Yenching (Yeonhaengrod).

Yi-Li WU 吳一立 is a Center Associate of the Lieberthal-Rogel Center for Chinese Studies at the University of Michigan, Ann Arbor (US) and an affiliated researcher of EASTmedicine, University of Westminster (UK). She earned a PHD in history from Yale University, and was previously a history professor at Albion College (Us) for 13 years. Her publications include Reproducing Women: Medicine, Metaphor, and Childbirth in Late Imperial China (University of California Press, 2010) and articles on medical illustration, forensic medicine, and Chinese views of Western anatomical science She is currently completing a book on the history of medicine for wounds in China.

Ronit YOELI-TLALIM is a Senior Lecturer in the History Department at Goldsmiths, University of London. Her research deals with the transmission of medical knowledge along the so-called 'Silk Roads'. Within this general scope, she has been working on the history of early Tibetan medicine, based primarily on manuscripts found in the Dunhuang caves. Her current research project, titled 'Re-Orienting Early Medicine: Bridges of Knowledge between 'east' and 'west", broadens the exploration of Eurasian transmissions of medical knowledge, with a focus on the Hebrew Book of Asaf.

ZHANG Qicheng 張其成, academic and practitioner, is director of the School of Chinese Cultural Studies at Beijing University of Chinese Medicine. He has published extensively on yangsheng ('nurturing life'), including, in English, Ten Thousand Things: Nurturing Life in Contemporary Beijing (2012) co-authored with Judith Farquhar. In 2011 he set up the Zhang Qicheng Foundation for Chinese cultural heritage studies 張其成國學基金. 
ZHANG Ruixian 張瑞賢 is a faculty member of the Institute of Chinese Materia Medica at the China Academy of Chinese Medical Sciences, a standing committee member of the Medical History section of the Chinese Medical Academy, and Director of the Popular Science section of the China Association of Chinese Medicine. He also serves on the editorial committee of four journals, including the Journal of Chinese Materia Medica 中國中藥雜誌) and Chinese Medicine and Drug Culture 中醫藥文化. His research interests include textual studies of Chinese medicine, with particular emphasis on Materia Medica, recipe texts, and composite medical texts.He has authored, edited and co-edited numerous monographs including Resolving Uncertainties around the Longmen Medical Recipes 龍門药方釋疑), Collected Editions of Famous Works on Materia Medica Through the Ages 歷代 本草名著集成, Introduction to the Bencao Gangmu School 走進<本草綱目>之門; and The Pictorial Study of Botanical Identity 植物名實圖考.

ZHENG Jinsheng 鄭金生 is an Emeritus Professor and former director of the China Institute for the History of Medicine and Medical Literature, China Academy of Chinese Medical Sciences. His research has focused on the history of Chinese medical literature, especially the Bencao literature. His major publications include The Great Dictionary ofChinese Culture: Pharmacy section 中華大典・藥學分典, Rare Ancient Texts of Chinese Medicine Held Abroad 海外中醫珍善本古籍趣 刊, An Unofficial History of the World of Medicine 藥林外 史, and Three Rare Northern Song Materia Medica Texts 南 宋珍稀本草三種

ZHOU Xun 周遜, lecturer in Modern History at the University of Essex, is the author of numerous books and articles on the social and cultural history of modern China. Over the past two decades, she has used newly available archival material and oral history interviews to research the history of the PRC. Her main publications include The Great Famine in China, 1958-1962: A Documentary History (2012) and Forgotten Voices: Mao's Great Famine, 1958-1961: An Oral History (2014). She is currently working on a book, Health for the Nation: Health Intervention and Delivery in the PRC under Mao, 1949-83, as part of a European-Commission funded project on Public Health Campaigns and Local Healing Practices in the PRC. 


\section{Index}

Acu-moxa, 2, 15, 33, 38-48, 54-6, 61, 79, 102, 105-9, 116, 161-4, 191-2, 271-8, 28o-88 et passim

Acupuncture, , xx, 4-7, 10-11, 15-16, 21, 38-43, $53-55,61-67,71,73,75^{-78}, 103,105-110,115$ 161-66, 167, 189, 190-91, 228, 257-9, 271288, 293, 297-9, 301, 308, 317-18, 328-35, 339-50, 378, 445-7 et passim

Advertising, 24, 467-70, 479-86, 489, 506

Animal Spirits, 389-96 et passim

Animals. See Veterinary Medicine

Arts of the bedchamber, 8-9, 18-19, 215-25, 268-9, 489. See also Fangzhong (shu) 房 中(術)

Aspirin, 23, 479, 482, 485

Astrology, 202, 259, 301, 430-2

Autopsy, 61

Baiyun guan 白雲觀, Beijing, 65-6; Shanghai, 359. See also White Cloud Temple

Baize jingguai tu 白澤精怪圖, 140

Bashiyi nanjing 八十一難經, 58, 295-7, 304, 316. See also Classic of Difficult Issues, Nanjing 難經

Bedchamber, arts of the, 8-9, 18-19, 215-25, 268-9, 489. See also Fangzhong (shu) 房 中(術)

Beiji qianjin yifang 備急千金翼方. See Qianjin yifang 千金翼方

Beiji qianqing yaofang, 備急千金要方. See Qianjin yaofang 千金翼方, Qianjinfang 千金方

Bencao beiyao 本草備要, 157

Bencao gangmu 本草綱目, 16, 36, 38, 135, 142-4, 146-7, 155-7, 266, 310-11, 507

Bencaojing jizhu 本草經集注, 33, 309

Bencao mengquan 本草蒙鉒, 36,157

Bencao pinhui jingyao 本草品彙精要, 16,18 , 20, 36-8, 143, 146, 156-8, 198-9, 201, 203-7, $213,305-7,311-2$

Bencao qiuzhen 本草求真, 155

Bencao tujing 本草圖經, 16, 34, 140, 151-2, 155-8, 200, 204, 310

Bencao tupu 本草圖譜, 38, 199, 204, 311

Bencao yuanshi 本草原始, 36, 154-5

Beriberi, 266. See also Jiaoqi 脚氣

Bian Que 扁鵲, 23, 36, 40, 287, 308, 335, 444-54

Bian she zhinan 辨舌指南, 174,182

Bladder, 48, 80, 113, 181-2, 191, 299-300, 330, $332,335,504$

Blue Beryl, 89, 98, 486, 409

Blue-green Dragon, 94, 177, 291, 296, 301, 302. See also Qing long 青龍

Bodhisattva of Offerings. See Gongyang pusa 供養菩薩

Bone-length measurement, 186. See also Gudu 骨度
Book of Odes, 243, 50o. See also Shijing 詩經

Borneol, 152, 305-7, 312, 471. See also Longnao 龍腦

Breast feeding, 119-20

Bright Hall, 5, 31, 164. See also Mingtang 明堂

Bronze Man, 42, 44-5, 55, 161-4, 293. See also Tongren 銅人

Browne, John, 342

Buddhism, 22, 91, 123, 251, 255-6, 263-4, 269, $279,292,303,315,378,380,400,403-5,407$, 409, 412-3, 418, 420-1, 430, 433, 453

Buyi Lei Gong paozhi bianlan 補遺雷公炮製 便覽, 18, 20, 37, 158, 198-9, 203-7, 209-14, 307, 311

Cartoons, 24, 497-506

Cautery, cauterisation, 271-2. See also Moxibustion

Chanjing 產經, 43, 101-2, 104-6, 108-10

Channel. See Jingluo 經絡, Jingmai 經脈, Mai 脈, Vessel

Chao Yuanfang 巢元方, 104

Chen Ziming 陳自明, 43, 118, 185

Childbirth, 43, 118, 185, 255, 281, 438

Children, 19, 25, 60, 103, 115-7, 119, 124, 126, 227, 309, 376, 469, 480-1, 488-9, 491-5. See also Paediatrics

Ch’onngsim pomyŏngdan 淸心保命丹, 471-2

Chungong hua 春宮畫, 215, 217-20, 224-5. See also Erotic art, Shunga 春画

Chunyu Yi 淳于意, 74-7, 80, 168, 38o, 446, 448

Churchill, James Morss, 345

Cinnabar Field, 96, 241, 261, 355, 357, 359, 368, 369. See also Dantian 丹田

Classic of Difficult Issues, 20, 44, 6o, 104, 167, 335-6. See also Bashiyi nanjing 八十一難 經, Nanjing 難經

Cliff Walker's Materia Medica, 34, 152, 157, 311. See also Lü Chanyan bencao 履㱱岩本草

Cold Damage, 46, 115, 167, 169, 172, 177-80, 380, 507. See also Shanghan 傷寒

Cold syndrome, 177-80. See also Hanzheng 寒證

Controller Vessel, 42, 127. See also Renmai 任脈

Coroner, 56, 61-62. See also Forensic medicine.

Corpse, 10, 56-7, 6o-1, 81, 259, 336, 414

Cunzhen huanzhong tu 存真環中圖, 58

Daguan bencao 大觀本草, 34, 142-3, 310

Dantian 丹田, 96, 171, 241, 261, 357, 368. See also Cinnabar Field

Dao, Daoist, Daoism. See also Tao, Taoist, Taoism

Dao 道, 64, 351 n. 1, 359, 367, 497-507

Daode jing 道德經, 24, 64, 351, 497-8
Daoism, 4, 7, 13, 22, 64, 67, 123, 124, 128, 215, 216, 241, 287, 294, 331, 333, 334, 389, 39o, 394, 400, 453

Daoist Canon. 22, 58, 6o, 62-3, 139, 296, 301, 327, 389. See also Daozang道藏

Daoist(s), 15, 20, 22-3, 31, 48, 53, 55, 57, 6o, $62-5,90,91,98,102,112,122,123,124-3$, 139, 202, 206, 215-16, 238-42, 287-92, 294-9, 301, 3०3, 306, 333-4, 357, 358, 364, $366,376-7,389-96,398,444,497$

Daoyin 導引, 22, 30, 53, 66, 70, 73, 76, 79, 82-7, 91, 98, 215, 261, 39o, 395, 397, 399, 40o-2. See also Gymnastics

Daoyin tu 導引圖, 8, 10, 12, 29, 70, 73, 82-7, 108

Daozang 道藏, 59, 62, 139, 327, 354-5. See also Daoist Canon

Death points, 399-401. See also Sixue 死穴

Dental hygiene, 251. See also Teeth cleaning, tooth cleaning

Dermatology, 190-1

Desi Sangye Gyatso, 403, 406, 409, 412-3, 420-1

Diamond Sutra, 11, 3o, 265. See also Vajracchedikā Prajñāpāramitā Sūtra

Diannan bencao 滇南本草, 36, 155-6

Divination, $5^{-6}, 15,21,92,136,138,140,259$, 282, 284, 316, 398, 402, 429-34, 438-9

Divine Farmer's Canon of Materia Medica, 33, 151. See also Shen Nong bencao jing 神農 本草經, Shen Nong's Canon of Materia Medica.

Douzhen 痘疹, 116. See also Pox, Smallpox

Dream of Red Mansions, 217. See also Honglou meng 紅樓夢

Du Qingbi 杜清碧, 169, 171, 173

Dumai 督脈, 42, 72, 127, 190. See also Governor Vessel.

Dunhuang 敦煌, 19-20, 21, 30-31, 41-2, 54, 69 $78,80,103,108-9,110,140,164-5,216,217$, 251-7०, 271-88, 292, 3०2, 3०3, 3०8-9, 315, $373,384,3^{8} 5^{-6}, 431,433^{-4}$

Dzogchen, 22, 403-8, 410-21. See also rDzogs chen

Eight Extraordinary Vessels, 40-1, 45-6. See also Eight Singular Vessels, Qijing ba mai 奇經八脈

Eight Singular Vessels, 127. See also Eight Extraordinary Vessels, Qijing ba mai 奇經 八脈

Emei shan tiangang zhixue fa 峨眉山天罡 指穴法, 397. See also Mount Emei Big Dipper Finger-Point Method

Emergency medicine, 31, 34, 41, 108, 154, 186, 200, 231, 251, 275, 329

Erotic art, 18, 119, 121, 215, 217-20. See also Chungong hua 春宮畫, Shunga 春画 
Erya 爾雅, 135, 139

Erya tuzan 爾雅圖讚, 139

Essence, 74, 75, 211, 216, 277, 295, 355, 394, 395, 400. See also Jing精

Eugenics, 24, 494

External alchemy, 291, 294-5. See also Waidan 外丹

External diseases, 112, 118, 126. See also External medicine, Waike 外科

External medicine, 17, 43, 127, 183-5, 188-90, 192-3, 309, 332. See also External diseases, Waike 外科

Famine Relief Herbal, 153, 203. See also Jiuhuang bencao 救荒本草

Famous physician, famous physicians, 189, 328, 453

Fangzhong shu 房中術, 8, 18, 215-25, 268. See also Arts of the Bedchamber

Five Agents, 5-7, 15, 20, 25, 59, 61, 63, 67, 178, 296, 301, 330, 333, 365, 389-9o, 430-1, 436-8. See also Wuxing 五行

Five Animal Frolics. See Wuqin xi 五离戲

Five Animal Mimes, 215. See also Wuqin xi 五 禽戲

Five viscera, 40-1, 48, 58, 61, 63, 228, 230, 241, 296, 301, 358, 389. See also Wuzang五藏, Zangfu臟腑

Floating world, 219. See also Ukiyo-e 浮世繪

Foetal poison, 116. See also Taidu 胎毒

Foetus, 14-5, 79, 101-110

Forensic medicine, $7,13,53,56-7,61-2,191$. See also Coroner.

Four (Medical) Tantras, 409, 432. See also Gyushi, rGyud bzhi

Fuke 婦科, 111, 114. See also Women's medicine, gynaecology

Funü zazhi 婦女雜誌, 479, 486. See also Ladies'Journal

Furen daquan liangfang 婦人大全良方, 118 Fuzhou 福州, 240

Gallbladder, 48, 181-2, 327, 330, 332, 334 389-90, 395-6

Ge Hong 葛洪, 39, 102, 294, 448

Gewu 格物, 16, 147. See also Investigation of things

Gender, 8-9, 11-12, 14-15, 24, 111-2, 114-5 $117-8,123,253,489,492$

Ginger, 103, 152, 178

Ginseng, 103, 201

Gold Manuscript, 408-9

Golden Lotus, 217. See also Jinping mei 金瓶梅

Gongyang pusa 供養菩薩, 261

Governor Vessel, 42, 127, 19o. See also Dumai 督脈

Grand Ultimate, 296. See also Taiji 太極

Greece, Greek, 5, 7, 67, 71, 300, 315, 491

Guangzhou 廣州, 152, 312, 443

Gudu 骨度. See Bone-length measurement

Guo $\mathrm{Pu}$ 郭璞, 139

Gymnastics, 70, 73, 79, 91, 261. See also Daoyin 導引
Gynaecology, 109. See Fuke 婦科, Women's medicine

Gyushi, 280, 432. See also Four (Medical) Tantras, See rGyud bzhi

Haiyao bencao 海藥本草, 294-5, 310

Hall of Brightness, 108. See also Mingtang 明 堂, Illuminated Hall, Bright Hall

Hama jing 蝦蟆經, 69, 286, 301. See also Huangdi hamajing 黃帝蛤蟆經, Toad Canon, Yellow Emperor's Toad Canon

Han Gan 韓幹, 324

Hancheng 韓城 (Shaanxi), 233, 236-42, 245, 246-7

Hangzhou 杭州, 16, 145, 153, 245

Hanxie 寒邪, 177. See also Malign cold

Hanzheng 寒證, 177. See also Cold syndrome

Happy Buddha, 269. See Huanxi Fo

Heyinyang 合陰陽, 77, 86, 108, 216

Heart, 48, 59, 61, 75, 78, 80, 85-6, 105, 113, 167, 179, 181-2, 191, 222, 230, 241, 266, 275, 291, 295, 299-303, 318, 320, 327, 329-30, 332-5 358 , 359, 368, 370, 374-5, 389-91, 399, $415-16,419,421-2,424,426,504$

Heart Connections, 299-300, 302-3

Heat evil, 177. See also Rexie 熱邪

Herbal medicine, 20, 34, 153, 156, 167, 316,397

Hetu 河圖, 21, 64, 296

Higashiyama Kuniyoshi 東山邦好, 180

Highest Clarity, 295. See also Shangqing 上清

Historical Records, 40, 102, 312. See also Records of the Historian, Shiji 史記

Honglou meng 紅樓夢, 114, 217. See also Dream of Red Mansions

Honzō wamyō 本草和名, 385-6

Horse, Horse Medicine, 20, 43-4, 71, 94, 137-8, $144,148,184-5,315-7,319-25,375$

Hospitals, 243, 379, 444, 465, 469-70

Hu Sihui 忽思慧, $35,143,311$

Hu Wenhuan 胡文煥, 53-4

Hu Yin 胡愔, 6o, 327, 334

Hua Tuo 華伦, 30, 57, 59, 6o, 448

Huangdi 黃帝. See Yellow Emperor

Huangdi hamajing 黃帝蛤蟆經, 77-8, 103, 108, 110. See also Hama jing 蛤蟆經, Toad Canon, Yellow Emperor's Toad Canon

Huangdi neijing 黃帝內經, 7-8, 38, 56, 76 n. $32,105,112,161,165,210,389$. See also Neijing 内經, Yellow Emperor's Inner Canon, Yellow Thearch's Inner Canon

Huangdi neijing lingshu黃帝内經靈樞See Lingshu 靈樞

Huangdi neijing suwen黄帝内經素問 See Suwen 素問

Huangdi neijing taisu黃帝内經太素 See Taisu 太素

Huangfu Mi 皇甫䍀, 161, 280, 293, 448

Huangting jing 黃庭經, 295, 301, 358, 389, 39o, 396

Huangting neijing wuzang liufu (buxie) tu 黃 庭內景五藏六腑 (補瀉) 圖, 48, 6o, 327, 389, 396
Huanxi Fo 歡喜佛, 269. See also Happy Buddha

Huayuan 畫院, 158. See also Imperial Academy of Art

Huihui yaofang 回回藥方, 20, 311, 312

Humane Elixir, 467-71. See also Jintan 仁丹

Hun 魂 soul, 77 n. 39, 78, 241, 282, 392

Illuminated Hall, 5, 31, 39-42, 78, 164, 19o, 333 . See also Bright Hall, Mingtang 明堂

Immortal, 9, 82, 122-30, 191, 215-6, 223, 258-9, 294-6, 306, 354-5, 358, 361, 369, 405. See also Transcendents

Imperial Academy of Art, 158. See also Huayuan 畫院

Imperial Academy of Medicine, 36, 46, 59, 61, 111, 161, 203-4, 206-7. See also Tai yiyuan 太醫院

Imperial Medical Office, 379. See also Taiyi shu 太醫署

Inner alchemy, 74, 90-1, 123, 125, 303, 355. See also Neidan 內丹

Inner landscape, 45, 58, 65, 67, 358, 389. See also Neijing 內景

Investigation of things, 16, 147. See also Gewu 格物

Inward contemplation, 58. See also Neiguan 內觀, Neizhao 內照

Ishimpo 醫心方, 14, 43, 101, 215, 309, 385

Japan, 101, 285-8, 336, 340-41, 343-4, 345, $467-78,479-83$, et passim

Japanese medicine, 101, 344, 48o-1

Jeon Yuhyeong 全有亨, 336

Jian guge 檢骨格, 61

Jian li mei 健力美 (magazine), 492

Jiaoqi 脚氣, 266. See also Beriberi

Jing 精, 9, 75, 216, 277, 295, 331, 400. See also Essence

Jingbian 經變, 252-5, 259, 263, 265, 270, 308. See also Sūtra painting

Jinghui bencao tu 精繪本草圖, 158, 198, 206

Jingluo 經絡, 71, 75, 340, 343-4. See also Jingmai 經脈, Mai 脈, Vessel

Jingmai 經脈, 73, 112, 167. See also Jingluo 經 絡,Mai脈, Vessel

Jingshizhenglei beiji bencao 經史證類備急 本草, 31, 34

Jingzhe zhuang 經摺裝, 29. See also Sutrafold binding)

Jinling 金陵, $36,44,144$

Jinping mei 金瓶梅, 217, 218, 224. See also Golden Lotus

Jinshi kunchong caomu zhuang 金石昆蟲草 木狀, 38, 199, 204, 206, 311

Jintan 仁丹, 467. See also Humane Elixir

Jiu nanchan xue tu 炎難產穴圖, 120

Jiuai tu 炎艾圖, 233. See also Moxibustion (painting)

Jiufa tu 众法圖, 108, 164. See also Moxibustion Chart, moxibustion charts

Jiugong 九宮. See Nine Palaces, Nine Mansions 
Jiuhuang bencao 救荒本草, 35, 153, 200, 203. See also Famine Relief Herbal

Jiujing 炎經, 31, 41, 43 47, 73, 76, 78, 108, 257

Jivvaka, 283,378

Kabuki 歌舞伎, 219, 220

Kaibao chongding bencao 開寶重定本草 200

Kajiwara Seizen 椲原性全, 59

Kangxi 康熙 (Emperor), 45, 47, 120, 143

Kidney, 48, 59, 75, 77, 105, 107, 113, 167, 181-2, 191, 229, 231, 274-5, 299-302, 320, 327, 330, $332-5,389-90,394,504$

Korea $20,200,316,321,327-37,467-78$, et passim

Korean medicine, 21, 328, 336, 470, 473, 477-8

Kublai Khan. See Qubilai Khan

Ladies'Journal, 23, 479-86. See also Funü zazhi 婦女雜誌

Lan Mao 蘭茂, 36, 155-6

Laoguanshan 老官山, 9, 39

Laozi 老子, $22,351^{1-72,497,500}$

Larynx, 173, 329-30, 332, 415

Lei Gong paozhi bianlan 雷公炮製便覽, 158 , 206. See also Buyi Lei Gong paozhi bianlan 補遺雷公炮製便覽

Lei Gong paozhi lun 雷公炮采論, 33, 206, 209, 213, 214

Lei Xiao 雷斅, 209

Leifa 雷法, 351, $35^{8}$

Leijing tuyi 類經圖翼, 45, 126

Li Chan 李梴, 327, 332-3

Li Jiong 李駧, 58, 296-7, 301-3

Li Shizhen 李時珍, 16, 36, 38, 135, 143-8 passim, 156-7, 266, 310, 311

Li Xun 李珣, 294, 310

Li Yuxing 李夷行, 58

Li Zhongli 李中立, 36, 154-5

Liangchengshan 兩城山, 446

Liangyou (huabao) 良友(畫報), 493. See Young Companion pictorial

Liao 疹, 83

Liaoma ji 療馬集, 316

Libailiu 禮拜六, 493. See also Saturday magazine

Liexian quanzhuan 列仙全傳, 122

Lin'an 臨安, 153

Lingjian 靈簡, 58

Lingshu 靈樞, $38,43,47,56,73,74,79,165,167$, 186, 282. See also Huangdi neijing lingshu 黃帝内經靈樞

Liquor, 35, 83, 103, 189, 201

Lithography, 498

Liu Juanzi guiweifang 劉涓子鬼遺方, 183

Liu Wentai 劉文泰, 36, 156, 204, 206, 305

Liu Yuansu 劉完素, 178

Liver, 48, 59, 61, 67, 74, 105, 113, 144, 167, 172, 181-2, 191, 222, 228-30, 299-302, 318, 327, $330,332-5,389-90,392,504$

Longevity, 15, 24, 30, 53-4, 6o, 118, 123, 153, 284, 309, 394, 476-7, 488-9o

Longmen (drug) recipes, 21, 373-86

Longmen 龍門 caves, 21, 373, 378, 383, 385-6
Longmen yaofang 龍門藥方, 385. See also Longmen (drug) recipes.

Longnao 龍腦, 152, 306-7, 312. See also Borneol

Lü Chanyan bencao 履㟴岩本草, 34, 152-3, 157, 311. See also Cliff Walker's Materia Medica

Lü Dongbin 呂洞賓, 238

Lu Xiujing 陸修靜, 370

Lukhang murals, 403, 406, 408, 410-14, 420-1

Lukhang temple, 22, 409, 416

Lung, 48, 6o, 74, 77, 83, 105, 113, 144, 179, 181, 191, 222, 230, 266, 299-302, 318, 320, 327, 329-30, 332-5, 389-91, 396, 5 O4

Luoli 瘵㾙, 114, 192. See also Scrofula

Luoshu 洛書, 64, 436-8.

Ma Huan 馬歡, 201

Ma Jixing 馬繼興, xix, xxi, 385, 443

Ma Kanwen 馬堪溫, 23, 443-54

Maeil Sinbo 每日申報, 467

Mai 脈, $57,70-77,78,83,86,87,90,98$, 165, 168, 256, 296. See also Jingluo 經絡, Jingmai 經脈, Vessel

Maijing 脈經, 168, 177, 293. See also Pulse Classic

Maishu 脈書, 72-6, 79, 80

Malign cold, 177, 179-80. See also Hanxie 寒邪

Malign Qi, 181, 227. See also Xieqi.

Manson, Patrick, 23, 457-65

Martial arts, 5, 14-6, 19, 22, 90-3, 95, 97-8, 123, $183,191,193,261,398-402$

Materia medica, $1-2,5,16,18,20,26,33-8$, $135-7,140-1,145,148,151-160,188-9,197-$ 200, 204, 206-7, 213, 243, 266, 280, 292-4, 305, 310-11, 339, 375, 471

Mawangdui 馬王堆, xx, 8-9, 12, 14, 15, 29, $30-31,39,53,69-73,75,79,80-1,86,102$, 104, 107-8, 138-9, 165, 167, 216, 217, 275, 436

Medicine Buddha, 19, 21, 259-6o, 432

Medicine King, 26o, 378, 447-9, 453. See also Yaowang 藥王

Michuan douzhen yusui 秘傳痘疹玉髓, 228

Microscope, 23, 457, 459-62, 465

Ming tang 明堂. See Mingtang

Ming wu 名物, 136

Mingmen 命門, 6, 181, 182, 191

Mingtang 明堂, 5, 31, 40, 163, 19o. See Illuminated Hall, Bright Hall

Mingtangjing 明堂經, 164

Mingtang tu 明堂圖, 108, 109, 190

Mingyi bielu 名醫別録, 33, 189

Mingyi leian 名醫類案, 177-8.

Modernity, 2-3, 6, 24, 173, 270, 445, 469, 471, $473-4,487,489,491,496$

Mogao Caves, xx, 10. See also Mogao Grottos

Mogao Grottos 莫高窟, 251-270 and others. See also Mogao caves

Mongol, Mongolian, 17, 20, 35, 143, 178, 269, 291, 292-4, 296, 303, 311, 317, 324, 325, 332, $398,412,437,453$

Mount Emei Big Dipper Finger-Point Method, 22, 397-402
Moxa-cautery. See Moxibustion

Moxibustion, 31, 38-46, 53-6, 69-80, 103, $107-8,112,115,161-6,184-5,190-91,233-5$, 243, 245-6, 257-8, 281, 293, 308, 327, 329, $331-5,341,343,345,375,378,446,449$, et passim. See also Cautery, cauterisation

Moxibustion (painting), 233, 235, 243, 245-6, 247. See also Jiuai tu 炎艾圖

Moxibustion Chart, moxibustion charts, 54, 69-86, 271-9o; Moxibustion Chart (Uyghur), 285. See also Jiufa tu 炎法圖

Moxibustion Classic. See Jiujing 炎經.

Mugwort, 76, 28o. See also Artemisia

Nam Dumin 南斗旻, 336

Nanjing 難經. See Bashiyi nanjing 八十一難 經, Classic of Difficult Issues

Neidan 內丹, 123, 359, 398. See also Inner alchemy

Neiguan 內觀, 58, 359. See also Inward Contemplation, Neizhao 內照

Neijing 内經, 7, 8, 13, 36, 40, 45, 56, 73, 74, 76 76 n. 32, 105, 112, 161, 65, 167, 168, 210, 282, 293, 335, 336, 389, 39०, 396, 5०4, 5०5, $5 \circ 7$

Neijing 內景, 45. See also Inner Landscape

Neijing tu 內景圖, 65, 295

Neizhao 內照, 59-6o. See also Inward Contemplation, Neiguan 內觀

Ni Zhumo 倪朱謨, 36, 155

Nine Mansions, 189. See also Nine Palaces

Nine Palaces, 189, 333, 436. See also Nine Mansions

Nobility, 82, 85, 87, 303, 489

Nourishing life, nurturing life. See Yangsheng 養生

Oenhaedu'chang'jip'yo 諺解痘瘡集要, 329

Omens, 140, 284, 324, 446. See also Divination

Organ. See Five viscera, Wuzang 五藏, Zangfu 藏腑

Ou Xifan 歐希范, 58, 59, 296

Paediatrics. See Children

Palace Medical Bureau, 379. See also Shangyao ju 尚藥局

Peony, 103, 236-7. See also Shaoyao 苟藥

Pharmaceutical Bureau, 379. See also Yaocang $j u$ 藥藏局

Pharmacopoeia, 16, 18, 31, 34-5, 135, 140, 142, $146,151,156,197,199-200,203-4,206-7$, 305, 309-10, 375

Pharynx, 173, 329-30, 332

Pingyang 平陽, 379

Po 魄 soul, 77 n. 39, 241, 296, 390-91, 392

Po Sou Xianren 婆藪仙人, 258

Pox, 115-7, 123, 126, 228-30. See also Douzhen 痘疹, Smallpox

Prescription, 9, 40, 54, 82-3, 102-5, 118, 128, 143, 164-5, 167-70, 172, 184, 200, 216, 218, 228, 251, $275,294,310,312,390,465,469,471$

Professionalism, 6

Pulse Classic, 168. See also Mai jing 脈經 
Qi 氣, 75 n. 26, et passim

Qianjin yaofang 千金要方, 14, 40, 54, 164, 218, 251, 261. See also Beiji qianqing yaofang 備急千金要方, Qianjinfang 千金方

Qianjin yifang 千金翼方, 40, 164

Qianjinfang 千金, 102-5, 108, 109. See also Beiji qianjin yaofang, Qianjin yaofang

Qigong 氣功, 93, 95, 123, 213, 397-402, 404

Qijing ba mai 奇經八脈, 40, 45, 46, 127. See also Eight Extraordinary Vessels, Eight Singular Vessels

Qimin yaoshu 齊民要術, 315

Qinding gujin tushu jicheng 欽定古今圖書 集成, 113

Qing long 青龍, 301. See also Blue-green Dragon

Qingming Scroll, 233-5, 240, 243-5, 246, 247. See also Qingming shanghe tu 清明上河 圖

Qingming shanghe tu 清明上河圖, 153, 233. See also Qingming Scroll

Qisun bayi 七損八益, 220. See also Seven Disadvantages and Eight Advantages

Quanzhou 泉州, 312

Qubilai Khan, 292

Rāksasa 羅刹, 255, 267

Rashīd al-Dīn, 20, 291-4, 296-300, 302-3, 437-8

rDzogs chen, 22, 403, 406, 421. See also Dzogchen

Records of the Historian, 161, 256, 446. See also Shiji 史記

Rectification of names, 16, 147. See also Zhengming 正名

Renmai 任脈, 42, 81, 127. See also Controller Vessel

Rexie 熱邪, 177. See also Heat evil

rGyud bzhi, 409, 432. See Gyushi, Four (Medical) Tantras

Rhubarb, 239-41

Roupu tuan 肉蒲團, 217, 218

San jiao 三角. See Triple Burner

Sancai tuhui 三才圖會, 113

Sanqing 三清, 358. See also Three Pure Ones, Three Pures

Saturday magazine, 493. See also Libailiu 禮 拜六

Scrofula, 114, 187, 192, 229. See also Luoli 瘰㾘

Seoul, 467, 469-73, 476-7.

Seven Disadvantages and Eight Advantages, 220. See also Qisun bayi 七損八益

Shaanxi 陝西, 233, 236, 444, 446, 449

Shanghan 傷寒, 169, 172, 380. See also Cold Damage

Shanghan lun 傷寒論, 168-9, 172, 177, 178, 179, 507

Shanghan shebian 傷寒舌辨, 179

Shangke 傷科, 17, 183-93. See also Traumatology

Shangqing 上清, 6o, 295, 355, 358. See also Highest Clarity
Shangyao ju 尚藥局, 379. See also Palace Medical Bureau

Shanhaijing 山海經, 139, 140

Shaoxing bencao 紹興本草, 34

Shaoyao 苟藥, 103. See also Peony

Shazhou dudufu tujing沙州都督府圖經, 258

Shen 神, 74, 75, 77-8, 80, 282, 295, 331, 355, 391, 400

Shen Gongchen 申拱宸, 114, 116, 118, 126, 184 , 188

Shen Gua 沈括, 58--9

Shen Jin'ao 沈金鳌, 127

Shen Nong bencao jing 神農本草經, 33, 151. See also Divine Farmer's Canon of Materia Medica, Shen Nong's Canon of Materia Medica

Shen Nong's Canon of Materia Medica, 33-4, 20o. See also Divine Farmer's Canon of Materia Medica, Shen Nong bencao jing 神 農本草經

Shenming 神明, 75 n. 27,81

Shenshi zunsheng shu 沈氏尊生書, 127

Shexiang 舌象, 180. See Tongue Image

Shiji 史記, 161, 168, 256, 446, 451. See also Records of the Historian

Shijing 詩經, 243, 390, 500. See also Book of Odes

Shiwu bencao 食物本草, 18, 37, 158, 198, 200, 204

Shizhang 屍帳, 56-7. See also Forensic medicine

Shouyang congshu 壽養叢書, 53

Shuangbaoshan 雙包山, 69

Shuihudi 睡虎地, 69, 80

Shunga 春画, 19, 215, 217-20, 224-5. See Chungong hua 春宮畫, Erotic art

Si xiang 四象, 291, 301

Sichuan 四川, 9, 22, 36, 39, 71, 156, 239, 310, $397-8$

Silk manuscript, 39, 102, 138-9

Sima Qian 司馬遷, 74, 161, 168, 256, 446

Sixue 死穴, 399. See also Death points

Sizhou 泗州, $5^{8}$

Skeleton, 13, 53, 57, 61-2, 67, 111, 144, 184, 187, 192, 236, 351, 354, 358-9, 371

Smallpox. 1, 3, 19, 116-7, 187, 227-32, 329. See also Douzhen 痘疹, Pox

Song Ci 宋慈, 61

Souls, 77 n. 39, et passim. See also Hun 魂 soul, $P o$ 魄 soul

Spirit. See Shen 神

Spirits, in Galenic medicine, 300-303

Spleen, 48, 72, 74, 103, 105, 113, 181-2, 191 , 222, 275, 284, 301-2, 342, 327, 330, 332-5 , 389-9o, 393, 504

Standardisation, 10-11, 13, 17, 25, 55, 141, 292, 445

Stomach, 48, 59, 61, 103, 105, 172, 181-2, 191, 24O, 244-5, 266, 320, 327, 330, 332, 335, $358,375,490,504$

Su Jing 蘇敬, 33, 140, 199, 309

Su Song 蘇頌, 34, 140, 151, 310

Suishu 隋書, 33, 39, 139, 151, 315

Sun Simiao 孫思邈, 11, 26, 40-41, 54, 102-5, 128, 218, 251, 275, 277, 330, 448
Sunü miaolun 素女妙論, 223

Superstition, 397, 402, 445

Surgery, 5, 20, 47, 67, 78, 86, 183, 185-93, 309, 318,465

Sūtra painting, 252-3, 259, 263-7, 308-9. See also Jingbian 經變

Sutra-fold binding, 29. See also Jingzhe zhuang 經摺裝

Suwen 素問, 38, 43, 44, 56, 74, 75, 163, 165, 168

Tai yiyuan 太醫院, 36, 203. See also Imperial Academy of Medicine

Taichanshu 胎產書, 79, 102-6, 108, 109

Taidu 胎毒, 116. See also Foetal poison

Taiji quan 太極拳, 30, 84, 92-3, 215

Taiji 太極, 359. See also Grand Ultimate

Taiping huimin ju 太平惠民局, 245

Taiping shenghuifang 太平聖惠方, 41-2, 235

Taisu, 40

Taiyi 太乙, 436

Taiyi shu 太醫署, 379.See also Imperial Medical Office

Tang Shenwei 唐慎微, 34, 35, 141, 305, 310

Tansūqnāma, 19, 291, 293-4, 296, 298-303, 437-8

Tao, Taoist, Taoism, 351-72. See also Dao, Daoist, Daoism

Tao Hongjing 陶弘景, 216, 294, 309, 445

Tao Yuanming 陶渊明, 139

Teeth cleaning, tooth cleaning, 19, 263-4. See also Dental hygiene

Ten Rhijne, Willem, 21, 339, 341-6

Three Kingdoms, 23, 125, 482

Three Pures, Three Pure Ones, 355, 358, 363, 368. See also Sanqing 三清

Tiansheng zhenjing 天聖針經, 161

Tibet, 89-98, 258, 268-9,271-88,292,403-26, 429-39, et passim

Tibetan medicine, 273-4, 279, 281, 409, 418, 430-2

Toad Canon, 39, 69, 103, 286, 301. See Hama jing 蝦蟆經, Huangdi hamajing 黃帝蛤 蟆經, Toad Canon, Yellow Emperor's Toad Canon

Tongren 銅人, 42, 44-5, 161-3, 293. See also Bronze Man

Tongren tujing 銅人圖經, 163

Tongue Diagnosis, 17, 47, 167-9, 171-4, 178-82

Tongue Image. See Shexiang 舌象

Ton-isho 頓醫鈔, 59

Traumatology, 62, 183-93. See also Shangke 傷科

Triple Burner, 48, 105, 113, 327, 334, 504

Tujing bencao 圖經本草, 140-42, 143, 310

Ui'bang'ryuchui醫方類聚, 327

Ukiyo-e浮世繪, 219. See also Floating World

Vajracchedikā Prajñāpāramitā Sūtra 金剛般 若波羅密經, 30, 265. See also Diamond Sūtra

Vermilion Bird, 4, 287, 291, 293, 296, 300-302, 303, 357-8, 365, 389-9o, 391. See also Zhu que 朱 
Vessel, 38, 40-46, 57, 59, 70, 72-6, 90, 103, $109-12,115,119,124,127,136-7,167-8,182$, 19o-91, 221, 244, 296, 299-30о, 330-343, et passim. See also Jingluo 經絡, Jingmai 經脈, Mai 脈

Veterinary Medicine, 315, 317, 322, 325. See also Animals

Viscera. See Five viscera, Wuzang 五臟, Zangfu藏腑

Waidan 外丹, 291, 294. See also External alchemy

Waike 外科, 17, 112, 126, 183-93. See also External medicine

Waike dacheng 外科大成, 184

Waike tushuo 外科圖說, 184, 188, 192

Waitai miyao 外臺秘要, 41, 78, 108

Wanbing huichun 萬病回春, 328, 332

Wang An 汪昂, 157

Wang Ji 汪機, 192

Wang Jie 王介, 16, 34, 152-3, 157, 311

Wang Kentang 王肯堂, 128, 178

Wang Qi 王圻, 113,147

Wang Qingren 王清任, 6o

Wang Shuhe 王叔和, 168, 293, 297, 299, 448

Wang Youhuai 王又槐, 61

Wang Zhizhong 王執中, 43, 163

Weisheng 衛生, 487,489

Wellcome, xix-xxi, 7, 21, 23, 420, 429, 433-4, $436,443,445,463$

Wenbing 溫病, 172, 175, 180

Wenyi lun 瘟疫論, 172

Western medicine, 5, 111, 173, 181, 340-1, 444, $465,471,479$

White Beryl, 431, 434

White Cloud Temple, Beijing, 65; Shanghai 359. See also Baiyun guan 白雲觀

Women's medicine, 111. See also Fuke 婦科, Gynaecology

Woodblock, woodcut, 29--45, 126, 128, 151-7 203-4, 242

$\mathrm{Wu}$ 巫, 7, 79, 444

Wu Jian 吳簡, 58

Wu Qijun 吳其雐, 16, 38, 155

Wuji tu 無極圖, 63

Wuqin xi 五禽戲, 30, 215

Wushi'er bingfang 五十二病方, 72, 165

Wuwei 武威, 39, 77-8, 161, 282, 286

Wuxing 五行, 389, 396. See also Five Agents

Wuyun liuqi 五運六氣, 129

Wuzang 五臟, 48, 58, 6o, 296, 302, 327, 389.

See also Five viscera, Zangfu 臟腑

Wuzang liufu 五臟六腑, 503

Wuzuo 仵作, 62. See also Coroner
Xian fo qi zong 仙佛奇路, 122

Xian zhang feixing sanjie zhi tu 獻章飛行三 界之圖, 351, 363

Xiantian tu 先天圖, 63

Xianyang 咸陽, 449

Xiao erfang 小兒方, 103

Xieqi 邪氣, 227. See also Malign Qi.

Xinke $m a$ shu 新刻馬書, 144, 316

Xinxi tu 心系圖, 299

Xinxiu bencao 新修本草, 16, 140, 199, 309, 384

Xiuzhen 修真, 295, 358

Xiuzhen shishu 修真十書, 58, 296

Xiyuan lu 洗冤錄, 57,61

$\mathrm{Xu}$ Dachun 徐大椿, 62

Xu Sidao 徐似道, 56

Xue Ji 薛己, 171

Xylographic images. See Woodblock, woodcut

Yang 陽, 17, 80, 83, 85, 86, 96, 357, 365

Yang Jie 楊介, $58-9$

Yang Jizhou 楊繼洲, 45, 55

Yangban 兩班, 329, 471

Yangke huicui 瘍科會粹, 191

Yangsheng 養生, 14, 18, 20, 29, 33, 123, 215-225 passim, 241, 336, 357, 359, 396, 399,498, 506

Yanluozi 煙蘿子, 58-9, 65, 191, 296, 303

Yaocang ju 藥藏局, 379. See also

Pharmaceutical Bureau

Yaoshifo 藥師佛. See Medicine Buddha

Yaotu 藥圖, 33, 140, 310

Yaowang 藥王, 378. See also Medicine King

Ye Hua 葉華, 267

Yellow Emperor, Yellow Thearch (Huangdi 黃帝), 1, 18, 38, 40, 47, 58, 79, 103, 191, 210, 280, 282, 296, 320-21, 336, 448, 477, 501504

Yellow Emperor's Inner Canon, 7, 38-40, 43-45, 293, 389, 498, 50o-1, 506. See also Huangdineijing 黃帝內經, Neijing 内經

Yellow Emperor's Toad Canon, 103. See also Hamajing 蛤蟆, Huangdi hamajing 黃帝 蛤蟆經

Yellow Thearch's Inner Canon. See Huangdi neijing, Neijing 内經, Yellow Emperor's Inner Canon

Yijing 易經, 316, 334, 432

Yilin gaicuo 醫林改錯, 60

Yin , 3-4, 9, 17, 74, 77, 79-83, 129, 169, 177, 178, 216, 223, 228, 241, 244, 393, 394

Yin and Yang, 3-7, 25, 63-7, 73-4, 79-80, 87, $89,104,108,111-12,123,129,130,167,175$, 19o, 217, 220, 223, 275, 287, 296, 303, 352, 40o, 446. See also Yin Yang

Yin Yang, 15, 20, 31, 41, 82, 84, 89, 96, 110, 215, 240, 26o. See also Yin and Yang

Yingxi tu 嬰戲圖, 117

Yingya shenglan 瀛涯勝覽, 201
Yinshan zhengyao 飲膳正要, 20, 35, 143, 311-12

Yinshu 引書, 70, 73, 76, 82-7

Yixue yuanshi 醫學原始, 60

Yizong jinjian 醫宗金鑑, 15, 46, 111, 183

Yoga, 22, 261, 268-9, 401, 403-26

Yongle dadian 永樂大典, 18, 201-3, 204, 305. See also Yongle Encyclopaedia

Yongle Encyclopaedia, 18, 201, 305. See also Yongle dadian 永樂大典

Young Companion pictorial, 493. See also Liangyou huabao 良友畫報

Yuan Heng liaoma ji 元亨療馬集, 144, 316

Yufang mijue 玉房秘訣, 223

Yunji qiqian 雲笈七籤, 301, 302

Yuzuan yizong jinjian 御纂醫宗金鑑, 15, 46, 111-30

Zangfu 臟腑, 42, 48, 112, 191, 329, 389, 390, 396, 504. See also

Zhang Guolao 張果老, 124

Zhang Ji 張機, 112, 168

Zhang Jiebin 張介賓, 45, 113, 126, 129

Zhang Lu 張璐, 401

Zhang shi yitong 張氏醫通, 401

Zhang Yuxi 掌禹錫, 140, 204, 310

Zhang Zeduan 張擇端, 153, 233, 243

Zhangjiashan 張家山, 39, 69, 70, 72, 73, 75, 81,217

Zheng He 鄭和, 18, 201

Zhenglei bencao 證類本草, 34, 35, 142, 144, 204, 205, 206, 305, 310

Zhengming 正名, 16, 147. See also Rectification of names

Zhengyi 正一, 22, 351, 355

Zhenjiu jiayi jing 針炎甲乙經, 38, 161, 280, 293

Zhenjiu juying 鍼尒聚英, 44, 334

Zhenjiu tu 鍼炎圖, 190

Zhigong tu 職貢圖, 308

Zhiwu mingshi tukao (changbian) 植物名實

圖考 (長編) , 16, 38, 155

Zhongli Quan 鍾離權, 122, 125, 127

Zhong-xiyi 中西醫, 444

Zhou Dunyi 周敦頣, 63, 129

Zhou Lüjing 周履靖, 53

Zhou Qianchuan 周潛川, 22, 397-402

Zhu Danxi 朱丹溪, 169,330

Zhu que 朱雀, 4, 301, 357, 391. See also Vermilion Bird

Zhu Su 朱橚, 35, 154, 203

Zhu Xi 朱喜, 129

Zhu Zhenheng 朱震亨, 129, 445, 454

Zhuangzi 莊子, 75, 76, 82, 135, 215, 315, 5 oo

Zhubing yuanhou lun 諸病源候論, 101, 102, 104 